Remote Sensing and Digital Image Processing

$92 / 2=0$ 


\section{Remote Sensing and Digital Image Processing}

\section{Volume 25}

\section{Series Editors}

Freek D. van der Meer, Faculty of Geo-Information Science and Earth Observation (ITC), Department of Earth Systems Analysis, University of Twente, Enschede, The Netherlands Anna Jarocińska, Department of Geoinformatics, Cartography and Remote Sensing, University of Warsaw, Warsaw, Poland 
Remote Sensing and Digital Image Processing book series. Remote sensing is the acquisition of Physical data of an object without touch or contact. Earth observation satellites have been used for many decades in a wide field of applications. With the advancements in sensor technology, earth imaging is now possible at an unprecedented level of detail. Imaging spectrometers and thermal multispectral systems acquire detailed spectroscopic information of physical properties of the earth's surface. Dynamic processes can now be studied with interferometric systems. SAR interferometry, laser altimetry and high-resolution imaging allow a very detailed, threedimensional reconstruction of the earth surface. With the advent of multi-sensor mission comes a new era of imaging, opening the possibility of integrating date from various sensor systems. The books published in the series explore these topics in remote sensing and provide a framework for related advanced digital image processing approaches.

Series Editorial Advisory Board:

Michael Abrams, NASA Jet Propulsion Laboratory, Pasadena, CA, USA

Paul Curran, City University London, UK

Arnold Dekker, CSIRO Land and Water Division, Canberra, Australia

Steven M. de Jong, Utrecht University, The Netherlands

Michael Schaepman, University of Zurich, Switzerland

Accepted for inclusion in Scopus.

More information about this series at http://www.springer.com/series/6477 
Irena Hajnsek • Yves-Louis Desnos

Editors

\section{Polarimetric Synthetic Aperture Radar \\ Principles and Application}

刑 Springer 


\section{Editors}

Irena Hajnsek

Microwaves and Radar Institute

German Aerospace Center (DLR)

Oberpfaffenhofen, Germany

Institute of Environmental Engineering

Swiss Federal Institute of Technology

Zurich (ETH Zurich)

Zürich, Switzerland
Yves-Louis Desnos

Science, Application and Future Technology

European Space Agency

Frascati, Roma, Italy

\section{(c) (}

ISSN 1567-3200

ISSN 2215-1842 (electronic)

Remote Sensing and Digital Image Processing

ISBN 978-3-030-56502-2

ISBN 978-3-030-56504-6 (eBook)

https://doi.org/10.1007/978-3-030-56504-6

(C) The Editor(s) (if applicable) and The Author(s) 2021. This book is published open access.

Open Access This book is licensed under the terms of the Creative Commons Attribution 4.0 International License (http://creativecommons.org/licenses/by/4.0/), which permits use, sharing, adaptation, distribution and reproduction in any medium or format, as long as you give appropriate credit to the original author(s) and the source, provide a link to the Creative Commons license and indicate if changes were made.

The images or other third party material in this book are included in the book's Creative Commons license, unless indicated otherwise in a credit line to the material. If material is not included in the book's Creative Commons license and your intended use is not permitted by statutory regulation or exceeds the permitted use, you will need to obtain permission directly from the copyright holder.

The use of general descriptive names, registered names, trademarks, service marks, etc. in this publication does not imply, even in the absence of a specific statement, that such names are exempt from the relevant protective laws and regulations and therefore free for general use.

The publisher, the authors, and the editors are safe to assume that the advice and information in this book are believed to be true and accurate at the date of publication. Neither the publisher nor the authors or the editors give a warranty, expressed or implied, with respect to the material contained herein or for any errors or omissions that may have been made. The publisher remains neutral with regard to jurisdictional claims in published maps and institutional affiliations.

This Springer imprint is published by the registered company Springer Nature Switzerland AG

The registered company address is: Gewerbestrasse 11, 6330 Cham, Switzerland 


\section{Foreword}

In the last 40 years, polarimetric Synthetic Aperture Radar (SAR) has evolved from an experimental concept to the implementation of many airborne and spaceborne SAR systems. In the meantime, many hard-working and dedicated researchers have developed theories and algorithms for efficient information extraction in relevant applications. These advancements have established SAR polarimetry as one of the principal remote sensing instruments for higher resolution imaging and more precise monitoring of Earth resources.

SAR is an active radar system, transmitting and receiving microwave pulses, and provides the desirable capability of all-weather, day and night imaging. For fully polarimetric SAR, also known as quad-polarization (HH, HV, VH, and VV) SAR, scatterers' dielectric properties and structures can be detected by their scattering mechanisms. With additional interferometric measurements, topography, building, and vegetation and forest height information can be retrieved. It is no wonder that a plethora of applications has flourished ranging from agriculture, forest, ocean, city buildings, terrain, cryosphere (glacier, ice, and snow), and environmental protection. Of course, military and other applications are too numerous to mention here. Some applications are well established and others are continuously advancing. Good survey papers on applications are scarce and in pressing demand.

This book contains five comprehensive survey papers written by well-known PolSAR researchers covering the application areas of forestry, agriculture and wetland, soil moisture retrieval, cryosphere, urban, and ocean. In addition, a chapter on the principle of polarimetry is given to provide a quick reference. The forest application chapter covers the techniques of forest classification, polarimetric SAR interferometry for forest height estimation, and polarimetric SAR tomography for 3D sensing. Due to the page limit, fine details could not be given. This chapter could be easily expanded to a book on polarimetric SAR forest sensing, and so can other chapters. The ocean application chapter details oil slick and ship detection. It could be extended to include the important and interesting topics of coastal remote sensing, and ocean current and wave measurements.

In summary, this book on polarimetric SAR applications is well written and timely. We congratulate the book's editors and authors for their dedicated effort and oversight in assembling such a long overdue book on polarimetric SAR applications.

Wolfgang-Martin Boerner Ph.D., IEEE, IEICE Life Fellow, OSA, SPIE, AAAS Fellow, Professor Emeritus \& Distinguished Radar Scientist, The University of Illinois at Chicago

Jong-Sen Lee, Ph.D., IEEE Life Fellow, co-author of Polarimetric Radar Imaging (jointly with Prof. Eric Pottier), published by CRC/Taylor and Francis 


\section{Preface}

Dear readers and colleagues,

Welcome to the scientific publication Principles and Applications of Polarimetric Synthetic Aperture Radar! This publication is open-access and has been co-authored by over 50 of the worlds' leading scientists, working in the relevant thematic domains. This book is also a comprehensive resource for training and education that includes theory, examples of key applications, and do-it-yourself tutorials. Following current trends in Earth Observation (EO) open science, the key application examples provided in the various chapters may be reproduced by the reader using the Synthetic Aperture Radar (SAR) data provided and the algorithms implemented in the open-source PolSARpro toolbox.

In order for the (young) readers to fully understand the background of this collective, collaborative and innovative publication, I would like to guide you through the genesis of ideas, initiatives and events that led to its preparation, which spans over the last 35 years, and can be summarised in several key periods presented below.

\section{Pioneering Space-Borne SAR Interferometry}

The first period of SAR interferometry started with the European Remote Sensing Satellite (ERS-1), developed by the European Space Agency (ESA), which was launched in July 1991. ERS-1 was equipped with a Synthetic Aperture Radar operating at C-band and single VV polarisation. Although not known at the time (and therefore not specified in ERS-1 radar development), scientists discovered, during preparation for the ERS-1 mission's scientific exploitation, that it was possible to exploit the phase difference from repeat pass acquisitions to measure topography and ground deformation. The technique, known as SAR interferometry, was demonstrated in orbit with ERS-1 data in August 1991 by the Politecnico di Milano team, allowing to generate interferometric fringes and to measure centimetre deformation of the terrain. ERS-1 was followed by the ERS-2 mission (launched in April 1995) after which scientists proposed the first tandem experiment to ESA. The objective of this experiment, which lasted 9 months, was to create a unique data set from highly coherent interferometric pairs in order to generate digital elevation models with high accuracy.

It was during this period that ESA prepared the Advanced Synthetic Aperture Radar (ASAR) to be put on-board ENVISAT. ASAR was an active array antenna with beam steering and multi-polarisation capability (HH/HV or VV/VH) and was launched in March 2002. As a lesson learned from the ERS-1 mission, the instrument was specified to perform repeat pass interferometry and the ground processor was developed for phase preservation of the ASAR signals. ASAR was the first active array SAR instrument that operated flawlessly for 10 consecutive years in space. The ASAR instrument, thanks to its experimental alternating polarisation mode, demonstrated the usefulness and importance of multi-polarisation radar for scientific and application purposes. In addition, the ASAR instrument, although not designed for it, demonstrated the capability of wide swath ScanSAR interferometry in orbit. 


\section{Organising Airborne Polarimetric SAR Campaigns and Scientific Studies}

During the ERS and ENVISAT missions period (1991-2012), airborne campaigns continued to play an important role in supporting the development of future SAR missions - helping the validation of new space-borne SAR products as well as providing key open data to the scientific community. ESA, NASA, ONERA and DLR organised several multifrequency SAR campaigns demonstrating the interest of full polarimetric and interferometric SAR data sets for scientific research (e.g. MAESTRO, INDREX, AGRISAR, BIOSAR, AfriSAR, etc.). In 2000 , reaping the full benefits of the available airborne campaign data, and in order to assess the benefits and capabilities of the newly published "polarimetric interferometry technique" by K. Papathanassiou and S. Cloude, ESA launched two SAR polarimetry studies. The objectives of these studies were to investigate radar capability to penetrate ground cover and to "see" the underlying terrain, as well as to assess the possible use of SAR imagery to characterise forest structure and height. Since then, the collection of multi-frequency and multi-baseline polarimetric SAR data via dedicated airborne campaigns continue to play an essential role in the demonstration and development of innovative algorithms for potential bio-/ geophysical product derivation and support the preparation for new space-borne missions.

\section{Dialoguing with POLinSAR Scientists and Training the Next Generation}

In 2003, the first POLinSAR workshop was organised to gather this new community, to present the findings of ESA-funded studies of SAR Polarimetry and Polarimetric Interferometry applications, and to prepare recommendations for future research work. ESA also implemented the first recommendations from the POLinSAR community to provide a state-of-the art, opensource educational software in the field of Polarimetric SAR data analysis. The Polarimetric SAR Data Processing and Educational Toolbox (PolSARpro) was developed under ESA contract by I.E.T.R at the University of Rennes 1, under the scientific leadership of Prof. E. Pottier. To date, PolSARpro provides the most comprehensive set of functions for the scientific exploitation of fully and partially polarimetric multi-data sets, and is used worldwide.

Additionally, as a second recommendation from the POLinSAR community, a multi-year programme of advanced thematic training courses and summer schools was organised for training the next generation of ESA Principal Investigators in SAR Polarimetry. These training events host about 60-70 scientists (i.e. Ph.D. students, young postdoctoral researchers) for a one week duration, that include formal lectures on theory to advanced concepts, as well as hands-on computing exercises, exposing students to EO data and the usage of ESA toolboxes. In 2019, ESA is organising the 5th Advanced Course on Radar Polarimetry dedicated to training the next generation of Earth Observation (EO) scientists to exploit dual and fully polarimetric data for science and applications development.

At the end of 2017, ESA launched, jointly with University of Jena and DLR, the first Massive Open Online Course (MOOC) on SAR that was followed by over 6000 participants and included modules on SAR polarimetry theory and applications using open-source toolboxes. In 2019, ESA organised the 9th International Workshop on Science and Applications of SAR Polarimetry and Polarimetric Interferometry, and the re-run of the SAR MOOC with the participation of the Canadian Space Agency.

\section{Pioneering Space-Borne SAR Polarimetric Interferometry}

Recently, satellites with SAR polarimetric capability and different frequencies have been launched (e.g. SIR-C/X-SAR, ALOS-2, RADARSAT-2 and TerraSAR-X, TanDEM-X) and 
operated successfully. Following the recommendations of scientists at POLinSAR, campaigns were organised by Space Agencies to collect SAR data time series over a wide range of validation sites. In addition, dedicated and successive Announcement of Opportunities to research on spaceborne SAR polarimetry at different frequencies were opened to European PIs by ESA for ALOS with JAXA (2005), with CSA for RADARSAT 2 SOAR EU (2009 and 2013), and with DLR for TerraSAR X -TanDEM-X.

Having acquired the suitable time series of space-borne data over proper validation tests sites, and benefiting from expertise developed in Europe, we were in a position to advance from demonstrations to applications and, as a result, identify key areas for which polarimetric data provides unique benefits. For instance, the polarimetric interferometric forest height measurements were demonstrated successfully using ALOS data in 2008, despite the long repeat pass intervals, and were validated using airborne data.

\section{Future Missions}

In 2013, the BIOMASS mission became the first ESA SAR mission with fully polarimetric capabilities selected to be the 7th Earth Explorer mission for launch in 2022. The primary scientific objectives of the mission are to determine the distribution of above-ground biomass in the world forests and to measure its annual changes, to help improve our understanding of the land carbon cycle. The BIOMASS radar sensor is a fully polarimetric space-borne SAR operating at P-band. During the first year of the mission, a tomographic phase will provide a full global coverage. The remaining 4 years will be an interferometric phase, providing full global coverage every 6 months. Though the prime objective is to collect data over forest, it is also foreseen to collect additional data over land, ice caps and the ocean, as appropriate. The data collected by this mission will allow advanced retrieval concepts based on polarimetric interferometry and polarimetric tomography, developed since 2003 via several ESA and DLR studies, to be put in practice.

Other SAR satellite missions are currently under study to provide fully polarimetric capabilities in future, such as the German Tandem-L, the European Commission Copernicus Sentinel-1 Next Generation and the Copernicus L-band candidate mission. The need for polarimetric SAR satellite missions and global coverage is now being well recognised.

\section{From Science to Applications}

In 2011, ESA launched the PolSAR-Ap project, aimed at evaluating and demonstrating the importance and unique benefits of fully polarimetric SAR data for a wide range of remote sensing applications. The project was awarded to DLR and coordinated by Prof. Irena Hajnsek. The main study objective was the identification of applications for which the availability of fully polarimetric SAR data brings unique benefits and/or significant performance improvements when compared to single or dual polarimetric data. In addition, the demonstration of the improvements induced by polarimetry was to be performed by implementing the applications algorithms, applying them on suitable space-borne SAR data (if available) and validating their performance against reference data available for each case. In order to cover a whole range of remote sensing applications, the study was organised into thematic domains: Forest, Agriculture, Cryosphere, Urban, Ocean and Hazard applications.

This publication aims to present the outcome of the PolSAR-Ap project and to introduce key research results, authored by scientific experts participating in the study. Some of the relevant applications are also implemented in the ESA open-source software PolSARpro allowing the easy generation and replication of key examples. 


\section{Outlook}

Today, ESA is developing fully polarimetric space-borne SAR missions at P-band, whilst studying L-band and C-band mission concepts and preparing for their scientific exploitation. ESA data are freely and openly available for further exploitation by the community. ESA is supporting the emerging EO "open science" practices, taking full advantage of the digital age and addressing new ways to do research for society and in society. ESA "open science" activities will include open access publications, open-source scientific toolboxes, new ways to educate via MOOCs, enhanced research using applications platforms and virtual research environments, increased collaboration using social networks, as well as innovative citizen science projects.

We sincerely wish the reader a good journey through the Principles and Applications of Polarimetric Synthetic Aperture Radar. You are kindly invited to use the sample data and the ESA toolbox for demonstration and educational purposes. We would like to acknowledge the contribution of all co-authors and special thanks to the Chapter Leads Dr. Carlos Lopez Martinez, Prof. Eric Pottier, Dr. Shane Cloude, Dr. Kostas Papathanassiou, Dr. Juan Manuel Lopez Sanchez, Prof. Maurizio Migliaccio and Dr Elise Colin Koeniger.

Sincerely,

Yves-Louis Desnos and Irena Hajnsek

\section{In Memoriam}

This book is dedicated to our beloved colleague and friend Prof. Wolfgang Boerner, age 80, who passed away on May 25, 2018, during the development of the book. Wolfgang was one of the strongest promoters for establishing a polarimetric SAR framework and applying it to a variety of applications. We have known Wolfgang attending countless workshops, conferences and meetings, promoting radar polarimetry and networking alongside scientists and researchers all around the world, with endless energy and passion. His presentations and talks were legendary, unpredictable, and nothing short of memorable!

For the upcoming scientists, Wolfgang was the one to introduce them to the famous polarimetry experts whose names could be found on papers they have read many times. He was integrating everyone into the "polarimetric family" and he appeared as the "grandfather" of this international family. In this way, he was opening a new perspective on the scientific world but also on the real world for us. He followed the progress of young scientists from workshop to workshop and from conference to conference. He praised the progress, encouraged the overcoming of problems and urged to continue to work hard. He never allowed us to resign in difficult moments. He himself, with his energetic and tireless promotion and his confidence on the importance of polarimetry, has been the best example of resilience to students and young professionals. He was a real mentor, at a time when the importance of mentoring was not yet well established. We - all his co-strugglers - got formed and stand by his idea that polarimetric radar and remote sensing collaboration will improve the world.

Oberpfaffenhofen, Germany

Irena Hajnsek

Frascati, Roma, Italy

Yves-Louis Desnos 


\section{Contents}

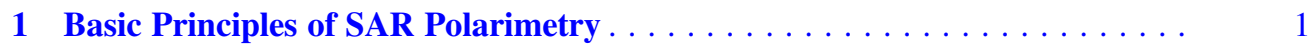

C. López-Martínez and E. Pottier

2 Forest Applications . . . . . . . . . . . . . . . . . .

K. P. Papathanassiou, S. R. Cloude, M. Pardini, M. J. Quiñones, D. Hoekman,

L. Ferro-Famil, D. Goodenough, H. Chen, S. Tebaldini, M. Neumann,

L. M. H. Ulander, and M. J. Soja

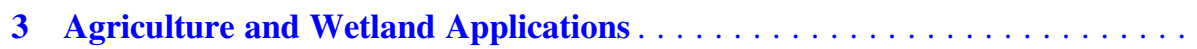

J. M. Lopez-Sanchez, J. D. Ballester-Berman, F. Vicente-Guijalba, S. R. Cloude,

H. McNairn, J. Shang, H. Skriver, T. Jagdhuber, I. Hajnsek, E. Pottier,

C. Marechal, L. Hubert-Moy, S. Corgne, S. Wdowinski, R. Touzi, G. Gosselin,

R. Brooks, Y. Yamaguchi, and G. Singh

4 Cryosphere Applications

I. Hajnsek, G. Parrella, A. Marino, T. Eltoft, M. Necsoiu, L. Eriksson, and M. Watanabe

5 Urban Applications . . . . . . . . . . . . . . . . . .

E. Colin-Koeniguer, N. Trouve, Y. Yamaguchi, Y. Huang, L. Ferro-Famil,

V. D. Navarro Sanchez, J. M. Lopez Sanchez, D. Monells, R. Iglesias,

X. Fabregas, J. Mallorqui, A. Aguasca, and C. López-Martínez

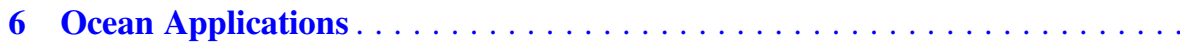

M. Migliaccio, F. Nunziata, A. Marino, C. Brekke, and S. Skrunes

Appendix: Test Sites and Data Sets . . . . . . . . . . . . . . . . . . . . . . . . . . 


\section{Symbols}

\begin{tabular}{|c|c|}
\hline$\alpha$ & Absolute angle (polarisation ellipse) \\
\hline$A$ & Anisotropy \\
\hline$\rho$ & Complex polarisation ratio \\
\hline $\mathbf{T}=E\left\{\mathbf{k}_{p} \mathbf{k}_{p}^{T *}\right\}$ & Coherency matrix \\
\hline $\mathbf{C}=E\left\{\mathbf{k}_{l} \mathbf{k}_{l}^{T *}\right\}$ & Covariance matrix \\
\hline DoP & Degree of polarisation \\
\hline$\lambda$ & Eigenvalue \\
\hline $\mathbf{u}$ & Eigenvector \\
\hline $\overrightarrow{\mathbf{E}}(z, t)$ & Electric field (real) \\
\hline $\overrightarrow{\mathbf{E}}(z, t)$ & Electric field (complex) \\
\hline$\tau$ & Ellipticity angle \\
\hline$H$ & Entropy \\
\hline$\{\widehat{\mathbf{x}}, \widehat{\mathbf{y}}, \widehat{\mathbf{z}}\}$ & Euclidian coordine system (unitary vectors) \\
\hline$E\{\cdot\}$ & Expectation \\
\hline$\omega$ & Frequency (angular) \\
\hline$f$ & Frequency (time) \\
\hline $\mathcal{N}\left(0, \sigma^{2}\right)$ & Gaussian/Normal real pdf \\
\hline $\operatorname{C\mathcal {N}}\left(0, \sigma^{2}\right)$ & Gaussian/Normal complex pdf \\
\hline $\mathcal{N}(\mathbf{0}, \mathbf{C}), \mathcal{C N}(\mathbf{0}, \mathbf{C})$ & Gaussian/Normal multidimensional pdf \\
\hline $\mathfrak{I}\{\cdot\}$ & Imaginary part \\
\hline$\gamma$ & Interferometric coherence \\
\hline$\underline{\mathbf{E}}$ & Jones vector \\
\hline $\mathbf{K}$ & Kennaugh matrix \\
\hline$\bigotimes$ & Kronecker product \\
\hline $\overrightarrow{\mathbf{H}}(z, t)$ & Magnetic field (real) \\
\hline$\left[\begin{array}{ll}x & x \\
x & x\end{array}\right]$ & Matrix \\
\hline$(\cdot)^{T}$ & Matrix/vector transposition \\
\hline$(\cdot)^{*}$ & Matrix/vector conjugate \\
\hline$(\cdot)^{T *}$ & Matrix/vector conjugate transpose (Hermitian) \\
\hline$(\cdot)^{-1}$ & Matrix/vector inverse \\
\hline $\bar{\alpha}$ & Mean alpha angle \\
\hline $\mathbf{M}$ & Mueller matrix \\
\hline$\phi$ & Orientation angle (polarisation ellipse) \\
\hline$\widehat{\mathbf{k}}$ & Propagation vector \\
\hline$\delta=\delta_{y}-\delta_{x}$ & Phase difference (polarisation ellipse) \\
\hline$\rho=|\rho| e^{j \phi_{x}}$ & Polarimetric coherence \\
\hline $\mathfrak{R}\{\cdot\}$ & Real part \\
\hline
\end{tabular}


InSAR

PolSAR

$\mathbf{S}=\left[\begin{array}{ll}S_{h h} & S_{h v} \\ S_{v h} & S_{v v}\end{array}\right]$

DInSAR

PolInSAR

TomoSAR

$S, S_{h h}, S_{h v}, S_{v h}, S_{v v}$

$S(x, r)$

SPAN

g

$\mathbf{k}$

$\mathbf{k}_{l}$

$\mathbf{k}_{p}$

$\left[\begin{array}{l}x \\ x \\ x \\ x\end{array}\right]$

$\mathbf{J}$

$\mathcal{W}(n \mathbf{C}, m)$
SAR interferometry

SAR polarimetry

Scattering matrix

Differential SAR interferometry

Polarimetric SAR interferometry

SAR tomography

SAR image

SAR image (azimuth - range)

Span

Stokes vector

Target vector

Target vector (Lexicographic basis)

Target vector (Pauli basis)

Vector

Wave coherence vector/matrix

Wishart pdf 


\title{
Basic Principles of SAR Polarimetry
}

\author{
C. López-Martínez and E. Pottier
}

\section{Abstract}

This chapter critically summarizes the main theoretical aspects necessary for a correct processing and interpretation of the polarimetric information towards the development of applications of synthetic aperture radar (SAR) polarimetry. First of all, the basic principles of wave polarimetry (which deals with the representation and the understanding of the polarization state of an electromagnetic wave) and scattering polarimetry (which concerns inferring the properties of a target given the incident and the scattered polarized electromagnetic waves) are given. Then, concepts regarding the description of polarimetric data are reviewed, covering statistical and scattering aspects, the latter in terms of coherent and incoherent decomposition techniques. Finally, polarimetric SAR interferometry and tomography, two acquisition modes that enable the extraction of the 3-D scatterer position and separation, respectively, and their polarimetric characterization, are described.

\subsection{Theory of Radar Polarimetry}

\subsubsection{Wave Polarimetry}

Polarimetry refers specifically to the vector nature of the electromagnetic waves, whereas radar polarimetry is the science of acquiring, processing and analysing the polarization state of an electromagnetic wave in radar applications. This section summarizes the main theoretical aspects necessary for a correct processing and interpretation of the polarimetric

C. López-Martínez $(\bowtie)$

Signal Theory and Communications Department, Universitat

Politècnica de Catalunya , Barcelona, Spain

e-mail: carlos.lopez@tsc.upc.edu

E. Pottier

Institut d'Électronique et de Télécommunications de Rennes, University of Rennes-1, Rennes, France information. As a result, the first part presents the so-called wave polarimetry that deals with the representation and the understanding of the polarization state of an electromagnetic wave. The second part introduces the concept of scattering polarimetry. This concept collects the topic of inferring the properties of a given target, from a polarimetric point of view, given the incident and the scattered polarized electromagnetic waves.

\subsubsection{Electromagnetic Waves and Wave Polarization Descriptors}

The generation, the propagation and the interaction with matter of the electric and the magnetic waves are governed by Maxwell's equations (Balanis 1989). For an electromagnetic wave that is propagating in the $\widehat{\mathbf{z}}$ direction, the real electric wave can be decomposed into two orthogonal components $\widehat{\mathbf{x}}$ and $\widehat{\mathbf{y}}$, admitting the following vector formulation:

$$
\overrightarrow{\mathbf{E}}(z, t)=\left[\begin{array}{c}
E_{x} \\
E_{y} \\
E_{z}
\end{array}\right]=\left[\begin{array}{c}
E_{0 x} \cos \left(\omega t-k z+\delta_{x}\right) \\
E_{0 y} \cos \left(\omega t-k z+\delta_{y}\right) \\
0
\end{array}\right]
$$

which may be also considered in a complex form

$$
\overrightarrow{\mathbf{E}}(z, t)=\left[\begin{array}{c}
\underline{E}_{x} \\
\underline{E}_{y} \\
\underline{E}_{z}
\end{array}\right]=\left[\begin{array}{c}
E_{0 x} e^{j \delta_{x}} e^{-j k z} e^{j \omega t} \\
E_{0 y} e^{j \delta_{y}} e^{-j k z} e^{j \omega t} \\
0
\end{array}\right],
$$

where $E_{0 x}$ and $E_{0 y}$ are the amplitudes of the waves in each coordinate. The electric wave in (1.1) and (1.2) presents a harmonic time dependence of the type $e^{j \omega t}$, where $\omega=2 \pi f$ is the angular frequency and $f$ is the time frequency. The propagation direction of an electromagnetic wave is determined by the propagation vector $\widehat{\mathbf{k}}$ that in case of (1.1) and (1.2) is considered parallel to $\widehat{\mathbf{z}}$. The amplitude of the propagation vector is represented by $k=2 \pi / \lambda$, where $\lambda$ is the wavelength. 
Finally, $\delta_{x}$ and $\delta_{y}$ represent the wave phases in each component. The magnetic wave $\overrightarrow{\mathbf{H}}(z, t)$ can be also represented in the same form.

According to the IEEE Standard Definitions for Antennas (IEEE standard number 145 1983), the polarization of a radiated wave is defined as that property of the radiated electromagnetic wave describing a time-varying direction and relative magnitude of the electric wave vector, specifically the figure traced as a function of time by the extremity of the vector at a fixed location in space and the sense in which it is traced as observed along the direction of propagation. Hence, polarization is the curve traced out by the end point of the arrow representing the instantaneous electric wave.

Let us consider the geometric locus described by the electric wave, as a function of time, for a particular point in space, which can be assumed $z=z_{0}$, without loss of generality. Under these hypotheses, the wave components $E_{x}$ and $E_{y}$ satisfy the following equation:

$$
\begin{gathered}
\left(\frac{E_{x}}{E_{0 x}}\right)^{2}-2 \frac{E_{x} E_{y}}{E_{0 x} E_{0 y}} \cos \left(\delta_{y}-\delta_{x}\right)+\left(\frac{E_{y}}{E_{0 y}}\right)^{2} \\
=\sin \left(\delta_{y}-\delta_{x}\right) .
\end{gathered}
$$

The previous equation describes an ellipse that is called polarization ellipse. As one may deduce from the previous equation, the electric wave, as a function of time, describes in the most general case an ellipse, whose shape does depend neither on time nor on space. The polarization ellipse, for some particular configurations, may reduce to a circle or to a line.

As it may be deduced from (1.3), the polarization state is completely characterized by three independent parameters: the wave amplitudes $E_{0 x}$ and $E_{0 y}$ and the phase difference $\delta=\delta_{y}-\delta_{x}$. Figure 1.1 presents the polarization ellipse for a general polarization state. In addition to the previous three parameters, it is also possible to describe the polarization ellipse by a different set of parameters:

- Orientation or tilt angle $\phi$. This angle gives the orientation of the ellipse major axis with respect to the $\widehat{\mathbf{x}}$ axis in such a way that $\phi \in[-\pi / 2, \pi / 2]$. This angle may be obtained as follows:

$$
\tan 2 \phi=2 \frac{E_{0 x} E_{0 y}}{E_{0 x}^{2}-E_{0 y}^{2}} \cos \delta .
$$

- Ellipticity angle $\tau$. This angle represents the ellipse aperture in such a way that $\tau \in[-\pi / 4, \pi / 4]$. This angle may be obtained as follows:

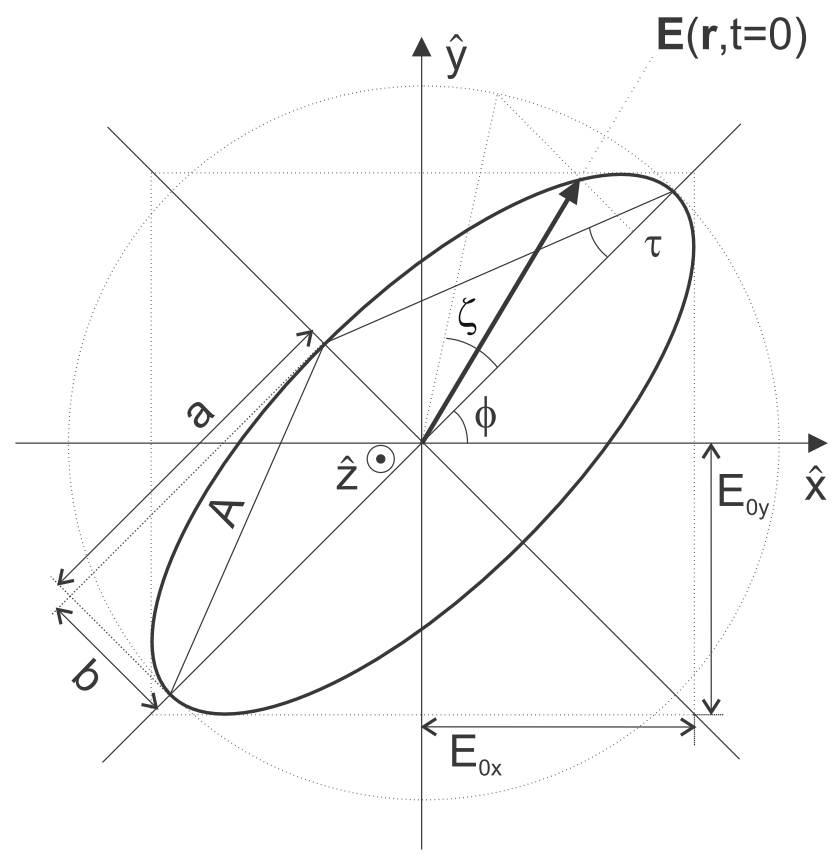

Fig. 1.1 Polarization ellipse

$$
|\sin 2 \tau|=2 \frac{E_{0 x} E_{0 y}}{E_{0 x}^{2}+E_{0 y}^{2}}|\sin \delta|
$$

- The polarization sense or handedness. This determines the sense in which the polarization ellipse is described. This parameter is given by the sign of the ellipticity angle $\tau$. Following the IEEE convention (IEEE standard number 145 1983), the polarization ellipse is right-handed if the electric vector tip rotates clockwise for a wave observed in the direction of propagation, given by $\widehat{\mathbf{k}}$. On the contrary, it is said to be left-handed. Therefore, for $\tau<0$ the polarization sense is right-handed, whereas for $\tau>0$ it is lefthanded.

- The polarization ellipse amplitude $A$. For a major and minor ellipse axes amplitudes $a$ and $b$, respectively, $A=$ $\sqrt{a^{2}+b^{2}}$. This amplitude may be also obtained as

$$
A=\sqrt{E_{0 x}^{2}+E_{0 y}^{2}}
$$

- The absolute phase $\zeta$. This phase represents the initial phase with respect to the phase origin for $t=0$ in such a way that $\zeta \in[-\pi, \pi]$. This term corresponds to the common phase in $\delta_{x}$ and $\delta_{y}$. This absolute phase cannot be directly measured as it corresponds to the exit phase from the radar system at $t=0$. 
Considering the previous sets of parameters describing the polarization state of a wave, one can identify some important polarization states that can be considered as canonical polarization states:

- Linear polarization state. Considering the expression for the real electric wave in (1.1), two canonical linear polarization states can be identified. Table 1.1 details the orientation and the ellipticity angles for these polarization states. These are the linear polarization states according to the $\widehat{\mathbf{x}}$ and to the $\widehat{\mathbf{y}}$ axes, respectively. The linear polarization states are characterized by presenting a phase difference of

$$
\delta=\delta_{y}-\delta_{x}=m \pi, m=0, \pm 1, \pm 2, \ldots
$$

As it may be seen, the linear nature of the polarization state is independent of the phase $\zeta$.

- Circular polarization state. In this particular case, also two canonical circular polarization states can be defined. Table 1.1 details the orientation and the ellipticity angles for these polarization states. When the ellipticity angle takes a value of $-\pi / 4$, the circular polarization state is right-handed, whereas this value is equal to $\pi / 4$ when it is left-handed. The circular polarization states are characterized by presenting a phase difference of

$$
\delta=\delta_{y}-\delta_{x}=m \frac{\pi}{2} \quad m= \pm 1, \pm 3, \pm 5, \ldots
$$

and equal amplitudes for the components of the electric wave $E_{0}=E_{0 x}=E_{0 y}$. Also for circular polarization states, the polarization state is independent of the absolute phase $\zeta$.

- Elliptical polarization state. When there are not restrictions on the orientation and ellipticity angle values, the electric wave is said to present an elliptical polarization state.

As observed, the polarization ellipse may be completely described by two equivalent sets of three independent parameters: the set of wave parameters $\left\{E_{0 x}, E_{0 y}, \delta\right\}$ or the set of ellipse parameters $\{\phi, \tau, A\}$. In addition to these, there exist additional equivalent descriptors that are detailed in the following.

Considering (1.1), the real electric wave vector can be directly obtained from the complex electric wave vector

$$
\begin{aligned}
\overrightarrow{\mathbf{E}}(z, t) & =\left[\begin{array}{c}
E_{0 x} \cos \left(\omega t-k z+\delta_{x}\right) \\
E_{0 y} \cos \left(\omega t-k z+\delta_{y}\right)
\end{array}\right]=\mathfrak{R}\left\{\left[\begin{array}{c}
E_{0 x} e^{j \delta_{x}} \\
E_{0 y} e^{j \delta_{y}}
\end{array}\right] e^{-j k z} e^{j \omega t}\right\}= \\
& =\mathfrak{R}\left\{\underline{\overrightarrow{\mathbf{E}}}(z) e^{j \omega t}\right\}
\end{aligned}
$$

where $\mathfrak{R}\{\cdot\}$ denotes the real part. The time dependence has been removed from the wave description. This is possible as the polarization state of the wave does not change with time. In order to derive a simple and concise description of the polarization state, it is also possible to remove the space dependence of $\overrightarrow{\mathbf{E}}(z)$ by considering the polarization state in a particular point of the space. Without loss of generality, this point can be $z=0$. Hence, $\underline{\overrightarrow{\mathbf{E}}}(0)$ reduces to

$$
\underline{\mathbf{E}}=\underline{\overrightarrow{\mathbf{E}}}(0)=\left[\begin{array}{l}
E_{0 x} e^{j \delta_{x}} \\
E_{0 y} e^{j \delta_{y}}
\end{array}\right] .
$$

The two-dimensional complex vector $\underline{\mathbf{E}}$ is referred to as the Jones vector, and it is a concise representation of a monochromatic, uniform plane wave with a constant polarization (Jones 1941a; Jones 1941b; Jones 1941c).

In the rectangular coordinate system, the Jones vector can be written as a function of the parameters that describe the polarization ellipse (Huynen 1970):

$$
\underline{\mathbf{E}}=A e^{j \zeta}\left[\begin{array}{cc}
\cos \phi & -\sin \phi \\
\sin \phi & \cos \phi
\end{array}\right]\left[\begin{array}{c}
\cos \tau \\
j \sin \tau
\end{array}\right]
$$

The Jones vector, considering the unitary vectors $\widehat{\mathbf{x}}$ and $\widehat{\mathbf{y}}$, may be also expressed as

$$
\begin{aligned}
\underline{\mathbf{E}}_{\{\widehat{\mathbf{x}}, \widehat{\mathbf{y}},\}}= & A\left[\begin{array}{cc}
\cos \phi & -\sin \phi \\
\sin \phi & \cos \phi
\end{array}\right]\left[\begin{array}{cc}
\cos \tau & j \sin \tau \\
j \sin \tau & \cos \tau
\end{array}\right] \\
& \times\left[\begin{array}{cc}
e^{j \zeta} & 0 \\
0 & e^{-j \zeta}
\end{array}\right] \widehat{\mathbf{x}}
\end{aligned}
$$

where the sub-index $\{\widehat{\mathbf{x}}, \widehat{\mathbf{y}}$,$\} indicates that the Jones vector is$ expressed in the linear basis $\{\widehat{\mathbf{x}}, \widehat{\mathbf{y}}$,$\} . The Jones vector$ describes completely the polarization ellipse shape, as well as the rotation sense of the electric wave vector. On the contrary, handedness information cannot be included within the Jones vector as propagation information has been removed. The use of the Jones vector to describe the polarization state is of enormous importance as it allows to define a polarization algebra that makes possible to perform a mathe- 
Table 1.1 Geometrical parameters of the polarization ellipse for canonical polarization states in the rectangular coordinate system

\begin{tabular}{l|l|l|l|l|l|l}
\hline & Linear $\mathbf{x}$ & Linear $\mathbf{y}$ & Linear $\pi / 4$ & Linear $3 \pi / 4$ & Right hand circ. & Left hand circ. \\
\hline$\varphi$ & 0 & $\pi / 2$ & $\pi / 4$ & $3 \pi / 4$ & {$[-\pi / 2, \pi / 2]$} & {$[-\pi / 2, \pi / 2]$} \\
\hline$\tau$ & 0 & 0 & 0 & 0 & $-\pi / 4$ & $\pi / 4$ \\
\hline
\end{tabular}

matical treatment and analysis of the wave polarization. This treatment allows, for instance, the correct definition of orthogonal polarization states. Finally, Table 1.2 details the Jones vector, in the rectangular basis, i.e. $\underline{\mathbf{E}}_{\{\widehat{\mathbf{x}}, \widehat{\mathbf{y}},\}}$, for some particular polarization states.

Another equivalent description of the wave polarization state is the so-called complex polarization ratio:

$$
\rho=\frac{E_{y}}{E_{x}}=\frac{E_{0 y}}{E_{0 x}} e^{j\left(\delta_{y}-\delta_{y}\right)} .
$$

As in the case of the Jones vector, the complex polarization ratio is not able to determine the handedness of the polarization state as propagation information is removed.

The Jones vectors, as well as the complex polarization ratio, are complex quantities that describe the polarization state of a wave. Sir G. Stokes introduced a wave polarization and wave amplitude description based on four real quantities in polarization wave optics (Stokes 1852). The Stokes vector, in the rectangular coordinate system, is defined as (Stokes 1852)

$$
\underline{\mathbf{g}}=\left[\begin{array}{l}
g_{0} \\
g_{1} \\
g_{2} \\
g_{3}
\end{array}\right]=\left[\begin{array}{c}
\left|E_{x}\right|^{2}+\left|E_{y}\right|^{2} \\
\left|E_{x}\right|^{2}-\left|E_{y}\right|^{2} \\
2 \mathfrak{R}\left\{E_{x} E_{y}^{*}\right\} \\
-2 \mathfrak{I}\left\{E_{x} E_{y}^{*}\right\}
\end{array}\right]
$$

where the elements of the vector $\underline{\mathbf{g}}$ are simply called Stokes parameters. Consequently, the Stokes vector is a fourdimensional real vector. Since the Stokes vector describes the polarization state of an electromagnetic wave, it can be directly obtained from the geometrical parameters that describe the polarization ellipse, i.e. $\{\phi, \tau, A\}$ :

$$
\underline{\mathbf{g}}=\left[\begin{array}{c}
A \\
A \cos (2 \phi) \cos (2 \tau) \\
A \sin (2 \phi) \cos (2 \tau) \\
A \sin (2 \tau)
\end{array}\right] .
$$

The polarization state of an electromagnetic wave is completely characterized by means of three independent parameters. These statements also hold for the Stokes parameters, since, as it may be deduced from (1.15), the following relation applies

$$
g_{0}^{2}=g_{1}^{2}+g_{2}^{2}+g_{3}^{2}
$$

Table 1.3 details the Stokes vector, in the rectangular basis, i.e. $\mathbf{g}_{\{\widehat{\mathbf{x}}, \widehat{\mathbf{y}},\}}$, for some particular polarization states.

\subsubsection{Totally and Partially Polarized Waves}

Single-frequency or monochromatic waves are completely polarized, that is, the tip of the electric wave vector describes an ellipse in the plane orthogonal to the propagation direction. The shape of this ellipse, neglecting attenuation propagation effects which affect only the overall power, does not change in time or space, and hence, the wave polarization is constant. Completely polarized waves appear when the different parameters of the wave $\omega, E_{0 x}, E_{0 y}, \delta_{x}$ and $\delta_{y}$ are constant. Nevertheless, many waves present in the nature are characterized by the fact that the previous parameters depend on time or on space randomly. Hence, the tip of the electric wave vector no longer describes an ellipse. These waves are referred to as partially polarized waves. This loss of polarization is due to the randomness of the illuminated scene, to the presence of noise, etc.

The different parameters that characterize the electric wave, i.e. $\omega, E_{0 x}, E_{0 y}, \delta_{x}$ and $\delta_{y}$, may vary randomly. This type of variation makes the electric wave to be modulated and therefore to present a finite bandwidth, so waves can no longer be considered as being monochromatic, but polychromatic. Under this circumstance, it would be also desirable to have a complex representation of the electromagnetic wave as shown in (1.10). Nevertheless, in most of the applications, we are interested into electromagnetic waves that will only have appreciable values in a frequency range which is small compared to the mean frequency $\omega$. Under this situation, waves are referred to as quasi-monochromatic waves. For such signals, the phase terms $\Theta_{x}(z, t)$ and $\Theta_{y}(z, t)$ change slowly when compared to the mean frequency. Then, one may represent the Jones vector of a quasi-monochromatic wave as

$$
\underline{\mathbf{E}}=\left[\begin{array}{l}
E_{x}(t) e^{j \Theta_{x}(\overrightarrow{\mathbf{r}}, t)} \\
E_{y}(t) e^{j \Theta_{y}(\overrightarrow{\mathbf{r}}, t)}
\end{array}\right]
$$


Table 1.2 Jones vector for some polarization states in the rectangular coordinate system, for $A=1$

\begin{tabular}{l|l|l|l|l|l|l}
\hline & Linear $\mathbf{x}$ & Linear $\mathbf{y}$ & Linear $\pi / 4$ & Linear $3 \pi / 4$ & Right hand circ. & Left hand circ. \\
\hline$\underline{\mathbf{E}}_{\{\widetilde{\mathbf{x}}, \mathbf{y}\}}$ & {$\left[\begin{array}{l}1 \\
0\end{array}\right]$} & {$\left[\begin{array}{l}0 \\
1\end{array}\right]$} & $\frac{1}{\sqrt{2}}\left[\begin{array}{l}1 \\
1\end{array}\right]$ & $\frac{1}{\sqrt{2}}\left[\begin{array}{c}1 \\
-1\end{array}\right]$ & $\frac{1}{\sqrt{2}}\left[\begin{array}{c}1 \\
-j\end{array}\right]$ & $\frac{1}{\sqrt{2}}\left[\begin{array}{l}1 \\
j\end{array}\right]$ \\
\hline
\end{tabular}

Table 1.3 Stokes vector for some polarization states in the rectangular coordinate system, for $A=1$

\begin{tabular}{|c|c|c|c|c|c|c|}
\hline & Linear $\mathbf{x}$ & Linear $\mathbf{y}$ & Linear $\pi / 4$ & Linear $3 \pi / 4$ & Right hand circ. & Left hand circ. \\
\hline $\mathbf{g}_{\{\widehat{\mathbf{x}, \mathbf{y}}\}}$ & {$\left[\begin{array}{l}1 \\
1 \\
0 \\
0\end{array}\right]$} & {$\left[\begin{array}{c}1 \\
-1 \\
0 \\
0\end{array}\right]$} & {$\left[\begin{array}{l}1 \\
0 \\
1 \\
0\end{array}\right]$} & {$\left[\begin{array}{c}1 \\
0 \\
-1 \\
0\end{array}\right]$} & {$\left[\begin{array}{c}1 \\
0 \\
0 \\
-1\end{array}\right]$} & {$\left[\begin{array}{l}1 \\
0 \\
0 \\
1\end{array}\right]$} \\
\hline
\end{tabular}

As one may see, the Jones vector of a quasimonochromatic electric wave depends on time and on space; thus, this vector is no longer constant. When the time dependence of the Jones vector is deterministic, the polarimetric properties of the wave also change in a deterministic way through time. In this case, the description of the wave polarization is not problematic and may be performed considering the different descriptors detailed in Sect. 1.1.1.1. Nevertheless, if the time dependence is random, the analysis of the polarization state of the electromagnetic wave must be carefully addressed, as this description must take into account the stochastic nature of the electric wave.

As previously mentioned, the variation of the parameters $E_{0 x}, E_{0 y}, \delta_{x}$ and $\delta_{y}$ may be random, so the Jones vector will be also random. In order to characterize the polarization of the quasi-monochromatic electromagnetic wave expressed by the variable Jones vector in (1.17), it is necessary to address this characterization from a stochastic point of view. In the frame of radar remote sensing, the wave transmitted by the radar system may be considered monochromatic and hence totally polarized. Nevertheless, the scattered wave represented by the Jones vector in (1.17) results from the combination of many different waves originated by the different elementary scatterers that form the scattering media. The complex addition of these elementary waves resulting from the scattering process for one component of the electric wave can be represented as

$$
A e^{j \theta}=\frac{1}{\sqrt{N}} \sum_{n=1}^{N} a_{n} e^{j \theta_{n}}
$$

where $\mathbf{A}$ represents the total wave and $a_{n} e^{j \theta_{n}}$ is originated from the scattering from every elementary scatterer. Under the assumption of $N$, i.e. the total number of scattered waves, to be large enough and certain relations that may be established between the amplitude and the phase of the elementary waves (Chandrasekhar 1960; Goodman 1976), it is possible to demonstrate that the mean value of the electric wave and the Jones vector are zero. Consequently, the Jones vector cannot be employed to characterize the polarization state of a quasi-monochromatic wave. This characterization shall be performed considering higher statistical moments.

The second-order moments may be arranged in a vector form, giving rise to the so-called coherency vector of a quasimonochromatic vector, which is defined in the following way:

$$
\mathbf{J}=E\left\{\underline{\mathbf{E}} \bigotimes \underline{\mathbf{E}}^{*}\right\}=\left[\begin{array}{c}
E\left\{E_{x} E_{x}^{*}\right\} \\
E\left\{E_{x} E_{y}^{*}\right\} \\
E\left\{E_{y} E_{x}^{*}\right\} \\
E\left\{E_{y} E_{y}^{*}\right\}
\end{array}\right]=\left[\begin{array}{c}
J_{x x} \\
J_{x y} \\
J_{y x} \\
J_{y y}
\end{array}\right]
$$

where $\mathbf{J}$ stands for the temporal averaging, assuming the wave is stationary, $\bigotimes$ is the Kronecker product, $(\cdot)^{*}$ represents complex conjugation and $E\{\cdot\}$ is the ensemble average. This vector is not zero for quasi-monochromatic waves. The arrangement of the second-order moments can be also done in a matrix, giving rise to the coherency matrix of the wave:

$$
\begin{aligned}
\mathbf{J} & =E\left\{\underline{\mathbf{E}} \cdot \underline{\mathbf{E}}^{T *}\right\}=\left[\begin{array}{ll}
E\left\{E_{x} E_{x}^{*}\right\} & E\left\{E_{x} E_{y}^{*}\right\} \\
E\left\{E_{y} E_{x}^{*}\right\} & E\left\{E_{y} E_{y}^{*}\right\}
\end{array}\right] \\
& =\left[\begin{array}{ll}
J_{x x} & J_{x y} \\
J_{y x} & J_{y y}
\end{array}\right]
\end{aligned}
$$

where the superscript $(\cdot)^{T}$ denotes vector transposition.

In the previous section, it was mentioned that monochromatic waves are completely polarized. This is not the case for quasi-monochromatic waves. Indeed, completely polarized waves present a polarization state that can be considered as a limit in the sense that it is constant. The opposed extreme is a completely unpolarized wave for which the polarization state is completely random. Between both extremes, waves are said to present a partial polarization state. In order to characterize the degree of polarization, one may consider the degree of polarization defined as a function of the trace of matrix $\mathbf{J}$ as 


$$
D o P=\left(1-4 \frac{|\mathbf{J}|}{\operatorname{trace}(\mathbf{J})}\right)^{\frac{1}{2}}
$$

\subsubsection{Change of Polarization Basis}

As seen in Sect. 1.1.1.1, an electromagnetic wave, considering the coordinate system $\{\widehat{\mathbf{x}}, \widehat{\mathbf{y}}, \widehat{\mathbf{z}}\}$, that propagates in $\widehat{\mathbf{z}}$ may be decomposed as the sum of two orthogonal components. Separately, the electromagnetic wave of each component can be considered as linearly polarized. Therefore, it is possible to consider that the total electromagnetic wave results from the sum of two orthogonal linear polarized waves. Indeed, this representation must be extended in the sense that any electromagnetic wave propagating in an infinite, lossless, isotropic media can be decomposed as the sum of two orthogonal elliptically polarized waves. The advantage of this representation is that the electric wave is decomposed in a pair of orthogonal polarization states, so it is possible, through a deterministic transformation, to obtain the electric wave for any other pair of orthogonal polarization states. This process is referred to as change of polarization basis or polarization synthesis.

Given two vectors $\mathbf{a}$ and $\mathbf{b}$, they are considered orthogonal if they verify

$$
\langle\mathbf{a}, \mathbf{b}\rangle=\mathbf{a}^{T} \cdot \mathbf{b}^{*}=0
$$

that is, the scalar (Hermitian) product of both vectors is zero. In case of two electromagnetic waves, expressed in terms of the corresponding Jones vectors, they are said to be orthogonal if the scalar product of the Jones vectors is zero, considering that both Jones vectors refer to waves propagating in the same direction and sense. The polarization ellipses corresponding to two orthogonal Jones vectors presents the same ellipticity angle, opposite polarization sense and mutually orthogonal polarization axis. That is, for a Jones vector representing a polarization state characterized by an orientation angle $\phi$, an ellipticity angle $\tau$ and an absolute phase $\zeta$, its orthogonal Jones vector presents an orientation angle of value $\phi+\pi$, an ellipticity angle of value $-\tau$ and an absolute phase $-\zeta$. In terms of (1.12), the corresponding orthogonal vector is

$$
\begin{aligned}
\underline{\mathbf{E}}_{\perp\{\widehat{\mathbf{x}}, \widehat{\mathbf{y}},\}}=A & {\left[\begin{array}{cc}
-\sin \phi & -\cos \phi \\
\cos \phi & -\sin \phi
\end{array}\right]\left[\begin{array}{cc}
\cos \tau & -j \sin \tau \\
-j \sin \tau & \cos \tau
\end{array}\right]\left[\begin{array}{cc}
e^{-j \zeta} & 0 \\
0 & e^{j \zeta}
\end{array}\right] \widehat{\mathbf{x}} } \\
= & A\left[\begin{array}{cc}
\cos \phi & -\sin \phi \\
\sin \phi & \cos \phi
\end{array}\right]\left[\begin{array}{cc}
\cos \tau & j \sin \tau \\
j \sin \tau & \cos \tau
\end{array}\right] \\
& \times\left[\begin{array}{cc}
e^{j \zeta} & 0 \\
0 & e^{-j \zeta}
\end{array}\right] \widehat{\mathbf{y}}
\end{aligned}
$$

The symbol $\perp$ denotes orthogonal Jones vector.
Considering what has been indicated, an electromagnetic wave propagating in an infinite, lossless, isotropic media may be described in the following way:

$$
\underline{\mathbf{E}}=E_{x} \widehat{\mathbf{x}}+E_{y} \widehat{\mathbf{y}}=E_{x} \widehat{\mathbf{u}}_{x}+E_{y} \widehat{\mathbf{u}}_{y}
$$

where the notation referring to the unitary vectors has been generalized. If (1.23) and (1.24) are considered, it may be seen that the unitary Jones vectors corresponding to the linear orthogonal polarization states $\widehat{\mathbf{x}}$ and $\widehat{\mathbf{y}}$ are transformed to the Jones vector of any polarization state and the corresponding orthogonal Jones vector through the transformation matrix $\mathbf{U}$ :

$$
\begin{gathered}
\left\{\widehat{\mathbf{u}}, \widehat{\mathbf{u}}_{\perp}\right\}=\left[\begin{array}{cc}
\cos \phi & -\sin \phi \\
\sin \phi & \cos \phi
\end{array}\right]\left[\begin{array}{cc}
\cos \tau & j \sin \tau \\
j \sin \tau & \cos \tau
\end{array}\right]\left[\begin{array}{cc}
e^{-j \zeta} & 0 \\
0 & e^{j \zeta}
\end{array}\right]\{\widehat{\mathbf{x}}, \widehat{\mathbf{y}},\} \\
=\mathbf{U}_{\left\{\widehat{\mathbf{u}}, \widehat{\mathbf{u}}_{\perp}\right\}}\{\widehat{\mathbf{x}}, \widehat{\mathbf{y}},\} .
\end{gathered}
$$

In the previous case, the matrix $\mathbf{U}_{\left\{\widehat{\mathbf{u}}_{\perp}, \widehat{\mathbf{u}}_{\perp}\right\}}$ indicates the transformation matrix from the orthogonal basis $\{\widehat{\mathbf{x}}, \widehat{\mathbf{y}}$,$\} to$ the arbitrary basis $\left\{\widehat{\mathbf{u}}, \widehat{\mathbf{u}}_{\perp}\right\}$. Considering (1.24), the electromagnetic wave expressed in the orthogonal basis $\left\{\widehat{\mathbf{u}}, \widehat{\mathbf{u}}_{\perp}\right\}$ takes the form

$$
\underline{\mathbf{E}}=E_{u} \widehat{\mathbf{u}}+E_{u \perp} \widehat{\mathbf{u}}_{\perp} .
$$

Therefore, the Jones vector in the new basis $\left\{\widehat{\mathbf{u}}, \widehat{\mathbf{u}}_{\perp}\right\}$, expressed in terms of the Jones vector in the basis $\{\widehat{\mathbf{x}}, \widehat{\mathbf{y}}$,$\} , is$

$$
\left[\begin{array}{c}
E_{u} \\
E_{u \perp}
\end{array}\right]=\mathbf{U}_{\left\{\widehat{\mathbf{u}}, \widehat{\mathbf{u}}_{\perp}\right\}}^{-1}\left[\begin{array}{c}
E_{x} \\
E_{y}
\end{array}\right]
$$

The previous equation indicates that if an electromagnetic wave has been measured in the linear orthogonal basis, it is possible to calculate the same electromagnetic wave, but measured in a different polarization basis, just multiplying by the matrix $\mathbf{U}_{\left\{\widehat{u}, \mathbf{u}_{\perp}\right\}}^{-1}$. That is, it is possible to synthesize the electromagnetic wave for any arbitrary polarization basis just measuring it in a particular polarization basis.

Table 1.4 and Table 1.5 detail the polarization ellipse parameters, the Jones vector and the Stokes vector for different polarization states for the rotated and the linear polarization bases, respectively.

\subsubsection{Scattering Polarimetry}

The previous section was concerned with the characterization and the representation of the polarization state of an electromagnetic wave. Although this characterization is important 
Table 1.4 Polarization states expressed in the rotated linear polarization basis $\left\{\widehat{\mathbf{u}}_{-\pi / 4}, \widehat{\mathbf{u}}_{\pi / 4}\right\}$, when $A=1$

\begin{tabular}{l|l|l|l|l|l|l}
\hline & Linear $\mathbf{x}$ & Linear $\mathbf{y}$ & Linear $\pi / 4$ & Linear $3 \pi / 4$ & Right hand circ. & Left hand circ. \\
\hline$\varphi$ & $-\pi / 4$ & $\pi / 4$ & 0 & $\pi / 2$ & $?$ & $?$ \\
\hline$\tau$ & 0 & 0 & 0 & 0 & $\pi / 4$ & $-\pi / 4$ \\
\hline$\underline{\mathbf{E}}_{\left\{\widehat{\mathbf{u}}_{-\pi / 4}, \widehat{\mathbf{u}}_{\pi / 4}\right\}}$ & $\frac{1}{\sqrt{2}}\left[\begin{array}{c}1 \\
-1\end{array}\right]$ & $\frac{1}{\sqrt{2}}\left[\begin{array}{l}1 \\
1\end{array}\right]$ & {$\left[\begin{array}{l}1 \\
0\end{array}\right]$} & {$\left[\begin{array}{l}0 \\
1\end{array}\right]$} & $\frac{1}{2}\left[\begin{array}{c}1+j \\
-1+j\end{array}\right]$ \\
\hline $\mathbf{g}_{\left\{\widehat{\mathbf{u}}_{-\pi / 4} \widehat{\mathbf{u}}_{\pi / 4}\right\}}$ & {$\left[\begin{array}{c}1 \\
0 \\
-1 \\
0\end{array}\right]$} & {$\left[\begin{array}{l}1 \\
0 \\
1 \\
0\end{array}\right]$} & {$\left[\begin{array}{l}1 \\
1 \\
0 \\
0\end{array}\right]$} & {$\left[\begin{array}{c}1 \\
-1 \\
0 \\
0\end{array}\right]$} & {$\left[\begin{array}{c}1 \\
0 \\
0 \\
1\end{array}\right]$} \\
\hline
\end{tabular}

Table 1.5 Polarization states expressed in the circular polarization basis $\left\{\widehat{\mathbf{u}}_{l c}, \widehat{\mathbf{u}}_{c c}\right\}$, for $A=1$

\begin{tabular}{l|l|l|l|l|l|l}
\hline & Linear $\mathbf{x}$ & Linear $\mathbf{y}$ & Linear $\pi / 4$ & Linear $3 \pi / 4$ & Right hand circ. & Left hand circ. \\
\hline$\varphi$ & $?$ & $?$ & $\pi / 4$ & $3 \pi / 4$ & 0 & $\pi / 2$ \\
\hline$\tau$ & $-\pi / 4$ & $\pi / 4$ & 0 & 0 & 0 & 0 \\
\hline$\underline{\mathbf{E}}_{\left\{\widehat{\mathbf{u}}_{c}, \widehat{\mathbf{u}}_{r c}\right\}}$ & $\frac{1}{\sqrt{2}}\left[\begin{array}{c}1 \\
-j\end{array}\right]$ & $\frac{1}{\sqrt{2}}\left[\begin{array}{c}-j \\
1\end{array}\right]$ & $\frac{1}{2}\left[\begin{array}{c}1-j \\
1-j\end{array}\right]$ & $\frac{1}{2}\left[\begin{array}{c}-1-j \\
1+j\end{array}\right]$ & {$\left[\begin{array}{c}1 \\
0\end{array}\right]$} \\
\hline $\mathbf{g}_{\left\{\widehat{\mathbf{u}}_{c c} \widehat{\mathbf{u}}_{r c}\right\}}$ & {$\left[\begin{array}{c}1 \\
0 \\
0\end{array}\right.$} & {$\left[\begin{array}{l}1 \\
0 \\
0 \\
-1\end{array}\right]$} & {$\left[\begin{array}{l}1 \\
0 \\
1\end{array}\right]$} & {$\left[\begin{array}{c}1 \\
1 \\
0\end{array}\right]$} & {$\left[\begin{array}{c}1 \\
0 \\
-1 \\
0\end{array}\right]$} & {$\left[\begin{array}{c}1 \\
0 \\
0\end{array}\right]$} \\
\hline
\end{tabular}

when a radar system is considered, as it transmits and receives electromagnetic waves, nevertheless, the interest is on the scattering process itself. The radar system transmits an electromagnetic wave, with a given polarization state, that reaches the scatterer of interest. The energy of the incident wave interacts with the scatterer, and as a result part of this energy is reradiated to the space. The way this energy is reradiated depends on the properties of the incident wave, as well as on the scatterer itself. Consequently, it is possible to infer some information of the scatterer under consideration considering the properties of the scattered electromagnetic wave with respect to the incident wave, which is basically the transmitted wave by the radar. One possibility that can be studied to characterize distant targets is to consider the change of the polarization state that a scatterer may induce to an incident wave.

In order to analyse the scattering problem, it is worth to start describing the scattering process that occurs when an incident wave reaches a flat transition between two dielectric, infinite, lossless and homogeneous media in oblique incidence. This scattering situation is exemplified in Fig. 1.2. In this case, the incident wave that propagates in the first media reaches the transition between media where part of the incident energy is scattered in the same media and part of the energy is transmitted to the second media. In order to characterize the scattering process, it is necessary to introduce the concept of plane of scattering, which is defined as the plane generated by the propagating vectors of the incident and the scattered waves.
In order to examine specifically reflections at oblique angles of incidence for a general wave polarization, it is convenient to decompose the electric wave into its perpendicular and parallel components, relative to the plane of scattering. The total scattered and transmitted waves will be the vector sum from each of these two polarizations. When the wave is perpendicular to the plane of scattering, the polarization of the wave is referred to as perpendicular polarization or horizontal polarization as the electric wave is parallel to the interface. When the electromagnetic wave is parallel to the plane of scattering, the polarization is referred to as parallel polarization or vertical polarization as the electromagnetic wave is also perpendicular to the interface. As indicated in Fig. 1.2, the total incident wave $\underline{\mathbf{E}}^{i}$ can be decomposed into two orthogonal components in the plane orthogonal to the incident propagation vector $\widehat{\mathbf{k}}^{i}$. These are the parallel $\underline{\mathbf{E}}_{\|}^{i}$ and the perpendicular $\underline{\mathbf{E}}_{\perp}^{i}$ components, which can be written as

$$
\begin{aligned}
& \mathbf{E}_{\|}^{i}=E_{\|}^{i} e^{-j\left\langle\widehat{\mathbf{k}}^{i}, \mathbf{r}\right\rangle} \widehat{\widehat{\mathbf{x}}}^{\prime}, \\
& \overrightarrow{\mathbf{E}}_{\perp}^{i}=E_{\perp}^{i} e^{-j\left\langle\mathbf{k}^{i}, \mathbf{r}\right\rangle} \widehat{\mathbf{y}}^{\prime} .
\end{aligned}
$$

As observed, the incident wave has been defined with respect to the coordinate system $\left\{\widehat{\mathbf{x}}^{\prime}, \widehat{\mathbf{y}}^{\prime}, \widehat{\mathbf{z}}^{\prime}\right\}$ in such a way 


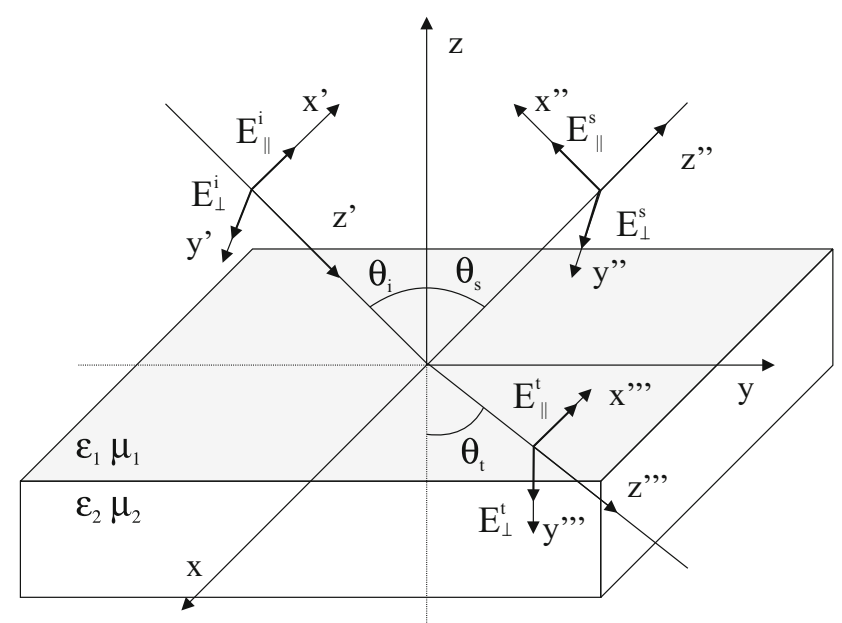

Fig. 1.2 Oblique incidence

that $\widehat{\mathbf{k}}^{i}=\widehat{\mathbf{z}}^{\prime}$. It may be shown that the scattered wave components can be written similarly

$$
\begin{aligned}
& \underline{\overrightarrow{\mathbf{E}}} \|=E_{\|}^{s} e^{-j\left(\mathbf{k}^{s}, \mathbf{r}\right)} \widehat{\mathbf{x}}^{\prime}, \\
& \underline{\mathbf{E}}_{\perp}^{s}=E_{\perp}^{s} e^{-j\left(\mathbf{k}^{s}, \mathbf{r}\right\rangle \widehat{\mathbf{y}}^{\prime}},
\end{aligned}
$$

but in this case according to $\left\{\widehat{\mathbf{x}}^{\prime \prime}, \widehat{\mathbf{y}}^{\prime \prime}, \widehat{\mathbf{z}}^{\prime}\right\}$.

Considering the equations of the incident and the scattered wave, the question rising at this point is to determine whether it is possible or not to express mathematically the scattering process that occurs at the interface between both media. First of all, it is of crucial importance to take into consideration where, in the space, the expressions of the incident and scattered waves are valid. The expressions in (1.28), (1.29), (1.30) and (1.31) make reference to uniform plane waves. In the case of the incident wave on the scatterer, such a description for the wave, i.e. the wave originated at the transmitting antenna, is only valid if the scatter is in the far-field zone of the transmitting antenna. In the case of the scattered wave, this wave admits a uniform plane wave formulation if the point where the wave is considered is in the far field of the scatterer. In both cases, the waves in the far-field zone may be considered spherical waves, which locally may be considered as uniform plane waves. Considering a spherical coordinate system centred in the scatterer and under the previous assumptions, the incident wave on the scatter can be expressed vectorially, in the far-field zone, as

$$
\mathbf{E}^{i}=\left[\begin{array}{c}
E_{\|}^{i} \\
\| \\
E_{\perp}^{i}
\end{array}\right], \mathbf{E}^{s}=\left[\begin{array}{c}
E_{\|}^{s} \\
E_{\perp}^{s}
\end{array}\right]
$$

As observed, there are different points that need to be considered in the analysis of this problem. The first one is the use of different coordinate systems to characterize, in an unambiguous way, the polarization state of the different waves involved in the scattering process. The second aspect, coupled to the previous one, is to determine the way the scatterer under study changes the different components of the wave. This section has studied this entire problem considering the analytical expressions of the waves.

\subsubsection{The Scattering Matrix}

This section will address the generalization of the previous scattering problem, and it will introduce those concepts necessary to address it in a vector form. The first aspect that needs to be fixed is to determine the different coordinate systems necessary to characterize the scattering problem and the description of the incident and the reflected waves. In the scattering problem, three coordinate systems must be chosen. The first one is the coordinate system located at the centre of the scatterer under consideration and referred to as $\{\widehat{\mathbf{x}}, \widehat{\mathbf{y}}, \widehat{\mathbf{z}}\}$. This coordinate system may be considered as a kind of absolute or global coordinate system. In addition to it, it is necessary to define two additional local coordinate systems in order to determine, in an unambiguous way, the polarization states of the incident and the scattered or reflected waves, respectively. These two coordinate systems, associated with the waves, are defined in terms of the global coordinate system.

Let us consider an object illuminated by an electromagnetic plane wave which may be described as

$$
\underline{\mathbf{E}}^{i}=E_{x} \widehat{\mathbf{x}}^{\prime}+E_{y} \widehat{\mathbf{y}}^{\prime}=E_{x} \widehat{\mathbf{h}}_{i}+E_{y} \widehat{\mathbf{v}}_{i}
$$

where the unitary vectors $\widehat{\mathbf{x}}^{\prime}$ and $\widehat{\mathbf{y}}^{\prime}$ are arbitrarily defined. Hence, the propagation direction of the incident wave is conveniently selected to be $\widehat{\mathbf{k}}^{i}=\widehat{\mathbf{z}}^{\prime}$. The incident wave reaches the object of interest and induces currents on it, which in turn reradiates a wave. This reradiated wave, as shown, is referred to as the scattered wave. In the far-field zone, the scattered wave is an outgoing spherical wave that in the area occupied by the receiving antenna can be

$$
\underline{\mathbf{E}}^{s}=E_{x} \widehat{\mathbf{x}}^{\prime \prime}+E_{y} \widehat{\mathbf{y}}^{\prime \prime}=E_{x} \widehat{\mathbf{h}}_{s}+E_{y} \widehat{\mathbf{v}}_{s}
$$

The propagation direction of the scattered wave is therefore $\widehat{\mathbf{k}}^{s}=\widehat{\mathbf{z}}^{\prime}$. The scattering process is finally analysed in terms of the plane of scattering, which is the plane that contains both the incident and the scattering propagating vectors. The concepts of perpendicular and parallel wave components, or horizontal and vertical wave components, are defined with respect to the plane of scattering. 
Consequently, and as indicated in (1.33), the perpendicular component of the wave admits to be considered as a horizontal component, i.e. $\widehat{\mathbf{x}}^{\prime}=\widehat{\mathbf{h}}_{i}$, whereas the parallel one admits to be considered as a vertical one, i.e. $\widehat{\mathbf{y}}^{\prime}=\widehat{\mathbf{v}}_{i}$. In the case of the scattered wave, the perpendicular component of the wave admits to be considered as a horizontal component, i.e. $\widehat{\mathbf{x}}^{\prime \prime}=\widehat{\mathbf{h}}_{s}$, whereas the parallel one admits to be considered as a vertical one, i.e. $\widehat{\mathbf{y}}^{\prime \prime}=\widehat{\mathbf{v}}_{s}$.

The incident and scattered waves in (1.33) and (1.34), respectively, may be also vectorially expressed by means of the Jones vectors:

$$
\underline{\mathbf{E}}^{i}=\left[\begin{array}{c}
E_{h}^{i} \\
E_{v}^{i}
\end{array}\right], \underline{\mathbf{E}}^{s}=\left[\begin{array}{c}
E_{h}^{s} \\
E_{v}^{s}
\end{array}\right]
$$

In the definition of the previous two Jones vectors, the coordinate systems defined previously are assumed. By using this vector notation for the electromagnetic waves, it is possible to relate the scattered wave with the one of the incident wave by means of a $2 \times 2$ complex matrix:

$$
\underline{\mathbf{E}}^{s}=\frac{e^{-j k r}}{r} \mathbf{S} \underline{\mathbf{E}}^{i}
$$

Here, $r$ is the distance between the scatterer and the receiving antenna, and $k$ is the wavenumber of the illuminating wave. The coefficient $1 / r$ represents the attenuation between the scatterer and the receiving antenna, which is produced by the spherical nature of the scattered wave. On the other hand, the phase factor represents the delay of the travel of the wave from the scatterer to the antenna. Equation (1.36) may be written as

$$
\left[\begin{array}{c}
E_{h}^{s} \\
E_{v}^{s}
\end{array}\right]=\frac{e^{-j k r}}{r}\left[\begin{array}{ll}
S_{h h} & S_{h v} \\
S_{v h} & S_{v v}
\end{array}\right]\left[\begin{array}{c}
E_{h}^{i} \\
E_{v}^{i}
\end{array}\right] .
$$

The matrix $\mathbf{S}$ is referred to as scattering matrix, whereas its components are known as complex scattering amplitudes. The arrangement of the scattering matrix indicates how these complex scattering amplitudes are measured. The first column of $\mathbf{S}$ is measured by transmitting a horizontally polarized wave and employing two antennas horizontally and vertically polarized to record the scattered waves. The second column is measured in the same form, but transmitting a vertically polarized wave.

It is worth mentioning that the scattering matrix characterizes the target under observation for a fixed imaging geometry and frequency. In addition, the four elements must be measured at the same time, especially in those situations where the scatterer is not static or fixed. If they are not measured at the same time, the coherency between the elements may be lost as the different elements may refer to a different scatterer.
As indicated, the scattering matrix represents the scattering process for particular incident and scattering directions, i.e. $\widehat{\mathbf{k}}^{i}$ and $\widehat{\mathbf{k}}^{s}$, respectively. In addition to that, it is also necessary to provide the horizontal and vertical unitary vectors, for the incident and the scattered waves, as they are necessary to define the polarization states of the waves.

In the most general case, which occurs in bistatic configurations where the transmitter and receiver antennas are located in different positions, the scattering matrix contains up to seven independent parameters to characterize the scatterer under observation. These parameters are the four amplitudes and three relative phases; see (1.38). Indeed, any absolute phase in the scattering matrix can be neglected as it does not affect the received power:

$$
\left[\begin{array}{c}
E_{h}^{s} \\
E_{v}^{s}
\end{array}\right]=\underbrace{\frac{e^{-j k r} e^{j \phi_{h h}}}{r}}_{\text {Absolute phase term }} \underbrace{\left[\begin{array}{cc}
\left|S_{h h}\right| & \left|S_{h v}\right| e^{j\left(\phi_{h v}-\phi_{h h}\right)} \\
\left|S_{v h}\right| e^{j\left(\phi_{v h}-\phi_{h h}\right)} & \left|S_{v v}\right| e^{j\left(\phi_{v v}-\phi_{h h}\right)}
\end{array}\right]}_{\text {Relative scattering matrix }}\left[\begin{array}{c}
E_{h}^{i} \\
E_{v}^{i}
\end{array}\right] .
$$

As it was already highlighted previously, the scattering coefficients depend on the direction of the incident and the scattered waves. When considering the matrix $\mathbf{S}$, the analysis of this dependence is of extreme importance since it also involves the definition of the polarization of the incident and the scattered waves. Since (1.37) considers the polarized electromagnetic waves themselves, it is mandatory to assume a frame in which the polarization is defined. There exist two principal conventions concerning the framework where the polarimetric scattering process is considered: Forward Scatter Alignment (FSA) and Backscatter Alignment (BSA); see Fig. 1.3. In both cases, the electric waves of the incident and the scattered waves are expressed in local coordinate systems centred on the transmitting and receiving antennas, respectively. All coordinate systems are defined in terms of a global coordinate system centred inside the target of interest.

The FSA convention (see Fig. 1.3), also called waveoriented since it is defined relative to the propagating wave, is normally considered in bistatic problems, that is, in those configurations in which the transmitter and the receiver are not located at the same spatial position.

The bistatic BSA convention framework (see Fig. 1.3) is defined, on the contrary, with respect to the radar antennas in accordance with the IEEE standard. The advantage of the BSA convention is that for a monostatic configuration, also called backscattering configuration, that is, when the transmitting and receiving antennas are collocated, the coordinate systems of the two antennas coincide; see Fig. 1.4. This configuration is preferred in the radar polarimetry community. In the monostatic case, the scattering matrix in the FSA convention, $\mathbf{S}_{F S A}$, can be related to the same matrix referenced to the monostatic BSA convention $\mathbf{S}_{B S A}$ as follows: 


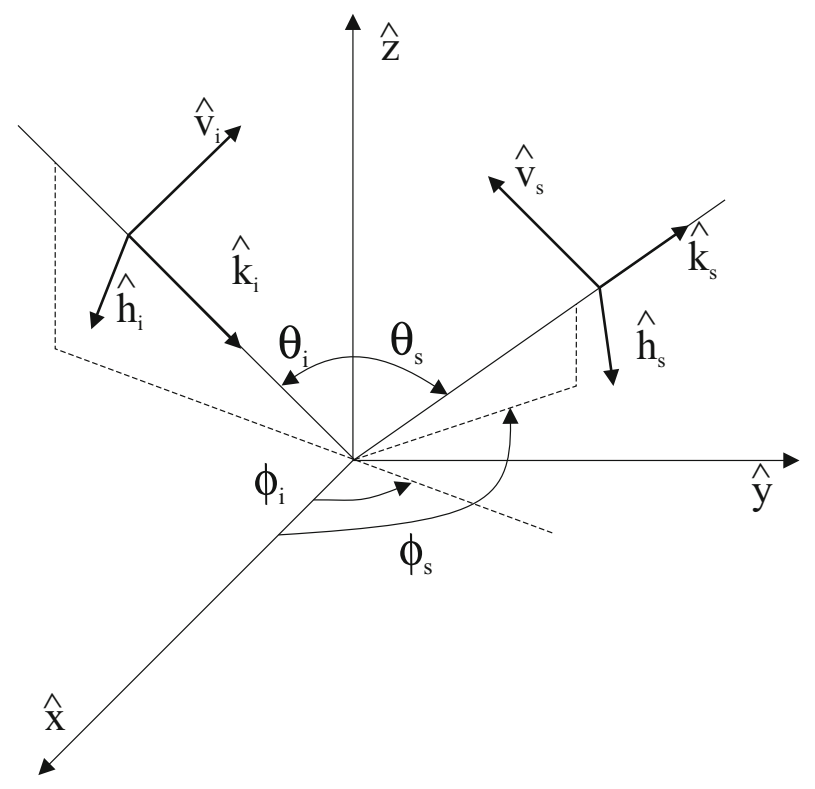

(a)

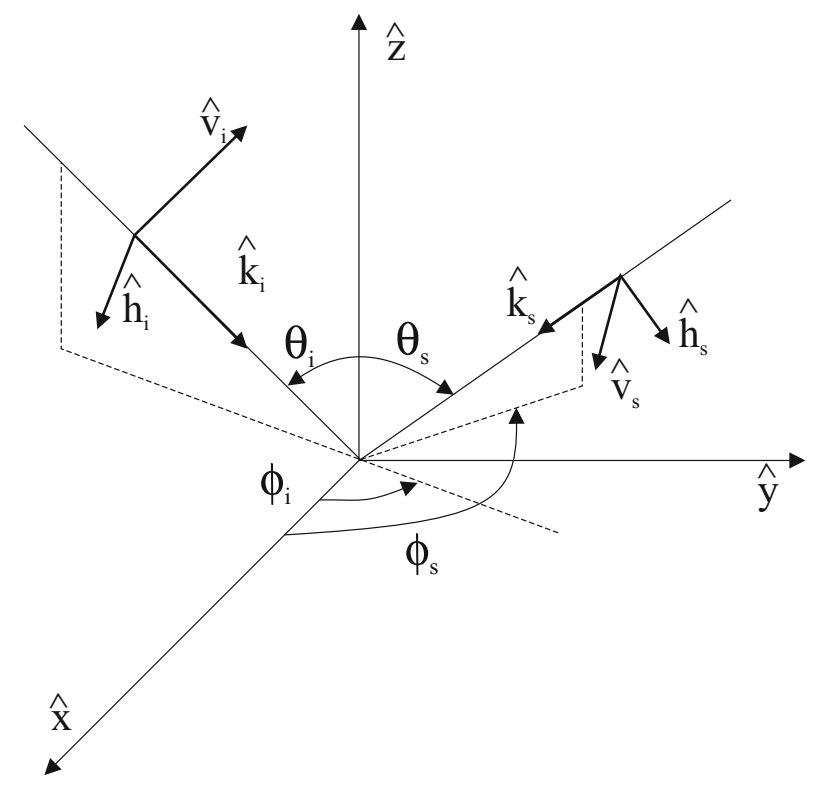

(b)

Fig. 1.3 (a) FSA and (b) BSA conventions

$$
\mathbf{S}_{B S A}=\left[\begin{array}{cc}
-1 & 0 \\
0 & 1
\end{array}\right] \mathbf{S}_{F S A}
$$

As it has been mentioned previously, in the radar polarimetry community, the monostatic BSA convention (backscattering) is considered as the framework to characterize the scattering process. The reason to select this configuration is due to the fact that the majority of the existing polarimetric radar systems operate with the same antenna for transmission and reception. One important property of this configuration, for reciprocal targets, is reciprocity, which states that

$$
\begin{gathered}
S_{h v_{B S A}}=S_{v h_{B S A}}, \\
S_{h v_{F S A}}=-S_{v h_{F S A}} .
\end{gathered}
$$

Then, the formalization of the scattering process given by (1.37), in the monostatic case under the BSA convention, reduces to

$$
\left[\begin{array}{c}
E_{h}^{s} \\
E_{v}^{s}
\end{array}\right]=\frac{e^{-j k r}}{r}\left[\begin{array}{ll}
S_{h h} & S_{h v} \\
S_{h v} & S_{v v}
\end{array}\right]\left[\begin{array}{c}
E_{h}^{i} \\
E_{v}^{i}
\end{array}\right] .
$$

In the same sense, Eq. (1.38) takes the form

$$
\left[\begin{array}{c}
E_{h}^{s} \\
E_{v}^{s}
\end{array}\right]=\underbrace{\frac{e^{-j k r} e^{j \phi_{h h}}}{r}}_{\text {Absolute phase }} \underbrace{\left[\begin{array}{cc}
\left|S_{h h}\right| & \left|S_{h v}\right| e^{j\left(\phi_{h v}-\phi_{h h}\right)} \\
\left|S_{h v}\right| e^{j\left(\phi_{h v}-\phi_{h h}\right)} & \left|S_{v v}\right| e^{j\left(\phi_{v v}-\phi_{h h}\right)}
\end{array}\right]}_{\text {Relative scattering matrix }}\left[\begin{array}{c}
E_{h}^{i} \\
E_{v}^{i}
\end{array}\right] .
$$

The main consequence of the previous equation is that in the backscattering direction, a given scatterer is no longer characterized by seven independent parameters, but by five. These are three amplitudes, two relative phases, and one additional absolute phase.

A central parameter when considering the scattering process occurring at a given scatterer consists of the scattered power. For single-polarization systems, the scattered power is determined by means of the radar cross section or the scattering coefficient. Nevertheless, a polarimetric radar has to be considered as a multichannel system. Consequently, in order to determine the scattered power, it is necessary to consider all the data channels, that is, all the elements of the scattering matrix. The total scattered power, in the case of a polarimetric radar system, is known as Span, being defined in the most general case as

$$
\begin{aligned}
\operatorname{SPAN}(\mathbf{S}) & =\operatorname{trace}\left(\mathbf{S S}^{T *}\right) \\
& =\left|S_{h h}\right|^{2}+\left|S_{h v}\right|^{2}+\left|S_{h v}\right|^{2}+\left|S_{v v}\right|^{2} .
\end{aligned}
$$

In the backscattering case, due to the reciprocity theorem, the Span reduces to

$$
\operatorname{SPAN}(\mathbf{S})=\left|S_{h h}\right|^{2}+2\left|S_{h v}\right|^{2}+\left|S_{v v}\right|^{2}
$$

The main property of the Span is that it is polarimetrically invariable, that is, it does not depend on the polarization basis employed to describe the polarization of the electromagnetic waves. 
Fig. 1.4 (a) FSA and (b) BSA conventions in the backscattering case
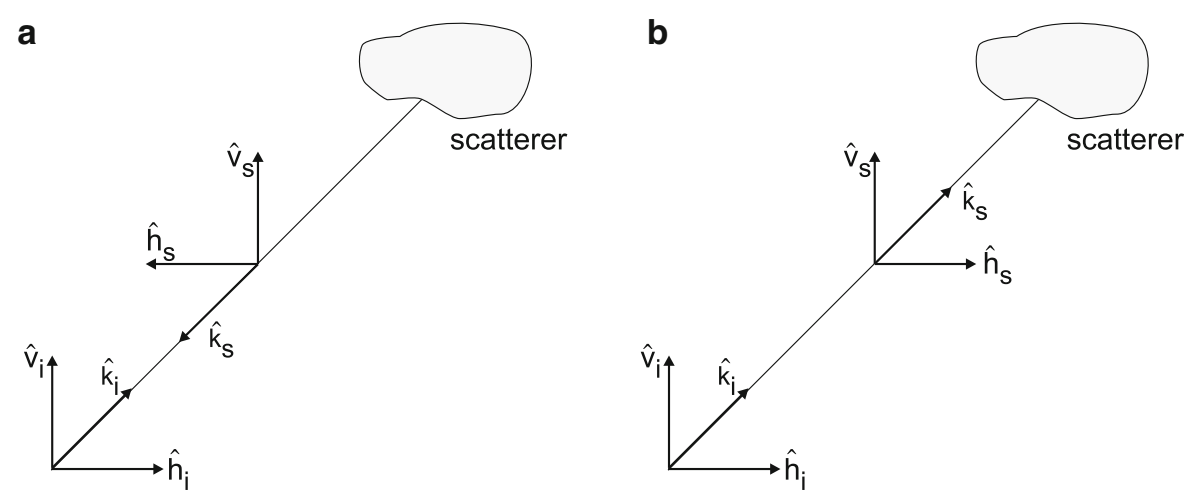

When the radar wave reaches a scatterer, part of the incident energy is reflected back to the system. If the incident wave is monochromatic, the target is unchanging and the radar-target aspect angle is constant, the scattered wave will be also monochromatic and completely polarized. Therefore, both the incident and the scattered waves can be characterized by their corresponding Jones vectors, and the scattering process can be characterized by the scattering matrix. These targets are referred to as point targets, single targets or deterministic targets, as when a radar images this type of scatterers, the scattered wave in the far-field zone appears to be originated by a single point. In other words, the target response is not contaminated by additional spurious, so it is possible to infer some information about the target from the single values of the scattering matrix. Table 1.6 shows the scattering matrix, expressed in the linear polarization basis, for some canonical bodies. These are referred to as canonical due to the simplicity of their scattering matrix.

\subsubsection{Scattering Polarimetry Descriptors}

The scattering matrix introduced in the previous section is indeed a scattering polarimetry descriptor that could be also included in this section. Nevertheless, it merits a separate section as this matrix represents the best vehicle to introduce the description of the scattering process when polarimetry is concerned, as the scattering matrix relates the Jones vectors of the involved electromagnetic waves. Section 1.1.1.1 introduced additional descriptors for the polarization state of an electromagnetic wave. As a consequence, some additional descriptors for the scattering process shall be introduced in the following.

The $2 \times 2$ complex scattering matrix, as indicated, describes the scattering matrix of a given target. Table 1.6 presented several examples for some simple or canonical scatterers. Nevertheless, a real target presents always a complex scattering response as a consequence of its complex geometrical structure and its reflectivity properties. Consequently, the interpretation of this response is obscure. As it shall be presented later on, a possible solution to interpret this response is to decompose the original scattering matrix into the response of canonical mechanisms. With this idea in mind, but also with the objective to introduce a new formulism to extract physical information, it is possible to transform the scattering matrix into a scattering vector that presents a clearer physical interpretation.

The construction of a target vector $\mathbf{k}$ is performed through the vectorization of the scattering matrix:

$$
\mathbf{k}=V(\mathbf{S})=\frac{1}{2} \operatorname{trace}(\mathbf{S \Psi})
$$

$\boldsymbol{\Psi}$ is a set of $2 \times 2$ complex basis matrices which are constructed as an orthonormal set under a Hermitian inner product. The interpretation of the target vector $\mathbf{k}$ depends on the selected basis $\boldsymbol{\Psi}$. The most common matrix bases employed in the context of the radar polarimetry are the so-called lexicographic ordering basis and the Pauli basis. The lexicographic ordering basis consists of the straightforward lexicographic ordering of the elements of the scattering matrix:

$$
\boldsymbol{\Psi}_{l}=\left\{2\left[\begin{array}{ll}
1 & 0 \\
0 & 0
\end{array}\right], 2\left[\begin{array}{ll}
0 & 1 \\
0 & 0
\end{array}\right], 2\left[\begin{array}{ll}
0 & 0 \\
1 & 0
\end{array}\right], 2\left[\begin{array}{ll}
0 & 0 \\
0 & 1
\end{array}\right]\right\}
$$

The Pauli basis consists of the set of Pauli spin matrices usually employed in quantum mechanics:

$\boldsymbol{\Psi}_{p}=\left\{\sqrt{2}\left[\begin{array}{ll}1 & 0 \\ 0 & 1\end{array}\right], \sqrt{2}\left[\begin{array}{cc}1 & 0 \\ 0 & -1\end{array}\right], \sqrt{2}\left[\begin{array}{ll}0 & 1 \\ 1 & 0\end{array}\right], \sqrt{2}\left[\begin{array}{cc}0 & -j \\ j & 0\end{array}\right]\right\}$.

Note that the multiplying factor in both bases is necessary in order to keep the total scattered power constant, i.e. trace $\left(\mathbf{S S}^{* T}\right)$.

The selection of the basis to vectorize the scattering matrix depends on the final purpose of the vectorization itself. When 
Table 1.6 Scattering matrix for canonical bodies in the linear polarization basis $\{\widehat{\mathbf{h}}, \widehat{\mathbf{v}}\}$

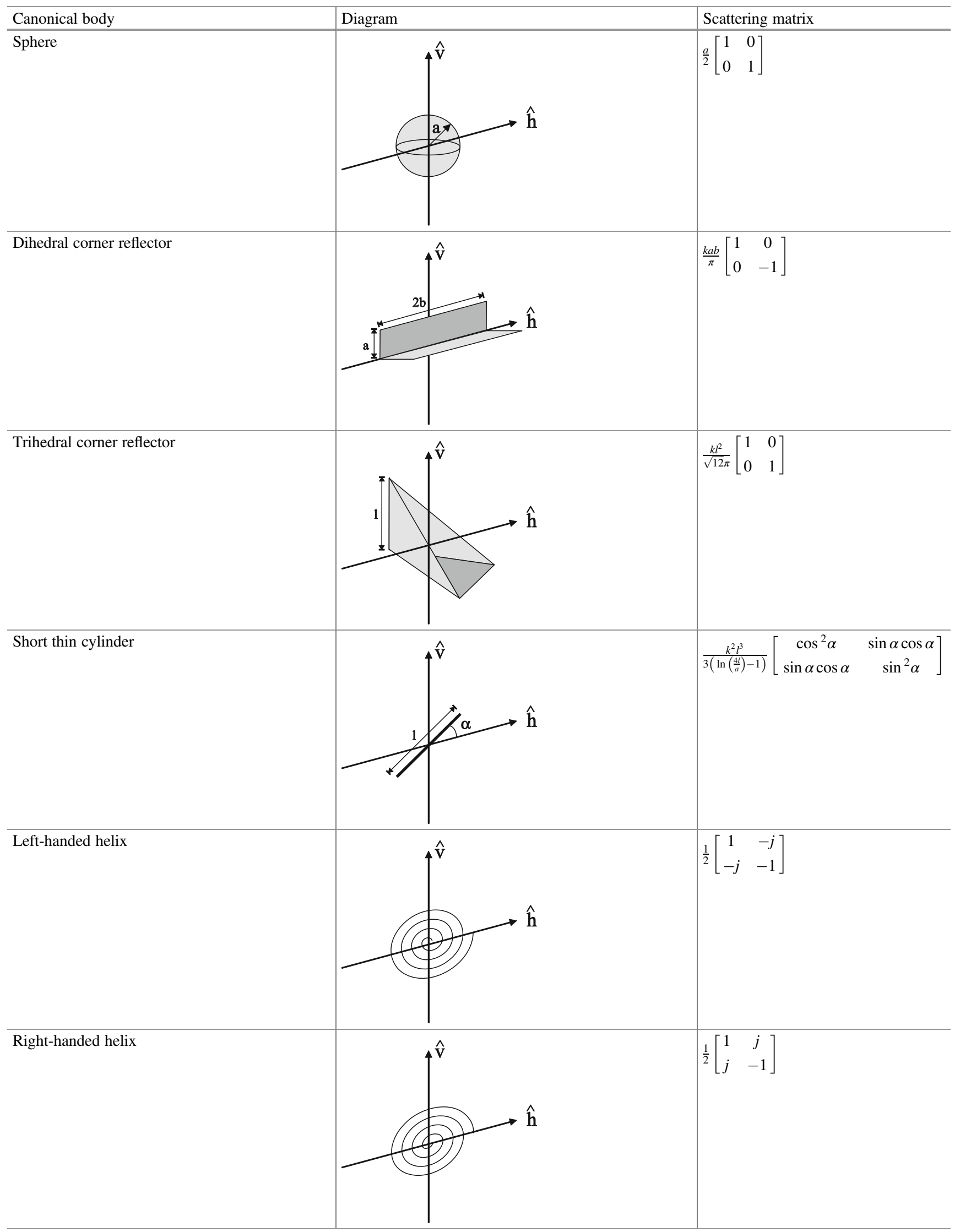


the objective is to study the statistical behaviour of the SAR data or the radar measurement, it is more convenient to consider the lexicographic basis due to its simplicity, as it shall be extended in the next sections. Nevertheless, when the objective is the physical interpretation of the scattering matrix, it is more convenient to consider the Pauli basis. Assuming the Pauli decomposition basis, an arbitrary $2 \times 2$ scattering matrix may be written in the following terms:

$$
\begin{aligned}
\mathbf{S}= & {\left[\begin{array}{cc}
a+b & c-j d \\
c+j d & a-b
\end{array}\right] } \\
= & a\left[\begin{array}{ll}
1 & 0 \\
0 & 1
\end{array}\right]+b\left[\begin{array}{cc}
1 & 0 \\
0 & -1
\end{array}\right]+c\left[\begin{array}{ll}
0 & 1 \\
1 & 0
\end{array}\right] \\
& +d\left[\begin{array}{cc}
0 & -j \\
j & 0
\end{array}\right] .
\end{aligned}
$$

It is worth noting that the elements $a, b, c$ and $d$ are complex. If one considers the decomposition of the scattering matrix as performed in (1.49), it is possible to identify the four elements of the Pauli basis with some of the scattering matrices of canonical bodies presented in Table 1.6. Therefore, the elements $a, b, c$ and $d$, i.e. the elements of the target vector $\mathbf{k}$, represent the contribution of every canonical mechanism to the final scattering mechanism. Therefore, the following interpretation is possible:

- $a$ corresponds to the single scattering from a sphere or plane surface.

- $b$ corresponds to dihedral scattering.

- $c$ corresponds to dihedral scattering with a relative orientation of $\pi / 4 \mathrm{rad}$ in the line of sight.

- $d$ corresponds to anti-symmetric, helix-type scattering mechanisms that transform the incident wave into its orthogonal circular polarization state (helix related).

All in all, what has been performed in (1.49) is a target decomposition. This concept shall be analysed in depth in the next. It is also worth to notice that the different components of the Pauli basis, or scattering components, are orthogonal. This means that from a practical point of view, the separation indicated in (1.49) is possible without ambiguities.

Finally, the explicit expressions of the target vector in the lexicographic and Pauli decomposition bases, considering the expression of the scattering matrix, in the most general case are:

$$
\mathbf{k}_{l}=\left[\begin{array}{c}
S_{h h} \\
S_{h v} \\
S_{v h} \\
S_{v v}
\end{array}\right], \mathbf{k}_{p}=\frac{1}{\sqrt{2}}\left[\begin{array}{c}
S_{h h}+S_{v v} \\
S_{h h}-S_{v v} \\
S_{h v}+S_{v h} \\
j\left(S_{h v}-S_{v h}\right)
\end{array}\right]
$$

In the backscattering case, under the BSA convention, the reciprocity property applies. Hence, the previous target vectors admit the following simplification:

$$
\mathbf{k}_{l}=\left[\begin{array}{c}
S_{h h} \\
\sqrt{2} S_{h v} \\
S_{v v}
\end{array}\right], \mathbf{k}_{p}=\frac{1}{\sqrt{2}}\left[\begin{array}{c}
S_{h h}+S_{v v} \\
S_{h h}-S_{v v} \\
2 S_{h v}
\end{array}\right]
$$

The different 2 and $\sqrt{2}$ factors that appear in the definition of the target vectors are necessary in order to maintain the total scattered power or Span. As it is evident, the Span must be constant and independent from the choice of the basis in which the scattering matrix is decomposed. This is known as total power invariance.

The concept of target vector, obtained as a vectorization of the scattering matrix, makes it possible to obtain a new formulation to describe the information contained in the scattering matrix by means of the outer product of the target vector with its conjugate transpose, or adjoint vector.

For a vectorization of the scattering matrix through the lexicographic basis, in the most general case, the outer product of the target vector with its transpose conjugate $\mathbf{k}_{l} \mathbf{k}_{l}^{T *}$ leads to the matrix:

$$
\mathbf{k}_{l} \mathbf{k}_{l}^{T *}=\left[\begin{array}{cccc}
\left|S_{h h}\right|^{2} & S_{h h} S_{h v}^{*} & S_{h h} S_{v h}^{*} & S_{h h} S_{v v}^{*} \\
S_{h v} S_{h h}^{*} & \left|S_{h v}\right|^{2} & S_{h v} S_{v h}^{*} & S_{h v} S_{v v}^{*} \\
S_{v h} S_{h h}^{*} & S_{v h} S_{h v}^{*} & \left|S_{v h}\right|^{2} & S_{v h} S_{v v}^{*} \\
S_{v v} S_{h h}^{*} & S_{v v} S_{h v}^{*} & S_{v v} S_{v h}^{*} & \left|S_{v v}\right|^{2}
\end{array}\right] .
$$

Due to a language abuse, the matrix $\mathbf{k}_{l} \mathbf{k}_{l}^{T *}$ is sometimes referred to as covariance matrix and represented by $\mathbf{C}$, but as it will be shown in Sect. 1.1.2.4, the covariance matrix presents a different definition. It is worth to observe that (1.52) is a $4 \times 4$, complex, Hermitian matrix. The construction of this matrix, through the outer product of the vector $\mathbf{k}_{l}$ and its transpose conjugate, makes the matrix $\mathbf{k}_{l} \mathbf{k}_{l}^{T *}$ have a rank equal to 1 . Consequently, $\mathbf{k}_{l} \mathbf{k}_{l}^{T *}$ presents exactly the same information as the scattering matrix, and hence it may have up to seven independent parameters. In the case of the backscattering direction under the BSA convention, and due to the fact that the reciprocity relation applies, $\mathbf{k}_{l} \mathbf{k}_{l}^{T *}$ can be written, considering (1.51), as

$$
\mathbf{k}_{l} \mathbf{k}_{l}^{T *}=\left[\begin{array}{ccc}
\left|S_{h h}\right|^{2} & \sqrt{2} S_{h h} S_{h v}^{*} & S_{h h} S_{v v}^{*} \\
\sqrt{2} S_{h v} S_{h h}^{*} & \left|S_{h v}\right|^{2} & \sqrt{2} S_{h v} S_{v v}^{*} \\
S_{v v} S_{h h}^{*} & \sqrt{2} S_{v v} S_{h v}^{*} & \left|S_{v v}\right|^{2}
\end{array}\right] .
$$

As in the previous case, the $\mathbf{k}_{l} \mathbf{k}_{l}^{T *}$ matrix presents a rank equal to 1 as it is obtained as the outer product of a vector and 
its transpose conjugate. Nevertheless, in this case, the covariance matrix may present up to five independent parameters, that is, the same number of independent parameters as the scattering matrix from which it derives.

A similar procedure can be applied when the scattering matrix is obtained considering the Pauli basis. In this case, the matrix is obtained from the outer product $\mathbf{k}_{p} \mathbf{k}_{p}^{T *}$. Due to a language abuse, this matrix is sometimes referred to as coherency matrix and represented by $\mathbf{T}$, but as it will be shown in Sect. 1.1.2.4, the coherency matrix presents a different definition. Under the most general imaging configuration, considering (1.65), the coherency matrix can be written as

$$
\mathbf{k}_{p} \mathbf{k}_{p}^{T *}=\left[\begin{array}{cccc}
\left|S_{h h}+S_{v v}\right|^{2} & \left(S_{h h}+S_{v v}\right)\left(S_{h h}-S_{v v}\right)^{*} & \left(S_{h h}+S_{v v}\right)\left(S_{h v}+S_{v h}\right)^{*} & \left(S_{h h}+S_{v v}\right)\left(j\left(S_{h v}-S_{v h}\right)\right)^{*} \\
\left(S_{h h}-S_{v v}\right)\left(S_{h h}+S_{v v}\right)^{*} & \left|S_{h h}-S_{v v}\right|^{2} & \left(S_{h h}-S_{v v}\right)\left(S_{h v}+S_{v h}\right)^{*} & \left(S_{h h}-S_{v v}\right)\left(j\left(S_{h v}-S_{v h}\right)\right)^{*} \\
\left(S_{h v}+S_{v h}\right)\left(S_{h h}+S_{v v}\right)^{*} & \left(S_{h v}+S_{v h}\right)\left(S_{h h}-S_{v v}\right)^{*} & \left|S_{h v}+S_{v h}\right|^{2} & \left(S_{h v}+S_{v h}\right)\left(j\left(S_{h v}-S_{v h}\right)\right)^{*} \\
j\left(S_{h v}-S_{v h}\right)\left(S_{h h}+S_{v v}\right)^{*} & j\left(S_{h v}-S_{v h}\right)\left(S_{h h}-S_{v v}\right)^{*} & j\left(S_{h v}-S_{v h}\right)\left(S_{h v}+S_{v h}\right)^{*} & \left|S_{h v}-S_{v h}\right|^{2}
\end{array}\right] .
$$

As in the case of $\mathbf{k}_{l} \mathbf{k}_{l}^{T *}, \mathbf{k}_{p} \mathbf{k}_{p}^{T *}$ presents a rank equal to 1 , and therefore, it may present up to seven independent parameters. Finally, if the backscattering direction is considered under the BSA convention, the coherency matrix reduces to

$$
\mathbf{k}_{p} \mathbf{k}_{p}^{T *}=\left[\begin{array}{ccc}
\left|S_{h h}+S_{v v}\right|^{2} & \left(S_{h h}+S_{v v}\right)\left(S_{h h}-S_{v v}\right)^{*} & 2\left(S_{h h}+S_{v v}\right) S_{h v}^{*} \\
\left(S_{h h}-S_{v v}\right)\left(S_{h h}+S_{v v}\right)^{*} & \left|S_{h h}-S_{v v}\right|^{2} & 2\left(S_{h h}-S_{v v}\right) S_{h v}^{*} \\
2 S_{h v}\left(S_{h h}+S_{v v}\right)^{*} & 2 S_{h v}\left(S_{h h}-S_{v v}\right)^{*} & 4\left|S_{h v}\right|^{2}
\end{array}\right]
$$

Again, the previous matrix presents a rank equal to 1 and may have up to five independent parameters.

The lexicographic and the Pauli target vector are just a different transformation of the scattering matrix into a vector. Hence, the covariance and coherency matrices are related by the following unitary transformation in the most general configuration:

$$
\begin{aligned}
\mathbf{k}_{p} \mathbf{k}_{p}^{T *}= & \frac{1}{2} \\
& \times\left[\begin{array}{cccc}
1 & 0 & 0 & 1 \\
1 & 0 & 0 & -1 \\
0 & 1 & 1 & 0 \\
0 & j & -j & 0
\end{array}\right] \mathbf{k}_{l} \mathbf{k}_{l}^{T *}\left[\begin{array}{cccc}
1 & 1 & 0 & 0 \\
0 & 0 & 1 & -j \\
0 & 0 & 1 & j \\
1 & -1 & 0 & 0
\end{array}\right] .
\end{aligned}
$$

In the case of the backscattering direction under the BSA convention, the previous transformation reduces to

$$
\mathbf{k}_{p} \mathbf{k}_{p}^{T *}=\frac{1}{2}\left[\begin{array}{ccc}
1 & 0 & 1 \\
1 & 0 & -1 \\
0 & \sqrt{2} & 0
\end{array}\right] \mathbf{k}_{l} \mathbf{k}_{l}^{T *}\left[\begin{array}{ccc}
1 & 1 & 0 \\
0 & 0 & \sqrt{2} \\
1 & -1 & 0
\end{array}\right]
$$

As it may be seen from all this section, the matrices $\mathbf{k}_{l} \mathbf{k}_{l}^{T *}$ and $\mathbf{k}_{p} \mathbf{k}_{p}^{T *}$ contain the same information as the scattering matrix, that is, they are rank 1 matrices. The necessity to introduce these matrices is that they will allow to define the covariance and coherency matrices.

The complex scattering matrix $\mathbf{S}$ is able to describe a single physical scattering process, as well as $\mathbf{k}_{l} \mathbf{k}_{l}^{T *}$ and $\mathbf{k}_{p} \mathbf{k}_{p}^{T *}$. All these descriptors are based on a wave representation of the data, which depend on the absolute phase from the scatterer. On the contrary, a power representation of the scattering process eliminates this dependence, as power parameters become incoherently additive parameters. In the most general case, assuming the BSA convention, one may define the $4 \times 4$ Kennaugh matrix as follows: 


$$
\mathbf{K}=\mathbf{A}^{*}(\mathbf{s} \bigotimes \mathbf{s}) \mathbf{A}^{-1}
$$

where

$$
\mathbf{A}=\left[\begin{array}{cccc}
1 & 0 & 0 & 1 \\
1 & 0 & 0 & -1 \\
0 & 1 & 1 & 0 \\
0 & j & -j & 0
\end{array}\right]
$$

The Kennaugh matrix can be written in the following form:

$$
\mathbf{K}=\left[\begin{array}{cccc}
A_{0}+B_{0} & C_{\psi} & H_{\psi} & F_{\psi} \\
C_{\psi} & A_{0}+B_{\psi} & E_{\psi} & G_{\psi} \\
H_{\psi} & E_{\psi} & A_{0}-B_{\psi} & D_{\psi} \\
F_{\psi} & G_{\psi} & D_{\psi} & -A_{0}+B_{0}
\end{array}\right]
$$

where

$$
\begin{aligned}
A_{0} & =\frac{1}{4}\left|S_{h h}+S_{v v}\right|^{2} \\
B_{0} & =\frac{1}{4}\left|S_{h h}-S_{v v}\right|^{2}+\left|S_{h v}\right|^{2} \\
B_{\psi} & =\frac{1}{4}\left|S_{h h}-S_{v v}\right|^{2}-\left|S_{h v}\right|^{2} \\
C_{\psi} & =\frac{1}{2}\left|S_{h h}-S_{v v}\right|^{2} \\
D_{\psi} & =\mathfrak{I}\left\{S_{h h} S_{v v}^{*}\right\} \\
E_{\psi} & =\mathfrak{R}\left\{S_{h v}^{*}\left(S_{h h}-S_{v v}\right)\right\} \\
F_{\psi} & =\mathfrak{I}\left\{S_{h v}^{*}\left(S_{h h}-S_{v v}\right)\right\} \\
G_{\psi} & =\mathfrak{R}\left\{S_{h v}^{*}\left(S_{h h}+S_{v v}\right)\right\} \\
H_{\psi} & =\mathfrak{I}\left\{S_{h v}^{*}\left(S_{h h}+S_{v v}\right)\right\}
\end{aligned}
$$

In the previous definition, the sub-index $\psi$ indicates that the different parameters are roll angle dependent, corresponding to the target rotation along the line of sight.

As detailed in Sect. 1.1.2.1, the scattering matrix relates the scattered wave to the incident Jones vector. The Kennaugh matrix is related to the associated Stokes vectors defined in Sect. 1.1.1.1. In the forward scattering case, where $\mathbf{S}$ is represented in the FSA coordinate formulation, this matrix is named the $4 \times 4$ Mueller matrix and is calculated by

$$
\mathbf{M}=\mathbf{A}(\mathbf{S} \bigotimes \mathbf{s}) \mathbf{A}^{-1}
$$

The main difference of $\mathbf{K}$ and $\mathbf{M}$, with respect to $\mathbf{k}_{l} \mathbf{k}_{l}^{T *}$ and $\mathbf{k}_{p} \mathbf{k}_{p}^{T *}$, is that the Kennaugh and the Mueller matrices are real matrices, whereas the covariance and coherency matrices are complex.

\subsubsection{Partial Scattering Polarimetry}

As indicated in Sect. 1.1.1.2, radar polarimetry is concerned with two types of waves. The first type is monochromatic, totally polarized electromagnetic waves where the polarization state is perfectly represented by the Jones vectors. Consequently, the scattering process can be completely represented by any of the scattering polarimetry descriptors detailed in the previous section, and especially the scattering matrix. This situation appears when the radar transmits a perfectly monochromatic wave and this wave reaches an unchanging scatterer, resulting in a perfectly polarized scattered wave. As mentioned, these targets are referred to as point targets or coherent targets. The most important point to be considered when coherent scattering is addressed is to determine the number of independent parameters necessary to represent the scattering process. That is, to determine the number of independent parameters necessary to represent the operator able to characterize the change of the polarization state of the scattered wave with respect to the incident wave that occurs in the scattering process. In a monostatic configuration, the scattering operator describing the scattering, i.e. any of the matrix operators indicated in Sections 1.1.2.1 and 1.1.2.2, may present up to five independent parameters. In the bistatic case, these descriptors may present up to seven independent parameters.

The situation changes when the scattering properties of the target being imaged by the radar system change in time, as it would be the case for a forest being affected by the wind conditions or, for instance, when the target presents more than one scattering centre (a point at which the incident wave can be considered to be reflected). Under this situation, although the radar system transmits a perfectly polarized wave, the wave scattered by the scatterer is partially polarized. A scatterer of this category is normally referred to as distributed scatterer, depolarizing scatterer or an incoherent scattering target. The change of the polarization state of the scattered wave makes not possible to use the scattering descriptors presented in Sects. 1.1.2.1 and 1.1.2.2 to describe the scattering process, as these descriptors are not able to describe the variation of the polarization state of the scattered wave.

In the case of partially polarized waves, the description of the polarization state must be addressed through polarization descriptors relying on the second-order moments of the electromagnetic wave. If a wave is decomposed into two orthogonal components in the plane perpendicular to the propagation direction, these second-order moments refer to the power of each orthogonal component and to the correlation between them. This information is perfectly represented by the vector and the wave coherency matrix or the Stokes vector. In the case of the description of the scattering process, this information can be perfectly represented by the covariance and coherency matrices as the mean values of these matrices are not zero. 


\subsubsection{Change of Polarization Basis}

The scattering properties of a given scatterer, as demonstrated, are contained within the scattering matrix $\mathbf{S}$, which, as shown previously, is measured in a particular polarization basis. Since there exist an infinite number of orthonormal polarization bases, the question rising at this point is whether it is possible or not to infer the polarimetric properties of the given target in any polarization basis from the response measured at a particular basis. This question presents an affirmative answer. The possibility to synthesize any polarimetric response of a given target from its measurement in a particular orthonormal basis represents the most important property of polarimetric systems in comparison with single-polarization systems. The most important consequence of this process is that the amount of information about a given scatterer can be increased, allowing a better characterization and study. This polarization synthesis process is based on the concept of change of polarization basis presented in Sect. 1.1.1.3.

Before describing the polarization synthesis process in the backscattering direction, it is necessary to analyse the scattering process given by (1.37) with respect to the direction of propagation of the incident and the scattered waves. It must be noticed that the incident wave propagates in the direction given by the unitary vector $\widehat{\mathbf{k}}^{i}$, whereas the scattered one propagates in the opposite direction, given by $-\widehat{\mathbf{k}}^{i}$. Consequently, this difference in the propagation direction must be taken into account when defining the polarization state of the wave. Given a Jones vector propagating in the direction $\widehat{\mathbf{k}}$, the Jones vector of a wave presenting the same polarization state but which propagates in the direction $-\widehat{\mathbf{k}}$ is obtained as

$$
\widehat{\mathbf{k}} \rightarrow-\widehat{\mathbf{k}}, \underline{\mathbf{E}}(\widehat{\mathbf{k}})=\underline{\mathbf{E}}^{*}(-\widehat{\mathbf{k}})
$$

where, as mentioned previously, the BSA convention is considered. Under this assumption, the scattering matrix is referred to as the coordinate system centred in the transmitting/receiving system. Consider a polarimetric radar system which transmits the electromagnetic waves in the following orthonormal basis $\left\{\widehat{\mathbf{u}}, \widehat{\mathbf{u}}_{\perp}\right\}$. In this particular basis, the incident and scattered waves are related by the scattering matrix as follows:

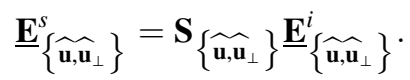

As shown in Sect. 1.1.1.3, given the Jones vector measured in a particular basis, for instance, $\left\{\widehat{\mathbf{u}}, \widehat{\mathbf{u}}_{\perp}\right\}$, it is possible to derive it in any other polarization basis $\left\{\widehat{\mathbf{u}}^{\prime}, \widehat{\mathbf{u}}_{\perp}^{\prime}\right\}$, which may be rewritten as follows:

$$
\underline{\mathbf{E}}_{\left\{\widehat{\mathbf{u}}, \widehat{\mathbf{u}}_{\perp}\right\}}=\mathbf{U}_{\left\{{\left.\widehat{\mathbf{u}}, \widehat{\mathbf{u}}_{\perp}\right\}}\right\} \rightarrow\left\{\widehat{\mathbf{u}}, \widehat{\mathbf{u}}_{\perp}\right\}} \underline{\mathbf{E}}_{\left\{\widehat{\mathbf{u}}, \widehat{\mathbf{u}}_{\perp}\right\}}
$$

Then, the incident and the scattered waves transformed in the new basis may be considered:

$$
\begin{aligned}
& \left.\underline{\mathbf{E}}_{\left\{\widehat{\mathbf{u}}, \widehat{\mathbf{u}}_{\perp}\right\}}^{i}=\mathbf{U}_{\left\{\widehat{\left.\mathbf{u}, \widehat{\mathbf{u}}_{\perp}\right\}}, \rightarrow\left\{\widehat{\left.\mathbf{u}, \widehat{\mathbf{u}}_{\perp}\right\}}\right.\right.}\right\} \underline{\mathbf{E}}_{\left\{\widehat{\mathbf{u}}_{\mathbf{u}_{\perp}}\right\}}^{i}, \\
& \left.\underline{\mathbf{E}}_{\left\{\widehat{\mathbf{u}}, \widehat{\mathbf{u}}_{\perp}\right\}}^{s}=\mathbf{U}_{\left\{\widehat{\left.\mathbf{u}, \mathbf{u}_{\perp}\right\}}\right.}\right\}\left\{\left\{\widehat{\left.\mathbf{u}, \widehat{\mathbf{u}}_{\perp}\right\}} \underline{\mathbf{E}}_{\left\{\widehat{\mathbf{u}}, \widehat{\mathbf{u}}_{\perp}\right\}} \cdot\right.\right.
\end{aligned}
$$

In order to apply the transformation basis procedure to the scattered waves $\underline{\mathbf{E}}_{\left\{\widehat{\mathbf{u}}_{\mathbf{u}_{\perp}}\right\}}$, we need to consider that it propagates in the opposite direction as the incident wave

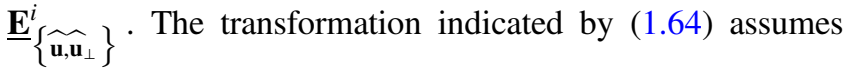
that the incident and the scattered waves propagate in opposite directions, but (1.66) and (1.67) assume that both waves propagate in the same direction. Consequently it is necessary to consider the transformation indicated by (1.63) in (1.67). As a result, the transformation basis procedure applies to the scattered wave as follows:

$$
\underline{\mathbf{E}}_{\left\{\widehat{\mathbf{u}}, \widehat{\mathbf{u}}_{\perp}\right\}}^{s}=\mathbf{U}_{\left\{\widehat{\mathbf{u}}, \widehat{\mathbf{u}}_{\perp}\right\}}^{*} \rightarrow\left\{\widehat{\left.\mathbf{u}, \widehat{\mathbf{u}}_{\perp}\right\}} \underline{\mathbf{E}}_{\left\{\widehat{\mathbf{u}}^{\mathrm{u}} \widehat{\mathbf{u}}_{\perp}\right\}}\right\}
$$

where now the wave in (1.68) is assumed to propagate in opposite direction with respect to the incident wave in (1.66). Now, it is possible to introduce (1.66) and (1.68) in (1.64):

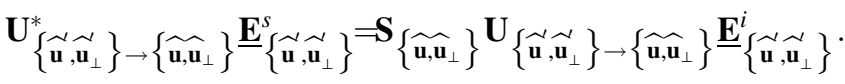

As the transformation matrix $\mathbf{U}$ is unitary, i.e. $\mathbf{U}^{-1}=\mathbf{U}^{* T}$,

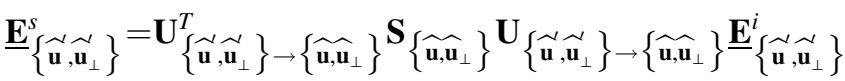

from where it can be clearly identified the following identity

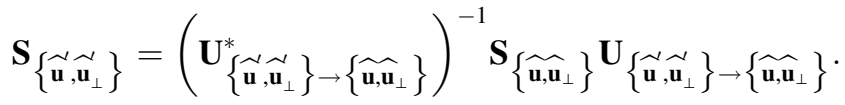

The transformation expressed in (1.71) receives the name of con-similarity transformation. This transformation allows to synthesize the scattering matrix in an arbitrary basis $\left\{\widehat{\mathbf{u}}^{\prime}, \widehat{\mathbf{u}}_{\perp}^{\prime}\right\}$ from its measure in the basis $\left\{\widehat{\mathbf{u}}, \widehat{\mathbf{u}}_{\perp}\right\}$.

\subsubsection{Scatterers Characterization by Single, Dual, Compact and Full Polarimetry}

The main objective behind the use of polarimetric diversity, also known as full polarimetry, when observing a particular scatterer is that this type of diversity allows a far more 
complete characterization of the scatterer than the characterization that could be obtained without polarimetric sensitivity, or simply single-polarization measurements. Although this improved characterization, if compared with singlepolarization data, the use of polarimetric diversity comes at a price, as the average transmitted power must be doubled and the swath width halved. In addition, a fully polarimetric SAR is technologically more complex than a singlepolarization SAR system. In order to understand the difference between these two philosophies and the improvement in the characterization of a scatterer provided by polarimetry, it is necessary to introduce two important concepts, since they will determine the way in which a target shall be characterized. It may happen the scatterer of interest to be smaller than the coverage of the radar system. In this situation, we consider the scatterer as an isolated scatterer, and from a point of view of power exchange, this target is characterized by the so-called radar cross section. Nevertheless, we can find situations in which the scatterer of interest is significantly larger than the coverage provided by the radar system. In these occasions, it is more convenient to characterize the target independently of its extent. Hence, in these situations, the target is described by the so-called scattering coefficient.

The most fundamental form to describe the interaction of an electromagnetic wave with a given scatterer is the so-called radar equation. This equation establishes the relation between the power the scatterer intercepts from the incident electromagnetic wave and the power reradiated by the same scatterer in the form of the scattered wave. The radar equation presents the following form:

$$
P_{r}=\frac{P_{t} G_{t}}{4 \pi r_{t}^{2}} \sigma \frac{A_{r}}{4 \pi r_{r}^{2}}
$$

where $P_{r}$ represents the power detected at the receiving system. The term

$$
\frac{P_{t} G_{t}}{4 \pi r_{t}^{2}}
$$

is determined by the incident wave, and it consists of its power density expressed in terms of the properties of the transmitting system. The different terms in (1.73) are the transmitted power $P_{t}$, the antenna gain $G_{t}$ and the distance between the system and the target $r_{t}$. On the contrary, the term

$$
\frac{A_{r}}{4 \pi r_{r}^{2}}
$$

contains the parameters concerning the receiving system: the effective aperture of the receiving antenna $A_{r}$ and the distance between the target and the receiving system $r_{r}$. The last term in (1.72), i.e. $\sigma$, determines the effects of the scatterer of interest on the balance of powers established by the radar equation. Since (1.73) is a power density, i.e. power per unit area, and (1.74) is dimensionless, the parameter $\sigma$ has units of area. Consequently, $\sigma$ consists of an effective area which characterizes the scatterer. This parameter determines which amount of power is intercepted from (1.73) by the scatterer and reradiated. This reradiated power is finally intercepted by the receiving system according to the distance $r_{t}$. An important fact which arises at this point is the way the scatterer reradiates the intercepted power in a given direction of the space. In order to be independent of this property, the radar cross section shall be referenced to an idealized isotropic scatterer. Thus, the radar cross section of an object is the cross section of an equivalent isotropic scatterer that generates the same scattered power density as the object in the observed direction:

$$
\sigma=4 \pi r^{2} \frac{|\overrightarrow{\mathbf{E}}|^{s}}{|\overrightarrow{\mathbf{E}}|^{2}}=4 \pi|S|^{2}
$$

where $|\overrightarrow{\mathbf{E}}|^{2}$ represents the intensity of the electromagnetic wave and $S$ is the complex scattering amplitude of the object. The final value of $\sigma$ is a function of a large number of parameters which are difficult to consider individually: the wave frequency, the wave polarization, the imaging geometry or the geometrical structure and the dielectric properties of the scatterer. Then, the radar cross section $\sigma$ is able to characterize the target being imaged for a particular frequency and imaging system configuration.

The radar equation, as given by (1.72), is valid for those cases in which the scatterer of interest is smaller than the radar coverage, that is, a point target or point scatterer. For those targets presenting an extent larger than the radar coverage, we need a different model to represent the scatterer. In these situations, a scatterer is represented as an infinite collection of statistically identical point scatterers. The resulting scattered wave $\overrightarrow{\mathbf{E}}^{s}$ results from the coherent addition of the scattered waves from every one of the independent scatterers which model the extended scatterer. In order to express the scattering properties of the extended target independently of its area extent, we consider every elementary target as being described by a differential radar cross section $d \sigma$. In order to separate the effects of the target extent, we consider $d \sigma$ as the product of the averaged radar cross section per unit area 
$\sigma^{0}$ and the differential area occupied by the target $d s$. Then, the differential power received by the systems due to an elementary scatterer can be written as

$$
d P_{r}=\frac{P_{t} G_{t}}{4 \pi r_{t}^{2}} \sigma^{0} d s \frac{A_{r}}{4 \pi r_{r}^{2}} .
$$

Hence, to find the total power received from the extended target, we need to integrate over the illuminated area $A_{0}$ :

$$
P_{r}=\iint_{A_{0}} \frac{P_{t} G_{t}}{4 \pi r_{t}^{2}} \sigma^{0} \frac{A_{r}}{4 \pi r_{r}^{2}} d s .
$$

It must be noted that the radar equation at (1.72) represents a deterministic problem, whereas (1.77) considers a statistical problem. Eq. (1.77) represents the average power returned from the extended target. Hence, the radar cross section per unit area $\sigma^{0}$, or simply scattering coefficient, is the ratio of the statistically averaged scattered power density to the average incident power density over the surface of the sphere of radius $r_{r}$ :

$$
\sigma^{0}=\frac{E\{\sigma\}}{A_{0}}=\frac{4 \pi r_{r}^{2}}{A_{0}} \frac{E\left\{\left|\vec{E}^{s}\right|^{2}\right\}}{|\vec{E}|^{2}} .
$$

The scattering coefficient $\sigma^{0}$ is a dimensionless parameter. As in the case of the radar cross section, the scattering coefficient is employed to characterize the scattered being imaged by the radar. This characterization is for a particular frequency $f$, polarization of the incident and scattered waves and incident and scattering directions.

As it has been shown, the characterization of a given scatterer by means of the radar cross section $\sigma$ or the scattering coefficient $\sigma^{0}$ depends also on the polarization of the incident wave $\overrightarrow{\mathbf{E}}^{i}$. As one can observe in (1.75) and (1.78), these two coefficients are expressed as a function of the intensity of the incident and scattered waves. Consequently, $\sigma$ and $\sigma^{0}$ shall be only sensitive to the polarization of the incident waves through the effects the polarization has over the power of the related electromagnetic waves. Hence, if we denote by $p$ the polarization of the incident wave and by $q$ the polarization of the scattered wave, we can define the following polarization-dependent radar cross section and scattering coefficient, respectively:

$$
\sigma_{q p}=4 \pi r^{2} \frac{\left|\overrightarrow{\mathbf{E}}_{q p}^{s}\right|^{2}}{\left|\overrightarrow{\mathbf{E}}_{q p}^{i}\right|^{2}}=4 \pi\left|S_{q p}\right|^{2},
$$

$$
\sigma_{q p}^{0}=\frac{E\left\{\sigma_{q p}\right\}}{A_{0}}=\frac{4 \pi r_{r}^{2}}{A_{0}} \frac{E\left\{\left|\overrightarrow{\mathbf{E}}_{q p}^{s}\right|^{2}\right\}}{\left|\overrightarrow{\mathbf{E}}_{q p}^{i}\right|^{2}} .
$$

As it has been shown, a given target of interest can be characterized by means of the radar cross section or the scattering coefficient depending on the nature of the scatterer itself; see (1.75) and (1.78). Additionally, in (1.79) and (1.80), it has been shown that these two coefficients depend also on the polarization of the incident and the scattered electromagnetic waves. A closer look to these expressions reveals that these two real coefficients depend on the polarization of the electromagnetic waves only through the power associated with them. Thus, they do not exploit, explicitly, the vectorial nature of polarized electromagnetic waves. A SAR system that measures $\sigma$ or $\sigma^{0}$ is usually referred to as single-polarization SAR systems as, normally, the same polarization is employed for transmission and for reception. In this case, the products delivered by the SAR system are real SAR images containing the information of $\sigma$ or $\sigma^{0}$.

In order to take advantage of the polarization of the electromagnetic waves, that is, their vectorial nature, the scattering process at the scatterer of interest must be considered as a function of the electromagnetic waves themselves. In Sect. 1.1.1.1, it was shown that the polarization of a plane, monochromatic, electric wave could be represented by the so-called Jones vector. Additionally, a set of two orthogonal Jones vectors form a polarization basis, in which any polarization state of a given electromagnetic wave can be expressed. Therefore, given the Jones vectors of the incident and the scattered waves, $\underline{\mathbf{E}}^{i}$ and $\underline{\mathbf{E}}^{s}$, respectively, the scattering process occurring at the target of interest is represented by the scattering matrix $\mathbf{S}$. In contraposition to a single-polarization SAR system, a fully polarimetric SAR system measures the complete scattering matrix $\mathbf{S}$. Therefore, the product delivered by this type of SAR systems corresponds to the $2 \times 2$ complex scattering matrix and not individual real SAR images.

As it can be observed, the polarimetric sensitivity of a measurement ranges from a complete absence of polarimetric sensitivity in the case of single-polarization SAR systems to a complete sensitivity in the case of fully polarimetric SAR systems. Polarimetric sensitivity comes to a price of a more complex system that implies, on the one hand, a heavier system and, on the other hand, the need to transmit a larger power. In addition, and due to the need to double the pulse repetition frequency to accommodate two polarizations in transmission, the radar swath is also reduced. Nevertheless, between both architectures, there exist other polarimetric radar configurations with may soften the previous limitations but at the cost of reducing the amount of acquired information. 
A single-polarization or mono-polarization SAR system is composed of one transmission and one reception chain that operate at a fixed polarization. In most of the cases, both chains operate at the same polarization providing a co-pol or co-polarized channel. In the particular case of the linear polarization basis, these channels would correspond to $\sigma_{h h}$ or $\sigma_{h h}^{0}$ and $\sigma_{v v}$ or $\sigma_{v v}^{0}$ for the horizontal and the vertical polarization states, respectively. As indicated, these simple imaging radars deliver real SAR images, proportional to $\sigma$ or $\sigma^{0}$, as products. One possibility to increase the amount of information is to consider a dual-polarized radar by including a second reception chain in the system, in such a way that it transmits in one polarization, for instance, $h$, and it receives simultaneously on the same polarization $h$ and also on the orthogonal one $v$, leading to one co-pol and the so-called co-polarized and the cross-polarized channels, respectively. A different alternative for a dual-polarized system is to consider a transmission chain that alternates between polarizations and a single reception chain. In all these cases, the polarimetric system provides images proportional to the radar brightness.

All the previous SAR systems present the limitation that the information that may be retrieved is restricted to the information that can be extracted from the real SAR images, proportional to $\sigma$ or $\sigma^{0}$, or their different combinations. Nevertheless, this limitation is overcome by allowing two simultaneous and coherent reception channels operating at orthogonal polarizations, making it possible to measure the relative phase between them. The coherent nature of the receiving channels allows measuring the different elements of the covariance or coherency matrix. The first option that may be considered is to assume a fixed polarization in transmission and orthogonal polarizations in reception. In the case of the transmission channel, the circular polarization and the $45^{\circ}$ linear polarizations have been proposed, whereas for reception the horizontal and vertical linear polarizations are assumed. This type of systems are collectively known as compact polarized systems as, although they allow to measure some of the elements of the covariance and coherency matrix, they do not allow to measure the complete matrices. Finally, by allowing the system to transmit alternatively between orthogonal polarizations and to receive coherently at the same two orthogonal polarizations, a system like this is able to measure coherently the scattering matrix and to produce the covariance and coherency matrices. In the case of a bistatic configuration, without any type of assumption, these

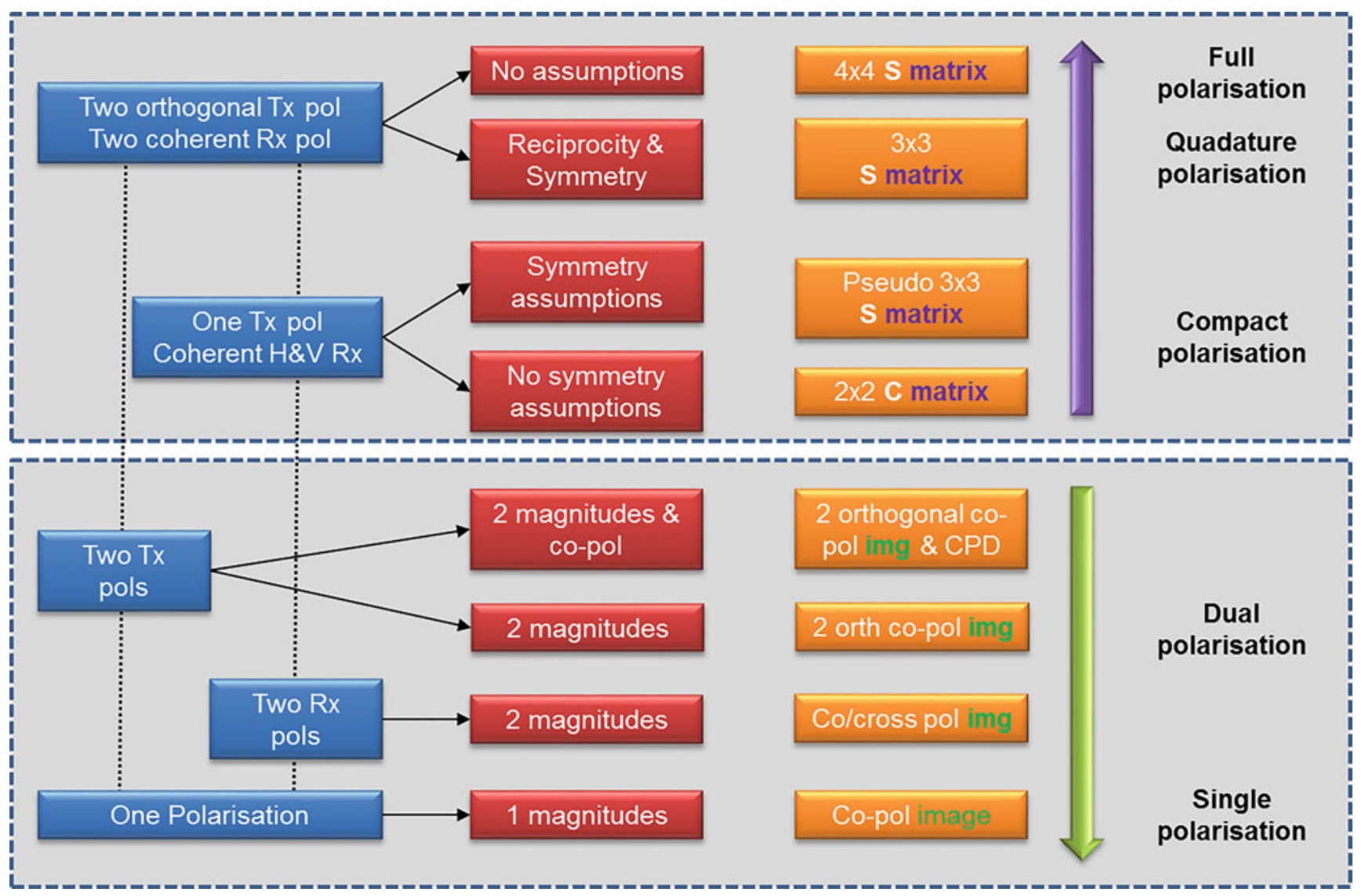

Fig. 1.5 The family of polarization diversity and polarimetric imaging radars. (Courtesy of Dr. R. K. Raney) 
will be $4 \times 4$ complex matrices, whereas in the case of a monostatic configuration, these will be $3 \times 3$ complex matrices. Figure 1.5 details the complete hierarchy of polarimetric SAR systems.

\subsection{SAR Data Statistical Description and Speckle Noise Filtering}

Most of geophysical media, for instance, rough surfaces, vegetation, ice, snow, etc., have a very complex structure and composition. Consequently, the knowledge of the exact scattered electromagnetic wave, when illuminated by an incident wave, is only possible if a complete description of the scene was available. This type of description of the scatterers is unattainable for practical applications. The alternative, hence, is to describe them in a statistical form. Such scatters are named, consequently, as distributed or partial scatterers (Ulaby et al. 1986a, b).

SAR systems are mainly employed for natural scenes observation. Owing to the complexity of these scatterers, the scattered wave has also a complex behaviour. Hence, the scattering process itself needs to be analysed stochastically. Most of the techniques focused on solving the scattered wave problem trying to find, hence, the statistical moments of the scattered wave as a function of the incident wave properties, as well as the scatterer features.

In order to derive a stochastic model for the observed SAR images in the case of distributed scatterers, it is necessary to consider a model for the SAR imaging process, a model for the scattering process and a model for the distributed scatter being imaged.

The SAR imaging process is divided into two main processes. The former consists of the acquisition of the scattered data, as a result of the illuminating wave, whereas the latter comprises the focusing process. The second, which is in charge of collecting all the contributions of a particular scatterer focusing it as good as possible, tries to remove the effects of the acquisition process. The SAR impulse response, or SAR system model, embracing the acquisition, as well as the focusing processes, can be assumed to be a rectangular low-pass filter (Curlander and McDonough 1991):

$$
h(x, r) \propto \operatorname{sinc}\left(\frac{\pi x}{\delta_{x}}\right) \operatorname{sinc}\left(\frac{\pi r}{\delta_{r}}\right)
$$

In the previous equation, $x$ and $r$ indicate the azimuth and slant-range (simply called range in the following) dimensions of the SAR image, respectively, whereas $\delta_{x}$ and $\delta_{r}$ indicate the spatial resolutions in the same spatial dimensions. Finally, a SAR image, i.e. $S(x, r)$, may be modelled as a two-dimensional low-pass filter, given by (1.81), applied to the scene's complex reflectivity $\sigma_{s}(x, r)$ (Curlander and McDonough 1991):

$$
S(x, r)=\int_{-\infty}^{\infty} \int_{-\infty}^{\infty} \sigma_{s}\left(x^{\prime}, r^{\prime}\right) h\left(x-x^{\prime}, r-r^{\prime}\right) \mathrm{d} x^{\prime} \mathrm{d} r^{\prime}
$$

Since the spatial resolutions of the SAR impulse response, $\delta_{x}$ and $\delta_{r}$, are not zero, it is possible to introduce the concept of resolution cell as the area given by $\delta_{x} \times \delta_{r}$. Qualitatively, in the absence of signal re-sampling, the information contained by an image pixel is basically determined by the average complex reflectivity $\sigma_{s}(x, r)$ within this resolution cell.

The resolution cell dimensions, $\delta_{x}$ and $\delta_{r}$, are larger than the wavelength of the illuminating electromagnetic wave $\lambda$. As a consequence, the resulting scattered wave is due to an elaborated scattering process. In order to arrive to a tractable mathematical model of the SAR image $S(x, r)$, it is convenient to find an approximation for the scattering process within the resolution cell. The most common simplification is the Born approximation or simple scattering approximation (Ulaby et al. 1986a). Under it, first, the distributed scatterer is considered to be composed of a set of discrete scatterers characterized by a deterministic response. This scatterer model might be reasonable for those cases in which the discrete scatterer description is valid, for instance, scattering from raindrops or vegetation-covered surfaces having leaves small compared with the wavelength. On the contrary, this assumption is not valid for continuous scatterers. In these cases, it is helpful to apply the concept of effective scattering centre (Ulaby et al. 1986a), in which the continuous scatterer is analysed in a discrete way, e.g. the facet model for surface scattering (Ulaby et al. 1986a; Beckmann and Spizzichino 1987). In a second step, the scattered wave from the resolution cell is supposed to be the linear coherent combination of the individual scattered waves of each one of the discrete scatters within the cell. The main limitation of the Born approximation is that it excludes attenuation or multiple scattering in the process.

Assuming the scattered wave from any distributed scatterer to be originated by a set of discrete sources, (1.82) can be considered in its discrete form:

$$
S(x, r)=\sum_{k=1}^{N} \sigma_{s}\left(x_{k}, r_{k}\right) h\left(x-x_{k}, r-r_{k}\right)
$$

where the sub-index $k$ refers to each particular discrete scatterer in the resolution cell and $N$ is the total number of these scatterers embraced by the response of the SAR system $h(x, r)$. Equation $(1.83)$ can be rewritten by using 


$$
\begin{gathered}
\sigma_{s}\left(x_{k}, r_{k}\right)=\sqrt{\sigma_{k}} \exp \left(j \theta_{s_{k}}^{\prime}\right), \\
h\left(x-x_{k}, r-r_{k}\right)=h_{k} \exp \left(j \varphi_{k}\right), \\
\theta_{s_{k}}=\theta_{s_{k}}^{\prime}+\varphi_{k},
\end{gathered}
$$

as follows:

$$
S(x, r)=\sum_{k=1}^{N} A_{k} \exp \left(j \theta_{s_{k}}\right)
$$

where $A_{k}=h_{k} \sqrt{\sigma_{k}}$. As observed in (1.87), the process to form a SAR image pixel consists of the complex coherent addition of the responses of each one of the discrete scatterers, which are not accessible individually. The sole available measure is the complex coherent addition itself. This coherent addition process receives the name of bi-dimensional random walk (McCrea and Whipple 1940; Doob et al. 1954).

At this point, it is necessary to consider certain assumptions about the elementary scattered waves $A_{k} \exp \left(j \theta_{S_{k}}\right)$ in order to derive a stochastic model for the observed SAR image (Beckmann and Spizzichino 1987; Goodman 1985):
- The amplitude $A_{k}$ and the phase $\theta_{s_{k}}$ of the $k$-th scattered wave are statistically independent of each other and from the amplitudes and phases of all other elementary waves. This fact states that the discrete scatterers are uncorrelated and that the strength of a given scattered wave bears no relation to its phase.

- The phases of the elementary contributions $\theta_{s_{k}}$ are equally likely to lie anywhere in the primary interval $[-\pi, \pi)$.

Under these conditions, (1.87) may be seen as an interference process, in which the interference itself is determined by the phases $\theta_{s_{k}}$. This interference can be constructive, as well as destructive. This effect can be clearly noticed in SAR images, as the amplitude or the intensity of (1.87) presents a salt and pepper or grainy aspect, as it may be observed in Fig. 1.6, which corresponds to $\left|S_{h h}\right|$ acquired with the RADARSAT-2 system over the city of San Francisco. Such a phenomenon is known as speckle (Goodman 1985; Lee 1981; Lopes et al. 1990; Raney 1983).

Speckle is a true electromagnetic measurement and has a complete deterministic nature, as shown in (1.87). Nevertheless, the information contained within speckle needs from two different analyses. In those cases in which there is a reduced number of discrete scatterers within the resolution cell, or its response is basically originated by a reduced set of

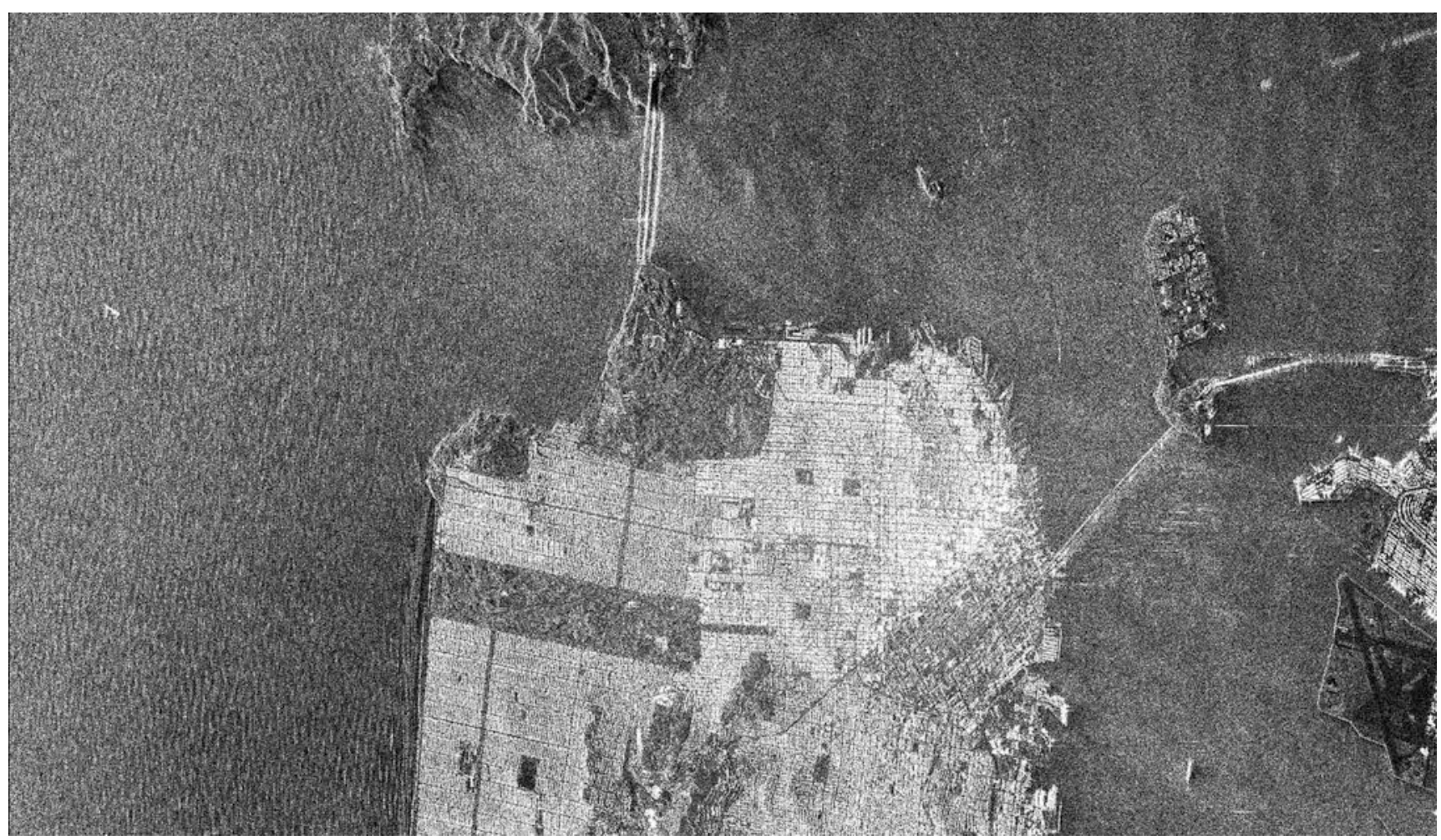

Fig. 1.6 RADARSAT-2 amplitude image of the scattering matrix element $S_{h h}$ over San Francisco (USA) 
dominant ones, speckle is said to be partially developed. Hence, the interference itself, i.e. the speckle, contains information about the scattering process. On the contrary, when there is a large number of discrete scatterers in the cell, without a dominant one, the interference process becomes so complex that it does contain almost no information about the scattering process itself (Oliver and Quegan 1998). This case is called fully developed speckle (Ulaby et al. 1986a), and the complexity of the interference process is overcome by analysing it by statistical means. Hence, speckle turns to be considered as a noise-like signal (Ulaby et al. 1986b; Lopes et al. 1990).

Summarizing, due to the lack of knowledge about the detailed structure of the distributed scatterer being imaged by the SAR system, it is necessary to discuss the properties of the scattered wave statistically. The statistics of concern are defined over an ensemble of objects, all with the same macroscopic properties, but differing in the internal structure. For a given SAR system imaging a particular scatterer, e.g. a rough surface, the exact value of each pixel cannot be predicted, but only the parameters of the distribution describing the pixel values. Therefore, for a SAR image, the actual information per pixel is very low as individual pixels are random samples from distributions characterized by a reduced set of parameters.

\subsubsection{One-Dimensional Gaussian Distribution}

Considering a SAR system to be described by a rectangular low-pass filter (see (1.81)) and the distributed scatterer to be modelled by a set of discrete deterministic scatterers, by means of the single or Born scattering approximation, a SAR image, $S(x, r)$, can be described by the model presented in (1.87).

The main parameter in the SAR image model is the number of discrete scatterers within the resolution cell, i.e. $N$. Depending on the nature of this parameter, different SAR image statistical models can be derived. On the one hand, if $N$ is considered as a constant value, provided that it is large enough, (1.87) leads to the complex, zero-mean, complex Gaussian pdf model, valid for homogeneous, non-textured SAR images (Beckmann and Spizzichino 1987; Goodman 1985; Papoulis 1984). On the other hand, to consider $N$ as a random variable makes (1.87) to lead to pdf models valid for textured areas description. In the following, the zero-mean, complex Gaussian distribution model shall be considered, although possible extensions to textured image models shall be indicated.

When the number of discrete scatterers inside the resolution cell $N$ is large, provided that $A_{k} \cos \left(j \theta_{k}\right)$ and $A_{k} \sin \left(j \theta_{k}\right)$ satisfy the central limit theorem (Oliver and Quegan 1998), the quantities $\mathfrak{R}\{S\}$ and $\mathscr{I}\{S\}$ are Gaussian distributed, that is, they follow a zero-mean, Gaussian probability density function (pdf). The parameters of this pdf can be obtained on the basis of the discrete scatterers model. The mean values of $\mathfrak{R}\{S\}$ and $\mathfrak{I}\{S\}$ are equal to zero, and the variances are $E\left\{\mathfrak{R}^{2}\{S\}\right\}=E\left\{\mathfrak{I}^{2}\{S\}\right\}=\frac{N}{2} E\left\{A_{k}^{2}\right\}$. Besides, the symmetry of the phase distribution of the discrete scatterers produces (Beckmann and Spizzichino 1987):

$$
\begin{aligned}
E\{\Re\{S\} \mathfrak{I}\{S\}\}= & \sum_{k=1}^{N} \\
& \times \sum_{l=1}^{N} E\left\{A_{k} A_{l}\right\} E\left\{\cos \left(\theta_{k}\right) \sin \left(\theta_{l}\right)\right\} \\
= & 0 .
\end{aligned}
$$

Under these conditions, $\mathfrak{R}\{S\}$ and $\mathfrak{I}\{S\}$, denoted in the following by $x$ and $y$, respectively, are described by means of zero-mean Gaussian pdfs:

$$
\begin{aligned}
& p_{x}(x)=\frac{1}{\sqrt{\pi \sigma^{2}}} \exp \left(-\left(\frac{x}{\sigma}\right)^{2}\right), \quad x \in(-\infty, \infty) \\
& p_{y}(y)=\frac{1}{\sqrt{\pi \sigma^{2}}} \exp \left(-\left(\frac{y}{\sigma}\right)^{2}\right), \quad y \in(-\infty, \infty)
\end{aligned}
$$

where the variance is $\sigma^{2}=(N / 2) E\left\{A_{k}^{2}\right\}$. The pdfs $p_{x}(x)$ and $p_{y}(y)$ are denoted in the following as $\mathcal{N}\left(0, \sigma^{2}\right)$. Consequently, a SAR image, $S=x+j y=A \exp (j \theta)$, is described by a zeromean, complex, Gaussian distribution, with uncorrelated real and imaginary parts, denoted next as $\mathcal{N}\left(0, \sigma^{2}\right)$. From (1.89) and (1.90), it is straightforward to derive $p_{A}(A)$ and $p_{\theta}(\theta)$, where $A=\sqrt{x^{2}+y^{2}}$ and $\theta=\arctan (y / x)$, as:

$$
\begin{gathered}
p_{A, \theta}(A, \theta)=\frac{A}{\pi \sigma^{2}} \exp \left(-\left(\frac{A}{\sigma}\right)^{2}\right) \\
p_{A}(A)=\frac{2 A}{\sigma^{2}} \exp \left(-\left(\frac{A}{\sigma}\right)^{2}\right), \quad A \in[0, \infty) \\
p_{\theta}(\theta)=\frac{1}{2 \pi}, \quad \theta \in[-\pi, \pi) .
\end{gathered}
$$

The amplitude pdf, i.e. $p_{A}(A)$, is known as Rayleigh distribution. In addition, if intensity, i.e. $I=A^{2}$, is considered, the Rayleigh pdf is transformed to an exponential pdf:

$$
p_{I}(I)=\frac{1}{\sigma^{2}} \exp \left(-\frac{I}{\sigma^{2}}\right), \quad I \in[0, \infty) .
$$


On the other hand, (1.93) shows that the SAR image phase has a uniform pdf. Consequently, this phase bears no information concerning the natural scene being imaged.

Given the SAR image amplitude pdf (1.92), the amplitude mean value is equal to $\sigma \sqrt{\pi} / 2$, whereas the variance equals $(1-(\pi / 4)) \sigma^{2}$. If the coefficient of variation, defined as the standard deviation divided by the mean, is calculated, it equals $\sqrt{(4 / \pi)-1}$. For the intensity $I$, it has a value equal to 1 as the mean and the variance are equal to $\sigma^{2}$. As a consequence, the intensity of a SAR image can be modelled as the product of two uncorrelated terms (Goodman 1985; Lee 1981; Lopes et al. 1990; Raney 1983), i.e.

$$
I=\sigma^{0} n
$$

The deterministic-like value is given by its mean, i.e. $\sigma^{0}$, which corresponds to the mean incoherent power of the area under study (1.78). The random process $n$, with mean and variance equal to 1 , is characterized by an exponential pdf and is defined as the speckle noise component. As it may be observed from the model (1.94) and (1.95), if the mean value of the intensity increases, the variance increases as well. Therefore, this model is known as the multiplicative speckle noise model. In other words, the signal to noise ratio of the image cannot be improved by increasing the transmitted power, as the variance of the data will increase proportionally.

The Gaussian model for SAR data, leading to (1.91) and (1.95), is able to characterize SAR data for homogeneous areas. In this case, useful information is described by a single degree of freedom, corresponding to the mean intensity. Nevertheless, for certain types of distributed scatterers, such a simple model cannot describe all the data variability. The reason behind this limitation is that this type of scatterers need from a more sophisticated model, with more than one degree of freedom, in order to be completely described. Collectively, these models are able to describe average intensity variations, which correspond to data texture (Oliver and Quegan 1998). A variety of two-degree of freedom pdf models have been proposed in the literature, for instance: K-distribution (Kong 1990), Weibull distribution or log-normal distribution (Oliver and Quegan 1998; Papoulis 1984; Kong 1990). All these models consist of assuming the number of scatterers $N$, within a resolution cell, no longer as a constant, but being described also by a certain distribution. Even so, there are situations in which these two-parameter models are not able to describe the scene. Hence, the solution goes into the direction of introducing more degrees of freedom, thus resulting in more elaborate image models (Oliver and Quegan 1998; Trunk and George 1970; Trunk 1972; Schleher 1975).

\subsubsection{Multidimensional Gaussian Distribution}

The previous section was concerned with the statistical description of one-dimensional complex data acquired by a complex SAR system, i.e. a single SAR image. As shown, despite the data's complex nature, only the amplitude, or the intensity, contains useful information concerning the distributed target under analysis. The amount of information can be increased by acquiring more than one SAR image, if one or more imaging parameters, e.g. system position, acquisition time, frequency or polarization, are varied. What it is pursued, hence, is the study of the variation of the scatterer's response to changes of the SAR system parameters. The volume of information is increased as more data channels are available but also because, if available, the multidimensional data correlation structure can be also exploited to extract information about the observed scatterer (Oliver and Quegan 1998; Cloude and Pottier 1996). The following list shows the most common multidimensional SAR configurations, as well as their main applications:

- SAR Interferometry (InSAR) (Bamler and Hartl 1998): In this configuration, two SAR systems image the same scene from slightly different positions in space, leading to two-dimensional SAR data. In this way, the phase difference between the two acquisitions is proportional to the terrain's topography. This configuration is extensively employed nowadays to obtain Digital Elevation Models (DEMs) of the terrain.

- Differential SAR Interferometry (DInSAR) (Gabriel et al. 1989): This SAR configuration admits several variants. On the one hand, a differential interferogram can be obtained through the difference of two interferograms acquired with a zero spatial baseline, but at different times. Consequently, the "residual" differential interferogram can contain small topographic deformations or even atmospheric effects. On the other hand, the same effect can be observed if the topography of a given interferogram is compensated for by means of an external DEM.

- SAR Polarimetry (PolSAR) (Ulaby and Elachi 1990): In this case, the parameters which vary between the different information channels are the polarization of the transmitted wave and the polarization with which the scattered wave by the terrain is collected. A set of two orthogonal polarization states are employed, being the most common the pair of horizontal and vertical polarizations. The most important property of polarimetry is that the polarimetric response to any polarization state of the incident wave of a given scatterer can be derived from the response to a pair of orthogonal polarization states. This SAR configuration exploits the fact that scatterers present different responses 
to different polarizations of the incident wave. For backscattering in which waves are transmitted and collected in the same position and by considering the reciprocity theorem, PolSAR leads to three-dimensional data. On the contrary, when scattered waves are collected in a different position with respect to the transmitted one, i.e. forward scattering, PolSAR data are four-dimensional data.

- Polarimetric SAR Interferometry (PolInSAR) (Cloude and Papathanassiou 1998): This technique tries to combine both the advantages of InSAR and PolSAR. On the one hand, the introduction of interferometric diversity makes possible the data to be sensible to the structure of the target in the vertical dimension. On the other hand, the data are related to different scattering mechanisms in the same resolution cell, thanks to the polarimetric capabilities of the acquisition system. Hence, PolInSAR data are sensible to different scattering mechanisms in the same image pixel, located at different heights. The introduction of simple scattering models allows to extract relevant information about the scatterer under study. Among the possible applications of this technique, the most important is the extraction of parameters related to the vegetation cover which allow biomass estimation. In terms of data dimensionality, PolInSAR data consist of six-dimensional data if backscattering is considered, whereas they are eight-dimensional data for forward scattering.

- SAR Tomography (TomoSAR) (Reigber and Moreira 2000): As shown in the previous point, PolInSAR represents a first step to resolve the vertical structure of the imaged scatterer. In this direction, SAR tomography is a technique directed to achieve a real three-dimensional reconstruction of the scene under observation. Both the SAR data acquisition and processing are based on the generation and processing of a synthetic aperture in the azimuth direction to reconstruct the object in this direction. In the same way, SAR tomography is based on the synthesis of an aperture in the dimension perpendicular to the plane formed by the azimuth and range dimensions, by acquiring several SAR images in the vertical dimension. Consequently, the phase information of these images can be employed to reconstruct, with enough spatial resolution, the vertical structure of the scatterer.

- Multifrequency SAR (Sarabandi 1997; Lee et al. 1991): As shown in the literature, the response of a given scatterer depends on frequency. Consequently, in order to extract the maximum amount of information concerning the scatterer, several SAR images can be acquired at different frequencies. Therefore, the dimensionality of the data depends on the number of acquired images.

From a general point of view, a multidimensional SAR system acquires a set of SAR images, represented by the complex vector

$$
\mathbf{k}=\left[S_{1}, S_{2}, \ldots, S_{m}\right]^{T}
$$

where $m$ represents the number of SAR images, i.e. the data dimensionality, according to the previous description. Each element of the vector $\mathbf{k}$, i.e. $S_{i}$ for $i=1,2, \ldots, m$, represents a single complex SAR image. In PolSAR, the $\mathbf{k}$ vector receives the name of scattering or target vector (in the straightforward lexicographic basis), and it represents a vectorization of the scattering matrix $\mathbf{S}$ as detailed in Sect. 1.1.2.2. The correlation structure of the vector $\mathbf{k}$, provided that its $m$ components $S_{i} \sim \mathcal{N}\left(0, \sigma^{2}\right)$, is completely characterized by the Hermitian covariance matrix $\mathbf{C}$, defined as follows:

$$
\begin{aligned}
\mathbf{C} & =E\left\{\mathbf{k k}^{H}\right\} \\
& =\left[\begin{array}{cccc}
E\left\{S_{1} S_{1}^{H}\right\} & E\left\{S_{1} S_{2}^{H}\right\} & \cdots & E\left\{S_{1} S_{m}^{H}\right\} \\
E\left\{S_{2} S_{1}^{H}\right\} & E\left\{S_{2} S_{2}^{H}\right\} & \cdots & E\left\{S_{2} S_{m}^{H}\right\} \\
\vdots & \vdots & \ddots & \vdots \\
E\left\{S_{m} S_{1}^{H}\right\} & E\left\{S_{m} S_{2}^{H}\right\} & \cdots & E\left\{S_{m} S_{m}^{H}\right\}
\end{array}\right] .
\end{aligned}
$$

In the particular case of PolSAR data, the data correlation structure can be also expressed by the Hermitian coherency matrix T (Cloude and Pottier 1996). Considering (1.97), one can see that the complex vector $\mathbf{k}$ is characterized by the following pdf (Lee et al. 1994; Tough et al. 1995):

$$
p_{\mathbf{k}}(\mathbf{k})=\frac{1}{\pi^{m}|\mathbf{C}|} \exp \left(-\mathbf{k}^{H} \mathbf{C}^{-1} \mathbf{k}\right)
$$

Hence, (1.98) represents the data pdf model for a set of $m$ correlated SAR images, which is denoted in the following as $\mathcal{N}(\mathbf{0}, \mathbf{C})$. Since $\mathbf{k} \tilde{\mathcal{N}}(\mathbf{0}, \mathbf{C})$, it is completely characterized by the first and the second central moments, i.e. the mean target vector and the covariance matrix, respectively.

At this point, it is important to consider, as presented before, the issue that the mean value of the real and imaginary parts of $\mathbf{k}$ equals zero. The main consequence is that it prevents the possibility to extract useful information via an estimation of this mean value. For instance, this circumstance determines the way PolSAR data has to be considered when distributed scatterers are of concern. A PolSAR system measures the $2 \times 2$ complex scattering matrix $\mathbf{S}$, which can be vectorized into the form presented by (1.96) (Cloude and Pottier 1996; Bebbington 1992); see also Sect. 1.1.2.2. On the one hand, when this scattering vector refers to a distributed scatterer, a given sample of it has almost no information concerning the scatterer itself, as $\mathbf{k}$ consists of a sample of the pdf given by (1.98) (Oliver and Quegan 1998). On the other hand, if the mean value of $\mathbf{k}$ is estimated, it turns out to be zero. Thus, as reported in the literature, when distributed scatterers are studied, the vector $\mathbf{k}$, or the scattering matrix $\mathbf{S}$ in the particular case of PolSAR, cannot completely describe the properties of the distributed scatterer. 
Therefore, it is necessary to characterize these properties by means of higher-order moments, i.e. through an estimation of the covariance matrix $\mathbf{C}$, or, additionally, the coherency matrix $\mathbf{T}$. These two matrices are derived through the outer product of the target vectors $\mathbf{k}_{l}$ and $\mathbf{k}_{p}$, respectively, as indicated in Sect. 1.1.2.2, so they are independent of the absolute phase of the scattering matrix $\mathbf{S}$ or the target vectors $\mathbf{k}_{l}$ and $\mathbf{k}_{p}$. Hence, the expected value in (1.97) needs to be estimated. The process to estimate the covariance matrix $\mathbf{C}$ is also referred to as the polarimetric speckle noise removal process, as the objective is to remove the variability of the data making it possible to retrieve the $\mathbf{C}$ matrix.

In the rest, the complex, multidimensional Gaussian model, presented by (1.98), is taken as the multidimensional SAR imagery model. As for the one-dimensional model for a single SAR image, the complex, multidimensional Gaussian model can be considered valid for homogeneous areas, that is, areas in which the statistical properties of the data remain constant. The main reason of this choice has to be found in the fact that the simplicity of the complex, multidimensional Gaussian pdf, makes it possible the analytical analysis of the information which can be extracted from the data. In addition, many studies reported in the literature support this model.

The multidimensional, zero-mean, complex Gaussian pdf model is based on the following assumptions:

- The distributed scatterer may be modelled as a collection of discrete or point scatterers, whereas the scattering process occurring at the surface, or within it, is considered under the Born or simple scattering approximation.

- The properties of the distributed scatterer remain constant in space, hence leading to homogeneous SAR data.

Thus, whenever any of the previous two suppositions are not fulfilled, SAR data can no longer be assumed to be described by the complex, multidimensional Gaussian pdf model. These departures have been noticed in the literature at high resolutions or high frequencies, giving rise to data texture. As for one-dimensional SAR imagery, some of these departures can be explained by considering $N$, the total number of scatterers in the resolution cell, to be described by a certain pdf. If the mean number of scatterers contributing to the measurement at each pixel is large, then whatever the pdf of the number of discrete scatterers, the vector $\mathbf{k}$ can be represented by the product of two independent processes:

$$
\mathbf{k}=T \mathbf{k}^{\prime}
$$

where $T$ is a positive scalar texture and $\mathbf{k}^{\prime}$ is a complex, multidimensional Gaussian distributed vector, with the same covariance as $\mathbf{k}$. When $T$ is considered to be described by a square-root gamma pdf, the data $\mathbf{k}$ in (1.99) is described by the so-called K-distribution (Kong 1990). Although (1.99) gives rise to textured data, an important result is that any model based on the fluctuation of the number of discrete scatterers within the resolution cell gives rise to data that is multivariate Gaussian at each pixel. That is, despite the texture, the data's correlation structure is still determined by the multidimensional Gaussian structure.

The main drawback of the model given by (1.99) is that, since the texture parameter $T$ is a scalar, the texture information is the same of all the channels of the vector $\mathbf{k}$. Nevertheless, recent results presented in the literature point out that, especially in the case of PolSAR data, the texture information could be different for every SAR data channel (Oliver and Quegan 1998; De Grandi et al. 2003). The physical reason that would explain this issue is that a scatterer presents different responses to different polarizations. Hence, these differences, of course considered in the covariance matrix $\mathbf{C}$, could be also be present within the texture information.

As noticed, in order to extract the useful information concerning the distributed scatterer under analysis from multidimensional SAR data, it is necessary to estimate the covariance matrix $\mathbf{C}$, or expressed in a different way, polarimetric speckle noise must be filtered out. The estimated value of the covariance matrix $\mathbf{C}$, which generally receives the name of sample covariance matrix and is denoted by $\mathbf{Z}$, is studied in detail in the following.

\subsubsection{The Wishart Distribution}

The nature of multidimensional SAR data, provided the zeromean, multidimensional, complex Gaussian pdf to be the right data model, makes it necessary to study the distributed scatterer properties through the estimation of the covariance matrix C. The maximum likelihood estimation (MLE) of C (Oliver and Quegan 1998) corresponds to

$$
\mathbf{Z}=\left[\begin{array}{cccc}
\frac{1}{n} \sum_{k=1}^{n}\left|S_{1 k}\right|^{2} & \frac{1}{n} \sum_{k=1}^{n} S_{1 k} S_{2 k}^{*} & \cdots & \frac{1}{n} \sum_{k=1}^{n} S_{1 k} S_{m k}^{*} \\
\frac{1}{n} \sum_{k=1}^{n} S_{2 k} S_{1 k}^{*} & \frac{1}{n} \sum_{k=1}^{n}\left|S_{2 k}\right|^{2} & \cdots & \frac{1}{n} \sum_{k=1}^{n} S_{2 k} S_{m k}^{*} \\
\vdots & \vdots & \ddots & \vdots \\
\frac{1}{n} \sum_{k=1}^{n} S_{m k} S_{1 k}^{*} & \frac{1}{n} \sum_{k=1}^{n} S_{m k} S_{2 k}^{*} & \cdots & \frac{1}{n} \sum_{k=1}^{n}\left|S_{m k}\right|^{2}
\end{array}\right] .
$$

If one considers the expectation of the MLE 


$$
E\{\mathbf{Z}\}=\frac{1}{n} E\left\{\mathbf{A A}^{H}\right\}=\mathbf{C},
$$

it can be demonstrated that the MLE of the Hermitian covariance matrix is an unbiased estimator. In addition, it can be shown that the variance of the different matrix components of $\mathbf{Z}$ decreases with the number of samples $n$.

In SAR applications, the MLE of the covariance matrix receives often the name of covariance matrix multilook estimator (Sarabandi 1997), whereas $\mathbf{Z}$ is known as the sample covariance matrix (Kay 1993). Here, look refers to each one of the independent averaged samples. Hence, it can be concluded from (1.101) that the performance of the covariance matrix estimation $\mathbf{Z}$, for homogeneous data, depends on the number of averaged samples or looks, in such a way that the larger the number of looks, the lower the variance and the better the estimation. As the $\mathbf{Z}$ matrix is estimated from random samples, this matrix is also a random matrix. Finally, the distribution of the sample covariance matrix $\mathbf{Z}$ corresponds to the Wishart distribution:

$$
p_{\mathbf{Z}}(\mathbf{Z})=\frac{|\mathbf{Z}|^{n-m}}{|\mathbf{C}|^{n} \widetilde{\Gamma}_{m}(n)} \operatorname{etr}\left(-\mathbf{C}^{-1} \mathbf{Z}\right)
$$

where $\operatorname{etr}(\cdot)$ is the exponential of the matrix trace and the complex multivariate gamma function is defined as

$$
\widetilde{\Gamma}_{m}(n)=\pi^{m(m-1) / 2} \prod_{i=1}^{m} \Gamma(n-i+1) .
$$

The distribution presented in (1.102) is denoted by $\mathbf{Z} \tilde{\mathcal{W}}(n \mathbf{C}, m)$. It can be observed from (1.102) that the Wishart distribution depends on three parameters: the number of data channels $m$, the number of averaged multidimensional data samples $n$ and the true covariance matrix $\mathbf{C}$. The expression of the Wishart distribution is only defined for $n \geq m$ in order to assure $\mathbf{Z}$ to be a full-rank matrix with a non-zero determinant.

As it has been highlighted, the Hermitian covariance matrix $\mathbf{C}$ represents the cornerstone in multidimensional SAR data processing, and especially in PolSAR, together with its counterpart, the coherency matrix $\mathbf{T}$. The final objective of estimating these matrices is the possibility to extract physical information to characterize the distributed scatterers being imaged by the SAR system. This task is performed by a collection of algorithms and techniques, collectively known as inversion algorithms. The aim of these techniques is the establishment of relations between the physical properties of the distributed scatterer and the observed SAR data, hence making it possible the inversion of this process in order to extract physical information from observed multidimensional SAR data. Most of these techniques have the covariance matrix $\mathbf{C}$, or certain information derived from it, as the main input of the inversion process. Since due to the intrinsic nature of SAR systems, direct access to the covariance matrix C is not possible, it must be estimated from the observed multidimensional SAR data.

As shown in Sect. 1.2.1, the estimation of incoherent power may be also understood as a filtering process. One alternative to define this filtering process is to assume a given noise model able to identify the information of interest and the noise sources that corrupt this information. In the case of single SAR images, this noise model corresponds to the multiplicative speckle noise model in (1.95). In the case of multidimensional SAR data, this model cannot be extended to the whole covariance matrix $\mathbf{Z}$ as it would imply uncorrelated SAR images. Nevertheless, the multiplicative speckle noise model can be extended to model the diagonal as well as the off-diagonal elements of $\mathbf{Z}$ (Lopez Martínez and Fabregas 2003). In this case, the nature of the speckle noise for a particular element of the covariance matrix depends on the correlation that characterizes this element. For low correlation, speckle noise presents an additive nature, whereas for high correlation, speckle noise is characterized by a multiplicative behaviour. Consequently, this model is referred to as the multiplicative-additive speckle noise model for multidimensional SAR data.

\subsubsection{The Polarimetric Covariance and Coherency Matrix}

As indicated in the previous section, the characterization of distributed scatterers must be performed through the covariance $\mathbf{C}$ or the coherency $\mathbf{T}$ matrices. In a bistatic configuration, and according to what has been presented in Sect. 1.1.2.2, these two matrices are defined as

$$
\begin{aligned}
\mathbf{C} & =E\left\{\mathbf{k}_{l} \mathbf{k}_{l}^{T *}\right\} \\
& =\left[\begin{array}{llll}
E\left\{\left|S_{h h}\right|^{2}\right\} & E\left\{S_{h h} S_{h v}^{*}\right\} & E\left\{S_{h h} S_{v h}^{*}\right\} & E\left\{S_{h h} S_{v v}^{*}\right\} \\
E\left\{S_{h v} S_{h h}^{*}\right\} & E\left\{\left|S_{h v}\right|^{2}\right\} & E\left\{S_{h v} S_{v h}^{*}\right\} & E\left\{S_{h v} S_{v v}^{*}\right\} \\
E\left\{S_{v h} S_{h h}^{*}\right\} & E\left\{S_{v h} S_{h v}^{*}\right\} & E\left\{\left|S_{v h}\right|^{2}\right\} & E\left\{S_{v h} S_{v v}^{*}\right\} \\
E\left\{S_{v v} S_{h h}^{*}\right\} & E\left\{S_{v v} S_{h v}^{*}\right\} & E\left\{S_{v v} S_{v h}^{*}\right\} & E\left\{\left|S_{v v}\right|^{2}\right\}
\end{array}\right]
\end{aligned}
$$

and 


$$
\begin{aligned}
\mathbf{T} & =E\left\{\mathbf{k}_{p} \mathbf{k}_{p}^{T *}\right\} \\
& =\left[\begin{array}{cccc}
E\left\{\left|S_{h h}+S_{v v}\right|^{2}\right\} & E\left\{\left(S_{h h}+S_{v v}\right)\left(S_{h h}-S_{v v}\right)^{*}\right\} & E\left\{\left(S_{h h}+S_{v v}\right)\left(S_{h v}+S_{v h}\right)^{*}\right\} & E\left\{\left(S_{h h}+S_{v v}\right)\left(j\left(S_{h v}-S_{v h}\right)\right)^{*}\right\} \\
E\left\{\left(S_{h h}-S_{v v}\right)\left(S_{h h}+S_{v v}\right)^{*}\right\} & E\left\{\left|S_{h h}-S_{v v}\right|^{2}\right\} & E\left\{\left(S_{h h}-S_{v v}\right)\left(S_{h v}+S_{v h}\right)^{*}\right\} & E\left\{\left(S_{h h}-S_{v v}\right)\left(j\left(S_{h v}-S_{v h}\right)\right)^{*}\right\} \\
E\left\{\left(S_{h v}+S_{v h}\right)\left(S_{h h}+S_{v v}\right)^{*}\right\} & E\left\{\left(S_{h v}+S_{v h}\right)\left(S_{h h}-S_{v v}\right)^{*}\right\} & E\left\{\left|S_{h v}+S_{v h}\right|^{2}\right\} & E\left\{\left(S_{h v}+S_{v h}\right)\left(j\left(S_{h v}-S_{v h}\right)\right)^{*}\right\} \\
E\left\{j\left(S_{h v}-S_{v h}\right)\left(S_{h h}+S_{v v}\right)^{*}\right\} & E\left\{j\left(S_{h v}-S_{v h}\right)\left(S_{h h}-S_{v v}\right)^{*}\right\} & E\left\{j\left(S_{h v}-S_{v h}\right)\left(S_{h v}+S_{v h}\right)^{*}\right\} & E\left\{\left|S_{h v}-S_{v h}\right|^{2}\right\}
\end{array}\right]
\end{aligned}
$$

respectively. In the case of a monostatic system configuration, the covariance and the coherency matrices are defined as

$$
\begin{aligned}
\mathbf{C} & =E\left\{\mathbf{k}_{l} \mathbf{k}_{l}^{T *}\right\} \\
& =\left[\begin{array}{ccc}
E\left\{\left|S_{h h}\right|^{2}\right\} & E\left\{\sqrt{2} S_{h h} S_{h v}^{*}\right\} & E\left\{S_{h h} S_{v v}^{*}\right\} \\
E\left\{\sqrt{2} S_{h v} S_{h h}^{*}\right\} & E\left\{\left|S_{h v}\right|^{2}\right\} & E\left\{\sqrt{2} S_{h v} S_{v v}^{*}\right\} \\
E\left\{S_{v v} S_{h h}^{*}\right\} & E\left\{\sqrt{2} S_{v v} S_{h v}^{*}\right\} & E\left\{\left|S_{v v}\right|^{2}\right\}
\end{array}\right]
\end{aligned}
$$

$$
\mathbf{T}=E\left\{\mathbf{k}_{p} \mathbf{k}_{p}^{T *}\right\}=\left[\begin{array}{ccc}
E\left\{\left|S_{h h}+S_{v v}\right|^{2}\right\} & E\left\{\left(S_{h h}+S_{v v}\right)\left(S_{h h}-S_{v v}\right)^{*}\right\} & E\left\{2\left(S_{h h}+S_{v v}\right) S_{h v}^{*}\right\} \\
E\left\{\left(S_{h h}-S_{v v}\right)\left(S_{h h}+S_{v v}\right)^{*}\right\} & E\left\{\left|S_{h h}-S_{v v}\right|^{2}\right\} & E\left\{2\left(S_{h h}-S_{v v}\right) S_{h v}^{*}\right\} \\
E\left\{2 S_{h v}\left(S_{h h}+S_{v v}\right)^{*}\right\} & E\left\{2 S_{h v}\left(S_{h h}-S_{v v}\right)^{*}\right\} & E\left\{4\left|S_{h v}\right|^{2}\right\}
\end{array}\right] .
$$

As demonstrated in the previous section, the maximum likelihood estimator of the expectation operator $E\{\cdot\}$ and therefore the maximum likelihood estimator of the covariance and coherency matrices correspond to the spatial averaging, referred to as multilook or boxcar filter. In this case, the estimated covariance and coherency matrices receive the names of sample covariance and sample coherency matrices, respectively.

Eqs. (1.106) and (1.107) represent the most general form of the covariance and coherency matrices, respectively, for a monostatic configuration. As these matrices are Hermitian, they contain up to nine independent parameters. Nevertheless, depending on the type of scatterer, the number of independent parameters can be lower leading to a particular form of the covariance $\mathbf{C}$ or the coherency $\mathbf{T}$ matrices. If the scatterer under study has reflection symmetry in a plane normal to the line of sight, then the covariance and the coherency matrices will have the following general forms:

$$
\begin{aligned}
& \mathbf{C}=\left[\begin{array}{ccc}
c_{11} & 0 & c_{13} \\
0 & c_{22} & 0 \\
c_{31} & 0 & c_{33}
\end{array}\right], \\
& \mathbf{T}=\left[\begin{array}{ccc}
t_{11} & t_{12} & 0 \\
t_{21} & t_{22} & 0 \\
0 & 0 & t_{33}
\end{array}\right],
\end{aligned}
$$


that is, the cross-polar scattering coefficient will be uncorrelated with the co-polar terms. Under this hypothesis, the covariance $\mathbf{C}$ or the coherency $\mathbf{T}$ matrices present up to five independent parameters. In addition to reflection symmetry, a medium may also exhibit rotation symmetry. This type of symmetry is referred to as azimuthal symmetry, leading to a coherency matrix presenting the following form:

$$
\mathbf{T}=2\left[\begin{array}{lll}
\alpha & 0 & 0 \\
0 & \beta & 0 \\
0 & 0 & \beta
\end{array}\right]
$$

which has only two independent parameters $\alpha$ and $\beta$.

\subsubsection{The Polarimetric Coherence}

From the expressions of the covariance and coherency matrices that were introduced in Sect. 1.2.4, one may see that the elements of these matrices may be divided into two types: on the one hand, the diagonal elements containing the power information and, on the other hand, the off-diagonal elements that contain the correlation information between the different channels of information. This correlation information may be considered in an absolute way by considering just the off-diagonal elements of these matrices. Nevertheless, this correlation information may be also considered in a relative way through the so-called complex correlation parameter, defined as

$$
\rho=\left|\rho e^{j \phi_{x}}\right|=\frac{E\left\{S_{k} S_{l}^{*}\right\}}{\sqrt{E\left\{\left|S_{k}\right|^{2}\right\} E\left\{\left|S_{l}\right|^{2}\right\}}} .
$$

This parameter contains the information of statistical resemblance between any two SAR images $S_{k}$ and $S_{l}$. Indeed, these SAR images correspond to the different elements of the target vector $\mathbf{k}$ defined in Sect. 1.1.2.2. The amplitude of the complex correlation coefficient, normally referred to as correlation $|\rho|$, presents a value in the range $[0,1]$. If $|\rho|=0$ it means that both SAR images are statistically independent, and the phase $\phi_{x}$ contains no information. For $|\rho|=1$, both SAR images are statistically equal, and the phase information, free of noise, is a delta function containing information about the scattering process. For any other value, $|\rho|$ establishes the correlation between both SAR images, and the phase information $\phi_{x}$ is contaminated by the effect of speckle noise.

In multidimensional SAR imagery, the complex correlation coefficient has been revealed as an important source of information. In particular, the correlation coefficient amplitude, named coherence, apart from depending on the SAR system characteristics, is also influenced by the physical properties of the area under study. The complex correlation coefficient is the most important observable for InSAR (Bamler and Hartl 1998). On the one hand, and considering the acquisition geometry, it has been demonstrated that its phase contains information about the Earth's surface topography. Therefore, InSAR phase data are employed to derive Digital Elevation Models (DEMs) of the terrain. On the other hand, although there is not a complete understanding about the parameters and the physical processes affecting the interferometric coherence, it has been shown that this parameter may be successfully employed to characterize the properties and the dynamics of the Earth's surface.

The coherence represents also an important source of information when PolSAR data are addressed. In particular, the complex correlation coefficient parameter derived from circularly polarized data has been employed to characterize rough surfaces (Mattia et al. 2003), to study the sea surface (Kasilingam et al. 2002) or to discriminate sea ice types (Wakabayashi et al. 2004). When obtained from linearly polarized data, the coherence has been also employed to characterize the forest cover in the Colombian Amazon (Hoekman and Quinones 2002). In conjunction with polarimetric techniques, i.e. polarimetric SAR interferometry (PolInSAR), the interferometric coherence is employed to retrieve the forest vegetation (Cloude and Papathanassiou 1998) or the crop plants heights (Ballester Berman et al. 2005).

All the techniques listed in the previous paragraph rely on a correct estimation of the coherence parameter. The estimated coherence values are overestimated, especially for low coherence values (Touzi et al. 1999). Under the homogeneity hypothesis, the coherence accuracy and bias depend on the extent of the averaging or estimation process, in such a way that the larger the number of averaged pixels, the higher the coherence accuracy and the lower the bias. Therefore, since coherence accuracy is achieved at the expense of spatial resolution and spatial details, this point represents a clear trade-off for coherence estimation. Coherence estimation techniques rely also on the hypothesis that all the signals involved in the estimation process are stationary and in particular locally stationary processes. When this is not the case, biased coherence values result (Touzi et al. 1999). Hence, a lack of signal stationarity can be considered as a second source of bias for coherence estimation. The departure of the stationarity condition may be induced by systematic phase variations mainly due to the terrain topography but also to atmospheric effects or to deformation gradients. The most reliable technique to eliminate this bias is to compensate for the topography by means of external DEMs. Nevertheless, the DEM may not be available for the scene under study, or its quality may be rather low for coherence estimation purposes. There exist alternative coherence estimation 
techniques aiming to solve these problems with different level of success.

\subsubsection{Polarimetric Speckle Noise Filtering}

As it has been explained previously, a PolSAR system measures the scattering matrix $\mathbf{S}$ for every pixel. In the case of deterministic or point scatterers, this matrix determines completely the scattering process, and it can be directly employed to retrieve physical information of the scatterer. Nevertheless, in the case of distributed scatterers, the scattering matrix $\mathbf{S}$ is no longer deterministic but random due to the complexity of the scattering process. As indicated, this random behaviour is referred to as speckle. Speckle is a true scattering measurement, but the complexity of the scattering process makes it necessary to consider it as a noise source. Consequently, the information of interest is no longer the scattering matrix, but the different stochastic moments necessary to specify completely the probability density function of the SAR data. These moments must be estimated from the measured data, or said in a different way, speckle noise must be filtered out or even eliminated to grant access to these statistical moments. In the case of PolSAR data, under the assumption of the vector $\mathbf{k}$ to be distributed according to the zero-mean, complex Gaussian distribution (1.98), these moments correspond to the covariance $\mathbf{C}$ or the coherency T matrices.

Section 1.2.3 already introduced the simplest approach to estimate the covariance or the coherency matrices, i.e. the multilook (1.100), which corresponds to an incoherent average or a spatial average. Although the multilook approach corresponds to the maximum likelihood estimator of the covariance or coherency matrices, it presents the drawback that the estimation of the data is obtained at the expense of degrading the spatial resolution and the spatial details of the data. Figure 1.7 shows an example of these effects.

Considering the limitations of the multilook filtering approach, it is necessary to define different filtering alternatives that improve the multilook approach in such a way that they are able to retain the spatial resolution and the spatial details of the image but also lead to a correct and unbiased estimation of the covariance and coherency matrices.

\subsubsection{PoISAR Speckle Noise Filtering Principles}

The objective of any PolSAR speckle noise filter to be defined is to estimate the covariance or the coherency matrix while retaining the spatial resolution and the spatial details of the data. From a general point of view, it would be necessary to determine the general principles a PolSAR filter should follow in order to perform a correct estimation of the information of interest. Different authors have addressed the necessity to specify the general principles of a PolSAR speckle filter and which are the potential limitations: Touzi et al. (Touzi and Lopes 1994), Lee et al. (Lee et al. 1999) and López-Martínez et al. (Lopez Martínez and Fabregas 2008).

In the previous three references, as the data is assumed to be characterized by the zero-mean, complex Gaussian pdf, the information to retrieve is on the second-order moments of the PolSAR data. In (Touzi and Lopes 1994), the authors propose the use of the Mueller matrix, although they also consider the covariance matrix. In (Lee et al. 1999; Lopez Martínez and Fabregas 2008), the filtering is performed on the covariance or on the coherency matrices. In any case, the use of the covariance, the coherency or the Mueller matrices to filter the data is equivalent as all these matrices contain the same information. For instance, as indicated previously in Sect. 1.1.2.2, the covariance and the coherency matrices are related by similarity transformations. Implicitly, the authors are considering that these matrices contain all the necessary information to characterize the PolSAR data. This assumption is only valid under the hypothesis of (1.98), which only applies in the case of stationary data. The presumption of more evolved stochastic data models that may take into account additional signal variability, for instance, texture, are always associated with the necessity to estimate additional stochastic moments associated with the texture information.

Another point in which all the previous three references are in agreement is the need to consider the estimation of the previous matrices locally, adapting to the stationarity or homogeneity of the PolSAR data. This requirement is justified from two different points of view. The first one refers, due to the stationarity of PolSAR data, to the need to maintaining the spatial resolution and the radiometric information in the case of point or deterministic scatterers, which may be extended to the idea of preserving the spatial resolution and the spatial details of the PolSAR data. The second refers to the fact that in the case of distributed scatterers, the covariance matrix must be estimated on stationary data, avoiding the mixture of different stationary areas. This idea implies that the PolSAR filter must adapt the filtering process to the morphology of the PolSAR data. The differences between the filtering principles for PolSAR data proposed by Touzi and Lopes (1994), Lee et al. (1999) and Lopez Martínez and Fabregas (2008) are on how to consider the information that may be provided by the off-diagonal elements of the covariance or coherency matrices and whether this information may be employed to optimize speckle noise reduction or not. The approaches proposed by Touzi and Lopes (1994) and Lee et al. (1999)) suggest an extension of the multiplicative speckle noise model that applies for the diagonal entries of the covariance matrix to the off-diagonal ones, although it is also admitted that this extension may not lead to an optimum filtering of the speckle 
a

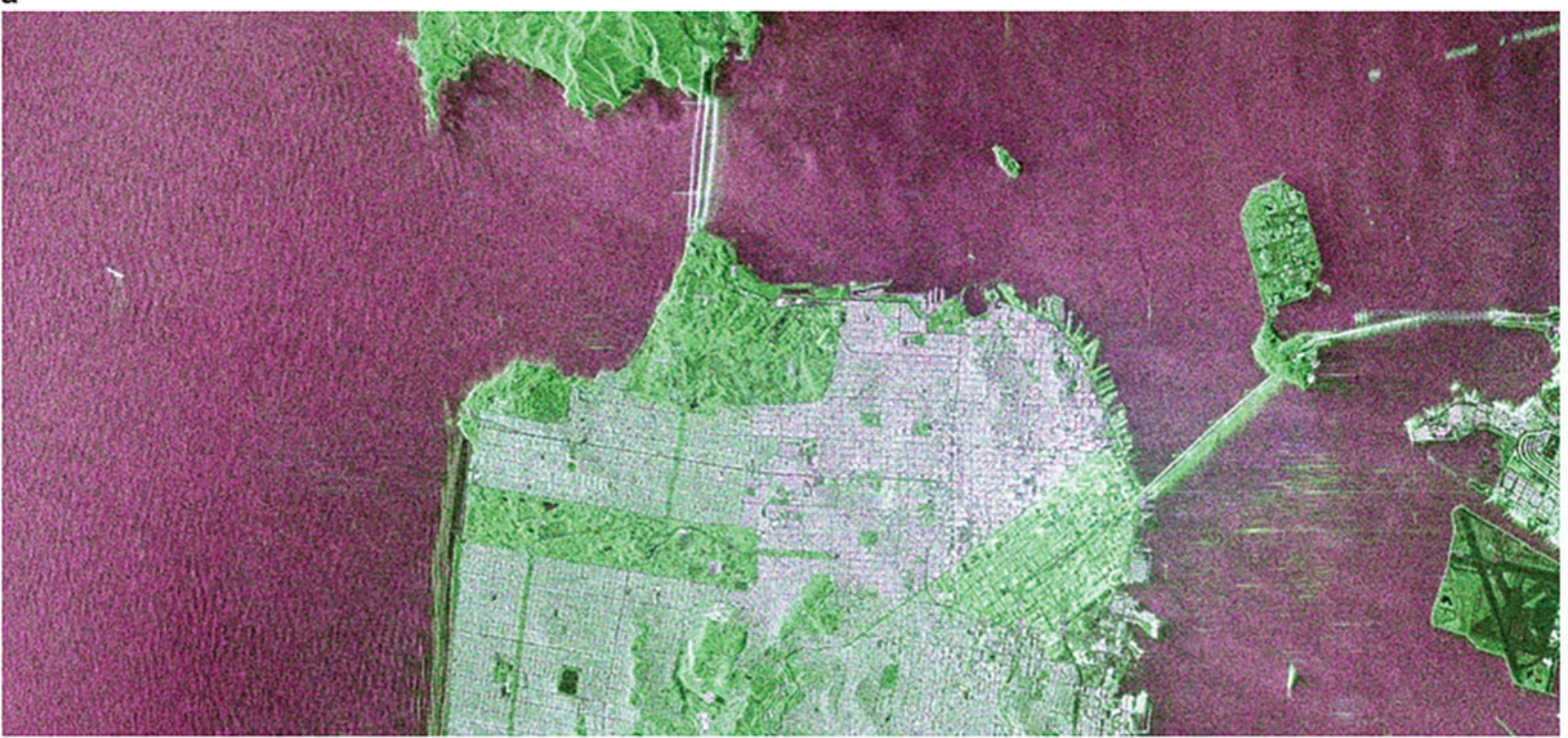

b

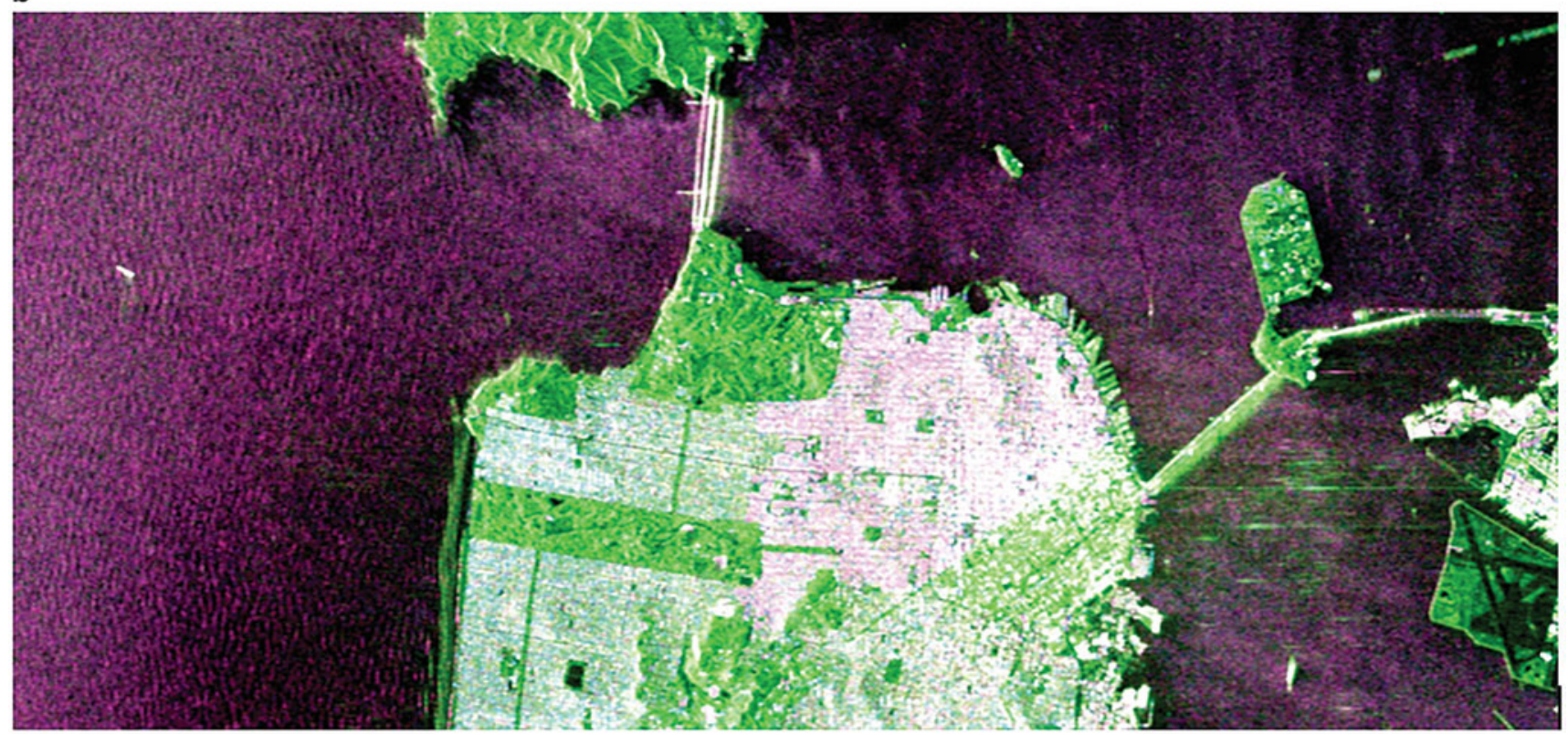

Fig. 1.7 RADARSAT-2 polarimetric RGB image over San Francisco (USA) where the colour code is $S_{h h}$ blue, $S_{v v}$ red and $S_{h v}$ green. (a) Original image and (b) filtered image with a $7 \times 7$ multilook filter

noise component. In (Lee et al. 1999), the authors even propose that the use of the degree of statistical independence between elements must be avoided in order to avoid crosstalk and that all the elements of the covariance matrix must be filtered by the same amount. These principles were extended in (Lopez Martínez and Fabregas 2008), based on a more accurate PolSAR speckle noise model for the off-diagonal elements of the covariance matrix (Lopez Martínez and Fabregas 2003). This model predicts that for a given off-diagonal element of the covariance matrix, speckle presents a complex additive nature for low coherence values, whereas speckle tends to be multiplicative in the case of high coherences. Consequently, an optimum speckle noise reduction should adapt to the type of noise for the off-diagonal elements of the covariance matrix, that is, filtering must adapt to the level of coherence (Lopez Martínez and Fabregas 2008). As it may be concluded, a PolSAR filter needs also to be adapted to the polarimetric information content of the data. Consequently, in connection with what has been explained previously, the way a PolSAR filter adapts to the 
local information must consider all the information provided by the covariance or coherency matrices.

\subsubsection{PolSAR Speckle Noise Filtering Alternatives}

As indicated in Sect. 1.2.3, the first alternative to estimate the covariance or the coherency matrices is to consider their maximum likelihood estimator that corresponds to an incoherent spatial average as expressed in (1.101). In this case, the estimation of the information is obtained at the expense of the loss of spatial resolution and spatial details. Consequently, in order to avoid the previous drawbacks, the PolSAR data filters should adapt to the morphology of the SAR image to retain the spatial details while leading to a correct and unbiased estimation of the covariance or coherency matrices.

PolSAR images are inherently heterogeneous as they reflect the heterogeneity of the Earth surface. Consequently, a first alternative to adapt to this heterogeneity, in order to avoid the loss of spatial resolution and spatial details, is to adapt locally to the signal morphology. One option to achieve this local adaptation is to consider edge aligned windows, as proposed in (Lee et al. 1999). Previously to the PolSAR data filtering process, the algorithm in (Lee et al. 1999), known as refined Lee filter, proposed the use of directional masks, within the analysis window, to determine the most homogeneous part of the sliding window where the local statistics have to be estimated. This spatial adaptation permits to preserve relatively sharp edges and local details. Once the directional mask defines the homogeneous pixels that have to be employed to estimate the covariance or the coherency matrices, these are estimated by means of the Local Linear Minimum Mean Square Error (LLMMSE) approach, i.e.

$$
\widehat{\mathbf{Z}}=\overline{\mathbf{Z}}+b(\mathbf{Z}-\overline{\mathbf{Z}})
$$

where $\widehat{\mathbf{Z}}$ is the estimated value of the covariance matrix, $\overline{\mathbf{Z}}$ is the local mean covariance matrix computed with the homogeneous pixels selected by the edge aligned window and $\mathbf{Z}$ corresponds to the covariance matrix of the central pixel. Finally, $b$ is a weighting function having a value between 0 and 1 derived from the statistics of the Span image. Over homogeneous areas, $b \approx 0$ so the estimated covariance matrix corresponds to the values of the local means as it would be expected in absence of spatial details. Nevertheless, in the case the central pixel of the analysis window corresponds to a deterministic scatterer, $b \approx 1$ producing $\widehat{\mathbf{Z}}$ to be the covariance matrix of the central pixel. Consequently, the pixel is not filtered and the spatial resolution is preserved, as observed in Fig. 1.8. In relation with the filtering procedure proposed in (Lee et al. 1999), the authors proposed also a filtering alternative where the pixels to be averaged within the analysis window are those with the same scattering mechanism as the central pixel, obtained through the Freeman-Durden decomposition (Lee et al. 2006).

As it may be observed, the previous filtering approach adapts to the signal morphology through a family of edge aligned windows. Hence, the adaptation to the signal morphology is restricted to a finite family of aligned windows. In (Vasile et al. 2006), the authors extended the ideas presented in (Lee et al. 1999), but instead of considering edge aligned windows, the authors introduced the concept of region growing to define an adaptive set of homogeneous pixels surrounding the pixel under analysis in order to adapt to the local morphology of the data. As in the case of Lee et al. (1999)), the adaptation to the signal morphology is achieved through the Span image. The region growing process is based on comparing a given pixel against its neighbours to determine their similarity by considering their corresponding covariance and coherency matrices. Since a PolSAR system provides for every pixel only the scattering matrix, an initial process of regularization that assures full-rank covariance or coherency matrices is necessary. This regularization process could be performed with the multilook filter, but it would introduce a loss of spatial resolution and spatial details. In (Lee et al. 1999), the authors propose the use of the median filter. Nevertheless, this alternative introduces a bias in the estimated data, as in the case of non-symmetric distributions, such as the one of the amplitude or the intensity of a SAR image or the one of the Span, the median does not correspond to the mean.

All the previous filtering approaches adapt to the signal morphology locally under the assumption that the pixels surrounding the pixel of analysis present a high probability to be statistically similar. Hence, these filters assume that the data are locally stationary. Nevertheless, it has been recently demonstrated that this idea of local stationary could be relaxed under the assumption that similar pixels to the one of analysis are available not only on the neighbourhood of the pixel of analysis but on the complete image (Deledalle et al. 2010).

In order to increase the filtering effect, one option, as shown in (Vasile et al. 2006), is to increase the number of homogeneous pixels to be averaged that are similar to the pixel under consideration. In (Vasile et al. 2006), as well as in (Lee et al. 1999), the similarity is measured considering only the information contained in the diagonal elements of the covariance or coherency matrices. Therefore, these approaches neglect the information provided by the off-diagonal elements of these matrices. The way to take into account all the information provided by the covariance or the coherency matrices is to consider the concept of the distance in the space defined by the matrices themselves. This approach has been considered in (Deledalle et al. 2010) as well as in (Alonso Gonzalez et al. 2012). In (Alonso Gonzalez et al. 2012), the authors propose to introduce the 


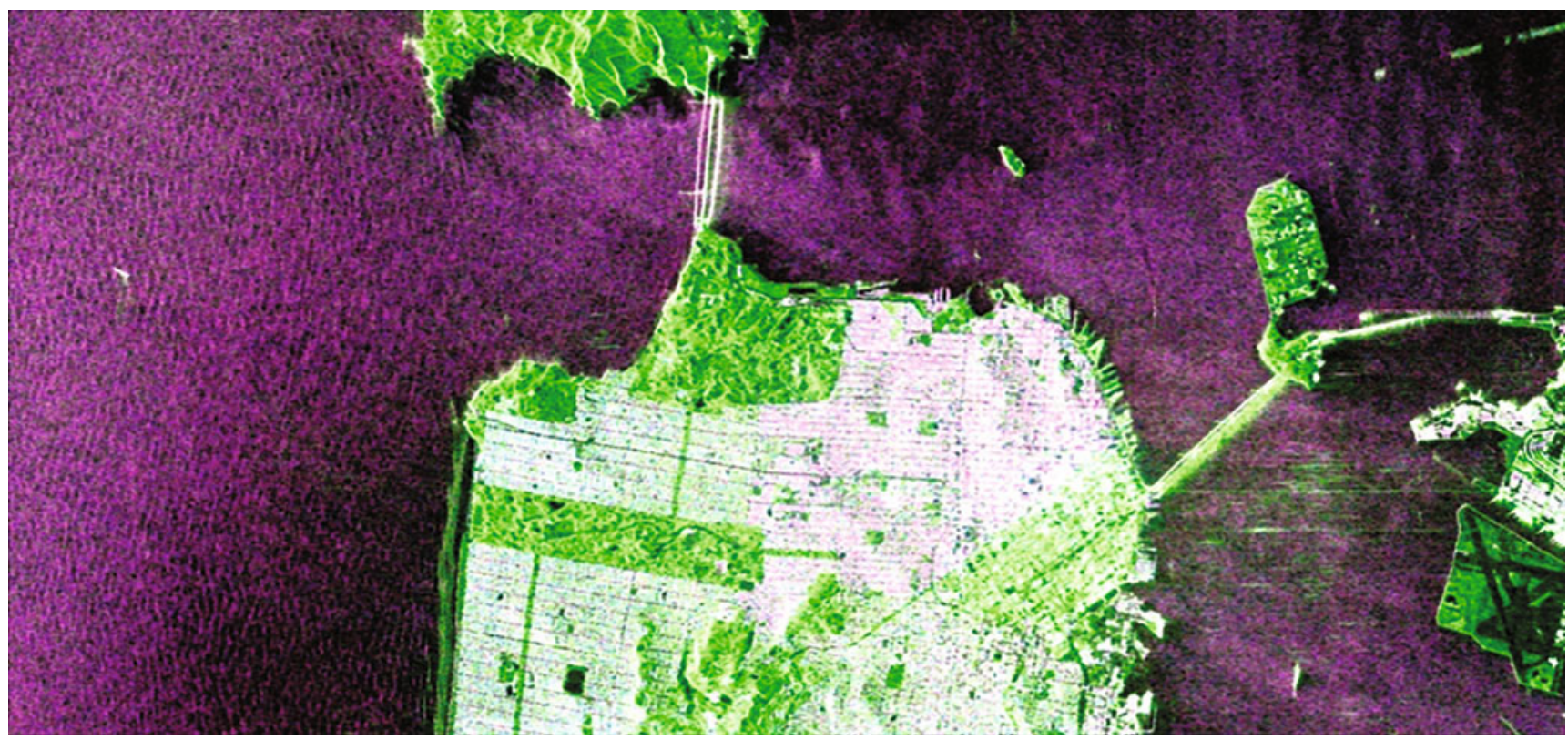

Fig. 1.8 RADARSAT-2 polarimetric RGB image over San Francisco (USA) where the colour code is $S_{h h}$ blue, $S_{v v}$ red and $S_{h v}$ green. Filtered image with the LLMMSE speckle filter

concept of binary partition tree (BPT) as a hierarchical structure to exploit the relations that may be established between similar pixels. In essence, the filtering alternative proposed in (Alonso Gonzalez et al. 2012) produces first a binary partition tree that establishes the relations between similar pixels on the basis of a distance that takes into account all the polarimetric information. In a second step, the binary partition tree is pruned to find the largest homogeneous regions of the image. This filtering alternative allows to filter large homogeneous areas while maintaining the spatial details of the data as observed in Fig. 1.9.

The objective of all the previous filtering techniques is to obtain the best estimate of the covariance or coherency matrices by means of increasing the number of averaged samples. Nevertheless, if the number of available homogeneous pixels is not large enough, the way to improve the estimation of the covariance and coherency matrices must be addressed by considering a better exploitation of the Wishart distribution. As shown in (Lopez Martínez and Fabregas 2003), the Wishart distribution allows defining the multiplicativeadditive speckle noise model for all the elements of the covariance or the coherency matrices. This model has been exploited for PolSAR data filtering in (Lopez Martínez and Fabregas 2008), where it is demonstrated that if the filtering process is adapted to the multiplicative or additive nature of speckle, depending on the correlation of a pair of SAR images, it may lead to an improved estimation of the different parametric parameters that characterize the covariance or coherency matrices.
Beyond all the PolSAR data filtering techniques presented in this section, there exist a wide variety of similar approaches in the related literature, where a comparison among some of them has been presented in (Foucher et al. 2012). Nevertheless, it may be concluded that reaching an optimal compromise of a joint preservation of the polarimetric and the spatial information, in the case of PolSAR data filtering, is still today a problem without an adequate solution. Consequently, the selection of a particular filtering alternative for PolSAR data must take into consideration the final application of the PolSAR data in order to determine the optimum filtering according to that application.

\subsection{Polarimetric Scattering Decomposition Theorems}

As shown in Sect. 1.1.2, the scattering matrix or the covariance and coherency matrices allow the characterization of a scatterer for a given frequency and a given imaging geometry. The information provided by these matrices, at a particular combination of transmitting and receiving polarization states, can be extended to any polarization state, thanks to the concept of polarization synthesis. Nevertheless, when facing real polarimetric SAR data, the interpretation of these matrices is not straightforward due to the complexity of the scattering process and the high variability of the scatterers. Polarimetric decomposition techniques appear as a solution to interpret the information provided by the 


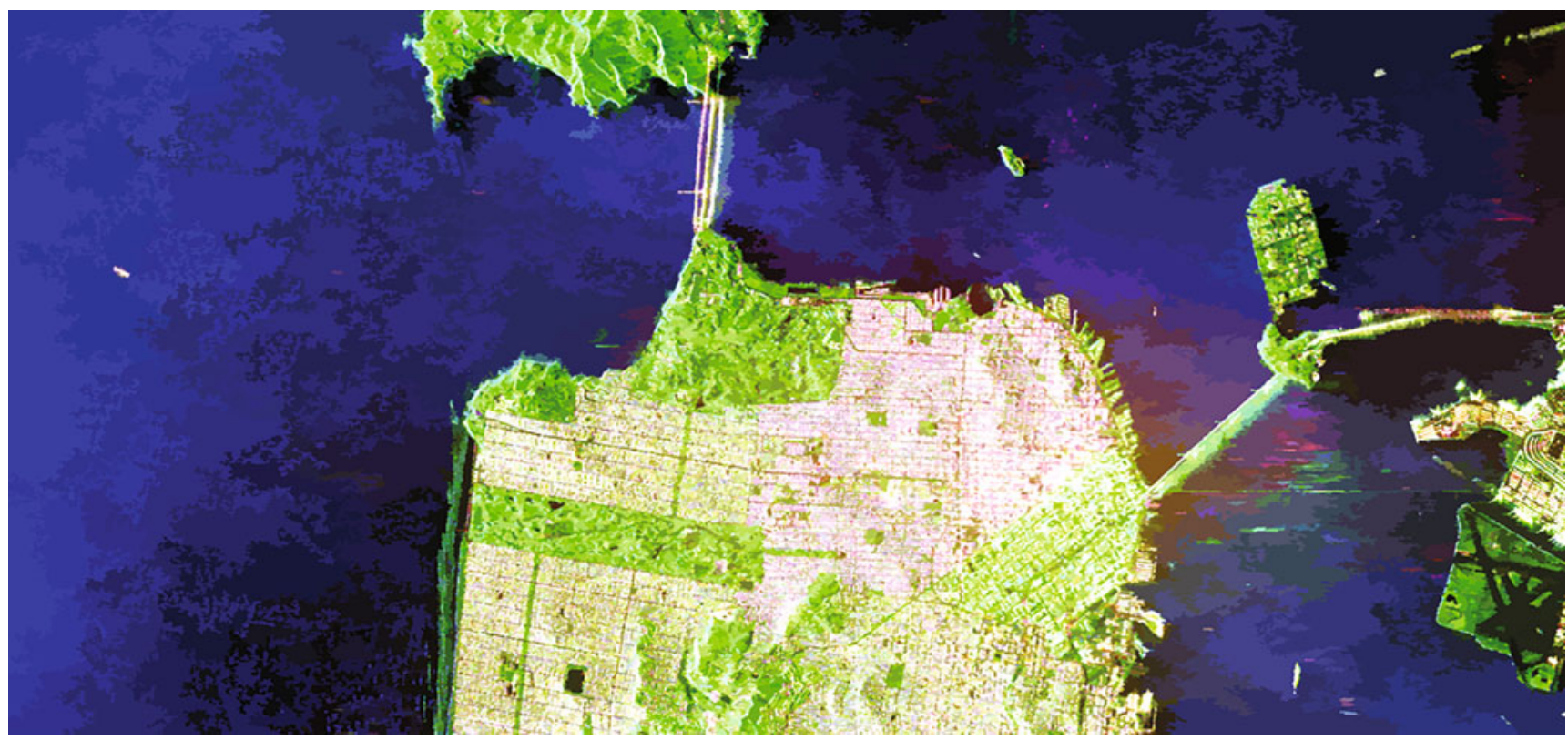

Fig. 1.9 RADARSAT-2 polarimetric RGB image over San Francisco (USA) where the colour code is $S_{h h}$ blue, $S_{v v}$ red and $S_{h v}$ green. Filtered image with the BPT speckle filter

scattering and the covariance and coherency matrices. These decomposition techniques must be divided into two main classes. The first one, referred to as coherent polarimetric decompositions, makes reference to those decomposition techniques applied to the scattering matrix. The validity of these decomposition techniques is restricted to point scatterers, that is, scatterers not affected by the speckle noise component. If applied to distributed scatterers, these decompositions would be random as they are not able to cope with the stochastic nature of the measurements. Distributed scatterers, on the contrary, can be analysed by the so-called incoherent polarimetric decompositions that base the analysis on the covariance or coherency matrices.

\subsubsection{Coherent Scattering Decomposition Techniques}

Section 1.1.2.1 introduced the $2 \times 2$ complex scattering matrix as a mathematical operator able to describe the scattering process that occurs when a wave reaches a given scatterer. As indicated, this matrix contains the necessary information to determine the far-field scattered wave by the scatterer as a function of the incident wave. Consequently, the scattering matrix characterizes the scatterer, for the employed imaging geometry and the working frequency. As indicated in Table 1.6, simple canonical scattering mechanisms may be recognized from the scattering matrix. Nevertheless, in real measurements, the scattering matrix usually presents a more complex structure that hinders the interpretation in physical terms. The objective behind coherent scattering decomposition techniques is to decompose the measured scattering matrix by the SAR system, i.e. $\mathbf{S}$, as a combination of the scattering matrices corresponding to simpler scatterers:

$$
\mathbf{S}=\sum_{i=1}^{k} c_{i} \mathbf{S}_{i}
$$

In (1.157), the symbol $\mathbf{S}_{i}$ corresponds to the response of every one of the simple or canonical scatterers, whereas the complex coefficients $c_{i}$ indicate the weight of $\mathbf{S}_{i}$ in the combination leading to the measured $\mathbf{S}$. As observed in (1.112), the term combination refers to the weighted addition of the $k$ scattering matrices. In order to simplify the understanding of (1.112), but also with the objective to make possible the decomposition itself, it is desirable that the matrices $\mathbf{S}_{i}$ present the property of independence among them to avoid a particular scattering behaviour to appear in more than one matrix $\mathbf{S}_{i}$. Often, the independence condition is substituted by the most restrictive property of orthogonality of the components $\mathbf{S}_{i}$. Orthogonality helps to eliminate possible ambiguities in the decomposition of the scattering matrix in those cases in which the elements $\mathbf{S}_{i}$ are not orthogonal.

The scattering matrix $\mathbf{S}$ characterizes the scattering process produced by a given scatterer and therefore the scatterer itself. This is possible only in those cases in which both the 
incident and the scattered waves are completely polarized waves. Consequently, coherent scattering decompositions can be only employed to study the so-called coherent scatterers. These scatterers are also known as point or pure targets.

In a real situation, the measured scattering matrix by the radar $\mathbf{S}$ corresponds to a complex coherent scatterer. Only in some occasions, this matrix will correspond to a simpler or canonical object, in which a good example is, for instance, the trihedrals employed to calibrate SAR imagery. Other simple scattering mechanisms may be observed in Table 1.6. Nevertheless, in a general situation, a direct analysis of the matrix $\mathbf{S}$, with the objective to infer the physical properties of the scatterer under study, is shown to be complex. Consequently, the physical properties of the target under study are extracted and interpreted through the analysis of the simpler responses $\mathbf{S}_{i}$ and the corresponding complex coefficients $c_{i}$ in (1.112).

The decomposition exposed in (1.112) is not unique in the sense that it is possible to find a number of infinite sets $\left\{\mathbf{S}_{i} ; i=1, \ldots, k\right\}$ in which the matrix $\mathbf{S}$ can be decomposed. Nevertheless, only some of the sets $\left\{\mathbf{S}_{i} ; i=1, \ldots, k\right\}$ are convenient in order to interpret the information contained in S. Two examples of these decomposition bases have been already shown in Sect. 1.1.2.2. Other examples of coherent scattering decompositions are the Krogager (Krogager 1990) or the Cameron decompositions (Cameron and Leung 1990).

\subsubsection{The Pauli Decomposition}

The most relevant coherent scattering decomposition is the Pauli decomposition that was already introduced in Sect. 1.1.2.2. The Pauli decomposition expresses the measured scattering matrix $\mathbf{S}$ in the so-called Pauli basis. If we considered the conventional orthogonal linear basis $\{\widehat{\mathbf{h}}, \widehat{\mathbf{v}}$,$\} , in a$ general case, the Pauli basis $\left\{\mathbf{S}_{a}, \mathbf{S}_{b}, \mathbf{S}_{c}, \mathbf{S}_{d}\right\}$ is given by the following four $2 \times 2$ matrices:

$$
\begin{aligned}
\mathbf{S}_{a} & =\frac{1}{\sqrt{2}}\left[\begin{array}{cc}
1 & 0 \\
0 & 1
\end{array}\right] \\
\mathbf{S}_{b} & =\frac{1}{\sqrt{2}}\left[\begin{array}{cc}
1 & 0 \\
0 & -1
\end{array}\right] \\
\mathbf{S}_{c} & =\frac{1}{\sqrt{2}}\left[\begin{array}{cc}
0 & 1 \\
1 & 0
\end{array}\right] \\
\mathbf{S}_{d} & =\frac{1}{\sqrt{2}}\left[\begin{array}{cc}
0 & -1 \\
1 & 0
\end{array}\right] .
\end{aligned}
$$

As mentioned, it has been always considered that $S_{h v}=S_{v h}$, since reciprocity applies in a monostatic system configuration under the BSA convention. In this situation, the Pauli basis can be reduced to a basis composed by the matrices (1.113), (1.114) and (1.115), that is, $\left\{\mathbf{S}_{a}, \mathbf{S}_{b}, \mathbf{S}_{c}\right\}$. Consequently, given a measured scattering matrix $\mathbf{S}$, this matrix can be expressed as follows:

$$
\mathbf{S}=\left[\begin{array}{ll}
S_{h h} & S_{h v} \\
S_{h v} & S_{v v}
\end{array}\right]=a \mathbf{S}_{a}+b \mathbf{S}_{b}+c \mathbf{S}_{c}
$$

where the complex coefficients that determine the contribution of every component of the basis can be obtained as

$$
a=\frac{S_{h h}+S_{v v}}{\sqrt{2}}, b=\frac{S_{h h}-S_{v v}}{\sqrt{2}}, c=\sqrt{2} S_{h v}
$$

From the previous equations, it can be shown that

$$
\operatorname{SPAN}(\mathbf{S})=|a|^{2}+|b|^{2}+|c|^{2} .
$$

The interpretation of the Pauli decomposition must be done according to the matrices $\left\{\mathbf{S}_{a}, \mathbf{S}_{b}, \mathbf{S}_{c}\right\}$ and their corresponding decomposition coefficients, i.e. $\{a, b, c\}$. In Sect. 1.1.2.1 it was seen that the matrices $\left\{\mathbf{S}_{a}, \mathbf{S}_{b}, \mathbf{S}_{c}\right\}$ correspond to the scattering behaviour of some canonical bodies.

The matrix $\mathbf{S}_{a}$ corresponds to the scattering matrix of a sphere, a plate or a trihedral; see Table 1.6. Generally, $\mathbf{S}_{a}$ is referred to as single- or odd-bounce scattering. Hence, the complex coefficient $a$ represents the contribution of $\mathbf{S}_{a}$ to the final measured scattering matrix. In particular, the intensity of this coefficient, i.e. $|a|^{2}$, determines the power scattered by scatterers characterized by single- or odd-bounce.

The second matrix $\mathbf{S}_{b}$ represents the scattering mechanism of a dihedral oriented at 0 degrees; see Table 1.6. In general, this component indicates a scattering mechanism characterized by double- or even-bounce, since the polarization of the returned wave is mirrored with respect to the one of the incident wave. Consequently, $b$ stands for the complex coefficient of this scattering mechanism, and $|b|^{2}$ represents the scattered power by this type of targets.

Finally, the third matrix $\mathbf{S}_{c}$ corresponds to the scattering mechanism of a diplane oriented at 45 degrees. As it can be observed in (1.115), and considering that this matrix is expressed in the linear orthogonal basis $\{\widehat{\mathbf{h}}, \widehat{\mathbf{v}}$,$\} , the$ scatterer returns a wave with a polarization orthogonal to the one of the incident wave. From a qualitative point of view, the scattering mechanism represented by $\mathbf{S}_{c}$ is referred to those scatterers which are able to return the orthogonal polarization, from which one of the best examples is the volume scattering produced by the forest canopy. The complex scattering that occurs in the forest canopy, characterized by multiple reflections, makes possible to return energy on the orthogonal polarization, with respect to the polarization 


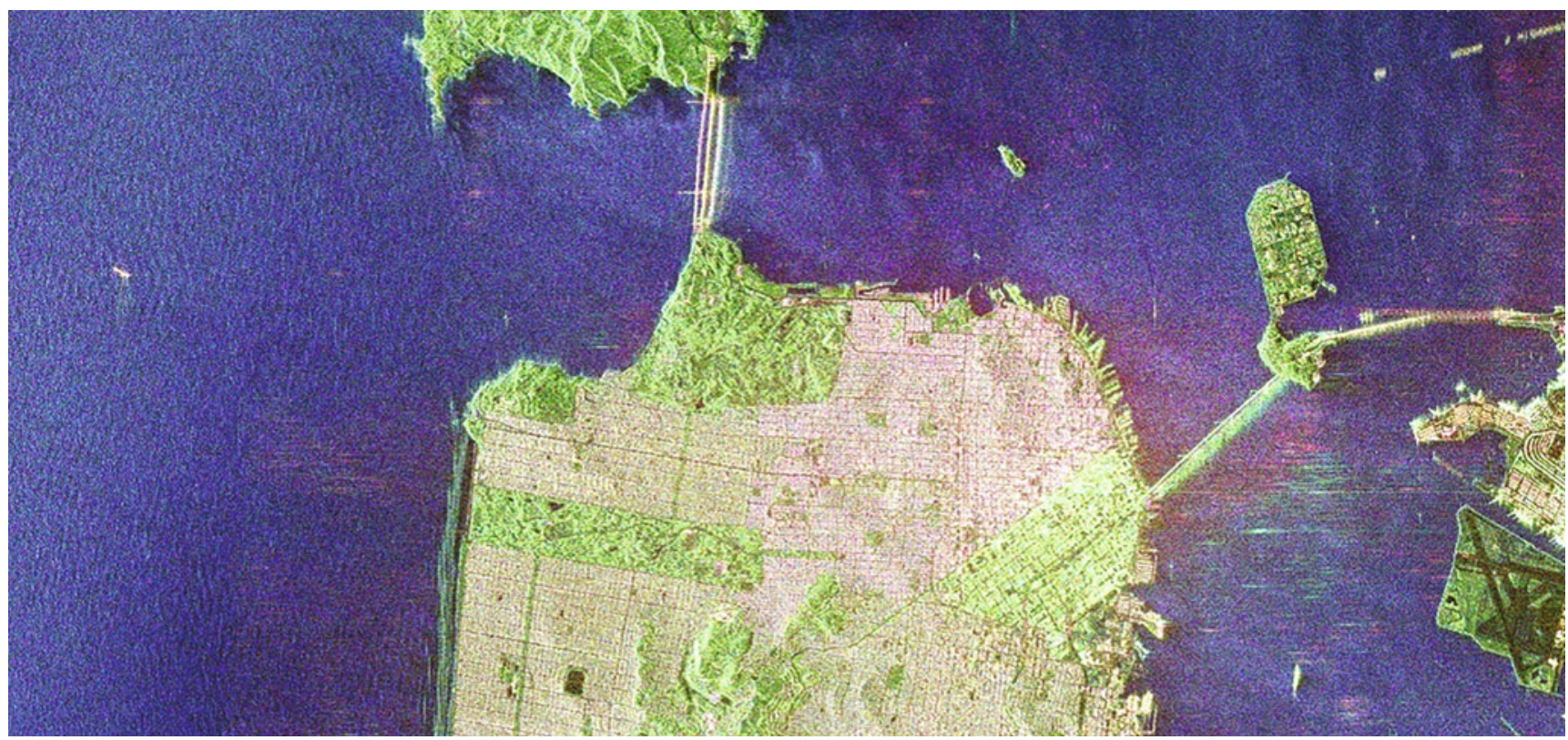

Fig. 1.10 RADARSAT-2 polarimetric RGB-Pauli image over San Francisco (USA) where the colour code is $\left|S_{h h}+S_{v v}\right|^{2}$ blue, $\left|S_{h h}-S_{v v}\right|^{2}$ red and $2\left|S_{h v}\right|^{2}$ green

of the incident wave. Consequently, this third scattering mechanism is usually referred to as volume scattering. The coefficient $c$ represents the contribution of $\mathbf{S}_{c}$ to $\mathbf{S}$, whereas I $\mathrm{cl}^{2}$ stands for the scattered power by this type of scatters.

The Pauli decomposition of the scattering matrix is often employed to represent the polarimetric information in a single SAR image. The polarimetric information of $\mathbf{S}$ could be represented with the combination of the intensities $\left|\mathbf{S}_{h h}\right|^{2}$, I $\left.\mathbf{S}_{v v}\right|^{2}$ and $2\left|\mathbf{S}_{h v}\right|^{2}$ in a single RGB image, i.e. each of the previous intensities coded as a colour channel. The main drawback of this approach is the physical interpretation of the resulting image in terms of $\left|\mathbf{S}_{h h}\right|^{2},\left|\mathbf{S}_{v v}\right|^{2}$ and $2\left|\mathbf{S}_{h v}\right|^{2}$. Consequently, a RGB image can be created with the intensities $|a|^{2},|b|^{2}$ and $|c|^{2}$, which, as indicated previously, correspond to clear physical scattering mechanisms. Thus, the resulting colour image can be employed to interpret the physical information from a qualitative point of view. The most employed codification corresponds to

$$
|a|^{2} \rightarrow \text { Blue, } \quad|b|^{2} \rightarrow \text { Red, } \quad|c|^{2} \rightarrow \text { Green. }
$$

Then, the resulting colour of the RGB image is interpreted in terms of scattering mechanism as given in (1.113)-(1.115); see Fig. 1.10.

\subsubsection{Incoherent Scattering Decompositions Techniques}

As explained in the previous sections, the scattering matrix $\mathbf{S}$ is only able to characterize the point or deterministic scatterers. In this case, the scattering process is completely determined by the five independent parameters the matrix S may present. On the contrary, this matrix cannot be employed to characterize, from a polarimetric point of view, the distributed scatterers, as the five independent parameters of the $\mathbf{S}$ matrix are insufficient to characterize the scattering process. As detailed in Sect. 1.2, distributed scatterers can be only characterized statistically due to the presence of speckle noise by means of higher-order descriptors. Since speckle noise must be reduced, only second-order polarimetric representations can be employed to analyse distributed scatterers. In the case of monostatic scattering under the BSA convention, these second-order descriptors are the $3 \times 3$ Hermitian covariance $\mathbf{C}$ or coherency $\mathbf{T}$ matrices.

The complexity of the scattering process makes extremely difficult the physical study of a given scatterer through the direct analysis of $\mathbf{C}$ or $\mathbf{T}$. Hence, the objective of the incoherent decompositions is to separate the $\mathbf{C}$ or $\mathbf{T}$ matrices as the combination of second-order descriptors corresponding to simpler or canonical objects, presenting an easier physical interpretation. These decomposition theorems can be expressed as

$$
\mathbf{C}=\sum_{i=1}^{k} p_{i} \mathbf{C}_{i}, \mathbf{T}=\sum_{i=1}^{k} q_{i} \mathbf{T}_{i}
$$

where the canonical responses are represented by $\mathbf{C}_{i}$ and $\mathbf{T}_{i}$ and $p_{i}$ and $q_{i}$ denote the coefficients of these components in $\mathbf{C}$ or $\mathbf{T}$, respectively. As in the case of the coherent decompositions, it is desirable that these components present some properties. First of all, it is desirable that the 
components $\mathbf{C}_{i}$ and $\mathbf{T}_{i}$ correspond to pure scatterers in order to simplify the physical study. Nevertheless, this condition is not absolutely necessary, and $\mathbf{C}_{i}$ and $\mathbf{T}_{i}$ may also represent distributed scatterers. In addition, the components $\mathbf{C}_{i}$ and $\mathbf{T}_{i}$ should be independent or, in a more restrictive way, orthogonal.

\subsubsection{Three-Component Freeman Decomposition}

The Freeman decomposition, also known as FreemanDurden decomposition (Freeman and Durden 1998), is the best exponent of the so-called model-based decompositions. In this type of decompositions, the canonical scattering mechanisms $\mathbf{C}_{i}$ and $\mathbf{T}_{i}$ in which the original matrices are decomposed into are fixed by the decomposition itself, i.e. the scattering mechanisms are imposed. In particular, the Freeman decomposition decomposes the original covariance or coherency matrices into the three following scattering mechanisms:

- Volume scattering, where a canopy scatterer is modelled as a set of randomly oriented dipoles

- Double-bounce scattering, modelled as a dihedral corner reflector

- Surface or single-bounce scattering, modelled as a firstorder Bragg surface scatterer

In the following, and without lack of generality, a formulation in terms of the covariance matrix $\mathbf{C}$ is considered.

The volume scattering component, mainly considered in forested areas, is modelled as the contribution from an ensemble of randomly oriented thin dipoles. If the set of randomly oriented dipoles are oriented according to a uniform phase distribution, the covariance matrix of the ensemble of thin dipoles corresponds to the following covariance matrix:

$$
\mathbf{C}_{v}=\frac{f_{v}}{8}\left[\begin{array}{lll}
3 & 0 & 1 \\
0 & 2 & 0 \\
3 & 0 & 3
\end{array}\right]
$$

where $f_{v}$ corresponds to the contribution of the volume scattering. The covariance matrix $\mathbf{C}_{v}$ presents a rank equal to 3 . Thus, the volume scattering cannot be characterized by a single scattering matrix of a pure scatterer. Finally, it is worth to indicate, as observed in (1.122), that the model assumed for forest scattering in the Freeman decomposition is fixed. In contrast, the other two scattering components of the decomposition, as it will be shown, admit a higher degree of flexibility.

The second component of the Freeman-Durden decomposition corresponds to double-bounce scattering. In this case, a generalized corner reflector is employed to model this scattering process. The diplane itself is not considered metallic. Hence, it is assumed that the vertical surface presents reflection coefficients $R_{t h}$ and $R_{t v}$ for the horizontal and the vertical polarizations, respectively, whereas the horizontal surface presents the coefficients $R_{g h}$ and $R_{g v}$ for the same polarizations. Additionally, two phase components for the horizontal and the vertical polarizations are considered, i.e. $e^{j 2 \gamma_{h}}$ and $e^{j 2 \gamma_{v}}$, respectively. The complex phase constants $\gamma_{h}$ and $\gamma_{h}$ account for any attenuation or phase change effect. Hence, the covariance matrix of the double-bounce scattering component, after normalization with respect to the $\mathbf{S}_{v v}$ component, can be written as follows:

$$
\mathbf{C}_{d}=f_{d}\left[\begin{array}{ccc}
|\alpha|^{2} & 0 & \alpha \\
0 & 0 & 0 \\
\alpha^{*} & 0 & 1
\end{array}\right]
$$

where

$$
\alpha=e^{j 2\left(\gamma_{h}-\gamma_{v}\right)} \frac{R_{g h} R_{t h}}{R_{g v} R_{t v}}
$$

and $f_{d}$ corresponds to the contribution of the double-bounce scattering to the $\left|\mathbf{S}_{v v}\right|^{2}$ component:

$$
f_{d}=\left|R_{g v} R_{t v}\right|^{2}
$$

As it can be observed, in this case the covariance matrix $\mathbf{C}_{d}$ presents a rank equal to 1 , and therefore it may be represented by a scattering matrix.

The third component of the Freeman-Durden decomposition consists of a first-order Bragg surface scattering modelling a surface rough scattering. Considering $R_{h}$ and $R_{v}$ the reflection coefficients for horizontally and vertically polarized waves, the covariance matrix corresponding to this scattering component is

$$
\mathbf{C}_{s}=f_{s}\left[\begin{array}{ccc}
|\beta|^{2} & 0 & \beta \\
0 & 0 & 0 \\
\beta^{*} & 0 & 1
\end{array}\right]
$$

where $f_{s}$ corresponds to the contribution of the double-bounce scattering to the $\left|\mathbf{S}_{v v}\right|^{2}$ component:

$$
f_{s}=\left|R_{v}\right|^{2}
$$

and

$$
\beta=\frac{R_{h}}{R_{v}}
$$


As in the case for the double-bounce scattering mechanism, $\mathbf{C}_{s}$ presents a rank equal to 1 .

Finally, it can be seen that the Freeman decomposition expresses the measured covariance matrix $\mathbf{C}$ as

$$
\mathbf{C}=\mathbf{C}_{v}+\mathbf{C}_{d}+\mathbf{C}_{s}
$$

that takes the expression

$$
\mathbf{C}=\left[\begin{array}{ccc}
\frac{3 f_{v}}{8}+f_{d}|\alpha|^{2}+f_{s}|\beta|^{2} & 0 & \frac{f_{v}}{8}+f_{d} \alpha+f_{s} \beta \\
0 & \frac{2 f_{v}}{8} & 0 \\
\frac{f_{v}}{8}+f_{d} \alpha^{*}+f_{s} \beta^{*} & 0 & \frac{3 f_{v}}{8}+f_{d}+f_{s}
\end{array}\right] .
$$

As one may deduce from (1.130), the Freeman decomposition presents five independent parameters $\left\{f_{v}, f_{d}, f_{s}, \alpha, \beta\right\}$ but only four equations. Consequently, some hypothesis must be considered in order to find the values of $\left\{f_{v}, f_{d}, f_{s}, \alpha, \beta\right\}$. Considering that the Span of the covariance matrix may be expressed as a function of the power scattered by each component of the decomposition $\left\{\mathbf{C}_{v}, \mathbf{C}_{d}, \mathbf{C}_{s}\right\}$, i.e.

$$
\begin{aligned}
\operatorname{SPAN}(\mathbf{C}) & =\left|S_{h h}\right|^{2}+\left|S_{v v}\right|^{2}+2\left|S_{h v}\right|^{2} \\
& =P_{v}+P_{d}+P_{s}
\end{aligned}
$$

the term $P_{v}$ corresponds to the contribution of the volume scattering of the final covariance matrix $\mathbf{C}$. Hence, the scattered power by this component may be written as

$$
P_{v}=f_{v} .
$$

The power scattered by the double-bounce component is expressed as

$$
P_{d}=f_{d}\left(1+|\alpha|^{2}\right) \text {, }
$$

whereas the power scattered by the surface component is

$$
P_{s}=f_{s}\left(1+|\beta|^{2}\right) .
$$

Consequently, the scattered power at each component $\left\{P_{v}, P_{d}, P_{s}\right\}$ may be employed to generate a RGB image, similarly as in the case of the Pauli decomposition, to present all the colour-coded polarimetric information in a unique image; see Fig. 1.11.

\subsubsection{Four-Component Yamaguchi Decomposition}

As it may be observed in (1.130), the three-component Freeman decomposition is based on the assumption that the analysed scatterer presents reflection symmetry, that is, the correlation of the co-polar channels, either $\mathbf{S}_{h h}$ or $\mathbf{S}_{v v}$, with the cross-polar one $\mathbf{S}_{h v}$ is zero, that is, $E\left\{S_{h h} S_{h v}^{*}\right\}=0$ and $E\left\{S_{h v} S_{v v}^{*}\right\}=0$. This type of symmetry in the scattering process appears normally in the case of natural distributed scatterers such as forests or grassland areas. Nevertheless, in the case of more complex scattering scenarios, for instance, man-made scatterers, this assumption is no longer true. In addition to the previous limitation, the Freeman decomposition, as detailed in the previous section, considers only one type of volume scattering, as reflected in (1.122), where the scattering at the co-polar channels are supposed equal, i.e. $E\{\mid$ $\left.\left.S_{h h}\right|^{2}\right\}=E\left\{\left|S_{v v}\right|^{2}\right\}$. The four-component Yamaguchi decomposition is proposed to overcome the previous two limitations of the Freeman decomposition (Yamaguchi et al. 2005).

If one considers the canonical scattering mechanisms presented in Table 1.6, it may be observed that only the rotated thin cylinder or the right- and left-handed helices are able to produce a covariance matrix such that $E\left\{S_{h h} S_{h v}^{*}\right\} \neq 0$ and $E\left\{S_{h v} S_{v v}^{*}\right\} \neq 0$ and therefore produce a covariance matrix without reflection symmetry. In the four-component Yamaguchi decomposition, the authors propose to take into account the absence of this type of symmetry by considering first the three scattering mechanisms considered by the Freeman decomposition, that is, volume, double-bounce and surface scattering, together with a fourth component composed by either the left- or the right-handed helix scattering (Krogager 1990). In particular, the helix scattering is characterized by generating a left-handed or a right-handed circular polarization for all incident linear polarizations, according to the scatterer helicity. The left-handed helix, whose scattering matrix is presented in Table 1.6, leads to the following covariance matrix:

$$
\mathbf{C}_{l h}=\frac{f_{c}}{4}\left[\begin{array}{ccc}
1 & -j \sqrt{2} & -1 \\
j \sqrt{2} & 2 & -j \sqrt{2} \\
-1 & j \sqrt{2} & 1
\end{array}\right]
$$

whereas the right-handed helix results in the following covariance matrix:

$$
\mathbf{C}_{r h}=\frac{f_{c}}{4}\left[\begin{array}{ccc}
1 & j \sqrt{2} & -1 \\
-j \sqrt{2} & 2 & j \sqrt{2} \\
-1 & -j \sqrt{2} & 1
\end{array}\right]
$$




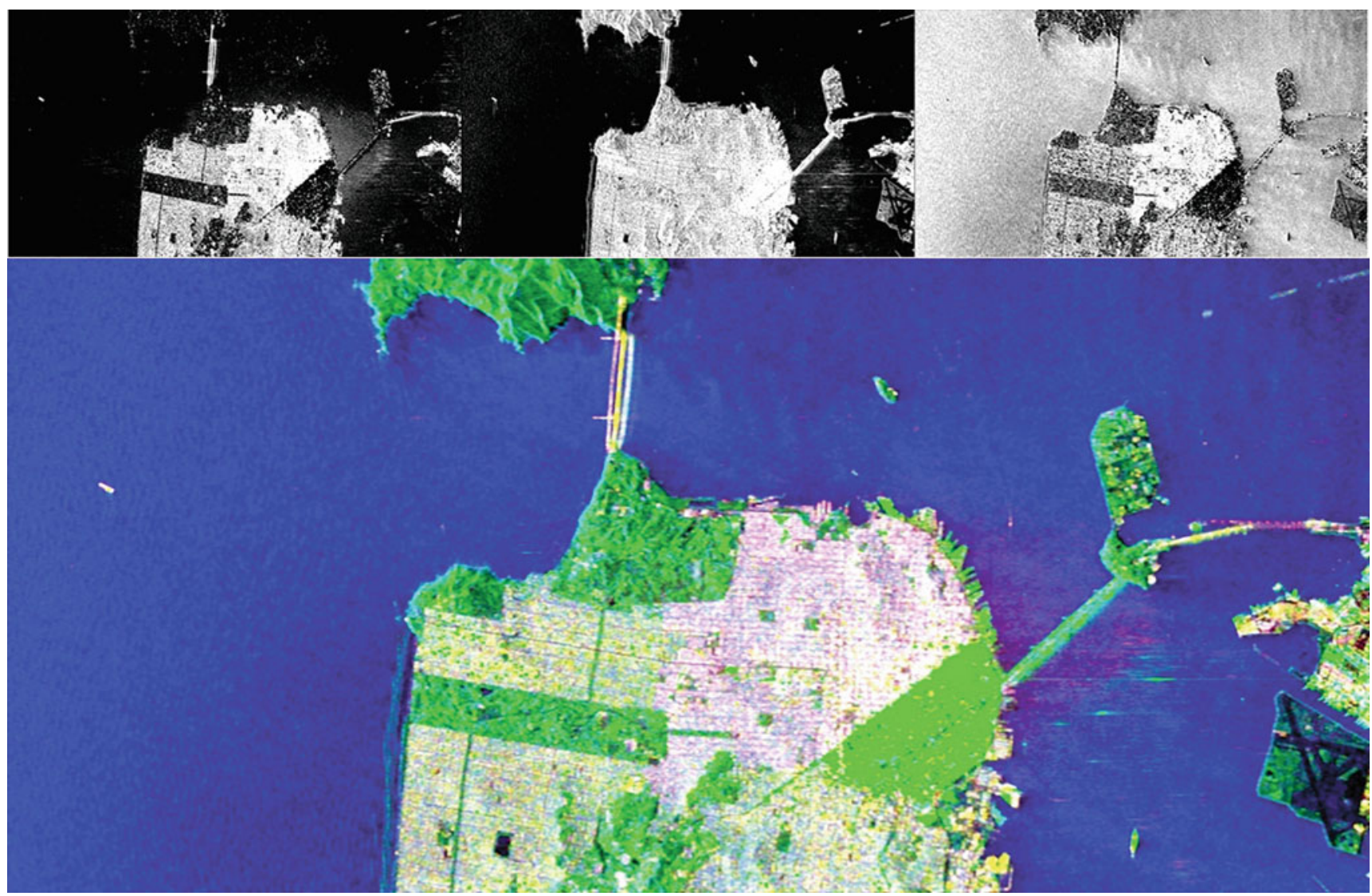

Fig. 1.11 Freeman decomposition of the RADARSAT-2 polarimetric RGB-Pauli image over San Francisco (USA). Top panel, from left to right: $P_{d}, P_{v}, P_{s}$. Bottom panel: RGB composition with $P_{d}$ red, $P_{v}$ green and $P_{s}$ blue

where $f_{c}$ accounts for the contribution of the helix component. As it may be observed in the previous two matrices, the inclusion of the helix component allows to consider a scattering mechanism without reflection symmetry. The selection of the left- or the right-handed helix will be determined by the sign of the imaginary part of $E\left\{S_{h h} S_{h v}^{*}\right\}$ or $E\left\{S_{h v} S_{v v}^{*}\right\}$.

In order to model the volume scattering, the Freeman decomposition considered a set of randomly oriented dipoles, oriented according to a uniform phase distribution. Nevertheless, when confronted to a real forest, the effect of the trunk and the branches, especially at high frequencies, may lead to a scattering from a cloud of oriented dipoles but with a non-uniform distribution. In this case, depending on the main orientation of these thin dipoles, the power associated with $E\left\{\left|S_{h h}\right|^{2}\right\}$ and $E\left\{\left|S_{v v}\right|^{2}\right\}$ may be different if the dipoles are preferably oriented horizontally or vertically, respectively. As it may be seen, the volume model considered by the Freeman decomposition (1.122) cannot take into account this effect. In order to account for this preference in the orientation, instead of considering a uniform distribution for the orientation of the thin dipoles, it is proposed to consider the following distribution:

$$
p(\theta)=\left\{\begin{array}{lll}
\frac{1}{2} \cos \theta & \text { for } & |\theta|<\pi / 2 \\
0 & \text { for } & |\theta|>\pi / 2
\end{array}\right.
$$

where $\theta$ is taken from the horizontal axis seen from the radar. When considering a cloud of randomly oriented, very thin horizontal dipoles, the volume scattering is represented by the following scattering matrix:

$$
\mathbf{C}_{v}=\frac{f_{v}}{15}\left[\begin{array}{lll}
8 & 0 & 2 \\
0 & 4 & 0 \\
2 & 0 & 3
\end{array}\right] .
$$

Otherwise, if the cloud of thin dipoles is considered to be composed of vertical dipoles, the covariance matrix representing the volume component is

$$
\mathbf{C}_{v}=\frac{f_{v}}{15}\left[\begin{array}{lll}
3 & 0 & 2 \\
0 & 4 & 0 \\
2 & 0 & 8
\end{array}\right] .
$$


In all the cases, $f_{v}$ corresponds to the contribution of the volume scattering.

Allowing the volume scattering to depend on the main orientation of the particles makes it necessary to introduce an additional step in the decomposition able to select the volume scattering most adapted to the data under observation. The four-component Yamaguchi decomposition proposes to select among (1.122), (1.138) and (1.139) according to the ratio $\chi=10 \log \left(E\left\{\left|S_{v v}\right|^{2}\right\} / E\left\{\left|S_{h h}\right|^{2}\right\}\right)$. Table 1.7 details the procedure to select the type of volume scattering proposed in (Yamaguchi et al. 2005).

Finally, the double-bounce and the surface scattering components of the four-component Yamaguchi decomposition are the same as the Freeman decomposition. Consequently, the Yamaguchi decomposition models the covariance matrix as

$$
\begin{aligned}
\mathbf{C}= & {\left[\begin{array}{ccc}
\frac{f_{c}}{4}+f_{d}|\alpha|^{2}+f_{s}|\beta|^{2} & \pm j \frac{\sqrt{2} f_{c}}{4} & -\frac{f_{c}}{4}+f_{d} \alpha+f_{s} \beta \\
0 j \frac{\sqrt{2} f_{c}}{4} & \frac{f_{c}}{2} & \pm j \frac{\sqrt{2} f_{c}}{4} \\
-\frac{f_{c}}{4}+f_{d} \alpha^{*}+f_{s} \beta^{*} & ⿴ 囗 ?) \frac{\sqrt{2} f_{c}}{4} & \frac{f_{c}}{4}+f_{d}+f_{s}
\end{array}\right] } \\
& +f_{v}\left[\begin{array}{lll}
a & 0 & d \\
0 & b & 0 \\
d & 0 & c
\end{array}\right]
\end{aligned}
$$

where the last matrix accounts for the volume scattering that has been selected according to Table 1.7. As one may deduce from (1.140), the four-component Yamaguchi decomposition presents six independent parameters $\left\{f_{v}, f_{d}, f_{s}, f_{c}, \alpha, \beta\right\}$. Considering that the Span of the covariance matrix may be expressed as a function of the power scattered by each component of the decomposition $\left\{\mathbf{C}_{v}, \mathbf{C}_{d}, \mathbf{C}_{s}, \mathbf{C}_{l h / r h}\right\}$, i.e.

$$
\begin{aligned}
\operatorname{SPAN}(\mathbf{C}) & =\left|S_{h h}\right|^{2}+\left|S_{v v}\right|^{2}+2\left|S_{h v}\right|^{2} \\
& =P_{v}+P_{d}+P_{s}+P_{c}
\end{aligned}
$$

the term $P_{v}$ corresponds to the contribution of the volume scattering of the final covariance matrix $\mathbf{C}$. Hence, the scattered power by this component may be written as

Table 1.7 Selection of the volume scattering covariance matrix

\begin{tabular}{l|l|l}
\hline$<-2 \mathrm{~dB}$ & $-2 \mathrm{~dB}<\chi<2 \mathrm{~dB}$ & $\chi>2 \mathrm{~dB}$ \\
\hline $\mathbf{C}_{v}=\frac{f_{v}}{15}\left[\begin{array}{lll}8 & 0 & 2 \\
0 & 4 & 0 \\
2 & 0 & 3\end{array}\right]$ & $\mathbf{C}_{v}=\frac{f_{v}}{8}\left[\begin{array}{lll}3 & 0 & 1 \\
0 & 2 & 0 \\
3 & 0 & 3\end{array}\right]$ & $\mathbf{C}_{v}=\frac{f_{v}}{15}\left[\begin{array}{lll}3 & 0 & 2 \\
0 & 4 & 0 \\
2 & 0 & 8\end{array}\right]$
\end{tabular}

$$
P_{v}=f_{v},
$$

the power scattered by the double-bounce component is expressed as

$$
P_{d}=f_{d}\left(1+|\alpha|^{2}\right),
$$

the power scattered by the surface component is

$$
P_{s}=f_{s}\left(1+|\beta|^{2}\right),
$$

whereas the power scattered by the helix component is

$$
P_{c}=f_{c} \text {. }
$$

Consequently, the scattered power at each component $\left\{P_{v}, P_{d}, P_{s}, P_{c}\right\}$ may be combined to generate a RGB image similarly as in the case of the Pauli decomposition, to present all the colour-coded polarimetric information in a unique image; see Fig. 1.12.

\subsubsection{Non-negative Eigenvalue Decomposition}

As indicated in the previous two sections, both the FreemanDurden and the Yamaguchi decomposition work under the hypothesis that the measured covariance matrix may be decomposed as the sum of a set of scattering mechanisms. Whereas the first decomposition assumes reflection symmetry for the scattering medium, this limitation is addressed by the second one by considering a fourth scattering component represented by either the left- or the right-handed helix scattering. All the scattering mechanisms in which the measured covariance matrix is decomposed into are represented by their corresponding covariance matrices. As shown in (Van Zyl et al. 2011), these matrices should correspond to physical scattering mechanisms, so all their eigenvalues must be larger than or equal to zero; in other words, the power received by any combination of transmitting and receiving polarizations should never be negative.

A close analysis of the Freeman-Durden decomposition shows that the contribution of the volume scattering component is directly estimated from the cross-polarized term, that is, the decomposition assumes that neither the double-bounce nor the surface scattering components contribute to it. This assumption is very strict as, for instance, the rotation of the polarization basis of the scattering matrix due to terrain slopes in the along-track dimension (Lee et al. 2002) or even rough surfaces may lead to significant cross-polarized power (Hajnsek et al. 2003). Consequently, if these effects are not taken into account, they may produce an overestimation of the volume component. Once this volume component is estimated from the data, it is extracted from the measured 


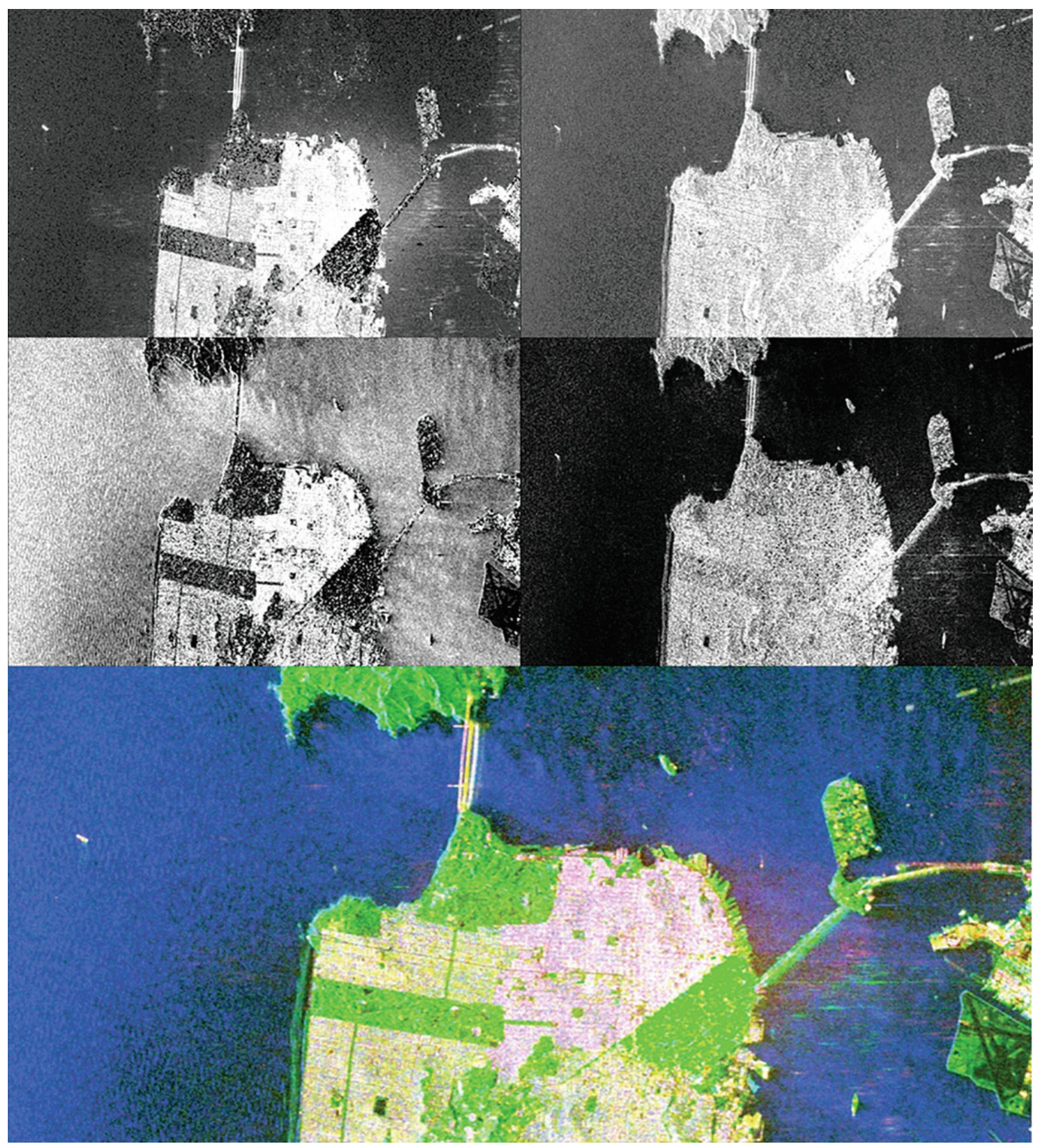

Fig. 1.12 Yamaguchi decomposition of the RADARSAT-2 polarimetric RGB-Pauli image over San Francisco (USA). From left to right, top panel: $P_{d}, P_{v}$; middle panel: $P_{s}, P_{c}$; bottom panel: RGB composition with $P_{d}$ red, $P_{v}$ green and $P_{s}$ blue

covariance matrix to estimate the double-bounce and the surface components as

$$
\mathbf{C}_{d+s}=\mathbf{C}-\mathbf{C}_{v}
$$

Consequently, if the volume component is not properly estimated, the previous subtraction may lead to a result in which the covariance matrix representing the double-bounce and the surface components $\mathbf{C}_{d}+s$ may present negative eigenvalues so it does not represent a physically possible 
scattering mechanism. The Yamaguchi decomposition also presents this drawback as the double-bounce and the surface like scattering components are estimated after the subtraction of the volume scattering component.

In order to correct the presence of negative eigenvalues when considering a decomposition based on (1.146), van Zyl et al. (Van Zyl et al. 2011) proposed the non-negative eigenvalue decomposition (NNED). The Freeman-Durden and the Yamaguchi decompositions assume that the measured covariance matrix results from the addition of a set of scattering mechanisms. Nevertheless, the NNED approach proposed to decompose the measured covariance matrix as

$$
\mathbf{C}=a \mathbf{C}_{\text {model }}+\mathbf{C}_{\text {remainder }}
$$

The matrix $\mathbf{C}_{\text {model }}$ represents the covariance matrix predicted by a theoretical model, for instance, the volume scattering component. The parameter $a$ is introduced in (1.147) to assure that all the matrices in (1.147) represent physically realizable scattering mechanism. Finally, the second matrix $\mathbf{C}_{\text {remainder will contain whatever is in the measured }}$ matrix $\mathbf{C}$ that is not consistent with the model matrix $\mathbf{C}_{\text {model }}$.

To find the value of $a$, (1.147) may be written as

$$
\mathbf{C}_{\text {remainder }}=\mathbf{C}-a \mathbf{C}_{\text {model }} \text {. }
$$

Consequently, the value of $a$ must assure that the eigenvalues of $\mathbf{C}_{\text {remainder }}$ must be positive. In the case of a scattering media with reflection symmetry, (1.147) may be written as

$$
\mathbf{C}_{\text {remainder }}=\left[\begin{array}{ccc}
\xi & 0 & \rho \\
0 & \eta & 0 \\
\rho^{*} & 0 & \zeta
\end{array}\right]-a\left[\begin{array}{ccc}
\xi_{a} & 0 & \rho_{a} \\
0 & \eta_{a} & 0 \\
\rho_{a}^{*} & 0 & \zeta_{a}
\end{array}\right] .
$$

Therefore, the maximum value of $a$ that assures that the eigenvalues of $\mathbf{C}_{\text {remainder }}$ are positive corresponds to

$$
a_{\max }=\min \left\{\eta / \eta_{a}, \frac{1}{2\left(\xi_{a} \zeta_{a}-\left|\rho_{a}\right|^{2}\right)}\left\{Z-\sqrt{Z^{2}-4\left(\xi_{a} \zeta_{a}-\left|\rho_{a}\right|^{2}\right) \xi \zeta-|\rho|^{2}}\right\}\right\},
$$

where $Z=\left(\xi \zeta_{a}+\zeta \xi_{a}\right)-\rho \rho_{a}^{*}-\rho^{*} \rho_{a}$. For the case of scattering media not presenting reflection symmetry, the process to derive the maximum value of $a$ is similar, but results in more complex expressions.

The volume scattering model employed for the canopy scattering is based on a cosine-squared distribution raised to the $n$th power for the vegetation orientation (Arii et al. 2011). Considering that the basic scatterer in the canopy is a dipole, it was shown that the covariance matrix can be written as

$$
\mathbf{C}_{v}\left(\theta_{0}, \sigma\right)=\mathbf{C}_{\alpha}+p(\sigma) \mathbf{C}_{\beta}+q(\sigma) \mathbf{C}_{\gamma}
$$

where

$$
\begin{gathered}
\mathbf{C}_{\alpha}=\frac{1}{8}\left[\begin{array}{lll}
3 & 0 & 1 \\
0 & 2 & 0 \\
3 & 0 & 3
\end{array}\right], \\
\mathbf{C}_{\beta}=\frac{1}{8} \\
\times\left[\begin{array}{ccc}
-2 \cos 2 \theta_{0} & \sqrt{2} \cos 2 \theta_{0} & 0 \\
\sqrt{2} \cos 2 \theta_{0} & 0 & \sqrt{2} \cos 2 \theta_{0} \\
0 & \sqrt{2} \cos 2 \theta_{0} & 2 \cos 2 \theta_{0}
\end{array}\right],
\end{gathered}
$$

$$
\begin{aligned}
\mathbf{C}_{\gamma}= & \frac{1}{8} \\
& \times\left[\begin{array}{ccc}
\cos 4 \theta_{0} & -\sqrt{2} \cos 4 \theta_{0} & -\cos 4 \theta_{0} \\
-\sqrt{2} \cos 4 \theta_{0} & -2 \cos 4 \theta_{0} & \sqrt{2} \cos 4 \theta_{0} \\
-\cos 4 \theta_{0} & \sqrt{2} \cos 4 \theta_{0} & \cos 4 \theta_{0}
\end{array}\right],
\end{aligned}
$$

and

$$
\begin{aligned}
& p(\sigma)=2.0806 \sigma^{6}-6.3350 \sigma^{5} \\
&+ 6.3864 \sigma^{4}-0.4431 \sigma^{3}-3.9638 \sigma^{2}-0.0008 \sigma \\
&+ 2.000 \\
& q(\sigma)= 9.0166 \sigma^{6}-18.7790 \sigma^{5}+4.9590 \sigma^{4} \\
&+14.5629 \sigma^{3}-10.8034 \sigma^{2}-0.1902 \sigma \\
&+1.000 .
\end{aligned}
$$

In the previous equations, the parameter $\theta_{0}$ represents the mean orientation angle of the thin dipoles, whereas $\sigma$ accounts for the randomness of the cloud of dipoles.

On the basis of the previous procedure to avoid the extraction of non-physical covariance matrices, Arii et al. (Arii et al. 2011) proposed an adaptive NNED decomposition 


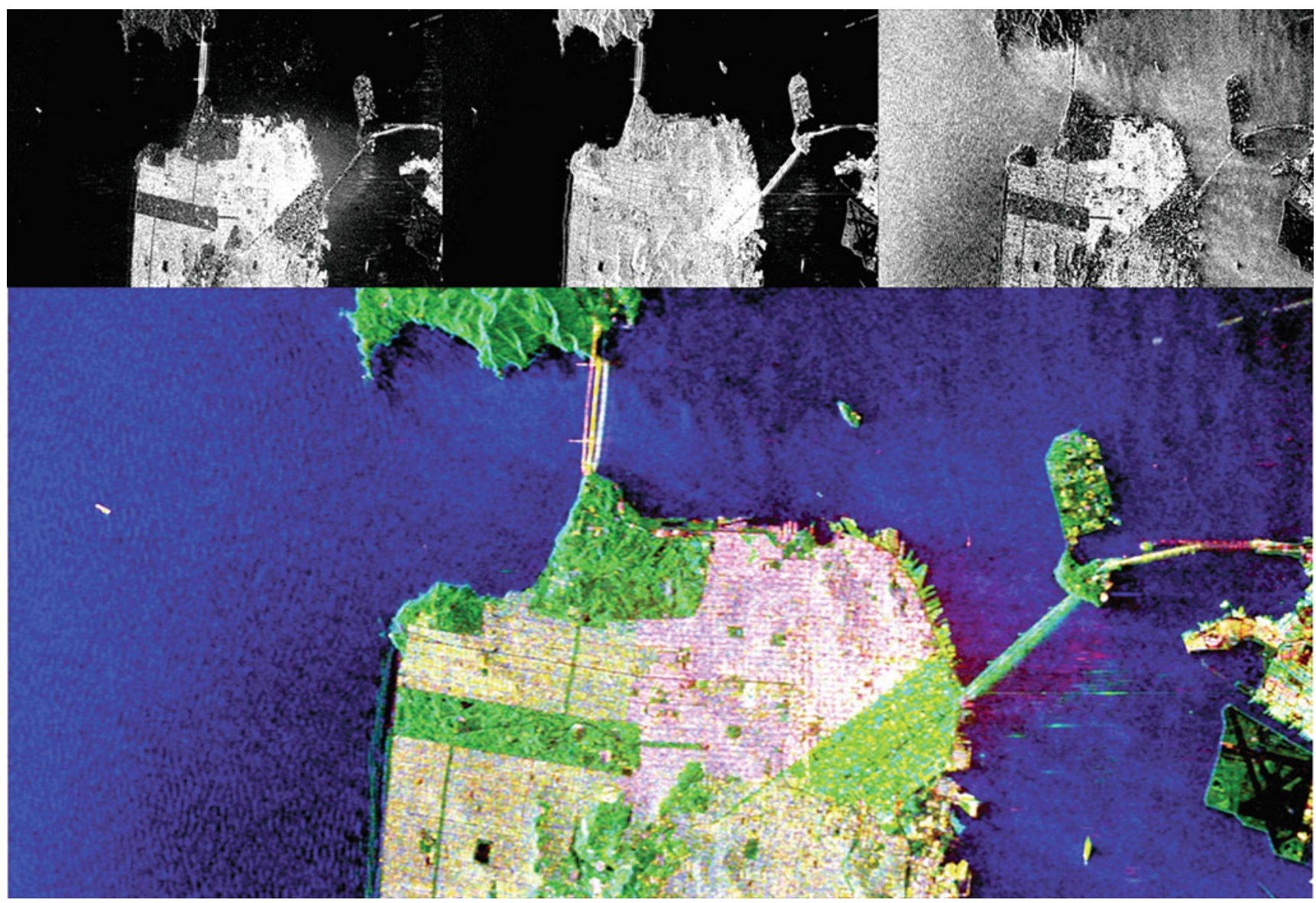

Fig. 1.13 Van Zyl decomposition of the RADARSAT-2 polarimetric RGB-Pauli image over San Francisco (USA). Top panel, from left to right: $f_{d}$, $f_{v}, f_{s}$. Bottom panel: RGB composition with $f_{d}$ red, $f_{v}$ green and $f_{s}$ blue

theorem, where also the previous extended model for volume scattering is considered. According to the NNED decomposition, a covariance matrix for the volume scattering is first subtracted from the measured covariance matrix as follows:

$$
\mathbf{C}_{\text {remainder }}=\mathbf{C}-f_{v} \mathbf{C}_{v}\left(\theta_{0}, \sigma\right) \text {. }
$$

As indicated previously, $f_{v}$ can be obtained analytically only under the assumption of reflection symmetry. In those cases in which the previous hypothesis does not apply, the maximum value of $f_{v}$ is obtained numerically by calculating the eigenvalues $\mathbf{C}_{\text {remainder }}$ at specific randomness $\sigma$ and mean orientation angle $\theta_{0}$ by varying $f_{v}$, and then, the maximum $f_{v}$ in which all three eigenvalues of $\mathbf{C}_{\text {remainder }}$ are nonnegative is selected. Once the volume component is extracted from the measured covariance matrix as specified in (1.157), the remainder matrix can be written as

$$
\mathbf{C}-f_{v} \mathbf{C}_{v}\left(\theta_{0}, \sigma\right)=f_{d} \mathbf{C}_{d}+f_{s} \mathbf{C}_{s}+\mathbf{C}_{\text {remainder }}^{\prime}
$$

where in this case $\mathbf{C}_{d}$ and $\mathbf{C}_{s}$ correspond to the doublebounce and surface scattering mechanisms already employed in the three-component Freeman-Durden decomposition. The parameters $f_{d}, f_{s}$ and $\mathbf{C}_{\text {remainder }}^{\prime}$ are obtained through an eigenvalue decomposition. This procedure shows how to find the parameters in the decomposition for a specific pair of randomness $\sigma$ and mean orientation angle $\theta_{0}$. To find the best fit decomposition, the power in the remainder matrix for all pairs of randomness and mean orientation angles is evaluated and then the set of parameters that minimize the power associated with $\mathbf{C}_{\text {remainder }}^{\prime}$ should be found.

Finally, the scattered power at each component $\left\{f_{v}, f_{d}, f_{s}\right\}$ may be combined to generate a RGB image similarly as in the case of the Pauli decomposition, to present all the colourcoded polarimetric information in a unique image; see Fig. 1.13.

\subsubsection{Eigenvector-Eigenvalue-Based Decomposition}

The previous incoherent decompositions were constructed on the assumption that the scattering of a given pixel was due to the combination of some predefined scattering mechanisms, hence assuming different properties of the scattering processes. These assumptions make these decompositions to be 
easy to interpret as the different scattering components present a clear physical interpretation. Nevertheless, as these decompositions consider only the predefined mechanisms, they are not able to identify additional scattering mechanisms when present. A way to circumvent this drawback is to decompose the covariance or coherency matrices based on their mathematical properties. Hence, contrary to the previous decompositions, the scattering mechanisms in which the original matrices are decomposed are not established a priori but given by the decomposition itself. The drawback of this approach is that the scattering mechanism found by the decomposition needs from a physical interpretation process.

The eigenvector-eigenvalue scattering decomposition, also known as Cloude-Pottier decomposition, is based on the eigendecomposition of the covariance $\mathbf{C}$ or coherency T matrices (Cloude and Pottier 1996). According to the eigendecomposition theorem, the $3 \times 3$ Hermitian matrix C may be decomposed as follows:

$$
\mathbf{T}=\mathbf{U} \mathbf{\Sigma} \mathbf{U}^{-1}
$$

The $3 \times 3$, real, diagonal matrix $\mathbf{\Sigma}$ contains the eigenvalues of $\mathbf{C}$ :

$$
\boldsymbol{\Sigma}=\left[\begin{array}{ccc}
\lambda_{1} & 0 & 0 \\
0 & \lambda_{2} & 0 \\
0 & 0 & \lambda_{3}
\end{array}\right]
$$

such that $\infty>\lambda_{1} \geq \lambda_{2} \geq \lambda_{3}>0$. The $3 \times 3$ unitary matrix $\mathbf{U}$ contains the eigenvectors $\mathbf{u}_{i}$ for $i=1,2,3$ of $\mathbf{C}$ :

$$
\mathbf{U}=\left[\begin{array}{lll}
\mathbf{u}_{1} & \mathbf{u}_{2} & \mathbf{u}_{3}
\end{array}\right]
$$

The eigenvectors $\mathbf{u}_{i}$ for $i=1,2,3$ of $\mathbf{C}$ can be reformulated, or parameterized, as

$$
\mathbf{u}_{i}=\left[\begin{array}{lll}
\cos \alpha_{i} & \sin \alpha_{i} \cos \beta_{i} e^{j \delta_{i}} & \sin \alpha_{i} \cos \beta_{i} e^{j \gamma_{i}}
\end{array}\right]^{T} .
$$

Considering (1.159), (1.160) and (1.161), the coherency matrix $\mathbf{C}$ may be written as

$$
\mathbf{C}=\sum_{i=1}^{3} \lambda_{i} \mathbf{u}_{i} \mathbf{u}_{i}^{* T} .
$$

As (1.163) shows, the rank-3 matrix $\mathbf{C}$ can be decomposed as the combination of three rank 1 coherency matrices which can be related to the pure scattering mechanisms given in (1.162). Consequently, the eigendecomposition is not able to produce scattering mechanisms in which the original matrix is decomposed into with a rank larger than 1 .

The eigenvalues (1.160) and the eigenvectors (1.161) of the decomposition are considered as the primary parameters of the eigendecomposition of $\mathbf{C}$. In order to simplify the analysis of the physical information provided by this eigendecomposition, three secondary parameters are defined as a function of the eigenvalues and the eigenvectors of $\mathbf{C}$ :

- Entropy:

$$
H=-\sum_{i=1}^{3} p_{i} \log _{3}\left(p_{i}\right) \quad p_{i}=\frac{\lambda_{i}}{\sum_{j=1}^{3} \lambda_{j}}
$$

where $p_{i}$ are known as the probabilities of the eigenvalue $\lambda_{i}$, respectively. These probabilities represent the relative importance of this eigenvalue with respect to the total scattered power, as

$$
\operatorname{SPAN}(\mathbf{S})=\sum_{i=1}^{3} \lambda_{i}
$$

- Anisotropy:

$$
A=\frac{\lambda_{2}-\lambda_{3}}{\lambda_{2}+\lambda_{3}}
$$

representing the relative importance of the second eigenvalue with respect to the third one:

- Mean alpha angle:

$$
\bar{\alpha}=\sum_{i=1}^{3} p_{i} \alpha_{i} .
$$

As it shall be shown, this parameter allows the physical interpretation of the scattering mechanism found by the eigendecomposition.

The eigendecomposition of the coherency matrix is usually referred to as the $H / A / \bar{\alpha}$ decomposition. An example of $H / A / \bar{\alpha}$ decomposition is shown in Fig. 1.14. The 
interpretation of the information provided by the eigendecomposition of the coherency matrix must be performed in terms of the eigenvalues and eigenvectors of the decomposition or in terms of $H / A / \bar{\alpha}$. Nevertheless, both interpretations have to be considered as complementary.

The interpretation of the scattering mechanisms given by the eigenvectors of the decomposition, $\mathbf{u}_{i}$ for $i=1,2,3$, is performed by means of a mean dominant mechanism which can be defined as follows:

$$
\mathbf{u}_{0}=\left[\begin{array}{lll}
\cos \bar{\alpha} & \sin \bar{\alpha} \cos \bar{\beta} e^{j \bar{\delta}} \quad \sin \bar{\alpha} \cos \bar{\beta} e^{\bar{\gamma}}
\end{array}\right]^{T},
$$

where the remaining average angles $\bar{\beta}, \bar{\delta}, \bar{\gamma}$ are defined in the same way as $\bar{\alpha}$.

The study of the mechanism given in (1.168) is mainly performed through the interpretation of the mean alpha angle $\bar{\alpha}$, since its value can be easily related to the physics behind the scattering process. The next list details the interpretation of $\bar{\alpha}$ :

- $\bar{\alpha} \rightarrow 0$ : the scattering corresponds to single-bounce scattering produced by a rough surface.

- $\bar{\alpha} \rightarrow \pi / 4$ : the scattering mechanism corresponds to volume scattering.

- $\bar{\alpha} \rightarrow \pi / 2$ : the scattering mechanism is due to doublebounce scattering.

The second part in the interpretation of the eigendecomposition is performed by studying the value of the eigenvalues of the decomposition. A given eigenvalue corresponds to the associated scattered power to the corresponding eigenvector. Consequently, the value of the eigenvalue gives the importance of the corresponding eigenvector or scattering mechanism. The ensemble of scattering mechanisms is studied by means of the entropy $H$ and the anisotropy $A$. The Entropy $H$ determines the degree of randomness of the scattering process, which can be also interpreted as the degree of statistical disorder. In this way

- $H \rightarrow 0$ :

$$
\lambda_{1}=S P A N, \lambda_{2}=0, \lambda_{3}=0
$$

As observed, in this case, the covariance matrix $\mathbf{C}$ presents rank 1, and the scattering process corresponds to a pure scatterer.

- $H \rightarrow 1$ :

$$
\lambda_{1}=\lambda_{2}=\lambda_{3}=\frac{S P A N}{3} .
$$

In this situation, the covariance matrix $\mathbf{C}$ presents rank 3 , that is, the scattering process is due to the combination of three pure targets. Consequently, $\mathbf{C}$ corresponds to the response of a distributed target. For instance, volume scattering for a forest canopy presents an entropy value very close to 1 .

- $0<H<1$ : In this case, the final scattering mechanism given by $\mathbf{C}$ results from the combination of the three pure targets given by $\mathbf{u}_{i}$ for $i=1,2,3$, but weighted by the corresponding eigenvalue.

The anisotropy $A,(1.166)$, is a parameter complementary to the entropy. The anisotropy measures the relative importance of the second and the third eigenvalues of the eigendecomposition. From a practical point of view, the anisotropy can be employed as a source of discrimination only when $H>0.7$. The reason is that for lower entropies, the second and third eigenvalues are highly affected by the SAR system noise.

In relation with the previous parameters, the Shannon entropy ( $S E$ ) was introduced in (Morio et al. 2007):

$$
S E=\log \left(\pi^{3} e^{3}|\mathbf{T}|\right)=S E_{I}+S E_{P}
$$

as the sum of two terms. The term $S E_{I}$ is the intensity contribution that depends on the total power

$$
S E_{I}=3 \log \left(\frac{\pi \cdot e \cdot I}{3}\right)=3 \log \left(\frac{\pi \cdot e \cdot \operatorname{trace}(\mathbf{T})}{3}\right)
$$

whereas $S E_{P}$ is the polarimetric contribution

$$
S E_{P}=\log \left(27 \frac{|\mathbf{T}|}{[\operatorname{trace}(\mathbf{T})]^{3}}\right)
$$

As indicated in Sect. 1.1.2.5, for some particular configurations, a polarimetric SAR system may not measure the complete polarimetric information. In this simpler configuration of dual polarization, the radar transmits only a single polarization and receives, either coherently or incoherently, two orthogonal components of the scattered signal. In this configuration, the covariance $\mathbf{C}$ and coherency $\mathbf{T}$ matrices are $2 \times 2$ Hermitian matrices. As it has been demonstrated (Cloude 2007a), these reduced matrices can be decomposed also considering their eigendecompositions. The sole particularity is that in this situation the matrices present only two eigenvalues.

\subsubsection{The Touzi Target Scattering Decompositions}

The Touzi decomposition (Touzi 2007) was introduced as an extension of the Kennaugh-Huynen coherent target scattering 


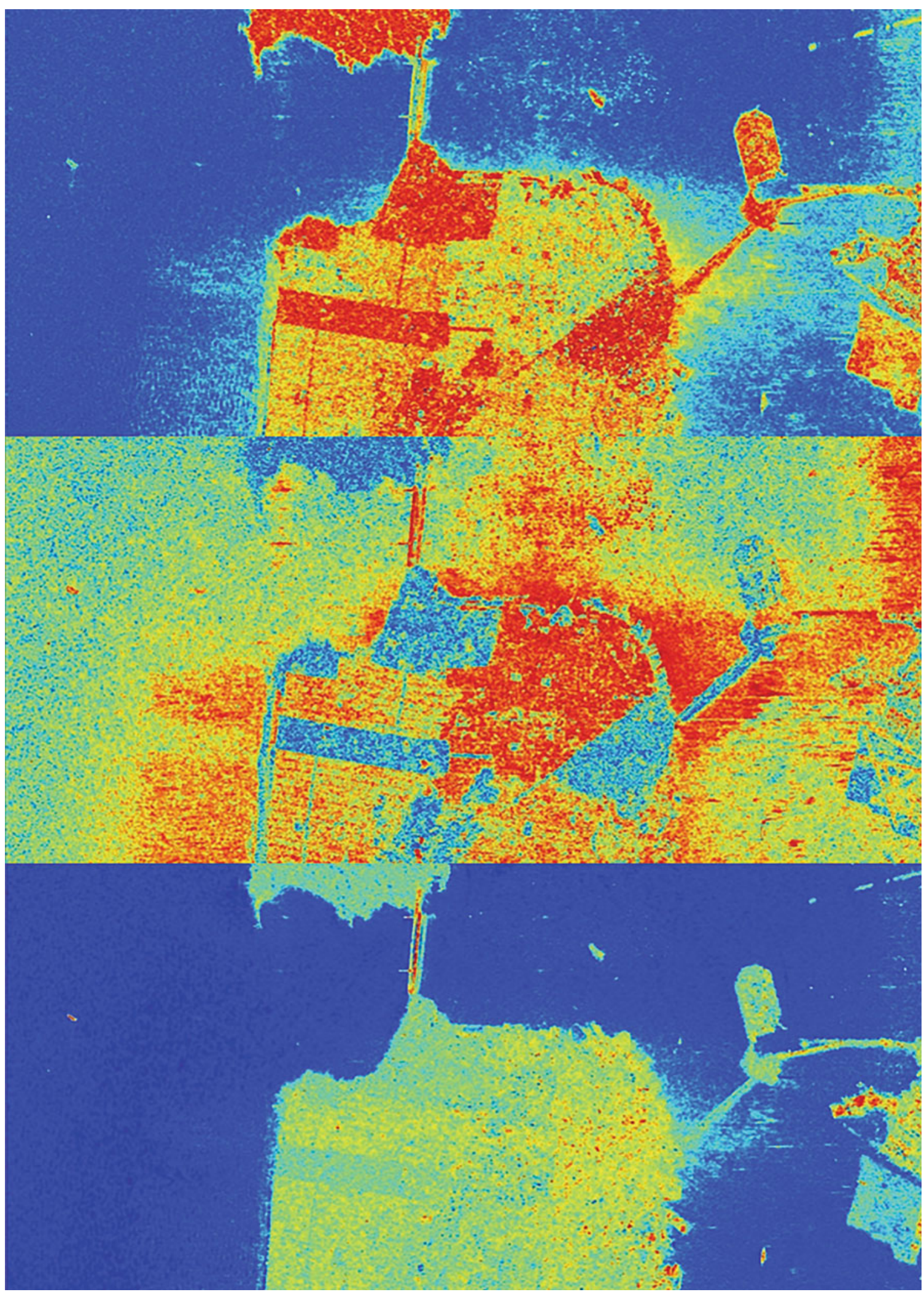

Fig. 1.14 $H / A / \bar{\alpha}$ decomposition of the RADARSAT-2 polarimetric RGB-Pauli image over San Francisco (USA). From top to bottom: entropy, anisotropy, mean alpha angle 
decomposition (Huynen 1970; Kennaugh 1951), for the characterization of both coherent and partially coherent target scattering. To characterize partially coherent scattering, Huynen introduced a target decomposition theorem in which he decomposed an average Mueller matrix into the sum of a Mueller matrix for a single scatter presented in terms of the Kennaugh-Huynen decomposition parameters and a noise or the N-target Mueller matrix (Huynen 1970). In 1988, Cloude (Cloude 1988) showed that the Huynen N-target decomposition was not polarization independent and introduced the eigenvector decomposition for a unique and roll-invariant incoherent decomposition. Following that, both Huynen's (N-target) incoherent decomposition and Huynen's fork decomposition were abandoned. Recently, the Kennaugh-Huynen decomposition has been reconsidered and integrated in Cloude's coherency eigenvector decomposition (Cloude 1988) for characterization of coherent and partially coherent scattering in terms of unique and polarization basis independent parameters (Touzi 2007).

The Kennaugh-Huynen decomposition, also named the Huynen fork, used to be the most popular method for decomposition of coherent target scattering (Touzi et al. 2004; Boerner et al. 1998). Huynen's fork was abandoned because of the nonuniqueness of certain fork parameters, and in particular the skip angle (scattering type phase), due to nonuniqueness of the con-eigenvalue phases (Luneburg 2002). To solve these ambiguities, the Kennaugh-Huynen scattering matrix con-diagonalization was projected into the Pauli basis (Touzi 2007), and a new target scattering vector model, the TSVM, was introduced in terms of target parameters that are not affected by the con-eigenvalue phase ambiguities (Touzi 2007). A complex entity, named the symmetric scattering type, was introduced for an unambiguous description of target scattering type. The polar coordinates of the symmetric scattering type, $\alpha_{s}$ and $\phi_{\alpha s}$, are expressed as a function of target scattering matrix polarization basis independent elements by (Touzi 2007)

$$
\tan \alpha_{s} \cdot e^{j \phi_{\alpha s}}=\frac{\mu_{1}-\mu_{2}}{\mu_{1}-\mu_{2}},
$$

where $\mu_{1}$ and $\mu_{2}$ are the con-eigenvalues of the target scattering matrix $\mathbf{S}$. The scattering vector of a symmetric scatterer can be expressed on the Pauli trihedral-dihedral basis $\left\{\mathbf{S}_{a}, \mathbf{S}_{b}\right\}$ as follows (Touzi 2007):

$$
\vec{V}_{s y m}=\left|\vec{V}_{s y m}\right| \cdot\left[\cos \alpha_{s} \cdot \mathbf{S}_{a}+\sin \alpha_{s} \cdot e^{j \phi_{\alpha s}} \mathbf{S}_{b}\right]
$$

where the scattering type magnitude $\alpha_{s}$ corresponds to the orientation angle of the symmetric scattering vector on the trihedral-dihedral $\left\{\mathbf{S}_{a}, \mathbf{S}_{b}\right\}$ basis. $\phi_{\alpha s}$ is the phase difference between the vector components in the trihedral-dihedral basis. The new scattering type phase entity introduced in (Touzi 2007) provides a measure of the phase offset between the trihedral and dihedral scattering components. The information provided by $\phi_{\alpha s}$ as complementary to $\alpha_{s}$ was shown to be essential for a better understanding of marsh wetland scattering variations between the spring run-off season and the fall using Convair 580 SAR data collected over the Mer Bleue wetland site (Touzi et al. 2007). The symmetric and asymmetric nature of target scattering was characterized using Huynen helicity $\tau$ (Touzi 2007). Notice that while the complex scattering parameters $\alpha_{s}$ and $\phi_{\alpha s}$ are independent of the basis of polarization (Touzi 2007; Paladini et al. 2012), Huynen's helicity characterizes the symmetric nature of target scattering in the $\{h, v\}$ polarization basis (Huynen 1970). Recently, a different expression of the helicity was derived at the circular polarization basis (Huynen 1970), and the complementary information it provides to the Huynen's helicity was demonstrated (Paladini et al. 2012).

The projection of the Kennaugh-Huynen coherent target decomposition on the Pauli polarization basis can be represented as a function of the complex scattering $\alpha_{s}$ and $\phi_{\alpha s}$ and the Huynen maximum polarization parameters $\psi$ and $m$ as

$$
\begin{aligned}
k=m & {\left[\begin{array}{ccc}
1 & 0 & 0 \\
0 & \cos 2 \psi & -\sin 2 \psi \\
0 & \sin 2 \psi & \cos 2 \psi
\end{array}\right] } \\
\times & {\left[\begin{array}{c}
\cos \alpha_{s} \cos 2 \tau \\
\sin \alpha_{s} \cdot e^{j \varphi_{\alpha s}} \\
-j \cos \alpha_{s} \sin 2 \tau
\end{array}\right] }
\end{aligned}
$$

where $\psi, \tau$ and $m$ are the Huynen orientation, the helicity and the maximum return of the maximum polarization, respectively.

It is worth noting that for a symmetric scattering $(\tau=0)$, $\alpha_{s}$ and $\phi_{\alpha s}$ are identical to the Touzi SSCM parameters $\eta$ and $\varphi_{S b}-\varphi_{S a} . \alpha_{s}$ and $\phi_{\alpha s}$ are also identical to the Cloude-Pottier parameters (Cloude and Pottier 1996) $\alpha$ and $\delta=\varphi_{2}-\varphi_{1}$, respectively (Touzi 2007). For scatterers of locally asymmetric scattering, such as urban areas, treed wetlands and forests, large divergence between $\phi_{\alpha s}$ and $\delta$ and $\alpha_{s}$ and $\alpha$ have been noted (Cloude and Pottier 1996). Unlike Cloude-Pottier parameters (Trunk and George 1970), the TSVM characterizes target scattering type with the complex entity $\left(\alpha_{s}\right.$ and $\left.\phi_{\alpha s}\right)$, which only depends on the scattering matrix eigenvalues. This leads to a unique and unambiguous description of target scattering in terms of parameters, which are polarization basis independent, for both symmetric and asymmetric targets as discussed in (Touzi 2007). 
For a unique characterization of coherent and partially coherent scattering, the TSVM (Touzi 2007) was integrated in Cloude's coherency eigenvector decomposition (Touzi 2007). Like Wiener's wave coherence characteristic decomposition (Wiener 1930), Cloude's characteristic decomposition of the coherency matrix, $\mathbf{T}$, permits the representation of $\mathbf{T}$ as the incoherent sum of coherency matrices that represent independent single scattering (Cloude 1988). Under the target reciprocity assumption, $\mathbf{T}$ is represented as the sum of up to three coherency matrices $\mathbf{T}_{i}$, each of them being weighted by its appropriate positive real eigenvalue $\eta_{i}$ :

$$
\mathbf{T}=\sum_{i=1,2,3} \eta_{i} \mathbf{T}_{i}
$$

In contrast to the Cloude-Pottier decomposition, the TSVM is used for the parameterization of each coherency eigenvector $\mathbf{T}_{i}$ (coherent single scattering) in terms of unique target parameters. In order to avoid any loss of information related to single scatterer parameters averaging, the target scattering decomposition is conducted through an in-depth analysis of each of the three single scattering eigenvectors $\mathbf{u}_{i}$, $i=1,2,3$ represented by the coherency eigenvector matrix $\mathbf{T}_{i}$ of rank 1 and the normalized positive real eigenvalues $\lambda_{i}=\eta_{i} /$ $\left(\eta_{1}+\eta_{2}+\eta_{3}\right)$. This leads to the representation of each single scattering $\mathbf{u}_{i}$ in terms of five roll-invariant and independent target scattering parameters $\left(\alpha_{s i}, \phi_{\alpha s i}, \tau_{i}, m_{i}, \lambda_{i}\right)$ and the Huynen orientation angle $\psi_{i}$.

\subsection{Polarimetric SAR Interferometry}

This section is devoted to the radar remote sensing technique called polarimetric interferometry (Cloude and Papathanassiou 1998). When used with synthetic aperture radar (SAR) systems, it is usually termed polarimetric interferometric SAR or PolInSAR for short (Papathanassiou and Cloude 2001). PolInSAR has important applications in the remote measurement of vegetation properties such as forest height (Papathanassiou et al. 2005a) and biomass (Mette et al. 2004), future applications (Williams and Cloude 2005), snow/ice thickness monitoring (Dall et al. 2003; Papathanassiou et al. 2005b) and urban height and structure applications (Schneider Zandona et al. 2005). As its name suggests, this technique combines two separate radar technologies, polarimetry and interferometry. The former, as detailed in the previous sections, involves switching the polarization state of transmit and receive channels to measure differences in backscatter due to orientation, shape and material composition (Cloude and Pottier 1996). This leads ultimately to measurement of the $2 \times 2$ complex scattering matrix $\mathbf{S}$, from which we can synthesize the response of the image pixel to arbitrary polarization combinations. On the other hand, radar interferometry (Bamler and Hartl 1998) involves coherently combining signals from two separated spatial positions (defining the so-called baseline of the interferometer) to extract a phase difference or interferogram. In radar this can be achieved in two main configurations, so-called along-track interferometry, which involves time displacements between separated antennas along the flight direction of the platform leading to velocity estimation. Alternatively, we can perform across-track interferometry, involving lateral separation of antennas and leading to spatial information relating to the elevation of the scatterer above a reference ground position. In PolInSAR, interest centres mainly on across-track geometries, but in principle it can be applied to along-track configurations as well.

PolInSAR differs from conventional interferometry in that it allows generation of interferograms for arbitrary transmit and receive polarization pairs. It turns out that the phase of an interferogram changes with the choice of polarization and consequently we can extract important biophysical and geophysical parameters by interpreting this change in the right way. It shall be seen that consequently the combination of interferometry with polarimetry is greater than the sum of its parts and that PolInSAR allows us to overcome severe limitations of both techniques when taken alone. This is especially true in the important area of remote sensing of vegetated land surface, where polarimetry suffers from the inherent high entropy problem (Cloude and Pottier 1996), while standard interferometry remains underdetermined, i.e. the interferogram depends on many possible physical effects, no one of which can be identified from the data itself (Treuhaft et al. 1996).

\subsubsection{SAR Interferometry}

PolInSAR algorithms make use of interferometric coherence, or equivalently phase and local phase variance, rather than backscattered power (Bamler and Hartl 1998; Zebker and Villasenor 1992). For this reason, it is necessary to introduce and to study the problems associated with the estimation of coherence from radar data, especially in the case of interferometric data. A similar introduction for polarimetric data was already seen in Sect. 1.2.5. Starting with any two co-registered single-look complex (SLC) data channels $S_{1}$ and $S_{2}$, the interferometric coherence is formally defined as

$$
\gamma=|\gamma| e^{i \phi}=\frac{E\left\{S_{1} S_{2}^{*}\right\}}{\sqrt{E\left\{S_{1} S_{1}^{*}\right\}} \sqrt{E\left\{S_{2} S_{2}^{*}\right\}}}
$$

where $0 \leq|\gamma| \leq 1$. In practice, the sample coherence is frequently used as a coherence estimate of Eq. (1.178): 


$$
\widehat{\gamma}=|\widehat{\gamma}| e^{i \chi}=\frac{\sum_{k=1}^{n} S_{1 k} S_{2 k}^{*}}{\sqrt{\sum_{k=1}^{n} S_{1 k} S_{1 k}^{*}} \sqrt{\sum_{k=1}^{n} S_{2 k} S_{2 k}^{*}}}
$$

where $k$ is the sample number and we have only a finite number $n$ independent signal measurements available. Eq. (1.179) represents the maximum likelihood estimate of coherence and under some general statistical assumptions provides an estimate that is asymptotically unbiased (see Sect. 1.2.3). For jointly complex Gaussian processes $S_{1}$ and $S_{2}$, the pdf of $|\widehat{\gamma}|$ can then be derived as a function of the true coherence value $|\gamma|$ and the number of samples $n$ (Touzi et al. 1999). The estimated coherence value $|\widehat{\gamma}|$ is consistently biased towards higher values (Touzi et al. 1999); in the extreme of single-look estimation, the coherence estimate is equal to unity and so always overestimated and without information. However, the bias decreases with increasing number of independent samples $n$ and with increasing underlying coherence $|\gamma|$. A second important parameter to estimate is the variance of the sample coherence magnitude. While the true estimated value would be desirable, often we assume zero bias, by using sufficient averaging, and estimate the variance by making use of simpler equations for speedier computations. In particular, the Cramér-Rao bounds provide lower limits on the variance for coherence and phase and have been derived in (Tabb and Carande 2001) to provide the simpler formulae:

$$
\operatorname{var}\{|\widehat{\gamma}|\} \geq \frac{\left(1-|\gamma|^{2}\right)^{2}}{2 n}, \operatorname{var}\{\chi\} \geq \frac{1-|\gamma|^{2}}{2 n|\gamma|^{2}}
$$

As it can be deduced for phase-based processing, it is always better to operate at high coherence and avoid low coherences; the latter involves not only increased variance but also severe bias issues that can distort the phase information. It is a key limitation of polarimetry that scattering by vegetation leads to low coherences for all polarization channels because of so-called depolarization. This severely limits the ability to use polarimetric phase information over vegetated land surfaces. Interferometry on the other hand allows to partially control coherence via baseline selection. PolInSAR exploits this advantage to obtain high coherence in multiple polarization channels.

The above considerations for coherence estimation are important in PolInSAR, the major distinguishing feature of which is that we add an extra stage in the construction of the two SLC channels $S_{1}$ and $S_{2}$. In general, for a fully polarimetric data set, we take as input the three calibrated SLC images $S_{h h}, S_{h v}$ and $S_{v v}$ and generate projections of these onto user-defined complex weight vectors $\mathbf{w}_{1}$ and $\mathbf{w}_{2}$ before calculating the coherence defined as

$$
\begin{aligned}
& s_{1}=w_{1}^{1} \frac{\left(S_{h h}^{1}+S_{v v}^{1}\right)}{\sqrt{2}}+w_{1}^{2} \frac{\left(S_{h h}^{1}-S_{v v}^{1}\right)}{\sqrt{2}}+w_{1}^{3} \sqrt{2} S_{h v}^{1}=\mathbf{w}_{1}^{T} \cdot \mathbf{k}_{1} \\
& \left.s_{2}=w_{2}^{1} \frac{\left(S_{h h}^{2}+S_{v v}^{2}\right)}{\sqrt{2}}+w_{2}^{2} \frac{\left(S_{h h}^{2}-S_{v v}^{2}\right)}{\sqrt{2}}+w_{2}^{3} \sqrt{2} S_{h v}^{2}=\mathbf{w}_{2}^{T} \cdot \mathbf{k}_{2}\right\} . \\
& \Rightarrow \gamma\left(\mathbf{w}_{1}, \mathbf{w}_{2}\right)=\frac{E\left\{S_{1} S_{2}^{*}\right\}}{\sqrt{E\left\{S_{1} S_{1}^{*}\right\}} \sqrt{E\left\{S_{2} S_{2}^{*}\right\}}}
\end{aligned}
$$

The weight vectors $\mathbf{w}_{1}$ and $\mathbf{w}_{2}$ define user-selected scattering mechanisms at ends 1 and 2 of the across-track baseline. In general, $\mathbf{w}_{1}$ and $\mathbf{w}_{2}$ can be different and both parameterized as complex unitary vectors of the form shown in (1.162) (Cloude and Pottier 1996). The weight vectors or scattering mechanisms in which the targets vectors could be projected could be the canonical mechanisms detailed in Table 1.6. However, it is a feature of PolInSAR algorithm development that use is often made of more general $\mathbf{w}$ vectors than those shown, derived, for example, as eigenvectors for coherence optimisation (Tabb and Carande 2001; Colin et al. 2003), or through prior model studies of scattering from vegetated terrain (Williams 1999). For this reason, we need to keep the more general notation of Eq. (1.162) so as to be able to consider arbitrary vectors in the formation of an interferogram. We now turn to consider such optimisation algorithms in more detail and to briefly assess their implications for coherence estimation and validation.

\subsubsection{Algorithms for Optimum Interferogram Generation}

Polarimetric interferometry is a special case of multichannel coherent radar processing (Reigber et al. 2000). Such problems are characterized by multidimensional covariance matrices (Lee et al. 1994, 2003). In PolSAR, for example, interest centres on the $3 \times 3$ Hermitian covariance matrix $\mathbf{C}$, unitarily equivalent to the coherency matrix $\mathbf{T}$ as shown in Sect. 1.1.2.2. This is the basic building block in polarimetric interferometry, and so it can be designated as $\boldsymbol{\Lambda}_{1}$ to indicate how it relates to fully polarimetric measurements but made at only one spatial position. In single baseline PolInSAR, a second measurement at a displaced position 2 is added. This is now characterized by a $6 \times 6$ coherency matrix $\boldsymbol{\Lambda}_{2}$ as shown in (1.182). The $6 \times 6$ matrix can be naturally partitioned into three sub-matrices each of size $3 \times 3$. This 
formulation then scales in a natural way for multibaseline PolInSAR by expansion of the governing coherency matrix $\Lambda_{N}$ to a $3 N \times 3 N$ complex system or $4 N \times 4 N$ for bistatic multibaseline PolInSAR, where $N$ is the number of baselines:

$$
\begin{aligned}
\boldsymbol{\Lambda}_{1} & =\mathbf{T} \rightarrow \boldsymbol{\Lambda}_{2}=\left[\begin{array}{cc}
\mathbf{T}_{11} & \boldsymbol{\Omega}_{12} \\
\boldsymbol{\Omega}_{12}^{H} & \mathbf{T}_{22}
\end{array}\right] \rightarrow \boldsymbol{\Lambda}_{N} \\
& =\left[\begin{array}{cccc}
\mathbf{T}_{11} & \boldsymbol{\Omega}_{12} & \cdots & \boldsymbol{\Omega}_{1 N} \\
\boldsymbol{\Omega}_{12}^{*} & \mathbf{T}_{22} & \cdots & \Omega_{2 N} \\
\vdots & \vdots & \ddots & \vdots \\
\boldsymbol{\Omega}_{1 N}^{*} & \boldsymbol{\Omega}_{2 N}^{*} & \cdots & \mathbf{T}_{N N}
\end{array}\right] .
\end{aligned}
$$

Returning now to the important case of $\boldsymbol{\Lambda}_{2}$, two of the sub-matrices $\mathbf{T}_{11}$ and $\mathbf{T}_{22}$ are Hermitian and relate to the polarimetry from positions 1 and 2, while the third $\boldsymbol{\Omega}_{12}$ is a complex $3 \times 3$ matrix that contains information about the variation of interferometric coherence and phase for all possible weight vectors $\mathbf{w}_{1}$ and $\mathbf{w}_{2}$ :

$$
\gamma\left(\mathbf{w}_{1}, \mathbf{w}_{2}\right)=\frac{\mathbf{w}_{1}^{H} \boldsymbol{\Omega}_{12} \mathbf{w}_{2}}{\sqrt{\mathbf{w}_{1}^{H} \mathbf{T}_{11} \mathbf{w}_{1}} \cdot \sqrt{\mathbf{w}_{2}^{H} \mathbf{T}_{22} \mathbf{w}_{2}}} .
$$

The previous relation leads to an important choice of approach to algorithm development in PolInSAR. In the first case, if the vectors $\mathbf{w}_{1}$ and $\mathbf{w}_{2}$ are known in advance, then the coherence can be directly estimated using (1.181) with the same InSAR fluctuation statistics and bias outlined in the previous section. However, often we wish to determine optimum weight vectors from the data themselves, and it follows from (1.183) that to do this we require estimates of the three $3 \times 3$ matrices, $\mathbf{T}_{11}$ and $\mathbf{T}_{22}$ and $\boldsymbol{\Omega}_{12}$. This opens up a much wider discussion about the fluctuation statistics and bias arising from the fact that only estimates and not true matrix values can be used in (1.183). For example, to estimate the sub-matrices, we must first estimate the full $6 \times 6$ coherency matrix $\boldsymbol{\Lambda}_{2}$. This estimate $\mathbf{Z}$ is obtained by means of the multilook estimator.

One important application of (1.183) is the calculation of the optimum coherences in PolInSAR. The most general formulation of this was first presented in (Cloude and Papathanassiou 1998) and is summarized in (1.184). Here, we first state the problem mathematically, which is to choose $\mathbf{w}_{1}$ and $\mathbf{w}_{2}$ so as to maximize the coherence magnitude, defined from the complex coherence as a function of the three sub-matrices $\mathbf{T}_{11}$ and $\mathbf{T}_{22}$ and $\boldsymbol{\Omega}_{12}$ as shown. This can be mathematically solved by using a Lagrange multiplier technique as shown and leads to the calculation of the required $\mathbf{w}$ vectors as eigenvectors of a pair of matrices, themselves defined as products of the composite matrices:

$$
\begin{gathered}
\max _{\mathbf{w}_{1}, \mathbf{w}_{2}} \frac{\mathbf{w}_{1}^{H} \mathbf{\Omega}_{12} \mathbf{w}_{2}}{\sqrt{\mathbf{w}_{1}^{H} \mathbf{T}_{11} \mathbf{w}_{1}} \cdot \sqrt{\mathbf{w}_{2}^{H} \mathbf{T}_{22} \mathbf{w}_{2}}} \\
\qquad=\mathbf{w}_{1}^{H} \mathbf{\Omega}_{12} \mathbf{w}_{2}+\lambda_{1}\left(\mathbf{w}_{1}^{H} \mathbf{T}_{11} \mathbf{w}_{1}-1\right)+\lambda_{2}\left(\mathbf{w}_{2}^{H} T_{22} \mathbf{w}_{2}-1\right) \\
\Rightarrow\left\{\begin{array}{l}
\frac{\partial L}{\partial \mathbf{w}_{1}^{H}}=\Omega_{12} \mathbf{w}_{2}+\lambda_{1} \mathbf{T}_{11} \mathbf{w}_{1}=0 \\
\frac{\partial L}{\partial \mathbf{w}_{2}^{H}}=\Omega_{12}^{* T} \mathbf{w}_{1}+\lambda_{2} T_{22} \mathbf{w}_{2}=0
\end{array}\right. \\
\Rightarrow\left\{\begin{array}{l}
\mathbf{T}_{22}^{-1} \mathbf{\Omega}_{12}^{H} \mathbf{T}_{11}^{-1} \mathbf{\Omega}_{12} \mathbf{w}_{2}=\lambda_{1} \lambda_{2}^{*} \mathbf{w}_{2} \\
\mathbf{T}_{11}^{-1} \mathbf{\Omega}_{12} \mathbf{T}_{22}^{-1} \mathbf{\Omega}_{12}^{H} \mathbf{w}_{1}=\lambda_{1} \lambda_{2}^{*} \mathbf{w}_{1}
\end{array}\right.
\end{gathered}
$$

As it was noted in Sect. 1.4.1, the estimated value of the coherence magnitude is biased with respect to the true value in such a way that the larger the number of averaged samples and the higher the coherence magnitude, the lower the bias. The previous hypothesis was based on considering (1.181) where the vectors $\mathbf{w}_{1}$ and $\mathbf{w}_{2}$ are known in advance. Nevertheless, if (1.184) is considered to obtain the coherence magnitude, the vectors $\mathbf{w}_{1}$ and $\mathbf{w}_{2}$ must also be estimated from the data, leading to a larger coherence magnitude bias.

In order to obtain an optimization approach that has less bias for a given number of samples, it is necessary to reduce the effective dimensionality of the problem. Several authors have proposed adopting the a priori assumption $\mathbf{w}_{1}=\mathbf{w}_{2}$, i.e. that the optimum coherence vector remains unknown but we assume that it doesn't change with baseline (Colin et al. 2003; Sagues et al. 2000; Flynn et al. 2002). This idea is supported on physical grounds for short baselines in the absence of temporal decorrelation, i.e. for single-pass or low-frequency sensors where the scattering does not change significantly over the effective angular width of the baseline. This approach calls for a new mathematical formulation of the optimization process. One approach is based on a straightforward extension of the Lagrange multiplier technique to constrain $\mathbf{w}_{1}=\mathbf{w}_{2}$. This leads by manipulation of (1.184) to a set of $\mathbf{w}$ vectors given as eigenvectors of the composite matrix:

$$
\left(\mathbf{T}_{11}+\mathbf{T}_{22}\right)^{-1}\left(\mathbf{\Omega}_{12}+\boldsymbol{\Omega}_{12}^{H}\right) \mathbf{w}=-\lambda \mathbf{w} .
$$

One problem with the previous equation is that the eigenvalue is not the coherence, but its real part, and so the optimization is phase sensitive. For this reason, a second related approach based on maximization of the phase difference as a function of polarization vector $\mathbf{w}$ has been developed. In this case, the optimum vector is found by solving a phase-parameterized eigenvalue problem (Colin et al. 2003; Flynn et al. 2002): 


$$
\boldsymbol{\Omega}_{H} \mathbf{w}=\lambda \mathbf{T w}\left\{\begin{array}{c}
\boldsymbol{\Omega}_{H}=\frac{1}{2}\left(\boldsymbol{\Omega}_{12} e^{i \phi_{1}}+\boldsymbol{\Omega}_{12}^{H} e^{-i \phi_{1}}\right) \\
\mathbf{T}=\frac{1}{2}\left(\mathbf{T}_{11}+\mathbf{T}_{22}\right)
\end{array} .\right.
$$

This has been shown to be equivalent to calculating the numerical radius of the complex matrix $\mathbf{A}=\mathbf{T}^{-1 / 2} \boldsymbol{\Omega}_{12} \mathbf{T}^{-1 / 2}$. A proposed algorithm for finding this optimum state has been presented in (Colin et al. 2003; Colin et al. 2005). One drawback in this approach is that $\phi_{1}$ is a free parameter, and so either search or iterative methods must be used to secure the global optimum. This adds to the computational complexity for each pixel.

A third related approach has been proposed based on a sub-space Monte Carlo searching algorithm (Sagues et al. 2000). This limits the search for the optimum (again assuming $\mathbf{w}_{1}=\mathbf{w}_{2}$ ) to the diagonal elements of $\boldsymbol{\Omega}_{12}$, i.e. to co-polarized or cross-polarized combinations across the whole Poincaré sphere. This again acts to effectively limit the dimensionality of the problem and demonstrates less bias than the full Lagrange multiplier method. Finally, phase centre super-resolution techniques based on the ESPRIT algorithm have also been proposed to find the optimum w vectors (Yamada et al. 2001).

In all these cases, a sub-optimum solution is obtained compared to the unconstrained Lagrange multiplier method but often with better numerical stability. Given the general increased processing overhead of employing optimization, it is always of interest to investigate the potential benefits of employing an optimization approach over simple linear, Pauli and circular options.

\subsubsection{Model-Based Polarimetric SAR Interferometry}

The previous section considered an important optimisation problem in PolInSAR, namely, to investigate the maximum variation of coherence with polarization by solving an eigenvalue problem. This section will be focused on some canonical problems of interest in the remote sensing of land surfaces and try and use the mathematical solutions obtained to conclude as to the potential of optimisation versus standard coherence estimation in PolInSAR. We consider three important problems, scattering from non-vegetated surfaces, random volume scattering and finally a 2-layer surface+volume mixture which more closely matches the behaviour of natural vegetated land surfaces.

\subsubsection{PollnSAR for Bare Surface Scattering}

The starting point will be to consider the simplest case of non-vegetated terrain. Under the assumption of surface scattering only, the polarimetry can then be characterized as a reflection symmetric random media with a coherency matrix $\mathbf{T}$ of the form shown in (1.187) (Cloude and Pottier 1996; Cloude et al. 2004). The interferometry, following range spectral filtering and assuming no temporal or SNR decorrelation, is characterized by a single parameter, i.e. the ground phase $\phi$ :

$\mathbf{K}=\mathbf{T}_{11}^{-1} \mathbf{\Omega}_{12} \mathbf{T}_{11}^{-1} \mathbf{\Omega}_{12}^{H}=\mathbf{T}_{11}^{-1} e^{i \phi} \mathbf{T}_{11} \mathbf{T}_{11}^{-1} e^{-i \phi} \mathbf{T}_{11}=\left[\begin{array}{lll}1 & 0 & 0 \\ 0 & 1 & 0 \\ 0 & 0 & 1\end{array}\right]$

From the previous equation, it follows that the optimum coherences are obtained as eigenvectors of the matrix $\mathbf{K}$ as shown. By multiplying terms we see that the matrix $\mathbf{K}$ is just the $3 \times 3$ identity matrix. This implies that all polarizations have the same interferometric coherence and PolInSAR plays no role in surface scattering problems. This is not quite true in practice for two important reasons: in practice there will be polarization-dependent SNR decorrelation. In fact, recently it has been suggested that such SNR coherence variations with polarimetry be used for quantitative InSAR surface parameter estimation. This formulation assumes that the scattering from the surface occurs within a thin layer. If there is significant penetration into the surface, then volume scattering effects can occur and this will lead to volume decorrelation effects. These effects have been observed for land ice (Treuhaft et al. 1996) and snow studies (Zebker and Villasenor 1992) where the surface is non-vegetated but covered by a low-loss scattering layer. Nonetheless, (1.187) demonstrates how for bare surface scattering PolInSAR plays only a secondary role. More interesting for application of natural land surfaces is to consider the presence of volume scattering due to vegetation cover.

\subsubsection{PollnSAR for Random Volume Scattering}

When considering scattering from a volume, interest centres on the special case of a random volume, i.e. one with macroscopic azimuthal symmetry (Cloude and Pottier 1996). In this case the polarimetric coherency matrix $\mathbf{T}$ is diagonal. However, more care is required over consideration of the interferometric phase in $\boldsymbol{\Omega}_{12}$. In this case one must include the effects of volume decorrelation due to the random vertical distribution of scatterers (Treuhaft et al. 1996). In this case, the interferometry must include a complex integral $I_{2}$ normalized by a real integral $I_{1}$ : 


$$
\mathbf{K}=\mathbf{T}_{11}^{-1} \boldsymbol{\Omega}_{12} \mathbf{T}_{11}^{-1} \boldsymbol{\Omega}_{12}^{H}=\left|\frac{I_{2}}{I_{1}}\right|^{2}\left[\begin{array}{ccc}
1 & 0 & 0 \\
0 & 1 & 0 \\
0 & 0 & 1
\end{array}\right]
$$

where

$$
\begin{aligned}
I_{1} & =e^{-\frac{2 \sigma h_{v}}{\cos \theta_{o}}} \int_{0}^{h_{v}} e^{\frac{2 \sigma z^{\prime}}{\cos \theta_{o}}} d z^{\prime}, I_{2} \\
& =e^{-\frac{2 \sigma h_{v}}{\cos \theta_{o}}} \int_{0}^{h_{v}} e^{\frac{2 \sigma z^{\prime}}{\cos \theta_{o}}} e^{i k_{z} z^{\prime}} d z^{\prime}
\end{aligned}
$$

where the vegetation is characterized by a height $h_{v}$ and mean extinction rate $\sigma$ and $\theta_{0}$ represents the mean incidence angle. In (1.188), it is also observed that $\mathbf{K}$ is proportional to the identity matrix, but this time the eigenvalues, all equal, are given by a ratio of integrals over the vertical distribution. This ratio is just the volume decorrelation displaying an increase in phase variance and a vegetation bias to the ground phase determined by $h_{v}$ and $\sigma$ :

$$
\begin{aligned}
& \gamma(\mathbf{w})=\frac{I_{2}}{I_{1}}=\frac{2 \sigma e^{i \phi\left(z_{o}\right)}}{\cos \theta_{o}\left(e^{2 \sigma h_{v} / \cos \theta_{o}}-1\right)} \int_{0}^{h_{v}} e^{i k_{z} z^{\prime}} e^{\frac{2 \sigma z^{\prime}}{\cos \theta_{o}}} d z^{\prime} \\
& =\frac{p}{p_{1}} \frac{e^{p_{1} h_{v}}-1}{e^{p h_{v}}-1}=\gamma_{v}
\end{aligned}
$$

where

$$
\begin{gathered}
p=\frac{2 \sigma}{\cos \theta_{0}}, \\
p_{1}=p+i k_{z}, \\
k_{z}=\frac{4 \pi \Delta \theta}{\lambda \sin \theta_{0}} \approx \frac{4 \pi B_{n}}{\lambda H \tan \theta_{0}} .
\end{gathered}
$$

Here the vertical interferometric wavenumber $k_{z}$ (Bamler and Hartl 1998) appears as a function of the normal baseline $B_{n}$, the wavelength $\lambda$ as well as the sensor height $H . \Delta \theta$ is the angular separation of the baseline end points from the surface pixel.

As it can be observed in (1.190), this coherence is independent of polarization, $\mathbf{K}$ has three degenerate eigenvalues and PolInSAR plays no role in the analysis of random volume scattering. This statement has to be modified in the presence of oriented volumes (Treuhaft and Cloude 1999), i.e. ones with a preferred orientation of scattering elements such as that occurring in some agricultural crops and even in forestry applications at low frequencies. In such cases
PolInSAR does indeed play a role for volume scattering, with $\mathbf{K}$ developing three distinct eigenvalues. However, for the treatment of forestry applications at L-band and above, such orientation effects are small and the random volume assumption is justified (Papathanassiou et al. 2000).

In conclusion, both bare surfaces and random volumes lead to a degenerate eigenvalue spectrum for the matrix $\mathbf{K}$. It is only when we combine these two effects together that we see the potential benefits of employing PolInSAR processing.

\subsubsection{PollnSAR Two-Layer Combined Surface and Random Volume Scattering}

In the general case when combined surface and volume scattering occurs, then PolInSAR coherence optimisation becomes useful as it is now demonstrated. In this two-layer case or Random-Volume-over-Ground (RVoG) model approach (Cloude and Papathanassiou 2003), the observed coherence is given by a mixture formula:

$$
\begin{aligned}
\gamma(\mathbf{w}) & =e^{i \phi} \frac{\widetilde{\gamma}_{v}+\mu(\mathbf{w})}{1+\mu(\mathbf{w})} \\
& =e^{i \phi}\left[\gamma_{v}+\frac{\mu(\mathbf{w})}{1+\mu(\mathbf{w})}\left(1-\gamma_{v}\right)\right] .
\end{aligned}
$$

Here, the ground phase $\phi$ and complex volume coherence $\gamma_{v}$ are combined with a new real parameter $\mu$, the ratio of effective surface, i.e. all scattering contributions with a phase centre located at $\phi$, to volume scattering. In effect, when $\mu=0$ the scattering reduces to the case of random volume scattering, while when $\mu$ tends to infinity, it reduces to the surface scattering case. Interest centres on the intermediate case because one has an unknown, but constant, complex contribution from the volume scattering combined with a polarization-dependent surface term. By isolating the polarization-dependent terms, the resulting coherence then lies along a straight line in the complex coherence plane as shown in (1.192).

This straight line model has been successfully tested on varied forest data sets and seems to be a good fit for L- and P-band PolInSAR forestry applications. It is interesting to note how the coherence varies as we adjust the single parameter $\mu$ along this line. Figure 1.15 illustrates three important cases. In all three we first note how the coherence starts for small $\mu$ at some value depending on the volume scattering contribution, 0.8 in the example. It then initially decreases with increasing surface contribution until reaching a turning point after which it increases with $\mu$, always approaching unity as $\mu$ tends to infinity.

In Fig. 1.15 three important special cases of the eigenvalue spectrum of $\mathbf{K}$ for this scenario are also superimposed. The top left shows the case when $\mu$ is always small, i.e. when there is strong volume scattering with high extinction masking the surface contributions. As polarization $\mathbf{w}$ is adjusted, then $\mu$ will also change, and the optimizer has an incentive to select the minimum $\mu$ channel to maximize coherence. At the other extreme, when $\mu$ is large and surface 

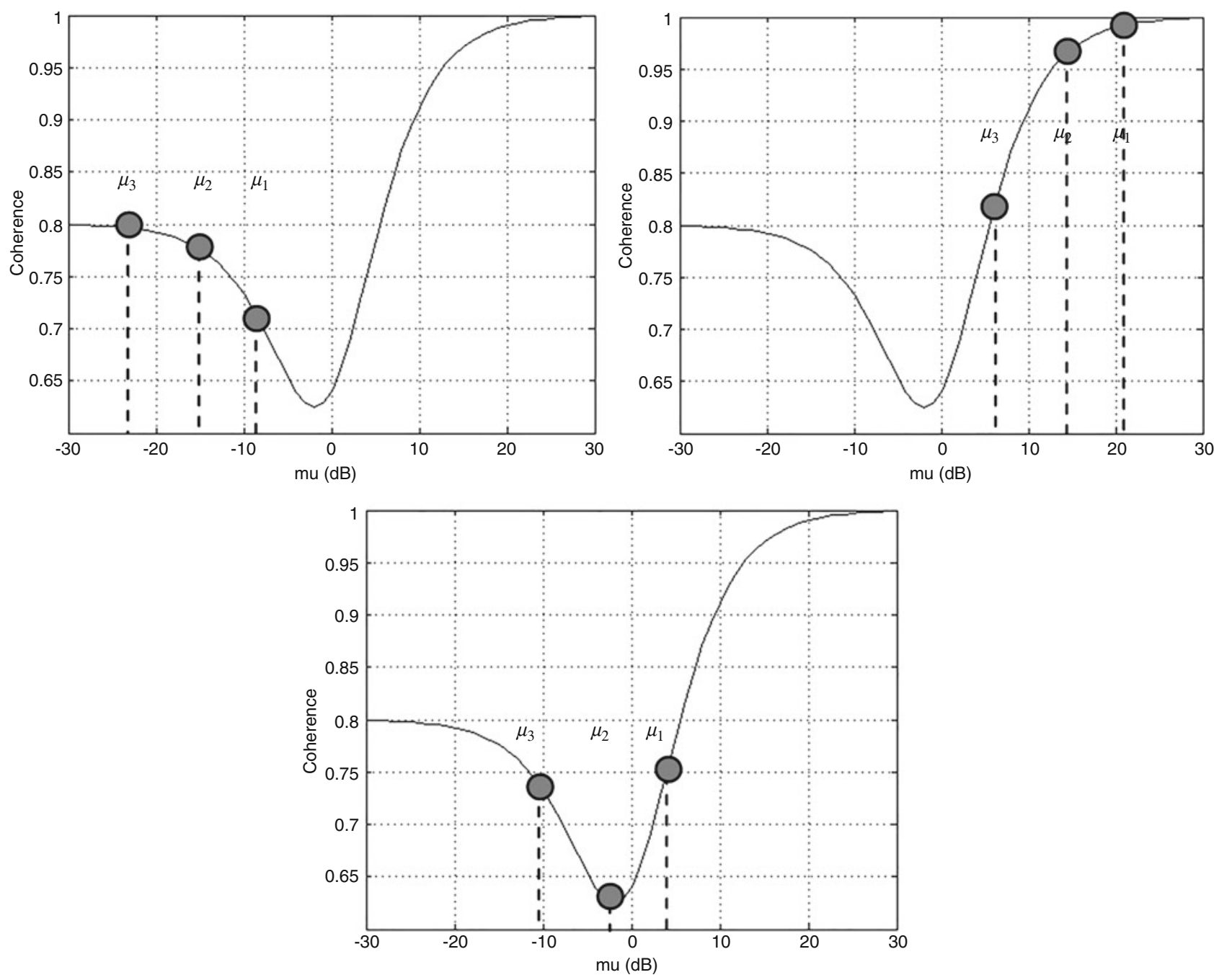

Fig. 1.15 Variation of coherence with small (top left), large (top right) and intermediate (lower) $\mu$ values

scattering dominates, we see that the optimizer has an incentive instead to maximize $\mu$ in order to maximize coherence. A more interesting case, and one that occurs often in practice for L-band forestry applications, is the intermediate zone when the variation of $\mu$ (the $\mu$ spectrum) includes the turning point. In this case the coherence can be maximized by either increasing or decreasing $\mu$ depending on circumstances.

Two important conclusions can be made from this. Firstly, in the mixed two-layer scattering case, the coherence varies with polarization and so optimisation plays a role in PolInSAR analysis. Secondly, we see that we cannot simply associate the maximum coherence with, for example, the maximum value of $\mu$. Both maxima and minima of $\mu$ can lead to the optimum coherence, depending on the circumstances. However, it follows that if we can estimate the $\mu$ spectrum for any problem, then we can compare the $\mathrm{max} / \mathrm{min}$ with the values for the standard channel (linear, Pauli, etc.) to quantify the potential benefits of employing optimisation techniques.

The determination of the extreme points of the $\mu$ spectrum is related to a classical problem in radar polarimetry, namely, contrast optimisation (Novak and Burl 1990). The solution to this is obtained as the eigenvalues of the product of the inverse volume times the surface polarimetric coherency matrices:

$$
\begin{gathered}
\mathbf{T}_{V}=m I_{1}\left[\begin{array}{ccc}
1 & 0 & 0 \\
0 & \kappa & 0 \\
0 & 0 & \kappa
\end{array}\right] \Rightarrow \mathbf{T}_{v}^{-1}=\frac{1}{m I_{1}}\left[\begin{array}{ccc}
1 & 0 & 0 \\
0 & \frac{1}{\kappa} & 0 \\
0 & 0 & \frac{1}{\kappa}
\end{array}\right] \\
\mathbf{T}_{s}=\left[\begin{array}{ccc}
t_{11} & t_{12} & 0 \\
t_{12}^{*} & t_{22} & 0 \\
0 & 0 & t_{33}
\end{array}\right] \\
\Rightarrow \mathbf{T}_{v}^{-1} \mathbf{T}_{s}=\frac{1}{I_{1} m}\left[\begin{array}{ccc}
\frac{t_{11}}{t_{12}^{*}} & \frac{t_{12}}{\kappa} & 0 \\
0 & 0 & \frac{t_{33}}{\kappa}
\end{array}\right]
\end{gathered}
$$


Under the assumption of a random volume and reflection symmetric surface scattering component, the eigenvalues of this matrix can be determined analytically as

$$
\begin{aligned}
& \left\{\begin{array}{c}
\mu_{1}=\frac{1}{2 I_{1} m}\left(t_{11}+\frac{t_{22}}{\kappa}+\sqrt{\left(t_{11}-\frac{t_{22}}{\kappa}\right)^{2}+\frac{4\left|t_{12}\right|^{2}}{\kappa}}\right) \\
\mu_{2}=\frac{1}{2 I_{1} m}\left(t_{11}+\frac{t_{22}}{\kappa}-\sqrt{\left(t_{11}-\frac{t_{22}}{\kappa}\right)^{2}+\frac{4\left|t_{12}\right|^{2}}{\kappa}}\right) \\
\mu_{3}=\frac{1}{I_{1} m}\left(\frac{t_{33}}{\kappa}\right)
\end{array}\right. \\
& \Rightarrow\left\{\begin{array}{l}
\left|\gamma_{1}\right| e^{i \delta_{1}}=\frac{e^{i \phi_{o}}\left(\gamma_{v}+\mu_{1}\right)}{1+\mu_{1}} \\
\left|\gamma_{2}\right| e^{i \delta_{2}}=\frac{e^{i \phi_{o}}\left(\gamma_{v}+\mu_{2}\right)}{1+\mu_{2}} . \\
\left|\gamma_{3}\right| e^{i \delta_{3}}=\frac{e^{i \phi_{o}}\left(\gamma_{v}+\mu_{3}\right)}{1+\mu_{3}}
\end{array}\right.
\end{aligned}
$$

Equally importantly, the eigenvectors of this matrix indicate the $\mathbf{w}$ vectors that should be employed in PolInSAR to secure these extreme coherence values. We note from Eq. (1.194) that the optimum contrast solutions are not generally the simple HH, HV and VV channels. This supports the investigation of optimisation techniques based on fully polarimetric data acquisition for PolInSAR processing.

\subsection{Polarimetric SAR Tomography}

3-D SAR Tomography (TomoSAR) is an experimental multibaseline (MB) interferometric mode achieving full 3-D imaging in the range-azimuth-height space through elevation beam forming, i.e. spatial (baseline) spectral estimation (Reigber and Moreira 2000). Thanks to TomoSAR, the resolution of multiple scatterers is made possible in height in the same range-azimuth cell, overcoming a limitation of the conventional InSAR processing and complementing PolInSAR. TomoSAR can add more features for the analysis of complex scenarios, e.g. for the estimation of forest structure and biomass, sub-canopy topography, soil humidity and ice thickness monitoring and extraction of heights and reflectivities in layover urban areas. In order to retrieve information on the nature of the imaged scatterers, TomoSAR has also been extended to include the polarimetric information (briefly, PolTomoSAR) (Guillaso and Reigber 2005). It jointly exploits multibaseline SAR data acquired with different polarization channels to improve the accuracy of the estimation of the vertical position of the imaged scatterers and to estimate a set of normalized complex coefficients characterizing the corresponding polarimetric scattering mechanism.
The very first demonstration of the tomographic concept was carried out in 1995 by processing single-polarization data acquired in an anechoic chamber of a two-layer synthetic target (Pasquali et al. 1995). TomoSAR was then experimented from an airborne platform a few years later by acquiring L-band data by means of the DLR E-SAR platform over the Oberpfaffenhofen site (Reigber and Moreira 2000). Although this experiment was successful in demonstrating the 3-D imaging capabilities of forest volumes and man-made targets at L-band, two main limitations of TomoSAR were apparent, namely, $(i)$ the usually low number of images available for processing to avoid large acquisition times and the consequent temporal decorrelation and (ii) the difficulty of obtaining ideal uniformly spaced parallel flight tracks due to navigation/orbital considerations.

In order to mitigate the effects of acquisition non-idealities, most of the subsequent research on (singlepolarization) TomoSAR investigated different imaging solutions, model-based and not. Many experiments have shown that the use of polarimetric information not only increments the number of observables, but it also allows to enhance the accuracy of height estimation of scatterers, to increase height resolution and to estimate a vector of complex coefficients describing the scattering mechanism at each height (Guillaso and Reigber 2005). In forest scenarios, the combination of multibaseline polarimetric data can be used to separate ground and canopy scattering and to estimate their vertical structures by following a relatively simple algebraic approach (Tebaldini 2009).

\subsubsection{TomoSAR and PolTomoSAR as Spectral Estimation Problems: Non-model-Based Adaptive Solutions}

As usual in SAR imaging and interferometry, after focusing on the range-azimuth plane, the $K$ SAR images available for processing are assumed to be co-registered and properly compensated for the flat-Earth phase. Moreover, $N$ independent looks (here multiple adjacent pixels) are used for processing. For each $n$-th look, the complex amplitudes of the pixels observed in the $K$ SAR images at the same range-azimuth coordinate are collected in the $K \times 1$ complex-valued vector $\mathbf{y}(n)$ (Lombardini and Reigber 2003). $\mathbf{y}(n)$ is characterized by its covariance matrix. It can be demonstrated that the generic $(l, m)$-th element of $\mathbf{R}$ can be written as

$$
[\mathbf{R}]_{l, m}=\int F(z) \exp \left\{j\left(k_{z, l}-k_{z, m}\right) z\right\} d z
$$

where $F(z)$ is the unknown vertical distribution of the backscattered power as a function of the height $z$ and $k_{z, m}$ is the vertical wavenumber at the $m$-th track. From (1.195), it 
is apparent the Fourier relationship existing between the MB covariances and the profile of the backscattered power, and it justifies the use of spectral estimation as a processing tool to estimate $F(z)$.

The inversion of (1.195) for the estimation of $F(z)$ cannot be carried out through a plain Fourier-based 3-D focusing as it suffers from inflated sidelobes and poor height resolution. Among the investigated alternatives, a state-of-the-art solution is the adaptive beam forming (shortly, $\mathrm{ABF}$ ), which is based on the Capon spectral estimator, and it has been demonstrated to have remarkable sidelobe rejection and resolution capabilities.

The single-polarization ABF spectral estimation problem can be equivalently stated as the problem of designing a complex-valued finite impulse response filter $\mathbf{h}$ of order $K$ that leaves undistorted the multibaseline signal component at the height under test, say $z$, while rejecting possible other components from noise and other heights (Lombardini and Reigber 2003). In formulas

$$
\min _{\mathbf{h}} \mathbf{h}^{T *} \widehat{\mathbf{R}} \mathbf{h} \text { subjectto } \mathbf{h}^{T *} \mathbf{a}(z)=1
$$

where $\mathbf{a}(z)$ is the so-called steering vector, with generic element $[\mathbf{a}(z)]_{k}=\exp \left\{j k_{z, k} z\right\}$ for $k=1, \ldots, K$, and $\widehat{\mathbf{R}}$ is the sample covariance estimate. Notice that the resulting $\mathrm{ABF}$ filter $\mathbf{h}$ depends on $\widehat{\mathbf{R}}$, and it varies with $z$; In particular, the dependency on $\widehat{\mathbf{R}}$ results in a null-placing at proper heights in the filtering operation, thus increasing resolution and sidelobe suppression in the final estimate of $F(z)$. The solution to the optimization problem (1.196) can be found in closed-form (Lombardini and Reigber 2003).

If fully polarimetric data are available, without losing generality, they can be combined in the Pauli basis. The resulting MB data vectors $\mathbf{y}_{1}(n), \mathbf{y}_{2}(n)$ and $\mathbf{y}_{3}(n)$ can then be stacked one on top of the other in order to form the $3 K$ dimensional multibaseline-polarimetric data vector $\mathbf{y}_{P}(n)$. As a consequence, a MB-polarimetric sample covariance matrix $\widehat{\mathbf{R}}_{P}$ can be calculated from $\mathbf{y}_{P}(n)$. Different from the single polarimetric case, the profile has now to be estimated also by considering the polarization state at the targeted height. In this sense, the definition of the steering vector can be extended to the polarimetric case by means of a three-dimensional target vector $\mathbf{w}$ whose elements are complex-valued coefficients describing the scattering mechanism in the Pauli basis, with $\|\mathbf{w}\|^{2}=1$. In formulas, the polarimetric steering vector $\mathbf{b}(z, \mathbf{w})$ is given by

$$
\mathbf{b}(z, \mathbf{w})=\mathbf{B}(z) \mathbf{w},
$$

$$
\mathbf{B}(z)=\left[\begin{array}{ccc}
\mathbf{a}(z) & \mathbf{0} & \mathbf{0} \\
\mathbf{0} & \mathbf{a}(z) & \mathbf{0} \\
\mathbf{0} & \mathbf{0} & \mathbf{a}(z)
\end{array}\right] .
$$

The ABF optimization problem (1.196) can be extended to the MB-polarimetric case as follows (Sauer et al. 2011):

$$
\min _{\mathbf{h}_{P}} \mathbf{h}_{P}^{T *} \widehat{\mathbf{R}}_{P} \mathbf{h}_{P} \text { subjectto } \mathbf{h}_{P}^{T *} \mathbf{b}(z, \mathbf{w})=1
$$

where $\mathbf{h}_{P}$ is the multibaseline-polarimetric $\mathrm{ABF}$ filter response. Now, $\mathbf{h}_{P}$ is optimized in order to place proper nulls in height and in the polarimetric space generated by $\mathbf{w}$. Notice that the dependence of $\mathbf{w}$ on $z$ has been formally dropped for easiness of notation. From (1.199), the power of the filtered signal is

$$
\widehat{F}_{A B F}(z, \mathbf{w})=\frac{1}{\mathbf{b}^{T *}(z, \mathbf{w}) \widehat{\mathbf{R}}_{P} \mathbf{b}(z, \mathbf{w})}
$$

which is still a function of $\mathbf{w}$. To estimate the vertical power distribution as a function of the only $z$, and the corresponding $\mathbf{w}$, one can maximize (1.200) over $\mathbf{w}$ to finally obtain

$$
\widehat{F}_{A B F}(z)=\frac{1}{\lambda_{\min }\left\{\mathbf{B}^{T *}(z) \widehat{\mathbf{R}}_{P}^{-1} \mathbf{B}(z)\right\}}
$$

where $\lambda_{\min }\{\cdot\}$ denotes the minimum eigenvalue operator. The resulting $\widehat{\mathbf{w}}_{A B F}(z)$ is the eigenvector associated with $\lambda_{\min }$. It is worth noting that the multibaseline-polarimetric ABF estimator (1.201) enhances the discrimination of particular scatterers or features. In other words, it is able to extract a rank 1 polarimetric information. This is generally the case of man-made targets like buildings in urban scenarios.

However, it can happen that the scatterers present at a given $z$ are characterized by a random polarimetric behaviour, and they are more properly described by a $3 \times 3$ polarimetric covariance matrix $\mathbf{T}(z)$ rather than by a deterministic target vector (Ferro-Famil et al. 2012). This is generally the case in natural scenarios like forests. In this way, a scattering mechanism at the generic $z$ will contribute to $\mathbf{R}_{P}$ with $\mathbf{T}(z) \otimes\left[\mathbf{a}(z) \mathbf{a}^{T *}(z)\right]$. In light of this, the polarimetric $\mathrm{ABF}$ estimator from the rank 1 formulation (1.201) can be extended in a full-rank sense (Ferro-Famil et al. 2012). The derivation of such estimate is based on the definition of a fullrank objective function which uses the polarimetric span instead of the intensity associated with a given scattering mechanism. The full-rank ABF estimator then is

where 


$$
\begin{gathered}
\widehat{F}_{A B F-F R}(z)=\operatorname{trace}\left(\left[\mathbf{B}^{T *}(z) \widehat{\mathbf{R}}_{P}^{-1} \mathbf{B}(z)\right]^{-1}\right), \\
\widehat{\mathbf{T}}_{A B F}(z)=\left[\mathbf{B}^{T *}(z) \widehat{\mathbf{R}}_{P}^{-1} \mathbf{B}(z)\right]^{-1} .
\end{gathered}
$$

The availability of the polarimetric coherence matrix makes possible the full exploitation of the polarimetric information for the characterization of the scattering, allowing the 3-D calculation of parameters like, e.g. entropy and degree of polarization, as well as the application of polarimetric decompositions.

\subsubsection{Model-Based PolTomoSAR}

As mentioned in Sect. 1.6.1, the non-model-based ABF possess some intrinsic degree of super-resolution, i.e. it is able to separate scatterers with a height difference lower than the Rayleigh resolution limit, which in turn depends on the maximum available track separation. However, a higher super-resolution could be needed in some applications. For this reason, a solution is to resort to model-based tomographic processors, which generally exploit the statistical description of the received signal or equivalently of the scattering behaviours present in the observed scene.

Several methods have been proposed for singlepolarization and then extended to full-polarization MB data sets. For instance, the MUSIC (multiple signal classification) is matched to point-like targets (Frey and Meier 2011), and it exploits the fact that the multibaseline response of each pointlike scatterer (i.e. the steering vector) in the backscattered radiation is orthogonal to the noise subspace. As a consequence, closed-form solution of the MUSIC PolTomoSAR functional can be found that outputs the scattering mechanism of each scatterer (Sauer et al. 2011). Still in the category of the eigen-based processors, the weighted signal subspace fitting can cope with more complex statistical descriptions of distributed and coherent scatterers, although a multidimensional optimization is required (Huang et al. 2011).

Alternatively to the eigen-based PolTomoSAR, a solution adaptive to both coherent and distributed scatterers, but possibly leading to a lower computational time, is the so-called covariance matching estimator (COMET). If the multibaseline data are jointly Gaussian distributed, the knowledge of the MB-multipolarimetric covariance matrix $\mathbf{R}_{P}$ is enough to perform a maximum likelihood (ML) estimation of the parameters describing the vertical distribution of the backscattered power. It can be demonstrated that the global ML problem can be decomposed by means of the extended invariance principle into a cascade of two ML problems (i.e. the estimation of $\mathbf{R}_{P}$ and the estimation of the parameters of interest from $\widehat{\mathbf{R}}_{P}$ ), leading to an asymptotically equivalent solution and with a non-negligible reduction of the computational complexity. Under the Gaussian hypothesis, the resulting COMET estimates can be obtained from the following minimization problem (Tebaldini and Rocca 2010):

$$
\widehat{\boldsymbol{\rho}}=\arg \min _{\boldsymbol{\rho}} \operatorname{trace}\left(\widehat{\mathbf{R}}_{P}^{-1}\left[\mathbf{R}_{P}(\boldsymbol{\rho})-\widehat{\mathbf{R}}_{P}\right] \widehat{\mathbf{R}}_{P}^{-1}\left[\mathbf{R}_{P}(\boldsymbol{\rho})-\widehat{\mathbf{R}}_{P}\right]\right)
$$

where $\boldsymbol{\rho}$ is the vector containing the parameters that describe the multibaseline covariances. Equation (1.204) can be seen as the weighted Frobenius norm of the approximation error $\mathbf{R}_{P}(\boldsymbol{\rho})-\widehat{\mathbf{R}}_{P}$ with weight $\widehat{\mathbf{R}}_{P}^{-1}$. Worth of notice, the COMET estimator can be used also when data are not Gaussian, although it is not asymptotically optimal anymore.

\subsubsection{Coherence Tomography}

Besides the development of spectral estimation- and modelbased PolTomoSAR, also the so-called (polarimetric) coherence tomography methods have been proposed which reconstruct the vertical distribution of scatterers from complex coherence measurements of volumetric scatterers. In a few words, the structure function is approximated through a weighted sum of a series of basis functions (Cloude 2007b). The individual parameterization has then to be inverted using a (limited) number of interferometric measurements at the same or different polarizations. In this class of "hybrid" algorithms, the different polarization channels can be used, e.g. to find a polarization state with lowest ground contribution.

\section{References}

Alonso Gonzalez A, Lopez Martinez C, Salembier P (2012) Filtering and segmentation of polarimetric SAR data based on binary partition trees. IEEE Trans Geosci Remote Sens 50:593-605

Arii M, Van Zyl JJ, Kim Y (2011) Adaptive model-based decomposition of polarimetric SAR covariance matrices. IEEE Trans Geosci Remote Sens 49:1104-1113

Balanis CA (1989) Advanced engineering electromagnetics. Wiley, New York

Ballester Berman JD, Lopez Sanchez JM, Fortuny Guasch J (2005) Retrieval of biophysical parameters of agricultural crops using polarimetric SAR interferometry. IEEE Trans Geosci Remote Sens 43:683-694

Bamler R, Hartl P (1998) Synthetic aperture radar interferometry. Inv Prob 14:R1-R54

Bebbington DHO (1992) Target vectors - spinorial concepts. In: Proc Second International Workshop on Radar Polarimetry

Beckmann P, Spizzichino A (1987) The scattering of electromagnetic waves from rough surfaces. Artech House, Norwood

Boerner WM et al (1998) Polarimetry in radar remote sensing: basic and applied concepts. In: Ryerson RA (ed) Manual of remote sensing: principles and applications of imaging radar, vol 3. Wiley, Hoboken, pp 271-356 
Cameron WL, Leung LK (1990) Feature motivated polarization scattering matrix decomposition. In: Proceedings of IEEE 1990 International Radar Conference

Chandrasekhar S (1960) Radiative transfer. Dover, New York

Cloude SR (1988) Uniqueness of target decomposition theorems in radar polarimetry. In: Proceedings of NATO Adv. Res. Workshop on Direct Inverse Methods in Radar Polarimetry

Cloude SR (2007a) The dual polarization H/alpha decomposition: a PALSAR case study. Paper presented at 2007 international workshop on science and applications of SAR polarimetry and Polarimetric interferometry (POLinSAR)

Cloude SR (2007b) Dual-baseline coherence tomography. IEEE Geosci Remote Sens Lett 4:127-131

Cloude SR, Papathanassiou KP (1998) Polarimetric SAR interferometry. IEEE Trans Geosci Remote Sens 36:1551-1565

Cloude SR, Papathanassiou KP (2003) A 3-stage inversion process for polarimetric SAR interferometry. IEE Proc Radar Sonar Navigation 150:125-134

Cloude SR, Pottier E (1996) A review of target decomposition theorems in radar polarimetry. IEEE Trans Geosci Remote Sens 34:498-518

Cloude SR, Corr DG, Williams ML (2004) Target detection beneath foliage using polarimetric SAR interferometry. Waves Random Media 14:393-414

Colin E, Titin Schneider C, Tabbara W (2003) Investigation of different interferometric coherence optimisation methods. Paper presented at 2003 International Workshop on Science and Applications of SAR Polarimetry and Polarimetric Interferometry (POLinSAR)

Colin E, Titin Schneider C, Tabbara W (2005) Coherence optimization methods for scattering centre separation in polarimetric interferometry. J Electrom Waves App 19:1237-1250

Curlander JC, McDonough RN (1991) Synthetic aperture radar: systems and signal processing. John Wiley \& Sons, New York

Dall J, Papathanassiou KP, Skriver H (2003) Polarimetric SAR interferometry applied to land ice: first results. In: Proceedings of IEEE 2003 International Geoscience and Remote Sensing Symposium (IGARSS)

De Grandi G, Lee JS, Schuler D, Nezry E (2003) Texture and speckle statistics in polarimetric SAR synthesized images. IEEE Trans Geosci Remote Sens 41:2070-2088

Deledalle CA, Tupin F, Denis L (2010) Polarimetric SAR estimation based on non-local means. In: Proceedings of IEEE 2010 International Geoscience and Remote Sensing Symposium (IGARSS)

Doob JL, Ornstein LS, Uhlenbeck GE, Rice SO, Kac M, Chandrasekhar S, Wax N (1954) Selected papers on noise and stochastic processes. Dover Publications, Mineola/New York

Ferro-Famil L, Huang Y, Reigber A (2012) High-resolution SAR tomography using full-rank polarimetric spectral estimators. In: Proceedings of IEEE 2012 International Geoscience and Remote Sensing Symposium (IGARSS)

Flynn T, Tabb M, Carande R (2002) Coherence region shape estimation for vegetation parameter estimation in POLINSAR. In: Proceedings of IEEE 2002 International Geoscience and Remote Sensing Symposium (IGARSS)

Foucher S, Landry T, Lopez Martinez C, Charbonneau F, Gagnon L (2012) An evaluation of PolSAR speckle filters on compact-pol images. In: Proceedings of IEEE 2012 International Geoscience and Remote Sensing Symposium (IGARSS)

Freeman A, Durden SL (1998) A three-component scattering model for polarimetric SAR data. IEEE Trans Geosci Remote Sens 36:963-973

Frey O, Meier E (2011) Analyzing tomographic SAR data of a forest with respect to frequency, polarization, and focusing technique. IEEE Trans Geosci Remote Sens 49:3648-3659

Gabriel AK, Goldstein RM, Zebker HA (1989) Mapping small elevation changes over large areas: differential radar interferometry. J Geo Res 94:9183-9191
Goodman JW (1976) Some fundamental properties of speckle. J Opt Soc Am 66:1145-1149

Goodman JW (1985) Statistical optics. John Wiley \& Sons, New York

Guillaso S, Reigber A (2005) Scatterer characterisation using polarimetric SAR tomography. In: Proceedings of IEEE 2005 International Geoscience and Remote Sensing Symposium (IGARSS)

Hajnsek I, Pottier E, Cloude SR (2003) Inversion of surface parameters from polarimetric SAR. IEEE Trans Geosci Remote Sens 41:727-744

Hoekman DH, Quinones MJ (2002) Biophysical forest type characterization in the colombian amazon by airborne polarimetric SAR. IEEE Trans Geosci Remote Sens 40:1288-1300

Huang Y, Ferro-Famil L, Reigber A (2011) Under-foliage object imaging using SAR tomography and polarimetric spectral estimators. IEEE Trans Geosci Remote Sens 50:2213-2225

Huynen JR (1970) Phenomenological theory of radar targets. Ph.D. thesis, Technical University of Delft, The Netherlands

IEEE standard number 145 (1983) IEEE standard definitions of terms for antennas

Jones RC (1941a) A new calculus for the treatment of optical systems. I Descrip Discussion J Opt Soc Am 31:488-493

Jones RC (1941b) A new calculus for the treatment of optical systems. II Proof of the three general equivalence theorems. J Opt Soc Am 31:493-499

Jones RC (1941c) A new calculus for the treatment of optical systems. III: The Stokes theory of optical activity. J Opt Soc Am 31:500-503

Kasilingam D, Schuler D, Lee JS, Malhotra S (2002) Modulation of polarimetric coherence by ocean features. In: Proceedings of IEEE 2002 International Geoscience and Remote Sensing Symposium (IGARSS)

Kay SM (1993) Fundamentals of statistical signal processing. Estimation theory. Prentice-Hall, Englewood Cliffs

Kennaugh K (1951) Effects of type of polarization on echo characteristics, Technical report. Ohio State University

Kong JA (ed) (1990) Polarimetric remote sensing. Elsevier, New York

Krogager E (1990) New decomposition of the radar target scattering matrix. Electron Lett 26:1525-1527

Lee JS (1981) Speckle analysis and smoothing of synthetic aperture radar images. Comp Graphics Im Proc 17:24-32

Lee JS, Grunes MR, Mango SA (1991) Speckle reduction in multipolarization, multifrequency SAR imagery. IEEE Trans Geosci Remote Sens 29:535-544

Lee JS, Hoppel KW, Mango SA, Miller AR (1994) Intensity and phase statistics of multilook polarimetric and interferometric SAR imagery. IEEE Trans Geosci Remote Sens 32:1017-1028

Lee JS, Grunes MR, De Grandi G (1999) Polarimetric SAR speckle filtering and its implication for classification. IEEE Trans Geosci Remote Sens 37:2363-2373

Lee JS, Schuler DL, Ainsworth TL, Krogager E, Kasilingam D, Boerner WM (2002) On the estimation of radar polarization orientation shifts induced by terrain slopes. IEEE Trans Geosci Remote Sens 40:30-41

Lee JS, Cloude SR, Papathanassiou KP, Grunes MR, Woodhouse IH (2003) Speckle filtering and coherence estimation of PolInSAR data for forest applications. IEEE Trans Geosci Remote Sens 41:2254-2263

Lee JS, Grunes MR, Schuler DL, Pottier E, Ferro-Famil L (2006) Scattering-model-based speckle filtering of polarimetric SAR data. IEEE Trans Geosci Remote Sens 44:176-187

Lombardini F, Reigber A (2003) Adaptive spectral estimators for multibaseline SAR tomography with airborne L-band data. In: Proceedings of IEEE 2003 International Geoscience and Remote Sensing Symposium (IGARSS)

Lopes A, Touzi R, Nezry E (1990) Adaptive speckle filters and scene heterogeneity. IEEE Trans Geosci Remote Sens 28:992-1000

Lopez Martínez C, Fabregas X (2003) Polarimetric SAR speckle noise model. IEEE Trans Geosci Remote Sens 41:2232-2242 
Lopez Martínez C, Fabregas X (2008) Model-based polarimetric SAR speckle filter. IEEE Trans Geosci Remote Sens 46:3894-3907

Luneburg E (2002) Aspects of radar polarimetry. Elektrik- Turkish J Electr Eng Comput Sci 10:219-243

Mattia F, le Toan T, Lee JS, Schuler DL (2003) On the sensitivity of polarimetric coherence to small and large scale surface roughness. In: Proceedings of IEEE 2003 International Geoscience and Remote Sensing Symposium (IGARSS)

McCrea WH, Whipple FJW (1940) Random paths in two and three dimensions. Proc R Soc Edinburgh 60:281-298

Mette T, Papathanassiou KP, Hajnsek I (2004) Biomass estimation from PolInSAR over heterogeneous terrain. In: Proc IEEE 2004 International Geoscience and Remote Sensing Symposium (IGARSS)

Morio J, Refregier P, Goudail F, Dubois Fernandez P, Dupuis X (2007) Application of information theory measures to polarimetric and interferometric SAR images. In: Proceedings of 2007 International Conference on Physics in Signal and Image Processing

Novak L, Burl MC (1990) Optimal speckle reduction in polarimetric SAR imagery. IEEE Trans Aerosp Electron Syst AES-26:293-305

Oliver C, Quegan S (1998) Understanding synthetic aperture radar images. Artech House, Boston

Paladini R, Ferro-Famil L, Pottier E, Martorella M, Berizzi F, Dalle Mese E (2012) Lossless and sufficient-invariant decomposition of random reciprocal target. IEEE Trans Geosci Remote Sens 50:3487-3501

Papathanassiou KP, Cloude SR (2001) Single baseline polarimetric SAR interferometry. IEEE Trans Geosci Remote Sens 39:2352-2363

Papathanassiou KP, Cloude SR, Reigber A (2000) Single and multibaseline polarimetric SAR interferometry over forested terrain. In: Proceedings of 2000 European Conference on Synthetic Aperture Radar (EUSAR)

Papathanassiou KP, Cloude SR, Liseno A, Mette T, Pretzsch H (2005a) Forest height estimation by means of polarimetric SAR interferometry: actual status and perspectives. Paper presented at 2005 International Workshop on Science and Applications of SAR Polarimetry and Polarimetric Interferometry (POLinSAR)

Papathanassiou KP, Hajnsek I, Nagler T, Rott H (2005b) Polarimetric SAR interferometry for snow cover parameter estimation. Paper presented at 2005 international workshop on science and applications of SAR polarimetry and polarimetric interferometry (POLinSAR)

Papoulis A (1984) Probability, random variables and stochastic processes. McGraw-Hill, New York

Pasquali P, Prati C, Rocca C, Seymour M, Fortuny J, Olmer E, Sieber AJ (1995) A 3D SAR experiment with EMSL data. In: Proceedings of IEEE 1995 International Geoscience and Remote Sensing Symposium (IGARSS)

Raney RK (1983) Transfer function for partially coherent SAR systems. IEEE Trans Aerosp Electron Syst AES-19:740-750

Reigber A, Moreira A (2000) First demonstration of airborne SAR tomography using multibaseline L-band data. IEEE Trans Geosci Remote Sens 38:2142-2152

Reigber A, Papathanassiou KP, Cloude SR, Moreira A (2000) SAR tomography and interferometry for the remote sensing of forested terrain. In: Proceedings of 2000 European Conference on Synthetic Aperture Radar (EUSAR)

Sagues L, Lopez Sanchez JM, Fortuny J, Fabregas X, Broquetas A, Sieber AJ (2000) Indoor experiments on polarimetric SAR interferometry. IEEE Trans Geosci Remote Sens 38:671-684

Sarabandi K (1997) $\Delta$ k-radar equivalent of interferometric SAR's: a theoretical study for determination of vegetation height. IEEE Trans Geosci Remote Sens 35:1267-1276

Sauer S, Ferro-Famil L, Reigber A, Pottier E (2011) Three-dimensional imaging and scattering mechanism estimation over urban scenes using dual-baseline polarimetric InSAR observations at L-band. IEEE Trans Geosci Remote Sens 49:4616-4629
Schleher DC (1975) Radar detection in log normal clutter. In: Proceedings of IEEE 1975 International Radar Conference

Schneider Zandona R, Papathanassiou K, Hajnsek I, Moreira A (2005) Polarimetric interferometry over urban areas: information extraction using coherent scatterers. In: Proc IEEE 2005 International Geoscience and Remote Sensing Symposium (IGARSS)

Stokes GG (1852) On the composition and resolution of streams of polarized light from different sources. Trans Cambridge Phil Soc 9:399-416

Tabb M, Carande R (2001) Robust inversion of vegetation structure parameters from low frequency polarimetric interferometric SAR. In: Proceedings of IEEE 2001 International Geoscience and Remote Sensing Symposium (IGARSS)

Tebaldini S (2009) Algebraic synthesis of forest scenarios from multibaseline PolInSAR data. IEEE Trans Geosci Remote Sens 47:4132-4142

Tebaldini S, Rocca F (2010) Single and multipolarimetric SAR tomography of forested areas: a parametric approach. IEEE Trans Geosci Remote Sens 48:2375-2387

Tough RJA, Blacknell D, Quegan S (1995) A statistical description of polarimetric and interferometric synthetic aperture radar data. Proc $\mathrm{R}$ Soc London 449:567-589

Touzi R (2007) Target scattering decomposition in terms of rollinvariant target parameters. IEEE Trans Geosci Remote Sens 45:73-84

Touzi R, Lopes A (1994) The principle of speckle filtering in polarimetric SAR imagery. IEEE Trans Geosci Remote Sens 32:1110-1114

Touzi R, Lopes A, Bruniquel J, Vachon PW (1999) Coherence estimation for SAR imagery. IEEE Trans Geosci Remote Sens 37:135-149

Touzi R, Boerner WM, Lee JS, Luneberg E (2004) A review of polarimetry in the context of synthetic aperture radar: concepts and information extraction. Can J Remote Sens 30:380-407

Touzi R, Deschamps A, Rother G (2007) Wetland characterization using polarimetric RADARSAT-2 capability. Can J Remote Sens 33:56-67

Treuhaft RN, Cloude SR (1999) The structure of oriented vegetation from polarimetric interferometry. IEEE Trans Geosci Remote Sens 37:2620-2624

Treuhaft RN, Madsen S, Moghaddam M, Van Zyl JJ (1996) Vegetation characteristics and underlying topography from interferometric data. Radio Sci 31:1449-1495

Trunk GV (1972) Radar properties of non-Rayleigh sea echo. IEEE Trans Aerosp Electron Syst AES-8:196-204

Trunk GV, George SF (1970) Detection of targets in non-gaussian sea clutter. IEEE Trans Aerosp Electron Syst AES-6:620-628

Ulaby FT, Elachi C (1990) Radar polarimetry for geoscience applications. Artech House, Norwood

Ulaby FT, Moore RK, Fung AK (1986a) Microwave remote sensing: active and passive, vol III. Artech House, Norwood

Ulaby FT, Moore RK, Fung AK (1986b) Microwave remote sensing: active and passive, vol II. Artech House, Norwood

Van Zyl JJ, Arii M, Kim Y (2011) Model-based decomposition of polarimetric SAR covariance matrices constrained for nonnegative eigenvalues. IEEE Trans Geosci Remote Sens 49:3452-3459

Vasile G, Trouve E, Lee JS, Buzuloiu V (2006) Intensity-driven adaptive-neighbourhood technique for polarimetric and interferometric SAR parameters estimation. IEEE Trans Geosci Remote Sens 44:1609-1621

Wakabayashi H, Matsuoka T, Nakamura K, Nishio F (2004) Polarimetric characteristics of sea ice in the sea of Okhotsk observed by airborne L-band SAR. IEEE Trans Geosci Remote Sens 42:2412-2425

Wiener N (1930) Generalized harmonic analysis. Acta Mathematica $5: 118-258$

Williams ML (1999) Prediction and observation of SAR clutter from vegetation canopies. In: Proc IEEE 1999 International Geoscience and Remote Sensing Symposium (IGARSS) 
Williams ML, Cloude SR (2005) Predictions of SAR polarimetry and InSAR coherence for a model wheat canopy. In: Proceedings of IEEE 2005 International Geoscience and Remote Sensing Symposium (IGARSS)

Yamada H, Yamaguchi Y, Rodriguez E, Kim Y, Boerner WM (2001) Polarimetric SAR interferometry for forest canopy analysis by using the super-resolution method. IEICE Trans Electron E84-C:1917-1924
Yamaguchi Y, Moriyama T, Ishido M, Yamada H (2005) Fourcomponent scattering model for polarimetric SAR image decomposition. IEEE Trans Geosci Remote Sens 43:1699-1706

Zebker HA, Villasenor J (1992) Decorrelation in interferometric radar echoes. IEEE Trans Geosci Remote Sens 30:950-959

Open Access This chapter is licensed under the terms of the Creative Commons Attribution 4.0 International License (http://creativecommons.org/ licenses/by/4.0/), which permits use, sharing, adaptation, distribution and reproduction in any medium or format, as long as you give appropriate credit to the original author(s) and the source, provide a link to the Creative Commons license and indicate if changes were made.

The images or other third party material in this chapter are included in the chapter's Creative Commons license, unless indicated otherwise in a credit line to the material. If material is not included in the chapter's Creative Commons license and your intended use is not permitted by statutory regulation or exceeds the permitted use, you will need to obtain permission directly from the copyright holder. 


\title{
Forest Applications
}

\author{
K. P. Papathanassiou, S. R. Cloude, M. Pardini, M. J. Quiñones, D. Hoekman, \\ L. Ferro-Famil, D. Goodenough, H. Chen, S. Tebaldini, M. Neumann, \\ L. M. H. Ulander, and M. J. Soja
}

\begin{abstract}
The application of polarimetric Synthetic Aperture Radar (SAR) to forest observation for mapping, classification and parameter estimation (especially biomass) has a relatively long history. The radar penetration through forest volume, and hence the multi-layer nature of scattering models, make fully polarimetric data the observation space enabling a robust and full inversion of such models. A critical advance came with the introduction of polarimetric SAR
\end{abstract}

K. P. Papathanassiou $(\bowtie) \cdot$ M. Pardini

Microwave and Radar Institute, German Aerospace Center (DLR),

Wessling, Germany

e-mail: kostas.papathanassiou@dlr.de

S. R. Cloude

AEL Consultants, Cupar, Fife, UK

M. J. Quiñones

SARvision, Wageningen, The Netherlands

D. Hoekman

Department of Environmental Sciences, Wageningen University,

Wageningen, The Netherlands

\section{Ferro-Famil}

Institut d'Électronique et de Télécommunications de Rennes, University of Rennes-1, Rennes, France

D. Goodenough

Department of Computer Science, University of Victoria, Victoria, Canada

H. Chen

Natural Resources Canada, Canadian Forest Service, Victoria, Canada

S. Tebaldini

Dipartimento di Elettronica, Informazione e Bioingegneria, Politecnico di Milano, Milan, Italy

M. Neumann

Google Cloud AI, Google, Sunnyvale, CA, USA

L. M. H. Ulander

Department of Space, Earth and Environment, Chalmers University of Technology, Gothenburg, Sweden

M. J. Soja

Horizon Geoscience Consulting, Belrose, Australia

University of Tasmania, Sandy Bay, TAS, Australia interferometry, where polarimetry provides the parameter diversity, while the interferometric baseline proves a userdefined entropy control as well as spatial separation of scattering components, together with their location in the third dimension (height). Finally, the availability of multiple baselines leads to the full 3-D imaging of forest volumes through TomoSAR, the quality of which is again greatly enhanced by the inclusion of polarimetry. The objective of this Chapter is to review applications of SAR polarimetry, polarimetric interferometry and tomography to forest mapping and classification, height estimation, 3-D structure characterization and biomass estimation. This review includes not only models and algorithms, but it also contains a large number of experimental results in different test sites and forest types, and from airborne and space borne SAR data at different frequencies.

\section{$2.1 \quad$ Introduction}

The application of radar polarimetry to forestry has a long history. Ever since the earliest days of airborne data trials with the JPL-AIRSAR system it was realized that forest scattering at microwave frequencies generates more linear cross-polarization (HV) than non-forest (especially at lower radar frequencies such as P- and L- bands). Since then, various groups have attempted to develop algorithms for the generation of imaging radar products based on forest mapping, classification and parameter estimation (especially biomass) requirements.

It was also quickly realized that improved products were obtained by using fully coherent scattering matrix or quadpol PolSAR systems. These then allow the application of target decomposition and multivariate classification techniques accounting not only for backscatter amplitude and ratios, but also for phase and coherence statistics. The physics behind these techniques is based on the idea that there is significant forest penetration of microwave radiation, 
Table 2.1 Remote sensing applications on forestry

\begin{tabular}{l|l|l}
\hline (End) Users & Application(s) & Interest/Motivation \\
\hline \multirow{2}{*}{ National International Authorities/Agencies } & Forest biomass & Carbon cycle \& environmental science \\
\cline { 2 - 3 } & Deforestration & Carbon cycle \& environmental science \\
\cline { 2 - 3 } & Forest type classification & Biodiversity \\
\hline \multirow{2}{*}{ Forest Management } & Forest (top) height & Inventory (selective) \\
\cline { 2 - 3 } & Species classification & Biodiversity, Forest monitoring \\
\cline { 2 - 3 } & Mapping of storm damage & Forest monitoring \\
\hline
\end{tabular}

even to the surface layer under the forest, and hence multilayer scattering theories are required to properly interpret the signatures. These multi-layer approaches in turn require multiple parameters for model based estimation and inversion and the use of quadpol data allows much more robust inversion of such models, providing as it does a wider set of observables than classical single or even dual-pol radars.

Still there remained a problem unique to forestry, namely high scattering entropy due to the complexity of the random media scattering environment generated by forests. This inevitably leads to lower accuracy and poorer resolution products. A significant advance therefore came with realization that lower entropy scattering could be obtained for forests by combining polarimetry with interferometry for PolInSAR. Here polarimetry provides again the parameter diversity, with the interferometric baseline now providing a user defined entropy control as well as spatial separation of scattering components.

This concept has also recently been extended to consider multiple baselines for multibaseline PolInSAR, which in the limit leads to 3-D imaging of forests through TomoSAR, while for limited baselines offers band-limited 3-D imaging, the quality of which is again greatly enhanced by the inclusion of polarimetry. This technology has now matured to the stage where several important products (especially forest height and vertical structure) can be accurately obtained at high spatial resolutions and with wide continuous coverage. Since 2006, with the launch of ALOS-PALSAR, such quad-pol capabilities have been available routinely from space imaging radars, enabling important developments in product maturity, as well as opening new possibilities by using time series analysis to capture dynamic changes in forests. A general classification of the applications is reported in Table 2.1.

\subsection{Forest Classification}

\subsubsection{Land Cover Classification in Tropical Lands Using PolSAR}

\subsubsection{Introduction, Motivation and Literature Review}

Recent radar space borne systems, like the C-band ENVISAT-ASAR, the C-band RADARSAT and the L-band ALOS-PALSAR systems, offer unique possibilities of mapping and monitoring the tropical forest, usually covered by clouds. Nevertheless it is still not clear which are the advantages of complex fully polarimetric systems over simple single or double polarized systems for certain applications. Also the specific use of frequencies and frequency combinations is still unclear: long radar wavelength like at L- and P- bands could add information to the short wavelengths at $\mathrm{C}$ - and $\mathrm{X}$ - bands due to the different scattering mechanisms involved in the wave interactions with the forest, the difference in canopy penetration, improving the classification of land cover classes or forest types.

Polarimetric radar classification simulations gave in the past insights into the accuracy of using certain band/polarization combination for land cover, forest type and biomass mapping (Hoekman and Quiñones 2000, 2002; Quiñones 2002). Nevertheless this information needed to be recreated on the frame of recent versatile, robust and computational efficient algorithms that can be applied over polarimetric and multi-frequency space borne data. In this Section, a pixel based unsupervised classification technique, developed in Hoekman et al. (2011), is used as a research tool to evaluate the NASA's AIRSAR, C-, L- and P-band radar data acquired in 1993 over the Guaviare site in the Colombian Amazon. A polarimetric decomposition algorithm, that preserves the full polarimetric information content into six different radar intensities is used. Results give indication on the added valued of certain frequency and polarization combination in a tropical land. The robustness of the algorithm is further demonstrated by its applications to fine beam dual-pol (FBD) L-band $\mathrm{HH} / \mathrm{HV}$ and wide beam (WB) L-band HH ALOSPALSAR data in central Kalimantan.

\subsubsection{Methodology}

Classification accuracy results from unsupervised segmentations applied to different combinations of polarimetric $\mathrm{C}, \mathrm{L}$ and $\mathrm{P}$ band data are used to evaluate the possible radar band combinations useful for tropical forest monitoring and mapping. Use is made of the unsupervised fully polarimetric SAR segmentation tool developed in Hoekman et al. (2011). The unsupervised approach consists of six processing steps extensively explained in Hoekman and Vissers (2003). The first step is a mathematical data transform which allows polarimetric data, without loss of any information, to be written in a form where classes are 
well approximated by multivariate normal distributions. This transform allows application of a wide class of mature image processing algorithms to polarimetric data, including unsupervised data clustering. The second step relates to unsupervised clustering encompassing a simple region-growing segmentation (incomplete and over-segmented), followed by model-based agglomerative clustering (Step 3), and expectation-maximization on the pixels of these segments (Step 4). Classification is achieved by Markov random field filtering on the original data (Step 5). The result is a series of segmented maps, which differ in the number of (unsupervised) classes.

For the analysis of the results three different accuracy percentages are used as indicators of the performance of a particular polarization/frequency combination in the classification of the four cover types. The first is the overall classification accuracy, calculated as the percentage of right and wrong classified pixels for all the classes, for a particular polarization/frequency combination. A Kappa statistic $(\widehat{K})$ was computed to evaluate significant differences between any pair of classification results (Lillesand and Kiefer 1994). A test statistic $\Delta \widehat{K}$ can be calculated as:

$$
\Delta \widehat{K}=\frac{\left|\widehat{K}_{1}-\widehat{K}_{2}\right|}{\sqrt{\widehat{\sigma}_{\infty}^{2}\left[\widehat{K}_{1}\right]+\widehat{\sigma}_{\infty}^{2}\left[\widehat{K}_{2}\right]}}
$$

where $\widehat{\sigma}_{\infty}^{2}[\widehat{K}]$ is the approximate large sample variance of $\widehat{K}$. At the $95 \%$ confidence level two results may be considered significantly different if $\Delta \widehat{K}>1.96$ (Benson and De Gloria 1985).

The second accuracy percentage is the users classification accuracy that indicates the percentages of pixels classified in a certain class given that the pixel was label into that class. This particular indicator is useful to evaluate the capacity of a certain combination to classify a class.
The third indicator is the percentage of confusion between two particular land cover types in the absence of other classes. This indicator is of particular interest for the evaluation of possible monitoring scenarios in a changing tropical forest. Monitoring scenarios are defined as the capacity to differentiate processes like deforestation, forest degradation and forest regeneration as explained in Hoekman and Quiñones (2000).

\subsubsection{Experimental Results}

Test sites and corresponding radar and validation data sets selected for the generation of showcases on land cover classification in tropical lands are summarized in Table 2.2 and further described in Appendix A. Figure 2.1 shows an overview of the radar data.

Fully polarimetric target properties for uniform distributed scatterers can be described by nine single-pol radar intensities as introduced in Hoekman and Vissers (2003). For the AIRSAR data the Stokes scattering operator matrix was set to zero for the four 'asymmetric' elements of the covariance matrix. For that reason it is assumed that the objects display azimuthal symmetry and that the asymmetrical information may be discarded. In this case the case of azimuthal symmetry (Freeman 1999) the covariance matrix simplifies to.

$$
\mathbf{C}_{r}=\left(\begin{array}{ccc}
\left\langle S_{h h} S_{h h}^{*}\right\rangle & 0 & \left\langle S_{h h} S_{v v}^{*}\right\rangle \\
0 & \left\langle S_{h v} S_{h v}^{*}\right\rangle & 0 \\
\left\langle S_{v v} S_{h h}^{*}\right\rangle & 0 & \left\langle S_{v v} S_{v v}^{*}\right\rangle
\end{array}\right)
$$

In the intensity representation introduced in Hoekman and Vissers (2003) it is possible to find several sets of 5 independent intensity values containing this and only symmetrical information. At least one (non-redundant) possibility is needed to represent the polarimetric data. A selection of 6 intensities were made using the conjugated Real and Imaginary parts of the HH-VV phase differences as follows:

$$
\left(\begin{array}{c}
\left\langle S_{h h} \cdot S_{h h}^{*}\right\rangle \\
\left\langle S_{v v} \cdot S_{v v}^{*}\right\rangle \\
\left\langle S_{h v} \cdot S_{h v}^{*}\right\rangle \\
\operatorname{Re}\left[\left\langle S_{h h} \cdot S_{v v}^{*}\right\rangle\right] \\
\operatorname{Im}\left[\left\langle S_{h h} \cdot S_{v v}^{*}\right\rangle\right]
\end{array}\right)=\mathbf{B}_{r}\left(\begin{array}{c}
\sigma_{h h}^{0} \\
\sigma_{v v}^{0} \\
\sigma_{h v}^{0} \\
\sigma_{+-45}^{0} \\
\sigma_{+45 l}^{0}+\sigma_{-45 r}^{0}
\end{array}\right) \text { with } \mathbf{B}_{r}=\frac{1}{4 \pi}\left(\begin{array}{ccc:cc}
1 & 0 & 0 & 0 & 0 \\
0 & 1 & 0 & 0 & 0 \\
0 & 0 & 1 & 0 & 0 \\
\hdashline \frac{1}{2} & \frac{1}{2} & 0 & -2 & 0 \\
\frac{1}{2} & \frac{1}{2} & 1 & 0 & -1
\end{array}\right)
$$


Table 2.2 Test sites and corresponding radar and validation data selected for the generation of showcases on land cover classification

\begin{tabular}{l|l|l}
\hline Application/product & Test site - Radar data & Reference data \\
\hline $\begin{array}{l}\text { Land cover classification } \\
\text { in tropical lands }\end{array}$ & $\begin{array}{l}\text { Guaviare, Colombian Amazon } \\
\text { AIRSAR P-, L-, and C-band data (1993), } \\
\text { incidence angle from } 20^{\circ} \text { to } 60^{\circ}\end{array}$ & $\begin{array}{l}\text { Direct field observations on vegetation and terrain characteristics } \\
\text { like drainage, flooding and slopes }\end{array}$ \\
\hline
\end{tabular}
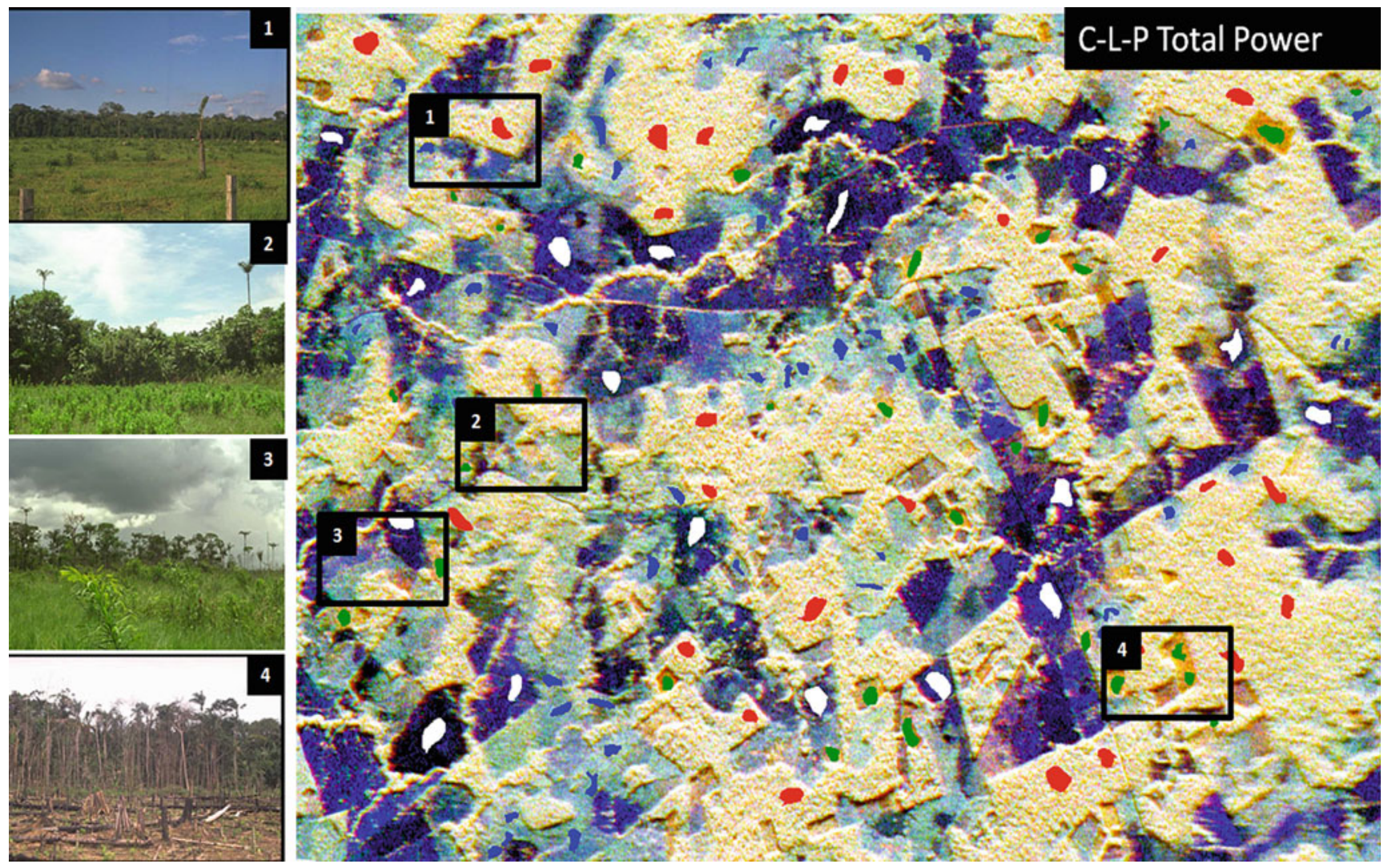

Fig. 2.1 Total power image of the P-, L- and C-band AIRSAR data, in the $45^{\circ}-50^{\circ}$ incidence angle range, over the Guaviare study site. Photographs illustrate the four vegetation cover types in this study. Polygons digitized over the visited field locations for all the four cover

Intensity images created for the C-, L-, and P-band data are shown in Fig. 2.2.

For each single frequency, comparisons were made between the combinations of dual-like polarizations $(\mathrm{HH} / \mathrm{VV})$, three linear polarizations $(\mathrm{HH} / \mathrm{VV} / \mathrm{HV})$ and all 6 intensity polarizations (Pol-6i) containing the polarimetric information (HH/VV/HV/MR/PL/PM). For the comparison between multi-frequency data, combinations where made using two and three frequencies (C-L), (L-P), (C-L-P) with three linear polarizations $(\mathrm{HH} / \mathrm{VV} / \mathrm{HV})$ and with all polarimetric data per frequency (HH/VV/HV/MR/PL/PM). Figure 2.3 shows the classification result from the unsupervised segmentation using different combinations. Table 2.3 shows all three accuracy percentages calculated for the studied frequency/polarizations combinations. Low overall classification accuracies and low users accuracy per class were types are illustrated: (1) primary forest (red): 27 polygons (4983 pixels); (2) secondary forest (blue): 49 polygons (4004 pixels); (3) recently deforested areas (green): 30 polygons (2878 pixels); and (4) grasslands (white): 18 polygons (4046 pixels)

found for all C-band combinations and for L- and P-band dual-pol (HH/VV)/single frequency combinations. High confusion between classes was also found for these channel combinations. For both L- and P-band the results by adding the HV channel to the dual-pol $(\mathrm{HH} / \mathrm{VV})$ were significantly higher $(94.5 \%$ to $85.5 \%$, for L-band and $93.5 \%$ to $61.2 \%$ for P-band, respectively) and improved both the users accuracy per class and the confusion between classes especially between primary and secondary forest and primary and recently cut areas in both cases. When these classes are confused the particular combination will not addressed the monitoring scenarios for detection of forest regeneration or deforestation processes, at least using only single-date data. The addition of HV polarization to the dual-pol (HH/VV) data, have significant impact on the classification accuracy for both L- and P-band data. 

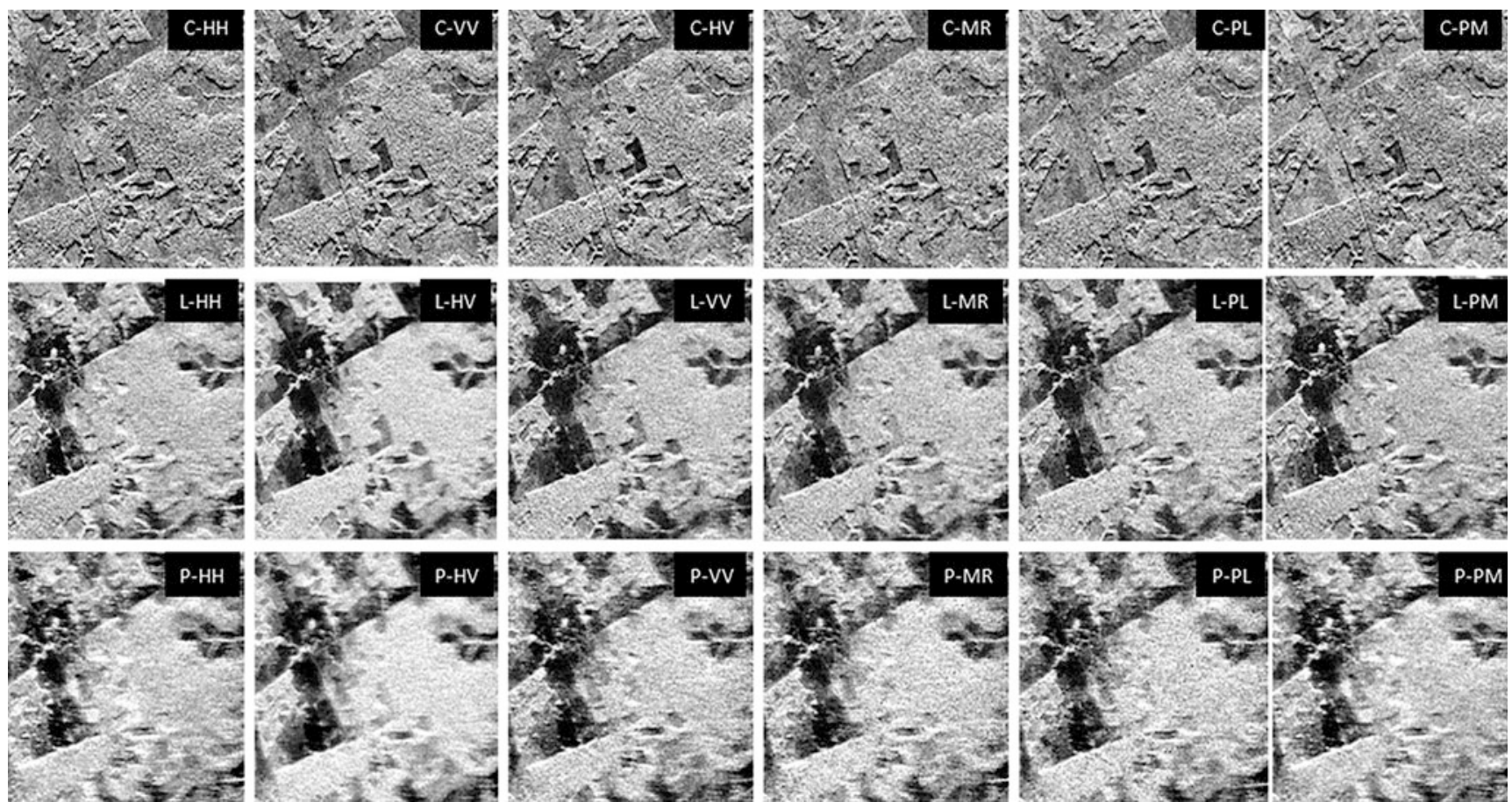

Fig. 2.2 Intensity images derived from the C-, L-, and P-band AIRSAR polarimetric decompositions into intensity images. Horizontal (h), vertical (v), left circular (l), right circular (r), $45^{\circ}$ linear (p) and $-45^{\circ}$ linear (m)
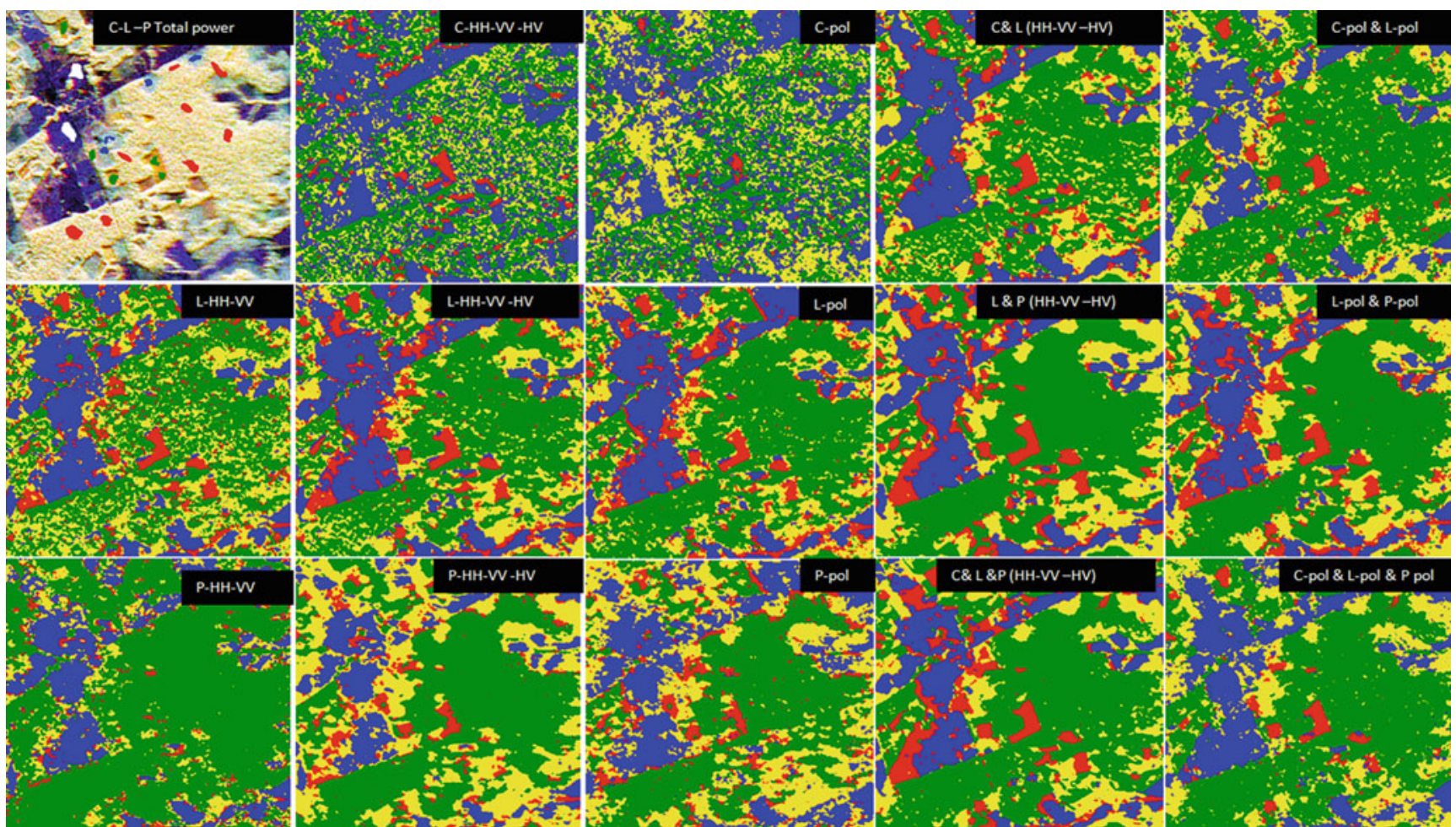

S. Lol \& P.pol

Fig. 2.3 A $400 \times 400$ pixels window, classification maps resulting from the unsupervised segmentation of images when using different frequency/polarization combinations. (1) primary forest (green); (2) secondary forest (yellow); (3) recently deforested areas (red); and

(4) grasslands (blue). In the top left corner, a total power image is shown with some of the validation polygons that have been used as a reference to evaluate the results 
Table 2.3 Accuracy classification results for different polarization combinations for all AIRSAR channels studied

\begin{tabular}{|c|c|c|c|c|c|c|c|c|c|c|c|c|c|}
\hline \multicolumn{4}{|c|}{$\begin{array}{l}\text { Overall classification accuracy calculated between } \\
\text { all classes }\end{array}$} & \multicolumn{4}{|c|}{$\begin{array}{l}\text { Users accuracy in percentage of right } \\
\text { classified pixels per class }\end{array}$} & \multicolumn{6}{|c|}{$\begin{array}{l}\text { Confusion between classes in absence of } \\
\text { other classes }\end{array}$} \\
\hline Band & Polariz. & Right $\%$ & Wrong \% & 1 & 2 & 3 & 4 & $1-2$ & $1-3$ & $1-4$ & $2-3$ & $2-4$ & $3-4$ \\
\hline $\mathrm{C}$ & HH/VV & 55.8 & 44.2 & 60.5 & 51.5 & 81.7 & 54.5 & 41.0 & 2.7 & 7.7 & 6.8 & 8.8 & 36.6 \\
\hline $\mathrm{C}$ & $\mathrm{HH} / \mathrm{VV} / \mathrm{HV}$ & 56.6 & 43.4 & 54.6 & 42.1 & 82.3 & 60.9 & 46.9 & 3.7 & 6.4 & 2.4 & 14.3 & 28.3 \\
\hline $\mathrm{C}$ & Pol-6i & 57.9 & 42.1 & 56.6 & 45.4 & 93.8 & 61.8 & 38.6 & 12.1 & 8.1 & 4.6 & 17.5 & 22.9 \\
\hline $\mathrm{L}$ & HH/VV & 85.5 & 14.5 & 78.1 & 77.8 & 87.7 & 99.1 & 17.5 & 9.0 & 0.0 & 3.6 & 0.3 & 3.6 \\
\hline $\mathrm{L}$ & $\mathrm{HH} / \mathrm{VV} / \mathrm{HV}$ & 94.5 & 5.5 & 91.7 & 91.2 & 96.3 & 99.1 & 7.6 & 0.8 & 0.0 & 2.0 & 0.3 & 0.3 \\
\hline L & Pol-6i & 90.7 & 9.3 & 81.0 & 91.3 & 97.0 & 99.3 & 13.4 & 1.1 & 0.0 & 2.9 & 0.6 & 0.6 \\
\hline $\mathrm{P}$ & HH/VV & 61.2 & 38.8 & 58.3 & 41.9 & 16.2 & 91.6 & 21.3 & 27.1 & 0.1 & 57.1 & 18.2 & 0.2 \\
\hline $\mathrm{P}$ & $\mathrm{HH} / \mathrm{VV} / \mathrm{HV}$ & 93.5 & 6.5 & 96.9 & 84.2 & 98.6 & 96.5 & 2.5 & 2.0 & 0.0 & 7.1 & 2.5 & 0.4 \\
\hline $\mathrm{P}$ & Pol-6i & 89.9 & 10.1 & 97.4 & 81.6 & 94.7 & 87.9 & 4.5 & 3.2 & 0.0 & 5.7 & 6.8 & 1.6 \\
\hline C-L & $\mathrm{HH} / \mathrm{VV} / \mathrm{HV}$ & 91.7 & 8.3 & 88.4 & 87.8 & 91.9 & 98.2 & 10.2 & 2.1 & 0.0 & 3.9 & 0.4 & 0.7 \\
\hline L-P & $\mathrm{HH} / \mathrm{VV} / \mathrm{HV}$ & 96.2 & 3.8 & 94.8 & 92.7 & 98.9 & 99.4 & 3.4 & 3.4 & 0.0 & 0.5 & 0.4 & 0.2 \\
\hline C-L & Pol-6i & 91.5 & 8.5 & 84.7 & 86.5 & 99.0 & 99.9 & 10.6 & 1.6 & 0.1 & 4.6 & 0.4 & 0.0 \\
\hline L-P & Pol-6i & 98.0 & 2.0 & 99.0 & 95.3 & 97.3 & 99.9 & 1.8 & 0.4 & 0.0 & 0.5 & 0.4 & 0.9 \\
\hline C-L-P & $\mathrm{HH} / \mathrm{VV} / \mathrm{HV}$ & 98.0 & 2.0 & 97.8 & 95.5 & 99.5 & 99.6 & 2.8 & 0.5 & 0.0 & 0.1 & 0.2 & 0.1 \\
\hline C-L-P & Pol-6i & 96.2 & 3.8 & 98.0 & 91.3 & 100. & 96.8 & 1.8 & 0.6 & 0.1 & 2.5 & 1.2 & 2.0 \\
\hline
\end{tabular}

The bold numbers indicate the best result plus the results that are not significantly different from the best result at the $95 \%$ level of confidence. (1) Primary forest; (2) Secondary forest; (3) Recently deforested areas; and (4) Grasslands

The addition of polarimetric (Pol-6i) data to the three polarization $(\mathrm{HH} / \mathrm{VV} / \mathrm{HV})$ combinations decreases the overall classification accuracy and in most of the cases increases the confusion between primary and secondary forest. In general the confusion between classes is below $10 \%$, for all the land cover pairs, when using L- (HH/VV/HV) or $\mathrm{P}-(\mathrm{HH} / \mathrm{VV} /$ $\mathrm{HV}$ ) and P- (Pol-6i) combinations. When comparing the results produced by the $\mathrm{L}$ - and the P-band combinations there are no significant differences in the results, meaning that both single L- or P- band data (HH/HV/VV) or (Pol-6i) are very good to assess the monitoring scenarios. All overall accuracies are above $90 \%$ for the frequency/polarizations combination. For the two frequencies combinations, the accuracy results of the C-L combination ( $91.5 \%$ and $91.7 \%$ ) are significantly lower than the combinations of the L-P combinations (96.2\% and 98\%) for (HH/VV/HV) and (Pol-6i) respectively. Lower percentages when using C-band are explained by the relatively lower "user's accuracy" classification results, for primary and secondary forest and the high confusion found between these same classes.

The combination of C-L-P (HH/VV/HV) and L-P (Pol-6i) were not significantly different from each other. These combinations also show high users accuracy for all the classes and low confusion percentages between all pairs of cover types, addressing all the monitoring scenarios.

\subsubsection{Discussion on the Role of Polarimetry, on the Maturity of the Application and Conclusions}

Most of the classifications for the combinations involving C-band channels appear to be very irregular affecting the accuracy results. The low classification accuracies and the high confusion between classes, found when using the C-band single frequency combinations and the C-L multifrequency combinations are obviously suffering from the effect of rough texture in the C-band images (high variance between neighboring pixels) due to the higher resolution of the C-band channels and also by the direct scattering occurring between the short $\mathrm{C}$-band waves with the leaves and branches of the rough canopy of the primary forest and secondary vegetation. On the other hand, the results involving C-band channels are also affected by the nature of the classification algorithm and the application of the Markov random field filter to the segmentation procedure. When there is much variance between neighbors the classification of a pixel might be more affected by system filter parameters than for channels with less texture.

For combinations involving L- and P-band channels the classifications are smoother and borders are better defined. Classification accuracy results are higher, which might be explained by the physical interactions, mostly double bounces and volume scattering, occurring between the longer wavelengths and the larger scatterers in this land cover classes. These frequencies are more sensitive to contrasting vegetation structure as is the case by the cover types selected for this study.

The use of polarimetric data for both single frequency and multi frequency combinations, for the L- and P-band channels, did not add significant information compared to the $(\mathrm{HH} / \mathrm{VV} / \mathrm{HV})$ combinations. For this contrasting vegetation structures polarimetric information is of no need, but 
might be of relevance for other applications like forest type mapping (Quiñones 2002).

In general, for addressing the monitoring scenarios in the tropical forest when using the land cover classes used in this study, the L- and P-band linear polarizations (HH/VV/HV) appear to be suitable, and there is no evidence that could show that any of this two frequencies should be preferred over the other.

What is certainly clear is that the use of only two like polarizations for the $\mathrm{L}$ and $\mathrm{P}$ band was not enough to differentiate this land cover classes and not good enough to address the monitoring scenarios in this study. The use of HV polarization significantly improved the overall classification accuracies and decreased the confusion between the cover classes. At that respect, the assessment of dual polarizations involving cross-polarized data (HV) is of interest for future studies.

With the launch of polarimetric space borne SAR systems like RADARSAT-2 (C-band) in December 2007 and ALOSPALSAR (L-band) in January 2006, the need for simple, robust and accurate polarimetric classification and biophysical parameter estimation algorithms for monitoring applications and research is of great importance. Ideally, algorithms should be sufficiently versatile to handle multiband, multi-polarization, multi-date and/or multi-sensor data sets. Moreover, it would be an important asset when algorithms could deal with situations were ground truth is sparse or incomplete. Combination of unsupervised with supervised approaches increases the accuracies of the classification as shown in Cao et al. (2010) so the possibility of using unsupervised segmentation algorithm as supervised segmentation procedure when classes are already being statistically described and labelled is very useful.

The current segmentation methodology applied for mapping and monitoring of tropical forest allows all the above mention possibilities. Until now, it is being extensively tested over images of the L-band WB and FBD-FBS ALOSPALSAR, and C-band ENVISAT-ASAR and RADARSAT. Some issues surrounding the application of the current algorithm, to these space borne images, are related to speckle and image texture. The use of a Markov random field filter in the classification procedure helps to overcome partly the effect of speckle, nevertheless it is being demonstrated that filtering of radar images previous segmentation can help in the better statistical definition of classes and in the final classification results. In addition, the use of the current algorithms in the high resolution RADARSAT-2 and (X-band) TerraSAR-X images can create very blurry classifications and re-sampling of the data is necessary before getting reasonable results. Also regarding the legend development process, the field information is still necessary and the interpretation of the radar signatures can be of great complexity. Nevertheless several maps have been created using this algorithm
(Hoekman et al. 2010). An example is a forest type map created for an ecologically complex area in Central Kalimantan. This map was created using a combination of space borne WB $(\mathrm{HH})$ and $\mathrm{FBD}(\mathrm{HV} / \mathrm{HH})$ polarizations for 2 years of ALOS-PALSAR acquisitions. The results are reported in Fig. 2.4. The overall classification accuracy calculated for the map is of $84 \%$ for 17 different vegetation cover types. This methodology has proven to be very robust to noise/outliers and overlapping clusters, is reasonably fast and is suitable for moderate to large images.

\subsubsection{Forest Mapping and Classification Using Polarimetric and Interferometric Data}

\subsubsection{Introduction, Motivation and Literature Review}

Forest remote sensing from SAR data has been intensively studied during the last 15 years. Various types of SAR data (single-, dual- and quad-pol, single- or multi-frequency) acquired in multi-temporal, multi-angular or interferometric modes were used to retrieve geophysical property estimates. All these studies demonstrated that SAR quantities (intensity, phase, correlation, coherence...) show particular behaviors over forested areas and may be used for classification purposes. Forest classification may be split into two complementary applications requiring different levels of accuracy and processing complexity:

- forest area mapping, which consists in delimiting the extent of forested areas within a SAR image;

- discrimination of vegetation categories, which aims to separate pixels belonging to different types of vegetated media.

This Section proposes to gather complementary aspects of polarimetric and interferometric data processing techniques to improve forest mapping and classification performance. If SAR polarimetry is particularly well adapted to the analysis and description of scattering mechanisms, and hence may be used to discriminate different environments, it is well known that PolSAR parameters tend to saturate over volumetric media with highly random response, like dense forest observed at L band or at higher carrier frequency. Oppositely, interferometric SAR measurements permit to further investigate volumetric media properties but suffer from a lack of contrast over areas showing more polarimetrically deterministic responses like agricultural fields and open surfaces.

This Section proposes simple processing schemes, based on both SAR signal statistical properties and physical interpretations of wave scattering, that combine PolSAR and PolInSAR analysis techniques into hierarchical, supervised or unsupervised classification approaches. It is 

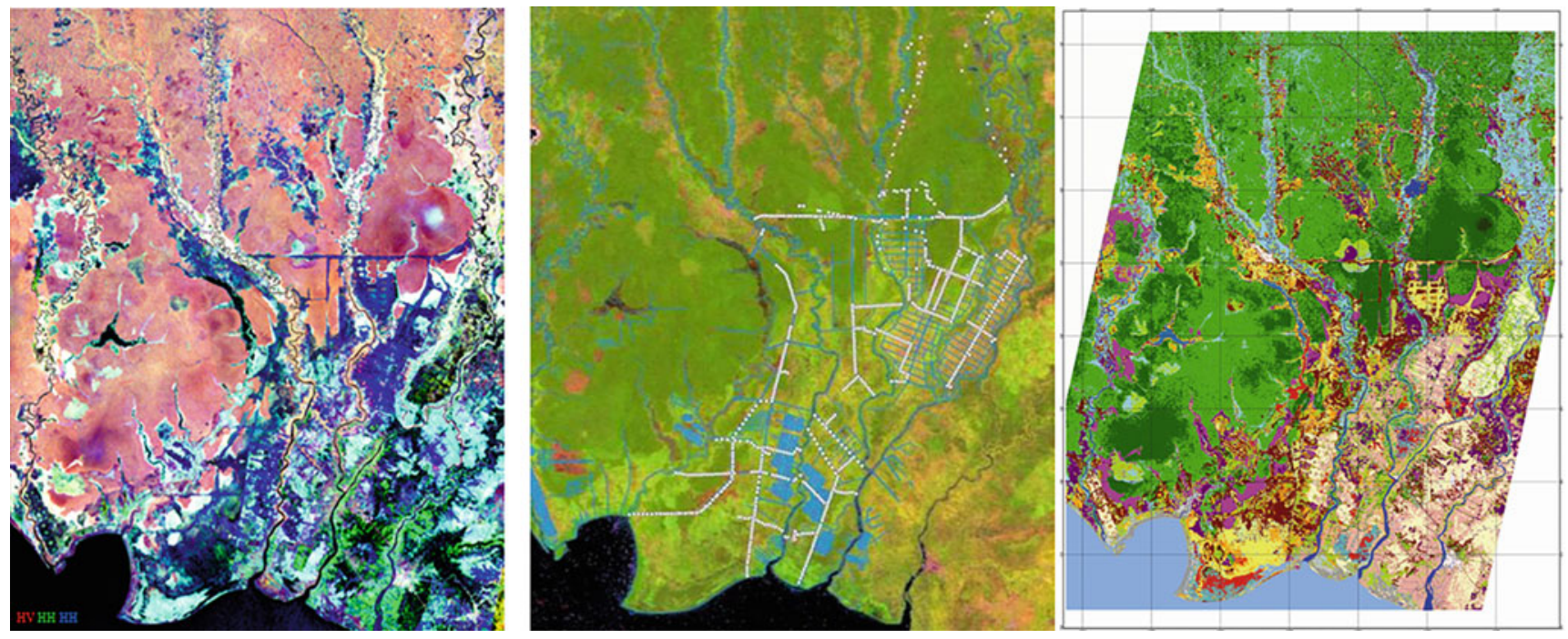

Fig. 2.4 Land cover Map (rightl) created using a combination of WB and FBD- L-band ALOS-PALSAR data (left). Accuracy assessment was done using field photographs taken on the points over long transects in the study area (centre). Calculated accuracy $84 \%$ for 17 classes

shown that forest mapping can be performed efficiently using PolSAR data processing and that refined segmentation results may be obtained by including POLinSAR information. Forest category identification is, in general, a significantly more complex task, since classical SAR indicators like reflectivity, or usual polarimetric parameters, can reveal highly misleading in the frame of forest classification, due to their saturation or high correlation with factors unrelated to the tree species under observation. As it is related hereafter, this serious limitation may be overcome by dealing with intrinsic PolInSAR parameters, that do not depend on forest radiometry and that are less affected by saturation effects.

Many studies report that SAR backscatter intensity value depends, up to a certain extent, on forest bio and geo-physical properties such as biomass, tree age (Lee et al. 2002). Nevertheless, high performance mapping or classification of forested areas can generally not be achieved by thresholding backscattered intensity due to the large variability of SAR image information. Single polarization data-based mapping techniques generally use additional modes of diversity, like texture (De Grandi et al. 2000; Wegmüller and Werner 1995), or time (seasonal variations, stability) (Grover et al. 1999; Lee et al. 1999; Paloscia et al. 1999). Partially or fully polarimetric SAR data may also be used to map forests, using cross-pol ratios or co-pol correlation (Hoekman and Quiñones 2000; Hoekman and Varekamp 2001) and combined with multi-frequency measurements (mainly P-, L- and C-bands) (Quegan et al. 2000; Ranson and Sun 1994; Ranson et al. 1995). However, the robustness of such supervised approaches has to be tempered by considering repeatability and generalization issues related to uncontrolled variations of polarimetric scattering patterns with time (year, season, month or even days) or depending on the geographical location or the investigated area (Ranson and Sun 1994; Le Toan et al. 2001; Cloude and Pottier 1997).

Unsupervised PolSAR approaches, related to the decomposition of polarimetric covariance matrices may be employed to determine the presence of forest from an interpretation of polarimetric scattering mechanisms (Le Toan et al. 2001; Cloude and Pottier 1997; Durden et al. 1989; Ferro-Famil et al. 2001, 2006). Such methods may meet some limitations over complex areas that cannot be separated from forests based on PolSAR information only (Ferro-Famil et al. 2003; Freeman and Durden 1998; Kurvonen and Hallikainen 1999). Single polarization interferometric coherence may be used to map forested areas (Askne et al. 1997; Dammert et al. 1999; Engdahl and Hyyppä 2003; Rignot et al. 1994a), but such techniques have to deal with exterior factors such as the spatial/temporal baselines compromises, forest density and topography that may affect the mapping accuracy and reliability. Finally, complementary aspects of both polarimetric and interferometric diversity modes may be combined in order to overcome intrinsic issues of each separate mode, and provide more reliable and accurate mapping results (Ferro-Famil et al. 2006).

An important number of studies have been led to discriminate different types of forest from single polarization SAR data (Dobson et al. 1996). Similarly to forest mapping applications, reasonable classification rates may be reached with supervised algorithms, particularly well adapted to one site or type of vegetation, but a systematic implementation may meet some problems of generalization, due to temporal variations and saturation of the basckscattered intensity (Lee et al. 2002; Hyypa et al. 1997; Mougin et al. 1999). The use of fully polarimetric and/or multifrequency data permit to further discriminate a large range of natural media (Hoekman 
and Quiñones 2000, 2002; Hoekman and Varekamp 2001; Ranson et al. 1995; Dobson et al. 1992) using supervised hierarchical classifiers, multi-frequency polarimetric acquisitions (Hoekman and Quiñones 2002; Dobson et al. 1992; Ferrazzoli et al. 1997; Hagberg et al. 1995), modelbased approaches (Hoekman and Quiñones 2000; Hoekman and Varekamp 2001; Ranson and Sun 1994; Lombardo and Macrì Pellizzeri 2002), or directly based on polarimetric measurements (Ranson et al. 1995) or on pre-processed polarimetric indicators, such as polarimetric decompositions results (Ferro-Famil et al. 2001; Kurvonen and Hallikainen 1999). One has to note that parameter saturation over forested areas may affect polarimetric indicators too and may then limit the performance of all the classification approaches mentioned above. Radar interferometry is an efficient tool for forest observation (Grover et al. 1999) and may overcome limitations due to polarimetric scattering coefficient saturation. Interferometric classification approaches generally rely on the modeling of SAR measurement coherence and an interpretation of its relation to the observed media nature and geophysical characteristics (Askne et al. 1997, 2003; Eriksson et al. 2003a; Imhoff 1995a; Strozzi et al. 2000; Van Zyl 1993; Wegmüller and Werner 1997). Statistical segmentation procedures adapted to inSAR data sets have been developed as well (Dammert et al. 1999; Engdahl and Hyyppä 2003; Rignot et al. 1994a). Interferometry based classification meet limitations similar to those enounced in the case of forest mapping, mainly linked to temporal-spatial baselines, topography and to the lack of polarimetric diversity. Quad polarization interferometric data, Pol-In-SAR, based classification is a powerful alternative to multifrequency data processing. The interpretation of this highdimensional information by the way of optimisation procedures permits to isolate different kinds of forested areas and constitutes a good solution to forest classification (Ferro-Famil et al. 2006). The introduction of joint polarimetric and interferometric information in an unsupervised classification scheme has shown the complementarity of both data types permits to discriminate refined features that cannot be observed from separate analysis (Ferro-Famil et al. 2003). The use of polarimetric interferometric representation statistics, derived in Ferro-Famil and Neumann (2008), in the frame of already existing robust and powerful supervised/ unsupervised classification algorithms (Ferro-Famil et al. 2006, 2003; Kurvonen and Hallikainen 1999), permit to reach higher levels of performance and robustness over a wide range of vegetation types (Ferro-Famil et al. 2006).

\subsubsection{Methodology}

The employed methodology for unsupervised forest mapping is illustrated in Fig. 2.5. The PolSAR image is first segmented using the Wishart $H / A / \bar{\alpha}$ statistical segmentation technique.
An identification of basic scattering mechanisms is then run over each pixel of the image, from specific polarimetric indicators derived from the eigenvector decomposition of coherency matrices, as described in Ferro-Famil et al. (2003): according to the number of scattering mechanisms detected within pixel using the $H$ and $A$ parameters, specific procedures are run to assign the pixel under observation to the volume diffusion, single- or bouble-bounce class. In order to reduce the random aspect of the mapping and increase its robustness with respect to arbitrarily fixed decision boundaries, a global decision is taken over statistically compact clusters obtained from the Wishart $H / A / \bar{\alpha}$ segmentation using a winner-takes-all decision strategy. As mentioned earlier, such a mapping approach may lead to some false alarms over complex and dense volumetric areas, like urban environments observed at L-band, and PolInSAR coherence optimization may be used to refine the PolSAR map. Parameters built from the PolInSAR optimal coherence set are used to determine the number of coherent scattering mechanisms from which is derived an indicator of the level of volumetric scattering. This information is then combined with the PolSAR result in order to obtain a refined forest map (Ferro-Famil et al. 2003, 2006).

Forest classification is performed here as a statistical supervised process which comprises two stages: a learning phase during which user-selected groups of data are used to learn the statistics of the different classes to be discriminated, and a classification phase which assigns a class label to each pixel on an image according to a statistical metric or to a specific decision rule whose parameters have been learned during the preceding phase. Here again, the random aspect of classification results may be reduced by taking global decisions over statistically compact clusters obtained from an unsupervised segmentation map.

This Section compares results obtained using the whole PolInSAR information, i.e. statistics of the $(6 \times 6)$ coherency matrix, or using reduced but more robust information consisting of the three optimal PolInSAR coherences.

\subsubsection{Experimental Results}

Test sites and corresponding radar and validation data sets selected for the generation of showcases on forest mapping and classifications are summarized in Table 2.4 and further described in the Appendix.

The complexity of the SAR scene over the Traunstein forest may be appreciated from the Pauli color-coded image shown in Fig. 2.6. This site is composed of forested areas, pasture fields with scattered farms and isolated buildings and an urban area at the center left part of the image. The unsupervised Wishart classification given in Fig. 2.6 provides some useful indications on the PolSAR properties of this data set: 


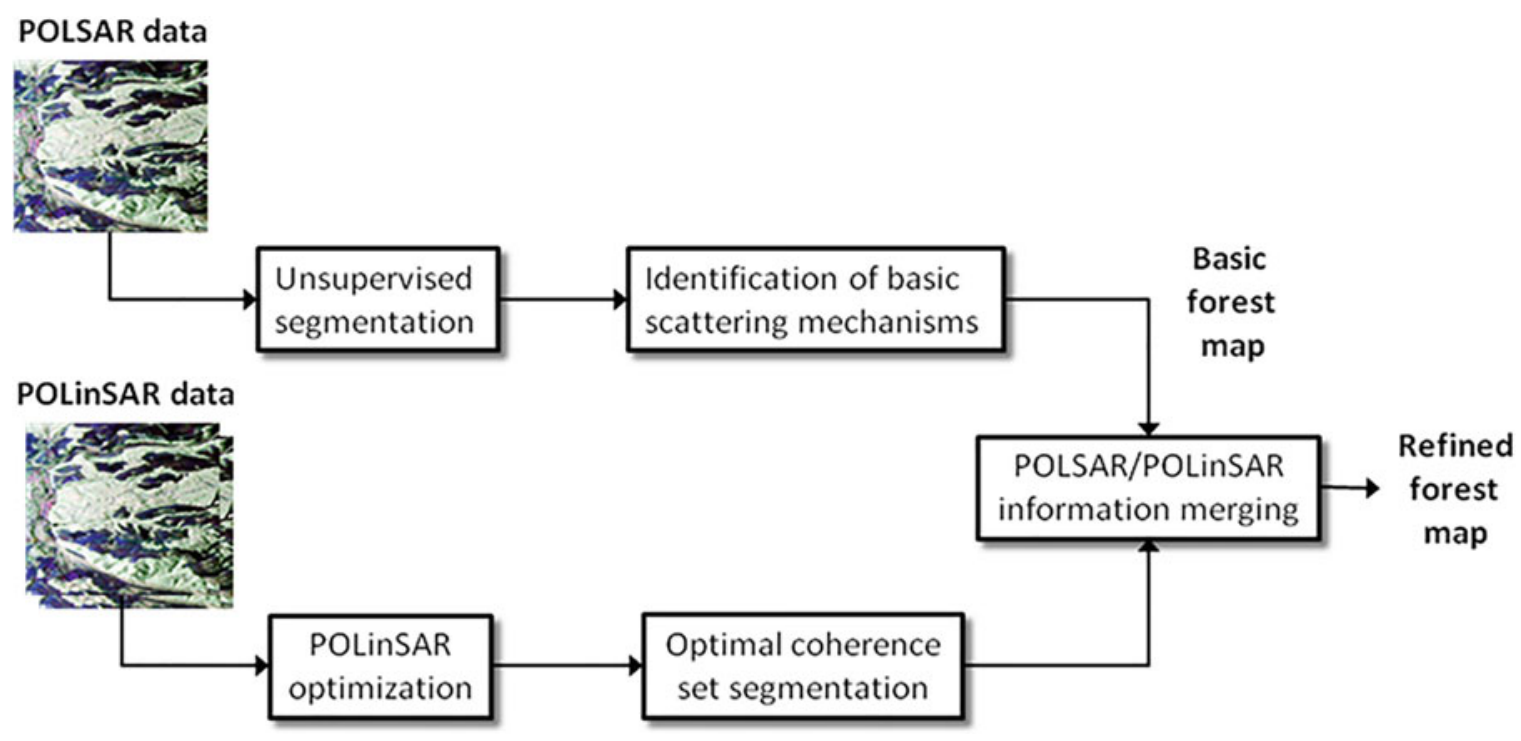

Fig. 2.5 Synopsis of the unsupervised forest mapping approach

Table 2.4 Test sites and corresponding radar and validation data selected for the generation of showcases on forest classification

\begin{tabular}{l|l|l}
\hline Application/product & Test site - Radar data & Reference data \\
\hline $\begin{array}{l}\text { Forest mapping and } \\
\text { classification }\end{array}$ & Traunstein forest, Germany & $\begin{array}{l}\text { Maps of tree species, growth state and biomass derived from ground } \\
\text { inventories }\end{array}$ \\
& $\begin{array}{l}\text { 5-image PolInSAR data set } \\
\text { Semporal baselines: } 10 \mathrm{~min},\end{array}$ & \\
& \begin{tabular}{l} 
15 days \\
\hline
\end{tabular}
\end{tabular}

- the different types of environments cannot be separated using their PolSAR statistics since many classes are spread over the whole image;

- one may observe a clear distribution of the classes in the range direction, related to the dependency of the backscattered intensity on the incidence angle, and to the predominance of the span over other polarimetric indicators. This aspect may be highly limiting for discriminating media located at different range positions.

Classification results shown in Figs. 2.6 and 2.7 indicate that PolInSAR data sets can be efficiently used in a supervised way to discriminate between different forest species and grow states or between different levels of biomass. The principal basic features of the ground information can be retrieved in the classification results whose spatial distribution is more heterogeneous than the provided reference map. This variability is mainly due to the fact that ground information is generally delivered under the highly simplified form of compact and homogeneous clusters, whereas forest stands are in general not homogeneous. A qualitative comparison between the ground information map and an aerial photograph revealed that some areas, considered as homogeneous in the ground maps, could indeed contain zones with varying tree densities, forest paths, clear cuts etc. On the other hand, some specific forest parcels belonging to slightly different types or having close biomasses may have very close PolInSAR responses that cannot be discriminated using statistical or hierarchical approaches. The overall performance of the biomass classification approach was evaluated over trusty locations, in terms of homogeneity, and a correct classification rate higher than $75 \%$ was found. The classification of forest type and growth states led to slightly lower rates.

As it has been mentioned earlier, single-pol techniques are mentioned in the literature for forest mapping and classification in the frame of marginal approaches, mainly based on texture and temporal analysis, in order to investigate the potential of existing spaceborne data sets for such applications. As reported in many studies, single polarization SAR data acquired at high frequency (L-band and higher) cannot be used in a robust way for mapping and classifying forested areas in general configurations, i.e. without a large amount of a priori information. The use of dual-pol data is not recommended either, due to the fact that the cross-pol HV channel is essential for accurately mapping and discriminating volumetric media with different physical features. Being this channel uncorrelated with other co-polarized measurements over the major part of natural environments, 

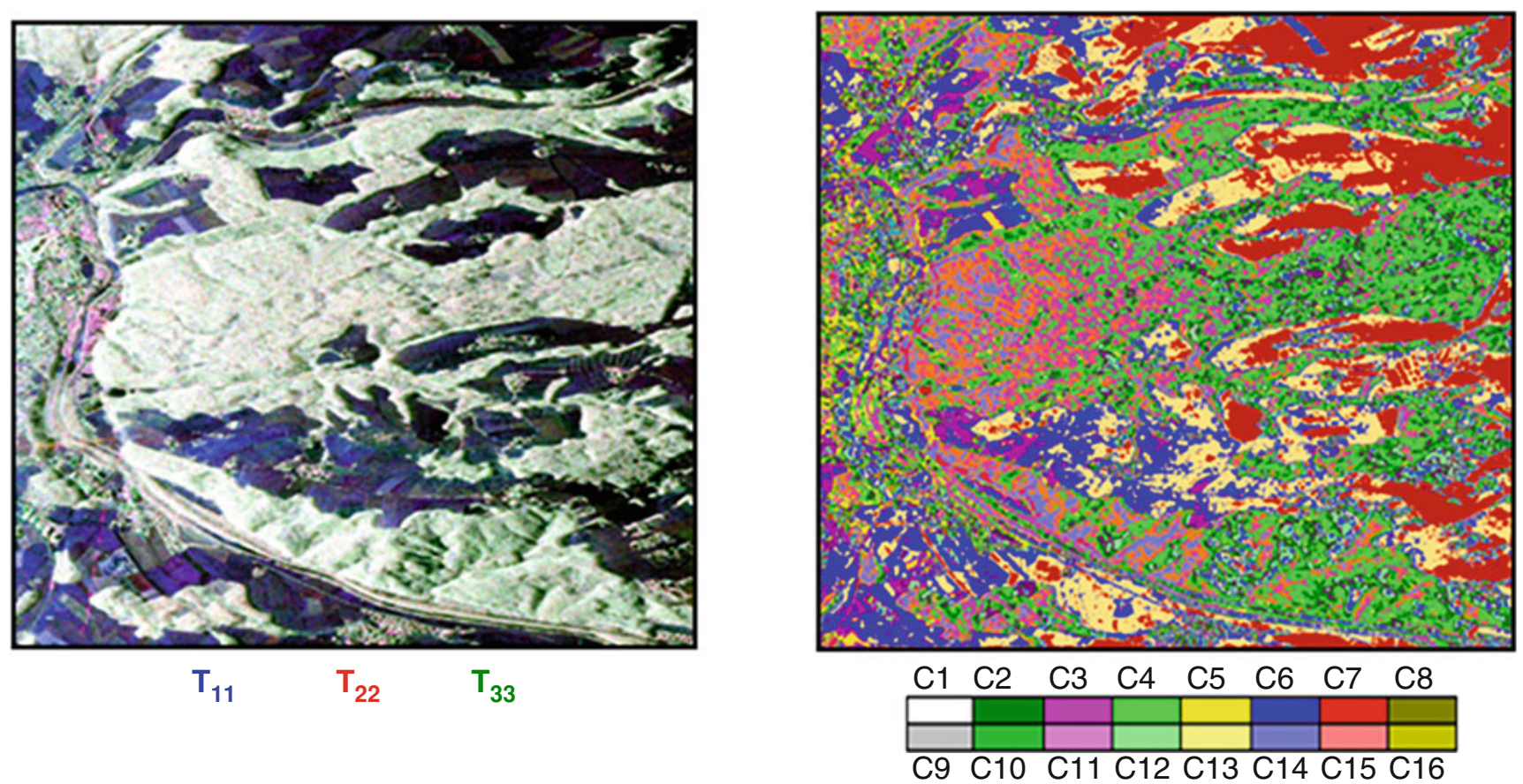

Fig. 2.6 Left: Pauli color-coded image of the Traustein site; right: result of the unsupervised PolSAR Wishart classification into 16 classes
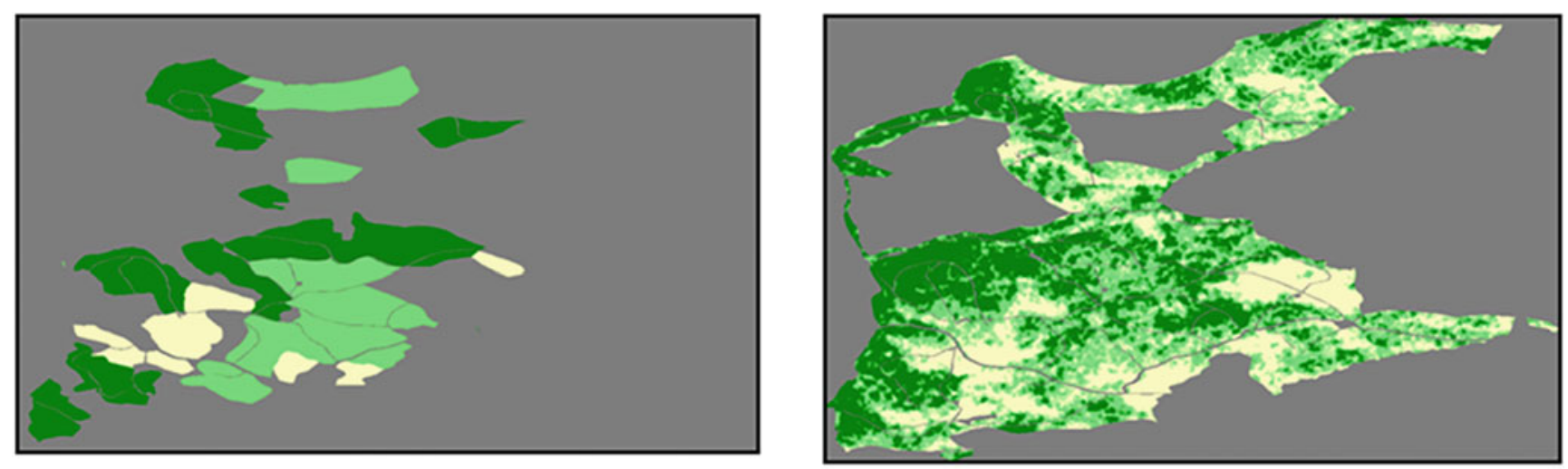

Fig. 2.7 Left: simplified forest biomass map, where medium biomass (B) means $200 \mathrm{t} / \mathrm{ha}<\mathrm{B} \leq 310 \mathrm{t} / \mathrm{ha}$; right: biomass classification result

volumes and surfaces, a $(2 \times 2)$ co- and cross-pol covariance matrix would not bring sufficient information for applying the technique proposed here. A co-pol covariance matrix, e.g. built from the HH and VV channels, can be used for forest mapping through the analysis of its eigenvalues, but with a significant loss of performance and additional ambiguities compared with the fully polarimetric case. Such a configuration leads to a significant reduction of the contrast between the elements of the optimal PolInSAR coherence set involving a severe loss of performance for classifying different types of forested areas or different levels of biomass.

The following comparison aims to show that over forested areas, single image polarimetry, i.e. classical SAR polarimetry, can be highly misleading for characterizing dense volumetric environments at L-band. This fact is due to the saturation of the polarimetric response, i.e. the covariance matrix tends to be proportional to the identity matrix, which strongly limits the potential of analysis of polarimetry and to the high dependence of the backscattered energy, i.e. the polarimetric span, on the scene geometry in general and the local incidence angle in particular. As one may note in Fig. 2.8, classification results obtained from PolSAR only data are largely influenced by the spatial distribution of the backscattered intensity over the whole scene, which may be appreciated over the Pauli color-coded image displayed in Fig. 2.6. Due to its interferometric aspect, PolInSAR-based 

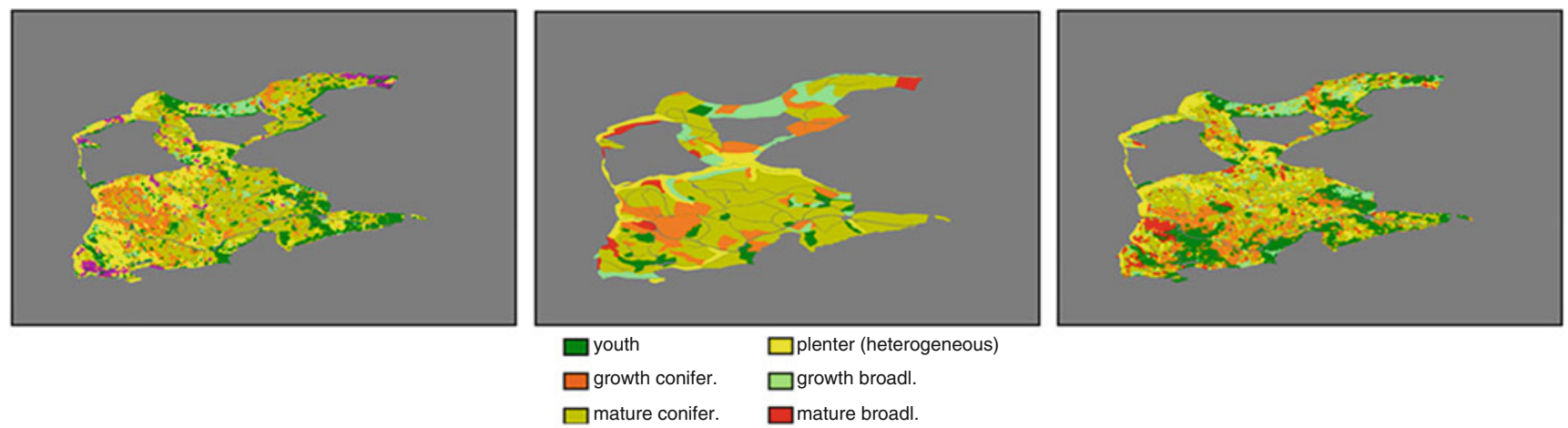

Fig. 2.8 Forest type classification results. Left: using the Wishart statistics of the PolSAR coherency matrix T; mid: reference map; right: using the proposed PolInSAR approach
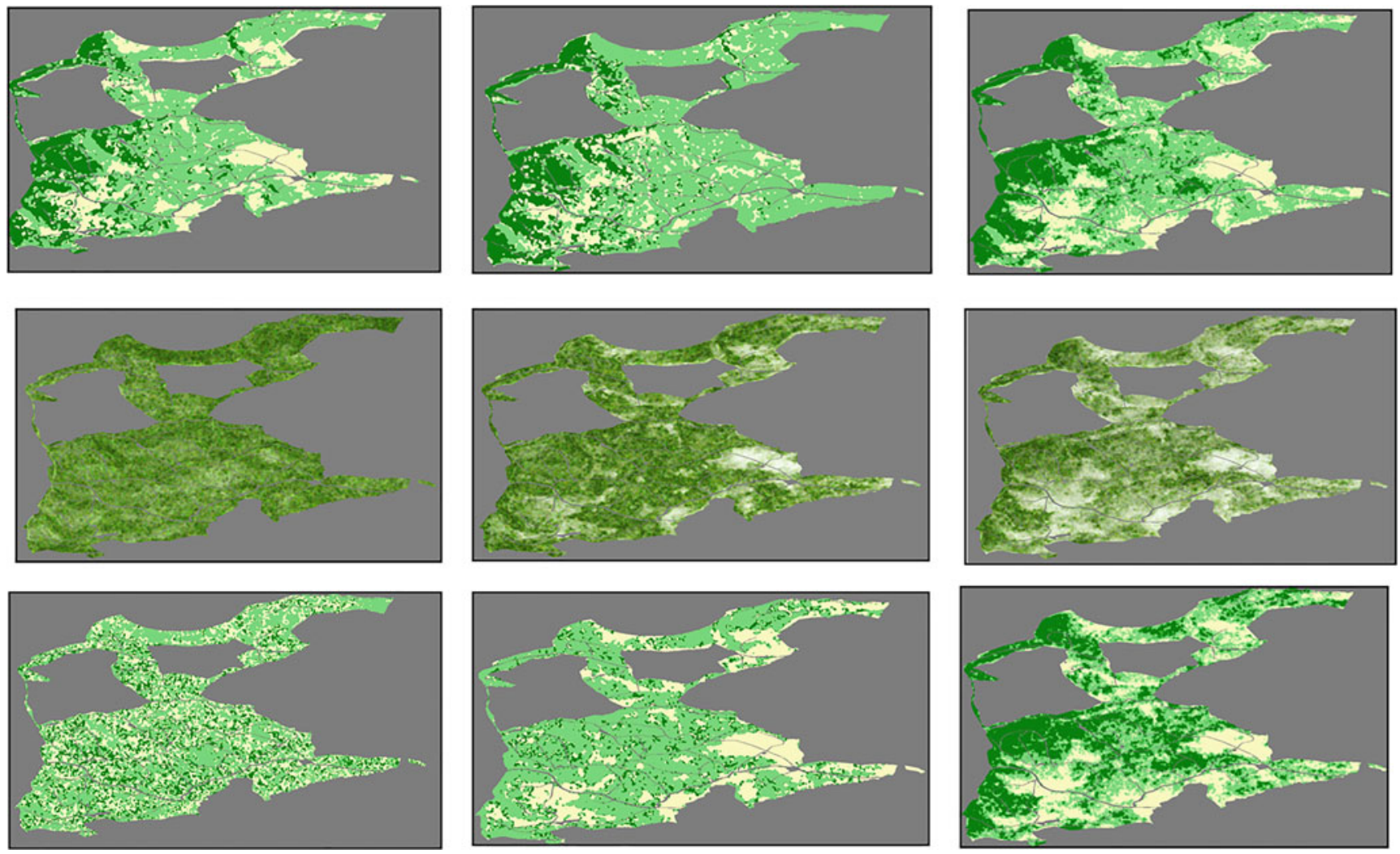

Fig. 2.9 Biomass classification using various temporal $\left(B_{t}\right)$ and spatial $\left(B_{s}\right)$ baseline configurations. Legend as in Fig. 2.7. Color coded images

Results obtained using the statistics of the full PolInSAR coherency matrix are plotted in the top panel, and the ones obtained by using the of the optimal Pol-inSAR coherence set are given (central panel). statistics of the optimal PolInSAR coherence set are in the bottom panel

technique permits to overcome this saturation effect. Being based on the statistics of span-independent quantities, it presents greatly enhanced features, whose distribution is tightly linked to the kind of forest under observation and not the angular dependence of the span.

Another illustration of this effect is given in Fig. 2.9 where classification results, obtained using the Wishart statistics of the full PolInSAR coherency matrix, are compared for various temporal and spatial baselines. The Wishart PolInSAR classifier output maps are quasi-insensitive to the level of interferometric correlation between the images. This observation shows that the volumetric analysis through interferometric coherence properties play a very little role during the classification, whereas the PolSAR part, saturated and strongly influenced by the span, predominates, leading to classification results poorly related to forest properties and perturbed by the scene topography or the acquisition geometry. Oppositely, the proposed approach, based on the 
statistics of the optimal coherence set, fully exploits the relative interferometric coherence information, and for a correct spatial and temporal configuration, provides results intrinsically related to forest properties and less affected by potential changes of incidence angle.

\subsubsection{Discussion on the Role of Polarimetry, on the Maturity of the Application and Conclusions}

Polarimetry plays a key role for applications related to forest mapping or classification using SAR images. Despite the fact that single polarization intensity value depends, up to a certain extent, on forest geophysical properties such as biomass or tree age, high performance mapping or classification of forested areas can generally not be achieved by thresholding backscattered intensity due to the large variability of SAR image information, to the potentially important influence of factors related to the scene topography or to the acquisition geometry, and to the saturation of the relation relating intensity to biomass at L- or higher frequency bands.

SAR polarimetry offers the possibility to measure this saturation from indicators related to the number of effective scattering mechanisms estimated within each pixel. Media with a polarimetrically saturated responses are associated to complex volumes, hence to forests. Such an approach works well over most environments, but may lead to false alarms over highly heterogeneous zones, mainly urban areas. This problem may be overcome by further measuring the presence of dense volume using PolInSAR parameters. Here again, polarization plays an essential role as it permits to separate media whose interferometric coherence may vary depending on the chosen polarimetric channel.

Due to the saturation of the polarimetric response of an environment in the presence of volume, classical SAR polarimetry, i.e. based on a single PolSAR image, cannot be used to classify forested areas with a sufficient accuracy, and one has to use PolInSAR data sets. However, this showcase clearly demonstrates that using the whole PolInSAR information for classifying forested areas can be counterproductive, as a significant part of this information can be dominated by factors unrelated to the nature of biomass features of the observed forest. Instead, using a set of elaborated parameters that concentrates the relative part of the PolInSAR information provides interesting results and limits the effects of artefacts encountered with usual or direct approaches.

In conclusion, polarimetry represents a very useful and efficient mode of diversity for forest mapping and classification, and needs to be coupled to interferometric measurements for characterizing complex volumetric environments. Pre-processing steps, aiming to separate sources of potential perturbations, linked to the acquisition geometry or other parameters unrelated to forest from the useful part of the signal should be implemented.

\subsubsection{Detection of Fire Scars}

\subsubsection{Introduction, Motivation and Literature Review}

Canada is home to $10 \%$ of the world's forests. Accounting of annual carbon emissions from forest fire events and monitoring changes in Canada's forests are important activities at Natural Resources Canada (NRCan). In 2004, NRCan initiated a joint project between the Canadian Forest Service (CFS) and the Canada Centre for Remote Sensing (CCRS) to create a system, the Canadian Wildland Fire Information System (CWFIS), used to estimate direct carbon emissions from Canadian wildfires (Groot et al. 2007). Accurate knowledge of burned areas is required to produce burned area estimates at the national level for post fire mapping. Currently, optical remote sensors, like SPOT-VGT and Landsat, are used to map burned areas at low resolution $(1 \mathrm{~km})$ and high resolution $(30 \mathrm{~m})$ respectively. A final burned-area output is used as an input to the National Forest Carbon Monitoring, Accounting and Reporting System (NFCMARS) (Kurz and Apps 2006) to estimate national carbon emissions. However, for producing such burned area estimates, the earliest possible cloud-free satellite images are critical. Because of adverse weather, cloud and illumination conditions in the Canada North, the limitation of remote sensing images from these optical sensors is evident.

Advanced space-borne Synthetic Aperture Radar (SAR) systems, such as Japanese ALOS-PALSAR, the German TerraSAR-X, and the Canadian RADARSAT-2, can contribute specially to this estimation effort. Over previous sensors, these offer better spatial resolution, shorter revisiting times, availability of polarimetry, and all-weather data acquisition capability. Therefore, considerable polarimetric SAR research in forest applications has been conducted at CFS with support of the Canadian Space Agency (CSA) and NRCan. One of the motivations is to determine whether polarimetric SAR information can be used to detect fire scars effectively in forest lands and offer alternative to traditional sketch mapping methods and optical sensors.

Historic burned area estimates, created from sketch mapping from small planes, GPS mapping from helicopters, and photo interpretation (Fraser et al. 2000, 2004), are available from provincial and territorial forest fire agencies. Because the management and protection of these data resources fall under provincial and territorial jurisdiction, GIS wildfire polygon data varies in quality due to the limitations of the traditional GIS technologies available at the time. Several Canadian provinces maintain GIS wildfire polygon data for managed forests from the 1940s to the 
present day. Generally, the older the data the less reliable it becomes. Fire perimeters derived from these traditional methods often include unburned "islands" and may overestimate burned areas. Moreover, the distribution of remote wild fire events and environmental conditions make them a challenge to accurately map.

Research has been conducted in mapping forest fire scar using SAR, which can be used to provide measurements of post-fire ecosystem changes in forest structure, ground surface exposure and soil moisture patterns (Landry et al. 1995; Bourgeau-Chavez et al. 1997). In Bourgeau-Chavez's studies based on radar backscatter analysis, he demonstrated that fire scars were detectable in a range of boreal ecosystems across the globe using C-band SAR and showed that the length of viewing time of fire scars with ERS or RADARSAT-1 data was between 3 and 7 years in Alaska and Canada (BourgeauChavez et al. 2002).

In previous studies, it was discovered that, using the airborne Convair $580 \mathrm{C}$-band quad-pol data, it is possible to detect a historical fire scar, more than 50 years old, over our study site in Hinton, Alberta (Goodenough et al. 2006). Here, we focus on the detection of two roughly 9 years old fire scars, using ALOS-PALSAR L-band and RADARSAT2 C-band quad-pol data data. The analysis includes data pre-processing, decomposition analysis and classification methodologies. The aim of the approach is to provide new fire-scar mapping methodologies from SAR quad-pol data in support of CWFIS and NFCMARS.

\subsubsection{Methodology}

Three techniques used to analyze space-borne quad-pol data for fire scar detection include polarimetric decomposition, scattering model, and classification. Decomposition approaches, such as the entropy-alpha decomposition (Cloude and Pottier 1997) and three component decomposition (Freeman and Durden 1998), provides various parameters showing different scattering characteristics of objects on the ground. Scattering model, such as the Oriented-Volume-over-Ground (OVOG) (Cloude 2009), estimates secondary parameters for volume and surface scattering components. The classification technique utilizes these scattering characteristics from the decomposition and modelling, performs image classification and extracts fire scars. Two latest classification methods employed here are a coherence-based geometrical detector described in Marino and Cloude (2010) and a data driven multi-dimensional clustering approach, i.e. the K-Nearest Neighbors (KNN) (Richardson et al. 2010).

Compact polarimetry (compact-pol) architecture is a new hybrid SAR mode and is proposed for the future Canadian RADARSAT Constellation Mission (RCM). The compactpol mode transmits single circular polarization (left/right) and receives simultaneous coherent orthogonal linear polarizations. The advantages of this mode are wide-swath coherent polarimetric information, low data rate, and relatively simple transmitter architecture (Raney 2007). These advantages plus shorter satellite revisiting intervals are particularly attractive for forest applications. Therefore, it has become essential to quantitatively assess this new mode. RADARSAT-2 quad-pol data were used to simulate RCM compact-pol data and new compact-pol parameters using compact-pol decomposition theories were introduced (Cloude et al. 2012) to investigate whether the loss of information through the compact-pol projection affects the quality of detection of forest fire scars. A rule-based classifier based on the physical interpretation of the compact parameters was constructed. A detailed description of this classification approach is provided in Cloude et al. (2013).

\subsubsection{Experimental Results}

Test sites and corresponding radar and validation data sets selected for the generation of showcases on fire scar detection are summarized in Table 2.5 and further described in the Appendix.

The L-band data set was first corrected for any Faraday rotation, a low frequency distortion arising from transionospheric propagation from the satellite to the ground. Because all data sets provided were in single-look complex format, multi-looking in azimuth and range directions were performed to reduce speckle. Next, a box car filter was used to generate the $3 \times 3$ PolSAR coherency matrix. To further reduce topography relief effects on the polarimetric SAR data, polarization orientation shifts introduced by terrain slopes in the azimuth direction were detected and corrected to generate reflection symmetry in the coherency matrix of the polarimetric SAR data for the next stage of analysis.

The coherence-based geometrical detector was performed on the PALSAR quad-pol data over the Keg River site. Figure 2.10 is a Pauli RGB composite of the PALSAR scene. The variety of colours indicates the wide diversity of

Table 2.5 Test sites and corresponding radar and validation data selected for the generation of showcases on fire scar detection

\begin{tabular}{l|l|l}
\hline $\begin{array}{l}\text { Application/ } \\
\text { product }\end{array}$ & Test site - Radar data & Reference data \\
\hline $\begin{array}{l}\text { Fire scar } \\
\text { detection }\end{array}$ & $\begin{array}{l}\text { Keg River, Alberta, Canada } \\
\text { ALOS-PALSAR (08.06.2009) } \\
\text { 6 RADARSAT-2 acquisitions (between Nov. 2010 and } \\
\text { June 2011) }\end{array}$ & $\begin{array}{l}\text { Fire scar polygons (government of Alberta), Google Earth maps, } \\
\text { field knowledge }\end{array}$ \\
\hline
\end{tabular}




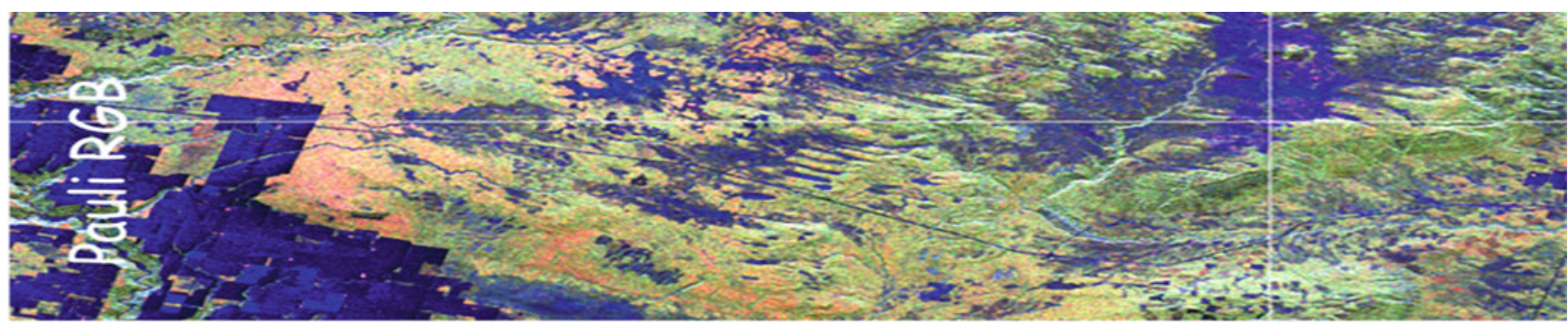

Fig. 2.10 Keg River, ALOS-PALSAR acquisition: Pauli RGB

Fig. 2.11 Keg River, ALOSPALSAR acquisition: supervised coherence detector

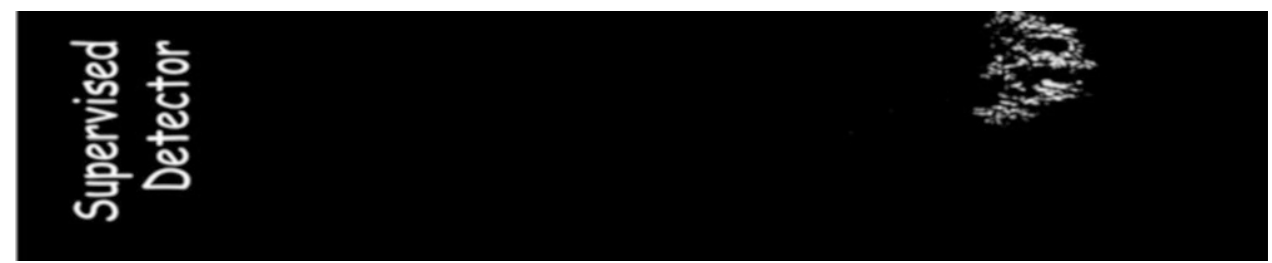

Fig. 2.12 Keg River, ALOSPALSAR acquisition: unsupervised coherence detector

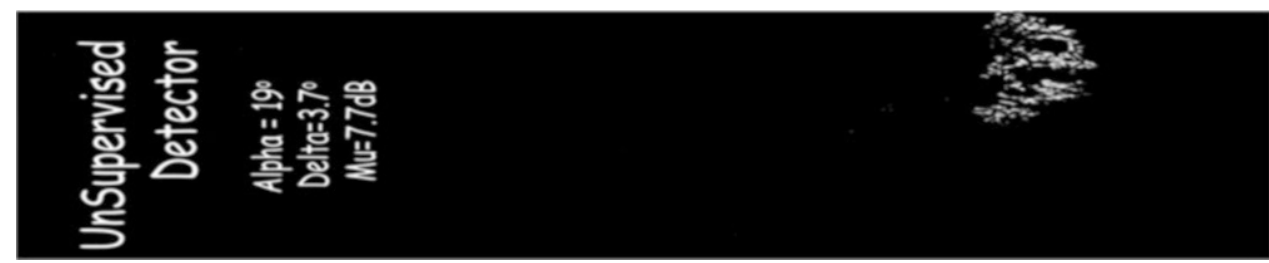

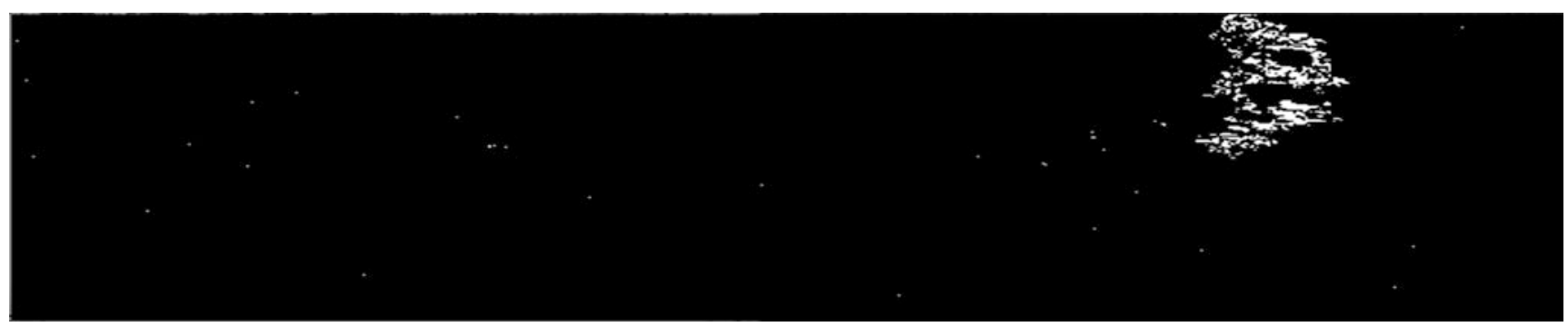

Fig. 2.13 Keg River, ALOS-PALSAR acquisition: $\mathrm{KNN}(K=55,22$ clusters)

landcover types in this area. Figure 2.11 is the result of a fire scar coherence detector using a supervised approach, i.e. with training on a known fire scar region. Figure 2.12 is the result for an unsupervised fire scar detector, both of which showed good detection and low false alarm rates. Figure 2.13 illustrates the fire scar detection and clustering result from the KNN classification, using $H / A / \bar{\alpha}$ from the entropy-alpha decomposition as input. Both of the coherence-based detector and the KNN classifier showed very good fire scar results for the PALSAR L-band Keg River scene.

A rule-based classifier was applied on simulated C-band compact-pol data with imagery dimension $29 \mathrm{~km} \times 27 \mathrm{~km}$, and the scene is shown in Fig.2.14. One issue was the temporal variability of the simulated compact data due to environmental changes. To avoid threshold values depending partly on the data set and meet the time-invariant classifier requirements, six simulated compact data sets in different seasons were combined to form a co-registered data stack. Each pixel was averaged in time across the whole stack to create a time averaged Stokes vector. Four decomposition parameters used for the rule-based classification were the compact minimum volume, the degree of polarization, the $\alpha$-angle and the compact phase. Figure 2.15 is a pseudo colour classification map of the averaged compact data. The fire scars are dark grey areas and outlined by the GIS fire polygons (red). The output fire scars from the rule-based classification are shown in Fig. 2.16. In Bourgeau-Chavez et al. (2002), Bourgeau-Chavez showed that fire scars between 3 and 7 years in Alaska and Canada were detectable with ERS or RADARSAT-1 single polarization data. 


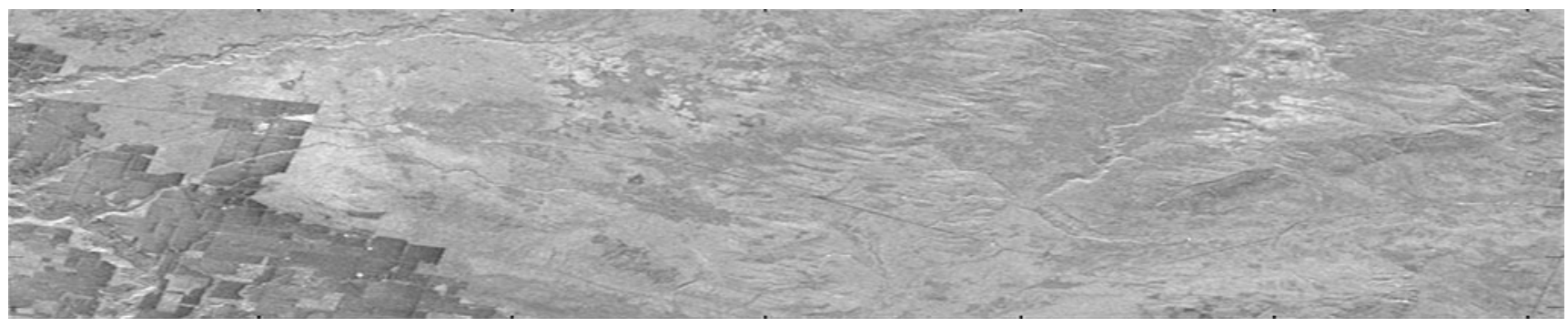

Fig. 2.14 Keg River, RADARSAT-2 average acquisition: HH single-pol amplitude

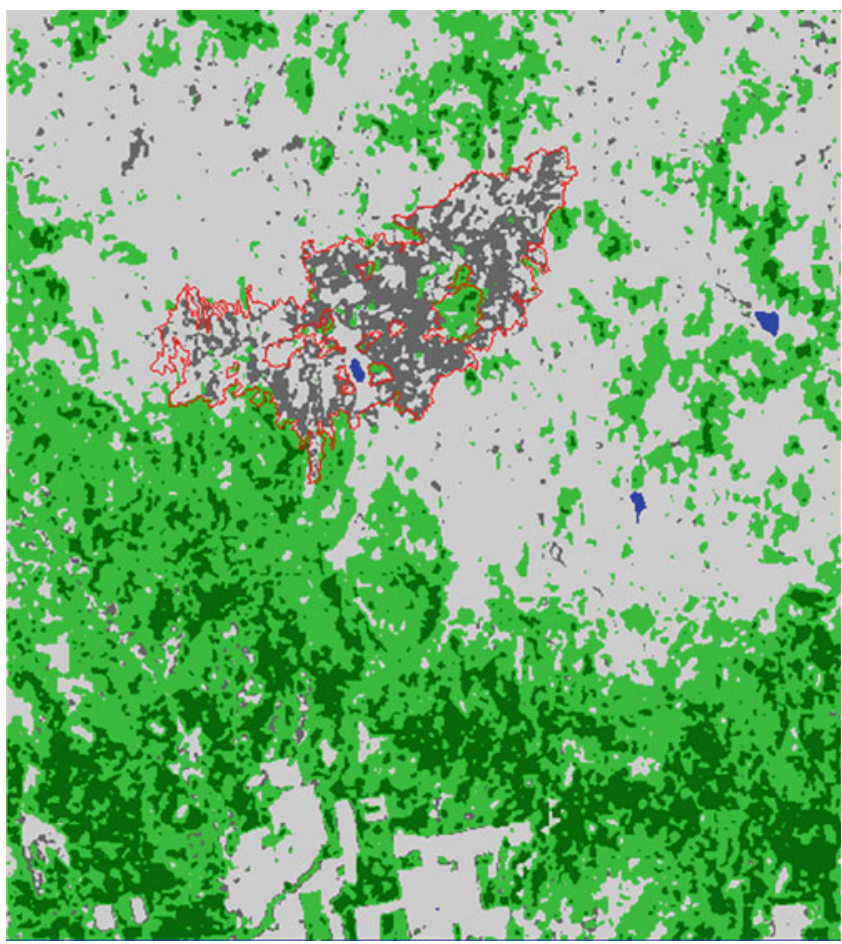

Fig. 2.15 Keg River, RADARSAT-2 average acquisition: rule-based compact classification

However, because their approach is a manual interpretation method based on the backscatter intensity, the accuracy of fire scar detection results were largely affected by human error, seasonal variations, topography effects, and environmental conditions.

In this Section, we demonstrated that the strong signature embedded in quad-pol SAR data provided much better capabilities for fire scar detection and monitoring. The fire scar detection results from quad-pol and simulated compactpol data showed the improved mapping of historical fire scars in this age category. With such method, the fire scar detection now is much more reliant on polarization information, tolerant of topographic variations and robust to absolute changes in backscatter due to environmental conditions, which is complementary to optical remote sensing and current fire scar mapping techniques.

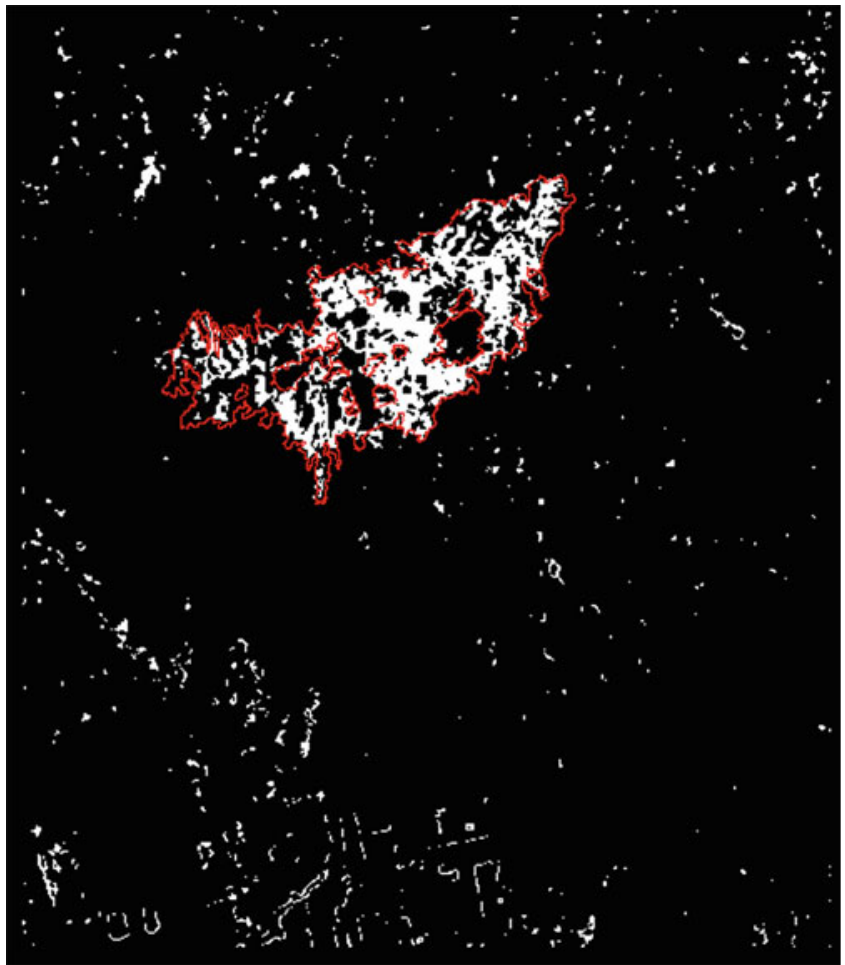

Fig. 2.16 Keg River, RADARSAT-2 average acquisition: fire scar identification

\subsubsection{Discussion on the Role of Polarimetry, on the Maturity of the Application and Conclusions}

This Section focused on utilizing the phase information contained in polarimetric SAR data to increase the sensitivity of SAR measurement for scar identification. ALOSPALSAR and RADARSAT-2 data were processed and analyzed over the study site in Keg River, Alberta. The results showed that it is feasible to clearly map historical fire scars of approximately 9 years of age with polarimetric SAR data from both sensors. The coherence-based geometrical detector and KNN classification results are encouraging, showing the potential and effectiveness of such methodology in segmenting and classifying polarimetric SAR data for fire scar detection. 
Compact polarimetry provides a new wide-swath multichannel coherent mode for radar imaging. However, the loss of information through projection and high entropy for vegetation scattering pose challenges for use in fire scar detection. Here, compact polarimetry decomposition and classification were employed. The degree of polarization, minimum-overtime-volume, and compact phase were very useful parameters for the rule-based classification, especially when supported by the $\alpha$-angle. With carefully defined threshold values and the use of extended time series of simulated compact data, the historical fire scars in this study area were clearly detected. The false positives outside the fire scar region in simulated compact-pol data can be further reduced by using area filters. These results support the idea that, in absence of an operational quad-pol mode, the compact mode would be a good mode to use for wide area land-use monitoring and change detection.

\subsection{Forest Height Estimation}

\subsubsection{Introduction, Motivation and Literature Review}

Forest height is one of the most important parameters in forestry along with basal area and tree species or species composition. It provides information about stand development and/or site index and describes dynamic forest development, modeling and inventory. Forest height is an (standard) indicator for the site dependent timber production potential of a stand, and is closely related (through allometric relations) to forest biomass (see Sect. 2.5.1). Furthermore, accurate forest height measurements allow concluding on the successional state of the forest and can be used to constrain model estimates of above ground biomass and associated carbon flux components between the vegetation and the atmosphere. The distribution of forest heights within a stand can be further used to characterize the disturbance regime while high (spatial and temporal) resolution forest height maps can be used for detecting selective logging activities (Köhler and Huth 2010; Dubayah et al. 2010; Thomas et al. 2008; Hurtt et al. 2010).

When it comes to characterize dynamic forest processes the (accurate) estimation of forest height change is even more important than static forest height measurements. Forest height change can be directly used to characterize forest growth, mortality and deforestration and to conclude about the associated carbon fluxes without the need of assumptions (or knowledge) about the successional status (Köhler and Huth 2010; Dubayah et al. 2010).

Being a standard parameter in forest inventories, forest height is hard to be measured on the ground and typical estimation errors are around 10\% accuracy, yet increasing with forest height and density. In terms of remote sensing techniques, lidar configurations have been today established as the reference (in terms of vertical and spatial resolution and/or accuracy) for measuring on local and regional scale vertical and horizontal distribution of vegetation structure components including vegetation height. Lidar estimation methodologies have been developed and validated through a variety of airborne and speceborne measurements and experiments (Lefsky 2010). However, the rather small footprints of spaceborne lidar configurations do not allow global forest height (and structure) monitoring with reasonable temporal resolution.

The introduction of polarimetric SAR interferometry (PolInSAR) at the end of the nineties was a decisive step towards developing remote sensing applications relevant to forest structure. The inherent sensitivity of the interferometric coherence to the vertical structure of volume scatterers combined with the potential of SAR polarimetry to interpret and characterise the individual scattering processes at different structural components allows a qualitative and quantitative determination of relevant (structure) parameters from SAR measurements. Today, PolInSAR is an established technique, allowing investigation of the 3-D structure of natural volume scatterers.

The fundamental interferometric measurement is the complex interferometric coherence, which comprises the interferometric correlation coefficient, as well as the interferometric phase. For a given spatial baseline (indicated by the vertical interferometric wavenumber $k_{z}$ ) and a given polarization (indicated by the unitary vector w (Cloude 2009; Marino and Cloude 2010)) the complex interferometric coherence is obtained by forming the (normalized) cross-correlation between the corresponding interferometric images $S_{1}(\mathbf{w})$ and $S_{2}(\mathbf{w})$ :

$$
\begin{aligned}
\gamma\left(k_{z}, \mathbf{w}\right) & =\frac{<S_{1}(\mathbf{w}) S_{2}^{*}(\mathbf{w})>}{\sqrt{<S_{1}(\mathbf{w}) S_{1}^{*}(\mathbf{w})><S_{2}(\mathbf{w}) S_{2}^{*}(\mathbf{w})>>}=\left(\left(k_{z}, \mathbf{w}\right)\right.} \\
& \mid \exp (j \varphi) .
\end{aligned}
$$

The measured coherence depends on the system and imaging geometry, as well as on the dielectric and structural parameters of the scatterers within the scene. A detailed discussion of system induced coherence contributions can be found in Lefsky (2010). After calibration of system induced decorrelation contributions and compensation of spectral decorrelation in azimuth and range the estimated interferometric coherence can be decomposed into three main decorrelation processes (Zebker and Villasenor 1992; Bamler and Hartl 1998; Moreira et al. 2013):

$$
\gamma\left(k_{z}, \mathbf{w}\right)=\gamma_{\mathrm{Temp}} \gamma_{\mathrm{SNR}} \gamma_{\mathrm{Vol}}\left(k_{z}, \mathbf{w}\right)
$$


- $\gamma_{S N R}$ known as the Signal-to-Noise Ratio (SNR) decorrelation is introduced by the additive white noise contribution on the received signal;

- $\gamma_{T e m p}$ is the temporal decorrelation caused by dynamic changes in the scene occurring in the time between the two acquisitions. It depends on the structure and the temporal stability of the scatterer, the temporal baseline of the interferometric acquisition and the dynamic environmental processes occurring in the time between the acquisitions;

- The volume decorrelation $\gamma_{V o l}\left(k_{z}, \mathbf{w}\right)$ is the decorrelation caused by the different projection of the vertical component of the scatterer reflectivity spectrum into the two interferometric SAR images. It contains therefore information about the vertical structure of the scatterer (Cloude 2009; Bamler and Hartl 1998). Indeed, $\gamma_{V o l}\left(k_{z}, \mathbf{w}\right)$ is directly related to the vertical distribution of scatterers $F$ $(z, \mathbf{w})$ in the medium through a (normalized) Fourier transformation relationship (Bamler and Hartl 1998; Papathanassiou and Cloude 2001)

$$
\gamma_{\mathrm{Vol}}\left(k_{z}, \mathbf{w}\right)=\exp \left(j k_{z} z_{0}\right) \frac{\int_{0}^{h_{V}} F\left(z^{\prime}, \mathbf{w}\right) \exp \left(j k_{z} z^{\prime}\right) d z^{\prime}}{\int_{0}^{h_{V}} F\left(z^{\prime}, \mathbf{w}\right) d z^{\prime}}
$$

where $h_{V}$ indicates the height (or depth) of the volume. $k_{z}=(m \cdot 2 \pi \cdot \Delta \theta) /\left[\lambda \cdot \sin \left(\theta_{0}\right)\right]$ is the effective vertical (interferometric) wavenumber that depends on the imaging geometry $(\Delta \theta$ is the incidence angle difference between the two interferometric images induced by the baseline and $\theta_{0}$ the local incidence angle) and the radar wavelength $\lambda . z_{0}$ is a reference height and $\varphi_{0}=k_{z} z_{0}$ the associated interferometric phase. For monostatic acquisitions $m=2$, while for bistatic acquisitions $m=1$.

Accordingly, $\gamma_{V o l}\left(k_{z}, \mathbf{w}\right)$ contains the information about the vertical structure of the scatterers and allows to estimate $F(z, \mathbf{w})$ (and/or associated structure parameters) from measurements of $\gamma_{V o l}\left(k_{z}, \mathbf{w}\right)$ (or $\gamma\left(k_{z}, \mathbf{w}\right)$ ). Indeed, for the estimation of $F(z, \mathbf{w})$ (and/or associated structure parameters) from $\gamma_{V o l}\left(k_{z}, \mathbf{w}\right)$ measurements at different polarisations, frequencies and/or (spatial) baselines two approaches have been explored in the literature:

1. The first one is to parameterise $F(z, \mathbf{w})$ in terms of geometrical and scattering properties and to use then $\gamma_{V o l}\left(k_{z}\right.$, w) measurements at different spatial baselines and/or different polarisations to estimate the individual model parameters. In this case, the scattering model is essential for the accuracy of the estimated parameters. On the one hand the model must contain enough physical structure to interpret the inreferometric measurements, while on the other hand it must be simple enough in terms of parameters in order to be determinable with the available (in general limited) number of observations (Cloude 2009; Papathanassiou and Cloude 2001; Cloude and Papathanassiou 2003).

2. The second approach to estimate $F(z, \mathbf{w})$ is to approximate it by a (normalized) polynomial series or another orthogonal function basis $P_{n}(z)$ (Cloude 2009; Cloude 2006):

$$
F(z, \mathbf{w})=\sum_{n} a_{n}(\mathbf{w}) P_{n}(z)
$$

and to use then $\gamma_{\mathrm{Vol}}\left(k_{z}, \mathbf{w}\right)$ measurements to estimate the coefficients $a_{n}(\mathbf{w})$ of the individual components. The advantage of this approach is that there is no assumption on the shape of $F(z, \mathbf{w})$ required, allowing the reconstruction of arbitrary vertical scattering distributions (Cloude 2006).

\subsubsection{Methodology}

\subsubsection{Random-Volume-Over-Ground Inversion}

For vegetation applications two layer statistical models, consisting of a vertical distribution of scatterers $F_{V}(z, \mathbf{w})$ that accounts for the vegetation scattering contribution, and a Dirac-like component $m_{G}(\mathbf{w}) \delta\left(z-z_{0}\right)$ that accounts for the scattering contribution(s) with the underlying ground (i.e. direct surface and dihedral vegetation-surface contributions) have been proven to be sufficient in terms of robustness and performance especially at lower frequencies (Cloude 2009; Moreira et al. 2013; Papathanassiou and Cloude 2001):

$$
F(z, \mathbf{w})=F_{V}(z, \mathbf{w})+m_{G}(\mathbf{w}) \delta\left(z-z_{0}\right)
$$

where $m_{G}(\mathbf{w})$ is the ground scattering amplitude. Substituting (2.8) into (2.6) leads to the model:

$$
\begin{aligned}
\widetilde{\gamma}_{\mathrm{Vol}}\left(k_{z}, \mathbf{w}\right)= & e^{j \varphi_{0}} \frac{\widetilde{\gamma}_{V}\left(k_{z}, \mathbf{w}\right)+\mu(\mathbf{w})}{1+\mu(\mathbf{w})}, \widetilde{\gamma}_{V}\left(k_{z}, \mathbf{w}\right) \\
= & \frac{\int_{0}^{h_{V}} F_{V}(z, \mathbf{w}) \exp \left(j k_{z} z^{\prime}\right) d z^{\prime}}{\int_{0}^{h_{V}} F_{V}(z, \mathbf{w}) d z^{\prime}} .
\end{aligned}
$$

The ratio $\mu(\mathbf{w})=m_{G}(\mathbf{w}) / \int_{o}^{h_{V}} F_{V}(z, \mathbf{w}) d z$ is the effective ground-to-volume amplitude ratio.

For modelling the vertical distribution of scatterers in the vegetation layer $F_{V}(z, \mathbf{w})$, or equivalently $\widetilde{\gamma}_{V}\left(k_{z}, \mathbf{w}\right)$, different models can be used. A widely and very successfully used 
model for $F_{V}(z, \mathbf{w})$ is an exponential distribution of scatterers (Moreira et al. 2013; Papathanassiou and Cloude 2001):

$$
F_{V}(z, \mathbf{w})=\exp \left[2 \sigma(\mathbf{w}) z / \cos \left(\theta_{0}\right)\right]
$$

where $\sigma(\mathbf{w})$ is a mean extinction value for the vegetation layer that defines the attenuation rate of the profile. Besides the exponential profile, that appears to fit better higher frequencies, Gaussian, or Linear scattering distributions have been proposed especially at lower frequencies (Garestier and Le Toan 2010a, b; Kugler et al. 2009).

Equation (2.9) comprises four unknowns: the forest height $h_{V}$, the extinction $\sigma(\mathbf{w})$, the ground topography phase $\varphi_{0}$, and the ground-to-volume amplitude ratio $\mu(\mathbf{w})$ and cannot be inverted by a single-channel (i.e. single polarisation) interferometric acquisition that provides only one (complex) $\gamma_{\mathrm{Vol}}\left(k_{z}\right.$, w) estimate. In order to invert (2.9), one has to increase the dimensionality of the observation space introducing:

- Baseline diversity: the dependency of $\gamma_{\mathrm{Vol}}\left(k_{z}, \mathbf{w}\right)$ on the vertical wave number is essential as it allows to increase the observation space in an effective way (i.e. without increasing the number of unknown parameters) as $F$ $(z, \mathbf{w})$ does not change with $k_{z}$ (Treuhaft and Siqueira 2000). At the same time, the choice of the vertical wave number allows to optimize the inversion performance (Krieger et al. 2005). However, the limitation of multibaseline inversion approaches arises when the acquisition of additional spatial baselines is associated with non-zero temporal decorrelations (i.e. when they are not acquired simultaneously).

- Polarimetric diversity: the variation of $\gamma_{\mathrm{Vol}}\left(k_{z}, \mathbf{w}\right)$ with polarization is due to the polarization dependency of $F$ $(z, \mathbf{w})$. The fact that certain components of $F(z, \mathbf{w})$ have a stronger polarised (scattering) behaviour than others allows to use the polarimetric dependency of $\gamma_{\mathrm{Vol}}\left(k_{z}, \mathbf{w}\right)$ for the estimation of $F(z, \mathbf{w})$ (Papathanassiou and Cloude 2001; Cloude and Papathanassiou 2003). Looking on the two layer model of (2.8), while the ground scattering component is strongly polarized and therefore has to be assumed to be polarization dependent, the volume scattering component can be both: in the case of oriented volumes $(\mathrm{OV})$ the vertical distribution of scatterers in the volume is polarization dependent, while in the case of random volumes $(\mathrm{RV})$, the vertical distribution of scatterers in the volume is the same for all polarisations, i.e. $F_{V}(z, \mathbf{w})=F_{V}(z)$.

In forest applications random volumes have been established so that a single polarimetric baseline allows the inversion of the Random-Volume-over-Ground (RVoG) model (Cloude 2009). Oriented volumes are more expected to be important in agriculture applications where the scatterers within the agriculture vegetation layer are in many cases characterized by an orientation correlation introducing anisotropic propagation effects and differential extinction (Treuhaft and Cloude 1999; Ballester-Berman et al. 2005).

In the absence of temporal decorrelation (i.e. $\gamma_{\mathrm{Temp}}=1$ ) and assuming a sufficient high SNR (i.e. $\gamma_{\mathrm{SNR}}=1$ ), from (2.5) follows:

$$
\gamma\left(k_{z}, \mathbf{w}\right)=\gamma_{\mathrm{Vol}}\left(k_{z}, \mathbf{w}\right) .
$$

The inversion problem for the quad-pol single-baseline case is balanced with six unknowns $\left(h_{V}, \sigma, \mu_{1}-3, \varphi_{0}\right)$ and three measured complex coherences $\left[\gamma\left(k_{z}, \mathbf{w}_{1}\right) \gamma\left(k_{z}, \mathbf{w}_{2}\right) \gamma\right.$ $\left.\left(k_{z}, \mathbf{w}_{3}\right)\right]$ each for any independent polarization channel

$$
\min _{h_{V}, \sigma, \mu_{i}, \varphi_{0}}\left\|\left[\begin{array}{l}
\gamma\left(k_{z}, \mathbf{w}_{1}\right) \\
\gamma\left(k_{z}, \mathbf{w}_{2}\right) \\
\gamma\left(k_{z}, \mathbf{w}_{3}\right)
\end{array}\right]-\left[\begin{array}{l}
\tilde{\gamma}_{V o l}\left(h_{V}, \sigma, \mu_{1} \mid k_{z}\right) \\
\tilde{\gamma}_{V o l}\left(h_{V}, \sigma, \mu_{2} \mid k_{z}\right) \\
\tilde{\gamma}_{V o l}\left(h_{V}, \sigma, \mu_{3} \mid k_{z}\right)
\end{array}\right]\right\| .
$$

with $\widetilde{\gamma}_{\mathrm{Vol}}\left(h_{V}, \sigma, \mu_{i} \mid k_{z}\right)$ modelled as in (2.9) and under the RVoG assumption.

However, Eq. (2.12) does not have a unique solution for a single baseline. Uniqueness can be established in terms of a single baseline only by regularisation (Cloude 2009; Flynn et al. 2002). A very efficient regularisation approach is to assume no response from the ground in one polarization channel (i.e., $\mu_{3}=0$ ) (Papathanassiou and Cloude 2001; Cloude and Papathanassiou 2003). This way, one obtains an inversion problem with five real unknowns $\left(h_{V}, \sigma\right.$, $\left.\mu_{1}-2, \varphi_{0}\right)$ and three measured complex coherences each for any independent polarization channel (Papathanassiou and Cloude 2001):

$$
\min _{h_{V}, \sigma, \mu_{i}, \varphi_{0}}\left\|\left[\begin{array}{c}
\gamma\left(k_{z}, \mathbf{w}_{1}\right) \\
\gamma\left(k_{z}, \mathbf{w}_{2}\right) \\
\gamma\left(z, \mathbf{w}_{3}\right)
\end{array}\right]-\left[\begin{array}{c}
\tilde{\gamma}_{\mathrm{Vol}}\left(h_{V}, \sigma, \mu_{1} \mid k_{z}\right) \\
\tilde{\gamma}_{\mathrm{Vol}}\left(h_{V}, \sigma, \mu_{2} \mid k_{z}\right) \\
\tilde{\gamma}_{\mathrm{Vol}}\left(h_{V}, \sigma, \mu_{3}=\left.0\right|_{z}\right)
\end{array}\right]\right\|
$$

Equation (2.13) has now a unique solution in terms of $h_{V}$ and $\sigma$ : each $\left\{h_{V}, \sigma\right\}$ pair for a given baseline (i.e. vertical wavenumber $\kappa_{z}$ ) and $\varphi_{0}$ phase is mapped through (2.10) into a unique $\widetilde{\gamma}_{\text {Vol }}\left(k_{z}\right)$ value. However, the validity of this assumption is not expected to be universal. Deviations of the real vertical structure from the modelled one degrade the inversion performance of (2.12) and (2.13). Two situations where such a reality/model mismatch becomes obvious are:

1. In low extinction forest scattering situations given in sparse forests the assumption that $\mu_{3}=0$ is not valid, 
i.e. all polarizations are affected by a significant ground scattering contribution. Also underlying topographic variations within the scene can increase the ground scattering contribution in all polarization channels. The presence of a residual $\mu_{3}$ in (2.13) biases the inversion results (usually causes an overestimation of forest height and/or an underestimation of the extinction coefficient). For a single-baseline inversion scenario, one way to still obtain a solution is to fix the extinction value. However, the relative strong variation of $\sigma$ in forest environments limits the inversion performance obtained in many forest environments.

2. Inverse scattering distributions, i.e. cases where more effective scatterers are located in the lower forest layers than on the higher ones. This can be the case in sparse forest environments with more or less distinct understorey, or at lower frequencies when the effective scatterers become larger and therefore located lower within the forest architecture. In this case, the exponential decay of $F_{V}(z)$ as assumed in (2.11) is no longer valid resulting in an underestimation of forest height and/or an overestimation of extinction.

However, quantitative model based estimation of forest height by means of (2.13) based on a single frequency, fully polarimetric, single baseline configuration has been successfully demonstrated at different frequencies, from P- up to X-band. Several space and airborne experiments demonstrated the potential of Pol-InSAR techniques to estimate with high accuracy forest height over a variety of natural and commercial; temperate, boreal and tropical sites characterized by different stand and terrain conditions (Lee et al. 2010, 2013; Lavalle et al. 2012).

\subsubsection{Non-volumetric Decorrelation Contributions}

Equation (2.9) accounts only for the volume decorrelation contribution while other non-volumetric decorrelation effects are ignored. Any decorrelation contribution reduces the interferometric coherence, and increases the variation of the interferometric phase. Furthermore, one has to distinguish between real and complex decorrelation contributions: while the expectation value of the interferometric phase remains invariant in the case of real decorrelation contributions, complex decorrelation biases the interferometric phase.

The most prominent decorrelation contribution in the case of non-simultaneous acquisitions (repeat pass system) is temporal decorrelation. It is caused by changes within the scene occurring in the time between (or even during) the two acquisitions. Such changes affect the location and/or the (scattering) properties of the scatterers within the scene inducing in the most general case a complex decorrelation (Lee et al. 2010, 2013; Lavalle et al. 2012).

In terms of the RVoG model (2.2), temporal decorrelation may affect both the volume component that represents the vegetation layer and the underlying ground layer and can be accounted for by introducing $\gamma_{\text {Temp }}(\vec{w})$ as complex temporal decorrelation coefficient in (2.11) (Lee et al. 2010):

$$
\begin{aligned}
\gamma\left(k_{z}, \mathbf{w}\right) & =\gamma_{\mathrm{Temp}}(\mathbf{w}) \gamma_{\mathrm{Vol}}\left(k_{z}, \mathbf{w}\right) \\
& =\exp \left(j k_{z} z_{0}\right) \gamma_{\mathrm{Temp}}(\mathbf{w}) \frac{\widetilde{\gamma}_{V}\left(k_{z}\right)+\mu(\mathbf{w})}{1+\mu(\mathbf{w})} .
\end{aligned}
$$

It is expected that decorrelation processes within the volume layer differs from temporal decorrelation of the ground layer, to account for this $\gamma_{\text {temp }}(\mathbf{w})$ needs to be split into a volume part and a ground part (Lee et al. 2010):

$$
\begin{aligned}
\gamma\left(k_{z}, \mathbf{w}\right)= & \exp \left(j k_{z} z_{0}\right) \\
& \times \frac{\gamma_{T V}(\mathbf{w}) \widetilde{\gamma}_{V}\left(k_{z}\right)+\gamma_{T G}(\mathbf{w}) \mu(\mathbf{w})}{1+\mu(\mathbf{w})} .
\end{aligned}
$$

$\gamma_{T V}(\mathbf{w})$ describes the temporal decorrelation of the volume layer and $\gamma_{T G}(\mathbf{w})$ the temporal decorrelation of the underlying surface scatterer. Note that in general the decorrelation processes within the volume layer occur at much smaller time scales than the decorrelation processes on the ground (which includes both surface and dihedral scattering) (Lee et al. 2010, 2013). As indicated, both coefficients may be polarisation dependent and complex. For example, changes in the dielectric properties of the canopy or ground layer lead to (complex) polarisation dependent temporal decorrelation contributions $\gamma_{T V}(\mathbf{w})$ and $\gamma_{T G}(\mathbf{w})$ (Lee et al. 2010). Changes in the vertical distribution of scattererers lead to complex decorrelation contributions.

From the parameter inversion point of view now, the RVoG model (2.14) with general temporal decorrelation contributions cannot be solved under any (repeat-pass) observation configuration. Any additional measurement of $\gamma\left(k_{z}, \mathbf{w}\right)$ at a different spatial baseline and/or polarisation introduces the same number of unknowns $\left(\gamma_{T V}\right.$ and $\left.\gamma_{T G}\right)$ as observation parameters. However, even if the general temporal decorrelation scenario leads to an underdetermined problem, special temporal decorrelation events may be accounted under certain assumptions. The most common temporal decorrelation over forested terrain is wind-induced decorrelation due to the (wind-induced) movement of the scatterers (e.g. leaves and/or branches) within the canopy layer. In terms of the RVoG model, this corresponds to a change of the position of the scattering particles within the volume in the two acquisitions that introduces a non-volumetric decorrelation. However, in this case the 
scattering amplitudes as well as the propagation properties of the volume remain the same. Assuming further that the scattering properties of the ground do not change in the time between the two acquisitions (2.12) reduces to:

$$
\widetilde{\gamma}_{\mathrm{Vol}}\left(k_{z}, \mathbf{w}\right)=\exp \left(j k_{z} z_{0}\right) \frac{\gamma_{\mathrm{Temp}} \widetilde{\gamma}_{V}\left(k_{z}\right)+\mu(\mathbf{w})}{1+\mu(\mathbf{w})}
$$

$\gamma_{\text {Temp }}$ describes the real temporal decorrelation of the volume scatterer. The inversion of PolInSAR coherences contaminated by temporal decorrelation using (2.13) leads to overestimated forest height as the RVoG model interprets the lower coherence by an increased forest height. Note that the estimation bias increases with increasing level of temporal decorrelation and is significantly larger for low(er) than for tall(er) forests stands. The effect of $\gamma_{\text {Temp }}$ decreases with increasing spatial baseline (Lee et al. 2013).

As indicated by $(2.5), \gamma\left(k_{z}, \mathbf{w}\right)$ may include several non volumetric decorrelation contributions $\gamma_{\text {Deco }}$ so that:

$$
\gamma\left(k_{z}, \mathbf{w}\right)=\gamma_{\text {Deco }} \gamma_{\mathrm{Vol}}\left(k_{z}, \mathbf{w}\right)
$$

In this sense, Eq. (2.9) becomes:

$$
\gamma\left(k_{z}, \mathbf{w}\right)=\exp \left(j \varphi_{0}\right) \gamma_{\text {Deco }} \frac{\tilde{\gamma}_{V}\left(k_{z}\right)+\mu(\mathbf{w})}{1+\mu(\mathbf{w})}
$$

The inversion of (2.18) requires apart from polarimetric also baseline diversity (Lee et al. 2010). Assuming $\gamma_{\text {Deco }}$ to be polarisation independent, at least a second baseline is required for height inversion. Each baseline provides three measured coherences $\left[\gamma\left(\mathbf{w}_{1} \mid k_{z, i}\right) \quad \gamma\left(\mathbf{w}_{2} \mid k_{z, i}\right) \quad \gamma\left(\mathbf{w}_{3} \mid k_{z, i}\right)\right]$ :

$$
\gamma\left(\mathbf{w} \mid k_{z, i}\right)=\exp \left(j \varphi_{0, i}\right) \gamma_{\text {Deco }, i} \frac{\widetilde{\gamma}_{V}\left(k_{z, i}\right)+\mu(\mathbf{w})}{1+\mu(\mathbf{w})}
$$

where $i=1,2$ indicate the two spatial baselines. Assuming $\mu\left(\mathbf{w}_{3}\right)=0$ the polarisation with the lowest ground contribution becomes:

$$
\gamma\left(\mathbf{w}_{3} \mid k_{z, i}\right)=\exp \left(j \varphi_{0, i}\right) \gamma_{\text {Deco }, i} \widetilde{\gamma}_{V}\left(k_{z, i}\right) .
$$

Equation (2.19) can be inverted in two steps. First, for each baseline all possible triplets $\left\{h_{V}, \sigma, \gamma_{\text {Deco, } i}\right\}$ fulfilling (2.20) are estimated. Then the triplets with common height/extinction pairs $\left\{h_{V}, \sigma\right\}$ are projected into each individual baseline and $h_{V}, \sigma$ are estimated according to

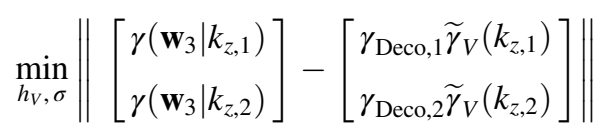

The advantage of (2.18) is that it can be inverted in a multi-baseline sense without requiring absolute (i.e. residual geometric, ionospheric and/or atmospheric) phase corrections.

\subsubsection{Experimental Results}

Test sites and corresponding radar and validation data sets selected for the generation of showcases on forest height estimation are summarized in Table 2.6 and further described in the Appendix.

The results achieved at L-band over the temperate Traunstein site are presented in Fig. 2.17. The L-band HV intensity image of the Traunstein forest site is shown on the left. In the middle and on the right of Fig. 2.17 forest height maps derived from Pol-InSAR data acquired at L-band in 2003 (middle) and 2008 (right) are shown. Comparing the two forest height maps a number of changes within the forest become visible: the logging of individual tall trees as a result of a change in forest management between 2003 and 2008 (marked by the green box); the damage caused in January 2007 by the hurricane Kyrill which blew down large parts of the forest (marked by the orange box); and finally forest growth on the order of 3-5 m over young stands as seen within the area marked by the white circle. The validation plot against the lidar reference data, shown in Fig. 2.18, indicates correlation coefficient of 0.95 and a root mean square error (RMSE) below $2 \mathrm{~m}$.

The inversion results achieved at P-band over the tropical Mawas site are shown in Fig. 2.19. The HH amplitude image is shown on the top. The river crosses the left part of the image embedded in secondary riverine forest. The lidar strip is superimposed on the amplitude image. Forest height along the Lidar strip is constant within $\pm 5 \mathrm{~m}$ around $27 \mathrm{~m}$ with lower heights in the parts close to the river and the disturbed forest areas. The PolInSAR forest height map is shown on the

Table 2.6 Test sites and corresponding radar and validation data selected for the generation of showcases on forest height estimation

\begin{tabular}{l|l|l}
\hline Application/product & Test site - Radar data & Reference data \\
\hline Forest height & Krycklan, Sweden & Airborne lidar measurements \\
& TanDEM-X dual-pol (HH VV) & \\
& Traunstein, Germany & \\
& Airborne E-SAR L-band repeat-pass quad-pol data 2003/2008 & \\
& Mawas, Indonesia & \\
& Airborne E-SAR P-band repeat-pass quad-pol data 2004 & \\
\hline
\end{tabular}



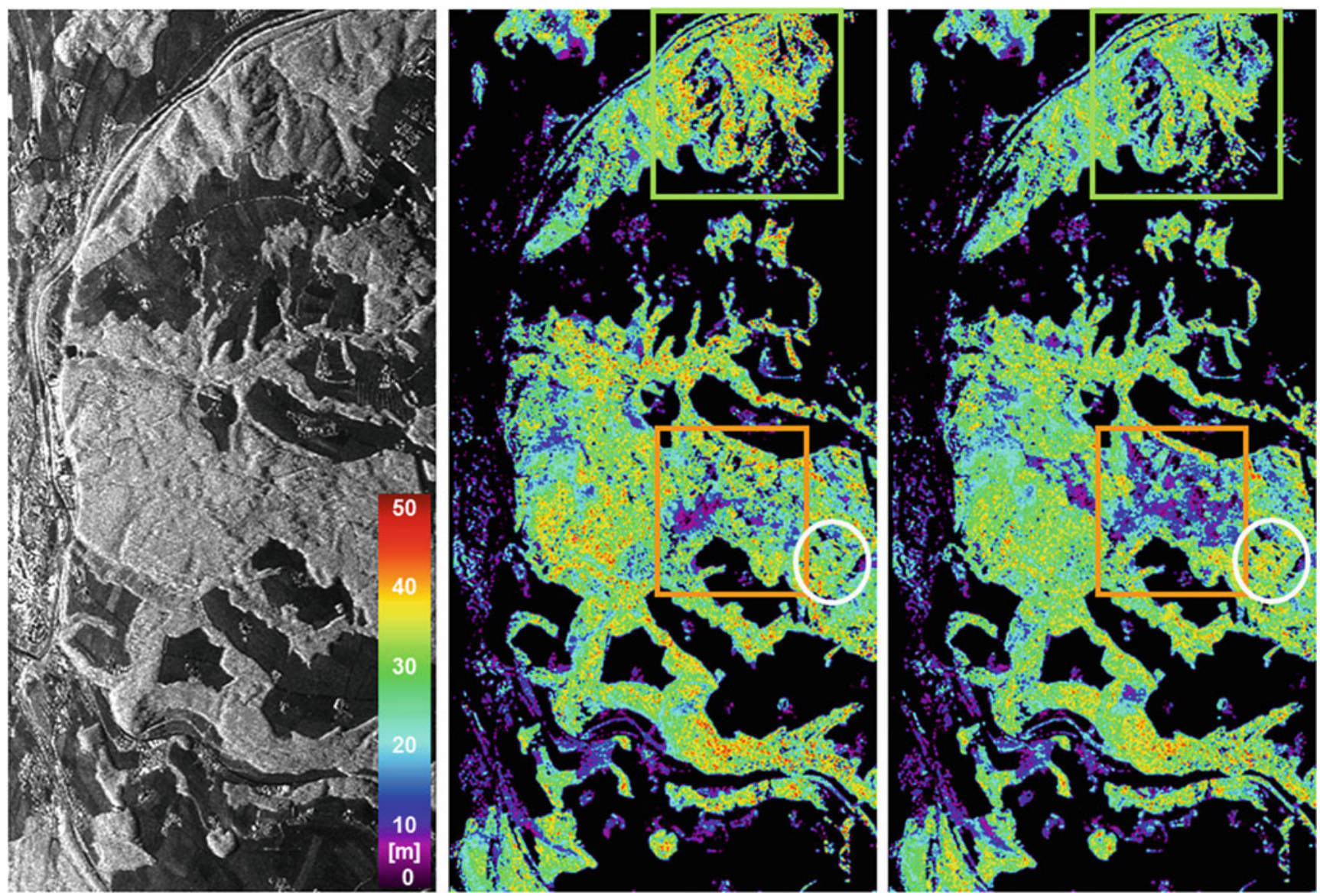

Fig. 2.17 E-SAR L-band HV intensity image of the Traunstein test site (left). Forest height map computed from PolInSAR data in 2003 (middle) and 2008 (right)

bottom. In the forested part the logging trails caused by logging activities $10-15$ years ago appear clearly. On the top right the validation plot against the lidar reference data is shown characterized by a correlation coefficient of 0.94 and a RMSE of clearly below $2 \mathrm{~m}$, indicating an estimation accuracy better than $10 \%$ of the mean forest height.

\subsubsection{Comparison with Single/Dual Polarimetric Data}

The RVoG model, as given in (2.9) and assuming $F_{V}(z$, $\mathbf{w})=F_{V}(z)$, can be inverted by means of a dual-polarimetric interferometric configuration that provides only two polarimetric channels $\mathbf{w}_{1}$ and $\mathbf{w}_{2}$. Assuming a zero ground-tovolume amplitude ratio for one polarization (i.e. $\mu\left(\mathbf{w}_{2}\right)=0$ ) leads to a balanced inversion problem with unique solutions for four unknowns, i.e. the forest height $h_{V}$, the extinction $\sigma$, the ground topography phase $\varphi_{0}$ and the ground-to-volume amplitude ratio $\mu\left(\mathbf{w}_{1}\right)$ :

$$
\min _{h_{V}, \sigma, \mu_{1}, \varphi_{0}}\left\|\left[\begin{array}{l}
\gamma\left(k_{z}, \mathbf{w}_{1}\right) \\
\gamma\left(k_{z}, \mathbf{w}_{2}\right)
\end{array}\right]-\left[\begin{array}{c}
\widetilde{\gamma}_{\mathrm{Vol}}\left(h_{V}, \sigma, \mu\left(\mathbf{w}_{1}\right) \mid k_{z}\right) \\
\widetilde{\gamma}_{\mathrm{Vol}}\left(h_{V}, \sigma, \mu\left(\mathbf{w}_{2}\right)=0 \mid k_{z}\right)
\end{array}\right]\right\|
$$

Indeed, the inversion scheme of Eq. (2.22) has been used to invert airborne but also space borne dual-pol interferometric configurations (Cloude 2009; Kugler et al. 2014). Compared to the quad-pol case the performance of the dualpolarimetric inversion has a reduced performance in terms of:

1. Biased estimation results which are obtained when the assumption of a zero ground-to-volume amplitude ratio is violated, i.e. when the ground scattering contribution is significant in all polarisations. This can be the case at lower frequencies and/or sparse forest conditions. With respect to the zero ground-to-volume amplitude ratio assumption, conventional dual-polarimetric configurations acquiring a co- and a cross-polarised channel are in favour when compared to dual-polarimetric configurations 


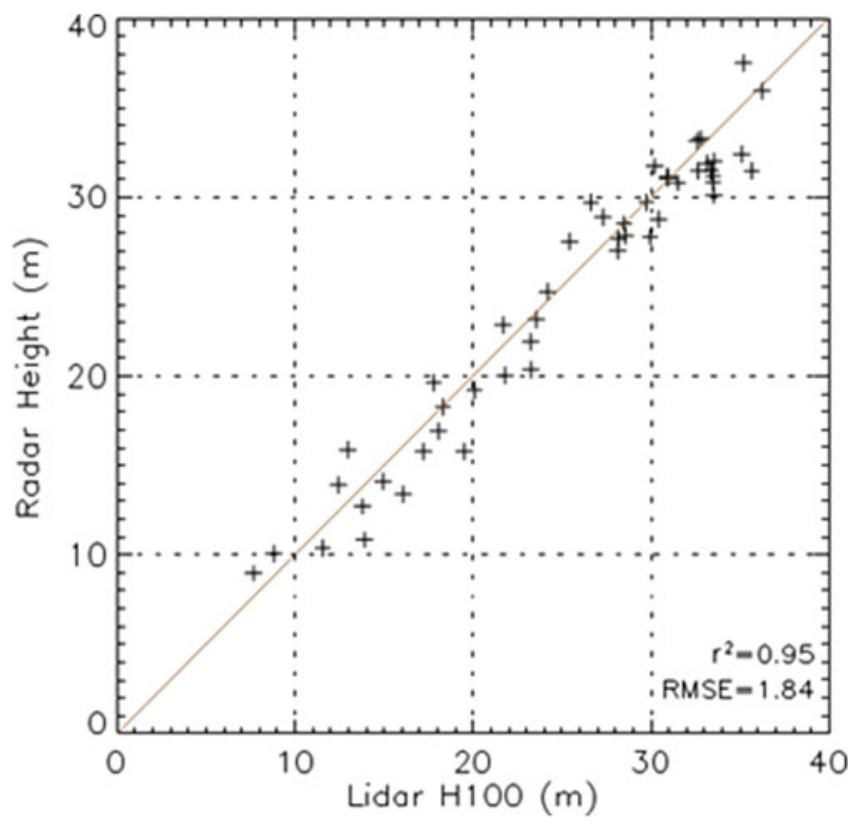

Fig. 2.18 Validation plot of forest height estimates at L-band over Traunstein (2008) against the lidar reference acquiring the two co-polarised channels. However, even the cross-polarised channel can be affected by a significant ground scattering contribution especially in the presence of terrain slopes.

2. Larger variance of the obtained forest height estimates when compared to the inversion results achieved by using the full polarimetric information as only a polarimetric subspace is available for performing the inversion. This affects the conditioning of the inversion problem and the accuracy of the obtained estimates. For the Traunstein site the performance of two dual-polarimetric interferometric configurations, $\mathrm{HH}$ and $\mathrm{HV}$ as well as $\mathrm{HH}$ and $\mathrm{VV}$ has been evaluated and compared to the quad-polarimetric case. While the forest height estimates obtained from both dual-polarimetric configurations do not show any significant bias, their variance is significantly higher across all validation stands than the variance of the forest height estimates obtained from the quad-polarimetric configuration as indicated in Fig. 2.20.

3. Larger amount of (forest) points with no RVoG solution. This is, in most cases, also the result of a non-zero ground scattering contribution in the estimated $\gamma_{V o l}\left(k_{z}, \mathbf{w}\right)=\gamma_{V}\left(k_{z}\right)$

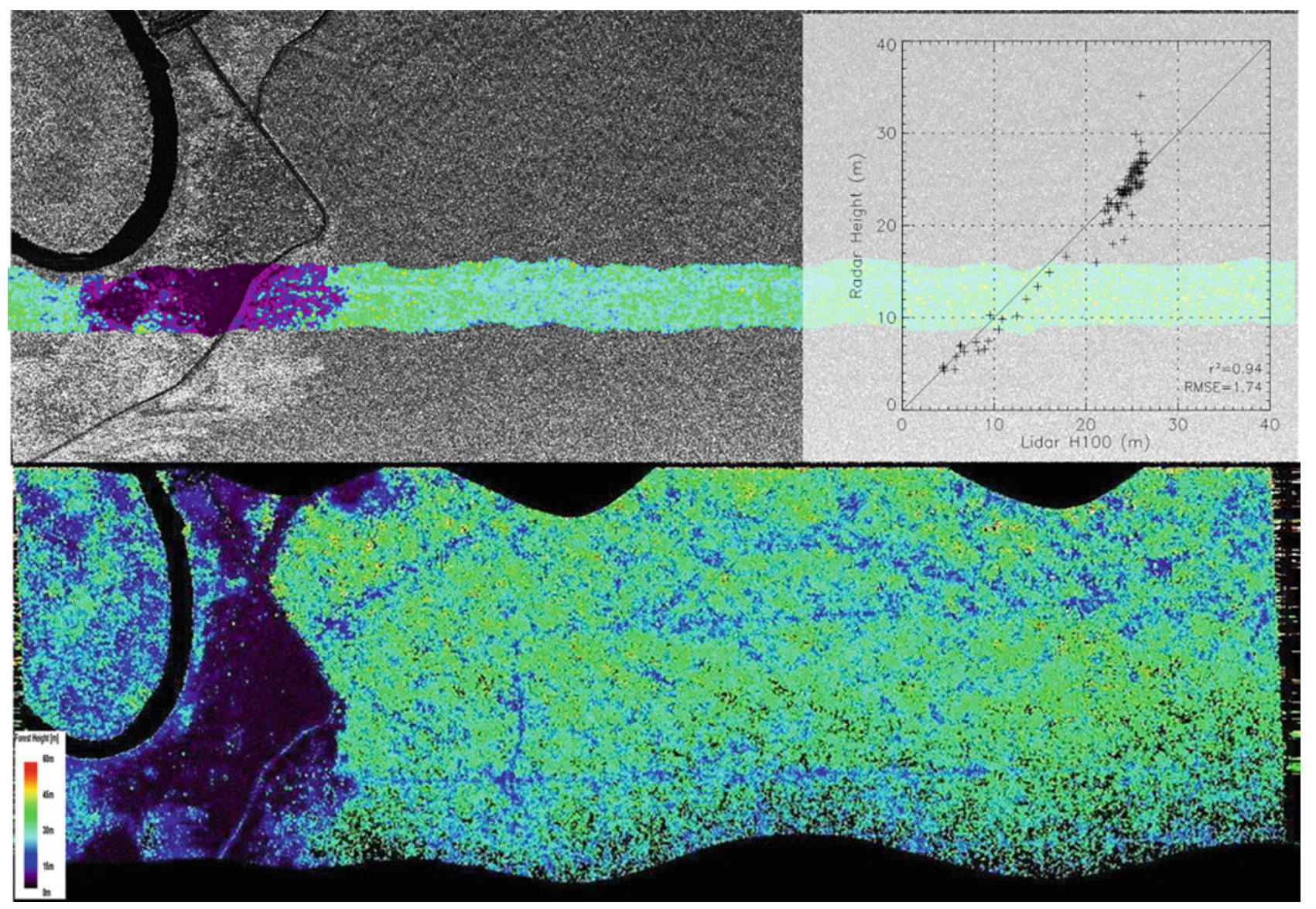

Fig. 2.19 Top: P-band HH intensity image of the Mawas test site. Bottom: Forest height map from PolInSAR data. Top right: associated validation plot against the lidar reference 


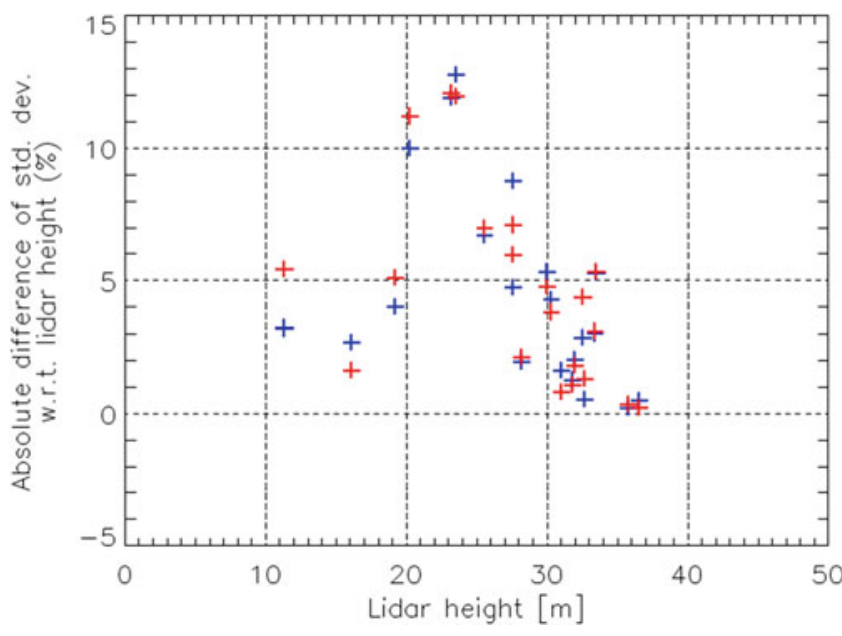

Fig. 2.20 Increase of forest height standard deviation (expressed in \% of absolute forest height) for the dual-pol inversion case (blue: HH and $\mathrm{HV}$, red: $\mathrm{HH}$ and VV) when compared to the quad-pol case for each validation stand of the Traunstein 2008 L-band

level that moves the $\gamma_{V}\left(k_{z}\right)$ values out of the $\widetilde{\gamma}_{V}\left(k_{z}, h_{V}, \sigma\right)$ solution space. In the case of Traunstein, both dualpolarimetric inversion configurations have $15 \%$ more non-invertible forest points than the quad-polarimetric configuration where $95 \%$ of all forest points could have been inverted.

In the case of a single-polarimetric interferometric configuration that provides a single polarimetric channel $\mathbf{w}$, the parameterisation of the measured interferometric coherence in terms of the RVoG model requires four parameters: forest height $h_{V}$, extinction $\sigma$, ground topography phase $\varphi_{0}$, and ground-tovolume amplitude ratio $\mu(\mathbf{w})$. Even neglecting the ground scattering contribution is not anymore sufficient to obtain a balanced inversion problem. In this case, the inversion relies on additional assumptions or external (a-priori) information on extinction or on ground topography. Fixing the extinction $\sigma$ has been proved to compromise the inversion performance as it restricts the ability of the RVoG model to interpret the spatial variability of forest structure (Hajnsek et al. 2009).

A significant better performance is achieved by using an external digital terrain model (DTM) to estimate the ground topography phase $\exp \left(i \varphi_{0}\right)$ (Kurvonen and Hallikainen 1999; Dobson et al. 1996). In this case $\gamma\left(\kappa_{z}, \vec{w}\right)$ can then be inverted for forest height $h_{V}$ and extinction $\sigma$ as.

$$
\min _{h_{V}, \sigma}\left\|\gamma\left(k_{z}, \mathbf{w}\right) \exp \left(-j \varphi_{0}\right)-\widetilde{\gamma}_{V}\left(h_{V}, \sigma, \mu=0 \mid k_{z}\right)\right\|
$$

Figure 2.21 compares the two approaches using singleand dual-polarimetric TanDEM-X data sets acquired over Kryclan. The lidar-derived reference forest height map is shown on the top left. The corresponding single- and dual- pol forest height maps are on top middle and top right respectively. The associated validation plots are shown on bottom middle and bottom right. Compared to the single-pol inversion characterized by a correlation coefficient equal to 0.91 and a RMSE of about $1.6 \mathrm{~m}$ the dual-pol estimates is noisier, in particular for the taller forest stands, but the overall correlation coefficient equal 0.86 and a RMSE of $2.02 \mathrm{~m}$ remains convincing. On the bottom left, the single-pol forest height estimates are plotted against the dual-pol forest heights estimates. A correlation coefficient equal to 0.93 in combination with a RMSE of 1.44 m underlines the consistency of the results obtained by the two approaches.

\subsubsection{Discussion on the Role of Polarimetry, on the Maturity of the Application and Conclusions}

Single-pol interferometric data at a single baseline do not provide enough measurables to invert forest height without a-priori information e.g. on terrain topography. A single-pol inversion is possible when multiple baselines are available, but the performance might be compromised by the presence of temporal decorrelation and the ratio of the spatial baselines. On the other hand, forest height inversion is possible with dualpol interferometric data taking into account a reduced estimation performance when compared to the quad-pol case. The availability of quad-pol interferometric measurements, that allows the implementation of adaptive optimisation technique, is critical when algorithm robustness and performance matters.

Forest height estimation matured and developed in the last years from a pre-operational to an operational PolInSAR product that has been validated in the frame of several campaigns over a wide range of forest, terrain and environmental conditions. The overall obtained estimation accuracy is on the order of $10 \%$ or better. Uncompensated non-volumetric decorrelation contributions, such as temporal or additive noise decorrelation, are the most critical error sources. The limitations of the individual inversion approaches and the error propagation through them are today well understood as well as the options available to compensate for them.

\subsection{Forest Vertical Structure Estimation Using Multi-baseline Polarimetric SAR Acquisitions}

\subsubsection{Polarimetric SAR Tomography}

\subsubsection{Introduction, Motivation and Literature Review}

The vertical structure of forest is widely recognized to be an indicator of the above-ground biomass, whose knowledge is crucial for understanding the carbon cycle. Moreover, the 


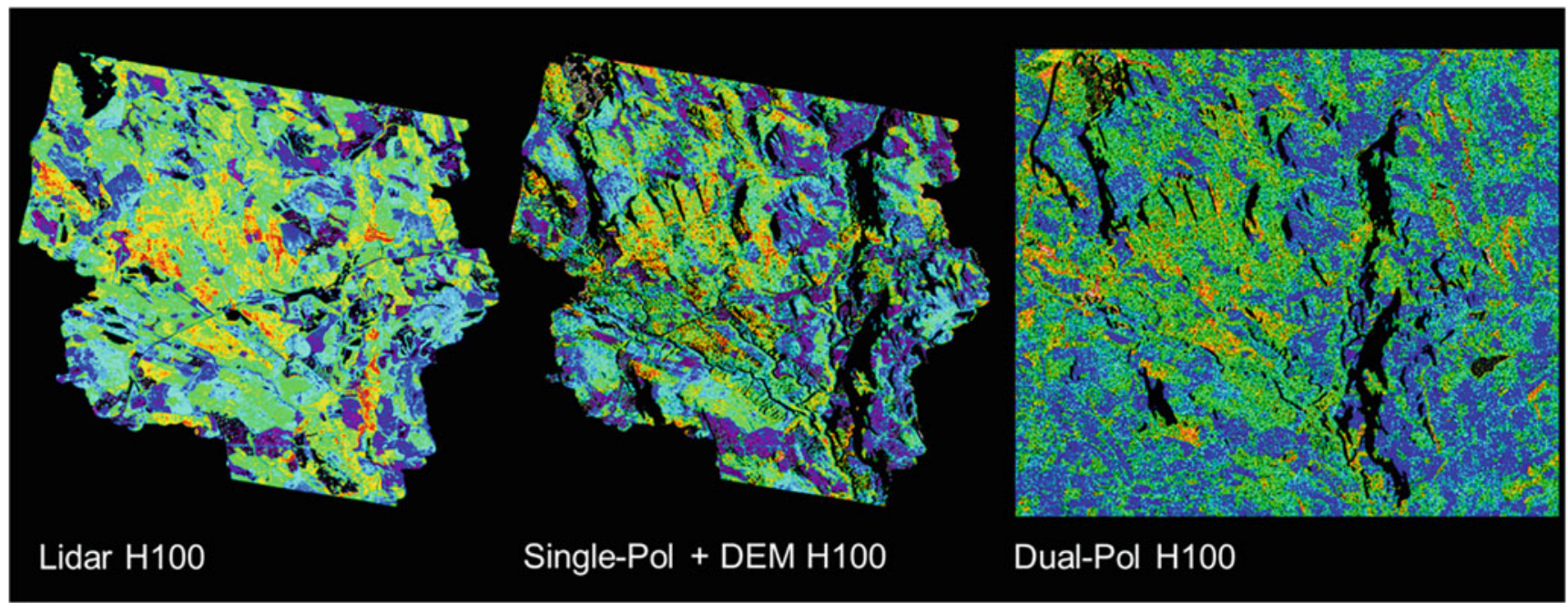

(a)
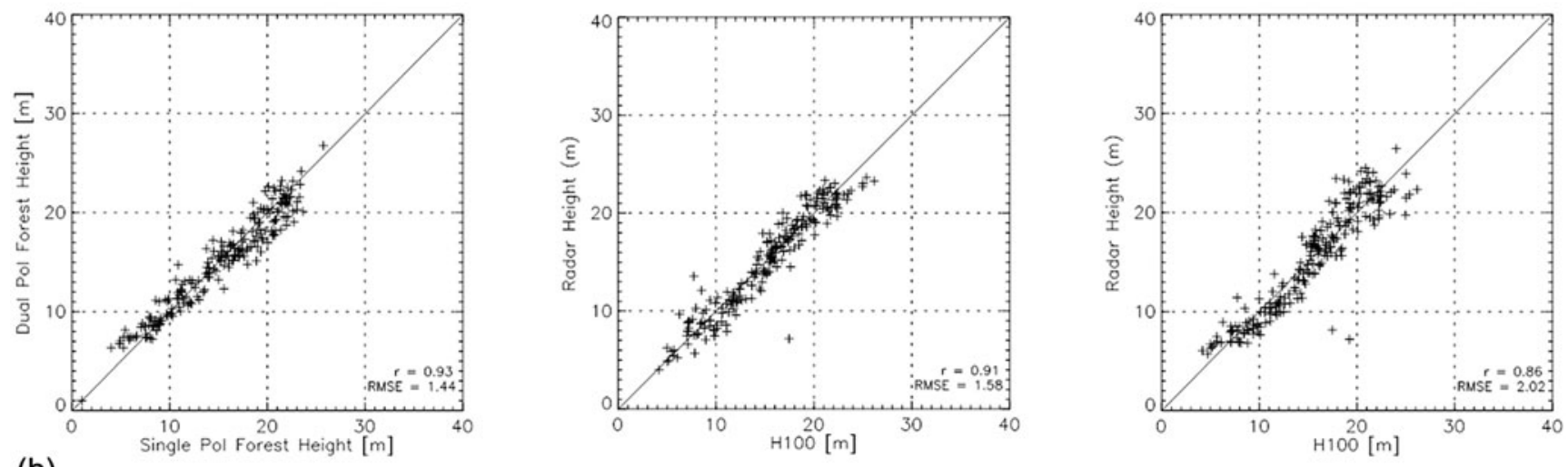

(b)

Fig. 2.21 X-band single and dual-polarimetric inversion results. Top: Forest height maps. Lidar H100 map (left), single-pol forest height map (middle), dual-pol forest height map (right). Bottom: Validation plots: dual-pol (right), single-pol (middle), single- vs dual-pol (left)

knowledge of the vertical structure can reduce effects of saturation in the measurement of biomass by means of remote sensing systems. Through vertical structure it is possible to characterize the state of a forest ecosystem, for instance relatively to local/global climate change. Biodiversity in a forest environment depends on its vertical structure (Pretszch 2009).

Forest structure and biomass are still too poorly quantified across most part of the planet. For this reason, the estimation of forest vertical structure has become a very important research topic within the remote sensing community. Much work has been done in recent years in order to characterize the 3-D structure of the vegetation layer, as demonstrated on the one hand by the employment of lidar sensors, and on the other hand by the attention paid by different research groups to SAR systems. Especially the processing of SAR data results to be particularly appealing in forest observation. In fact, the unique ability of low frequency (P-/L-band) waves to penetrate into and through even dense vegetation and thus to interact with the different vegetation layers, provides sensitivity to vegetation structure. On the other hand, space borne SAR systems can acquire data continuously and provide the related products with high spatial and temporal resolution at a global scale.

A way to obtain the vertical structure of forests from SAR data consists in making use of SAR tomography (TomoSAR) techniques, which combine coherently (i.e. amplitude and phase) more than two acquisitions separated by horizontal and/or vertical displacements (baselines). The resulting cross-track baseline diversity allows to produce an aperture synthesis along the vertical plane to get full 3-D imaging through elevation beamforming for each given range-azimuth cell. The main result of this imaging process is a profile of the backscattered power as a function of the height. The size of the 3-D resolution cell is determined by pulse bandwidth along the slant range direction, and by the lengths of the synthetic apertures in the azimuth and cross-range directions. This concept is sketched in Fig. 2.22. It is worth noting that, provided a sufficient number of baselines, while polarimetric diversity per se is not required for obtaining forest profiles, 


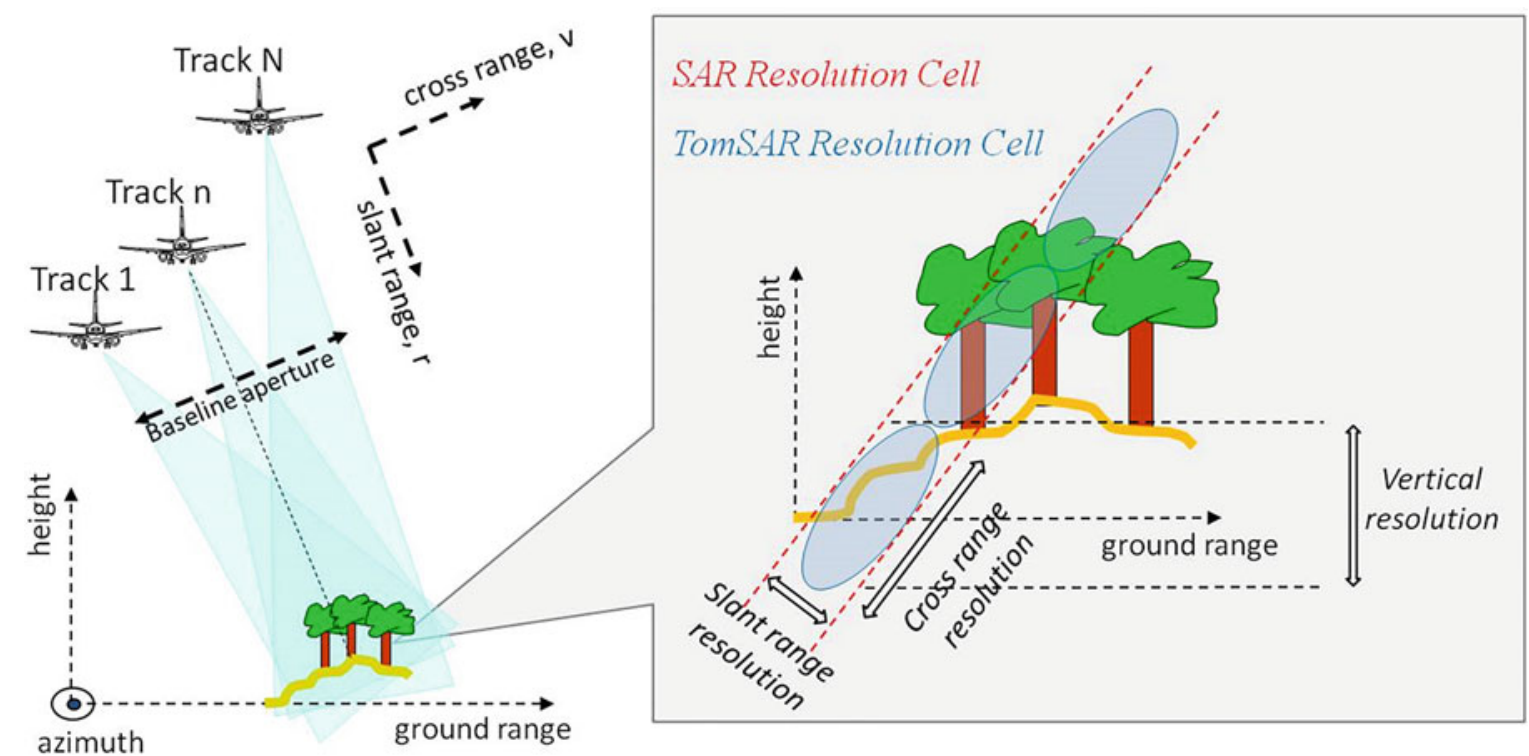

Fig. 2.22 Left panel: pictorial view of a tomographic SAR system; right panel: cross range and slant range extent of the TomSAR resolution cell

polarimetric information is essential to characterize the individual scattering processes resolved in height. Such an enhanced representation results in the possibility to (1) extend from 2-D to 3-D all the classes of polarimetric estimation/ decomposition techniques; (2) provide new estimation/ decomposition techniques that are explicitly based on the joint exploitation of tomographic and polarimetric data.

The development of TomoSAR started with single-pol data. In parallel to the development of PolInSAR, TomoSAR for forest observation was demonstrated with an L-band airborne multibaseline (MB) experiment (Reigber and Moreira 2000) carried out by the DLR E-SAR platform over the Oberpfaffenhofen site. The possibility was shown to gain insights about the 3-D nature of the scattering mechanisms. However, two main limitations of SAR Tomography for forests were apparent since the beginning, namely the usually low number of images available for processing to avoid large acquisition times and the consequential temporal decorrelation, and the difficulty of obtaining ideal uniformly spaced parallel flight tracks due to navigation/orbital considerations. The baseline non-uniformity causes distortions in the imaging point-spread function along height, preventing from employing a simple Fourier-based focusing due to anomalous side and quasi-grating lobes.

In order to mitigate the effects of acquisition non-idealities, most of the subsequent research on (singlepol) TomoSAR investigated different imaging solutions. With particular reference to forest scenarios, a significant model-free advancement was reported in Lombardini and Reigber (2003), in which an adaptive beam forming technique based on the Capon spectral estimator was proposed and tested. The adaptive beam forming allows a reamarkable improvement in terms of both sidelobe level and resolution, and it has become a state-of-the-art technique in TomoSAR. Model-based alternatives were investigated as well, such as the multiple signal classification (MUSIC) (Nannini et al. 2009) and multi-baseline inversion approaches based on the covariance matching principle (Tebaldini 2010). These techniques have been extended in order to handle different polarimetric channels (Sauer et al. 2011; Huang et al. 2012; Ferro-Famil et al. 2012; Frey and Meier 2011). Many experiments carried out with real data have shown that the use of polarimetric information not only increments the number of observables, but it also improves the accuracy of height estimation of scatterers, increase height resolution, and allows to estimate a vector of complex coefficients describing the scattering mechanism at each height. Moreover, the combination of multibaseline polarimetric data can also be used to separate ground and canopy scattering, and to estimate their vertical structures by following a relatively simple algebraic approach based on the sum of Kronecker products (Tebaldini 2009) that extends with continuity the PolInSAR concept. Other authors have also shown the possibility to separate different contributions in the multibaseline PolInSAR coherences in order to estimate structural parameters associated e.g. to tree morphology (Neumann et al. 2010).

In this Section, the application of TomoSAR techniques to the estimation of the forest vertical structure is demonstrated with real data acquired in the framework of relevant (repeatpass) airborne SAR campaigns. It is shown that the employed methodologies can separate different scattering layers and can estimate the distribution of the radar backscattered power along height, being such profile a proxy for a biomass 
distribution profile. Moreover, the role of polarimetry in improving and enriching the tomographic processing output will be emphasized and discussed.

\subsubsection{Methodology}

In order to estimate a vertical profile of the backscattered power, as already mentioned in Sect. 1.6, a plain Fourierbased 3-D focusing suffers from inflated sidelobes and poor height resolution (Lombardini and Reigber 2003). For this reason, in this Section the adaptive beam forming (from here on indicated with the acronym ABF for brevity) solution is considered. In this Section, we recall the ABF principle, while details can be found in Chap. 1 .

In the classical single-pol TomoSAR, it is assumed to process data from an equivalent cross-track array of $K$ phase centers, each of them corresponding to one of the $K$ repeated flight tracks of the SAR sensor over the area of interest. As usual in SAR imaging and interferometry, after focusing in the range-azimuth plane, the images are assumed to be coregistered and properly compensated for the flat-earth phase. Moreover, $N$ independent looks (here formed from multiple adjacent pixels) are used for processing. For each $n$ th look, the complex amplitudes of the pixels observed in the $K$ SAR images at the same range-azimuth coordinate are collected in a number of $K \times 1$ complex-valued vectors corresponding to the number of polarimetric channels (Sauer et al. 2011). If fully polarimetric data are available, without losing generality, they can be combined in the Pauli basis. The resulting MB data vectors can then be stacked one on top of the other in order to form the $3 K$-dimensional MB-polarimetric data vector $\mathbf{y}_{P}(n)$, which is statistically characterized by the MB-polarimetric covariance matrix $\mathbf{R}_{P}$.

In forest scenarios, the scatterers at a given height $z$ are characterized by a random polarimetric behaviour, and they are more properly described by means of a $3 \times 3$ polarimetric covariance matrix $\mathbf{T}(z)$ rather than by a deterministic (coherent) target vector (Ferro-Famil et al. 2012). In this way, the scattering mechanism at the generic $z$ will contribute to $\mathbf{R}_{P}$ with $\mathbf{T}(z) \otimes\left[\mathbf{a}(z) \mathbf{a}^{T *}(z)\right]$, in which " $\bigotimes$ " denotes the Kronecker product and $\mathbf{a}(z)$ is the $K \times 1 \mathrm{MB}$ steering vector. At the generic $z$, the ABF processor maximizes the total polarimetric power, and at the same time minimizes the interfering power contributions coming from heights different than $z$. By repeating this procedure for all the heights of interest, a stack of estimated polarimetric covariance matrices $\mathbf{T}(z)$ can be obtained. In this way, the polarimetric information can be exploited for a full 3-D characterization of the scattering, allowing for instance the use of incoherent polarimetric decompositions, as well as the application of physical scattering models for the 3-D identification of the single scattering components.

The Kronecker product representation discussed before can be specialized to express two-layer scattering models like the ones that are commonly used to describe model scenarios in the PolInSAR framework. A ground and a volume scattering layer will then contribute to $\mathbf{R}_{P}$ with $3 \times 3$ polarimetric covariance matrices $\mathbf{T}_{G}$ and $\mathbf{T}_{V}$ and with structure matrices (i.e. baseline-dependent only) $\mathbf{R}_{G}$ and $\mathbf{R}_{V}$, respectively. As a consequence, under the RVoG assumption, the MB-polarimetric covariance matrix $\mathbf{R}_{P}$ can be modelled as a sum of Kronecker products as follows (Tebaldini 2009):

$$
\mathbf{R}_{P}=\mathbf{C}_{G} \bigotimes \mathbf{R}_{G}+\mathbf{C}_{V} \bigotimes \mathbf{R}_{V} .
$$

It is worth noting that if only one baseline is available, then Eq. (2.24) reduces to the classical PolInSAR model of (2.9), and therefore it constitutes its consistent multibaseline extension. The form of (2.24) allows to separate ground and volume scattering by means of algebraic techniques (Tebaldini 2009).

\subsubsection{Experimental Results}

Test sites and corresponding radar and validation data sets selected for the generation of showcases on forest structure estimation are summarized in Table 2.7 and further described in the Appendix.

To begin with, Fig. 2.23 shows a tomographic profile obtained in the range-height plane by processing both the L- and P-band Traunstein data with the full rank ABF at the same (fixed) azimuth coordinate. Polarimetric TomoSAR results have been derived from the selected data set by using a square multilook cell in the range-azimuth plane

Table 2.7 Test sites and corresponding radar and validation data selected for the generation of showcases on forest structure estimation

\begin{tabular}{l|l|l}
\hline $\begin{array}{l}\text { Application/ } \\
\text { product }\end{array}$ & Test site - Radar data & Reference data \\
\hline $\begin{array}{l}\text { Forest structure } \\
\text { estimation }\end{array}$ & $\begin{array}{l}\text { Remningstorp, Sweden } \\
\text { Airborne E-SAR P-band repeat-pass } \\
\text { quad-pol data 2007 } \\
\text { Krycklan, Sweden } \\
\text { Airborne E-SAR P-band repeat-pass } \\
\text { quad-pol data 2008 } \\
\text { Traunstein, Germany } \\
\text { Airborne E-SAR L/P-band repeat-pass } \\
\text { quad-pol data 2009 }\end{array}$ & $\begin{array}{l}\text { Airborne lidar measurements (ground topography and forest height), biomass } \\
\text { profiles extracted from ground measurements }\end{array}$ \\
\hline
\end{tabular}



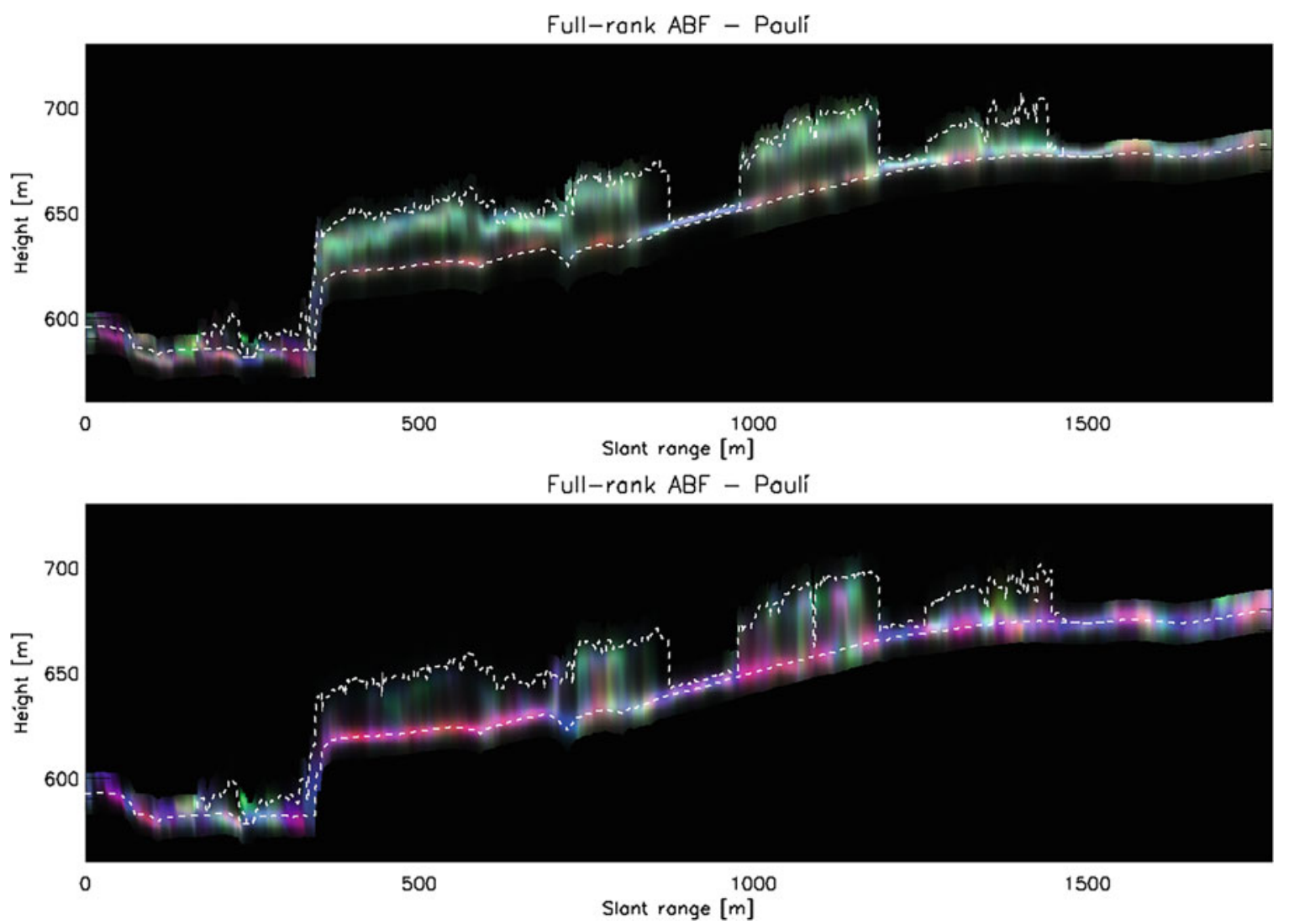

Fig. 2.23 Traunstein data set: tomographic slices obtained in the range-height plane at a fixed azimuth with the full-rank ABF. Estimated powers in the three Pauli channels are RGB-coded (Red: HH-VV, Green: 2 HV, Blue: HH + VV). Top panel: L-band; bottom panel: P-band

measuring around $25 \mathrm{~m} \times 25 \mathrm{~m}$. This cell corresponds to more than 100 independent looks, which is enough to guarantee a well-conditioned inversion of the MB-polarimetric covariance matrix. The slant range coordinate spans an interval of approximately $1.7 \mathrm{~km}$, while the height varies in an interval of $160 \mathrm{~m}$ around $650 \mathrm{~m}$ above the sea level. The powers estimated in the three Pauli channel (i.e. the values on the diagonal of the estimated $\mathbf{T}(z))$ have been RGB-coded, as usually done for polarimetric SAR images. Height resolution capabilities are apparent, allowing to distinguish different scatterers at different heights in the same cell, and do not worsen significantly from near to far range, being robust to the variation of the incidence angle. Bare ground areas can be distinguished from forested areas. Moreover, the ground scattering is located in correspondence of the lidar ground height, and it is more powerful at P-band than at L-band. In addition to height position, from the profiles of Fig. 2.23 a first insight of the occurring scattering mechanisms can be gained. For instance, in the forested areas, it is possible to distinguish mixed surface and double-bounce components at the ground level, as it is reasonable to expect from direct ground backscattering and tree trunk-ground interactions. Moreover, double-bounces are clearer at P-band, while at L-band their polarimetric signature looks more affected by the propagation through the canopy and/or the presence of understorey, although some estimation bias can have been induced by the volume due the limited height resolution and number of baselines. At the increase of the height, volumetric contributions (in green) start to appear at some range coordinates, sometime mixed to multiple (even or odd) bounce scattering contributions. They are always visible at L-band, while they are semi-transparent for many stands at P-band.

The following examples show how ground and volume scattering can be separated. One way to achieve this, is to exploit the ABF PolTomoSAR focusing. Using simple iterative MB-polarimetric techniques (Pardini and Papathanassiou 2013), the ground height has been retrieved from the data with an estimation accuracy of around $2 \mathrm{~m}$ at L-band and $1.5 \mathrm{~m}$ at P-band. Once the ground height is available, a simple separation between ground and volume can be performed. Figure 2.24 shows the Pauli RGB image of the ABF powers in correspondence of the estimated ground height at both Land P-band. Double-bounce and direct surface scattering contributions are predominant, as expected, with a few contributions in the third Pauli channel due to residual understorey vegetation and possible orientation effects due to azimuth slopes. Notice that the ground scattering is more 

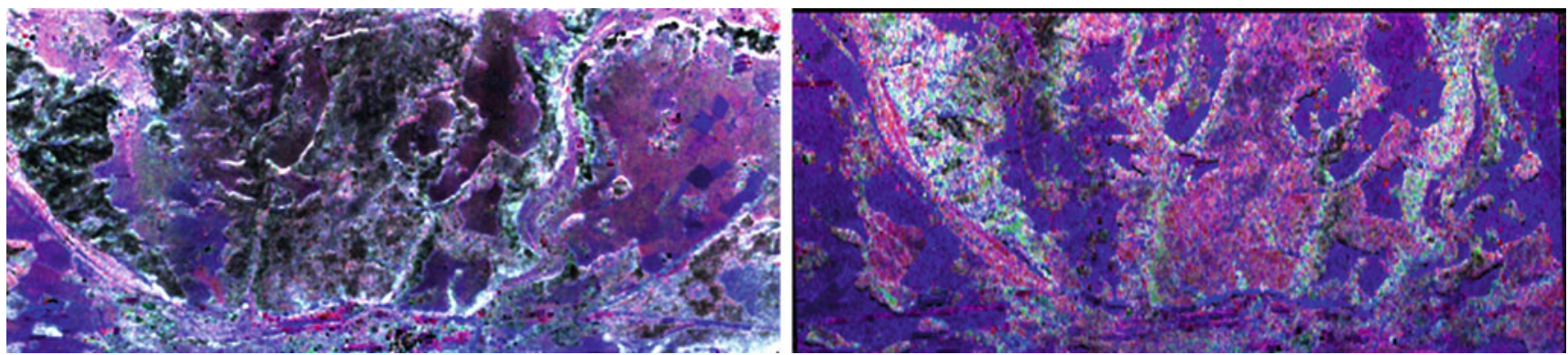

Fig. 2.24 Traunstein data set: Pauli images of the ground scattering isolated from the canopy (volume) scattering. Azimuth coordinate is on the horizontal axis. Color coding is as in Fig. 2.23. Left panel: L-band; right panel: P-band

powerful at P-band than at L-band, as it is reasonable to expect. The availability of the polarimetric covariance matrix $\mathbf{T}_{\mathrm{ABF}}(z)$ at each imaged height allows also to characterize the scattering by means of conventional incoherent decomposition tools developed in SAR polarimetry. A demonstration of this capability can be found in Fig. 2.25, in which the joint distribution of the entropy and $\bar{\alpha}$ angle are plotted in correspondence of the forested areas and for both ground and canopy contributions in isolation. The majority of the ground scattering (Fig. 2.25a, b) presents in general moderate entropy, with $\bar{\alpha}$ angles around $45^{\circ}$, as it typical for vegetated surface imaged after propagation through a forest canopy with relatively small baseline aperture, therefore with some volume-induced bias. Notice that the P-band ground scattering shows an increased number of low-entropy surface contributions with respect to L-band. Concerning the volume (Fig. $2.25 \mathrm{c}, \mathrm{d}$ ), an entropy $/ \bar{\alpha}$ diagram characteristic of a random volume is obtained, although with entropy values lower at P-band than at L-band.

Ground and volume scattering can also be extracted by referring to model (2.24) and by using algebraic techniques. This possibility is shown experimentally here after processing the P-band Remningstorp and Krycklan data sets. With reference to the Remingstorp data set, the two profiles in the left panels of Fig. 2.26 were obtained by processing the $\mathrm{HH}$ (top left) and $\mathrm{HV}$ (bottom left) polarizations independently. The most apparent feature in these panels is that the strongest scattering contributions appear at the ground level, not only in $\mathrm{HH}$ polarization but in HV polarization as well. This observation was interpreted as a result of double bounce interactions between the vegetation and the terrain, which determined cross-pol contributions at the ground level. In this situation, the vertical resolution capabilities granted by TomoSAR do not suffice for the aim of separating ground and volume scattering, as both occur at the ground level. The results of the separation between ground and volume scattering are shown in the right panels of Fig. 2.26. In the profile relative to ground scattering the backscattered power is associated with the ground level only, as witnessed by the excellent agreement with lidar terrain height. The profile relative to volume scattering yields a much more uniform backscattered power distribution as compared to HV, and also provides an excellent agreement with lidar forest height.

The same procedure was applied to the aim of a large scale characterization of the forested area within the Krycklan river catchment. Figure 2.27 displays maps of the heights at which the estimated backscattered power for volume-only scattering undergoes a loss of $0 \mathrm{~dB}$ (i.e., no loss), $3 \mathrm{~dB}, 5 \mathrm{~dB}$, and $7 \mathrm{~dB}$ with respect to the peak value (which corresponds by definition to the phase center height). Lidar heights are displayed in Fig. 2.27b to help the interpretation of the results. Forest height was assessed through a direct investigation of the shape of the backscattered power distribution. The estimation was found to be unbiased for forest height values larger than about $8 \mathrm{~m}$. Standard deviation was assessed in about $3 \mathrm{~m}$. Results are shown in Fig. 2.27c. Another effective way to characterize the forest vertical structure at large scale is to display the ground-to-volume backscattered power ratio at different polarizations, see Fig. 2.28. These maps are sensitive to both forest height and terrain topography, and may be used to assess the impact of multiple reflections on SAR images.

The analysis carried out up to now has assessed the quality and the information content of tomographic products. Moreover, some simple examples have been shown about the validity of the resulting polarimetric information for scattering interpretation. A further qualitative experiment can be carried out to understand if a correspondence exists between the ABF spectral components and the biomass components at different heights. For this validation, a few representative biomass profiles calculated from ground measurements have been selected, and the ABF full-rank profiles have been estimated in the same coordinates.

Figure 2.29a shows six different biomass profiles (first row) which refer to stands taller than $30 \mathrm{~m}$. These profiles mainly present a biomass contribution close to the top (tree crown and stems) and an additional one close to the ground (tree trunk). Starting from the left, in the third and the last two profiles some understory vegetation can be noticed. In 

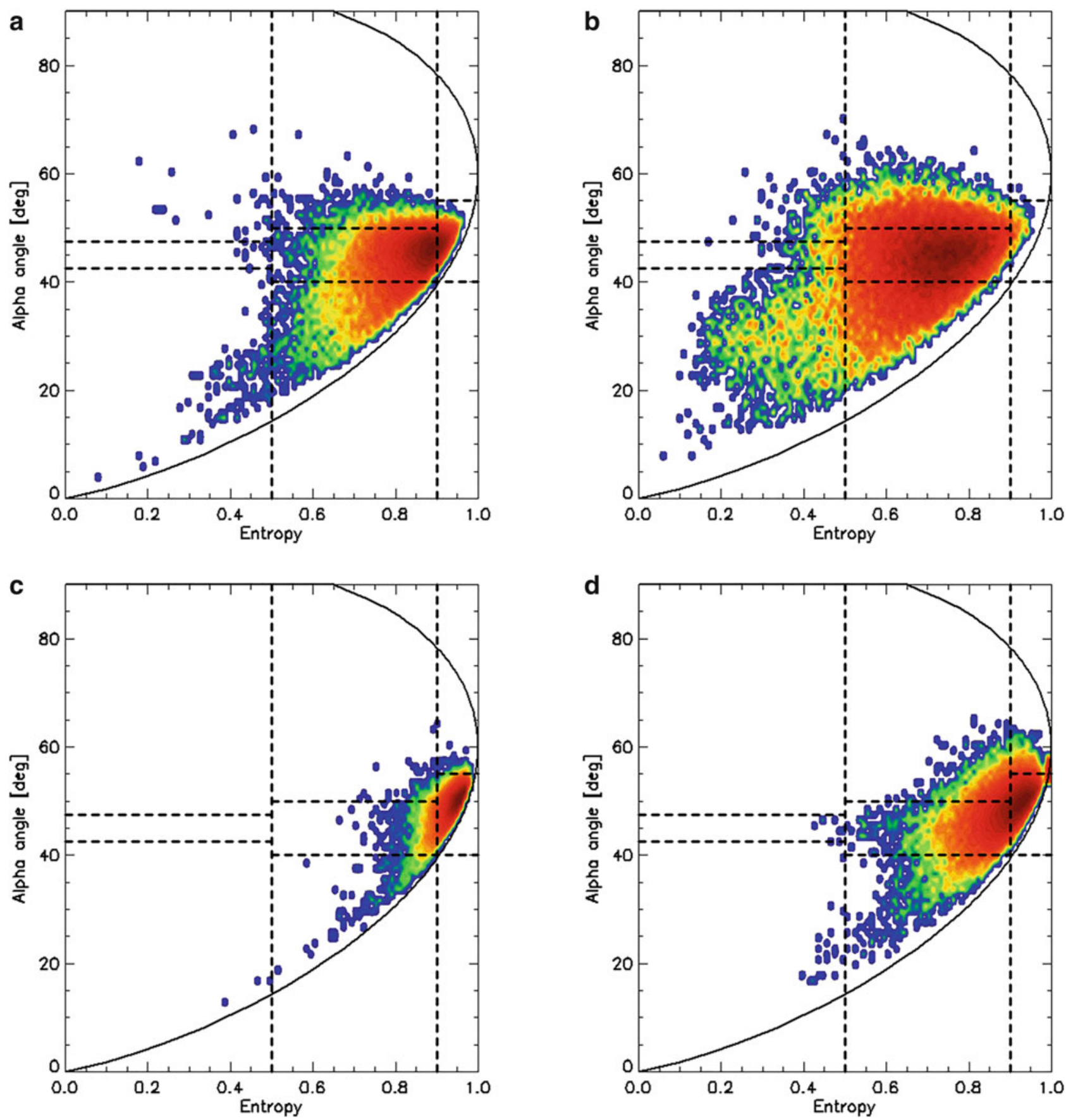

Fig. 2.25 Traunstein data set: 2-D histograms of entropy and $\bar{\alpha}$ angle of forested areas for both ground and volume scattering in isolation after the ABF polarimetric TomoSAR processing. (a) Ground, L. (b) Ground, P. (c) Volume, L. (d) Volume, P

Fig. 2.29b the L-band ABF profiles are reported in the three Pauli channels. Qualitatively, it is apparent the agreement between the backscattered radar power and the biomass distributions already by processing a relatively low number of images (7) with a short total baseline $(30 \mathrm{~m})$, therefore resulting into a vertical Rayleigh resolution around $15 \mathrm{~m}$ for the profiles under analysis. It is worth noting that different polarimetric channels are sensitive to (slightly) different biomass components, as the profiles show variations from channel to channel. From this example, it is therefore possible to conclude that polarimetric diversity is crucial for a complete characterization of the mapping of biomass components into radar scattering components. 

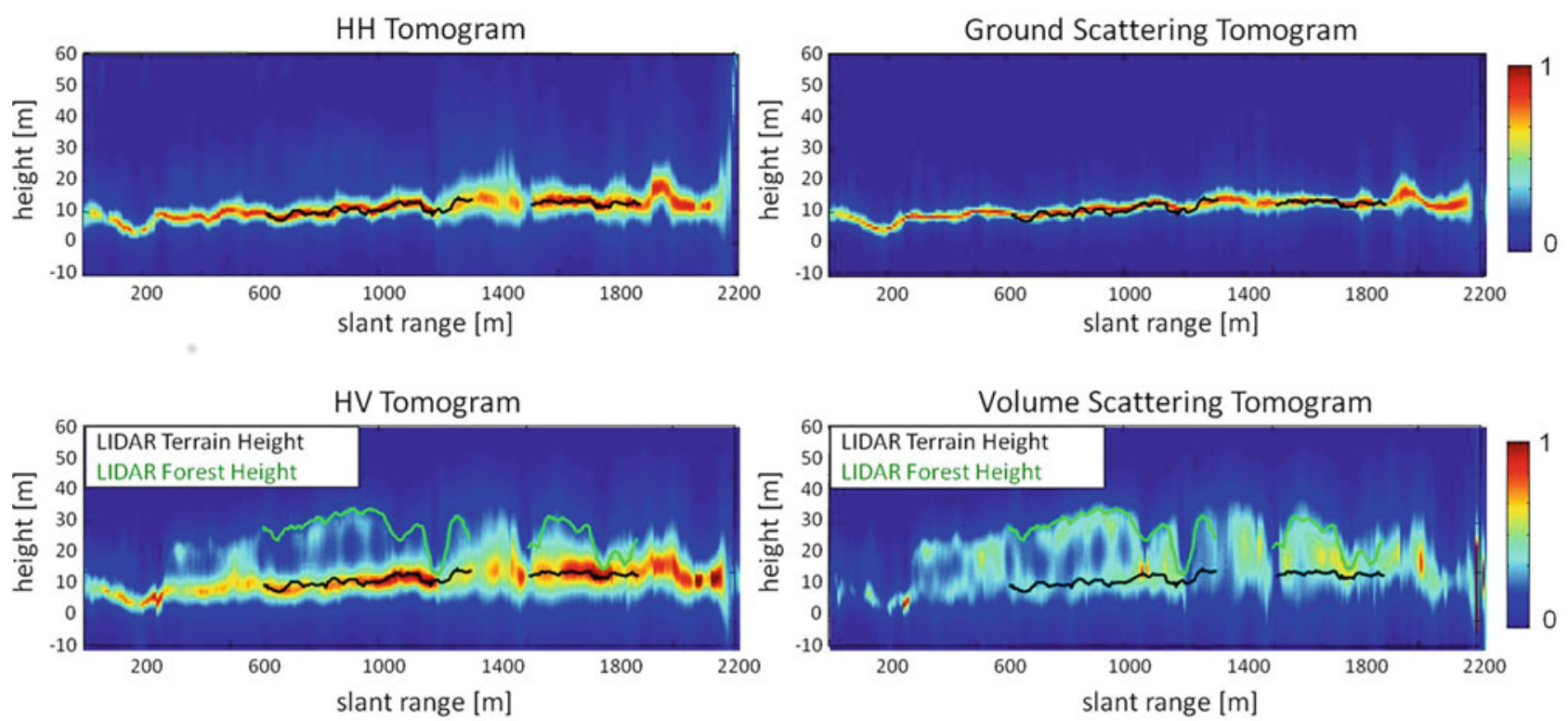

Fig. 2.26 Remningstorp data set. Left panels: HH and HV profiles. Right panels: ground and volume Tomograms. All panels are normalized such that the sum along each column is one. The black and green lines are relative to terrain and forest height as estimated by lidar
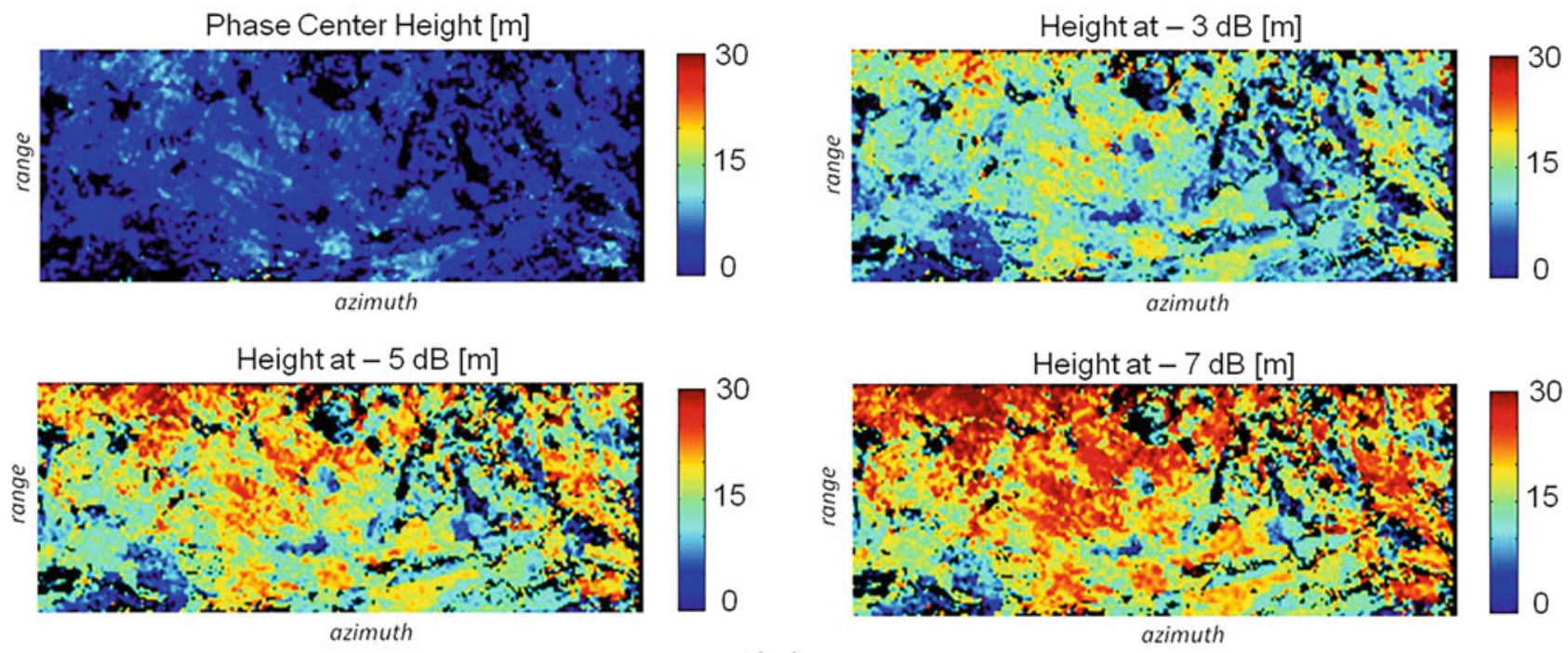

(a)
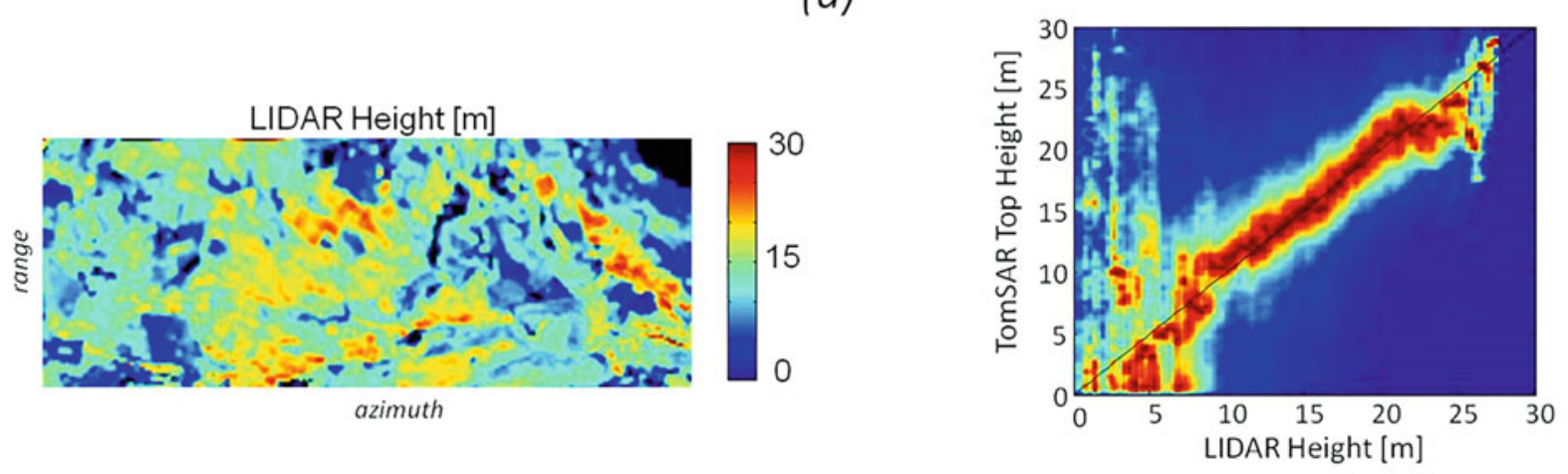

(b)

(c)

Fig. 2.27 Krycklan data set. (a) Tomographic heights relative to volume-only scattering. (b) lidar forest heights. (c) 2D histograms relative to TomSAR and lidar forest height estimates 
Fig. 2.28 Krycklan data set: Ground-to-volume power patio [dB] at $\mathrm{HH}$ (left) and HV (right)

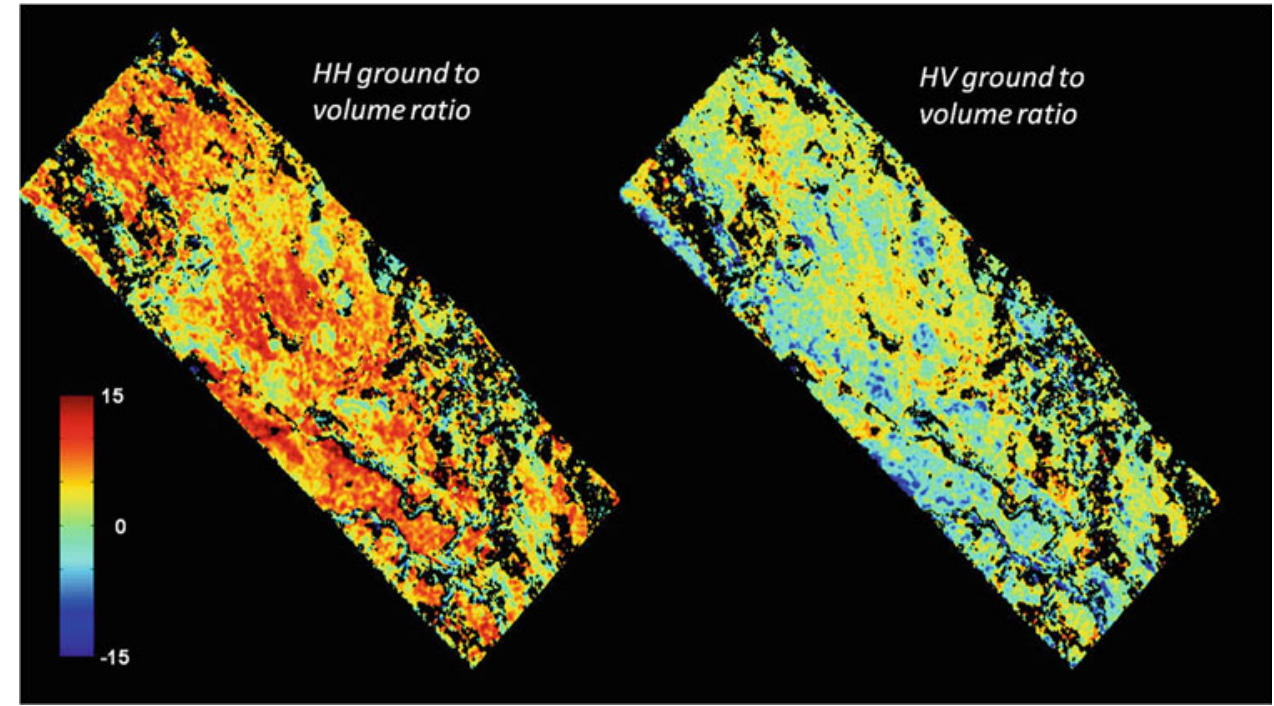

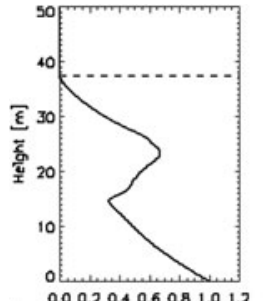

(a)

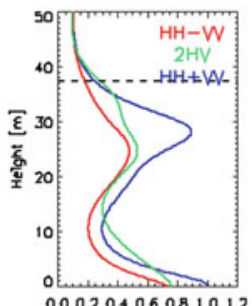

(b)

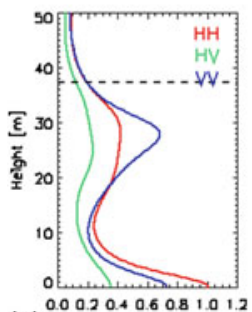

(c)

[-]

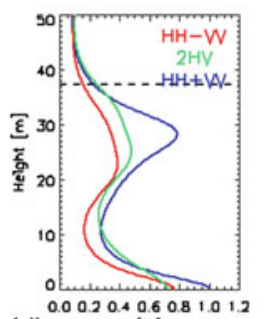

(d)

[-]

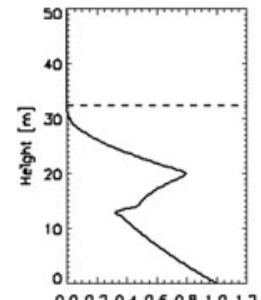

$[-1$

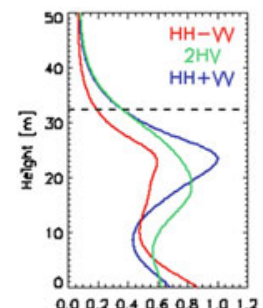

[-]

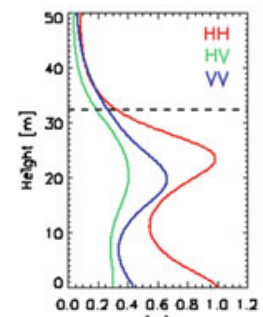

$[-]$

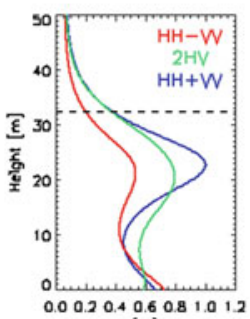

$[-1$

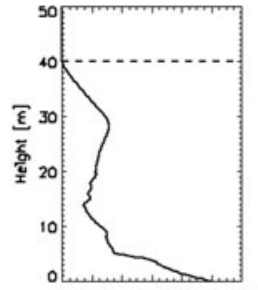

[-]

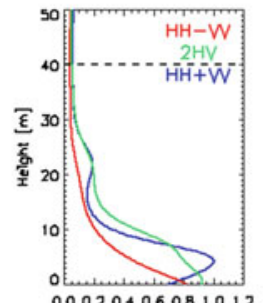

[-]

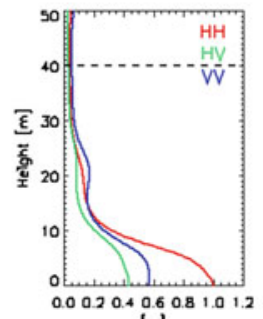

[-]

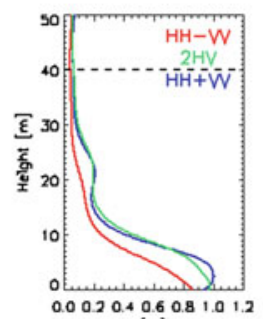

$[-]$

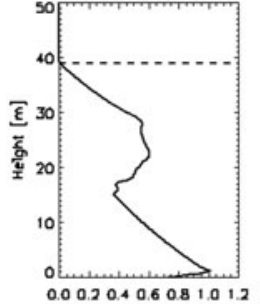

[-]

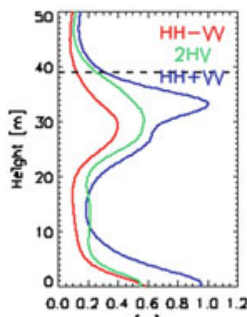

[-]

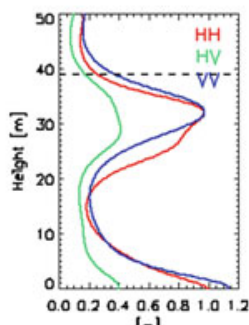

[-]

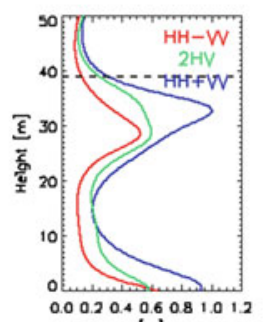

[-]

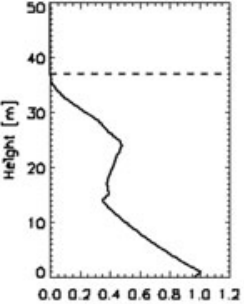

$[-1$
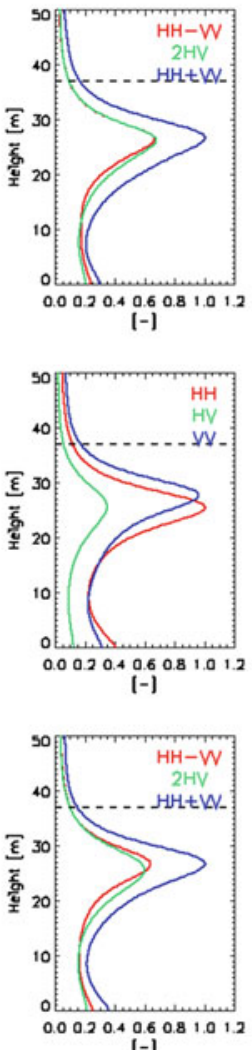

[-]

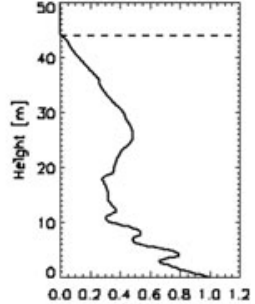

[-]

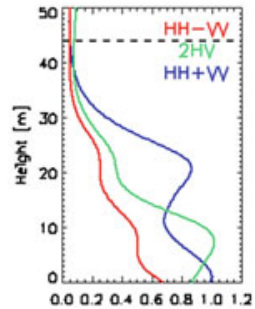

$1-1$

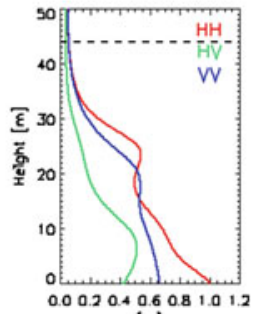

$[-]$

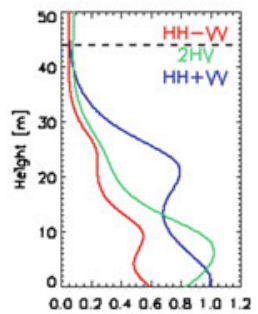

$[-1$
Fig. 2.29 Traunstein data set: reference biomass profiles and radar profiles estimated with the ABF processor from the L-band acquisitions. Profiles amplitudes are normalized with respect to the maximum. The ground is located at $0 \mathrm{~m}$. The dashed horizontal lines indicate the Lidar stand height. (a) Biomas profiles estimated from ground measurements. (b) Polarimetric $\mathrm{ABF}$ profiles, Pauli basis, full-rank coherent processing. (c) ABF profiles, lexicographic basis, each channel processed independently. (d) Polarimetric ABF profiles, Pauli basis, each channel processed independently 


\subsubsection{Comparison with Single/Dual Polarization Data}

The ABF profiles obtained by processing single polarization MB data in the lexicographic basis are plotted in Fig. 2.29c. It is apparent that one single power profile makes difficult the interpretation of the scattering from the different vegetation components. For instance, with this (realistic) baseline distribution it is not possible to discriminate the presence of understory vegetation without ambiguities. A way to circumvent this inconvenient could be to increase the number of baselines and at the same time to increase the height resolution in order to be able to separate many more power components. However, this may not be feasible depending on navigation/orbital considerations and temporal decorrelation problems. As a consequence, the polarimetric information, beyond the increased number of outputs, becomes crucial (1) to relax the acquisition requirements, and (2) to enhance profile interpretation by the application of physical scattering models.

A second comparison is in order. Supposing to have full polarization data available, one could also select a basis different from the lexicographic one and to process the new MB data independently from channel to channel. An example is shown (again in correspondence of the reference profiles) in Fig. 2.29d. Comparing them with the profiles in Fig. 2.29b, the polarization-coherent full-rank ABF can resolve slightly better different scattering components by keeping fixed the total baseline length. Clearly, the presence of volume scatterers reduces the contribution of polarimetry to any increase of vertical resolution, differently from e.g. urban scenarios. Not to be forgotten is of course the difference in the output. While a polarization-incoherent processing only outputs three real-valued profiles, the polarization-coherent approach outputs an entire polarimetric coherence matrix.

\subsubsection{Discussion on the Role of Polarimetry, on the Maturity of the Application and Conclusions}

In this Section the application of PolTomoSAR algorithms has been demonstrated for the estimation of the vertical structure of forests. The scattering can therefore be described in 3-D by means of a stack of polarimetric covariance matrices estimated at each height of interest, or by focusing on certain layers. Experiments have been carried out with a Pand an L-band MB-fully polarimetric data sets acquired by the DLR E-SAR platform. Both data sets are characterized by a relatively low number of baselines and an height resolution in the order of magnitude of $10 \mathrm{~m}$. The goodness of the extracted polarimetric signatures has been qualitatively assessed, and the estimated power distributions have been compared with the corresponding biomass profiles derived from ground measurements. A general agreement has been found.
To summarize, from the results of the experimental analysis, the crucial role of polarimetry in the coherent processing of MB data has been individuated in the following aspects:

1. Increased amount of structural-dependent information in output, allowing the physical interpretation of the estimated profile;

2. Enhanced possibility to separate scattering contributions at different heights beyond the height resolution limit imposed by both the total baseline length and the tomographic processor;

3. Higher height resolution in the profiles extracted in each channel of the polarimetric basis;

4. Higher accuracy in the estimation of vertical structure parameters.

Point (2), in turn, allows to relax constraints on the relative acquisition tracks displacements, and might be of help for forest vertical structure monitoring with non-optimal baseline distributions. Point (3) has been shown here also by comparing the coherent $\mathrm{MB}$-polarimetric $\mathrm{ABF}$ with an adaptive processing incoherent in the polarization channels. Point (4) is out of the scope of this Section, but experimental examples can be found e.g. in Sauer et al. (2011) and Huang et al. (2012).

3-D (Pol)TomoSAR imaging requires additional efforts compared to traditional 2-D SAR products and PolInSAR, due to the need of gathering multiple viewpoints and to employ more sophisticated processing techniques. Also, compare to single-pol TomoSAR, a small disadvantage of TomoSAR is the need for a larger multilook cell in order to obtain a reliable and well-conditioned estimate of the MB-polarimetric covariance matrix. Nevertheless, this issue is mitigated in SAR sensors with very high range-azimuth resolution, for which a satisfactory multilook degree for a stable $\mathrm{ABF}$ inversion can be achieved without increasing dramatically the horizontal resolution cell of the output products. With reference to forest monitoring and applications, this has an impact on the resolution of forest structure products, and on the way in which they are able to describe (dynamic) phenomena that may occur at very different scales depending on the kind of forest.

Another limitation peculiar of forest scenarios is the temporal decorrelation of the scattering. This factor has limited until now the tomographic forest monitoring from space borne platforms due to the large revisit times. Very recently, the possibility to track scattering changes has been shown in the so-called differential tomographic framework, which combines in a synergic way spatial and temporal spectral analysis. However, although promising and extended in a polarimetric sense in Lombardini et al. (2010), these techniques are in a very early stage of experimentation. Nevertheless, space borne implementations of 
(Pol)TomoSAR will benefit from repeated single-pass (Pol)InSAR implementations, in which a temporal-decorrelationfree PolInSAR coherence is available for each satellite pass.

PolTomoSAR is quite well developed and mature from the signal processing point of view. Many alternative techniques are continuously being investigated and their performance characterized. In particular, the adaptive processing in general represents a state-of-the-art, model-free, yet wellperforming algorithm. Modern techniques based on compressive sensing are being investigated as well. A still underdeveloped element is the physical interpretation of profiles. The link between the obtained profiles that depend in general on frequency, polarization and acquisition geometry and physical forest structure parameters is essential for establishing potential applications and is today not well understood, although intensively investigated.

\subsubsection{Estimation of Vegetation Structure Parameters}

\subsubsection{Introduction, Motivation and Literature Review}

Forest structure and biomass are important components in terrestrial ecosystem carbon and water cycles and provide significant controls on land-atmosphere interactions. The present estimate by IPCC (Intergovernmental Panel on Climate Change) is that deforestation amounts to between $10 \%$ and $30 \%$ of the total anthropogenic carbon flux. The range of uncertainty is large due to the lack of accurate measurement techniques. SAR observations can help to better quantify terrestrial carbon stocks and fluxes in forest biomass, providing several advantages over other techniques: independence of the time of the day (in comparison to optical remote sensing), cloud cover penetration (using selected low frequencies), large coverage (in comparison to lidar), and high resolution (in comparison to radiometers).

Early remote sensing attempts to estimate biomass were originally based on regression from microwave backscatter signal (possibly combining multiple frequencies and polarizations). However, methods based on radar backscatter signals are limited by saturation and loss of sensitivity to biomass for high biomass levels (maximum at about 80-200 tons/ha), depending on the wavelength, polarization, and incidence angle. In addition, the saturation is dependent on forest type, ground topography, and environmental conditions. Due to spatial and temporal forest variability and the signal dependence on instrument parameters, the regression approach poses difficulties to generalize over extended areas.

Recent advances in Lidar and InSAR enabled the estimation of vertical forest structure. To a large extent, forest biomass is determined by tree structure, which makes the vertical structure information a powerful indicator for biomass. Lidar illuminates the forest with laser rays and allows the reconstruction of vertical structure profile from the returned signal (waveform). Especially the recently developed small-footprint lidars provide unprecedented means for high-resolution structure estimation. However, lidar coverage is limited, disallowing global forest monitoring based on this technology alone.

On the other hand, the developments in InSAR, PolInSAR and TomoSAR enable large coverage and independence of cloud cover. Acquiring SAR signals from two (or more) slightly displaced flight trajectories (possibly using the same platform in a repeat pass configuration) and exploiting the effect of interference of coherent waves enables InSAR to estimate several phase centers inside of the forest, and thus the important parameters of forest structure extent. Polarimetry plays an important role by helping to decompose different scattering processes from the ground and the canopy. In order to further improve the accuracy of forest structure and biomass estimation, multibaseline (MB) approaches are considered. This showcase demonstrates the application of MB-PolInSAR to airborne SAR data over temperate and boreal forests estimating forest structure, biomass, tree morphology and ground scattering related parameters.

Interferometric decorrelation in volumetric media such as canopy, snow and ice has been modeled to measure vegetation depth and extinction for the first time in 1995-1996 (Hagberg et al. 1995; Treuhaft et al. 1996). The model resembles a simple sparse-medium water cloud model (Treuhaft et al. 1996). In 1997-1998 time frame, authors in Cloude and Papathanassiou (1998) and Papathanassiou and Cloude (2001) proposed to combine polarimetry with interferometry to discriminate ground scattering from volume scattering in the canopy. This enables to estimate forest height by differentiating the InSAR phase centers from different polarizations, or to invert the complex coherence model accounting for the extinction. PolInSAR coherence optimization in single- and multi-baseline configurations has also been used to reduce phase uncertainty and to determine the polarization with lowest/highest InSAR phase noise (Cloude and Papathanassiou 1998). Also extensive PolInSAR scattering models for random and oriented scattering in the canopy and single- and double-bounce scattering at the ground level were derived and evaluated in Treuhaft and Siqueira (2000). Since then, the PolInSAR approach to measure volumetric media properties has been extended and applied to other volumetric media (such as snow, ice, and agriculture (Lopez-Sanchez et al. 2007)) and to complex multi-scattering environments in urban areas. Polarimetry demonstrated to be a key factor in distinguishing physically different scattering centers inside individual resolution cells.

Alternative radar approaches to estimate forest structure are based on tomography (Reigber and Moreira 2000), multi- 
baseline InSAR estimation (Treuhaft et al. 2009), and polarization coherence tomography (Cloude 2006). The first aims to reconstruct the reflectivity profile of the volume by using conventional or adaptive beam forming techniques (see Sect. 2.4.1). The last two rely on structure models, which are fitted using coherences from different baselines, and which corresponds to probing the medium with different Fourier frequencies.

Another approach (Neumann et al. 2010), as presented in this Section, aims to further decompose the MB-PolInSAR data into ground and volume sources related to polarimetric scattering, vertical profile structure and temporal change. On the one hand, this improves the understanding of the involved processes, and on the other, providing e.g. tree morphology parameters would enhance tree species characterization and biomass estimation.

The limitations of PolInSAR are governed by the geometric configuration of the interferometer (structure sensitivity) and the ambiguities related to vertically non-homogeneous structure, spatial variability of the forest, temporal decorrelation in repeat pass acquisitions, and possibly other minor (but usually well understood) noise sources (thermal noise and other instrument/system induced effects) (Krieger et al. 2005).

Height sensitivity and accuracy is varying with environmental and acquisition configuration parameters. On one side, airborne acquisition geometry causes variation in height sensitivity along the swath, and on the other side, the optimal acquisition geometry depends on the actual forest height. One proposed approach was to combine height estimates from multiple single-baseline PolInSAR data sets, if they are available (Hajnsek et al. 2009).

Acquiring SAR data in repeat pass configuration leads to illuminating possibly changed targets. Recent airborne and ground-based studies confirmed several common sources of temporal change in PolInSAR observables, including wind, growth, and changes in soil and vegetation water content. Initially, temporal decorrelation has been modeled by a Gaussian process. Over a short temporal scale (sub-hour level), wind effects appear to be the dominating source of decorrelation. Therefore, in Askne et al. (1997), the authors incorporate change in the Gaussian motion with height, arguing that thinner branches (higher in the canopy) are stronger effected by the wind than thicker branches and the trunk (closer to the ground). Soil moisture effects have been observed to affect the interferometric coherence and phase. Diurnal variability of vegetation water content is as well expected to affect the temporal decorrelation, as recently observed in ground-based radar measurements (Hamadi et al. 2013).

Recent important contributions to PolInSAR statistical properties and estimation approaches promise further advances. Tests of common physical assumptions, such scattering symmetries and stationary behavior, were initially derived in Ferro-Famil and Neumann (2008). The singular value decomposition based "Sum of Kronecker Products" (SKP) provides an elegant way to decompose the MB-PolInSAR covariance matrix into a parametric form of independent contributions (Tebaldini 2009). This work was recently updated by deriving a maximum likelihood estimator for the linear structure of two independent layers, as presented in [R7].

\subsubsection{Methodology}

The MB-PolInSAR approach of this Section consists of combining a physical model-based polarimetric decomposition with the PolInSAR Random Volume over Ground (RVoG) coherence model for the vertical forest structure. The goal is to enhance vertical structure estimation and to permit the retrieval of morphological vegetation parameters as well as ground parameters under the vegetation. The temporal change is taken into account in order to compensate for the caused decorrelation.

The models used in PolInSAR are usually based on the two-layer model, where it is assumed that the signal can be decomposed into independent and distinctive layers, representing the ground and the volume. The "distinctiveness" can be based on three orthogonal principles: (1) polarimetric scattering, (2) vertical extent, and (3) temporal processes (compare Fig. 2.30). Though the layers are considered independent, they can affect each other. For instance, the volume layer attenuates the ground scattering, possibly modifying the ground scattering polarization by means of refraction. The double-bounce is caused by scattering in both layers, though structurally it is attributed to the ground layer.

The modeled ground contribution consists of attenuated surface scattering from the soil, the double-bounce scattering between the soil and tree elements such as trunk and branches, and volumetric scattering from a low layer of understory. The volume layer is dominated by diffuse volume scattering from the canopy elements, including multiple scattering effects. Forest canopy is expected to be a sparse medium, attenuating the signal with the distorted Born approximation. The vertical profile is represented by an effective scattering profile $F(z)$, which takes into account extinction and the distribution of the scattering elements. Different forms were proposed for $F(z)$ in the literature, including a uniform attenuated, Gaussian, or represented by Fourier or Legendre series. In this example, to represent the temperate and boreal forests, $F(z)$ is characterized by two parameters, extinction $\sigma_{x}$ and the canopy-fill-ratio $r_{h}$, as outlined in Fig. 2.31.

The polarimetric coherency matrix of the volume component is characterized by two parameters related to the polarization entropy in the canopy (called orientation randomness 
Fig. 2.30 Information content of PolInSAR data, separated into polarimetric and interferometric sources in ground and volume layers

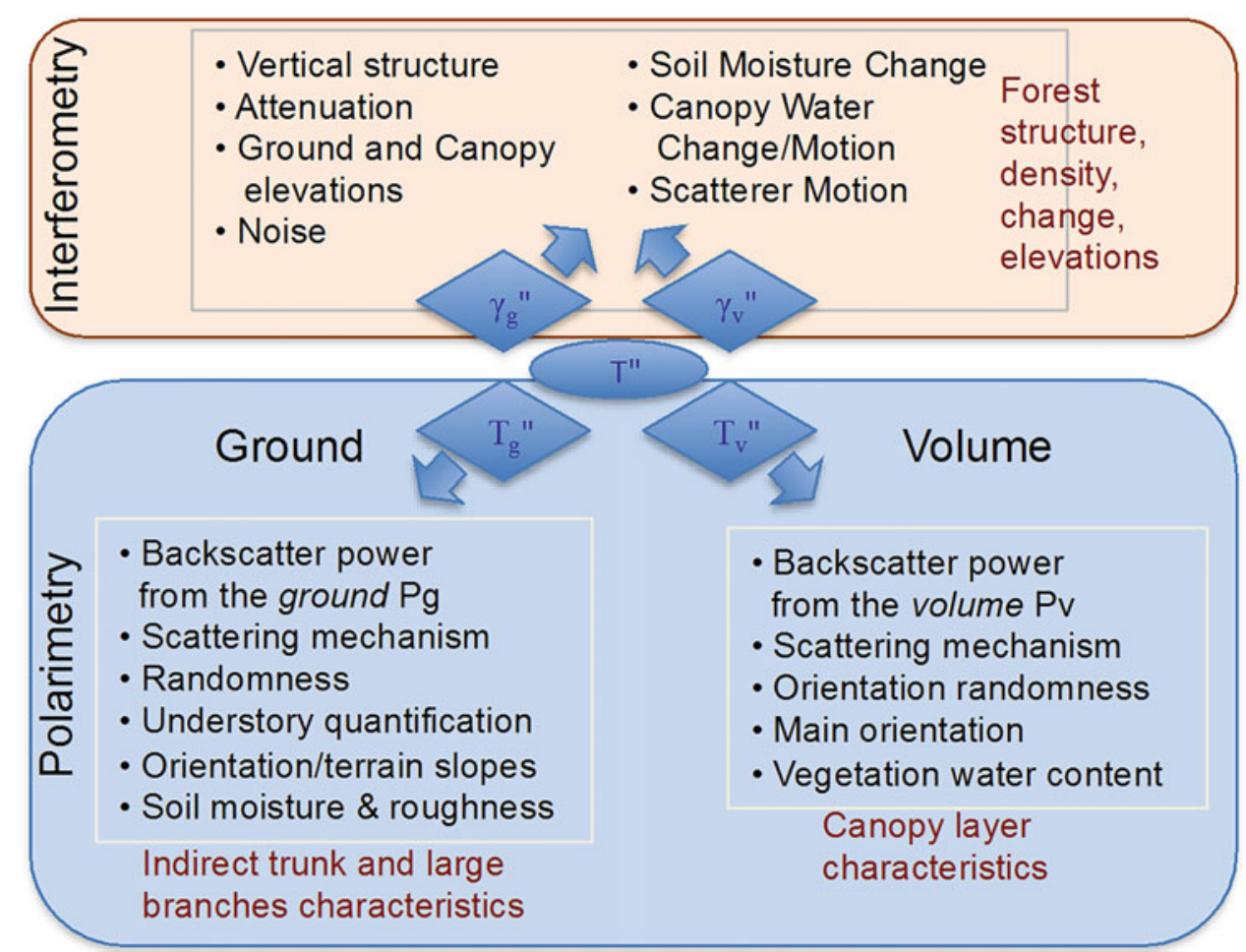

Fig. 2.31 PolInSAR model and retrieval representation in the coherence plane
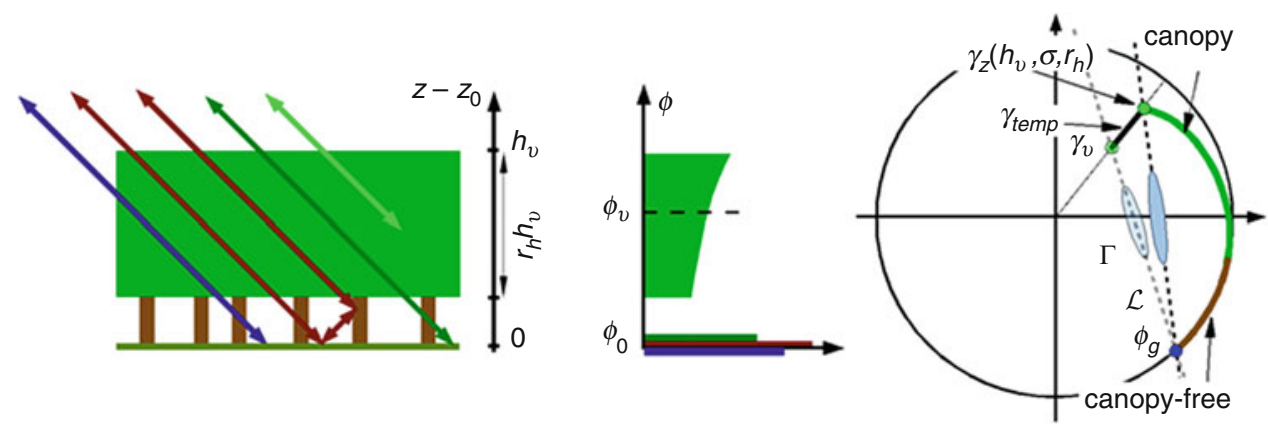

in Neumann et al. (2010), as it is more constrained than the generic entropy) and the effective scattering mechanism type. The particle anisotropy characterizes the effective shape of the average particle in the polarization plane in dependence of the particle and background permittivities The orientation randomness characterizes the width of the distribution of particle orientation angles in the polarization plane (Neumann et al. 2010). The polarimetry of the volume component can range from purely randomly oriented collection of scatterers with high polarization entropy to a less diffuse form with ordered branch structure.

Prior to parameter retrieval, data preparation consists of the following steps, starting with a stack of single-look complex (SLC) data sets for multiple acquisitions and polarizations: flat earth removal, range spectral filtering, polarimetric calibration, MB-PolInSAR covariance matrix estimation including pre-summing and speckle filtering, radiometric calibration, SNR compensation, polarimetric stationary calibration, local orientation angle compensation, ground topography phase shift, natural media (reflection symmetry) assumption, forest/non-forest pre-classification.

In the retrieval phase, most crucial is the estimation of the ground elevation phases and the linear ground-volume structure. Using one of the recently proposed least squares or maximum likelihood approaches (Tebaldini 2009) provides a parametric decomposition of the MB-PolInSAR covariance matrix. The determination of the decomposition parameters (which characterize the ground to volume ratio and the polarization responses at both layers) is based on a set of assumptions. Common assumptions are based on physical insights into the ground-volume distinctiveness and can include metrics on polarization entropy, scattering mechanism type, ranges for forest height and ground elevation, and temporal effects. The constrained optimization problem 
is solved in a least squares sense determining the decomposition of the MB-PolInSAR covariance matrix into two layers, providing the ground and volume PolSAR matrices, and a MB series of coherences for the ground and volume components. The physical structure indicators are extracted from the PolSAR matrices, such as canopy orientation randomness, scattering mechanism types, ground-to-volume ratio, etc. The final estimation of the vertical structure profile is performed in an additional step by fitting the coherence sets for the ground and volume to a model of the verticals structure distribution.

For the demonstration of biomass estimation, different regression frameworks (multiple linear regression (LR), support vector machines (SVM), and random forests (RF)) were evaluated by using field measurements of above-ground-biomass. Validation is performed using leave-one-out approach.

\subsubsection{Experimental Results}

Test sites and corresponding radar and validation data sets selected for the generation of showcases on forest parameter estimation are summarized in Table 2.8 and further described in the Appendix.

MB-PolInSAR modeling and inversion provide many retrievable parameters including: forest height, ground elevation topography, vertical structure characterizing parameters (attenuation, canopy-fill-factor, differential extinction), temporal decorrelation, ground-to-volume ratio, individual power contributions, ground and volume scattering mechanism types and orientation randomness. For the Traunstein 2003 data, only the forest height can be related to the validation data, as projected from the 1998 inventory. In the following, we will present several retrieved forest structure parameters from the Traunstein 2003 data as obtained in the study (Neumann et al. 2010). Estimates are plotted in Fig. 2.32.

1. Vertical forest structure: forest heights are estimated close to projected field measurements with an average stand-wise root mean square error (RMSE) of about $5 \mathrm{~m}$ and standard deviation of $4.3 \mathrm{~m}$. The heights were underestimated by $1.5 \mathrm{~m}$. However, in some stands, the forest heights vary continuously, indicating non-homogeneity inside of the stands. Figure 2.33a shows the forest heights and the individual canopy layer depths for the 20 evaluation stands. The red and green lines in this plot represent the field measured and PolInSAR estimated forest heights, respectively. The brown line delimits the canopy layer from the non-canopy layer above the ground and represents the canopy-fill-ratio, normalized to the total vegetation height. It is notable that the smallest error corresponds to the most homogeneous forest stand \#2, which is largely dominated by a single species, Northern Spruce (bias: $-0.3 \mathrm{~cm}$; RMSE: $1.5 \mathrm{~m}$ ). The highest errors correspond to stand \#20: the heights are underestimated by $5 \mathrm{~m}$ with an RMSE of $7.6 \mathrm{~m}$. However, examining the optical data, one can observe several clear cuts and roads inside of this forest stand, which we initially assumed to be homogenous. This indicates significant changes in the spatial structure of this forest stand between the forest inventory in 1998 and the date of radar acquisition in 2003.

2. Temporal decorrelation: using the full model inversion allows us to estimate the total amount of temporal decorrelation, which varied significantly with forest stand and baseline between 0.5 and 0.95 , as presented in Fig. 2.33b. The temporal decorrelation of the volume at these time scales is mostly caused by wind, which is non-stationary, neither temporally nor spatially. These results indicate that the temporal decorrelation is more correlated with the forest height and the spatial baseline and less with the time separation at the given sub-hour level.

3. Orientation randomness: Fig. $2.32 \mathrm{~d}$ shows the estimated degree of orientation randomness over the whole scene. The standard deviation of orientation randomness is low, and the distinctiveness is high, which permits discriminating between the different evaluation stands. As discussed in the theoretical part, this parameter depends mostly on the morphology of the tree structures and the incidence angle. The incidence angle dependence is clearly observable in the scatter plot in Fig. 2.33d. Forest species type effects on orientation randomness were well visible. While coniferous tree canopies caused lower orientation randomness, down to 0.65 , the highest orientation randomness was observed for deciduous trees, and in particular for the maple dominated forest stand \#3 in the center of the image.

Table 2.8 Test sites and corresponding radar and validation data selected for the generation of showcases on forest structure estimation

\begin{tabular}{l|l|l}
\hline Application/product & Test site - Radar data & Reference data \\
\hline Vegetation parameter & Krycklan, Sweden & Airborne lidar measurements (ground topography and forest height), \\
estimation & Airborne E-SAR L-band repeat-pass quad- & forest inventory data \\
& pol data 2008 & \\
& Traunstein, Germany & \\
& Airborne E-SAR L-band repeat-pass quad- & \\
& pol data 2003 & \\
\hline
\end{tabular}



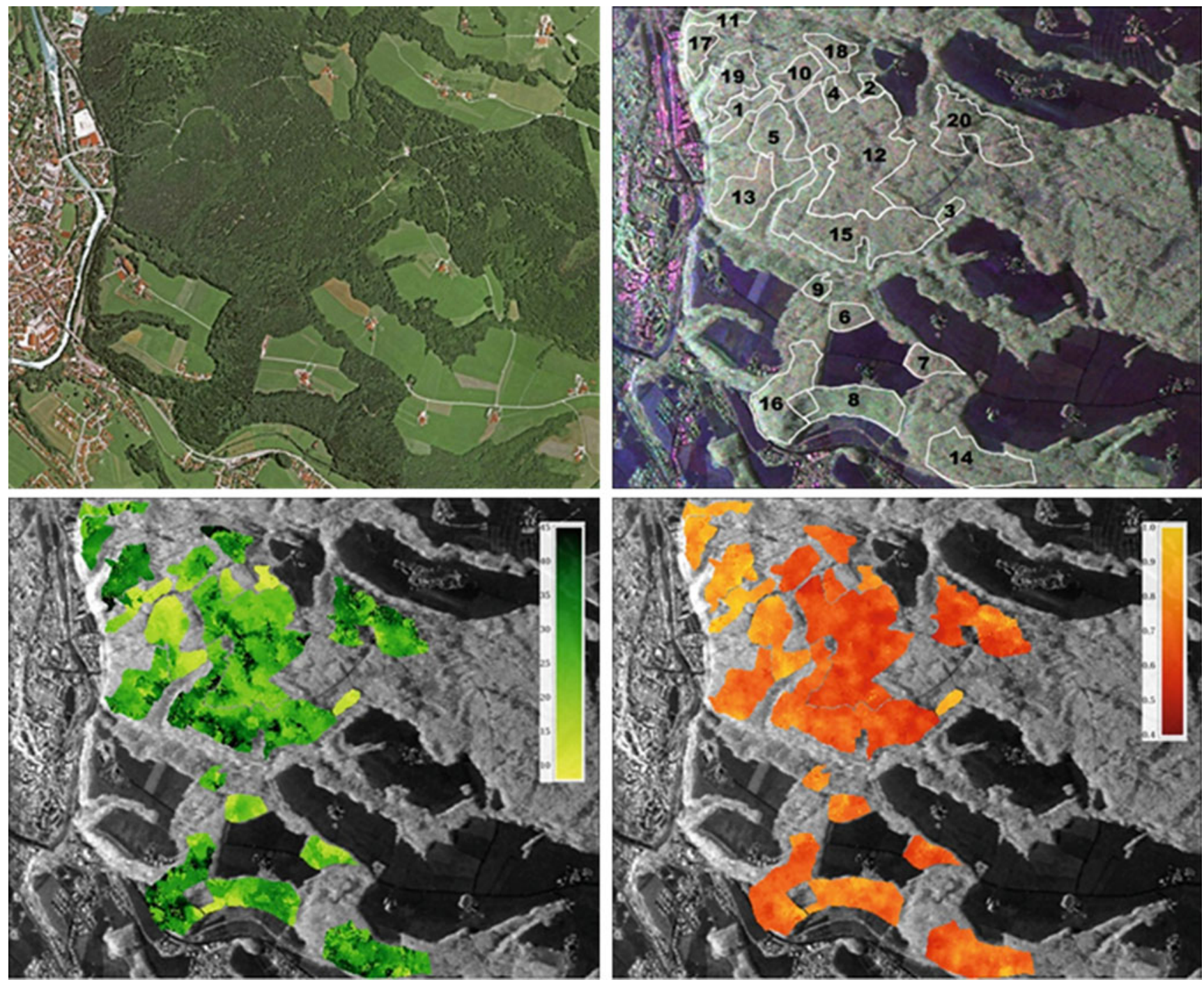

Fig. 2.32 Traunstein test site: optical image, PolSAR image with delineated forest stands, estimated forest height, and orientation randomness

4. Particle scattering anisotropy: The effective particle scattering mechanism type seems to be insensitive to the evaluation stand and the incidence angle. In contrast, the particle anisotropy phase has a large dynamic range of about 70 degrees over the different evaluation stands. These findings were surprising and still need to be confirmed by rigorous analysis.

5. Extinction and canopy fill factor: Extinction $\sigma_{x}$ (average at $0.15 \mathrm{~dB} / \mathrm{m}$ ) and the canopy-fill-ratio (average at 0.6 ) provide additional degrees of freedom for the adaptation of the model to the given forest structure. However, these estimates were found to be partly correlated with each other, which indicates that the used forest structure model and inversion approach are still ambiguous and a better representation should be found.

6. Differential extinction and coherence: Based on the estimated structure (extinction) and the PolSAR covariance matrix, we estimated the differential extinction in the canopy between $\mathrm{HH}$ and VV polarizations to be up to $0.06 \mathrm{~dB} / \mathrm{m}$. This tree canopy orientation induced effect changes the PolInSAR coherence for the canopy. The induced effect on coherence magnitude and phase is at the order of $0.005^{\circ}$ and $1^{\circ}$, respectively.

7. Ground level contributions: Next to ground topography elevation, PolInSAR allows to estimate the ground-to-volume ratio (estimated to be in average about 0.2 ) and the individual PolSAR covariance matrices for the ground and volume contributions. We observed noteworthy crosspolarized contributions (about 20\%) at the ground level, but the major scattering power at the ground was observed in the Pauli HH-VV component (about 50\%). Though $\mathrm{HH}-\mathrm{VV}$ is dominant, these results suggest that a simple first-order models for the ground component are insufficient to reliably represent the ground scattering inside the forest. 

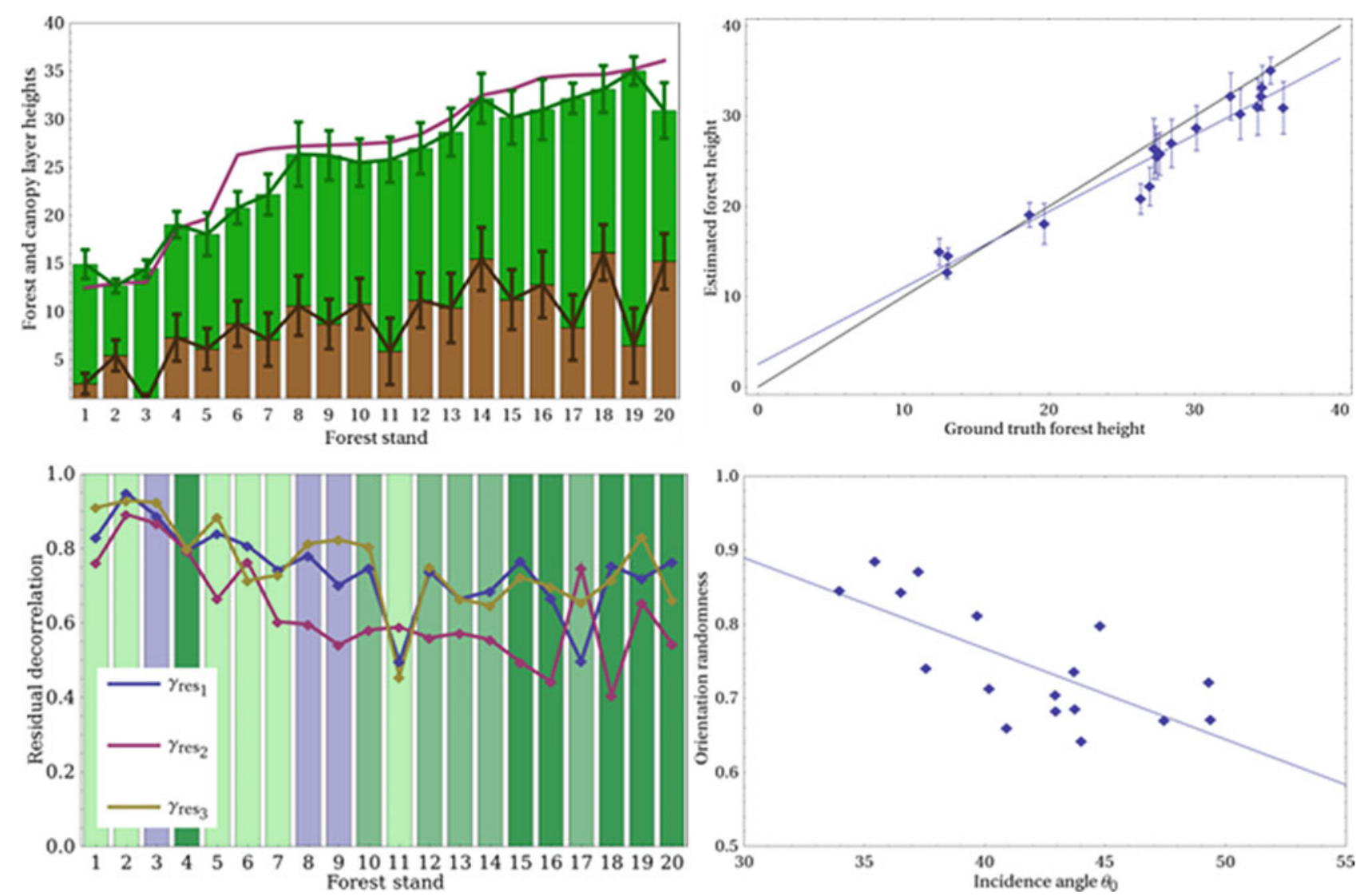

Fig. 2.33 Traunstein test site: Estimated forest structure, scatterplot of estimated height, temporal decorrelation, orientation randomness over incidence angle

Figures 2.34 and 2.35 represent the Krycklan catchment area and show forest structure parameters retrieved from MB-PolInSAR data: polarimetric image in Pauli matrix basis (2.34a), lidar top forest height (h100) (2.34b), forest height (2.34c), biomass using SVM regression (2.34d), ground and volume orientation randomness $(2.35 \mathrm{a}, \mathrm{b})$ and ground and volume scattering mechanism types $(2.35 \mathrm{c}, \mathrm{d})$. Investigating regression techniques using a combination of PolInSAR retrieved forest structure parameters allowed us to improve biomass estimation at both, L- and P-band frequencies. On the one hand, providing more structurerelated input parameters naturally improves the regression. However, due to spatial and temporal forest variability this regression usually does not generalize over large forest areas. We evaluated three approaches (Multiple Linear Regression (LR), Support Vector Machines (SVM), and Random Forests (RF)) using up to 14 parameters, independently at both frequencies. Using "leave-one-out" cross-validation approach, the best biomass RMSE were 19.7 and $22.7 \mathrm{t} / \mathrm{ha}$ at L- and P-band, respectively, using either SVM or LR. For comparison, without providing any forest structure information, the best RMSE values were 27.3 and $28.5 \mathrm{t} / \mathrm{ha}$ at L- and P-bands (both using SVM).

These were initial results on biomass regression from selected MB-PolInSAR forest structure estimates. It is to expect, that the results can be improved by more systematically evaluating the entire available parameter space and by performing a better regularization of the regression frameworks.

\subsubsection{Discussion on the Role of Polarimetry, on the Maturity of the Application and Conclusions}

This Section demonstrated a MB-PolInSAR approach for forest structure and biomass estimation on two airborne data sets at L- and P-band frequencies. We presented a model to characterize polarimetric interferometric radar response from vegetation. For the volume component, a novel polarimetric model was used to allow varying degrees of orientation randomness and scattering anisotropy inside the canopy.

The forest height estimation performance has been evaluated on real airborne L-band SAR data over the 


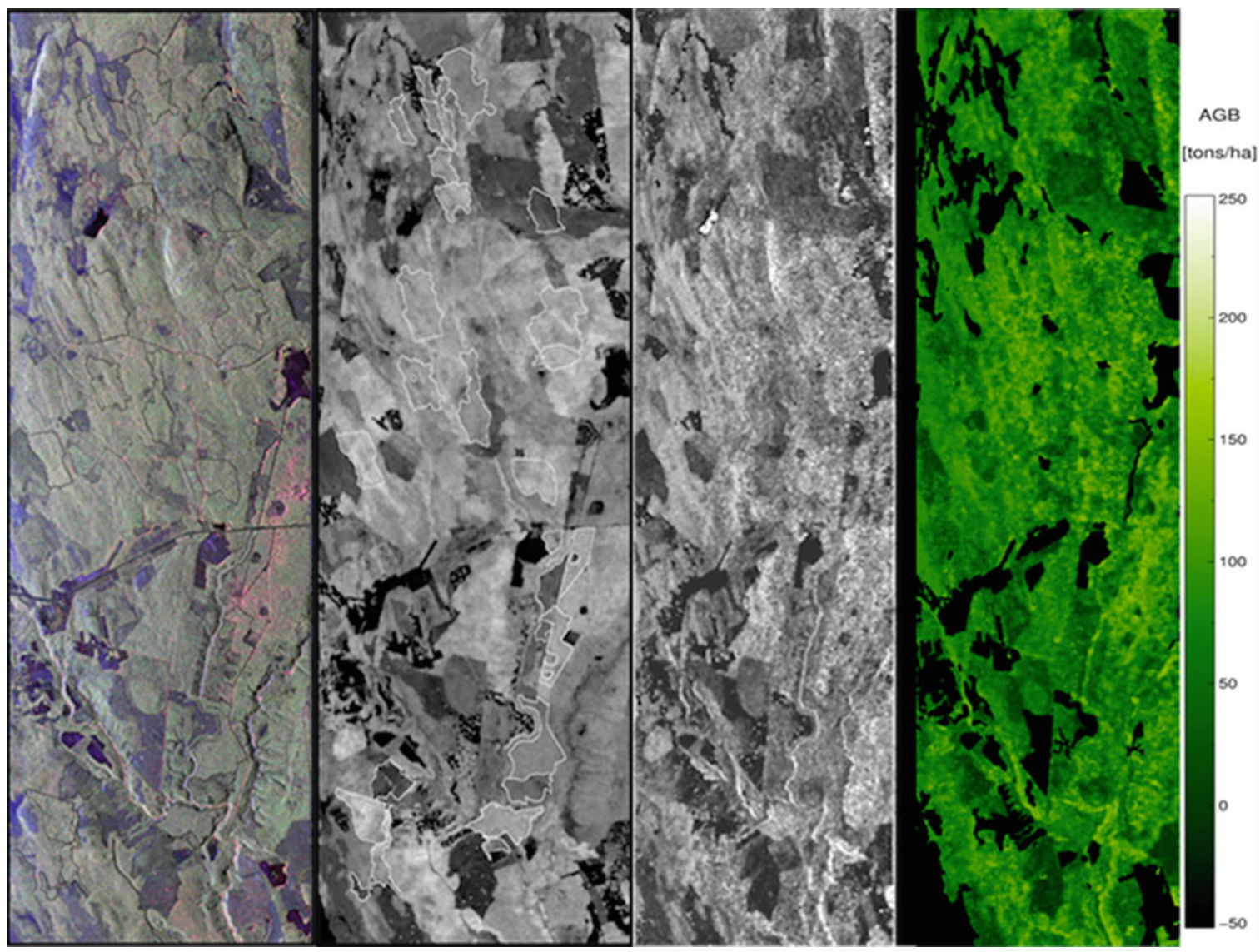

Fig. 2.34 Krycklan catchment. From left: L-band PolSAR image, Lidar height (rh100), PolInSAR height and biomass estimates

Traunstein forest. Using three baselines, the forest height has been underestimated with an average bias of $1.51 \mathrm{~m}$ and RMSE of $4.97 \mathrm{~m}$. In addition, polarimetric parameters and backscattered powers were estimated for both layers, the ground and the canopy. On the other hand, the estimations of the extinction and the canopy-fill-factor were not satisfactory, as there seems to rest a level of ambiguity and correlation between these two parameters. Furthermore, differential extinction and temporal decorrelation were estimated.

The same methodology was applied to boreal forest in the Krycklan Catchment to estimate forest structure parameters and to successively estimate biomass via regression. The combination of polarimetric canopy and ground indicators with estimated forest structure information (consisting of forest height and ground-to-volume ratio) improved the RMSE of biomass estimation up to $27 \%$ and $43 \%$ at $\mathrm{L}$ - and P-bands, respectively.

The limitations for the presented parameter retrieval framework are determined by model assumptions. Only a simplified vegetation structure is taken into account assuming vertically uniform and horizontally homogeneous layers.
Next it is assumed, that the average effective particle shape is representative for all particles, and independent of height and polarization orientation. The variation of extinction with polarization is assumed to be insignificant for parameter retrieval. Also, a plane of reflection symmetry is assumed to exist for the illuminated vegetation area. The possibility to estimate the degree of temporal coherence over vegetation provides new opportunities for PolInSAR time series analysis, which might lead to competitive multi-temporal monitoring of ecosystem dynamics. However, further theoretical and experimental investigations need to be conducted to improve the understanding and to examine the possibilities and limits of radar remote sensing of the temporal behavior and the forest structure.

Interferometry and polarimetry provide complementary information on the illuminated forest and we only start to explore the potentials for forest monitoring using these techniques. Though, it is an actively developing field and over the last few years several important breakthroughs in information content understanding and processing were reported which should help making this technique operational. 

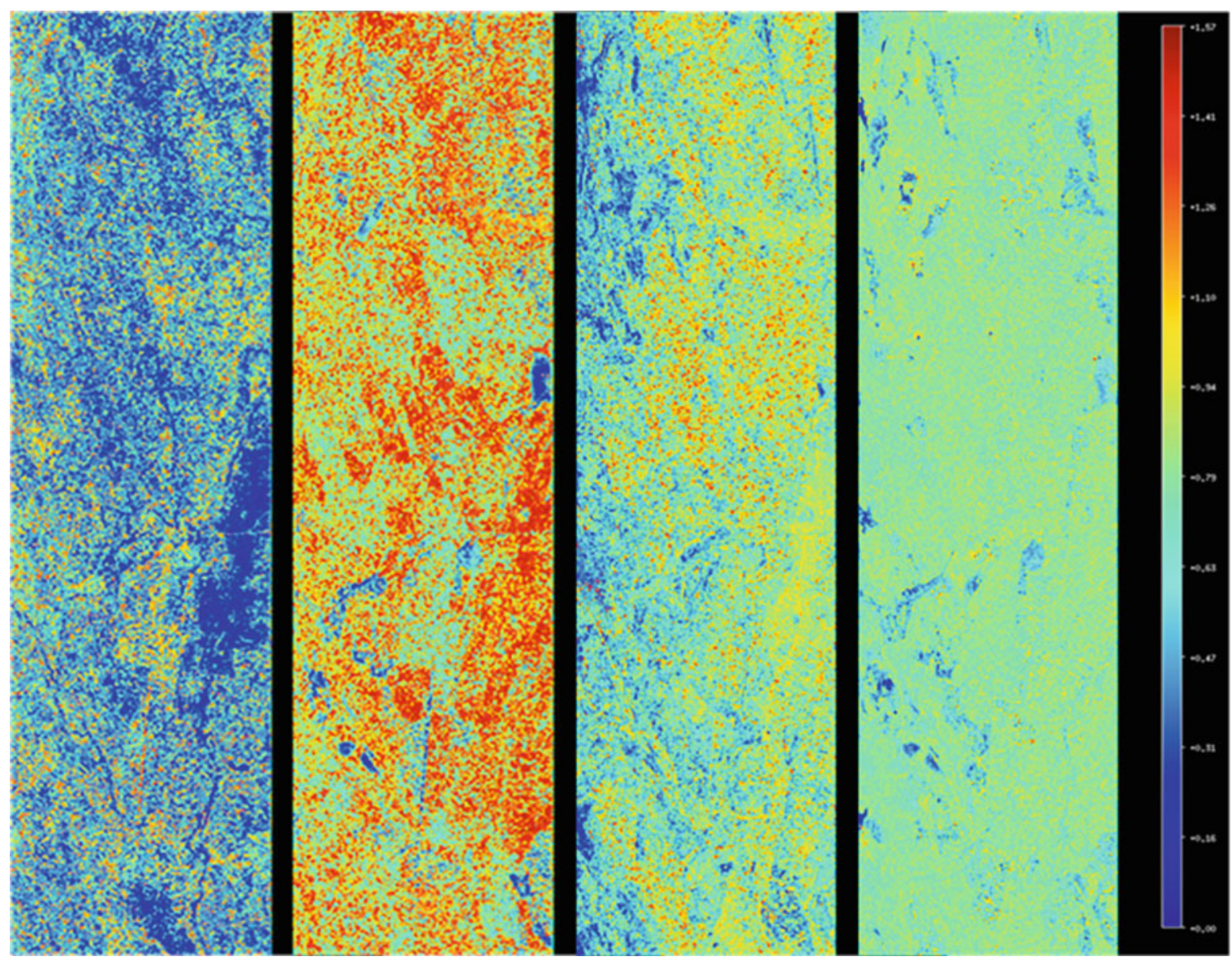

Fig. 2.35 Krycklan catchment. From left: Ground and volume orientation randomness, and ground and volume scattering mechanism types

\subsection{Biomass Estimation}

\subsubsection{Biomass Estimation: A Review}

\subsubsection{Introduction, Motivation}

Biomass is defined as the quantity of living organic matter per unit volume and is usually given as a mass, or a mass per unit area. Forest biomass refers to living and dead organic material of plants and trees in the forest including roots, stem, stump, branches, bark, seeds and foliage. Above ground forest biomass defines to the forest biomass above the soil (e.g. excluding the roots). About half of the forest biomass is carbon, so that biomass is often used as proxy for carbon and its spatial distribution characterises the distribution of carbon in the biosphere (Houghton et al. 2009; Houghton 2005; IPCC good practice guidance for land use, land use change and forestry 2003). Dynamic variations in the biomass are therefore a direct measure of the exchange of carbon between the terrestrial ecosystem and the atmosphere (GCOS 2003, 2004; GOFC-GOLD 2016). In this context, biomass was identified by the UNFCCC as an Essential Climate variable (GCOS 2003), whose determination is of great importance to reduce the uncertainties in our knowledge of the climate system. The importance of biomass manifests itself not only due to its role as a carbon sink or carbon source (Canadell et al. 2007; DeFries et al. 2002), but also due to its relevance for characterising forest or ecosystem productivity (Keeling and Phillips 2007).

Biomass inventories and biomass dynamics in local, regional and global scales are essential, though, up to now, largely unknown initialisation parameters of current climate models. A precise determination (on the order of 20\%) of the spatial distribution of forest biomass would be sufficient to match the inaccuracy of terrestrial carbon fluxes to the other components of the carbon cycle (IPCC good practice guidance for land use, land use change and forestry 2003).

The significance of an accurate knowledge of biomass is further underlined by the increased importance of two other aspects: (1) the increasing use of biomass for generating energy and the resulting increase in the proportion of greenhouse gases emitted from biomass (Fargione et al. 2008), and (2) the increased interest in reducing greenhouse gases by preventing deforestation and forest damage (UNFCCC 2016; UN-REDD Programme 2008; Herold and Johns 2007). 
Despite its importance, estimations of biomass at local, regional and global scales are today very inaccurate. One reason for this is the large effort required for a precise estimation of forest biomass. Direct biomass measurements are destructive and therefore costly in terms of resources, effort and time (Chave et al. 2003). Usually, biomass is estimated on a single tree basis from a number of measurements such as tree height and/or diameter and the use of appropriate allometric relationships (Chave et al. 2005). The stand biomass levels are then obtained by summing up the single tree biomass levels up. In heterogeneous forests, this procedure is very arduous and often inaccurate, meaning that ground measurements of biomass in natural forests are never better than $20 \%$ (Chave et al. 2003). Particularly in tropical forest ecosystems there are large deviations also induced by allometric deviations. On larger scales, apart from the uncertainties arising from forest complexity, the different definitions, measurement protocols and used generalisation methods introduce additional inconsistencies in regional and national inventories and data bases. Finally, the Intergovernmental Panel on Climate Change (IPCC) recognises the lack of exact spatial forest biomass data (the measurement errors often exceed the estimated mean value) as one of the largest uncertainties in the global carbon budget.

Figure 2.36 demonstrates the actual stand of uncertainty. It compares four biomass maps of the Brazilian Amazon basin: on top left the Saatchi et al. 2007 map (Saatchi et al. 2007a), top right the Saatchi et al. 2011 map (Saatchi et al. 2011a), bottom left the Nogueira et al. 2008 map (Nogueira et al. 2008) and bottom right the Baccini et al. 2012 map (Baccini et al. 2012). All four are generated by combining ground and remote sensing data. However, each of them is using a different set of remote sensing data, different approaches to interpolate ground and remote sensing data, and/or different allometric relations. It is quickly evident that the four maps deliver strongly deviating mean biomass values, spatial distribution patterns and spatial biomass trends.

Biomass is an integrative forest parameter that depends on multiple tree or stand attributes. Starting from a single tree, the above ground stem biomass $\mathrm{AGB}_{T}$ is given by the product of the stem volume $V_{T}$ with the (species specific) wood density $\rho_{T}$ :

$$
\mathrm{AGB}_{T}=V_{T} \rho_{T}=\frac{1}{4} H_{T} \mathrm{DBH}_{T}^{2} F_{T} \rho_{T}
$$

where $H_{T}$ is the (top) tree height, $\mathrm{DBH}_{T}$ the diameter at breast height, $F_{T}$ is a (species dependent) form-factor accounting for the deviation from a pure cylinder shape. The wood density $\rho_{T}$ typically varies between 0.5 and $0.69 \mathrm{~g} / \mathrm{cm}^{-3}$ (GOFCGOLD 2016). The so-called biomass expansion factor $(\mathrm{BEF})$ is used to account for the total above ground biomass including branches, understorey, etc. contributions (Canadell et al. 2007).

However, single-tree measurements are ineffective for large areas and global applications. In contrast, forest stand parameters are an option with great potential as remote sensing systems can measure them globally. In order to develop methodologies that use forest stand parameter to estimate aboveground biomass, new allometric relationships accounting for stand rather single tree parameters need to be derived and investigated. In this sense, moving from a single tree to a stand, the above ground stand biomass AGB is obtained by summing up the biomasses of the $\mathrm{N}$ individual trees in the stand

$$
\begin{aligned}
\mathrm{AGB} & =\sum_{i=1}^{N} \mathrm{AGB}_{T i}=\sum_{i=1}^{N} V_{T i} \rho_{T i} \\
& =\sum_{i=1}^{N} \frac{1}{4} H_{T i} \mathrm{DBH}_{T i}^{2} F_{T i} \rho_{T i}
\end{aligned}
$$

Substituting in (2.26) the basal area BA, that describes the amount of the stand area occupied by tree stems, the mean top height $H_{M}$ and a mean wood density $\rho_{M}$

$$
\begin{aligned}
\mathrm{BA} & =\frac{1}{4} \sum_{i=1}^{N} \mathrm{DBH}_{T i}^{2}, H_{M}=\frac{1}{N} \sum_{i=1}^{N} \boldsymbol{F}_{T i}, \\
& =\frac{1}{N} \sum_{i=1}^{N} F_{T i}, \rho_{M}=\frac{1}{N} \sum_{i=1}^{N} \rho_{T i}
\end{aligned}
$$

follows

$$
\mathrm{AGB}=\mathrm{BAH}_{M} F_{M} \rho_{M}
$$

Equation (2.28) makes clear that an accurate estimation of stand biomass relies on the knowledge of three key parameters: the tree height and density (expressed for example by the basal area in (2.28)) which both together define the woody volume of the stem and the wood density. Today there is no remote sensing configuration able to measure directly all three parameters.

Radar, and particular SAR remote sensing techniques for the estimation of above ground forest biomass, can be divided into three categories: (1) direct, (2) model-based, and (3) allometric techniques. Direct techniques use empirical or statistical relationships to relate directly the radar observables (primarily radar backscatter measurements at a single or different polarisations but also polarimetric or interferometric correlations) to above ground forest biomass values. Model based approaches rely on electromagnetic scattering models to interpret the SAR data and to establish 

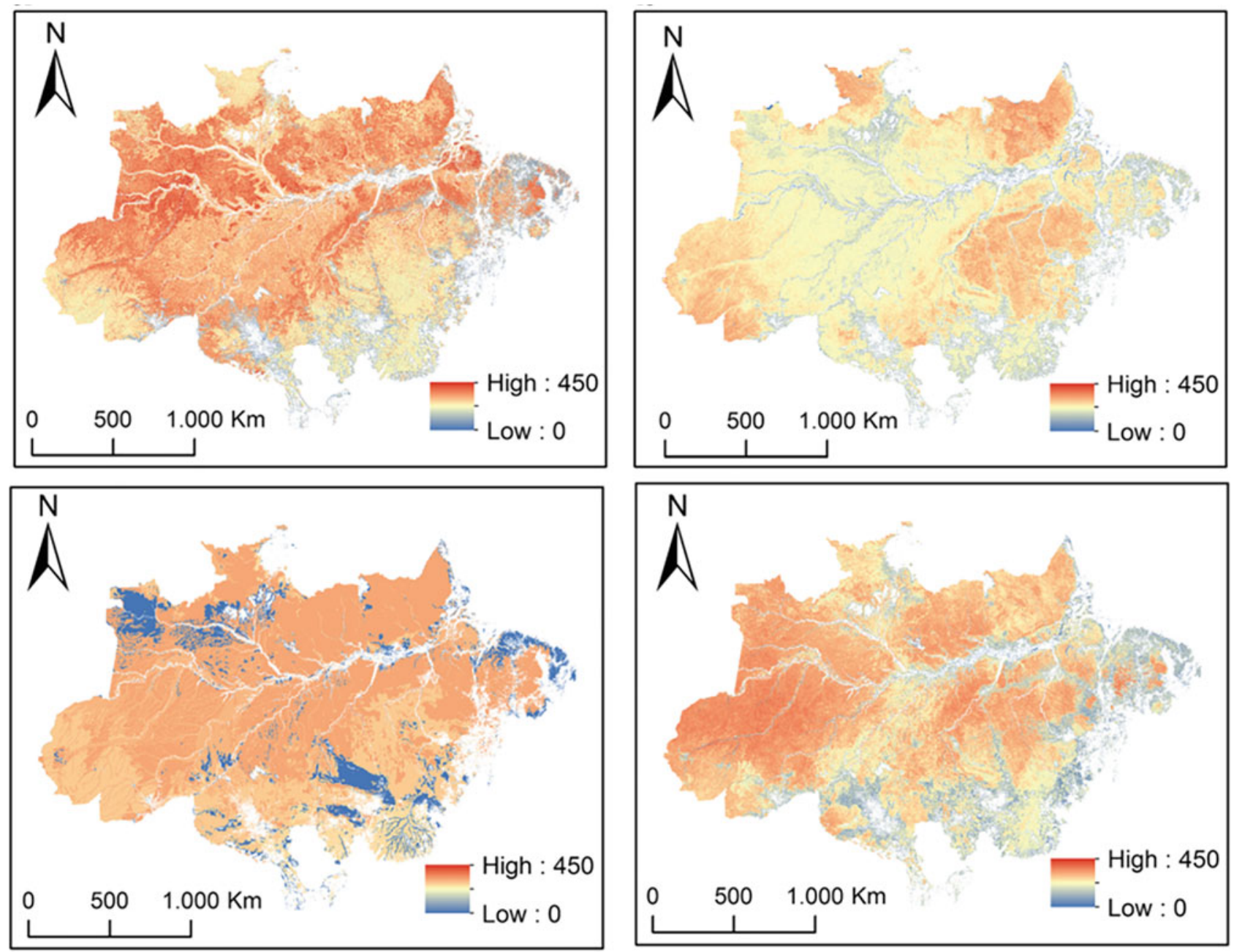

Fig. 2.36 Four different biomass classification maps for the Brazilian Amazon. Top left: Saatchi et al. (2007a), top right: Saatchi et al. (2011a), bottom left: Nogueira et al. (2008), bottom right: Baccini et al. (2012)

the relationship to biomass. Finally, in allometric techniques, the radar observables are used for the estimation of one or more physical forest parameters, as for example forest height, and then these parameters are related to forest biomass through allometric relations. The different approaches and techniques will be reviewed in the following.

\subsubsection{Methodology}

\subsection{Direct Biomass Estimation}

The first approaches to estimate forest biomass directly from backscatter measurements at different frequencies and polarisations date back to the early days of SAR remote sensing, and are today still an active research area. The basis of these approaches is the fact that with increasing forest biomass the (measured) backscatter intensity increases. Accordingly the backscattered intensity from a young forest with low biomass is in general weaker than the backscattered intensity from an old taller forest with high biomass. This has been observed, investigated and reported in the frame of airborne SAR experiments (at different frequencies and polarisations) already back in the eighties - early nineties (Le Toan et al. 1992; Rignot et al. 1994b; Imhoff 1995b; Dobson et al. 1995; Kasischke et al. 1995; Rauste et al. 1994; Harrell et al. 1995) These early results initiated a big interest in exploring SAR measurements for forest biomass estimation. Following experiments and work focused on studying and establishing (empirical) relations between biomass and SAR intensity measurements at the conventional HV, VV and HV polarisations.

A number of experiments performed over different forests and at different frequencies, indicated that the HV polarised (back-) scattering coefficient is strongly correlated to biomass, that this is often the case also for the $\mathrm{HH}$ scattering coefficient, while the correlation between biomass and VV scattering coefficient is often weak. However, at lower frequencies and/or sparse forest conditions the $\mathrm{HH}$ scattering coefficient is contaminated by dihedral scattering 
contributions (characterised by a strong directional backscattering behaviour) and is therefore stronger affected by topographic variations. Accordingly, $\mathrm{HV}$ is identified as the best polarisation for biomass inversion because it is less affected by the disturbing ground scattering contributions. Figure 2.37 shows P-band HV backscattering coefficient plotted against aboveground biomass for four different sites. Calibration factors of $5 \mathrm{~dB}$ and $-3 \mathrm{~dB}$ have been added to the datasets from La Selva and Remningstorp, respectively. The solid line is the regression curve derived from the combined data (Le Toan et al. 2011).

However, even if the experimental results appear in many cases convincing the theoretical interpretation relationships used where rather weak and largely based on simple forest scattering models. Today is known that that there are three critical limitations common to these studies/approaches:

1. At every frequency (and polarisation) the sensitivity of the backscattered SAR intensity decreases with increasing biomass level. This effect is often referred as "saturation" and imposes a maximum limit for which a biomass estimation is possible within a given accuracy. The sensitivity and with it the saturation limit increases with increasing wavelength (decreasing frequency). Authors generally agree upon that L-Band saturates between $40 \mathrm{t} / \mathrm{ha}$ and $100 \mathrm{t} / \mathrm{ha}$, and P-band between $120 \mathrm{t} / \mathrm{ha}$ and $200 \mathrm{t} / \mathrm{ha}$. Longer wavelengths like VHF as provided by the Swedish VHF sensor CARABAS are sensitive to biomass levels beyond $200 \mathrm{t} / \mathrm{ha}$.

2. The empirical and semi-empirical relationships between backscattered SAR intensity and biomass depend on site conditions, and forest type and structure.
3. The fact that the backscattered SAR intensity depends also on the acquisition geometry, terrain topography, seasonal and environmental (dielectric) conditions make the interpretation of the backscattered SAR intensity in terms of biomass levels ambiguous.

In order to overcome these limitations model-based approaches have been developed.

\subsection{Model-Based Estimation}

Model-based approaches use electromagnetic scattering models to establish the relation between SAR observables, primarily the (back-) scattering coefficients at one or more polarisations, and biomass. The main motivation behind their development was twofold: to better control the dependency of the biomass relation on the individual site characteristics by parameterising propagation and scattering processes and to reduce the distortion due to non-vegetation scattering contributions by decomposing the total scattering into elementary scattering contributions.

While they have been many approaches investigated and proposed addressing model based biomass estimation one can distinguish two different model families: The first family of models develop from the so called Water Cloud Model (Attema and Ulaby 1978), are in general two layer models (i.e. volume and ground layer) and are applicable primarily at higher frequencies where canopy attenuation is relevant (Henderson and Lewis 1998); The second one is derived by means of radiative transfer modelling, accounting in general up to three layers (i.e. grown, trunk and ground layer) and appears more suited for the lower frequency range (Saatchi and McDonnald 1997; Saatchi and Moghaddam 2000;
Fig. 2.37 P-band HV backscattering coefficient plotted against aboveground biomass for four different sites. (Le Toan et al. 2011)

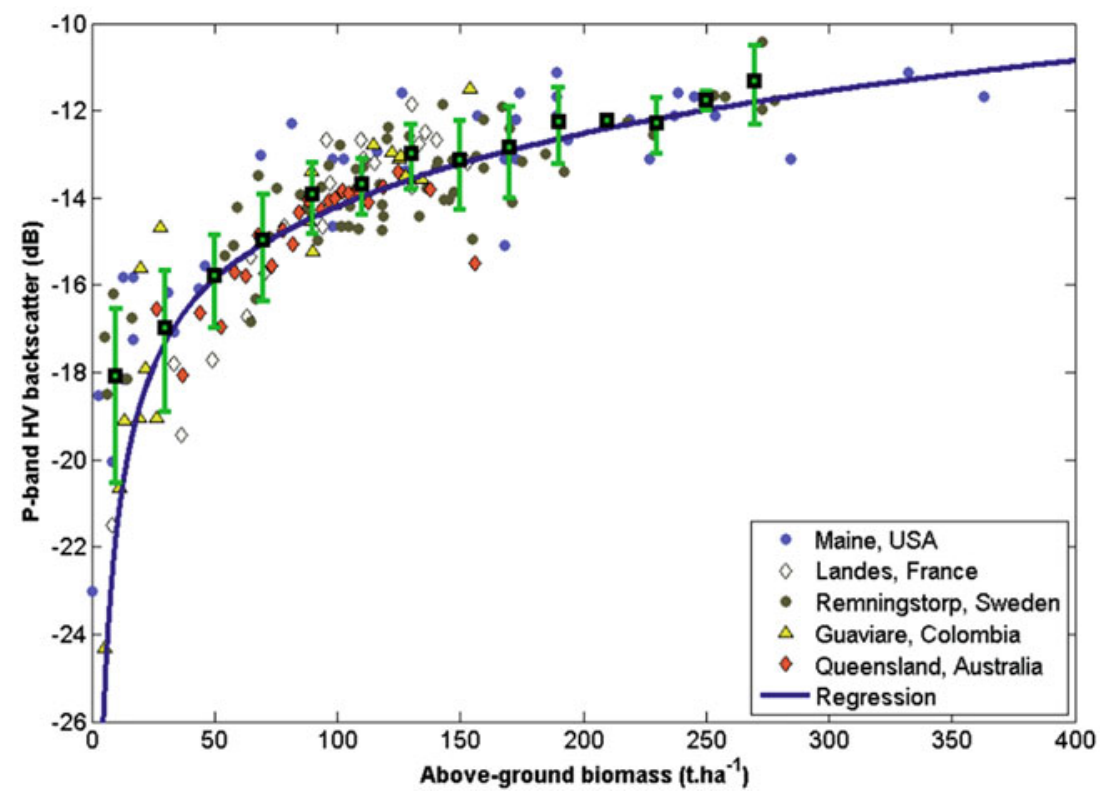


Mitchard et al. 2011). Both approaches rely on the separation (or interpretation) of volume and ground (including surface and/or dihedral) scattering contributions either by means of polarimetric scattering coefficients or covariance matrices. This allows, depending on the implementation, to incorporate topographic corrections.

However, there are two critical points that constrain the applicability and/or performance of these models: the first one is the large number of model parameters required in relation to the low dimensionality of conventional SAR observation spaces. This results in a large number of parameters/ coefficients that have to be a-priori known or estimated from forest biomass reference data. The second one is the rather poor integration of the effect of forest biomass into EM scattering models at relevant frequencies. This is primarily because of our inability to describe in a realistic way the complex scattering interactions occurring within a forest under different conditions and at different scales. The fact that forest biomass by itself depends on a number of forest parameters makes this task even more complex.

The advantage of using multitemporal SAR acquisitions to improve the performance of biomass estimators was already recognized at a very early stage (Kurvonen et al. 1999). The availability of multiple SAR acquisitions increases the sensitivity of the SAR backscattered intensity to biomass and allows an estimation even beyond the saturation point as recent studies demonstrated (Santoro et al. 2011, 2013). More than 100 acquisitions per year are used to derive biomass estimates from C-band radar backscatter. Averaging across large number of acquisitions, performed under slightly different incidence angles in different environmental (i.e. weather) and seasonal conditions allows to overcome the saturation appearing in single image relationships. The price to be paid is the low spatial resolution resulting from the large number of samples required to obtain robust estimates. This has been demonstrated in Santoro et al. (2011) with a stock volume map of the boreal zone from ENVISAT-ASAR data with a resolution of $1 \mathrm{~km} \times 1 \mathrm{~km}$. Up to $300 \mathrm{~m}^{3} / \mathrm{ha}$ (approx. 150 tons/ha) no saturation effect could be detected.

The introduction of SAR interferometry in the late nineties had a significant impact on forest applications by improving the performance of existing applications when introducing both interferometric coherences and/or interferometric phases or triggering the development of new ones.

The interferometric coherence has been explored in terms of its temporal as well as in terms of its volumetric contributions. As the temporal decorrelation contribution dominated the early C-band repeat-pass interferometric spaceborne data available the first attempts where based on empirically relating the amount of change (i.e. temporal decorrelation) estimated in the interferograms to the biomass (or the stock volume, or the height) of the individual stands (Koskinen et al. 2001; Pulliainen et al. 2003; Eriksson et al. 2003b). In Wagner et al. (2003) ERS interferometric coherence in combination with JERS backscattering was used with success to classify the biomass levels across Siberia. The availability of repeat-pass space borne interferometric acquisitions with smaller temporal baselines and/or at longer wavelengths (L-band) with weaker temporal decorrelation contributions allowed the development of more physical interpretation and inversion models for the interferometric coherence able to distinguish between temporal and volume decorrelation contributions. This helped to improve biomass estimation and to obtain more robust estimates, especially in boreal forests (Santoro et al. 2002; Askne and Santoro 2009; Askne et al. 2013).

Besides the interferometric coherence, interferometric phase measurements have been also used to support biomass estimation in cases where the coherence level was allowing meaningful phase reconstruction. The so-called scattering center height, i.e. the height of the interferometric phase center with respect to the underlying ground has been used as a proxy for forest height in different biomass estimation schemas (Solberg et al. 2010, 2013; Treuhaft et al. 2015). However, the dependency of the phase center location on the acquisition geometry and terrain topography as well as on seasonal and environmental variation constrains its robustness especially at lower frequencies.

\subsection{Allometric Biomass Estimation}

Accurate forest height measurements can be used as a proxy for aboveground biomass estimates, especially in high biomass regions (Mette et al. 2003, 2004a, b). Differently than the conventional biomass estimates at stand level obtained by multiplying stock volume with mean wood density, biomass estimates from forest height measurements rely on the use of allometric relationships (Mette et al. 2003). A first approach in this direction was introduced in Mette et al. (2003) where a simple power law allometric relationship was proposed

$$
\mathrm{AGB}=l_{a}(1.66 H)^{b}
$$

where $\mathrm{AGB}$ is the stand biomass ( $\mathrm{Mg} / \mathrm{ha}), H$ is the forest dominant (top) height or H100 (height of the 100 largest trees per hectare (Reigber and Moreira 2000)), $b$ is the allometric exponent and $l_{a}$ a scaling factor known as allometric level. The allometric level accounts primarily for differences in stand densities but also tree species and site conditions and decreases from climax tree species to pioneer tree species. For estimating AGB from height measurements in the context of (2.29) the allometric level needs to be known. On regional (i.e. landscape) scale a mean allometric level can be assumed (under the assumption of similar tree species composition, logging practices, management concepts and growth conditions).

Height-to-biomass allometric relationships proved to be robust and able to provide accurate AGB estimates from 
forest height in homogenous forest conditions. This has been demonstrated for a number of boreal, and temperate sites with different conditions. However, the approach meets its limitations in forest of heterogeneous structure and density. This is shown in Fig. 2.38 where the height (i.e. H100) to biomass relationship for four European forest test sites. The results have been achieved by using a common allometric exponent of 0.52 and site adapted values for the allometric
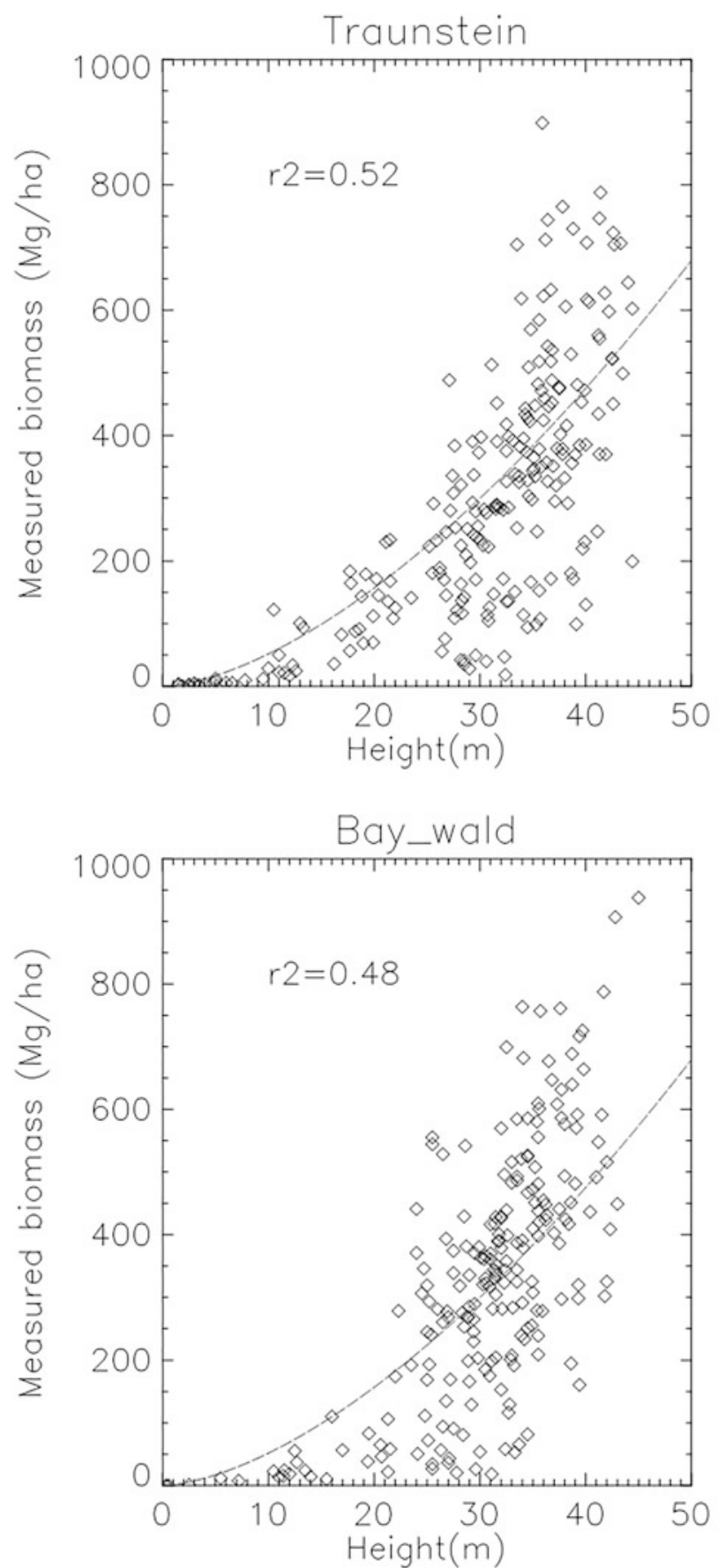

level. The three more heterogeneous sites, namely Traunstein, Ebersberger Forst and the Nationalpark Bayrischer Wald, are characterised by a lower height-biomass correlations indicating the insufficiency of a fixed allometric level to represent the height to biomass relationship. On the other hand, the more homogeneous, in terms of horizontal and vertical structure, boreal forest in Krycklan is well described by a single allometric level. The
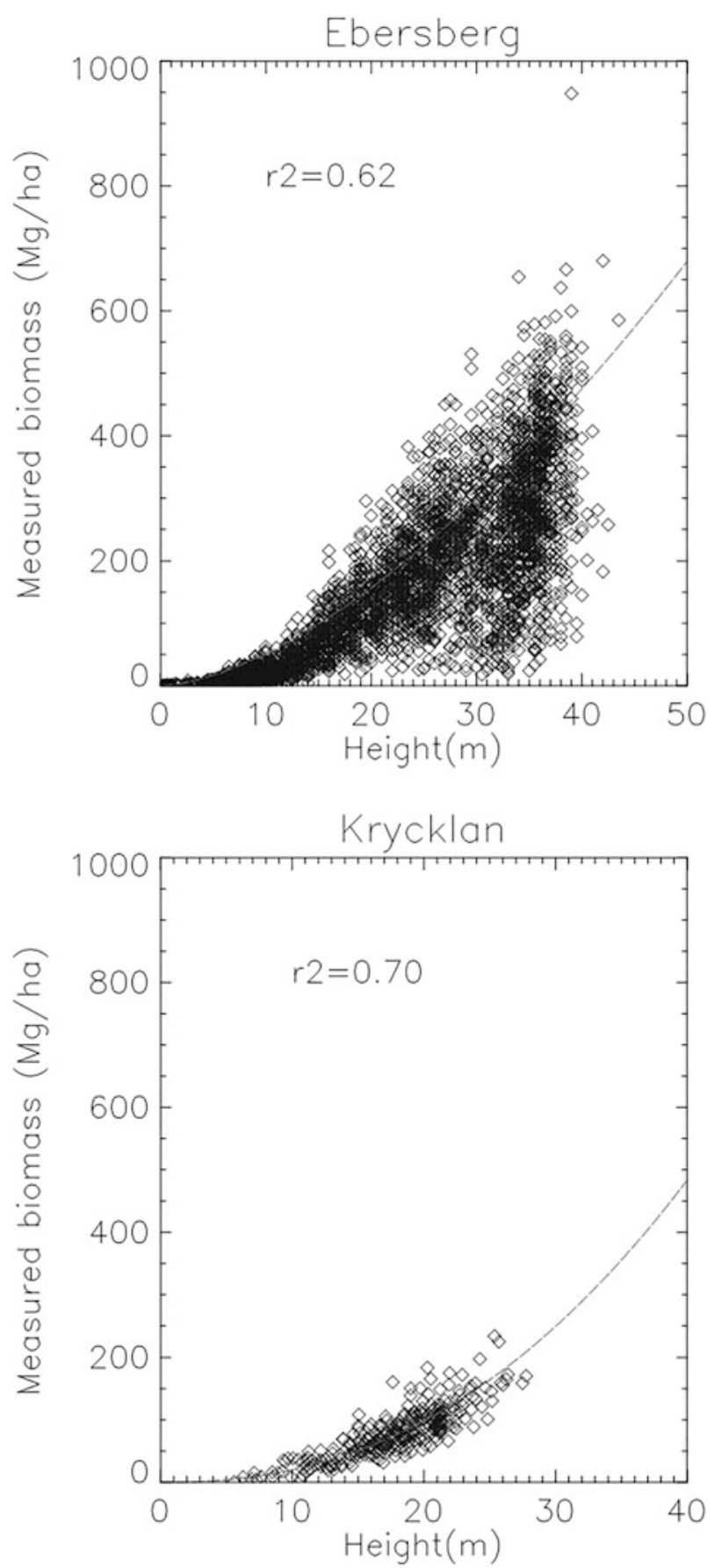

Fig. 2.38 Height to biomass allometry for four European forest test sites. Top left: Traunstein Site in Germany; top right: Ebersberger Forst in Germany; bottom left: Bayrischer Wald in Germany; bottom right: Krycklan in Sweden 
correspondences obtained for the four test sites between the biomass estimated from ground measurements and using the height to biomass allometry are shown in Fig. 2.39.

The high correlation between forest height and biomass has been explored in Toraño-Caicoya et al. (2016a) to use forest height estimated from TanDEM-X interferometric data to estimate successfully biomass at boreal forests by means of (2.29).

In order to improve the performance of the conventional forest height to biomass allometry that degrades in structurally heterogeneous forests the direct or indirect use of forest structure information in (2.29) has been proposed and is currently investigated by several groups. The forest structure information can be obtained from inventory data or even more relevant from 3-D radar reflectivity reconstructed from tomographic measurements. One such example has been recently presented in Toraño-Caicoya et al. (2015) where the use of vertical structure information derived from 3-D radar reflectivity reconstructed at L-band by means of tomographic SAR techniques has been used to improve the performance of the height to biomass allometry. First the vertical reflectivity profile $F(z)$ is expressed in terms of a Legendre polynomial series

$$
\begin{aligned}
F(z) & =\sum_{n} a_{n} P_{n}(z) \text { where } a_{n} \\
& =\frac{2 n+1}{2} \int_{-1}^{1} F(z) P_{n}(z) d z
\end{aligned}
$$

where $P_{n}(z)$ are the Legendre polynomials and $a_{n}$ the associated Legendre coefficients. The first four Legendre coefficients $\left(a_{1}, \ldots a_{4}\right)$ are then used to define a structure ratio $S_{\text {rat }}$ that allows to distinguish between different allometric levels

$$
S_{\mathrm{rat}}=\frac{\left|a_{1}\right|}{\left|a_{2}+a_{3}+a_{4}\right|} .
$$

The lower frequency contribution, expressed by the first Legendre coefficient $\left(a_{1}\right)$, is associated to the stem compartment while the higher frequency components, represented by the higher order coefficients $a_{2}, a_{3}$ and $a_{4}$ are associated to the crown/canopy compartment. Accordingly, the structure ratio $S_{\text {rat }} S_{\text {rat }}$ may be used as a proxy to stand density to improve the allometric biomass estimation (Toraño-Caicoya et al. 2016b)

$$
\mathrm{AGB}=7 S_{\text {rat }}^{0.8} H^{b} .
$$

Thus, for a constant height, if the numerator of the ratio increases (indicating a higher proportion of stem biomass), biomass increases, and if the denominator increases (indicating a higher proportion of crown biomass) biomass decreases. Figure 2.46 shows the improvement achieved by using (2.35) instead of (2.32). The high variance produced by the "height to biomass" allometric relation in (2.32) can be corrected with the inclusion of forest structure information.

\subsubsection{Experimental Results}

Test sites and corresponding radar and validation data sets selected for the generation of showcases on biomass estimation are summarized in Table 2.9 and further described in the Appendix.

Biomass maps have been obtained in the two selected test sites in two steps: first by estimating forest height from interferometric TanDEM-X and second by estimating biomass from height using following allometric relation provided by the Swedish National Forest Service Inventory (SLU)

$$
\mathrm{AGB}=0.3 H^{2} .
$$

Results are shown in Fig. 2.40.

The accuracy of the estimated biomass depends on two error sources: the accuracy of the estimated height-to-biomass relationship ( $\triangle \mathrm{AGB})$ and the accuracy of the forest height estimates $(\Delta H) . \Delta \mathrm{AGB}$ is defined by the forest conditions and can only be improved when additional forest parameters (like forest density, structural parameters, etc.) are included in the allometric estimation process. Under the assumption of homogenous stand conditions across the boreal region, $\triangle \mathrm{AGB}$ can be assumed constant and it is estimated from the bias observed in the height-to-biomass relationship applied to the reference data. $\Delta H$ depends on forest height estimation methodology and performance. Airborne lidar measurements provide height estimates with sub-meter accuracy and are therefore used as a reference.

For forest heights obtained from TanDEM-X data, $\Delta H$ depends, in general, on the acquisition mode (single-pol, dual-pol, dual-baseline) the number of available acquisitions (see Sect. 2.2) and on the associated range of spatial baseline.

Accordingly, the total biomass estimation sensitivity $\left(\Delta \mathrm{ABG}_{\mathrm{tot}}\right)$ can be expressed as

$$
\Delta \mathrm{AGB}_{\mathrm{tot}}=\frac{\partial A G B}{\partial H} \Delta H+\Delta \mathrm{AGB} .
$$

Using (2.33) in (2.34) it follows:

$$
\Delta \mathrm{AGB}_{\text {tot }}=0.6 H \Delta H+\Delta \mathrm{AGB} .
$$

$\Delta H$ and $\Delta \mathrm{AGB}$ determine the accuracy of biomass estimation. However, assuming the allometric error $\triangle \mathrm{AGB}$ to be 

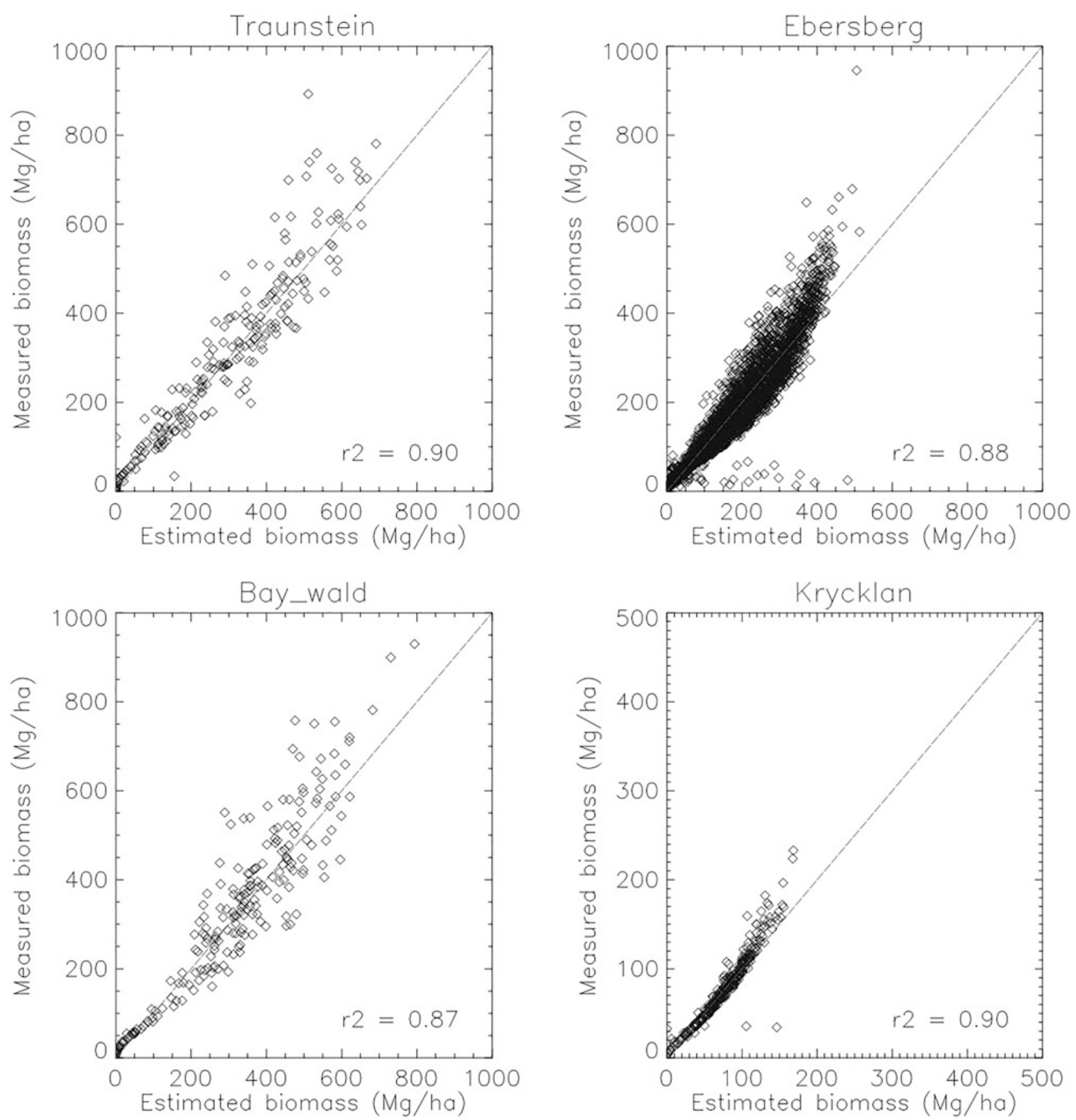

Fig. 2.39 Height and structure to biomass allometry for four European forest test sites. Top left: Traunstein Site in Germany; top right: Ebersberger Forst in Germany; bottom left: Bayrischer Wald in Germany; bottom right: Krycklan in Sweden

independent from $H$, the biomass estimation accuracy is driven by $\Delta H$ only.

Figure 2.41 shows classification performance obtained by means of (2.33) for a relative height error of $0 \%$ (top left) and $10 \%$ (top right), and 1 (bottom left) and $3 \mathrm{~m}$ (bottom right) absolute errors. The real biomass is plotted on the y-axis, the estimated biomass, for a $90 \%$ confidence interval, is plotted on the $\mathrm{x}$-axis in $10 \mathrm{Mg} / \mathrm{ha}$ biomass steps. The blue dashed lines indicate the class boundaries, estimated at the point in which two biomass intervals do not overlap. Assuming an error free height measurement $(\Delta H=0)$, up to 9 biomass classes can be obtained. This is the best performance that can be achieved, due to the residual error in $\triangle \mathrm{AGB}$. In case of a $10 \%$ height error five classes can be distinguished. For height errors larger than $20 \%$ only two biomass classes can be separated reducing the classification to a forest/non forest classification. 
The analysis above makes clear that the biomass estimation performance reacts very sensitive to height errors. Consequently, reliable and robust biomass estimates require accurate and robust height estimates which again strongly depend on the polarimetric configuration of the observation space. The performance shortcomings of dual-polarimetric observations in terms of:

1. biased estimation results due to the lack of appropriate observation dimensionality to account for ground depolarisation (especially at lower frequencies and/or sparse forest conditions);

2. larger amount of forest types/samples with model mismatch (due to the over constrained model);

3. larger variance of the obtained forest height estimates when compared to the inversion results achieved by using the full polarimetric information;

can be widely compensated by using quad-polarimetric observations.

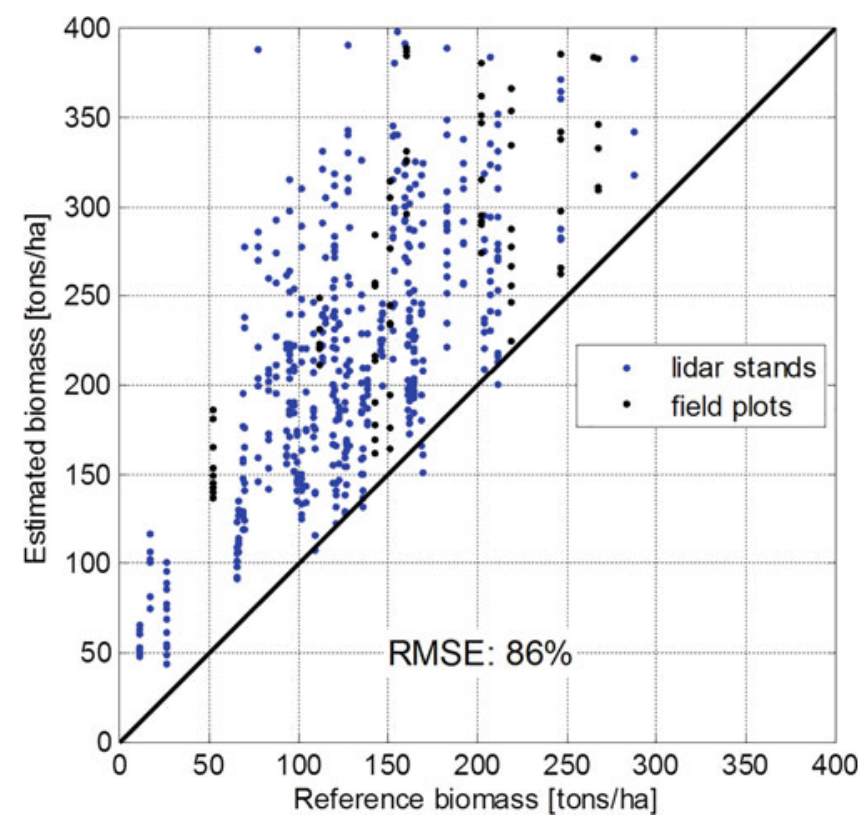

Fig. 2.46 Scatter plot for model R1. For each stand/plot, all available acquisitions are shown

\subsubsection{Discussion on the Role of Polarimetry, on the Maturity of the Application and Conclusions}

Single-pol data interferometric data at a single baseline do not provide enough measurable to invert forest height from interferometric data without a-priori information on terrain topography. A single-pol inversion is possible when multiple baselines are available but the performance is compromised by the presence of temporal decorrelation and the ratio of the spatial baselines. On the other hand, forest height inversion is possible with dual-polarimetric interferometric data taking into account a reduced estimation performance when compared to the quad-polarimetric case. The availability of quadpolarimetric interferometric measurements - that allows the implementation of adaptive optimisation techniques - is critical when algorithm robustness and performance matters.

\subsubsection{Biomass Estimation from Semi-empirical Relationships}

\subsubsection{Introduction, Motivation and Literature Review}

Understanding the terrestrial carbon cycle and predicting future climate changes are important topics in climate research. One of the major uncertainties in the current carbon cycle models lies in terrestrial ecosystems, mainly forests (Solomon et al. 2007). Rather than estimating forest carbon directly, biomass can be used instead since about $50 \%$ of biomass is carbon. Furthermore, above-ground biomass $B$ [t/ha] is often used as a proxy indicator which is estimated from remote sensing measurements, e.g. using P-band (ca $450 \mathrm{MHz}$ ) SAR as discussed in this Section. B is here defined as dry weight and includes stem, bark, branches, and needles/leaves, but excludes stump and roots.

Several studies of using P-band SAR for forest biomass retrieval have been performed in the past. The early studies (Ranson and Sun 1994; Imhoff 1995a; DeFries et al. 2002; Rauste et al. 1994; Beaudoin et al. 1994; Rignot et al. 1995) concluded that HV-polarized backscatter shows highest correlation with biomass. In later studies (Hoekman and Quiñones 2000; Rignot et al. 1995; Santos et al. 2003), retrieval models were extended to other polarisation channels, showing improved retrieval results. In Saatchi et al. (2007b, 2011b) and Sandberg et al. (2011), the need for topographic corrections was pointed out and models were

Table 2.9 Test sites and corresponding radar and validation data selected for the generation of showcases on biomass estimation with allometric relationships

\begin{tabular}{l|l|l}
\hline Application/product & Test site - Radar data & Reference data \\
\hline Biomass estimation with allometric relationships & $\begin{array}{l}\text { Krycklan and Remningstorp, Sweden } \\
\text { TanDEM-X dual-pol data HH-VV }\end{array}$ & $\begin{array}{l}\text { Inventory biomass maps } \\
\text { Lidar forest height measurements }\end{array}$ \\
\hline
\end{tabular}


Fig. 2.40 Biomass estimation using forest height-tobBiomass allometry. Left panel: Krycklan, TanDEM-X (27.02.2011), $13.5 \times 18.8 \mathrm{~km}$; right panel: Remningstorp, $9.3 \times 11.3 \mathrm{~km}$, 30.12.2011. From top to bottom: single look complex amplitude, coherence scaled from 0 (black) to 1 (white), forest height map scaled from 0 to $40 \mathrm{~m}$, and biomass (classification) map. The 5 biomass classes are colored from light to dark green according to the mean biomass (center value) of each class
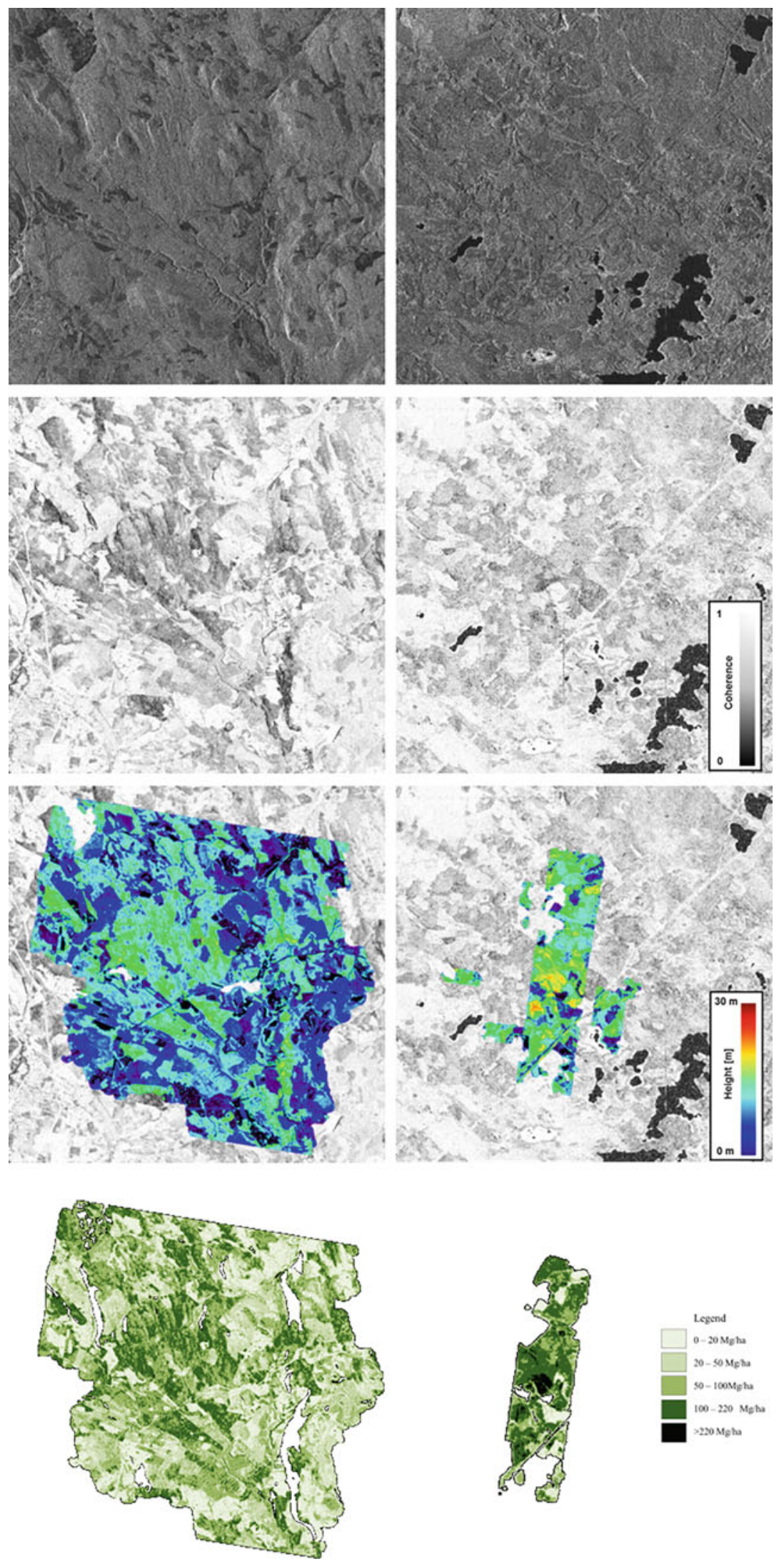

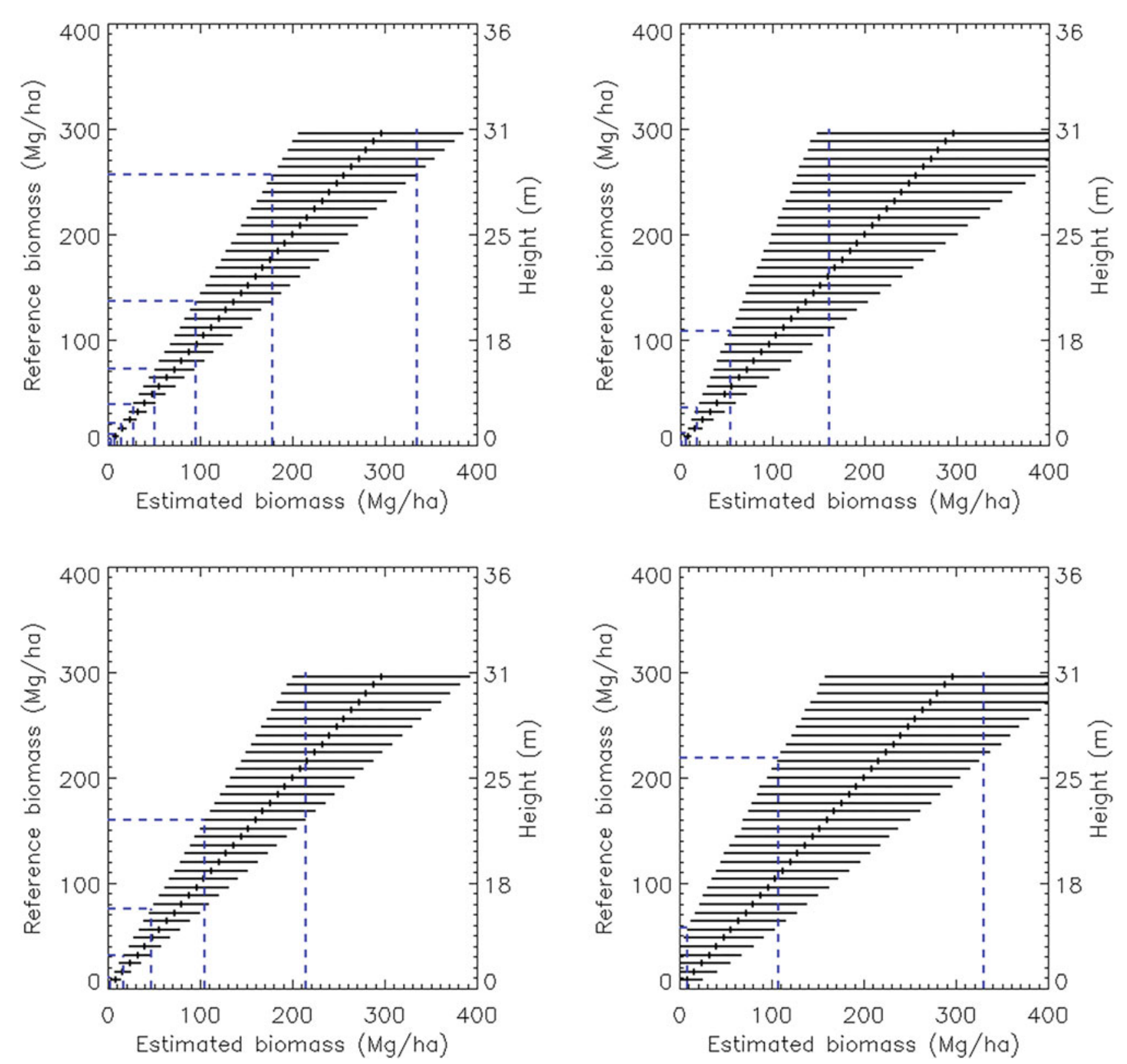

Fig. 2.41 Biomass estimation performance based on (2.36) for a 90\% confidence interval for relative height errors on the top panel (0 left and $10 \%$ right) and absolute height errors on the bottom (1 $\mathrm{m}$ left and $3 \mathrm{~m}$ right) panel

improved. In Neumann et al. (2012), polarimetric and interferometric SAR observables were also used. Complementary approaches include polarimetric SAR interferometry (Cloude and Papathanassiou 1998; Papathanassiou and Cloude 2001; Cloude and Papathanassiou 2003; Neumann et al. 2010) or SAR tomography (Cloude 2006; Reigber and Moreira 2000; Tebaldini 2010). Although the models presented in the mentioned articles show promising results, the analysis were limited to a single test site and the diversity of the data was low. Therefore, the extrapolation capabilities of the models remain untested.

\subsubsection{Methodology}

Biomass retrieval using the following two polarimetric SAR models is analysed: 
Table 2.10 Test sites and corresponding radar and validation data selected for the generation of showcases on biomass estimation

\begin{tabular}{l|l|l}
\hline $\begin{array}{l}\text { Application/ } \\
\text { product }\end{array}$ & Test site - Radar data & Reference data \\
\hline Biomass & Krycklan, Sweden & In-situ measurements (stem diameters, stem volume maps, tree \\
estimation & Airborne E-SAR P-band repeat-pass quad-pol data & heights) \\
& 2008 & \\
& Remningstorp, Sweden & \\
& Airborne E-SAR P-band repeat-pass quad-pol data & \\
& 2007 & \\
\hline
\end{tabular}

(M1) $\ln B=3.280+0.138\left[\gamma_{H V}^{0}\right]_{d B}+0$

(M2) $\ln B=3.129+0.093\left[\gamma_{H V}^{0}\right]_{d B}+(C$

The coefficients were estimated by least squares using the 97 circular plots in Krycklan (for each plot, four data points representing each flight heading were used). The first model is a linear combination of the three like-pol backscatter channels. The second model is a model proposed in Soja et al. (2013), which includes HH/VV ratio (which was found to be less susceptible to temporal and topographic changes), and the ground-slope angle $u$, which introduces a first-order topographic correction. The models were tested in Solomon et al. (2007) for sensitivity to site, topography and temporal change. Furthermore, the models were evaluated using two sets of test data from two different test sites. The test sites are located $720 \mathrm{~km}$ apart and represent two different cases of boreal forest. Models fitted to data from one test site are evaluated on the other. In this manner, the model is validated independently of the training data set.

Biomass retrieval using a third model is also analysed, i.e. the single-pol model according to

$$
\text { (R1) } \ln B=C_{0}+C_{1}\left(\left[\gamma_{H V}^{0}\right]_{d B}-0.766\right)
$$

where $C_{0}=3.8914$ and $C_{1}=0.1301$ (Le Toan et al. 2011).

\subsubsection{Experimental Results}

Test sites and corresponding radar and validation data sets selected for the generation of showcases on direct biomass estimation with semi-empirical relationships are summarized in Table 2.10 and further described in the Appendix.

Averaged, stand-wise backscatter data were extracted from the geocoded SAR images for all stands and plots in both Remningstorp and Krycklan. A $50 \times 50 \mathrm{~m}$ DEM was used for geocoding and normalisation. Although highresolution lidar DEMs were also available for both test sites, they were not used because the evaluation scenario would be less realistic as comparable DEM resolutions are

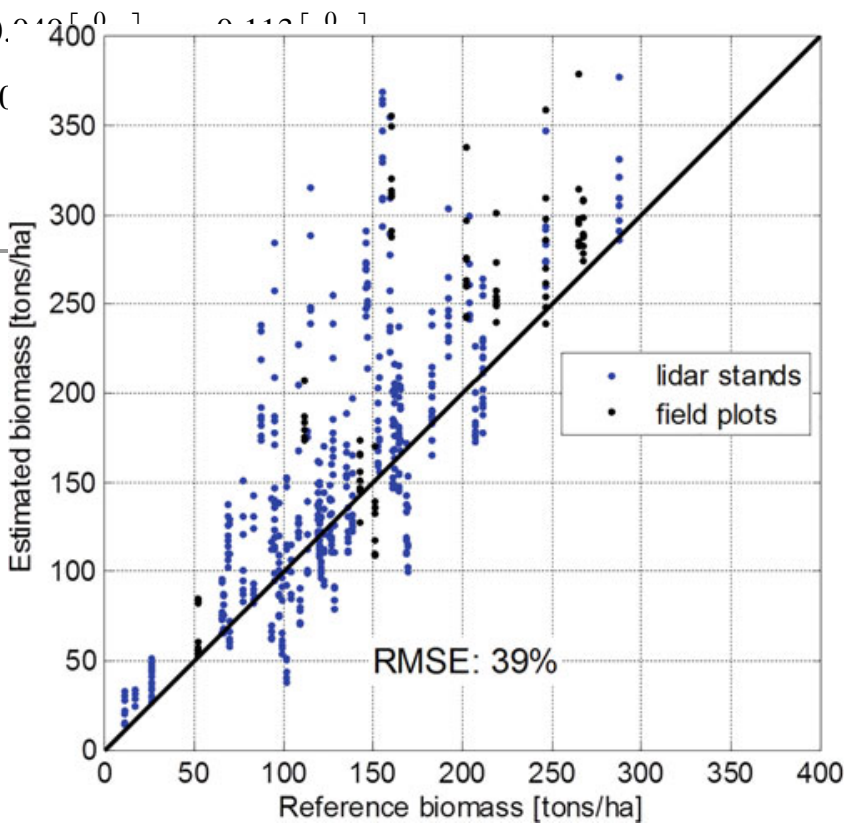

Fig. 2.42 Scatter plot for model M1. For each stand/plot, all available acquisitions are shown

not available on global scale. All normalisation procedures were performed before averaging, that is on high-resolution SAR data. A buffer zone of $10 \mathrm{~m}$ was also added to avoid border effects.

Quantitative results for model M1 are shown in Fig. 2.42. Scatter plots for biomass for the 58 lidar stands and 10 field plots in Remningstorp are shown. Model parameters were extracted in Krycklan. As it can be seen in the figure, biomass is overestimated by $25-50$ t/ha. Root mean square error (RMSE) for all data (all acquisitions at all headings and all dates) is measured to $39 \%$ of the mean biomass of 181 tons/ ha (RMSE is estimated for field plots, for which biomass estimation error is a few per cent). For model M4, the corresponding results are shown in Fig. 2.44. RMSE is $25 \%$, and bias is much lower than for model M1. It is thus concluded, that the inclusion of the HH/VV ratio and topography notably improves retrieval performance.

Biomass maps for model M1 and M4 are shown in Figs. 2.43, 2.44, and 2.45. For M1, overestimation can be observed in many places in the maps. For M4, overestimation can be observed in some limited regions. This overestimation 
Fig. 2.43 Remningstorp: biomass maps for model M1. One single image for the 179-degree heading was used for each date to create SAR-based biomass maps (IDs: 0110, 0206, and 0412, March to May, respectively)

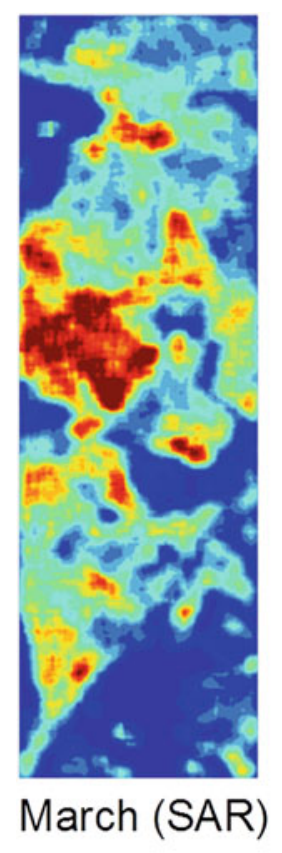

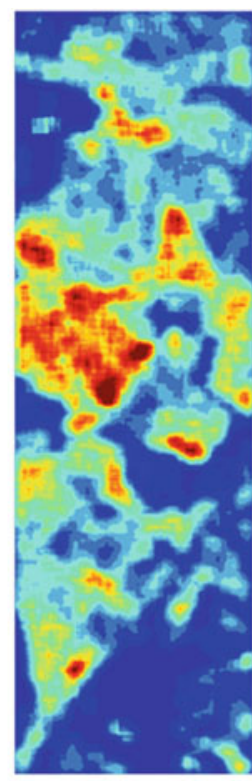

April (SAR)

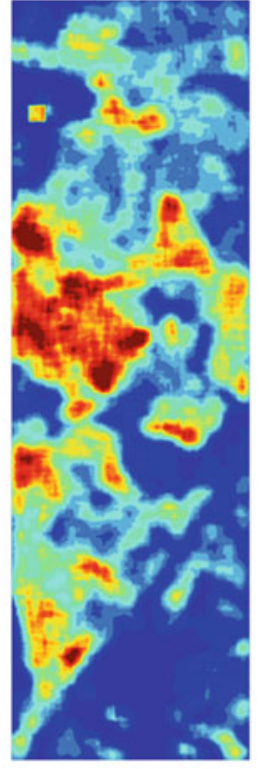

May (SAR)

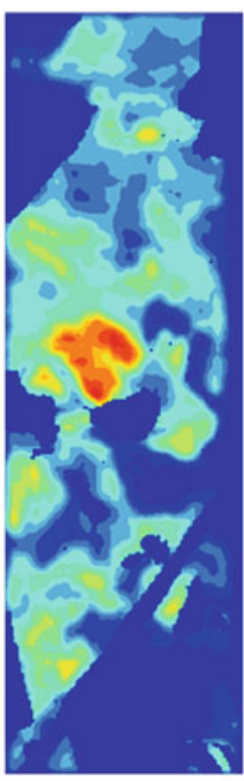

Lidar

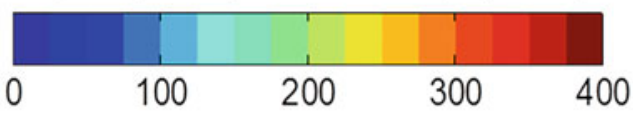

Biomass [tons/ha]

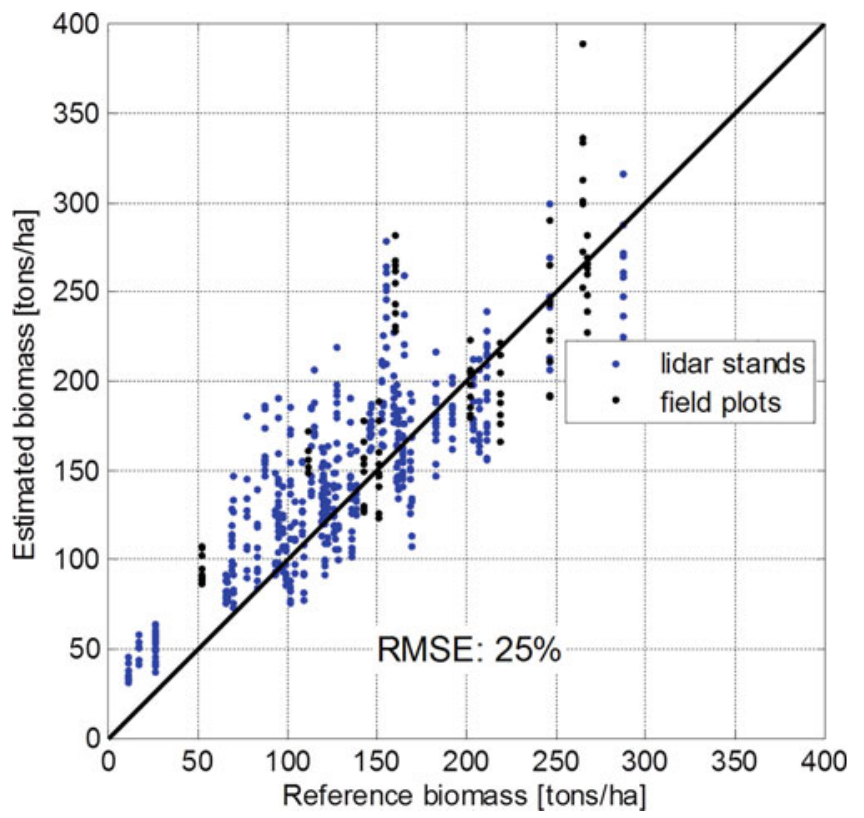

Fig. 2.44 Scatter plot for model M4. For each stand/plot, all available acquisitions are shown

can been explained by physical scattering properties; see (Le Toan et al. 2011).
In Figs. 2.46 and 2.47, quantitative results and biomass maps are shown for single-pol model R1. The model overestimates biomass gravely for almost all stands and is thus not suitable for across-site biomass retrieval.

\subsubsection{Discussion on the Role of Polarimetry, on the Maturity of the Application and Conclusions}

The inclusion of polarimetry significantly improves biomass retrieval performance for regression-based models. Using the $\mathrm{HH} / \mathrm{VV}$-ratio, the geophysical variability common for both $\mathrm{HH}$ and $\mathrm{VV}$ is eliminated (for example, moisture, topography 
Fig. 2.45 Remningstorp:

biomass maps for model M4. One single image for the 179-degree heading was used for each date to create SAR-based biomass maps (IDs: 0110, 0206, and 0412, March to May, respectively)

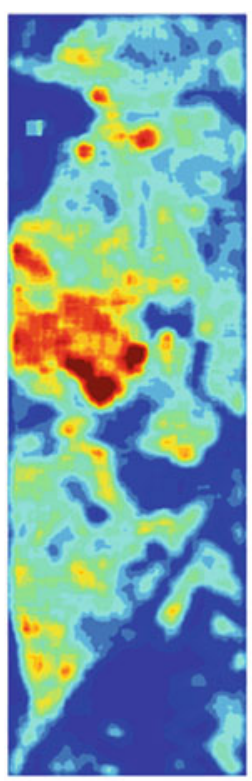

March (SAR)
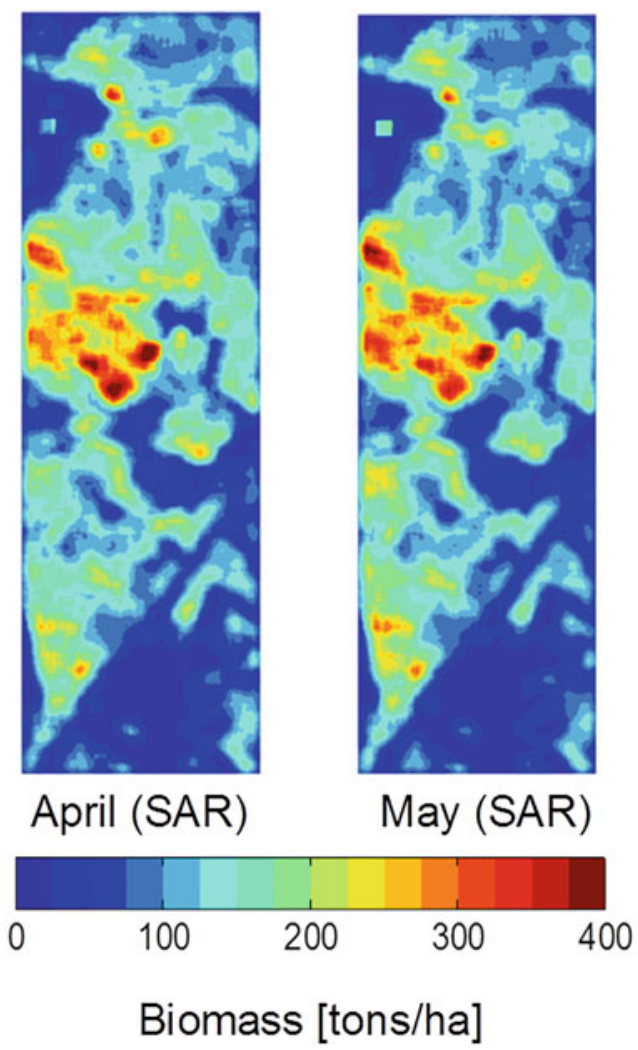

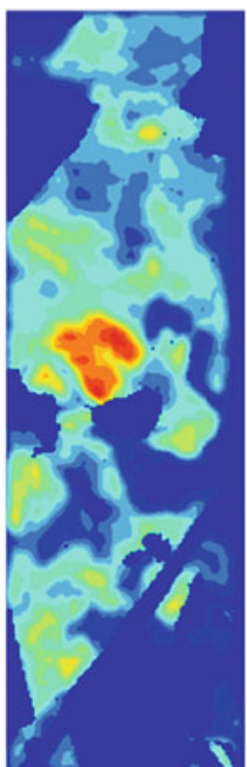

Lidar
Fig. 2.47 Remningstorp: biomass maps for model R1. One single image for the 179-degree heading was used for each date to create SAR-based biomass maps (IDs: 0110, 0206, and 0412, March to May, respectively)
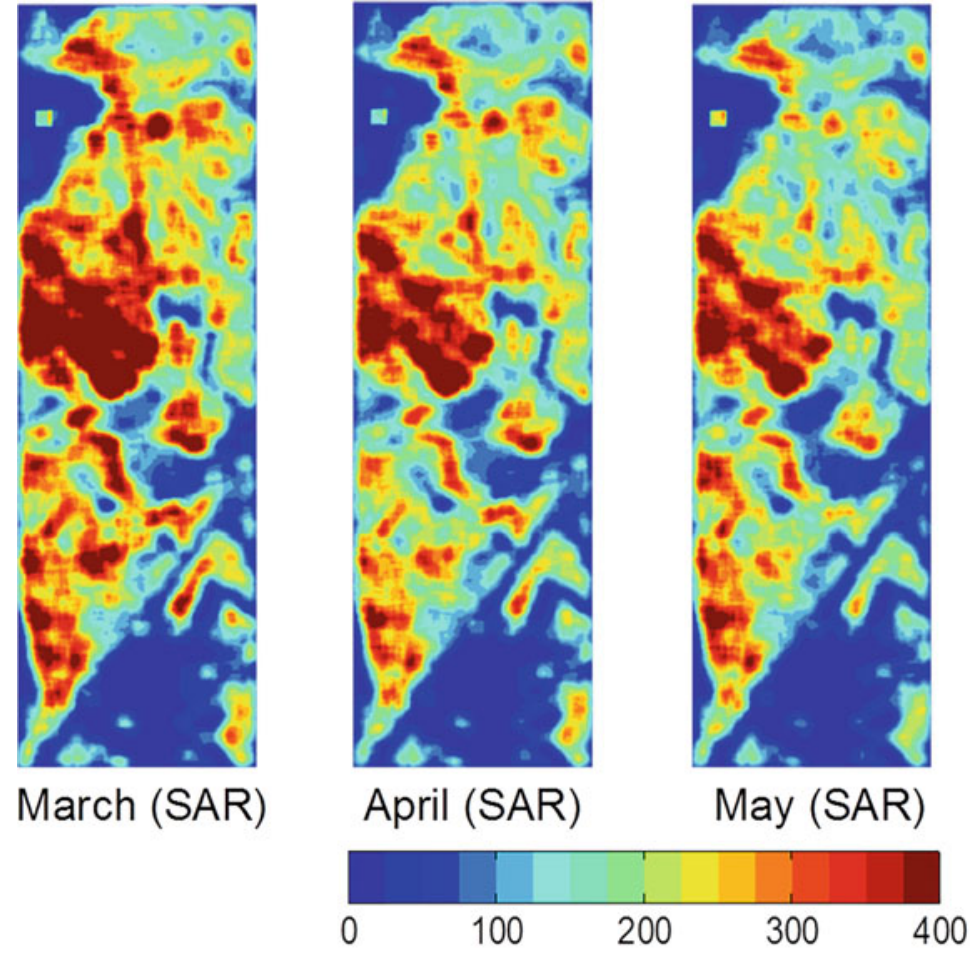

Biomass [tons/ha]

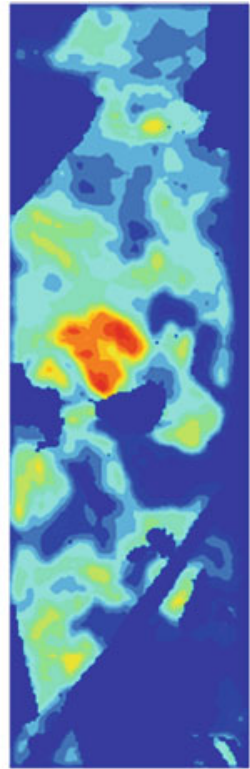

Lidar 
Table 2.11 Summary of presented application, methods and preferred system configurations for forest monitoring

\begin{tabular}{|c|c|c|}
\hline Application & Methods and used frequency (P/L/C/X) & Radar data preference/requirements/comments \\
\hline \multirow[t]{2}{*}{ Classification } & $\begin{array}{l}\text { Using PolSAR observables, Hoekman } \\
\text { decomposition }(\mathrm{P} / \mathrm{L} / \mathrm{C})\end{array}$ & $\begin{array}{l}\text { Preferred frequency: } \mathrm{P} / \mathrm{L} \\
\text { Inc. angle } \sim 45^{\circ} \\
\text { Linear polarization suffice } \\
\text { HV needed }\end{array}$ \\
\hline & Using PolInSAR coherences (L) & $\begin{array}{l}\text { HV needed } \\
\text { Single-pass/short revisit time }\end{array}$ \\
\hline $\begin{array}{l}\text { Fire scar } \\
\text { detection }\end{array}$ & Using polarimetric decompositions (L/C) & High resolution and wide swath desirable - compact-pol is a good trade-off \\
\hline Stand height & PolInSAR dual-baseline inversion (P/L/X) & \\
\hline \multirow[t]{3}{*}{$\begin{array}{l}\text { Vertical } \\
\text { structure }\end{array}$} & $\begin{array}{l}\text { Estimation of 3-D power distribution and } \\
\text { polarimetric scattering characterization }(\mathrm{P} / \mathrm{L})\end{array}$ & \multirow{3}{*}{$\begin{array}{l}\text { Preferred frequency: P/L } \\
\text { Space borne implementation: single-pass systems are preferred. Repeat-pass } \\
\text { configuration benefit from long wavelengths like at P-band } \\
\text { (e.g. BIOMASS) but also L-band depending on time differences among } \\
\text { acquisitions } \\
\text { Multilook needed: high resolution desirable }\end{array}$} \\
\hline & $\begin{array}{l}\text { Ground/Volume separation and parameter } \\
\text { estimation }(\mathrm{P})\end{array}$ & \\
\hline & $\begin{array}{l}\text { Estimation of structure parameters from } \\
\text { PolInSAR scattering models (L) }\end{array}$ & \\
\hline \multirow{2}{*}{$\begin{array}{l}\text { Above- } \\
\text { ground } \\
\text { biomass }\end{array}$} & $\begin{array}{l}\text { Allometric relationships from forest height }(\mathrm{P} / \mathrm{L} / \\
\mathrm{X})\end{array}$ & \\
\hline & $\begin{array}{l}\text { Semi-/Empirical relationships from } \\
\text { backscattering }(\mathrm{P})\end{array}$ & Preferred frequency: $\mathrm{P}$ \\
\hline
\end{tabular}

and structure). Using the slope angle $u$, the influence of topography is reduced.

\subsection{Summary}

\section{References}

Askne J, Santoro M (2009) Automatic model-based estimation of boreal forest stem volume from repeat pass C-band InSAR coherence. IEEE Trans Geosci Remote Sens 47:513-516

Askne JIH, Dammert PBG, Ulander LMH, Smith G (1997) C-band repeat-pass interferometric SAR observations of the forest. IEEE Trans Geosci Remote Sens 35:25-35

Askne J, Santoro M, Smith G, Fransson JES (2003) Multitemporal repeat-rass SAR interferometry of boreal forests. IEEE Trans Geosci Remote Sens 41:1540-1550

Askne JIH, Fransson JES, Santoro M, Soja MJ, Ulander LMH (2013) Modelbased biomass estimation of a hemi-boreal forest from multitemporal TanDEMX acquisitions. Remote Sens 5 (11):5574-5597

Attema EPW, Ulaby FT (1978) Vegetation modeled as a water cloud. Radio Sci 13:357-364

Baccini A, Goetz SJ, Walker WS, Laporte NT, Sun M, Sulla-Menashe D, Hackler J, Beck PSA, Dubayah R, Friedl MA, Samanta S, Houghton RA (2012) Estimated carbon dioxide emissions from tropical deforestation improved by carbon-density maps. Nat Clim Chang 2:182-185

Ballester-Berman JD, Lopez-Sanchez JM, Fortuny-Guasch J (2005) Retrieval of biophysical parameters of agricultural crops using polarimetric SAR interferometry. IEEE Trans Geosci Remote Sens 43:683-694

Bamler R, Hartl P (1998) Synthetic aperture radar interferometry. Inv Probl 14:R1-R54
Beaudoin A, Le Toan T, Goze S, Nezry E, Lopes A, Mougin E, Hsu CC, Han HC, Kong JA, Shin RT (1994) Retrieval of forest biomass from SAR data. Int J Remote Sens 15:2777-2796

Benson AS, De Gloria SD (1985) Interpretation of Landsat-4 thematic mapper and multispectral scanner data for forest surveys. Photogrammetric Eng Remote Sens 51:1281-1289

Bourgeau-Chavez LL, Harrell PA, Kasischke ES, French NHF (1997) The detection and mapping of Alaskan wildfires using a space borne imaging radar system. Int J Remote Sens 18:355-373

Bourgeau-Chavez LL, Kasischke ES, Brunzell S, Mudd JP (2002) Mapping fire scars in global boreal forests using imaging radar data. Int J Remote Sens 23:4211-4234

Canadell JG, Le Quere C, Raupach MR, Field CB, Buitenhuis ET, Ciais P, Conway TJ, Gillett NP, Houghton RA, Marland G (2007) Contributions to accelerating atmospheric $\mathrm{CO} 2$ growth from economic activity, carbon intensity, and efficiency of natural sinks. Proc Natl Acad Sci USA 104:18866-18870

Cao F, Hong W, Pottier E (2010) An improvement for the unsupervised Wishart Freeman classification with fully polarimetric SAR data In: Proceedings of the IEEE 2010 International Geoscience and Remote Sensing Symposium (IGARSS)

Chave J, Condit R, Lao S, Caspersen JP, Foster RB, Hubbell SP (2003) Spatial and temporal variation in biomass of a tropical forest: results from a large census plot in Panama. J Ecol 91:240-252

Chave J, Andalo C, Brown S, Cairns MA, Chambers JQ, Eamus D, Folster H, Fromard F, Higuchi N, Kira T, Lescure JP, Nelson BW, Ogawa H, Puig H, Riera B, Yamakura T (2005) Tree allometry and improved estimation of carbon stocks and balance in tropical forests. Oecologia 145(1):87-99

Cloude SR (2006) Polarisation coherence tomography. Radio Sci. https://doi.org/10.1029/2005RS003436

Cloude SR (2009) Polarisation: applications in remote sensing. Oxford University Press, Oxford

Cloude SR, Papathanassiou KP (1998) Polarimetric SAR interferometry. IEEE Trans Geosci Remote Sens 36:1551-1565

Cloude SR, Papathanassiou KP (2003) Three-stage inversion process for polarimetric SAR interferometry. IEE Proc Radar Sonar Navigation 150:125-134

Cloude SR, Pottier E (1997) An entropy-based classification scheme for land applications of polarimetric SAR. IEEE Trans Geosci Remote Sens 35:68-78 
Cloude SR, Goodenough DG, Chen H (2012) Compact decomposition theory. IEEE Geosci Remote Sens Lett 9:28-32

Cloude SR, Goodenough DG, Chen H, Leckie DG, Hill DA (2013) Time series decomposition analysis for compact polarimetry. Paper presented at 2013 international workshop on science and applications of SAR polarimetry and polarimetric interferometry (POLinSAR)

Dammert PBG, Askne JIH, Kuhlmann S (1999) Unsupervised segmentation of multitemporal interferometric SAR images. IEEE Trans Geosci Remote Sens 37:2259-2271

De Grandi G, Mayaux P, Rauste Y, Rosenqvist A, Simard M, Saatchi SS (2000) The global rain forest mapping project JERS-1 radar mosaic of tropical Africa: development and product characterization aspects. IEEE Trans Geosci Remote Sens 38:2218-2233

DeFries RS, Houghton RA, Hansen MC, Field CB, Skole D (2002) Carbon emissions from tropical deforestation and regrowth based on satellite observations for the 1980s and 1990s. Proc Natl Acad Sci USA 99:14256-14261

Dobson MC, Ulaby FT, Le Toan T, Beaudoin A, Kasischke E, Christensen N (1992) Dependence of radar backscatter on coniferous forest biomass. IEEE Trans Geosci Remote Sens 30:412-415

Dobson MC, Ulaby FT, Pierce LE, Sharik TL, Bergen KM, Kellndorfer J, Kendra JR, Li E, Lin YC, Nashashibi A, Sarabandi K, Siqueira P (1995) Estimation of forest bio physical characteristics in Northern Michigan with SIR-C/X-SAR. IEEE Trans Geosci Remote Sens 33:877-895

Dobson MC, Pierce LE, Ulaby FT (1996) Knowledge-based land-cover classification using ERS-1/JERS-1 SAR composites. IEEE Trans Geosci Remote Sens 30:83-99

Dubayah RO, Sheldon S, Clark DB, Hofton MA, Blair JB, Hurtt GC, Chazdon RL (2010) Estimation of tropical forest height and biomass dynamics using lidar remote sensing at La Selva, Costa Rica. J Geophys Res Biogeosci. https://doi.org/10.1029/2009JG000933

Durden S, Van Zyl JJ, Zebker HA (1989) Modeling and observation of the radar polarization signature of forested area. IEEE Trans Geosci Remote Sens 27:290-301

Engdahl ME, Hyyppä JM (2003) Land-cover classification using multitemporal ERS-1/2 InSAR data. IEEE Trans Geosci Remote Sens 41:1620-1628

Eriksson LEB, Santoro M, Wiessman A, Schmullius CC (2003a) Multitemporal JERS repeat-pass coherence for growing-stock volume estimation of Siberian forest. IEEE Trans Geosci Remote Sens 41:1561-1570

Eriksson LEB, Santoro M, Wiesmann A, Schmullius CC (2003b) Multitemporal JERS repeat-pass coherence for growing-stock volume estimation of Siberian forest. IEEE Trans Geosci Remote Sens 41(7):1561-1570

Fargione J, Hill J, Tilman D, Polasky S, Hawthorne P (2008) Land clearing and the biofuel carbon debt. Science 319:5867

Ferrazzoli P, Paloscia S, Pampaloni P, Schiavon G, Sigismondi S, Solimini D (1997) The potential of multifrequency polarimetric SAR in assessing agricultural and arboreous biomass. IEEE Trans Geosci Remote Sens 35:5-17

Ferro-Famil L, Neumann M (2008) Recent advances in the derivation of POL-inSAR statistics: study and applications. In: Proceedings of the 2008 European conference on synthetic aperture radar (EUSAR)

Ferro-Famil L, Pottier E, Lee JS (2001) Unsupervised classification of multifrequency and fully polarimetric SAR images based on the H/ A/Alpha-Wishart classifier. IEEE Trans Geosci Remote Sens 39:2332-2342

Ferro-Famil L, Pottier E, Lee JS (2003) Unsupervised classification of natural scenes from polarimetric interferometric SAR data. In: Chen $\mathrm{CH}$ (ed) Frontiers of remote sensing information processing. World Scientific Publishing, Singapore

Ferro-Famil L, Pottier E, Kugler F, Lee JS (2006) Forest mapping and classification at L-band using POLINSAR optimal coherence set statistics. In: Proceedings of the 2006 European conference on synthetic aperture radar (EUSAR)

Ferro-Famil L, Huang Y, Reigber A (2012) High-resolution SAR tomography using full-rank polarimetric spectral estimators. In: Proceedings of the IEEE 2012 International Geoscience and Remote Sensing Symposium (IGARSS)

Flynn T, Tabb M, Carande R (2002) Coherence region shape extraction for vegetation parameter estimation in polarimetric SAR interferometry. In: Proceedings of the IEEE 2002 International Geoscience and Remote Sensing Symposium (IGARSS)

Fraser RH, Li Z, Cihlar J (2000) Hotspot and NDVI differencing synergy (HANDS): a new technique for burned area mapping over boreal forest. Remote Sens Environ 74:362-376

Fraser RH, Hall RJ, Landry R, Lynham TJ, Lee BS, Li Z (2004) Validation and calibration of Canada-wide coarse-resolution satellite burned area maps. Photogrammetric Eng Remote Sens 70:451-460

Freeman A (1999) Fitting a two-component scattering model to polarimetric SAR data. In: Proceedings of the IEEE 1999 International Geoscience and Remote Sensing Symposium (IGARSS)

Freeman A, Durden SL (1998) A three-component scattering model for polarimetric SAR data. IEEE Trans Geosci Remote Sens 36:963-973

Frey O, Meier E (2011) Analyzing tomographic SAR data of a forest with respect to frequency, polarization, and focusing technique. IEEE Trans Geosci Remote Sens 49:3648-3659

Garestier F, Le Toan T (2010a) Estimation of the backscatter vertical profile of a pine forest using single baseline P-band (Pol-)InSAR data. IEEE Trans Geosci Remote Sens 48:3340-3348

Garestier F, Le Toan T (2010b) Forest modeling for height inversion using single baseline (Pol-)InSAR data. IEEE Trans Geosci Remote Sens 48:1528-1539

GCOS (2003) Second report on the adequacy of the global observing system for climate in support of the UNFCCC. GCOS-82 (WMO/TD no. 1143). World Meteorological Organization, 2003

GCOS (2004) Implementation plan for the global observing system for climate in support of the UNFCCC. GCOS-92, WMO technical document no. 1219

GOFC-GOLD (2016) A sourcebook of methods and procedures for monitoring and reporting anthropogenic greenhouse gas emissions and removals associated with deforestation, gains and losses of carbon stocks in forests remaining forests, and forestation. GOFCGOLD Report version COP22-1, (GOFC-GOLD Land Cover Project Office, Wageningen University, The Netherlands

Goodenough DG, Chen H, Dyk A (2006) Evaluation of Convair-580 and simulated RADARSAT-2 polarimetric SAR for forest change detection. In: Proceedings of the IEEE 2006 International Symposium on Geoscience and Remote Sensing (IGARSS)

Groot WJ, Landry R, Kurz WA, Anderson KR, Englefield P, Fraser RH, Hall RJ, Banfield E, Raymond DA, Decker V, Lynham TJ, Pritchard JM (2007) Estimating direct carbon emissions from Canadian wildland fires. Int J Wildland Fire 16:593-606

Grover K, Quegan S, da Costa FC (1999) Quantitative estimation of tropical forest cover by SAR. IEEE Trans Geosci Remote Sens 37:479-490

Hagberg JO, Ulander LMH, Askne J (1995) Repeat-pass SAR interferometry over forested terrain. IEEE Trans Geosci Remote Sens 33:331-340

Hajnsek I, Kugler F, Lee SK, Papathanassiou KP (2009) Tropical forest parameter estimation by means of Pol-InSAR: the INDREX II campaign. IEEE Trans Geosci Remote Sens 47:481-493

Hamadi H, Villard L, Borderies P, Koleck T, Le Toan T (2013) Impacts of geophysical changes on the P-band Pol-InSAR phase center of tropical dense forests: EM simulations and TropiScat experimental results. Paper presented at 2013 International Workshop on Science and Applications of SAR Polarimetry and Polarimetric Interferometry (POLinSAR) 
Harrell PA, Bourgeau-Chavez LL, Kasischke ES, French NHF, Christensen NL (1995) Sensitivity of ERS-1 and JERS-1 radar data to biomass and stand structure in Alaskan boreal forest. Remote Sens Environ 54:247-260

Henderson FM, Lewis AJ (1998) Principles and applications of imaging radar. Manual of remote sensing, vol 2, 3rd edn. Wiley, New York

Herold M, Johns T (2007) Linking requirements with capabilities for deforestation monitoring in the context of the UNFCCC-REDD process. Environ Res Lett 2(4):045025

Hoekman DH, Quiñones MJ (2000) Land cover type and biomass classification using AirSAR data for evaluation of monitoring scenarios in the Colombian Amazon. IEEE Trans Geosci Remote Sens 38:685-696

Hoekman DH, Quiñones MJ (2002) Biophysical forest type characterisation in the Colombian Amazon by airborne polarimetric SAR. IEEE Trans Geosci Remote Sens 40:1288-1300

Hoekman DH, Varekamp C (2001) Observation of tropical rain forest trees by airborne high resolution interferometric radar. IEEE Trans Geosci Remote Sens 39:584-594

Hoekman DH, Vissers MAM (2003) A new polarimetric classification approach evaluated for agricultural crops. IEEE Trans Geosci Remote Sens 41:2881-2889

Hoekman DH, Vissers MAM, Wielaard NJ (2010) PALSAR wide-area mapping methodology and map validation of Borneo. IEEE J Sel Topics Appl Earth Observ Remote Sensing 3:605-617

Hoekman DH, Vissers MAM, Tranh T (2011) Unsupervised fullpolarimetric SAR data segmentation as a tool for classification of agricultural areas. IEEE Trans Geosci Remote Sens 49:402-411

Houghton RA (2005) Aboveground forest biomass and the global carbon cycle. Glob Chang Biol 11:945-958

Houghton RA, Hall F, Goetz SJ (2009) Importance of biomass in the global carbon cycle. J Geophys Res 114:G00E03

Huang Y, Ferro-Famil L, Reigber A (2012) Under-foliage object imaging using SAR tomography and polarimetric spectral estimators. IEEE Trans Geosci Remote Sens 50:2213-2225

Hurtt GC, Fisk J, Thomas RQ, Dubayah RO, Moorcroft PR, Shugart HH (2010) Linking models and data on vegetation structure. J Geophys Res Biogeosci. https://doi.org/10.1029/2009JG000937

Hyypa J, Pulliainen J, Hallikainen M, Saatsi A (1997) Radar-derived standwise forest inventory. IEEE Trans Geosci Remote Sens 35:392-404

Imhoff ML (1995a) Radar backscatter and biomass saturation: ramifications for global biomass inventory. IEEE Trans Geosci Remote Sens 33:511-518

Imhoff ML (1995b) A theoretical analysis of the effect of forest structure on synthetic aperture radar backscatter and the remote sensing of biomass. IEEE Trans Geosci Remote Sens 33:341-352

Penman P, Gytarsky M, Hiraish T, Krug T, Kruger D, Pipatta R, Buendia L, Miwa K, Ngara T, Tanabe K, Wagner F (eds) (2003) IPCC good practice guidance for land use, land use change and forestry. Published by the Institute for Global Environmental Strategies (IGES) for the IPCC, Hayama

Kasischke ES, Christensen NL, Bourgeau-Chavez LL (1995) Correlating radar backscatter with components of biomass in loblolly pine forests. IEEE Trans Geosci Remote Sens 33:643-659

Keeling C, Phillips OL (2007) The global relationship between forest productivity and biomass. Glob Ecol Biogeogr 16:618-631

Köhler P, Huth A (2010) Towards ground-truthing of spaceborne estimates of above-ground life biomass and leaf area index in tropical rainforests. Biogeosciences 7:2531-2543

Koskinen JT, Palliainen JT, Hyyppa JM, Engdahl ME, Hallikainen MT (2001) The seasonal behavior of interferometric coherence in boreal forest. IEEE Trans Geosci Remote Sens 39(4):820-829

Krieger G, Papathanassiou KP, Cloude SR (2005) Spaceborne polarimetric SAR interferometry: performance analysis and mission concepts. EURASIP J Applied Sig Proc 20:3272-3292
Kugler F, Lee SK, Papathanassiou KP, Hajnsek I (2009) Estimation of forest vertical structure parameter by means of multi-baseline Pol-InSAR. In: Proceedings of the IEEE 2009 International Geoscience and Remote Sensing Symposium (IGARSS)

Kugler F, Schulze D, Hajnsek I, Pretzsch H, Papathanassiou KP (2014) TanDEM-X Pol-InSAR performance for forest height estimation. IEEE Trans Geosci Remote Sens 52:6404-6422

Kurvonen L, Hallikainen M (1999) Textural information of multitemporal ERS-1 and JERS-1 SAR images with applications to land and forest type classification in boreal zone. IEEE Trans Geosci Remote Sens 37:680-689

Kurvonen L, Pulliainen J, Hallikainen M (1999) Retrieval of biomass in Boreal forests from multitemporal ERS-1 and JERS-1 SAR images. IEEE Trans Geosci Remote Sens 37:198-205

Kurz WA, Apps MJ (2006) Developing Canada's national forest carbon monitoring, accounting and reporting system to meet the reporting requirements of the Kyoto protocol. Mitigation Adapt Strat Global Change 11:33-43

Landry R, Ahern FJ, O’Neil R (1995) Forest burn visibility on C-HH radar images. Can J Remote Sens 21:204-206

Lavalle M, Simard M, Hensley S (2012) A temporal decorrelation model for polarimetric radar interferometers. IEEE Trans Geosci Remote Sens 50:2880-2888

Le Toan T, Beaudoin X, Riom J, Guyon D (1992) Relating forest biomass to SAR data. IEEE Trans Geosci Remote Sens 30:403-411

Le Toan T, Picard G, Martinez JM, Melon P, Davidson M (2001) On the relationship between radar measurements and forest structure and biomass. Paper presented at 2001 Workshop on Retrieval of Bio- and Geophysical Parameters from SAR

Le Toan T, Quegan S, Davidson MWJ, Balzter H, Paillou P, Papathanassiou KP, Plummer S, Rocca F, Saatchi S, Shugart H, Ulander LMH (2011) The BIOMASS mission: mapping global forest biomass to better understand the terrestrial carbon cycle. Remote Sens Environ 115:2850-2860

Lee JS, Grunes MR, Ainsworth TL, Du LJ, Schuler DL, Cloude SR (1999) Unsupervised classification using polarimetric decomposition and the complex Wishart classifier. IEEE Trans Geosci Remote Sens 37:2249-2258

Lee JS, Grunes MR, Pottier E, Ferro-Famil L (2002) Segmentation of polarimetric SAR images that preserves scattering mechanisms. In: Proceedings of the IEEE 2002 International Geoscience and Remote Sensing Symposium (IGARSS)

Lee SK, Kugler F, Papathanassiou KP, Hajnsek I (2010) Multi-baseline Pol-InSAR forest height estimation in the presence of temporal decorrelation. In: Proceedings of the 2010 European conference on synthetic aperture radar (EUSAR)

Lee SK, Kugler F, Papathanassiou KP, Hajnsek I (2013) Quantification of temporal decorrelation effects at L-band for polarimetric SAR interferometry applications. IEEE Trans Geosci Remote Sensing IEEE J Sel Topics Appl Earth Observ Remote Sensing 6:1351-1367

Lefsky MA (2010) A global forest canopy height map from the moderate resolution imaging spectroradiometer and the geoscience laser altimeter system. Geophys Res Lett. https://doi.org/10.1029/ 2010GL043622

Lillesand T, Kiefer RW (1994) Remote sensing and image interpretation. Wiley, New York

Lombardini F, Reigber A (2003) Adaptive spectral estimators for multibaseline SAR tomography with airborne L-band data. In: Proceedings of IEEE 2003 International Geoscience and Remote Sensing Symposium (IGARSS)

Lombardini F, Pardini M, Cai F (2010) Polarimetric differentialTomoSAR imaging. In: Proceedings of the 2010 European conference on synthetic aperture radar (EUSAR)

Lombardo P, Macrì Pellizzeri T (2002) Maximum likelihood signal processing techniques to detect a step pattern of change in 
multitemporal SAR images. IEEE Trans Geosci Remote Sens 40:853-870

Lopez-Sanchez JM, Ballester-Berman JD, Marquez-Moreno Y (2007) Model limitations and parameter-estimation methods for agricultural applications of polarimetric SAR interferometry. IEEE Trans Geosci Remote Sens 45:3481-3493

Marino A, Cloude SR (2010) Detecting depolarizing targets using a new geometrical perturbation filter. In: Proceedings of the 2010 European conference on synthetic aperture radar

Mette T, Papathanassiou KP, Hajnsek I (2003) Height-biomass allometry in temperate forests - performance accuracy of height-biomass allometry. In: Proceedings of the IEEE 2003 Geoscience and Remote Sensing Symposium (IGARSS)

Mette T, Papathanassiou KP, Hajnsek I (2004a) Biomass estimation from polarimetric SAR interferometry over heterogeneous forest terrain. In: Proceedings of the IEEE 2004 Geoscience and Remote Sensing Symposium (IGARSS)

Mette T, Papathanassiou KP, Hajnsek I, Pretzsch H, Biber P (2004b) Applying a common allometric equation to convert forest height from Pol-InSAR data to forest biomass In: Proceedings of the IEEE 2004 Geoscience and Remote Sensing Symposium (IGARSS)

Mitchard E, Saatchi S, Lee W, Abernethy K, Jeffery K. Lewis S, Collins M, Lefsky MA, Leal M, Woodhouse I, Mier P (2011) Mapping tropical forest biomass with radar and spaceborne LiDAR in Lopé National Park, Gabon: overcoming problems of high biomass and persistent cloud. Biogeosciences 8:1-13

Moreira A, Prats-Iraola P, Younis M, Krieger G, Hajnsek I, Papathanassiou KP (2013) A tutorial on synthetic aperture radar. IEEE Geosci Remote Sens Mag 1:6-43

Mougin E, Proisy C, Marty G, Fromard F, Puig H, Betoulle JL, Rudant JP (1999) Multifrequency and multipolarization radar backscattering from mangrove forests. IEEE Trans Geosci Remote Sens 37:94-102

Nannini M, Scheiber R, Moreira A (2009) Estimation of the minimum number of tracks for SAR tomography. IEEE Trans Geosci Remote Sens 47:531-543

Neumann M, Ferro-Famil L, Reigber A (2010) Estimation of forest structure, ground, and canopy layer characteristics from multibaseline polarimetric interferometric SAR data. IEEE Trans Geosci Remote Sens 48:1086-1104

Neumann M, Saatchi SS, Ulander LMH, Fransson JES (2012) Assessing performance of $\mathrm{L}$ - and $\mathrm{P}$-band polarimetric interferometric SAR data in estimating boreal forest above-ground biomass. IEEE Trans Geosci Remote Sens 50:714-726

Nogueira EM, Fearnside PM, Nelson BW, Barbosa RI, Keizer EWH (2008) Estimates of forest biomass in the Brazilian Amazon: new allometric equations and adjustments to biomass from wood-volume inventories. For Ecol Manage 256(11):1853-1867

Paloscia S, Macelloni G, Pampaloni P, Sigismondi S (1999) The potential of C- and L-band SAR in estimating vegetation biomass: the ERS-1 and JERS-1 experiments. IEEE Trans Geosci Remote Sens 37:2107-2110

Papathanassiou KP, Cloude SR (2001) Single-baseline polarimetric SAR interferometry. IEEE Trans Geosci Remote Sens 39:2352-2363

Pardini M, Papathanassiou KP (2013) Sub-canopy ground estimation from multi-baseline SAR data: experiments with a polarimetric RELAX-based solution at L-band. Paper presented at 2013 International Workshop on Science and Applications of SAR Polarimetry and Polarimetric Interferometry (POLinSAR)

Pretszch H (2009) Forest dynamics, growth and yield: from measurement to model. Springer, Berlin/Heidelberg

Pulliainen J, Engdahl M, Hallikainen M (2003) Feasibility of multitemporal interferometric SAR data for stand-level estimation of boreal forest stem volume. Remote Sens Environ 85:397-409
Quegan S, Le Toan T, Jiong Yu J, Ribbes F, Floury N (2000) Multitemporal ERS SAR analysis applied to forest mapping. IEEE Trans Geosci Remote Sens 38:741-753

Quiñones MJ (2002) Polarimetric data for tropical forest monitoring, studies at the Colombian Amazon. Ph.D. thesis, Wageningen University, The Netherlands

Raney RK (2007) Hybrid-polarimetry SAR architecture. IEEE Trans Geosci Remote Sens 11:3397-3404

Ranson KJ, Sun G (1994) Mapping biomass of a northern forest using multifrequency SAR data. IEEE Trans Geosci Remote Sens 32:388-396

Ranson KJ, Saatchi SS, Sun G (1995) Boreal forest ecosystem characterization with SIR-C/ X SAR. IEEE Trans Geosci Remote Sens 33:867-876

Rauste Y, Hame T, Pulliainen J, Heiska K, Hallikainen M (1994) Radarbased forest biomass estimation. Int J Remote Sens 15:2797-2808

Reigber A, Moreira A (2000) First demonstration of airborne SAR tomography using multibaseline L-band data. IEEE Trans Geosci Remote Sens 38:2142-2152

Richardson A, Goodenough DG, Chen H, Hobart G, Moa B, Myrvold W (2010) Unsupervised nonparametric classification of polarimetric SAR data using the k nearest neighbour graph. In: Proceedings of the IEEE 2010 International Symposium on Geoscience and Remote Sensing (IGARSS)

Rignot EJM, Williams CL, Way J, Viereck LA (1994a) Mapping of forest types in Alaskan boreal forests using SAR imagery. IEEE Trans Geosci Remote Sens 32:1051-1059

Rignot EJ, Way J, Williams C, Viereck L (1994b) Radar estimates of aboveground biomass in boreal forests of interior Alaska. IEEE Trans Geosci Remote Sens 32:1117-1124

Rignot EJ, Zimmermann R, Van Zyl JJ (1995) Spaceborne applications of P-band imaging radars for measuring forest biomass. IEEE Trans Geosci Remote Sens 33:1162-1169

Saatchi S, McDonnald K (1997) Coherent effects in microwave backscattering models for forest canopies. IEEE Trans Geosci Remote Sens 35:585-598

Saatchi SS, Moghaddam M (2000) Estimation of crown and stem water content and biomass of boreal forest using polarimetric SAR imagery. IEEE Trans Geosci Remote Sens 38(2):697-709

Saatchi S, Houghton RA, Dos Santos Alval RC, Soares JV, Yu Y (2007a) Distribution of aboveground live biomass in the Amazon basin. Glob Chang Biol 13(4):816-837

Saatchi S, Halligan K, Despain D, Crabtree R (2007b) Estimation of forest fuel load from radar remote sensing. IEEE Trans Geosci Remote Sens 45:1726-1740

Saatchi S, Harris NL, Brown S, Lefsky M, Mitchard ETA, Salas W, Zutta BR, Buermann W, Lewis SL, Hagen S, Petrova S, White L, Silman M, Morel A (2011a) Benchmark map of forest carbon stocks in tropical regions across three continents. Proc Natl Acad Sci USA 108(24):9899-9904

Saatchi S, Marlier M, Chazdon RL, Clark DB, Russell AE (2011b) Impact of spatial variability of tropical forest structure on radar estimation of aboveground biomass. Remote Sens Environ 115:2836-2284

Sandberg G, Ulander LMH, Fransson JES, Holmgren J, Le Toan T (2011) L- and P-band backscatter intensity for biomass retrieval in hemiboreal forest. Remote Sens Environ 115:2874-2886

Santoro M, Askne J, Smith G, Fransson J (2002) Stem volume retrieval in boreal forests from ERS-1/2 interferometry. Remote Sens Environ 81:19-35

Santoro M, Beer C, Cartus O, Schmullius C, Shvidenko A, McCallum I, Wegmüller U, Wiesmann A (2011) Retrieval of growing stock volume in boreal forest using hyper-temporal series of Envisat ASAR ScanSAR backscatter measurements. Remote Sens Environ 115(2):490-507 
Santoro M, Cartus O, Fransson J, Shvidenko A, McCallum I, Hall RJ, Beaudoin A, Beer C, Schmullius C (2013) Estimates of forest growing stock volume for Sweden, Central Siberia, and Québec using Envisat advanced Synthetic Aperture Radar backscatter data. Remote Sens 5(9):4503-4532

Santos JR, Freitas CC, Araujo LS, Dutra LV, Mura JC, Gama FF, Soler LS, Sant'Anna SJ (2003) Airborne P-band SAR applied to the aboveground biomass studies in the Brazilian tropical rainforest. Remote Sens Environ 87:482-493

Sauer S, Ferro-Famil L, Reigber A, Pottier E (2011) Three-dimensional imaging and scattering mechanism estimation over urban scenes using dual-baseline polarimetric InSAR observations at L-band. IEEE Trans Geosci Remote Sens 49:4616-4629

Soja MJ, Sandberg G, Ulander LMH (2013) Regression-based retrieval of boreal forest biomass in sloping terrain using P-band SAR backscatter intensity data. IEEE Trans Geosci Remote Sens 51:2646-2665

Solberg S, Astrup R, Gobakken T, Næsset E, Weydahl DJ (2010) Estimating spruce and pine biomass with interferometric X-band SAR. Remote Sens Environ 114:2353-2360

Solberg S, Astrup R, Breidenbach J, Nilsen B, Weydahl DJ (2013) Monitoring spruce volume and biomass with InSAR data from TanDEM-X. Remote Sens Environ 139:60-67

Solomon S, Qin D, Manning M, Marquis M, Averyt K, Tignor MMB, Miller HL (2007) Climate change 2007: the physical science basis. Contribution of Working Group I to the fourth assessment report of the Intergovernmental Panel on Climate Change. Cambridge University Press, Cambridge

Strozzi T, Dammert PBG, Wegmüller U, Martinez JM, Askne JIH, Beaudoin A, Hallikainen MT (2000) Landuse mapping with ERS SAR interferometry. IEEE Trans Geosci Remote Sens 38:766-775

Tebaldini S (2009) Algebraic synthesis of forest scenarios from multibaseline PolInSAR data. IEEE Trans Geosci Remote Sens 47:4132-4142

Tebaldini S (2010) Single and multipolarimetric SAR tomography of forested areas: a parametric approach. IEEE Trans Geosci Remote Sens 48:2375-2387

Thomas RQ, Hurtt GC, Dubayah RO, Schilz MH (2008) Using lidar data and a height-structured ecosystem model to estimate forest carbon stocks and fluxes over mountainous terrain. Can J Remote Sens 34:351-363

Toraño-Caicoya A, Pardini M, Hajnsek I, Papathanassiou KP (2015) Forest above-ground biomass estimation from vertical reflectivity profiles at L-band. IEEE Geosci Remote Sens Lett 12:2379-2383
Toraño-Caicoya A, Kugler F, Hajnsek I, Papathanassiou KP (2016a) Large scale biomass classification in boreal forests with TanDEM-X data. IEEE Trans Geosci Remote Sens 54:5935-5951

Toraño-Caicoya KF, Pretzsch H, Papathanassiou KP (2016b) Forest vertical structure characterization for estimating above-ground biomass. Can J Forest Resources 46:25-38

Treuhaft RN, Cloude SR (1999) The structure of oriented vegetation from polarimetric interferometry. IEEE Trans Geosci Remote Sens 37:2620-2624

Treuhaft RN, Siqueira PR (2000) The vertical structure of vegetated land surfaces from interferometric and polarimetric radar. Radio Sci 35:141-177

Treuhaft RN, Madsen SN, Moghaddam M, Van Zyl JJ (1996) Vegetation characteristics and underlying topography from interferometric radar. Radio Sci 31:1449-1485

Treuhaft RN, Chapman BD, Dos Santos JR, Gonçalves FG, Dutra LV, Graça PMLA, Drake JB (2009) Vegetation profiles in tropical forests from multibaseline interferometric synthetic aperture radar, field, and lidar measurements. J Geophys Res Atmos. https://doi.org/10. 1029/2008JD011674

Treuhaft R, Gonçalves F, dos Santos JR, Keller M, Palace M, Madsen SN, Sullivan F, Graça PMLA (2015) Tropical forest biomass estimation at X-Band from the spaceborne TanDEM-X interferometer. IEEE Geoscience and Remote Sensing letters 12(2):239-243

UNFCCC (2016) Key decisions relevant for reducing emissions from deforestation and forest degradation in developing countries (REDD + ), Decision booklet REDD+, UNFCCC Secretariat

UN-REDD Programme (2008) UN collaborative programme on reducing emissions from deforestation and forest degradation in developing countries (UN-REDD). FAO, UNDP, UNEP

Van Zyl JJ (1993) The effect of topography on radar scattering from vegetated areas. IEEE Trans Geosci Remote Sens 31:153-160

Wagner W, Luckman A, Vietmeier J, Tansey K, Balzter H, Schmullius C, Davidson M, Gaveau D, Gluck M, Le Toan T, Quegan S, Shvidenko A, Wiesmann A, Yu JJ (2003) Large-scale mapping of boreal forest in SIBERIA using ERS tandem coherence and JERS backscatter data. Remote Sens Environ 85:125-144

Wegmüller U, Werner CL (1995) SAR interferometric signatures of forest. IEEE Trans Geosci Remote Sens 33:1153-1161

Wegmüller U, Werner C (1997) Retrieval of vegetation parameters with SAR interferometry. IEEE Trans Geosci Remote Sens 35:18-24

Zebker HA, Villasenor J (1992) Decorrelation in interferometric radar echoes. IEEE Trans Geosci Remote Sens 30:950-959

Open Access This chapter is licensed under the terms of the Creative Commons Attribution 4.0 International License (http://creativecommons.org/ licenses/by/4.0/), which permits use, sharing, adaptation, distribution and reproduction in any medium or format, as long as you give appropriate credit to the original author(s) and the source, provide a link to the Creative Commons license and indicate if changes were made.

The images or other third party material in this chapter are included in the chapter's Creative Commons license, unless indicated otherwise in a credit line to the material. If material is not included in the chapter's Creative Commons license and your intended use is not permitted by statutory regulation or exceeds the permitted use, you will need to obtain permission directly from the copyright holder.

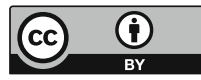




\title{
Agriculture and Wetland Applications
}

\author{
J. M. Lopez-Sanchez, J. D. Ballester-Berman, F. Vicente-Guijalba, S. R. Cloude, \\ H. McNairn, J. Shang, H. Skriver, T. Jagdhuber, I. Hajnsek, E. Pottier, \\ C. Marechal, L. Hubert-Moy, S. Corgne, S. Wdowinski, R. Touzi, G. Gosselin, \\ R. Brooks, Y. Yamaguchi, and G. Singh
}

\section{Abstract}

Based on experimental results, this chapter describes applications of SAR polarimetry to extract relevant information on agriculture and wetland scenarios by exploiting differences in the polarimetric signature of different

J. M. Lopez-Sanchez $(\varangle) \cdot$ J. D. Ballester-Berman · F. Vicente-Guijalba Institute of Computing Research, University of Alicante, Alicante,

Spain

e-mail: juanma.lopez@ua.es

S. R. Cloude

AEL Consultants, Cupar, Fife, UK

H. McNairn · J. Shang

Science and Technology Branch, Agriculture and Agri-Food Canada, Ottawa, ON, Canada

\section{H. Skriver}

Microwaves and Remote Sensing, National Space Institute, Technical University of Denmark, Lyngby, Denmark

T. Jagdhuber

Microwave and Radar Institute, German Aerospace Center (DLR),

Wessling, Germany

I. Hajnsek

Microwaves and Radar Institute, German Aerospace Center (DLR), Oberpfaffenhofen, Germany

Institute of Environmental Engineering, Swiss Federal Institute of Technology Zurich (ETH Zurich), Zürich, Switzerland

E. Pottier · C. Marechal

Institut d'Électronique et de Télécommunications de Rennes, University of Rennes-1, Rennes, France

L. Hubert-Moy $\cdot$ S. Corgne

LETG Rennes, University of Rennes-2, Rennes, France

S. Wdowinski

Division of Marine Geology and Geophysics, University of Miami, Miami, FL, USA

R. Touzi · G. Gosselin · R. Brooks

Canada Center for Remote Sensing, Natural Resources Canada, Ottawa, ON, Canada

Y. Yamaguchi · G. Singh

Institute of Science and Technology, Niigata University, Niigata, Japan scatterers, crop types and their development stage depending on their physical properties. Concerning agriculture, crop type mapping, soil moisture estimation and phenology estimation are reviewed, as they are ones with a clear benefit of full polarimetry over dual or single polarimetry. For crop type mapping, supervised or partially unsupervised classification schemes are used. Phenology estimation is treated as a classification problem as well, by regarding the different stages as different classes. Soil moisture estimation makes intensive use of scattering models, in order to separate soil and vegetation scattering and to invert for soil moisture from the isolated ground component. Then, applications of SAR polarimetry to wetland monitoring are considered that include the delineation of their extent and their characterisation by means of polarimetric decompositions. In the last section of the chapter, the use of a SAR polarimetric decomposition is shown for the assessment of the damages consequential to earthquakes and tsunamis.

\subsection{Introduction}

There exists a wide variety of remote sensing applications in the context of agriculture. A possible classification is summarised in Table 3.1, including the end-users and their motivation for such applications.

Most of these applications have made use of quad-pol data, but recently several studies have been carried out using compact polarimetry, where a single transmit (usually circular) and dual linear coherent receive is used. These have shown some promising advances in agriculture applications, for instance, combining high classification accuracy from the polarimetric information with wide swath coverage. Among all these applications, the following ones demonstrate the role of polarimetry and the benefits of quad-pol data over singlepol or dual-pol approaches: 
Table 3.1 Remote sensing applications on agriculture

\begin{tabular}{|c|c|c|}
\hline (End) users & Application(s) & Interest/motivation \\
\hline \multirow{3}{*}{$\begin{array}{l}\text { National international } \\
\text { authorities/agencies }\end{array}$} & Crop type mapping or classification & Justification of subsidies and fraud detection, acreage \\
\hline & Water resources consumption & $\begin{array}{l}\text { Control in regions suffering droughts or with scarce water } \\
\text { resources }\end{array}$ \\
\hline & Yield prediction & Economic and market predictions, price regulations, etc. \\
\hline \multirow[t]{3}{*}{ Forest management } & $\begin{array}{l}\text { Timely information about crop condition } \\
\text { and phenology }\end{array}$ & $\begin{array}{l}\text { Planning and triggering of farming practices according to specific } \\
\text { phenological stages }\end{array}$ \\
\hline & Water requirements/soil moisture & Irrigation optimisation: only when and where necessary \\
\hline & Final crop productivity & Benefits \\
\hline
\end{tabular}

- Crop type mapping: By means of supervised or partially unsupervised classification schemes, different crop types can be classified in PolSAR images provided that they exhibit different polarimetric signatures as a result of different physical properties.

- Soil moisture retrieval: Using target decomposition approaches, the response from the vegetation and the underlying ground can be separated, as well as the dependence of the radar signal on soil roughness and moisture.

- Phenology monitoring: When plants change their physical characteristics as they develop, different stages can be regarded as different classes, hence being separated by their polarimetric signatures.

Regarding PolInSAR, the rapid rate of growth and change in most crops has undermined so far the development of operational applications based on space-borne SAR systems, since they only provide repeat-pass interferometry (with the notable exception of the recently launched TanDEM-X sensor, which has polarimetric modes available for future use) and agriculture requires single-pass acquisitions. To date, the only successful application based on PolInSAR data is the retrieval of crop height, as an indicator of crop condition or phenology, which has been demonstrated with airborne data.

Wetlands constitute an application scenario with increasing interest in the remote sensing community, especially due to their ecological importance and their clear role in biodiversity. The main applications of PolSAR in wetlands consist in their delineation and characterisation (i.e. condition assessment and vegetation-type classification), for which some approaches have been successfully tested recently. In addition, PolInSAR has been employed to monitor changes in the water level by exploiting the high coherence provided by the double-bounce mechanism produced by the interaction between the water layer and the plants.

Finally, PolSAR has been applied to assess the effect of earthquakes and tsunamis, thanks to the detection of changes in the physical properties of the scene when these events happen.

\subsection{Crop Type Mapping}

\subsubsection{Evaluation of C-Band Polarimetric SAR for Crop Classification}

\subsubsection{Introduction, Motivation and Literature Review}

As the global population increases at a rate of about 80 million per year (United Nations Population Fund 2011), the agriculture and agri-food sector must continue to innovate if it is to meet this demand with an ever-decreasing land base. Recognising the importance of timely information to support accurate national, regional and global agricultural production forecasts, the G20 Group on Earth Observations Global Agricultural Geo-Monitoring Initiative (GEO-GLAM) was launched in 2011 (European Space Agency 2012). Under this initiative, data from Earth observing satellites will be fundamental to characterise crop types and estimate acreages in order to monitor global food production. Yet as the sector adapts to meet the needs of this growing population, society will demand that food be grown in an environmentally sustainable manner. Thus, monitoring how crops are being grown and effects on the landscape and surrounding ecosystems is also important.

Synthetic aperture radars (SARs) can contribute significantly to this global monitoring effort. This is particularly true given improved access in the last decade to satellites with SARs which acquire data at multiple polarisations as well as those that are fully polarimetric-capable. And when agriculture monitoring agencies consider accessing data at multiple frequencies by tasking satellites from various international data providers, a radar-only solution to delivery of crop inventories is possible. Yet research and development are required to adapt existing classification methodologies to use data from SARs. In addition, global cropping systems vary significantly, and consequently, research is required to determine the optimal SAR configuration for different mixes of crops grown under diverse growth cycles. 
In 2004, Agriculture and Agri-Food Canada (AAFC) began developing a classification method to inventory Canada's crops. This research led to the implementation of AAFC's annual crop inventory with the first national inventory available in 2011. Reliability in delivery of the AAFC inventory has been facilitated by acquiring RADARSAT2 dual-polarisation (VV, VH) C-band data to fill gaps when adequate optical data are unavailable (Fig. 3.1). Although the proportion of SAR-to-optical data used in the inventory is growing each year, optical data are still required to meet individual crop accuracy targets. Thus AAFC research continues to explore how multi-frequency and polarimetric SAR data can help to move the inventory towards increased reliance on SAR sensors and less dependence on optical satellites.

SAR satellite data are attractive for mapping and monitoring agriculture as microwave frequencies are unaffected by cloud and haze, unlike their optical counterparts. Agricultural targets (soils and crops) are very dynamic, and thus optical sensors can miss crucial periods in the growing season when information is critically required. Early studies examined the use of single frequency-single polarisation SAR data and reported that these data had to be integrated with optical satellite images if adequate crop classification accuracies were to be achieved. This included integration of $\mathrm{C}-\mathrm{HH}$ (Brisco and Brown 1995), C-VV (Ban 2003; Schotten et al. 1995) or C-HH and C-VV (Blaes et al. 2005) images with at least one optical image. Even when ENVISAT-ASAR and RADARSAT-1 were integrated to create a data set with all three noncoherent linear polarisations $(\mathrm{C}-\mathrm{VV}, \mathrm{C}-\mathrm{VH}$ and C-HH), optical images were still needed to successfully classify individual crops in complex cropping systems (McNairn et al. 2009a). Yet these studies also reported that optically based classification accuracies benefited by the inclusion of SAR data (Brisco and Brown 1995; Ban 2003; McNairn et al. 2009a)

Of all the linear polarisations, many studies have confirmed that the cross-polarisation ( $\mathrm{HV}$ or $\mathrm{VH}$ ) is the single most important polarisation to identify the majority of crops (McNairn et al. 2000, 2009a, b; Foody et al. 1994; Lee et al. 2001). This polarisation is responsive to the multiple scattering from within the vegetation volume, and because

\begin{tabular}{|c|c|c|}
\hline Color & Values & Description \\
\hline & 0 & Unclassified \\
\hline & 20 & WaterWater \\
\hline & 30 & Barren \\
\hline & 34 & Urban \\
\hline & 50 & Shrubland \\
\hline & 80 & Wetland \\
\hline & 110 & Grassland \\
\hline & 122 & Forage Crops \\
\hline & 131 & Fallow \\
\hline & 133 & Barley \\
\hline & 136 & Oats \\
\hline 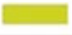 & 137 & Rye \\
\hline & 139 & Triticale \\
\hline & 146 & Wheat \\
\hline & 147 & Com \\
\hline 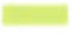 & 153 & Canola/Rapeseed \\
\hline & 154 & Flaxseed \\
\hline & 157 & Sunflowers \\
\hline & 158 & Soybeans \\
\hline 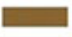 & 162 & Peas \\
\hline 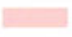 & 177 & Potatoes \\
\hline 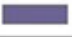 & 179 & Other Vegetables \\
\hline & 181 & Beries \\
\hline$\square$ & 194 & Nursery \\
\hline$\square$ & 220 & Broadleaf \\
\hline
\end{tabular}

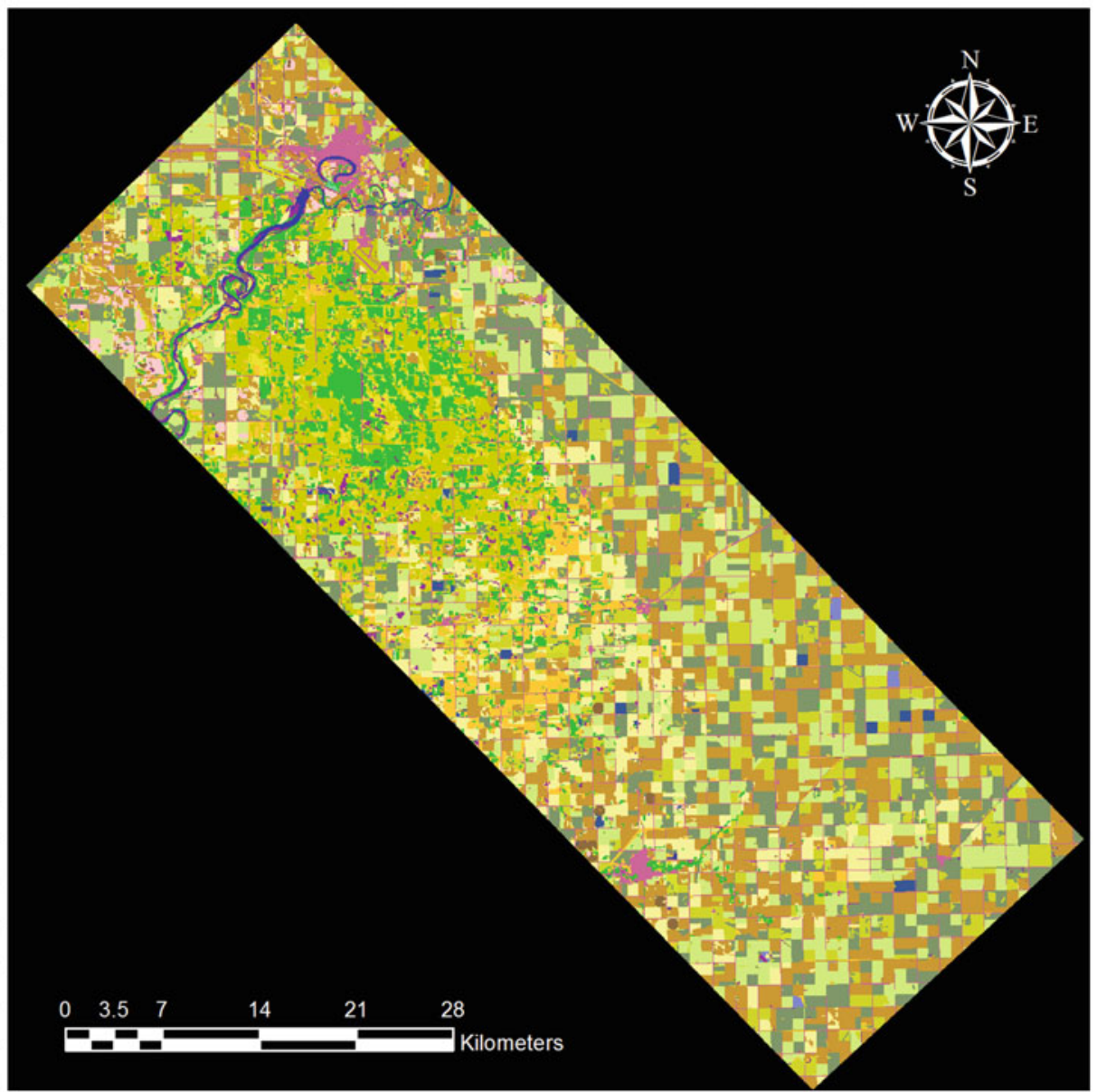

Fig. 3.1 Example of product from Agriculture and Agri-Food Canada's 2012 crop inventory which uses an integration of optical and RADARSAT2 data 
vegetation structure varies greatly among crops, crosspolarised backscatter provides the best discrimination. Nevertheless, classification accuracies are increased substantially with the inclusion of additional polarisations. The greatest incremental increase in accuracy is observed when adding a second polarisation (Foody et al. 1994; McNairn et al. 2000; Hoekman and Vissers 2003), yet a third polarisation will still result in additional improvements to the classification for some crops (McNairn et al. 2000, 2009a; Hoekman and Vissers 2003).

Less research has been completed on the additional benefit of coherent polarimetric parameters for crop classification. Integrating all three linear polarisations and several polarimetric parameters (the co- and cross-polarised pedestal and variation coefficients, e.g.) (Foody et al. 1994) reported a small increase in accuracies. This small incremental increase in accuracy was confirmed in a study where parameters generated from scattering decompositions provided slightly higher accuracies (McNairn et al. 2009b). The HH-VV correlation coefficient and the RR-RL ratio, integrated with $\mathrm{HV}$, have also proven useful in classifying crops (Quegan et al. 2003).

A multi-frequency multi-polarisation approach is the best option for crop discrimination. Ground-based scatterometer and airborne SAR research campaigns describe improvements in vegetation discrimination using multi-frequency data. Researchers reported that relative to single frequency data, higher crop classification accuracies are achieved using $\mathrm{X}$ and C-band (Thomson et al. 1990); C- and L-band (Skriver 2012); X-, C- and L-band (Brisco and Protz 1980; Guindon et al. 1984); as well as C- and L- and P-band (Chen et al. 1996; Ferrazzoli et al. 1997, 1999; Hill et al. 2005; Hoekman et al. 2011) data. The advantage of a multi-frequency data set for separating vegetation types has also been demonstrated using data acquired from multiple satellite platforms, specifically ERS (C-band) and JERS (L-band) (Bouman and Uenk 1992; Dobson et al. 1996). Lower-frequency (i.e. L-band) microwaves penetrate larger biomass crops, and the scattering from within the canopy where structure is quite different aids in separating these crops (McNairn et al. 2009b). Yet for smaller biomass crops, lower frequencies can penetrate too far into the canopy and result in significant soil contributions. For these crops, discrimination is achieved using higherfrequency microwaves where most interaction is limited to within the canopy.

\subsubsection{Methodology}

The evaluation of C-band SAR for crop classification was accomplished using RADARSAT-2 data acquired for three study sites - two in Canada's western Prairie region and a third in eastern Canada. Field observations of crop type were collected to train the supervised classification model and to test the classification accuracy. Fields were randomly split with half used for training and the remainder used to independently evaluate the classification results. A $7 \times 7$ boxcar speckle filter was applied to the single-look complex RADARSAT- 2 data for the eastern site. For the two western sites, a $9 \times 9$ boxcar filter was selected due to the larger field sizes typical of this region of Canada. The covariance matrix was then converted to a symmetrised 3 by 3 covariance matrix from which radar intensity backscatter $(\mathrm{HH}$, HV/VH, VV) was generated. In addition, the Cloude-Pottier (Cloude and Pottier 1997) decomposition was applied generating entropy $(H)$, anisotropy $(A)$ and alpha angle $(\bar{\alpha})$ parameters. With the Freeman-Durden decomposition, the total power was partitioned into contributions from three scattering mechanisms: single-bounce, double-bounce and volume scattering (Freeman and Durden 1998). After generating these radar parameters, the data were orthorectified using platform ephemeris information and models of the internal sensor distortion, a set of ground control points (GCPs) and digital elevation models. Prior to classification an enhanced Lee filter was applied with a window size of $5 \times 5$ for the eastern site and $9 \times 9$ for the western sites.

Fully polarimetric sensors are limited in terms of swath coverage, making their use in large area monitoring a challenge. Specific to RADARSAT-2 the maximum swath for quad-pol (QP) is approximately $50 \mathrm{~km}$, achieved with the wide QP mode. With compact polarimetric (CP) configurations, a larger swath is maintained relative to fully polarimetric SAR systems (Charbonneau et al. 2010), making CP an attractive option to assist with the production of national crop inventories. Although CP offers this operational advantage, the information generated by $\mathrm{CP}$ configurations is not well understood particularly as it relates to applications such as crop classification. To investigate the potential of CP for this application, a circular right transmitting-coherent linear receiving compact polarimetric mode (CL-pol) was simulated from the RADARSAT-2 fully polarimetric data acquired for the Casselman site. The simulated CL-pol data were stored in the Stokes vector format, and the Stokes vector parameters were subsequently used in the classification. A detailed description of the CP simulation in provided in (Charbonneau et al. 2010).

Upon evaluation of several classification models (McNairn et al. 2009a), AAFC chose a decision tree (DT) supervised classifier as it provided the most consistent and reliable classification accuracies particularly when SAR data were used in the classification. Decision boundaries and coefficients for the linear discriminate function used in the classification were estimated empirically from the training data. The DT was run using boosting over five trials to force the classifier to focus on poorly classified cases. To avoid over-fitting, the classifier used a global pruning of the model of $25 \%$. The DT classifier was implemented with the software See5 (Rulequest Research 2008) and is currently the basis of AAFC's operational annual crop inventory. 


\subsubsection{Experimental Results}

Test sites and corresponding radar and validation data sets selected for crop classification are summarised in Table 3.2 and further described in the Appendix.

When a multitemporal data set with all linear polarisations $(\mathrm{HH}, \mathrm{VV}, \mathrm{HV} / \mathrm{VH})$ is available, high overall accuracies are achievable. With the simple crop mix (corn, soybeans, wheat, pasture) of the eastern site, accuracies of $90 \%$ are reached. Slightly lower accuracies are found in more complex cropping systems, such as those of Canada's western region. Here overall accuracies of $85.5 \%$ are reported for both the Manitoba and Saskatchewan sites. Using either the CloudePottier (western sites) or Freeman-Durden (all three sites) decomposition parameters resulted in higher accuracies although gains were small. A classification using CloudePottier entropy, alpha and anisotropy inputs increased overall accuracies by less than a percentage for the Manitoba site $(86.3 \%)$ and less than $2 \%$ for the Saskatchewan site (87.3\%). With the simple cropping mix in the eastern site, the linear polarisations outperformed the accuracies achieved with Cloude-Pottier (87.5\%). The scattering parameters generated by the Freeman-Durden decomposition provided the best results for all three sites (Ontario, 91.3\%; Manitoba, 87.1\%; Saskatchewan, $88.7 \%$ ) although increases in accuracy were limited to between 1 and 3\%. For the Casselman site, the CL-pol Stokes vector parameters produced an overall accuracy $(91.1 \%)$ similar to that achieved with the FreemanDurden decomposition (91.3\%).

Although high overall accuracies are an important indicator of a successful classification, crops must also be well classified individually in order to provide accurate production estimates. Reaching these accuracies has been a challenge for SAR-only classifications. For the Canadian inventory, achieving these individual crop accuracies has required integration of optical data particularly where the cropping mix is more complex. As presented in Fig. 3.2, when the three linear polarisations achieve high crop-specific accuracies (greater than $90 \%$ ), little is gained in using fully polarimetric or $\mathrm{CP}$ parameters. However, the Cloude-Pottier or Freeman-Durden decompositions can improve accuracies when the linear polarisations are unable to reach accuracies above $90 \%$. The results from the decompositions $(\mathrm{QP})$ and the Stokes vector parameters $(\mathrm{CP})$ were comparable with differences of less than one percent observed. The one exception was the pasture class, where the Freeman-Durden outperformed the Stokes vectors by about $3 \%$. It should be noted that small incremental increases in accuracies are important as these improved accuracies lead to more precise acreage estimates when considering the generation of national, regional or global production assessments.

\subsubsection{Comparison with Single-/ Dual-Polarisation Data}

As expected, the use of a single polarisation does not achieve satisfactory classification results. For the more complex cropping mix found in Carman, single VV and VH polarisations produced overall accuracies of $75.6 \%$ and $75.1 \%$, respectively. Slightly higher accuracies were reached in the eastern site (77.9\% for VV polarisation and $79.4 \%$ for $\mathrm{VH})$. Only 62.5\% (Casselman) and 68.1\% (Carman) were attained with the HH polarisation. For the western cropping mix, while the VV polarisation provided better classification of pasture, corn and cereal crops, the remaining crops (canola, flax and beans) were best identified with the cross-polarisation. In Ontario, VH outperformed VV for all crops except corn. For both sites, $\mathrm{HH}$ always produced the lowest crop-specific accuracies.

As previously reported, the addition of a second polarisation (in this case adding $\mathrm{VV}$ to $\mathrm{VH}$ ) provided significant improvements for many crops. For a more complex cropping mix (Carman), with these two polarisations, accuracies for corn, beans and cereals increased by $17.8 \%$, $13.5 \%$ and $8.0 \%$, respectively (Fig. 3.3). The addition of $\mathrm{HH}$ to these first two polarisations increased accuracies by less than $2 \%$. In the eastern site, the integration of $\mathrm{VV}$ with $\mathrm{VH}$ increased individual accuracies by $7-8 \%$ for all crops except soybeans. For soybeans this increase was limited to less than $2 \%$. For the Casselman site, there was value in adding the third $\mathrm{HH}$ polarisation for pasture and wheat where accuracies rose by a further $10-11 \%$ (Fig. 3.3).

In regions of Western Canada, approximately threequarters of the annual cropland is planted in cereals and canola. In the eastern part of the country, this is true for soybeans and corn. Early estimates of these specific crop acreages are important for production and yield forecasting.

Table 3.2 Test sites and corresponding radar and validation data selected for the generation of showcases on crop classification

\begin{tabular}{|c|c|c|}
\hline Application/product & Test site - radar data & Reference data \\
\hline \multirow[t]{7}{*}{ Crop classification } & Casselman, Ontario (2008) & \multirow[t]{2}{*}{274 fields surveyed for crop type } \\
\hline & RADARSAT-2 FQ19 June 22, July 16, Aug. 9, Sept. 2 & \\
\hline & Carman, Manitoba (2009) & \multirow[t]{2}{*}{219 fields surveyed for crop type } \\
\hline & RADARSAT-2 FQ15 June 22, July 16, Aug. 9, Sept. 2 & \\
\hline & Indian Head, Saskatchewan ('09) & \multirow[t]{3}{*}{610 fields surveyed for crop type } \\
\hline & RADARSAT-2 FQ2 June 8, July 2, July 26, Aug. 19, Sept. 12 & \\
\hline & RADARSAT-2 FQ19 June 4, July 22, Aug. 15, Sept. 8 & \\
\hline
\end{tabular}



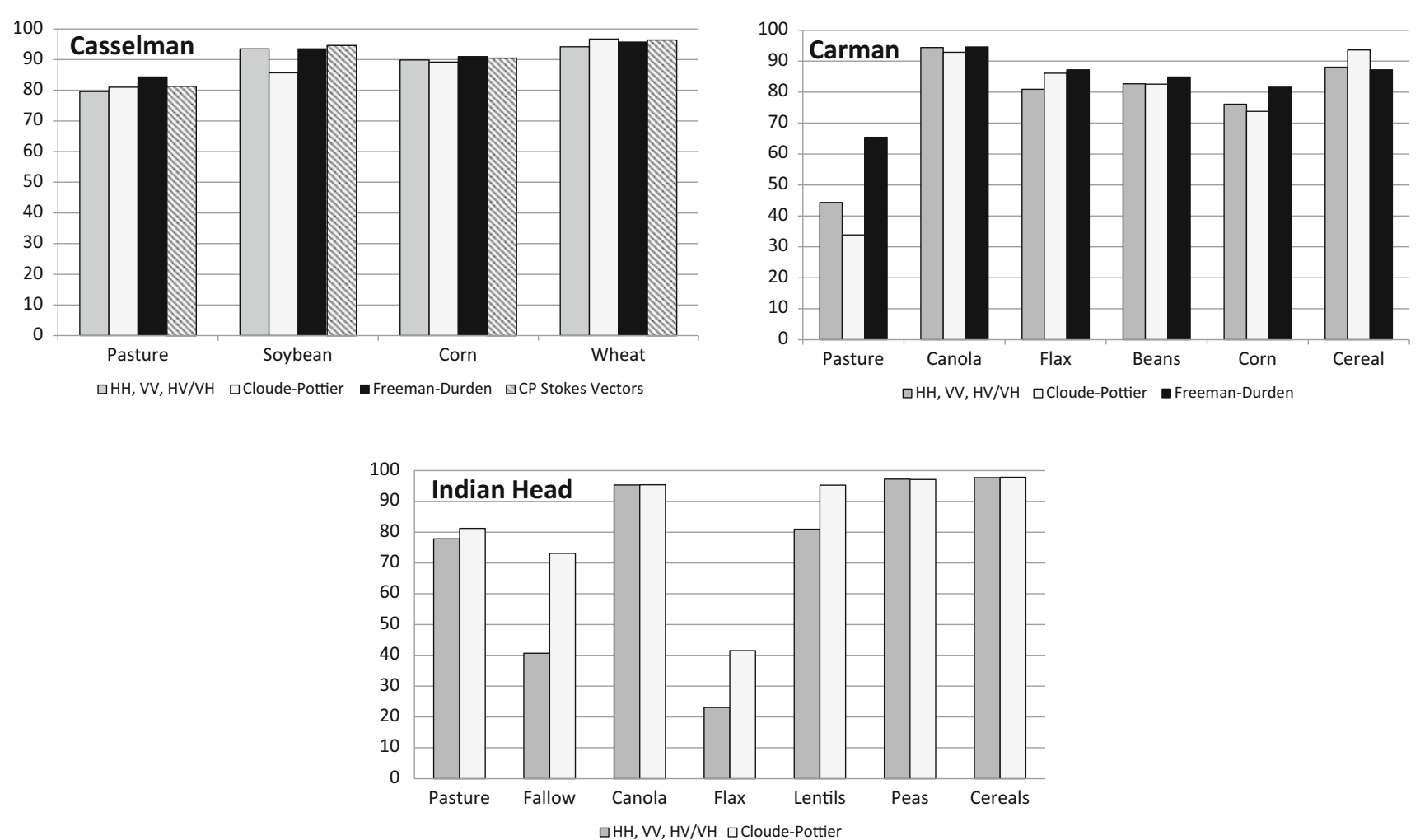

Fig. 3.2 Producer's accuracies for the three study sites
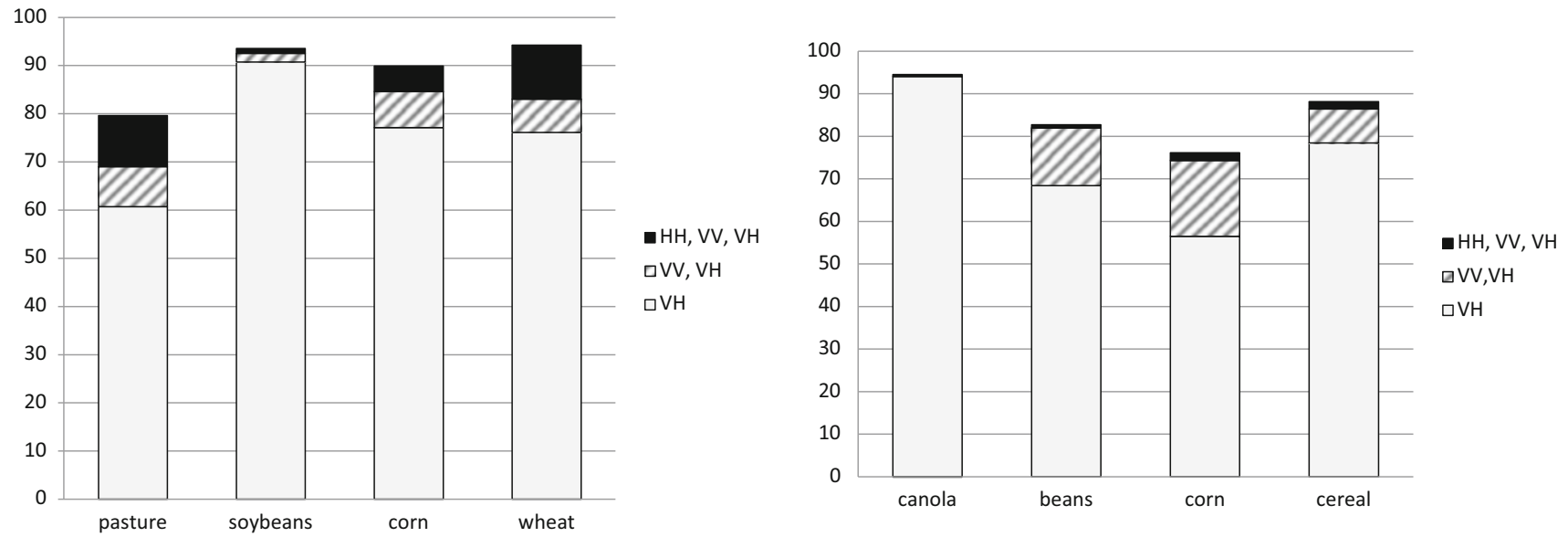

Fig. 3.3 Comparison of crop-specific producer's accuracies using single, dual and multiple polarisations for Casselman (left) and Carman (right)

By mid-July, using only two RADARSAT-2 images (June 22 and July 16), accuracies above 70\% (canola) and 80\% (cereals) could be reached if either the Cloude-Pottier or Freeman-Durden decompositions were used in the classification of the western site. This represents a significant improvement in accuracies reached relative to those generated using single $(\mathrm{VH})$, dual $(\mathrm{VV}, \mathrm{VH})$ or multiple $(\mathrm{HH}, \mathrm{VV}, \mathrm{VH})$ polarisations (Fig. 3.4). For the eastern Ontario site, mid-season estimates (as well as by July 16) were significantly improved using the $\mathrm{QP}$ or $\mathrm{CP}$ configurations. Using either the simulated CL-pol data or one of the decompositions, accuracies close to $80 \%$ were achieved for corn and $93 \%$ for wheat. The Stokes vector parameters were far superior for mid-season identification of soybeans (79.9\%), about $10 \%$ better than accuracies reached with either of the decompositions and $6 \%$ better than accuracies produced using the three linear polarisations. 

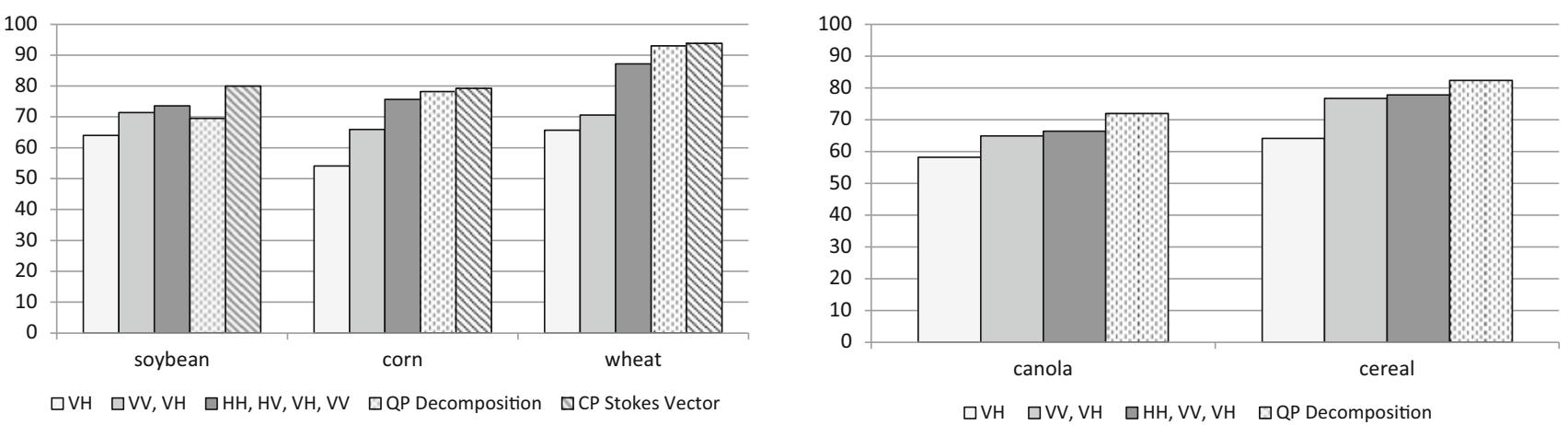

Fig. 3.4 Mid-season classification accuracies for Casselman (left) and Carman (right)

\subsubsection{Discussion on the Role of Polarimetry, on the Maturity of the Application and Conclusions}

Accurate crop classification is the basis for generating production estimates and yield forecasts. Regardless of the source of satellite data, multitemporal acquisitions are necessary. These map products have traditionally been generated using optical satellite data. However, with improved access to SAR satellites with greater polarisation diversity, and in some cases polarimetric capabilities, the potential of this technology for agriculture monitoring is evident. The use of SAR for crop classification requires multitemporal acquisitions of dual polarisations (at a minimum) to meet acceptable overall classification accuracies. However, cropspecific accuracies still require the integration of additional data which has often come from optical sensors. Yet with increased access to polarimetric satellites, results are demonstrating that parameters generated from these complex data, such as radar decompositions, can improve cropspecific identification. In particular, fully and compact polarimetric data can assist in crop identification mid-season which is important if production estimates are required before end of season.

Repetition in results by many international researchers has brought confidence to the idea of radar-assisted crop identification. This has been put into practice by Agriculture and Agri-Food Canada where RADARSAT-2 (VV, VH) data are integrated with optical imagery to produce that country's annual inventory. Increasing the ratio of radar-to-optical data in such an inventory is now limited primarily by access to polarimetric swaths deemed to be operationally appropriate for national mapping. Missions which include wide swath compact polarimetry modes may provide a partial solution. Further, researchers have repeatedly demonstrated that a radar-only approach, with no optical data requirement, is possible with the integration of multi-frequency SAR. The primary impediment to implementing this approach is access to multi-frequency data over swaths appropriate for large area mapping. Without a single multi-frequency satellite, coordinating access from multiple sensors (from multiple agencies) is required. If this challenge can be met, monitoring agencies would have the classification tools and data to monitor national, regional and global agriculture production exclusively with SAR sensors.

\subsubsection{Crop Classification Using Multitemporal L- and C-Band Airborne Polarimetric SAR}

\subsubsection{Introduction, Motivation and Literature Review}

Remote sensing has the potential to provide estimates of inputs for land process models, for instance, the land cover type as well as quantitative geophysical parameters such as soil moisture and leaf area index (LAI). Such models can provide information that is crucial for a number of applications such as flood and drought prediction, crop irrigation scheduling and meteorology (Moran et al. 2004). Also, land cover maps provide fundamental information for many aspects of land use planning and policy development (Bocco et al. 2001), as a prerequisite for monitoring and modelling land use and environmental change (Shalaby and Tateishi 2007) and as a basis for land use statistics at all levels. The European Environmental Agency (EEA) has produced the CORINE Land Cover, and the requirement is a classification accuracy of $85 \%$ for the classification performance of the so-called level 3 classes, which are relatively broad classes (EEA 2007). An important part of land cover is the distribution of agricultural crops, and it is well-known that synthetic aperture radar (SAR) is able to perform such classification. The main advantage of SAR compared with optical and infrared sensors is its all-weather mapping capability. The discrimination potential of SAR data is based on the sensitivity of the radar backscatter to dielectric properties of the objects and to object structure (i.e. the size, shape and orientation distribution of the scatterers) (Skriver et al. 1999). The possibility of identifying individual classes is based on the fact that the dielectric properties and structure of different 
crop types vary. A distinct variation is also seen for these properties through the growing season due to the development of crops (Skriver et al. 1999). Therefore, the discrimination capabilities may vary through the year, and it may also be improved by performing multitemporal classification (Schotten et al. 1995; Skriver 2012; Skriver et al. 2011). The radar backscatter is also sensitive to other parameters, e.g. dielectric properties of the soil, surface roughness, terrain slope and vegetation canopy structure (e.g. row direction and spacing and cover fraction) (Moran et al. 2004). These properties are not necessarily specific for the individual classes and may therefore cause variability of the backscatter and other polarimetric observables within the classes. Also, differences in the development stages at a specific point in time due to, for instance, differences in sowing time may cause such variability (Skriver et al. 1999).

Polarimetric SAR measures all polarimetric information for a target in the form of the scattering matrix. Therefore, such data may be used to assess the capabilities of SAR for crop classification in various configurations, including single-polarisation, dual-polarisation and fully polarimetric modes. Various methods have been used for such assessments: statistical methods based on the Wishart distribution (Lee et al. 1994; 2001; Skriver 2012; Skriver et al. 2011) or covariance matrix elements transformed into backscatter coefficients (Lee et al. 2001; McNairn et al. 2009b; Hoekman and Vissers 2003; Skriver 2012; Skriver et al. 2011; Foody 1988; Freeman et al. 1994), methods based on scattering mechanisms (Lee et al. 1999, 2004) and knowledge-based methods (Ferrazzoli et al. 1999; Pierce et al. 1994; Skriver 2001). In the latter approaches, it is possible to include scattering model results and common knowledge about the targets. Hence, such methods are normally relatively robust and easy to adjust to different growing conditions caused by, for instance, different sowing time, soil and weather conditions. The number of different classes that can be determined is normally relatively small using this type of method. The statistically based methods, on the other hand, will normally provide a larger number of classes, but the classifiers will then normally be specifically adjusted to the data set at hand, and it is difficult to adapt the classifier to other environmental conditions and/or geographical locations.

\subsubsection{Methodology}

The basis for our analysis and processing is the multi-look covariance matrix, which is formed from the coherent scattering vector made up of the backscatter responses at the linear polarisations, $\mathrm{HH}, \mathrm{VV}$ and cross-polarisation. The diagonal backscatter elements in the covariance matrix are then normalised so they correspond to the $\gamma^{0}$ backscatter coefficient in order to reduce the influence of the incidence angle.
All acquisitions have been co-registered by identifying ground control points in the images. The covariance matrix data, corresponding to the original one-look scattering matrix data, have been averaged to reduce the speckle by a cosinesquared weighted 9 by 9 filter. After averaging the equivalent number of looks is estimated to be 9-11 from homogenous areas in the images in the Foulum data set and 10 in the AgriSAR06 data set.

Three different SAR modes are simulated using the same data set, i.e. single-polarisation, dual-polarisation and fully polarimetric data. For the polarimetric results, the full covariance matrix data has been used. For the single- and dual-polarisation modes, subsets of the covariance matrix data have been used, e.g. for the single-polarisation backscatter coefficients, $\gamma_{h h}^{0}$ and $\gamma_{v v}^{0}$, the corresponding elements from the diagonal of the covariance matrix have been used, and for the dual-polarisation backscatter coefficients, pairs of these elements have been used, as well as the cross-polarised backscatter, $\gamma_{x p}^{0}$. For the single- and dual-pol modes, the backscatter intensities have been used, and hence not the complex scattering matrix elements, in order to simulate satellite systems, like ENVISAT, RADARSAT, ALOS and Sentinel-1, where such modes are used to optimise the spatial and temporal coverage. Hence, the possibility of a coherent combination of the $\mathrm{HH}$ and VV polarisations has not been considered for this application.

For the single- and dual-polarisation data, the backscatter coefficients have been used in the classification. Backscatter coefficients follow the Gamma pdf for a multi-look SAR image. When the number of looks is large, the Gamma pdf can, however, be approximated by the Gaussian pdf. The number of looks in our case is at least 10 . Hence, the Gaussian pdf is a valid approximation, and the multivariate Gaussian pdf is used for both single- and dual-polarisation data. The classification method used in all cases is the standard Bayesian maximum likelihood (ML) classifier for multivariate Gaussian statistics.

For the fully polarimetric cases, two methods are used: the standard Bayesian maximum likelihood Wishart classifier originally proposed by Lee et al. (1994) and the method introduced by Hoekman and Vissers using a reversible transform of the covariance matrix into backscatter intensities (Hoekman and Vissers 2003). The complex Wishart classifier is based on the classical Bayesian approach to classification, and the distance measure is derived based on the pdf of the covariance (or coherency) matrix for polarimetric SAR data, i.e. the complex Wishart pdf (Lee et al. 1994). Hoekman and Vissers (2003) introduced a reversible transform of the covariance matrix into backscatter intensities. The advantage is that the fully polarimetric information can be described by backscatter intensities alone. This description will, for instance, better than the Wishart distribution, describe the statistics of a collection of homogeneous areas for the same 
class but with some variability of the mean parameters due to, e.g. slightly different development stages for crops.

The methodology for the assessment of the classification accuracy that has been used is the following: one relatively large and representative field (more than 500 pixels) has been picked for each of the crop classes, and these areas have been used as training areas for the classifiers. A large number of test areas for the classes have been selected, and the classification accuracy has been estimated using these areas. The classification errors estimated are based on the number of pixels in all these test areas, and hence no results are shown using the same data set for training and for testing.

\subsubsection{Experimental Results}

Test sites and corresponding radar and validation data sets selected for crop classification are summarised in Table 3.3 and further described in the Appendix.

The results of classifying the crops for different modes of the SAR acquisitions and for the multitemporal configurations are shown in Figs. 3.5 and 3.6. For each frequency and test site, all single-polarisation and incoherent dual-polarisation configurations have been assessed, as well as the two polarimetric classification methods when fully polarimetric data were available. In the two figures, only the configuration with the best performance, i.e. the lowest classification error, within these groups of configurations is shown. For the Foulum data set, it is seen in Fig. 3.5 that the polarimetric data always provide the best classification result, especially at L-band, where there is a relatively large improvement compared to the incoherent dual-polarisation results. At C-band, the difference between polarimetric results and the dual-polarisation results is smaller. At both frequencies, the Hoekman and Vissers classifier performs best, and the classification error is down to approximately $20 \%$. Also at both frequencies, a clear improvement is seen using the multitemporal acquisitions.

At the AgriSAR06 test site, polarimetric data were available only at L-band, and in this case, as can be seen from Fig. 3.6, there is a clear advantage when only a few temporal acquisitions are available, whereas the performance is worse than the incoherent dual polarisation for more acquisitions. The best classification result is about $11 \%$.

\subsubsection{Comparison with Single-/ Dual-Polarisation Data}

As mentioned above, also single- and incoherent dualpolarisation results are shown in Figs. 3.5 and 3.6. For both test sites at C-band, it is seen that the best single polarisation is $\mathrm{VV}$ and the best dual polarisation is VVXP, whereas at L-band the best single polarisation is $\mathrm{HH}$ and the best dual polarisation is HHXP.

Table 3.3 Test sites and corresponding radar and validation data selected for the generation of showcases on crop classification

\begin{tabular}{l|l|l}
\hline Application/product & Test site - radar data & Reference data \\
\hline Crop classification & Foulum & Crop maps \\
\hline & C- and L-band fully polarimetric multitemporal data \\
\hline & AgriSAR06 & \\
\hline & C-band dual pol., multitemporal \\
\hline & L-band fully pol., multitemporal & \\
\hline
\end{tabular}

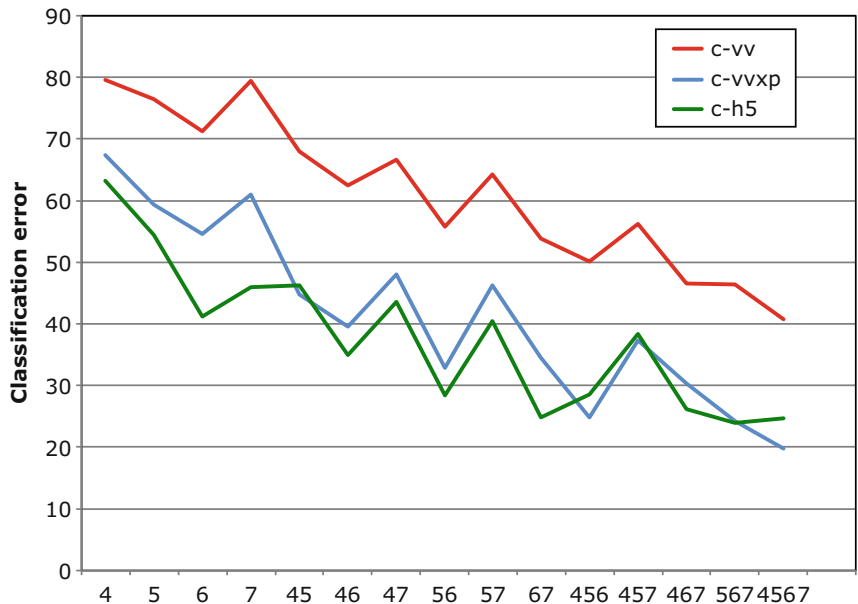

Fig. 3.5 Classification errors for Foulum at C-band (left) and L-band (right) for the best modes for the three cases, single polarisation (red), dual polarisation (blue) and full polarimetry (green), as a function of the

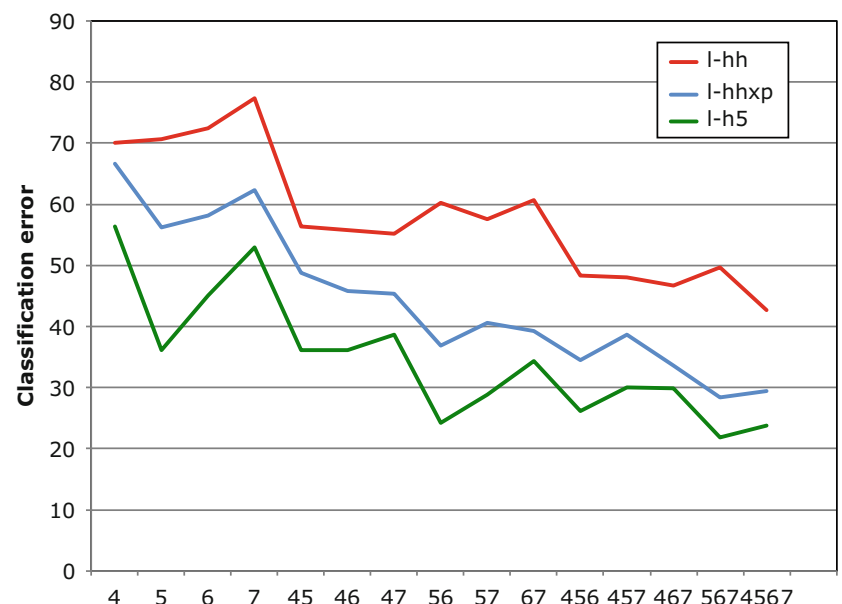

multitemporal acquisition mode, where 4, 5, 6 and 7 correspond to April, May, June and July, respectively 


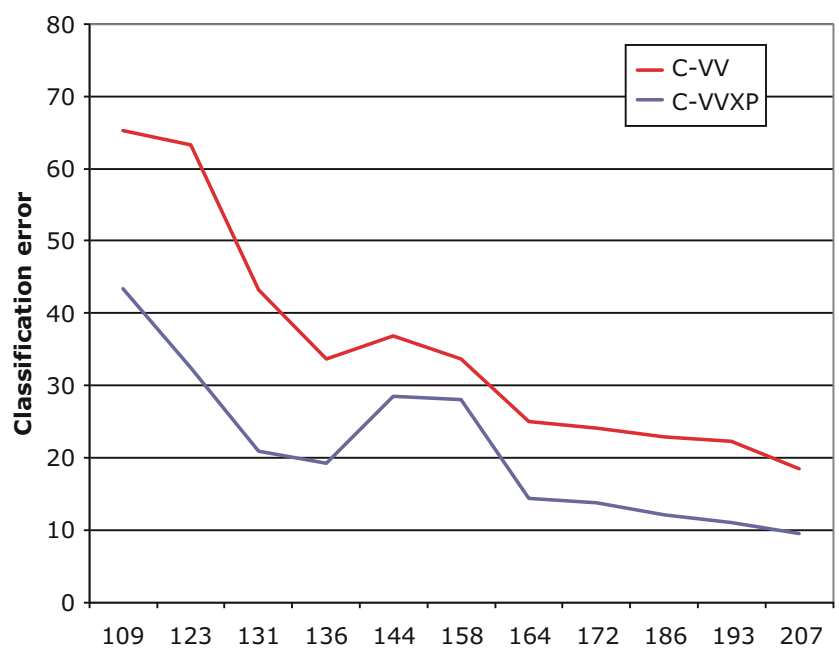

Fig. 3.6 Classification errors for AgriSAR06 at C-band (left) and L-band (right) for the best modes for the three cases, single polarisation (red), dual polarisation (blue) and full polarimetry (green) (only

For the Foulum test site, the smallest classification error for the C-band results is $41 \%$ for VV and $20 \%$ for VVXP, and for the L-band results, it is $43 \%$ for $\mathrm{HH}$ and $28 \%$ for HHXP (Skriver 2012). For the AgriSAR06 test site, the results are at C-band $19 \%$ for VV and $10 \%$ for VVXP, and at L-band $15 \%$ for $\mathrm{HH}$ and 3\% for HHXP (Skriver et al. 2011). It is observed that the differences in performance between the C- and the L-band configurations are relatively small. For the Foulum test site, the C-band performs better than L-band, whereas it is the other way around for the AgriSAR06 test site. This probably has to do with the different set of crop types present at the two test sites.

In all cases, there is a large improvement from the singlepolarisation to the dual-polarisation results. It is clearly seen that the multitemporal acquisitions are necessary for these configurations in order to obtain a reasonable performance. Especially, the dual-polarisation modes including the crosspolarisation perform well, and at C-band they provide comparable results with the fully polarimetric data.

\subsubsection{Discussion on the Role of Polarimetry on the Maturity of the Application and Conclusions}

From the presented results, it is clear that polarimetry has a role in crop classification from SAR. Especially, for L-band an improvement is seen compared to the single and incoherent dual polarisations. At C-band, the polarimetric SAR still performs best, but the improvement compared to the simpler modes is small. The classification results for C-and L-band are relatively similar, and there is no clear preference for one or the other, except that L-band probably requires the polarimetric modes to obtain a sufficient classification performance, whereas simpler modes may be sufficient at C-band.

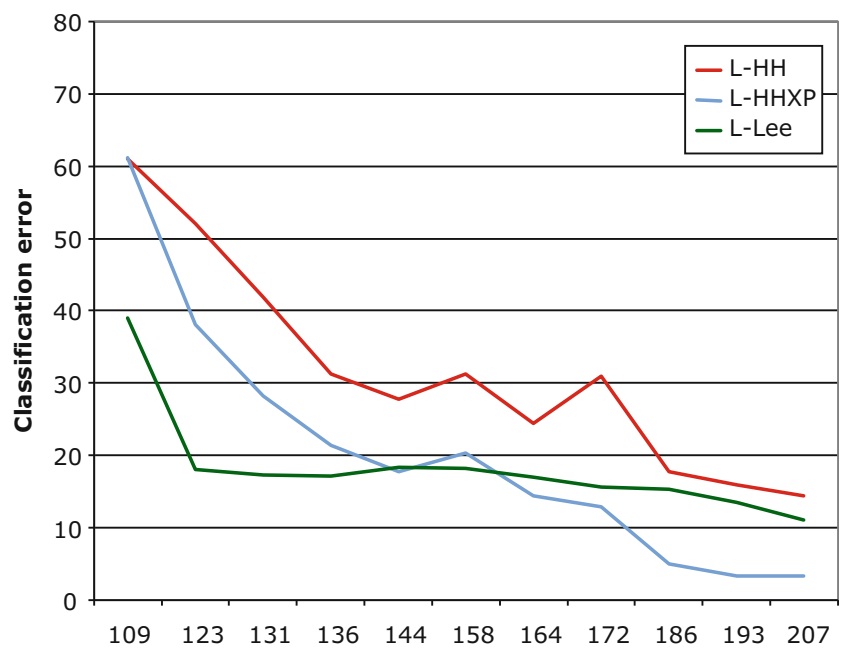

L-band), as a function of the multitemporal series, where the day number corresponds to a series of acquisitions up to and including that day

The application of using multitemporal SAR for crop classification is considered to be relatively mature. Multitemporal acquisitions are considered to be the most important requirement for this application, whether polarimetric or simpler modes are available or not. For the polarimetric modes, it may be less critical if a lot of multitemporal acquisitions are not available, whereas it is essential with multitemporal acquisitions for the single- and dual-polarisation modes.

In conclusion, the performance of the multitemporal SAR data is seen to be very close and even better for some of the modes, compared with the requirements on, e.g. the CORINE land cover of $85 \%$ classification accuracy on level 3 classes. Hence, it will be possible with future polarimetric and/or short revisit systems to obtain a sufficient classification performance. This could, for instance, be possible with the future Sentinel-1 satellites, where single- and dualpolarisation acquisitions at C-band will be available with short revisit times for large parts of the continents, or with future L-band polarimetric missions, such as ALOS-2.

\subsection{Soil Moisture Estimation Under Vegetation Using SAR Polarimetry}

\subsubsection{Introduction, Motivation and Literature Review}

Several hydrological processes, like surface runoff, infiltration, plant water uptake and groundwater drainage, are affected by the spatial and temporal variations of soil moisture within the pedosphere (Bronstert et al. 2012; Krajewski et al. 2006). These moisture changes are still very difficult to 
simulate by environmental/hydrological models due to the multiple and multi-dimensional dependencies of this highly varying soil parameter. Therefore they require a continuous and long-term monitoring (Meehl et al. 2007).

Monitoring of soil moisture content reveals, for instance, the retention capabilities of the soil to store the infiltrated precipitation for a delayed and uncritical release in the discharge system (Bronstert and Bardossy 1999). In this way hazard precautions, like flood forecasting and dam management, can be supported to identify critical catchment states before flooding events (Minet et al. 2011). Besides flood prevention in humid regions, also agricultural management practices in semi-arid regions, like irrigation, benefit from the incorporation of soil moisture information leading to crop yield optimisation in terms of precision farming (Robinson et al. 2008).

In practice, information about the soil moisture status is mostly acquired by single point measurements (Robinson et al. 2003), which might be combined to wireless sensor networks in order to enlarge the monitoring to field scale (Cardell-Oliver et al. 2005). In addition, soil moisture sensing on this scale can be also approached by geophysical methods, like ground-penetrating radar (GPR) (Huisman et al. 2003) or ground albedo neutron sensing (GANS) with cosmic ray probes (Zreda et al. 2008). As the spatial upscaling from point or field measurements to large scales is highly non-linear (Merz and Plate 1997), remote sensing extends soil information retrieval from local point or field studies to spatial investigations on sub-catchment or entire catchment areas, including also very isolated regions (Western et al. 2002).

Since optical remote sensing of soil properties (Katra et al. 2006) relies on daylight and clear weather conditions, RADAR-based methods turn out to be weather- and illumination-independent supporting a continuous soil monitoring strategy. Therefore passive microwave sensors (radiometers) (Jackson 1993) as well as active microwave sensors (scatterometers (Wagner et al. 2007), synthetic aperture radars (SAR) (Ulaby et al. 1982)) are used on airborne or space-borne platforms.

Space-borne radiometers and scatterometers, like MIRAS on SMOS (Kerr et al. 2001) and AMI on ERS, operate with low spatial resolution in the order of kilometres and high temporal resolution in the range of one acquisition every second or third day (in mid-latitudes), which is favourable for global monitoring purposes.

In contrary, SAR sensors, like PALSAR on ALOS (Rosenqvist et al. 2007) or ASAR on ENVISAT, acquired data in the order of decametres with a temporal resolution in the range of one acquisition every first to second week (in mid-latitudes), which fits to regional imaging purposes (Wagner et al. 2008). In order to combine both spatial scales for a spatially enhanced soil moisture retrieval on a global scale, an active-passive microwave sensor will be launched for the SMAP mission (Entekabi et al. 2010).

However, only SAR remote sensing enables a monitoring of the soil and its variability down to the local scale due to its imaging capabilities in high spatial resolution compared to the other microwave sensors. The sensitivity of SAR for soil characteristics is based on the fact that the transmitted electromagnetic (EM) wave interacts with two key parameters of the soil system: soil moisture and soil roughness. SAR sensors, used for soil moisture retrieval, acquire in different wavelengths (ranging from X-band $(\lambda=3 \mathrm{~cm}$, TerraSAR-X (Anguela et al. 2010)), C-band ( $\lambda=5 \mathrm{~cm}$, ENVISAT-ASAR (Löw et al. 2006)), L-band ( $\lambda=23 \mathrm{~cm}$, ALOS (Koyama and Schneider 2011)) to P-band ( $\lambda=80 \mathrm{~cm}$, E-SAR (Jagdhuber et al. 2012a)).

Moreover, SAR remote sensing has the capability to penetrate through natural media, like vegetation layers, especially with increasing wavelength (Cloude et al. 2004). Hence, also agricultural regions, which are most of the year covered by vegetation, can be investigated for their soil moisture conditions. This enables a continuous soil monitoring along the entire plant growth cycle, if the increasing scattering contribution from the vegetation is considered (Hajnsek et al. 2009).

Concerning SAR remote sensing approaches, the first soil moisture analyses were carried out on single-polarisation intensities over bare soils leading to ill-posed inversion problems, because the soil moisture and roughness influences are enclosed in one acquired signal (Bernard et al. 1982). With the emerging establishment of fully polarimetric SAR sensors, the observation space has been enlarged allowing to separate soil roughness from soil moisture influence for an unambiguous inversion of both soil parameters over bare soils (Hajnsek et al. 2003).

For bare soils, three different categories of EM scattering models, which relate the natural soil conditions with the characteristics of the scattered RADAR signature, can be distinguished: empirical models, semi-empirical models and theoretical models. As remote sensing relies on the understanding of electromagnetic (EM) scattering on ground, only theoretical models can be considered as test site independent and form the basis of the analyses. Among these models, the integral equation method (IEM) is widely applied for EM scattering of bare soils because of its broad range of applicability in terms of soil roughness classes (Fung 1994).

So far the investigation of soil parameters, like soil moisture, with polarimetric SAR remote sensing was constrained on bare soil areas due to the lack of retrieval algorithms including the influence of a vegetation cover (Hajnsek et al. 2003). The dielectric discontinuities in vegetation volumes, which can be, for example, stems, branches, twigs and leaves, scatter the waves in a complex manner (Ulaby and Wilson 1985). 
In order to incorporate the influence of vegetation cover, different EM models have been developed to describe vegetation scattering (Lang and Sidhu 1983), whereby the level of detail varies from a simple layer of uniformly shaped spheres (Attema and Ulaby 1978) until sophisticated threedimensional models of the vegetation volume, including cylinders, dipoles and discs with their respective orientation distributions to simulate stems, branches and leaves (Karam et al. 1992). Especially for the latter models, the amount of required input parameters exceeds by far the potential observable space of SAR remote sensing for direct inversion.

Unlike traditional retrieval approaches evaluating the total backscattered signature, an innovative soil information retrieval under vegetation cover should focus on the separation of the individual scattering contributions from the soil and the vegetation in order to extract only the soil properties for inversion. Polarimetric decompositions represent an established technique for separation of elementary scattering processes and have quite a history in polarimetric SAR remote sensing. Originally, these decomposition methods were designed for forested environments including scattering interactions with the ground, with the tree trunk and the ground as well as with the tree canopy. Recently, the description of the polarimetric vegetation volume component was considerably extended by Freeman (Freeman 2007), Neumann (Neumann et al. 2010) and Arii (Arii et al. 2010) using shape parameters for the scattering components (leaves, twigs, branches) and different orientation distributions within the tree canopy to characterise the polarimetric volume scattering of various tree species in a more detailed way. Besides the polarimetric decomposition models for forests, several (multi-angular) polarimetric decomposition methods for the more complex scattering scenario of agriculture, including a depolarising surface component due to soil roughness or an oriented vegetation component due to plant structure, are under development and provide the methodical foundation for this application showcase (Hajnsek et al. 2009; Jagdhuber et al. 2012b, 2013a).

\subsubsection{Methodology}

The retrieval of soil characteristics by polarimetric SAR remote sensing is investigated for agricultural areas using C-band wavelength $(\lambda=5 \mathrm{~cm})$ to assess a continuous soil parameter estimation with a growing vegetation cover along the phenological cycle. Due to polarimetric decomposition techniques, the separation of vegetation from soil/ground information should allow a continuous monitoring of soil moisture under vegetation cover. A modular polarimetric decomposition for retrieval of soil moisture is introduced to provide a profound basis for representation of different natural scattering conditions.

The received SAR signal of almost any natural media is a mixed response of different scattering processes occurring within the resolution cell. Therefore polarimetric decomposition techniques have been developed and used to separate the different scattering contributions. Equation (3.1) describes a generic example of an incoherent decomposition using the coherency matrix notation (Cloude 2009). A simple and generic case of model-based decompositions considers a non-penetrable surface superimposed by a volume of particles. This can be modelled with three components defined as surface $\mathbf{T}_{S}$, dihedral $\mathbf{T}_{D}$ and volume $\mathbf{T}_{V}$ scattering. The first component in Eq. (3.1) represents direct backscatter from a smooth surface $(k s<0.3)$, which can be expressed by the SPM or Bragg scattering model (Yamaguchi et al. 2006):

$$
\begin{aligned}
& \mathbf{T}_{S}=f_{S}\left[\begin{array}{ccc}
1 & \beta_{S}^{*} & 0 \\
\beta_{S} & \left|\beta_{S}\right|^{2} & 0 \\
0 & 0 & 0
\end{array}\right] \text { with } \\
& f_{S}=\frac{m_{S}^{2}}{2}\left|R_{h}+R_{v}\right|^{2} \text { and } \beta_{S}=\frac{R_{h}-R_{v}}{R_{h}+R_{v}}
\end{aligned}
$$

where the coefficients $R_{h}$ and $R_{v}$ are the horizontal and vertical Bragg scattering coefficients and $m_{S}$ represents the soil roughness influence on the intensity component $f_{S}$. Both Bragg scattering coefficients depend only on the dielectric constant of the soil $\varepsilon_{S}$ and the local incidence angle $\theta_{l}$.

The dihedral scattering component can be modelled as a double Fresnel reflection on smooth dielectric media leading to the following rank-1 coherency matrix (Yamaguchi et al. 2006) and including the dihedral scattering intensity $f_{D}$ and the dihedral scattering mechanism ratio $\alpha_{D}$ :

$$
\begin{aligned}
& \mathbf{T}_{D}=f_{D}\left[\begin{array}{ccc}
\left|\alpha_{D}\right|^{2} & \alpha_{D} & 0 \\
\alpha_{D}^{*} & 1 & 0 \\
0 & 0 & 0
\end{array}\right], \text { with } \\
& f_{D}=\frac{m_{D}^{2}}{2}\left|R_{s h} R_{t h}+R_{s v} R_{t v} e^{i \varphi_{D}}\right|^{2} \text { and } \\
& \alpha_{D}=\frac{R_{s h} R_{t h}-R_{s v} R_{t v} e^{i \varphi_{D}}}{R_{s h} R_{t h}+R_{s v} R_{t v} e^{i \varphi_{D}}}
\end{aligned}
$$

where the horizontal and vertical Fresnel coefficients of the soil plane $\left(R_{s h}, R_{s v}\right)$ and of the trunk plane $\left(R_{t h}, R_{t h}\right)$ depend on the soil and trunk dielectric constant $\left(\varepsilon_{S}\right.$ and $\left.\varepsilon_{t}\right)$ and the respective incidence angle $\theta_{s}=\theta_{l}$ and $\theta_{t}=\pi / 2-\theta_{l}$. The phase $\varphi_{D}$ incorporates differential propagation in the case of an orientated vegetation layer, while $m_{D}$ represents the scattering loss on the intensity component $f_{D}$. 
The most challenging component with respect to EM modelling is the vegetation volume. A widely used approach deals with the vegetation volume as a cloud of equally shaped particles with a certain orientation distribution. In one of the simplest ways, the volume component is modelled as randomly oriented cloud of dipoles. The initial vertical dipole can be expressed with the following scattering matrix $\mathbf{S}_{v d i}$ :

$$
\mathbf{S}_{v d i}=\left[\begin{array}{ll}
0 & 0 \\
0 & 1
\end{array}\right]
$$

After expansion to the coherency matrix, the matrix is rotated around the LoS by an angle of $2 \psi$ to account for the orientation of the scattering particles:

$$
\mathbf{T}_{r o t}=\mathbf{R}_{2} \mathbf{T}_{v d i} \psi \mathbf{R}_{2 \psi}^{T}=\left[\begin{array}{ccc}
1 & 0 & 0 \\
0 & \cos 2 \psi & \sin 2 \psi \\
0 & -\sin 2 \psi & \cos 2 \psi
\end{array}\right]\left[\begin{array}{ccc}
1 / 2 & -1 / 2 & 0 \\
-1 / 2 & 1 / 2 & 0 \\
0 & 0 & 0
\end{array}\right]\left[\begin{array}{ccc}
1 & 0 & 0 \\
0 & \cos 2 \psi & -\sin 2 \psi \\
0 & \sin 2 \psi & \cos 2 \psi
\end{array}\right]
$$

In order to respect all the different orientations occurring within vegetation, the rotated coherency matrix $\mathbf{T}_{r o t}$ is integrated together with the specific probability density function $p_{\psi}(\psi)$ of orientations assuming a uniform angular distribution $p_{\psi}(\psi)=1 /(2 \pi)$ and a distribution width $\Delta \psi=\psi_{2}-\psi_{1}=2 \pi$ in the case of a randomly oriented vegetation volume (Yamaguchi et al. 2006):

$$
\begin{aligned}
\mathbf{T}_{V} & =f_{V} \int_{\psi_{1}}^{\psi_{2}} p_{\psi}(\psi) \mathbf{T}_{r o t} d \psi \\
& =f_{V}\left[\begin{array}{ccc}
1 / 2 & 0 & 0 \\
0 & 1 / 4 & 0 \\
0 & 0 & 1 / 4
\end{array}\right] .
\end{aligned}
$$

In a first step, the modelled vegetation volume component $\mathbf{T}_{V}$ with its volume intensity $f_{V}$ is subtracted from the measured polarimetric SAR data $\mathbf{T}$ to separate the volume $\mathbf{T}_{V}$ and the ground $\mathbf{T}_{G}$ components $\left(\mathbf{T}_{S}+\mathbf{T}_{D}\right)$ :

$$
\langle\mathbf{T}\rangle-f_{V} \mathbf{T}_{V}=\mathbf{T}_{G}=\mathbf{T}_{S}+\mathbf{T}_{D} .
$$

However, if the volume modelling is imperfect, Eq. (3.6) leads to biased or even non-physical results for the ground component $\mathbf{T}_{G}$. To avoid this effect, a mathematical method using the positive semi-definiteness of the Hermitian ground component was proposed by $\mathrm{Van} \mathrm{Zyl}$ et al. to correct the volume intensity $f_{V c o r r}$ (Van Zyl et al. 2008). With this corrected volume intensity, the linear system of Eq. (3.2) is solved for the single ground components $\left(\alpha_{D}, \beta_{S}, f_{S}, f_{D}\right)$ of the three scattering contributions obeying the dominance criterion of Freeman et al. for the ground components (Freeman and Durden 1998).

\subsubsection{Experimental Results}

Test sites and corresponding radar and validation data sets selected for crop classification are summarised in Table 3.4. The Indian Head test site is further described in the Appendix.

The presented polarimetric methodology was employed on the fine quad-polarimetric RADARSAT-2 data sets of Flevoland and Indian Head in order to decompose the SAR data into their respective scattering components (surface, dihedral, volume) and afterwards to invert the volumetric soil moisture from the ground components (surface, dihedral).

Figure 3.7 displays exemplarily the RGB composite of the normalised decomposed scattering power components from the three-component model-based decomposition introduced in Sect. 3.3.2 for the two test sites and two different acquisition dates (Flevoland, in the beginning of the observation cycle, 11th of June 2009, towards the end of the observation cycle, 29th of August 2009; Indian Head, 4th of August and 4th of September 2009). For the Flevoland scenes, the water bodies in blue colour and the urban areas predominantly in red colour are clearly visible in both scenes due to their temporal scattering stability. However, the agricultural areas are mostly indicated by the rectangular "patch work" structures across the centre of the scenes, which change significantly between the two acquisitions. For the Indian Head scenes, the agricultural fields show two different patterns for each date (August, September) due to the phenological plant development along the growing cycle. While the strongly vegetated fields exhibit a strong volume scattering, the low vegetated (catch crops) or bare fields (after harvest) indicate predominantly surface scattering. 
Table 3.4 Test sites and corresponding radar and validation data selected for the generation of showcases on soil moisture estimation under vegetation

\begin{tabular}{|c|c|c|}
\hline Application/product & Test site - radar data & Reference data \\
\hline \multirow{6}{*}{$\begin{array}{l}\text { Soil moisture estimation } \\
\text { under vegetation }\end{array}$} & Flevoland, the Netherlands & \multirow{3}{*}{$\begin{array}{l}\text { Two continuously recording soil moisture stations (volumetric soil moisture } \\
\text { content, hourly sampling from } 26 / 05-06 / 10 / 2009 \text { ) }\end{array}$} \\
\hline & $\begin{array}{l}\text { RADARSAT-2, C-band - fine quad- } \\
\text { polarimetric SLC data sets }\end{array}$ & \\
\hline & $\begin{array}{l}\text { Time series from April } 2009 \text { to } \\
\text { August } 2009\end{array}$ & \\
\hline & Indian Head, Canada & \multirow{3}{*}{$\begin{array}{l}\text { One continuously recording soil moisture station (volumetric soil moisture } \\
\text { content, hourly sampling from 17/07-13/09/2009) }\end{array}$} \\
\hline & $\begin{array}{l}\text { RADARSAT-2, C-band - fine quad- } \\
\text { polarimetric SLC data sets }\end{array}$ & \\
\hline & $\begin{array}{l}\text { Time series from July } 2009 \text { to } \\
\text { September } 2009\end{array}$ & \\
\hline
\end{tabular}

In June, after the start of the crop cycle (Fig. 3.7 left), the fields appear in all three colours, indicating a mixture of surface (bare soil), dihedral (stalk/leave-ground) and volume (complex vegetation) scattering due to the different phenologies of the different crop types, but also the different sowing dates on different fields.

In August, within the final stage of the crop cycle (Fig. 3.7 right), a part of the agricultural fields (mostly the winter crops) are already harvested and appear in blue colour indicating predominantly surface scattering, while the other part of the agricultural fields (summer crops, like corn) still exhibit green colours, indicating complex volume scattering before harvesting.

Figure 3.8 exhibits the trends of the three normalised scattering power components (red, dihedral; green, volume; blue, surface) for Flevoland and Indian Head test sites. The general trend is similar for both test sites at Flevoland, where volume is dominant most of all times with values around 0.6-0.7 peaking in July/August during the maturity stage of the vegetation. The ground components always stay below 0.4-0.5 except for the April dates in Flevoland.

Moreover, dihedral scattering is mostly present within the June dates in Flevoland, when the vegetation is active and fresh for a distinct double bounce and almost vanishes afterwards, while surface scattering dominates over dihedral scattering for Flevoland in July and August 2009. For the Indian Head test site, the time series in Fig. 3.8 reveals a clear dominance of volume scattering (level $=0.8$ along all August) due to a strong agricultural vegetation cover of canola crops (vegetation height $>1.0 \mathrm{~m}$ ) on the test field, where some minor ground scattering occurs as single-bounce surface and/or as double-bounce dihedral scattering (level $<0.2$ ). Hence, the dry moisture regime (mean soil moisture level lower than $8 \mathrm{vol} . \%$ ) together with the thick vegetation layer of canola will be a big challenge for soil moisture inversion under vegetation at $\mathrm{C}$-band.

In conclusion, the decomposition led to a physically meaningful separation of the scattering contributions within the C-band RADARSAT-2 scenes, which reflects the agricultural scattering scenario reasonably well. After the model-based polarimetric decomposition into the single scattering components, the surface and dihedral components were inverted for soil moisture retrieval under vegetation cover as described in Sect. 3.3.2. Only for Flevoland very low inverted moisture ranges (soil moisture $<5.6 \mathrm{vol} . \%$ ) compared to the in situ measured moistures ( 30-40vol.\%) are not representative and are filtered out in the end of the inversion process.

Figure 3.9 presents exemplarily the combination of inverted soil moisture values from both ground components (surface, dihedral) for the Flevoland (11th of June 2009 and 29th of August 2009) and Indian Head (4th of August and 4th of September 2009) test sites. The inversion rates for the whole Flevoland scene are $33.12 \%$ for the June date and $32.70 \%$ for the August date, while the inversion rates for both test sites and all scenes are displayed in Fig. 3.10. For Flevoland the inversion rates vary between $22 \%$ and $33 \%$ along time, whereby the minimum is reached in July, when the agricultural vegetation is in its maturity stage. For Indian Head the inversion rate varies between $11 \%$ and $25 \%$ growing slightly higher in the end of the vegetation growth period. Hence, in all cases, the inversion exhibits gaps of non-invertible points (white areas in Fig. 3.9). The inversion mostly failed within the agricultural regions due to the variety of scattering scenarios (typical field sizes at Flevoland: 10-15 ha), which are difficult to describe in detail with one type of model-based polarimetric decompositions assuming just a randomly oriented vegetation volume.

However, distinct field heterogeneities are visible within the soil moisture results, which fit the expectations of a highly varying soil moisture content on the agricultural fields. For Indian Head, a higher inversion rate can be stated for the scene acquired in September due to a diminished vegetation cover caused by harvesting activities and senescence.

In general, the inversion result at Flevoland for the 11th of June 2009 reveals a higher soil moisture level on the dihedral dominant fields than on the fields with surface scattering. In addition, the dominance of surface scattering for the 29th of 
a
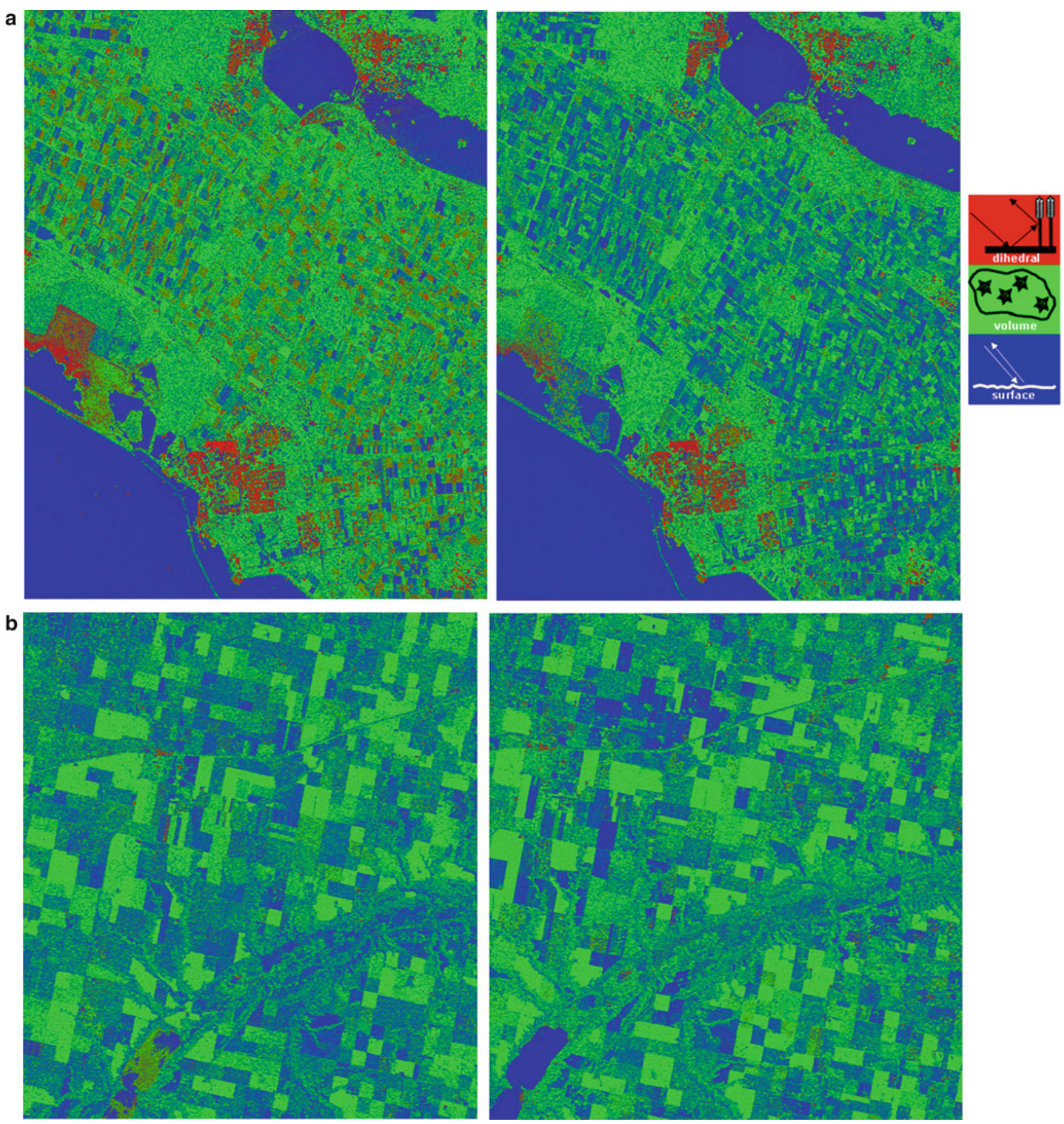

Fig. 3.7 RGB composite of normalised decomposed scattering power components from model-based polarimetric decomposition for the beginning of the observation cycle at 11th of June 2009 (Top left) and the end of the observation cycle at 29th of August 2009 (Top right) at the Flevoland test site and for 4th of August in the main growth period

(Bottom left) and 4th of September 2009 in the end of the main growth period (Bottom right) at the Indian Head test site (red, even bounce/ dihedral scatterers; green, volume scatterers; blue, odd bounce/surface scatterers)

August 2009 seems to result in a lower moisture level on most of the fields. In contrast to Flevoland, the Indian Head test site exhibits generally a lower soil moisture level, but also states relatively a higher moisture regime for fields with dihedral compared to surface scattering.

Figure 3.11 presents the validation of the inverted soil moisture from the ground scattering components (surface, dihedral) with the in situ measurements of the 2 test sites and all continuously recording moisture stations for 11 different dates from 11th of June until the 29th of August 2009 at 

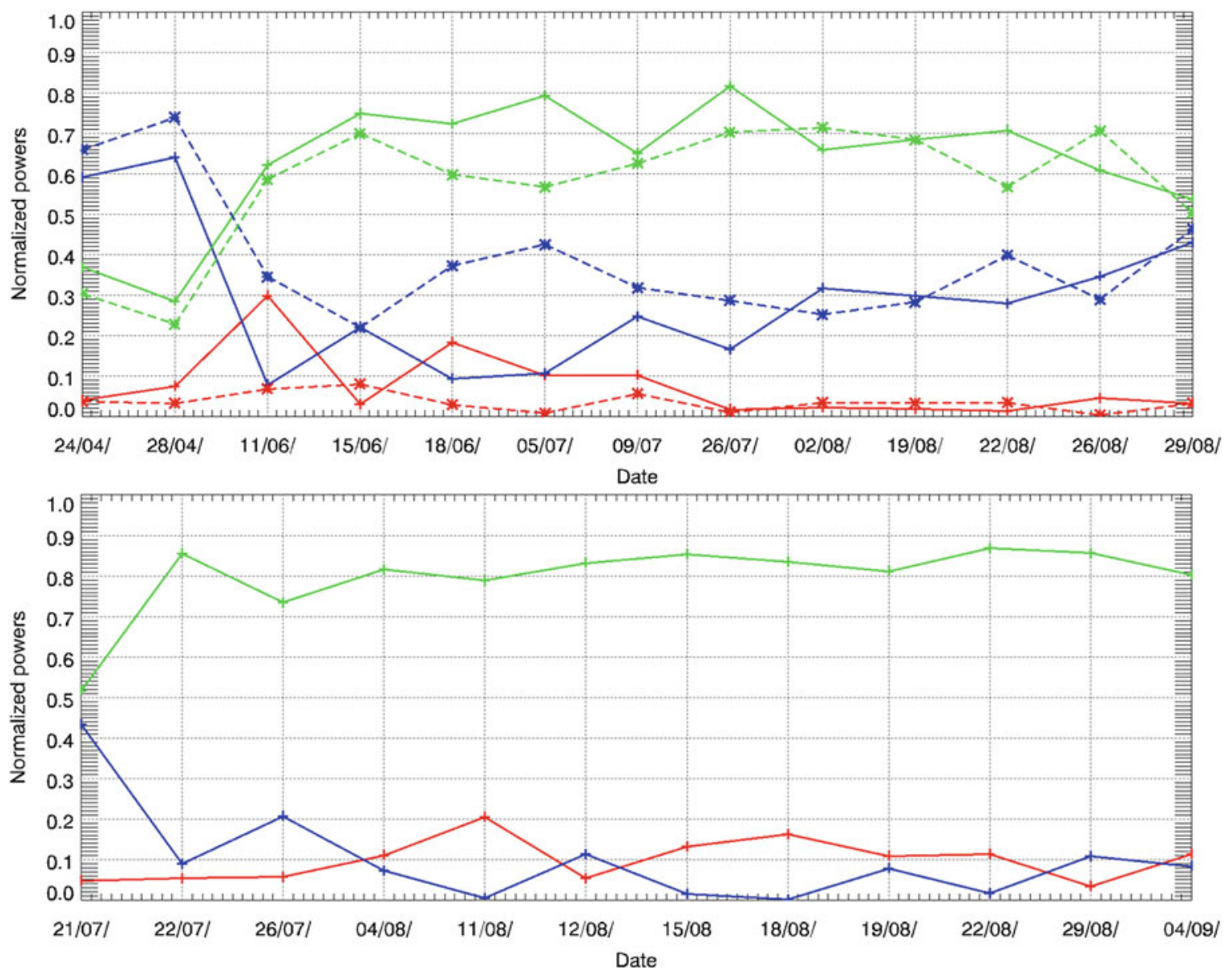

Fig. 3.8 Normalised decomposed scattering power components (red, dihedral; green, volume; blue, surface) from the model-based polarimetric decomposition for the Flevoland test sites (site 1, Plus sign; site 2 , Star sign from the beginning of the observation cycle at 11th of June

Flevoland and for 9 dates from 4th of August until 4th of September 2009 at Indian Head. A clear underestimation has to be stated at Flevoland for all dates of the observation cycle. In contrary, a distinct overestimation is reported by the comparison with in situ measurements at Indian Head. The estimated soil moisture values for the two measurement locations at Flevoland are underestimating the measured soil moisture values by a RMSE of 13.32 vol. $\%\left(r^{2}=0.12\right)$, whereas the comparison with the field measurements for Indian Head overestimates with a RMSE of 22.84vol.\% $\left(r^{2}=0.02\right)$ including all analysed dates.

In the past, the soil moisture retrieval method was developed and implemented for L-band data (see Jagdhuber et al. 2012b, 2013a, b), and with the transfer to the C-band scattering scenario, the algorithm needs further adaptions especially concerning the ground scattering models. First analyses on the surface component inversion blame the low moisture values at Flevoland on the low $\mathrm{T}_{12}$ correlation term, which
2009 until the end of the observation cycle at 29th of August 2009) and for the Indian Head test site (site 3, Plus sign) (R, even bounce/dihedral scatterers; G, volume scatterers; B, odd bounce/surface scatterers)

leads to small $\beta_{S}$ values and the underestimation of the soil moisture level.

In addition, the measured soil moisture of the two in situ stations at Flevoland ranges between $24 \mathrm{vol} \%$ and $42 \mathrm{vol} \%$. This represents a significantly high soil moisture level, which is an additional challenge for the inversion algorithm, because the sensitivity of the inversion models decreases with increasing soil moisture level (Jagdhuber 2012).

For Indian Head, the distinct overestimation of the soil moisture might be traced back to a very challenging scattering scenario of a large canola vegetation cover $(>100 \mathrm{~cm})$ and a very dry soil underneath, which might be hardly detectable at C-band (wavelength: $5 \mathrm{~cm}$ ).

Moreover, it has to be stated that the validation could only be conducted over time (continuously recording moisture stations) and not over space (no soil moisture network or spatial sampling), since only three measurement locations were available for Flevoland and Indian Head in 2009. 

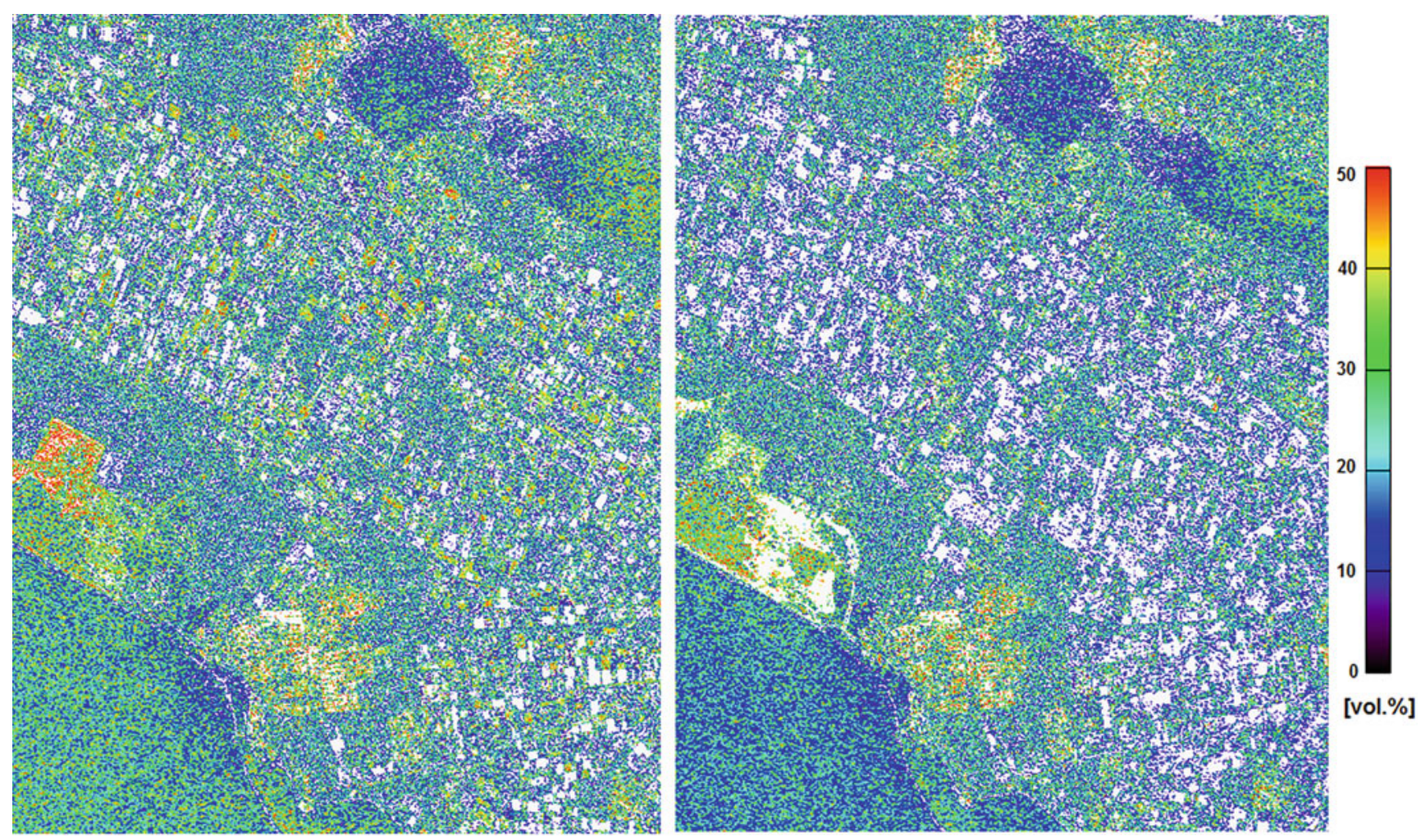

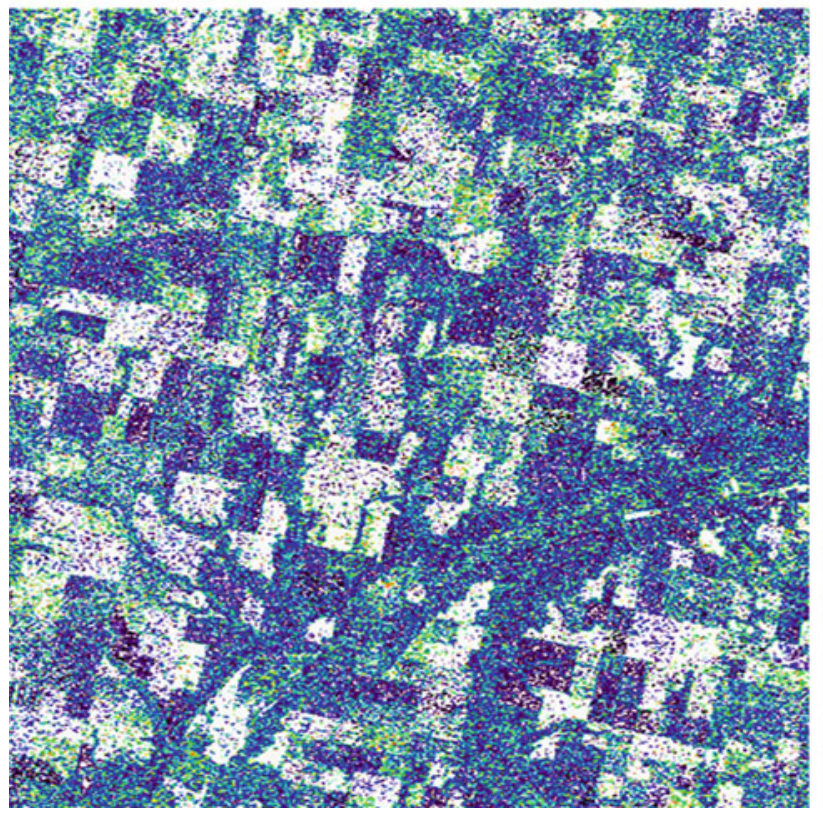

Fig. 3.9 Inverted soil moisture [vol.\%] from both ground scattering components (surface, dihedral) for the Flevoland test site (at the beginning of the observation cycle at 11th of June 2009 (Top left) and the end of the observation cycle at 29th of August 2009 (Top right)) and for the

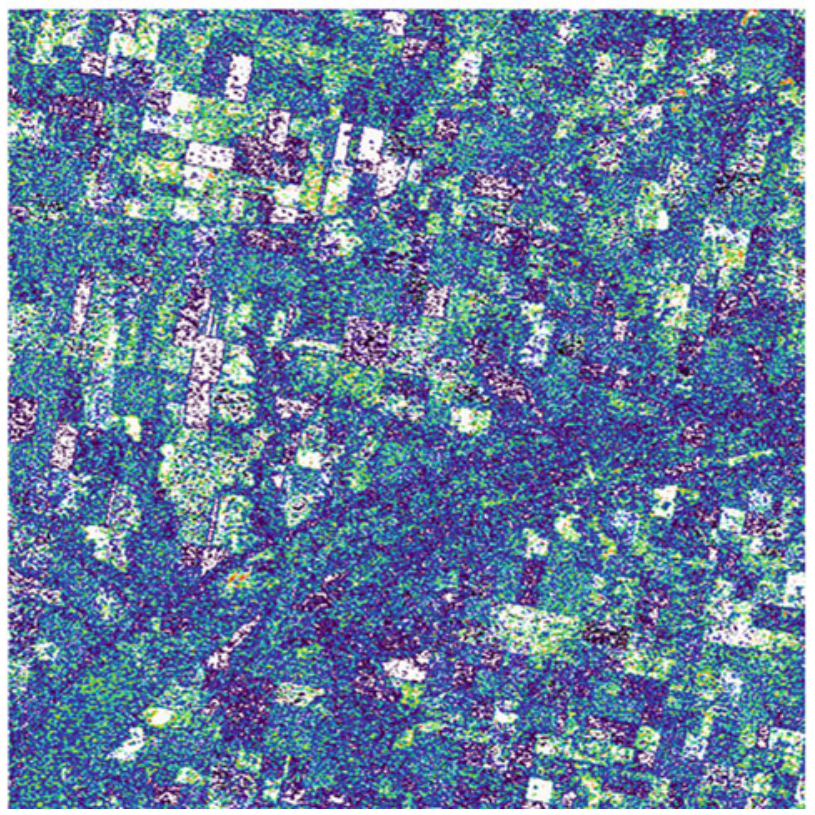

Indian Head test site (on the 4th of August (Bottom left) and the 4th of September (Bottom right) 2009); non-invertible areas are masked white; image smooth: $4 \times 4$ 


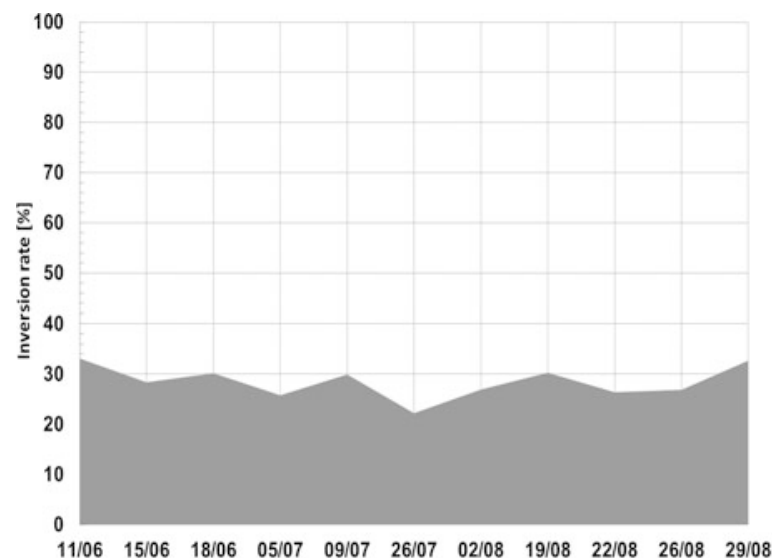

(a)

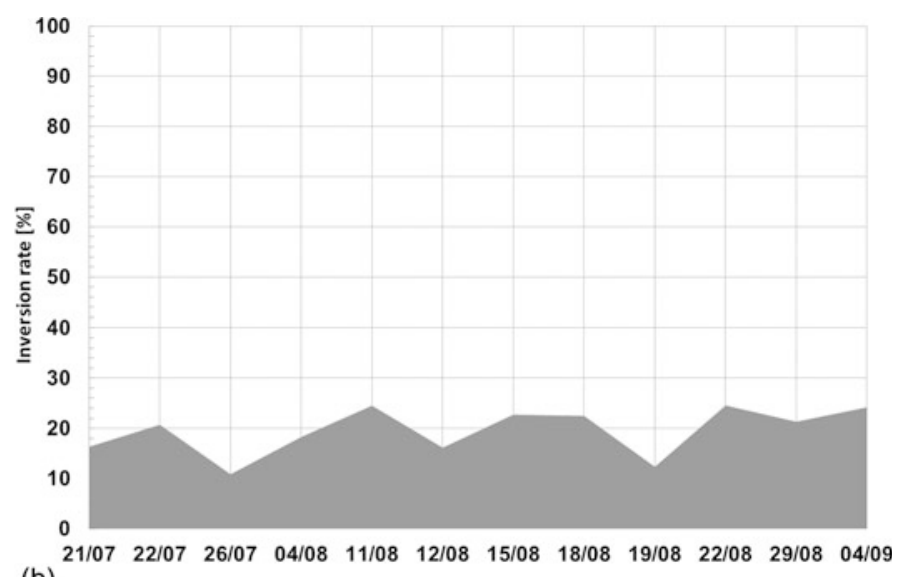

(b)

Fig. 3.10 Inversion rate [\%] for soil moisture retrieval of both ground scattering components (surface, dihedral) at Flevoland (left) from the beginning of the observation cycle at 11th of June 2009 until the end of

the observation cycle at 29th of August 2009 and at Indian Head (right) from 21st of July until 4th of September 2009

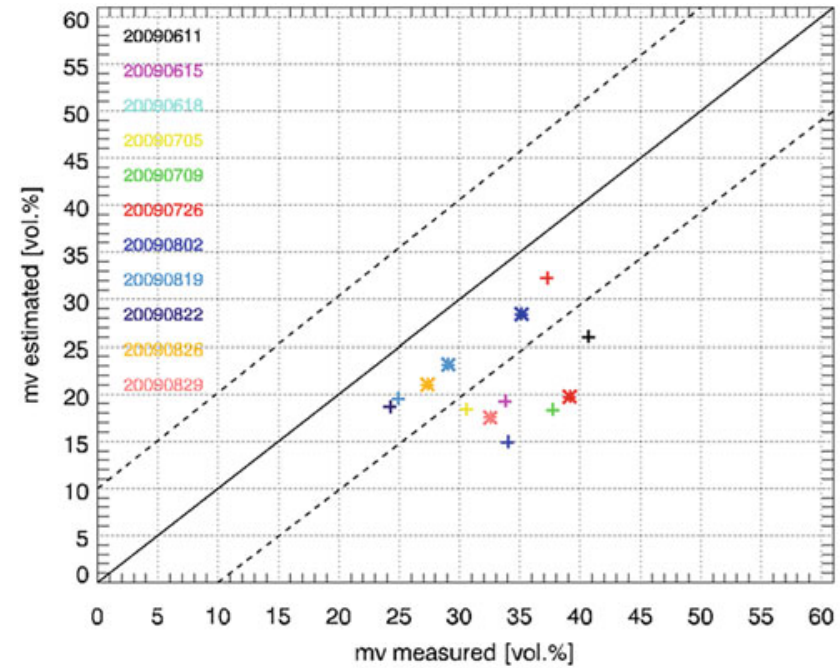

Fig. 3.11 Validation of inverted soil moisture [vol.\%] from ground scattering components (surface, dihedral) with in situ measurements of soil moisture at the two continuously recording moisture stations (site 1, Plus sign; site 2, Star sign) at Flevoland (left) for 11 different dates

\subsubsection{Comparison with Single-/Dual-Pol Data}

The developed algorithm is directly and solely based on the fully polarimetric observation space and therefore directly affected by the reduction to single- or dual-polarimetric data. This means that only in the case of fully polarimetric data, the vegetation- and/or soil roughness-induced depolarisation can be assessed, quantified and removed in the best way. A decomposition, separating the vegetation

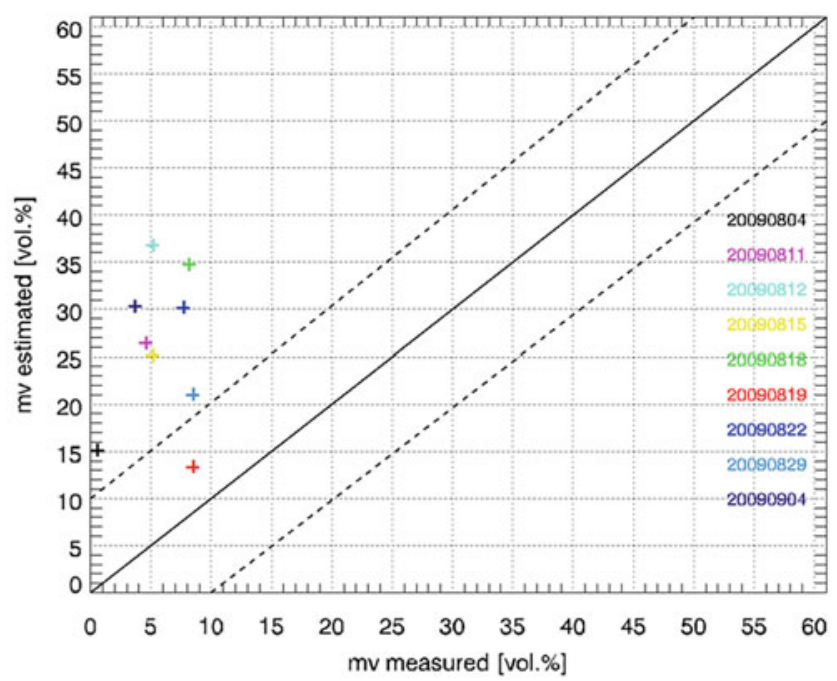

from 11th of June until the 29th of August 2009 (colours) and at Indian head (right) for 12 dates from 4th of August until 4th of September 2009; validation box: $7 \times 7$ pixels

volume and the soil ground scattering components, is not/only partly feasible with single-/dual-polarimetric approaches.

Hence, there is a trade-off between decomposing fully polarimetric data and then solving a lower parameterised inversion problem or using single-/dual-polarimetric data and modelling the whole backscatter signal by solving an ambiguous, highly parameterised inversion problem. 

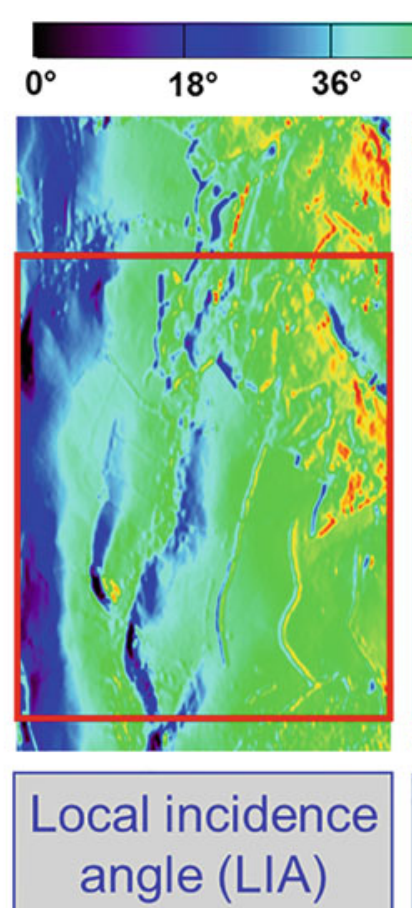

master

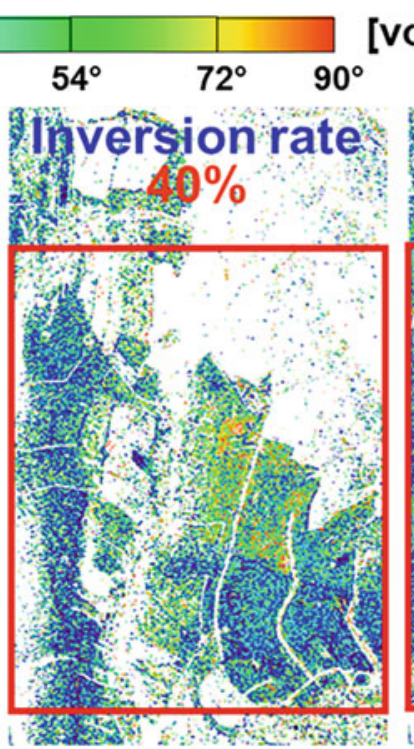

Single angular

1 acquisition

master $(\mathrm{m})$

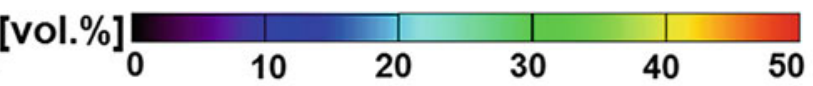
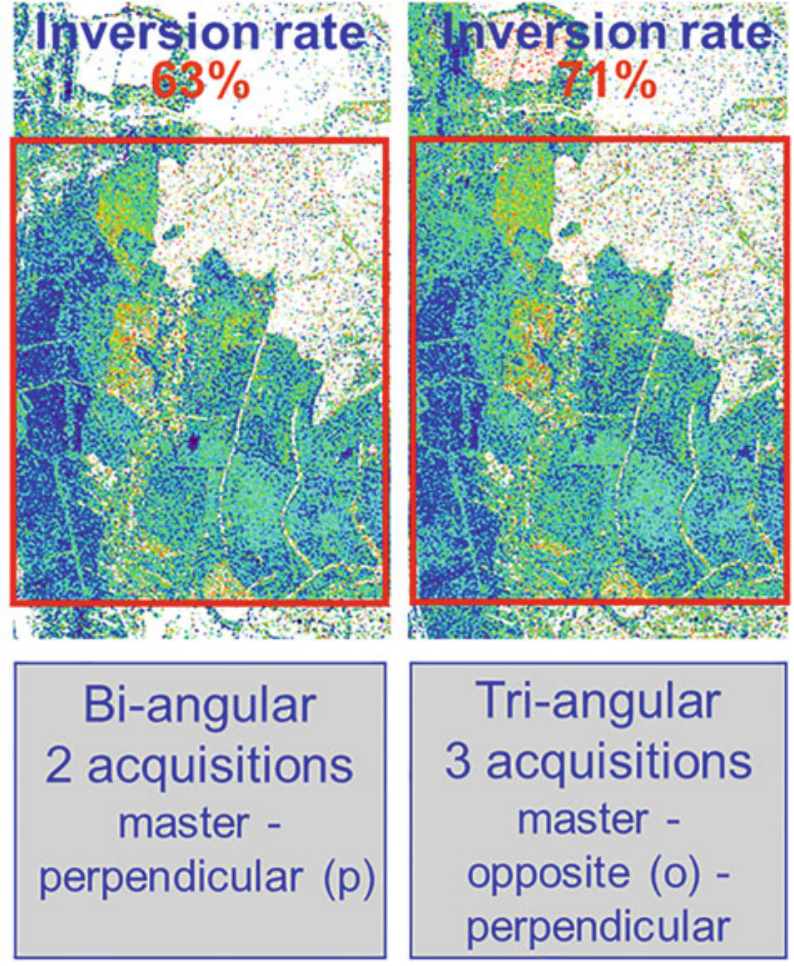

Tri-angular 3 acquisitions master opposite (o) perpendicular

\section{Tri-angular: Master-opposite-perpendicular}
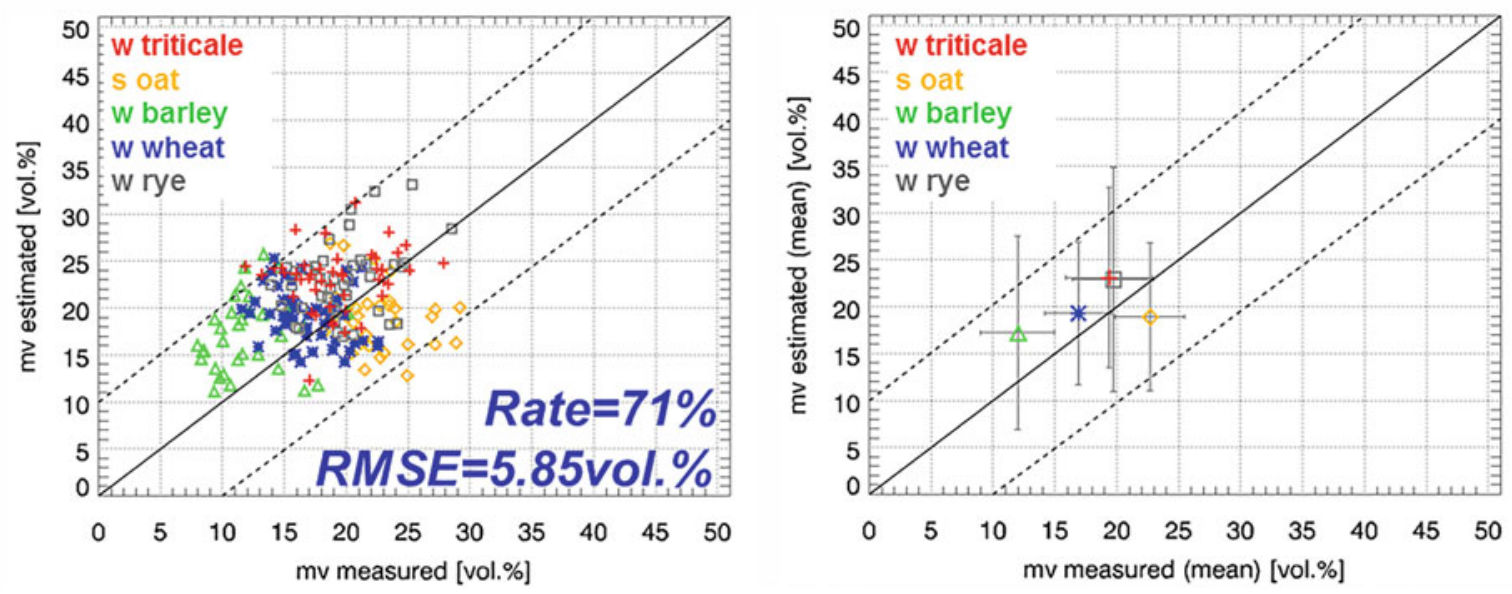

Fig. 3.12 Multi-angular polarimetric decomposition for soil moisture retrieval under vegetation cover at L-band applied to E-SAR data of the OPAQUE 2008 campaign: (Top) Local incidence angle and soil moisture inversion results for a single-, bi- and tri-angular model-based decomposition and inversion are depicted. The inversion rate constantly

increases, when moving to a multi-angular decomposition and inversion. (Bottom) Validation of the SAR-based moisture estimates with in situ measurements from different crop types for each single measurement location (left) and for the mean of field values (right) (Jagdhuber et al. 2013a) 

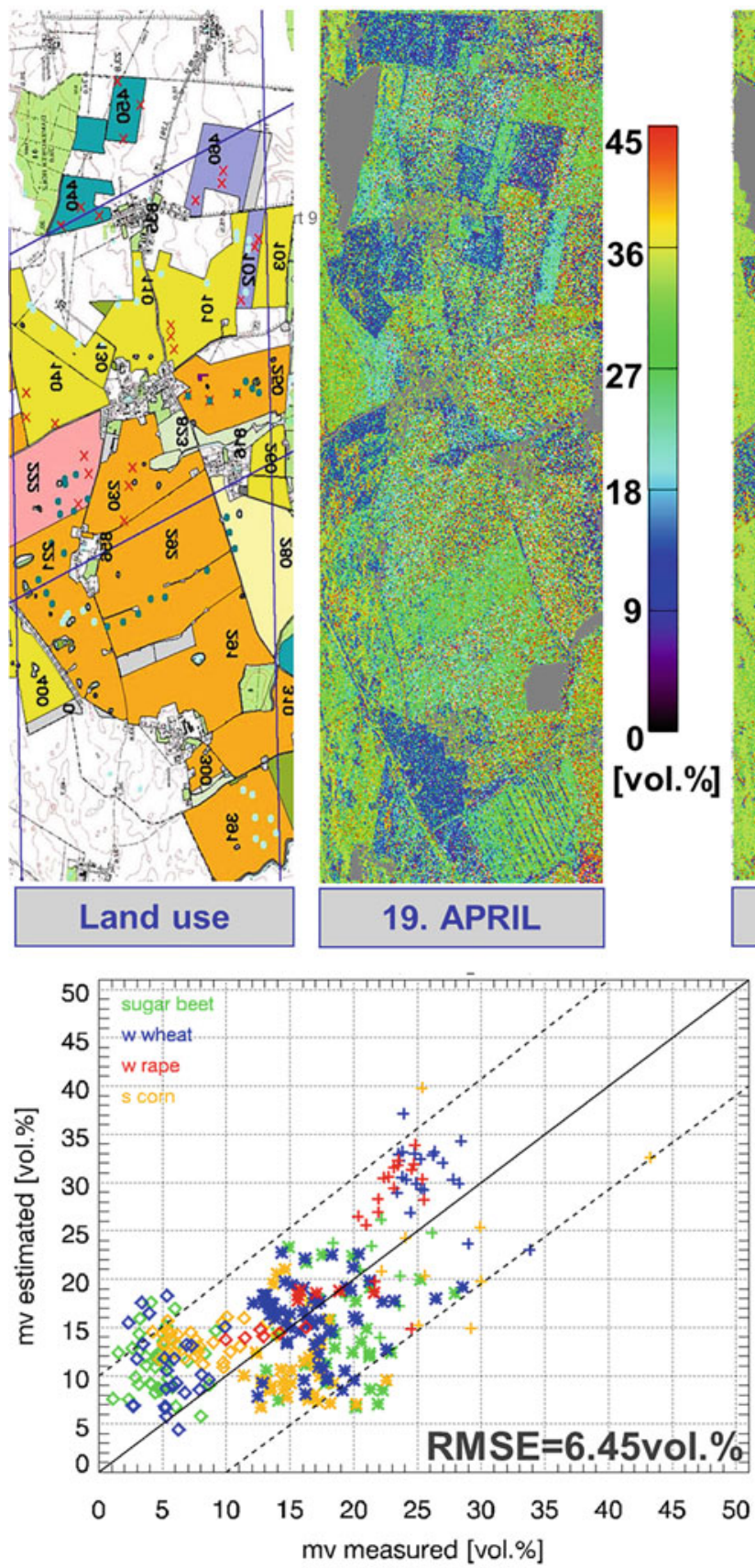

Fig. 3.13 Hybrid polarimetric decomposition for soil moisture retrieval under vegetation cover at L-band applied to E-SAR data of the AgriSAR 2006 campaign: (Top) Land use and soil moisture inversion results for a time series covering the entire vegetation cycle (April-July 2006) are depicted. Urban and forested areas are masked grey. (Bottom)

\subsubsection{Discussion on the Role of Polarimetry, on the Maturity of the Application and Conclusion}

Polarimetry and the acquisition of fully polarimetric SAR data are essential for the retrieval of soil moisture under vegetation. Only the polarimetric observation space with its
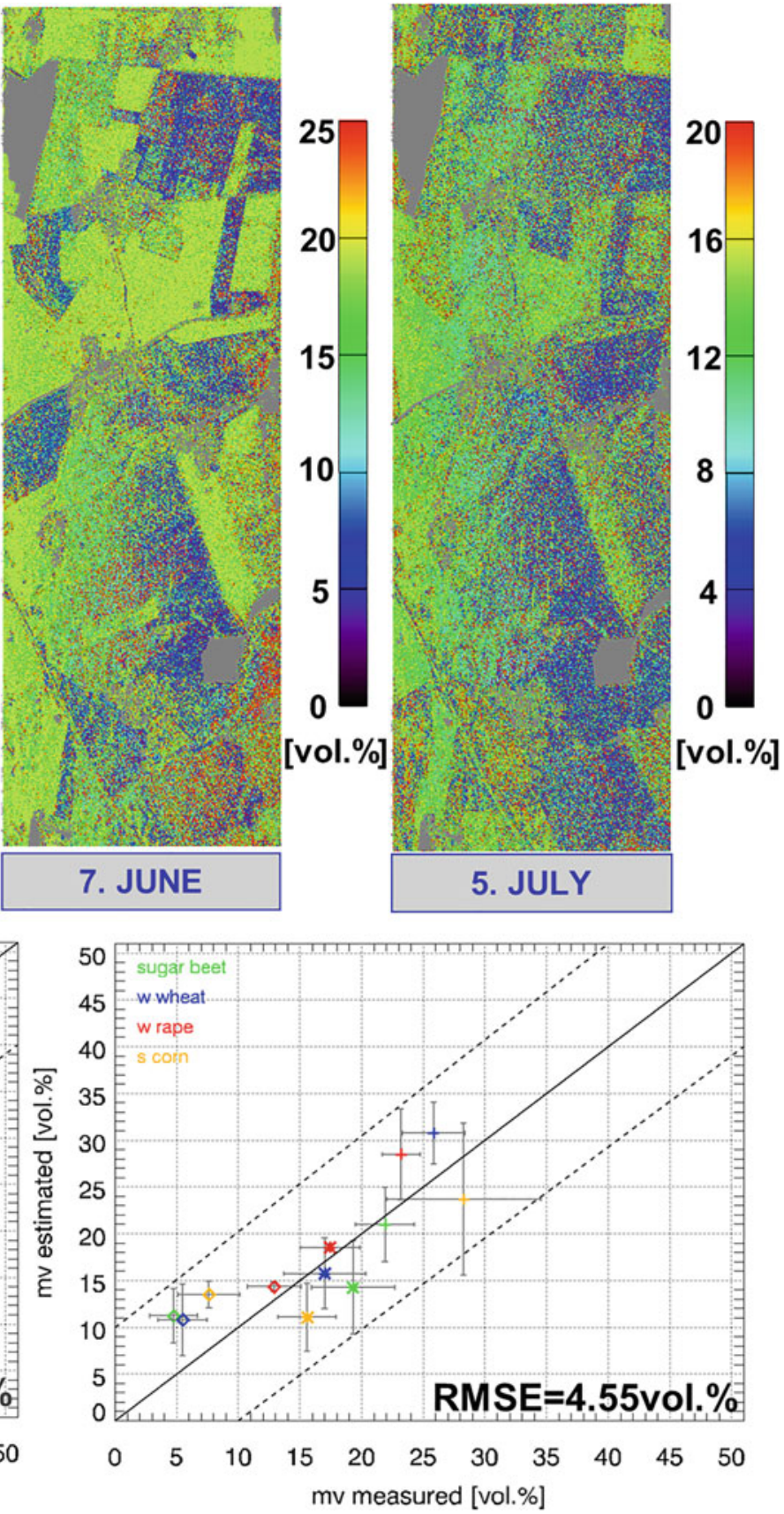

Validation of the SAR-based moisture estimates with in situ measurements from different crop types for each single measurement locations (left) and for the mean of field values (right). Symbology: Diamond, 5th of July; Stars, 7th of June; Plus, 19th of April) (Jagdhuber et al. 2013b, 2014)

capability of polarimetric decompositions provides and reveals the different properties (dielectric content, object geometry) of the scatterers on ground in the most complete way compared to backscatter intensity analyses or interferometric techniques. Therefore SAR polarimetry plays an essential role in this application. 
In the last years, the application of soil moisture retrieval under vegetation advanced to a distinct level of maturity by using longer wavelength SAR (L-band) (Jagdhuber et al. 2012b, 2013a, b). Compared to C-band, L-band provides a deeper penetration into the vegetation canopy together with a convenient signal-to-noise ratio (SNR) for reliable polarimetric signal analyses (Jagdhuber 2012). Figures 3.12 and 3.13 show exemplarily two novel and innovative types of polarimetric decompositions for soil moisture inversion under vegetation at longer wavelength (L-band): a multi-angular, model-based decomposition and a hybrid (combined model-/eigen-based) decomposition. The multi-angular decomposition in Fig. 3.12 leads to the increase in inversion rate from $40 \%$ to $71 \%$, when moving from a single-angular to a tri-angular model-based decomposition, which also reflects in the quality of the inversion with an RMSE of 5.85vol.\% in contrast to the C-band results (in Fig. 3.11) (Jagdhuber et al. 2013a). In comparison to all presented inversion approaches, Fig. 3.12 reveals the best inversion and validation results of the novel hybrid decomposition technique using a modelbased decomposition for the volume removal and an eigenbased decomposition to retrieve the single ground scattering components for soil moisture inversion under vegetation cover for an entire vegetation growth period sensed during the AgriSAR 2006 campaign (Jagdhuber et al. 2013b). The inversion rate is always higher than $95 \%$ including the entire vegetation cycle. Moreover, the RMSE for all acquisition dates (April-July 2006) lies in the favourable range of $4,6 \mathrm{vol} . \%$ (mean of field values) to $6.5 \mathrm{vol} . \%$ (single scattering locations) for ecosystem applications.

Furthermore, first attempts were also made for retrieving soil moisture under boreal forest using P-band in order to enlarge the moisture retrieval to forested environments (Jagdhuber et al. 2012a). In addition, also X-band fully polarimetric data of TerraSAR-X are currently investigated for soil moisture retrieval on bare and sparsely vegetated soils for shorter wavelength SAR (Jagdhuber et al. 2013c, d). In this way, the fully polarimetric soil moisture estimation using decomposition techniques is approached covering the frequency range from $\mathrm{X}$ - to $\mathrm{P}$-band considering the wavelength-dependent sensitivity for vegetation cover and the soil below.

\subsection{Crop Phenology Estimation Using SAR Polarimetry}

\subsubsection{Introduction, Motivation and Literature Review}

All agricultural crops present a continuous development, from sowing or transplanting to harvest, in which they grow and evolve in accordance with their biophysical characteristics and the farming practices applied to them. Phenology denotes such a succession of stages during the cultivation cycle and is commonly expressed using numerical scales (Meier 2001).

Tracking phenology of agricultural fields by remote sensing is useful for farmers with extensive fields because it provides key information for planning and triggering cultivation practices, so the main application of this EO product is precision farming. These cultivation practices (e.g. irrigation, fertilisation, effective germination counting, harvest, etc.) require timely inputs about the status of the plants and, specifically, about their condition or situation along the expected cultivation cycle.

Besides precision farming, timely information of phenology can contribute to agencies and institutions involved in market predictions, insurance policies, subsidies claims, etc. since such information complements their own data sources and provides a temporal schedule for the crop production and yield calendar.

Most of the applications require phenology information at parcel (field) level, but in some cases it may be necessary to provide values at pixel (sub-parcel) level, especially when dealing with detection of heterogeneities produced by cultivation problems (e.g. water salinity used of irrigation) and plant diseases (e.g. pests and plagues). Unfortunately phenology monitoring by satellite remote sensing has not attracted much attention in the past due to both the lack of time series of images and the cost of the ground campaigns. Nevertheless, this situation has changed in recent times, thanks to the launch of satellites with shorter revisit times (e.g. 11 days for TerraSAR-X) and reconfigurable acquisitions (different beams can be operated for more frequent observation of a particular area). Importantly for us these are also available in multiple polarisation channels, which prompted this research into phenology retrieval using polarimetric imaging radar (PolSAR).

Algorithms for phenology monitoring have to be devised individually for each crop type, based on the expected response of each crop to the sensor at its different stages, which can be extracted from the data themselves (training sets) or from previous experiments, models and simulations. In addition, approaches based on the availability of time series of images can improve importantly the performance of these algorithms, since phenology is intrinsically related to time.

The starting point of this application is the knowledge of which crop type or plant species is cultivated at the monitored fields, which is provided by the users or can be obtained from a crop type map. Then, the general objective of this product consists in estimating the current phenological stage of the plants in the parcel by exploiting a single PolSAR acquisition.

The topic of vegetation phenology monitoring by means of remote sensing has been mostly addressed in the past by 
analysing temporal variations of vegetation indices acquired by optical sensors. These vegetation indices are formed by combining different spectral bands which exhibit different sensitivities to biochemical plant constituents and canopy biophysical parameters. These indices are then used as a proxy for monitoring the plant growth stage by means of empirical relationships with structural parameters. Among all of them, the most important one is LAI (leaf area index) which plays a key role in the design of crop models for crop condition assessment and final yield prediction (Bach and Mauser 2003).

In addition to different airborne sensors operating worldwide (CASI, ROSIS, DAIS-7915), there are several operative satellite missions which have been providing multispectral data for large-scale Earth dynamics studies. Some of these instruments are the MODerate-resolution Imaging Spectroradiometer (MODIS) by NASA, the Advanced Very High Resolution Radiometer (AVHRR) by NOAA and the VEGETATION sensor on board SPOT satellite by CNES. In general, the main purpose of all these projects has been primarily focused on crop yield prediction and crop mapping/classification, being these tasks directly related to the estimation of phenology (Nellis et al. 2009).

Early studies by Badhwar and Henderson in 1981 (Badhwar and Henderson 1981) made use of Landsat Multispectral Scanners (MSS) in order to design a crop model able to determine development stage of corn and soybean. Further improvement was proposed by Tilton and Hollinger in 1982 (Tilton and Hollinger 1982) when they proposed an algorithm to predict the development stages early in the growing season by means of a principal component analysis of Landsat images. In 1990, Lloyd exploited the approximately linear relationship between solar energy absorption of plants and NDVI measurements acquired by NOAA-AVHRR in order to describe phenological events (Lloyd 1990). Likewise, in 1994 Reed et al. (1994) analysed time series of NOAA-AVHRR acquisitions as well, and they found a high correlation between NDVI temporal trends and phenological variations in case of spring wheat at a continental scale.

The work by Tucker in 1979 (Tucker 1979) is considered as one of the first contributions that proposed the systematic use of NDVI for characterising vegetation. Since then, NDVI has been the most widely used spectral index for vegetation monitoring. It combines the reflectance values of plants in red (low reflectance) and near-infrared (high reflectance) bands. In general, temporal variations of NDVI exhibit a high correlation with the main growth stages of plants, i.e. vegetative, reproductive, maturity and senescence stages.

The combination of other spectral bands has led to the development of refined optical indices that could yield different results depending upon the type and conditions of vegetation. Studies conducted in (Haboudane et al. 2004) demonstrated that LAI values for soybean, corn and wheat can be consistently estimated from vegetation indices at different phenological stages.

Further improvements have been proposed, such as the enhanced vegetation index (EVI) which was designed in order to increase the sensitivity to vegetation changes by considering the canopy background contributions as well as the atmosphere influences (Huete et al. 2006).

Contrary to the valuable and long experience acquired in hyperspectral systems, the use of active microwave remote sensing for agriculture monitoring activities is a relatively new topic (Lopez-Sanchez and Ballester-Berman 2009), also due to the scarcity of well-established and long-term research programmes developing both air- and space-borne missions capable of providing the required radar data sets. Fortunately, this situation has entirely changed in recent years after the initiation of a large number of radar remote sensing campaigns promoted by different aerospace agencies and institutions around the world such as ESA, CSA, NASA, DLR and JAXA.

After some pioneer works on the potential of radar for characterising natural targets (see, e.g. Ulaby et al. 1987), the interest on the use of active microwave sensors was greatly stimulated during the 1990s (Henderson and Lewis 1998) as a consequence of the launch of three satellites operating at C-band, i.e. ERS, ENVISAT-ASAR and RADARSAT-1.

Among other applications, vegetation phenology monitoring has been also tackled, but the literature on this topic is still very scarce. First contributions demonstrating the relationship between radar backscatter and growth stages of rice were presented by Le Toan et al. (1989), Kurosu et al. (1995) and Le Toan et al. (1997) in 1989, 1995 and 1997, respectively. Other follow-up works to this topic were also based on the analysis of incoherent radar measurements and can be found in (Inoue et al. 2002; Chen and McNairn 2006; Koay et al. 2007; Bouvet et al. 2009). These works have shown the potential of $\mathrm{C}$-band $\mathrm{HH} / \mathrm{VV}$ ratio for rice field discrimination and that a clear intensity signature appears as a consequence of phenological development of rice plants. More recently in (Lopez-Sanchez et al. 2012a), an algorithm to estimate the phenological stages throughout the whole growing season was designed on the basis of a coherent approach. However, the phenology retrieval issue has not been explicitly studied for other crops in the literature. Indeed, the only clear example we have found on this topic is the work by Wegmüller and Werner in 1997 (Wegmüller and Werner 1997) where they described the sensitivity of interferometric coherence to the fraction of vegetation cover and the potential for tracking phenological events for corn, sugar beets, potatoes and rapeseed.

Alternatively, the interest has been focused on estimating parameters related to crop growth, such as biomass and LAI (which in turn are intrinsically related to each other), as well as crop condition and type assessment. Early contributions by 
Brown et al. (1992) and Bouman and Hoekman (1993) made use of different frequency bands for separating crop types. Also the estimation of biomass from olive groves, sunflower, corn, sorghum, rape, wheat and alfalfa was studied in (Ferrazzoli et al. 1997). Estimation of LAI with saturation effect at $2-3 \mathrm{~m}^{2} / \mathrm{m}^{2}$ has been also reported in (Ferrazzoli et al. 1992) by means of radar backscatter at C-band. The use of time series of backscattering coefficients, together with electromagnetic models and neural networks, for such a purpose has been also addressed in the literature (Del Frate et al. 2004). Backscattering coefficients at different polarimetric channels were also correlated with phenology and cultivation practices (e.g. irrigation) for several crop types in (Moran et al. 2012).

As an alternative way to retrieve information on the phenology of crops, other works have been focused on crop height estimation, given that plant height could be used as a proxy for phenology at least for certain growth stages (mainly during the vegetative phase). The applied retrieval strategy has evolved from the design of empirical relationship between coherence and height as shown in (Engdahl and Borgeaud 1998; Engdahl et al. 2001) for sugar beet, potato and winter wheat to more elaborated and robust approaches consisting of electromagnetic modelling of PolInSAR observables (Treuhaft et al. 1996; Treuhaft and Siqueira 2000; Cloude and Papathanassiou 2003) which have yielded successful results in crops such as maize and rice in laboratory conditions (Ballester-Berman et al. 2005) and maize and winter rape in the framework of an airborne campaign (Lopez-Sanchez et al. 2012b).

In summary, it can be concluded that the topic of crop phenology is an incipient research field which recently has been stimulated by the potential positive socio-economic impact that remote sensing tools offer in terms of monitoring and management tasks on crop fields at large scale.

\subsubsection{Methodology}

As a matter of fact, the problem of identifying the phenological stage of an agricultural crop can be regarded as a classification problem, where each stage corresponds to a class, and hence can be approached in a similar way to crop type mapping. This application is better suited to algorithms based on hierarchical trees or simple decision planes, since they can be tailored to match specifically the different features of the plants that change or appear as they develop. This type of rule-based algorithms facilitates the physical interpretation of the phenology retrieval procedure, since the criteria are based on scattering mechanisms (e.g. surface, dihedral, volume) and properties (e.g. extinction, depolarisation, etc.) in correspondence to the crop structure and features at each stage and in contrast with other algorithms based on the full covariance statistics (e.g. Wishart classifier). Hierarchical tree algorithms have been widely used for classification purposes in the literature and specifically for crop type mapping with PolSAR data, so they can be considered as mature since they provide consistent accuracies above $85 \%$ in crop type mapping. Therefore, hierarchical tree algorithms will be implemented and tested for this product.

The starting point is the multi-look processing or filtering of the available PolSAR images, providing the covariance or coherency matrix for each multi-looked pixel. A sliding boxcar filter has been employed since the monitored parcels are wide enough and homogeneous for applying such a filtering type. Then, all images have been geocoded. If all images were acquired with the same beam and pass, a coregistration of the whole set to a common master image could be used instead of geocoding. Once geocoded, all available PolSAR images are studied for each crop type by restricting the region of interest (ROI) to the interior of all fields of each crop. In all cases, an analysis of a large number of polarimetric observables is carried out in order to extract the most meaningful set for the crop under study. The available observables are backscatter powers and correlations (linear, Pauli and circular basis and for compact polarimetry as well), backscattering ratios for different channels and for various polarisation bases, eigenvector/eigenvalue decomposition parameters, compact polarimetry decomposition parameters and the Freeman-Durden and Touzi decomposition outputs.

This analysis is based on the representation of the observables as a function of phenology, so the reference data recorded at each acquisition date are used to define the $\mathrm{x}$-axis of their representation. For each observable, the mean and standard deviation within the parcel at every radar acquisition are obtained and plotted.

From the analysis of the evolution of all observables for each crop type, a reduced set of them will be selected for the retrieval algorithm by identifying the ones that define with more distinction particular phenological stages. Specifically, those with wider dynamic ranges and less presence of ambiguities will be chosen. Moreover, observables with easy physical interpretations will be preferred to those with less clear explanations in terms of scattering physics. With the selected set of observables, a hierarchical tree is defined by setting manually thresholds based on the previous analysis.

An important question to address for the definition of the final product concerns the required spatial resolution since phenology can be provided either at pixel level (one value per multi-looked pixel) or at parcel level (one value per parcel). In the first case, we could detect areas with different degrees of development within the same parcel, hence being also useful for localised farming practices such as irrigation and fertilisation. In the second case, we would be interested in the 
Table 3.5 Test sites and corresponding radar and validation data selected for the generation of showcases on crop phenology estimation under vegetation

\begin{tabular}{l|l|l}
\hline Application/product & Test site - radar data & Reference data \\
\hline $\begin{array}{l}\text { Crop phenology } \\
\text { estimation }\end{array}$ & Indian Head & $\begin{array}{l}\text { Intensive campaign of } \\
\text { AgriSAR2009 }\end{array}$ \\
\cline { 2 - 3 } & $\begin{array}{l}57 \text { quad-pol RADARSAT-2 images, from which 20 are used in this } \\
\text { showcase }\end{array}$ & \\
\hline
\end{tabular}

global development of the crop field, which would be considered as homogeneous.

According to the available reference data (see Appendix), we know the phenology at several ground points of each field, but (1) they are mostly coincident for the same parcel; and (2) available values are maximum and minimum values of phenological stages, instead of single values. Therefore, we have considered in the analysis that phenological data at each field and each date are the same for the whole field, being the mean the value adopted.

The inversion algorithm however has been applied at both pixel and parcel levels. This option increases the usefulness of the product for potential end-users since retrieved information is provided at different scales, i.e. at pixel level any possible heterogeneity within a field can be detected and, in addition, at parcel level an overall conclusion on the status of the field is obtained. For the estimation at parcel level, it will be computed as the mode of the estimates within the parcel in accordance with the available reference data.

\subsubsection{Experimental Results}

Test sites and corresponding radar and validation data sets selected for crop phenology estimation are summarised in Table 3.5 and further described in the Appendix.

\subsubsection{Analysis}

First we describe and justify the evolution as a function of phenology of different parameters. To this end, all parameters presenting similar evolutions are grouped. In general, from the five crop types analysed (barley, oat, wheat, field peas and canola), we have found only three main signatures, since all three cereals (barley, oat and wheat) behave similarly.

Although in principle we expected clear differences in the radar responses as a function of incidence angle (there are images acquired with angles from $22^{\circ}$ to $39^{\circ}$ ), such differences are only evident in some parameters and especially for certain crop conditions (e.g. during the vegetative phase in cereals, since ground dominates the radar response). Consequently, a joint analysis of all angles has been carried out. In some extreme situations, like two images acquired on consecutive days with $22^{\circ}$ and $39^{\circ}$, a discontinuity is expected, so this will be commented when necessary.

Finally, and according to the discussion in the previous sections, we have computed the evolutions of all parameters at pixel level after a $9 \times 9$ multi-look. The plots of the evolutions show the average and the standard deviation computed over the whole field.

In this report, we will focus our analysis on the results on cereals (which benefit most strongly from polarimetry), and some additional short comments will be given on canola and pea fields. Only observables with some trends or sensitivity to phenology will be commented for each crop type.

Sample results are shown in Fig. 3.14.

\subsection{Cereals}

Parameters with high sensitivity:

- Linear cross-polar backscatter $\left(S_{h v}\right)$ presents an increase at both the early stages (6-10 dB from stages 10 to 25-30) and the late ones (4-5 $\mathrm{dB}$ from stage 75$)^{*}$, being quite constant in the middle. Similar parameters are $S_{h h}-S_{v v}, S_{r r}$ and $S_{l l}$ backscatter and $P_{v}$ of Freeman decomposition. * Note that the increase in late stages is not present for oat (but at one single acquisition at $22^{\circ}$ ).

- HHVV correlation decreases clearly during the vegetative phase (stages 10-50) and then remains around 0.4 with important differences between acquisitions. Similar parameters:

- Average alpha increases from $0^{\circ}$ to $45^{\circ}$ only during the vegetative phase.

- $S_{r l} / S_{r r}$ and $S_{l r} / S_{l l}$ ratios show a decrease of $10 \mathrm{~dB}$ during the vegetative phase.

- Correlations RRRL and LLRL behave similarly, especially for wheat.

- Entropy shows a sudden increase from 0.2 at stage 10 to $0.7-0.8$ at stages $20-30$, and then it remains around 0.8 all the time.

- Dominant alpha $\left(\alpha_{1}\right)$ increases monotonically during the whole cycle, but it is slightly saturated after the vegetative phase (see Fig. 1.1).

- $\tau$ of Touzi decomposition is always close to zero, so the corresponding dominant alpha is like $\alpha_{1}$ from the conventional eigen-analysis.

\subsection{Canola}

The most remarkable result in this case is that the cross-polar backscatter follows a clear monotonic increasing trend for the whole growth period. This enables the phenology estimation in a straightforward way by using one single channel, HV. Indeed, coherent polarimetry does not contribute much to this crop type. 

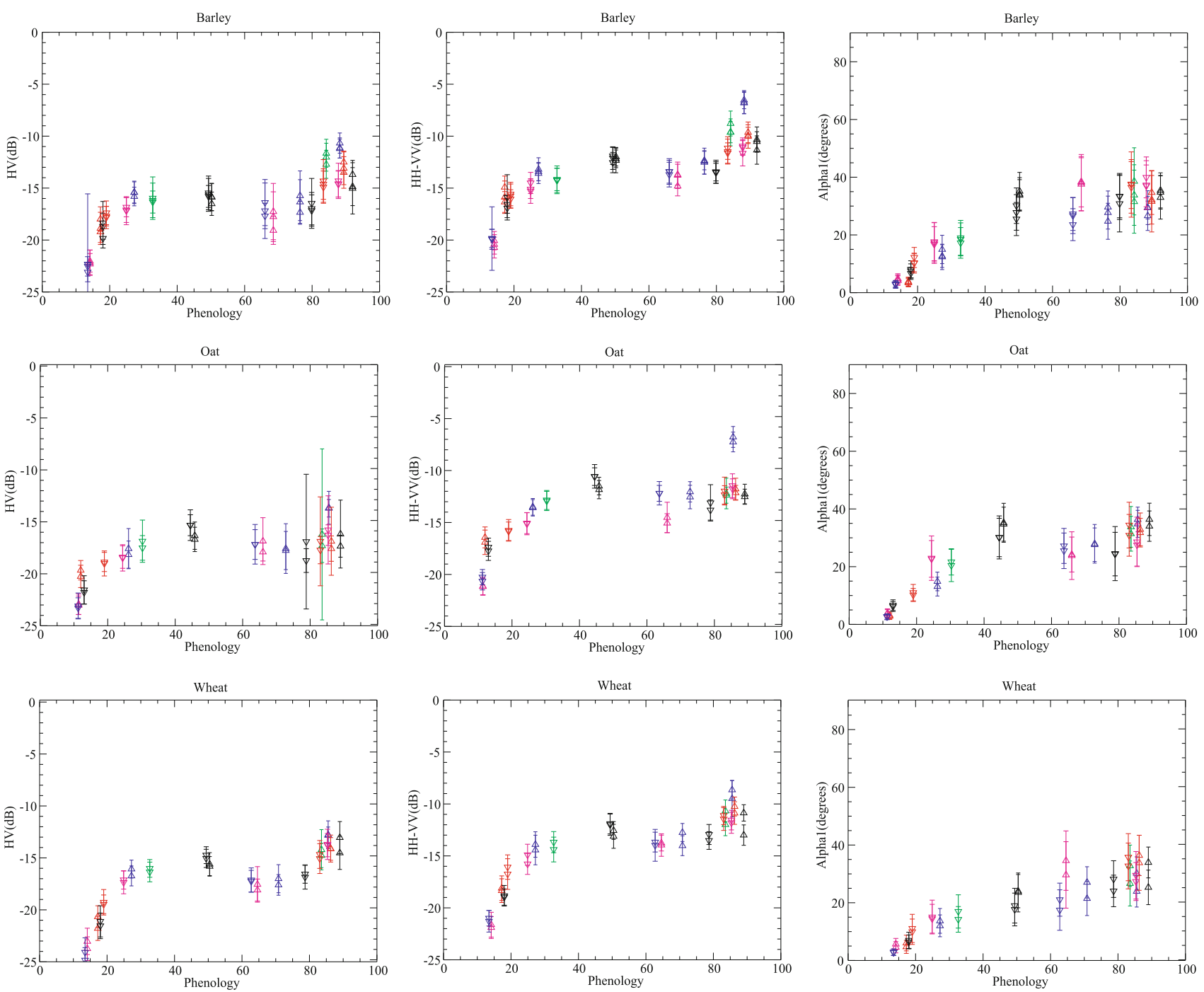

Fig. 3.14 Evolution of $\mathrm{HV}\left(t_{33}\right), \mathrm{HH}-\mathrm{VV}\left(t_{22}\right)$ and dominant alpha $\left(\alpha_{1}\right)$ as a function of phenology for barley (top row), oat (middle row) and wheat (bottom row)

\subsection{Field Pea}

In general we found that many observables here are symmetrical with respect to stages 20-25, hence making it difficult to break the ambiguity between early and late stages without any auxiliary information (e.g. time coordinate). Plants are always very short, so there is not much development or clear changes in terms of structure.

\subsubsection{Retrieval Algorithms}

In the following, details on the retrieval algorithm for cereals are given. After a close inspection of Fig. 3.14, a feasible algorithm could be designed to distinguish four different phenological intervals, i.e. early vegetative (stages 0-19), advanced vegetative (stages 20-39 or 20-44), late vegetative, reproductive and early maturation (stages from 40 or 45 to 79) and, finally, maturation (stages 80+).
The physical description of each interval is the following:

1. Surface scattering dominates the radar echo: very low entropy, alpha and alpha1 close to zero, very low $S_{h v}$ and high correlation between $S_{h h}$ and $S_{v v}$.

2. Vegetation starts to be present in the radar response, hence increasing entropy. Dominant alpha $\left(\alpha_{1}\right)$ is low (less than $20^{\circ}$ ), but alpha has already reached $40^{\circ}$. Backscatter power will remain quite constant at all channels during this stage and the next one.

3. This corresponds to a moment of fast development in terms of phenology (buds, flowers, etc.), but not much change in terms of structure and, thus, radar response at least at C-band. Backscatter powers remain constant and both alpha and alpha1 too. 
4. Finally, backscatter power increases at $S_{h v}$ and $S_{h h}-S_{v v}$ channels as a result of an increase of the randomness of the structure of the plants (but for oat, which remains as in previous stages since its morphology does not change).

A basic hierarchical tree algorithm can be defined in terms of just two parameters: dominant alpha angle $\left(\alpha_{1}\right)$ and backscatter power at $S_{h v}$ or $S_{h h}-S_{v v}$ ( $t_{33}$ or $t_{22}$ entries of the coherency matrix). The proposed algorithm is depicted in Fig. 3.15.

\subsubsection{Results and Validation}

The results obtained by applying the algorithm proposed in the previous section are analysed here for each crop type separately. As mentioned above, we will concentrate in cereals where the benefits of polarimetry are well evident. A figure showing the output of the retrieval algorithm applied at pixel level for wheat fields and for the 20 images will be presented. The statistics of the retrieved values and their comparison against the reference data will be also shown in form of a table. Results on oat and barley are just summarised in the text.

\subsection{Wheat}

Figure 3.16 shows the retrieved results for wheat. We can appreciate how the most frequent phenology value at each data is in perfect agreement with the reference data at all dates but for one image acquired on 2nd of July (with $22^{\circ}$ incidence). This acquisition corresponds to an extreme incidence angle, so the proposed algorithm (common for all incidences) is more likely to fail. Nevertheless, the first images provide a $100 \%$ of pixels with the right value. In later acquisitions, the transitions between successive stages are, in general, quite smooth in terms of the amount of pixels estimated to be at each stage around the transitions.

For some images there is a non-negligible amount of pixels (more than 25\%) assigned to wrong stages. These cases correspond to either dates of transition between successive stages or cases where the particular incidence angle affects more clearly the radar response. Anyway, the overall result demonstrates that the proposed algorithm is quite reliable despite its simplicity and it provides right estimates for 19 of the 20 images.

\subsection{Oat}

Results for oat (not shown here) are not as good as for wheat after the early vegetative phase. In this case, the radar response does not change significantly from the sixth acquisition date onwards. Consequently, it is virtually impossible to distinguish the two last intervals, from stage 45 to the end of the season, and also separating the advance vegetative (interval 2) from the later stages. With the proposed approach, the most frequent value of retrieved phenology from the sixth to the last image corresponds to interval 3 but in five of the images, hence demonstrating the lack of sensitivity for this crop type. The overall validation provides 13 right estimates at parcel scale from the 20 cases.

Attending at the physical characteristics of oat, the vegetation volume, it is less dense and tall than other cereals (e.g. wheat and barley), so the radar response does not exhibit clear variations after the end of the vegetative phase, and, moreover, the ground contribution is more present than for other cereals.

\subsection{Barley}

The overall performance of the proposed algorithm for barley (tables not shown here) is quite similar to that of wheat, so the same comments apply. In this case the algorithm provides right estimates in all 20 images.

\subsubsection{Comparison with Single- $/$ Dual-Polarisation Data}

The analysis of the evolutions of radar observables as a function of phenology suggests that the dimensionality of the polarimetric space influences the number of different stages that could be identified for each crop type. As in the previous lines, the following analysis is focused on cereals since in this case polarimetry does play a substantial role for monitoring purposes.

For single polarisation, $\mathrm{HH}$ and VV exhibit low sensitivity to phenology and large dependence on incidence angle and even on ascending/descending mode (probably due to row orientation w.r.t. radar) in early stages. HV shows three stages in its evolution as a function of phenology, i.e. initial fast increase at early vegetative, slow decrease at central part
Fig. 3.15 Basic hierarchical tree algorithm for cereals. $t_{22}$ can be equivalently substituted by $t_{33}$ setting the threshold in $-15 \mathrm{~dB}$

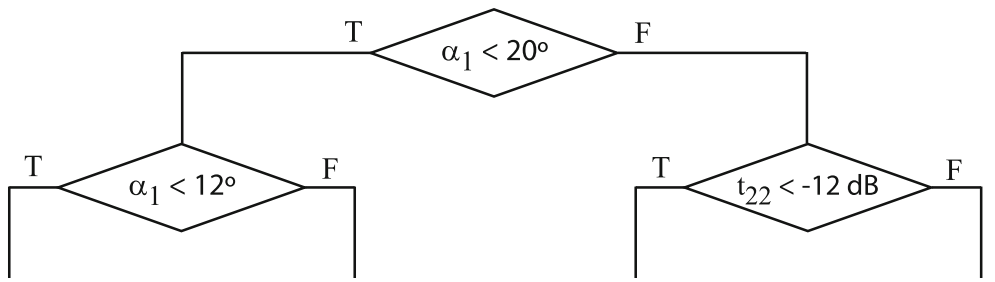

1. Early vegetative (stages 0-19)
2. Advanced vegetative (stages 20-44)
3. Reproductive

(stages 45-79)
4. Maturation (stages $80+$ ) 

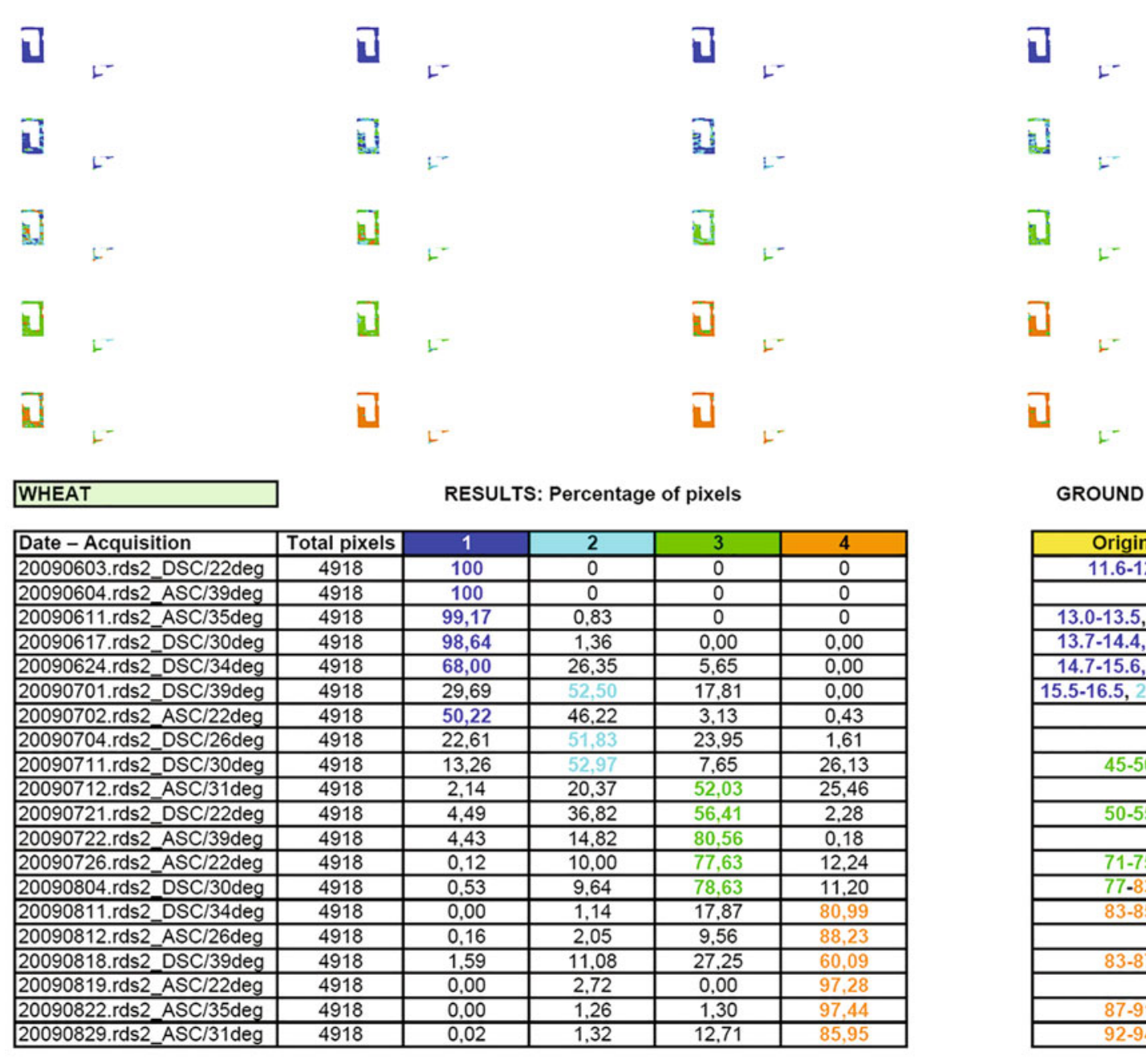

GROUND DATA

\begin{tabular}{|c|c|}
\hline Original & Acq. Date \\
\hline $11.6-12.3$ & 20090601 \\
\hline & \\
\hline $13.0-13.5,20-22$ & 20090610 \\
\hline $13.7-14.4,20-22$ & 20090617 \\
\hline $14.7-15.6,20-24$ & 20090624 \\
\hline $15.5-16.5,21-24,31$ & 20090630 \\
\hline & \\
\hline & \\
\hline $45-50$ & 20090708 \\
\hline & \\
\hline $50-55$ & 20090715 \\
\hline & \\
\hline $71-75$ & 20090724 \\
\hline $77-83$ & 20090730 \\
\hline $83-85$ & 20090806 \\
\hline & \\
\hline $83-87$ & 20090813 \\
\hline & \\
\hline $87-91$ & 20090821 \\
\hline $92-94$ & 20090829 \\
\hline & \\
\hline &
\end{tabular}

Fig. 3.16 Results obtained for wheat: Percentage of pixels assigned to each stage at each image and available reference data. The most frequent value at each date is coloured according to the scale employed in the map

from 20 to 80 stages and late fast increase. Therefore, the number of stages to be separated is smaller than with full-pol, and there is more uncertainty between stages $10-20$ and $20+$. Moreover, the same threshold would not fit equally all cereals and should be adapted to each type.

In case of dual-pol, and provided the mentioned lack of sensitivity of $S_{h h}$ and $S_{v v}$, typical dual-pol systems gathering $\left[S_{h h}, S_{v h}\right]$ or $\left[S_{v v}, S_{h v}\right]$ data do not show a clear improvement with respect to $S_{h v}$. Anyway, the joint use of $S_{h v}$ and any of the $S_{h v} / S_{v v}$ or $S_{h v} / S_{h h}$ ratios provides enough information for barley and wheat to classify correctly the phenology for the three intervals mentioned in the previous paragraph (i.e. stages below 20 , from 20 to 80 and above 80 ). Intermediate stages, however, are not separable in this observation space.

A $\left[S_{h h}, S_{v v}\right]$ coherent measurement, instead, provides similar performance to full-pol, since $\alpha_{1}$ is quite similar to the $\alpha_{1}$ gathered with full polarimetry and $S_{h h}-S_{v v}$ is already used by the proposed algorithm. Anyway, such acquisitions suffer the same drawback of full polarimetry in terms of spatial coverage, due to the reduced swath required by doubling the PRF of the radar system.

Compact polarimetry (Charbonneau et al. 2010; BallesterBerman and Lopez-Sanchez 2012; Cloude et al. 2012) is expected to offer a slightly lower performance than full polarimetry but with a wider swath capability may suffice in some applications. Note that $t_{33}, t_{22}$ and $\alpha_{1}$, used for cereals in this study, are mapped approximately in an equivalent way by compact-pol using $P_{v}, P_{d}$ and $\alpha_{s}$.

\subsubsection{Discussion on Role of Polarimetry, on the Maturity of the Application and Conclusions}

Figure 3.17 summarises the useful parameters for each crop type according to the retrieval results presented previously. We confirm that the sensitivity of C-band polarimetry to crop 


\begin{tabular}{ll}
\hline Crop type & Useful observables \\
\hline Barley & \\
Oat & $\begin{array}{l}\alpha_{1}, S_{h h^{-}} S_{v v}\left(t_{22}\right), S_{h v}\left(t_{33}\right), P_{v} \text { of Freeman decomp., } S_{r r}, S_{l l}, S_{r l} / S_{r r}, S_{r l} / S_{l l}, \text { cor- } \\
\text { relations: HHVV, RRRL and LLRR }\end{array}$ \\
Wheat & \\
Canola & $S_{h h^{-}} S_{v v}\left(t_{22}\right), S_{h v}\left(t_{33}\right), P_{v}$ of Freeman decomp., $S_{r r}, S_{l l}$ \\
Pea & $\begin{array}{l}S_{h h^{-}} S_{v v}\left(t_{22}\right), \text { Std.Dev. }\left\{S_{r r}\right\}, \text { HHVV correlation, entropy, average alpha, } P_{v} \\
\text { of Freeman decomp., } S_{r r}, S_{l l}, S_{r l} / S_{r r}, S_{r l} / S_{l l}\end{array}$ \\
\hline
\end{tabular}

FULL POLARIMETRY

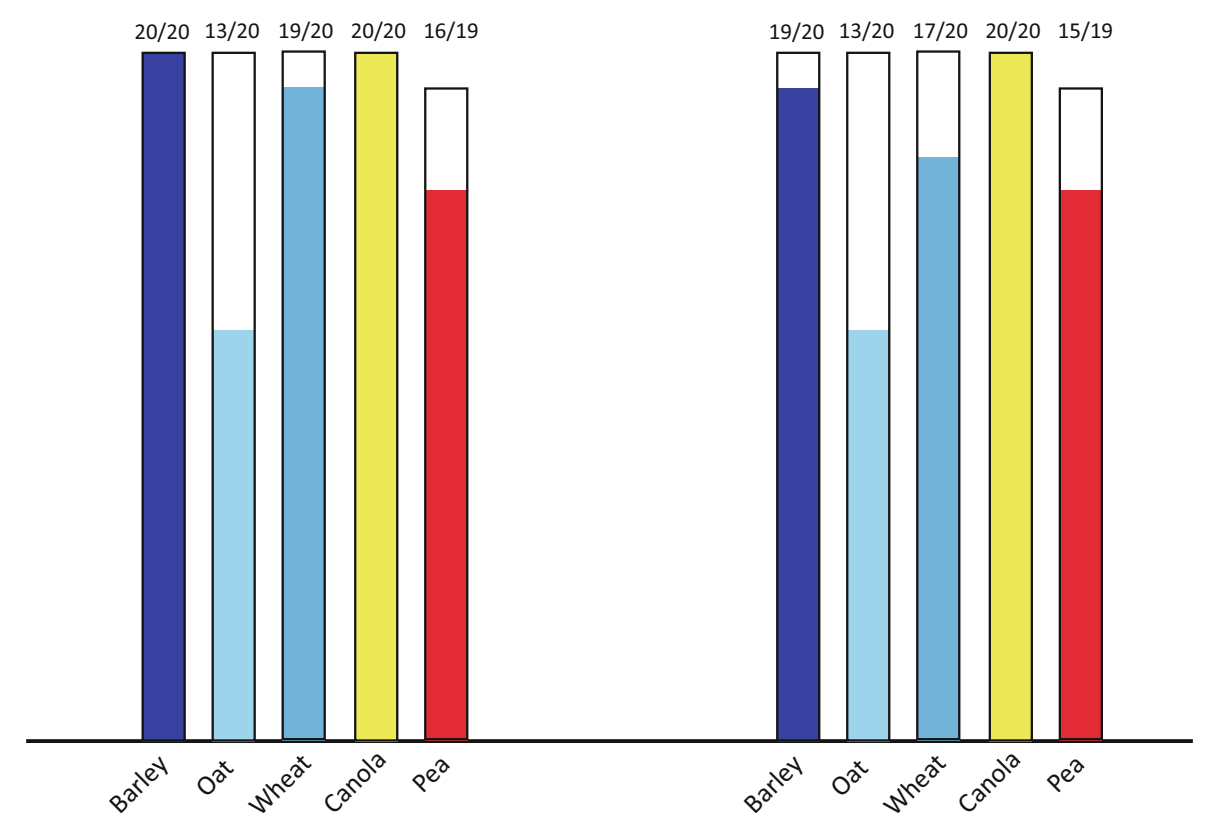

COMPACT POLARIMETRY

Fig. 3.17 Top: summary of useful parameters for each crop type. Bottom: overall performance

phenology is defined by the presence of different morphologies of plants and parcels as they develop along the cultivation cycle. For cereals with distinct plant structures at different stages, polarimetry enables the estimation of their growth stage (from a set of 4 significant intervals) by exploiting just a single radar acquisition, without any other additional information. It is also pointed out that the use of time coordinate, enabled by the availability of time series of radar data from current SAR sensors, will definitely improve the estimation accuracy even more.

The conclusions from this study can be extrapolated to other crop types by taking into account the physical rationale employed to establish the retrieval algorithms. Hence, phenology is likely to be retrieved for every crop with development features analogous to those analysed here. In addition, the wide range of incidence angles employed in this study, despite their influence in the observables, demonstrates the robustness of this application.

According to the most important observables found in this study $\left(t_{33}, t_{22}\right.$ and $\left.\alpha_{1}\right)$, a compact-pol sensor would be able to provide most of the polarimetric sensitivity required for this application and wide swath coverage. It is noted that only in case of canola fields, because of its particular morphology, dual-pol and single-pol systems would suffice.

It should be noted that all approaches validated in this study make use of backscattering coefficients (echo power) at some stages, which may limit its applicability in case of radiometric fluctuations due to diverse causes. In this sense, a phenology retrieval algorithm based on amplitudeindependent polarimetric observables has been demonstrated for rice fields at X-band (Lopez-Sanchez et al. 2012a) and C-band (Lopez-Sanchez et al. 2014). 


\subsection{Wetland Observation}

\subsubsection{C-Band Polarimetric Time Series for Delineating and Monitoring Seasonal Dynamics of Wetlands}

\subsubsection{Introduction, Motivation and Literature Review}

Land use changes, especially in agricultural landscapes, are considered as the major cause of negative environmental impacts, which can be aggravated by climate change. Drainage of wetlands for land reclamation and extraction of groundwater to irrigate agricultural land have caused degradation of soil and water quality and biodiversity loss in some parts of the world. Wetlands are complex ecosystems, permanently or seasonally flooded that provide many ecological, biological and hydrologic functions (Hubert-Moy 2006); this natural resource plays an important role in the regulation of water flow, in the protection of the water quality and in the sustainable management of ecosystems (Maltby 2009). Damaged in the past because considered as unsanitary and unfarmable areas, this ecotone or transition area is a fragile and threatened ecosystem. Preventing the wetland reduction and degradation has now become a priority. Therefore, both inventory and characterisation of wetlands constitute an important stake from an environmental but also socioeconomical point of view. A recent study proposed a method to inventory wetlands based on the functional analysis of potential, existing and effective wetlands (Mérot et al. 2006). The authors have shown that it is important to localise existing and potential wetlands because the area between them can be considered as negotiation areas for restoration purposes. It has also been demonstrated that the length of the contact between existing wetlands and the dry land - including potential wetlands - plays an important role in the denitrification process. For these reasons, a precise determination of the limits of existing and potential wetlands is an important environmental issue. Moreover, little attention has been devoted to our knowledge to the evaluation of wetlands functionalities (for hydrologic processes but also for bio-geochemical and ecological functions) on large areas. In these last years, intensive research efforts have focused on the identification and broad delineation of wetlands (Technical Report Y-87-1 1987). Remotely sensed data are currently used to identify, delineate and characterise wetlands (Ozesmi and Bauer 2002; Hubert-Moy et al. 2006). Optical data provide interesting information to inventory vegetation and agricultural practices in wetlands but are limited to cloud-free periods. For these reasons it is not possible to precisely delineate saturated areas extent as well as water cycles and water levels in these areas with passive remote sensing techniques. Although radar is not sensitive to visibility conditions and can be obtained day or night and through smoke and cloud cover, the spatial resolution of radar imagery that has been used until now was too low to investigate wetlands with a sufficient level of precision. For this purpose, quad-polarimetric and fine-resolution SAR data sets show great potential for mapping wetlands. The objective of this showcase is to address the issue of evaluating fully polarimetric RADARSAT-2 time series data sets for monitoring the seasonal dynamics of saturated areas extent in wetlands. To that end, the development and validation of a general PolSAR segmentation including multitemporal analysis of wetland evolution and polarimetric decomposition are presented.

One fundamental task in wetland monitoring is the regular mapping of temporarily flooded areas. Monitoring and mapping wetlands on a large scale is becoming increasingly more important, and satellite remote sensing provides today a practical approach. Remotely sensed data are widely used to identify, delineate and characterise wetlands. Optical data provide interesting information of improved spectral characterisation of vegetation and soil types to inventory vegetation and agricultural practices in wetlands but are limited to cloud-free periods. For this reason it is not possible to precisely delineate saturated areas extent as well as water cycles and water levels in these areas with passive remote sensing techniques. Due to the independence of weather and illumination conditions, SAR sensors could provide a suitable data base and have been proved today to be an effective tool offering great potential for quantitative monitoring and mapping of wetlands.

The topic of wetland monitoring by means of SAR remote sensing has been mostly addressed in the past by analysing temporal variations of the backscattering coefficient. The use of single-polarisation SAR data has been shown to be important for wetland water extent characterisation. However, single-polarisation SAR in vegetation-type discrimination which is necessary for wetland mapping presents a limited capability. A good overview of SAR wetland monitoring is given in (Bourgeau-Chavez et al. 2009) and (Lang and Kasischke 2008). A multi-polarised attempt is reported in (Henderson and Lewis 2008) where the amplitudes in horizontal and vertical polarisation, but no phase information, are interpreted and correlated with certain wetland characteristics.

Until today just a few publications are concerned with the use of fully polarimetric SAR data sets for wetland monitoring. The operational use of polarimetric SAR is not yet really demonstrated and is still at a study level. The results shown in different publications are always at a preliminary result stage and had never been confirmed when applied on other test sites or under different acquisition configurations (multifrequency or multi-angular). The most important publications 
in that topic are mainly based on a change detection analysis or trying to show that there exist relationships between some specific polarisation parameter and the related scattering mechanism type.

In (Liao and Wang 2010), fully polarimetric RADARSAT-2 data sets are investigated for wetland classification. The target decomposition is used for optimum characterisation of wetland target scattering, and it is shown that the polarimetric information permits the discrimination of eight classes of land surface, based on the use of the entropy and alpha parameters, and leads to an effective classification of two wetland classes: closed and open.

In (Park et al. 2010), an effective method of extracting geophysical information of tidal wetlands (zone of interaction between marine and terrestrial environments ) is proposed, based on the use of fully polarimetric forward/inverse scattering models for quantitative estimation of intertidal mudflats including surface geometric characteristics such as the roughness of the scattering surface.

In (Koch et al. 2012) multi-frequency (RADARSAT2 and ALOS-PALSAR) analysis is conducted for characterising and mapping wetland conditions in a semiarid environment in Central Spain. The results suggest that the fully polarimetric SAR data enables a better separation of the vegetation structure and fragmentation than with the optical data.

In (Brisco et al. 2011), (Schmitt et al. 2012) and (Schmitt and Brisco 2013), three polarimetric decomposition techniques (Cloude-Pottier, Freeman-Durden and normalised Kennaugh elements) are compared to each other in terms of identifying the wetland flooding extent as well as its temporal change. The image comparison along the time series is performed with the help of a novel curvelet-based change detection method for the enhancement of polarimetric decomposition channels, as well as temporal differences in these channels. The results indicate that the decomposition algorithm has a strong impact on the robustness and reliability of the change detection.

In (Patel et al. 2009), scattering models based on physical principles have been applied to characterise the wetland targets like open water habitat and various types of aquatic vegetation with or without standing water, along with various species of forested areas. Entropy, alpha angle and anisotropy have been derived, thus enabling to understand the differences in wetland targets in terms of their scattering behaviour at the L- and P-bands. A significant outcome of this study is that it explores and demonstrates the potential of the state-of-the-art technique of polarimetric SAR for characterising scattering behaviour of various components of a wetland ecosystem.

In (Yajima et al. 2008) is proposed an analysis of the seasonal changes of a wetland by a modified polarimetric four-component scattering power decomposition method. It is shown by the modified scheme that the seasonal changes and features of the vegetation of a lagoon can be clearly detected and observed clearly.

At last, in (Touzi and Gosselin 2010), the TSVM decomposition is investigated when applied for wetland monitoring. Different polarimetric decompositions are studied and compared, and some new polarimetric parameters are extracted that characterise uniquely the scattering type with a complex entity, whose both magnitude and phase have been shown very promising for wetland class characterisation and peatland characterisation. It has been shown, for example, that the scattering type phase permits the detection of water flow variations beneath the peat surface.

Until today no publications are concerned with the use of fully polarimetric SAR series data sets to identify and locate the seasonal dynamics of saturated areas in wetlands. To that end, the development and validation of a supervised PolSAR segmentation including multitemporal analysis of wetland evolution and polarimetric decomposition method are presented in this showcase. The proposed methodology is based on the segmentation of a polarimetric descriptor, the Shannon entropy, which has been shown to be a very sensitive parameter to the temporal variability of flooded areas.

\subsubsection{Methodology}

The employed methodology is presented on Fig. 3.18. The RADARSAT-2 quad-polarimetric SAR image pre-processing and processing has been conducted using the PolSARpro software. The first step consists in extracting the polarimetric $3 \times 3$ T3 coherency matrix which is independent of the polarimetric absolute phase, in order to apply the geocoding process directly on the elements of the T3 matrix. The geocoding of the RADARSAT-2 quad-polarimetric slantrange SAR images to the geographic ground range coordinate system is performed using 46 precise ground control points, uniformly distributed over the subset image, selected from the ortho-rectified ALOS-PRISM and ALOS-AVNIR-2 images used as reference images (Marechal et al. 2010). Due to the fact that the topography of the investigated area is relatively flat, the three inherent and specific geometrical distortion sources in SAR images (foreshortening, layover and radar shadow) do not occur in such a case, and one can conclude that the polarimetric data quality is preserved after the geocoding process (Toutin et al. 2013). To validate the rectification procedure, corner reflectors have been set in the investigated area during the RADARSAT-2 acquisitions. As these corner reflectors have been precisely located with a differential GPS, it has been possible to assess the geocoding accuracy, thus leading to an RMSE error lower than 1 pixel (Marechal et al. 2012).

Concerning the RADARSAT-2 quad-pol SAR image processing, different polarimetric decomposition methods (Huynen, Cloude, Barmes, Holm, Freeman, Van Zyl, 


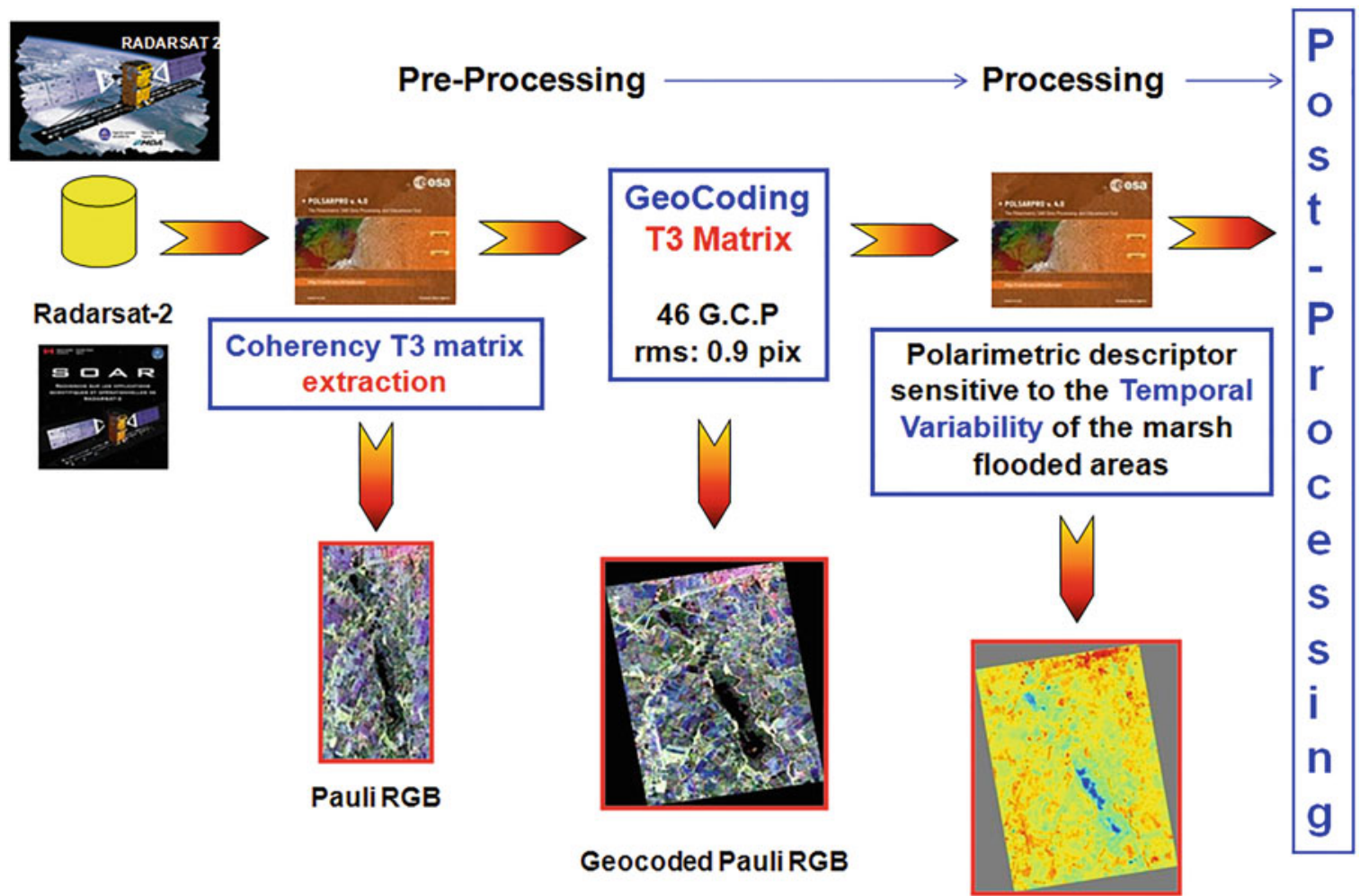

Fig. 3.18 Proposed methodology

Yamaguchi, Cloude-Pottier and Shannon entropy decompositions) (Lee and Pottier 2009) or specific PolSAR image analysis when applied on wetland characterisation and interpretation (Yajima et al. 2008; Touzi and Gosselin 2010) has been investigated in order to extract one polarimetric descriptor that could be very sensitive to the temporal variability of the marsh flooded areas. The temporal coefficient of variation along the 15 RADARSAT-2 quad-polarimetric SAR images has been derived for each of the polarimetric parameters extracted from the polarimetric decompositions, and among all of them, the Shannon entropy (SE) (Lee and Pottier 2009), defined in equation (1.171), has shown the most pronounced contrast between the marsh flooded areas and the surrounding, allowing a segmentation of the data to extract only the water table.

Figure 3.19 shows the normalised Shannon entropy (SE) parameter when applied on the Pleine-Fougères PolSAR image. It is important to point out that the investigated area is a permanent windy area and the open water area becomes a slightly rough surface. Consequently and despite the quite low intensity level, the backscattering mechanism corresponds to a single-bounce scattering mechanism with an associated Shannon entropy low value. Figure 3.20 shows the temporal coefficient of variation $\operatorname{CV}(x)=\frac{\sqrt{\operatorname{var}(x)}}{E\{x\}}$ of the Shannon entropy parameter over the 1-year time series. It can then be seen that this polarimetric descriptor is a very sensitive parameter to the temporal variability of the marsh flooded area.

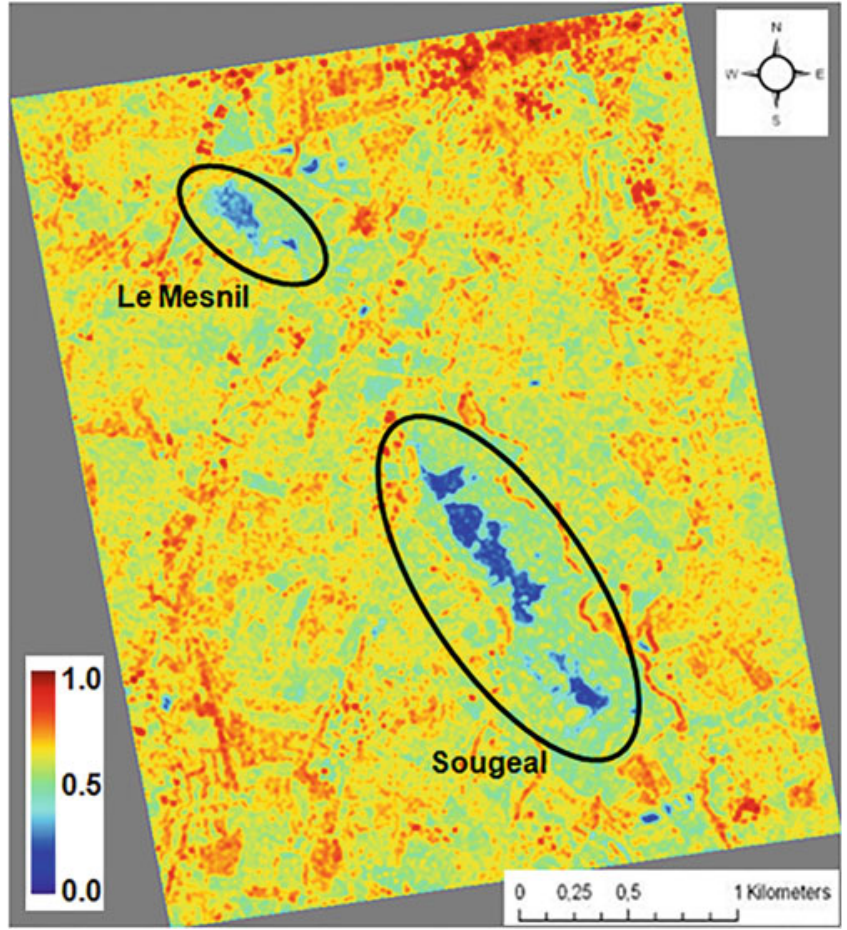

Fig. 3.19 The normalised Shannon entropy (SE) image

The original concept proposed in this methodology is based on the post-processing which is based on a segmentation of the Shannon entropy image in order to detect and 
extract the limits of the open water area and its evolution during the time period under study. Indeed, the extent of the flooding area and its timing exert a strong influence on ecological patterns such as the distribution and diversity as well as the amount of plant community in the wetland (Bourgeau-Chavez et al. 2009). The segmentation-based image procedure that has been used is based on the following steps: calculation of gradient values map, computation of global statistics (cumulative relative histogram) and application of watershed transform on the modified gradient map to detect homogeneity sets of pixels. This segmentation-based

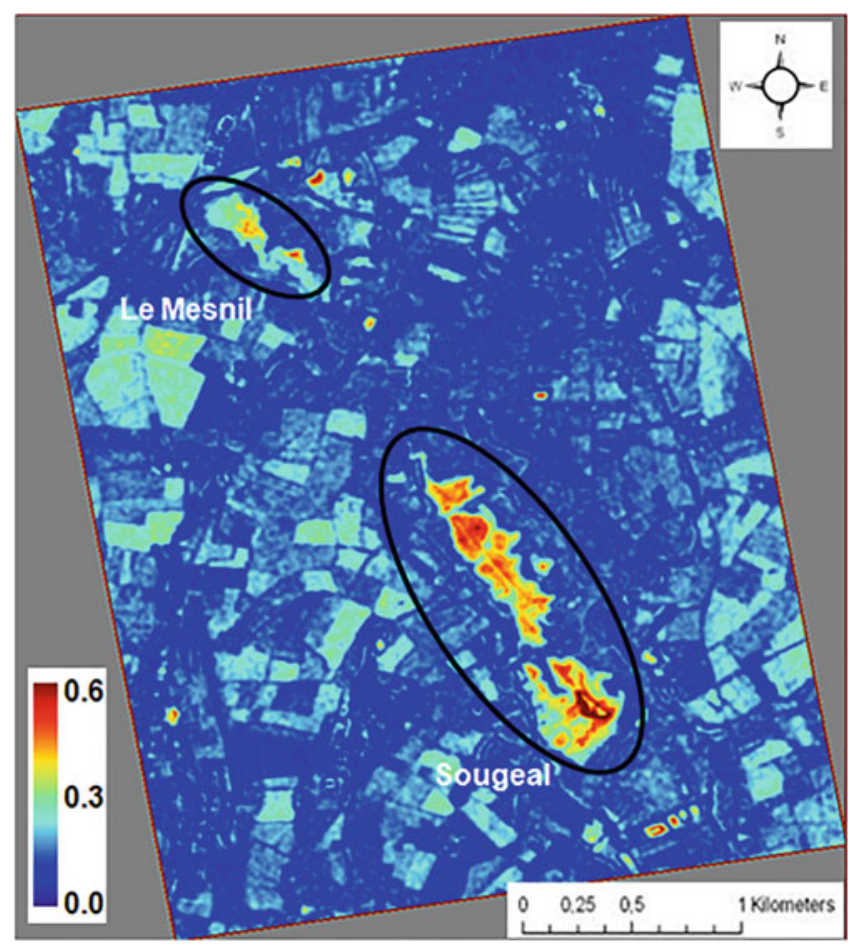

Fig. 3.20 Temporal coefficient of variation of the Shannon entropy parameter image processing system is implemented in ENVI software (ITT Visual Information Solutions 2007, Boulder, USA), and details on this patented application can be found in (Xiaoying 2009).

The Shannon entropy image has been segmented to identify open water and saturated areas. For this, segmentation parameters, like Shannon entropy threshold values, pixel population and neighbourhood limit, have been set up from the first RADARSAT-2 quad-pol SAR image acquired on 22nd of February 2010. Due to both the pixel size $\left(5 \times 6 \mathrm{~m}^{2}\right)$ of the SAR image, versus the area of the investigated study site, and the ground truth observations, a good compromise in the setting method has been to fix the pixel population to 30 for open water to 40 for saturated area and to fix the neighbourhood limit to 8 pixels in both cases. The threshold used during the segmentation procedure has been set up from a histogram analysis of the Shannon entropy value distribution and has been fixed to 0.4 to separate the open water case to the saturated area case as shown in Fig. 3.21. Once trained on this first image, the same resulting and fixed parameters have been applied for the segmentation of all the 14 other SAR images, without applying any new training on each image.

\subsubsection{Experimental Results}

Test sites and corresponding radar and validation data sets selected for wetland delineation and monitoring are summarised in Table 3.6 and further described in the Appendix.

The segmented image corresponding to the first RADARSAT-2 quad-pol SAR image acquired on 22nd of February 2010 is shown in Fig. 3.22. The validation of the segmentation of the Shannon entropy ( $S E)$ image has been conducted using the methodology presented in Sect. 3.5.1.2. On Fig. 3.23 are displayed the results of the segmentations corresponding to the maximum (2010/12/31) and minimum (summer acquisitions from 2010/07/16 to 2010/09/26)
Fig. 3.21 Histogram analysis of the Shannon entropy value distribution and corresponding thresholds

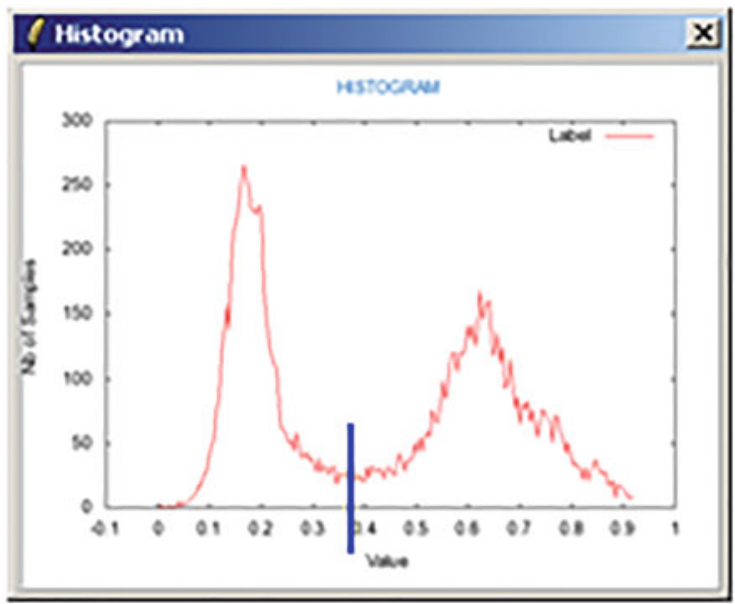


Table 3.6 Test sites and corresponding radar and validation data selected for the generation of showcases on wetland delineation and monitoring

\begin{tabular}{l|l|l}
\hline Application/product & Test site - radar data & Reference data \\
\hline $\begin{array}{l}\text { Wetland delineation } \\
\text { and monitoring }\end{array}$ & Pleine-Fougères, Brittany, France & $\begin{array}{l}\text { ALOS-PRISM } \\
(16 / 03 / 2010)\end{array}$ \\
\cline { 2 - 3 } & 16 RADARSAT-2 Fine Quad-Pol (FQ23) & $\begin{array}{l}\text { ALOS-AVNIR- } \\
2(16 / 03 / 2010)\end{array}$ \\
\cline { 2 - 4 } & $\begin{array}{l}22 / 02 / 2010,16 / 03 / 2010,11 / 04 / 2010,05 / 05 / 2010,29 / 05 / 2010,22 / 06 / 2010,16 / 07 / 2010,09 / 08 / \\
\text { Lidar }(05 / 04 / 2009)\end{array}$ \\
\cline { 2 - 4 } & $2010,02 / 09 / 2010,26 / 09 / 2010,20 / 10 / 2010,13 / 11 / 2010,07 / 12 / 2010,31 / 12 / 2010,24 / 01 / 2011$, & $0.5 \mathrm{~m} \mathrm{DTM}$ \\
\cline { 2 - 4 } & $17 / 02 / 2011$ & Ground truth data \\
\hline
\end{tabular}
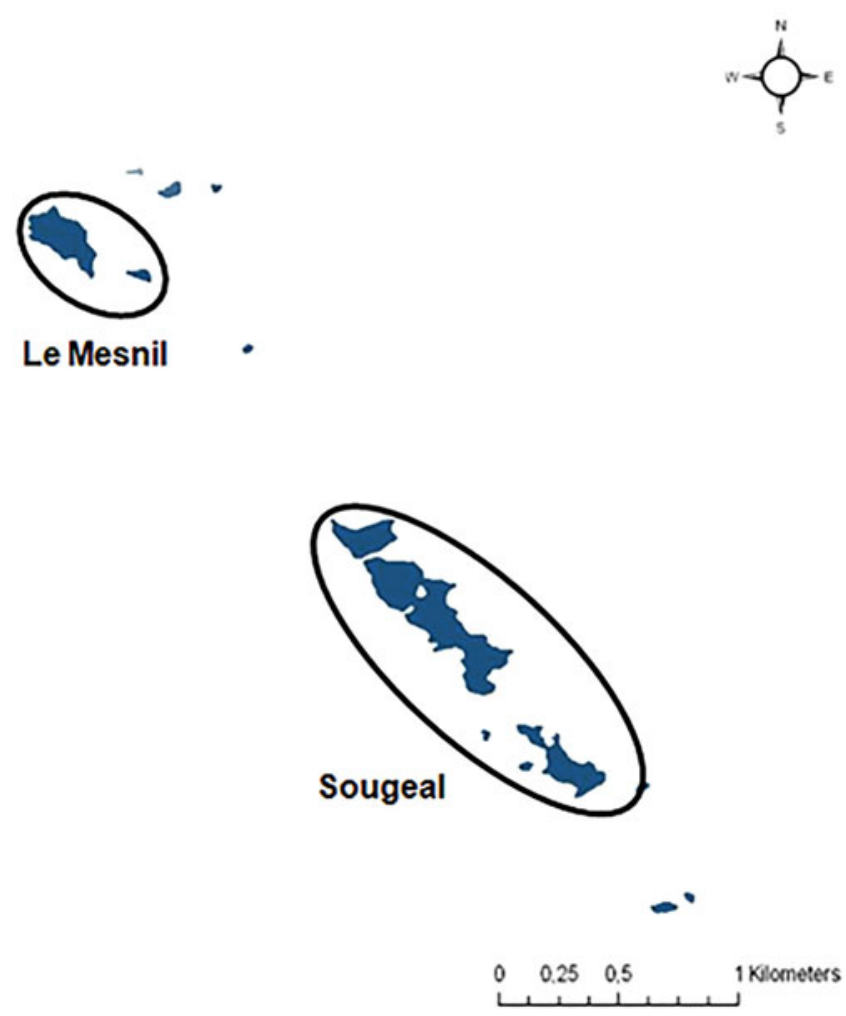

Fig. 3.22 The segmented Shannon entropy (SE) image with the open water in blue

extension of the water table. The segmentation result for the acquisition of March (2010/03/18) is also shown as a classical example. The measurement stations, located along the transect, are represented with the symbol $\boldsymbol{O}$, and the symbol t represents the reference fix point. The detected limits of the water table for the three examples are represented with the symbols (December), $\boldsymbol{X}$ (March) and $\boldsymbol{*}$ (summer). As it can be seen, there exists a prefect correspondence between the results of the segmentation and the limit of the water table detected during the different ground truth campaigns.

More of that, to assess the results, the spatial distribution of the open water, extracted from the segmented image, is displayed over the lidar image. The analysis of the altimetric profile, obtained from the digital elevation model, confirms the shape of the limit of the open water according to the local microtopography (presence of little canal with deposits or presence of two holes, artificial depression on the ground, etc.).

This 1-year time series of segmented Shannon entropy images is merged in a geographic information system in order to create a precise map of permanent and temporary water. Figure 3.24 provides an overview of the variations of the water table over the wetland site from February 2010 to February 2011, in showing the minimum and maximum water extent of the water table, including open water bodies and inundated vegetation and soils, during one hydrological year.

\subsubsection{Comparison with Single-I Dual-Polarisation Data}

The fully polarimetric RADARSAT-2 data of wetlands are used to simulate dual-polarimetric mode data from the upcoming RADARSAT Constellation Mission (CSA) and Sentinel-1 (ESA). The simulated dual-polarimetric SAR data are then used to evaluate the Shannon entropy for wetland monitoring, and the results are compared with the fully polarimetric data. In the dual-polarimetric case, the two terms of the Shannon entropy are given by:

$$
\begin{aligned}
& \mathrm{SE}_{I}=2 \log \left(\frac{\pi e I_{T}}{2}\right)=2 \log \left(\frac{\pi e \operatorname{trace}\left(\mathbf{C}_{2}\right)}{2}\right) \\
& \mathrm{SE}_{P}=\log \left(1-p_{T}^{2}\right)=\log \left(4 \frac{\left|\mathbf{C}_{2}\right|}{\operatorname{trace}\left(\mathbf{C}_{2}\right)^{2}}\right)
\end{aligned}
$$

where $\mathbf{C}_{2}$ stands for the $2 \times 2$ covariance matrix of one of the possible dual-polarimetric target vector: $\left[S_{h h}, S_{v h}\right]^{T},\left[S_{h v}\right.$, $\left.S_{v v}\right]^{T}$ or $\left[S_{h h}, S_{v v}\right]^{T}$.

Figures 3.25 and 3.26 show the normalised Shannon entropy (SE) parameter when derived from the dualpolarimetric target vectors $\left[S_{h h}, S_{v h}\right]^{T}$ and $\left[S_{h v}, S_{v v}\right]^{T}$.

As it can be seen, the results are consistent for both dualpolarisation Shannon entropy and the fully polarimetric one (Fig. 3.19). This result is very interesting and mainly promising in the sense that the methodology developed for the fully polarimetric case can be applied without any restriction to the dual-polarimetric case as the input feature seems to be the same. Delineating and monitoring the seasonal 
Fig. 3.23 Validation of the limits of the water table extracted from the segmented images with the limits detected during the corresponding ground truth campaigns

Fig. 3.24 Variations of the water table over the wetland site from February 2010 to February 2011
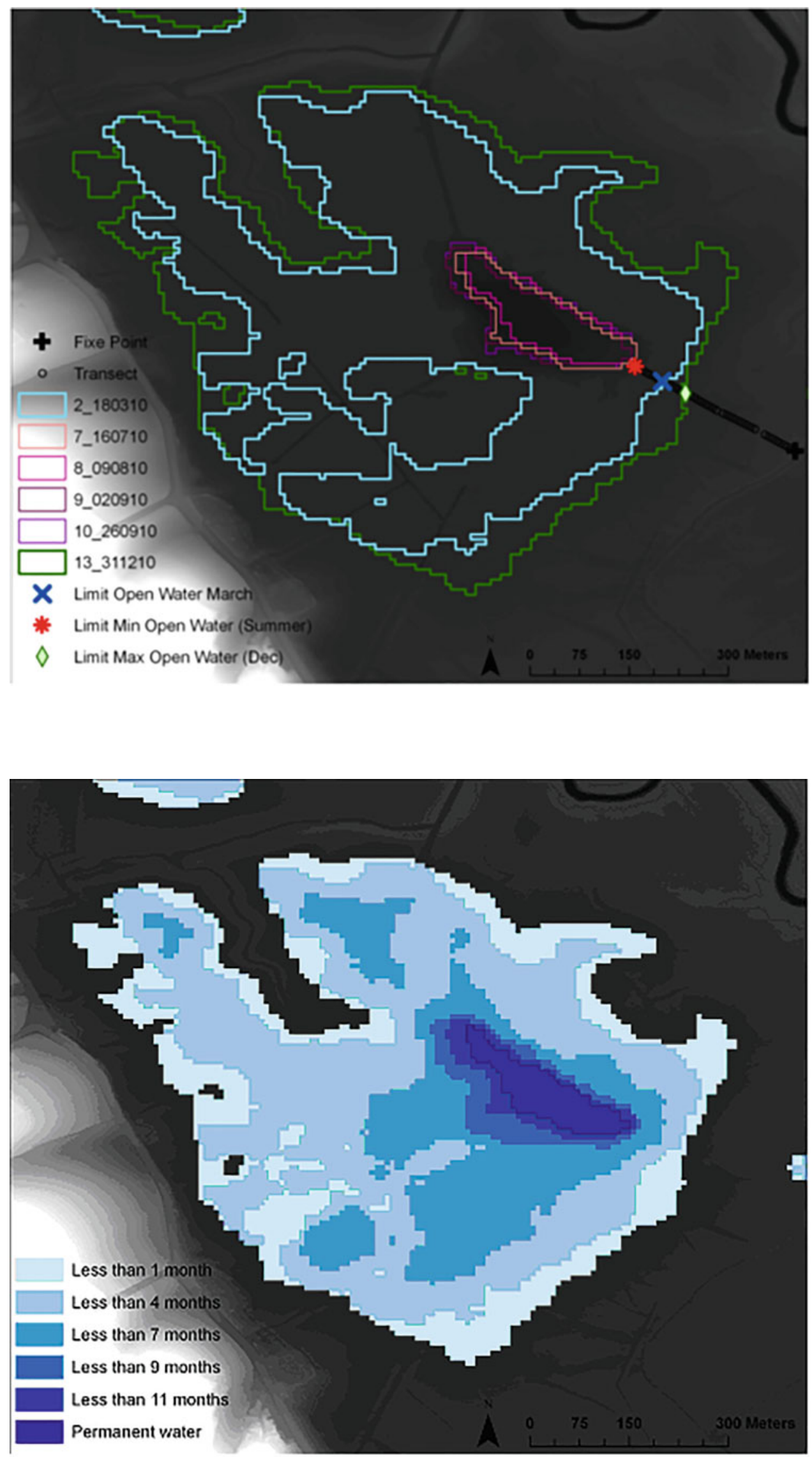


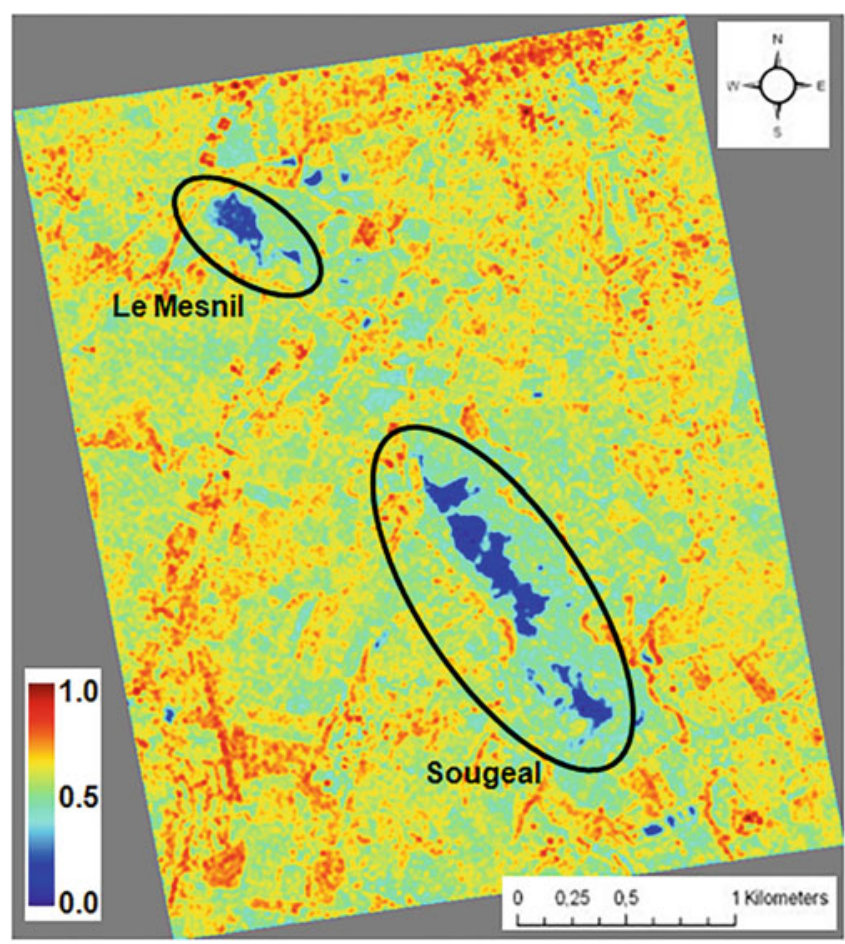

Fig. 3.25 The normalised Shannon entropy (SE) image in the case of the target vector $\left[S_{h h}, S_{v h}\right]^{T}$

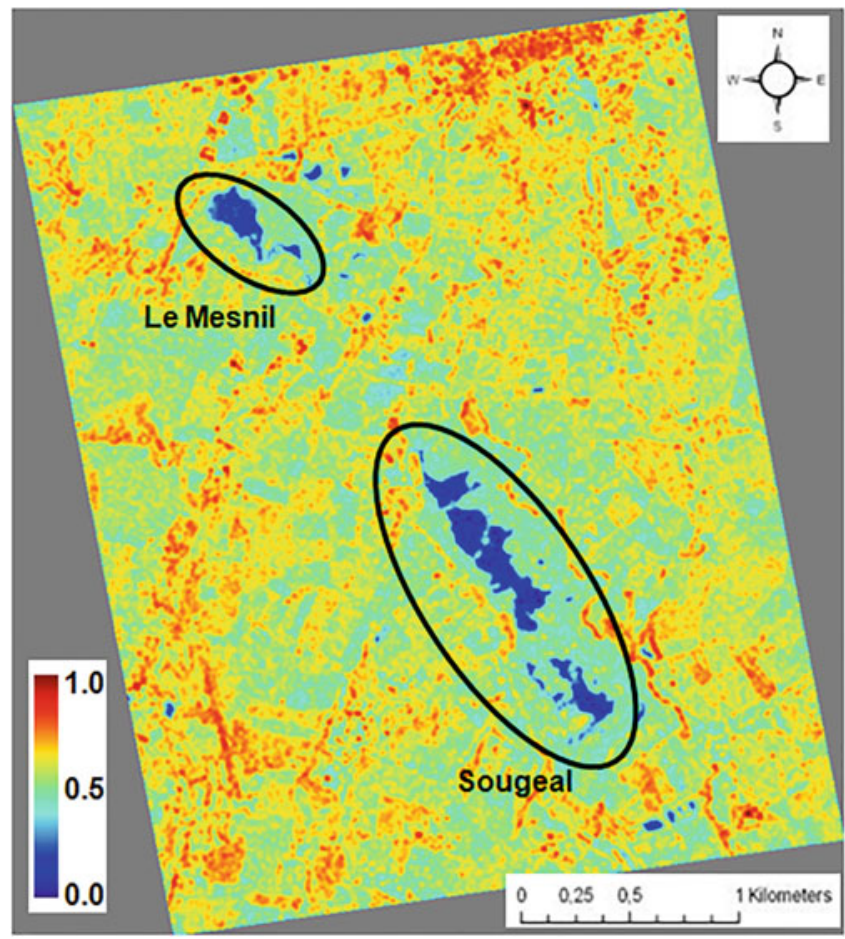

Fig. 3.26 The normalised Shannon entropy (SE) image in the case of the target vector $\left[S_{h v}, S_{v v}\right]^{T}$

dynamics of wetlands can be seriously considered as a concrete application for the RADARSAT-RCM and Sentinel-1 missions.

\subsubsection{Discussion on the Role of Polarisation, on the Maturity of the Application and Conclusions}

The aim of this showcase is to determine the capabilities of fully polarimetric SAR time series data sets to delineate and monitor wetland ecosystems. This study was set from February 2010 to February 2011 (15 images) over a wetland area located in France (Brittany). Every 24 days, simultaneously with the image acquisition in a Fine Quad-Pol mode (beam FQ23, 42.6 ${ }^{\circ}$ ), a 1-year ground truth measurement campaign to measure the distance between a reference fix point and the limit of the water table has been conducted. It has been established in this study that the Shannon entropy is a parameter very sensitive to the temporal variability of flooded areas. Maps of the fluctuated trend of the open water from a 1-year RADARSAT-2 quad-pol time series have been achieved, in an automatic procedure, from the segmentation of this polarimetric descriptor. A lidar image of the investigated area, providing very accurate elevation and precise microtopography information, has corroborated these results, leading to a very fine analysis of the obtained results. The limits of the water bodies extracted from segmented images highlight inundation extend oscillation, which is a key environmental factor controlling ecological, hydrological and chemical processes. This product, when generated over several years, can provide useful information to characterise the water cycle and identify variations that may affect the overall ecosystem. The results presented here show already the important potential of fully polarimetric SAR data. However, a very promising result concerns the very good correlation between the dual-polarimetric Shannon entropy and the fully polarimetric one, which means that it will also be possible to extract the open water and its spatial distribution when this proposed methodology will be applied on the future dual polarimetry RADARSAT-RCM and Sentinel-1 time series data sets. At last, it is important to note that this proposed methodology cannot be applied to single-polarisation data. Indeed, single-pol data processing is often and/or only based on the analysis of the backscattering level, and in such a case, single-pol data become very ambiguous for the delineation of wetlands since the same backscatter level can be reached at land and water, depending on the wind conditions. Water bodies become a rougher surface and so return a stronger signal in presence of wind.

\subsubsection{Tropical Wetland Characterisation with Polarimetric SAR}

\subsubsection{Introduction, Motivation and Literature Review}

Tropical wetlands are very productive ecosystems located mainly in the tropics, but can also be found in sub-tropical regions, where temperatures sometimes drop below freezing 
point. They consist of both inland freshwater ecosystems and coastal, mangrove systems. Both ecosystems serve as a critical habitat for a wide variety of plant and animal species. Tropical wetlands also have a valuable economical importance, as they serve as a large carbon reservoir, filter nutrients and pollutants and provide aquatic habitats for outdoor recreation, tourism and fishing (Barbier 1994). Globally, many tropical wetlands are under severe environmental stress, mainly from agricultural and urban development, pollution and rising sea level.

In order to generate an inventory of tropical wetlands and assess their health, it is important to map, characterise and monitor these fragile ecosystems. Such tasks are best conducted using remote sensing observations, because of the remoteness and large extent of many tropical wetlands. Optical remote sensing observations are fairly limited in the tropics, because of frequent cloud coverage. The all-weather capability synthetic aperture radar (SAR) observations are very useful for the characterisation and monitoring tasks, especially quadruple-polarimetric (quad-pol) data that contains more spatial information related to physical characteristics than single- or dual-polarimetric data.

A common method for characterising landscapes with polarimetric SAR (PolSAR) observations is decomposition analysis, which maps the landscape according to the surface's scattering mechanisms. In this contribution we use four decomposition types, Pauli, Freeman, Yamaguchi and Hong and Wdowinski. The first three decompositions, Pauli, Freeman and Yamaguchi, have been applied successfully to characterise landscapes in various environments. The fourth decomposition by Hong and Wdowinski (H\&W) is a new decomposition, which was developed in accordance with new SAR phase observations (interferograms) in tropical wetlands. The contribution includes a brief description of the new decomposition and the application of all four decompositions to the following four quad-pol data sets acquired over the Everglades: three satellites, TerraSAR-X, RADARSAT-2 and ALOS-PALSAR, and one airborne sensor UAVSAR. The results of our decomposition analyses indicate that quad-pol SAR data is very useful for tropical wetland characterisation, in particular the X- and L-band data sets.

Quad-pol SAR data have been used over the past two decades to study and characterise tropical wetlands in various locations worldwide. The early quad-pol PolSAR studies relied on airborne $\mathrm{C}$-, L- and P-band observations acquired by NASA's AIRSAR mission over tropical wetlands in Belize, French Guiana, Australia and the USA. Most of these studies focused on the interaction of the SAR signal with mangrove vegetation and the possibility to retrieve quantitative estimates of biophysical parameters such as tree height and biomass (Pope et al. 1994; Proisy et al. 2000; Lucas et al. 2007; Trisasongko 2009). The AIRSAR data was also used to map sub-environments of coastal herbaceous wetlands (Slatton et al. 1996). Another more advanced airborne sensor that acquired quad-pol data over tropical wetlands is NASA's UAVSAR, which operates at L-band. Fatoyinbo and Simard (2011) used quad-pol UAVSAR combined with ALOS polarimetric data to estimate biomass of mangrove forests in various remote locations in the tropics.

The use of space-borne quad-pol data for tropical wetland studies has been, so far, fairly limited due to the limited amount of space-borne quad-pol data acquired over tropical wetlands. The shuttle imaging radar-C (SIR-C) was the first space-borne sensor to acquire quad-pol SAR data. The SIR-C data were used to detect a seasonal flooding component in Yucatan wetlands (Pope et al. 1997) and for land cover classification in India (Turkar and Rao 2011). Insofar only three satellites acquired quad-pol SAR data, two of them in an experimental mode (TerraSAR-X and ALOS-PALSAR) and one, RADARSAT-2, as part of its regular operation. Ullmann et al. (2012) used quad-pol TerraSAR-X data acquired over Indonesia and analysed them using Cloude and Yamaguchi decompositions. Their analyses revealed land classification in which wetlands and other flooded vegetation could easily be identified by the double-bounce scattering component. Hong et al. (2010) and Hong and Wdowinski (2011) used RADARSAT-2 quad-pol data acquired over the Everglades wetlands in south Florida. Interferometric processing of the data revealed a very similar fringe pattern in all polarisation interferograms suggesting that both co- and cross-pol signals sample the water surface beneath the vegetation. Based on the phase observations, Hong and Wdowinski (2013) developed a new PolSAR decomposition analysis with a double-bounce component from cross-polar channel.

Decomposition analyses are common methods for characterising landscape with polarimetric SAR observations. The widely used Pauli decomposition is a simple method that represents the main three scattering mechanisms: singlebounce, double-bounce and volume scattering (Cloude 2009; Cloude and Pottier 1996; Lee and Pottier 2009). A threecomponent scattering model proposed by Freeman and Durden (1998) has been successfully applied to decompose polarised SAR data into three components according to the main scattering mechanisms under reflection symmetry conditions. In order to account for non-reflection symmetry conditions, Yamaguchi et al. $(2005,2006)$ added a fourth helix component to their decomposition. Various methods were proposed to estimate the volume scattering component considering non-reflection symmetry condition (Arii et al. 2010, 2011; Neumann et al. 2009). Recently an extended volume scattering model was discussed using randomly orientated diplane scatterers (Sato et al. 2012a). More recent decomposition studies also included mathematical operations on the decomposed coherency matrix in order to resolve anomalous 
Table 3.7 List of decompositions used in this study and their main characteristics

\begin{tabular}{l|l|l}
\hline Decomposition & Characteristics & References \\
\hline Pauli & 3 components: HH-VV, HH + VV, HV & Cloude and Pottier (1996) \\
\hline Freeman & Model based, 3 components: single bounce, double bounce, volume & Freeman and Durden (1998) \\
\hline Yamaguchi & Model based, 4 components: single bounce, double bounce, volume, helix & Yamaguchi et al. (2005) \\
\hline H\&W & Model based, 3 components: single bounce, double bounce (co- and cross-pol), volume & Hong and Wdowinski (2013) \\
\hline
\end{tabular}

values generated by the previous three- and fourdecomposition methods (Yamaguchi et al. 2011; van Zyl et al. 2011; Lee and Ainsworth 2011). Touzi et al. (2009) proposed a phase of target scattering decomposition for wetland characterisation, and Hong and Wdowinski (2013) developed a new decomposition analysis with a double-bounce component from cross-pol.

\subsubsection{Methodology}

Our methodology for characterising tropical wetlands with quad-pol SAR data consists of four decomposition analyses, Pauli, Freeman, Yamaguchi and H\&W (Table 3.7). The first decomposition we considered, Pauli, is a simple method that represents the main three scattering mechanisms. The other three decompositions are model-based, accounting for three or four scattering mechanisms. The Freeman decomposition accounts for three mechanisms (single bounce, double bounce and volume), whereas the Yamaguchi decomposition includes a fourth helix scattering component. The H\&W decomposes the cross-pol signal into volume and doublebounce scattering components, which increases the doublebounce scattering component on the account of volume scattering. The inclusion of a cross-pol double-bounce scattering is based on cross-pol SAR phase observations in wetlands showing coherent interferometric signal backscattered from the water surface beneath the vegetation. Touzi et al. (2009) presented a non-model, roll-invariant target scattering decomposition for the characterisation of temperate wetlands. We did not include Touzi decomposition in this study, because we focus on model-based decompositions.

The full description and mathematical formulation of the four decompositions can be found in the references listed in Table 3.7. The first three listed decompositions are wellknown and do not need much introduction. However, the fourth decomposition, $\mathrm{H} \& \mathrm{~W}$, is fairly new. Hence we provide a brief description of the decomposition, including key equations. Our decomposition is based on phase observations from wetlands indicating that the cross-pol signal samples the water surface beneath the vegetation. The simplest scattering mechanism that accounts for scattering in the cross-pol signal is a rotated dihedral, which we included in our formulation. Following the formulation of Yamaguchi et al. (2005), we utilise a $3 \times 3$ coherency matrix to derive each scattering component mathematically as shown in Eq. (3.8):

$$
\begin{aligned}
& \langle\mathbf{T}\rangle^{H V}=\left[\begin{array}{ccc}
T_{11} & T_{12} & T_{13} \\
T_{21} & T_{22} & T_{23} \\
T_{31} & T_{32} & T_{33}
\end{array}\right]= \\
& =f_{s} \mathbf{T}_{\text {single }}^{h v}+f_{d} \mathbf{T}_{\text {double }}^{h v}+f_{v} \mathbf{T}_{\text {volume }}^{h v}+f_{d} \mathbf{T}_{\text {rotated_diplane }}^{h v}
\end{aligned}
$$

where \langle\rangle denotes the ensemble average of a few pixels in the data processing and $f_{s}, f_{d}$ and $f_{v}$ are the coefficients related to the powers of single-bounce, double-bounce and volume scattering components, respectively.

Following Yamaguchi et al. (2005) four-component decomposition formulation, we adopted the same scattering coherency matrices for the single-bounce, double-bounce and volume scattering as follows:

$$
\begin{aligned}
\langle\mathbf{T}\rangle_{\text {single }}^{h v}= & {\left[\begin{array}{ccc}
1 & \beta^{*} & 0 \\
\beta & |\beta|^{2} & 0 \\
0 & 0 & 0
\end{array}\right] } \\
\langle\mathbf{T}\rangle_{\text {double }}^{h v} & =\left[\begin{array}{ccc}
|\alpha|^{2} & \alpha & 0 \\
\alpha^{*} & 1 & 0 \\
0 & 0 & 0
\end{array}\right] \\
\langle\mathbf{T}\rangle_{\text {volume }}^{h v} & =\frac{1}{4}\left[\begin{array}{lll}
2 & 0 & 0 \\
0 & 1 & 0 \\
0 & 0 & 1
\end{array}\right]
\end{aligned}
$$

We introduce a double-bounce scattering model of a rotated dihedral for the cross-pol signal, with the following matrix which is integrated with the probability density function similar to formulations of Yamaguchi et al. (2005):

$$
\begin{aligned}
\mathbf{S}^{H V} & =\left[\begin{array}{cc}
1 & 0 \\
0 & -1
\end{array}\right] \text { or } \mathbf{S}^{H V}=\left[\begin{array}{cc}
-1 & 0 \\
0 & 1
\end{array}\right] \\
& \Rightarrow\langle\mathbf{T}\rangle_{\text {rotated_diplane }}^{h v}=\frac{1}{2}\left[\begin{array}{lll}
0 & 0 & 0 \\
0 & 1 & 0 \\
0 & 0 & 1
\end{array}\right]
\end{aligned}
$$

Equation (3.10) allows us to estimate the doublebounce component in the cross-pol signal. Thus, the 
coherency matrix in Eq. (3.8) can now be written as a combination of single-bounce, double-bounce from both co- and cross-pol and volume scattering components as follows:

$$
\begin{aligned}
& \langle\mathbf{T}\rangle^{H V}=f_{s} \mathbf{T}_{\text {single }}^{h v}+f_{d} \mathbf{T}_{\text {double }}^{h v}+f_{v} \mathbf{T}_{\text {volume }}^{h v}+f_{d} \mathbf{T}_{\text {rotated_diplane }}^{h v}= \\
& =f_{s}\left[\begin{array}{ccc}
1 & \beta^{*} & 0 \\
\beta & |\beta|^{2} & 0 \\
0 & 0 & 0
\end{array}\right]+f_{d}\left[\begin{array}{ccc}
|\alpha|^{2} & \alpha & 0 \\
\alpha^{*} & 1 & 0 \\
0 & 0 & 0
\end{array}\right]+\frac{f_{v}}{4}\left[\begin{array}{lll}
2 & 0 & 0 \\
0 & 1 & 0 \\
0 & 0 & 1
\end{array}\right]+\frac{f_{d}}{2}\left[\begin{array}{ccc}
0 & 0 & 0 \\
0 & 1 & 0 \\
0 & 0 & 1
\end{array}\right]
\end{aligned}
$$

We have four observables and five unknown parameters in Eq. (3.11). We reduce the number of unknown parameters by assuming $\alpha=0$ in the areas dominated by surface scattering and $\beta^{*}=0$ in areas dominated by double-bounce scattering. Further details of the decomposition are provided by (Hong and Wdowinski 2013).

\subsubsection{Experimental Results}

Test sites and corresponding radar and validation data sets selected for tropical wetland characterisation and monitoring are summarised in Table 3.8 and further described in the Appendix.

We applied the 4 decompositions listed in Table 3.7 to the 4 quad-pol data sets listed in Table 3.8 and obtained 16 different decompositions that characterise our study area in the Everglades. In order to evaluate the decomposition results, we first present the four decompositions applied to the RADARSAT-2 data set and compare the results with Landsat image and detailed vegetation map (Fig. 3.27). We then compare three decomposition results applied to three data sets that were acquired with three different SAR frequencies (X-, C- and L-bands), covering the same area (Fig. 3.27). In this comparison we applied the $\mathrm{H} \& \mathrm{~W}$ decomposition to all data sets. Again, we compare the decomposition results with an optical Landsat image and a vegetation map.

The study of the RADARSAT-2 data set (Fig. 3.27) shows that all four decompositions yield similar results indicating double-bounce scattering (red) in the northeast corner of the data set, combined volume and single-bounce scattering (blue-green) in the southwest corner and mostly volume scattering (green) in a wide area located between the two corners. There are some variations in the decompositions. The most noticeable difference is the high volume scattering of the Freeman decomposition (Fig. 3.27b). The issue of high volume scattering (too much green) in urban environments led (Yamaguchi et al. 2005) to develop their four-component decomposition with helix scattering, which indeed shows less volume scattering. The H\&W decomposition also shows less volume scattering than the Freeman one, because some of the cross-pol signal contributes to the double-bounce component. We validate the decomposition results by comparing them to an optical Landsat image (Fig. 3.27e) and vegetation map of the study area (Fig. 3.27f). A visual comparison between the decompositions and vegetation map indicates that all four decompositions characterised well the mangrove forests in the southwest corner and the sawgrass in the northeast corner, as both areas contain fairly homogeneous vegetation. The decompositions' spotty patterns in the transition zone between the saltwater mangroves and freshwater sawgrass reflect the mixture of vegetation types in that transition zone. Both prairies and scrub vegetation types consist of a variety of grass, bushes and trees, which results in a spotty pattern in the decomposition.

The multi-frequency decomposition study (Fig. 3.28) yields very interesting results, as in each frequency the radar signal interacts differently with the vegetation. In this study we show results of the Hong and Wdowinski decomposition; similar results were obtained by the other

Table 3.8 Test sites and corresponding radar and validation data selected for the generation of showcases on tropical wetland characterisation

\begin{tabular}{l|l|l}
\hline Application/product & Test site - radar data & Reference data \\
\hline \multirow{2}{*}{ Tropical wetland characterisation } & Shark River Slough & Optical data, vegetation maps \\
\cline { 2 - 2 } & TerraSAR-X, 16/04/2010 & \\
\cline { 2 - 3 } & RADARSAT-2, 23/09/2008 & \\
\cline { 2 - 3 } & ALOS-PALSAR, 19/04/2011 & \\
\cline { 2 - 3 } & Coastal mangrove forests & \\
\cline { 2 - 3 } & UAVSAR, L-band, 22/06/2010 & \\
\hline
\end{tabular}




\section{(a) Pauli}

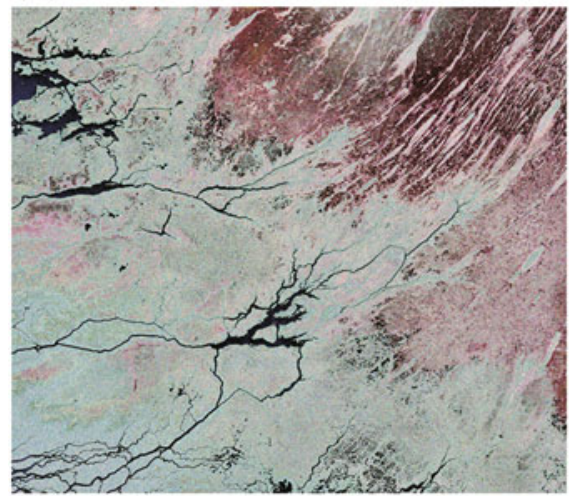

(d) H\&W

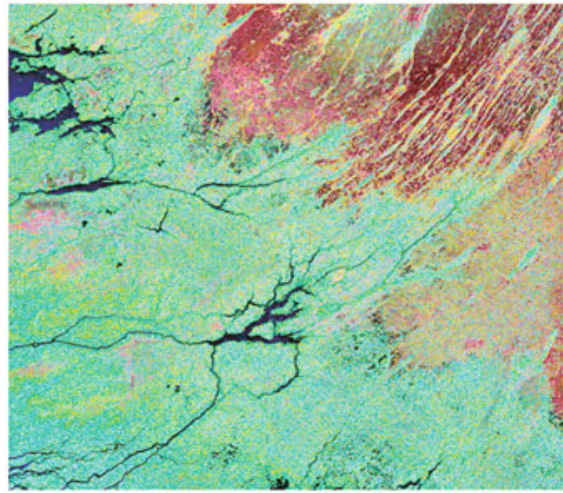

\section{(b) Freeman}

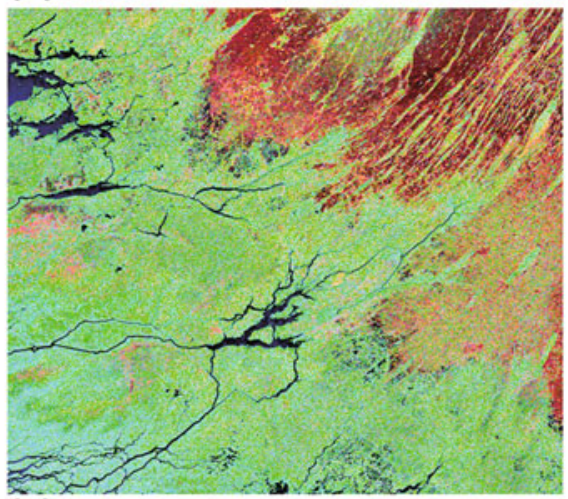

(e) Landsat

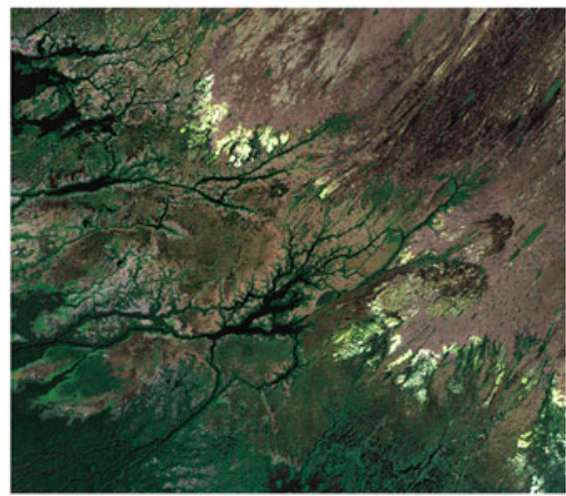

(c) Yamaguchi

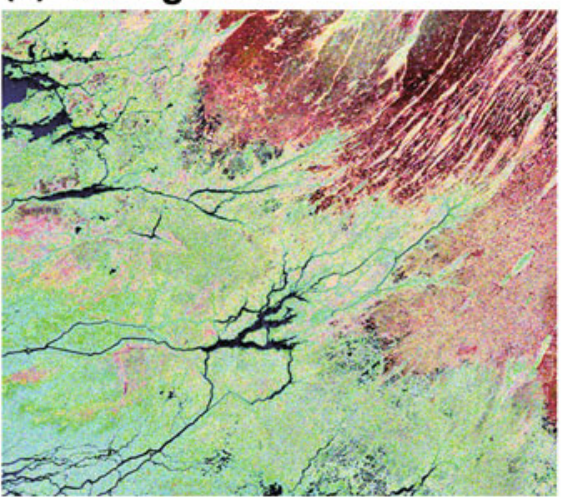

(f) Vegetation map

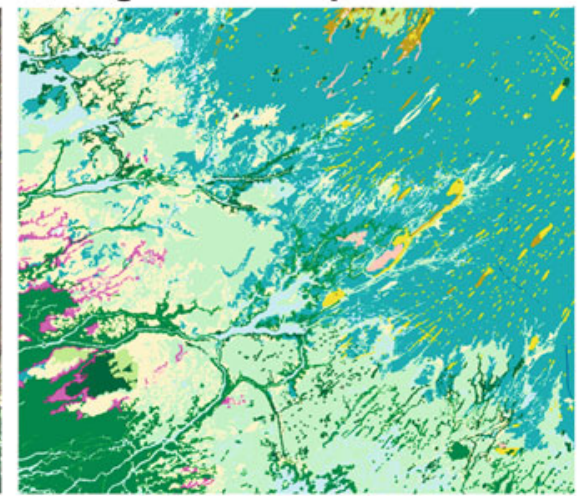

Fig. 3.27 Polarimetric decomposition results of the C-band RADARSAT-2 data, acquired on 23rd of September 2008. (a) Pauli decomposition presented as colour composite image with the following decomposition components: $\mathrm{HH}-\mathrm{VV}$ (red), $\mathrm{HH}+\mathrm{VV}$ (blue) and $\mathrm{HV}$ (green). (b) Freeman's three-component decomposition with the following components: blue, single bounce; red, double bounce; and green,

decompositions. The decomposition of the TSX data set shows large colour variability reflecting a high sensitivity level of the X-band $(3.1 \mathrm{~cm})$ to distinguish between the various vegetation types. The decomposition shows in great details the distribution of mangroves along the tidal channels as volume scattering, the sawgrass in the northeast corner as double-bounce scattering and a mixture of all three scattering mechanisms in this vegetation transition zone between freshwater (sawgrass) and saltwater (mangroves) ecosystems. The decomposition of the RADARSAT-2 data set shows a dominance of volume scattering (green) throughout the image and, hence, low resolution to distinguish between the various vegetation types in the transition zone. Nevertheless, the decomposition still distinguishes well the sawgrass (red in the northeast corner) from the rest of the vegetation types. The L-band decomposition also shows high variability and sensitivity to different vegetation types.

The most interesting difference between the ALOS and the other two decompositions is the sawgrass characterisation in northeast corner. The ALOS decomposition characterises the sawgrass area as single-bounce scattering (blue), whereas volume scattering. (c) Yamaguchi's four-component decomposition with same colour scheme as in (b). (d) Hong and Wdowinski's threecomponent decomposition with cross-pol double bounce. (e) Landsat-7 $\mathrm{ETM}+$ optic colour composite image. (f) Vegetation map of the study area (Florida Coastal Everglades, Long Term Ecological Research (FCE LTER, http://fcelter.fiu.edu)). Legend is shown in Fig. 3.28 the other two decompositions as double bounce. The different scattering mechanism reflects a different interaction of the relatively thin sawgrass (a few $\mathrm{cm}$ wide) with the different wavelengths of the radar signal. The sawgrass serves as good scatterers for the X- and C-band signals $(3.1$ and $5.6 \mathrm{~cm}$, respectively), but is transparent to the longer wavelength L-band signal $(24.1 \mathrm{~cm})$.

The validation of the three decompositions with the Landsat image and vegetation maps indicates a very good sensitivity of the TSX and ALOS decompositions to characterise this vegetation transition zone. Furthermore, the two decompositions show higher variability than the vegetation map, which can be used for a better vegetation classification of tropical wetlands. The lower performance of the RADARSAT-2 decomposition is surprising, because the C-band data corresponds to an intermediate frequency/wavelength between the X-and L-band data, which both revealed high sensitivity to tropical wetland vegetation. In order to verify these results, we checked five different RADARSAT2 quad-pol data sets acquired over the same area at different times with all four decompositions. The results of all 


\section{(a) TerraSAR-X}

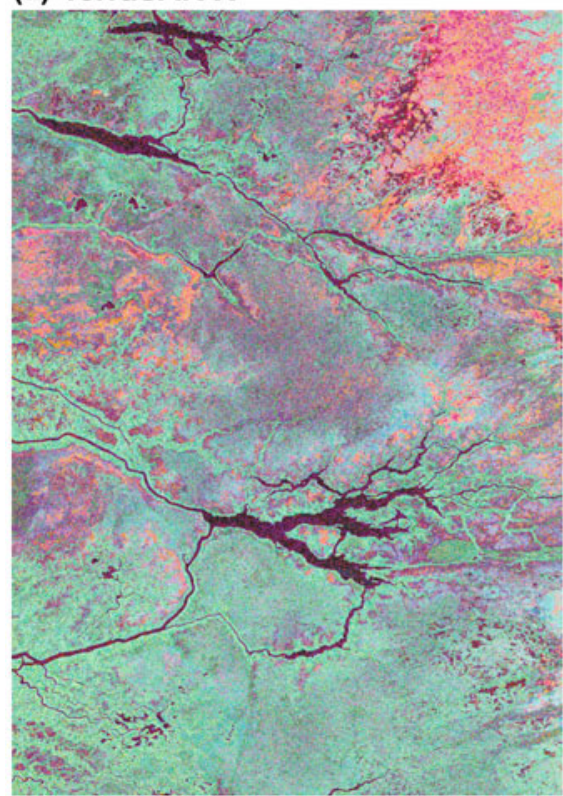

(d) Landsat

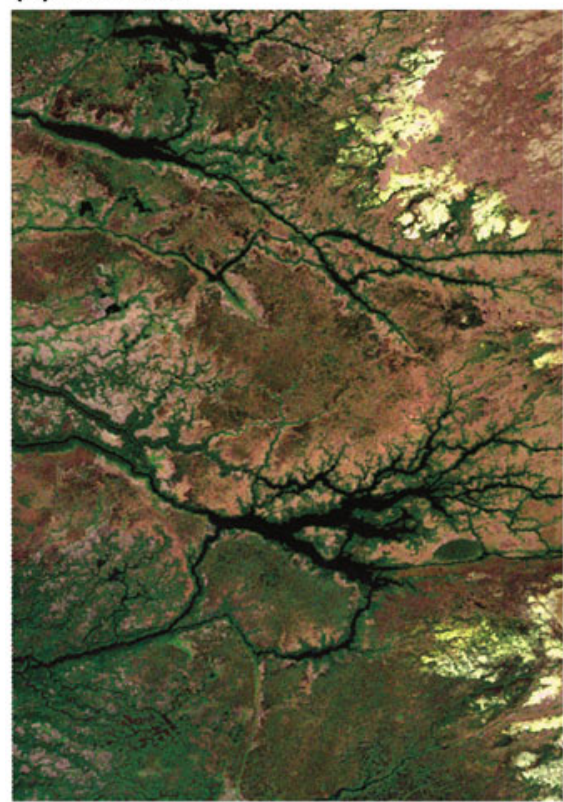

(b) Radarsat-2

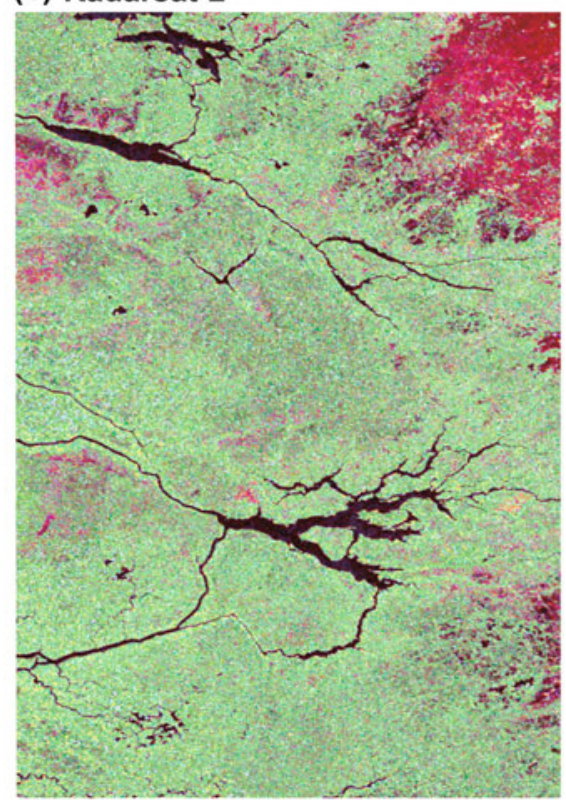

(e) Vegetation map

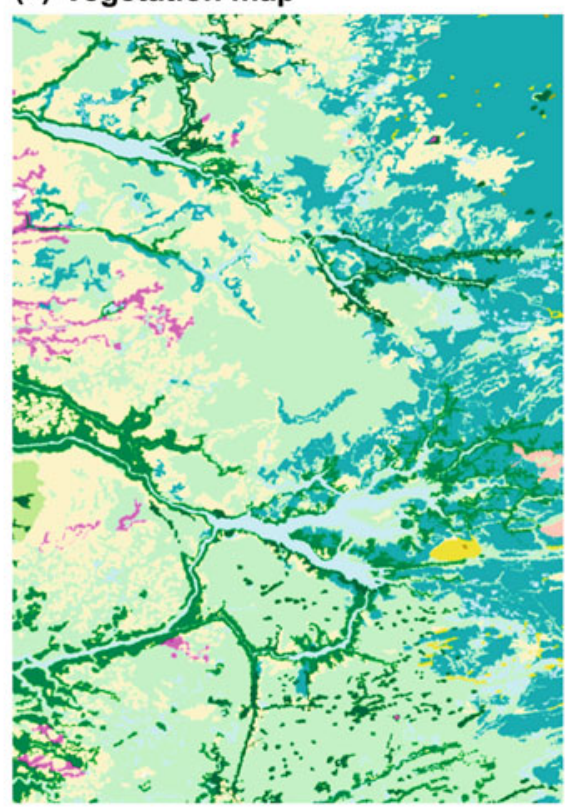

(c) ALOS

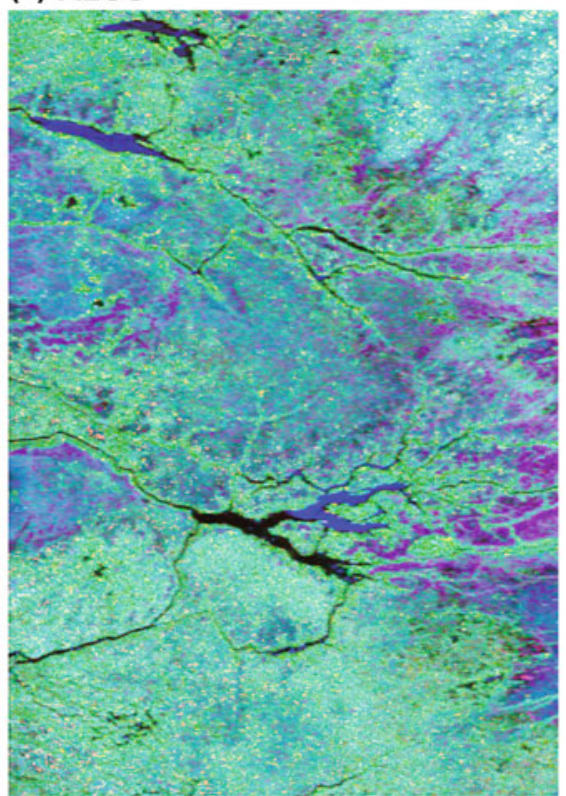

Fig. 3.28 Decomposition colour composite image of the Shark River Slough site in the Everglades using Hong-Wdowinski's approach (blue, single bounce; red, double bounce (both from the co- and the cross-pol); and green, volume scattering) of the quad-pol data sets: (a) X-band
TerraSAR-X, (b) C-band RADARSAT-2 and (c) L-band ALOSPALSAR. (d) Landsat-7 ETM+ optic colour composite image and (e) vegetation maps (Florida Coastal Everglades, Long Term Ecological Research (FCE LTER), http://fcelter.fiu.edu) decompositions are very similar to those presented here in Fig. 3.28b. A possible explanation for the lower performance of the C-band data is the vegetation wavelength that promotes Bragg scattering in C-band, as suggested by Atwood et al. (2013).

The analysis of the UAVSAR data set with four decompositions is presented in Fig. 3.29. The data covers a long swath of mainly mangrove forest located along the western coast of south Florida. The decompositions yielded similar results to that presented in Fig. 3.27, in which the Yamaguchi and $\mathrm{H} \& \mathrm{~W}$ decompositions show a better fit to the vegetation map.

\subsubsection{Comparison with Single-/ Dual-Polarisation Data}

The use of single- or dual-polarimetric data to characterise tropical wetlands yielded variable quality results, depending the radar frequency, image resolution, hydrological 
(a) Pauli

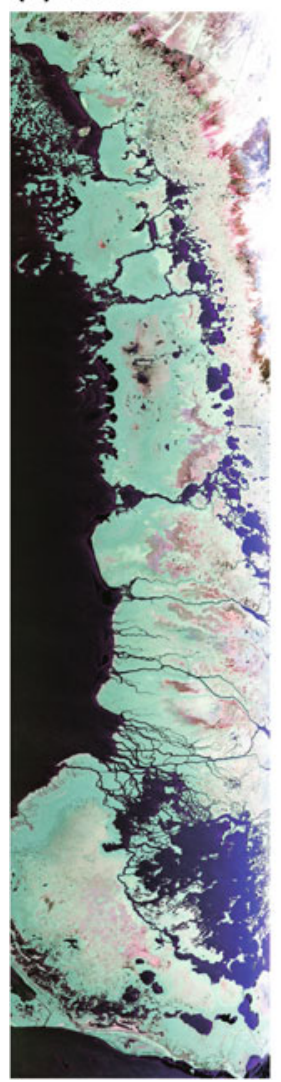

(b) Freeman

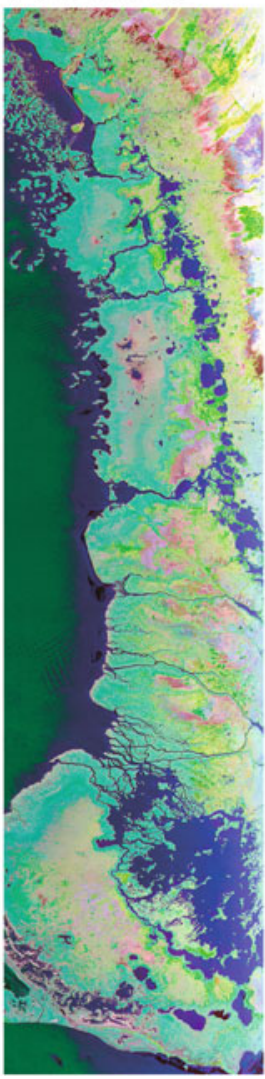

(c) Yamaguchi

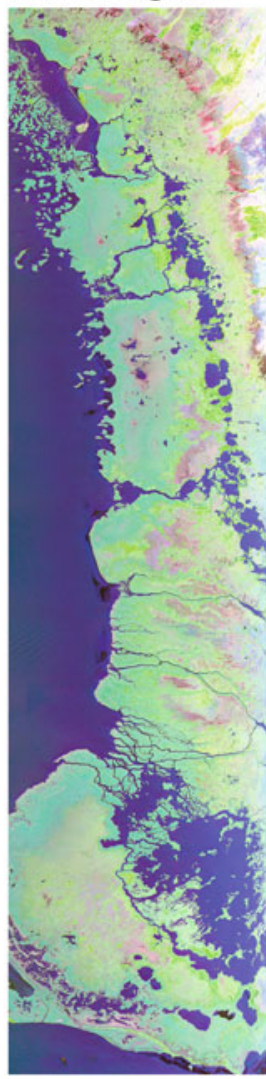

(d) H\&W

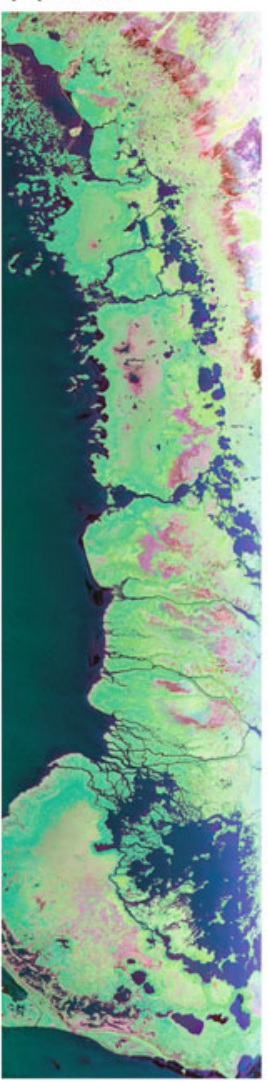

(e) Landsat

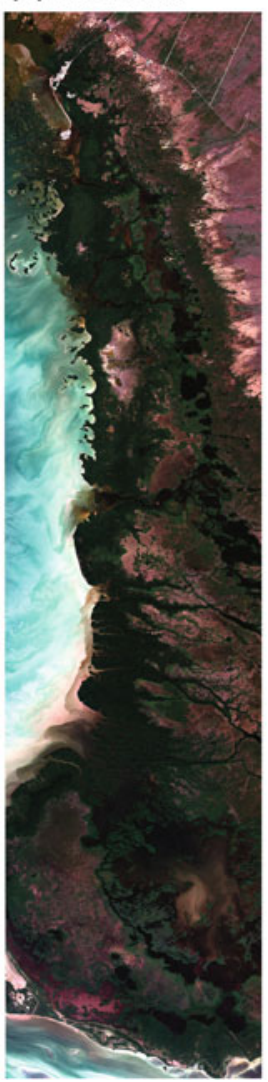

(f) Vegetation map

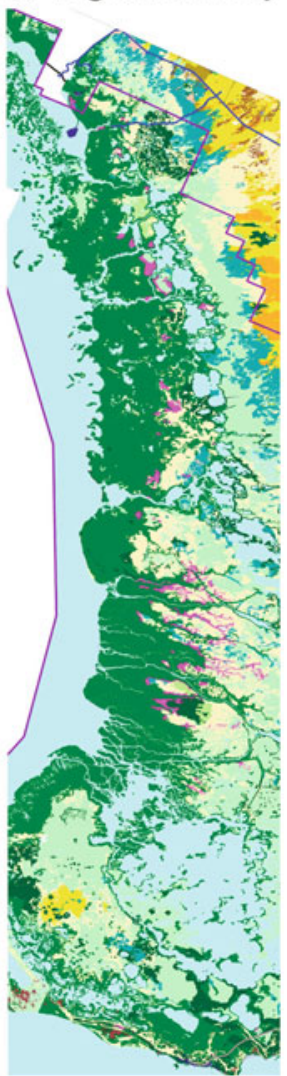

Fig. 3.29 Polarimetric decomposition results of the L-band UAVSAR data, acquired on 19th of April 2011. (a) Pauli decomposition presented as colour composite image with the following decomposition components: $\mathrm{HH}-\mathrm{VV}$ (red), $\mathrm{HH}+\mathrm{VV}$ (blue), and $\mathrm{HV}$ (green). (b) Freeman's three-component decomposition with the following components: blue, single bounce; red, double bounce; and green,

conditions and vegetation type (e.g. Bourgeau-Chavez et al. 2005). Single- and dual-pol can distinguish fairly well between woody and herbaceous vegetation and was successfully used to map mangrove forest distribution (e.g. Lucas et al. 2007). However, single- and dual-pol data are often not sufficient to distinguish between non-homogeneous vegetation distributions. Thus recent studies suggest the use of single- and dual-pol data combined with optical data for wetland characterisation (Bourgeau-Chavez et al. 2009; Evans and Costa 2013). Because quad-pol data contains twice or quadruple amount of data than dual- or single-pol data, the usage of quad-pol data might be sufficient for characterising tropical wetlands.

The examples presented in this study support the idea that quad-pol data can be sufficient for precise wetland characterisation, as the examples indicate high sensitivity of the various decomposition results to the different vegetation types. To illustrate the strength of the quad-pol characterisation, we compared our decomposition results with single- and dual-pol representations of the same wetland

volume scattering. (c) Yamaguchi's four-component decomposition with same colour scheme as in (b). (d) Hong and Wdowinski's threecomponent decomposition with cross-pol double bounce. (e) Landsat-7 ETM+ optic colour composite image. (f) Vegetation map of the study area. Legend is shown in Fig. 3.29

area (Fig. 3.30). The comparison shows that the single- and dual-pol images have limited ability to distinguish between the different vegetation types. The grey scale of single-pol image allows us to distinguish between 3 and 4 shades of grey corresponding to different vegetation types. The dualpol image that combines two ranges of colours (red and green) can distinguish 6-7 areas of different scattering characteristics. The quad-pol decomposition uses three ranges of colours (red, blue and green), which provide sufficient sensitivity to distinguish ten or more areas of different scattering characteristics, which are sufficient to characterise the main vegetation types in tropical wetlands.

\subsubsection{Discussion on the Role of Polarimetry, on the Maturity of the Application and Conclusions}

Decomposition analyses have been used for more than a decade to characterise landscapes according to scattering mechanisms. In that sense, it is a mature application for most landscape characterisation. However, the use of 


\section{(a) Single-pol: $\mathrm{HH}$}

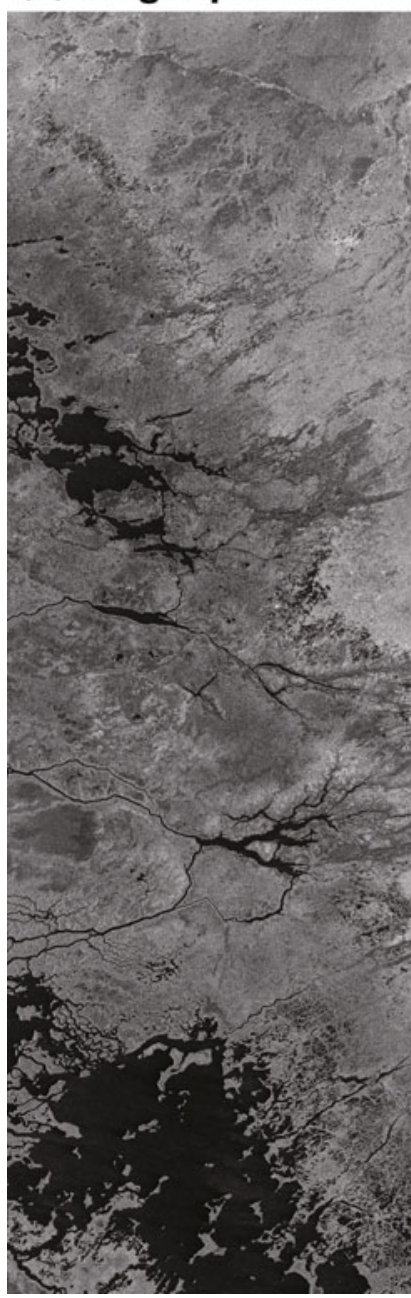

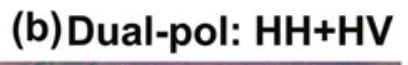

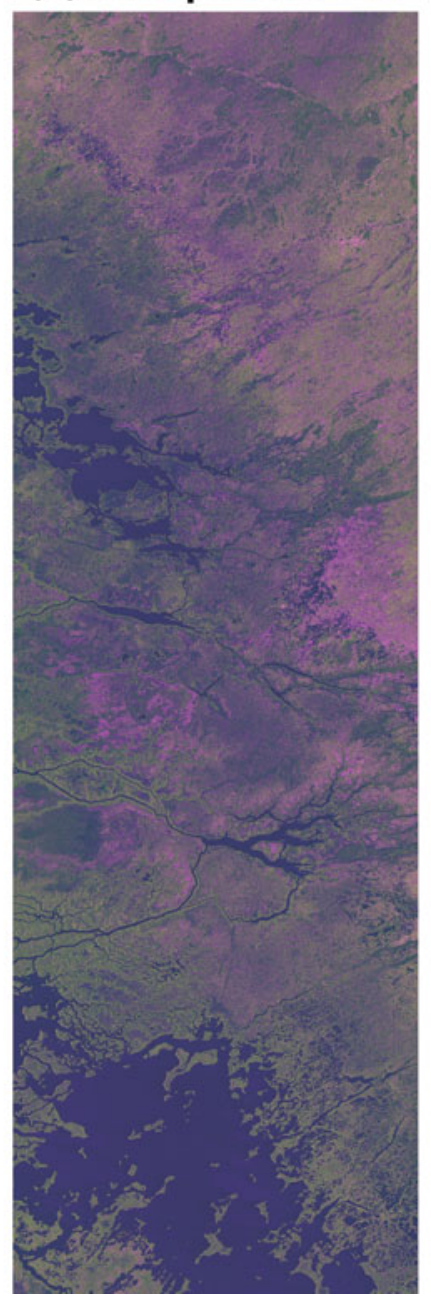

(c) Quad-pol: Pauli

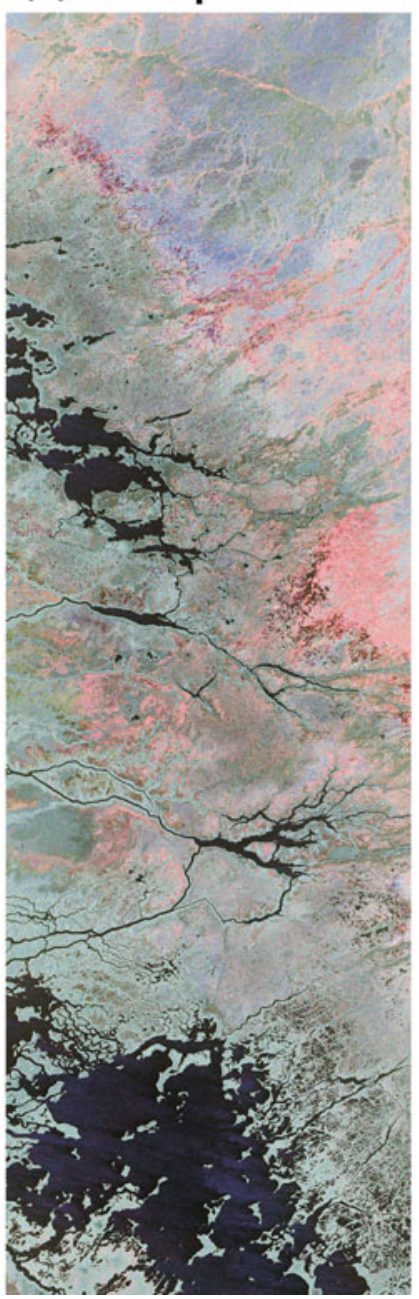

(d)Quad-pol: H\&W

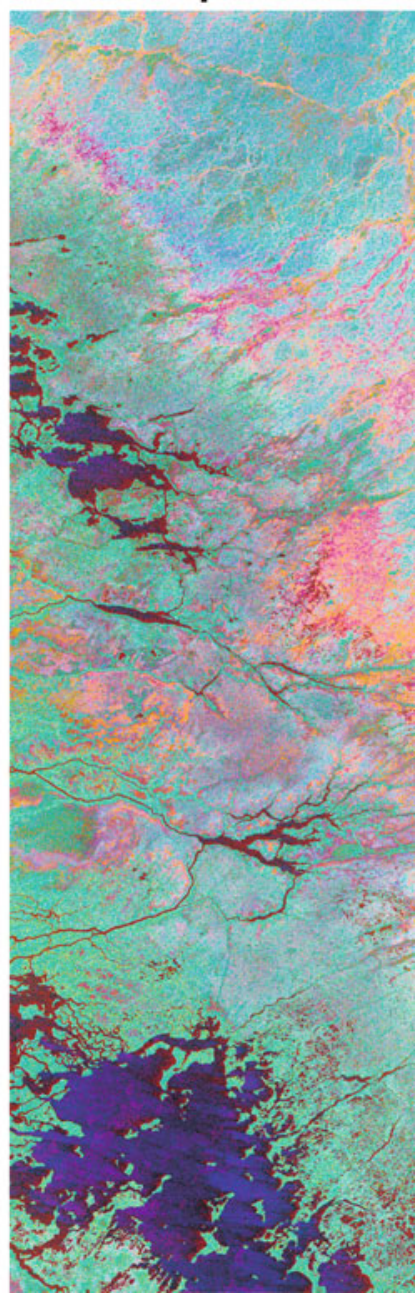

Fig. 3.30 Comparison between single-, dual- and quad-pol characterisation of tropical wetland area. (a) Amplitude image of single-polarimetric data of TerraSAR-X. (b) Colour composite image of dual-polarimetric data: HH (red), HV (green) and HH-HV (blue). (c) decompositions for characterising tropical wetlands was rather limited due to the limited available quad-pol data acquired over these wetlands. In this study we demonstrated that quad-pol decompositions are very useful methods for the characterisation of tropical wetlands. We analysed four quadpol data sets using four decomposition types. All decompositions were able to distinguish well between the main vegetation types, which include saltwater mangrove forests, freshwater sawgrass swamp and a transition zone with a mixture of vegetation types. Our analyses indicate that the TSX and ALOS quad-pol decompositions are more sensitive to vegetation variation in the transition zone than the RADARSAT-2 decomposition. Both TSX and ALOS decompositions show more details of vegetation distribution that the available vegetation cover map.
Pauli decomposition colour composite image: $\mathrm{HH}-\mathrm{VV}$ (red), $\mathrm{HH}+\mathrm{VV}$ (blue) and HV (green). (d) Hong and Wdowinski's three-component decomposition: double bounce (red), surface bounce (blue) and volume scattering (green)

\subsubsection{Subarctic Peatland Characterisation and Monitoring}

\subsubsection{Introduction, Motivation and Literature Review}

Wetlands play a key role in regional and global environments and are critically linked to major issues such as climate change, water quality, the hydrological and carbon cycles and wildlife habitat and biodiversity. Wetlands with at least 30-40 centimetres of peat accumulated on the surface represent an important class of wetland named peatland. Although peatlands globally only cover $3 \%$ of the land, they store $30 \%$ of the terrestrial carbon (Gorham 1995). Therefore, it is important to maintain and protect peatlands to reduce greenhouse gases. 
Unfortunately, major peatland transformations have been detected in the boreal and subarctic peatland regions. While it is well established that fens change naturally into bogs over time and that bogs can revert to fens, the observations over the last 50 years indicate that the rate of these changes has been significantly altered by various sources of stress (climate change, isostatic uplift, fire and anthropogenic activities) and this could have important implications (Gorham 1995; Brook and Kenkel 2002; Brook 2006; Jano et al. 1998). This has been noted in the Hudson Bay Lowlands of Manitoba, Canada, which contain the most extensive wetlands and thickest peat deposits in Canada (Brook and Kenkel 2002; Brook 2006; Jano et al. 1998). This region is home to unique concentrations of wildlife, most notably polar bears and caribous. Bears rely on inland denning habitat in the peatlands, and caribous are tied to peatland vegetation (bogs in winter and fens in summer). The loss of bogs will have important implications for polar bear denning habitat which is entirely within bogs with thick peat deposits (Brook and Kenkel 2002; Brook 2006; Jano et al. 1998). This is in addition to the impact on global warming that would occur due to the release of the carbon stored by peatlands. Therefore, there is an immediate need for cost-effective tools that permit accurate classification and mapping of peatlands, as well as long-term monitoring of their (bog-fen) transformations.

Cost-effective wetland characterisation and monitoring are now possible due to advances in the technology of earth observation satellites that provide the possibility of efficient monitoring of wetland status over large and remote areas (Fournier et al. 2007; Grenier et al. 2007). Recently, the visible near-infrared (VNIR) satellites (and Landsat in particular) have become the most popular source of information for wetland mapping. In Canada, Landsat 7 data combined with RADARSAT1 HH-polarisation SAR data have been used as the basis source of information for building the Canadian Wetland Inventory (Fournier et al. 2007; Grenier et al. 2007). The use of the additional all-weather single polarisation $(\mathrm{HH})$ RADARSAT1 information permits better delineation and monitoring of wetland water extent (Touzi et al. 2009; Grenier et al. 2007). Unfortunately, the combination with VNIR optical and SAR satellite information cannot clearly discriminate bogs from poor (shrubs and sedges) fens (Fournier et al. 2007; Grenier et al. 2007; Touzi et al. 2007). As a result, bogs are generally confused with fens in the North, and the bog to fen transformations related to climate change or anthropogenic activities may not be detected.

Since bog and fen vegetation can hardly be discriminated by optic and conventional SAR sensors, it might be interesting to use their different hydrological properties to discriminate these two peatland classes. In fact, fens and bogs differ in the way that they receive water and therefore receive nutrients differently. A bog is a peat-forming ecosystem that lacks any significant groundwater inflow and is therefore ombrotrophic. The bog soils are not affected by the mineralenriched ground waters from surrounding soils, since precipitation, fog and snow are the primary water sources. In contrast to the ombrotrophic bogs, the minerotrophic fens are connected to small streams and may also receive water from surrounding uplands (Ingram 1982; Zoltai and Vitt 1995). In this study, we will show that earth observation satellites equipped with L-band polarimetric SAR could provide the required information for cost-effective peatland mapping and monitoring in the boreal and subarctic peatlands. The unique capability of polarimetric SAR for the detection of peatland subsurface water flow, first demonstrated in (Touzi et al. 2007), could permit an enhanced discrimination of bog from fen, which will result in better monitoring of bog-fen transformations related to the various (natural and anthropogenic) sources of stress. This will be confirmed herein using polarimetric L-band ALOS collected over a boreal peatland, La Baie des Mines, and subarctic peatlands in the Wapusk National Park in Canada.

Several studies have been published on the investigation of polarimetric SAR for wetland classification (Pope et al. 1994, 1997; Hess et al. 1995; Sokol et al. 2004). Pope et al. $(1994,1997)$ have shown that the phase difference between the $\mathrm{HH}$ and $\mathrm{VV}$ polarisations, $\phi_{\mathrm{HH}}-\phi_{\mathrm{VV}}$, was the most useful parameter for flooded wetland classification and detection of seasonal flooding wetland changes. $\phi_{\mathrm{HH}}-\phi_{\mathrm{VV}}$, which characterises wetland scattering in terms of odd and even bounce interactions (Van Zyl 1989), permits an enhanced identification of marsh classes generally dominated by double-bounce interactions. However, they raised the point that the high variability of $\phi_{\mathrm{HH}}-\phi_{\mathrm{VV}}$ might limit its efficiency in wetland classification (Pope et al. 1994, 1997; Hess et al. 1995).

In the $1990 \mathrm{~s}, \phi_{\mathrm{HH}}-\phi_{\mathrm{VV}}$ was among the most used polarimetric parameters for natural target characterisation (Ulaby and Elachi 1990; Touzi et al. 2004). Currently, target scattering decomposition has become the standard method for the extraction of natural target geophysical parameters from polarimetric SAR data (Touzi et al. 2004; Boerner et al. 1998). The objective of incoherent target scattering decomposition (ICTD) theory is to express natural target average scattering as the sum of independent elements in order to associate a physical mechanism with each component (Cloude 1988; Van Zyl 1992). Several techniques have been proposed during the past two decades to decompose the scattering average covariance matrix. The first class (Van Zyl 1992; Touzi 2007a; Touzi and Shimada 2009) is based on the eigenvector-based decomposition (Cloude 1988) introduced by Cloude in the context of radar imaging. The latter (Cloude 1988) was an extension of Wiener's characteristic decomposition of wave coherence matrix to target coherency matrix (Touzi and Shimada 2009; Wiener 1930). The 
characteristic decomposition of the averaged target coherency matrix (Cloude 1988) permits the characterisation of global target scattering with two parameters, the CloudePottier "averaged" scattering type $(\alpha)$ and the entropy $(H)$. This served as the basis of the very popular Cloude-Pottier $\alpha /$ $H$ classification (Cloude and Pottier 1997). The second class corresponds to model-based decompositions (Freeman and Durden 1998; Yamaguchi et al. 2005; van Zyl et al. 2011), which are based on Freeman's basic idea (Freeman and Durden 1998), supposing that target observed scattering can be modelled as the linear sum of scattering that can be represented by models of the physical scattering process. Freeman's decomposition assumes that target scattering can be modelled as the linear sum of surface, double-bounce and volume scattering (Freeman and Durden 1998).

In this study, the Touzi decomposition (Touzi 2007a; Touzi et al. 2007) will be assessed for peatland characterisation using L-band polarimetric ALOS data. This method, which exploits Cloude's eigenvector-based decomposition (Freeman and Durden 1998), permits a highresolution decomposition of target scattering in terms of roll-invariant and unique target characteristics (Touzi 2007a; Touzi et al. 2009). In contrast to Cloude-Pottier ICTD (Cloude and Pottier 1997), the new decomposition uses a complex entity, $\left(\alpha_{s}, \phi_{\alpha s}\right)$ for an unambiguous description of target scattering type (Touzi et al. 2009; Touzi 2007a). The information provided by both the scattering type magnitude $\alpha_{s}$ and phase $\phi_{\alpha s}$ has been shown to be important for wetland classification (Touzi et al. 2007, 2009). The analysis of C-band SAR data collected with the Convair-580 over the RAMSAR Mer Bleue peatland, near Ottawa, Canada, permits the demonstration of the unique potential of the scattering type phase, $\phi_{\alpha s}$, for bog-fen discrimination (Touzi et al. 2007, 2009). These two classes could not be separated using the multi-polarisation ( $\mathrm{HH}, \mathrm{HV}, \mathrm{VV})$ intensity or the $\mathrm{HH}-\mathrm{VV}$ phase difference information (Touzi et al. 2007, 2009). These very promising results obtained with the scattering type phase are confirmed in the following using polarimetric L-band ALOS data collected over boreal and subarctic peatlands (Touzi and Gosselin 2010). We will show in the following that the phase of the dominant scattering type $\phi_{\alpha s}$ is sensitive to peatland subsurface water flow variations, and this permits an efficient discrimination of bog from poor fens. The use of the additional information provided by the scattering type magnitude $\alpha_{s}$ is also required for effective wetland classification.

\subsubsection{Experimental Results}

Test sites and corresponding radar and validation data sets selected for subarctic peatland characterisation and monitoring are summarised in Table 3.9 and further described in the Appendix.

\subsection{La Baie des Mines}

\section{Peatland Hydrology Characteristics for Bog-Fen}

Discrimination

Open (sedge and shrub) bogs and poor (sedge) fens are regrouped in the peatland class of Fig. 9.1a. Traditional definitions of peatlands denote that a minimum peat layer of $30-40 \mathrm{~cm}$ is needed for an ecosystem to be considered as a peatland (Ingram 1982; Zoltai and Vitt 1995). Both bogs and fens in the peatland study site satisfy this criterion with peat thickness varying from $1 \mathrm{~m}$ to $3 \mathrm{~m}$ according to the field data. Shrub bog, which is the dominant class, has a complete ground cover of sphagnum moss with a shrub canopy dominated by ericaceous shrubs (such as Labrador tea). Poor fen areas contain vegetation that is composed primarily of sedges and an understory of sphagnum mosses. Since bog and fen vegetation can hardly be discriminated by optic and SAR sensors, their different hydrological properties can be used to discriminate them, as demonstrated in the following. In particular, the depth of peatland subsurface water will be shown to be a key parameter for bog-fen discrimination. A bog consists of two layers: one is the upper thin layer about $45 \mathrm{~cm}$ deep, known as acrotelm, through which rain sinks rapidly. Below the acrotelm, there is a much thicker layer of peat, the catotelm, of dramatically reduced hydraulic conductivity and through which water movement is very slow, which is typically less than $1 \mathrm{~m}$ /day (Ingram 1982; Zoltai and Vitt 1995; Fraser et al. 2001). Unlike bogs, a poor fen peat has a higher capacity of water retention, and water moves slowly through the fen. The field measurements in La Baie des Mines site reveal a water table $20 \mathrm{~cm}$ below the poor fen peat surface, whereas the bog subsurface water lies on the bottom of the acrotelm at about $40 \mathrm{~cm}$ below the peat surface. This is in agreement with other studies, which show that bog water remains generally $30-40 \mathrm{~cm}$ below the peat

Table 3.9 Test sites and corresponding radar and validation data selected for the generation of showcases on subarctic peatland characterisation

\begin{tabular}{l|l|l}
\hline Application/product & Test site - radar data & Reference data \\
\hline Subarctic peatland characterisation & La Baie des Mines Peatland, Quebec, Canada & Classification based on Ikonos images and forest inventory \\
\cline { 2 - 3 } & ALOS-PALSAR, 10/11/2006, 13/05/2007 & Fieldwork data \\
\cline { 2 - 3 } & Wapusk National Park, Manitoba, Canada & Brook's classification (Brook and Kenkel 2002) \\
\cline { 2 - 3 } & ALOS-PALSAR, 08/06/2010, 24/07/2010 & Landsat-5 images, 18/07/2010, 04/09/2010 \\
\hline
\end{tabular}




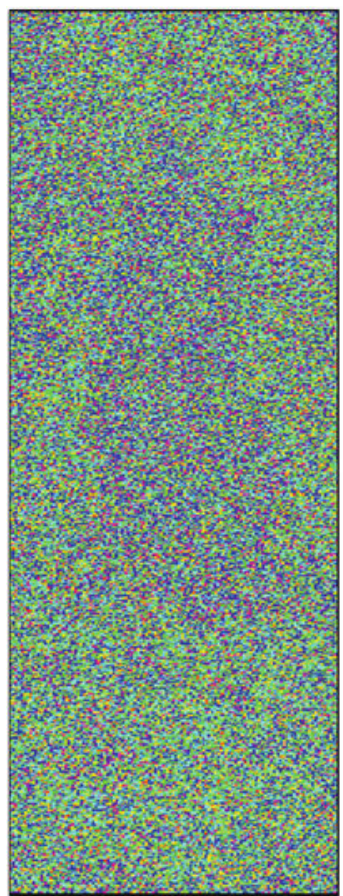

(a)

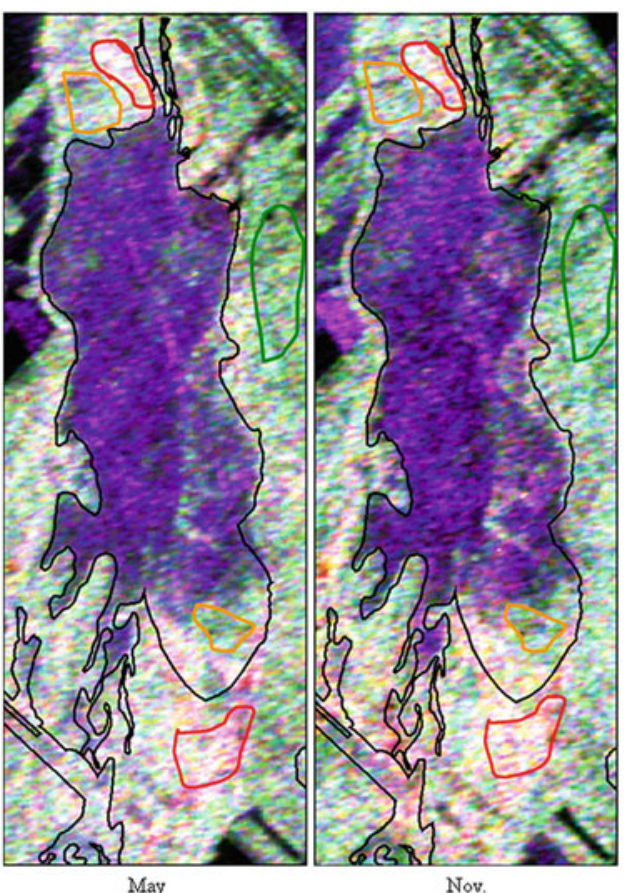

(b)

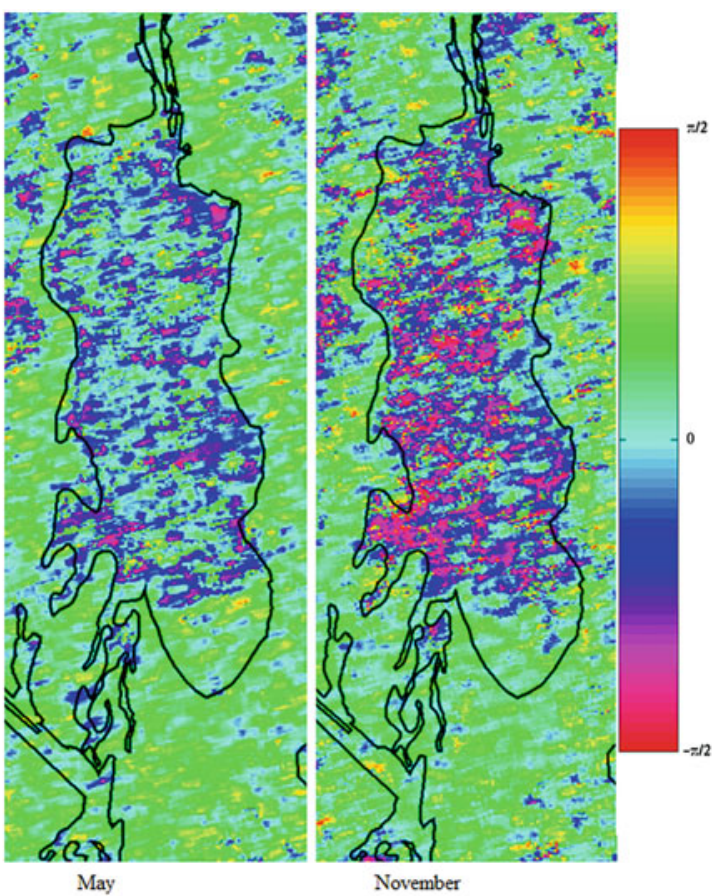

(c)

Fig. 3.31 La Baie des Mines Peatland. (a) 1-look scattering type phase (May). (b) Multi-polarisation images for May and November acquisitions (HH in red, $\mathrm{HV}$ in green and VV in blue). Wetland samples

are delineated: swamp (red), upland forest (green), treed bog (brown), open peatland (black). (c) Scattering type phase images

surface (at the catotelm layer) (Ingram 1982; Touzi et al. 2007; Fraser et al. 2001)).

\section{Application of the Touzi Decomposition to Polarimetric ALOS Data: Required Processing Window Size for Unbiased ICTD}

As mentioned above, our decomposition may be considered as an extension of Kennaugh-Huynen decomposition, which solves for con-eigenvalue phase ambiguities and can be applied for the decomposition of both coherent (presented in terms of the $[S]$ matrix) and partially coherent (presented in terms of the coherency $[T]$ matrix) target scattering. Since bog scattering might be highly coherent, the application of the decomposition under coherent conditions (1-look image) should preserve the spatial resolution. In that case, the parameters provided by the Touzi decomposition would be equivalent to the ones obtained with the Kennaugh-Huynen CTD after phase ambiguities removal. Under the assumption of coherent scattering, the decomposition is applied with $1 \times 1$ processing window on the May acquisition. Figure 3.31a presents the 1-look scattering type $\phi_{\alpha s}$. As can be noted, the phase is random because of the presence of speckle, and no useful information can be extracted. The fact that each resolution cell is not dominated by a single coherent scatterer does not permit the generation of a meaningful scattering decomposition using the CTD. We have previously shown that a processing window with a minimum of
60 independent samples is required for unbiased estimation of the ICTD parameters (Touzi 2007b). A 3-look image is firstly generated in Mueller matrix with a square pixel by replacing each pixel's Mueller matrix with the Mueller matrix averaged over the 3 pixel azimuth segment centred on the pixel. The ICTD is then applied to the 3-look image using a $7 \times 7$ processing window. The decomposition is applied under the target reciprocity assumption, and the cross-polarisation magnitude is taken as the average of $\mathrm{HV}$ and VH magnitude to increase the signal-to-noise ratio of $3 \mathrm{~dB} \mathrm{HV}=e^{j \phi_{H V}}(|\mathrm{HV}|+|\mathrm{VH}|) / 2$ (where $\phi_{\mathrm{HV}}$ is the phase of $\mathrm{HV}$ ).

\section{Analysis of the ALOS Acquisitions}

The May acquisition, in dry conditions, provides the most suitable data set for wetland classification. The dominant scattering parameters are generated with a processing window that includes more than 60 independent samples. A colour wheel with equally spaced bins between $-\pi / 2$ and $\pi /$ 2 is used to represent the scattering type phase $\phi_{\alpha s 1}$ of the dominant scattering. $\phi_{\alpha s 1}$ image of the May acquisition is presented in Fig. 3.31c, and the scattering type magnitude $\alpha_{s 1}$ image is presented in Fig. 3.32a. The classification and the field data are used to compute the statistics of the scattering type parameters and $\sigma^{0}$ for the $\mathrm{HH}, \mathrm{VV}$ and $\mathrm{VV}$ polarisation, for the various wetland classes: (poor) fen, open (sedge and shrub) bog, treed bog, swamp and upland forests. Table 3.10 
presents for each class the average and variation of each parameter for each class using the samples outlined in Fig. 3.31b. Analysis of Figs. 3.31c and 3.32a and the dominant scattering type statistics of Table 3.10 lead to the following conclusions:

1. $\phi_{\alpha s}$ permits a clear separation of poor fen $\left(\phi_{\alpha s}\right.$ about $\left.-60^{\circ}\right)$ from open bog $\left(\right.$ about $\left.-18^{\circ}\right)$. $\phi_{\alpha s}$ has similar values (about $30^{\circ}$ ) for the various treed areas: swamp, treed bog and upland forests.

2. Even though the scattering magnitude $\alpha_{s 1}$ cannot separate poor fen from bogs, $\alpha_{s 1}$ discriminates well the swamp class from the upland forest and treed bog. $\alpha_{s 1}$ is very efficient in detecting the quasi-dipole scattering $\left(\alpha_{s 1}=40^{\circ}\right)$ due to the wave interactions of water and trees in the swamp. The fact that the contribution of the trihedral and dihedral scattering is not added in phase $\left(\phi_{\alpha s}=31^{\circ}\right)$ leads to a quasi-dipole scattering instead of the perfect-dipole scattering (with $\alpha_{s 1}=45^{\circ}$ and $\phi_{\alpha s}=0^{\circ}$ ) (Touzi et al. 2007).

3. It is worth noting that the swamp quasi-dipole scattering can also be detected with $\mathrm{HH}$ (much larger than VV and HV) as can be expected.
Therefore, we can conclude that the information provided by both the scattering type magnitude and phase is required for wetland characterisation. To validate the unique potential of the scattering type phase for discrimination of poor fens from bogs, in situ field measurements were collected. The areas that appear in pink $\left(\phi_{\alpha s 1}\right.$ about $\left.-60^{\circ}\right)$ on Fig. 3.31c were effectively identified as fens. They are dominated by herbs, and the water level lies between 10 and $20 \mathrm{~cm}$ under the peat surface. The sites that look dark blue on Fig. 3.31c were identified as bogs. They are dominated by Labrador tea and a very thick peat (from $1.6 \mathrm{~m}$ to $3 \mathrm{~m}$ deep). The water table is much deeper $(40-50 \mathrm{~cm}$ under the peat surface) in the bog samples, as expected according to hydrologic properties of bogs (Fraser et al. 2001).

\section{Peatland Subsurface Water Flow Monitoring Using \\ Polarimetric May and November ALOS Acquisitions: \\ Multi-polarisation Versus Polarimetric Information}

The spring acquisition took place under dry conditions; no rain for 14 days and warm weather with a temperature between $10^{\circ}$ and $20^{\circ}$. The fall image was collected under cool weather (with a temperature below $5^{\circ}$ ) and wet

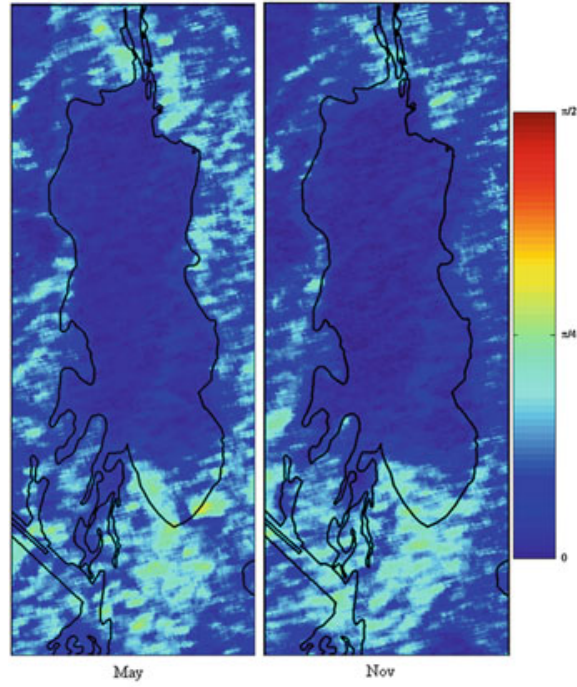

(a)

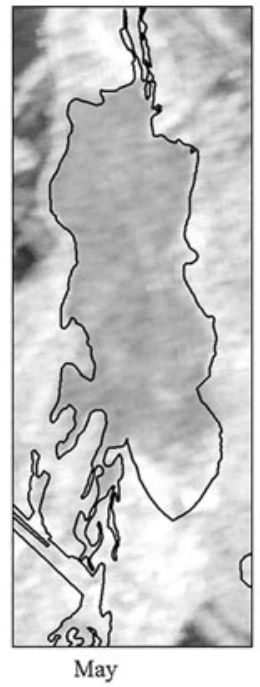

(b)

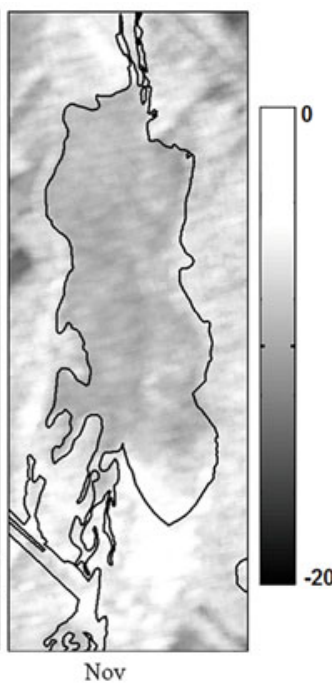

b)

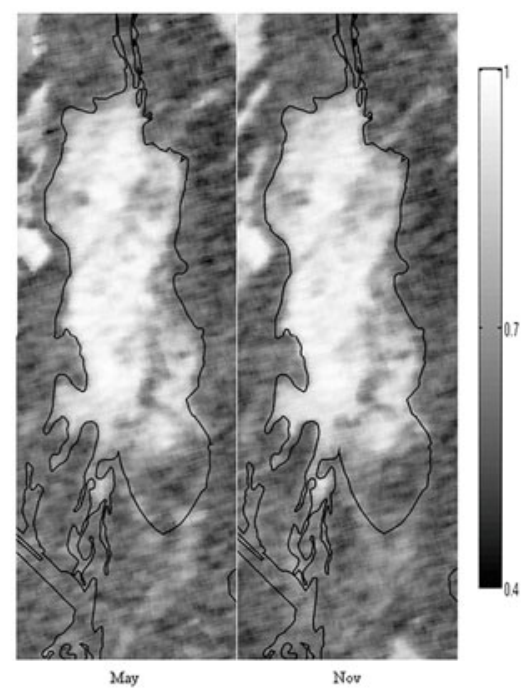

(c)

Fig. 3.32 May and November images: (a) $\alpha_{s 1} ;$ (b) $m_{1} ;$ (c) $\lambda_{1}$

Table 3.10 Scattering type parameters estimates and HH, HV and VV for May acquisition

\begin{tabular}{|c|c|c|c|c|c|}
\hline Class & $\phi_{\alpha s 1} \mathrm{Z}(\mathrm{deg})$ & $\alpha_{s 1} \mathrm{Z}(\mathrm{deg})$ & $\mathrm{HH}(\mathrm{dB})$ & VV (dB) & $\mathrm{HV}(\mathrm{dB})$ \\
\hline Open bog & $-18^{\circ} \pm 3^{\circ}$ & $6^{\circ} \pm 3^{\circ}$ & $-6.8 \pm 1$ & $-7.1 \pm 1$ & $-17.5 \pm 1$ \\
\hline Poor fen & $-60^{\circ} \pm 3^{\circ}$ & $6^{\circ} \pm 3^{\circ}$ & $-6.8 \pm 1$ & $-7.2 \pm 1$ & $-17 \pm 1$ \\
\hline Treed bog & $30^{\circ} \pm 3^{\circ}$ & $15^{\circ} \pm 3^{\circ}$ & $-5 \pm 1$ & $-6.5 \pm 1$ & $-12 \pm 1$ \\
\hline Swamp & $31^{\circ} \pm 3^{\circ}$ & $40^{\circ} \pm 3^{\circ}$ & $-2.5 \pm 1$ & $-5.6 \pm 1$ & $-11 \pm 1$ \\
\hline Forest & $30^{\circ} \pm 3^{\circ}$ & $25^{\circ} \pm 3^{\circ}$ & $-5 \pm 1$ & $-6.5 \pm 1$ & $-12 \pm 1$ \\
\hline
\end{tabular}


conditions; about $10 \mathrm{~mm}$ rain accumulated during the 2 days that precede the acquisition. Since the L-band ALOS penetrating wave is sensitive to wetland groundwater conditions, we should expect a significant change in radar backscattering at $\mathrm{HH}, \mathrm{HV}$ and $\mathrm{VV}$ polarisations. Figure $3.31 \mathrm{~b}$ presents the composite colour of $\mathrm{HH}, \mathrm{HV}$ and $\mathrm{VV}$ for both acquisitions. Water-level change can be noted in the swamps outlined in Fig. 3.31b. However, no change can be detected in the open peatland. The multi-polarisation information looks similar for the two acquisitions even though we might expect significant changes in the peatland subsurface water flow between the dry and wet acquisitions. These changes cannot also be detected using the dominant scattering type parameters $\alpha_{s 1}, m_{1}$ and $\lambda_{1}$ as can be noted in Fig. 3.32. Analysis of all the other intensity parameters (span, $\lambda_{i}, m_{i}, i=1, \ldots, 3$ ) and Cloude-Pottier's parameters ( $\alpha$, entropy and anisotropy) leads to similar conclusions. None of them are sensitive to water flow changes beneath the peat surface.

Like in (Touzi 2007a), the scattering type phase $\phi_{\alpha s 1}$ seems to be the only polarimetric parameter that can detect peatland subsurface water flow changes, as can be noted in Fig. 3.31c. Major changes (pink to dark blue) in $\phi_{\alpha s}$ can be noted when the phase images of May and November are compared. These significant variations (larger than $40^{\circ}$ ) represent the significant variations of the water flow beneath the peat surface between the dry and wet (May-November) peatland conditions. The pink colour, which indicates the presence of subsurface water, is dominant in the November acquisition. The latter acquisition took place shortly after the rain stopped, and this does not give enough time for the rain water to sink deeply into the acrotelm. This makes fen-bog discrimination difficult since the L-band wave reaches the subsurface water in both fens and bogs. Bog-fen discrimination is easier with the May data set collected under dry conditions, as discussed previously.

It is worth noting that similar observations regarding the higher sensitivity of the radar signal phase to subsurface features in comparison with the detected intensity were brought out by Lasne et al. (2004). Using the airborne L-band RAMSES SAR, they showed that the phase difference $\phi_{\mathrm{HH}}-\phi_{\mathrm{VV}}$ permits the detection of pale soils buried in a bare sandy area near Bordeaux (France), at a depth greater than $5.2 \mathrm{~m}$. These pale soils cannot be detected with the radar signal intensity at $\mathrm{HH}, \mathrm{HV}$ or VV polarisation, which is only sensitive to the presence of pale soil that is not deeper than $3.5 \mathrm{~m}$ (Lasne et al. 2004). One might expect that the phase difference $\phi_{\mathrm{HH}}-\phi_{\mathrm{VV}}$ of the like-polarisations is also sensitive to the peatland subsurface water flow. The phase $\phi_{\mathrm{HH}}-\phi_{\mathrm{VV}}$ and the Pauli phase difference, i.e. phase of $(\mathrm{HH}-\mathrm{VV}) /(\mathrm{HH}+\mathrm{VV})$, are presented in Fig. 3.33a, b, respectively. As can be seen, both $\mathrm{HH}-\mathrm{VV}$ phase difference

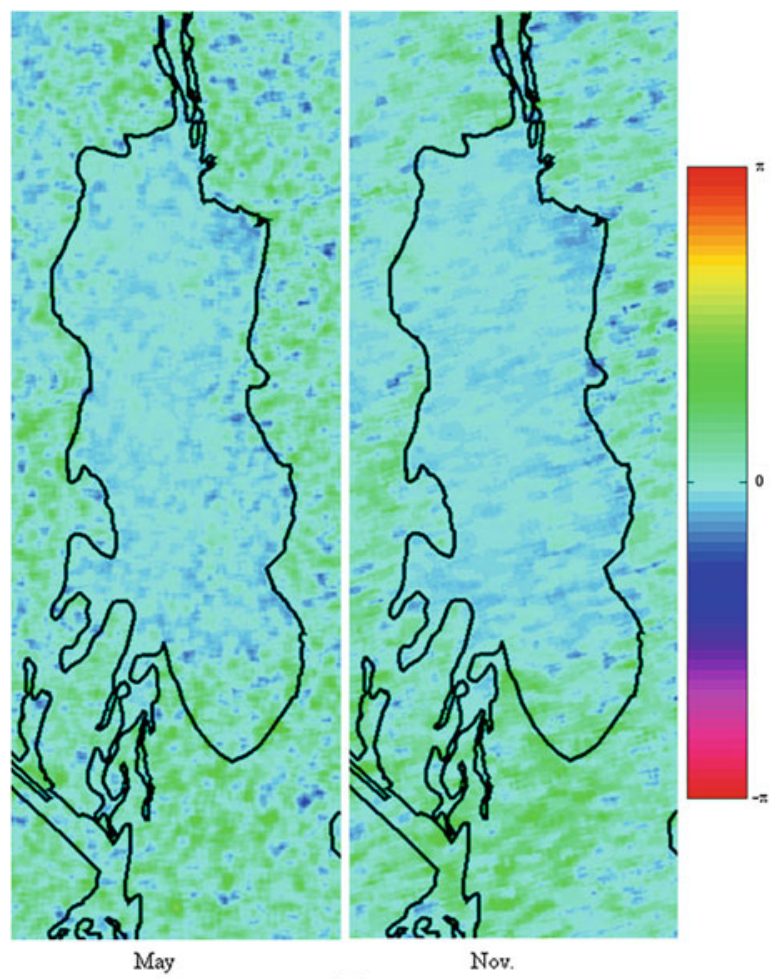

(a)

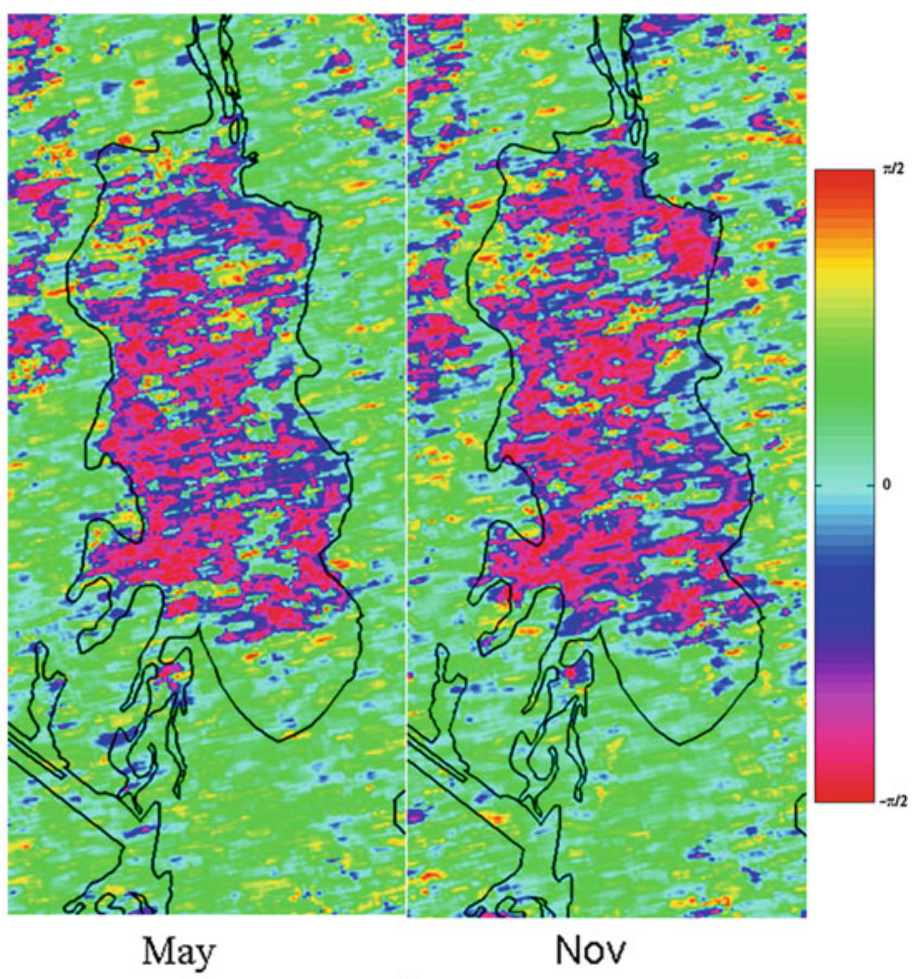

(b)

Fig. 3.33 Phase and orientation images: (a) $\phi_{\mathrm{HH}}-\phi_{\mathrm{VV}}$; (b) Pauli phase difference 
and the Pauli phase are not able to detect peatland subsurface water flow changes. This is in agreement with the results of a previous study on the Mer Bleue wetland (Touzi 2007a). The optimum phase $\phi_{\alpha s 1}$, which is sensitive to peatland subsurface water flow, is generated with a non-zero orientation angle in a polarisation basis different from the conventional $(\mathrm{H}-\mathrm{V})$ linear-polarisation basis. The Kennaugh-Huynen diagonalisation of the coherent dominant scattering matrix leads to the generation of the maximum polarisation, whose phase $\phi_{\alpha s 1}$ permits the detection of peatland subsurface water flow. It is worth noting that such promising results obtained with $\phi_{\alpha s 1}$ could only be derived, thanks to the excellent quality of ALOS HV measurement in terms of calibration and high signal-to-noise HV measurement (Touzi and Shimada 2009). An accurate measurement of HV is required to generate the optimum polarisation whose scattering type phase $\phi_{\alpha s 1}$ is sensitive to the water beneath the peat surface. This makes this unique application suitable with only fully polarimetric SAR. The reconstruction of HV from dualpolarisation RH-RV Compact (Souyris et al. 2005; Raney 2007) SAR measurements (using Souyris's method (Souyris et al. 2005)) does not permit the generation of the optimum $\phi_{\alpha s}$ required for peatland subsurface water flow monitoring, as demonstrated in (Touzi 2013).

\subsection{Wapusk National Park}

The area of study is dominated mainly by a sedge bulrush poor fen (dark orange) in an area that includes lichen melt pond bog, peat plateau bog, spruce bog and sedge-rich fens. During the spring and summer active layer melting season, we should expect important changes in the peat subsurface water flow in the fen class. A thermistor cable was installed in a pond bog (Dyke and Wendy 2010). The active layer thickness was $13 \mathrm{~cm}$ in June at the start of the melting season, $27 \mathrm{~cm}$ in July and more than $80 \mathrm{~cm}$ in September. Figure 3.34 presents the colour composite of HH (red), HV (green) and VV (blue) for the June and July acquisitions, respectively. As can be noted, the radiometric information provided by the multi-polarisation information cannot detect any change in the peatland hydrology between the June start of the melting season and the late part of July.

Our ICTD is applied to the ALOS images as described in Sect. 9.5.1.3, and the various ICTD parameters are analysed. Again, the scattering type phase $\phi_{\alpha s}$ of Fig. 3.35 is the only target scattering decomposition parameter that has revealed peatland subsurface flow variations. Major changes in $\phi_{\alpha s}$ can be noted when the phase images of early June and late July are compared. The bulrush sedge poor fen area, in particular, outlined in Fig. 3.35a (and denoted (2)) shows significant changes. This area is dominated in the June image by the pink colour, as might be expected. $\phi_{\alpha s}$ is sensitive to the fen shallow subsurface water lying on the permafrost surface (about $13 \mathrm{~cm}$ deep). In July, the fen is still irrigated by shallow subsurface water that can be detected (in pink) by the L-band wave, whereas a large part of the fen subsurface water either has been evaporated or was too deep (more than $25 \mathrm{~cm}$ ) to be detected by $\phi_{\alpha s 1}$. No change can be noted on the bogs (in dark blue) between June and July acquisition, and the recent field trip allowed us to explain the phase behaviours in these bog areas, as discussed in the following.

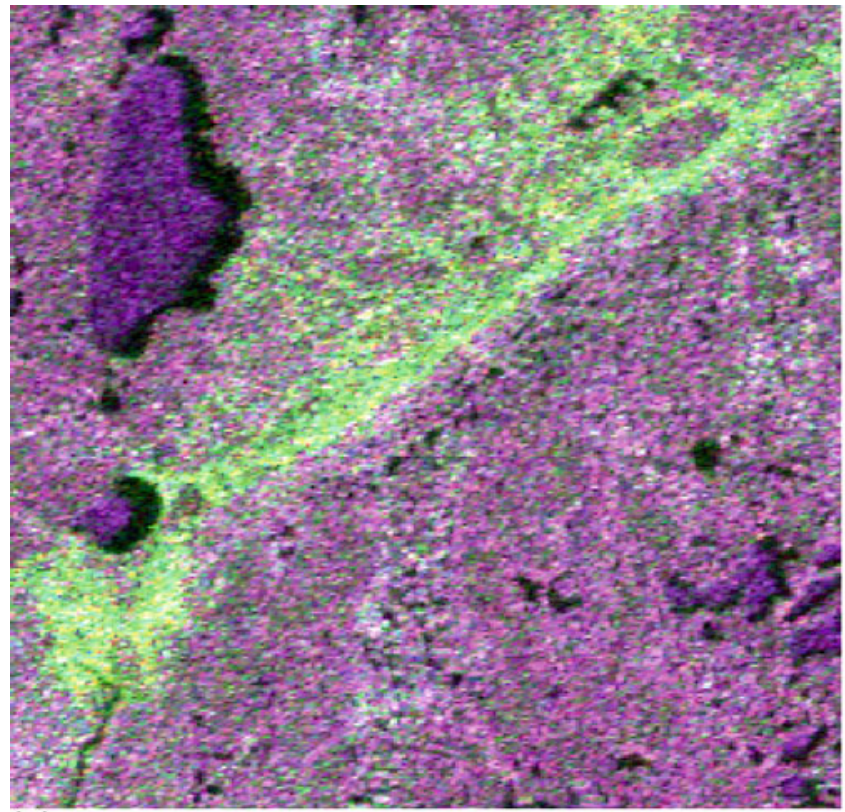

(a)

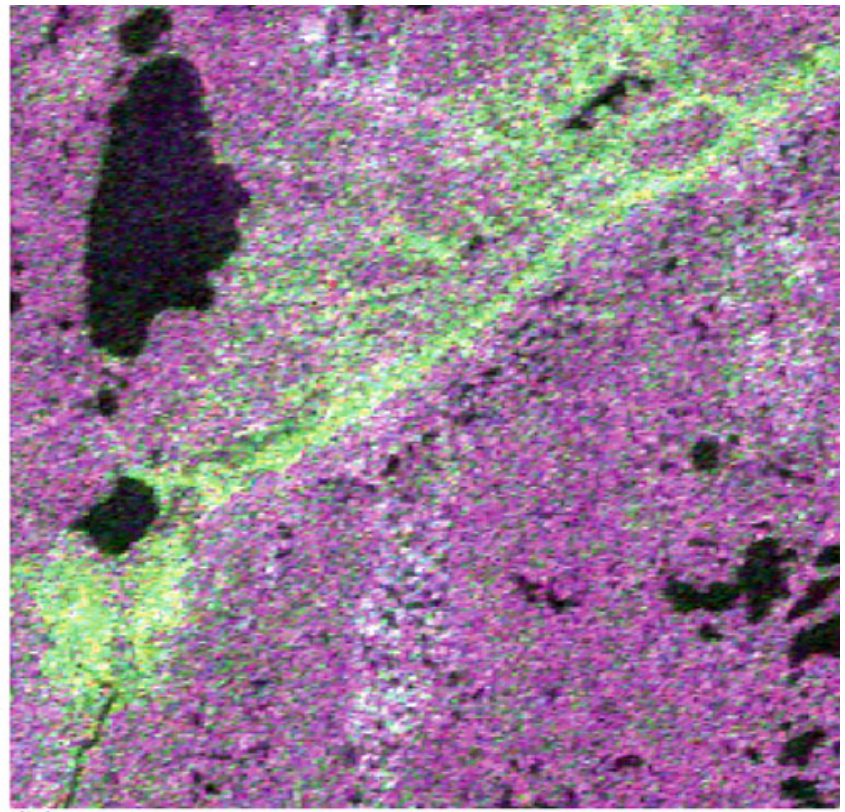

(b)

Fig. 3.34 Multi-polarisation (HH, HV, VV) images for the bog-fen peatland in Wapusk National Park (June and July 2010) 


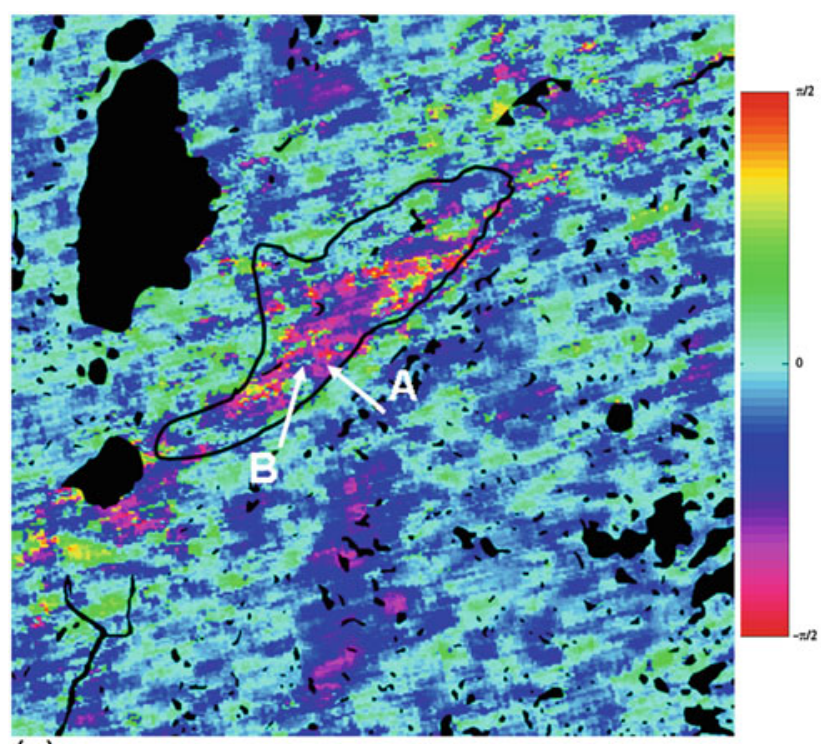

(a)

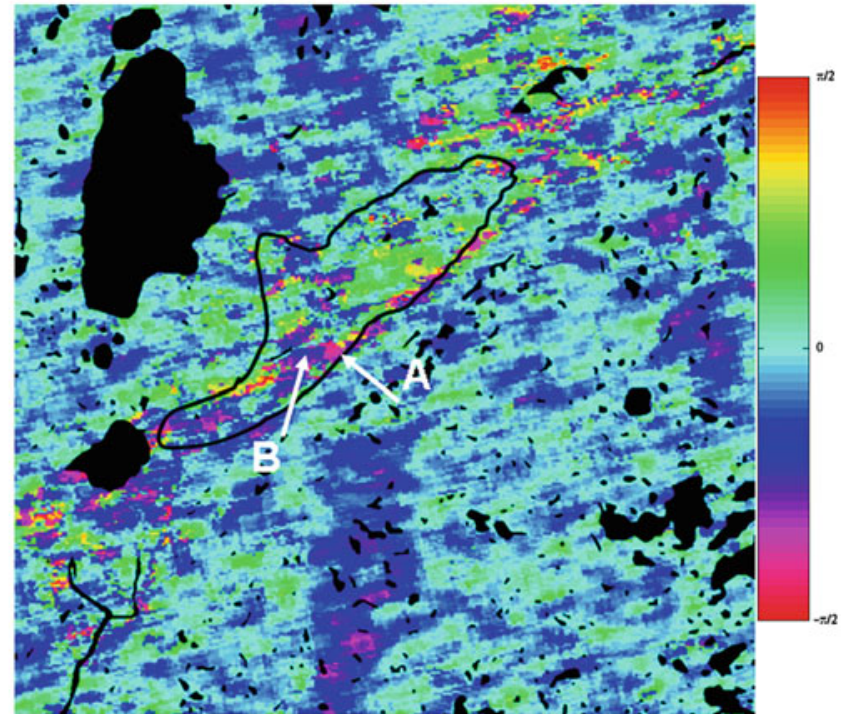

(b)

Fig. 3.35 Scattering type phase image for the bog-fen peatland in Wapusk National Park: (a) June; (b) July

In the first week of July 2012, additional in situ field measurements were collected in the Wapusk National Park peatland for further validation of the results above. Helicopter was used to visit various samples in the areas 1 and 2 outlined in Fig. 3.35a. These areas were assigned to the sedge bulrush fen and lichen melt pond bog classes, respectively, according to Brook's classification. Water-level measurements, vegetation species characterisation, active layer and peat thickness were collected in the various sites. The average of active layer thickness in the bogs and fens visited during the field trip in July is deeper than $25 \mathrm{~cm}$. Areas in pink were identified as fens with herb vegetation and water level 10 to $20 \mathrm{~cm}$ beneath the peat surface. Samples in dark blue were identified as bogs. No water was detected under the peat surface or at the frozen ground about $25 \mathrm{~cm}$ under the peat surface. The water coming to bogs from precipitations has evaporated, while the fens are continuously irrigated by subsurface and runoff water. The absence of subsurface water in bogs should explain the stability of the phase (dark blue) between the June and July acquisitions, in particular in the area (1) on the bottom of in Fig. 3.35. This area (1) outlines a fen site A (pink in the phase image) and a bog site B (dark blue in the phase image) that were visited. Pictures of the corresponding peat samples are shown in Fig. 3.36. While no water can be seen under the bog peat surface (at the frozen ground interface), the water level at the shrub fen was about $15 \mathrm{~cm}$ under the peat surface, as can be seen on the peat sample pictures of Fig. 3.36c, d. These observations confirm the promising potential of $\phi_{\alpha s}$ for bog-fen discrimination. Since no water lies beneath bog peat surface, $\phi_{\alpha s}$ can easily identify poor fens of shallow subsurface water. The sensitivity of polarimetric L-band scattering phase to peatland subsurface water should provide an efficient tool for monitoring bog-fen transformations.

\subsubsection{Discussion on the Role of Polarimetry, on the Maturity of the Application and Conclusions}

This study confirms that the scattering type phase $\phi_{\alpha s}$ extracted from fully polarimetric L-band ALOS data can reveal the seasonal changes in poor fen subsurface water flow and permits the separation of poor fen from shrub bogs. Such information cannot be obtained with the multi-polarisation $\mathrm{HH}, \mathrm{HV}$ and $\mathrm{VV}$ intensities nor with the conventional polarimetric decomposition parameters such as the Cloude-Pottier $\alpha$, the entropy and the extrema of the intensities provided by the coherency eigenvalues. $\phi_{\alpha s}$, which cannot detect deep ( $45 \mathrm{~cm}$ below the peat surface) water in a boreal bog, seems to be more sensitive to the shallower water $(10-20 \mathrm{~cm})$ beneath the surface in fen, and this makes possible the separation of poor fens from shrub bogs. These results have recently been confirmed in a boreal peatland in the Athabasca region in the context of an investigation on the long-term monitoring of oil sand exploration on surrounding peatland. The use of polarimetric L-band SAR and $\phi_{\alpha s}$ could also be very promising for the detection of new fens in subarctic bogs. Recent field work in the Wapusk National Park peatlands revealed the absence of water (on the surface of the permafrost) beneath the peat bog surface. Fens, which are continuously irrigated by subsurface water 10-20 cm deep, could be clearly identified with $\phi_{\alpha s}$. Even though we are convinced that further experiments are needed to validate these results with other peatland sites, the results obtained so far look very promising for the operational 
a
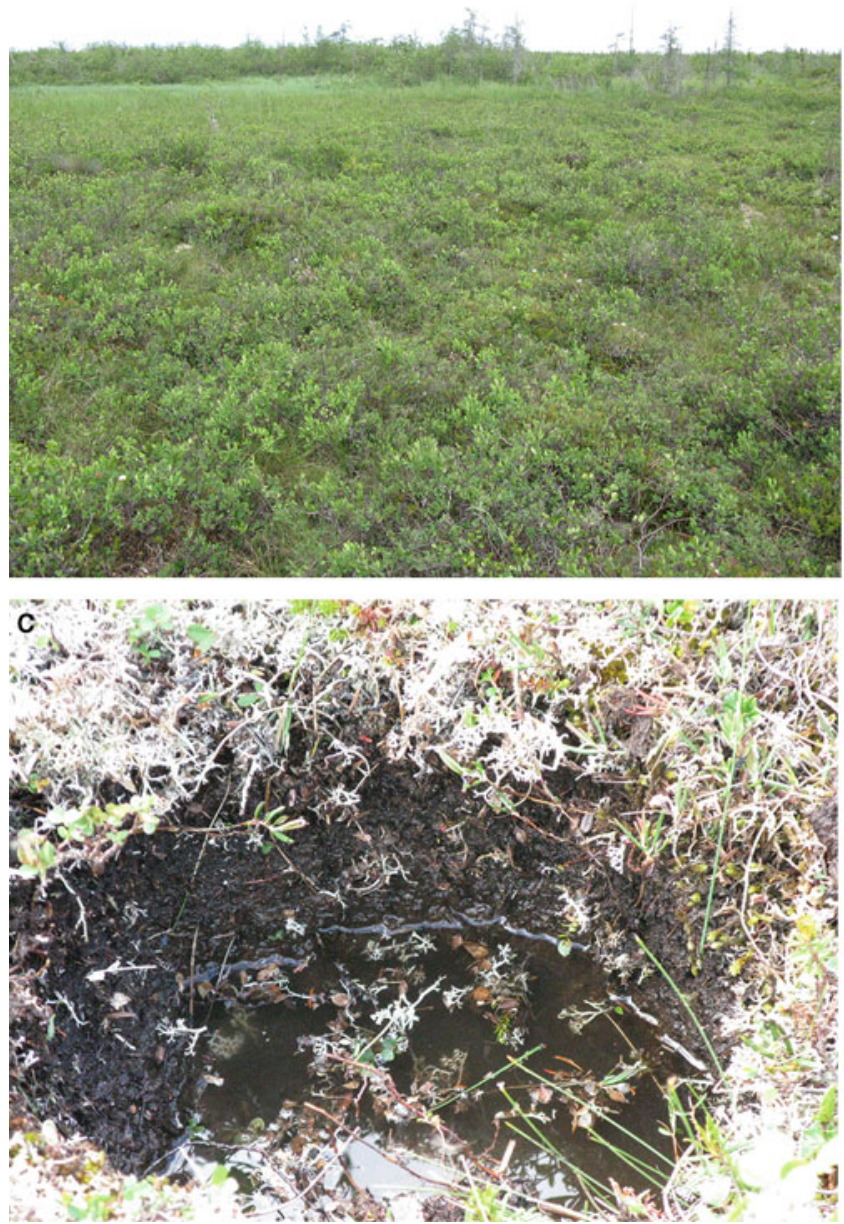

b
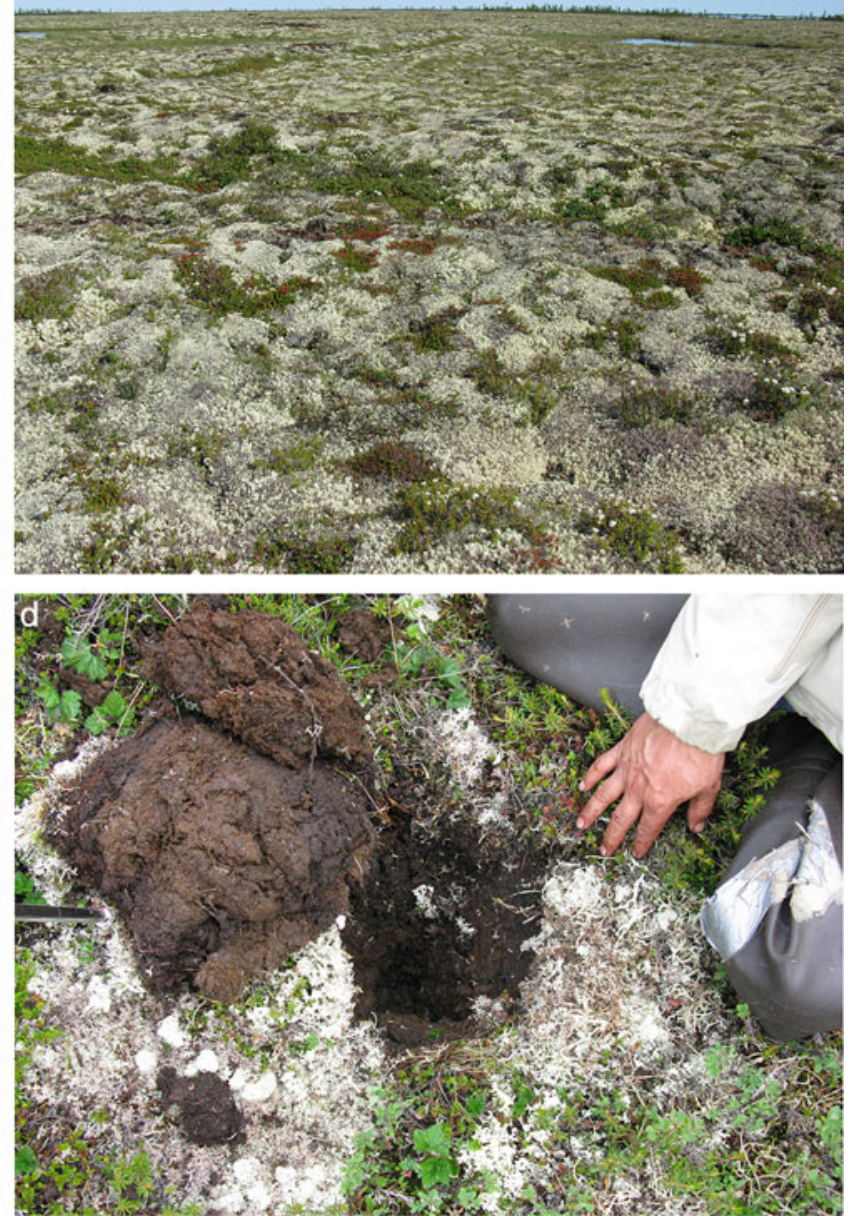

Fig. 3.36 Field data collection. (a) Sedge fen A. (c) Water-level picture in sedge fen A. (b) Open bog B. (d) Water-level picture in bog B; no water at the frozen ground interface

use of the upcoming polarimetric L-band SAR satellite missions, and ALOS2 in particular, as an essential source of information for mapping peatlands and monitoring bog to fen transformations. Operational windows of polarimetric L-band SAR acquisitions are required from the spring runoff water to the fall season, and over a long period of time, for efficient peatland monitoring.

\subsubsection{Acknowledgement}

The authors would like to thank S. Kowalchuk, M. Gibbons, J. Larkin and H. Stewart from the Wapusk National Park for their help in organising and carrying out field data collection. S. Nedelcu and K. Omari from CCRS are thanked for their help in data processing. We would also like to thank JAXA for having provided the ALOS-PALSAR data under the ALOS-PI project and the Canadian Space Agency for having partially funded the present study under the Government Related Initiative Program.

\subsection{Monitoring Change Detection Produced by Tsunamis and Earthquakes by Using a Fully Polarimetric Model-Based Decomposition}

\subsubsection{Introduction, Motivation and Literature Review}

Natural disasters occur frequently, causing significant loss of life and leading to major geo-/bio-environmental and socioeconomic costs. Therefore, the monitoring of the disaster damages over the globe is an urgent need. It is quite difficult to obtain an immediate response of large-scale earthquakes and tsunami disaster areas by ground survey methods. Although the ground survey is accurate, it is also highly time-consuming and manpower extensive, and, consequently, this causes delays in assessment responses to rescue 
teams. Satellite remote sensing has great potential in the monitoring of disaster damages because of its repetitive capability and synoptic coverage. The aim of this work is to present a methodology to generate information from highresolution polarimetric SAR images to identify directly the differences or damages between pre- and post-tsunami and earthquake conditions on the affected regions. This methodology is based on scattering decomposition techniques for polarimetric data. We take the advantage of the excellent quad-polarisation data sets acquired with the Japanese Advanced Land Observing Satellite-Phase Array type L-band Synthetic Aperture Radar (ALOS-PALSAR) imaging system using its high-resolution PolSAR mode to produce colour-coded images for easily interpreting earth surface features and monitoring the earthquake and tsunami damage along the Miyagi coast affected by the 11th of March 2011 tsunami.

Assessment of earthquake and tsunami disaster damages in urban areas has been investigated based on visual interpretation or change detection methods using remotely sensed imagery. Hitherto, several methods are available to monitor earthquake and tsunami damages by using multispectral and monochromatic optical images as well as mono/dualpolarisation SAR images (Matsuoka and Koshimura 2010; Chini et al. 2009). High-resolution multispectral optical images enable direct visual interpretation of the damages and are rather straightforward and simple to interpret by users. However, optical remote sensing fails under cloudy, foggy and hazy as well as severe rainy conditions for monitoring near-real-time damage. Single-wavelength SAR images obtained from fixed single- and/or dual-polarisation sensors are independent of meteorological conditions, but are difficult to be interpreted and require tedious computational analyses for assessments. Most recently, the potential and advantages of fully polarimetric SAR data to monitor the natural disasters, including tsunami and earthquake, were demonstrated in (Yamaguchi 2012) and (Sato et al. 2012b).

Scattering power decompositions have been a research topic in radar polarimetry for the analysis of fully polarimetric synthetic aperture radar data (Freeman and Durden 1998; Yajima et al. 2008; Yamaguchi et al. 2005; 2011; Arii et al. 2011; Sato et al. 2012a; van Zyl et al. 2011; Lee and Ainsworth 2011; Touzi 2007a; Singh et al. 2012). There exist nine real independent observation parameters in the $3 \times 3$ coherency or covariance matrix with respect to the second-order statistics of polarimetric information (Freeman and Durden 1998; Yajima et al. 2008; Yamaguchi et al. 2005; Touzi 2007a). There are several decomposition methods to retrieve information from the coherency matrix. Physical scattering model-based decompositions are straightforward to interpret the final imaging result because the experimental evidence is incorporated in the model-based approach. The pioneering work of the model-based decomposition was presented by Freeman and Durden (Freeman and Durden 1998) by introducing the three-component decomposition. To date, a significant amount of research has been carried out on the model-based decomposition techniques (Arii et al. 2011; Sato et al. 2012a; Yamaguchi et al. 2011; van Zyl et al. 2011; Lee and Ainsworth 2011; Singh et al. 2012).

The original three-component decomposition was proposed by Freeman and Durden (1998) under the reflection symmetry condition, i.e. the cross-correlation between the co- and cross-polarised scattering elements are close to zero for natural distributed objects. This method decomposes the observation matrix into the surface, double bounce and volume scattering terms based on the physical scattering models and accounts for five terms out of nine independent parameters. Then, Yamaguchi et al. (2005) added a helix scattering term and proposed the four-component decomposition. Then, by using the rotation of coherency matrix, Yamaguchi et al. (2011) reduced the number of polarimetric parameters from 9 to 8. The Yamaguchi et al. (2011) method yielded better decomposition results by accounting for six parameters out of eight. The un-accounted parameters are the real and imaginary part of $T_{13}$ in the coherency matrix, and they still remained un-accounted in any of the known physical scattering model-based decompositions. Finally, a general four-component decomposition (G4U) method has been proposed recently by Singh et al. (2012) using a special unitary transformation to the rotated coherency matrix, which has been used in the existing four-component decomposition. Since unitary transformations do not change any information included in the coherency matrix, the rotated coherency matrix is transformed to eliminate the $T_{23}$ element. This four-component decomposition finally accounts for seven terms out of seven polarimetric parameters.

\subsubsection{Methodology}

Figure 3.37 shows the general four-component scattering power decomposition procedure (G4U). The number of independent parameters in the coherency matrix is reduced from 9 to 7 by the rotation and the special unitary transformations. This decomposition scheme describes the total scattering power into surface scattering power $P_{s}$, double-bounce scattering power $P_{d}$, volume scattering power $P_{v}$ from dipole and/or oriented dihedral and helix scattering power $P_{c}$. This recently developed four-component scattering power decomposition scheme also includes the complete fully polarimetric relative phase information and the extended volume scattering model for oriented dihedral structures (Singh et al. 2012) (which are the most convenient in urban areas to estimate the appropriate volume scattering as compared to threecomponent decomposition (Freeman and Durden 1998)). The decomposition starts by retrieving the helix scattering 


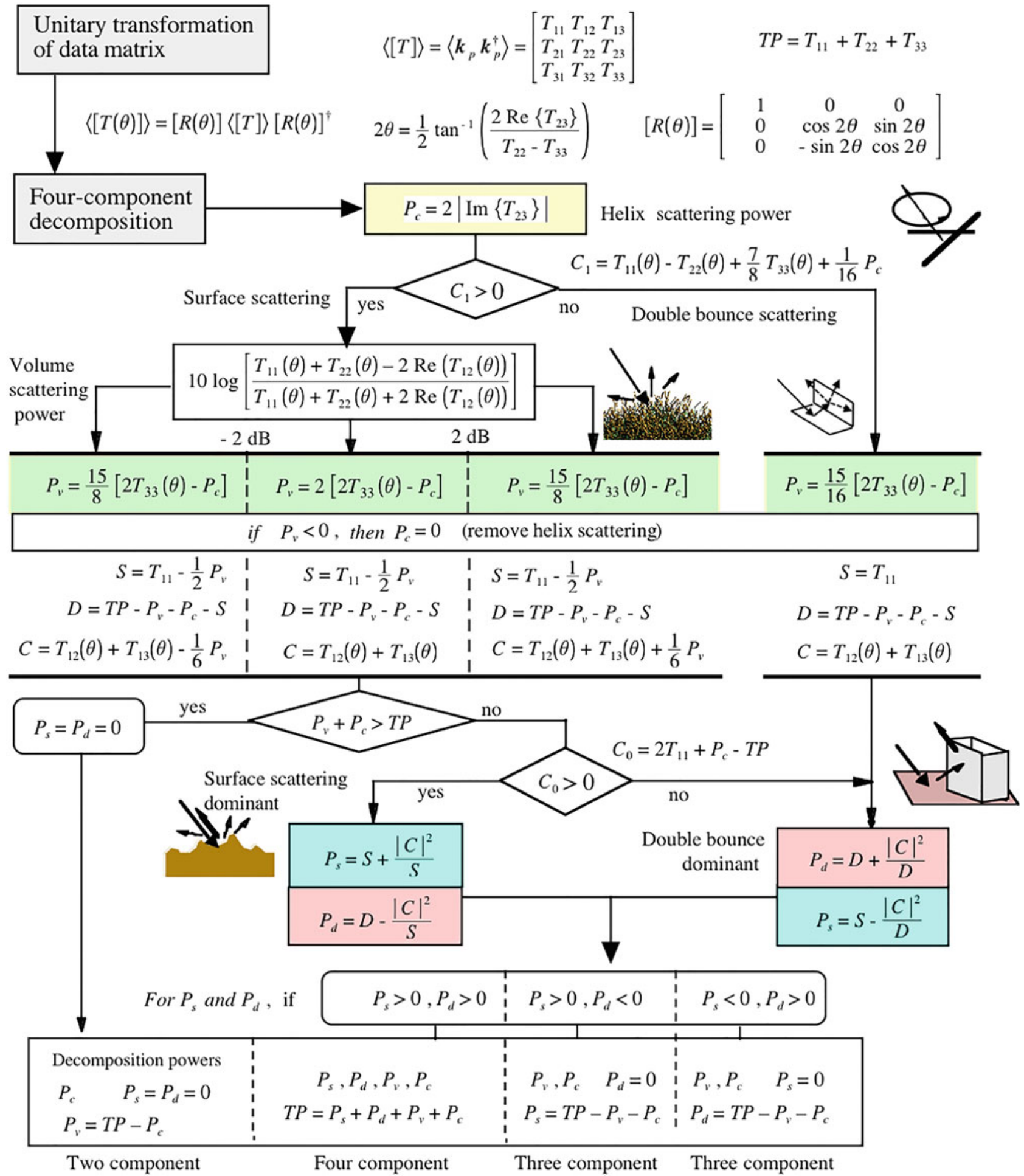

Fig. 3.37 General four-component scattering power decomposition algorithm (G4U) (Singh et al. 2012)

power. The branch condition for $\mathrm{C} 1$ is used to retrieve double-bounce scattering caused by oriented dihedrals. The second branch condition for $\mathrm{C} 0$ is to select dominant scattering mechanism. The outputs of the G4U are used to examine the disaster areas.
Colour composite images of Fig. 3.38 were generated with multi-look factors of 18 times in azimuth direction and 3 times in range direction for the PALSAR data sets in Table 3.9. After the decomposition of ALOS-PALSAR data over the earthquake/tsunami disaster site, the four scattering 

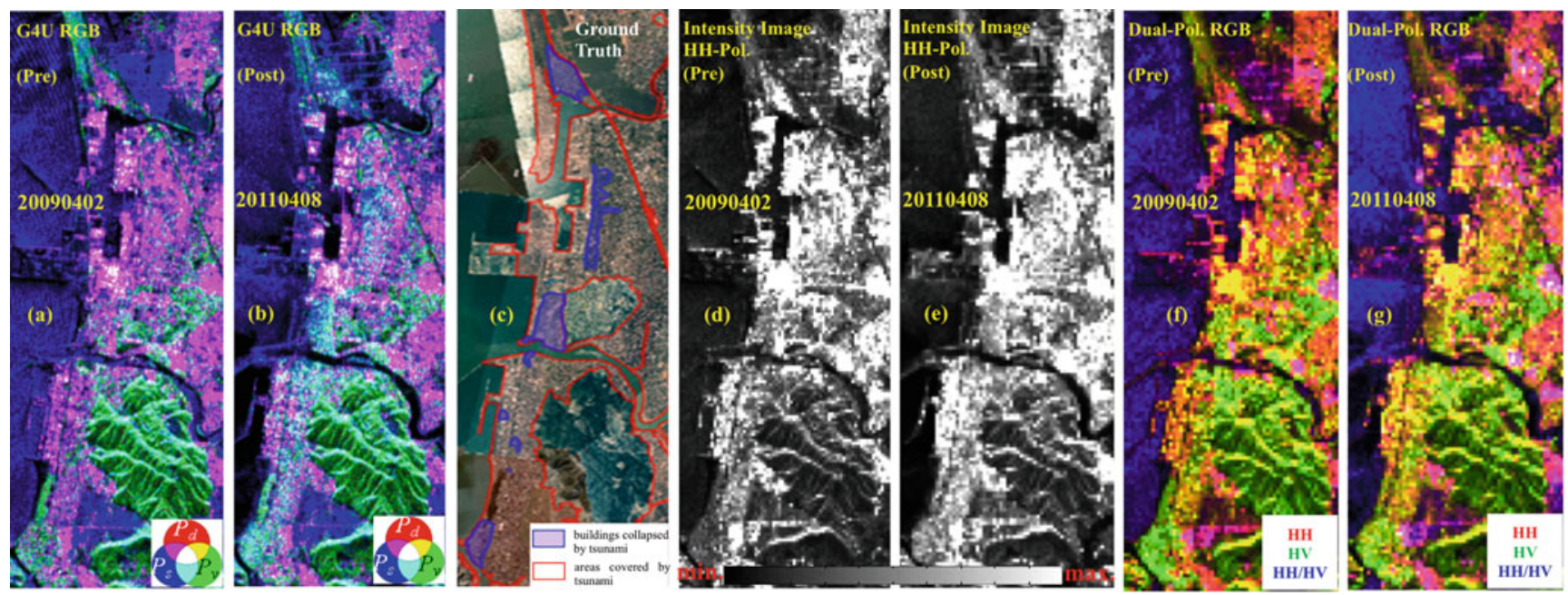

Fig. 3.38 (a) Pre- and (b) post-earthquake/tsunami G4U colour-coded images for PALSAR data. (c) Ground truth information, (d) pre- and (e) post-earthquake/tsunami HH-pol intensity images for PALSAR data and (f) pre- and (g) post-earthquake/tsunami dual-pol RGB images of

PALSAR data. In all images, the flight direction of ALOS-PALSAR is from left to right, and PALSAR illumination direction is from top to bottom

Table 3.11 Test sites and corresponding radar and validation data selected for the generation of showcases on monitoring of post-disaster effects in urban areas

\begin{tabular}{l|l|l}
\hline Application/product & Test site - radar data & Reference data \\
\hline $\begin{array}{l}\text { Monitoring of post-disaster } \\
\text { effects in the urban areas }\end{array}$ & $\begin{array}{l}\text { Ishinomaki, Miyagi } \\
\text { Prefecture, Japan }\end{array}$ & $\begin{array}{l}\text { Ground truth information provided by the Association of Japanese Geographers } \\
\text { and Geospatial Information Authority of Japan }\end{array}$ \\
\cline { 2 - 2 } & $\begin{array}{l}\text { ALOS-PALSAR data } \\
\text { set \#20090402 }\end{array}$ & \\
\cline { 1 - 2 } & $\begin{array}{l}\text { ALOS-PALSAR data } \\
\text { set \#20110408 }\end{array}$ & \\
\hline
\end{tabular}

components $\left(P_{s}, P_{d}, P_{v}\right.$ and $\left.P_{c}\right)$ were normalised by total power $(T P)$ for further analysis. A mean filter with $3 \times 3$ window size is applied on these normalised scattering power component images before analysing the images in more detail (Singh et al. 2013).

\subsubsection{Experimental Results}

We have selected parts of the coastal areas within the Miyagi Prefecture affected by the 11th of March 2011 magnitude 9.0 Honshu, Japan, earthquakes (38.322 N, 142.369 E, depth $32 \mathrm{~km}$ ) that struck off Japan's northeastern coast and triggered a historical super-tsunami. We used the ALOSPALSAR fully polarimetric, single-look complex (SLC), level 1.1 (ascending orbit) images acquired over study areas before (2nd of April 2009) and after the earthquake/tsunami (8th of April 2011) struck; see Table 3.11.

Two (pre- and post-disaster) images are processed by implementing the G4U scheme (Singh et al. 2012) to the data sets in Table 3.11. These decomposition RGB colourcoded images of 20090402 and 20110408 are presented in Fig. 3.39. Since man-made structures such as building and bridges orthogonal to radar illumination are categorised into double-bounce scatterer types in the G4U scheme, the double-bounce $\left(P_{d}\right)$ scattering component in urban area is caused by right angle scattering between building block walls and road surfaces. The volume scattering $\left(P_{v}\right)$ and surface scattering $\left(P_{s}\right)$ components are small for orthogonally illuminated man-made structures. However, damaged or collapsed urban blocks or man-made structures resulting from the earthquake/tsunami impact that do not appear to be orthogonal to radar direction, and which corresponding main scattering centre is at an oblique direction with respect to radar illumination, generate no double-bounce type response in the G4U images after the tsunami struck. Due to multiple scattering, these red areas (pre-tsunami image) turn into green (volume scattering) in post-tsunami images. In cases of buildings washed out and/or eliminated by the tsunami, these areas appear as blue (i.e. surface scattering types of the G4U scheme) in the post-tsunami images. These effects in post-tsunami images, compared to pre-tsunami images, provide a simple straightforward tool for interpreting collapsed buildings in tsunami-affected areas (Singh et al. 2013). 
The changes produced by the disaster are shown in Fig. 3.39 using the difference in the normalised scattering powers $p_{s}\left(=P_{s} / T P\right), p_{v}\left(=P_{v} / T P\right)$ and $p_{d}\left(=P_{d} / T P\right)$. Table 3.12 shows the quantitative mean statistics of the normalised difference scattering parameters $\Delta p_{s}, \Delta p_{v}, \Delta p_{d}$ and $\Delta p_{c}$ corresponding to surface, volume, double bounce and helix scattering powers, for patch A (vegetated area, Fig. 3.39), patch B (agricultural area/wiped-out houses debris-deposited area, Fig. 3.39) and patch C (urban region,
Fig. 3.39). The mean statistics including \pm standard deviation of the pixel distributions over all patches were analysed, and the basic behaviours of scattering parameters $p_{s}, p_{v}$ and $p_{d}$ were observed as follows: (1) the surface scattering is increased in tsunami-affected areas (vegetation-damaged area and wiped-out/collapsed urban block area), but it can be decreased in the areas with deposition of wiped-out houses by the retreating tsunami and for the floating houses on bay areas; (2) the volume scattering is decreased in tsunami-
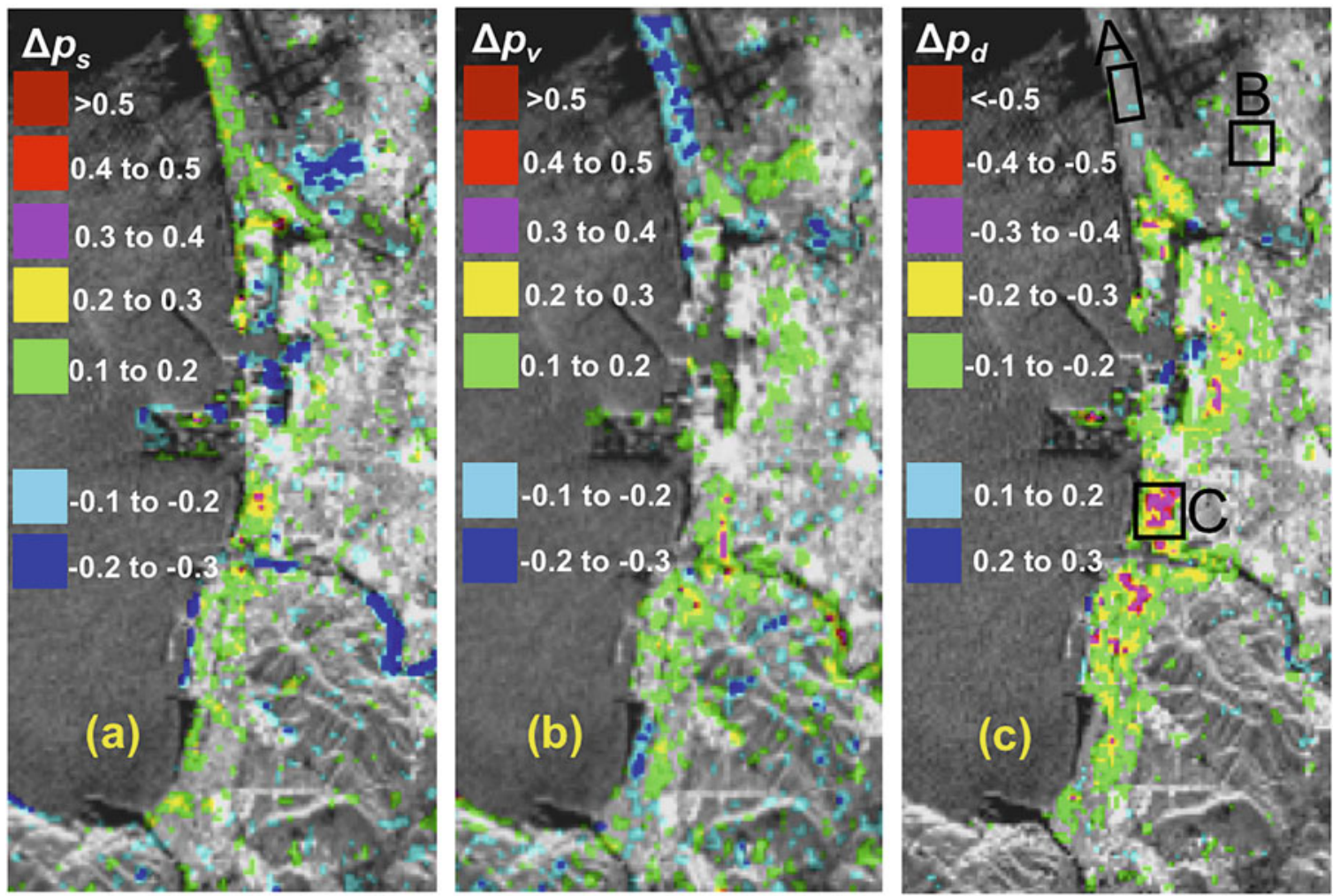

Fig. 3.39 The difference images in between pre-earthquake/tsunami (20090402) and post-earthquake/tsunami (20110408) for decomposition parameters (a) $\Delta p_{s}$, (b) $\Delta p_{v}$ and (c) $\Delta p_{d}$ are superimposed on $T P$ image of 20090402. Patch A shows the vegetation area on relatively flat surface areas, patch B represents the agricultural area over a relatively flat ground, and patch $\mathrm{C}$ illustrates urban areas of Ishinomaki city

Table 3.12 Statistics of the difference values of pixels for patches A, B and C in between pre- earthquake/tsunami (20090402) and postearthquake/tsunami (20110408) decomposition parameters

\begin{tabular}{l|l|l|l|l|}
\hline & Scattering component & Mean & Std. Dev. & Number of pixels \\
\hline \multirow{3}{*}{ Patch A } & $\Delta p_{s}$ & 0.107 & 0.070 \\
\cline { 2 - 4 } & $\Delta p_{v}$ & -0.154 & 0.066 \\
\cline { 2 - 4 } & $\Delta p_{d}$ & 0.047 & 0.054 \\
\cline { 2 - 4 } & $\Delta p_{c}$ & -0.000 & 0.015 \\
\hline \multirow{5}{*}{ Patch B } & $\Delta p_{s}$ & -0.222 & 0.050 \\
\cline { 2 - 4 } & $\Delta p_{v}$ & 0.145 & 0.046 \\
\cline { 2 - 4 } & $\Delta p_{d}$ & 0.061 & 0.031 \\
\cline { 2 - 4 } & $\Delta p_{c}$ & 0.016 & 0.008 \\
\hline \multirow{5}{*}{ Patch C } & $\Delta p_{s}$ & 0.136 & 0.132 \\
\cline { 2 - 4 } & $\Delta p_{v}$ & 0.150 & 0.093 \\
\cline { 2 - 4 } & $\Delta p_{d}$ & -0.280 & 0.075 \\
\cline { 2 - 4 } & $\Delta p_{c}$ & -0.006 & 0.018 \\
\hline
\end{tabular}


affected or damaged vegetation areas and increased in damaged or collapsed, deposited and/or floating (on bays water surface) urban blocks or man-made structures by the earthquake/tsunami; and (3) the double-bounce scattering is decreased in earthquake/tsunami collapsed or damaged urban areas and increased in areas with deposited (in agricultural fields or other type scattering dominated areas) wiped-out urban blocks or man-made structures, mainly caused by the force of tsunami. More detailed analysis and discussion are given in (Singh et al. 2013).

\subsubsection{Comparison with Single-/ Dual-Polarisation Data}

SAR images obtained from fixed single- and/or dualpolarisation sensors are independent of meteorological conditions, but are difficult to interpret and require tedious computational analyses for at most incomplete assessments only. A single observation using conventional SAR images makes it difficult to generate desirable images ready for direct visual interpretation. The features that can be identified in the fully polarimetric case cannot be distinguished in single and dual-pol images (see Fig. 3.38). However, high-resolution fully polarimetric SAR images are straightforward to identify the differences or damages between pre- and post-tsunami conditions of the affected regions.

\subsubsection{Discussion on the Role of Polarimetry, on the Maturity of the Application and Conclusions}

Since microwave radar remote sensing is a suitable tool for monitoring the near-real-time earthquake and tsunami damage at large scales, at anytime of day or night, its implementation becomes of vital relevance to governmental and other agencies for initiating swift and well-orchestrated rescue operations. In this showcase, we have explored the role of polarimetry in tsunami and earthquake disaster monitoring. Fully polarimetric high-resolution L-band image data sets with the implementation of the G4U scheme provide a straightforward simple tool for interpreting as well as identifying collapsed buildings caused by earthquake/tsunami disasters. This method also holds other types of natural (typhoon or tornado) and man-made disaster assessment application. It is found that the double-bounce scattering power is the most promising parameters to detect automated disaster-affected urban areas at pixel level. It is also observed that the very-high-resolution PolSAR images are required for superior urban area monitoring over the oriented urban blocks with respect to the illumination of radar.

\subsection{Summary (Table 3.13)}

Table 3.13 Summary of presented application, methods and preferred system configurations for monitoring and characterisation of agriculture and wetland scenarios

\begin{tabular}{|c|c|c|}
\hline Application & Methods and used frequency (P/L/C/X) & $\begin{array}{l}\text { Radar data preference/requirements/ } \\
\text { comments }\end{array}$ \\
\hline \multirow[t]{3}{*}{ Crop type mapping } & $\begin{array}{l}\text { Decision tree supervised classification applied to PolSAR } \\
\text { observables from incoherent decompositions, multitemporal data (C) }\end{array}$ & $\begin{array}{l}\text { Preferred frequency: } \mathrm{C}-\text { simpler } \\
\text { polarimetric modes could suffice }\end{array}$ \\
\hline & \multirow[t]{2}{*}{$\begin{array}{l}\text { Statistical classification applied to PolSAR backscattering, } \\
\text { multitemporal data (L/C) }\end{array}$} & $\begin{array}{l}\text { Need of multitemporal data: short revisit } \\
\text { time needed }\end{array}$ \\
\hline & & $\begin{array}{l}\text { Radiometric stability in time may be } \\
\text { needed }\end{array}$ \\
\hline $\begin{array}{l}\text { Soil moisture retrieval under } \\
\text { vegetation }\end{array}$ & PolSAR decomposition and inversion of scattering models (L/C) & Preferred frequency: L \\
\hline \multirow[t]{3}{*}{ Phenology monitoring } & \multirow[t]{3}{*}{ Hierarchical classification based on PolSAR observables (C) } & Short revisit time \\
\hline & & Radiometric stability in time \\
\hline & & $\begin{array}{l}\text { High-resolution and/or wide swath } \\
\text { desirable - compact-pol is a good trade- } \\
\text { off }\end{array}$ \\
\hline \multirow{4}{*}{$\begin{array}{l}\text { Wetland and peatland } \\
\text { delineation and } \\
\text { characterisation }\end{array}$} & Segmentation of the Shannon entropy image (C) & $\begin{array}{l}\text { Dual-pol could suffice (e.g. for } \\
\text { RADARSAT-2 and Sentinel-1) }\end{array}$ \\
\hline & PolSAR model-based decompositions (L/C/X) & Preferred frequencies: L/X \\
\hline & \multirow[t]{2}{*}{ Touzi decomposition (L) } & Preferred frequency: L \\
\hline & & Good SNR in HV needed \\
\hline $\begin{array}{l}\text { Effect of tsunami and } \\
\text { earthquakes (change } \\
\text { detection) }\end{array}$ & Four-component decomposition (L) & High resolution needed \\
\hline
\end{tabular}




\section{References}

Anguela TP, Zribi M, Baghdadi N, Loumange C (2010) Analysis of local variation of soil surface parameters with TerraSAR-X radar data over bare agricultural fields. IEEE Trans Geosci Remote Sens 48:874-881

Arii M, van Zyl JJ, Kim Y (2010) A general characterization for polarimetric scattering from vegetation canopies. IEEE Trans Geosci Remote Sens 48:3349-3357

Arii M et al (2011) Adaptive model-based decomposition of polarimetric SAR covariance matrices. IEEE Trans Geosci Remote Sens 49:1104-1113

Attema E, Ulaby FT (1978) Vegetation modelled as a water cloud. Radio Sci 13:357-364

Atwood D et al (2013) Proceedings of the 2013 POLinSAR workshop

Bach H, Mauser W (2003) Methods and examples for remote sensing data assimilation in land surface process modelling. IEEE Trans Geosci Remote Sens 41:1629-1637

Badhwar GD, Henderson KE (1981) Estimating development stages of corn from spectral data - an initial model. Agron J 73:748-755

Ballester-Berman JD, Lopez-Sanchez JM (2012) Time series of hybridpolarity parameters over agricultural crops. IEEE Geosci Remote Sens Lett 9:139-143

Ballester-Berman JD, Lopez-Sanchez JM, Fortuny-Guasch J (2005) Retrieval of biophysical parameters of agricultural crops using polarimetric SAR interferometry. IEEE Trans Geosci Remote Sens 43:683-694

Ban Y (2003) Synergy of multitemporal ERS-1 SAR and Landsat TM data for classification of agricultural crop. Can J Remote Sens 29:518-526

Barbier EB (1994) Valuing Environmental functions: tropical wetlands. Land Econ 70:155-173

Bernard R, Martin P, Thony JL, Vauclin M, Vidal-Madjar D (1982) C-band radar for determining surface soil moisture. Remote Sens Environ 12:189-200

Blaes X, Vanhalle L, Defourny P (2005) Efficiency of crop identification based on optical and SAR image time series. Remote Sens Environ 96:352-365

Bocco G, Mendoza M, Velázquez A (2001) Remote sensing and GIS-based regional geomorphological mapping - a tool for land use planning in developing countries. Geomorphology 39:211-219

Boerner WM et al (1998) Chapter 5: Polarimetry in radar remote sensing: basic and applied concepts. In: Ryerson RA (ed) Manual of remote sensing: principles and applications of imaging radar, vol 3. Wiley, pp 271-356

Bouman BAM, Hoekman D (1993) Multi-temporal, multi-frequency radar measurements of agricultural crops during the Agriscatt- 88 campaign in the Netherlands. Int J Remote Sens 14:1595-1614

Bouman BAM, Uenk D (1992) Crop classification possibilities with radar in ERS-1 and JERS-1 configuration. Remote Sensing of Environment 40:1-13

Bourgeau-Chavez LL et al (2005) Remote monitoring of regional inundation patterns and hydroperiod in the greater everglades using synthetic aperture radar. Wetlands 25:176-191

Bourgeau-Chavez LL, Riordan K, Powell RB, Miller N, Nowels M (2009) Improving wetland characterization with multi-sensor, multi-temporal SAR and optical/infrared data fusion. In: Jedlovec $\mathrm{G}$ (ed) Advances in geoscience and remote sensing. InTech, pp 679-708

Bouvet A, Le Toan T, Lam-Dao N (2009) Monitoring of the rice cropping system in the Mekong Delta using ENVISAT/ASAR dual polarization data. IEEE Trans Geosci Remote Sens 47:517-526

Brisco B, Brown RJ (1995) Multidate SAR/TM synergism for crop classification in Western Canada. Photogram Eng Remote Sens 61:1009-1014
Brisco B, Protz R (1980) Corn field identification accuracy using airborne radar imagery. Can J Remote Sens 6:15-24

Brisco B, Kapfer M, Hirose T, Tedford B, Liu J (2011) Evaluation of $\mathrm{C}$-band polarization diversity and polarimetry for wetland mapping. Can J Remote Sens 37:82-92

Bronstert A, Bardossy A (1999) The role of spatial variability of soil moisture for modelling surface runoff generation at the small catchment scale. Hydrol Earth Syst Sci 3:505-516

Bronstert A et al (2012) Potentials and constraints of different type of soil moisture observations for flood simulations in headwater catchments. Nat Hazar 60:879-914

Brook RK (2006) In: Riewe R, Oakes J (eds) Forest and tundra fires in the Hudson Bay Lowlands of Manitoba. Climate Change: Linking Traditional and Scientific Knowledge. Aboriginal Issues Press, Winnipeg

Brook RK, Kenkel NC (2002) A multivariate approach to vegetation mapping of Manitoba's Hudson Bay Lowlands. Int J Remote Sens 23:4761-4776

Brown RJ, Manore MJ, Poirier S (1992) Correlations between X-, Cand L-band imagery within an agricultural environment. Int $\mathbf{J}$ Remote Sens 13:1645-1661

Cardell-Oliver R, Kranz M, Smettem K, Mayer K (2005) A reactive soil moisture sensor network: design and field evaluation. Int J Distrib Sens Netw 1:149-162

Charbonneau FJ et al (2010) Compact polarimetry overview and applications assessment. Can J Remote Sens 36:298-315

Chen C, McNairn H (2006) A neural network integrated approach for rice crop monitoring. Int J Remote Sens 27:1367-1393

Chen KS, Huang WP, Tsay DH, Amar F (1996) Classification of multifrequency polarimetric SAR imagery using a dynamic learning neural network. IEEE Trans Geosci Remote Sens 34:814-820

Chini M, Pierdicca N, Emery WJ (2009) Exploiting SAR and VHR optical images to quantify damages caused by the 2003 Bam earthquake. IEEE Trans Geosci Remote Sens 47:145-152

Cloude SR (1988) Uniqueness of target decomposition theorems in radar polarimetry. In: Boerner WM et al (eds) Proceedings of NATO Advanced research workshop on direct and inverse methods in radar polarimetry, vol 350. Kluwer, pp 267-296

Cloude SR (2009) Polarisation: applications in remote sensing. Oxford University Press

Cloude SR, Papathanassiou KP (2003) Three-stage inversion process for polarimetric SAR interferometry. IEEE Proc Radar Sonar Navig 150:125-134

Cloude SR, Pottier E (1996) A review of target decomposition theorems in radar polarimetry. IEEE Trans Geosci Remote Sens 34:498-518

Cloude SR, Pottier E (1997) An entropy based classification scheme for land applications of polarimetric SAR. IEEE Trans Geosci Remote Sens 35:68-78

Cloude SR, Corr DG, Williams ML (2004) Target detection beneath foliage using polarimetric synthetic aperture radar interferometry. Waves Random Media 14:393-414

Cloude SR, Goodenough DG, Chen H (2012) Compact decomposition theory. IEEE Geosci Remote Sens Lett 9:28-32

Del Frate $\mathrm{F}$ et al (2004) Wheat cycle monitoring using radar data and a neural network trained by a model. IEEE Trans Geosci Remote Sens 42:35-44

Dobson MC, Pierce LE, Ulaby FT (1996) Knowledge-based land-cover classification using ERS-1/JERS-1 SAR composites. IEEE Trans Geosci Remote Sens 34:83-99

Dyke D, Wendy ES (2010) Permafrost and peatland evolution in the Northern Hudson Bay lowland, Manitoba. Arctic 63:429-441

EEA (2007) CLC2006 technical guidelines. Technical report, no. $17 / 2007$

Engdahl M, Borgeaud M (1998) ERS-1/2 Tandem interferometric coherence and agricultural crop height. In: Proceedings of retrieval 
of bio- and geo-physical parameters from SAR data for land applications workshop 121-127, ESA-ESTEC

Engdahl M, Borgeaud M, Rast M (2001) The use of ERS-1/2 tandem interferometric coherence in the estimation of agricultural crop heights. IEEE Trans Geosci Remote Sens 39:1799-1806

Entekabi D et al (2010) The soil moisture active passive (SMAP) mission. Proc IEEE 98:704-716

European Space Agency (2012) The earth observation handbook: 2012 special edition for Rio+20, Noordwijk

Evans TL, Costa M (2013) Landcover classification of the Lower Nhecolandia subregion of the Brazilian Pantanal Wetlands using ALOS/PALSAR, RADARSAT-2 and ENVISAT/ASAR imagery. Remote Sens Environ 128:118-137

Fatoyinbo T, Simard M (2011) Remote sensing of mangrove structure and biomass. In: Proceedings of the 2011 workshop on Tropical Wetland Ecosystems of Indonesia: Science Needs to Address Climate Change Adaptation and Mitigation

Ferrazzoli P et al (1992) Sensitivity of microwave measurements to vegetation biomass and soil moisture content: a case study. IEEE Trans Geosci Remote Sens 30:750-756

Ferrazzoli P, Paloscia S, Pampaloni P, Schiavon G, Sigismondi S, Solimini D (1997) The potential of multifrequency polarimetric SAR in assessing agricultural and arboreous biomass. IEEE Trans Geosci Remote Sens 35:5-17

Ferrazzoli P, Guerriero L, Schiavon G (1999) Experimental and model investigation on radar classification capability. IEEE Trans Geosci Remote Sens 37:960-968

Foody GM (1988) Crop classification from airborne synthetic aperture radar data. Int J Remote Sens 9:655-668

Foody GM, McCulloch MB, Yates WB (1994) Crop classification from C-Band polarimetric radar data. Int J Remote Sens 15:2871-2885

Fournier R, Grenier M, Lavoie A, Helie R (2007) Towards a strategy to implement the Canadian wetland inventory using satellite remote sensing. Can J Remote Sens 33:1-16

Fraser CJD, Roulet NT, Lafleur M (2001) Groundwater flow patterns in a large peatland. J Hydrol 246:142-154

Freeman A (2007) Fitting a two component scattering model to polarimetric SAR data from forests. IEEE Trans Geosci Remote Sens 45:2583-2592

Freeman A, Durden SL (1998) A Three-component scattering model for polarimetric SAR data. IEEE Trans Geosci Remote Sens 36:963-973

Freeman A, Villasenor J, Klein JD, Hoogeboom P, Groot J (1994) On the use of multi-frequency and polarimetric radar backscatter features for classification of agricultural crops. Int J Remote Sens 15:1799-1812

Fung AK (1994) Microwave scattering and emission models and their applications. Artech House, Boston

Gorham E (1995) Northern peatlands: role in the carbon budget and probable 7 responses to global warming. Ecol Appl 1:182-188

Grenier M, Demers AM, Labrecque S, Benoit M, Fournier R, Drolet B (2007) An object-based method to map wetland using RADARSAT1 and Landsat ETM images: test case on two sites in Quebec, Canada. Can J Remote Sens 33:28-45

Guindon B, Teillet PM, Goodenough DG, Palimaka JJ, Sieber A (1984) Evaluation of the crop classification performance of X, L and C-band SAR Imagery. Can J Remote Sens 10:4-16

Haboudane D, Miller J, Pattey E, Zarco-Tejada PJ, Strachan I (2004) Hyperspectral vegetation indices and novel algorithms for predicting green LAI of crop canopies: modeling and validation in the context of precision agriculture. Remote Sens Environ 90:337-352

Hajnsek I, Pottier E, Cloude SR (2003) Inversion of surface parameters from polarimetric SAR. IEEE Trans Geosci Remote Sens 4:727-744

Hajnsek I, Jagdhuber T, Schön H, Papathanassiou KP (2009) Potential of estimating soil moisture under vegetation cover by means of PolSAR. IEEE Trans Geosci Remote Sens 47:442-454
Henderson FM, Lewis AJ (eds) (1998) Manual of remote sensing: principles and applications of imaging radar, vol 2, 3rd edn. Wiley

Henderson FM, Lewis AJ (2008) Radar detection of wetland ecosystems: a review. Int J Remote Sens 29:5809-5835

Hess LL, Melack JM, Filoso S, Wang Y (1995) Delineation of inundated area and vegetation along the Amazon floodplain with SIR-C synthetic aperture radar. IEEE Trans Geosci Remote Sens 33:896-904

Hill MJ, Ticehurst CJ, Lee J-S, Grunes MR, Donald GE, Henry D (2005) Integration of optical and radar classifications for mapping pasture type in Western Australia. IEEE Trans Geosci Remote Sens 43:1665-1681

Hoekman DH, Vissers AM (2003) A new polarimetric classification approach evaluated for agricultural crops. IEEE Trans Geosci Remote Sens 41:2881-2889

Hoekman DH, Vissers AM, Tran TN (2011) Unsupervised fullpolarimetric SAR data segmentation as a tool for classification of agricultural areas. IEEE J Sel Topics Appl Earth Observ Remote Sens 4:402-411

Hong SH, Wdowinski S (2011) Evaluation of the quad-polarimetric RADARSAT-2 observations for the wetland InSAR application. Can J Remote Sens 37:484-492

Hong SH, Wdowinski S (2013) Double bounce component in crosspolarimetric SAR from new scattering target decomposition. In: Proceedings of the 2013 POLinSAR workshop

Hong SH et al (2010) Evaluation of TerraSAR-X observations for wetland InSAR application. IEEE Trans Geosci Remote Sens 48:864-873

Hubert-Moy L (2006) Wetlands. In: Geist H (ed) Our Earth's changing land: an encyclopedia of land-use and land-cover change, vol 2. Greenwood Press, pp 665-671

Hubert-Moy L, Michel K, Corpetti T, Clément B (2006) Object-oriented mapping and analysis of wetlands using SPOT 5 data. In: Proceedings of the IEEE Geoscience and Remote Sensing Symposium (IGARSS), pp 3447-3450

Huete AR et al (2006) Amazon rainforests green-up with sunlight in dry season. Geophys Res Lett 33

Huisman JA, Snepvangers JJJC, Bouten W, Heuvelink GBM (2003) Monitoring temporal development of spatial soil water content variation: comparison of ground penetration radar and time domain reflectometry. Vadose Zone J 2:519-529

Ingram HAP (1982) Size and shape in raised mire ecosystems: a geophysical model. Nature 297:300-303

Inoue $\mathrm{Y}$ et al (2002) Season-long daily measurements of multifrequency $(\mathrm{Ka}, \mathrm{Ku}, \mathrm{X}, \mathrm{C}$, and $\mathrm{L})$ and full-polarization backscatter signatures over paddy rice field and their relationship with biological variables. Remote Sens Environ 81:194-204

Jackson TJ (1993) Measuring surface soil moisture using passive microwave remote sensing. Hydrol Process 7:139-152

Jagdhuber T (2012) Soil parameter retrieval under vegetation cover using SAR polarimetry. PhD thesis, University of Potsdam. http:// opus.kobv.de/ubp/volltexte/2012/6051/

Jagdhuber T, Hajnsek I, Sauer S, Papathanassiou KP, Bronstert A (2012a) Soil moisture retrieval under forest using polarimetric decomposition techniques at P-band. In: Proceedings of European conference on Synthetic Aperture Radar (EUSAR)

Jagdhuber T, Hajnsek I, Papathanassiou KP, Bronstert A (2012b) Soil moisture retrieval under agricultural vegetation using fully polarimetric SAR. In: Proceedings of IEEE 2012 international symposium on Geoscience and Remote Sensing (IGARSS)

Jagdhuber T, Hajnsek I, Papathanassiou KP, Bronstert A (2013a) Soil moisture estimation under low vegetation cover using a multiangular polarimetric decomposition. IEEE Trans Geosci Remote Sens 51:2201-2215

Jagdhuber T, Hajnsek I, Papathanassiou KP (2013b) Refined soil moisture estimation by means of L-band polarimetry. In: Proceedings of 
IEEE international geoscience and remote sensing symposium (IGARSS)

Jagdhuber T, Hajnsek I, Papathanassiou KP (2013c) Polarimetric soil moisture retrieval at short wavelength. In: Proceedings of international workshop on Science and Applications of SAR Polarimetry and Polarimetric Interferometry (PolINSAR)

Jagdhuber T, Hajnsek I, Caputo M, Papathanassiou KP (2013d) Soil moisture estimation using dual-polarimetric coherent $(\mathrm{HH} / \mathrm{VV})$ TerraSAR-X and TanDEM-X data. In: Proceedings of TSX/TDX science meeting

Jagdhuber T, Hajnsek I, Papathanassiou KP (2014) An iterative generalized hybrid decomposition for soil moisture retrieval under vegetation cover using fully polarimetric SAR. IEEE J Sel Topic Appl Earth Observ Remote Sens. https://doi.org/10.1109/JSTARS. 2014.2371468

Jano AP, Jefferies RL, Rockwell R (1998) The detection of vegetational change by multitemporal analysis of LANDSAT data: The effects of goose foraging. J Ecol 86:93-99

Karam MA, Fung AK, Lang RH, Chauhan NS (1992) A microwave scattering model for layered vegetation. IEEE Trans Geosci Remote Sens 30:767-784

Katra I, Blumberg DG, Lavee H, Sarah P (2006) A method for estimating the spatial distribution of soil moisture of arid microenvironments by close range thermal infrared imaging. Int $\mathrm{J}$ Remote Sens 27:2599-2611

Kerr Y, Waldteufel P, Wigneron J-P, Martinuzzi J-M, Font J, Berger M (2001) Soil moisture retrieval from space: the soil moisture and ocean salinity (SMOS) mission. IEEE Trans Geosci Remote Sens 39:1729-1735

Koay J-Y et al (2007) Paddy fields as electrically dense media: theoretical modeling and measurement comparisons. IEEE Trans Geosci Remote Sens 45:2837-2849

Koch M, Schmid T, Reyes M, Gumuzzio J (2012) Evaluating full polarimetric $\mathrm{C}$ - and $\mathrm{L}$-band data for mapping wetland conditions in a semi-arid environment in central Spain. IEEE J Sel Topic Appl Earth Observ Remote Sens 5:1033-1044

Koyama CN, Schneider K (2011) Soil moisture retrieval under vegetation using polarized PALSAR data. In: Proceedings of IEEE 2011 international symposium on Geoscience and Remote Sensing (IGARSS)

Krajewski WF et al (2006) A remote sensing observatory for hydrologic sciences: a genesis for scaling to continental hydrology. Water Resour Res 42. https://doi.org/10.1029/2005WR004435

Kurosu T, Fujita M, Chiba K (1995) Monitoring of rice crop growth from space using the ERS-1 C-band SAR. IEEE Trans Geosci Remote Sens 33:1092-1096

Lang MW, Kasischke ES (2008) Using C-band synthetic aperture radar data to monitor forested, wetland hydrology in Maryland's coastal plain, USA. IEEE Trans Geosci Remote Sens 46:535-546

Lang RH, Sidhu JS (1983) Electromagnetic backscattering from a layer of vegetation: a discrete approach. IEEE Trans Geosci Remote Sens 21:62-71

Lasne Y, Paillou P, August-Bernex T, Ruffie G, Grandjean G (2004) A phase signature for detecting wet subsurface structures using polarimetric L-band SAR. IEEE Trans Geosci Remote Sens 42:1683-1694

Le Toan T, Laur H, Mougin E, Lopes A (1989) Multitemporal and dual polarization observations of agricultural vegetation covers by X-band SAR images. IEEE Trans Geosci Remote Sens 27:709-718

Le Toan $T$ et al (1997) Rice crop mapping and monitoring using ERS-1 data based on experiment and modeling results. IEEE Trans Geosci Remote Sens 35:41-56

Lee J-S, Ainsworth TL (2011) The effect of orientation angle compensation on coherency matrix and polarimetric target decompositions. IEEE Trans Geosci Remote Sens 49:53-64

Lee J-S, Pottier E (2009) Polarimetric radar imaging: from basics to applications. CRC Press, Taylor \& Francis
Lee JS, Grunes R, Kwok R (1994) Classification of multi-look polarimetric SAR imagery based on complex Wishart distribution. Int J Remote Sens 15:2299-2311

Lee JS, Grunes MR, Ainsworth TL, Du LJ, Schuler DL, Cloude SR (1999) Unsupervised classification using polarimetric decomposition and the complex Wishart classifier. IEEE Trans Geosci Remote Sens 37:2249-2258

Lee J-S, Grunes MR, Pottier E (2001) Quantitative comparison of classification capability: fully polarimetric versus dual and singlepolarization SAR. IEEE Trans Geosci Remote Sens 39:2343-2351

Lee JS, Grunes MR, Pottier E, Ferro-Famil L (2004) Unsupervised terrain classification preserving polarimetric scattering characteristics. IEEE Trans Geosci Remote Sens 42:722-731

Liao J, Wang Q (2010) Wetland characterization and classification using polarimetric RADARSAT-2 data. Proc SPIE 7841. https://doi.org/ $10.1117 / 12.873258$

Lloyd D (1990) A phenological classification of terrestrial vegetation cover using shortwave vegetation index imagery. Int J Remote Sens 11:2269-2279

Lopez-Sanchez JM, Ballester-Berman JD (2009) Potentials of polarimetric SAR interferometry for agriculture monitoring. Radio Sci 44. https://doi.org/10.1029/2008RS004078

Lopez-Sanchez JM, Cloude SR, Ballester-Berman JD (2012a) Rice phenology monitoring by means of SAR polarimetry at X-band. IEEE Trans Geosci Remote Sens 50:2695-2709

Lopez-Sanchez JM, Hajnsek I, Ballester-Berman JD (2012b) First demonstration of agriculture height retrieval with PolInSAR airborne data. IEEE Geosci Remote Sens Lett 9:242-246

Lopez-Sanchez JM, Vicente-Guijalba F, Ballester-Berman JD, Cloude SR (2014) Polarimetric response rice fields at C-band: analysis and phenology retrieval. IEEE Trans Geosci Remote Sens 52:2977-2993

Löw A, Ludwig R, Mauser W (2006) Derivation of surface soil moisture from ENVISAT ASAR wide swath and image mode data in agricultural areas. IEEE Trans Geosci Remote Sens 44:889-899

Lucas RM et al (2007) The potential of L-band SAR for quantifying mangrove characteristics and change: case studies from the tropics. Aquat Conserv Mar Freshw Ecosyst 17:245-264

Maltby E (ed) (2009) The functional assessment wetland ecosystems: towards evaluation of ecosystem services. Woodhead Publ, Boston

Marechal C, Pottier E, Hubert-Moy L, Corgne S, Allain-Bailhache S, Meric S (2010) Spaceborne fully polarimetric time-series datasets for land cover analysis. In: Proceedings of the IEEE International Geoscience and Remote Sensing Symposium (IGARSS), pp 859-862

Marechal C, Pottier E, Hubert-Moy L, Rapinel S (2012) One year wetland survey investigations from quad-pol RADARSAT-2 timeseries SAR images. Can J Remote Sens 38:240-252

Matsuoka M, Koshimura S (2010) Tsunami damaged area estimation for the 2010 Maule, Chile earthquake using ASTER DEM and PALSAR images on the geo grid. In: 8th international workshop on Remote Sensing and Disaster Response, Tokyo, Japan

McNairn H, van der Sanden JJ, Brown RJ, Ellis J (2000) The potential of RADARSAT-2 for crop mapping and assessing crop condition. In: Proceedings of the second international conference on Geospatial Information in Agriculture and Forestry

McNairn H, Champagne C, Shang J, Holmstrom D, Reichert G (2009a) Integration of optical and synthetic aperture radar (SAR) imagery for delivering operational annual crop inventories. ISPRS J Photogram Remote Sens 64:434-449

McNairn H, Shang J, Jiao X, Champagne C (2009b) The contribution of ALOS PALSAR multipolarization and polarimetric data to crop classification. IEEE Trans Geosci Remote Sens 47:3981-3992

Meehl GA et al (2007) Global climate projections. In: Climate change 2007: the physical science basis. Contributions of Working Group I to the Fourth Assessment Report of the Intergovernmental Panel on Climate Change. Cambridge University Press, Cambridge 
Meier U (ed) (2001) Growth stages of mono- and dicotyledonous plants. BBCH Monograph. http://www.jki.bund.de/fileadmin/dam_ uploads/_veroeff/bbch/BBCH-Skala_englisch.pdf

Mérot P et al (2006) A method for improving the management of controversial wetland. Environ Manag 37:258-270

Merz B, Plate EJ (1997) An analysis of the effects of spatial variability of soil and soil moisture on runoff. Water Resour Res 33:2909-2922

Minet J, Laloy E, Lambot S, Vanclooster M (2011) Effect of highresolution spatial soil moisture variability on simulated runoff response using a distributed hydrological model. Hydrol Earth Syst Sci 15:1323-1338

Moran MS, Peters-Lidard CD, Watts JM, McElroy S (2004) Estimating soil moisture at the watershed scale with satellite-based radar and land surface models. Can J Remote Sens 30:805-826

Moran MS et al (2012) A RADARSAT-2 quad-polarized time series for monitoring crop and soil conditions in Barrax, Spain. IEEE Trans Geosci Remote Sens 50:1057-1070

Nellis MD, Price KP, Rundquist D (2009) Remote sensing of cropland agriculture. Papers in Natural Resources 217

Neumann M et al (2009) A general model-based polarimetric decomposition scheme for vegetated areas. In: Proceedings of the 2009 POLInSAR workshop

Neumann M, Ferro-Famil L, Reigber A (2010) Estimation of forest structure, ground and canopy layer characteristics from multibaseline polarimetric interferometric SAR data. IEEE Trans Geosci Remote Sens 48:1086-1104

Ozesmi SL, Bauer M (2002) Satellite remote sensing of wetlands. Wetl Ecol Manag 10:381-402

Park SE, Kimb D, Lee H-S, Moon WM, Wagner W (2010) Tidal wetland monitoring using polarimetric synthetic aperture radar. In: Wagner W, Székely B (eds) ISPRS TC VII symposium - 100 years ISPRS, vol XXXVIII. IAPRS, Vienna

Patel P, Srivastava HS, Navalgund RR (2009) Use of synthetic aperture radar polarimetry to characterize wetland targets of Koladeo National Park, Bharatpur, India. Curr Sci 97:529-537

Pierce LE, Ulaby FT, Sarabandi K, Dobson MC (1994) Knowledgebased classification of polarimetric SAR images. IEEE Trans Geosci Remote Sens 32:1081-1086

Pope KO et al (1994) Radar remote-sensing of forest and wetland ecosystems in the Central-American tropics. Remote Sens Environ 48:205-219

Pope KO et al (1997) Detecting seasonal flooding cycles in marshes of the Yucatan Peninsula with SIR-C polarimetric radar imagery. Remote Sens Environ 59:157-166

Proisy C et al (2000) Interpretation of polarimetric radar signatures of mangrove forests. Remote Sens Environ 71:56-66

Quegan S, Le Toan T, Skriver H, Gomez-Dans J, Gonzalez-Sampedro MC, Hoekman DH (2003) Crop classification with multitemporal polarimetric SAR data. In: Proceedings of POLinSAR2003 workshop, Frascati, 2003

Raney RK (2007) Hybrid-polarity SAR architecture. IEEE Trans Geosci Remote Sens 45:3397-3404

Reed BC et al (1994) Measuring phenological variability from satellite imagery. J Veg Sci 5:703-714

Robinson DA, Jones SB, Wraith JM, Or D, Friedman SP (2003) A review of advances in dielectric and electrical conductivity measurement in soils using time domain reflectometry. Vadose Zone J 2:444-475

Robinson DA et al (2008) Soil moisture measurement for ecological and hydrological watershed-scale observatories: A review. Vadose Zone J 7:358-389

Rosenqvist A, Shimada M, Ito N, Watanabe M (2007) ALOS PALSAR: a pathfinder mission for global-scale monitoring of the environment. IEEE Trans Geosci Remote Sens 45:3307-3316

Rulequest Research (2008) Rulequest research, data mining tools See5 and C5.0. http://www.rulequest.com/see5-info.html
Sato A et al (2012a) Four-component scattering power decomposition with extended volume scattering model. IEEE Geosci Remote Sens Lett 9:166-170

Sato M, Chen S-W, Makoto S (2012b) Polarimetric SAR analysis of tsunami damage following the March 11, 2011 East Japan earthquake. Proc IEEE 100:2861-2875

Schmitt A, Brisco B (2013) Wetland monitoring using the curveletbased change detection method on polarimetric SAR imagery. Water 5:1036-1051

Schmitt A, Brisco B, Kaya S, Murnaghan K (2012) Polarimetric change detection for wetlands, remote sensing and hydrology. Proc IAHS 352:375-379

Schotten CGJ, Van Rooy WWL, Janssen LLF (1995) Assessment of the capabilities of multi-temporal ERS-1 SAR data to discriminate between agricultural crops. Int J Remote Sens 16:2619-2637

Shalaby A, Tateishi R (2007) Remote sensing and GIS for mapping and monitoring land cover and land-use changes in the Northwestern coastal zone of Egypt. Appl Geogr 27:28-41

Singh G, Yamaguchi Y, Park S-E (2012) General four-component scattering power decomposition with unitary transformation of coherency matrix. IEEE Trans Geosci Remote Sens 50

Singh G, Yamaguchi Y, Boerner WM, Park S-E (2013) Monitoring of the 2011 March 11 Off-Tohoku 9.0 earthquake with super-tsunami disaster by implementing fully polarimetric high resolution PolSAR techniques. Proc IEEE 101:831-846

Skriver H (2001) Land-cover map information from polarimetric SAR using knowledge-based techniques. In: Proceedings of 3rd international symposium on Retrieval of Bio- and Geophysical Parameters from SAR data for Land Applications, 2001

Skriver H (2012) Crop classification by multitemporal C- and L-band single- and dual-polarization and fully polarimetric SAR. IEEE Trans Geosci Remote Sens 50:2138-2149

Skriver H, Svendsen MT, Thomsen A (1999) Multitemporal C- and L-band polarimetric signatures of crops. IEEE Trans Geosci Remote Sens 37:2413-2429

Skriver H, Mattia F, Satalino G, Balenzano A, Pauwels VRN, Verhoest N, Davidson M (2011) Crop classification using shortrevisit multitemporal SAR data. IEEE J Sel Topics Appl Earth Observ Remote Sens 4:423-431

Slatton KC et al (1996) Modeling wetland vegetation using polarimetric SAR

Sokol J, McNairn H, Pultz T (2004) Case studies demonstrating hydrological applications of C-band multi-polarized and polarimetric SAR. Can J Remote Sens 30:470-483

Souyris JC, Imbo P, Fjortoft R, Mingot S, Lee J-S (2005) Compact polarimetry based on symmetry properties of geophysical media: The pi/4-mode. IEEE Trans Geosci Remote Sens 43:634-646

Technical Report Y-87-1 (1987) Environmental laboratory, corps of engineers wetlands delineation manual. U.S. Army Engineer Waterways Experiment Station, Vicksburg

Thomson KPB, Edwards G, Landry R, Jaton A, Cadieux SP, Gwyn QHJ (1990) SAR Applications in agriculture: multiband correlation and segmentation. Can J Remote Sens 16:47-54

Tilton JC, Hollinger SE (1982) Estimating crop development stages from multispectral data. LARS technical report, Purdue University/ Laboratory for Applications of Remote Sensing

Toutin T, Wang H, Chomaz P, Pottier E (2013) Orthorectification of full-polarimetric RADARSAT-2 data using accurate lidar DSM. IEEE Trans Geosci Remote Sens 51

Touzi R (2007a) Target scattering decomposition in terms of rollinvariant target parameters. IEEE Trans Geosci Remote Sens 45

Touzi R (2007b) Speckle effect on polarimetric target scattering decomposition of SAR imagery. Can J Remote Sens 33:60-68

Touzi R (2013) Compact-hybrid versus linear-dual and fully polarimetric SAR. In: Proceedings of POLinSAR workshop 
Touzi R, Gosselin G (2010) Peatland subsurface water flow monitoring using polarimetric L-band PALSAR. In: Proceedings of the IEEE International Geoscience and Remote Sensing Symposium (IGARSS), pp 750-753

Touzi R, Shimada M (2009) Calibration of polarimetric PALSAR. IEEE Trans Geosci Remote Sens 47:3911-3913

Touzi R, Boerner WM, Lee J-S, Luneberg E (2004) A review of polarimetry in the context of synthetic aperture radar: concepts and information extraction. Can J Remote Sens 30:380-407

Touzi R, Deschamps A, Rother G (2007) Wetland characterization using polarimetric RADARSAT-2 capability. Can J Remote Sens 33:56-67

Touzi R, Deschamps A, Rother G (2009) Phase of target scattering for wetland characterization using polarimetric C-band SAR. IEEE Trans Geosci Remote Sens 47:3241-3261

Treuhaft RN, Siqueira PR (2000) Vertical structure of vegetated land surfaces from interferometric and polarimetric data. Radio Sci 35:141-177

Treuhaft RN, Madsen SN, Moghaddam M, van Zyl JJ (1996) Vegetation characteristics and underlying topography from interferometric radar. Radio Sci 31:1449-1485

Trisasongko BH (2009) Tropical mangrove mapping using fullypolarimetric radar data. ITB J Sci 41:98-109

Tucker CJ (1979) Red and photographic infrared linear combinations for monitoring vegetation. Remote Sens Environ 8:127-150

Turkar V, Rao YS (2011) Analysis of multi-frequency SIR-C data over India. In: Proceeding of the 2011 international conference ICWET

Ulaby FT, Elachi C (1990) Radar polarimetry for geoscience applications. Artech House, Norwood

Ulaby FT, Wilson EA (1985) Microwave attenuation properties of vegetation canopies. IEEE Trans Geosci Remote Sens 23:746-753

Ulaby FT, Moore RK, Fung AK (1982) Microwave remote sensing: active and passive: Volume II: Radar remote sensing and surface scattering and emission theory. Artech House, Boston

Ulaby FT, Held DN, Dobson MC, McDonald KC, Senior TBA (1987) Relating polarization phase difference of SAR signals to scene properties. IEEE Trans Geosci Remote Sens 25:83-92

Ullmann T et al (2012) Application of quadpolarimetric TerraSAR-X data for landcover characterization in tropical regions - a case study in south Kalimantan, Indonesia. In: Proceedings of the IEEE International Geoscience and Remote Sensing Symposium, pp 5133-5136

United Nations Population Fund (2011) The state of world population 2011, New York

Van Zyl JJ (1989) Unsupervised classification of scattering behavior using radar polarimetry data. IEEE Trans Geosci Remote Sens $27: 36-45$
Van Zyl JJ (1992) Application of Cloude's target decomposition theorem to polarimetric imaging radar data. In: Mott $\mathrm{H}$, Boerner WM (eds) Proceedings of SPIE, vol 1748, San Diego, pp 184-191

Van Zyl JJ, Kim Y, Arii M (2008) Requirements for model-based polarimetric decompositions. In: Proceedings of IEEE 2008 International Symposium on Geoscience and Remote Sensing (IGARSS)

van Zyl JJ et al (2011) Model-based decomposition of polarimetric SAR covariance matrices constrained for nonnegative eigenvalues. IEEE Trans Geosci Remote Sens 49:3452-3459

Wagner W et al (2007) Operational readiness of microwave remote sensing of soil moisture for hydrologic applications. Nord Hydrol 28:1-20

Wagner W, Pathe C, Doubkova M et al (2008) Temporal stability of soil moisture and radar backscatter observed by the advanced synthetic aperture radar (ASAR). Sensors 8:1174-1197

Wegmüller U, Werner CL (1997) Retrieval of vegetation parameters with SAR interferometry. IEEE Trans Geosci Remote Sens 35:18-24

Western AW, Grayson RB, Blöschl G (2002) Scaling of soil moisture: a hydrologic perspective. Annu Rev Earth Planet Sci 30:149-180

Wiener N (1930) Generalized harmonic analysis. Acta Mathematica $5: 118-258$

Xiaoying J (2009) Segmentation-based image processing system. US patent 20090123070, filed Nov. 14, 2007, and issued May 14, 2009

Yajima Y, Yamaguchi Y, Sato R, Yamada H, Boerner W-M (2008) PolSAR image analysis of wetlands using a modified fourcomponent scattering power decomposition. IEEE Trans Geosci Remote Sens 46:1667-1673

Yamaguchi Y (2012) Disaster monitoring by fully polarimetric SAR data acquired with ALOS-PALSAR. Proc IEEE 100:2851-2860

Yamaguchi Y et al (2005) Four-component scattering model for polarimetric SAR image decomposition. IEEE Trans Geosci Remote Sens 43:1699-1706

Yamaguchi Y, Yajima Y, Yamada H (2006) A four component decomposition of PolSAR images based on the coherency matrix. IEEE Geosci Remote Sens Lett 3:292-296

Yamaguchi Y et al (2011) Four-component scattering power decomposition with rotation of coherency matrix. IEEE Trans Geosci Remote Sens 49:2251-2258

Zoltai SC, Vitt DH (1995) Canadian wetlands: environmental gradients and classification. Vegetation 118:131-137

Zreda M, Desilets D, Ferre TPA, Scott RL (2008) Measuring soil moisture content non-invasively at intermediate spatial scale using cosmic ray neutrons. Geophys Res Lett 35:1-5

Open Access This chapter is licensed under the terms of the Creative Commons Attribution 4.0 International License (http://creativecommons.org/ licenses/by/4.0/), which permits use, sharing, adaptation, distribution and reproduction in any medium or format, as long as you give appropriate credit to the original author(s) and the source, provide a link to the Creative Commons license and indicate if changes were made.

The images or other third party material in this chapter are included in the chapter's Creative Commons license, unless indicated otherwise in a credit line to the material. If material is not included in the chapter's Creative Commons license and your intended use is not permitted by statutory regulation or exceeds the permitted use, you will need to obtain permission directly from the copyright holder.

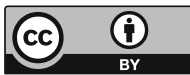




\title{
Cryosphere Applications
}

\author{
I. Hajnsek, G. Parrella, A. Marino, T. Eltoft, M. Necsoiu, L. Eriksson, \\ and M. Watanabe
}

\begin{abstract}
Synthetic aperture radar (SAR) provides large coverage and high resolution, and it has been proven to be sensitive to both surface and near-surface features related to accumulation, ablation, and metamorphism of snow and firn. Exploiting this sensitivity, SAR polarimetry and polarimetric interferometry found application to land ice for instance for the estimation of wave extinction (which relates to sub surface ice volume structure) and for the estimation of snow water equivalent (which relates to snow density and depth). After presenting these applications, the Chapter proceeds by reviewing applications of SAR polarimetry to sea ice for the classification of different ice types, the estimation of thickness, and the characterisation of its surface. Finally, an application to the characterisation of permafrost regions is
\end{abstract}

\footnotetext{
I. Hajnsek ( $₫)$

Microwaves and Radar Institute, German Aerospace Center (DLR), Oberpfaffenhofen, Germany

Institute of Environmental Engineering, Swiss Federal Institute of Technology Zurich (ETH Zurich), Zürich, Switzerland

e-mail: irena.hajnsek@dlr.de

G. Parrella

Microwave and Radar Institute, German Aerospace Center (DLR), Wessling, Germany
}

A. Marino

Biological and Environmental Sciences, University of Stirling, Stirling, UK

T. Eltoft

UiT the Arctic University of Norway, Troms $\emptyset$, Norway

\section{Necsoiu}

Surveillance and Geolocation Department, Southwest Research Institute, San Antonio, TX, USA

\section{Eriksson}

Department of Space, Earth and Environment, Chalmers University of Technology, Gothenburg, Sweden

M. Watanabe

Department of Life Science and Engineering, Tokio Denki University, Tokio, Japan considered. For each application, the used (model-based) decomposition and polarimetric parameters are critically described, and real data results from relevant airborne campaigns and space borne acquisitions are reported.

\subsection{Introduction}

Over the last 30 years, the use of air- and space borne remote sensing has revolutionised glaciology through dramatic improvements in the scale and in the temporal and spatial resolutions of cryospheric observations. Remote sensing offers the possibility to cover large areas quickly and often at a low cost compared to more traditional methods. Many types of remote sensing can be useful for glacier studies including aerial photography and space borne sensors operating in the visible spectrum, gravimetry, passive microwave technology, and radar including satellite altimetry, scatterometry, and other active imaging systems.

However, the greatest advance has been the successful implementation of synthetic aperture radar (SAR). Over iceand snow-covered terrain, SAR has been proven to be sensitive to both surface and near-surface features related to accumulation, ablation, and metamorphism of snow and firn (material in the intermediate stage between snow and ice).

Land Ice In the last decade, the coherent combination of both interferometric and polarimetric observations has established PolInSAR as a viable technique for the extraction of geophysical parameters. However, the use of PolInSAR over glaciers to date is restricted to a small number of airborne studies due to the complexity of glacier environments and the inherent difficulties in validation. Temporal decorrelation and the limited polarimetric modes of spaceborne sensors limited the role of space-borne SAR data.

Nevertheless, the high spatial and temporal resolution and the (sensitive) observation space provide a considerable potential in PolSAR and PolInSAR applications over land ice. 
Land Ice - Snow Characterisation For land ice, the main issue today is the characterisation of the snow layer at the surface and the underlying ice volume. The snow layer can be characterised by means of two main parameters, the snow depth and density, which can be jointly measured through the snow water equivalent. Polarimetry can help to isolate the scattering contribution of the snow volume from the one of the underlying ground and with this it allows to establish a relation with the snow water equivalent.

Land Ice - Ice Extinction Another important parameter is the ice extinction that provides information about the ice volume structure and is, therefore, also an important indicator for changes in the ice volume. The main sensitivity is concentrated in the first 10-20 m of depth of the ice volume. The introduction of polarimetry in combination with interferometry represented a breakthrough for the retrieval of ice extinction.

Sea Ice The ice coverage of oceans plays a sensitive role in the interaction between the atmosphere and the water surface, while, at the same time, it affects sea traffic and human activities. In the northern hemisphere, significant economic components drive the need to study sea ice, in addition to the scientific reasons, while climatological and environmental aspects predominate the need for knowledge about the ice situation in the southern hemisphere.

The main interest lies in the information about sea ice extent, movement, and conditions. The formation of sea ice depends on a variety of environmental factors like temperature, wind, current, and water salinity. At the same time, sea ice undergoes a seasonal cycle and has several distinct stages of development. Polarisation diversity has shown to increase the information content of SAR imagery significantly and the benefits for sea ice monitoring have been recognised early; despite this, the amount of polarimetric SAR data of sea ice available today remains limited. Most of the research work in this domain has been based on airborne SAR data and later on images from ALOS-PALSAR and RADARSAT-2. Many of the early studies based on polarimetric SAR focused on the physics of scattering from sea ice and the resulting polarimetric signatures of ice types. Major investigations were made for sea ice-type classification using different algorithms.
Sea Ice - Classification The most used approach for sea ice characterisation is based on image segmentation algorithms applied on single, dual, or fully polarimetric SAR data. With the introduction of RADARSAT-2, fully polarimetric SAR images have been used on a wider basis in classification procedures improving segmentation performance of different ice types.

Sea Ice - Thickness For thin sea ice thickness is a sensitive parameter affecting the heat exchange between atmosphere and ocean. There is an increasing interest to better understand and quantify this relation within the climate system as well as to monitor and to model its state. During the last two decades, the sensitivity of active microwave sensors and their potential for thin sea ice thickness retrieval was intensively investigated, both empirically and theoretically. A simplified scattering solution for the rough surface case of polarised backscattering of thin sea ice has been established. In addition, a theoretical base for the relation of co-polarised polarimetric coefficients (co-pol ratio and phase difference, complex correlation coefficient) - being independent of surface roughness - to the thickness of thin sea ice was developed in L- and X-band.

Sea Ice - Surface Characterisation Sea ice surface roughness is another important parameter together with sea ice thickness for the thermodynamic exchange between atmosphere and ocean. Although not yet fully understood and quantified, sea ice surface roughness is used as an input parameter for weather prediction modelling. Several works consider surface roughness algorithms for bare surface applications, but only few studies investigate their use on sea ice surfaces. However, with fully polarimetric airborne sensors, first data sets were acquired to test and develop this application.

There are two main parties interested into cryosphere applications for either ecosystem change research or industrial interest (see Table 4.1). The main motivation is to infer the measured snow and ice parameters into ecosystem change models and use them as an input for climate change model predictions or have a commercial interest as for example securing continuous ship traffic roads.

Table 4.1 Polarimetric SAR applications in cryosphere: use and motivation

\begin{tabular}{l|l|l}
\hline (End) users & Application(s) & Interest/motivation \\
\hline \multirow{2}{*}{ Authorities/agencies research } & Snow characterisation & Justification of subsidies and fraud detection, acreage \\
\cline { 2 - 3 } & Retreat of glaciers & Control in regions suffering droughts or with scarce water resources \\
\cline { 2 - 3 } & Sea ice characterisation & Economic and market predictions, price regulations, etc. \\
\hline Commercial & Sea ice extend, movement, condition & Ship traffic road mapping \\
\hline
\end{tabular}




\subsection{Land Ice Extinction Estimation Using PollnSAR}

\subsubsection{Introduction, Motivation, and Literature Review}

Monitoring glaciers and ice sheets is a primary task to understand and quantify climate change. Mass balance is the most important descriptor of the health of a glacier and is the final objective of nearly all glacier monitoring studies. It expresses the total mass change of a glacier in 1 year and is computed by the algebraic sum of accumulation (all processes by which material is added to a glacier) and ablation (all processes by which snow or ice is lost from a glacier). Hence, mass balance is an important indicator of a glacier's likely response to climate change. There are several reasons why direct observations of mass balance are either impractical or too inaccurate to be of value, in which case it may be useful to measure a proxy variable that is qualitatively or quantitatively related to mass balance or to a change in mass balance. The two main existing approaches are to monitor or track changes in the extent of the various glacier facies (characterised by differing degrees of summer melt) and to make repeated observations of changes in the areal extent and terminus position of a glacier over time. Areal extent is still today best measured with optical imagery, although radar imagery has shown significant promise for facies classification.

Because dry snow is relatively transparent at microwave frequencies, end-of-summer conditions are preserved in winter SAR images and the different melting and freezing patterns of the various zones result in distinct radar backscatter properties. SAR imagery has been used to help delineate facies boundaries, although interpretation can be difficult as variations in backscatter are also caused by changes in the surface dielectric properties, roughness, and topography. The additional information provided by PolInSAR can help eliminate this ambiguity by separating surface and volume contributions and thus lead to a more accurate characterisation of melt extent. PolInSAR-derived glacier properties such as facies boundaries and the presence of melt features could thus be used as proxy indicators of changes in glacier mass balance and regional climate. Both facies and melt structures are related to the electromagnetic ice extinction through a variation of vertical density.

The estimation of a parameter such as extinction is of high relevance for glaciologists since it contains information on the density and internal structure of the ice:

- It is useful for characterising regions of greater or lesser volume scatter (Hoen 2001), and in turn increasing the accuracy of facies delineation and classification.
- It provides a better understanding of glacier dynamics, monitoring the extent of the various melt zones (facies) and mass balance fluctuations that may be occurring in the Polar Regions as a result of climatic changes (Davis and Poznyak 1993).

- Extinction also has implications on the accuracy of existing radar products, as knowledge of its temporal and spatial variability could help in correcting InSAR (Dall 2007) and radar altimeter-derived elevation maps which suffer from an extinction-dependent penetration bias (Jezek et al. 1994).

Despite the significant progress in determining the spatial extent of glaciers, in mapping surface features and in determining surface elevation, large uncertainties remain in estimating reliable glacier accumulation rates, ice thickness, subsurface structures and discharge rates. Consequently, even today it is challenging to grasp the structure of the ice volume with SAR remote sensing methods.

The first SAR images, taken in 1978 by Seasat (L-band), revealed the potential of SAR for identifying surface and subsurface features and in classifying the glacier zones (Bindschadler et al. 1987; Rott 1984). A significant step forward was done with the introduction of experimental fully polarimetric SAR systems. PolSAR data over alpine glaciers were provided by the SIR-C (1994) at C- and L-band (Mätzler et al. 1984).

SAR interferometry has been widely applied to measure glacier topography and displacements at accuracies of centimetres to metres (Dowdeswell et al. 1999; Massonnet and Feigl 1998; Mohr and Madsen 1996; Joughin et al. 1998). Preliminary investigations into the relation between interferometric coherence and the rate of extinction of the radar signal in ice were conducted in (Hoen 2001; Hoen and Zebker 2000) in which the ice was modelled as a homogeneous, lossy, and infinite scattering volume. However, polarimetric effects were not included in the coherence model.

The use of PolInSAR over glaciers is restricted to a small number of airborne studies (Dall et al. 2003, 2004; Stebler et al. 2005) due to temporal decorrelation and to the limited polarimetric modes of space-borne sensors, as well as to the complexity of glacier environments and to difficulties in validation. In (Stebler et al. 2005), the authors describe PolInSAR signatures at L- and P-band over an alpine glacier, although no model is suggested to explain polarisation dependencies in the backscatter and interferometric coherence. PolInSAR coherences at L-band over Greenland are presented in (Dall et al. 2003), and several models are considered in (Dall et al. 2004), although no physically model consistent with observed coherence magnitude and phase was found. Given the high resolution and multiple observables offered by PolInSAR on a pixel-by-pixel basis, there is 
considerable potential for its use in glaciological applications if a model relating observables to ice properties could be found.

In the recent literature, great attention has been given to model-based decomposition techniques. One of the first model-based polarimetric decomposition for ice was proposed in (Sharma et al. 2011). The main scattering contributions were assumed to come from a shallow snow-ice interface, the underlying possibly oriented ice volume (of dipole-shaped particles) and an oriented sastrugi field at the surface.

\subsubsection{Methodology}

The ice extinction estimation method used for this application was proposed in (Sharma 2010) and can be seen as made of two parts: model-based polarimetric decomposition and PolInSAR inversion procedure. The principal objective of the polarimetric decomposition is to break down the total scattering within one resolution cell into a sum of elementary scattering contributions and to associate a physical mechanism to each component. The advantage of the model-based approach is that it is based on the physics of radar scattering; hence the interpretation of its results is relatively straightforward (Van Zyl et al. 2008).

The considered decomposition technique assumes, in a more general case, that the total backscattering consists of three components: surface, volume, and oriented sastrugi field. Assuming that the mentioned contributions are uncorrelated, the combined covariance matrix is the sum of the three individual matrices plus a diagonal noise matrix $\mathbf{N}$ :

$$
\mathbf{C}_{\text {tot }}=\mathbf{C}_{g}+\mathbf{C}_{v}+\mathbf{C}_{s}+\mathbf{N}
$$

where $\mathbf{C}_{g}, \mathbf{C}_{v}$, and $\mathbf{C}_{s}$ indicate the covariance matrices of the surface, volume, and sastrugi contribution, respectively.

The surface scattering is postulated to originate from a slightly rough snow-ice interface and modelled using the first-order Small Perturbation Model (SPM). The overlying dry snow layer is assumed to be transparent at long wavelength (L- and P-band) and only responsible for refraction of the incident wave.

The ice volume component is believed to be due to a dominant scattering mechanism related to ice crystals or ice inclusions (ice pipes and lenses). Consequently, the ice mass is modelled as an infinite homogeneous volume of identically shaped and sized scatterers. For simplicity, the scatterers are assumed to be thin randomly oriented dipoles. Transmission and propagation effects are also introduced to model a more realistic scenario.
A third relevant scattering contribution is expected to originate from a shallow oriented sastrugi field. This consists of streamlined snow dunes formed by wind erosion and deposition on the snow surface. It is generally oriented parallel to the main wind direction, and its size ranges from one to a few metres. An oriented sastrugi field is then modelled as an oriented volume of dipoles where all scatterers are contained in the plane of the air-snow interface.

The importance of possible multiple scattering mechanisms has been investigated by analysing circular ratio values (RR/RL and LL/RL) observed in the dataset selected for this showcase. The available L-band and P-band acquisitions show low circular ratio, with values ranging between 0.2 and 0.4 . Only in some cases at L-band, it reaches values higher than 1.0 in the extreme far range region. This general behaviour can be interpreted as an indicator of the low importance of multiple scattering, which was consequently neglected in the model.

Decomposition results are used to estimate surface-tovolume ratios $\mu$ (where the surface contribution includes the sastrugi component) for each available polarimetric channel in order to isolate the volume backscattering contribution.

In a second step, the outcome of the polarimetric decomposition is used as input in an interferometric coherence model, which is finally inverted to retrieve ice extinction for each polarimetric channel. In detail, surface-to-volume ratios are employed to estimate the volumetric coherence $\gamma_{\mathrm{Vol}}$ from the total InSAR coherence $\gamma$ of the corresponding channel using the following relations:

$$
\begin{gathered}
\gamma=e^{j \phi} \gamma_{\mathrm{SNR}} \gamma_{\text {range }} \gamma_{z} \\
\gamma_{z}=\frac{\gamma_{\mathrm{Vol}}\left(k_{e}\right)+\mu}{1+\mu}
\end{gathered}
$$

where $\phi$ is a topographic phase term, $\gamma_{\mathrm{SNR}}$ is the coherence term due to SNR decorrelation, $\gamma_{\text {range }}$ represents the coherence due to range spectral decorrelation, and $\gamma_{z}$ the term dependent only on the vertical distribution of the scatterers. In (4.3), $\mu$ is the estimated surface-to-volume ratio, and $\gamma_{\mathrm{Vol}}\left(k_{e}\right)$ the volume decorrelation expressed as function of the extinction coefficient $k_{e}$ to be estimated. In order to account for the propagation within the ice volume, the vertical wavenumber $k_{z}$ has been modified by considering the dielectric constant of ice.

The range spectral decorrelation $\left(\gamma_{\text {range }}\right)$ and SNR decorrelation $\left(\gamma_{\mathrm{SNR}}\right)$ can be separately estimated according to (Hoen and Zebker 2000; Zebker and Villasenor 1992), respectively. At this point, the extinction coefficient can be inverted from $\gamma_{\mathrm{Vol}}$ for each polarisation and baseline. 


\subsubsection{Experimental Results}

Test sites and corresponding radar and validation data sets selected for the generation of showcases on land ice extinction estimation are summarised in Table 4.2 and further described in the Appendix.

As shown in Fig. 4.1, surface-to-volume scattering ratios estimated by means of the polarimetric decomposition are used in combination with PolInSAR coherences and the infinite-uniform-volume-underground model to determine the ice extinction coefficient. The PolInSAR inversion procedure is separately applied to each polarimetric channel. A spatial averaging window of 100 effective looks is used to compute interferometric coherences, corresponding to an approximately square window of $20 \times 20 \mathrm{~m}$ in ground range - azimuth geometry. Results from multiple baselines are combined by eliminating solutions from extremely small baselines (which have no interferometric sensitivity) and from longer baselines more susceptible to insufficiencies in modelling. Results are then averaged from the remaining valid baselines on a pixel-by-pixel basis. For brevity, results of polarimetric decomposition and extinctions inversion are shown in the following only for ascending acquisitions for the Summit test site. Figures 4.2 and 4.3 show the

Table 4.2 Test sites and corresponding radar and validation data selected for the generation of showcases on land ice extinction estimation

\begin{tabular}{l|l|l}
\hline Application/product & Test site - Radar data & Reference data \\
\hline \multirow{2}{*}{ Land ice extinction estimation } & Summit, Austfonna, Svalbard & GPR profiles, meteorological data, snow pit \\
& Etonbreen, Austfonna, Svalbard & \\
\cline { 2 - 3 } & ICESAR 2007, E-SAR, L-/P-band full-pol & \\
\hline
\end{tabular}

Fig. 4.1 Workflow for extinction inversion using PolInSAR observables (Sharma 2010)

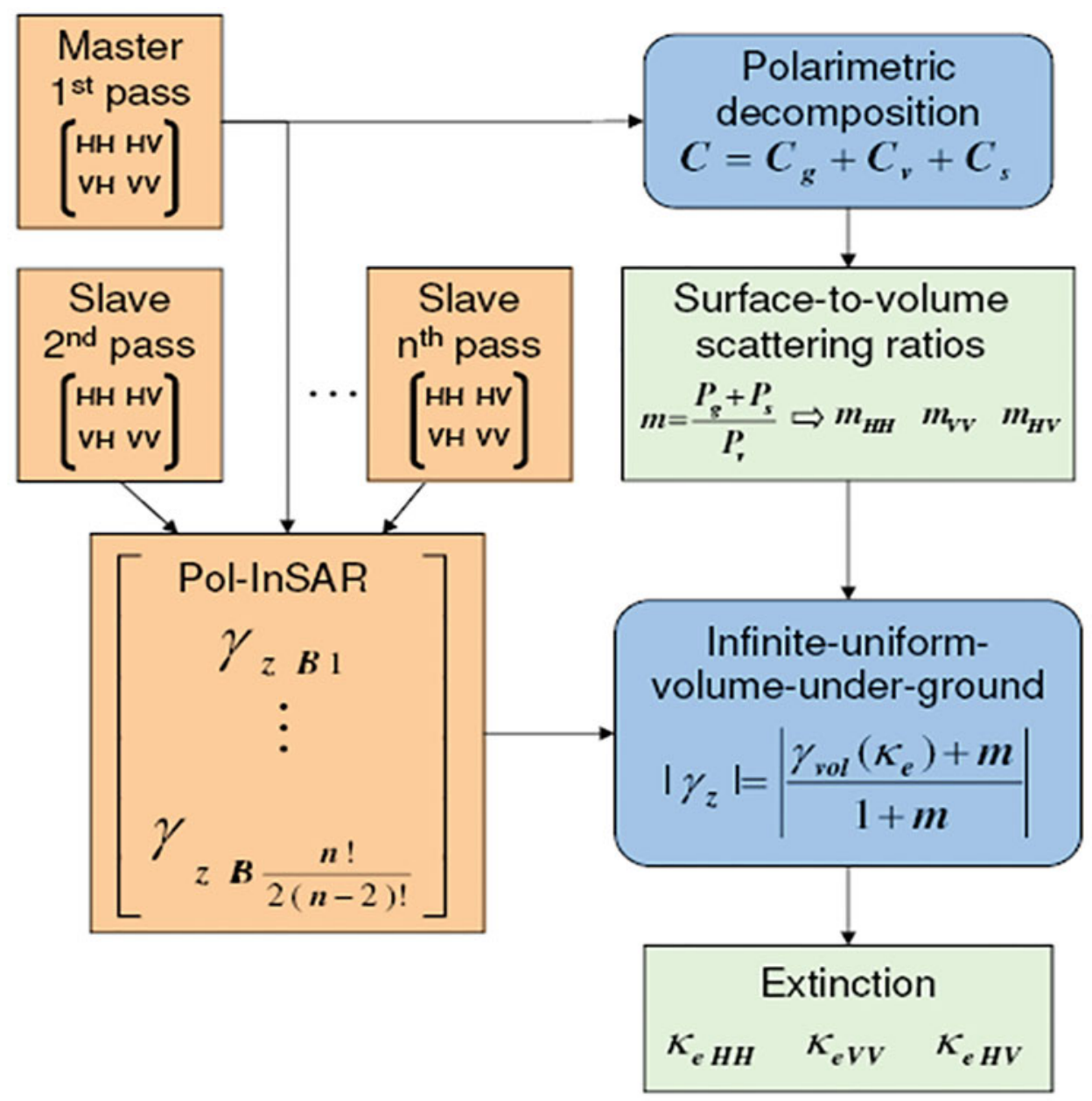

\section{SAR Data Models Derived}



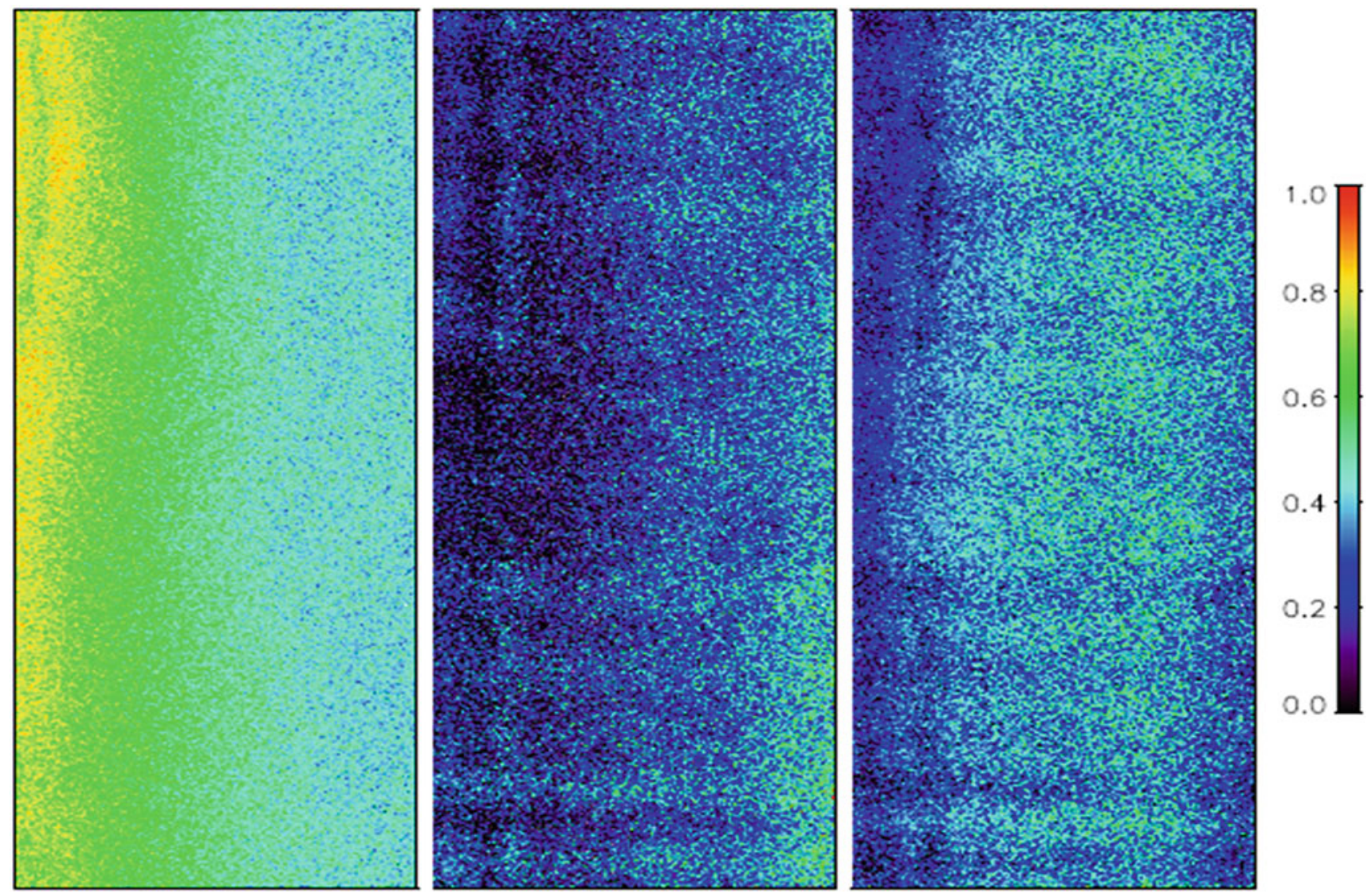

Fig. 4.2 Decomposed power contributions from L-band ascending acquisitions of March 2007, for the Summit test site. From left to right, surface, volume, and oriented sastrugi contributions are shown
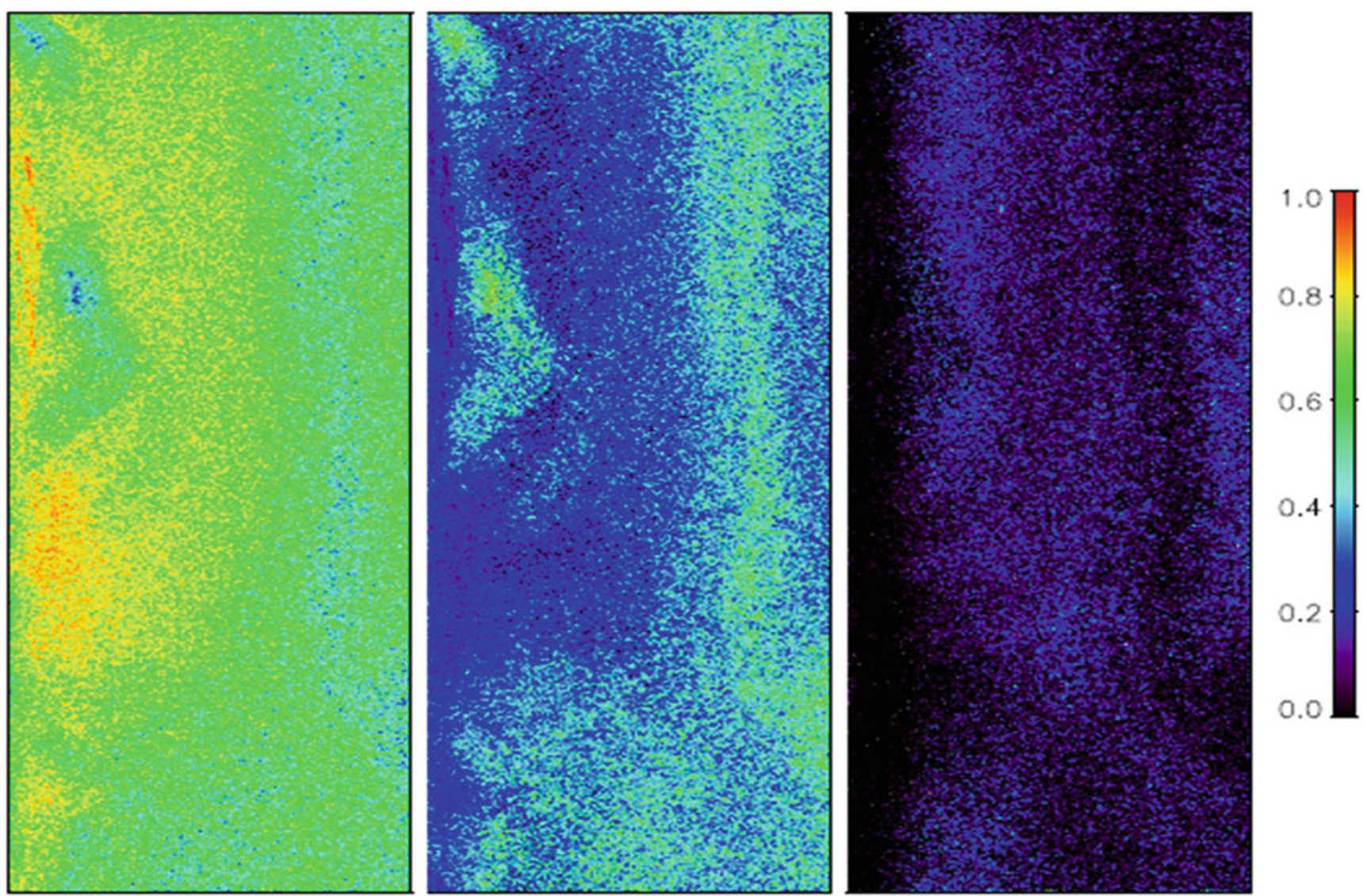

Fig. 4.3 Decomposed power contributions from P-band ascending acquisitions of March 2007, for the Summit test site. From left to right, surface, volume and oriented sastrugi contributions are reported 
decomposed powers for the surface, volume, and sastrugi contributions at L- and P-band, respectively, for the Summit test site. As expected, the surface contribution is generally higher in the near range, due to the steeper incidence angle, while it decreases for higher incidence angle at both frequencies. Interestingly, the estimated volume power is rather different at L- and P-band. In the first case, Fig. 4.2 shows a quite homogeneous image, with slight increase from the near to the far range, consistent with the behaviour of the surface component described above. Figure 4.3 depicts a rather different scenario for P-band as the volume component varies more within the scene. Areas characterised by higher volume contribution might be related to the presence of abundant melt features (ice pipes, lenses) located deeper in the firn layer, that can only be detected at P-band due to the enhanced penetration capability. Finally, decomposed sastrugi powers point out a stronger influence on L-band measurements than at P-band, confirming that longer wavelengths are more sensitive to the underlying ice volume.

A first validation of the estimated extinction values has been carried out by comparison with published laboratory experiments on pure ice from (Tiuri et al. 1984; Ulaby et al. 1986a; Dowdeswell and Drewry 2004; Warren and Brandt
2008). These studies report absorption extinction coefficients of 0.02 to $0.09 \mathrm{~dB} / \mathrm{m}$ at $\mathrm{L}$ - and P-band for temperatures ranging from -8 to $-5{ }^{\circ} \mathrm{C}$, with L-band extinctions slightly larger than P-band values. Extinctions derived at L-band from glacier ice samples include one study from the dry-zone of Antarctica yielding $0.29 \mathrm{~dB} / \mathrm{m}$ at $1.5 \mathrm{GHz}$ and $0.04 \mathrm{~dB} / \mathrm{m}$ at $0.9 \mathrm{GHz}$ (Holmlund et al. 2000) and a second study from the Canadian Arctic quoting a value of $0.05 \mathrm{~dB} / \mathrm{m}$ at $1.3 \mathrm{GHz}$ (Uratsuka et al. 1996). At P-band an extinction rate of $0.03 \mathrm{~dB} / \mathrm{m}$ was derived from GPR data in the percolation zone of central Greenland (Paden et al. 2007). Comparing these values to the experimentally derived results from the firn zone at Summit (see Fig. 4.4), the averaged L-band results from the Pol- InSAR model of around $0.1 \mathrm{~dB} / \mathrm{m}$ are reasonable when compared to (Holmlund et al. 2000), falling between the extinctions derived for 0.9 and $1.5 \mathrm{GHz}$. The L-band extinctions at Summit are somewhat higher than that quoted in (Uratsuka et al. 1996), although the glacier faces for (Uratsuka et al. 1996) is unknown, such that it may correspond to relatively pure ice such as in the ablation or superimposed ice zones without ice inclusions to introduce scattering loss. At P-band, there are higher extinctions $(>0.15 \mathrm{~dB} / \mathrm{m})$ in areas of concentrated potential melt
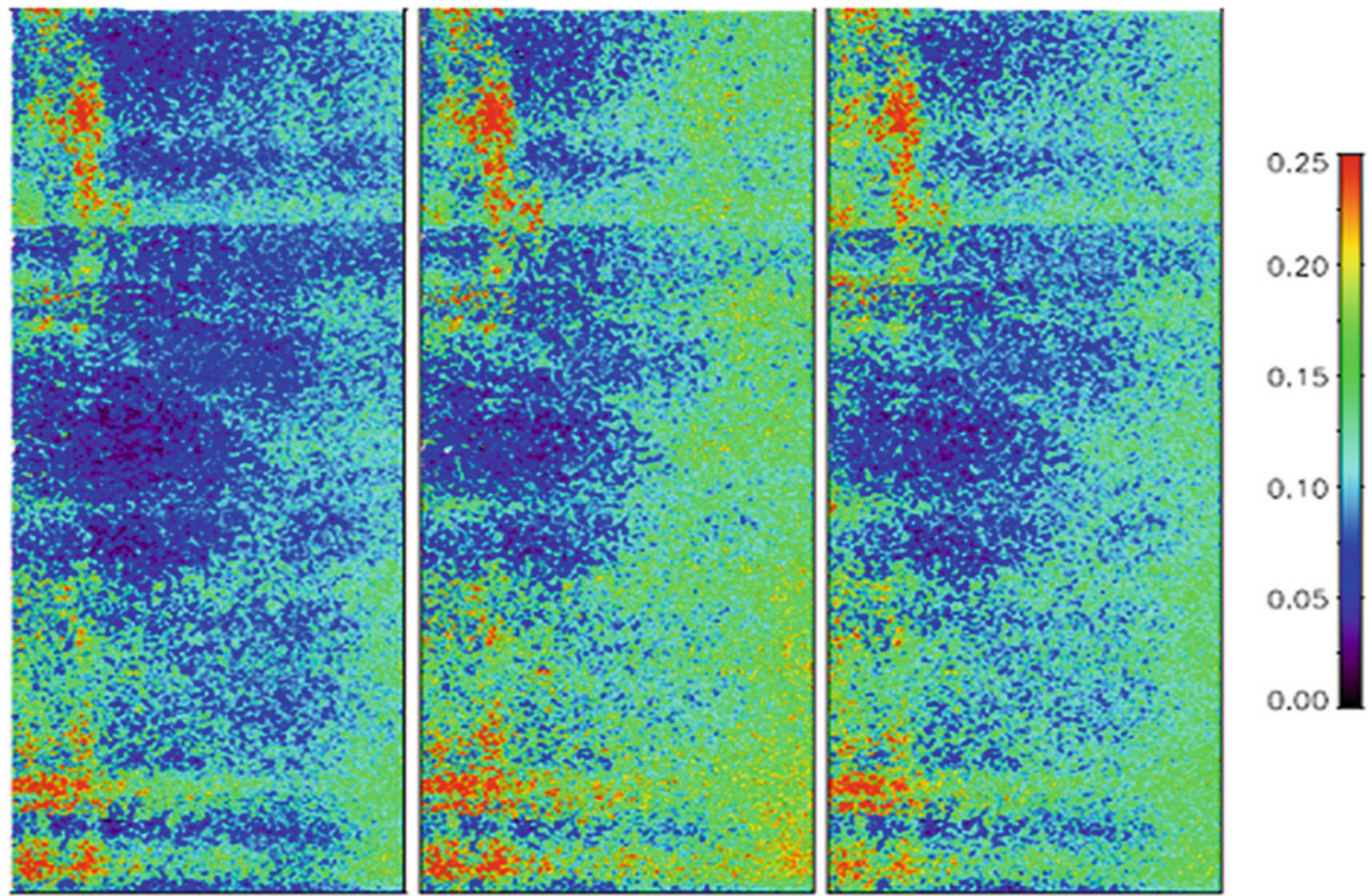

Fig. 4.4 Inverted extinctions $k_{e}[\mathrm{~dB} / \mathrm{m}]$ for $\mathrm{HH}$ (left), $\mathrm{VV}$ (middle), and $\mathrm{HV}$ (right) polarisations, from ascending L-band acquisitions of March 2007 over Summit 

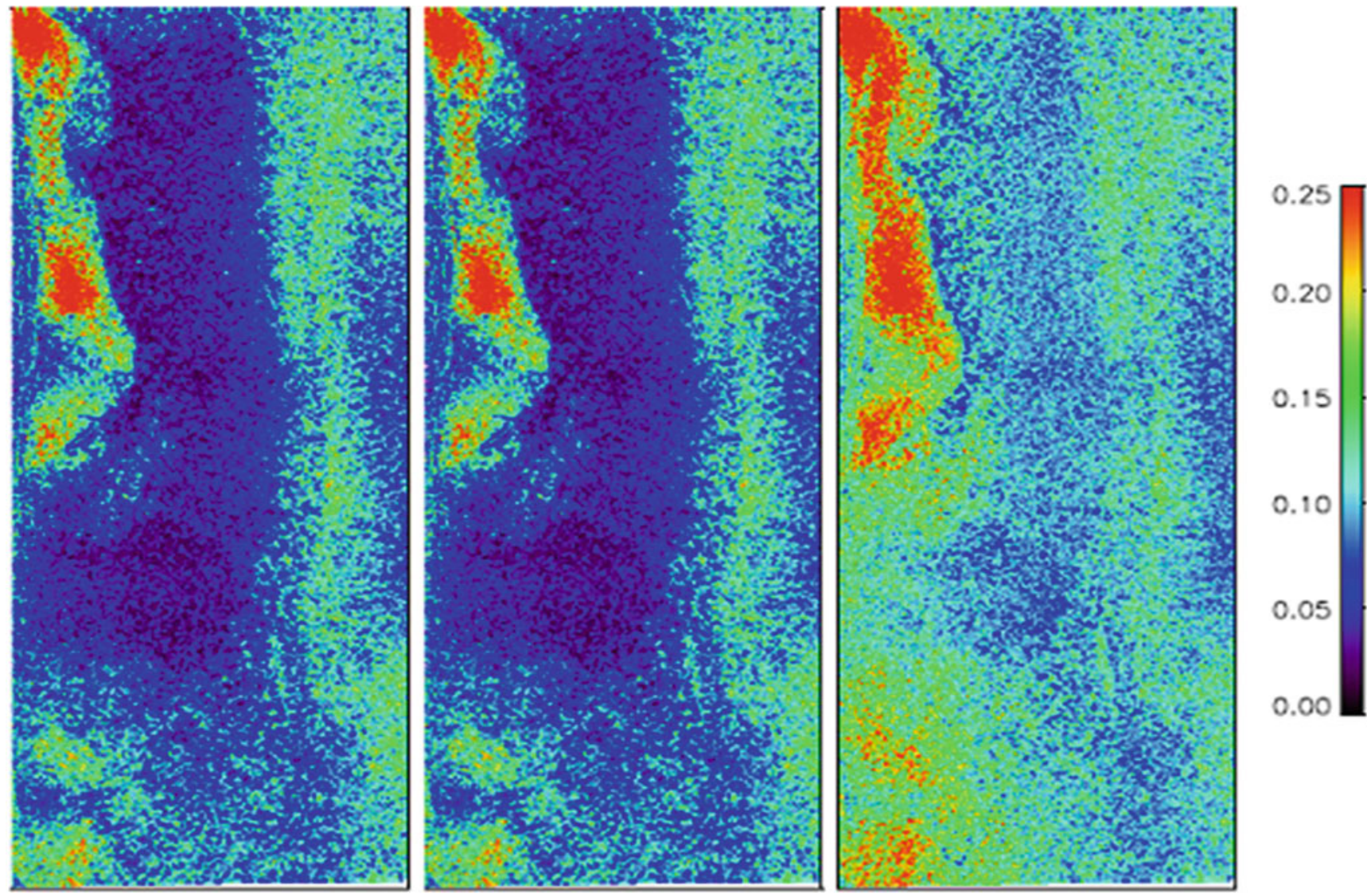

Fig. 4.5 Inverted extinctions $k_{e}[\mathrm{~dB} / \mathrm{m}]$ for $\mathrm{HH}$ (left), $\mathrm{VV}$ (middle) and $\mathrm{HV}$ (right) polarisations, from ascending P-band acquisitions of March 2007 over Summit

structures at Summit, but background values are approximately $0.05 \mathrm{~dB} / \mathrm{m}$ in Fig. 4.5 for the co-polarisations, in rough agreement with (Paden et al. 2007). At Etonbreen in the superimposed ice zone, extinctions at both L-band and P-band are lower than at Summit, with values close to $0.05 \mathrm{~dB} / \mathrm{m}$ at L-band and $0.04 \mathrm{~dB} / \mathrm{m}$ at P-band for the co-polarisations.

Only a relative comparison between the GPR and SAR data is possible, because of the differences in frequency, depth of integration (only around $10 \mathrm{~m}$ for the GPR data compared to the much deeper SAR penetration depths inverted from the estimated extinctions), and most significantly, differences in acquisition geometry. In fact, the GPR is nadir-looking and thus has a strong surface reflection component compared with the SAR side-looking backscatter. Nevertheless, a relative comparison of GPR backscattering coefficients with the SAR data was carried out in (Sharma 2010) to verify whether the volume scattering seen by the GPR is related to that from the inverted extinctions. However, better agreements were observed between inverted extinctions and GPR backscatter at L-band. This could indicate that the polarimetric decomposition was able to remove the surface contribution from the SAR data. At P-band, a worse agreement was found, probably because of the SAR's deeper penetrations, well below the range of the GPR data.

\subsubsection{Comparison with Single/Dual Polarisation Data}

For this application, a comparison with a dual-polarimetric case is not possible. As seen in Sect. 4.2.2, the employed methodology is based on a polarimetric-decomposition technique for which fully polarimetric SAR data are needed. Moreover, the complexity of the scattering mechanisms involved in an ice scenario still represents a very limiting factor for the development of further electromagnetic models. Consequently, there is still a lack of studies addressing ice extinction by means of SAR, and the adopted methodology represents one of the very few published works in this field.

\subsubsection{Discussion on the Role of Polarimetry, on the Maturity of the Application, and Conclusions}

The potential of SAR for ice extinction retrieval is due to its capability to penetrate into the ice masses for several tens of metres at long wavelengths. In addition, the typical sidelooking acquisition geometry of SAR sensors avoids strong echoes from glaciers surface like in the case of GPR, and the total backscatter results to be more related to the ice volume 
structure. In this sense, polarimetry has a crucial role in the understanding of the scattering mechanisms involved in this kind of scenario, and polarimetric model-based decomposition techniques are actually a very powerful tool to model and interpret SAR signatures. The advantage of model-based approaches is that they are based on the physics of the radar scattering and the characteristics of the investigated scenario; hence their interpretation turns very straightforward.

\subsection{Snow Water Equivalent Retrieval}

\subsubsection{Introduction, Motivation, and Literature Review}

Snow is a basic reservoir of water and a significant part of the Earth's population relies on seasonal snow-packs and glacier for their water supply (Barnett et al. 2005). Snow also represents a critical component of the global water cycle and climate system. Consequently, the global warming could have a severe impact on several aspects of our society. For this reason, spatially distributed information on snow accumulation rate, glaciers mass balance, lake ice, and sea ice are strongly required. In fact they became essential for the development of large-scale hydrological and climate models, in order to better understand the on-going changes and to predict future climate dynamics. In this sense, satellite missions are capable to provide global and systematic observations also over the Polar Regions, where the extreme climate makes in situ studies very challenging. For snow cover monitoring, snow water equivalent (SWE) is the primary parameter to estimate. SWE can be defined in units of $\mathrm{kg} / \mathrm{m}^{2}$ as the product of snow depth and density, or in units of $\mathrm{m}$ by normalising it to the water density $\left(\rho_{\text {water }}=1 \mathrm{~kg} / \mathrm{m}^{3}\right)$.

Microwave remote sensing overcomes several limiting factors, like weather and sun illumination dependence, affecting optical and infrared techniques. Its potential in monitoring snow cover properties is related to retrieval of the electromagnetic properties (e.g. dielectric constant) of snow. In the microwave range, snow exhibits well-defined spectral and polarimetric signatures related to its special dielectric proprieties which, in turn, depend on the micro- and macroscopic structure and geometry of the snowpack (Maetzler 1998). These characteristics make SAR suitable for studying and monitoring snow cover.

Due to the high penetration capability of microwaves (for frequencies up to X-band) and low attenuation of dry snow, a snowpack behaves as a quasi-transparent medium. In this case, the major scattering source is the snow/ground interface (Shi et al. 1993). Hence, X-band or higher frequency (e.g. Ku-band) SAR sensors are needed for snow depth measurement and snowpack characterisation because of their higher sensitivity (shorter wavelength) to the snowpack structure (Shi and Dozier 1993). Considering a typical snow cover scenario, several parameters influence the measured backscattering. The most significant include snow density and depth, grain size distribution, wetness, and their variation along depth as well as snow surface roughness underlying soil conditions (permittivity, roughness, and topography).

First investigations concerning snow parameter estimation by means of SAR have been addressed with single frequency and single polarisation (both $\mathrm{VV}$ and $\mathrm{HH}$ ) data mainly due to the restricted operation modes of early airborne and space borne SAR sensors (Guneriussen et al. 2001; Shi and Dozier 1995, 2000a, b). Several methods have been developed for snow cover mapping using multi-temporal SAR data. Time series C-band SAR data have been used to estimate SWE of a snow cover in the Appalachian Mountains in Southern Quebec (Bernier and Fortin 1998). The study revealed that the scattering from a shallow snow layer $(\mathrm{SWE}<20 \mathrm{~cm})$ is undetectable at C-band.

Several experiments have been conducted by using multifrequency (L-, C-, and X-band) and multi-polarisation radar backscatter data from the Shuttle Imaging Radar-C (SIR-C) mission to estimate snow density, depth, and particle size (Shi and Dozier 2000a, b). The retrieval approach was based on a physical model and provided reasonable estimates of SWE, validated with in situ measurements. Nevertheless, the estimation uncertainty remained large, probably due to the already mentioned smaller sensitivity of low frequencies to SWE than higher microwave frequencies. Recent modelling approaches indicate that combined observations at X-and $\mathrm{Ku}$-bands are more suitable for remote sensing of SWE (Shi 2006). In this sense, the dual-frequency COld REgions Hydrology High-resolution Observatory (CoReH2O) Candidate Mission was selected from the European Space Agency (ESA) for feasibility studies in the frame of the Earth Explorer Programme (European Space Agency 2008). The CoReH2O SWE retrieval procedure is based on the inversion of a two-layer (snow-over-ground) radiative transfer model, using measured dual polarisation ( $\mathrm{VV}$ and $\mathrm{VH}$ ) backscattering as input together with some a priori information (European Space Agency 2008). This procedure is specifically developed for open areas under the assumption of a dry snow cover.

In the last years, the availability of fully polarimetric space-borne X-band data (e.g. from TerraSAR-X) made possible first investigations on the potential of polarimetry in estimating snow cover properties from space. For this, a first polarimetric decomposition technique for a snow-cover scenario was proposed in (Pisciottano et al. 2011) to discriminate the scattering contribution of the snow-pack from the underlying ground, in order to extract the snow layers characteristics independently from the ground conditions. 


\subsubsection{Methodology}

The SWE estimation procedure developed for this application is based on a two-step procedure. In the first stage, the polarimetric decomposition proposed in (Pisciottano et al. 2011) is applied on fully polarimetric data acquired at $\mathrm{X}$ and C-band to separate volume and ground scattering contribution, then an entropy-based criterion is used to mask out vegetated areas. As second step, the decomposed VV and VH backscattered power components are used in the place of the respective backscattering coefficient as input for the CoReH2O inversion algorithm. For this, the original estimation procedure has been adapted to the single-frequency case (X- or C-band) considering only the modelling of the snowvolume backscattering. Some a priori information (from ground measurements) is also needed (e.g. snow density and temperature) as input for the estimation procedure.

The main advantage of using polarimetric decomposition for this application is the possibility to work with the only snow-volume scattering component. In this way, the SWE inversion is not depending on the ground layer conditions (dielectric constant, soil moisture, and roughness) anymore and the modelling of a ground scattering component becomes unnecessary. Consequently, the number of a-priori information needed for the inversion algorithm is considerably reduced.

\subsubsection{Polarimetric Decomposition for Snow-Covered Areas}

The polarimetric decomposition proposed in (Pisciottano et al. 2011) is used for the developed SWE retrieval procedure over open areas. It consists in a two-component decomposition, derived as adaptation of the model proposed in (Freeman 2007), originally developed for a volume of vegetation covering the ground. In the case of a snow cover scenario, the volume scattering component is attributable to the snow cover, acting as a volume of particles (snow grains), whereas the surface-like scattering contribution is due to the underlying ground. Both volume and ground component can be modelled by means of their respective coherency matrices.

The snow layer is assumed to be a volume of uniformly distributed and randomly oriented particles. An additional parameter is the shape factor $\rho$, ranging from $\rho=1 / 3$ for the case of dipoles to $\rho=1$ for spheres. Intermediate values of $\rho$ indicate spheroidal particles (Nghiem et al. 1992). Under this assumption, the volume coherency matrix can be expressed as (Hajnsek et al. 2007):

$$
\mathbf{T}_{v}=f_{v}\left[\begin{array}{ccc}
1+\rho & 0 & 0 \\
0 & 1-\rho & 0 \\
0 & 0 & 1-\rho
\end{array}\right]
$$

where $f_{v}$ corresponds to the intensity of the volume scattering component.

The surface scattering component is modelled according to the Bragg model and its coherency matrix results to be:

$$
\mathbf{T}_{s}=f_{s}\left[\begin{array}{ccc}
1 & \beta * & 0 \\
\beta & |\beta|^{2} & 0 \\
0 & 0 & 0
\end{array}\right]
$$

where $f_{s}$ is the intensity of the surface scattering component and $\beta$ is defined as:

$$
\beta=\frac{R_{h}-R_{v}}{R_{h}+R_{v}}
$$

with $R_{h}$ and $R_{v}$ representing the Bragg scattering coefficients for horizontally and vertically polarised wave, respectively.

The power contribution of the two components to the total backscattered power $P_{\text {tot }}$ can now be written as:

$$
\begin{gathered}
P_{s}=f_{s}\left(1+|\beta|^{2}\right) \\
P_{v}=f_{v}(3-\rho)
\end{gathered}
$$

corresponding to the trace of the associated coherency matrices. Using the transformation from coherency to covariance matrix (Hajnsek 2001) the VV and VH power contribution of the volume scattering component can be derived and written as:

$$
\begin{gathered}
P_{v}^{\mathrm{VV}}=f_{v} \\
P_{v}^{\mathrm{VH}}=f_{v} \frac{1-\rho}{2}
\end{gathered}
$$

The quantities $f_{v}$ and $\rho$ can be estimated from the coherency matrix of the real data as suggested in (Pisciottano et al. 2011).

\subsubsection{Snow Water Equivalent Retrieval Algorithm}

The original SWE inversion procedure proposed in (European Space Agency 2008) for the CoReH2O candidate mission is based on a dual frequency (X-and Ku-band) and dual polarisation approach and is developed for dry snow cover in open areas. The core of the procedure is a two-layer Dense Medium Radiative Transfer (DMRT) backscatter model. It relates the physical properties of the snow layer and the underlying ground to the SAR backscattering in the $\mathrm{VV}$ and $\mathrm{VH}$ polarisations. In particular, the snow volume is modelled as a layer of uniformly distributed spherical 
particles (snow grains), which have same size. Under this hypothesis, the volume backscatter contribution can be written as (Rott et al. 2008; Ulaby et al. 1984):

$$
\begin{aligned}
\sigma_{p q}^{v}\left(\vartheta_{t}\right)= & T_{p q}^{2}\left(\vartheta_{t}\right) \\
& \times\left[\frac{\omega_{p q}}{2}\left(1-\exp \left(-2 \frac{k_{e} \mathrm{SWE}}{\rho_{s} \cos \left(\vartheta_{t}\right)}\right)\right) \cos \left(\vartheta_{t}\right)\right]
\end{aligned}
$$

where $T_{p q}$ is the transmission coefficient at the air-snow interface; $p$ and $q$ the transmitted and received polarisation, respectively; and $\vartheta_{t}$ the transmitted angle through the snowpack. The snow parameters involved are the density $\rho_{s}$, the extinction coefficient $k_{e}$, the scattering albedo $\omega$, and the SWE.

The entire estimation algorithm can be split in two steps. First, VV and VH backscatter coefficients are simulated using the DMRT model, together with a set of a priori information and initialisation values of the parameters to estimate: SWE and grain size. Successively, a constrained optimisation method is applied pixel by pixel to iteratively match the simulated $\mathrm{X}$ - and Ku-band VV/VH backscatter coefficients to the measured values. The input values of SWE and grain size ensuring the reached optimum condition are finally assumed as estimates.

As already mentioned, this procedure is strongly dependent on the required a priori information about the ground and snowpack conditions. This kind of information are usually obtained through in situ measurements which are rather expensive, time-consuming, and can be conducted only on very small scale. Consequently, the dependency on a priori information represents a strong limitation when deriving SWE maps (and not single-point values) over a certain area.

In this sense, the modified SWE inversion procedure based on polarimetric decomposition results to be much more robust since the dependency on most of the a priori information is eliminated. First, the $\mathrm{CoReH} 2 \mathrm{O}$ algorithm has been adapted for a single-frequency case (X- or C-band). Then, the capability of the polarimetric decomposition to isolate the snow volume scattering component allows to focus the DMRT modelling on the only volume component. In fact, assuming that the volume backscattering is uncorrelated to the underlying ground layer (no second order scattering considered), there is no need to know soil conditions. This represents a significant simplification of the entire procedure since the ground contribution strongly influences the total backscattering. In addition, the sensitivity analysis of the CoReH2O DMRT model to the snow properties performed in (Pisciottano et al. 2011) showed that the backscattering contribution from the snow layer is mainly influenced by SWE and grain size, and only slightly dependent on other parameters, like snow temperature and density. This means that the latter two quantities need to be known for a rigorous SWE inversion, whereas a slightly rougher estimate can be obtained by assuming fixed values of snow temperature and density over the processed SAR scene. The scheme in Fig. 4.6 shows the working flow-line of the proposed SWE estimation procedure. The final products are SWE maps with a spatial resolution of about $20 \times 20 \mathrm{~m}$ in the case of TerraSAR-X data and $40 \times 40 \mathrm{~m}$ for RADARSAT- 2 .

\subsubsection{Experimental Results}

Test sites and corresponding radar and validation data sets selected for the generation of showcases on snow water equivalent retrieval are summarised in Table 4.3 and further described in the Appendix.

The SWE estimation procedure described before has been applied over different subsets of the available TerraSAR-X and RADARSAT- 2 dataset. A validation could be carried out only over the small areas where the ground measurements took place. For brevity, the focus is on test site of Sodankylae. In particular, the results shown in the following are obtained in correspondence of the Intensive Observation
Fig. 4.6 Scheme of the SWE estimation procedure based on polarimetric decomposition and modified $\mathrm{CoReH} 2 \mathrm{O}$ algorithm

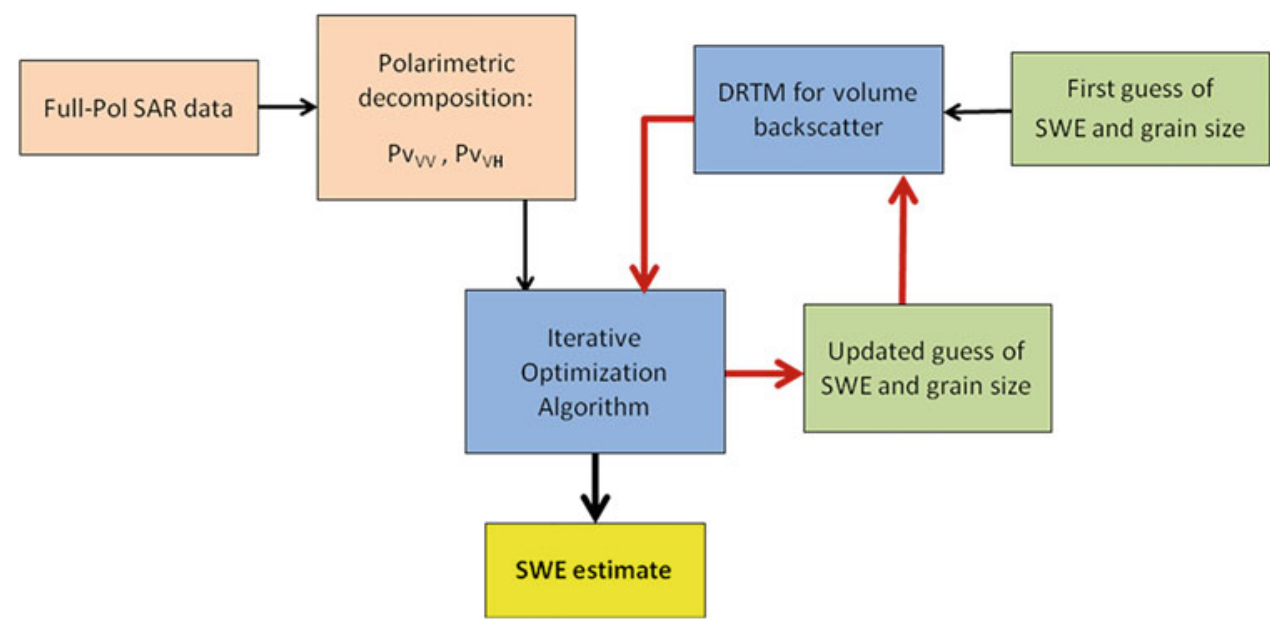


Table 4.3 Test sites and corresponding radar and validation data selected for the generation of showcases on snow water equivalent retrieval

\begin{tabular}{l|l|l}
\hline Application/product & Test site - Radar data & Reference data \\
\hline \multirow{2}{*}{$\begin{array}{l}\text { Snow water equivalent } \\
\text { retrieval }\end{array}$} & Sodankylae, Finland & $\begin{array}{l}\text { Ground measurements (CASIX } \\
\text { experiment) }\end{array}$ \\
\cline { 2 - 3 } & $\begin{array}{l}\text { 3 TerraSAR-X quad-pol scenes (2010), 8 RADARSAT-2 quad-pol scenes } \\
(2011-2012)\end{array}$ & \\
\cline { 2 - 3 } & $\begin{array}{l}\text { Churchill, Canada } \\
\text { 3 TerraSAR-X quad-pol scenes (2010) 5 RADARSAT-2 quad-pol scenes } \\
(2011-12)\end{array}$ & \\
\hline
\end{tabular}

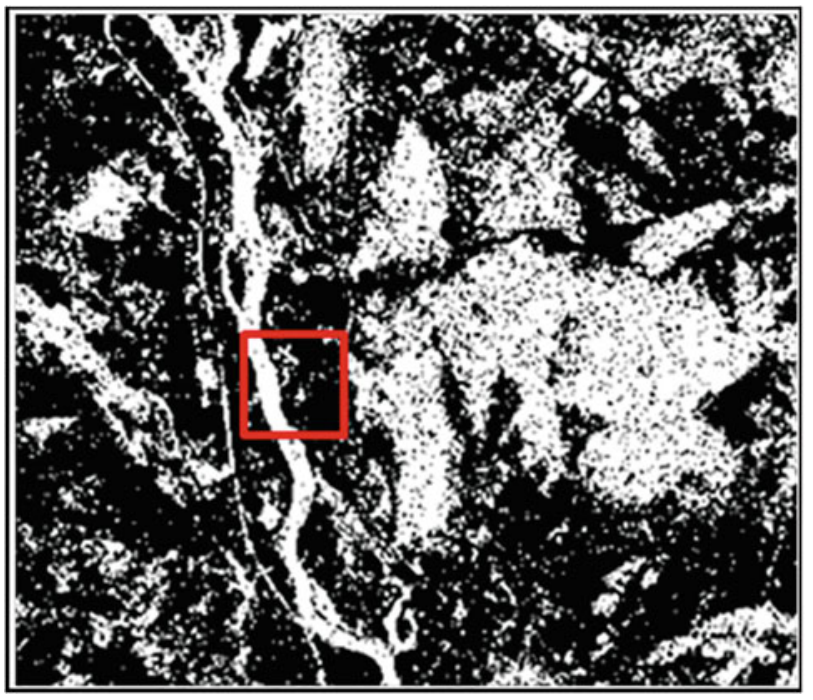

Fig. 4.7 The image on the left shows the entropy-based mask for open areas obtained from the TerraSAR-X acquisition of the 11.04.2010 over Sodankylae. The red box indicates the subset including the IOA where a

Area (IOA), where continuous SWE measurements are available for the same period of the SAR acquisitions.

The three TerraSAR-X acquisitions available for this test site were acquired on the 11th and 22nd of April and the 3rd of May 2010. In these dates, point measurements of SWE in the IOA reported values of $205 \mathrm{~mm}, 191 \mathrm{~mm}$, and $164 \mathrm{~mm}$, respectively. The corresponding retrieved values obtained from the inversion procedure, using snow density and temperature measurements as a priori information, are shown in Fig. 4.7, where a validation is also performed by comparison with the respective measured values of SWE. As clearly shown in Fig. 4.8, for the acquisition of April, the SWE inversion performs very well; estimated values of $211 \mathrm{~mm}$ and $193 \mathrm{~mm}$ correspond to measured values of $205 \mathrm{~mm}$ and $191 \mathrm{~mm}$, respectively. These results definitely satisfy the accuracy requirement established for the $\mathrm{CoReH} 2 \mathrm{O}$ mission. For the case of May, the worse performance can be due to the raising of air temperature reported in the ground measurements for that period. This may have led to a change in the snow-pack structure, moving away from the validity hypothesis of the used DMRT model. For the case of RADARSAT-2, preliminary results (see Fig. 4.9) seem to confirm the conclusions of (Bernier and Fortin 1998) since

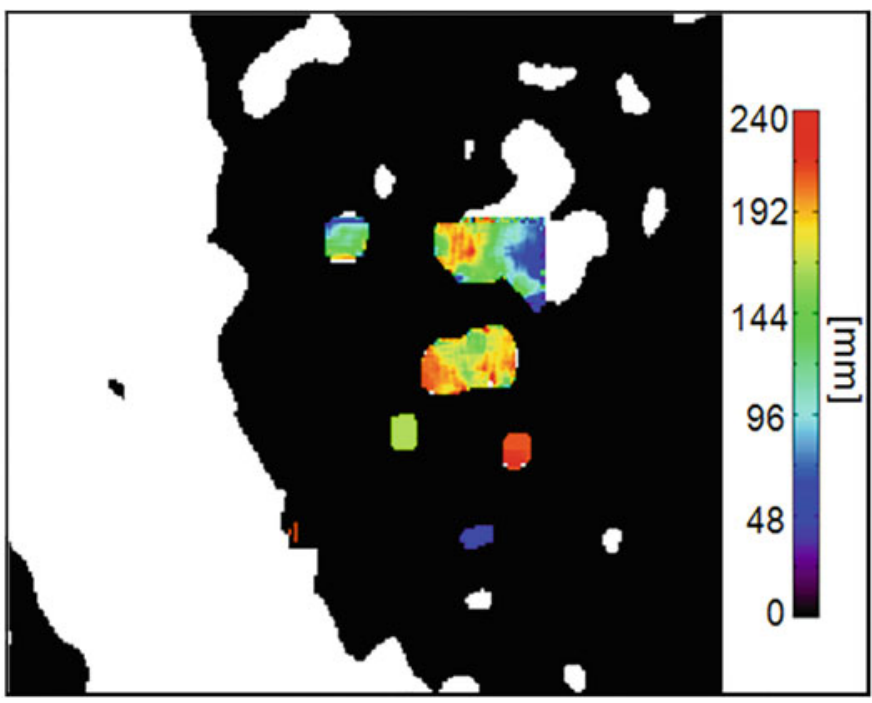

demonstration of SWE estimation has been performed (image on the right). SWE values are retrieved over a $60 \times 60$ pixels subset and range from $0 \mathrm{~mm}$ (black) to $240 \mathrm{~mm}$ (red)

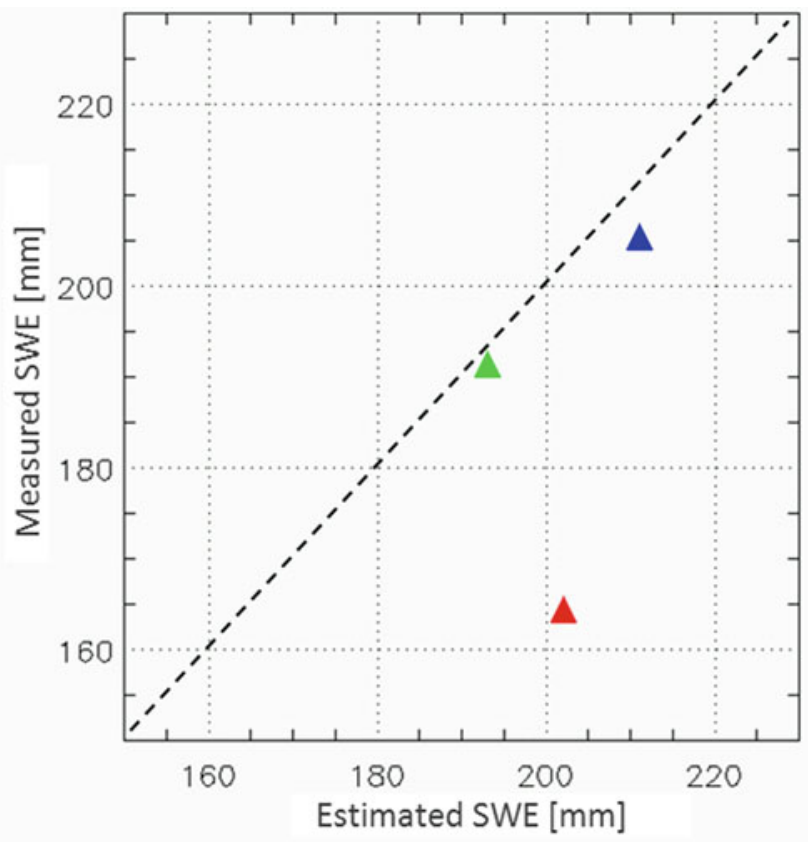

Fig. 4.8 Validation of estimated SWE over the IOA of the Sodankylae test site using the three TerraSAR-X acquisitions. Blue, green, and red symbols are referred to the dates of 11-04-2010, 22-04-2010 and 03-052010, respectively 


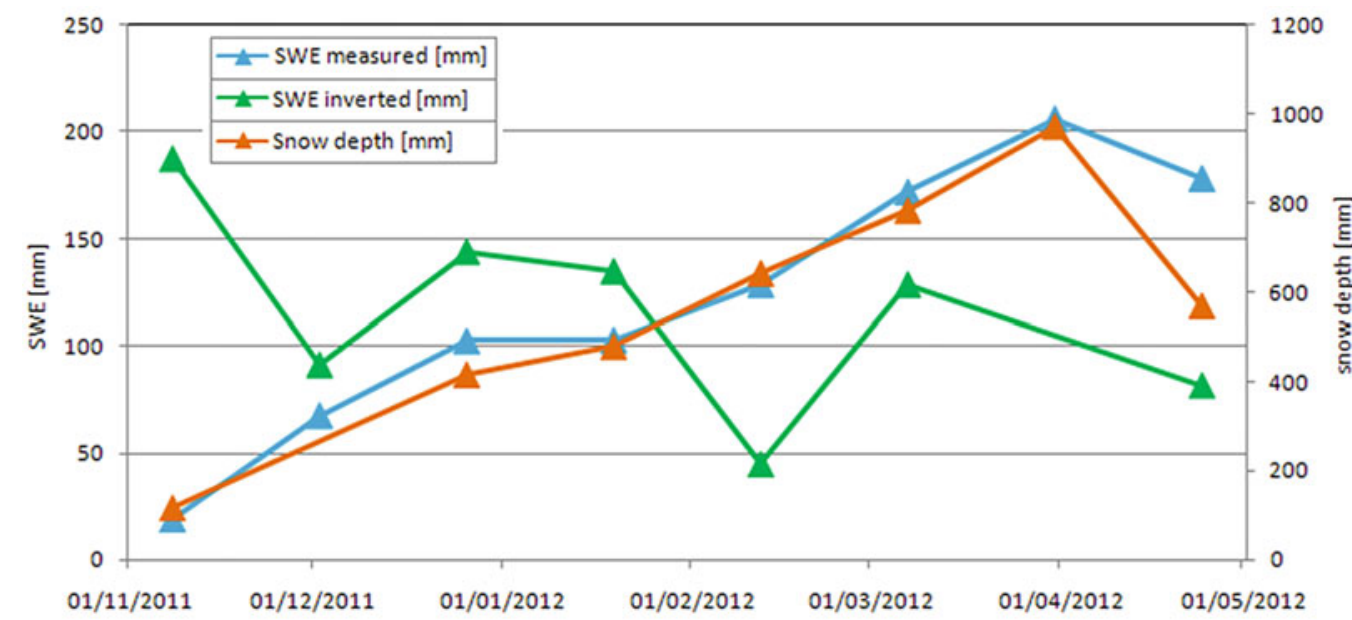

Fig. 4.9 Time series of measured SWE (blue), snow depth (orange), and inverted SWE (green) using RADARSAT-2 data for the Sodankylae IOA test site

almost no sensitivity can be observed along time in presence of the shallow snow cover (SWE always lower than $20 \mathrm{~cm}$ ) reported for Sodankylae.

\subsubsection{Comparison with Single/Dual Polarisation Data}

Some comparisons have been carried out between the employed methodology, based on fully polarimetric data, and the original $\mathrm{CoReH} 2 \mathrm{O}$ dual polarimetric retrieval procedure. Preliminary results show that the procedure based on polarimetric decomposition is more robust than the dual-pol procedure, especially when tested over different ground conditions (bare soil, swamp). This is attributable to the capability of the polarimetric-decomposition to extract the volume scattering component quite efficiently over different kinds of ground, while the efficiency of DRTM model for the ground scattering proposed in the dual-pol approach is severely compromised when soil condition are not known (Pisciottano et al. 2011). Nevertheless, these results have to be confirmed by further investigations.

\subsubsection{Discussion on the Role of Polarimetry, on the Maturity of the Application and Conclusions}

The importance of polarimetry in this kind of applications is well established, as already discussed in the literature review. Single polarisation studies have shown a very limited potential and were mainly employed for snow cover mapping. The introduction of polarimetry, even if with a dual-pol configuration, allowed deeper investigations about the scattering mechanisms and their relative contributions to the total scattering. They also contributed significantly for the development of more detailed physical models. Nevertheless, as observed for the case of the CoReH2O SWE inversion, a dual polarisation approach requires a significant a priori knowledge to be able to estimate snow properties (e.g. SWE, grain size, etc.). The introduction of fully polarimetric data makes possible the development of decomposition techniques for snow cover scenarios and has the big potential to reduce (almost eliminate) the need of any a priori information about the investigated test site.

\subsection{Sea Ice Characterisation}

\subsubsection{Sea Ice Observation with Quad-Polarimetric SAR}

\subsubsection{Introduction, Motivation, and Literature Review}

The observation of sea ice is a major topic in remote sensing due to the difficulty of performing frequent in situ expeditions (Elachi and Van Zyl 2006; Gareth Rees 2006; Jackson and Apel 2004). Monitoring of sea ice is important for many environmental issues (Sandven et al. 2006). First of all, it is a sensitive climate indicator, and it plays an important role in global climate systems. It restricts the exchange of heat and chemical constituents between ocean and atmosphere acting as an insulator. Moreover, it influences the global climate system with effects related to its elevated 
albedo, reducing the amount of solar radiation absorbed at the Earth's surface. On the other hand, sea ice affects oceanic circulation directly by the rejection of salt to the underlying ocean during ice growth, which is responsible for deep water formation. Besides these, the possibility and safety of navigation in Polar Regions is severely influenced by the presence of sea ice.

Microwave sensors and synthetic aperture radar (SAR) are very valuable for monitoring sea ice since they can acquire information in absence of solar illumination (i.e. during Polar nights) and with almost any weather conditions (Franceschetti and Lanari 1999). Unfortunately, the description of the backscattering behaviour of sea ice is particularly challenging. For this reason, many scientists moved towards systems able to increase the amount of information acquired. In this context, polarimetry plays a key role, because it is able to enhance dramatically the discrimination capability of the observed target, solving many ambiguities revealed in single polarisation images (Cloude 2009; Lee and Pottier 2009; Van Zyl 2011). Specifically, sea ice could be modelled as a layered media showing several interfaces: air-snow, snowice, and (eventually) ice-water (Elachi and Van Zyl 2006; Jackson and Apel 2004; Drinkwater et al. 1992; Nghiem et al. 1995a, b; Ulaby et al. 1986b).

An introduction to polarimetry is provided in the first chapters of this book. In this section we only concentrate on the polarimetric models introduced to characterise sea ice. Several models were proposed to predict the polarimetric behaviour of sea ice (Nghiem et al. 1995a, b; Ulaby et al. 1986b; Carlström 1997; Tjuatja et al. 1992). A thorough description of them is outside the purpose of this Section.

\subsection{Polarimetric Models}

To understand the backscattering from ice, it is important to know the physical differences between different ice types. Here, only a very short inventory is reported and the reader is redirected to the World Meteorological Organization (WMO) Sea Ice Nomenclature document for further details. Following its formation, the ice can be defined new ice (frazil ice, grease ice, slush and shuga), nilas (below $10 \mathrm{~cm}$ thick), young ice (10-30 cm thick), first year ice $(30 \mathrm{~cm}$ to $2 \mathrm{~m}$ thick), and old or multi-year ice (that has survived at least one melting season). The floating structures are generally called floes (that can have extensions from few metres to several kilometres). Another structure commonly seen during ice formation is pancakes (generally smaller than $3 \mathrm{~m}$ ) that may present raised rims and draft. With deformed ice, it is designed ice that suffered deformations due to pressure or melting. Some classical structures are ridges and hummocks. Another interesting feature of sea ice areas are leads, which are openings (cracks) in the ice tens of metres large and up to several kilometres long. Each of the ice formations or features will have a characteristic scattering behaviour.
Sea ice is generally modelled as a multi-layer medium:

1. The first interface is between air and snow cover (Tjuatja et al. 1992; Beaven et al. 1995). The behaviour of this first layer is strongly dependent on the dielectric properties of the snow, which are dependent on factors as water content and temperature. If the dielectric constant is small enough (i.e. dry and cold conditions), this interface will not scatter much, and the snow will be transparent to the electromagnetic wave. It is important to keep in mind that in some conditions as during snow melting this layer can mask completely the underneath ice.

2. The second interface is snow-ice. The behaviour of this interface depends on the difference in dielectric constants between snow and ice (Nghiem et al. 1995a, b; Ulaby et al. 1986b). If the ice is highly saline, its dielectric constant is quite high, and the most of the backscattering will come from the surface. On the other hand, if the ice is less saline, the electromagnetic wave can penetrate, and a consistent volume scattering will be observed (Beaven et al. 1995). Another important factor in the balance of surface and volume scattering is the surface roughness. As a role of thumb, higher roughness (compared with the wavelength) will provide higher backscattering (Ulaby et al. 1986b). The Bragg model is generally exploited to characterise the surface scattering, but when the roughness is very large, an extended version of the model should be used (Cloude 2009).

3. Ice volume: Characterising the backscattering of the ice volume is particularly challenging due to the large differences showed by ice types (strongly depending on their formation process). From experiments and modelling, it appears that the backscattering mainly comes from brine inclusions which represent small discontinuities in the volume. Polarimetry is expected to be useful to acquire some insight on the typology of ice, since it is sensitive to particles anisotropy and orientation (Nghiem et al. 1995a, b; Ulaby et al. 1986b).

Besides a simple layered structure, ice generally presents a large variety of deformation features due to the action of sea and wind that compress and crack floes (Carlström 1997; Isleifson et al. 2009; Onstott et al. 1998). Ridges, hummocks, and areas with broken ice are generally (but not necessarily) bright in the SAR images. This makes possible, in some instances, the identification of floes edges (Dierking and Dall 2007). Polarimetry combined with heterogeneity analysis may play a role in detecting such features, since their polarimetric behaviour is expected to be different from the one of homogeneous floes.

Another feature observable under some circumstances is the frost flowers. They form in calm and windless conditions when the atmosphere temperature is much lower than the one 
of the ice (Isleifson et al. 2010). They may present a very large backscattering which may be eventually misinterpreted by some models.

\subsection{Validating Models and Experiments}

Many experiments were carried out in order to test the polarimetric models. Some of them consider laboratory conditions, where all the parameters are under control and can be accurately monitored. A famous series of experiments were the CORRELEX (Nghiem et al. 1995b; Beaven et al. 1995). Although in a lab it is possible to control accurately the experiment, there may be problems in replicating some of the characteristics of sea ice formations that are only achievable in the field. For instance, the mechanical effect of waves is central in ice formation. One experiment that tried to capture this characteristic is presented in Onstott et al. (1998).

Another way to test models is to use a scatterometer and collect data over actual sea ice (Nghiem et al. 1995b; Isleifson et al. 2009; Dierking 1999; Geldsetzer et al. 2007; Kern et al. 2006; Partington et al. 2010). Clearly this approach may lack control compared to a lab experiment, and it is generally limited to a reduced number of ice types; however, it is often preferred to lab experiments because it is able to picture more realistic scenarios.

\subsubsection{Methodology}

In this section the employed methodologies and polarimetric observables are briefly listed. Details about them can be found in the introductory chapters, here only the applicability to sea ice observation is examined.

\subsection{Co-polarisation Ratio}

This is defined as the averaged intensities of $\mathrm{VV}$ over $\mathrm{HH}$ polarisations. In the literature, many authors have used this observable (Geldsetzer et al. 2007; Kern et al. 2006; Drinkwater et al. 1990; Geldsetzer and Yackel 2009; Kwok et al. 1991) for several reasons. (1) To detect open water and leads: it is a useful discriminator between Bragg and volume scattering (Geldsetzer and Yackel 2009). A complication is when water in very calm conditions has a very low signal-tonoise ratio (SNR), which makes the two Co-Polarisations more similar. (2) To analyse volume scattering: due to particles anisotropy, it is sometimes observed that the volume can have HH power higher than VV (Geldsetzer et al. 2007). (3) Ice thickness: the balance of surface and volume scattering in sea ice is ruled by dielectric constant and roughness. When these two are high, the sea ice behaves more as a surface; otherwise a volume is observed (Kern et al. 2006). Interestingly, the dielectric constant is strongly dependent on the ice salinity, and the latter exhibits some dependency on ice thickness (thicker ice is less saline due to expulsion and washing up during melting seasons). Therefore, thicker ice should have a lower ratio, and several attempts were made to find a regression curve to extract thickness from this ratio (Kim et al. 2012; Wakabayashi et al. 2004).

\subsection{Cross-Polarisation Ratio (or Depolarisation Ratio)}

This is the ratio between a cross-pol and a co-pol channel (Kim et al. 2012; Thomsen et al. 1998). This can be extended using the sum of cross-polarisations over the sum of co-polarisations (Geldsetzer et al. 2007). It is mainly employed to:

1. Detect open water: water is expected to have low CrossPolarisation backscattering.

2. Ice thickness: for similar reasoning like the Co-Polarisations Ratio (Kim et al. 2012).

\subsection{Co-polarisation Coherence}

Many authors exploited the coherence between the two co-polarisations $\gamma_{C o}$ for sea ice monitoring (Nghiem et al. 1995b; Isleifson et al. 2009; Geldsetzer et al. 2007; Drinkwater et al. 1990; Kwok et al. 1991; Israelsson and Askne 1991), for the following reason. Analysing surface and volume scattering, both magnitude and phase provide information regarding the scattering process. For instance, a zero phase difference with small standard deviation is an indicator of surface scattering. Regarding volume scattering, isotropic (spherical or randomly oriented) particles provide again zero phase difference but with a standard deviation generally larger compared to surfaces. Finally, different phase behaviours can be observed depending on the particles anisotropy and orientation (Nghiem et al. 1995a, b; Ulaby et al. 1986b).

\subsection{Circular Polarisation Coherence}

This observable is very sensitive to: (1) surface roughness (Wakabayashi et al. 2004), using the magnitude; (2) orientation of the observed targets (Lee et al. 2000, 2002), using the phase. In the case of ice floes, a large scale surface variation can introduce a large phase standard deviation (Wakabayashi et al. 2004). Moreover, target (i.e. particles or surface features) orientation can be analysed and it can be used to solve misclassification with volume scattering.

\subsection{Cross-Polarisation Coherence}

In some situations (especially in melting conditions), the cross-polarisation backscattering from ice is particularly low; consequently, the signal-to-noise ratio, SNR, (defined as the ratio between averaged intensity of signal over intensity of noise) is low. This coherence can be used to check the reliability of cross-channel measurements over dark areas. 


\subsection{Cloude-Pottier Decomposition}

Several examples can be found where the Cloude-Pottier decomposition is employed for sea ice observation, and they are mainly related to (1) classification and (2) features extraction (Wakabayashi et al. 2004; Scheuchl et al. 2002, 2003a; Wesche and Dierking 2012).

\subsection{Noise Estimation/Mitigation}

Every time a coherence is estimated, it is important to understand whether it represents a physical decorrelation effect or not. Quad-polarimetry allows a very powerful methodology for estimating the value of the noise floor (Cloude 2009). Thermal noise decorrelation can be easily estimated based on the SNR of the specific channels (e.g. the two co-polarisations). Also some noise mitigation procedure can be exploited (Cloude 2009).

\subsubsection{Experimental Results}

Test sites and corresponding radar and validation data sets selected for the generation of showcases on sea ice observation are summarised in Table 4.4 and further described in the Appendix.

In this Section the results obtained using polarimetric observables are presented. For the sake of brevity, only few observables will be displayed. Figure 4.10 presents the Storfjord dataset, while Fig. 4.11 shows the Barents Sea dataset. In all the images, the horizontal axis is azimuth and the vertical is range (near range at the bottom and far range at the top of the image). The average (filtering) used considers a boxcar of $7 \times 28$ pixels. Such window should provide an equivalent number of looks of around 86. Moreover, only for visualisation purposes, the images consider the multi-look of 4 pixels in azimuth, which makes the pixels on the ground

Table 4.4 Test sites and corresponding radar and validation data selected for the generation of showcases on sea ice observation

\begin{tabular}{l|l|l}
\hline Application/product & Test site - Radar data & Reference data \\
\hline \multirow{2}{*}{ Sea ice observation } & Fram strait, Storfjord, Barents Sea (Svalbard) & No ground measurement available \\
\cline { 2 - 2 } & E-SAR L-band & \\
\hline
\end{tabular}
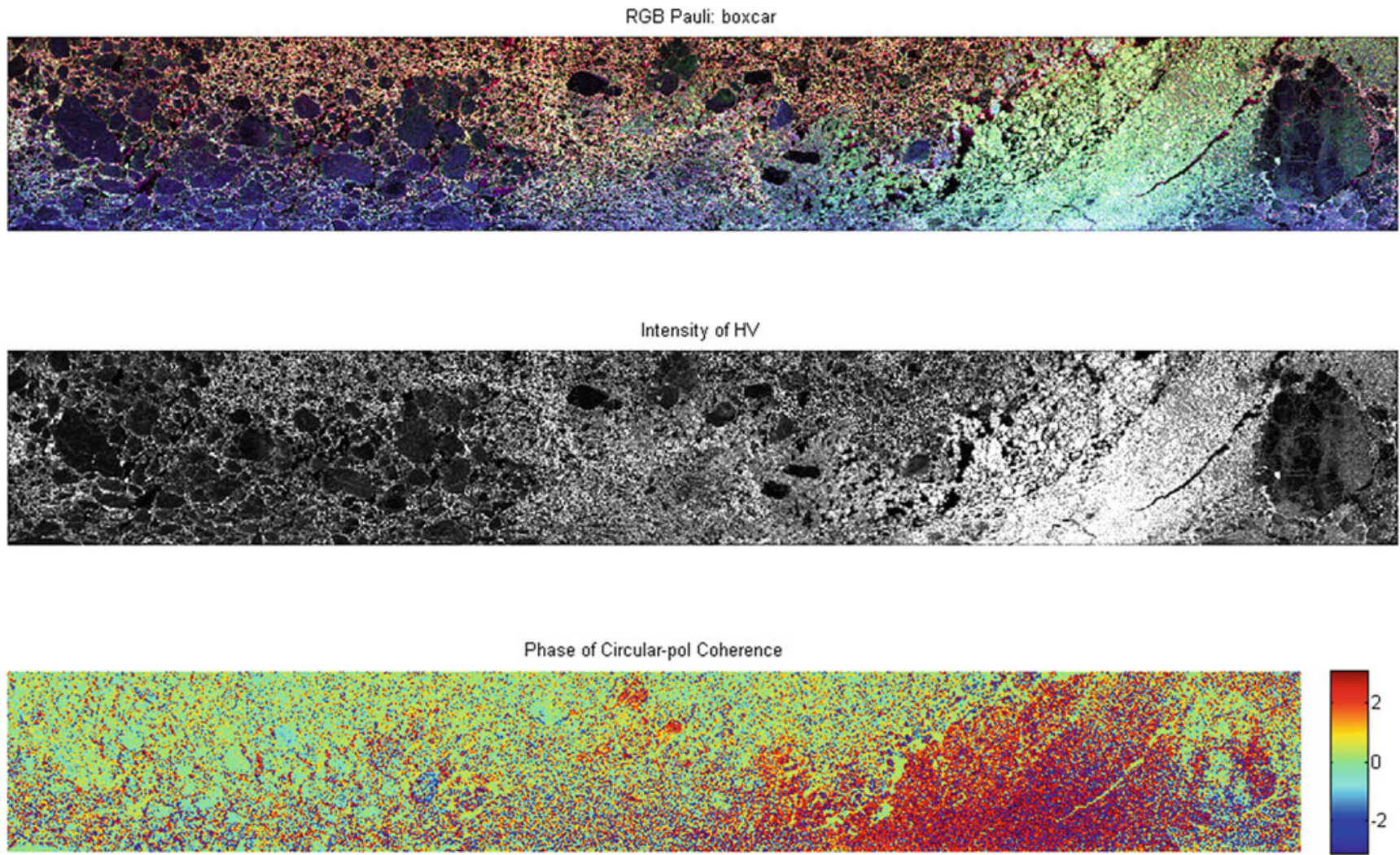

Fig. 4.10 Polarimetric analysis of L-band ICESAR data: Storfjord (16.03.2007). RGB Pauli composite image (Red, $|\mathrm{HH}-\mathrm{VV}|^{2}$; Green, $|2 \mathrm{HV}|^{2}$; Blue, $\mid \mathrm{HH}+\mathrm{VVI}^{2}$ ); intensity of cross-polarisation; phase of circular-polarisations coherence 


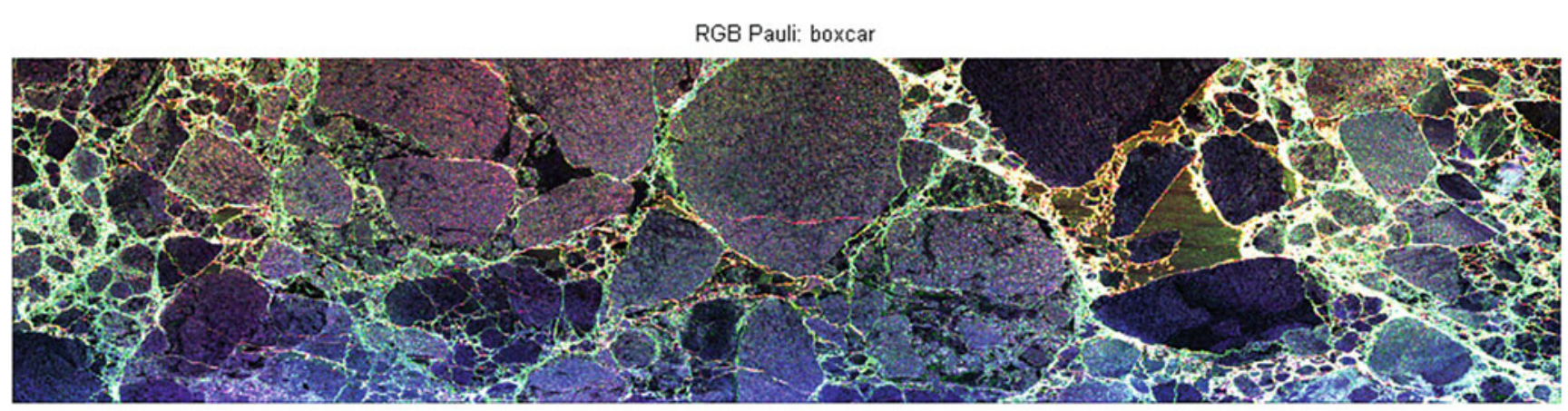

CoPolarisations Ratio

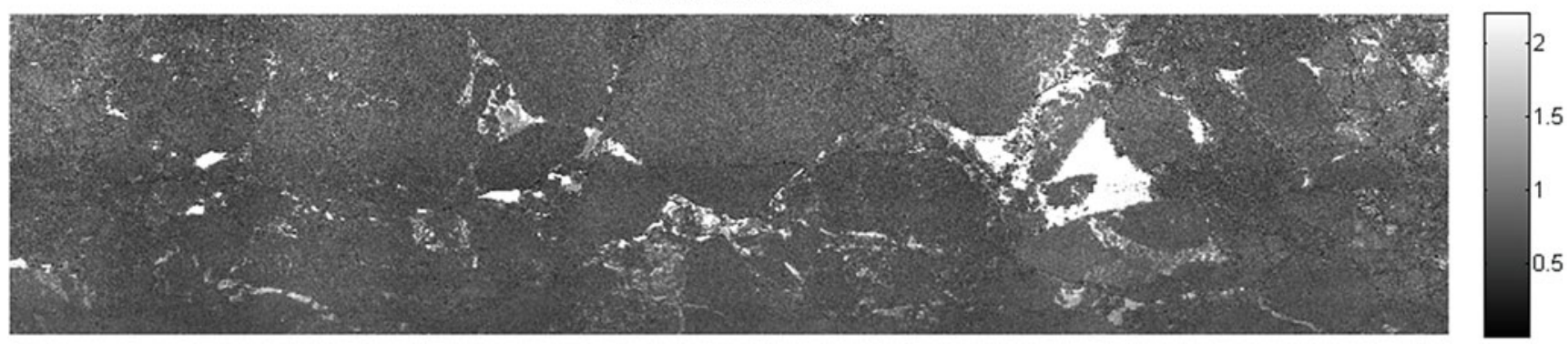

Magnitude of $\mathrm{CoP}$ ol coherence
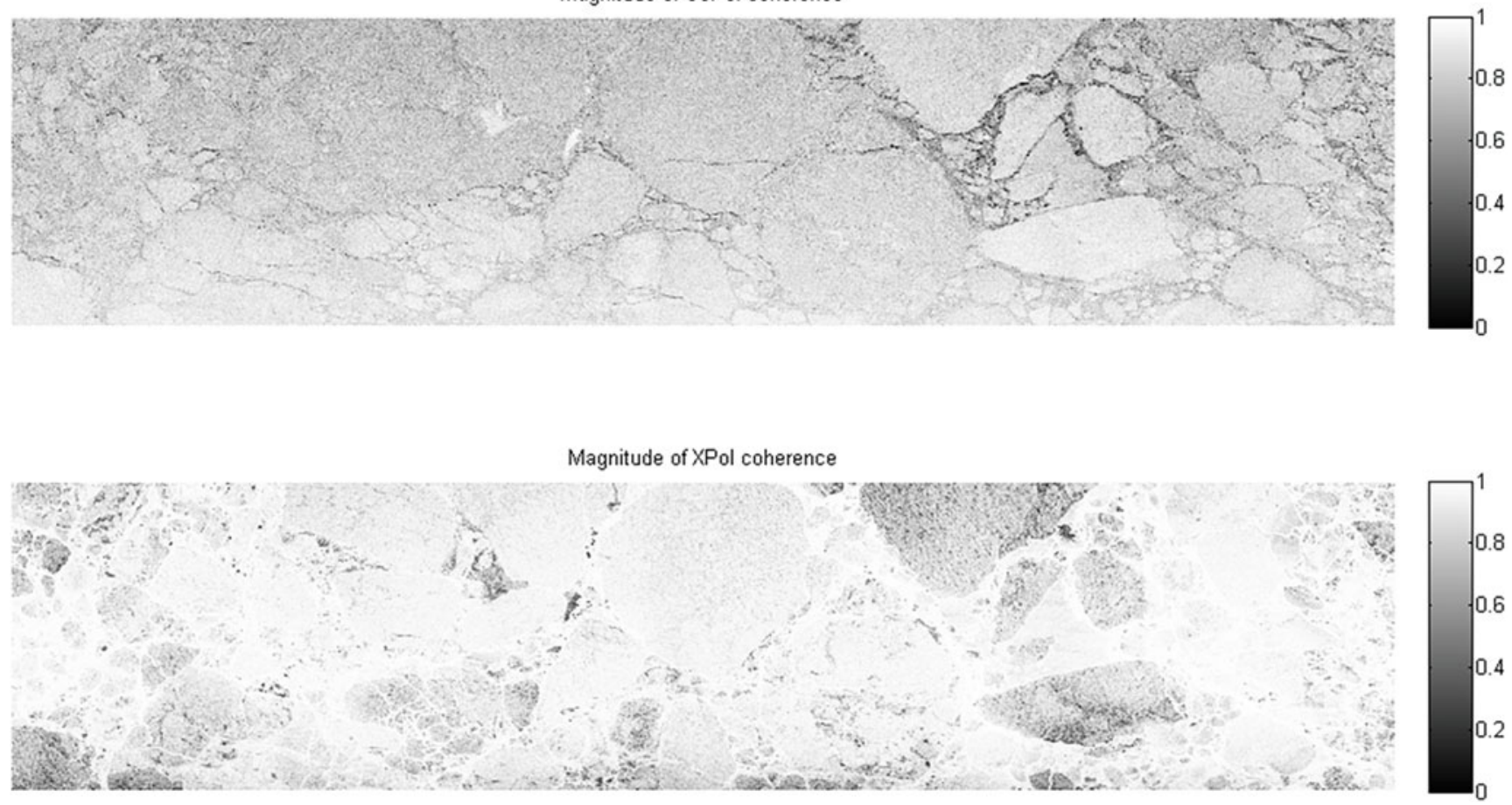

Fig. 4.11 Polarimetric analysis of L-band ICESAR data: Barents Sea (18.03.2007). RGB Pauli composite image (Red, $\mid \mathrm{HH}-\mathrm{VVI}^{2}$; Green, $|2 \mathrm{HV}|^{2}$; Blue, $\mid \mathrm{HH}+\mathrm{VVI}^{2}$ ); co-polarisations ratio; magnitude of co-polarisations coherence; magnitude of cross-polarisations coherence 
more squared (please note, this depends on the range location in the image).

Results can be interpreted as follows:

1. Pauli RGB: It can be noticed that the simple visualisation of the Pauli RGB image can help significantly to distinguish between different sea ice types. Interestingly, it appears that the colour of the images is bluer in the near range (bottom: around $26^{\circ}$ of incidence angle) than in far range (top: about $65^{\circ}$ of incidence angle). This is in line with many models that predict a stronger surface contribution for small incidence angles and a larger volume contribution for larger incidence angles (Nghiem et al. 1995b; Carlström 1997; Tjuatja et al. 1992).

2. Phase of circular-polarisation: in Fig. 4.10, a green (i.e. HV) bright area is visible. Strong backscattering in the cross-channel is often associated with volume scattering (e.g. multiyear ice, MYI). However there is no MYI in the scene. Only by analysing this phase it is possible to understand that the strong cross return is due to orientation effects (i.e. it is not MYI). The reason of such orientation is not completely known; nevertheless the correction allowed solving this ambiguity.

3. Co-polarisation ratio: This observable is very powerful to highlight areas of open water (Geldsetzer and Yackel 2009). In Fig. 4.11, the bright areas correspond to open water (this was validated with a photograph captured during the flight pass).

4. Co-polarisation coherence: This is used to discriminate between different kind of scattering mechanisms and volume types. Figure 4.11 represents an example where the magnitude of this observable can be used to discriminate between floes. Additionally, some studies described correlation between this observable and ice thickness (Nghiem et al. 1995b).

5. Cross-polarisations coherence: From Fig. 4.11, it can be seen that some floes present a low value (i.e. low SNR), and therefore care has to be given when the crosspolarisation is used to retrieve parameters over such areas.

\subsubsection{Discussion on the Role of Polarimetry, on the Maturity of the Application and Conclusions}

Few conclusions could be drawn on the benefits of using polarimetric data for sea ice observation.

1. Higher discrimination: The use of four images instead of one enhances the capability to characterise the scattering process. For instance, the co-polarisation ratio can be used to detect open water, and the co-polarisations coherence tells about the scattering mechanism. If our interest is focused on detecting ice features as ridges or hummocks, then the depolarisation ratio or the Cloude-Pottier decomposition can be useful.

2. Avoiding misclassification (correction for orientation angle): Another powerful methodology only possible with quad-pol data is the correction for orientations of observed targets. The latter can produce misclassification (in this case with multi-year ice).

3. Noise estimation/mitigation: With quad-pol data, it is possible to estimate locally the noise floor and understand where the measurements are less reliable due to noise (and therefore they should not be used for quantitative analysis).

As a final remark, from the literature it seems that polarisation has a valuable role in sea ice observation (even if just with dual-polarimetric data). It is generally quite agreed that at least a dual-polarimetric mode is necessary in order to understand the behaviour of different kinds of sea ice. In some instances, the refrain in exploiting polarimetric modes is the impossibility to acquire very large swaths (as ScanSAR images) that in many sea ice applications are needed to cover vast areas in short time. Fortunately, many of the new generations of satellites will be able to acquire polarimetric data with wide swaths.

\subsubsection{Sea Ice Segmentation and Classification from Fully Polarimetric SAR Imaging}

\subsubsection{Introduction, Motivation and Literature Review}

SAR images of sea ice reveal large variability in appearance as function of imaging geometry (incidence angle, polarisation, frequency), physical properties (surface roughness, ice type, and other surface properties), and meteorological conditions. Wave propagation effects, such as attenuation, emission, and scattering, are strongly affected by physical properties like salinity, temperature, snow cover, wetness, volume structure, and surface roughness (Kong 1986). As a result, the physical structure of sea ice leads to radar signatures that may enable image segmentation and ice type classification.

Sea ice is in general divided into two major categories, first-year ice (FY) and multi-year ice (MY). MY ice has survived at least one summer melt and is discriminated from FY-ice on the basis of properties such as deformation (roughness, surface topography), thickness, salinity, and snow cover (Nghiem et al. 1995a). The extent of deformation is exploited when trying to discriminate ice types. The most commonly referred sea ice types are level ice, rafted ice, ridged ice, rubble fields, and hummocked ice. Level ice is ice with a relatively flat surface, which has not been deformed 
to any extent. Rafted ice arises when ice sheets collide and override one another; it occurs usually on new and FY-ice. Pressure processes cause the ice to pile up both above and below the surface. A ridge is the result of such processes and can be described as a long line of piled up, cracked ice. Repeated ridging causes rubble fields. Leads are open water channels in areas of predominantly sea ice. In addition, there exist several ice types representing young and thin sea ice.

The emergence of dual- and fully polarimetric spaceborne SAR systems gives prospects for enhancement of the amount of information about sea ice that can be obtained from satellite-borne sensors.

The goal of this note is to present an example of the improvements gained in characterisation of sea ice by utilising polarimetric observations instead of just singlepolarisation SARs. We will here focus on how polarimetry may improve segmentation and classification.

From previous studies of multi-polarisation measurements of sea ice, the knowledge status can currently be summarised as follows:

- Polarimetry is promising for the identification of the early stages of new (thin) ice formation and the discrimination of ice and open water (Dierking et al. 2003).

- The role of phase in polarimetric classification is unclear.

- The optimal frequency for polarimetric classification remains unclear and varies with application and region (Dierking et al. 2004). Overall, L-band appears to be more effective for full polarimetric ice classification than C-band.

- Multi-frequency is superior to single-frequency polarimetry. This would require improved methods for data integration and data fusion processing.

Full polarimetric SAR observations allow for the decomposition of radar signals into the contributions from the various scattering mechanisms. Even if it is generally known that scattering from sea ice is dominated by surface scattering, the backscattered signals may include contributions from several mechanisms. The relative contributions of rough surface scattering, specular reflections, volume scattering, and multiple scattering processes depend on thickness, degree of deformation, size of deformed structures, amount of snow on the ice, salinity, and compactness of the ice fragments (Dierking et al. 1997).

Several polarimetric parameters have been evaluated for first year sea ice discrimination in C-band RADARSAT-
2 data in (Gill and Yackel 2012), and the authors obtained a classification accuracy greater than $90 \%$. Extracting polarimetric information from sea ice scenes was the main subject of (Eltoft et al. 2012), and it was noted that the multitude of decomposition parameters are yet to be fully evaluated for sea ice discrimination.

\subsubsection{Methodology}

The workflow for retrieval of sea ice information undertaken in this Section includes two major steps. The first step is an unsupervised segmentation of the image, which subdivides it into a given number of segments based on variations in statistical and polarimetric properties. This step is followed by a polarimetric analysis, where the objective is to infer the polarimetric properties of each image segment. These properties may be interpreted in terms of physical characteristics, which may help label the segments into ice types.

The PolSAR image is segmented using a mixture of Gaussian models for the global probability density function (pdf). We assume reciprocity, and the segmentation algorithm uses the following 6 parameters generated from the general $(3 \times 3)$ C-matrix, as described in (Doulgeris and Eltoft 2010; Doulgeris 2013). These 6 parameters are geometric brightness, co-polarisation ratio, cross-polarisation ratio, co-polarisation, correlation magnitude, and co-polarisation correlation angle. The six features are nonlinearly transformed such that the marginal pdfs have a Gaussian-like appearance at the peaks. We then model the global pdf as a multivariate Gaussian mixture distribution and segment the image into a given number of unlabeled segments using the expectation maximisation algorithm, as described in (Doulgeris and Eltoft 2010). The proper number of classes is currently manually estimated based on optical images, the Pauli image, the sea ice observation log, and the segmentation results obtained with different number of classes.

\subsubsection{Experimental Results}

Test sites and corresponding radar and validation data sets selected for the generation of showcases on sea ice segmentation and classification are summarised in Table 4.5.

The smoothed, geocoded segmentation result is shown in Fig. 4.12. The image has been segmented into five classes. Sea ice experts interpreted the segmentation result aided by thickness measurements, optical photos, and the Pauli image. According to their interpretation, the yellow class is thin ice or open water; the red class is young, thin first year ice with

Table 4.5 Test sites and corresponding radar and validation data selected for the generation of showcases on sea ice segmentation and classification

\begin{tabular}{l|l|l}
\hline Application/product & Test site - Radar data & Reference data \\
\hline $\begin{array}{l}\text { Sea ice segmentation and } \\
\text { classification }\end{array}$ & $\begin{array}{l}\text { Arctic Sea, north of } \\
\text { Svalbard }\end{array}$ & $\begin{array}{l}\text { Ice thickness measurements (EM-bird), optical photos, roughness } \\
\text { measurements }\end{array}$ \\
\cline { 2 - 2 } & $\begin{array}{l}\text { RADARSAT-2, April } \\
2012\end{array}$ & \\
\hline
\end{tabular}




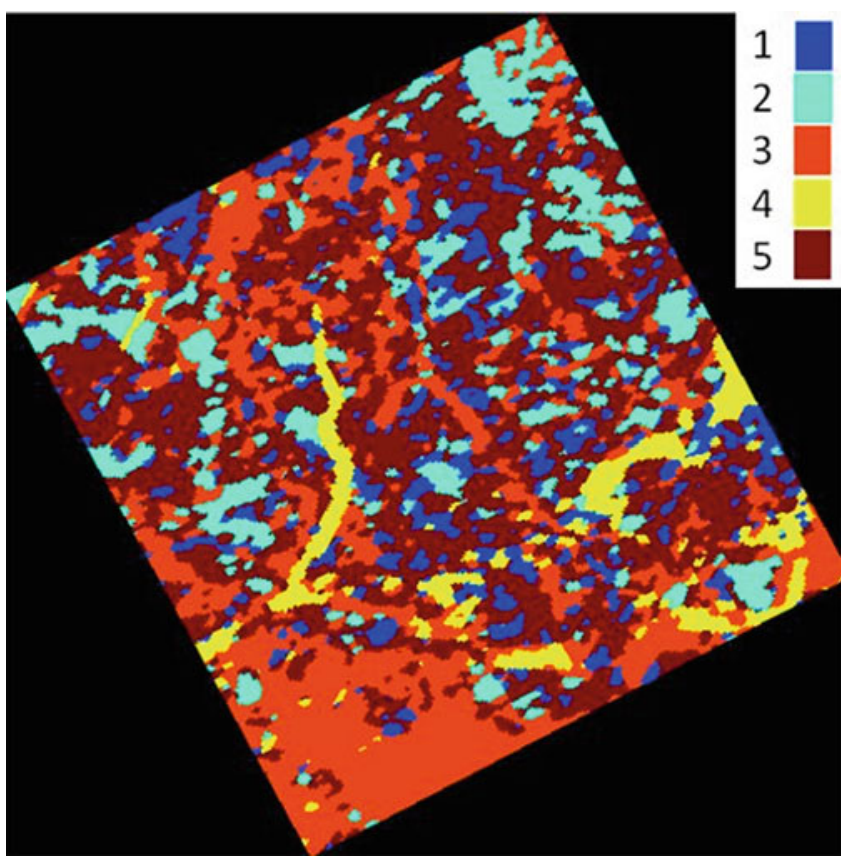

Fig. 4.12 Image segmented by the automated segmentation algorithm into five classes snow cover and some deformation; the blue, brown, and cyan classes are various stages of first year ice.

Figure 4.13 displays the thickness estimates along the helicopter track. As can be noted, the thickness varies between 0 and 6-7 m. Below the thickness curve, we have plotted the image segments along the flight track. By comparing the thickness and the coloured segments, we can conclude that the yellow class certainly corresponds to thin, new frozen ice, or open leads. We also see strong fluctuations in thickness in some classes, indicating deformed ice. We note that the cyan class seems to have the least thickness variations, indicating that this is a smooth ice type. This observation is in agreement with the fact that this class has very low backscatter. However, this class consists of relatively thick ice, around $1.5 \mathrm{~m}$ according to the EM-bird estimates.

\subsubsection{Comparison with Single/Dual Polarisation Data}

As illustrated in Fig. 4.14, radar polarimetry will in general increase the dimension of the feature space and enable retrieval of more information from a scene. Whereas single polarisation SAR images basically allow for image

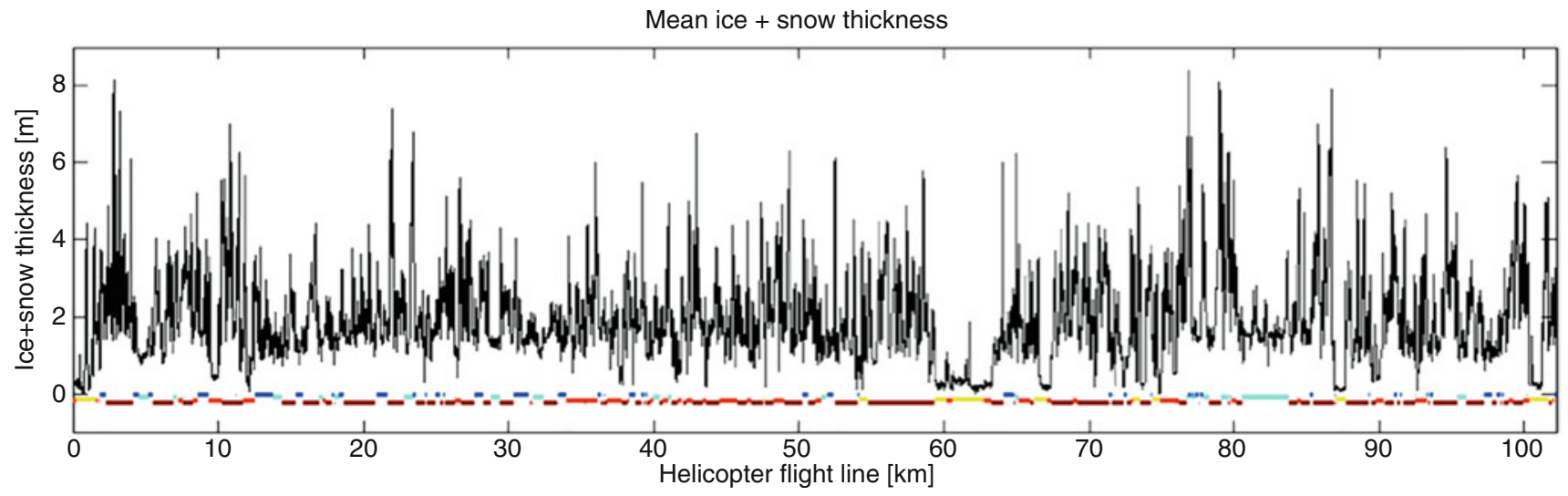

Fig. 4.13 Thickness estimates from EM-bird measurements. The colour segments at the bottom corresponds to the segments along the flight track

Fig. 4.14 The added feature space dimension by radar polarimetry

\section{Single polarization}

$$
\begin{aligned}
s_{l} & =\operatorname{Re}\left\{s_{l}\right\}+j \operatorname{Im}\left\{s_{l}\right\} \\
I_{l} & =\left|s_{l}\right|^{2} \\
I_{L} & =\frac{1}{L} \sum_{i=1}^{L} s_{i}
\end{aligned}
$$

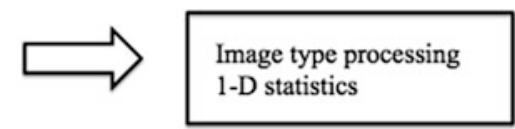

Multiple polarizations

$\mathbf{S}=\left[s_{h h}, s_{h v}, s_{v h}, s_{v v}\right]^{t}$
$C=\frac{1}{L} \sum_{i=1}^{L} \mathbf{s}_{i} \mathbf{s}_{i}^{*}$$\longrightarrow$\begin{tabular}{l|}
$\begin{array}{l}\text { Multidimensional } \\
\text { statistical analysis }\end{array}$ \\
\hline
\end{tabular}


processing type analysis, polarimetry will allow for both polarimetric analysis, to infer scattering information, as well as the use of advanced multivariate statistical methods. With respect of the current application showcase, we note that a single polarisation image will be restricted to only two parameters, the multi-looked intensity and a measure of non-Gaussianity. Polarimetry allows for much more, and it is a subject for future research to optimise the feature selection. From our experience, polarimetry will result in more detailed segmentation.

\subsubsection{Discussion on the Role of Polarisation, on the Maturity of the Application and Conclusions}

This Section has presented the result of segmenting a RADARSAT-2 PolSAR scene of Arctic sea ice, collected north of Svalbard in the Spring 2011. The scene shows various stages of first year ice, plus leads, some open, and some refrozen. The data set also comprised in situ data such as EM-bird thickness measurements, and optical photos along the helicopter tracks covered by the SAR image.

The analysis shows that the feature set consisting of six statistical and polarimetric parameters has discrimination power and enables segmentation of this sea ice scene into five proper segments. Some of these can be identified as distinct ice types. The added information associated with full polarimetry allows for a more detailed segmentation of the scene, as well as providing scattering mechanism information, which may subsequently help labelling the segments into ice types.

\subsubsection{Antarctic Sea Ice Thickness Using Sea Ice Surface Measurements and TerraSAR-X Data}

\subsubsection{Introduction, Motivation and Literature Review}

Sea ice thickness data is critical to the long-term assessment of climate change in the Polar Regions. The validation of sea ice remote sensing products in Antarctica has been complicated by remote location, limited extent, and infrequency of direct measurements. Estimates of sea ice thickness are particularly important in the Antarctic due to the limited opportunities for direct physical measurements, even when access is available.

Research objectives described in this Section are:

- Obtain spatially and temporally coincident ice measurements and TerraSAR-X (TSX) satellite measurements of Antarctic sea ice floes in the Bellingshausen Sea, an area that has undergone significant change.
- Examine polarimetric descriptors derived from TSX related to sea ice and snow characteristics for sea ice floes.

- Assess statistical relationships between surface elevation, snow depth, freeboard, ice thickness, and roughness with the ultimate goal of classifying sea ice types and calculating sea ice thickness from active radar returns.

This research benefited from direct collaboration with the British Antarctic Survey (BAS), Scottish Association of Marine Science (SAMS), and other international participants in obtaining in situ sea ice measurements during the IceBell field campaign in November 2010. The results of these efforts were used to derive sea ice and snow cover thickness relationships in the Antarctic which ultimately could improve satellite remote sensing products, allowing improved long term monitoring of the ice mass balance in the Antarctic sea ice zone.

Both passive and active microwave remote sensing have provided useful information on the extent and area of sea ice in both Polar Regions and their trends of change over approximately 30 years. Techniques for monitoring ice thickness from space have used altimetry measurements from lasers and the buoyancy of the snow and ice relative to local sea level reference (Zwally et al. 2008; Yi et al. 2011; Kurtz et al. 2009; Kurtz and Markus 2012). An alternate approach uses empirical relationships developed from in situ field data to derive ice thickness from snow freeboard (Xie et al. 2011).

Previous studies of the potential of polarimetric SAR data for sea ice monitoring and characterisation have concentrated on thin-ice due to limited data available (Scheuchl et al. 2004) which resulted in part from a lack of coincident surface measurements with SAR acquisitions. Attempts to obtain thin-ice thickness from airborne radar imagery have been made using full polarimetric data acquired by JPL's AIRSAR (Nghiem et al. 1995a; Rignot and Van Zyl 1992; Rignot and Drinkwater 1994; Kwok et al. 1995; Winebrenner et al. 1995), Danish EMISAR (Thomsen et al. 1998; Dierking et al. 2004; Skriver and Pedersen 1995), Canadian Convair580 SAR (Livingstone et al. 1996; Scheuchl et al. 2003b), German HELISCAT (Kern et al. 2006), and Japanese PiSAR (Wakabayashi et al. 2004; Matsuoka et al. 2002; Nakamura et al. 2005). Fully polarimetric spaceborne SAR data were first acquired over sea ice in 1994 by the SIR-C mission, with first results reported by Eriksson et al. (1998). Other results based on high-resolution polarimetric data were reported using ALOS-PALSAR (e.g. Wakabayashi and Sakai 2010), RADARSAT-2 (e.g. Kim et al. 2012), and TerraSAR-X (e.g. Kim et al. 2012; Busche et al. 2009).

Scheuchl et al. (2005) indicate that swath widths for fully polarimetric modes are limited and will not provide sufficient coverage for operational sea ice monitoring. This research allowed for multiple acquisitions of X-band dual-polarisation 
SAR for tracking distinct ice floes, in conditions of rapid drift, typically over $100 \mathrm{~m} / \mathrm{h}$, with $80 \%$ success. The proposed ice-thickness method improved on existing techniques showing high potential in operational sea ice monitoring.

\subsubsection{Methodology}

Radar backscatters are affected by thick snow cover (volume scattering), snow wetness, presence of ice layers, age (grain size/shape), and sea water flooding at the snow-ice interface. Due to differences in structure and composition, different types of sea ice have different polarimetric scattering behaviours. The unique coherent dual-polarimetric X-band capability of TSX imagery was used to emphasise the volume scattering through the parameters derived from entropy/alpha decomposition developed by Cloude and Pottier (1997). This method does not depend on the assumption of a particular underlying statistical distribution and is free from the physical constraints imposed by multivariate models (Singh et al. 2010).

While the conceptual theory of target decomposition was developed for quad-pol radar backscatter, it can also be applied to dual-polarised data (Cloude 2007). To derive the polarimetric decomposition parameters, we first generated the covariance matrix $\mathrm{C} 2$ of the images and then applied a $(9 \times 9$ kernel) refined Lee Filter (Lee 1981) to eliminate speckle but preserve the edge sharpness. A target decomposition technique was performed to derive the mean Alpha angle $(\bar{\alpha})$ and Entropy $(H)$. The Shannon Entropy (SE), characterised as the sum of intensity, degrees of polarisation, and the intrinsic degrees of coherence, was based on the method described by Morio et al. (2007). Correlation between SE estimates of the sea ice floe derived from TSX data acquired on November 27 and field-based surface elevation, snow depth, and freeboard was performed to discriminate the freeboard condition. For ice thickness calculation, we segregated sea ice and snow cover into generalised classes as they may impact the buoyancy model employed but not necessarily to discriminate between all the ice conditions present at that time. The sea ice thickness algorithm was based on empirical equations presented in (Xie et al. 2011) and 4 classes (i.e. deep slush layer, shallow slush layer, ice block with little snow cover, and snow cover with positive freeboard) based on the physical measurements of surface elevation, snow depth, and ice freeboard (Lewis et al. 2013). Finally, co-registration was performed on TSX imagery acquired on Nov. 27, Dec. 01, and Dec. 13. The co-registration could not be performed using floe boundaries, as these are dynamic and change rapidly, so ice blocks with very high SE values within the floe provided the tie points in the co-registration process. The root mean square (RMS) error in the co-registration of these images was less than 2 pixels (i.e. $<10 \mathrm{~m}$ ).

\subsubsection{Experimental Results}

Test sites and corresponding radar and validation data sets selected for the generation of showcases on sea ice thickness are summarised in Table 4.6.

\subsection{D Eigenvector Analysis}

The results of the entropy-alpha decomposition applied to a TSX image acquired on December 1, 2010, are shown in Fig. 4.15. Areas of low entropy commonly have a single dominant mechanism for radar backscatter as compared to areas of high entropy where multiple scattering mechanisms contribute. The alpha angle, derived from eigenvectors of the decomposition, is indicative of the average or dominant scattering mechanism. The majority of the alpha angle values are near $45^{\circ}$, suggesting that the scattering mechanism is mainly volume scattering. The extreme degrees of randomness over the open water portions of the image, which also show increased alpha angle values, are indicative of a low signalto-noise ratio.

\subsection{Sea Ice Classification and Ice Thickness Estimates}

Several data sources defined classes of SE from the Nov. 27 TSX data (coincident with the IceBell field surveys of Floe 6). These sources included the alpha channel image of entropy-alpha decomposition, the conventional $\mathrm{HH}$, and $\mathrm{VV}$ backscattering coefficients image, and the reflectivity ratio image corresponding to the ratio between $\mathrm{HH}$ and $\mathrm{VV}$ intensities. The range of SE was important in identifying areas of negative and positive freeboard where different buoyancy models were applied in sea ice thickness calculation. Figure 4.16 shows the range of SE classes as applied to the IceBell survey grid for Floe 6 for the three TSX images of the time series. A decrease in SE values over time is observed within the boundaries of the survey grid area. The declining SE relates to changing distribution of SE classes over time, thus implying that additional areas of sea water flooding (slush) were present later in the season. The ice bottom calculated from our SE-based model follows the trend of

Table 4.6 Test sites and corresponding radar and validation data selected for the generation of showcases on sea ice thickness

\begin{tabular}{l|l|l}
\hline $\begin{array}{l}\text { Application/ } \\
\text { product }\end{array}$ & Test site - Radar data & Reference data \\
\hline $\begin{array}{l}\text { Sea ice } \\
\text { thickness }\end{array}$ & Bellingshausen Sea & $\begin{array}{l}\text { Surface and snow depth surveys, electromagnetic induction thickness surveys, drilled hole } \\
\text { thickness profiles, terrestrial scanning LiDAR surveys, airborne scanning LiDAR surveys, and } \\
\text { ground penetrating radar surveys on sea ice floes. }\end{array}$ \\
\cline { 2 - 3 } & $\begin{array}{l}\text { TerraSAR-X dual-pol } \\
\text { Nov.2010-Jan. 2011 }\end{array}$ & \\
\hline
\end{tabular}




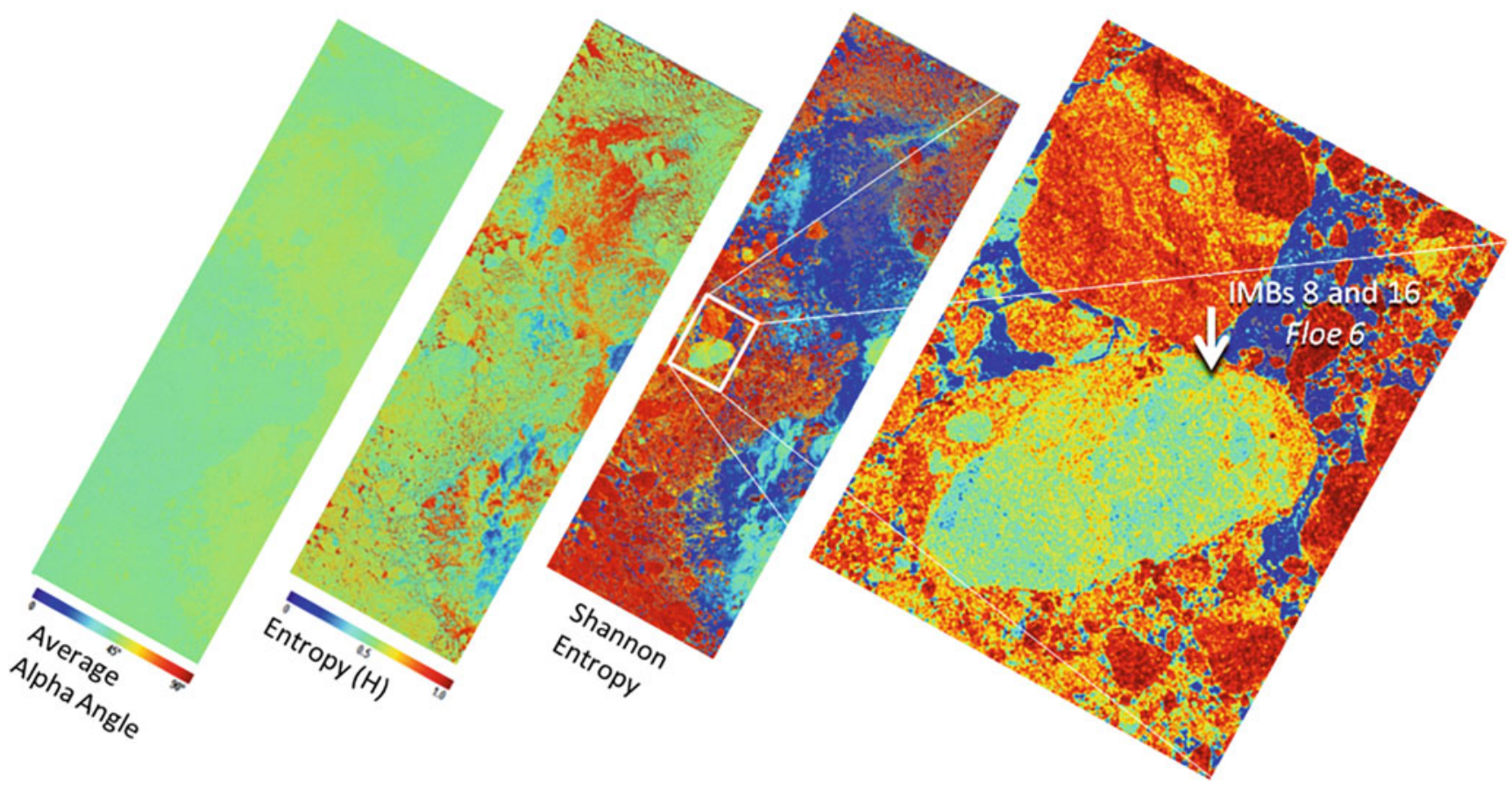

Fig. 4.15 The average Alpha angle, Entropy, and computed SE for the TSX image acquired on Dec 01, 2010. Higher SE is apparent in smaller broken floes and areas of brash ice (red) with interspersed areas of open water (dark blue). After Dec 13, the Floe 6 broke with the IMBs separated on different pieces (after Necsoiu et al. 2011; Lewis and Necsoiu 2011)
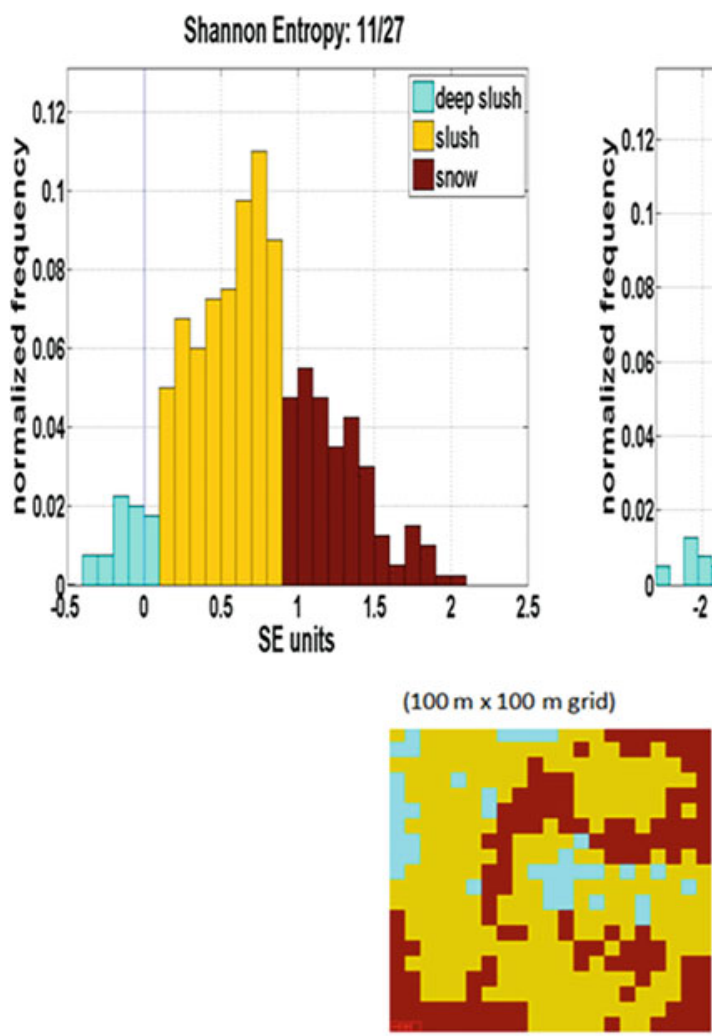

$11 / 27$

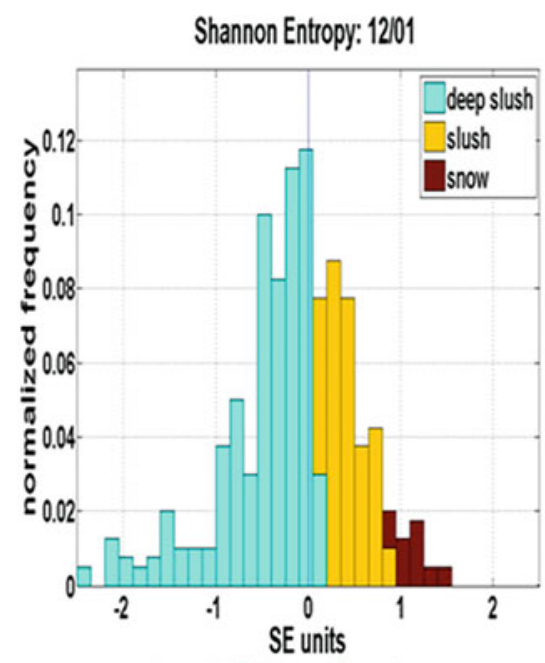

Spatial Distribution

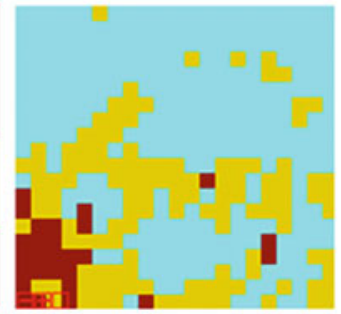

$12 / 01$

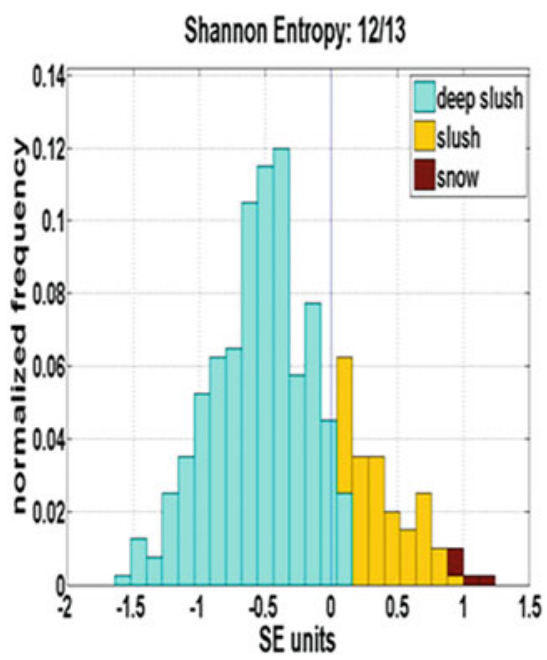

Fig. 4.16 Time series and distribution of SE for the $100 \mathrm{~m} \times 100 \mathrm{~m}$ grid survey area shown by SE class. Notice the absence of ice block class 4 present on the floe but not within the grid boundaries (this class is present elsewhere on Floe 6) 
Fig. 4.17 A 2-D profile comparison of snow and ice surface elevations and ice bottom derived from EMI measurements with ice bottom calculated from both standard buoyancy relationships and the SE-based model ice bottom (Lewis et al. 2013)

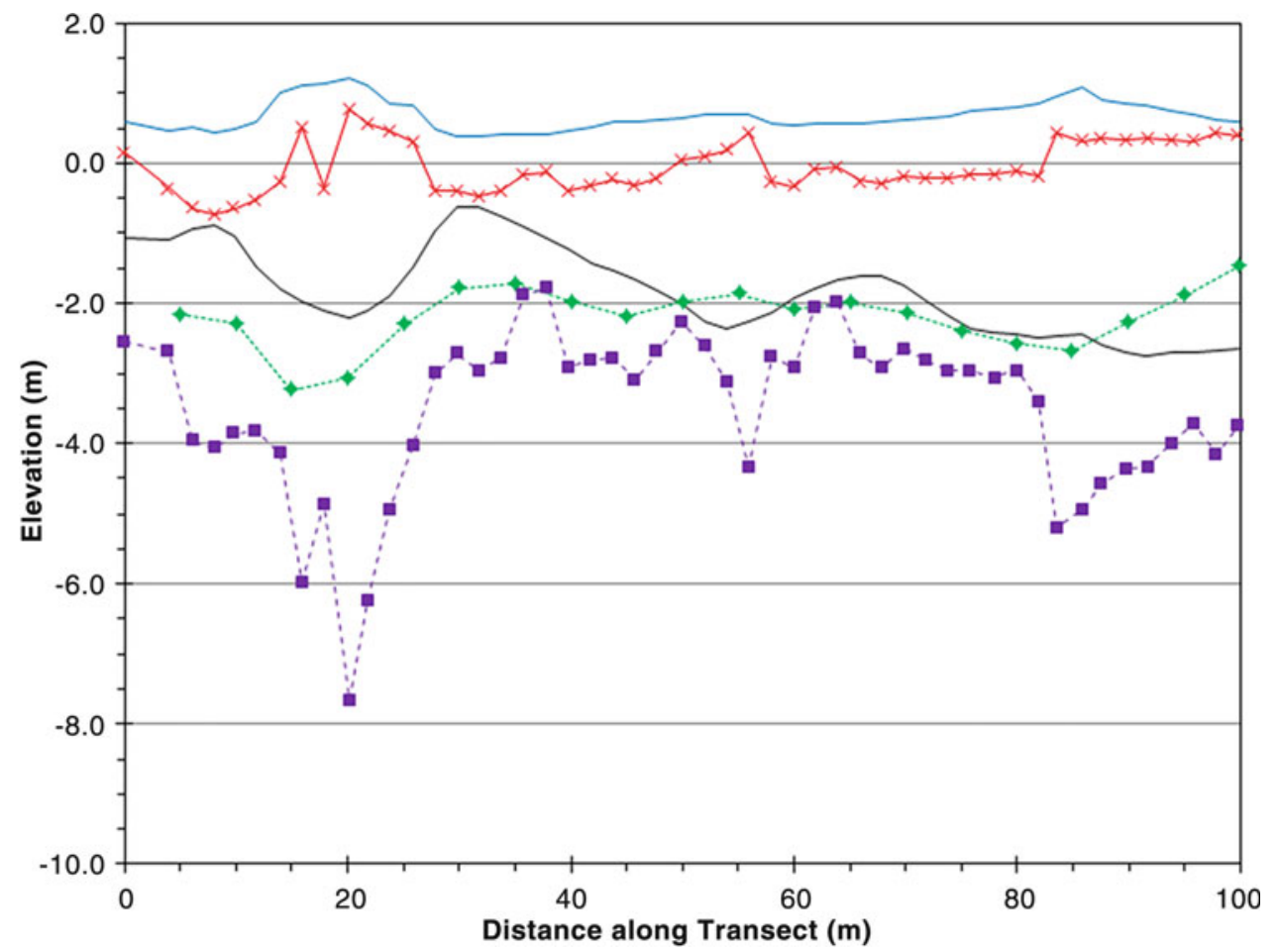

the EM-31 derived ice bottom much more closely than the simple buoyancy model used in other studies (Fig. 4.17).

\subsubsection{Discussion on the Role of Polarisation, on the Maturity of the Application and Conclusions}

Based on preliminary results, TSX dual-pol HHVV data have the potential to derive sea ice type, snow and ice thickness, and surface roughness features. Regarding sea ice interpretations, it is crucial that a detailed and direct survey of ice characteristics is performed on the sea ice floes. Essential in monitoring sea ice characteristics is the capability, provided by location of the drifting buoys, to follow sampled floes with high-resolution, narrow-swath satellite data acquisitions during the months following surface sampling. IMB sensors, with their capability to track changing surface conditions through temperature and other measurements, such as snow depth and flooding, are also essential to provide field calibration over an extended period. The methodology presented here marks an advance towards an integrated sea ice algorithm based on surface sea ice measurements (including IMBs) and TSX imagery; however, more study of polarimetric descriptors and detailed analysis of field measurements will be needed to increase the information content and to validate this approach.

\subsubsection{Acknowledgements}

This research was funded by the SwRI ${ }^{\circledR}$ internal research and development program (project 20-R8181). TerraSAR-X data was provided by DLR proposal (OCE0997). The IceBell campaign was supported by Ted Maksym (BAS) and Jeremy Wilkinson (SAMS). Special credits to Steve Ackley and Blake Weissling (University of Texas at San Antonio), Dana Floricioiu, Mihai Datcu (DLR), Shane Cloude (AEL Consultants), and Phil Hwang (SAMS).

\subsubsection{Polarimetric SAR for Classification of Sea Ice in the Baltic Sea}

\subsubsection{Introduction, Motivation, and Literature Review}

The Baltic Sea is a semi-enclosed brackish sea in Northern Europe. The average ice season extends from December into May and reaches a maximum ice extent of approximately $150,000 \mathrm{~km}^{2}$. The region is steadily busy with shipping traffic for which the ice conditions are of great importance. Conditions where wind drives the ice, causing it to form ridges or pressing it against the shoreline or entrances to harbours, are of particular concern as such regions are difficult to break into.

During 2007 to 2009, Chalmers University of Technology and the Swedish Meteorological and Hydrological Institute (SMHI) carried out the project "Improved sea ice monitoring for the Baltic Sea" (Eriksson et al. 2010), with the goal to evaluate the usefulness of new space-borne SAR instruments for the purpose of operational sea ice monitoring. Within this project, a number of SAR scenes were acquired in the northern Baltic Sea. The images were evaluated, for instance, in terms of how well different ice types could be separated, and 
how differences appear between single and dual polarisation data and between different frequency bands. The Swedish Ice Service at the SMHI participated in parts of the evaluation.

In the context of the above-mentioned project, fully polarimetric SAR data were ordered from two SAR satellite systems: The Canadian RADARSAT-2 (RS2) which utilises a C-band SAR and the Japanese Advanced Land Observing Satellite (ALOS) which carried an L-band SAR.

Fully polarimetric SAR data were collected with the NASA airborne AIRSAR system over sea ice in the Beaufort, Bering, and Chukchi Seas already in 1988. These data have been evaluated in several studies and results have been presented by a number of authors, e.g. Drinkwater et al. (1991), Ngheim et al. (1995b), and Weinbrenner et al. (1995). These studies have mainly focused on the possibilities to separate different ice types or determine sea ice thickness. Various combinations of co-polar (HH or VV) and cross-polar ( $\mathrm{HV}$ or $\mathrm{VH}$ ) backscatter values and ratios, co-polar phase, or magnitude and phase of the complex correlation between the backscatter components were evaluated. Similar studies were later done on polarimetric datasets collected at other locations with various airborne SAR systems, e.g. Greenland Sea with EMISAR in 1995 (Thomsen et al. 1998), Sea of Okhotsk with PiSAR in 1999 (Wakabayashi et al. 2004) and during the SIR-C mission with the Space Shuttle over the Weddell Sea in 1994 (Eriksson et al. 1998). In a study of the improvement of sea ice classification by means of radar polarimetry, which was published by Dierking et al. in 2004 (Dierking et al. 2004), the authors came to the conclusion that the goal of a robust, fully automated sea ice classification scheme by means of polarimetric SAR was not yet achieved.

The first satellite with a fully polarimetric SAR was ALOS, which was launched in 2006. In the last 10-15 years it also became common to include various decomposition methods in the analysis of polarimetric SAR data of sea ice. These methods often make it possible to identify how individual scattering mechanisms contribute to the total received signal. Studies that have used these methods for separation of sea ice types are, e.g., presented by Wakabayashi et al. 2004, Scheuchl et al. 2002, and more recently Gill and Yackel (2012), Doulgeris (2012), and Dierking and Wesche (2013).

The most extensive comparison between ice type classification accuracies for different combinations of polarimetric parameters is the one presented by Gill and Yackel (2012). Their evaluation for three ice types (smooth first year ice, rough first year ice, and deformed first year ice) and open water for polarimetric RS2 images indicate that no single parameter discriminates significantly $(>60 \%)$ between all these ice types, but with a combination of three parameters, an overall accuracy of up to $91 \%$ was achieved.

\subsubsection{Methodology}

In order to make a qualitative evaluation of the SAR images, a field campaign was organised to collect field data. Flights with helicopter were carried out from the Umeå Airport in Sweden. Optical and thermal infrared photos were captured by two cameras directed in the nadir direction. The optical camera had a $114^{\circ}$ diagonal angle of view, whereas the infrared camera spanned $30^{\circ}$.

The fully polarimetric SAR images were studied using $H / A / \bar{\alpha}$ decomposition (Lee and Pottier 2009; Cloude and Pottier 1997). First, we formed local estimates of the coherency matrix and multi-looked to square pixel size (in groundrange coordinates). The decomposition was performed with a $9 \times 9$ boxcar filter to minimise biases in the entropy and anisotropy estimation. A polarimetric signature analysis was carried out and was used to assess the credibility of the employed segmentation.

\subsubsection{Experimental Results}

Test sites and corresponding radar and validation data sets selected for the generation of showcases on sea ice classification in the Baltic sea are summarised in Table 4.7.

We will demonstrate the results by using examples from one RS2 image and one ALOS-PALSAR image.

Site $\mathrm{A}$ is located in image \#7, at the border between the fast ice and dense drift ice seen in Fig. 4.18. The fast ice was rugged and covered by a snow layer between 0 and $45 \mathrm{~cm}$ thick. The ice thickness was measured at one point to $110 \mathrm{~cm}$. The drift ice was densely packed and attached to the fast ice. It had a level, glossy surface with a thin snow layer of less than $2 \mathrm{~cm}$ thickness. The thickness of the drift ice was measured at two locations, to 30 and $37 \mathrm{~cm}$.

An $H / A / \bar{\alpha}$ decomposition was made on the RADARSAT- 2 image. The result shows that both the fast ice and the drift ice in this location are subject to low entropy surface scattering. The alpha parameter is slightly lower over the fast ice $\left(\sim 10^{\circ}\right.$ as compared to $\left.\sim 20^{\circ}\right)$, thus being closer to the geometrical optics limit. The entropy is lower over the fast ice as well (0.2 as compared to 0.5$)$, indicating more random scattering from the drift ice. Specular reflection, away from the radar, is presumed to occur on the flat surface of the drift ice. The returned signal is instead dominated by reflection from brine pockets and fractures in the uppermost

Table 4.7 Test sites and corresponding radar and validation data selected for the generation of showcases on sea ice classification in the Baltic sea

\begin{tabular}{l|l|l}
\hline Application/product & Test site - Radar data & Reference data \\
\hline \multirow{2}{*}{ Sea ice classification in the Baltic Sea } & Baltic Sea (Bay of Bothnia, Kvarken) & Optical and thermal infrared photos \\
\cline { 2 - 2 } & ALOS-PALSAR 2007, 2009 & \\
\cline { 2 - 2 } & RADARSAT-2 2009 & \\
\hline
\end{tabular}



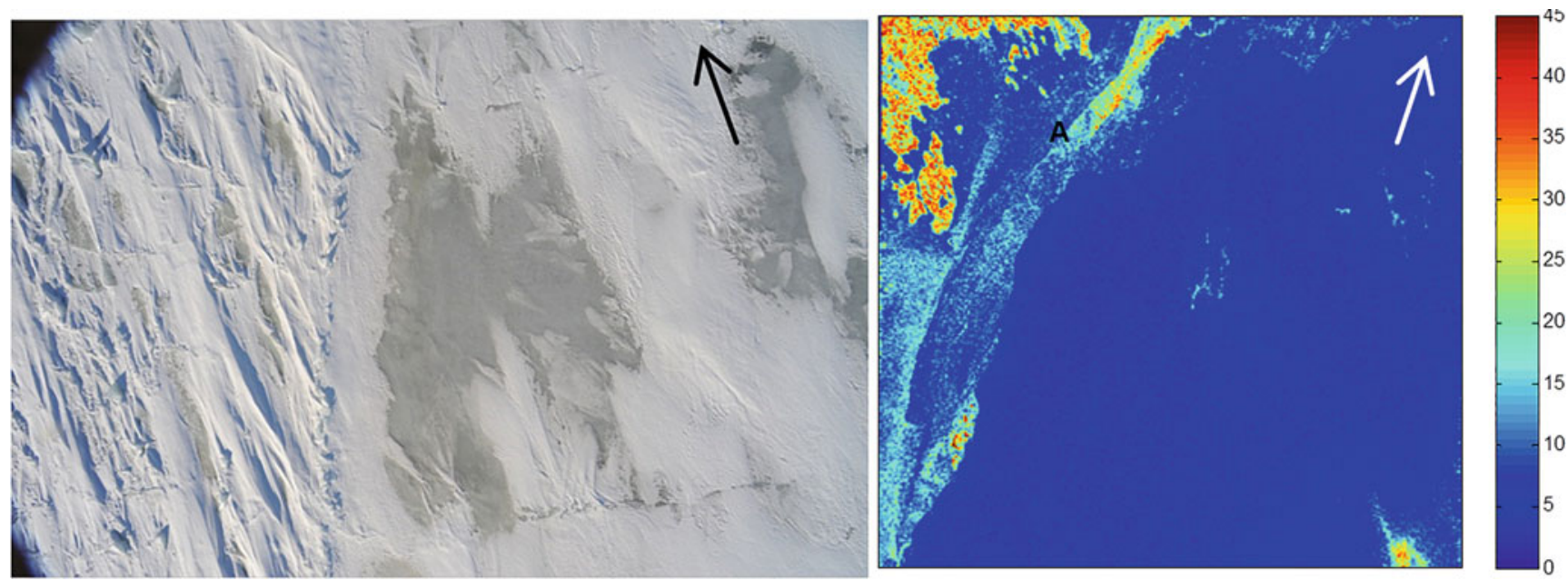

Fig. 4.18 Scene \#7. Left: Optical photo taken from helicopter in the nadir direction. The photo covers an area of $50 \times 30 \mathrm{~m}$ and shows fast ice to the left and dense drift ice to the right. Right: The alpha-parameter

from the $H / A / \alpha$-decomposition. The scene covers an area of $24 \times 24 \mathrm{~km}$. Site A is marked with letter A and is also the location of the optical photo. Arrows indicate North

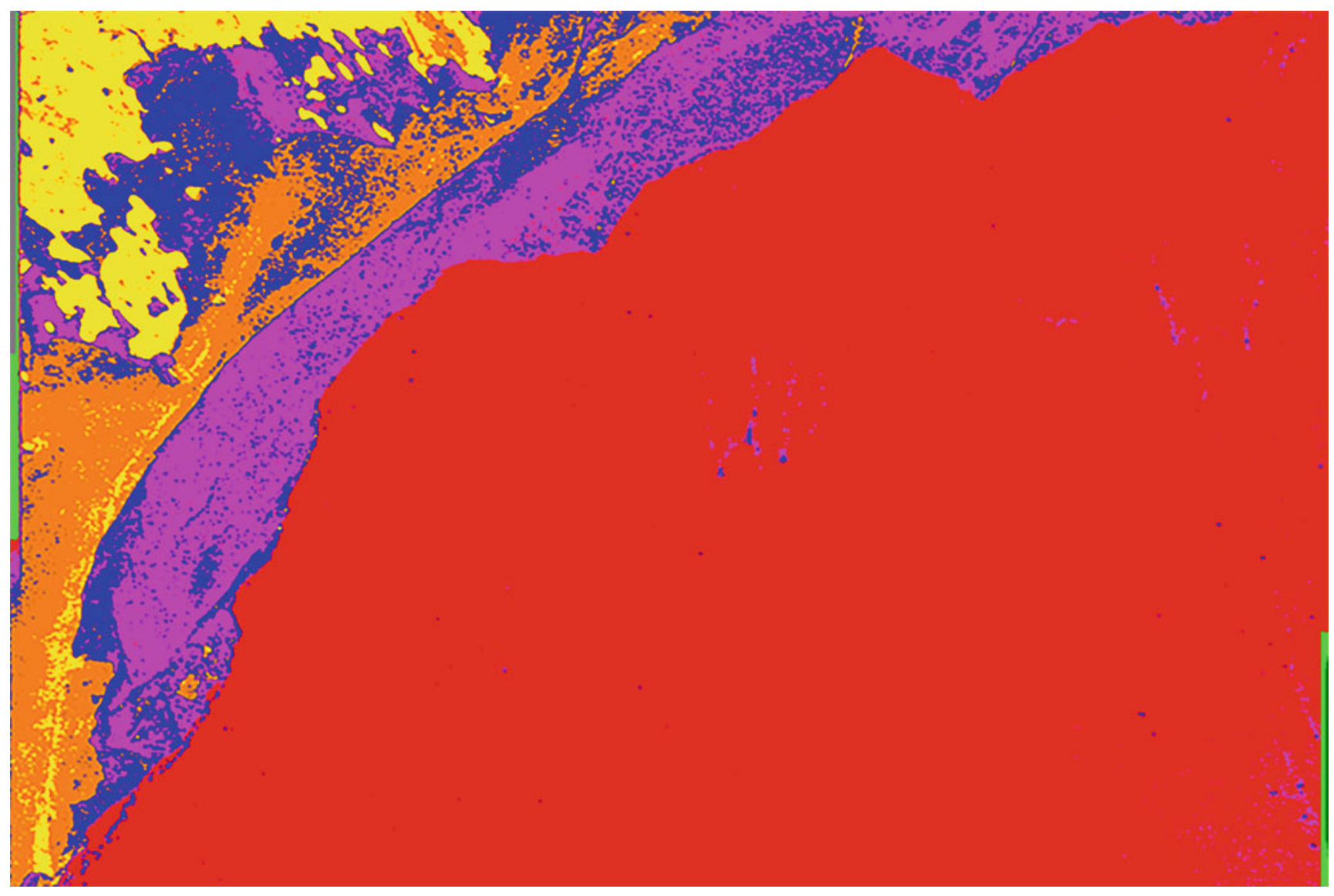

Fig. 4.19 Scene \#7. Wishart $H / \alpha$ classification where orange corresponds to fast ice, purple to drift ice, red to open water, and yellow to land areas

layer of the low-saline ice, which then explains the higher entropy and alpha values. The two ice types found in site A are believed to be representative for a much larger area, judging from the optical helicopter images. This is also in agreement with the results from a classification, shown in Fig. 4.19. The figure shows an unsupervised $H / \bar{\alpha}$ classification with eight classes, where the fast and drift ice are well distinguished. 

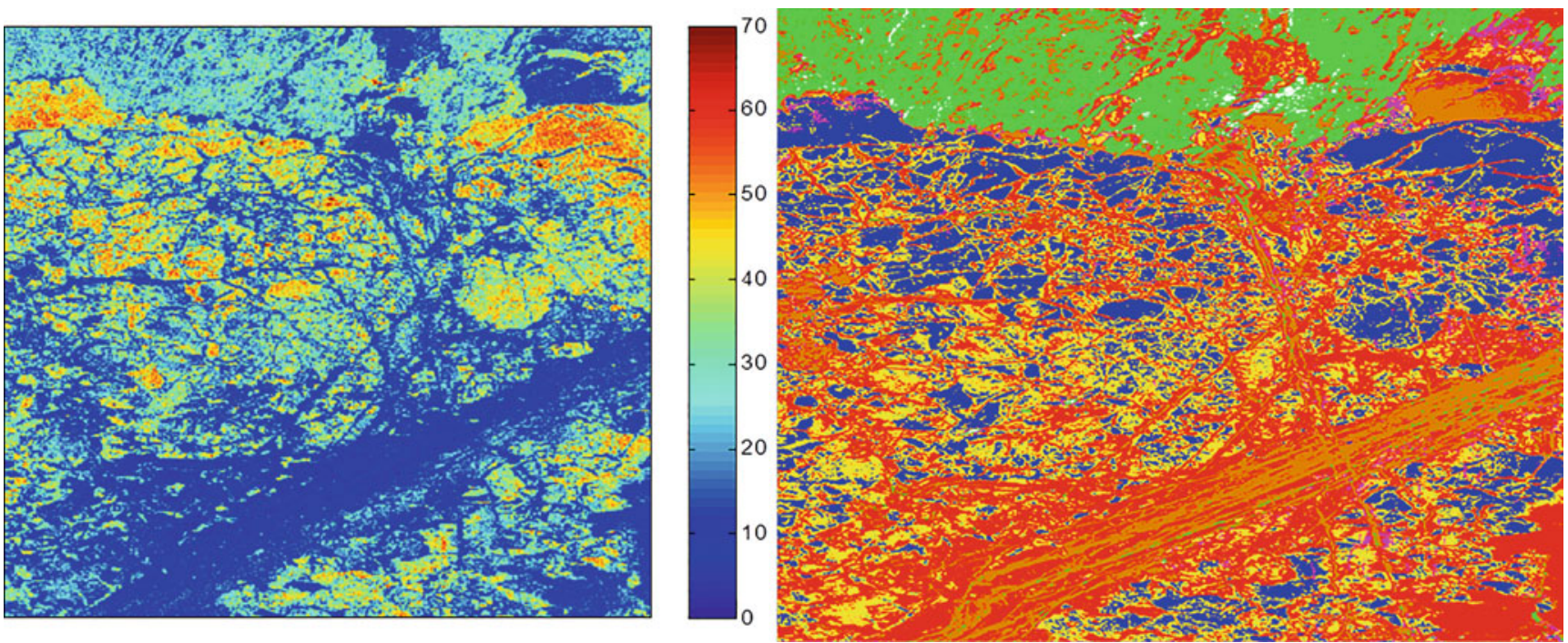

Fig. 4.20 Scene \#10. Left: The alpha-parameter from the $H / A / \alpha$-decomposition. Right: Wishart $H / \alpha$ classification (green colour is land, other colours sea ice). The scene covers an area of approximately $30 \times 50 \mathrm{~km}$ (width $\times$ height)

Figure 4.20 shows the alpha parameter obtained for scene $\# 10$ along with the Wishart $H / \alpha$ classification. The scene covers a diverse ice field with an ice concentration of approximately $90 \%$. It can be observed that volume scattering is more common in the northern parts of the scene. The Wishart $H / \bar{\alpha}$ classification identifies four major classes of ice types (blue, red, yellow, and orange). The red and orange classes are characterised by low alpha and entropy values, clearly acting as surface scatterers. The blue class is defined by alpha values in the range between $40^{\circ}$ and $50^{\circ}$, relating to volume scattering, whereas the yellow class is similar but with slightly more weight towards surface scattering. The optical helicopter images reveal that these classes are mostly level ice surfaces with no snow layer, whereas the red/yellow classes consist of snow-covered or rough ice of densely packed or consolidated floes.

\subsubsection{Comparison with Single/Dual Polarisation Data}

Sea ice is fairly well studied with single and dual polarisation data using L- and C-band SAR sensors. Fully polarimetric data may be used to study in detail the scattering mechanisms for different ice types or to improve sea ice classification for the purpose of ice charting. An experienced ice analyst can use dual polarisation data to map the ice conditions with high precision, but for automated classification this remains difficult. With fully polarimetric data, the automated classification will have an advantage because the many information channels will make manual interpretation complex.

\subsubsection{Discussion on the Role of Polarisation, on the Maturity of Application and Conclusions}

SAR polarimetry has shown potential for sea ice classification and segmentation. It is still in the early development phase, not only because of the limited spatial coverage that is insufficient for traditional operational sea ice charting services but also due to the non-trivial task to accurately relate classes with their respective ice type. The classifier must either aim for a reliable segmentation into few classes or for a detailed segmentation into many ice types with the secondary task to label each class with its actual ice type.

\subsubsection{Acknowledgements}

Data from ALOS were provided by the European Space Agency within the framework of the ALOS Data European node category-1 proposal titled Improved sea ice monitoring for the Baltic Sea (AOALO.3562). Data from RADARSAT2 were granted within the Canadian program for Science and Operational Applications Research for RADARSAT2 (SOAR), project number 3924. The Umeå Marine Science Centre in Norrbyn and Lapplandsflyg AB in Umeå are acknowledged for logistical support during the field campaigns.

\subsection{Investigation of Permafrost with Fully Polarimetric Data}

\subsubsection{Introduction, Motivation, and Literature Review}

Satellite-borne synthetic aperture radar (SAR) data are useful for estimating soil moisture and surface roughness over large areas. However, few studies have been conducted using SAR to examine permafrost areas, which have surfaces covered by low vegetation, including areas with tussocks, mosses, and low shrubs; whereas SAR data has been widely used to study mineral soil surfaces. Knowledge of the moisture levels in permafrost regions is important for monitoring the seasonal 
variability of the active layer, which is a seasonally unfrozen layer that occurs in upper part of the permafrost. The North Slope in Alaska has experienced significant changes in vegetation greenness over the past two decades (Goetz et al. 2005; Verbyla 2008), and it has been suggested that variations in soil moisture may be partly responsible for these changes. Rignot and Way (1994) showed that freeze-thaw cycles in high-latitude terrestrial ecosystems, which include permafrost regions, can be monitored using ERS-1 SAR data. Several studies have examined surface soil moisture in firedisturbed forests in Alaska using C-band SAR data; variations in soil moisture were detected by variations in the backscattering coefficient $\left(\sigma^{0}\right)$ (Kasischke et al. 2007).

PALSAR was launched in 2006 onboard ALOS. PALSAR was the first Earth observing satellite to carry a full polarimetry mode, and it provides complete radar backscattering information on both the intensity and phase of the signal. This is a significant advantage for deriving a complete understanding of the factors controlling radar backscattering in areas, especially those with complex scattering mechanisms, such as low vegetation, for which the impact would be expected to be minimal in the L-band. This is also essential for deriving robust algorithms for estimating soil moisture levels, as well as other parameters.

Here, we demonstrate how to analyse field measurement data and L-band full polarimetry data. This Section is based on (Watanabe et al. 2012).

\subsubsection{Methodology}

\subsubsection{Single-Layer Model}

Three parameters describe the primary factors affecting radar scattering mechanisms: soil moisture $(M v)$ or dielectric constant $(\varepsilon)$, root mean square (RMS) height of the soil surface $(s)$, and correlation length $(l)$. The parameters $s$ and $l$ are usually multiplied by the wave number $k(k=2 \pi / \lambda$, where $\lambda$ is the radar wavelength) to give the derived parameters $k s$ and $\mathrm{kl}$.

Several models have been suggested for estimating $M v$ from SAR data. One of the popular models is the integral equation method (IEM) (Fung 1994), which describes the behaviour of $\sigma^{0}$ for both the co-polarisation and crosspolarisation terms. For cases where $k s, k l<1.2 \sqrt{\varepsilon}$, the backscattering coefficient can be calculated using the following analytical equation:

$$
\begin{aligned}
\sigma_{q p}^{0}= & \frac{k^{2}}{2} \exp \left[-2\left(\mathrm{k}_{\mathrm{z}} \cdot s\right)^{2}\right] \\
& \times \sum_{n=1}^{\infty} s^{2 n}\left|I_{q p}^{n}\right|^{2} \frac{W^{(n)}\left(-2 k_{x, 0}\right)}{n !}
\end{aligned}
$$

where $\theta$ is the local incidence angle, $k_{z}=k \cos \theta, k_{x}=k \sin \theta$, and

$$
\begin{aligned}
I_{q p}^{n} & =\left(2 k_{z}\right)^{n} f_{q p} \exp \left(-s^{2} k_{z}{ }^{2}\right)+\frac{k_{z}{ }^{n}\left[F_{q p}\left(-k_{x}, 0\right)+F_{q p}\left(k_{x}, 0\right)\right]}{2} \\
f_{v v} & =\frac{2 R_{v v}}{\cos \theta}, f_{h h}=-\frac{2 R_{h h}}{\cos \theta} \\
F_{v v}\left(-k_{x}, 0\right)+F_{v v}\left(k_{x}, 0\right) & =\frac{2 \sin ^{2} \theta\left(1+R_{v v}\right)^{2}}{\cos \theta}\left[\left(1-\frac{1}{\varepsilon}\right)+\frac{\mu \varepsilon-\sin ^{2} \theta-\varepsilon \cos ^{2} \theta}{\varepsilon^{2} \cos ^{2} \theta}\right] \\
F_{h h}\left(-k_{x}, 0\right)+F_{h h}\left(k_{x}, 0\right) & =-\frac{2 \sin ^{2} \theta\left(1+R_{h h}\right)^{2}}{\cos \theta}\left[\left(1-\frac{1}{\mu}\right)+\frac{\mu \varepsilon-\sin ^{2} \theta-\mu \cos ^{2} \theta}{\mu^{2} \cos ^{2} \theta}\right]
\end{aligned}
$$

where $\mu$ is the magnetic permeability, $R_{h h}$ and $R_{v v}$ are Fresnel reflection coefficient, and $p$ and $q$ represent the polarisations corresponding to $h$ and $v . W^{(n)}$ is the Fourier transform of the $n$-th power of the surface correlation function; the exponential, Gaussian, and 1.5-power forms of this function are well known. Many curves observed in the field appear to follow an exponential shape generated by the exponential correlation function (Fung 1994), represented by

$$
W^{(n)}(K)=\left(\frac{l}{n}\right)\left[1+\left(\frac{K l}{n}\right)^{2}\right]^{-1.5} .
$$

Oh (2004) proposed a semi-empirical model in which some parameters were tuned using ground-based
(GB) polarimetric scatterometers and AIRSAR data obtained for various soil conditions. Three parameters are defined in this model, and the following one is used in our analysis

$$
\sigma_{V H}^{0}=0.1 M_{v}^{0.7}(\cos \theta)^{2.2}\left[1-\exp \left(-0.32(k s)^{1.8}\right)\right]
$$

The applicable range of the model is less than $3 \cdot k s$.

\subsubsection{Two-Layer Model}

The two-layer model was developed to describe more complex ground phenomena, such as the presence of vegetation, or snow on ice. Fung (1994) used four terms to represent the co-polarisation surface and volume backscattering of the two layers (see Fig. 4.21): 


\section{Two layer model}

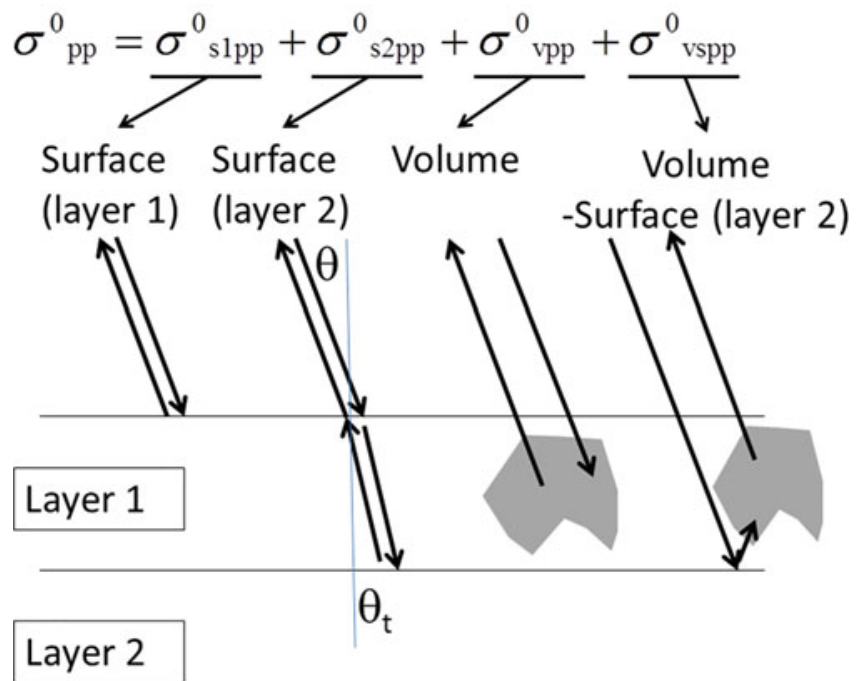

Fig. 4.21 An illustration of the terms in the 2-layer scattering model

$$
\sigma_{\mathrm{pp}}^{0}=\sigma_{\mathrm{s} 1 \mathrm{pp}}^{0}+\sigma_{\mathrm{s} 2 \mathrm{pp}}^{0}+\sigma_{\mathrm{vpp}}^{0}+\sigma_{\mathrm{vspp}}^{0}
$$

where $\sigma_{\text {s1pp }}^{0}$ describes surface scattering from layer 1 and is represented by Eq. (4.12), and $\sigma_{\text {s2pp }}^{0}$ describes surface scattering from layer 2 and is represented by

$$
\begin{aligned}
\sigma_{\mathrm{s} 2 \mathrm{pp}}^{0}= & T_{1 t}\left(\theta, \theta_{t}\right) T_{t 1}\left(\theta_{t}, \theta\right) \exp \left(\frac{-2 \tau}{\cos \theta_{t}}\right) \\
& \times \frac{\cos \theta}{\cos \theta_{t}} \sigma_{\mathrm{spp}}^{0}\left(\theta_{t}\right)
\end{aligned}
$$

where $T_{1 t}$ and $T_{t 1}$ are the transmissivity from layer 1 to 2 and from layer 2 to 1 , respectively, $\tau$ is the optical depth, and $\sigma_{\text {spp }}^{0}$ is the surface scattering at layer 2, represented by Eq. (4.12). $\sigma_{\text {vpp }}^{0}$ describes the volume scattering in layer 1 and is represented by

$\sigma_{\mathrm{vpp}}^{0}=0.5 a T_{1 t} T_{t 1} \cos \theta\left[1-\exp \left(\frac{-2 \tau}{\cos \theta_{t}}\right)\right] P_{p p}\left(\cos \theta_{t},-\cos \theta_{t} ; \pi\right)$

where $a$ is the albedo and $P_{p p}$ is element $p p$ of the phase matrix. A Rayleigh phase matrix is assumed, and $P_{h h}=P_{v v}=1.5$. The fourth term, $\sigma_{\text {vspp}}^{0}$, describes interactions between volume scattering in layer 1 and surface scattering from layer 2 . This term is very small and is negligible in most cases.

In the case of cross-polarisation, Eq. (4.15) of the Oh model is used to describe the backscattering from layer 1. Additionally, Eq. (4.16) can be modified to describe the
Table 4.8 Test sites and corresponding radar and validation data selected for the generation of showcases on permafrost characterisation

\begin{tabular}{l|l|l}
\hline $\begin{array}{l}\text { Application/ } \\
\text { product }\end{array}$ & Test site - Radar data & $\begin{array}{l}\text { Reference } \\
\text { data }\end{array}$ \\
\hline $\begin{array}{l}\text { Permafrost } \\
\text { characterisation }\end{array}$ & $\begin{array}{l}\text { Arctic National Wildlife Refuge } \\
\text { (ANWR), Alaska, USA }\end{array}$ & \\
\cline { 2 - 2 } & ALOS-PALSAR & \\
\hline
\end{tabular}

backscattering from layer 2 by inserting Eq. (4.16) for $\sigma_{\text {spp }}^{0}$, which is represented by

$$
\begin{aligned}
\sigma_{q p}^{0}= & \sigma_{\mathrm{slpq}}^{0(\text { Oh model })} \\
& +T_{1 t}\left(\theta, \theta_{t}\right) T_{t 1}\left(\theta_{t}, \theta\right) \exp \left(\frac{-2 \tau}{\cos \theta_{t}}\right) \\
& \times \frac{\cos \theta}{\cos \theta_{t}} \sigma_{s 2 q p}^{0(\text { Oh model })}\left(\theta_{t}\right) .
\end{aligned}
$$

\subsubsection{Experimental Results}

Test sites and corresponding radar and validation data sets selected for the generation of showcases on permafrost characterisation are summarised in Table 4.8.

PALSAR data acquired for the test sites (Arctic National Wildlife Refuge (ANWR), Alaska, USA) were used in this analysis. Full polarimetry mode $(\mathrm{HH}, \mathrm{HV}, \mathrm{VH}$, and VV) data was acquired on July 29, 2007, and July 31, 2008, in descending orbit. Dual polarisation mode ( $\mathrm{HH}$ and $\mathrm{HV}$ ) data was observed on August 17, 2007, 2 weeks after the observation of full polarimetry data for the test sites in ascending orbit. The off-nadir angle was $21.5^{\circ}$ for the full polarimetry mode and $34.3^{\circ}$ for the dual polarisation mode. The position of the field test sites and the physical characteristics are summarised in Table 4.8. The test sites are in the coastal tundra ecosystem of the Alaskan Arctic coastal plain. Trees were absent because of the high-latitude location $\left(70^{\circ} \mathrm{N}\right) . \mathrm{HH}$ and $\mathrm{VV}$ polarisation was dominant in the permafrost area. This means that surface backscattering was dominant. Strong radar reflections were observed for very wet areas along the small stream, and high moisture values were observed in this area (A-5 and A-6). Field observations were conducted at 6 sites in 2007 (A-1 to A-6) and 4 sites in 2008 (A-1 to A-3 and A-7). Tussocks and polygons, which are structures typical of permafrost, were observed in many tussocks in our test sites was 3 to $10 \mathrm{~cm}$ in diameter. On a larger scale, thermal contraction cracks form polygonal (mainly tetragonal) nets that cover extensive areas of arctic and subarctic regions; the polygons typically range in size from 15 to $40 \mathrm{~m}$. 
Table 4.9 Parameters derived from field measurements and from the satellite data

\begin{tabular}{|c|c|c|c|c|c|c|c|c|c|}
\hline \multirow[b]{2}{*}{ Site } & \multirow[b]{2}{*}{ Lat/Lon } & \multicolumn{7}{|c|}{ Parameters from field data collection and PalSAR } & \multirow[b]{2}{*}{ Notes } \\
\hline & & Year & $M v$ & ks & $k l$ & $\sigma_{H H}^{0}$ & $\sigma_{V H}^{0}$ & $\sigma_{V V}^{0}$ & \\
\hline \multirow[t]{2}{*}{ A-1 } & -143.66 & 2007 & 34.7 & \multirow[t]{2}{*}{0.82} & \multirow[t]{2}{*}{2.8} & -9.3 & -20.5 & -9.6 & \multirow[t]{2}{*}{ Covered with polygons well-developed tussock } \\
\hline & 69.72 & 2008 & 25.2 & & & -10.2 & -22.0 & -12.2 & \\
\hline \multirow[t]{2}{*}{ A-2 } & -143.63 & 2007 & 39.8 & \multirow[t]{2}{*}{0.68} & \multirow[t]{2}{*}{2.0} & -10.2 & -22.4 & -10.2 & \multirow[t]{2}{*}{ No polygonal structures observed } \\
\hline & 69.72 & 2008 & 46.6 & & & -11.1 & -24.1 & -9.9 & \\
\hline \multirow[t]{2}{*}{ A-3 } & -143.61 & 2007 & 41.6 & \multirow[t]{2}{*}{0.44} & \multirow[t]{2}{*}{3.4} & -11.0 & -22.8 & -11.0 & \multirow[t]{2}{*}{ No polygonal structures observed } \\
\hline & 69.72 & 2008 & 38.8 & & & -10.4 & -24.6 & -11.6 & \\
\hline \multirow[t]{3}{*}{ A-4 } & -143.62 & \multirow[t]{3}{*}{2007} & \multirow[t]{3}{*}{47.7} & \multirow[t]{3}{*}{0.71} & \multirow[t]{3}{*}{3.1} & \multirow[t]{3}{*}{-10.1} & \multirow[t]{3}{*}{-22.2} & \multirow[t]{3}{*}{-11.2} & Numerous shrub patches \\
\hline & 69.71 & & & & & & & & No polygonal structures observed \\
\hline & & & & & & & & & Well-developed tussocks \\
\hline \multirow[t]{2}{*}{ A-5 } & -143.63 & \multirow[t]{2}{*}{2007} & \multirow[t]{2}{*}{63.8} & \multirow[t]{2}{*}{0.70} & \multirow[t]{2}{*}{2.5} & \multirow[t]{2}{*}{-9.0} & \multirow{2}{*}{-21.3} & \multirow[t]{2}{*}{-9.3} & Covered with polygons \\
\hline & 69.71 & & & & & & & & Well-developed tussocks \\
\hline \multirow[t]{3}{*}{ A-6 } & -143.64 & \multirow[t]{3}{*}{2007} & \multirow[t]{3}{*}{78.9} & 1.13 & 3.2 & -8.8 & -19.3 & -8.8 & No polygonal structures observed \\
\hline & 69.71 & & & & & & & & Well-developed tussocks \\
\hline & & & & & & & & & Very wet \\
\hline A-7 & -143.60 & 2009 & 29.2 & 0.48 & 5.6 & -13.5 & -25.3 & -10.0 & \\
\hline & 69.72 & & & & & & & & \\
\hline
\end{tabular}

Mv measured in inorganic layer

\subsubsection{Data Processing}

Parameters derived from the PALSAR data and field experiment are summarised in Table 4.9. We used PALSAR data processed by the Earth Observation Center of the Japan Aerospace Exploration Agency (JAXA) and calculated the backscattering coefficient $\left(\sigma^{0}\right)$ for each polarisation. We measured $k s$ and $k l$ using a needle profilometer; the profilometer was $1-\mathrm{m}$ long for the measurements, which is 10 times longer than the typical $10-\mathrm{cm}$ correlation length at our test sites.

The 2-cm interval of needles on the profilometer was sufficiently small to have an insignificant effect on the measurement results. The surface correlation functions measured for our test sites matched well with the exponential form represented by Eq. (4.14).

The vertical cross section of the soil at site A-1 is presented in Fig. 4.22. This is a typical permafrost structure, and the soil moisture for the organic layer was measured using time-domain reflectometry (TDR-type sensor; TRIME-FM2) in 2007 and frequency-domain reflectometry (FDR-type sensor; Decagon) in 2008. The length of the probes was $15 \mathrm{~cm}$. These systems directly measured the $\varepsilon$ value, and $\varepsilon$ was converted to soil moisture using the Topp equation (Topp et al. 1980),

$$
\varepsilon_{r}=3.03+9.3 M_{v}+146 M_{v}^{2}-76.7 M_{v}^{3}
$$

The system only displays the moisture value. The value of the dielectric constant originally measured by the device was determined using this relation. The soil moisture of the sphagnum moss layer was measured for several points in site A-1 and had a value of $10.6 \%$.

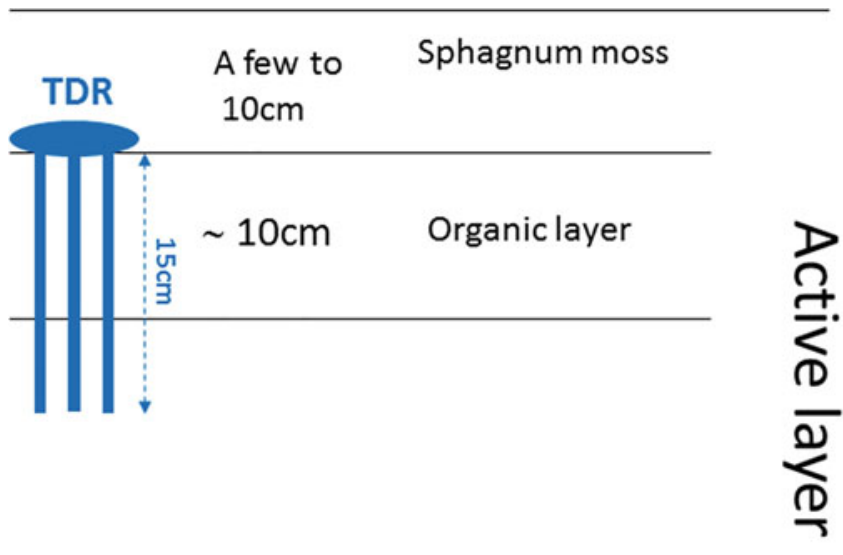

$\sim 35 \mathrm{~cm} \quad$ Minerallayer

\section{Frozen layer}

Fig. 4.22 Vertical cross section of the soil at site A-1

\subsubsection{Comparing PALSAR Data with Single-Layer Models}

The observed $\sigma^{0}$ values were compared with those estimated using the IEM/Oh model, which included the $M v$ (organic layer) and surface parameters measured in the field. The $\sigma_{\text {co- }}^{0}$ pol values were 5 to $7 \mathrm{~dB}$ lower than those calculated by the IEM model, while the $\sigma_{V H}^{0}$ values were moderately matched to values calculated using the Oh model.

Next, we calculated the $\sigma^{0}$ value assuming a $10 \%$ soil moisture value (sphagnum moss layer). The $\sigma_{\text {co-pol values }}^{0}$ 
were well matched to the IEM model, while the $\sigma^{0}{ }_{\mathrm{VH}}$ values were 3 to $6 \mathrm{~dB}$ less than those in the Oh model. There is no single-layer model that simultaneously accounts for the $\sigma_{\text {co-pol }}^{0}$ and $\sigma_{\text {cross-pol observed with PALSAR. }}^{0}$

\subsubsection{Comparing PALSAR Data with Two-Layer Models}

The 2-layer model (sphagnum moss, layer 1; organic layer, layer 2) was used to calculate $\sigma^{0}$ at the A-1 site, where a data logger was deployed to monitor moisture levels. Albedo and optical depth values were changed from 0.1 to 1 , and $\sigma^{0}$ was calculated from the two-layer model; these results were compared with PALSAR data taken with off-nadir angles of $21.5^{\circ}$ and $34.3^{\circ}$. As the sphagnum moss layer may smoothen surface roughness, a value of half the surface roughness was adopted for $k s$ in layer $1 ; k l$ was estimated from its correlation with $k s$.

Several combinations of albedo and optical depth values yielded $\sigma^{0}$ discrepancies of $<2 \mathrm{~dB}$ between PALSAR data and the results of the 2-layer model. One of the best results is presented in Fig. 4.23, which was obtained using the parameters listed in Table 4.10. The $\sigma^{0}$ values derived from the PALSAR data are plotted as squares for the full polarisation mode (off-nadir angle of $21.5^{\circ}$; incidence angle of $24^{\circ}$ ) and as circles for the dual polarisation mode (off-nadir angle of $34.3^{\circ}$; incidence angle of $38^{\circ}$ ). In this case, the 2-layer model for both co-polarisation and cross-polarisation fit the data to within $1 \mathrm{~dB}$. In the case of co-polarisation with an incident angle of $24^{\circ}$, surface scattering from layer 1 was dominant with smaller contributions expected from layer 2 and from volume scattering. In the case of crosspolarisation, scattering from layer 2 was dominant, and scattering from layer 1 (soil moisture of 10\%) was negligible.

The entropy $(\mathrm{H})$ and $\alpha$ were also calculated from the full polarimetry data. The $\alpha$ angle ranged from $13.5^{\circ}$ to $23.6^{\circ}$, and $\mathrm{H}$ ranged from 0.28 to 0.47 . These values are categorised as
Zone 9 in the $\mathrm{H} / \alpha$ classification scheme, which represents low entropy scattering processes such as surface scattering. The entropy values observed at Ulaanbaatar were 0.14 , and smaller than those observed in ANWR, although the sites have almost the same surface parameters and $M v$. A larger entropy value indicates the complexity of the scattering mechanism; therefore, the two-layer scattering model for ANWR may generate a large entropy value.

\subsubsection{Discussion on the Role of Polarimetry, on the Maturity of the Application and Conclusions}

The simultaneous collection of field data and ALOS-PALSAR fully polarimetric observations was performed in Alaska, USA (2007 and 2008). The ground surface in Alaska is covered by an active layer of permafrost consisting of a few to $10 \mathrm{~cm}$ of sphagnum moss layer and deeper organic and mineral layers.

From the analysis of field data and PALSAR data, we compiled the following results:

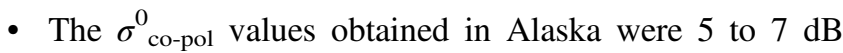
lower than those predicted by the IEM model.

- Unlike $\sigma_{\text {co-pol }}^{0}$ values, $\sigma_{\mathrm{VH}}^{0}$ values estimated from the $\mathrm{Oh}$ model are moderately well matched to those derived from the PALSAR data.

Table 4.10 Parameters for the two-layer model

\begin{tabular}{l|l|l}
\hline Parameters & Layer 1 & Layer 2 \\
\hline$M v$ & $10 \%(\varepsilon=5.3)$ & $34.7 \%(\varepsilon=20.6)$ \\
\hline$k s$ & 0.41 & 0.82 \\
\hline$k l$ & 3.88 & 2.75 \\
\hline Albedo $(a)$ & 0.1 & \\
\hline Optical depth $(\tau)$ & 0.5 & \\
\hline Frequency & $1.27 \mathrm{GHz}$ &
\end{tabular}

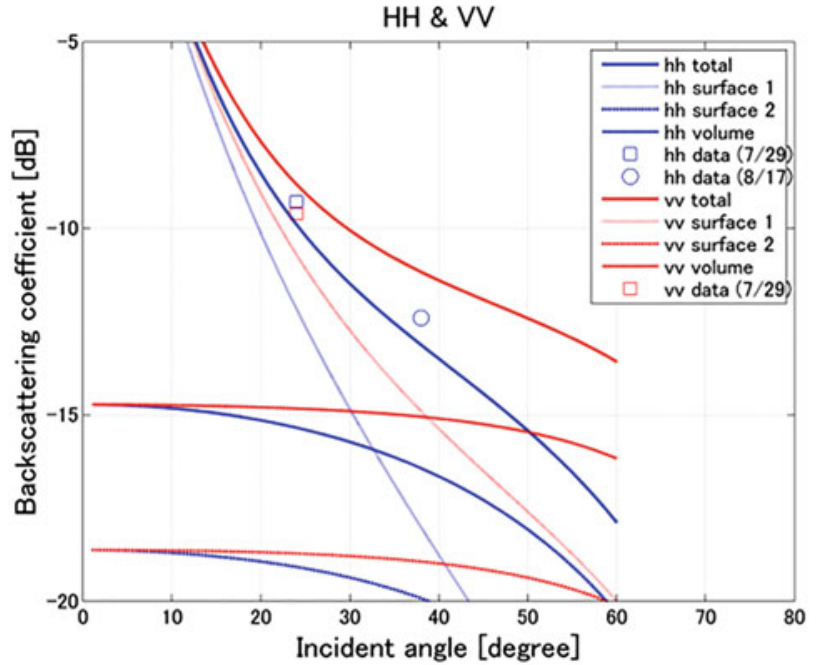

Fig. 4.23 $\sigma^{0}$ derived from the two-layer model is plotted against incidence angle. (Left panel) Co-polarisation. (Right panel) Cross-polarisation. PALSAR data are represented by squares (July 29, 2007, observations) and circles (August 17, 2007, observations) 
- Vertical depth dependency of moisture levels was observed in the field data from permafrost regions. Moisture levels of the sphagnum layer, located above the organic layer, were estimated to be about $10 \%$, while moisture levels of the underlying organic and mineral layers were $25 \%$ to $79 \%$, respectively.

From these observations, we concluded that the sphagnum moss layer plays an important role in radar backscattering processes in permafrost regions and is a main contributor to the $\sigma_{\text {co-pol }}^{0}$ backscattering component; the underlying organic and mineral layers, on the other hand, contribute to the $\sigma_{\text {cross- }}^{0}$ pol component. A two-layer model, which was applied to one of the test sites in Alaska, provided a good prediction of $\sigma^{0}$ values derived from PALSAR data obtained with off-nadir angles of $21.5^{\circ}$ and $34.3^{\circ}$, for both co-polarisation and cross-polarisation results.

Full polarimetry data describe the complete radar backscattering from a target, and it is essential for understanding the scattering mechanism from the target. If full polarimetry data is not used, it is difficult to conclude whether the two-layer model is necessary for the description of $\sigma^{0}$ in the permafrost region.

\subsubsection{Acknowledgements}

We are grateful to the graduate and undergraduate students at Sato Laboratory in Tohoku University for their assistance with field data collection. The field measurements in Alaska were funded by JAXA. This work was supported by Japan Society for the Promotion of Science (JSPS) Grants-in-Aid for Scientific Research, (S) 18106008, (B) 22404001, and (C) 21510004 .

\subsection{Summary (Table 4.11)}

\section{References}

Barnett TP, Adam JC, Lettenmaier DP (2005) Potential impacts of a warming climate on water availability in snow-dominated regions. Nature 438:303-309

Beaven SG, Lockhart GL, Gogineni SP, Hossetnmostafa AR, Jezek K, Gow AJ, Perovish DK, Fung AK, Tjuauja S (1995) Laboratory measurements of radar backscatter from bare and snow-covered saline ice sheets. Int J Remote Sens 16:851-876

Bernier M, Fortin JP (1998) The potential of time series of C-band SAR data to monitor dry and shallow snow cover. IEEE Trans Geosci Remote Sens 36:226-243

Bindschadler RA, Jezek KC, Crawford J (1987) Glaciological investigations using the synthetic aperture radar imaging system. Ann Glaciol 9:11-19

Busche T, Hajnsek I, Papathanassiou KP, Krumpen T, Rabenstein L, Hölemann J, Haas C, Willmes S (2009) Comparison of helicopterborne thin sea ice thickness profiles with polarimetric signatures of dual-pol TerraSAR-X data. In: Proceedings of IEEE 2009 International Geoscience and Remote Sensing Symposium (IGARSS)

Carlström A (1997) A microwave backscattering model for deformed first-year sea ice and comparisons with SAR data. IEEE Trans Geosci Remote Sens 35:378-391

Cloude SR (2007) The dual polarization entropy/alpha decomposition: a PALSAR case study. Paper presented at the 2007 international workshop on Science and Applications of SAR Polarimetry and Polarimetric Interferometry (POLinSAR)

Cloude SR (2009) Polarisation: applications in remote sensing. Oxford University Press, Oxford

Cloude SR, Pottier E (1997) An entropy based classification scheme for land application of polarimetric SAR. IEEE Trans Geosci Remote Sens 35:68-78

Dall J (2007) InSAR elevation bias caused by penetration into uniform volumes. IEEE Trans Geosci Remote Sens 45:2319-2324

Dall J, Papathanassiou KP, Skriver H (2003) Polarimetric SAR interferometry applied to land ice: first results. In: Proceedings of IEEE 2003 International Geoscience and Remote Sensing Symposium (IGARSS)

Dall J, Papathanassiou KP, Skriver H (2004) Polarimetric SAR interferometry applied to land ice: modelling. In: Proceedings of 2004 European Conference on Synthetic Aperture Radar (EUSAR)

Davis CH, Poznyak VI (1993) The depth of penetration in Antarctic firn at $10 \mathrm{GHz}$. IEEE Trans Geosci Remote Sens 31:1107-1111

Dierking W (1999) Multifrequency scatterometer measurements of Baltic Sea ice during EMAC-95. Int J Remote Sens 20:349-372

Table 4.11 Summary of presented application, methods and preferred system configurations for cryosphere monitoring

\begin{tabular}{l|l|l}
\hline Application & Methods and used frequency (P/L/C/X) & $\begin{array}{l}\text { Radar data preference/requirements/ } \\
\text { comments }\end{array}$ \\
\hline $\begin{array}{l}\text { Land ice extinction (internal } \\
\text { structure) }\end{array}$ & PollnSAR decomposition and inversion (P/L) & Preferred frequencies: P/L \\
\hline Snow water equivalent & PolSAR decomposition and inversion (C/X) & Preferred frequencies: X/Ku \\
\cline { 2 - 3 } & Pea ice: Observation & Dual-pol may suffice \\
\hline Sea ice: Thematic mapping & Statistical image segmentation based on PolSAR descriptors (C) & Wide swath needed \\
& $\begin{array}{l}\text { Wishart classification applied to PolSAR entropy/alpha } \\
\text { decomposition (L/C) }\end{array}$ & \\
\hline Sea ice thickness & PolSAR decompositions, segmentation and empirical models (X) & Wide swath needed \\
\hline Permafrost observation & Based on a PolSAR two-layer scattering model (L) & At X-band, dual-pol may suffice \\
\hline
\end{tabular}


Dierking W, Dall J (2007) Sea-ice deformation state from synthetic aperture radar imagery - Part I: comparison of C- and L-band and different polarization. IEEE Trans Geosci Remote Sens 45:3610-3622

Dierking W, Wesche C (2013) C-band radar polarimetry - useful for detection of icebergs in sea ice? IEEE Trans Geosci Remote Sens 52:25-37

Dierking W, Carlstrom A, Ulander LMH (1997) The effect of inhomogeneous roughness on radar backscattering from slightly deformed sea ice. IEEE Trans Geosci Remote Sens 35:147-159

Dierking W, Skriver H, Gudmandsen P (2003) SAR polarimetry for sea ice monitoring. Paper presented at the 2003 International Workshop on Science and Applications of SAR Polarimetry and Polarimetric Interferometry (POLinSAR)

Dierking W, Skriver H, Gudmandsen P (2004) On the improvement of sea ice classification by means of radar polarimetry. In: Goossens $\mathrm{R}$ (ed) Remote sensing in transition. Millepress, Rotterdam

Doulgeris AP (2012) Rethinking statistical based segmentation of sea ice. Paper presented at the ESA SeaSAR 2012 workshop

Doulgeris AP (2013) A simple and extendable segmentation method for multi-polarisation SAR images. Paper presented at the 2013 International Workshop on Science and Applications of SAR Polarimetry and Polarimetric Interferometry (POLinSAR)

Doulgeris AP, Eltoft T (2010) Scale mixture of Gaussian modelling of polarimetric SAR data. EURASIP J Adv Sig Proc 2010:1-12

Dowdeswell JA, Drewry DJ (2004) Investigations of the form and flow of ice sheets and glaciers using radio-echo sounding. Publ Rep Progr Phys 67:1821-1861

Dowdeswell JA, Unwin B, Nuttall AM, Wingham DJ (1999) Velocity structure, flow instability and mass flux on a large Arctic ice cap from satellite radar interferometry. Earth Planet Sci Lett 167:131-140

Drinkwater MR, Kwok R, Rignot E (1990) Synthetic aperture radar polarimetry of sea ice. In: Proceedings of IEEE 1990 International Geoscience and Remote Sensing Symposium (IGARSS)

Drinkwater MR, Kwok R, Winebrenner DP, Rignot E (1991) Multifrequency polarimetric synthetic aperture radar observations of sea ice. J Geophys Res 96:20679-20698

Drinkwater M, Kwok R, Rignot R, Israelsson H, Onstott RG, Winebrenner DP (1992) Potential applications of polarimetry to the classification of sea ice. Geophys Monogr Ser 68:419-430

Elachi C, Van Zyl JJ (2006) Introduction to the physics and techniques of remote sensing. Wiley, New York

Eltoft T, Fors A, Moen MA, Renner A, Doulgeris A, Gerland S, FerroFamil L (2012) A multi-polarization study of Arctic sea ice in C-band and X-band. Paper presented at the ESA SeaSAR 2012 Workshop

Eriksson L, Drinkwater M, Holt B, Valjavek E, Nortier O (1998) SIR-C polarimetric radar results from the Weddell Sea, Antarctica. In: Proceedings of IEEE 1998 International Geoscience and Remote Sensing Symposium (IGARSS)

Eriksson LEB, Borenäs $\mathrm{K}$, Dierking $\mathrm{W}$, Berg A, Santoro $\mathrm{M}$, Pemberton P, Lindh H, Karlson B (2010) Evaluation of new spaceborne SAR sensors for sea-ice monitoring in the Baltic Sea Can J Remote Sens 36:56-73

European Space Agency (2008) CoReH2O: candidate earth explorer core missions- reports for assessment. Mission Science Division, ESA-ESTEC, Noordwijk, The Netherlands, ESA SP-1313(3)

Franceschetti G, Lanari R (1999) Synthetic aperture radar processing. CRC Press, Boca Raton

Freeman A (2007) Fitting a two-component scattering model to polarimetric SAR data from forests. IEEE Trans Geosci Remote Sens 45:2583-2592

Fung AK (1994) Microwave scattering and emission models and their applications. Artech House, Norwood

Gareth Rees W (2006) Remote sensing of snow and ice. Taylor \& Francis, Boca Raton
Geldsetzer T, Yackel JJ (2009) Sea ice type and open water discrimination using dual co-polarised C-band SAR. Can J Remote Sens 35:73-84

Geldsetzer T, Mead JB, Yackel JJ, Scharien RK, Howell SEL (2007) Surface-based polarimetric C-band scatterometer for field measurements of sea ice. IEEE Trans Geosci Remote Sens 45:3405-3416

Gill JP, Yackel JJ (2012) Evaluation of C-band SAR polarimetric parameters for discrimination of first- year sea ice types. Can J Remote Sens 38:306-323

Goetz SJ, Bunn AG, Fiske GJ, Houghton RA (2005) Satellite-observed photosynthetic trends across boreal North America associated with climate and fire disturbance. Proc Natl Acad Sci U S A 102:13521-13525

Guneriussen T, Hogda KA, Johnsen H, Lauknes I (2001) InSAR for estimation of changes in snow water equivalent of dry snow. IEEE Trans Geosci Remote Sens 39:2101-2108

Hajnsek I (2001) Inversion of surface parameters using polarimetric SAR. Ph.D. thesis, Friedrich-Schiller Universität, Jena

Hajnsek I, Schoen H, Jagdhuber T, Papathanassiou KP (2007) Estimation of soil moisture under vegetation using PolInSAR: a comparison of methods. Paper presented at the AgriSAR workshop

Hoen EW (2001) A correlation-based approach to modeling interferometric radar observations of the Greenland ice sheet. Ph.D. thesis, Stanford University

Hoen EW, Zebker HA (2000) Penetration depths inferred from interferometric volume decorrelation observed over the Greenland ice sheet. IEEE Trans Geosci Remote Sens 38:2572-2583

Holmlund P, Nyman M, Petterson R, Stenberg M, Gjerde K, Gundestrup N, Hansson M, Isaksonn E, Karloef L, Winther JG, Pinglot F, Reijmer C, Thomassen M, Van de Wal R, Van der Veen C, Wilhelms F (2000) Spatial gradients in snow layering at $10 \mathrm{~m}$ temperatures at two EPICA-dronning Maud Land (Antarctica) pre-site-survey drill sites. Ann Glaciol 30:13-19

Isleifson D, Langlois A, Barber DG, Shafai L (2009) C-Band scatterometer measurements of multiyear sea ice before fall freezeup in the Canadian Arctic. IEEE Trans Geosci Remote Sens 47:1651-1661

Isleifson D, Hwang B, Barber DG, Scharien RK, Shafai L (2010) C-band polarimetric backscattering signatures of newly formed sea ice during fall freeze-up. IEEE Trans Geosci Remote Sens 48:3256-3267

Israelsson H, Askne J (1991) Analysis of polarimetric SAR observations of sea ice. In: Proceedings of IEEE 1991 International Geoscience and Remote Sensing Symposium (IGARSS)

Jackson CR, Apel JR (2004) Synthetic aperture radar marine user's manual. National Oceanic and Athmospheric Administration (NOAA)

Jezek KC, Gogineni P, Shanableh M (1994) Radar measurements of melt zones on the Greenland ice sheet. Geophys Res Lett 21:33-36

Joughin IR, Kwok R, Fahnestock MA (1998) Interferometric estimation of three-dimensional ice-flow using ascending and descending passes. IEEE Trans Geosci Remote Sens 36:25-37

Kasischke ES, Bourgeau-Chavez LL, Johnstone JF (2007) Assessing spatial and temporal variations in surface soil moisture in firedisturbed black spruce forests in Interior Alaska using spaceborne synthetic aperture radar imagery - implications for post-fire tree recruitment. Remote Sens Environ 108:42-58

Kern S, Gade M, Haas C, Pfaffling A (2006) Retrieval of thin-ice thickness using the L-band polarization ratio measured by the helicopter-borne scatterometer HELISCAT. Ann Glaciol 44:275-280

Kim JW, Kim DJ, Hwang BJ (2012) Characterization of arctic sea ice thickness using high-resolution spaceborne polarimetric SAR data. IEEE Trans Geosci Remote Sens 50:13-22

Kong JA (1986) Electromagnetic wave theory. Wiley, New York 
Kurtz NT, Markus T (2012) Satellite observations of Antarctic Sea ice thickness and volume. J Geophys Res Oceans. https://doi.org/10. 1029/2012JC008141

Kurtz NT, Markus T, Cavalieri DJ, Sparling LC, Krabill WB, Gasiewski AJ, Sonntag JG (2009) Estimation of sea ice thickness distributions through the combination of snow depth and satellite laser altimetry data. J Geophys Res Oceans. https://doi.org/10.1029/2009JC005292

Kwok R, Drinkwater M, Pang A, Rignot E (1991) Characterization and classification of sea ice in polarimetric SAR data. In: Proceedings of IEEE 1991 International Geoscience and Remote Sensing Symposium (IGARSS)

Kwok R, Nghiem SV, Yueh SH, Huynh DD (1995) Retrieval of thin ice thickness from multifrequency polarimetric SAR data. Remote Sens Environ 51:361-374

Lee JS (1981) Refined filtering of image noise using local statistics. Comput Vis Graph Image Proc 15:380-389

Lee JS, Pottier E (2009) Polarimetric radar imaging: from basics to applications. CRC Press, Boca Raton

Lee JS, Schuler DL, Ainsworth TL (2000) Polarimetric SAR data compensation for terrain azimuth slope variation. IEEE Trans Geosci Remote Sens 38:2153-2163

Lee JS, Schuler DL, Ainsworth TL, Krogager E, Kasilingam D, Boerner WM (2002) On the estimation of radar polarization orientation shifts induced by terrain slopes. IEEE Trans Geosci Remote Sens 40:30-41

Lewis MJ, Necsoiu M (2011) Seeing sea ice. In: Technology Today, SwRI

Lewis MJ, Necsoiu M, Parra J, Maksym T (2013) Evolution of Antarctic Sea ice characteristics using TerraSAR-X data. TBD

Livingstone CE, Barber DG, Spiring F, Liu W (1996) The SIMMS'93 SAR polarimetry experiment: combined surface and airborne radar measurements of winter sea ice. In: Proceedings of IEEE 1996 International Geoscience and Remote Sensing Symposium (IGARSS)

Maetzler C (1998) Microwave properties of ice and snow. In: Shmitt B et al (eds) Solar system ices. Astrophys Space Sci Library 227:241-257. Kluwer Academic Publisher

Massonnet D, Feigl KL (1998) Radar interferometry and its application to changes in the Earth's surface. Rev Geophys 36:441-500

Matsuoka T, Uratsuka S, Satake M, Nadai A, Umehara T, Maeno H, Wakabayashi H, Nishio F, Fukamachi Y (2002) Deriving Sea-ice thickness and ice types in the Sea of Okhotsk using dual-frequency airborne SAR (Pi-SAR) data. Ann Glaciol 34:429-434

Mätzler C, Strozzi T, Weise T, Floricioiu D, Rott H (1984) Microwave snowpack studies made in the Austrian Alps during the SIR-C/XSAR experiment. Int J Remote Sens 18:2505-2530

Mohr JJ, Madsen SN (1996) Application of interferometry to studies of glacier dynamics. In: Proceedings of IEEE 1996 International Geoscience and Remote Sensing Symposium (IGARSS)

Morio J, Refregier P, Goudail F, Dubois-Fernandez P, Dupuis X (2007). Application of information theory measures to polarimetric and interferometric SAR images. In: Proceedings of 2007 International Conference on Physics in Signal and Image Processing

Nakamura K, Wakabayashi H, Naoki K, Nishio F, Moriyama T, Uratsuka S (2005) Observation of sea-ice thickness in the Sea of Okhotsk by using dual-frequency and fully polarimetric airborne SAR (Pi-SAR) data. IEEE Trans Geosci Rem Sens 43:2460-2469

Necsoiu M, Lewis MJ, Parra J, Hwang P, Wilkinson J, Maksym T, Floricioiu D, Weissling B, Xie H, Ackley S (2011) Studies of Antarctic sea ice characteristics using surface sea ice measurements and TerraSAR-X data. Paper presented at the 2011 TerraSAR-X DLR Science Meeting no. 4

Nghiem SV, Yueh SH, Kwok R, Li FK (1992) Symmetry properties in polarimetric remote sensing. Radio Sci 27:693-711

Nghiem SV, Kwok R, Yueh SH, Drinkwater MR (1995a) Polarimetric signatures of sea ice -1 . Theoretical model. J Geophys Res 100:665-679
Nghiem SV, Kwok R, Yueh SH, Drinkwater MR (1995b) Polarimetric signatures of sea ice -2 . Experimental observations. J Geophys Res 100:681-698

Oh Y (2004) Quantitative retrieval of soil moisture content and surface roughness from multipolarized radar observations of bare soil surfaces. IEEE Trans Geosci Remote Sens 42:596-601

Onstott RG, Gogineni P, Gow AJ, Grenfell TC, Jezek KC, Perovich DK, Swift CT (1998) Electromagnetic and physical properties of sea ice formed in the presence of wave action. IEEE Trans Geosci Remote Sens 36:1764-1783

Paden JD, Allen CT, Gogineni S, Jezek KC, Dahl-Jensen D, Larsen LB (2007) Wideband measurements of ice sheet attenuation and basal scattering. IEEE Geosci Remote Sens Lett 2:164-168

Partington KC, Flach JD, Barber DG, Isleifson D, Meadows PJ, Verlaan P (2010) Dual-polarization C-band radar observations of sea ice in the Amundsen Gulf. IEEE Trans Geosci Remote Sens 48:2685-2691

Pisciottano I, Jagdhuber T, Hajnsek I (2011) First analysis on snow cover change using fully polarimetric TerraSAR-X data. Paper presented at the 2011 international workshop on Science and Applications of SAR Polarimetry and Polarimetric Interferometry (POLinSAR)

Rignot E, Drinkwater MR (1994) Winter Sea-ice mapping from multiparameter synthetic aperture radar. J Glaciol 40:31-45

Rignot E, Van Zyl JJ (1992) Analysis of scattering behavior and radar penetration in AIRSAR data. In: Proceedings of IEEE 1992 International Geoscience and Remote Sensing Symposium (IGARSS)

Rignot E, Way JB (1994) Monitoring freeze-thaw cycles along northsouth Alaskan transects using ERS-1 SAR. Remote Sens Environ 49:131-137

Rott H (1984) The analysis of backscattering properties from SAR data of mountain regions. IEEE J Ocean Eng 9:347-355

Rott H, Cline D, Haas C, Duguay C, Essery R, Macelloni G, Malnes J, Pulliainen J, Rebhan H, Yueh S (2008) Scientific preparations for $\mathrm{CoReH} 2 \mathrm{O}$, a dual frequency SAR mission for snow and ice observations. In: Proceedings of IEEE 2008 International Geoscience and Remote Sensing Symposium (IGARSS)

Sandven S, Johannessen OM, Kloster K (2006) Sea ice monitoring by remote sensing. Wiley, New York

Scheuchl B, Hajnsek I, Cumming IG (2002) Model-based classification of polarimetric SAR sea ice data. In: Proceedings of IEEE 2002 International Geoscience and Remote Sensing Symposium (IGARSS)

Scheuchl B, Hajnsek I, Cumming IG (2003a) Classification strategies for polarimetric SAR sea ice data. Paper presented at the 2003 International Workshop on Science and Applications of SAR Polarimetry and Polarimetric Interferometry (POLinSAR)

Scheuchl B, Cumming IG Hajnsek I (2003b) Classification of ice types from Convair-580 data of Northumberland Strait, PEI. In: Proceedings of CSA 2003 advanced SAR workshop

Scheuchl B, Caves R, Flett D, De Abreu R, Arkett M, Cumming I (2004) The potential of cross-polarization information for operational sea ice monitoring. Paper presented at the 2004 Envisat/ERS symposium

Scheuchl B, Cumming I, Hajnsek I (2005) Classification of fully polarimetric single-and dual-frequency SAR data of sea ice using the Wishart statistics. Can J Remote Sens 31:61-72

Sharma J (2010) Estimation of glacier ice extinction coefficients using long-wavelength polarimetric interferometric synthetic aperture radar. Ph.D. thesis, Karlsruhe Institute for Technology

Sharma J, Hajnsek I, Papathanassiou KP, Moreira A (2011) Polarimetric decomposition over glacier ice using long-wavelength airborne PolSAR. IEEE Trans Geosci Remote Sens 49:519-535

Shi J (2006) Snow water equivalence retrieval using $\mathrm{X}$ and $\mathrm{Ku}$ band dual-polarization radar. In: Proceedings of IEEE 2006 International Geoscience and Remote Sensing Symposium (IGARSS) 
Shi J, Dozier J (1993) Modelling and observation of polarimetric SAR response to dry snow. In: Proceedings of IEEE 1993 International Geoscience and Remote Sensing Symposium (IGARSS)

Shi J, Dozier J (1995) Inferring snow wetness using C-band data from SIRC's polarimetric synthetic aperture radar. IEEE Trans Geosci Remote Sens 33:905-914

Shi J, Dozier J (2000a) Estimation of snow water equivalent using SIR-C/X-SAR. I. Inferring snow density and subsurface properties. IEEE Trans Geosci Remote Sens 38:2465-2474

Shi J, Dozier J (2000b) Estimation of snow water equivalent using SIR-C/X-SAR. II. Inferring snow depth and particle size. IEEE Trans Geosci Remote Sens 38:2475-2488

Shi J, Davis RE, Dozier J (1993) Stereological determination of dry snow parameters for discrete microwave modelling. Ann Glaciol 17:295-299

Singh G, Yamaguchi G, Park SE, Venkataraman G (2010) Identification of snow using SAR polarimetry techniques. International Archives of the Photogrammetry, Remote Sensing and Spatial Information Science, Volume XXXVIII, Part 8, Kyoto

Skriver H, Pedersen LT (1995) Polarimetric signatures of sea ice in the Greenland Sea. In: Proceedings of IEEE 1995 International Geoscience and Remote Sensing Symposium (IGARSS)

Stebler O, Schwerzmann A, Luthi M, Meier E, Nuesch D (2005) PolInSAR observation from an alpine glacier in the cold infiltration zone at L- and P- band. IEEE Geosci Remote Sens Lett 2:357-361

Thomsen BB, Nghiem SV, Kwok R (1998) Polarimetric C-band SAR observations of sea ice in the Greenland sea. In: Proceedings of IEEE 1998 International Geoscience and Remote Sensing Symposium (IGARSS)

Tiuri M, Sihvola A, Nyfors E, Hallikaiken M (1984) The complex dielectric constant of snow at microwave frequencies. IEEE J Ocean Eng 9:377-382

Tjuatja S, Fung AK, Bredow J (1992) A scattering model for snowcovered sea ice. IEEE Trans Geosci Remote Sens 30:804-810

Topp GC, Davis JL, Annan AP (1980) Electromagnetic determination of soil water content. Water Resour Res 16:574-582

Ulaby FT, Stiles WH, Abdelrazik M (1984) Snow cover influence on backscattering from terrain. IEEE Trans Geosci Remote Sens 22:126-133

Ulaby FT, Moore RK, Fung AK (1986a) Microwave remote sensing, active and passive. Volume III: From theory to applications. Artech House, Norwood

Ulaby FT, Moore RK, Fung AK (1986b) Microwave remote sensing: active and passive. Artech House, Norwood
Uratsuka S, Maeno H, Suitz T, Fisher DA, Goto-Azuma K, Mae S (1996) Internal layering detected by microwave ice-radar in the Arctic ice cap. Nat Inst Polar Res 51:387-394

Van Zyl JJ (2011) Synthetic aperture radar polarimetry. Wiley, New York

Van Zyl JJ, Arii M, Kim Y (2008) Requirements for model-based polarimetric decompositions. In: Proceedings of 2008 European Conference on Synthetic Aperture Radar (EUSAR)

Verbyla D (2008) The greening and browning of Alaska based on 1982-2003 satellite data. Glob Ecol Biogeogeogr 17:547-555

Wakabayashi H, Sakai S (2010) Estimation of sea ice concentration in the Sea of Okhotsk using PALSAR polarimetric data. In: Proceedings of IEEE 2010 International Geoscience and Remote Sensing Symposium (IGARSS)

Wakabayashi H, Matsuoka T, Nakamura K, Nishio F (2004) Polarimetric characteristics of sea ice in the sea of okhotsk observed by airborne L-band SAR. IEEE Trans Geosci Remote Sens 42:2412-2425

Warren SG, Brandt RE (2008) Optical constants of ice from the ultraviolet to the microwave: a revised compilation. J Geophys Res Atmosp. https://doi.org/10.1029/2007JD009744

Watanabe M, Kadosaki G, Kim Y, Ishikawa M, Kushida K, Sawada Y, Tadono T, Fukudah T, Sato M (2012) Analysis of the sources of variation in L-band backscatter from terrains with permafrost. IEEE Trans Geosci Remote Sens 50:44-54

Wesche C, Dierking W (2012) Polarimetric analysis of iceberg and sea ice radar backscattering mechanisms for iceberg detection. In: Proceedings of IEEE 2012 International Geoscience and Remote Sensing Symposium (IGARSS)

Winebrenner DP, Farmer LD, Joughin IR (1995) On the response of polarimetric SAR signatures at $24 \mathrm{~cm}$ wavelength to sea ice thickness in arctic leads. Radio Sci 30:373-402

Xie H, Ackley SF, Yi D, Zwally HJ, Wagner P, Weissling B, Lewis M, Ye K (2011) Sea ice thickness distribution of the Bellingshausen Sea from surface measurements and ICESat altimetry. Deep Sea Res Part II. Topic Stud Oceanogr 58:1039-1051

Yi D, Zwally HJ, Robbins J (2011) ICESat observations of seasonal and interannual variation of sea-ice freeboard and estimated thickness in the Weddell Sea. Ann Glaciol 52:43-51

Zebker HA, Villasenor J (1992) Decorrelation in interferometric radar echoes. IEEE Trans Geosci Remote Sens 30:950-959

Zwally HJ, Yi D, Kwok R, Zhao Y (2008) ICESat measurements of sea ice freeboard and estimates of sea ice thickness in the Weddell Sea. J Geophys Res Oceans. https://doi.org/10.1029/2007JC004284

Open Access This chapter is licensed under the terms of the Creative Commons Attribution 4.0 International License (http://creativecommons.org/ licenses/by/4.0/), which permits use, sharing, adaptation, distribution and reproduction in any medium or format, as long as you give appropriate credit to the original author(s) and the source, provide a link to the Creative Commons license and indicate if changes were made.

The images or other third party material in this chapter are included in the chapter's Creative Commons license, unless indicated otherwise in a credit line to the material. If material is not included in the chapter's Creative Commons license and your intended use is not permitted by statutory regulation or exceeds the permitted use, you will need to obtain permission directly from the copyright holder.

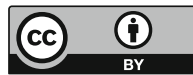




\title{
Urban Applications
}

\author{
E. Colin-Koeniguer, N. Trouve, Y. Yamaguchi, Y. Huang, L. Ferro-Famil, \\ V. D. Navarro Sanchez, J. M. Lopez Sanchez, D. Monells, R. Iglesias, \\ X. Fabregas, J. Mallorqui, A. Aguasca, and C. López-Martínez
}

\begin{abstract}
The experimental result reported in this chapter review the application of (high resolution) Synthetic Aperture Radar (SAR) data to extract valuable information for monitoring urban environments in space and time. Full polarimetry is particularly useful for classification, as it allows the detection of built-up areas and to discriminate among their different types exploiting the variation of the polarimetric backscatter with the orientation, shape, and distribution of buildings and houses, and street patterns. On the other hand, polarimetric SAR data acquired in interferometric configuration can be combined for 3-D rendering through coherence optimization techniques. If multiple baselines are available, direct tomographic imaging can be employed, and polarimetry both increases separation performance and characterizes the response of each scatterer. Finally, polarimetry finds also application in differential interferometry for subsidence monitoring, for instance, by improving both the number of resolution cells
\end{abstract}

\section{E. Colin-Koeniguer $(\bowtie) \cdot N$. Trouve}

Office National d'Etudes et de Recherches Aérospatiales, University of Paris-Saclay, Palaiseau, France

e-mail: elise.koeniguer@onera.fr

Y. Yamaguchi

Institute of Science and Technology, Niigata University, Niigata, Japan

Y. Huang $\cdot$ L. Ferro-Famil

Institut d'Électronique et de Télécommunications de Rennes, University of Rennes-1, Rennes, France

V. D. N. Sanchez

Starlab, Barcelona, Spain

J. M. L. Sanchez

Institute of Computing Research, University of Alicante, Alicante, Spain

D. Monells · R. Iglesias · X. Fabregas · J. Mallorqui · A. Aguasca

Signal Theory and Communications Department, Universitat

Politecnica de Catalunya, Barcelona, Spain

C. López-Martínez

Environmental Research and Innovation, Luxembourg Institute of

Science and Technology, Esch-sur-Alzette, Luxembourg in which the estimate is reliable, and the quality of these estimates.

\subsection{Introduction}

Cities and urban places grow fast, especially in the developing countries. As most countries rapidly become urbanized, environmental change, including climate change, is becoming a leading development challenge. The impacts of weather variability and climatic changes on cities and urban areas are many and complex. Major cities situated along the coast are likely to be or are already affected by sea- level rise, increased storm flooding, inundation, coastal erosion, rising coastal water tables, and obstructed drainage. Displacement of people, especially to or from urban areas, destruction of property, and loss of livelihoods are other common impacts, which often contribute to and perpetuate stresses to the system.

Remote sensing is particularly well adapted to monitor urban land expansion and urbanization. Indeed, remotely sensed data are inherently suited to provide information on urban land cover characteristics, and their changes over time, at various spatial and temporal scales. Synthetic Aperture Radar (SAR) is an active remote sensing technique capable to gather data independently of time and weather conditions.

Urban scenes are composed of a variety of natural and artificial scatterers. In this sense, polarimetric information is useful for classification, because the polarimetric backscatter from man-made targets varies highly with orientation, shape and distribution of buildings and houses, and street patterns. The first application linked to polarimetric data is then the classification methods for detecting built-up areas and discriminating their different types. Several classification schemes can effectively extract the urban structures by mapping urban related classes with better accuracy than with single-polarimetric data. Some works identify building characteristics through the polarimetric mechanisms such as 
orientation effects and dihedral effects (Iribe and Sato 2007) or through time-frequency analysis (Ferro-Famil and Pottier 2007). Other ones propose the estimation of the dielectric constant of buildings (Cloude 2009).

PolInSAR data have also been proven to help classification in urban scenarios, as demonstrated in (Moriyama et al. 2005). It is hence possible by using the PolInSAR coherence to remove some volume scattering ambiguities remaining in PolSAR scenario. The three dimensional rendering over urban areas has been recently found to be another possible product. At this state of the art, the PolInSAR performances that have been shown in this context have only been demonstrated on airborne high-resolution data. The height estimations are based on coherence optimization (Colin et al. 2006; Colin-Koeniguer and Trouve 2014) or a phase scatter separation methods such as ESPRIT (Guillaso et al. 2005). Then the potential of polarimetry for this application is double: it can first be used for pre-segmentation algorithms and then for improving the estimation of the heights or separating phase centers. Another technique dedicated to 3-D rendering is the tomographic approach that is the extension of the conventional two-dimensional SAR imaging principle to three dimensions. Full three-dimensional imaging of a scene is achieved by the formation of an additional synthetic aperture in elevation by a coherent combination of images acquired from several parallel flight tracks. Once again, the polarimetric extension of this technique has been applied only to airborne data (Huang et al. 2012; Sauer et al. 2007).

On the other hand, land subsidence is a major geological disaster in urban areas. Monitoring land subsidence efficiently will not only help people to identify the spatial and temporal pattern of this kind of disaster but also help people minimize the hazard ahead. Persistent Scatterer Interferometry (PSI) has been recognized as the most powerful tool to monitor the land subsidence in long time series and on large scale (Ferretti et al. 1999, 2001). In PSI approaches, PS selection is a decisive stage because the number and quality of PS directly affect the computed deformation results. In this context, it becomes relevant to assess the use of polarimetry associated with PSI to improve the PS selecting algorithm (Pipia et al. 2009; Navarro-Sanchez et al. 2010).

\subsection{Classification of Urban Areas}

\subsubsection{Polarimetry for Urban Classification}

\subsubsection{Introduction and Motivation}

An urban area is characterized by complex man-made structures with heterogeneous scattering objects. When sensed by radar, it exhibits strong backscattering if the radar illumination is orthogonal to buildings. The scattering magnitude from an urban area, in general, is much larger than those from other areas such as rural, agricultural, vegetation, or forest region. It is rather easy to recognize urban areas using the backscattering coefficient even with a single-polarimetric radar. If the detailed information is desired for urban area application, we need to use fully polarimetric data (Lee and Pottier 2009; Yamaguchi et al. 2005). Fully polarimetric data, i.e., the scattering matrix $\mathbf{S}$, can be expressed in the horizontal $(\mathrm{H})$ and vertical $(\mathrm{V})$ polarization basis.

Polarimetric data analyses on urban areas, up to now, have revealed the following results as shown in Fig. 5.1. If the polarimetric radar illumination is orthogonal to buildings or building blocks, the scattering mechanism is characterized by the double bounce scattering caused by the vertical building wall surface and horizontal road surface. The co-polarized backscattering $\left(\left|S_{h h}\right|^{2}\right.$ and $\left.\left|S_{v v}\right|^{2}\right)$ are strong enough compared to the cross-polarized $\left(\left|S_{h v}\right|^{2}\right)$ component. On the other hand, if the radar illumination is not orthogonal or parallel to building or building blocks, i.e., in the case of oblique incidence to building facets, the scattering magnitude significantly reduces, and the scattering mechanism changes from double bounce to single bounce with the generation of the cross-polarized component. In this case, the scattering characteristics with small RCS and with a rather big contribution of the cross-polarized $\left|S_{h v}\right|^{2}$ component impose a difficult problem to distinguish between oriented buildings against vegetation. Since the scattering characteristics become similar to those of forest in this case, it is difficult to classify them.

The purpose of this section is to show the effectiveness of polarimetry for urban classification considering the effect of scattering characteristics in urban structures. There are, at least, two effective methods for this urban classification:

1. The correlation coefficient in the circular polarization basis (Moriyama et al. 2005; Lee et al. 2002; Schuler et al. 2006; Yamaguchi et al. 2008)

2. The scattering power decomposition with de-orientation (Yamaguchi et al. 2005), polarization orientation compensation (Lee and Ainsworth 2011), or minimization of the cross-polarized component (Yamaguchi et al. 2011; Arii et al. 2011; Sato et al. 2012; Singh et al. 2013)

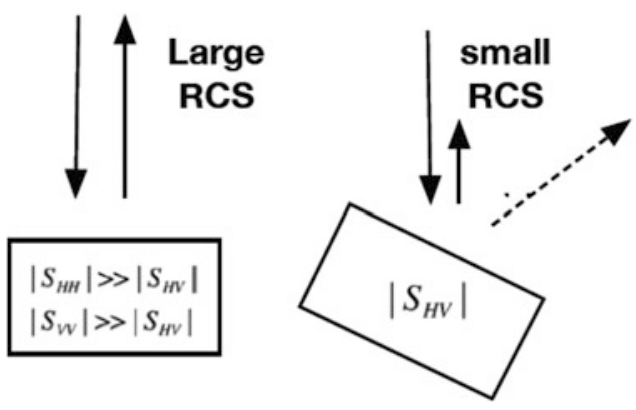

Fig. 5.1 Scattering from buildings 
In the following, some classification results using both methods are explained with high-resolution data sets.

\subsubsection{Literature Review and Methodology}

\subsection{The Correlation Coefficient}

The utilization of the correlation coefficient in the circular polarization basis dates back more than a decade (Moriyama et al. 2005; Lee et al. 2002). The advantage of circular polarization basis seems to be insensitive to object orientation. The original application was proposed by D.L. Schuler et al. for the detection of man-made structures (Guillaso et al. 2005). Similar works can be found in (Yamaguchi et al. 2008). The key point is an enhancement of urban areas against surrounding areas using the correlation coefficient.

The correlation coefficient can be written in terms of scattering elements as:

$$
\begin{aligned}
\gamma_{L L-R R}= & \left|\gamma_{L L-R R}\right| \angle \gamma_{L L-R R} \\
= & \frac{\left\langle 4\left|S_{h v}\right|^{2}-\left|S_{h h}-S_{v v}\right|^{2}\right\rangle-j \operatorname{Re}\left\langle S_{h v}^{*}\left(S_{h h}-S_{v v}\right)\right\rangle}{\sqrt{\left\langle\left|S_{h h}-S_{v v}+2 j S_{h v}\right|^{2}\right\rangle\left\langle\left|S_{h h}-S_{v v}-2 j S_{h v}\right|^{2}\right\rangle}},
\end{aligned}
$$

where the symbol $\langle\cdot\rangle$ denotes ensemble average in an image window.

It is experimentally known that the cross-correlation between the co- and cross-polarized scattering elements are close to zero for natural distribute objects,

$$
\left\langle S_{h h} S_{h v}^{*}\right\rangle=\left\langle S_{v v} S_{v h}^{*}\right\rangle \approx 0
$$

This is the so-called reflection symmetry condition. Under this condition, the correlation coefficient (5.1) becomes realvalued, and it is expressed as:

$$
\gamma_{L L-R R}(0)=\frac{\left\langle 4\left|S_{h v}\right|^{2}-\left|S_{h h}-S_{v v}\right|^{2}\right\rangle}{\left\langle 4\left|S_{h v}\right|^{2}+\left|S_{h h}-S_{v v}\right|^{2}\right\rangle}
$$

If we calculate the correlation coefficient (5.1) in an urban area which exhibits non-reflection symmetry condition, the values become larger than (5.3). If we normalize (5.1) by (5.3):

$$
\gamma_{L L-R R}^{\prime}=\frac{\left|\gamma_{L L-R R}\right|}{\left|\gamma_{L L-R R}(0)\right|}
$$

The value of $\gamma_{L L-R R}^{\prime}$ will be close to unity for the reflection symmetry condition and will be larger than unity for the non-reflection symmetry case. We denote (5.4) as an extended correlation coefficient for discrimination versus (5.1).

If we examine the distribution of the correlation coefficient (5.1) for typical areas shown in rectangular boxes of Fig. 5.2, the values exhibit specific features (see Fig. 5.3). Sea (Patch A) and forest areas (Patch B) are typical reflection symmetry scatterers. The mean value of the coefficient is close to -0.8 for sea and 0 for the forest. The values of oriented urban areas (Patch $\mathrm{C}$ ) are widely spread within the unit circle in the complex plane, while the mean values of orthogonal blocks (Patch D) are concentrated around -1 on the plane. These specific distributions are important for urban classification.

\subsection{Extraction of Oriented Urban Area by Extended Correlation Coefficient}

The mean values of extended correlation coefficients of specific scattering structures in Fig. 5.2 are shown in Table 5.1. It is seen that the oriented houses exhibit large values of more than 2 compared to other areas. Orthogonal urban areas exhibit values similar to those of the reflection symmetry scatterer. Therefore, it is possible to extract oriented urban blocks using the extended correlation coefficient only. The detection result by the extended correlation coefficient is shown in Fig. 5.4, where typical oriented residential houses are highlighted in circles.

\subsection{Tree Area Detection}

Clusters of trees or forests exhibit volume or diffuse scattering with relatively small backscattering power. If trees are mixed within complex urban areas, the detection of trees

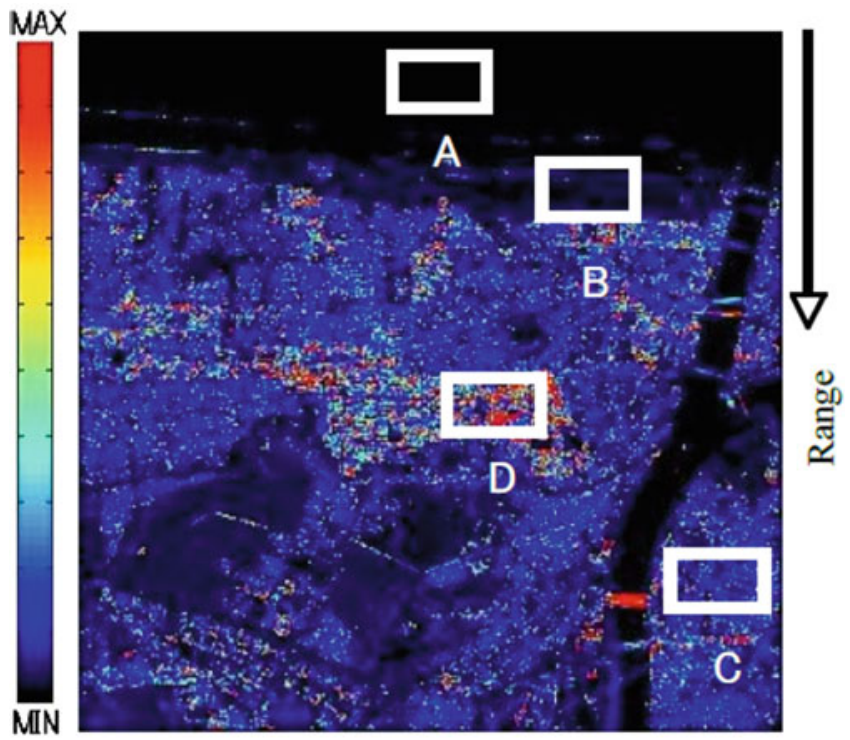

Fig. 5.2 The total power image, X-band Pi-SAR data 
Fig. 5.3 The distribution of the correlation coefficient for specific areas in Fig. 5.2
Reflection Symmetry

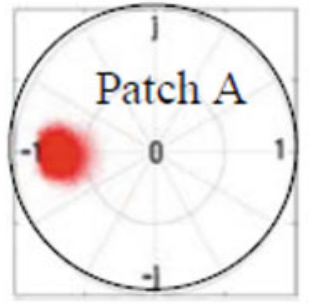

Sea

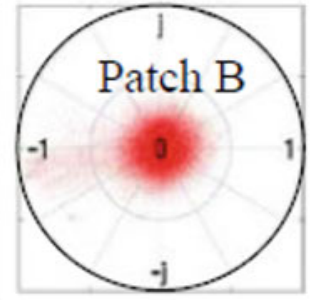

Forest
Non-Reflection Symmetry
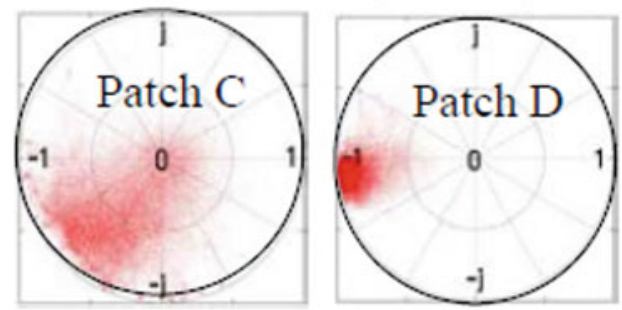

Non-orthogonal Orthogonal to radar illumination urban area

Table 5.1 Values of extended correlation coefficient

\begin{tabular}{l|l}
\hline Category & $\gamma_{L L-R R}$ \\
\hline C: Non-orthogonal urban area & 2.54 \\
\hline D: Orthogonal urban area & 1.12 \\
\hline B: Forest (pine trees) & 1.44 \\
\hline Crop field & 1.22 \\
\hline Paddy rice field & 1.11 \\
\hline Seashore & 1.10 \\
\hline A. Sea & 1.01
\end{tabular}

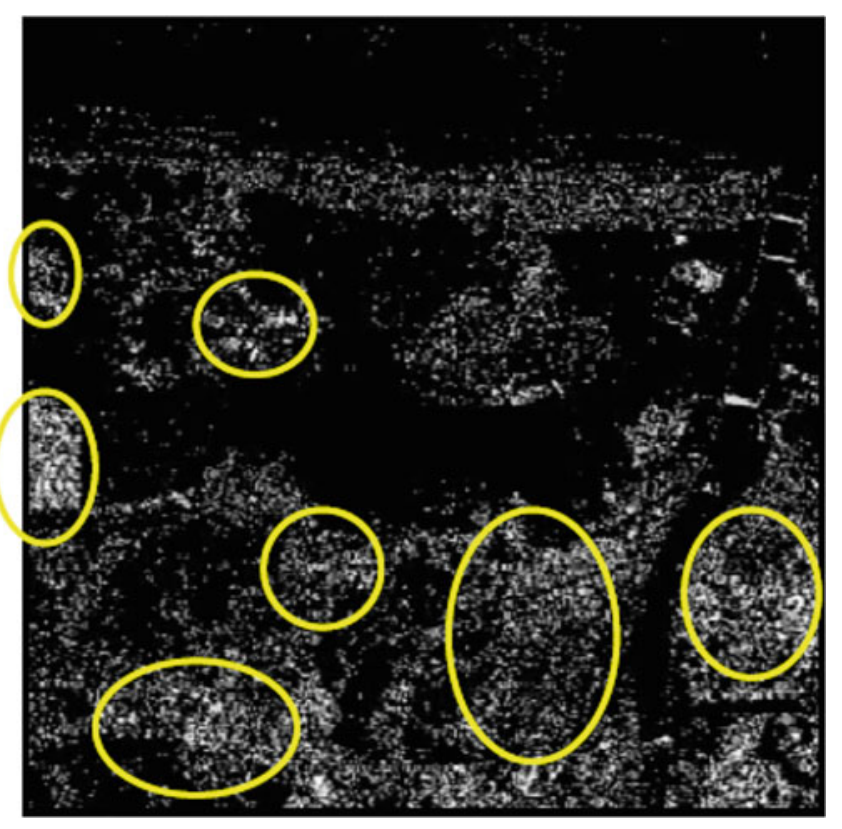

Fig. 5.4 Detection result of oriented building blocks

becomes difficult because strong total powers from man-made structures mask the tree echo.

If we pay attention to forest areas in Fig. 5.3, the mean value of the correlation coefficient is concentrated around 0 . This situation serves to extract forested areas in a very simple way. If we take the reciprocal of (5.1), the value becomes very large for the tree or forested area. Figure 5.5

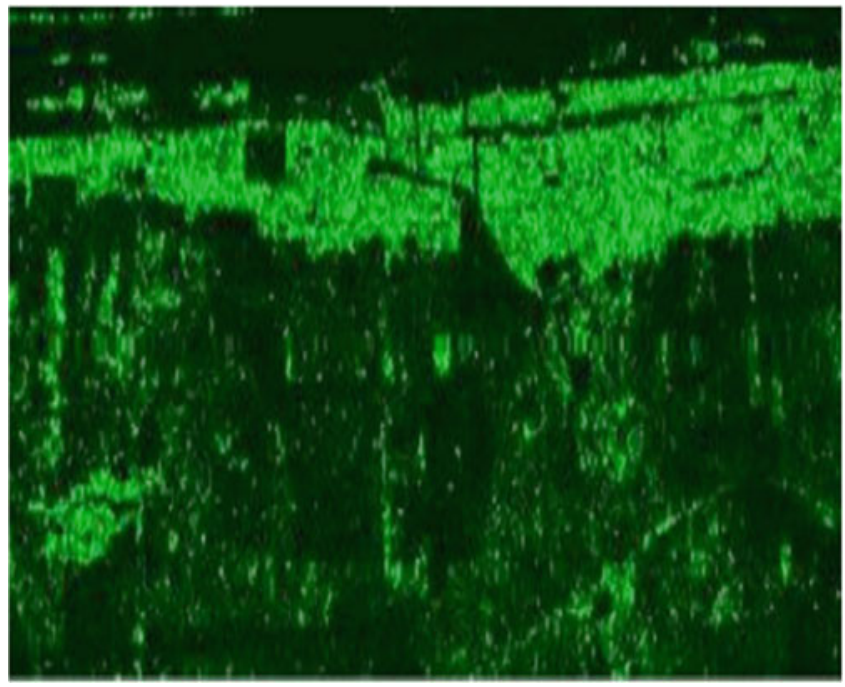

Fig. 5.5 Tree clusters highlighted by $1 / \mid \gamma_{L L}-R R$

shows the value of which indicates the tree or forested area in Fig. 5.2. For the sake of comparison, an aerial photo of the same area is shown in Fig. 5.6. The bright areas in Fig. 5.5 are in good agreement with trees in the actual photo image. As can be deduced, it is possible to identify small forests along the seashore and cluster of trees in urban residential areas. They perfectly match between Figs. 5.5 and 5.6.

\subsection{Classification of Terrain by Total Power and the Correlation Coefficients}

Since the total power is the essential radar parameter, and the correlation coefficient in the circular polarization basis provides useful information, it is possible to use these parameters together for the identification and classification of complex urban terrain. One of the algorithms for urban classification is shown in Fig. 5.7.

The algorithm uses the total power and the correlation coefficient and its extension (5.4). The total power below $-13 \mathrm{~dB}$ is assigned to sea or water area for the exclusion of spiky noise in that area even if $\left|\gamma_{L L}-R R\right|$ is large. The total power larger than $-5.2 \mathrm{~dB}$ with $\left|\gamma_{L L}-R R\right|>0.6$ is assigned to 


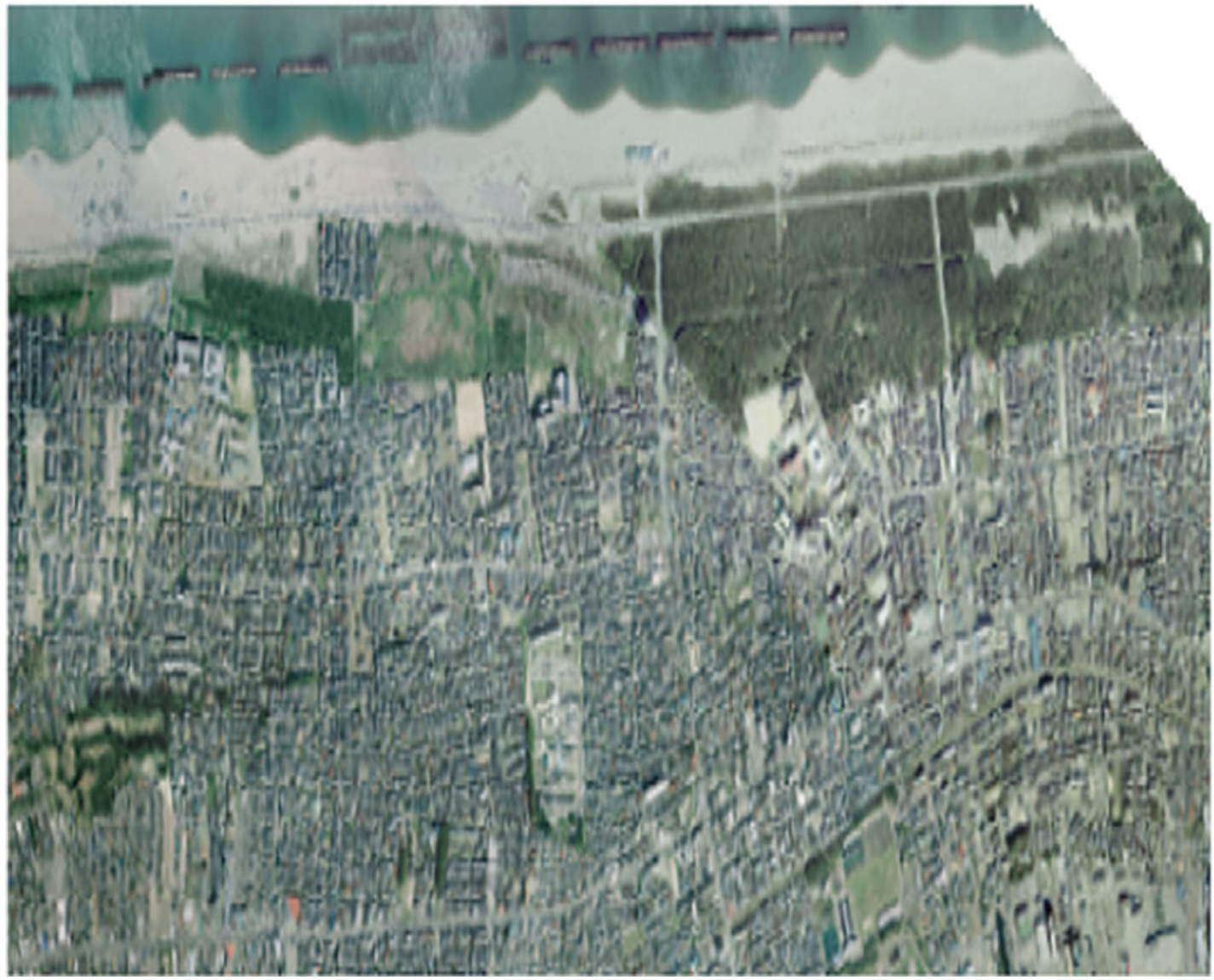

Fig. 5.6 An aerial photo image of the area

orthogonal urban scatterer (orthogonal to radar illumination), because the magnitudes of the total power and the correlation coefficient are large for man-made structures (see Figs. 5.3 and 5.4, respectively). In the range of $-13<\mathrm{TP}<-5.2 \mathrm{~dB}$, the appropriate values of the correlation coefficients $\left|\gamma_{L L}-R R\right|$ and the extended $\left|\gamma_{L L-R R}^{\prime}\right|$ are employed to discriminate areas based on Table 5.1 and Fig. 5.4. The final classification result for the Pi-SAR image is shown in Fig. 5.8. Since the radiometric and polarimetric calibrations have been carried out in the Pi-SAR data sets, these criterion values can be applied to other scenes.

\subsection{Scattering Power Decomposition}

Scattering power decompositions provide tools for the analysis of fully polarimetric images (Yamaguchi et al. 2005). The decomposition images based on the physical scattering model are easy to interpret because experimental pieces of evidence are incorporated. The pioneering work in the model-based decomposition was presented by Freeman and Durden (Freeman and Durden 1998) by introducing the three-component decomposition. To date, a significant amount of research has been carried out on the model-based decomposition techniques (Arii et al. 2011; Sato et al. 2012; Singh et al. 2013).

This section presents the four-component scattering power decomposition by rotation of coherency matrix (Yamaguchi et al. 2011) for urban classification. The advantage of this method is the final imaging performance compared to other schemes. It provides the most natural and beautiful imaging results when RGB color-code is used for double bounce, surface, volume scattering power plus additional Yellow for helix scattering (see Fig. 5.10). Since the helix scattering decreases with an increasing number of averaging pixels, yellow color is assigned to this helix scattering. The yellow color makes RGB images more vivid. It fades away when the number of ensemble averaging pixels increases. On the other hand, we sometimes need high-resolution images using a small number of averaging pixels, $3 \times 3$ for example, for urban area classification. In such a case the yellow color looks bright for man-made structures and fits for human eye recognition, although the statistics may not be accurate due to a small number of averaging. 


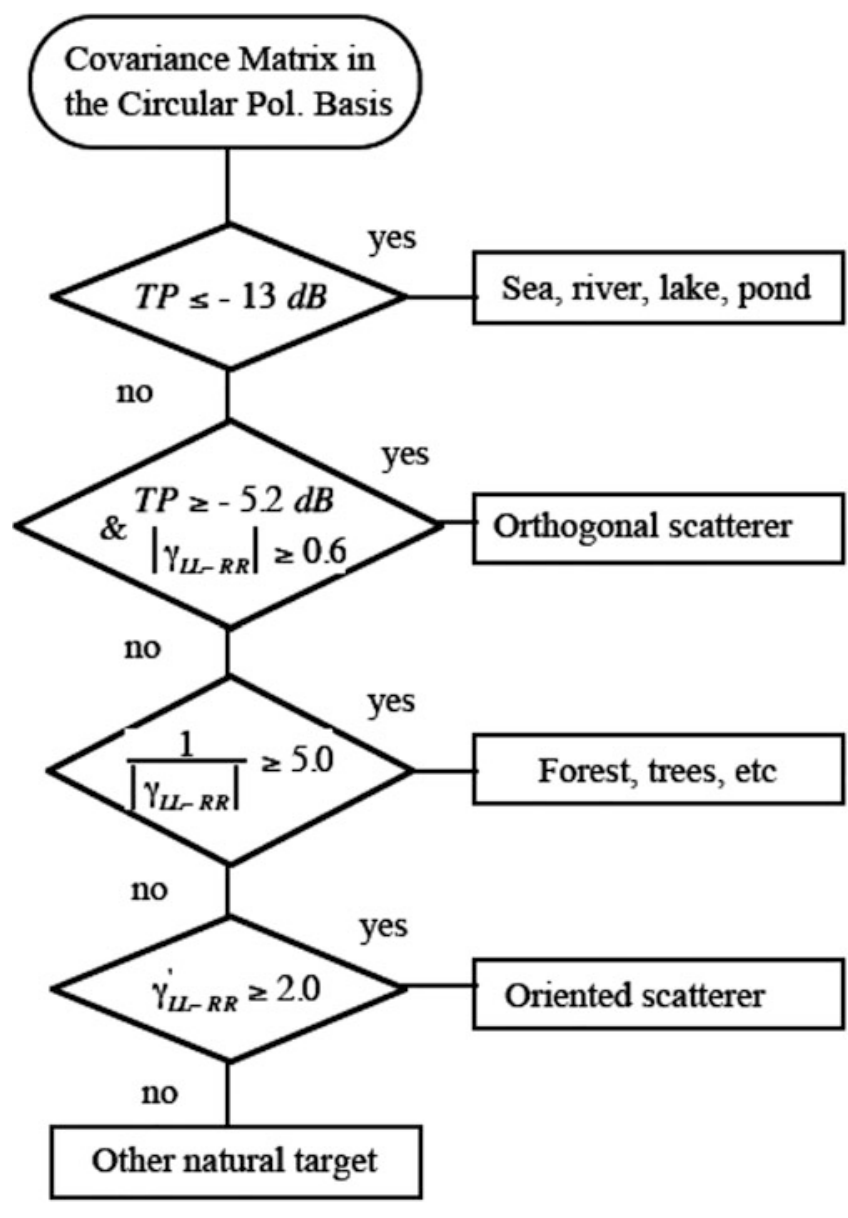

Fig. 5.7 Classification algorithm

\subsection{Decomposition Algorithm}

The procedures for the four-component scattering power decomposition have been already shown in Fig. 3.37. This method first rotates the coherency matrix in an imaging window so as to minimize the cross-polarized component. Then it decomposes the observation matrix into the surface, double bounce, volume, and helix scattering terms based on the physical scattering models and determines the corresponding scattering powers. This method accounts for 6 terms out of 8 independent polarimetric parameters.

\subsubsection{Experimental Results}

Test sites and corresponding radar and validation data sets selected for the generation of showcases on urban classification are summarized in Table 5.2.

The decomposition algorithm in Fig. 3.37 can be directly applied to fully polarimetric data sets. Once the scattering powers are obtained, they are assigned to RGB color-code with the double bounce scattering power Pd to Red, the volume scattering power $\mathrm{Pv}$ to Green, the surface scattering power Ps to Blue, and the helix scattering Pc to Yellow as shown in Fig. 5.9. The magnitude is assigned to color brightness. The general tendencies of scattering powers are displayed as a function of imaging window size.

The decomposition results are shown in Fig. 5.10. The window size for the ensemble average was chosen as $5 \times 5$, $7 \times 7$, and $9 \times 8$. The images become vivid with an increasing number of pixels as shown in Fig. 5.10. The double bounce scattering power $\mathrm{Pd}$ (Red) and volume scattering power $\mathrm{Pv}$ (Green) increase with an increasing number of pixels from $5 \times 5$ to $9 \times 9$. The decomposition results can be compared
Fig. 5.8 Classified area by correlation coefficient and total power

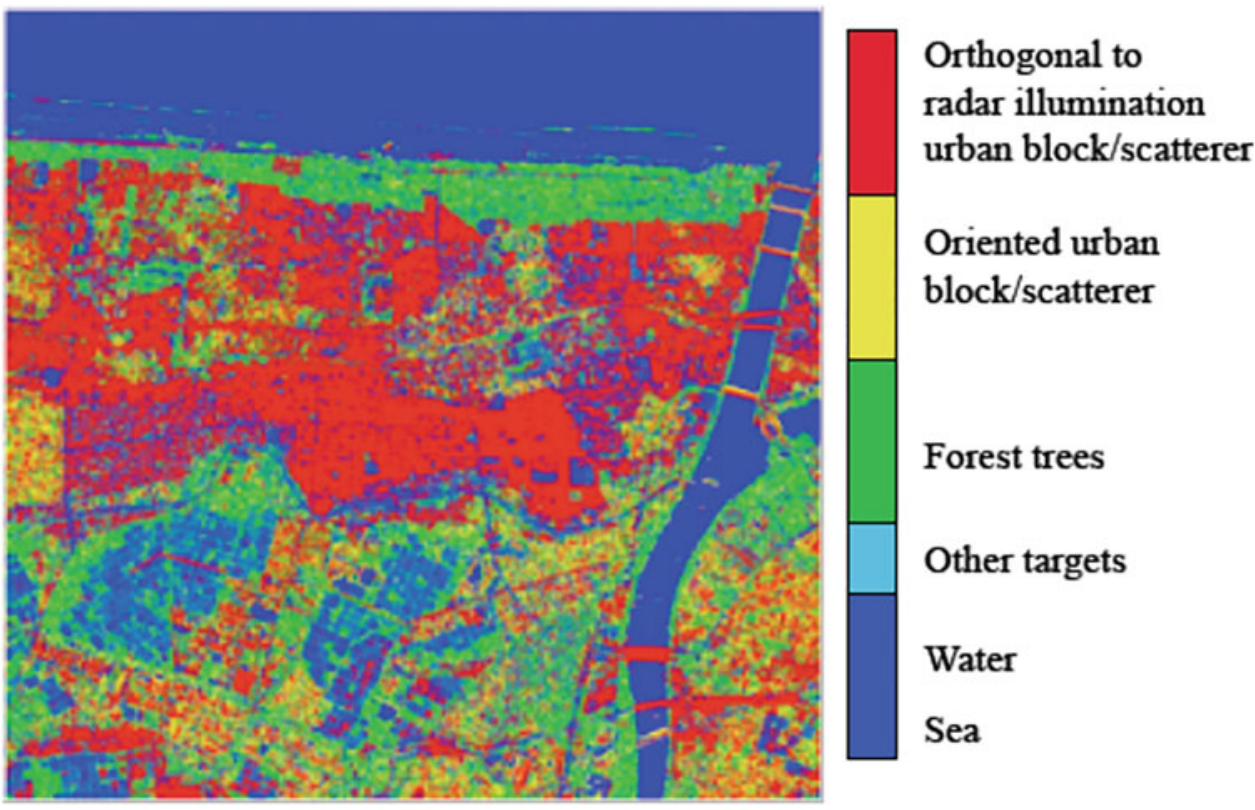


Table 5.2 Test sites and corresponding radar and validation data selected for the generation of showcases on urban classification

\begin{tabular}{l|l|l}
\hline Application/product & Test site - Radar data & Reference data \\
\hline Urban classification (PolSAR) & Western Niigata city, Japan & Google photo images \\
\cline { 2 - 2 } & PiSAR-X airborne data, X-band, $1.5 \mathrm{~m}$ resolution & \\
\hline
\end{tabular}

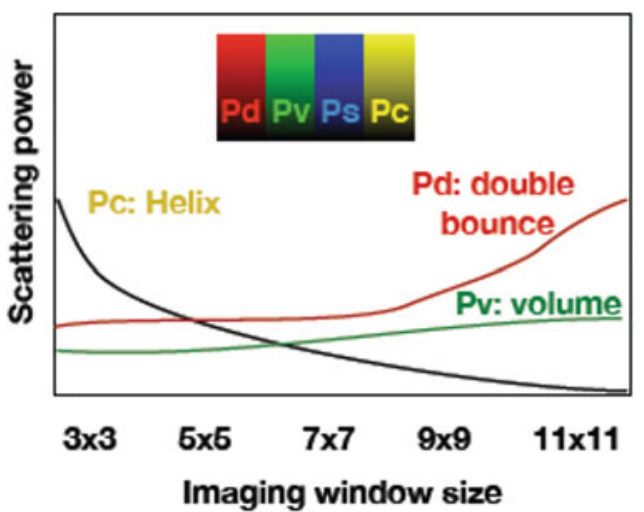

Fig. 5.9 Color-code for the four-component scattering power decomposition. The color-code are used with Red for the double bounce scattering power Pd, Blue for the surface scattering power Ps, Green for the volume scattering power Pv, and Yellow for the helix scattering Pc. The magnitude corresponds to the brightness of each color. The general scattering power behavior is displayed as a function of imaging window size

with the Google photo image in Fig. 5.10, where perfect matching can be seen. Residential areas orthogonal to radar illumination exhibit pink color $(\mathrm{R}+\mathrm{B})$, whereas oblique areas show yellow or orange color. At the time of data acquisition, the rice paddy field was full of rice stem with $50 \mathrm{~cm}$ long, which caused a mixture of volume and double bounce scattering mechanisms. If we need a high-resolution image in urban areas, we need to reduce the averaging number in order to avoid blurring. This causes an adverse effect in polarimetric statistics itself; however, the complementary behavior of Pc and Pd serves good performance in urban classification. Lower left of Fig. 5.10 are buildings not orthogonal to the radar beam. They exhibit yellow color in the $5 \times 5$ image and gradually become a mixture of yellow and red in $7 \times 7$ and $9 \times 9$ images. Bright yellow color best fits for man-made structure indicator.

\subsubsection{Comparison with Single/ Dual-Polarization Data}

Since the correlation coefficient in the circular polarization basis and the four-component scattering power decomposition are based on fully polarimetric data, they do not apply to single-/dual-polarimetric data sets. These methods make use of fully polarimetric data and bring full color image by polarization. If single-/dual-polarimetric data are provided, the backscattering information only will be available, resulting in mono-color image.

\subsubsection{Discussion on the Role of Polarimetry and on the Maturity of the Application and Conclusions}

The correlation coefficient in the circular polarization basis contains useful information on objects. It can be used for classification, derivation of surface slope, and polarization orientation angle, among others. Since the correlation value is dependent on neighboring pixels, the value becomes large if there are similar scatterings and small if the scattering is random. Important is the independence from backscattering power. For high-resolution data sets, polarimetry and this polarimetric index will be a key parameter for the classification of objects.

Scattering power is one of the most essential radar parameters. Polarimetric decomposition powers provide us with an easy way to interpret the radar scene for everybody. Therefore polarimetry seems an essential monitoring tool for radar remote sensing.

If scattering power decomposition results are combined together with the correlation coefficient results, they will serve the most efficient tool for polarimetric analyses of objects.

\subsubsection{Detection of Built-Up Areas}

\subsubsection{Introduction, Motivation, and Literature Review}

In the context of rapid global urbanization, urban environments represent one of the most dynamic regions on earth. Even in developed countries, the yearly conversion of natural or agricultural space into residential, industrial, or transport areas frequently exceeds 100 ha. The current increase in population has resulted in widespread spatial changes, the particularly rapid development of built-up areas, in the city, and its environs. Due to these rapid changes, up-to-date spatial information is required for the effective management and mitigation of the effects of builtup dynamics.

Various studies have shown the potential of highresolution optical satellite data for the detection and classification of urban areas. Nevertheless optical satellite imagery is characterized by a high dependency in weather conditions and daytime. Thus, particularly in case of regional and national surveys within a short period of time, disaster management, or when data have to be acquired at specific dates, radar systems are more valuable. 


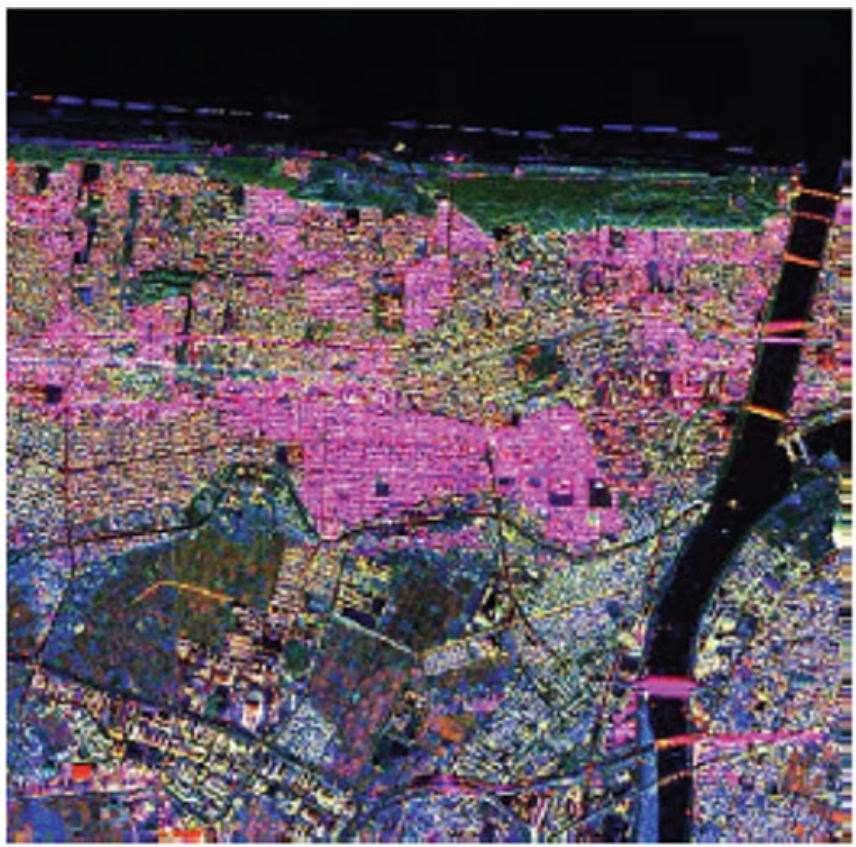

(a) $5 \times 5$

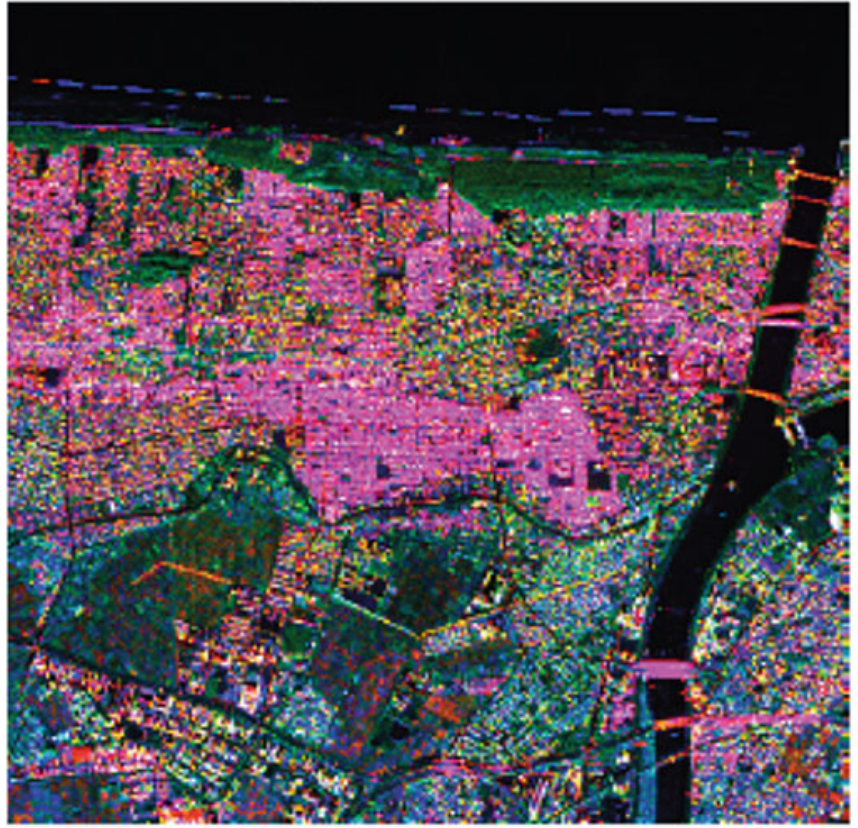

(c) $9 \times 9$

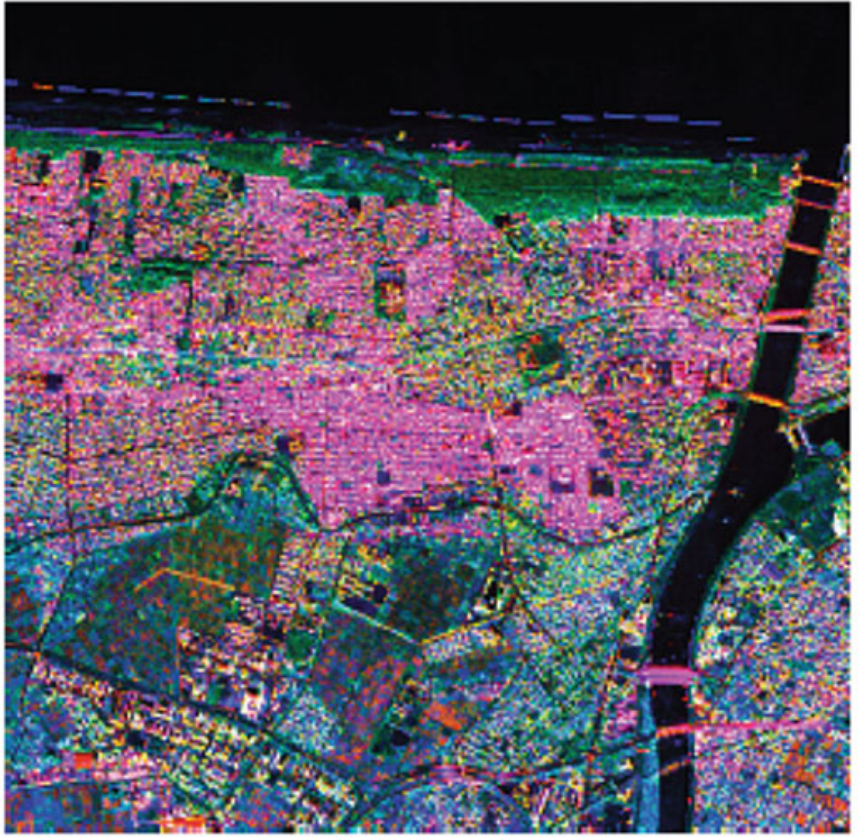

(b) $7 \times 7$

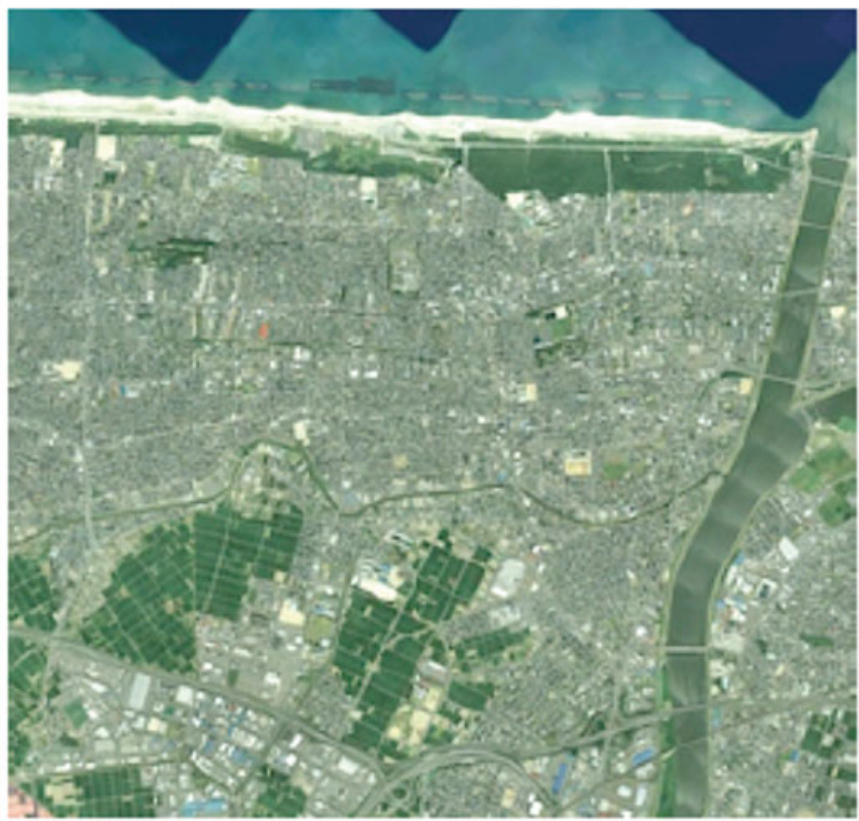

(d) Google earth image

Fig. 5.10 Four-component power decomposition to Pi-SAR-X data set. The effect of imaging window size can be seen and compared in the decomposition image

Thus, the new generation of civil space borne Synthetic Aperture Radar (SAR)-Systems with short revisit can serve as a valuable resource. Promising approaches toward the classification of urban areas include the analysis of multipolarized image analysis. However, to ensure a detailed mapping of urban structures, we need high ground resolution. Indeed, the emergence and recognition of urban remote sensing appear to be linked to the continuous improvement of the spatial resolution offered by generation sensors (Boehm and
Schenkel 2003). Unfortunately, satellite sensors using the polarimetric mode are often in a degraded resolution. That is why to evaluate the benefits of polarimetry in the urban areas, we try to use here the satellite data with the best achievable resolution, which are achieved today by the TerraSAR-X system.

Among the algorithms to detect buildings in polarimetric SAR image, we must distinguish between what is purely statistical (Pellizzeri et al. 2003; Cao et al. 2011; Deledalle 
et al. 2015) or imaging techniques (Wang et al. 2010; Liu et al. 2010) from what is of interest of polarimetry. Some works are only effective exploitation of spatial characteristics of the image such as line detection, analysis of speckle, region-based and edge-based information, etc. Others focus on the best way of estimating PolSAR or PolInSAR coherence matrices or to use dedicated statistical tools as fuzzy logic, neuronal networks, and maximum likelihood classifiers. Others deal with performance evaluation of different polarimetric parameters in their contributions to classification or segmentation algorithm and consider their ability to provide a physical interpretation. The latter is more related to the benefit of polarimetry itself.

Polarimetric decompositions can provide physical interpretations of the PolSAR observations such as scattering mechanisms or polarimetric properties. Parameters obtained by the decomposition methods can be directly used as classification features in non-parametric classifiers. In this framework, various polarimetric SAR parameters can be evaluated for urban land cover detection. They include the Pauli parameters, Freeman and Cloude-Pottier decompositions, Freeman or Yamaguchi decompositions, coherency matrix, intensities of each polarization, and their logarithms.

The principal algorithms proposed in the literature for the extraction of buildings in SAR images using polarimetric information are summarized in the following.

\subsection{Main Scattering Mechanisms Occurring for Buildings}

The contribution of urban polarimetry can be justified by the diversity and complexity of the interpretation of the different mechanisms involved. Briefly, the recorded observations are summed by the scatterings from the targets on the same wave front. For example, the layover areas contain the scatterings from the roof, wall, and ground. A mixture of volume scattering by vegetation and double-bounce scattering from buildings can also be observed in low-density areas. The total scattering is strongly influenced by the looking directions and the alignment of structures: man-made structures which are arranged perpendicularly to the illumination direction increase the oriented double bounce contribution.

Among the polarimetric parameters that can be used in urban areas, some are from coherent decompositions, others from incoherent decompositions. Generally, the former are dedicated to the analysis of targets called deterministic whose statistical fluctuation of the polarimetric response is neglected. This could be the case of manufactured targets such as buildings. The latter take into account the sources of decorrelation.

\subsection{A Classical Polarimetric Parameter: Entropy}

Generally, the polarimetric entropy can precisely distinguish the degree of randomness in the mixture of different polarimetric mechanisms taking place within a resolution cell. Thus, this parameter, and its alternatives such as depolarization or scattering diversity (Praks et al. 2009), seem therefore appropriate to discriminate man-made targets from natural targets.

However, the use of entropy for classification purposes is not so simple. Firstly, entropy requires a statistical estimation, and it is well-known that the results depend on how this estimation is performed and on the number of samples. Furthermore, it is known that entropy is connected to numerous factors related to the design of the sensor, such as the resolution, the noise level, the wavelength, and the geometrical configuration.

Thus, for the classification algorithms based on the use of polarimetric entropy over several images of San Francisco, two main issues have been raised. First, entropy strongly depends on the orientation of the buildings with respect to the sensor axis. This correlation has been also analyzed using a UAVSAR image of New Orleans (Colin-Koeniguer et al. 2015). In this image, several different neighborhoods with various orientations were selected, and the mean entropy has been calculated for each of them. Entropy increases very rapidly with street orientations. Since San Francisco contains some district, the SOMA, with a specific orientation, all classical parameters failed to classify correctly both the buildings of this district and the neighboring districts with different orientations. The second problem is that entropy is high all over the TerraSAR-X image and does not provide sufficient contrast for the detection of built-up areas. This high entropy is the result both of the strong effect of the orientation at $\mathrm{X}$-band and the metric resolution. As soon as the orientations of the streets are not equal to the sensor trajectory orientation, all mechanisms have comparable amplitudes. The different mechanisms are mixed together in the estimation of the coherence matrix, and therefore, the estimated entropy is high.

\subsection{Temporal Estimation}

We have seen that for oriented districts, and for some resolution ranges, entropy is high when estimated spatially, and therefore, it cannot be used efficiently for built-up detection or land classification. In this case, we can estimate entropy temporally. If a few years ago, access to polarimetric SAR data on the same site was rare, today the number of revisits over the same site increases and can be used for statistical estimation of second-order parameters.

The potential of a temporal estimation has been shown on part of SAR images of San Francisco. We calculated the entropy of parts of the image for three types of estimation:

- A spatial average over 3 pixels

- A spatial average using a classical $5 \times 5$ pixels

- A temporal average using 3 passes 
The resulting estimation of entropy is presented over an extract of a TerraSAR-X image in Fig. 5.11 and over an extract of a UAVSAR image in Fig. 5.12. Results on the TerraSAR-X image show that a spatial estimation gives poor results, whereas a better contrast seems to appear between deterministic and non-deterministic targets using a temporal estimation over only 3 pixels. On the UAVSAR image, when we increase the number of pixels used in the spatial estimation, the contrast between districts with different orientations increases also. When we use the temporal estimation, then the contrast between these different districts decreases, while the contrast between deterministic and non-deterministic targets increases. These results have been confirmed on larger temporal image stacks and are very promising for the benefit of temporal estimates in urban polarimetric images.
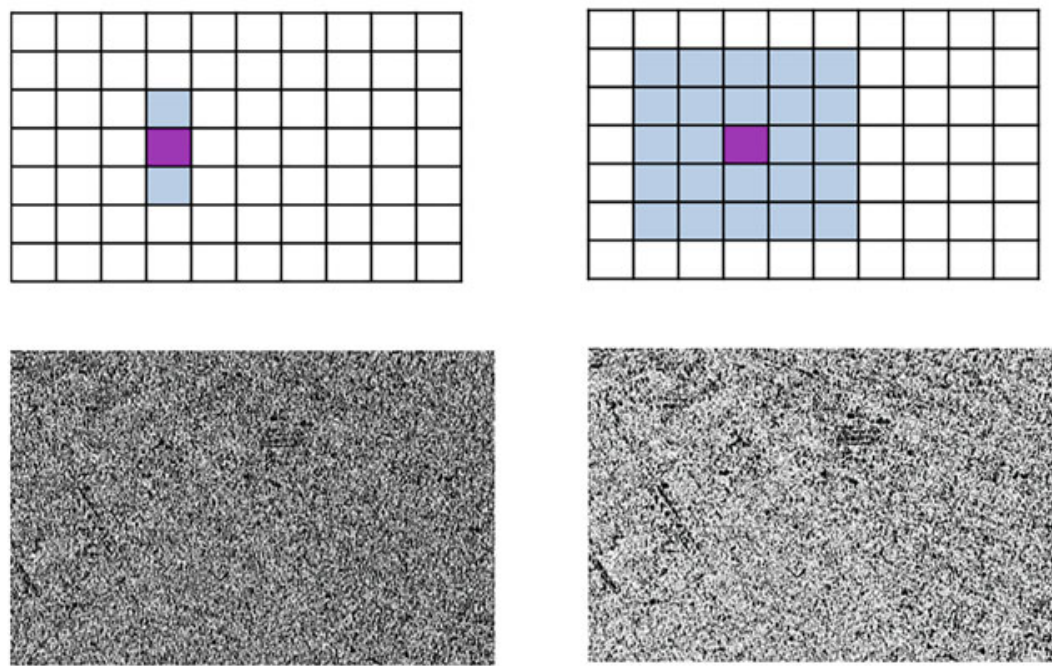

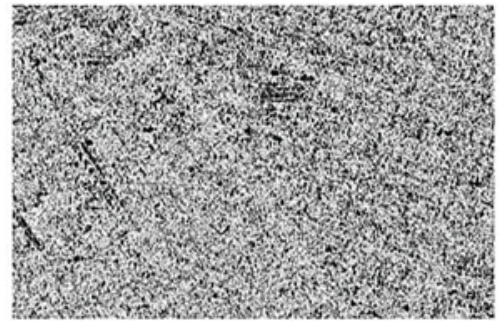

Fig. 5.11 Different entropy maps obtained using different estimation methods over an extract of TerraSAR-X data set
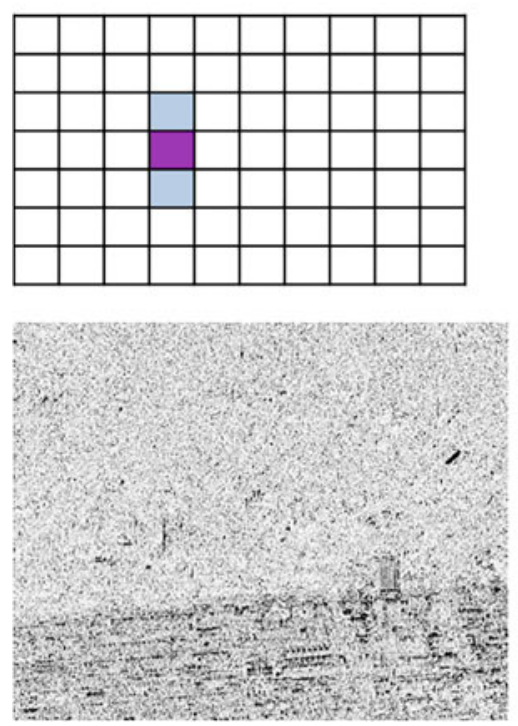
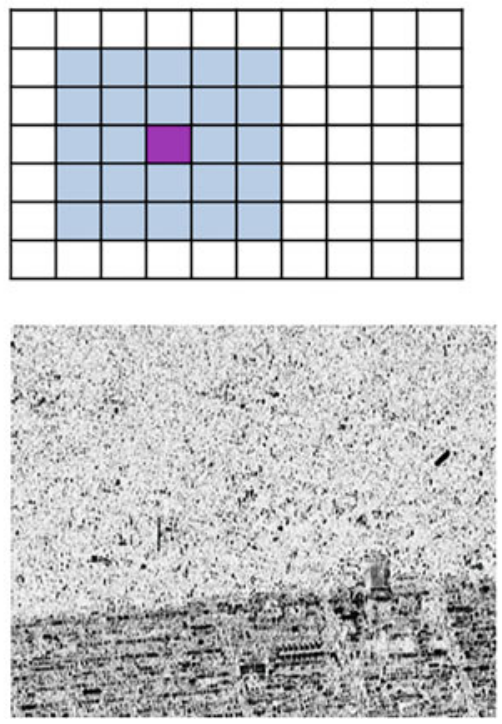
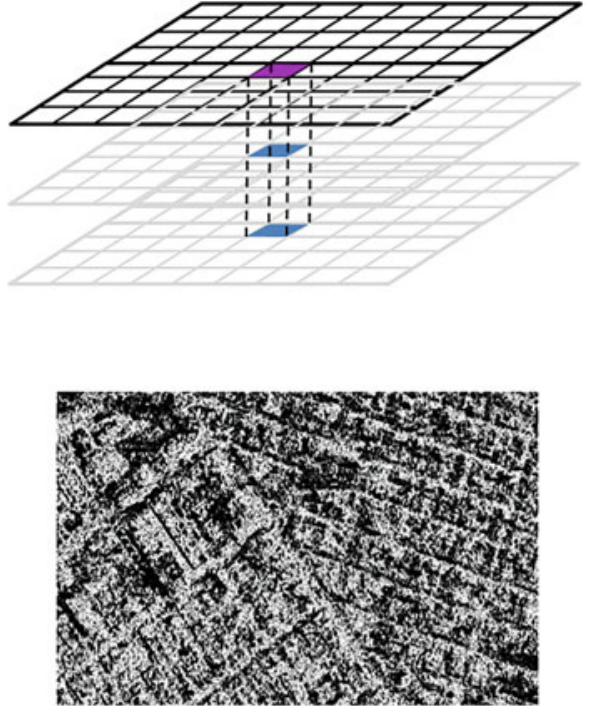

Fig. 5.12 Different entropy maps obtained using different estimation methods over an extract of UAVSAR data set 

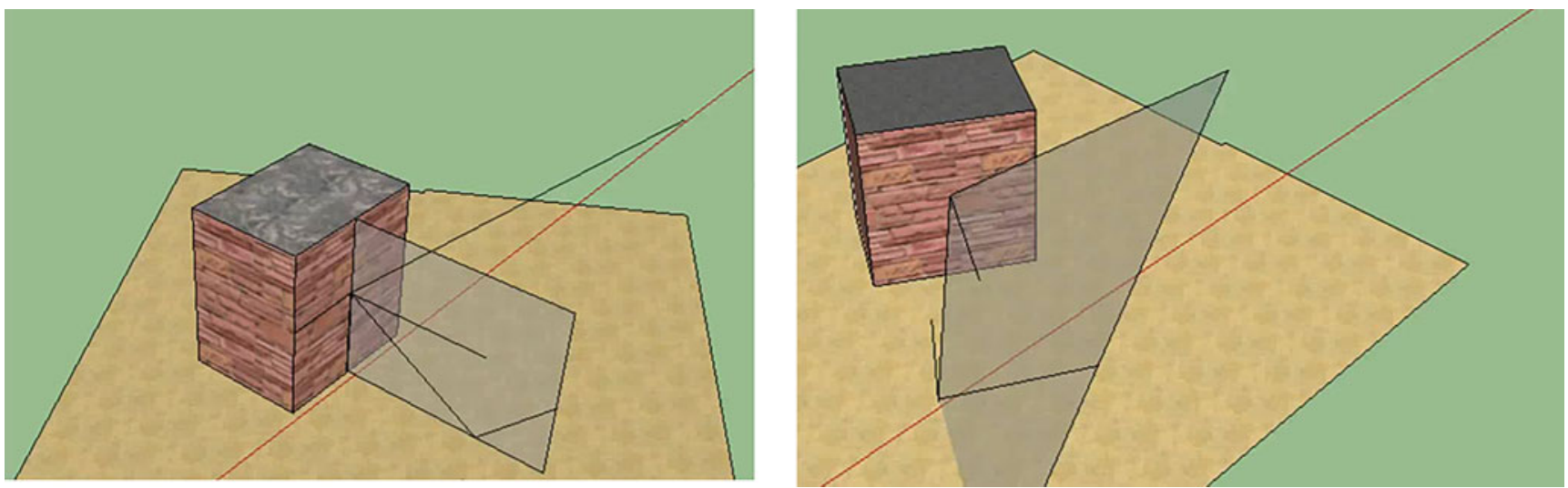

Fig. 5.13 Representation of mechanisms for the classical dihedral effect and the disoriented dihedral effect

\subsection{The Polarization Orientation Angle and Disorientation}

The orientation angle has also been deeply investigated in polarimetry in built-up areas (Lee and Ainsworth 2011). The polarization orientation angle (POA) is defined by the angle of rotation about the line of sight. It has been shown that the polarization orientation angle shifts are induced by surfaces with buildings that are not aligned in the along-track direction. Classical disorientation algorithms aim at:

- Estimating the orientation angle of the target under study

- Applying a rotation of the polarimetric basis to align the axis of the target on the horizontal axis and therefore obtain zero cross-polarization returns

However, in the case of a real dihedral mechanism, this operation is not so simple, for many reasons:

- First, we do not deal with metallic canonical effects, but with dielectric ones. The Fresnel coefficients on dielectric materials are not equal in amplitude for the $\mathrm{HH}$ and the VV polarization, and so the corresponding scattering matrix is not strictly equal to the second Pauli matrix (Thirion-Lefevre et al. 2020).

- Second, the rotation is applied in the wave plane. As a consequence, a dihedral effect whose corner is horizontal with a given orientation has not necessarily a polarization orientation angle (POA) equal to its corner orientation.

- Finally, the double bounce mechanism related to a vertical wall with a non-zero orientation angle cannot involve two successive specular scattering mechanisms. Generally, it is assumed that the vertical wall implies a specular one, whereas the ground does not, as represented in Fig. 5.13. Therefore, this real double bounce effect has a very smaller return in the co-polarization channels that the classical ones. Small elements of the facade can now also contribute to the polarimetric return and can have comparable amplitudes. In this case, the double bounce effect is not more dominant for a building.

\subsection{The Use of the Generalized Interferometric Coherence}

At X-band, as the traditional parameter of entropy does not seem satisfactory, we propose to use polarimetry contribution to a repeat-pass interferometric mode. Indeed, the phenomena of temporal decorrelation will be very fast in this frequency band, again sensitive to displacements of the order of a centimeter. A HIS colored representation of the interferogram obtained over the whole image of San Francisco is given in Fig. 5.14.

Thus, the interferometric correlation image exhibits a contrast much better than the intensity image between natural and artificial targets, as shown in Fig. 5.15 on a detail of the image. It is clear on these images that areas of parks and urban vegetation whose intensity is very high present a high decorrelation that allows distinguishing buildings.

The proposed solution is therefore to use optimized repeat-pass coherent polarimetry as an essential criterion for an unsupervised 2-class classification that will eventually be improved by shape criteria extracted from the span image. The optimized coherence will be obtained by one of the optimization procedures described in (Colin et al. 2006; Cloude and Papathanassiou 1998).

\subsubsection{Experimental Results}

Test sites and corresponding radar and validation data sets selected for the generation of showcases on urban classification are summarized in Table 5.3 and further described in the Appendix. 


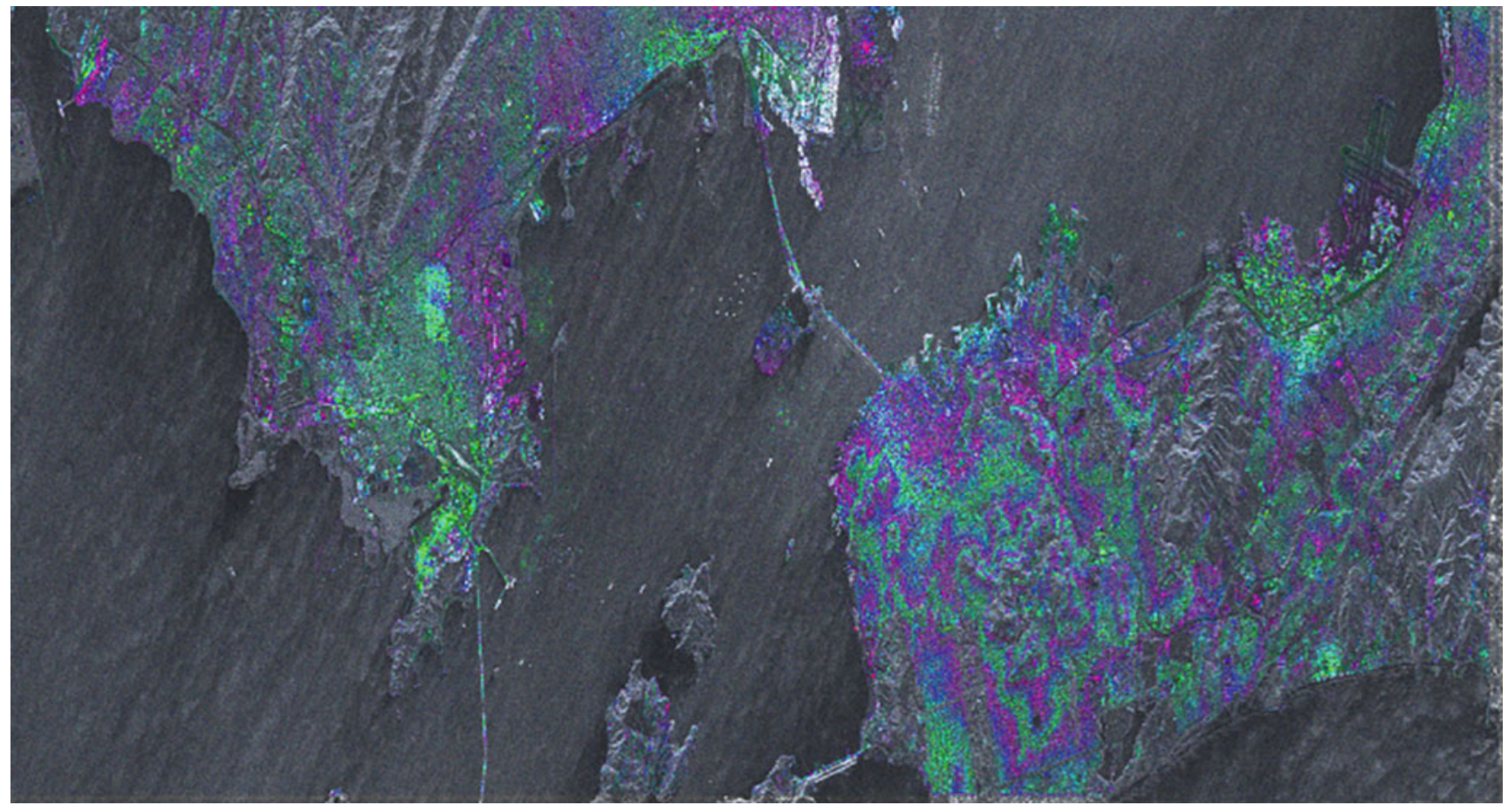

Fig. 5.14 Interferogram obtained over the whole image of San Francisco in HH polarization with a HSI representation. Hue: interferometric angle, Saturation: coherence level, Intensity: span
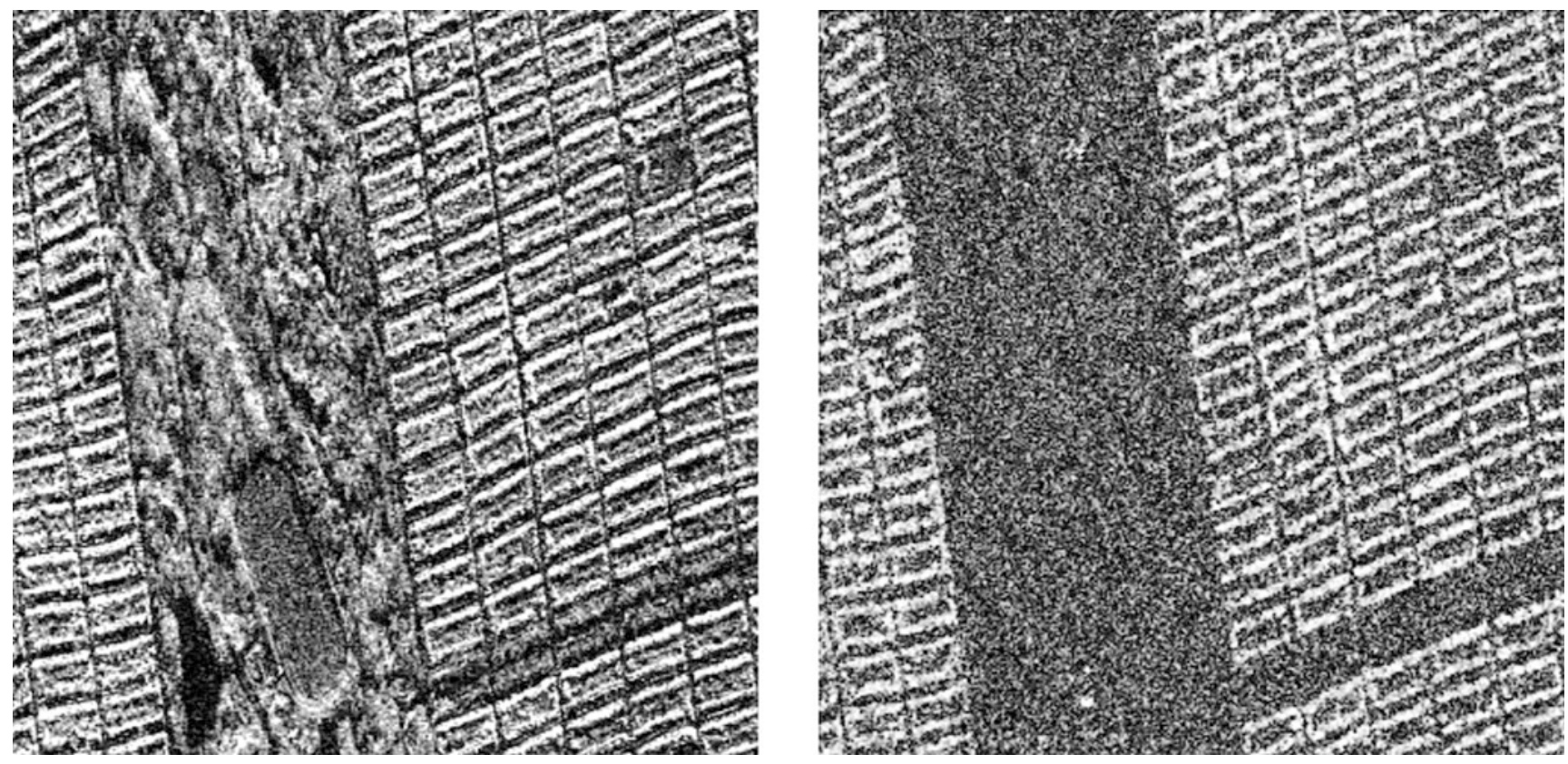

Fig. 5.15 Image of the masks obtained from the ground truth files and applied to the performance evaluation of the different classification methods. Left: intensity on a detail of the image; right: optimum coherence on a detail of the image

Table 5.3 Test sites and corresponding radar and validation data selected for the generation of showcases on urban classification

\begin{tabular}{l|l|l}
\hline Application/product & Test site - Radar data & Reference data \\
\hline Urban classification (optimized coherence) & San Francisco, USA & Shapefile with building footprints \\
& TerraSAR-X HH/HV/VV & \\
\cline { 2 - 2 } & TerraSAR-X HH/VV & \\
\cline { 2 - 3 } & Toulouse, France & \\
\cline { 2 - 3 } & TerraSAR-X HH/VV & \\
\hline
\end{tabular}


To use polarimetric parameters in the framework of builtup areas detection, four main features of the polarimetric analysis in the context of urban areas can be used:

- Polarimetry can distinguish between deterministic (man-made targets) and non-deterministic (natural targets).

- Built-up areas contain a lot of orientation effects that induce a non-zero polarization orientation angle (Moriyama et al. 2004). This polarization orientation angle is defined by the angle of rotation about the line of sight. It has been shown that the polarization orientation angle shifts are induced either by dihedral effects between the ground and a vertical wall that are not aligned in the along-track direction or by tilted roofs. However, the evaluation of this orientation angle is very noisy as soon as this angle becomes high.

- Double bounce effects between vertical walls and the ground give often very strong echoes in the SAR image.

- Lack of azimuthal symmetry implies that the correlation coefficient between cross-polarization and co-polarization is not equal to zero on the contrary to a flat surface or vegetated areas.

To quantify the contribution of polarimetry for building detection, we plotted ROC (Receiver Operating Characteristic) curves that are a plot of the true positive rate against the false-positive rate for the different possible cut points of a diagnostic test. These curves analyze the efficiency of using various input parameters and various distances between the two classes defined by the ground truth (built-up and natural). The closer the curve follows the left-hand border and then the top border of the ROC space, the more accurate is the test. Different distances can be calculated using different parameters: entropy, Yamaguchi, the different polarimetric correlations existing in case of non-symmetry.

The results are presented in Fig. 5.16. Then these curves clearly show that the best discriminating parameter among the tested ones is the Yamaguchi double bounce component. However, we must keep in mind that the Yamaguchi parameter does not only depend on the polarimetric content but also the powers. When we compare this parameter to the polarimetric intensity channels in Fig. 5.17, we see that it is always less efficient than the polarimetric amplitudes. That proves that polarimetric parameters that are independent of the span (entropy, correlation) give not good results here to identify alone built-up areas.

Since at X-band traditional polarimetric parameter fails to identify man-made targets, we propose to use polarimetry contribution to a repeat pass interferometric mode. Indeed, the phenomena of temporal decorrelation will be very high in this frequency band, because it will be sensitive to displacements of the order of a few centimeters. Thus, the interferometric correlation image exhibits a contrast much

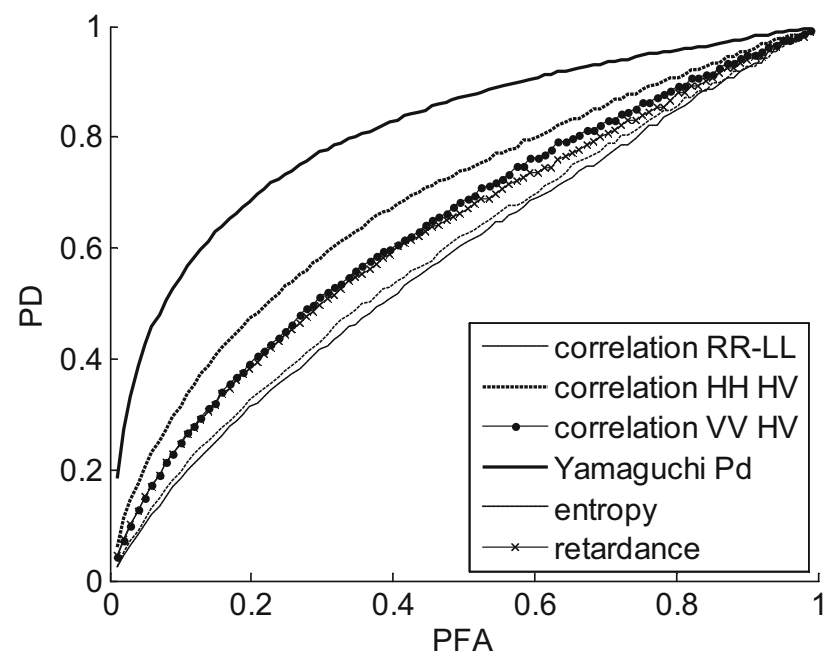

Fig. 5.16 Efficiency (ROC) of different polarimetric parameters

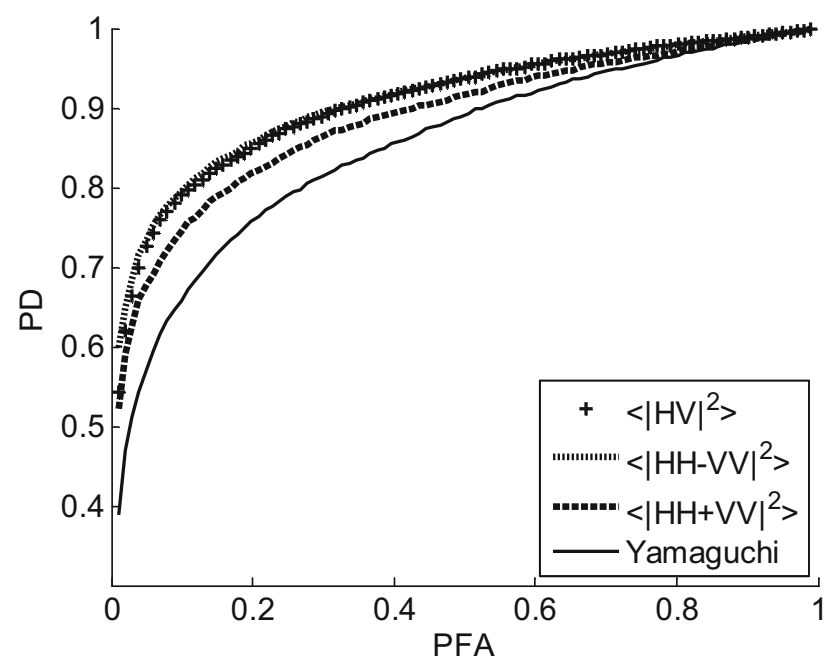

Fig. 5.17 ROC: Yamaguchi and intensity

better than the intensity image between natural and artificial targets.

The ROC curves presented in Fig. 5.18 clearly show the benefit to use interferometric coherence for discriminating buildings, at least at $\mathrm{HH}$ and VV polarization. Then, the benefit of polarimetry can be also considered through the use of a coherence optimization The ROC curves estimated in Fig. 5.19 also show the following points:

- It is clear that the contribution of polarimetry to optimization allows for improving the detection performance.

- Coherence optimized on a single mechanism gives similar results to the coherence optimized on two mechanisms.

- The map of HV interferometric coherence gives poor results. It is actually very noisy, maybe due to the lower signal to noise ratio that exists in this experimental polarimetric channel. 


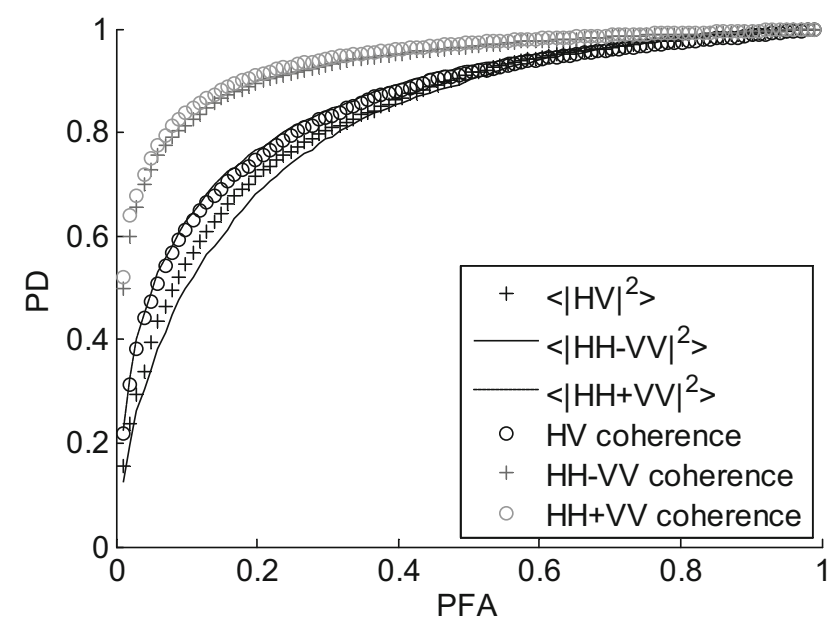

Fig. 5.18 ROC: comparison of intensity and interferometric coherence

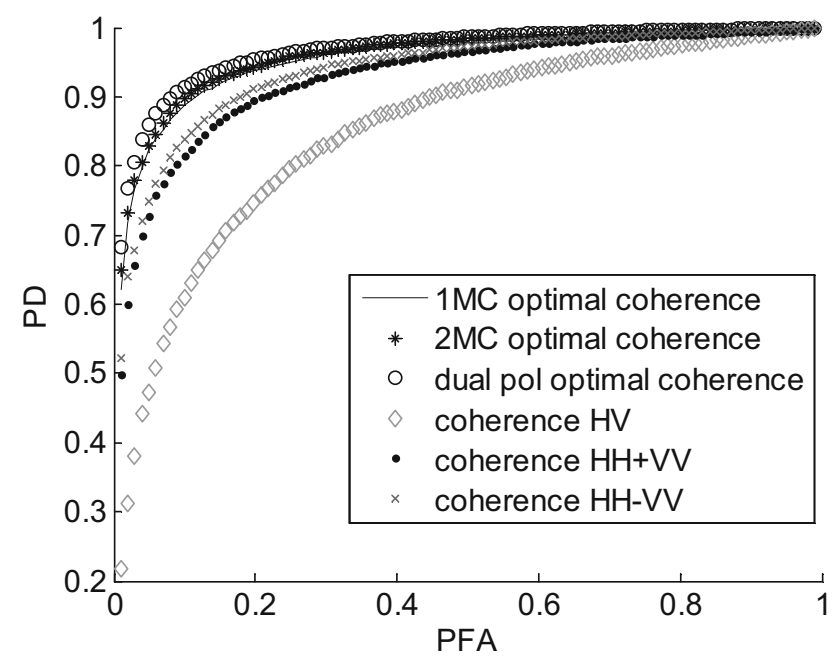

Fig. 5.19 ROC: Benefit of a coherence optimization

- The polarimetric coherence matrix provides also some interesting information for classification, which can outperform some pure interferometric information (e.g., in $\mathrm{HV})$.

- The dual-polarimetric optimization seems to outperform slightly the full polarimetric optimization.

However, the contribution of the HV should be highlighted differently. Indeed, it is clear that the contribution can be shown only in cases where the SNR is sufficient. The preliminary results of classification obtained images of San Francisco and Toulouse are given in Fig. 5.20. They will be further improved by taking into account shape criteria, for example, effective forms as the rectilinear contours of buildings.

\subsubsection{Comparison with Single-/Dual-Pol Data}

The contribution of polarimetric data in comparison with single-pol data has been demonstrated in the previous section. As regards the contribution of $\mathrm{HV}$ versus dual-pol mode $\mathrm{HH} / \mathrm{VV}$, the situation is less clear. The ROC curves comparing the benefits of full polarimetric optimization compared to dual-pol mode presented in Fig. 5.21 show that

- For small false-positive rates $(<0.5)$, the dual-pol optimization seems to outperform slightly the full polarimetric optimization.

- For high false-positive rates higher than 0.5 , the full polarization outperforms the dual-pol mode.

However, the contribution of the HV should be highlighted differently. Indeed, it is clear that the contribution can be shown only in cases where the SNR is sufficient.

The ROC curves estimated over Toulouse in dualpolarimetric mode are presented in Fig. 5.21. They also show that the second optimal coherence gives better contrast than the first one. Indeed, we should remember that Toulouse contains only bare soil and buildings, whereas San Francisco contains also vegetation and ocean. The water surface decorrelates more than soil, and then the contrast in decorrelation remains high even after optimization. Optimization can be sometimes not as efficient as expected, for example, in presence of bare soil whose optimization can improve coherence or when a polarimetric channel has an inefficient level (HV). However, averaging the optimized coherence appears to present the best performance for the detection of built-up areas. The proposed solution is therefore to use optimized repeat-pass coherent polarimetry as an essential criterion for unsupervised 2-class classification. This classification can be eventually improved by shape criteria extracted from the Span image.

\subsubsection{Discussion on the Role of Polarimetry and on the Maturity of the Application and Conclusions}

The detection of man-made targets using polarimetry has a few years now. The contribution of polarimetry to distinguish natural areas from artificial targets is well-known in classification.

This application at X-band using satellite data is far more recent with the launch of TerraSAR-X. In this context, polarimetry seems to become less effective for discriminating built-up areas. Indeed, the roughness of roofs and walls, sensitive to this scale, the different materials, and different elements that compose them, all seem to contribute to the depolarization effects or mixtures of mechanisms. In this context, it becomes difficult to predict and interpret the polarimetric answer of the urban areas. 

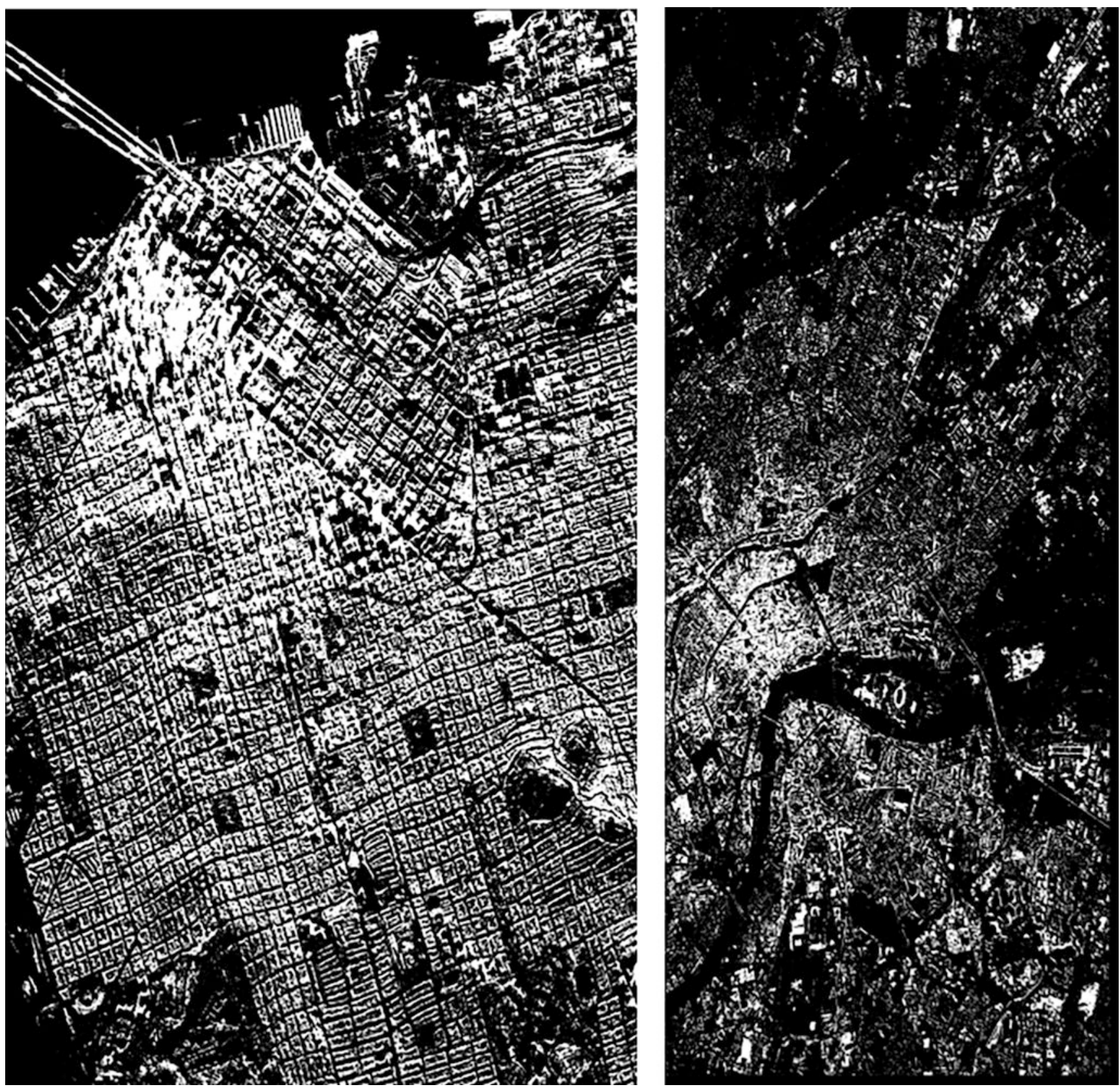

Fig. 5.20 Results of classification of built up areas in white, over a full polarimetric image of San Francisco on the left, and over a dual-polarimetric image over Toulouse on the right

The processing of these data at this wavelength is indeed relatively recent. There is still a lot of effort to do, especially from the point of view of modeling tools, to be able to better understand the polarimetric response at these wavelengths. In particular, there is a major effort to carry out about the influence of the wavelength related to the size of the resolution cell.

Still at X-band, it appears that the essential contribution of polarimetry is the optimization of the interferometric coherence and its use to discriminate targets based on their speed of temporal decorrelation.

\subsection{3-D Rendering Over Urban Areas}

\subsubsection{3-D Rendering Using Coherence Optimization}

\subsubsection{Introduction, Motivation, and Literature Review}

3-D rendering is a logical extension to the classification of buildings proposed in the previous section, to enrich the data necessary to monitor the growth of the urban extension. But it 


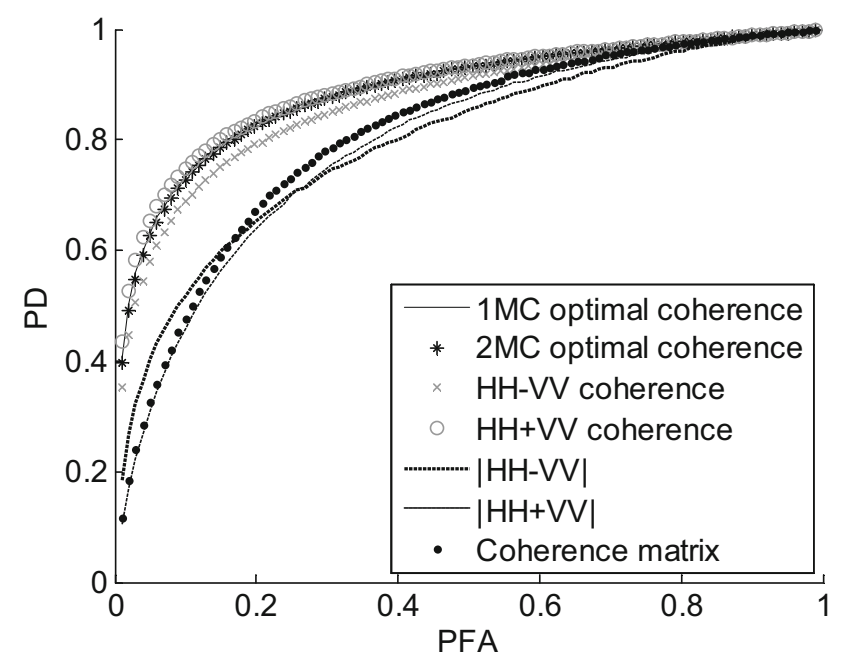

Fig. 5.21 ROC curves over dual-polarimetric images of Toulouse

can also be considered as part of the diagnostic of urban areas after natural disasters such as tsunamis or earthquakes. Natural disaster monitoring and evaluation of their effects is a complex problem in urban areas because a lot of parameters can be investigated. Areas of significant changes can be detected on the basis of the exploitation of high-resolution satellite data, areas where a 3-D model can be established on a large scale by interferometry. In this context, the contribution of radar is its immediate use regardless of weather conditions or smoking out due to fires.

Polarimetry combined with interferometry can improve the product of the latter either by separation of scattering phase center, i.e., by being able to distinguish different heights in a single resolution cell or by improving the interferometric correlation map, i.e., by enhancing its value and reducing the noise level of the interferometric phase. In the latter case, to assess the benefit of polarimetry, we simply need to compare the use of the interferometric phase before and after optimization.

\subsection{Scattering Phase Center Separation}

For the separation of phase centers, the goal is to get the ground height jointly with the elevation of the roof. In this framework, the benefits of polarimetry are often compared with the benefits of pure technical image processing.

The results of techniques for phase separation will obviously depend on three factors:

- The resolution of the images. This is even the essential criterion. For instance, if the resolution is low, a vertical wall will be found synthesized in a single resolution cell. This wall will include several scattering centers, and potentially different polarimetric returns with different heights will therefore be mixed.
- The frequency. At low frequencies, the wave will not necessarily be sensitive to details. Thus, even a large resolution cell will see a limited number of mechanisms.

- The height of ambiguity for the interferometric process. The distribution of heights observed depends on this parameter: if the ambiguity height is small, then the angular diversity of the generalized coherence will be very important.

Among the techniques of phase separation, two main techniques exist:

- The ESPRIT method (Guillaso et al. 2005)

- The coherence optimization using a single mechanism that has been shown in (Colin et al. 2006) to be able to separate different phase scattering centers, under some assumptions (absence of temporal or volume decorrelation)

\subsection{Coherence Optimization}

There are different possible definitions of a generalized coherence for polarimetry and therefore other possible methods to perform an optimization. For example, we can distinguish between:

- The one mechanism optimization, proposed in (Colin et al. 2006; Qong et al. 2005).

- The initial two mechanism optimization, where the generalized coherence measures the resemblance between the response of an electromagnetic mechanism at the first antenna and another electromagnetic mechanism at the second antenna. The optimization problem has been introduced and solved to obtain the optimum scattering mechanism (Cloude and Papathanassiou 1998).

- The so-called polarization subspace method (PSM) is based on finding local maxima of the co-polar or cross-polar coherence functions. Physically, the mechanisms must be represented as an elliptic polarization transformation. The approach of the polarization state conformation (PSC) algorithm is very similar: it is based on the knowledge of the polarimetric basis transformation along with the polarization signatures of both interferometric images (Pascual et al. 2002).

All these methods can be generalized to the multibaseline case (Neumann et al. 2008).

\subsection{Limitations}

Limitations of these techniques for three-dimensional reconstruction are:

- At present, the technical phase scattering separation can distinguish up to three different heights. Practically, with 
current resolutions images of buildings, they are effective for a mixture of two main contributions. To be able to separate more scattering phase centers and to obtain volumetric images, it is possible to consider multibaseline approaches or tomography techniques.

- The effects of layover and interactions between buildings by the existence of urban canyons are rarely treated together on large images in a systematic way.

- The effects of statistical averaging are very influential in this type of image. A satisfactory 3-D rendering requires preliminary stages of image segmentation.

- We have seen that at X-band, the temporal decorrelation of the images is very fast.
Particularly in the resolution cells of the San Francisco images containing layover phenomenon, it is clear that the phase of the roof is mixed with the ground phase. However the ground seems to induce a high decorrelation in the mixture, and therefore its elevation cannot be estimated satisfactorily.

Thus, concerning the TerraSAR-X images of San Francisco, if the estimate of the height of the roof is possible, at present we have no satisfactory estimate of the height of the associated ground. The following image in Fig. 5.22 shows a HSI representation of the interferogram obtained over San Francisco where the interferometric phase is in hue and the optimized coherence level is in saturation. Even
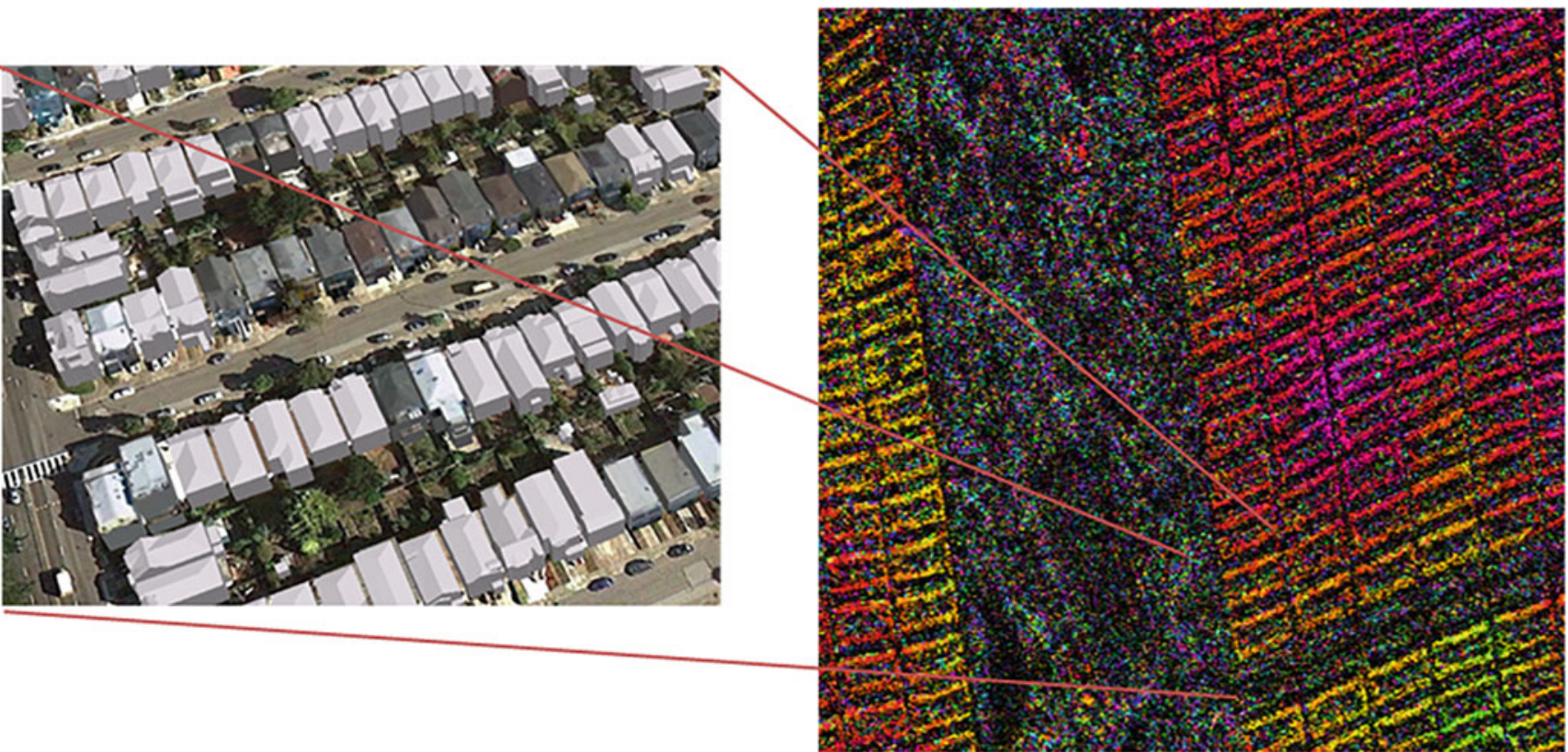

\section{Hue: interferometric phase}

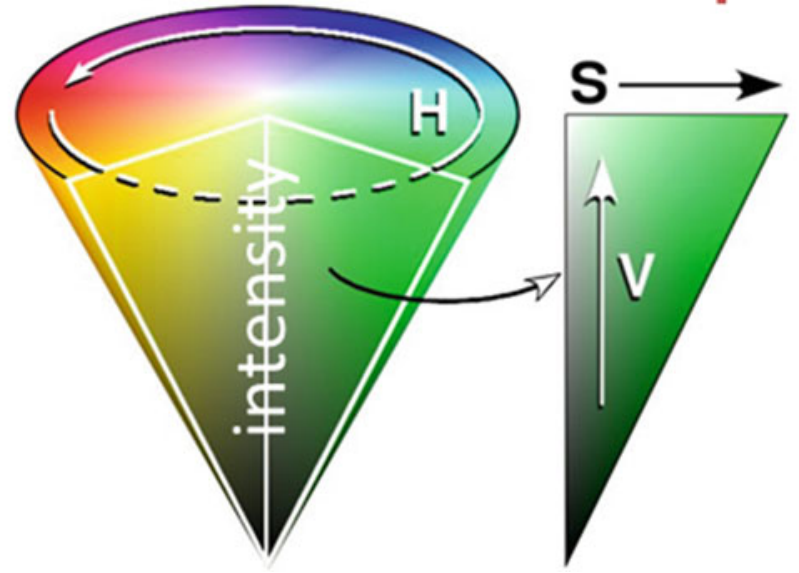

Saturation: coherence level

Fig. 5.22 Details of an interferogram obtained in the optimum polarization 
after an optimization, no pertinent elevation can be found on the ground. We conclude that at X-band, it is necessary to have single pass data or smaller temporal baselines to be able to provide a 3-D rendering over the whole image.

\subsubsection{Methodology}

To perform a 3-D rendering over the entire image using a PolInSAR data set in a single pass mode, we use the following method:

- First, a prior segmentation method is proposed. It is a method of using hierarchical segmentation criteria form (essentially linear edge detection and size criteria).

- Then, an optimization method is applied to the whole image, using the calculation of coherence matrices of all pixels belonging to the same segment. We use the coherence optimization described in (Colin et al. 2006)

- Locally, for building elevation estimation, we use a scattering phase center separation method, based on the assumption that the resolution cell contains only two main scatterers at two different elevations. The algorithm is described in (Colin-Koeniguer and Trouve 2014).

Concerning this last point, we can use here the methodology that has achieved the best results. Roofs are expected to correspond either to the case of one bright point or to the case of two bright points when layover is assumed and that the resolution cell contains scatterers of the roofs mixed to scatterers from the ground. We have shown that the coherence set corresponding to the top of the roof mixed with the ground is a narrow ellipse. If polarimetric decorrelation between the two interferometric signals is very low, then the major axis of this ellipse will intersect the unitary circle into the interferometric phase of the roof and the interferometric phase of the ground. Most of the time, the ground alone is not necessarily visible. As the extension of the major axis of the ellipse is not always sufficient to ensure a robust regression, we choose to estimate separately the phase of the ground through the optimization applied to pixels belonging to the ground. This optimization enables us to find the point exp $\left(j \varphi_{0}\right)$ where $\varphi_{0}$ corresponds to the interferometric phase of the ground. Then we find the intersection of the segment joining $\exp \left(j \varphi_{0}\right)$ and the optimized coherence of the roof with the unitary circle. This intersection corresponds to exp $\left(j \varphi_{1}\right)$. The total height is deduced from $\varphi_{1}-\varphi_{0}$.

\subsubsection{Experimental Results}

The 3-D rendering applications are conditioned mainly by two parameters:

- The single-pass or multipass mode of acquisitions

- The resolution

In terms of resolution, it is unlikely to achieve satisfactory results with images of resolutions higher than 3 meters. Thus we will restrict satellite data in case of the dual-pol mode of TerraSAR-X. In this context, we can evaluate the contribution of this partial polarization mode. This data will also allow us to quantify the impact of temporal decorrelation on this type of applications. We will also use data from RAMSES airborne single-pass mode and will be able to quantify the impact of multipass or single-pass mode. In both cases, we select the site of Toulouse city in southwestern France. The Toulouse metropolitan area is the fifthlargest in France, one of the bases of the European aerospace industry.

Test sites and corresponding radar and validation data sets selected for the generation of showcases on 3-D urban rendering are summarized in Table 5.4 and further described in the Appendix.

To validate our estimations over Toulouse with the singlepass mode, we have used a file describing building footprints and their elevation as ground truth for this application. It is a file shapefile, organized as a structure containing a list of polygonal elements. These polygons define the footprint of each building on the ground, and for each element, the minimum and maximum elevation data are given. We select the buildings of our ground truth over Toulouse that are in our PolInSAR image and that are high $(>6 \mathrm{~m})$ and big (> $10 \mathrm{~m}^{2}$ ) enough. That gives us 140 buildings whose elevation is given with a precision of $1 \mathrm{~m}$, represented in Fig. 5.23. The ambiguity height lies between $90 \mathrm{~m}$ for minimum ranges to $120 \mathrm{~m}$ for far range. In order to automatically select the pixels belonging to the building or on the ground nearby, we have registered the footprints of each building on our SAR image. An excerpt of this coregistration is given in Fig. 5.24.

The different heights so found are evaluated in terms of the mean error in the measurement compared with heights given by ground truth, and the root mean square error. The ambiguity height on this image is about 100 meters. The three methods are also compared to the estimation computed in the

Table 5.4 Test sites and corresponding radar and validation data selected for the generation of showcases on 3-D urban rendering

\begin{tabular}{l|l|l}
\hline Application/product & Test site - Radar data & Reference data \\
\hline \multirow{3}{*}{ 3-D urban rendering (optimized coherence) } & Toulouse & Shapefile with elevations of all buildings \\
\cline { 2 - 2 } & RAMSES (airborne), single-pass, HH/HV/VV; & \\
\cline { 2 - 2 } & TerraSAR-X HH/VV, repeat-pass mode & \\
\hline
\end{tabular}




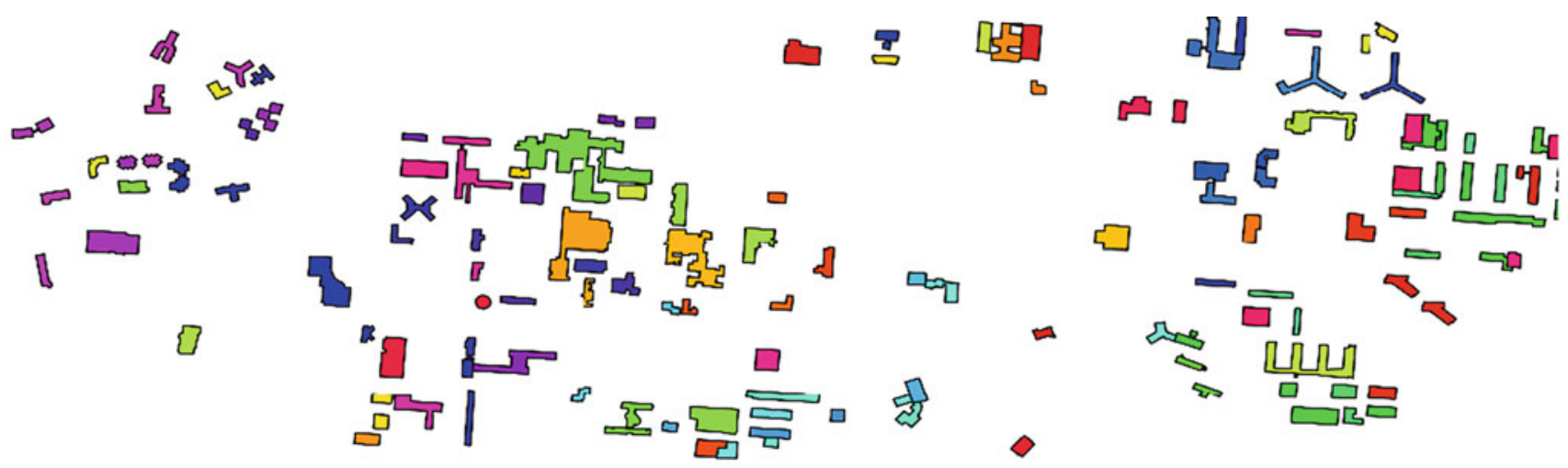

Fig. 5.23 The set of buildings for elevation estimation evaluation

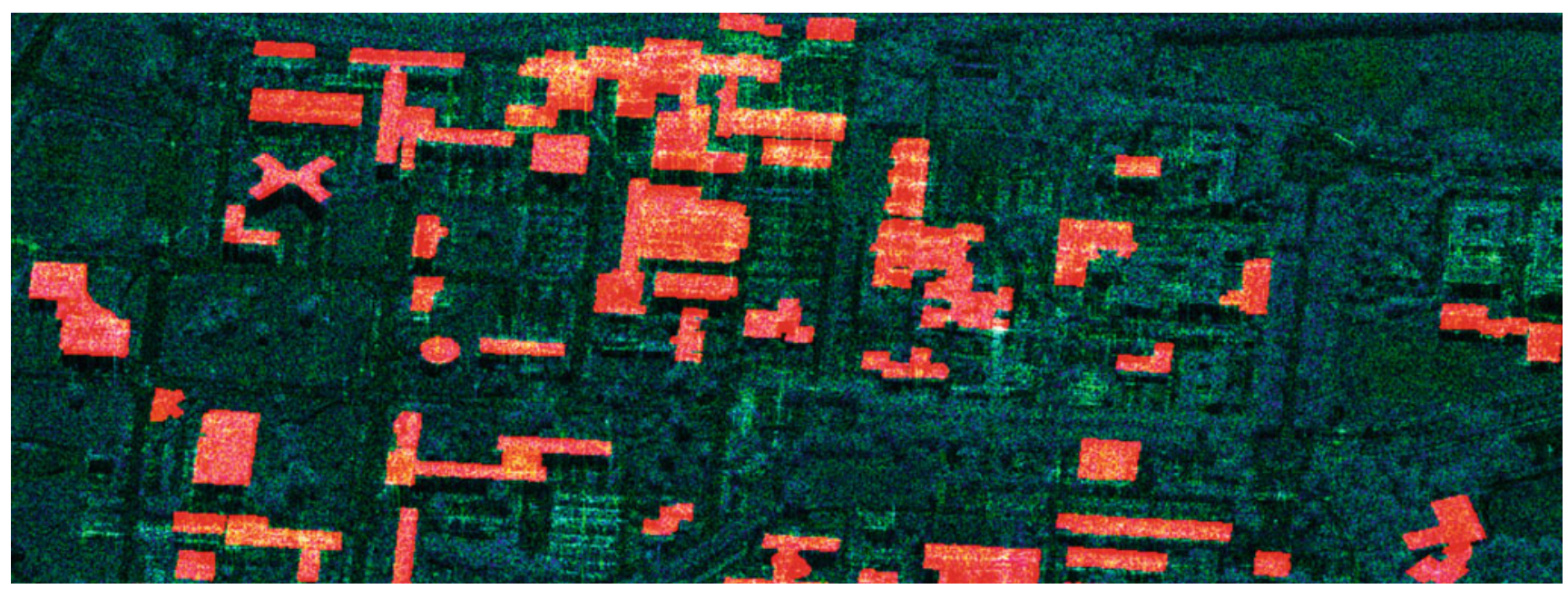

Fig. 5.24 Coregistration of the ground truth and the PolInSAR image

Table 5.5 Results of building height estimation

\begin{tabular}{l|l|l}
\hline Estimation: & Ground truth height - estimated height $(\mathrm{m})$ & Root mean square error $(\mathrm{m})$ \\
\hline $\mathrm{HH}+\mathrm{VV}$ & 2.57 & 3.89 \\
\hline $\mathrm{HH}-\mathrm{VV}$ & 2.76 & 4.60 \\
\hline HV & 2.23 & 3.79 \\
\hline \# 3 - Linear regression, optimal coherence & 1.20 & 2.87 \\
\hline
\end{tabular}

single-polarimetric channels of the Pauli basis. The results are presented in Table 5.5. The best result is obtained for Method 3 . Method 2 is the only one that overestimates the heights. Note that the best single-polarimetric channel for the estimation of interferometric heights is the HV. Results obtained for a 3-D rendering over the whole image are shown in Fig. 5.25.

\subsubsection{Comparison with Single/ Dual-Polarization Data}

Results indicate that estimation using a single polarization is better for $\mathrm{HV}$ polarization whose level is high at this frequency over a wide variety of roof surfaces. When using the partial coherence matrices obtained from $\mathrm{HH}$ and VV polarization only, we still can follow the same algorithm and obtain the results reported in Table 5.6. Results obtained show that the mean error is equal for dual-pol and full-pol case; however, the root mean square error is higher in the dual-pol case.

\subsubsection{Discussion on the Role of Polarization and on the Maturity of the Application and Conclusions}

Within the 3-D reconstructions field, to obtain a visually readable reconstruction thanks to SAR data, it is necessary to perform segmentation before the height reconstruction. In this context, polarimetry can be used at two levels:

- In the process of segmentation

- In the improvement of the height estimation 


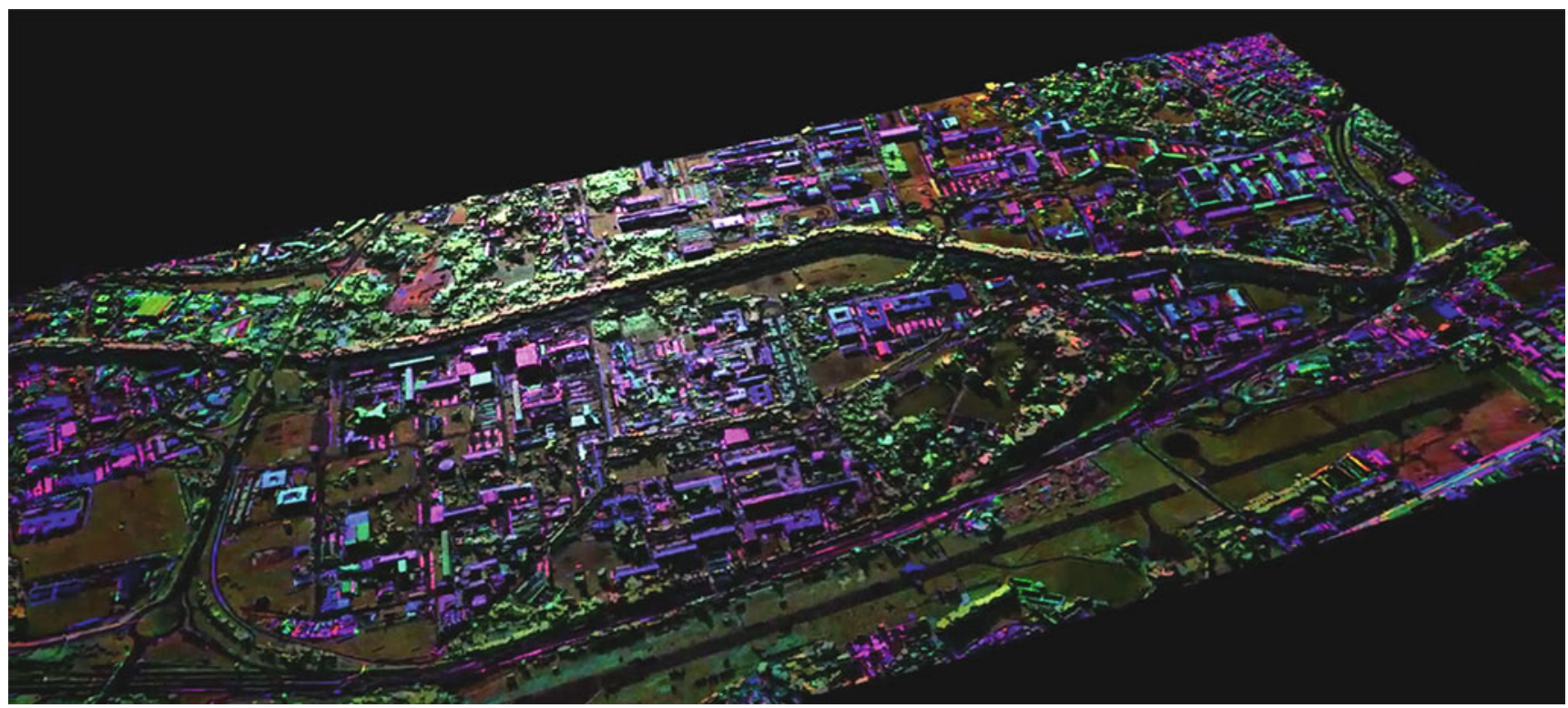

Fig. 5.25 Three-dimensional reconstruction performed over X-band airborne data over Toulouse. The colors result from the Pauli decomposition

Table 5.6 Results of building height estimations: comparison full/dual/single pol

\begin{tabular}{l|l|l}
\hline Estimation: & Ground truth height - estimated height $(\mathrm{m})$ & Root mean square error $(\mathrm{m})$ \\
\hline HH+VV & 2.57 & 3.89 \\
\hline HH-VV & 2.76 & 4.60 \\
\hline HV & 2.23 & 3.79 \\
\hline Dual pol & 1.20 & 3.76 \\
\hline Full pol & 1.20 & 2.87 \\
\hline
\end{tabular}

The application described here is still at an early stage in terms of performance evaluation. Note that unlike the applications of classification, most of the work in this area has concerned X-band. At this frequency, the crosspolarization returns seem to be particularly high. Indeed, all the polarization channels play an important role, because at this scale, numerous oriented objects will create HV returns, and depolarization effects will be high everywhere. As at $\mathrm{X}$-band, the estimation of elevation requires a single-pass interferometric mode. Despite the early stage of development, this application seems to be promising as it will benefit from the improving resolutions of the new generation of satellites.

\subsubsection{Building Height Estimation Using Polarimetric SAR Tomography with a Minimal Set of Images}

\subsubsection{Introduction, Motivation, and Literature Review}

SAR Polarimetry (PolSAR) provides valuable information about the type of soil and urban object geometry, especially over buildings. SAR Interferometry (InSAR) may be used to determine either digital elevation models and surface deformation or the radial velocity of objects (e.g., cars). However, SAR information over dense urban environments is particularly complex due to: geometric distortions caused by the layover and shadowing phenomena, described in Fig. 5.26, complex scattering patterns within the same resolution cell (e.g., single/double-bounce scattering, volume diffusion), random aspect due to speckle effects, etc.

SAR tomography is the extension of conventional two-dimensional SAR imaging into three dimensions. 3-D imaging of a scene is achieved by the formation of an additional synthetic aperture in elevation and the coherent combination of images acquired from several parallel flight tracks using tomographic imaging. This technique directly retrieves the distribution of the backscattered power in the vertical direction and may be applied to estimate forest structure, building height, or layover areas induced by strong terrain slopes or discontinuities in the imaged scene.

3-D SAR focusing using tomographic processing of multibaseline interferometric data sets may be considered as a spectral estimation problem. A wide variety of spectral analysis techniques can be used to perform tomography, ranging from classical Fourier-based methods to highresolution (HR) approaches. A recent study by (Sauer et al. 2011) proposed to apply polarimetric versions of spectral 
Fig. 5.26 Layover and shadow phenomena in urban areas

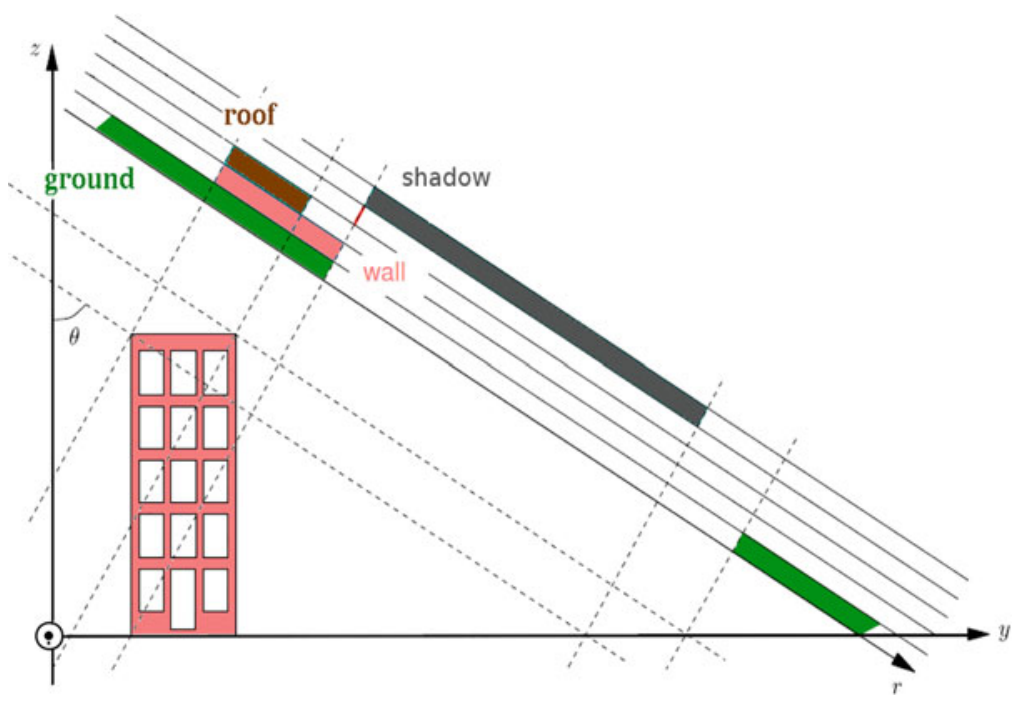

estimation methods to the airborne dual-baseline PolInSAR data sets at L-band. Results showed that using polarimetric data could improve building height estimation, both in terms of discrimination of mixed scattering responses (layover, vegetation, etc.) and determination of physical characteristics of observed media. Despite undeniable performance improvements, such an approach may have some limitations, due to the lack of statistical adaptivity of the commonly used spectral estimation methods. Firstly, as shown by (FerroFamil and Pottier 2007), scatterers in urban areas may have very different statistical properties that are not optimally handled by the methods proposed by (Sauer et al. 2011) and may involve estimation errors and instability. Over urban areas, backscattered signals have diverse statistical properties, e.g., coherent scatterers (e.g., point-like or double bounce scatterers) or distributed scatterers with speckle affected responses (e.g., surface or vegetation), respectively. Therefore the Conditional and Unconditional model assumptions (CM and UM) (Stoica and Nehorai 1990) may be used to estimate optimally both types of source signals.

Maximum Likelihood (ML) estimation performed under these hypotheses lead to the deterministic (Determ-ML) and stochastic (Stocha-ML) solutions, the former being statistically less efficient than the later. However, Stocha-ML achieves an optimal estimation performance at the cost of exceedingly complicated computation. Moreover, the complex scattering response from a dense urban environment leads to a mixture of various scatterers with different statistical properties that can be handled using a hybrid signal model introduced in (Sauer et al. 2011). A source signal under the hybrid assumption presents statistical properties originating partially from the UM and CM models. The performance of the MUSIC estimator degrades significantly in case of correlated signals or closely spaced signals. Moreover, processing tomographic data acquired from irregularly distributed baselines can cause ambiguous responses and sidelobe effects that may lead to erroneous interpretations and estimations.

\subsubsection{Methodology}

In order to overcome these limitations, weighted subspace techniques are of great interest, since they apply to arbitrary array structures and have a prominent performance even for highly correlated signals that are often encountered in urban areas. With an appropriate choice of weighting matrices, subspace fitting estimators possess an estimation accuracy similar to the one of conventional ML techniques (Sauer et al. 2011), at a modest computational cost. Depending on the nature of the considered subspace, different estimators may be obtained, SSF (on signal subspace) or NSF (on noise subspace), respectively, (Huang et al. 2012; Viberg and Ottersten 1991) extended the NSF estimator from the dualpolarization case (Swindlehurst and Viberg 1993) to the Fully Polarimetric (FP) case and also provided an analytic solution that maintains its optimization complexity to the one of the single-polarization (SP) case (Huang et al. 2012). Using a critical and minimal tomographic configuration consisting of only 3 PolSAR data sets, this FP-NSF estimator is applied to estimate building heights and scattering mechanisms over dense urban areas.

\subsubsection{Experimental Results}

The application data set was acquired by the DLR's experimental SAR (E-SAR) system over the city of Dresden (see Table 5.7) in a dual-baseline fully polarimetric interferometric configuration with a small baseline equal to $10 \mathrm{~m}$ and a large one of $40 \mathrm{~m}$, which form a small-size irregular array. The acquired SAR images are of intermediate resolution $(3 \mathrm{~m}$ in azimuth and $2.2 \mathrm{~m}$ in range), leading to a sum of diverse polarimetric and statistical contributions within each 
Table 5.7 Test sites and corresponding radar and validation data selected for the generation of showcases on building height estimation with PolTomoSAR

\begin{tabular}{l|l|l}
\hline Application/product & Test site - Radar data & Reference data \\
\hline Building height estimation with PolTomoSAR & Dresden, Germany (2000) & Lidar DTM and DSM \\
\cline { 2 - 2 } & Dual-baseline E-SAR data & \\
\end{tabular}

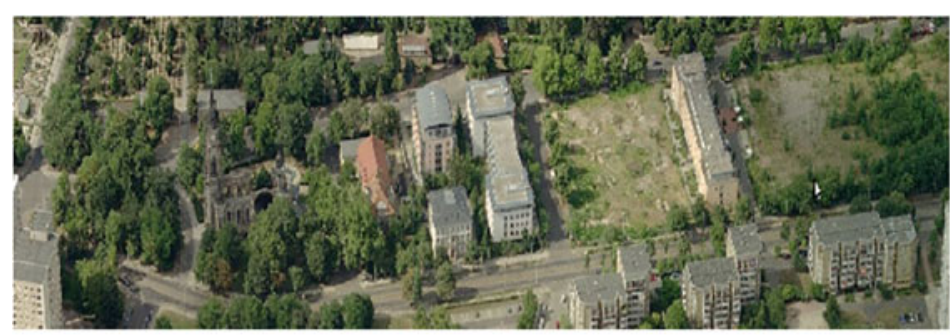

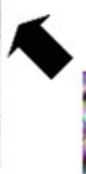
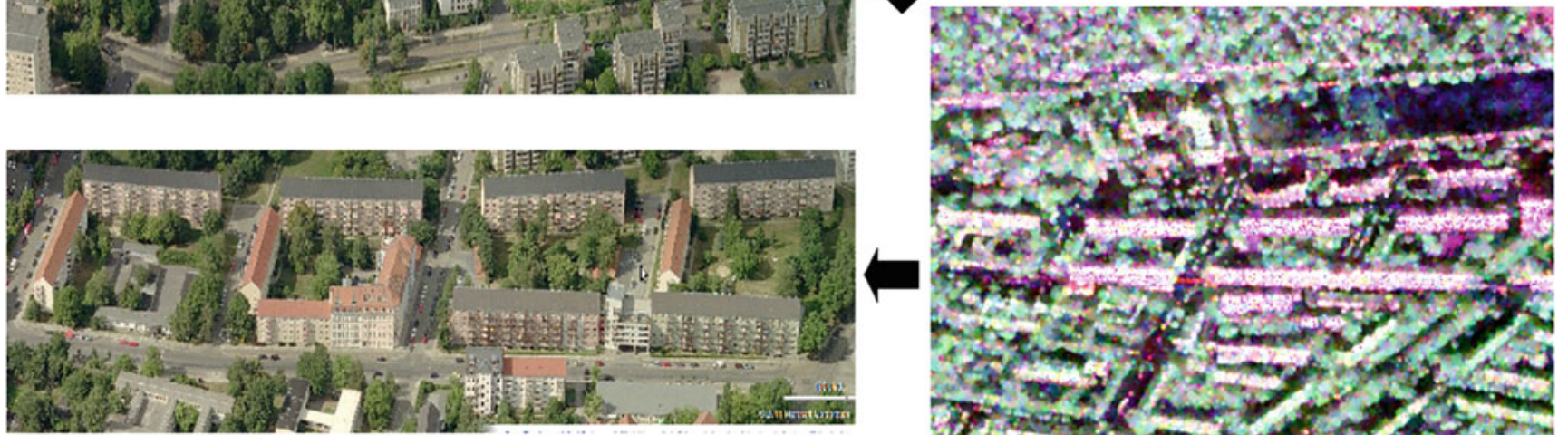

\section{.}
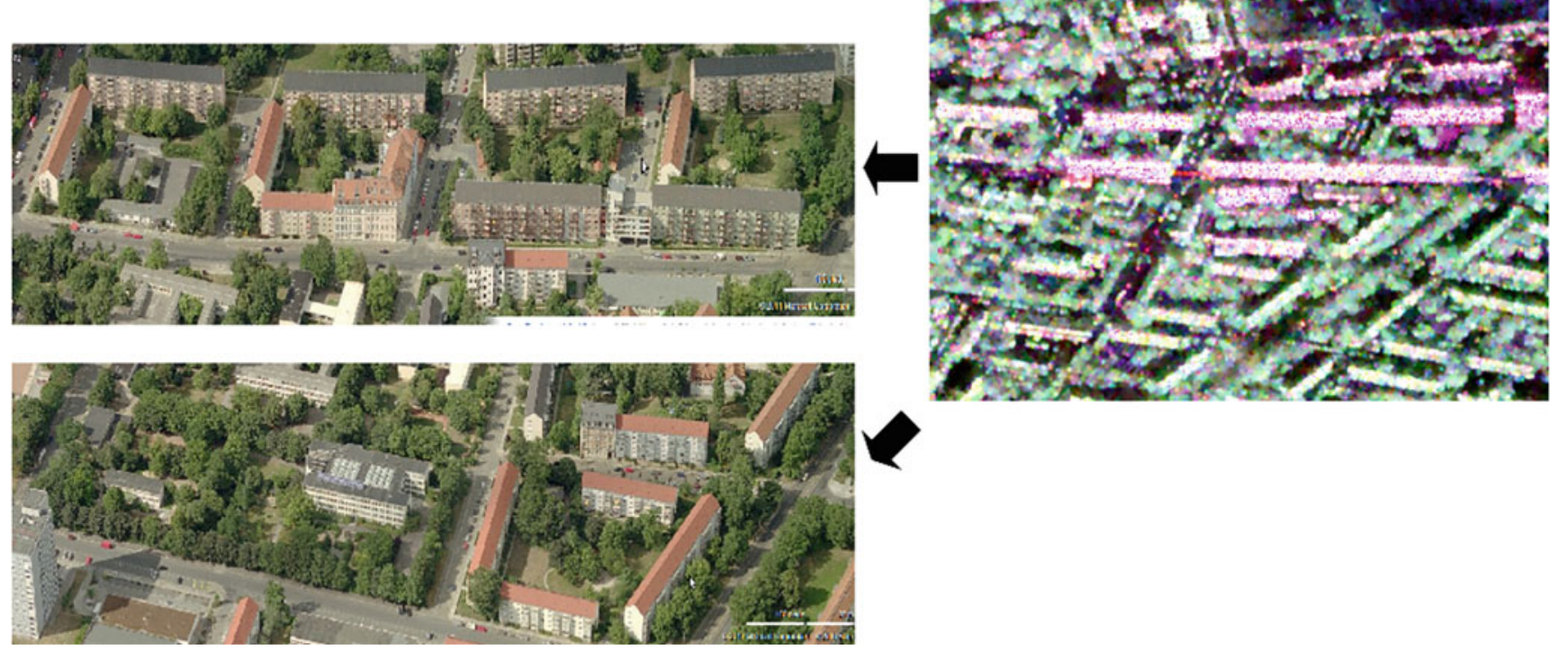

Fig. 5.27 Optical and SAR images of the city of Dresden. Optical images: Copyright Bing Maps

resolution cell. The scene consists of buildings, trees, parks, grassland, and some bare surfaces like sports fields, as depicted in Fig. 5.27.

Two buildings facing the radar flight track are studied over a set of range bins corresponding to the yellow bar in Fig. 5.28. Tomograms over this test line are computed by using the dual-baseline Fully Polarimetric (FP) data sets and the FP NSF method and then projected in ground range in Fig. 5.29. Due to the very low dimension of the observation space, conventional model order selection techniques may fail to accurately determine the number of scatterers within one resolution cell. For this reason, the number of scatterers is fixed to 2 over the selected range bins. The resulting tomograms depict the building shape and scattering patterns using the reflectivity in Fig. 5.29 (left) and $\alpha$ values in Fig. 5.29 (right). Compared with the lidar profile (black line), the building height and its shape are quite well estimated based on this dual-baseline intermediate-resolution data set. At the wall-ground interaction, strong reflectivities and high $\alpha$ values are due to the powerful double-bounce reflection. Over the roofs and surfaces, the $\alpha$ value decreases indicating surface scattering.

The 3-D reconstruction over an urban zone shown in Fig. 5.30 has been run using the FP-NSF tomographic estimator with model order equal to 2 , and the corresponding results are depicted in Fig. 5.31. From the $\alpha$ values in Fig. 5.31 (right), scattering mechanisms can be distinguished in the vertical direction (unlike conventional 2-D polarimetric analysis) that allows to discriminate for instance double bounce scattering at the wall-ground interaction as well as over some of roofs with complex structures. Over the whole test zone, the surface elevation is estimated by the FP-NSF estimator considering two sources, which matches very well with lidar elevation data, as can be observed in Fig. 5.32. A 3-D reconstruction of a group of buildings is validated against lidar in Fig. 5.33. 

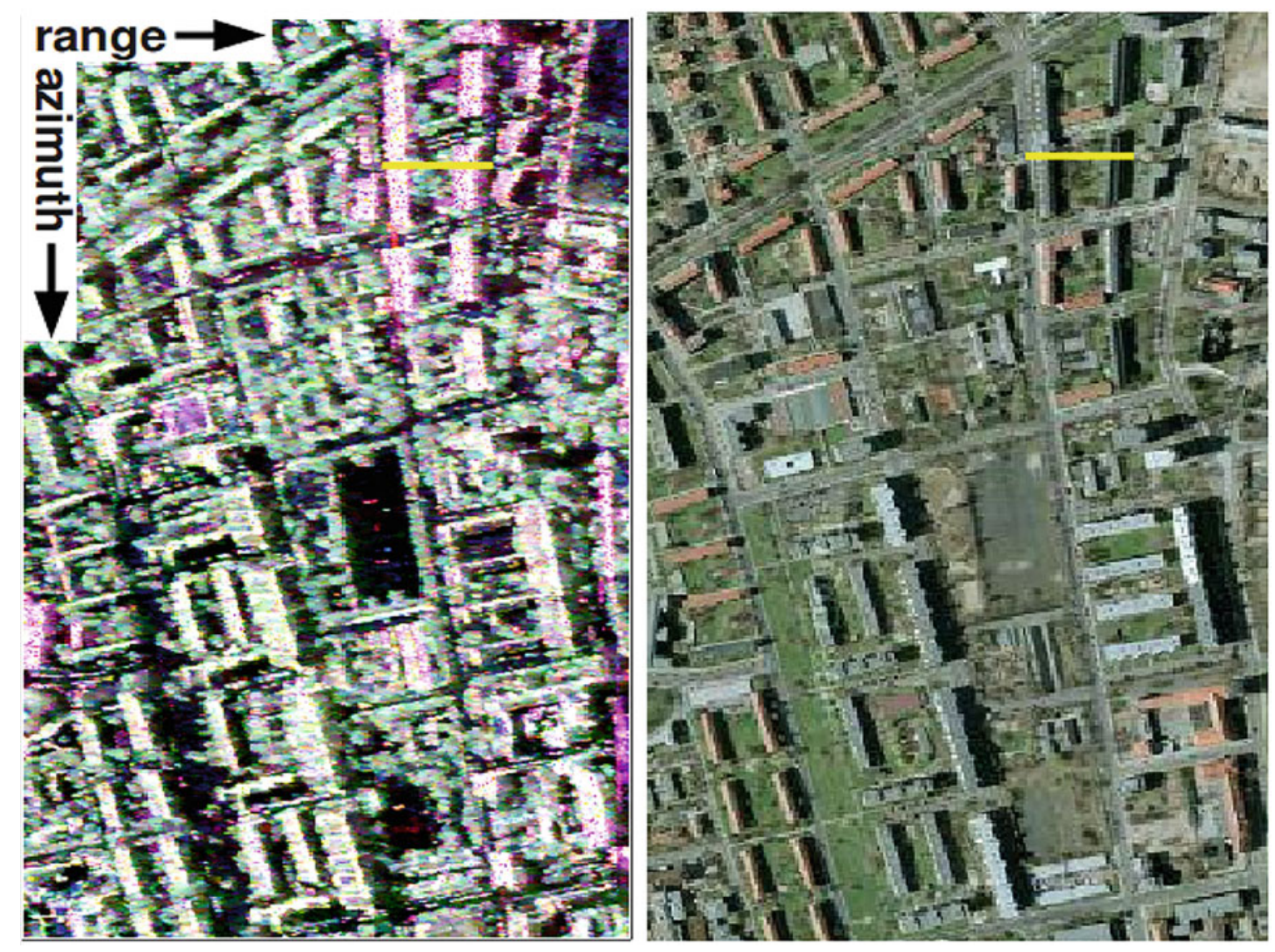

Fig. 5.28 Test area containing buildings facing the acquisition flight path: optical image (left) and Pauli-coded SAR image (right)
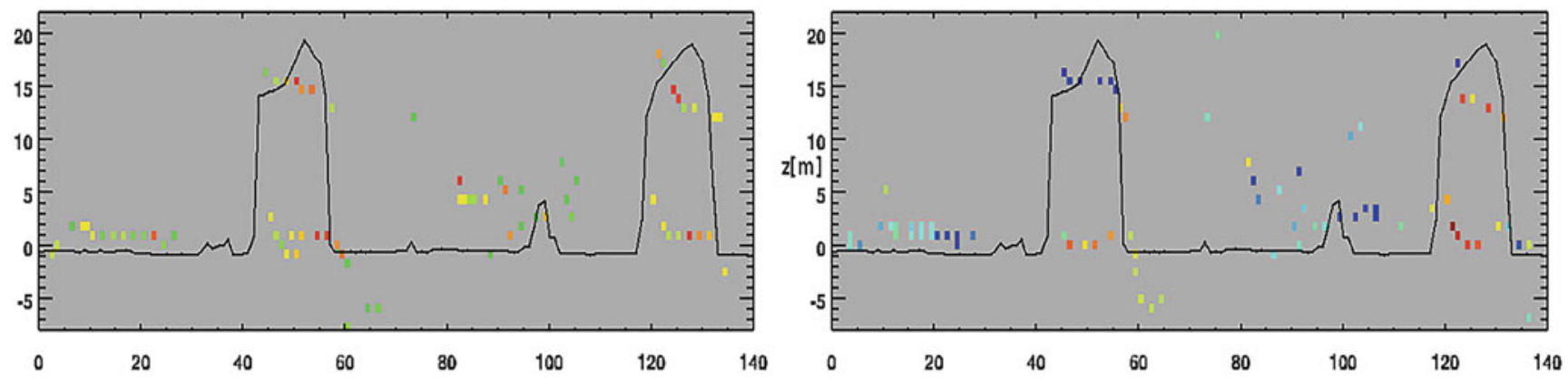

Fig. 5.29 Tomograms estimated by FP-NSF method: reflectivity tomogram (left) with scale: $25-110 \mathrm{~dB}$ and $\alpha$ tomogram with scale: $0-90^{\circ}$ (right)

\subsubsection{Comparison with Single/ Dual-Polarization Data}

The VV reflectivity tomogram in Fig. 5.34 (left) shows an incomplete building shape especially on the top of the buildings, leading to an inaccurate estimation of the building height due to missed patterns. The HH tomogram is affected by spurious sidelobes which degrade building height estimation too. However, the tomographic profile obtained from fully polarimetric data set permits to guess correctly building shapes with a consistent height estimation compared to the lidar profile. This fact reveals that fully polarimetric dual-baseline configuration improves significantly the tomographic accuracy, compared with single-polarization ones, and provides additional information, related to scattering mechanisms, which helps to better characterize building features, like geometrical shapes as well as dielectrical properties, etc. 

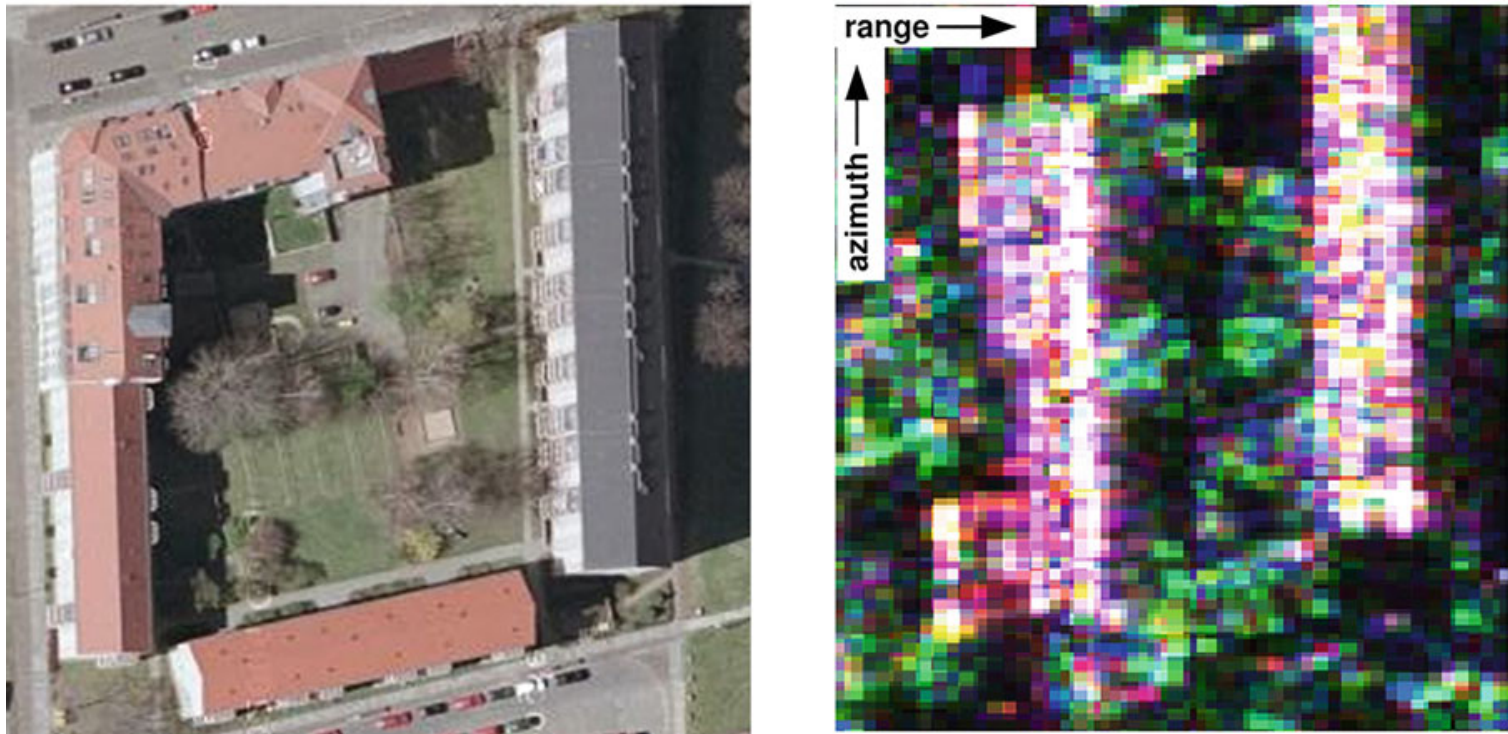

Fig. 5.30 Another urban area under study. Optical image (left) and Pauli-coded SAR image (right)
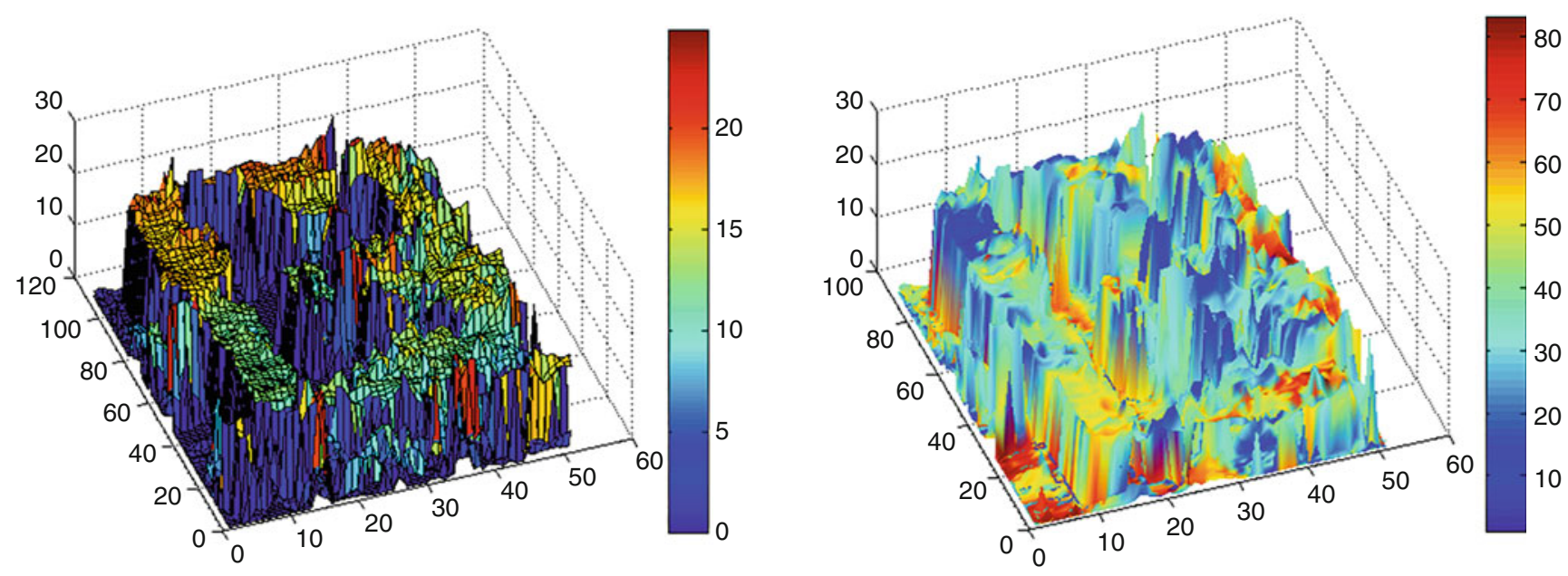

Fig. 5.31 3-D tomographic reconstruction using dual-baseline PolInSAR data sets, shaded by surface elevation (left) and $\alpha$ value (right)

\subsubsection{Discussion on the Role of Polarization and on Maturity of the Application and Conclusions}

Polarimetric SAR tomography (PolTomoSAR) is a very interesting approach to analyze complex 3-D environments, like urban environments, forested areas, etc. As it is demonstrated in this section, powerful spectral analysis techniques can be used to efficiently separate responses from scatterers located at different elevations in very severe scenarios, i.e., with only three images. Combining tomographic processing with polarimetric diversity provides a significant gain in performance as the 3-D imaging process adapts to the polarimetric properties of the scatterers to be imaged, i.e., adaptively maximizes SNR and estimation accuracy. Fully polarimetric tomography permits to further discriminates closely spaced scatterers having diverse polarimetric responses and is less sensitive to artifacts and spurious sidelobes, compared to singlepolarization approaches. Moreover, PolTomoSAR results can be processed through usual polarimetric approaches, like polarimetric decompositions and others, in order to characterize, identify scatterers, and provide interpretation of scattering mechanisms.

PolTomoSAR analysis of urban environments has been conducted over nearly a decade, with very different spectral estimation approaches and acquisition configurations, and may be considered as mature. The approach used here 

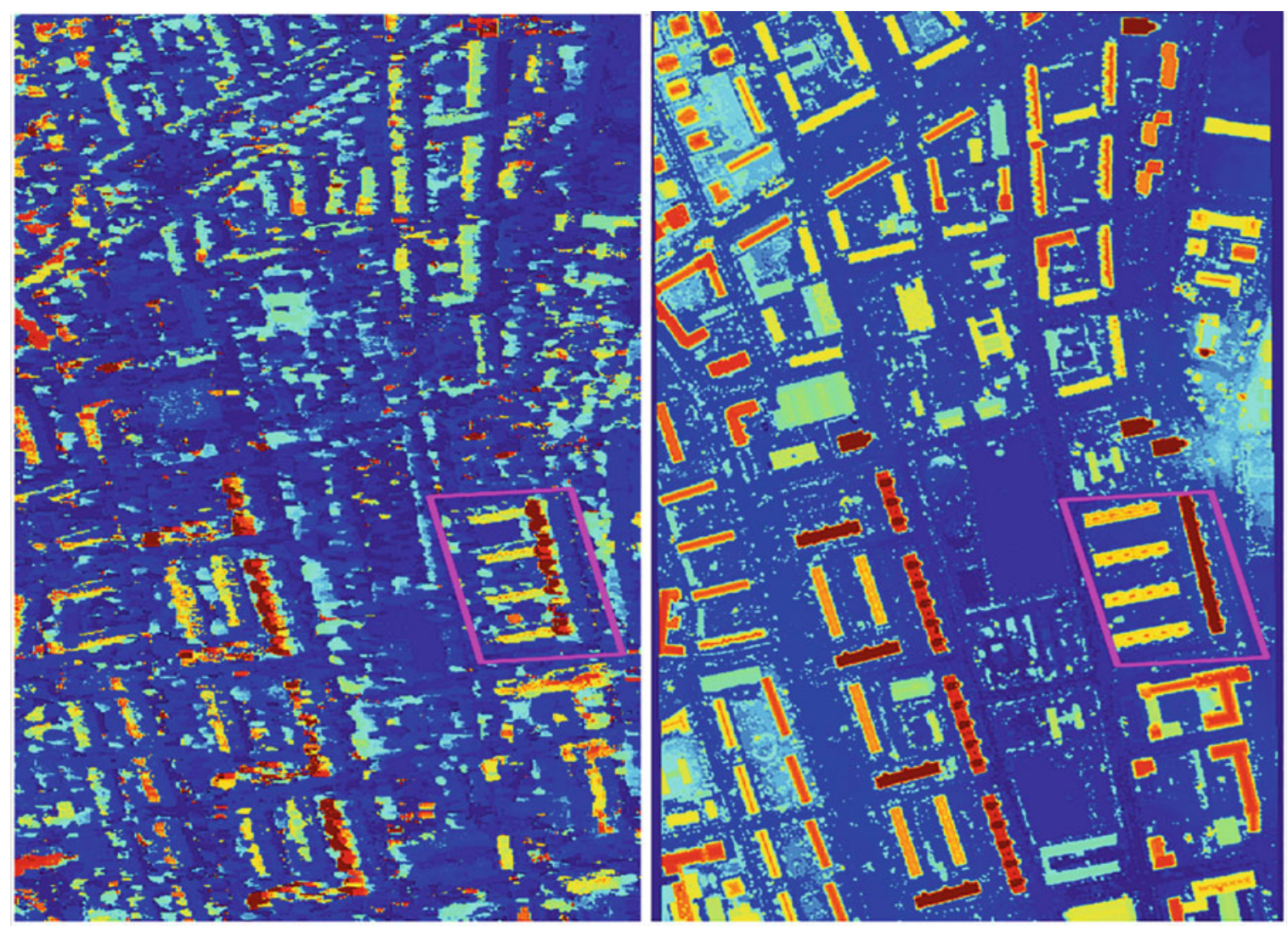

Fig. 5.32 Lidar surface elevation (right) and estimated surface elevation by FP-NSF estimator (left)

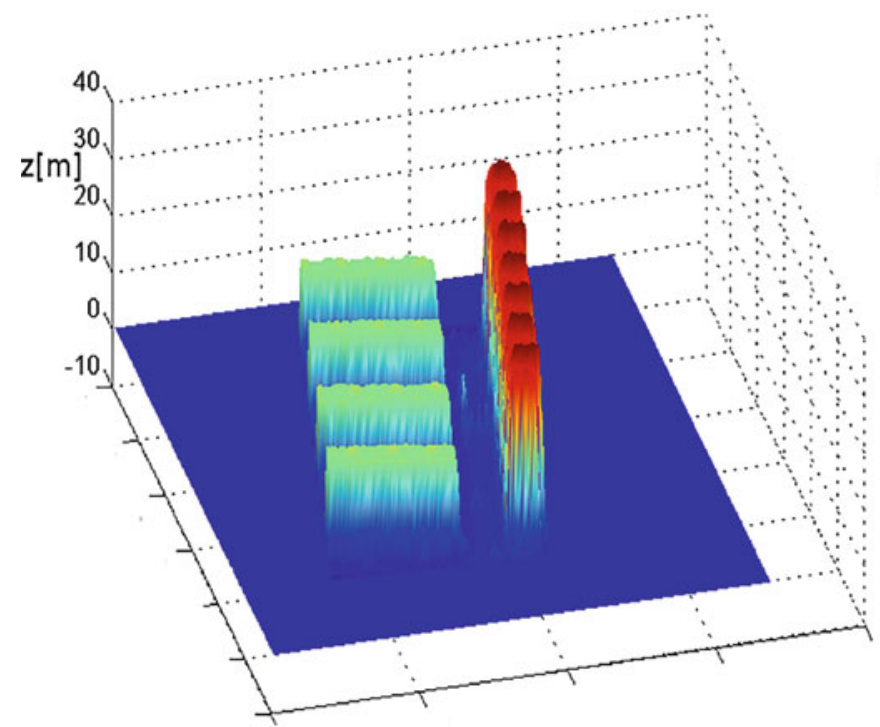

Fig. 5.33 3-D reconstruction: lidar (left) and FP-NSF estimator (right)

provides very high vertical resolution and a robust estimation of different parameters of urban environments, like building heights and scattering mechanisms. PolTomoSAR

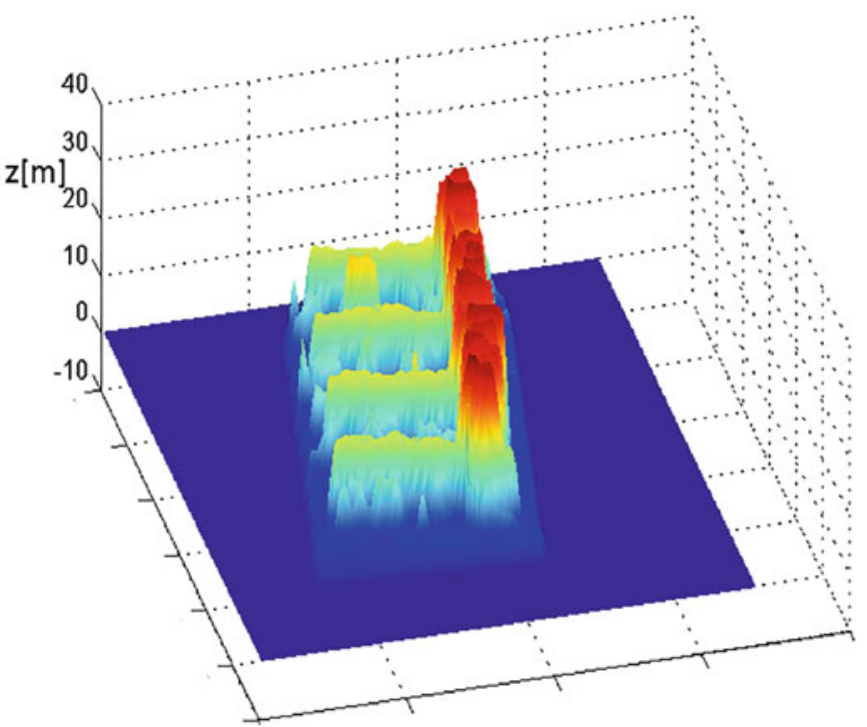

techniques can be extended to different applications such as subsurface observation and forestry remote sensing, under foliage imaging and object detection (Huang et al. 2012). 

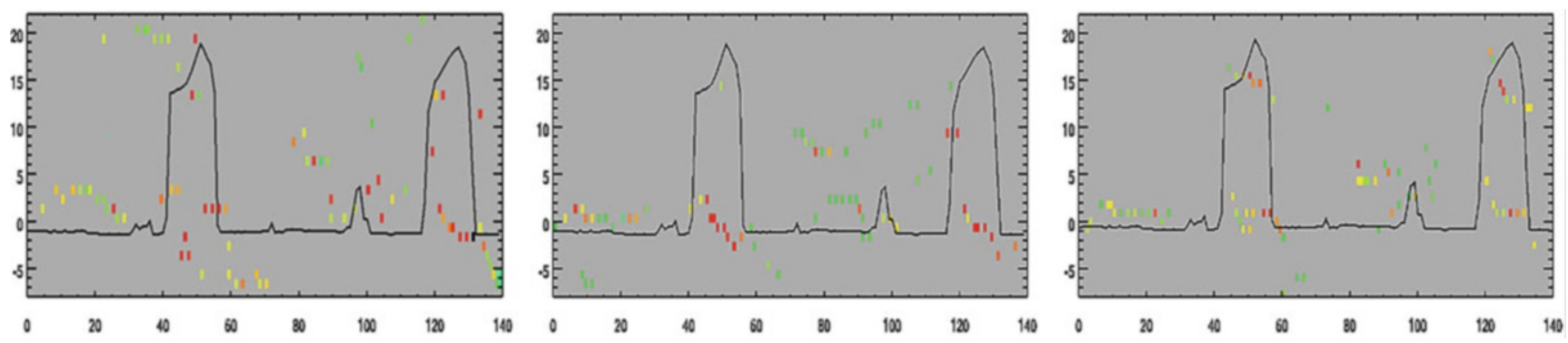

Fig. 5.34 Single-polarization HH (left), VV (middle) and fully polarimetric (right) reflectivity tomograms with scale: $25-110 \mathrm{~dB}$

\subsection{Subsidence Monitoring}

Since its conception in the late 1990s, differential SAR Interferometry (DInSAR) is an established technique useful for the monitoring of deformation episodes over wide areas (Ferretti et al. 1999, 2001). PSI techniques exploit the phase information of a stack of interferograms, obtained from a set of SAR images acquired at different dates, to retrieve accurate information of the ground deformation evolution along time. In this framework and mainly due to decorrelation phenomena, any advanced DInSAR technique is constrained by the number and the quality of trustful points from where reliable information of deformation can be retrieved. Two main criteria are available in the literature to perform adequate pixel quality estimation. In the first approach, the phase quality is assessed through the coherence estimator applied to each interferometric pair (Mora et al. 2003; Berardino et al. 2002). In the second approach, the phase quality is associated with the amplitude dispersion index DA of the images (Ferretti et al. 1999), often used in urban environments, where it is common to find point-like, deterministic scatterers (called Persistent Scatterers, PS) associated with strong and stable backscattering from man-made structures. The higher the interferometric coherence or, accordingly, the lower the DA, the better the phase quality and, thus, the most reliable the deformation process estimation.

Due to the lack of polarimetric SAR (PolSAR) data sets, the use of DInSAR techniques has been traditionally limited to the single-polarization approach.

However, the scenario has changed with the launch of new satellites with polarimetric capabilities, such as TerraSAR-X, RADARSAT-2, ALOS-PALSAR, and the upcoming launch of Sentinel-1, ALOS-2, and RADARSAT Constellation Mission. The new polarimetric diversity can be exploited in order to enhance the performance of conventional PSI approaches.

\subsubsection{Improvement of Differential SAR Interferometry for Subsidence Monitoring with Polarimetric Optimization Techniques}

\subsubsection{Introduction, Motivation, and Literature Review}

The application of polarimetric optimization methods has led to an improvement in the density but also in the quality of the deformation process retrieval (Pipia et al. 2009; NavarroSanchez et al. 2010; Navarro-Sanchez and Lopez-Sanchez 2011a, b, 2012; Iglesias et al. 2012; Monells et al. 2012) .

This section describes the three polarimetric optimization methods available in the literature when PolSAR data sets are available. The methods are referred as Best (Pipia et al. 2009), Equal Scattering Mechanism (ESM) (Colin et al. 2006) and Sub-Optimum Scattering Mechanism (SOM) (Sagues et al. 2000). Their exploitation for DInSAR applications is addressed with both the coherence stability and DA pixel selection criteria. The objective is to improve the quality of the interferograms through the proper combination of the available polarimetric channels. The optimization techniques will use the phase quality estimators as figures of merit. Deformation maps obtained from fully polarimetric data sets will be compared with those obtained with the traditional single-polarimetric approaches in order to show the benefits of the former. In addition, the performances of dual-polarimetric data are also evaluated.

\subsection{Classical Differential SAR Interferometry}

DInSAR processing aims at obtaining the temporal evolution of deformation episodes, together with the topographic error and atmospheric artifacts, from a stack of multi-temporal differential interferograms. In this framework, a usual approach, among others, is the Coherent Pixels Technique (CPT) (Mora et al. 2003; Blanco et al. 2008). CPT can work 
with either the coherence or the amplitude-based pixel selection criteria. Similarly to other DInSAR algorithms, a linear model, which includes the linear deformation term and the topographic error of the DEM used to generate the differential interferograms, is adjusted to the interferometric data through a minimization process (Blanco et al. 2008). Once the linear deformation term and the topographic error have been determined for the selected pixels, in a second step the deformation time series and the atmospheric phase screen are derived for all selected pixels leading to a complete characterization of the deformation process.

\subsection{Polarimetric Differential SAR Interferometry}

Working at the PolSAR acquisition level, the scattering matrix $\mathbf{S}$, which indicates the polarimetric information associated to each pixel of the scene, can be defined, in the orthogonal horizontal and vertical polarization basis $\{\mathrm{H}, \mathrm{V}\}$, as (Lee and Pottier 2009).

$$
\mathbf{S}_{\left(\widehat{\mathbf{u}_{\mathrm{H}}, \widehat{\mathbf{u}}}\right)}=\left[\begin{array}{ll}
S_{\mathrm{HH}} & S_{\mathrm{HV}} \\
S_{\mathrm{HV}} & S_{\mathrm{VV}}
\end{array}\right]
$$

In this context, it is possible to indicate the scattering matrix $\mathbf{S}$ in another generic orthogonal basis $\{\mathrm{X}, \mathrm{Y}\}$ through a unitary transformation (Lee and Pottier 2009; Kostinski and Boerner 2009).

$$
\mathbf{S}_{(\widehat{\mathbf{x}}, \mathbf{y})}=\left[\begin{array}{ll}
S_{\mathrm{XX}} & S_{\mathrm{XY}} \\
S_{\mathrm{XY}} & S_{\mathrm{YY}}
\end{array}\right]=\mathbf{U}_{2}^{T} \mathbf{S}_{\left(\widehat{\mathbf{u}}_{\mathrm{H}}, \widehat{\mathbf{u}}_{\mathbf{v}}\right)} \mathbf{U}_{2}
$$

where $(\cdot)^{T}$ refers to the vector transposition and the matrix transformation $\mathbf{U}_{2}$ can be expressed in terms of the orientation and ellipticity angles $(\psi, \chi)$ of the polarization ellipse by

$$
\begin{aligned}
\mathbf{U}_{2}= & {\left[\begin{array}{cc}
\cos \psi & -\sin \psi \\
\sin \psi & \cos \psi
\end{array}\right]\left[\begin{array}{cc}
\cos \chi & j \sin \chi \\
j \sin \chi & \cos \chi
\end{array}\right] } \\
& \times\left[\begin{array}{cc}
e^{+j \phi_{0}} & 0 \\
0 & e^{-j \phi_{0}}
\end{array}\right] .
\end{aligned}
$$

From an interferometric point of view, if two PolSAR acquisitions obtained at different times $i=1,2$ are available, the so-called scattering vector $\mathbf{k}_{i}$ can be defined as a vectorization of the scattering matrix $\mathbf{S}$ as

$$
\mathbf{k}_{i}=\frac{1}{\sqrt{2}}\left[S_{\mathrm{HH}, i}+S_{\mathrm{VV}, i}, S_{\mathrm{HH}, i}-S_{\mathrm{VV}, i}, 2 S_{\mathrm{HV}, i}\right]^{T} .
$$

The scattering vector $\mathbf{k}_{i}$ can be projected onto an unitary projection vector $\mathbf{w}$ obtaining a generic scattering coefficient $S_{i}=\mathbf{w}_{i}^{* T} \mathbf{k}_{i}$ for each pair of images $i=1,2$, where ${ }^{* T}$ is the conjugate transpose operator. At this stage, the PolInSAR vector between two PolSAR acquisitions is defined by (Cloude 2009; Qong et al. 2005)

$$
\mathbf{k}_{6}=\left[\mathbf{k}_{1}^{T}, \mathbf{k}_{2}^{T}\right]^{T}
$$

Once the PolInSAR vector $\mathbf{k}_{6}$ is defined, under the assumption of spatial homogeneity and ergodicity, the $6 \times 6$ PolInSAR coherency complex matrix $\mathbf{T}_{6}$ is defined as

$$
\mathbf{T}_{6}=E\left\{\mathbf{k}_{6} \mathbf{k}_{6}^{* T}\right\}=\left[\begin{array}{ll}
\mathbf{T}_{11} & \boldsymbol{\Omega}_{12} \\
\mathbf{\Omega}_{12}^{* T} & \mathbf{T}_{22}
\end{array}\right]
$$

$\mathbf{T}_{11}$ and $\mathbf{T}_{22}$ refer to the coherency matrices of each PolSAR data set and $\boldsymbol{\Omega}_{12}$ indicate the polarimetric interferometric coherency matrix. In this context, the expression of the classical interferometric coherence can be generalized taking into account its polarimetric dependence

$$
\gamma\left(\mathbf{w}_{1}, \mathbf{w}_{2}\right)=\frac{\mathbf{w}_{1}^{* T} \mathbf{\Omega}_{12} \mathbf{w}_{2}}{\sqrt{\left(\mathbf{w}_{1}^{* T} \mathbf{T}_{11} \mathbf{w}_{1}\right)\left(\mathbf{w}_{2}^{* T} \mathbf{T}_{22} \mathbf{w}_{2}\right)}}
$$

Notice that different projection vectors between the different acquisitions of the interferogram, $\mathbf{w}_{1} \neq \mathbf{w}_{2}$, may lead to the introduction of a polarimetric contribution in the interferometric phase, due to a phase center change within the same resolution cell. Thus, ensuring the same projection vector along the whole stack of interferograms, $\mathbf{w}=\mathbf{w}_{1} \neq \mathbf{w}_{2}$, is mandatory for Polarimetric Differential SAR Interferometry (PolDInSAR) applications. Under this restriction, (5.11) can be rewritten as

$$
\gamma(\mathbf{w})=\frac{\mathbf{w}^{* T} \mathbf{\Omega}_{12} \mathbf{w}}{\sqrt{\left(\mathbf{w}^{* T} \mathbf{T}_{11} \mathbf{w}\right)\left(\mathbf{w}^{* T} \mathbf{T}_{22} \mathbf{w}\right)}} .
$$

In the framework of PolDInSAR, polarimetric optimization methods seek to optimize the generalized expression of the coherence (5.12). The first approach explores the whole space of projection vectors $\mathrm{w}$ looking for the one providing the highest value of coherence. The second one explores all the polarimetric transformations given by (5.6) and again looks for the one providing the maximum value of coherence. Meanwhile, when working with point-like scatterers, the expression of the $D_{A}$ can be also generalized (NavarroSanchez et al. 2010; Navarro-Sanchez and Lopez-Sanchez 2011a, b, 2012, 2013; Navarro-Sanchez et al. 2014) as

$$
\begin{gathered}
D_{A}(\mathbf{w})=\frac{\sigma_{A}}{m_{A}}=\frac{1}{\left\langle\left|\mathbf{w}^{* T} \mathbf{k}\right|\right\rangle} \sqrt{\frac{1}{N} \sum_{i=1}^{N}\left(\left|\mathbf{w}^{* T} \mathbf{k}_{i}\right|-\left\langle\left|\mathbf{w}^{* T} \mathbf{k}\right|\right\rangle\right)^{2}} \\
\text { where }\left\langle\left|\mathbf{w}^{* T} \mathbf{k}\right|\right\rangle=\frac{1}{N} \sum_{i=1}^{N}\left|\mathbf{w}^{* T} \mathbf{k}_{i}\right|
\end{gathered}
$$

where $\sigma_{A}$ is the standard deviation and $m_{A}$ is the mean of the amplitude time series. In this case the objective is, as stated in 
the coherence case, to find the projection vector or the polarimetric transformation that minimizes the generalized expression of $D_{A}$.

\subsubsection{Methodology}

\subsection{Stability Optimization Methods}

In this section, the basis of the different polarimetric optimization methods for PolDInSAR applications with the coherence stability pixel selection approach is addressed.

The first approach, referred as Best, is based on selecting the polarimetric channel providing the highest temporally averaged coherence value for each pixel along the whole stack of interferograms. Consequently, the original three interferograms (one per polarimetric channel) are mixed in a new interferogram where the phase of each pixel corresponds to the channel providing the highest temporally averaged coherence. In order to avoid changes in the phase centers, the polarization mechanism for each pixel has to be equal in all the interferograms of the data set.

The second approach, which is referred as Equal Scattering Mechanism (ESM), consists on finding the projection vector $\mathbf{w}$ that maximizes the generalized expression of the coherence (5.12). The solution must be obtained using numerical methods since the maximization problem has no analytical solution. The simplest approach consists on parameterizing the projection vector $\mathbf{w}$ to obtain all the possible values of the generalized coherence. The parameterization presented in (Cloude and Papathanassiou 1998) can be used to ensure the unitarity of the projection vector

$$
\mathbf{w}=\left[\begin{array}{c}
\cos \alpha \\
\sin \alpha \cos \beta e^{j \delta} \\
\sin \alpha \sin \beta e^{j \gamma}
\end{array}\right] \quad\left\{\begin{array}{c}
0 \leq \delta \leq \pi,-\pi \leq \gamma<\pi, \\
0 \leq \alpha \leq \frac{\pi}{2}, 0 \leq \beta \leq \pi
\end{array}\right.
$$

The optimum projection vector will be the one providing the maximum coherence. The main drawback of this approach is its high computational cost. To face this problem, the solution presented in (Colin et al. 2006) which makes use of an iterative process to find the optimum projection vector $\mathrm{w}$ is proposed. This approach assumes that the two coherency matrices $\mathbf{T}_{11}$ and $\mathbf{T}_{22}$ are similar, which is accomplished when polarimetric stability applies. Under this hypothesis, the estimated complex differential coherence is approximated by

$$
\widehat{\gamma}(\mathbf{w})=\frac{\underline{\mathbf{w}}^{* T} \mathbf{\Omega}_{12} \mathbf{w}}{\underline{\mathbf{w}}^{* T} \mathbf{T} \underline{\mathbf{w}}} \quad \text { where } \mathbf{T}=\frac{\mathbf{T}_{11}+\mathbf{T}_{22}}{2},
$$

where $|\widehat{\gamma}|<|\gamma|$ and the interferometric phase provided by both estimators is preserved. In the framework of
PolDInSAR applications, an extension of the method introduced by Colin et al. in (Colin et al. 2006) to the multi-temporal case may be considered. The extension presented by Neumann et al. in (Neumann et al. 2008) aims to optimize the temporally averaged coherence instead of the coherence of each interferogram separately. Once the optimum projection vector $\mathbf{w}_{\text {opt }, E S M}$ is found, the coherence is obtained through (5.12), and the interferometric phase is given by

$$
\phi_{E S M}=\arg \left(\mathbf{w}_{\text {opt }, E S M}^{* T} \boldsymbol{\Omega}_{12} \mathbf{w}_{\text {opt }, E S M}\right) .
$$

On the other hand, when polarimetric stability does not apply the optimized differential phase may be affected by the difference of polarimetric behavior, leading to an erroneous phase value. An alternative method referred as Sub-Optimum Scattering Mechanism (SOM) (Sagues et al. 2000) is proposed in order to overcome these restrictions. The algorithm is based on exploring the entire possible polarimetric basis, departing from $S_{h v}$ and sweeping all the values of ellipticity and orientation angles. This will consider all the possible polarization states of the propagating wave. The key step resides in looking for the polarization basis transform providing the highest temporally averaged coherence value among all the co-polar and cross-polar realizations

$$
\left|\bar{\gamma}_{S O M}\right|=\max _{(\psi, \chi)}\left\{\left|\bar{\gamma}_{\mathrm{XX}}(\psi, \chi)\right|,\left|\bar{\gamma}_{\mathrm{XY}}(\psi, \chi)\right|\right\}
$$

The subscripts XX and XY refer to the co-polar and crosspolar channels in the new $(\psi, \chi)$ polarization basis, respectively. This method could be seen as a subspace of the ESM approach.

\subsection{Amplitude Dispersion Optimization Methods}

In this section, the basis for the adaptation of the three optimization methods presented before will be particularized for the $D_{A}$ pixel selection criterion approach.

As in the coherence case, the Best approach is the simplest way to face the polarimetric optimization problem. It is based on selecting the interferometric phases of the polarimetric channel providing the minimum $D_{A}$.

The second approach, ESM, explores the whole polarimetric space looking for the projection vector $\mathbf{w}$ that minimizes the generalized $D_{A}$ expression (5.13). To solve this problem, there is no analytical solution. Hence, the optimization problem must be solved by brute force, parameterizing the projection vector as seen in (5.12). As in the coherence case, the main drawback of this approach is the computational cost since a 4-D space needs to be explored. For this reason, the adaptation of the SOM approach is an appropriate alternative. 
Table 5.8 Test sites and corresponding radar and validation data selected for the generation of showcases on urban subsidence monitoring

\begin{tabular}{l|l|l}
\hline Application /product & Test site - Radar data & Reference data \\
\hline \multirow{2}{*}{ Urban subsidence monitoring } & Barcelona, Spain & No ground measurements \\
\cline { 2 - 3 } & 34 RADARSAT-2 Fine Quad-Pol images, 2010-2012 & \\
\hline
\end{tabular}

As seen in the coherence stability case, the method consists in sweeping all the possible orientation and ellipticity angles in order to reach a scattering matrix in a new polarization basis, which provides a minimum $D_{A}$ value among all the co-polar $D_{A, a a}$ and cross-polar $D_{A, a b}$ indices. With this approach, the computational load is highly reduced since the solution now consists in exploring a 2-D space corresponding to all possible orientation and ellipticity angles.

\subsubsection{Experimental Results}

The PolSAR data set used in this work consists of 34 fully polarimetric RADARSAT-2 images, from January 2010 to May 2012, that correspond to the metropolitan area of Barcelona, Northeastern Spain. RADARSAT-2 works at C-Band, with a resolution of 5 meters in both range and azimuth directions and a revisiting time of 24 days. Selected test sites and data sets are summarized in Table 5.8 and further described in the Appendix.

This section shows the PolDInSAR results obtained using the different polarization optimization techniques described previously with both the coherence and amplitude approaches. Once the phase optimization is performed in the pixel selection step, the classical DInSAR processing can be applied to the new stack of optimized interferograms, since there are no differences from the single-polarimetric case.

The pixel selection with the coherence approach has been performed establishing a threshold of 0.75 , which corresponds to a phase standard deviation of $5^{\circ}$ with the $9 \times 5$ multi-look window used (Hanssen 2001). To highlight the performances of the different optimization methods, a quite restrictive threshold has been established. Table 5.9 shows the pixel density obtained for each method. Notice how with polarimetric optimization techniques, it is possible to reach an increase in the number of selected pixels of a 2.7 factor compared with the single-polarization case.

Figure 5.35 shows a comparison of final DInSAR deformation maps obtained using the $S_{h h}$ single-polarimetric channel and the three different optimization methods. The deformation pattern is almost identical in all cases, and the main differences lay in the pixel densities shown in Table 5.9. Different deformation bowls can be observed in the figure; the most severe is in the airport and in the harbor area. Some weaker deformations are observed in the city. In the NorthEast part of the image, interesting subsidence that follows the track of a metro line under construction is identified. Since the affected area is narrow, it is difficult to detect when the density of pixels is low, such as in the single-polarimetric
Table 5.9 Coherence stability pixel selection statistics for each method. (\%) refers to the total number of pixels

\begin{tabular}{l|c}
\hline Method & Number of pixels \\
\hline hh & $6431(3.3 \%)$ \\
\hline hv & $5026(2.6 \%)$ \\
\hline vv & $5014(2.6 \%)$ \\
\hline Best & $11,931(6.1 \%)$ \\
\hline SOM & $13,894(7.1 \%)$ \\
\hline ESM & $17,281(8.9 \%)$
\end{tabular}

case. The optimization methods largely increase the number of selected pixels leading thus to a better determination of the deformation bowl extension and its characterization.

Regarding the amplitude-based approach, a $D_{A}$ threshold of 0.25 , which corresponds to a phase standard deviation of $15^{\circ}$ (Hanssen 2001), has been used. Notice how the differences among the different methods are more substantial for this case; see Table 5.10. The ESM approach increases in a factor of seven the number of pixels from the singlepolarimetric case. As in the coherence stability case, this pixels' density increase justifies the use of PolSAR data in the DInSAR framework.

Finally, Fig. 5.36 shows the linear deformation maps using each optimization approach in the area within the city of Barcelona commented before. All approaches are able to determine the deformation bowls with similar values of terrain deformation. The main differences come from the larger pixels' densities obtained when using the advanced polarimetric optimization methods. ESM provides the larger improvement in number of pixels, and consequently the deformation map allows precisely determining the extension of the deformation bowl along the new underground line. The different deformation bowls observed in the figure match the path followed by the tunnel, and the widest bowl, also with the highest subsidence, is located over a new underground station.

\subsubsection{Comparison with Single/ Dual-Polarization Data}

The usage of fully polarimetric data imposes some limitations in the sensors regarding the swath extension, basically reduced by a factor of two if compared with the single or dual-pol cases. This section presents a comparison between the performances of full and dual-pol data in terms of pixels' density. Fully polarimetric data entails covering the entire polarimetric space; hence the optimum value in terms of the 


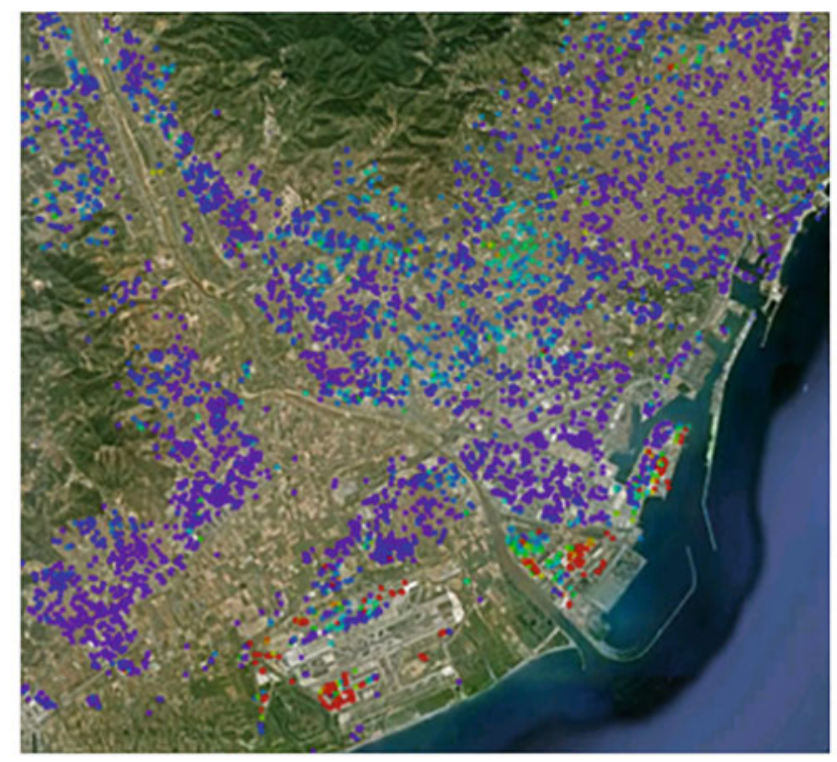

(a)

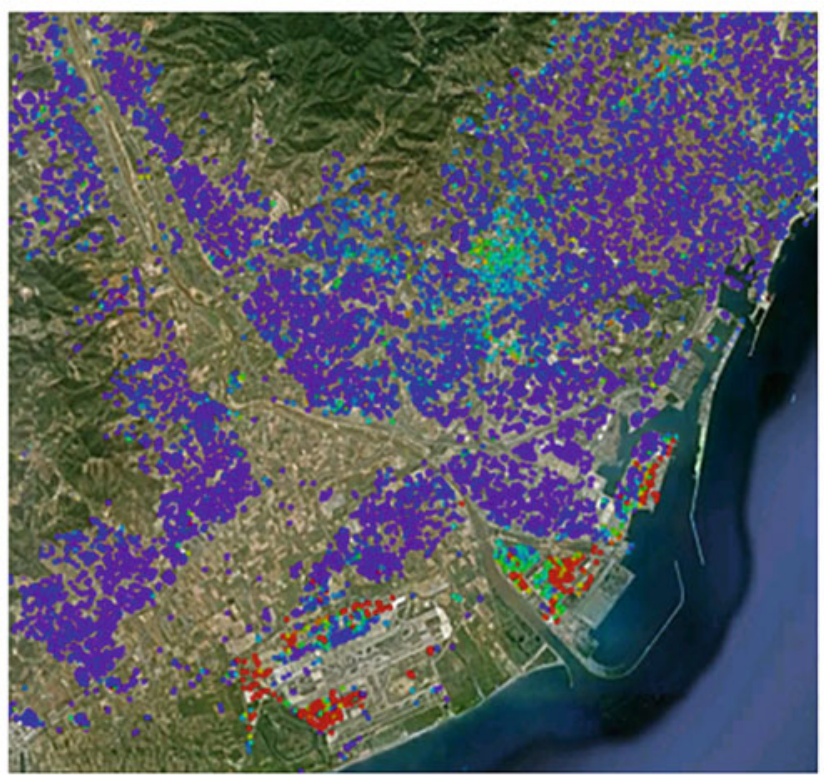

(c)

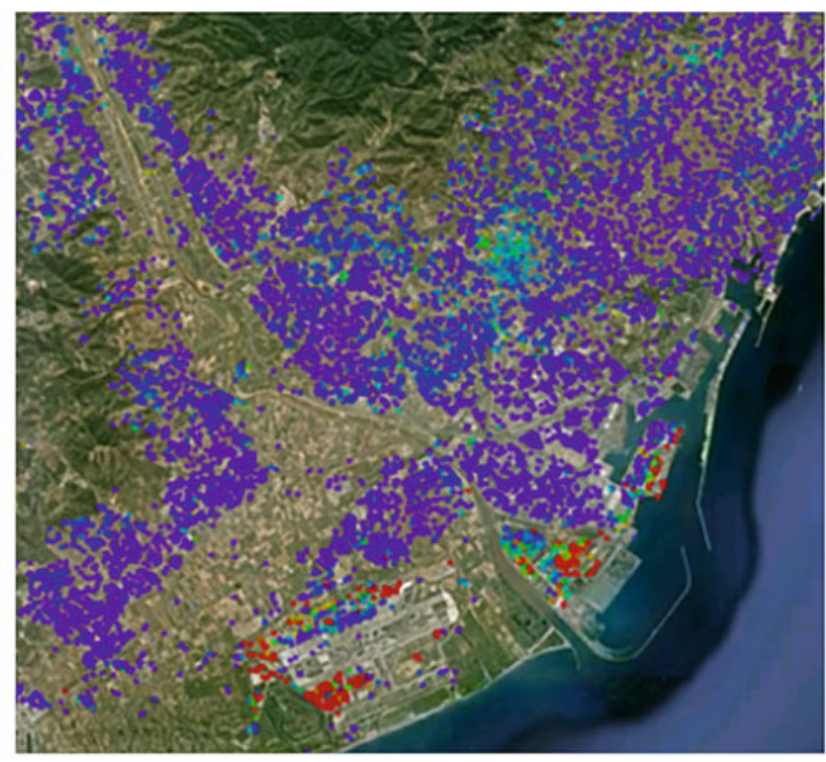

(b)

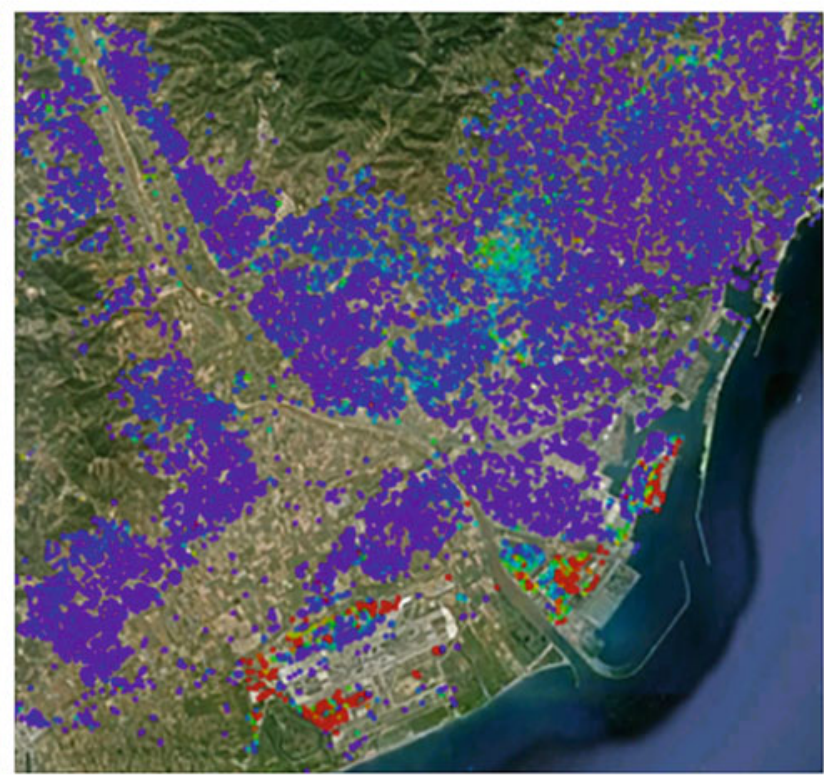

(d)

\section{$0 \mathrm{~cm} /$ year}

Fig. 5.35 Linear velocity retrieved over Barcelona, from January 2010 to May 2011. Using the hh polarimetric channel (a) or the Best (b), SOM (c) and ESM (d) mean coherence stability optimization methods

phase quality can be reached. If only dual-pol data are available, the result is obviously suboptimum, but the complexity of the optimization process is significantly reduced. Moreover, dual-pol configurations are in general more efficient in terms of coverage.
To simulate the performance of dual-polarimetric products, the fully polarimetric data set available has been narrowed down selecting either the two direct channels $(\mathrm{HH} / \mathrm{VV})$ or a direct channel and a cross-polar channel $(\mathrm{HH} / \mathrm{HV}$ or $\mathrm{VV} / \mathrm{VH})$. The first option presents the same 
swath restrictions than the full-pol case. The comparison is based on the optimization process performance, so swath differences are omitted. The ESM approach, under the DA pixel selection criterion, is selected for the comparison since it provides the best performance. Table 5.11 shows the results after the pixel selection step. Results reveal that both fully

Table 5.10 $D_{A}$ pixel selection statistics for each method. (\%) refers to the total number of pixels

\begin{tabular}{l|c}
\hline Method & Number of pixels \\
\hline hh & $9398(1.9 \%)$ \\
\hline hv & $8522(1.7 \%)$ \\
\hline vv & $9927(2.0 \%)$ \\
\hline Best & $21,721(4.4 \%)$ \\
\hline SOM & $40,032(8.1 \%)$ \\
\hline ESM & $71,702(14.6 \%)$ \\
\hline
\end{tabular}

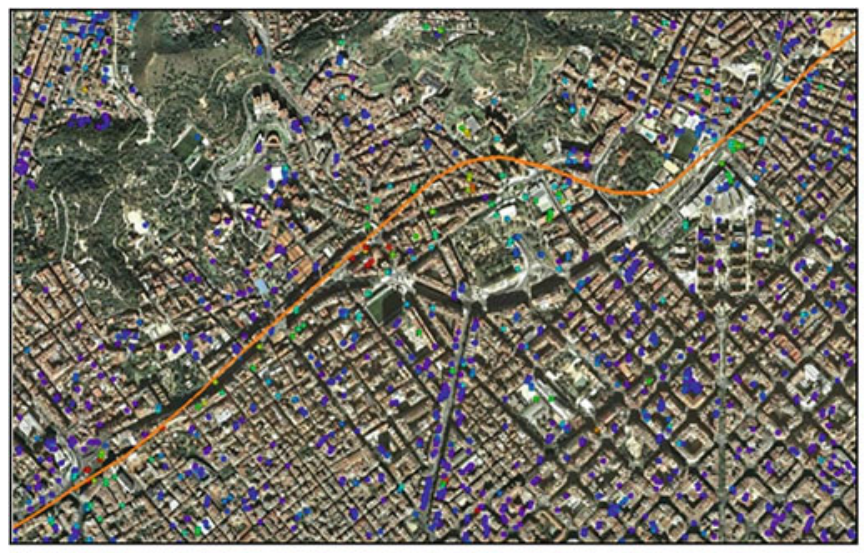

(a)

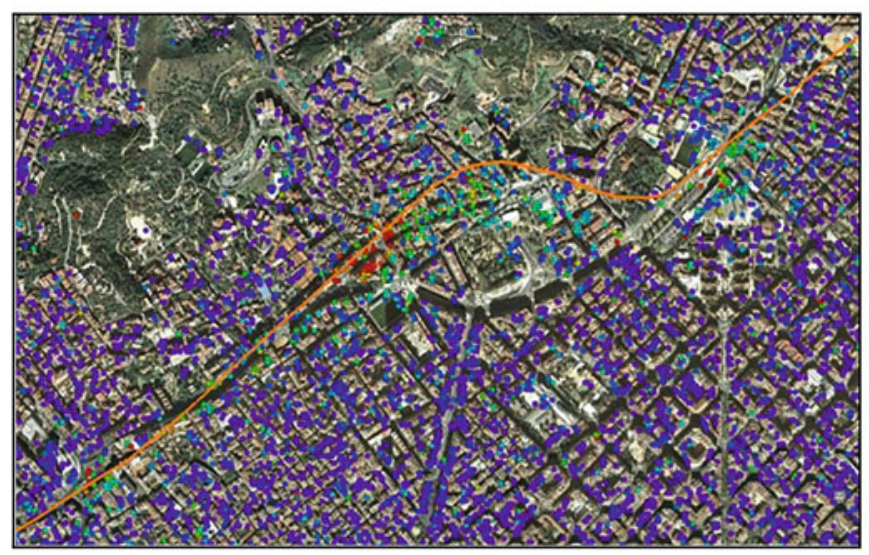

(c) polarimetric and dual-polarimetric data are suitable for phase quality optimization techniques since they clearly outperform the single-polarimetric case. As expected, the dual-pol optimization does not produce the same improvement in pixels density than the full-pol case. For this particular data set,

Table 5.11 Amplitude dispersion pixel selection for dual VS fully polarimetric configurations. The $\%$ of pixels is over the total pixels considered

\begin{tabular}{l|c}
\hline Method & Number of pixels \\
\hline hh & $9398(1.9 \%)$ \\
\hline Dual-Pol HH-VV & $35,410(7.2 \%)$ \\
\hline Dual-Pol HH-VV & $33,697(6.9 \%)$ \\
\hline Dual-Pol HH-VV & $33,179(6.8 \%)$ \\
\hline Full-Pol & $71,702(14.6 \%)$ \\
\hline hh & $9398(1.9 \%)$ \\
\hline
\end{tabular}

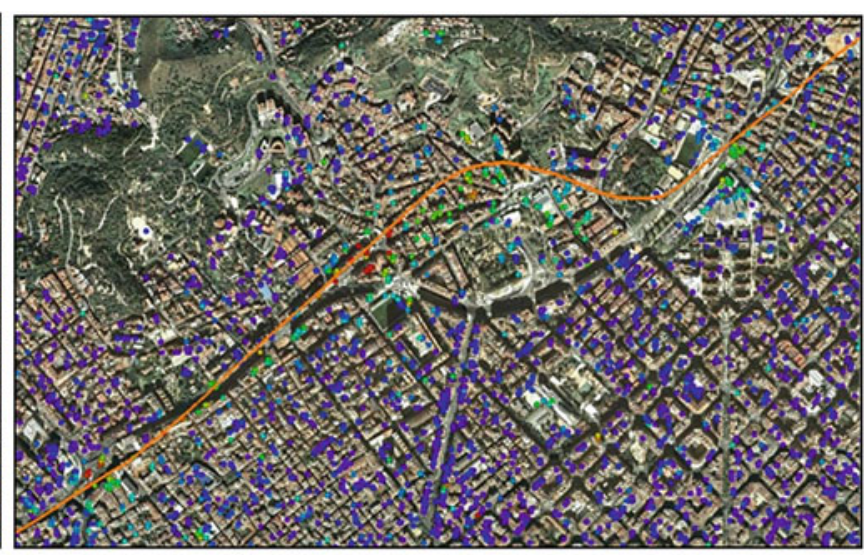

(b)

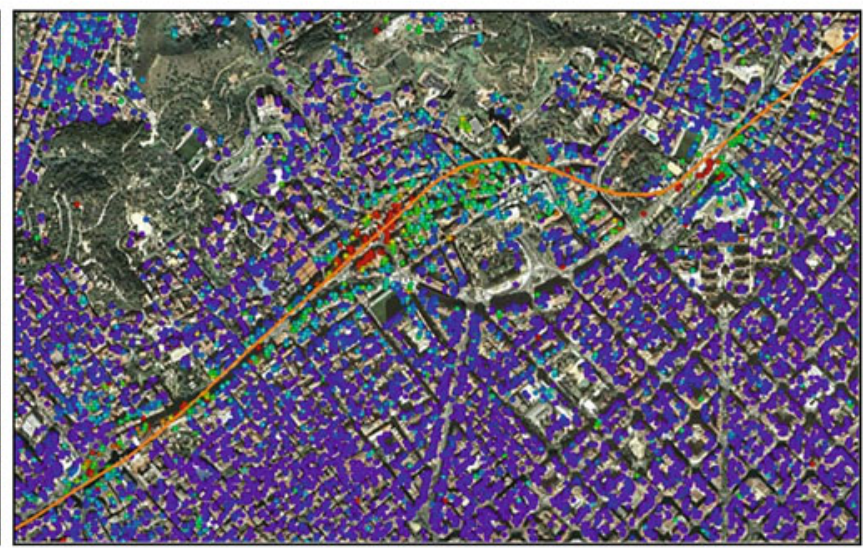

(d)

\section{$0 \mathrm{~cm} /$ year}

\section{$0.5 \mathrm{~cm} /$ year}

Fig. 5.36 Linear velocity retrieved from the RADARSAT-2 PolSAR data set. Using the hh polarimetric channel (a) and the Best (b), SOM (c) or ESM (d) DA-based optimization methods for the DInSAR processing. The orange line shows the path of the new underground line 
there is roughly a factor of two between the dual-pol and the full-pol densities. On the other hand, the performance among the dual-polarimetric configurations is rather similar, being the HH-VV combination slightly better than the ones involving the cross-polar channel. For this reason, despite the major computational load and the higher complexity in the algorithms, the use of fully polarimetric data is strongly recommended.

\subsubsection{Discussion on the Role of Polarization and on the Maturity of the Application and Conclusions}

General polarimetric optimization methods for its application in DInSAR processing have been evaluated. The three different optimization techniques have been put forward using RADARSAT-2 fully polarimetric data and working with both the coherence stability and the amplitude-based pixel selection criteria.

With the proper combination of the available polarimetric channels, the proposed polarimetric optimization methods demonstrate their capability to enhance the quality of DInSAR results. First, it is possible to obtain a higher density of pixels compared with the single-polarization case. Moreover, the quality of the interferometric phase is also improved, leading to more precise deformation maps. This fact provides major robustness in DInSAR algorithms and is a key factor to better determine and characterize deformation bowl extensions.

Concerning the comparison of fully polarimetric and dualpolarimetric data, the use of fully polarimetric data is strongly recommended since it clearly improves the DInSAR results.

\subsubsection{Acknowledgment}

This research work received partial support from the SafeLand project funded by the Commission of the European Communities, grant agreement 226479, from the Big Risk project, contract number BIA2008-06614, the project TEC2011-28201-C02-01 and the grant BES-2009015990 associated with the project TEC2008-06764-C0201, both funded by the Spanish MICINN and FEDER funds. The RADARSAT-2 images were provided by MDA in the framework of the scientific project SOAR-EU 6779.

\subsubsection{Ground Deformation Estimation Using Polarimetric Persistent Scatterers Interferometry}

\subsubsection{Introduction, Motivation, and Literature Review}

In this section, we present a general framework for improving PSI through polarimetry. The proposed approach seeks the optimization of the parameters used as quality criteria for the initial pixel selection, with the consequent improvement in terms of density of points with valid deformation results. Moreover, disposing of denser distributions of PS or CP constitutes an advantage for the data processing itself, since many stages (e.g., phase unwrapping, atmospheric phase screen removal, interpolations, etc.) are carried out more robustly and accurately than with sparse distributions of pixels. Consequently, final deformation values are also more accurate in such conditions.

First works on single-pol PSI can be found in (Ferretti et al. 1999, 2001) which make use of an amplitude dispersion criterion $\left(\mathrm{D}_{\mathrm{A}}\right)$ for persistent scatterers selection. $\mathrm{D}_{\mathrm{A}}$ criterion may not be suitable for non-urban areas, where strong pointlike scatterers are less common, or when only a small set of SAR images of the area under study is available; hence $D_{A}$ estimates may be biased. To overcome these drawbacks, many techniques based on average interferometric coherence $(|\bar{\gamma}|)$ were proposed (Mora et al. 2003; Berardino et al. 2002). Points selected using this method are usually referred as coherent pixels (CP) and correspond to stable distributed scatterers, rather than point-like ones. Notice that maximum likelihood estimation of interferometric coherences generally requires averaging of neighboring samples, consequently spatial resolution is degraded. Alternative PSI approaches can also be found in (Hooper et al. 2004, 2007), where a phase coherence analysis is used in order to refine the initial PS selection, and (Ferretti et al. 2011) where joint analysis of PS and CP is addressed. A reference book in this area is (Kampes 2006).

The first application of polarimetry to urban PSI was proposed in (Perissin and Ferretti 2007) which made use of ENVISAT incoherent dual-pol data to recognize target physical features and to classify PS. Another PS classification strategy supported by polarimetry was also presented in (Dheenathayalan and Hansen 2011) in the context of building-versus-ground relative movement estimation, using TerraSAR-X coherent dual-pol data.

To further exploit polarimetric diversity at initial PSI processing stages, an algorithm aiming to increase the quality and number of pixels pre-selected for PSI processing was introduced in (Pipia et al. 2009). The proposed algorithm consisted in selecting, from the set of polarimetric channels provided by the sensor, the one that optimizes the average interferometric coherence for each pixel. The algorithm was tested using dual-pol (HH, VV) ground-based SAR data, achieving a significant improvement in the density of selected CP and demonstrating for the first time the potentials of polarimetry for PSI. Extending that idea, in (NavarroSanchez and Lopez-Sanchez 2012; Navarro-Sanchez et al. 2014), a generic method for polarimetric PSI optimization was proposed and tested using a set of TerraSAR-X dual-pol images. For each pixel, the algorithm finds the optimum channel as a linear combination of the set of channels 
measured by the sensor. That algorithm, which is employed in this work, can be adapted to optimize any parameter used as quality criterion and can be used as a pre-processing step to any known PSI technique. Following this line, an efficient search of the optimum channel was also addressed, and results using fully polarimetric data were presented in (Navarro-Sanchez et al. 2014; Monells and Mallorqui 2013), showing a remarkable improvement compared to dual-pol data in terms of density of PS and CP.

Concerning other pixel selection criteria, the normalized average Polarization Phase Difference (PPD) was proposed in (Samsonov and Tiampo 2011) for selecting only those pixels clearly dominated by odd and even bounce scattering mechanisms. In (Navarro-Sanchez and Lopez-Sanchez 2011b), a polarimetric stationarity test was proposed as a means to refine PS and CP selection. Similarly, a complete study of the temporal evolution of the polarimetric behavior of an urban area was carried out in (Pipia et al. 2012) using ground-based SAR data, and a filtering strategy was proposed to minimize the presence of non-stationary backscattering processes. Finally, in (Navarro-Sanchez and LopezSanchez 2013), an adaptive spatial speckle filtering approach driven by polarimetric temporal statistics is exploited as a pre-processing stage before polarimetric optimization, which allows to process simultaneously both optimized PS (pointlike targets) and CP (homogeneous, distributed scatterers), obtaining a significant increase in the final density of points and spatial coverage of deformation maps.

\subsubsection{Methodology}

The main objective of the polarimetric approach used here for PSI is to maximize the quality and number of PS or CP selected as reliable a priori, by optimizing the parameters used as a selection criterion. In (46), (48) we proposed a general framework for PSI polarimetric optimization, starting from the concept of polarimetric (or vector) interferometry introduced in (Cloude and Papathanassiou 1998). Let $\mathbf{k}$ be a pixel's target vector obtained by projecting its scattering matrix $\mathbf{S}$ onto the Pauli basis. For fully polarimetric data, it is given by:

$$
\mathbf{k}=\frac{1}{\sqrt{2}}\left[\begin{array}{c}
H H+V V \\
H H-V V \\
2 H V
\end{array}\right]
$$

where $H H$ and $V V$ stand for the horizontal and vertical co-polar channels, respectively, and $H V$ is the cross-polar channel. Notice that we assume $H V=V H$ due to reciprocity. In order to generate an interferogram, $\mathbf{k}$ can be projected onto a unitary complex column vector $\boldsymbol{\omega}$, resulting in:

$$
\mu=\omega^{T *} \mathbf{k}
$$

where and $\mu$ is a scalar complex scattering coefficient. As a scalar complex, $\mu$ is analogous to single-pol data, so we can make use of any known PSI technique by applying it to $\mu$. Therefore, the proposed PSI optimization approach consists in finding, for each pixel, the projection vector $\boldsymbol{\omega}$ that optimizes the parameter considered as the quality criterion when computed for $\mu$.

To facilitate the search of the optimum projection vector, we can parameterize $\omega$ in a way we ensure it is unitary, and we take into account all possible unambiguous combinations:

$$
\boldsymbol{\omega}=\left[\begin{array}{c}
\cos (\alpha) \\
\sin (\alpha) \cos (\beta) e^{i \delta} \\
\sin (\alpha) \sin (\beta) e^{i \psi}
\end{array}\right], \quad\left\{\begin{array}{c}
0 \leq \alpha \leq \frac{\pi}{2} \\
0 \leq \beta \leq \frac{\pi}{2} \\
-\pi \leq \delta<\pi \\
-\pi \leq \psi<\pi
\end{array}\right.
$$

Therefore, the problem is reduced to find four real parameters, $\alpha, \beta, \delta$, and $\psi$ whose range is finite and known and which value is related to the geometric and electromagnetic properties of the target (Cloude 2009). We have adapted this formulation to the dual-pol case, as the TerraSAR-X data at our disposal. For dual-pol data, the target vector is given by:

$$
\mathbf{k}=\frac{1}{\sqrt{2}}\left[\begin{array}{l}
H H+V V \\
H H-V V
\end{array}\right]
$$

and the projection vector can be parameterized as:

$$
\boldsymbol{\omega}=\left[\begin{array}{c}
\cos (\alpha) \\
\sin (\alpha) e^{i \psi}
\end{array}\right], \quad\left\{\begin{array}{c}
0 \leq \alpha \leq \frac{\pi}{2} \\
-\pi \leq \psi<\pi
\end{array}\right.
$$

so in this case the search is reduced to just two real parameters, $\alpha$ and $\psi$.

The optimization approach is tested here for the two most commonly used criteria of selection: the average interferometric coherence and the amplitude dispersion index. In the context of polarimetric interferometry, the average coherence $\overline{|\gamma|}$ can be expressed as:

$$
\begin{aligned}
\overline{|\gamma|} & =\frac{1}{K} \sum_{k=1}^{K}\left|\gamma_{k}\right|, \quad \text { with } \gamma_{k}(\boldsymbol{\omega}) \\
& =\frac{\boldsymbol{\omega} \boldsymbol{\Omega}_{i j} \boldsymbol{\omega}}{\sqrt{\boldsymbol{\omega} \mathbf{T}_{i i} \boldsymbol{\omega} \sqrt{\boldsymbol{\omega} \mathbf{T}_{j j} \boldsymbol{\omega}}}}
\end{aligned}
$$

where subscript $k$ denotes the $k$-th interferogram obtained by combining images $i$ and $j$. Polarimetric coherency matrices $\mathbf{T}_{i i}, \mathbf{T}_{j j}$ and polarimetric interferometric cross-correlation matrix $\boldsymbol{\Omega}_{i j}$ are defined as in (7): 


$$
\begin{aligned}
\mathbf{T}_{i i} & =E\left\{\mathbf{k}_{i} \mathbf{k}_{i}^{T *}\right\}, \quad \mathbf{T}_{j j}=E\left\{\mathbf{k}_{j} \mathbf{k}_{j}^{T *}\right\}, \quad \boldsymbol{\Omega}_{i j} \\
& =E\left\{\mathbf{k}_{i} \mathbf{k}_{j}^{T *}\right\}
\end{aligned}
$$

where $E\{\cdot\}$ is the expectation operator. As these expectations cannot be computed in practice, they are usually replaced by their maximum-likelihood estimates, given by the empirical mean evaluated using $L$ samples of the target vectors (multilook). Despite a larger number of looks generally implies better estimates (if the averaged area is sufficiently homogeneous), averaging also degrades spatial resolution, so a tradeoff is required, especially in urban, highly heterogeneous environments.

As for the amplitude dispersion index, it can be expressed as (Navarro-Sanchez VD et al. 2010):

$$
D_{A}=\frac{\sigma_{a}}{\bar{a}}=\frac{1}{\overline{\left|\boldsymbol{\omega}^{T *} \mathbf{k}\right|} \sqrt{N}} \sqrt{\sum_{i=1}^{N}\left(\left|\boldsymbol{\omega}^{T *} \mathbf{k}_{i}\right|-\overline{\left|\boldsymbol{\omega}^{T *} \mathbf{k}\right|}\right)^{2}}
$$

where $N$ is the total number of images and the overline indicates empirical mean value.

Note that different scattering mechanisms may correspond to different heights inside the resolution cell, so allowing the projection vector $\boldsymbol{\omega}$ to vary along time could introduce a variation in the phase term which might be misinterpreted as deformation. In order to avoid this, we constrain the optimum $\boldsymbol{\omega}$ obtained for each pixel to be the same along all the stack of images. This constraint is usually referred in the literature to as ESM (Equal Scattering Mechanisms) (Neumann et al. 2008).

\subsubsection{Experimental Results with Dual-Polarimetric Data and Comparison with Single-Polarimetric Data}

A set of 41 dual-pol, Single-look Slant-range Complex (SSC) images acquired by TerraSAR-X from February 19, 2009, to May 27, 2010, over the urban area of Murcia (Spain) has been used to test the proposed algorithms. All images have been acquired using StripMap mode (SM) at $\mathrm{HH}$ and VV channels, along ascending passes, with a mean incidence angle of 37.8 degrees. Azimuth and slant range resolutions are $6.6 \mathrm{~m}$ and $1.17 \mathrm{~m}$, whereas pixel dimensions are $2.44 \mathrm{~m}$ and $0.91 \mathrm{~m}$, respectively. Therefore, the resulting oversampling factors are 2.7 and 1.28 in azimuth and range. The processing has been applied over a part of the image with $2000 \times 2000$ pixels.

For comparison purposes, we have also computed deformation maps using a set of 41 single-pol SSC TerraSAR-X images, acquired at $\mathrm{VV}$ channel using SM mode, along descending passes, with a mean incidence angle of 35.3 degrees, from February 1, 2009, to May 20, 2010 (i.e., during the same period as the dual-pol images). These images have azimuth and slant-range resolutions of $3.0 \mathrm{~m}$ and $1.17 \mathrm{~m}$, with a pixel spacing of $1.89 \mathrm{~m}$ and $0.91 \mathrm{~m}$, respectively. We have selected a crop of $2582 \times 2000$ pixels corresponding approximately to the same area considered for the dual-pol images.

The test site and corresponding radar and validation data sets selected for the generation of this showcase on urban ground deformation monitoring are presented in Table 5.12. Despite the advantage of fully polarimetric data over dual-pol for this application, as stated later in the text, this test site was preferred due to the availability of reference ground data for validation and the granted access to the TerraSAR-X images for this purpose.

In this section, we present results obtained for dual-pol and single-pol data, using PSI based on both PS (selection by amplitude dispersion index) and CP (selection by average interferometric coherence). Note that for average coherence computation, a $7 \times 7$ multi-look scheme has been considered for both dual-pol and single-pol sets. Taking into account the oversampling factors presented before, this corresponds to an equivalent number of looks (ENL) of approximately 22 for single-pol images and 14 for dual-pol images. These ENLs are sufficient to ensure a negligible bias in the coherence estimation for coherence values over 0.6 for both data sets (Touzi et al. 1999). In order to minimize decorrelation issues and to keep a reasonable stack size, a $100 \mathrm{~m}$ limit for the perpendicular baseline and 100 days for the temporal baseline have been defined, resulting in the generation of 166 interferograms from the dual-pol images and 140 interferograms from the single-pol images. Table 5.13 shows, for the dual-pol data set, the percentage of PS and CP selected for the linear and the Pauli channels, as well as for the optimum channel, considering different thresholds for $D_{A}$ and $|\bar{\gamma}|$. The column labeled as Union corresponds to a simple optimization algorithm based on selecting the best channel

Table 5.12 Test sites and corresponding radar and validation data selected for the generation of showcases on urban subsidence monitoring

\begin{tabular}{l|l|l}
\hline Application/product & Test site - Radar data & Reference data \\
\hline $\begin{array}{l}\text { Urban subsidence } \\
\text { monitoring }\end{array}$ & Murcia & $\begin{array}{l}\text { Extensometer network (19 extensometers). Data provided by IGME (Instituto } \\
\text { Geológico y Minero de España) }\end{array}$ \\
\cline { 2 - 2 } & $\begin{array}{l}\text { 41 TerraSAR-X dual-pol images } \\
{[\mathrm{HH}, \mathrm{VV}]}\end{array}$ & \\
\cline { 1 - 2 } & $\begin{array}{l}\text { 41 TerraSAR-X single-pol } \\
\text { images [VV] }\end{array}$ & \\
\hline
\end{tabular}


Table 5.13 Percentage of pixel candidates selected for each channel, considering different and DA thresholds

\begin{tabular}{l|l|l|l|l|l|l}
\hline$|\bar{\gamma}|$ threshold & HH & VV & HH+VV & HH-VV & Union & OPT \\
\hline 0.6 & $39.15 \%$ & $35.18 \%$ & $31.74 \%$ & $41.45 \%$ & $47.25 \%$ & $48.81 \%$ \\
\hline 0.7 & $26.43 \%$ & $22.17 \%$ & $19.46 \%$ & $28.51 \%$ & $34.18 \%$ & $36.06 \%$ \\
\hline 0.8 & $13.93 \%$ & $10.52 \%$ & $9.19 \%$ & $15.44 \%$ & $19.28 \%$ & $20.94 \%$ \\
\hline 0.9 & $4.19 \%$ & $2.84 \%$ & $2.50 \%$ & $4.63 \%$ & $6.13 \%$ & $6.78 \%$ \\
\hline$D_{A}$ threshold & HH & VV & HH+VV & HH-VV & Union & OPT \\
\hline 0.3 & $6.27 \%$ & $5.17 \%$ & $5.06 \%$ & $6.52 \%$ & $10.97 \%$ & $6.38 \%$ \\
\hline 0.25 & $3.60 \%$ & $2.77 \%$ & $2.77 \%$ & $3.77 \%$ & $14.52 \%$ \\
\hline 0.2 & $1.73 \%$ & $1.24 \%$ & $1.28 \%$ & $1.78 \%$ & $3.11 \%$ & \\
\hline 0.15 & $0.58 \%$ & $0.39 \%$ & $0.43 \%$ & $0.58 \%$ & $4.34 \%$ \\
\hline
\end{tabular}

among all the copolar and the Pauli channels (similar to the algorithm described in (Pipia et al. 2009)), instead of searching over the whole polarimetric space, whereas the column OPT corresponds to the optimization approach described in Sect. 5.4.2. A clear improvement in terms of percentage of points selected as reliable is achieved for both criteria of selection. In the case of $\mathrm{CP}$ selection, if we compare the best-performing copolar channel, $\mathrm{HH}$, with the optimum channel OPT, we observe a significant increase of about $25 \%$ more pixels selected for the least restrictive threshold $(0.6)$. For more demanding thresholds $(0.8,0.9)$, the increment becomes more noticeable, up to a $60 \%$ increase in comparison with $\mathrm{HH}$. In this case, the simple algorithm consisting in choosing the best from a reduced set of channels (denoted as Union) achieves a suboptimal solution at a considerably lower computational cost. Also note that, in this urban scenario, the HH-VV channel, generally associated with double-bounce reflections like the ones originated by building façade-ground dihedrals, is the best choice from the set of copolar and Pauli channels. The increase in the number of selected pixels is more spectacular for PS selection since in this case the optimization is applied to single-look data (i.e., not spatially averaged), which are more sensitive to the geometrical and polarimetric features of dominant scatterers inside the resolution cell. Consequently, improvement ranges in this case from $130 \%$ (more than 2 times more pixels selected) for the least restrictive threshold (0.3) to $170 \%$ (2.7 times more pixels) for the most restrictive threshold (0.15), when comparing the optimum channel to the reference single-pol channel HH. Notice that in this case, the Union approach is far from providing the same improvement, yielding results at midway between single-pol and optimized ones.

For comparison, Table 5.14 shows results obtained for the single-pol data set, at VV channel. Given the different acquisition geometry (descending vs ascending), the selected area of study is not exactly the same as for the dual-pol set, but results are still quite similar to those obtained for the dual-pol set at VV channel in terms of selected pixels density. Note that the higher azimuth resolution of the single-pol images implies that we dispose of more pixels for the same area, but
Table 5.14 Percentage of pixel candidates selected for the single-pol VV images set, for different and DA thresholds

\begin{tabular}{l|l|l|l|l}
\hline$|\bar{\gamma}|$ threshold & 0.6 & 0.7 & 0.8 & 0.9 \\
\hline VV & $32.92 \%$ & $21.83 \%$ & $11.08 \%$ & $3.35 \%$ \\
\hline$D_{A}$ threshold & 0.3 & 0.25 & 0.2 & 0.15 \\
\hline VV & $5.70 \%$ & $3.30 \%$ & $1.65 \%$ & $0.65 \%$ \\
\hline
\end{tabular}

the ratio between selected and total pixels remains similar. Therefore, for improving the total area coverage, it is better to opt for polarimetrically optimized data (see more comments on that at the end of this section).

In Figs. 5.37 and 5.38, we present maps of the deformation velocity obtained using PSI based on CP and PSI based on PS, respectively. Both single-pol and dual-pol sets have been processed. We easily appreciate the increase in the density of pixels with output deformation estimates and the appearance of new details that did not show up in single-pol channels. Concerning the estimated deformation values, we also observe that for the dual-pol set (for both optimized and not optimized channels), an area of slow ground subsidence is found in the north of the city, which appears as stable for the single-pol data set.

An attempt to validate the obtained results with the available extensometer measures has been carried out, but unfortunately neither the spatial sampling (19 extensometers in total and only 12 inside the area of interest) nor the temporal sampling (only 3 measures per extensometer inside the time span) were sufficient for a proper deformation characterization. In addition, available extensometers data exhibit unexpected uplift measures that do not match the general deformation trend of the area, which has been studied in several works (Herrera et al. 2010; Monells et al. 2010).

Finally, Fig. 5.39 illustrates in a more precise way the increase of area coverage achieved by the polarimetric optimization procedure. An area of $1 \mathrm{~km}^{2}$ has been selected, and the actual coverage has been computed by taking into account projected pixel sizes: approximately $2.44 \mathrm{~m}$ azimuth and $1.48 \mathrm{~m}$ range for dual-pol images and $1.89 \mathrm{~m}$ azimuth and $1.56 \mathrm{~m}$ range for single-pol. Note that in the 


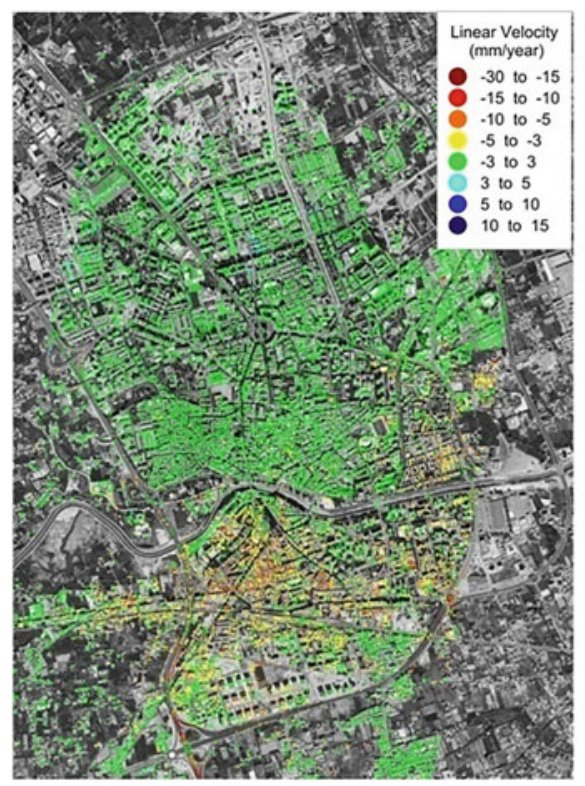

VV (single-pol set), $11.08 \%$

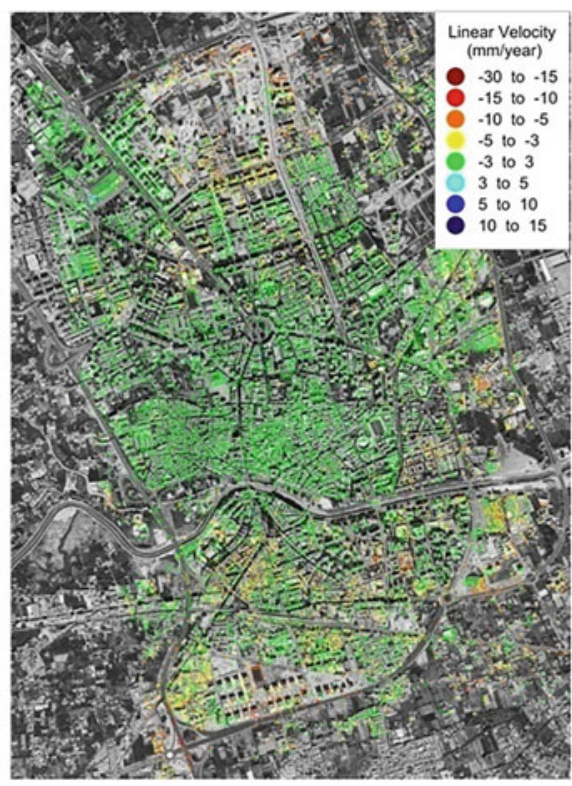

VV (dual-pol set), $10.52 \%$

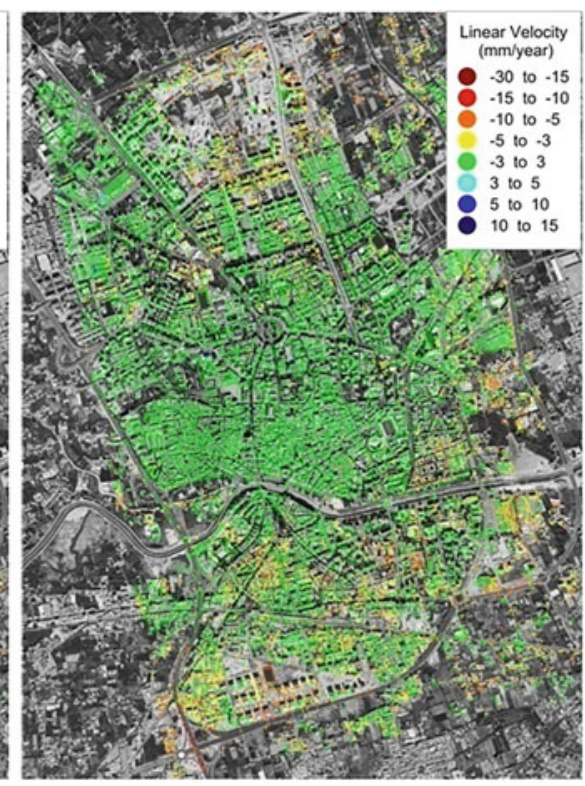

OPT, CP $20.94 \%$

Fig. 5.37 Deformation velocity maps obtained for CP-PSI, considering an average coherence threshold of 0.8. The percentage of candidate pixels is reported

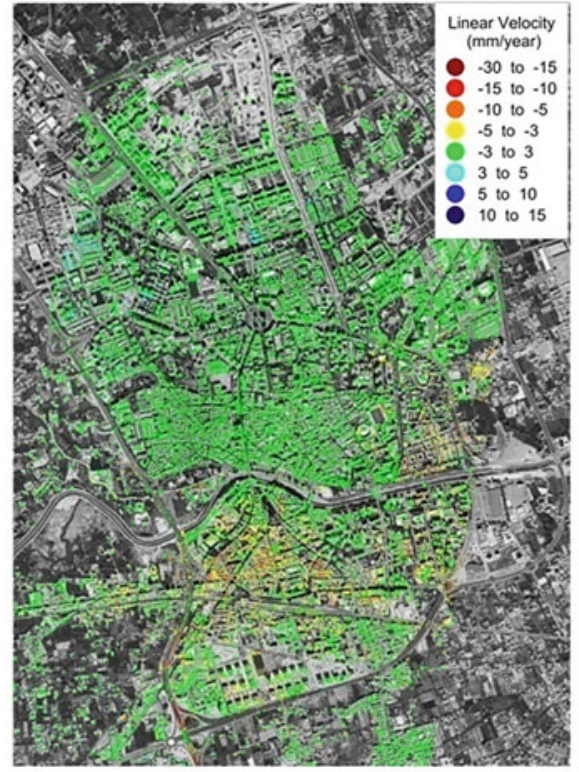

VV (single-pol set), $3.30 \%$

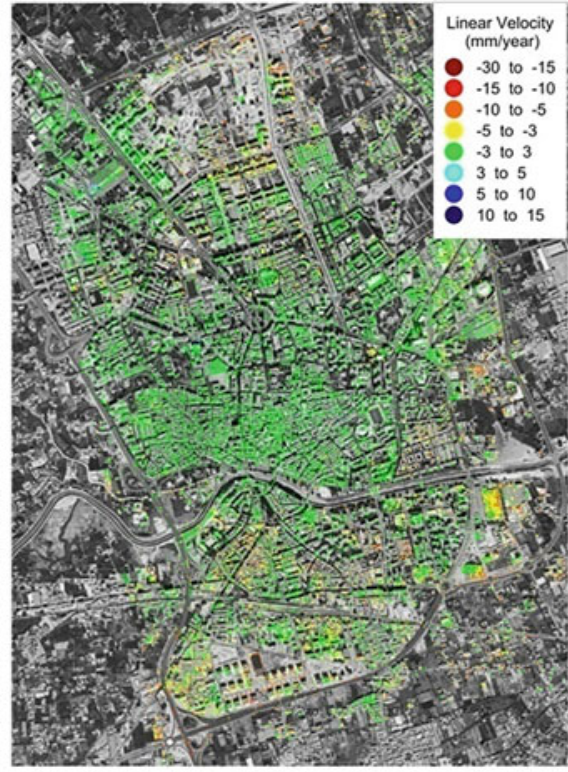

VV (dual-pol set), $2.77 \%$

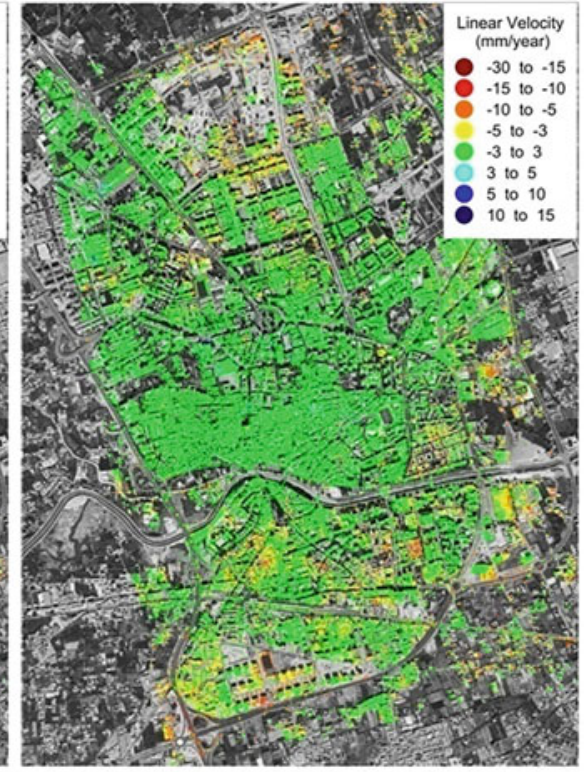

OPT, $8.63 \%$

Fig. 5.38 Deformation velocity maps obtained for PS-PSI, considering a $D_{A}$ threshold of 0.25

case of $\mathrm{CP}$, pixel size is increased by the multi-look factor $(7 \times 7)$, so in general we will have large area coverage but poorer resolution. As extracted from the figure, the better resolution of single-pol images does not affect actual coverage as significantly as the polarimetric optimization procedure. 

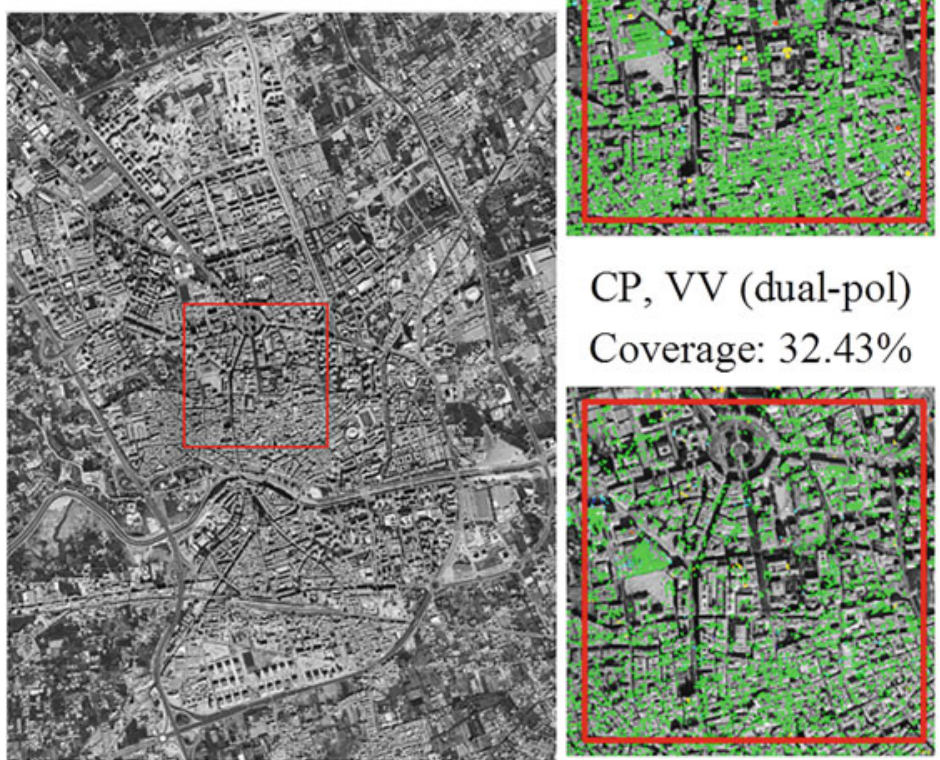

PS, VV (dual-pol)

Coverage: $4.41 \%$

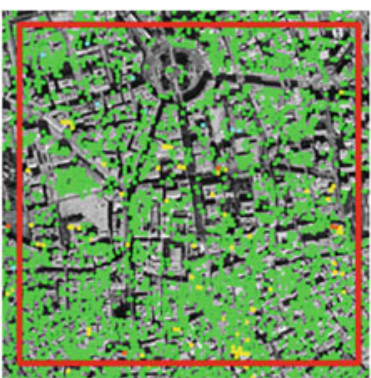

CP, VV (single-pol)

Coverage: $36.38 \%$

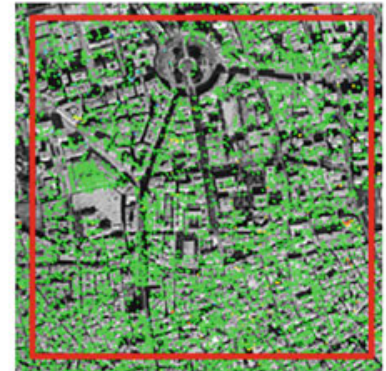

PS, VV (single-pol)

Coverage: $5.58 \%$

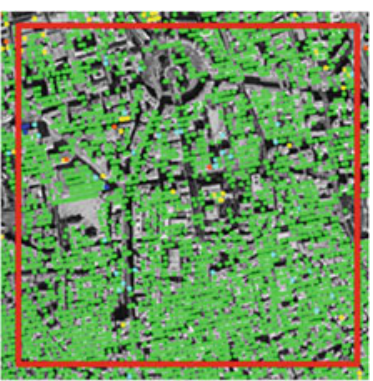

$\mathrm{CP}, \mathrm{OPT}$

Coverage: $51.63 \%$

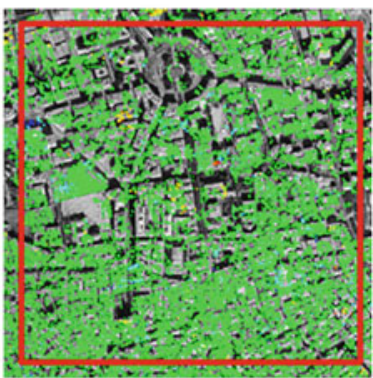

PS, OPT

Coverage: $14.04 \%$

Fig. 5.39 PSI area coverage comparison, for CP and PS modes

\subsubsection{Discussion on the Role of Polarimetry and on the Maturity of the Application, and Conclusions}

In this study we demonstrate how polarimetric diversity can enhance the performance of conventional PSI techniques without introducing significant changes to the processing chain, getting an important improvement in terms of deformation maps density and spatial coverage. In addition, polarimetry can be of use for PS/CP characterization, allowing us to assign in a more precise way each PS/CP to actual targets, hence widening the range of applications of these techniques.

In its current status, polarimetric PSI can be regarded as a sufficiently mature approach to be used successfully with a variety of polarimetric configurations. It is important to mention that first experiments with full-pol data (NavarroSanchez and Lopez-Sanchez 2013) show a more significant improvement than dual-pol, increasing the density of selected pixels up to twice compared to dual-pol optimized data, and more than four times compared to single-pol. Compared to the copolar dual-pol data analyzed here, the HV channel adds a great deal of information, given the important crosspolar response coming from tilted dihedrals in urban areas (oriented buildings). Note that the described optimization procedure based on parameter search, though robust, can be computationally costly for fully polarimetric data, so more efficient optimization methods are currently under development. Finally, additional efforts have yet to be made in the validation of results with ground truth data.

\subsection{Summary}

For each application concerning urban monitoring, the methods presented are summarized, with their acquisition modes and the required frequency bands in Table 5.15. 
Table 5.15 Summary of presented application, methods, and preferred system configurations for urban areas monitoring

\begin{tabular}{|c|c|c|}
\hline Application & Methods and used frequency (P/L/C/X) & $\begin{array}{l}\text { Radar data preference/requirements/ } \\
\text { comments }\end{array}$ \\
\hline \multirow[t]{3}{*}{ Classification } & Detection of built-up areas after optimization of PolInSAR coherence (X) & $\begin{array}{l}\text { Preferred frequency: } \mathrm{X} \text { (more sensitive } \\
\text { to details) }\end{array}$ \\
\hline & \multirow{2}{*}{$\begin{array}{l}\text { Based on PolSAR parameters: LL/RR correlation coefficient and four-components } \\
\text { decomposition }(\mathrm{X})\end{array}$} & High resolution \\
\hline & & Single-pass or short revisit time \\
\hline \multirow[t]{7}{*}{ 3-D rendering } & \multirow[t]{5}{*}{ Based on PolInSAR coherence region (X) } & $\begin{array}{l}\text { Preferred frequency: } \mathrm{X} \text { (more sensitive } \\
\text { to details) }\end{array}$ \\
\hline & & High resolution \\
\hline & & Single-pass or short revisit time \\
\hline & & Large enough ambiguity height \\
\hline & & Good SNR in HV \\
\hline & \multirow[t]{2}{*}{ Based on polarimetric tomography $(\mathrm{L})$} & Relatively short revisit time \\
\hline & & High resolution desired \\
\hline \multirow[t]{2}{*}{$\begin{array}{l}\text { Subsidence } \\
\text { monitoring }\end{array}$} & $\begin{array}{l}\text { DInSAR after PolInSAR coherence optimization with selection of reliable pixels } \\
\text { (C) }\end{array}$ & \\
\hline & $\begin{array}{l}\text { Persistent scatterer interferometry after a model-based PolInSAR coherence } \\
\text { optimization }(\mathrm{X})\end{array}$ & \\
\hline
\end{tabular}

\section{References}

Arii M et al (2011) Adaptive model-based decomposition of polarimetric SAR covariance matrices. IEEE Trans Geosci Remote Sens 49:1104-1113

Berardino P et al (2002) A new algorithm for surface deformation monitoring based on small baseline differential SAR interferograms. IEEE Trans Geosci Remote Sens 40:2375-2383

Blanco P et al (2008) The coherent pixels technique (CPT): an advanced DInSAR technique for non-linear deformation monitoring. Pure Appl Geophys 165:1167-1193

Boehm C, Schenkel R (2003) Analysis of high resolution polarimetric SAR in urban areas. In: Proceedings of IEEE 2003 workshop advances in techniques for analysis of remotely sensed data

Cao F et al. (2011) Influence of speckle filtering of polarimetric SAR data on different classification methods. In: Proceedings of IEEE 2011 International Geoscience and Remote Sensing Symposium (IGARSS)

Cloude SR (2009) Polarisation: applications in remote sensing. Oxford University Press, Oxford

Cloude SR, Papathanassiou KP (1998) Polarimetric SAR interferometry. IEEE Trans Geosci Remote Sens 36:1551-1565

Colin E et al (2006) An interferometric coherence optimization method in radar polarimetry for high-resolution imagery. IEEE Trans Geosci Remote Sens 44:167-175

Colin-Koeniguer E, Trouve N (2014) Performance of building height estimation using high-resolution PolInSAR images. IEEE Trans Geosci Remote Sens 52:5870-5879

Colin-Koeniguer E, Weissgerber F, Trouvé N, Nicolas JM (2015) A new light on misclassification results on the SOMA district in San Francisco due to the difficulty to predict entropy. Paper presented at the International Workshop on Science and Applications of SAR Polarimetry and Polarimetric Interferometry (POLinSAR)

Deledalle C et al (2015) NL-SAR: a unified non-local framework for resolution-preserving (Pol)(In)SAR denoising. IEEE Trans Geosci Remote Sens 53:2021-2038
Dheenathayalan P, Hansen, R (2011) Target characterization and interpretation of deformation using persistent scatterer interferometry and polarimetry. Paper presented at 2011 International Workshop on Science and Applications of SAR Polarimetry and Polarimetric Interferometry (POLinSAR)

Ferretti A et al (1999) Permanent scatterers in SAR interferometry. In: Proceedings of IEEE 1999 International Geoscience and Remote Sensing Symposium (IGARSS)

Ferretti A et al (2001) Permanent scatterers in SAR interferometry. IEEE Trans Geosci Remote Sens 39:8-20

Ferretti A et al (2011) A new algorithm for processing interferometric data-stacks: SqueeSAR. IEEE Trans Geosci Remote Sens 49:3460-3470

Ferro-Famil L, Pottier E (2007) Urban area remote sensing from L-band PolSAR data using time-frequency techniques. In: Proceedings of IEEE 2007 Joint Urban Remote Sensing Event (JURSE)

Freeman A, Durden S (1998) A three-component scattering model for polarimetric SAR data. IEEE Trans Geosci Remote Sens 36:963-973

Guillaso S et al (2005) Building characterization using L-band polarimetric interferometric SAR data. IEEE Geosci Remote Sens Lett 2:347-351

Hanssen RF (2001) Radar interferometry. Data interpretation and error analysis. Kluwer Academic Publishers, Dordrecht

Herrera G et al (2010) Analysis of subsidence using TerraSAR-X data: Murcia case study. Eng Geol 116:284-295

Hooper A et al (2004) A new method for measuring deformation on volcanoes and other natural terrains using InSAR persistent scatterers. Geophys Res Lett. https://doi.org/10.1029/ 2004GL021737

Hooper A et al (2007) Persistent scatterer interferometric synthetic aperture radar for crustal deformation analysis, with application to Volcán Alcedo, Galápagos. J Geophys Res Solid Earth. https://doi. org/10.1029/2006JB004763

Huang Y et al (2012) Under-foliage object imaging using SAR tomography and polarimetric spectral estimators. IEEE Trans Geosci Remote Sens 50:2213-2225 
Iglesias R et al (2012) Polarimetric optimization for DInSAR pixel selection with ground-based SAR. In: Proceedings of IEEE 2012 International Geoscience and Remote Sensing Symposium (IGARSS)

Iribe K, Sato M (2007) Analysis of polarization orientation angle shifts by artificial structures. IEEE Trans Geosci Remote Sens 45:3417-3425

Kampes BM (2006) Radar interferometry. Persistent scatterer technique. Springer, Berlin/Heidelberg

Kostinski A, Boerner W (2009) On foundations of radar polarimetry. IEEE Trans Antennas Prop 34:1395-1404

Lee JS, Ainsworth TL (2011) The effect of orientation angle compensation on coherency matrix and polarimetric target decompositions. IEEE Trans Geosci Remote Sens 49:53-64

Lee JS, Pottier E (2009) Polarimetric radar imaging - from basics to applications. CRC Press, Boca Raton

Lee JS et al (2002) On the estimation of radar polarization orientation shifts induced by terrain slopes. IEEE Trans Geosci Remote Sens 40:30-41

Liu Y et al (2010) Urban area extraction from polarimetric SAR imagery using only positive samples. In: Proceedings of IEEE 2010 International Conference on Acoustic, Speech and Signal Processing (ICASSP)

Monells D, Mallorqui JJ (2013) Performance comparison between dual polarimetric and fully polarimetric data for DInSAR subsidence monitoring. Paper presented at 2013 International Workshop on Science and Applications of SAR Polarimetry and Polarimetric Interferometry (POLinSAR)

Monells D et al (2010) Application of TerraSAR-X data to the monitoring of urban subsidence in the city of Murcia. In: Proceedings of IEEE 2010 International Geoscience and Remote Sensing Symposium (IGARSS)

Monells D et al (2012) Phase quality optimization in orbital differential SAR interferometry with fully polarimetric data. In: Proceedings of IEEE 2012 International Geoscience and Remote Sensing Symposium (IGARSS)

Mora $\mathrm{O}$ et al (2003) Linear and nonlinear terrain deformation maps from a reduced set of interferometric SAR images. IEEE Trans Geosci Remote Sens 41:2243-2253

Moriyama $T$ et al (2004) A study on extraction of urban areas from polarimetric Synthetic Aperture Radar images. In: Proceedings of IEEE 2004 International Geoscience and Remote Sensing Symposium (IGARSS)

Moriyama T et al (2005) A study on polarimetric correlation coefficient for feature extraction of polarimetric SAR data. IEICE Trans Commun E88-6:235-236

Navarro-Sanchez VD, Lopez-Sanchez JM (2011a) Subsidence monitoring using polarimetric persistent scatterers interferometry. In: Proceedings of IEEE 2011 International Geoscience and Remote Sensing Symposium (IGARSS)

Navarro-Sanchez VD, Lopez-Sanchez JM (2011b) Polarimetric stationarity criteria applied to the selection of pixel scatterers candidates. Paper presented at 2011 International Workshop on Science and Applications of SAR Polarimetry and Polarimetric Interferometry (POLinSAR)

Navarro-Sanchez VD, Lopez-Sanchez JM (2012) Improvement of persistent-scatterer interferometry performance by means of a polarimetric optimization. IEEE Geosci Remote Sens Lett 9:609-613

Navarro-Sanchez VD. Lopez-Sanchez JM (2013) Polarimetric adaptive speckle filtering driven by temporal statistics for PSI applications. Paper presented at 2013 International Workshop on Science and Applications of SAR Polarimetry and Polarimetric Interferometry (POLinSAR)
Navarro-Sanchez VD et al (2010) A contribution of polarimetry to satellite differential SAR interferometry: increasing the number of pixel candidates. IEEE Geosci Remote Sens Lett 7:276-280

Navarro-Sanchez VD et al (2014) Polarimetric approaches for persistent scatterers interferometry. IEEE Trans Geosci Remote Sens 52:1667-1676

Neumann M et al (2008) Multibaseline polarimetric SAR interferometry coherence optimization. IEEE Geosci Remote Sens Lett 5:93-97

Pascual $\mathrm{C}$ et al. (2002) The equivalence between the polarization subspace method (PSM) and the coherence optimization in polarimetric radar interferometry. In: Proceedings of 2002 European Conference on Synthetic Aperture Radar (EUSAR)

Pellizzeri TM et al (2003) Model-based processing of multifrequency polarimetric SAR images of urban areas. In: Proceedings of GRSS/ ISPRS 2003 Joint Workshop Remote Sensing and Data Fusion over Urban Areas

Perissin D, Ferretti A (2007) Urban-target recognition by means of repeated spaceborne SAR images. IEEE Trans Geosci Remote Sens 45:4043-4058

Pipia L et al (2009) Polarimetric differential SAR interferometry: first results with ground-based measurements. IEEE Geosci Remote Sens Lett 6:167-171

Pipia L et al (2012) Polarimetric temporal analysis of urban environments with a ground-based SAR. IEEE Trans Geosci Remote Sens 51:2343-2360

Praks J et al (2009) Alternatives to target entropy and alpha angle in SAR polarimetry. IEEE Trans Geosci Remote Sens 47:2262-2274

Qong M et al (2005) Coherence optimization using the polarization state conformation in PolInSAR. IEEE Geosci Remote Sens Lett 2:301-305

Sagues L et al (2000) Indoor experiments on polarimetric SAR interferometry. IEEE Trans Geosci Remote Sens 38:671-684

Samsonov S, Tiampo K (2011) Polarization phase difference analysis for selection of persistent scatterers in SAR interferometry. IEEE Geosci Remote Sens Lett 8:331-335

Sato A et al (2012) Four-component scattering power decomposition with extended volume scattering model. IEEE Geosci Remote Sens Lett 9:166-170

Sauer S et al (2007) 3-D visualisation and physical feature extraction of urban areas using multibaseline POL-InSAR data at L-Band. In: Proceedings of IEEE 2007 Joint Urban Remote Sensing Event (JURSE)

Sauer S et al (2011) Three-dimensional imaging and scattering mechanism estimation over urban scenes using dual-baseline polarimetric InSAR observations at L-Band, Geoscience and Remote Sensing. IEEE Trans Geosci Remote Sens 49:4616-4629

Schuler DL et al. (2006) Polarimetric SAR detection of man-made structures using normalized circular-pol correlation coefficients. In: Proceedings of IEEE 2006 International Geoscience and Remote Sensing symposium (IGARSS)

Singh G et al (2013) General four-component scattering power decomposition with unitary transformation of coherency matrix. IEEE Trans Geosci Remote Sens 51:3014-3022

Stoica P, Nehorai A (1990) Performance study of conditional and unconditional direction-of-arrival estimation. IEEE Trans Acoust Speech Signal Proc ASSP-38:1783-1795

Swindlehurst AL, Viberg M (1993) Subspace fitting with diversely polarized antenna arrays. IEEE Trans Antennas Prop 41:1687-1694

Thirion-Lefevre L et al (2020) The combined effect of orientation angle and material on PolSAR images of urban areas. Remote Sens 12 (10): 1632

Touzi R et al (1999) Coherence estimation for SAR imagery. IEEE Trans Geosci Remote Sens 37:135-149 
Viberg M, Ottersten B (1991) Sensor array processing based on subspace fitting. IEEE Trans Signal Proc 39:1110-1121

Wang Y et al (2010) PolSAR data segmentation by combining tensor space cluster analysis and Markovian framework. IEEE Geosci Remote Sens Lett 7:210-214

Yamaguchi Y et al (2005) Four-component scattering model for polarimetric SAR image decomposition. IEEE Trans Geosci Remote Sens 43:1699-1706
Yamaguchi Y et al (2008) Classification of terrain by implementing the correlation coefficient in the circular polarization basis using X-band PolSAR data. IEICE Trans Commun E91-B:297-301

Yamaguchi Y et al (2011) Four-component scattering power decomposition with rotation of coherency matrix. IEEE Trans Geosci Remote Sens 49:2251-2258

Open Access This chapter is licensed under the terms of the Creative Commons Attribution 4.0 International License (http://creativecommons.org/ licenses/by/4.0/), which permits use, sharing, adaptation, distribution and reproduction in any medium or format, as long as you give appropriate credit to the original author(s) and the source, provide a link to the Creative Commons license and indicate if changes were made.

The images or other third party material in this chapter are included in the chapter's Creative Commons license, unless indicated otherwise in a credit line to the material. If material is not included in the chapter's Creative Commons license and your intended use is not permitted by statutory regulation or exceeds the permitted use, you will need to obtain permission directly from the copyright holder. 


\title{
Ocean Applications
}

\author{
M. Migliaccio, F. Nunziata, A. Marino, C. Brekke, and S. Skrunes
}

\begin{abstract}
In this chapter, the most promising techniques to observe oil slicks and to detect metallic targets at sea using polarimetric synthetic aperture radar (SAR) data are reviewed and critically analysed. The detection of oil slicks in SAR data is made difficult not only by the presence of speckle but also by the presence of, e.g. biogenic films, low-wind areas, rain cells, currents, etc., which increase the false alarm probability. The use of polarimetric features has been shown to both observe oil slicks and distinguish them from weak-damping look-alikes but also to extract some of their properties. Similarly to oil slicks, the same factors can hamper the detection of metallic targets at sea. The radiometric information provided by traditional single-channel SAR is not generally sufficient to unambiguously detect man-made metallic targets over the sea surface. This shortcoming is overcome by employing polarimetry, which allows to fully characterize the scattering mechanism of such targets.
\end{abstract}

\subsection{Introduction}

The two main ocean applications analysed are related to oil slicks and metallic target detection.

SAR oil slick observation is physically possible under low-to-moderate wind conditions $(\sim 3-12 \mathrm{~m} / \mathrm{s})$, because an oil slick damps the short gravity and capillary waves and reduces the friction velocity generating a region of low

M. Migliaccio $(\bowtie) \cdot$ F. Nunziata

Dipartimento di Ingegneria, University of Naples "Parthenope", Naples, Italy

e-mail: m.m@uniparthenope.it

A. Marino

Biological and Environmental Sciences, University of Stirling, Stirling, UK

C. Brekke $\cdot$ S. Skrunes

UiT the Arctic University of Norway, Troms $\varnothing$, Norway backscatter area in the SAR image. However, SAR oil slick detection is not an easy task, since SAR images are affected by multiplicative noise, known as speckle, which hampers interpretability of such images. Furthermore, there are other physical phenomena, known as look-alikes, which can generate dark areas in SAR images not related to oil slicks, such as biogenic films (e.g. slicks produced by animals and plankton), low-wind areas, areas of wind-shadow near coasts, rain cells, currents, zones of upwelling, internal waves and oceanic or atmospheric fronts. Tailored filtering techniques must be developed in order to minimize the number of false alarms. Within such a context, single-polarization SAR oil slick detection procedures can be generally divided into three phases: dark area detection, feature extraction and oil slick/ look-alike classification. On the basis of the estimated features and some a priori knowledge, it is possible to assign a probability that a dark area is an oil slick. Furthermore, the usefulness of additional external data is recognized to enhance the ability to distinguish between oil slicks and look-alikes, such as local wind field information (to sort out low-wind areas) and optical data to identify biogenic films. Recently, the superiority of polarimetric SAR measurements for sea oil slick observation purposes has been demonstrated. New quad- and dual-polarimetric approaches have been recently developed for sea oil slick observation, under lowto-moderate wind conditions, and are here considered.

Polarimetric models and analysis tools have been developed to observe sea oil slicks and have shown that some polarimetric features, namely, HH-VV correlation, unpolarized backscattered energy and scene depolarization capabilities, can be successfully used to both observe oil slicks and distinguish them from weak-damping look-alikes (Nunziata et al. 2012a; Solberg 2012).

With respect to the state of the art related to SAR observation of man-made metallic targets at sea, both image- and physically based approaches have been developed. The electromagnetic wave scattered off man-made metallic targets at sea is physically determined by several scattering 
mechanisms that cause a high coherent microwave response depending on the construction material and the characteristics of the radar instrument, such as incidence angle, frequency, polarization, resolution and speckle. Accordingly, ships and oil rigs, hereinafter man-made metallic targets, appear in SAR images as bright spots over a dark marine background. Following this rationale, many image-based techniques have been developed which seek for anomalies in SAR images. However, SAR observation of man-made metallic targets at sea is a nontrivial task due to speckle and natural physical processes, e.g. atmospheric fronts, internal waves, current boundaries, breaking waves, outlying rocks, shoals, sea currents, coastal effects, etc., which may generate false alarms. Accordingly, man-made metallic targets detection is a complex topic that can hardly be optimized with conventional single-polarization SARs. Radiometric information provided by traditional single-channel SAR is not generally sufficient to unambiguously detect man-made metallic targets over the sea surface, since it does not fully characterize the scattering mechanism of such targets. Hence, new polarimetric approaches have been developed for man-made metallic targets observation at sea that perform better than the singlepolarization ones.

In this chapter the most promising polarimetric techniques to observe oil slicks and to detect metallic targets at sea are reviewed and critically analysed.

\subsection{Oil at Sea Observation}

\subsubsection{Introduction, Motivation and Literature Review}

Oil at sea observation has received considerable attention in recent literature due to its impact on marine ecosystems, fisheries, wildlife, etc. SAR has proven to be a fundamental remote sensing tool for oil slick mapping, under low-tomoderate wind conditions $(3-15 \mathrm{~m} / \mathrm{s})$, due to its almost all-weather and its all-day imaging capabilities. In very simple terms, the physical rationale underpinning SAR oil slick observation resides on its viscoelastic properties that damp the short Bragg resonant waves resulting in a low backscatter area, i.e. a dark patch in the SAR image plane.

Marine slicks are composed of two major types of hydrocarbons, mineral oils including petroleum-based material and films from biological processes. Mineral oils are typically due to spills from ships and offshore drilling platforms and pipelines, natural hydrocarbon seeps and discharge from storm-water urban run-off. Mineral oils spread into thin layers through gravity and surface tension and evaporate through weather over time. Biogenic films, also called surfactants (surface-active agents) or natural films, are a viscous by-product of ocean plant and animal growth and decay.
Traditionally, SAR oil slick observation is based on single-polarization VV or $\mathrm{HH}$ intensity images, and manual inspection is very often still needed (Solberg 2012).

With polarimetric SAR sensor capabilities now operationally available, several polarimetric techniques have been proposed for oil slick monitoring showing superiority with respect to single-polarization SAR cases (Nunziata et al. 2012a; Solberg 2012). Here, a brief review of the most upto-date polarimetric techniques to observe oil at sea is provided. Then, some of the most promising polarimetric techniques are applied to selected test cases in order to clearly show the benefit of polarimetric SAR data for oil at sea observation.

Polarimetric models and analysis tools have been developed to observe sea oil slicks and have shown that some polarimetric features, namely, the correlation between the co-polarized (HH and VV) channels, unpolarized backscattered energy and scene depolarization capabilities, can be successfully used to both observe oil slicks and distinguish them from weak-damping look-alikes (Nunziata et al. 2012a). All these approaches share a common physical rationale that relies on the fact that, under low-to-moderate wind conditions $(3-12 \mathrm{~m} / \mathrm{s})$ and at intermediate incidence angles, both slick-free and weak-damping slick-covered sea surfaces call for Bragg scattering, whereas in the case of oil-covered sea surfaces, a completely different one, i.e. non-Bragg scattering mechanism, is in place. Following this rationale, these polarimetric features, which can be considered as measures of the departure from Bragg scattering, have been estimated by either the scattering $\mathbf{S}$, the Kennaugh $\mathbf{K}$ or the coherency $\mathbf{T}$ matrices and have been successfully used to observe sea oil slicks in polarimetric SAR data (Nunziata et al. 2012a). A brief description of the state of the art related to polarimetric SAR data for oil at sea observation is provided as follows.

In Migliaccio et al. (2011a)), the benefits of polarimetric RADARSAT-2 SAR data to observe the Deepwater Horizon oil spill were shown using polarimetric features. In Minchew et al. (2012)) and Migliaccio and Nunziata (2014)), polarimetric features were extracted from L-band UAVSAR fullpolarimetric SAR data to observe the oil slicks due to the Deepwater Horizon accident in Gulf of Mexico. It was shown that the dominant scattering mechanism, both over the slickfree and the slick-covered sea surface, is the tilted-Bragg scattering. In Jones et al. (2011)), polarimetric UAVSAR data collected over the polluted area of the Gulf of Mexico were exploited to observe oil-affected wetlands in Barataria Bay, Louisiana. In Nunziata et al. (2008)), a model to interpret the elements of the Mueller matrix in terms of sea surface scattering with and without oil slicks was developed. In Nunziata et al. (2011)), the co-polarized signature, i.e. the plot of the synthesized normalized radar cross section (NRCS) as a function of ellipticity and orientation angle, was exploited to observe oil at sea. The pedestal of the 
signature is shown to be both able to observe oil at sea and distinguish them from weak-damping look-alikes. In Migliaccio et al. (2009a)), multi-polarization features were exploited to conceive a polarimetric processing chain to both observe oil slicks and distinguish them from weak-damping look-alikes. In Zhang et al. (2011)), full-polarimetric SAR data were used to observe oil seeps in the Gulf of Mexico. In Migliaccio et al. (2009b) and Velotto et al. (2011)), the standard deviation of the co-polarized phase difference (CPD) was shown to result in larger values over oil-covered areas and in very low values over both free sea surface and over biogenic slicks. In Migliaccio et al. (2011b)), the CPD approach was used together with a target detection approach, based on HH-HV SAR data, to conceive a processing chain able to observe both oil slicks and metallic targets at sea; i.e. oil field, in an unsupervised way.

\subsubsection{Methodology}

In this section the theoretical background behind the operational procedures is briefly described, starting from quadpolarimetric approaches and, then, moving to the dualpolarimetric one. To provide an effective physical description, the section presents a brief description of the polarimetric model that describes sea surface scattering with and without oil slicks, which is the backbone of the selected polarimetric approaches.

Under low-to-moderate wind conditions and at intermediate incidence angles, two reference scenarios must be distinguished: sea surface with and without oil slicks.

Slick-free sea surface scattering calls for Bragg or tiltedBragg scattering. It is a single-reflection scattering mechanism that, being quasi-deterministic, is expected to be characterized by a low polarimetric entropy, a high correlation between likepolarized channels and a low unpolarized backscattered energy, as demonstrated in Nunziata et al. (2012a)). Similar polarimetric scattering features apply when dealing with weakdamping surfactants; e.g. biogenic slicks, some marine features, etc. In real cases, illegal oil discharges are typically heavy oils that do not belong to this class.

An oil-covered sea surface, due to the strong damping properties of oil slicks, dampens the short Bragg scattering waves generating a low backscatter area (i.e. a dark area in single-pol SAR images). From a polarimetric viewpoint, this means a large departure from the Bragg scattering mechanism (Nunziata et al. 2012a). In detail, a low correlation between like-polarized channels and a large unpolarized backscattered energy (which indicates that a high random scattering mechanism is in place) is expected, as demonstrated in Nunziata et al. (2012a)).

Following this rationale, polarimetric features, which can be considered as measures of the departure from Bragg scattering, have been successfully used to observe sea oil slicks in polarimetric SAR data. All the polarimetric features can be derived by the second-order products of the scattering matrix; i.e. either the coherency $\mathbf{T}$ or the covariance $\mathbf{C}$ matrix can be used. In this study the following polarimetric features are used: polarimetric entropy $(H)$, modified anisotropy $\left(A_{12}\right)$ (Wenguang et al. 2010), the mean scattering parameter $\bar{\alpha}$, normalized pedestal height (NP) (Nunziata et al. 2012a), conformity coefficient (Zhang et al. 2011) $\mu$ and standard deviation of the co-polarized phase difference $(\sigma)$ (Migliaccio et al. 2009b). According to theoretical modelling, those polarimetric features are expected to follow the behaviour synthesized in Table 6.1.

\subsubsection{Experimental Results}

The Gulf of Mexico site includes both oil slicks related to the Deepwater Horizon accident and oil seeps. Test sites and corresponding radar and validation data sets selected for the generation of showcases are summarized in Table 6.2 and further described in the appendix.

In this sub-section the polarimetric approaches described in Sect. 6.2.2 are applied to actual polarimetric SAR data

Table 6.1 Expected behaviour of the polarimetric features

\begin{tabular}{l|l|l}
\hline Polarimetric feature & Slick-free & Oil-covered \\
\hline$H$ & Low & High \\
\hline$A_{12}$ & High & Low \\
\hline $\bar{\alpha}$ & Low & Intermediate \\
\hline$N P$ & Low & High \\
\hline$\mu$ & Positive & Negative \\
\hline$\sigma$ & Low & High \\
\hline
\end{tabular}

Table 6.2 Test sites and corresponding radar and validation data selected for the generation of showcases on oil at sea observation

\begin{tabular}{l|l|l}
\hline Application/Product & Test site - radar data & Reference data \\
\hline \multirow{2}{*}{ Oil at sea observation } & Gulf of Mexico & Deepwater Horizon oil slick \\
\cline { 2 - 3 } & 3 RADARSAT-2 fine quad-pol & Oil seeps \\
\cline { 2 - 3 } & Gulf of Mexico & Deepwater Horizon oil slick \\
\cline { 2 - 3 } & 2 RADARSAT-2 fine quad-pol & \\
\cline { 2 - 3 } & Gulf of Mexico & 1 UAVSAR MLC quad-pol \\
\hline
\end{tabular}


where verified oil slicks are present to clearly show the added-value information that can be obtained by PolSAR data. All the polarimetric features provide similar results; hence, to save space, only the outputs obtained using NP and $\mu$, evaluated over the whole image by using a $7 \times 7$ average moving, are shown.

The first experiment is relevant to the RADARSAT2 SAR scene collected on May 15, 2010. The wind speed is 4-7 $\mathrm{m} / \mathrm{s}$, and the incidence angle varies in the range $29.1^{\circ}$ $30.9^{\circ}$. The imaged scene contains few slicks, related to the Deepwater Horizon accident, that result in areas darker than the background sea both in the Pauli and in the singlepolarization grey-tones VV image; see Fig. 6.1a, b. There are also few bright spots that are due to metallic targets at sea. It can be noted that the slick-covered sea area is very heterogeneous and presents some holes that, although present also in the VV-polarized image, are better visible in the Pauli image.

The outputs of $N P$ and $\mu$ are shown in Fig. 6.1c, d. The $N P$ image [see Fig. 6.1c] clearly shows the slick-covered areas that result in $N P$ values larger than the background sea. Note that also metallic targets result in lager $N P$ values. A deeper analysis on $N P$ values reveals that slick-free and slickcovered sea surface is not well-separated. NP results in very low values both for slick-free and slick-covered sea surface

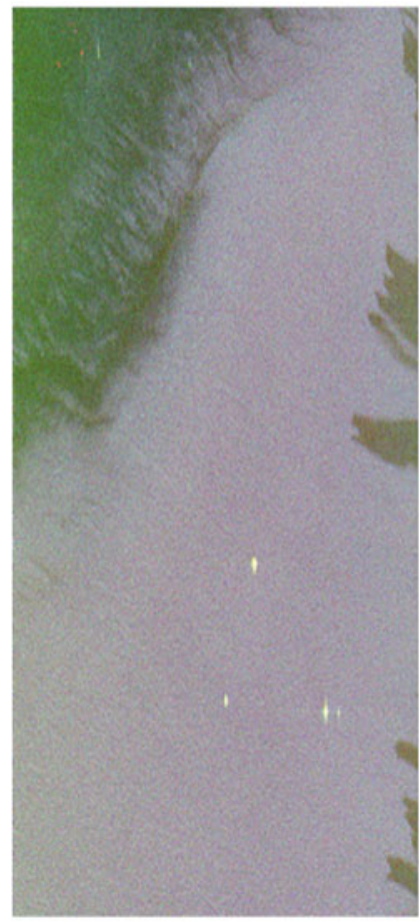

(a)

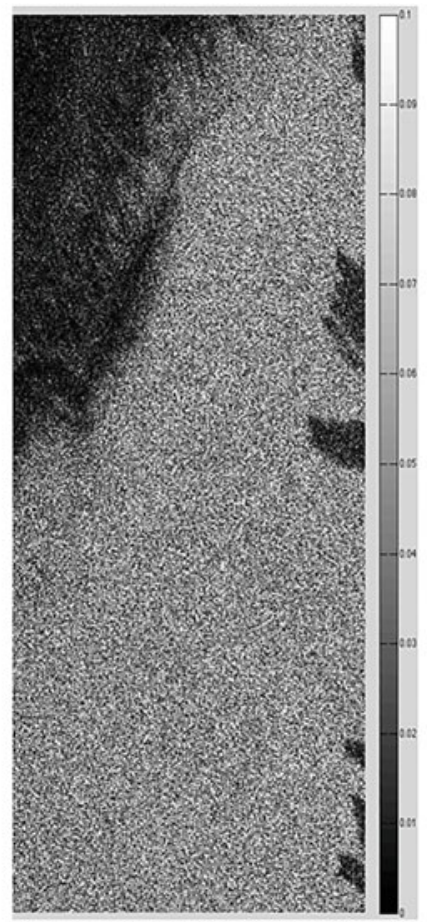

(b)

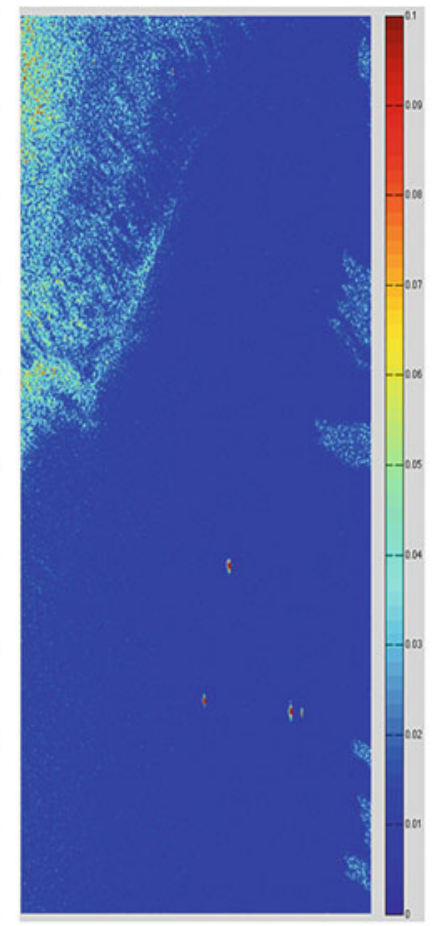

(c)

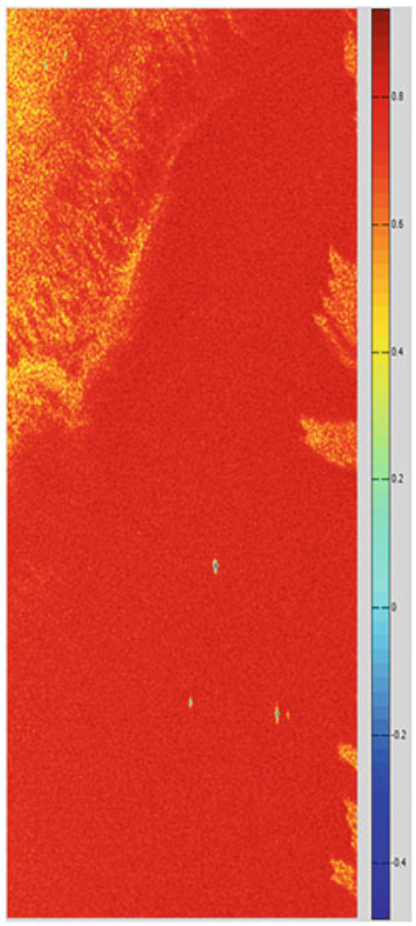

(d)

Fig. 6.1 RADARSAT-2 SAR data collected on May 15, 2010 over the Gulf of Mexico. (a) Pauli image. (b) Squared-modulus VV-polarized SAR data. Outputs of the $N P(\mathbf{c})$ and $\mu(\mathbf{d})$ approaches 
the oil slick or to the kind of surfactant that is present. It must be explicitly pointed out that this information cannot be extracted from single-polarization intensity SAR data.

The second experiment is relevant to the RADARSAT2 SAR scene collected on May 8, 2010. The wind speed is $6 \mathrm{~m} / \mathrm{s}$, and the incidence angle varies in the range $41.9^{\circ}$ $43.3^{\circ}$. The imaged scene contains few slicks, related to a well-known oil seep in the Gulf of Mexico. Those slicks result in "green" areas in the Pauli image and in areas darker than the background sea in the single-polarization grey-tones VV image; see Fig. 6.2a, b.

The outputs of the $N P$ and $\mu$ approach are shown in Fig. 6.3a, b, respectively. It can be noted that, unlike the previous experiment, in this case, all the polarimetric approaches result in good separation between oil-covered and slick-free sea surface. From an electromagnetic viewpoint, one can say that this scene is everywhere dominated by Bragg scattering but within the oil-covered sea surface. With respect to NP, it can be noted that oil-covered sea surface results in a significant amount of unpolarized backscattered energy $(N P>0.5)$, which is compatible with a non-Bragg scattering mechanism, while a significantly lower unpolarized energy applies in case of slick-free sea surface. It can be also noted that $\mu$ results in positive (negative) values over slick-free (oil-covered) sea surface, allowing a clear and unambiguous distinction between the two scattering mechanisms.

Since in this case a "conventional" oil slick is in place, it is worth analysing the performance of the polarimetric features in terms of the detection capability. Within this context, the most attractive polarimetric feature is the conformity coefficient (or, equivalently, the approach proposed in Nunziata et al. (2008))), since it is able to separate in two non-overlapped regions: Bragg and non-Bragg scatterings without any external threshold. The logical true (sea) and false (oil) output obtained using $\mu$ is shown in Fig. 6.3c. As expected, positive (true) $\mu$ values result from sea surface Bragg scattering, while negative (false) $\mu$ values result from the oil-covered sea surface non-Bragg scattering. It must be explicitly pointed out that no empirical threshold must be set, and this is a key advantage of PolSAR approaches with respect to single-polarization ones.

These results demonstrate the unique benefit of PolSAR data for oil at sea observation with respect to singlepolarization SAR data. In fact, polarimetric data result in unprecedented information that in no way can be extracted from single-polarization intensity data.
Fig. 6.2 RADARSAT-2 SAR data collected on May 8, 2010 over the Gulf of Mexico. (a) Pauli image. (b) Squared-modulus VV-polarized SAR data

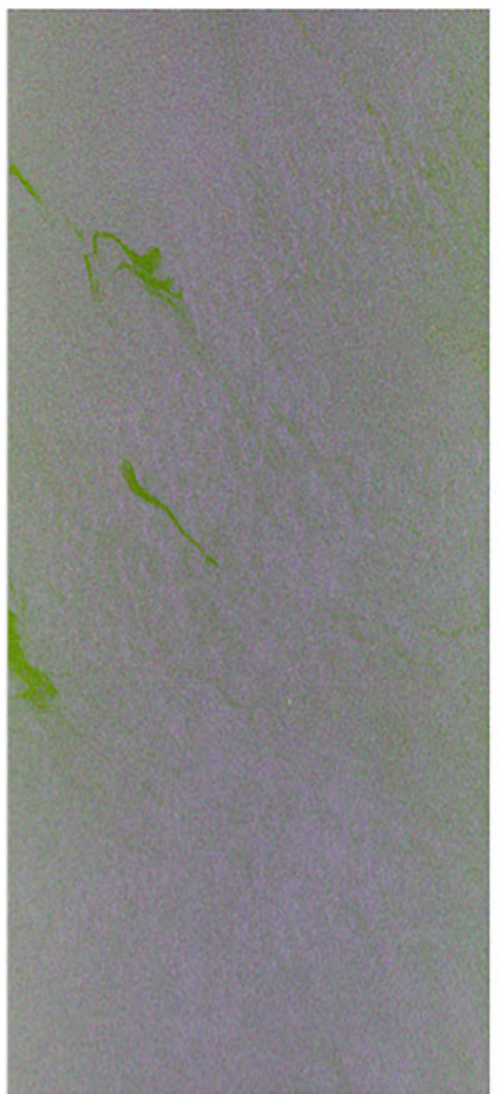

(a)

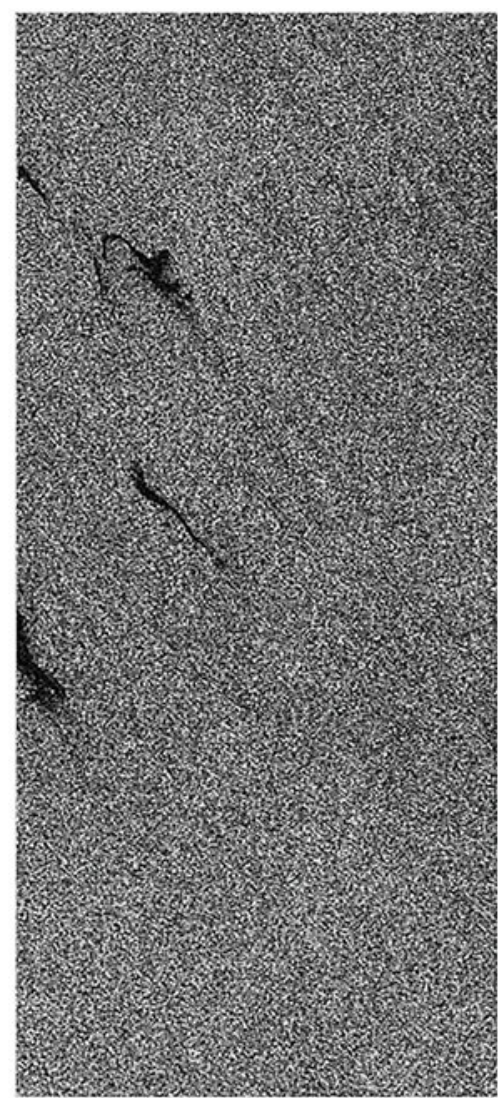

(b) 


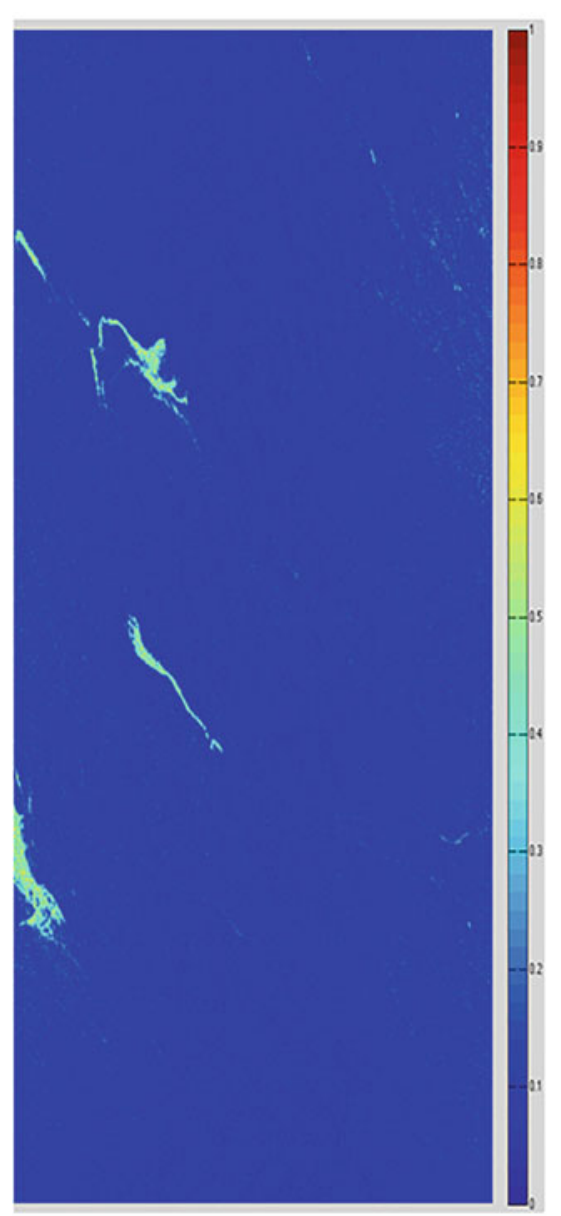

(a)

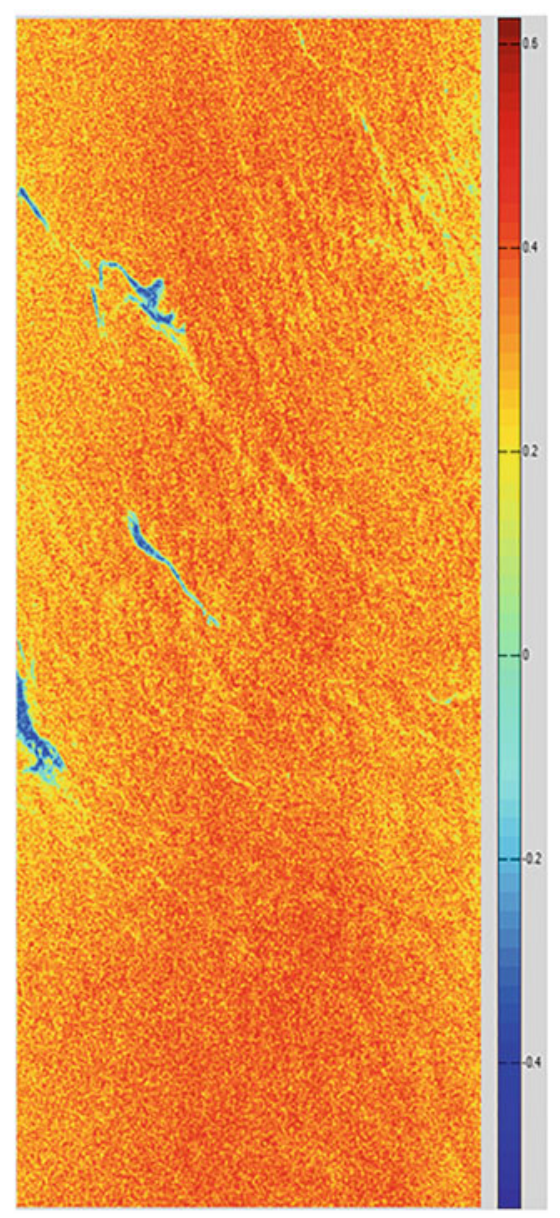

(b)

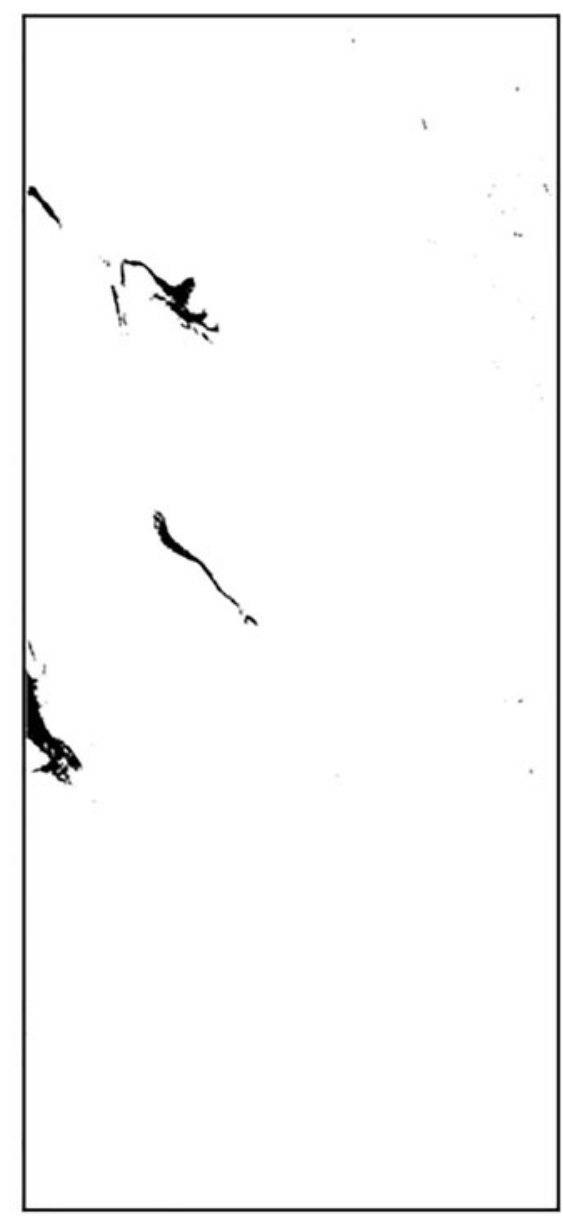

(c)

Fig. 6.3 Output of the $N P$ (a) and $\mu$ (b) approach, binary $\mu$ image (c)

\subsubsection{Comparison with Single/Dual Polarization Data}

In this sub-section the dual-polarimetric $\sigma$ approach is verified against actual polarimetric SAR data to discuss its performance with the above-described quad-polarimetric approaches. Note again that $\sigma$ is the standard deviation of the co-polarized phase difference.

The output of the $\sigma$ approach applied to the RADARSAT2 SAR scenes of Figs. 6.1, and 6.2 is shown in Fig. 6.4a, b, respectively. The slick-covered areas are emphasized by the $\sigma$ approach, as expected. With respect to Fig. 6.4a, the mean $\sigma$ value related to slick-free and slick-covered sea surface is $30^{\circ}$ and $45^{\circ}$, respectively. Hence, as far as for the quadpolarimetric approaches, the scattering mechanism that characterizes slick-covered sea surface is compatible with a Bragg mechanism. With respect to Fig. 6.4b, even in this case, the $\sigma$ approach provides results similar to the quadpolarimetric ones. The output witnesses that a highly non-Bragg scattering mechanism characterizes the oil-covered sea surface, resulting in larger $\sigma$ values $\left(\sim 110^{\circ}\right)$. A different scattering mechanism characterizes the surrounding sea, where the mean $\sigma$ value is $\sim 55^{\circ}$. Even in this case, as far as for the quad-polarimetric approaches, $\sigma$ values larger than expected are measured over sea surface that results to be very heterogeneous in terms of $\sigma$ values, showing that, probably, a larger oil-affected area is present. Moreover, the area that surrounds the slicks is characterized by intermediate $\sigma$ values, witnessing that low oil concentration/mixing phenomena apply.

\subsubsection{Discussion on the Role of Polarization, on the Maturity of the Application and Conclusions}

Experiments clearly witness that the extra information inherently carried on polarimetric SAR data allows not only detecting oil at sea but also (and often more importantly) to provide a rough information on the kind of surfactant and on 
Fig. 6.4 $-\sigma\left[{ }^{\circ}\right]$ map related to the RADARSAT-2 SAR scene of

Figs. 6.1 (a) and 6.2 (b)

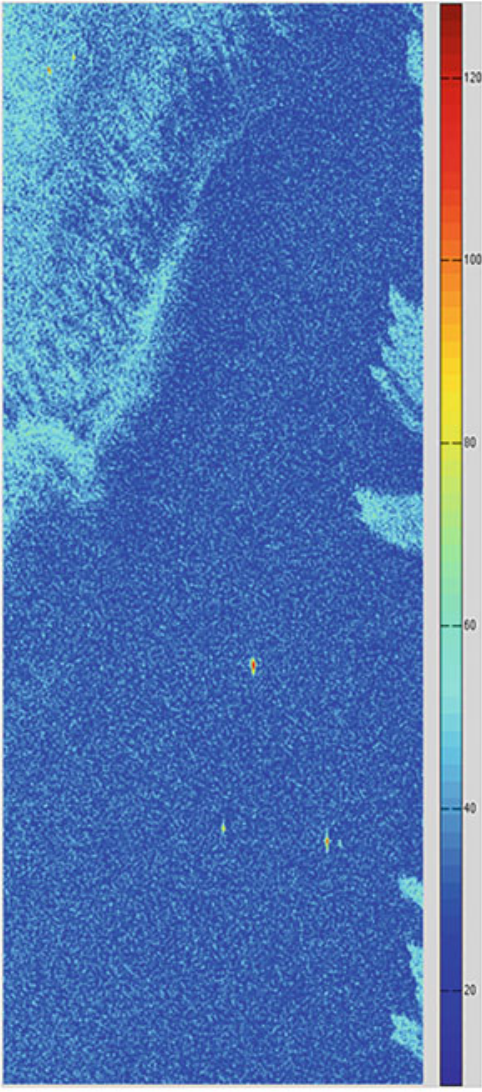

(a)

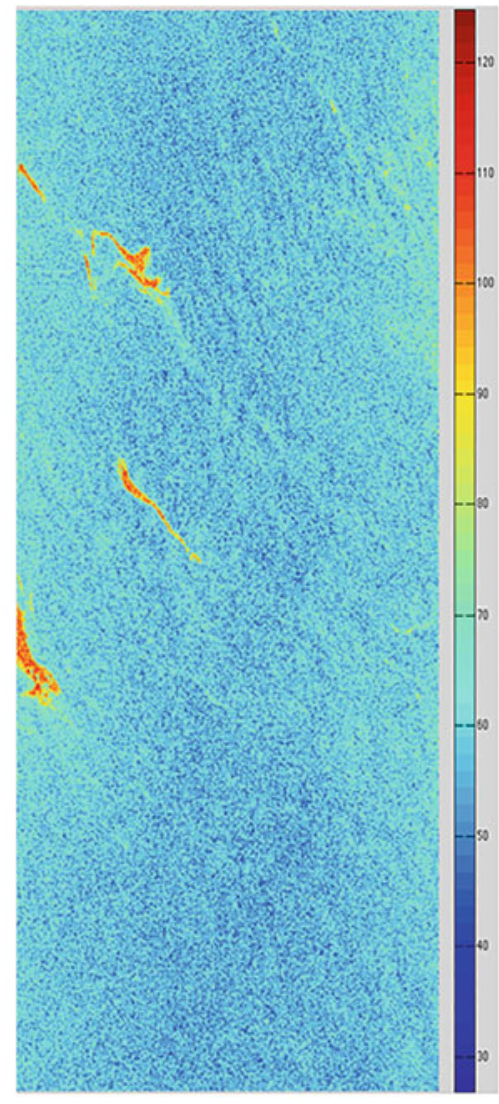

(b) its damping properties. Hence, SAR polarimetry is able to offer an unprecedented level of scattering details that can be used to assist classical "large swath" single-polarization procedure providing extra information on the surfactant. The application is mature: the proposed techniques have been successfully verified against actual polarimetric L-, C- and $\mathrm{X}$-band SAR data collected in a broad range of incidence angles and sea state conditions.

\subsection{Targets at Sea Observation}

\subsubsection{Introduction, Motivation and Literature Review}

Sea metallic targets, i.e. oil rigs and ships, observation is nowadays a topic of great applicative interest. Both trafficrelated and surveillance applications need a synoptic, all-weather and day and night observation system. The SAR is the key remote sensing tool for this application. However, SAR metallic targets observation is not an easy task. Specifically, one of the main issues to be tackled is the presence of false alarms versus missed targets.
In this section, the benefits of using fully polarimetric SAR data for targets at sea observation are carefully analysed. Accordingly, the most up-to-date state-of-the-art detectors able to deal with full- and dual-polarimetric single look complex (SLC) SAR data are briefly reviewed, and their performance is analysed using actual full-polarimetric SAR data collected in selected test sites. Then, a fair intercomparison of the detectors is accomplished in order to clearly show the benefits related to the use of fully polarimetric SAR data. As a benchmark, a single-polarization HV detector, based on the generalized-K (GK) distribution, is also applied to the HV SLC SAR data to clearly point out the benefits of radar polarimetry for targets at sea observation.

Metallic targets, being characterized by structures large with respect to the electromagnetic wavelength, call for a backscatter signal stronger than the background sea. Hence, they appear as bright spots in the SAR image plane. Following this rationale, several radiometric-based approaches have been developed to observe targets in single-polarization SAR data (Crisp 2004). However, the information provided by the backscattered intensity collected by a single-polarization SAR is not always sufficient to effectively observe metallic targets. To conceive more effective observation techniques, 
great attention to polarimetric approaches has been paid. In fact, new high-performance polarimetric SARs, e.g. the ones operated onboard the Advanced Land Observing Satellite2 (ALOS-2), RADARSAT-2, TerraSAR-X and COSMOSkyMed missions, are of particular interest.

In Liu et al. (2005)), a ship detector, that applies a likelihood ratio test with the Neyman-Pearson criterion to define a pixel-based detection criterion, is developed. Gaussian distributions for the scattering matrix components were assumed in order to derive an approximate decision variable. Even though the Gaussian distribution does not fit perfectly the data, the test shows good accuracy on real data. In Yeremy et al. (2001)), two studies have been accomplished using airborne C-band polarimetric SAR data. The first study compares target-to-clutter ratios (TCRs) for various polarimetric channels, demonstrating that the best polarimetric channel for ship observation purposes depends on the incidence angle. Co-polarized horizontal channel $(\mathrm{HH})$ performs better than cross-polarized one (HV) for larger incidence angles. Moreover, a comparative study on the suitability of two polarimetric target decomposition techniques, i.e., the coherent target decomposition (CTD) and Van Zyl decomposition (VZD), is also undertaken, demonstrating that CTD performs better than VZD in terms of false alarm rate. In Touzi and Charbonneau (2002)), the CTD is enhanced to extend its range of applicability, and the symmetric scattering characterization method (SSCM) is introduced to better exploit the information provided by the symmetric scattering component in the frame of coherent scattering. In Ferrara et al. (2011)), a speckle-based multi-polarization study is developed to define a physically based single-polarization filter. It is shown that a proper combination of speckle-related parameters, when evaluated over the cross-polarized channel, is able to observe targets at sea with a very low false alarm rate. In Novak et al. (1989)), a linear polarimetric detector that maximizes the target-to-clutter contrast, the so-called polarimetric matched filter (PMF), is derived, and the structure of this detector is related to simple polarimetric targets types.

These approaches are meant to identify a set of polarimetric features peculiar to the metallic targets (e.g. high polarimetric entropy, large coherent component, etc.), but, unfortunately, there is a strong dependence on the scattering mechanism of both target and surrounding background. A different approach is proposed in Marino (2013)) where a notch filter for target detection purposes is developed. Experiments on C-band RADARSAT-2 full-polarimetric SAR data demonstrate the capability of the approach to highlight features, which are polarimetrically different from the background sea in a broad range of sea state conditions and almost independently of look angle. In Nunziata et al. (2012b)), a polarimetric detector that exploits the different symmetry properties that characterize sea surface with and without metallic targets is proposed. The detector works on dual-polarimetric $\mathrm{HH} / \mathrm{HV}$ (VV/VH) pairs, and it exploits the cross-correlation between co- and cross-polarized channels to detect targets at sea in a robust and effective way. Experiments accomplished using L-band ALOS-PALSAR, C-band RADARSAT- 2 and X-band TerraSAR-X data confirmed the effectiveness of the proposed approach. In Nunziata and Migliaccio (2013)), X-band dual-polarization Cosmo-SkyMed PingPong SAR data are exploited to observe metallic targets at sea in a very effective way.

\subsubsection{Methodology}

In this section the operational procedures used are briefly described, starting from quad- and dual-polarimetric detectors, and, finally, a single-pol detector is considered as a benchmark.

\subsubsection{Liu Et al. Detector}

The detector is fully described in Liu et al. (2005)). It assumes that ocean and target backscatter follow a zeromean multivariate Gaussian distribution; hence, a likelihood ratio test is proposed, based on the Neyman-Pearson criterion to maximize the detection probability subject to the constraints that the probability of false alarm is less than a prefixed value. Moreover, typically the elements of covariance matrix of the target, $\mathbf{C}_{t}$, are much larger than the ones related to the sea, $\mathbf{C}_{0}$; hence, a simplified decision rule can be used:

$$
\Lambda=\mathbf{k}^{*} \mathbf{C}_{o}^{-1} \mathbf{k}= \begin{cases}>T_{l} & \text { target } \\ \leq T_{l} & \text { ocean }\end{cases}
$$

where $\mathbf{k}$ is the target scattering vector. This detector is suboptimal; the magnitude of the measured data over sea surface typically does not strictly follow a Gaussian distribution, showing significant tails. In the following the value of probability of false alarm used for setting the threshold is $P_{f}=10^{-5}$.

\subsubsection{Geometrical Perturbation Polarimetric Notch Filter}

This ship detector shares the same general methodology of the geometrical perturbation partial-target detector (GP-PTD), and physical justification of the algorithm can be found in Marino (2013)). The idea behind the GP-PNF is to build an algorithm that is able to identify any partial target, which is different from the background clutter. In the case of ship detection, the background is the sea. Hence, the following decision rule is proposed (Marino 2013): 


$$
\gamma_{n}=\frac{1}{\sqrt{1+\frac{\operatorname{RedR}}{\mathbf{t}^{* T} \mathbf{t}-\mid \mathbf{t}^{*} \widehat{\mathbf{t}_{\text {sea }} \mid}}}}>T_{n}
$$

where $\mathbf{t}$ is the partial vector extracted from the scene (observables), $T_{n}$ is the threshold, and $\operatorname{RedR}$ is a detector parameter that can be set using a rationale based on the signal-to-clutter ratio (SCR) [R17]. The solution followed in this report is to set the threshold to $T_{n}=0.98$ and choose $R e d R$ based on the intensity of the minimum target of interest $P_{T}^{\min }$. In this study $P_{T}^{\min }$ is about 0.2 (linear scale).

\subsubsection{Polarimetric Match Filter}

This detector was firstly introduced in Novak et al. (1989)), and it considers an optimization of the power ratio between the quadratic forms of the covariance matrices of target and clutter. The optimization is easily carried out exploiting the Lagrangian method:

$$
\begin{aligned}
L & =\boldsymbol{\omega}^{* T} \mathbf{T}_{s e a} \boldsymbol{\omega}-\lambda\left(\boldsymbol{\omega}^{* T} \mathbf{T}_{t} \boldsymbol{\omega}-C\right) \\
& \Rightarrow \frac{\partial L}{\partial \boldsymbol{\omega}^{* T}}=\mathbf{T}_{\text {sea }} \boldsymbol{\omega}-\lambda \mathbf{T}_{t} \boldsymbol{\omega}=0 \Rightarrow \mathbf{T}_{\text {sea }}^{-1} \mathbf{T}_{t} \boldsymbol{\omega}=\lambda \boldsymbol{\omega}
\end{aligned}
$$

The latter says that the optimum scattering mechanisms $\boldsymbol{\omega}$ are the ones that diagonalize the matrix $\mathbf{A}=\mathbf{T}_{\text {sea }}{ }^{-1} \mathbf{T}_{t}$. Even though $\mathbf{A}$ is not Hermitian, it can be proved that it admits real positive eigenvalues. The maximum eigenvalue represents the maximum contrast; therefore the detector is finalized in setting a threshold on this. In this report a value of contrast equal to 9 is considered.

\subsubsection{Reflection Symmetry Detector}

In this sub-section the theoretical rationale that lies at the basis of the polarimetric detector (Nunziata et al. 2012b) is briefly outlined. The underpinning idea is that natural distributed targets (sea surface) satisfy reflection symmetry, while man-made metallic targets call for larger departure from this condition. To highlight the different target/sea behaviour in dual-polarized SAR data, the modulus of the correlation between like- and cross-polarized scattering amplitudes is used (Nunziata et al. 2012b):

$$
X=\left|\left\langle S_{h h} S_{h v}^{*}\right\rangle\right|>T
$$

To obtain a binary mask, a threshold $T$ is used. Here, $T=0.04$ is used.

\subsubsection{GK Filter}

In this sub-section the single-polarization detector proposed in Ferrara et al. (2011)) is briefly outlined. An electromagnetic model, based on the generalized-K (GK) distribution, is proposed in Ferrara et al. (2011)) that allow observing the scattering features associated with metallic targets at sea in full-resolution single-look-complex (SLC) SAR data. Hence, a simple and effective approach to observe targets at sea in HV-polarized SLC SAR data is proposed. In Ferrara et al. (2011)), the following index is shown:

$$
\gamma=\left(\frac{1}{2 \eta}\right)^{2}
$$

where $\eta$ is related to the rice factor (Ferrara et al. 2011) and, when evaluated over the HV channel, is able to sort targets and sea areas in two nonoverlapping sets, i.e. $\gamma<1$ and $\gamma>1$, respectively. Following this theoretical rationale, a simple filter that implements (6.5) is developed that operates on SLC SAR data using two nested windows, to estimate the coherent and incoherent backscattered field components, whose sizes are $8 \times 8$ and $18 \times 18$, respectively.

\subsubsection{Experimental Results}

Test sites and corresponding radar and validation data sets selected for the generation of showcases on targets at sea observation are summarized in Table 6.3 and further described in the appendix.

In this sub-section the full-polarimetric detectors, i.e. Liu et al., geometrical perturbation-polarimetric notch filter (GP-PNF) and the polarimetric match filter (PMF), are verified against actual polarimetric RADARSAT-2 SAR data (see Sect. 6.3.2).

One meaningful experiment, relevant to the scene collected on May 15, 2010, is detailed. The scene consists of six targets: one oil rig and five visually inspected targets. The Pauli image and the HH-polarized squared-modulus SAR data are shown in Fig. 6.5a, b, respectively. The location of the targets was derived by a map (i.e. ground truth), and it is shown as red boxes in the image. Moreover, a visual inspection of the radar image was carried out trying to interpret the points in the image that may represent vessels. The latter are marked by yellow circles.

Table 6.3 Test sites and corresponding radar and validation data selected for the generation of showcases on targets at sea observation

\begin{tabular}{l|l|l}
\hline Application/Product & Test site - radar data & Reference data \\
\hline \multirow{2}{*}{ Targets at sea observation } & Gulf of Mexico & Oil/gas rigs/platform Google Earth map \\
\cline { 2 - 2 } & 3 RADARSAT-2 fine quad-pol & \\
\hline
\end{tabular}


Fig. 6.5 RADARSAT-2 SAR

data collected on May 15, 2010, over the Gulf of Mexico. (a) Pauli image. (b) Squared-modulus HH-polarized SAR data. Yellow circles, visually inspected vessels; red boxes, verified metallic targets. Dark areas are related to the oil spilled during the Deepwater Horizon accident

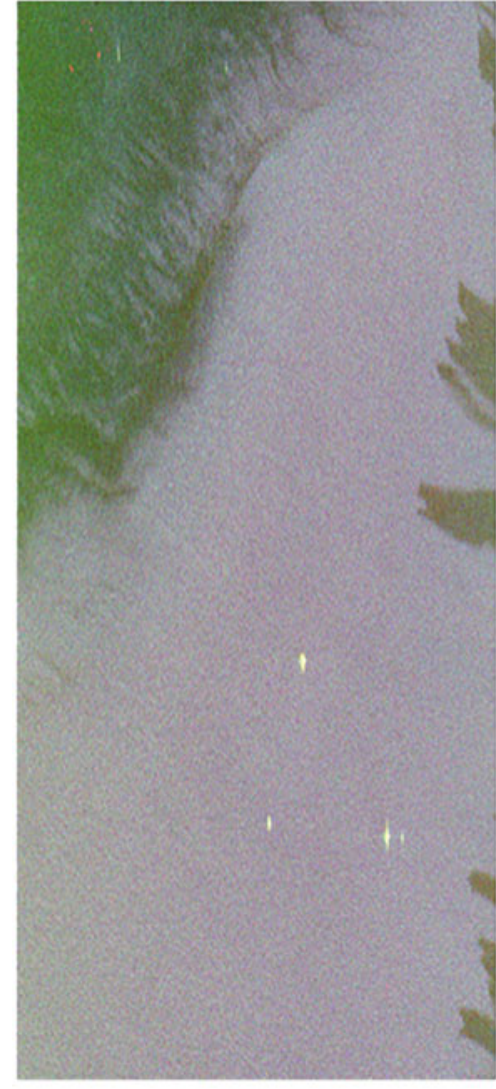

(a)

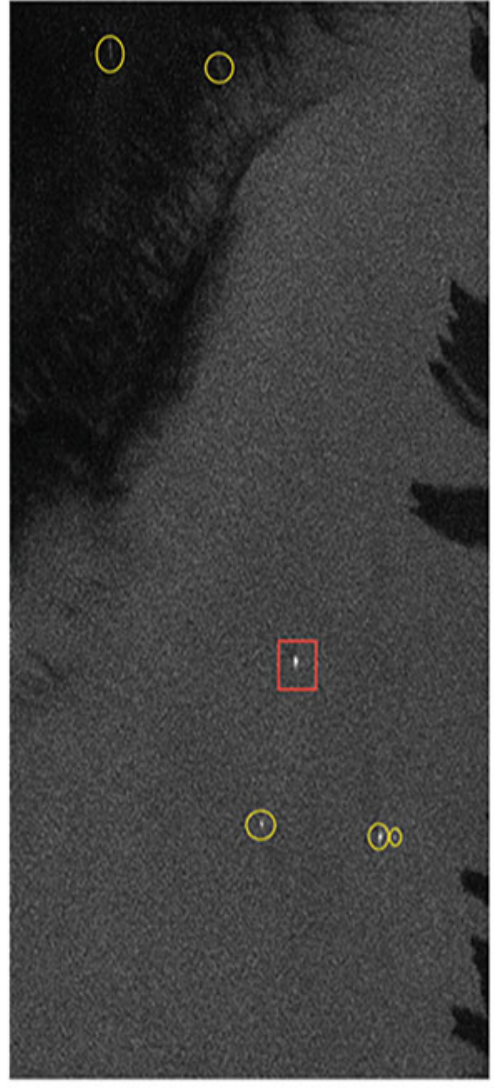

(b)
The output of the full-polarimetric Liu et al., GP-PNF and PMF detectors is shown in Fig. 6.6a, c, respectively. The detection masks show that all the targets identified in the scene are detected. Moreover, all the quad-polarimetric detectors seem to detect two points in the upper left corner. These appear in the Pauli image [see Fig. 6.5a] as bright red spots, and, hence, they can be associated to dihedral structures. It is not clear what the points are since their brightness is relatively low (therefore we did not consider them belonging to vessels in the visual inspection). However, their polarimetric behaviour is particularly well-defined (and repeated among them), and therefore we do not believe that they are common false alarms. It may be speculated that they are small vessels or probes beside the larger vessel or just ambiguities/artefacts. In the absence of accurate ground measurements, it is not possible to provide any specific conclusion. Finally, the PMF seems to be particularly sensitive to changes of the polarimetric background, and it is able to detect one of the ambiguities in the image that appears very faint in the Pauli RGB. This is clearly not a genuine target; however, it is an illustration of the power of these algorithms in picking up anomalies on the background. In general, azimuth ambiguities can be somehow filtered out by some algorithms; therefore it should be possible to remove this typology of false alarms with some post-processing. In general, more tests should be carried out in order to understand if the PMF high sensitivity may constitute a drawback in practical application (i.e. leading to irremovable false alarms).

\subsubsection{Comparison with Single/Dual Polarization Data}

In this sub-section the dual-polarimetric symmetry detector and the single-polarization (HV) GK filter are verified against actual polarimetric RADARSAT-2 SAR data.

The symmetry detector and the GK filter outputs obtained processing the SAR scene of Fig. 6.5 are shown in Fig. 6.7a, $\mathrm{b}$, respectively.

The symmetry detector succeeds in observing all the targets highlighted in Fig. 6.5b. The two "potential" targets observed by all the quad-pol detectors are not present in the symmetry output. The single-polarization detector misses one target (the one on the upper left side of the image). Moreover, even this detector does not observe the two "potential" targets. These results clearly witness the superiority of polarimetric detectors with respect to the singlepolarization $\mathrm{HV}$ one. 
Fig. 6.6 Output of the fullpolarimetric detectors. (a) Liu et al., (b) GP-PNF, (c) PMF

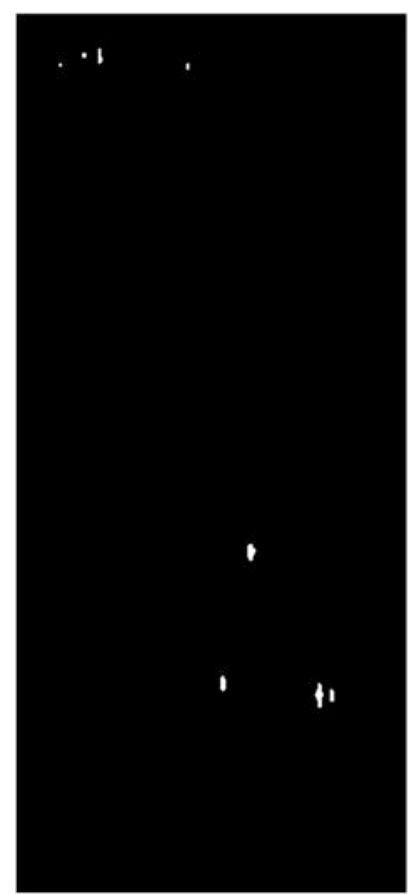

(a)

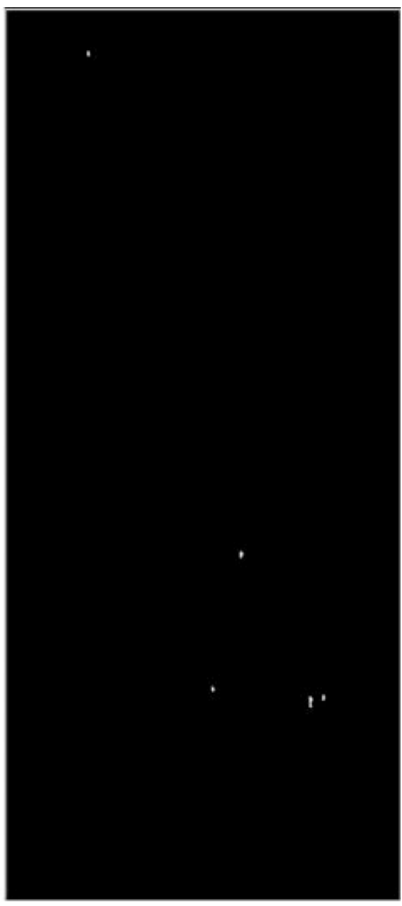

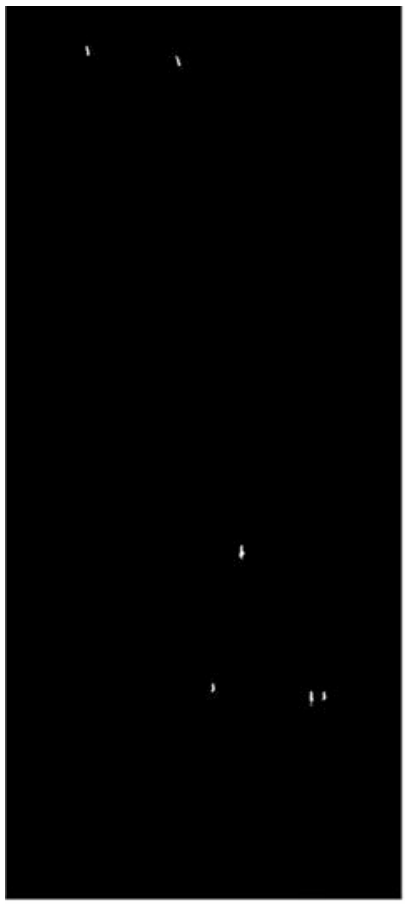

(b)

(a)

Fig. 6.7 RADARSAT-2 SAR scene of Fig. 6.5. (a) Dual-polarimetric symmetry detector output. (b) Single-polarization HV GK filter output

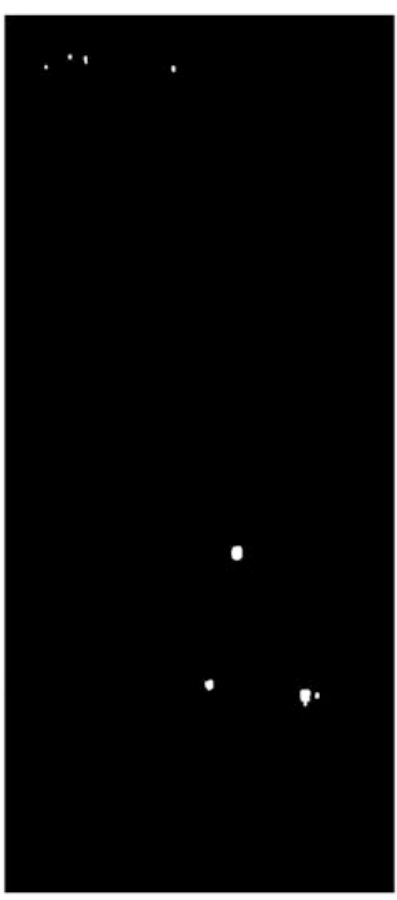

(b)

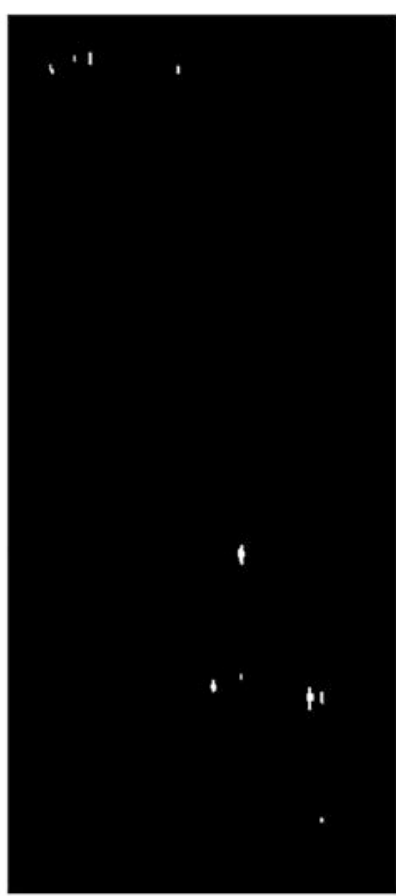

(c)

\subsubsection{Discussion on the Role of the Polarization, on the Maturity of the Application and Conclusions}

In this section, some of the most up-to-date polarimetric detectors to observe metallic targets at sea in PolSAR images are reviewed, and their performance is discussed using actual RADARSAT-2 SAR data where both visually inspected and verified targets are present.

Experimental results clearly witness that the extra information inherently carried on polarimetric detectors allows a better discrimination of the "anomalies" in the polarimetric backscattering of sea surface related to targets. This results in a better target/sea discrimination, even when very challenging scenes are considered, e.g. the one showed in Fig. 6.5, where few targets are present within a low backscatter area. Hence, SAR polarimetry is able to offer an unprecedented level of scattering details that can be used to detect targets that cannot be observed when using conventional singlepolarization detectors. On this purpose, it must be explicitly noted that some of the "potential" targets detected only by the quad-pol detectors are very complicated to be visually inspected from the HH or HV SAR image. Hence, if confirmed they would clearly witness the benefits of quad-pol detectors in detecting even targets characterized by low signal-to-noise (SNR) ratio. These results pushed toward a fair intercomparison of the above-mentioned approaches using a SAR data set where verified small targets are present (see external contributor document). 
The application is mature: the proposed techniques have been successfully verified against actual polarimetric L-, Cand X-band SAR data collected in a broad range of incidence angles and sea state conditions.

\subsection{Polarimetric Synthetic Aperture Radar Measurements of Oil Pollution at Sea}

\subsubsection{Introduction, Motivation and Literature Review}

Full-polarimetric SAR systems allow us to link scattering processes and variations in the backscattered signal to the physics of the medium measured. Recent advances in the field show a potential in polarimetric SAR measurements with respect to discrimination between different types of oil or surfactants and in deriving valuable information about oil slicks physical properties. In this showcase, we demonstrate that multi-polarization SAR can be used to obtain key information for pollution response authorities about detected slicks at sea (Skrunes et al. 2015).

The experimental work described in this chapter has been conducted by University of Troms $\varnothing$ in collaboration with Norwegian Clean Seas Association for Operating Companies (NOFO), with funding from Total E \& P Norge AS and the Norwegian Space Centre (NSC).

Occasionally, large amounts of oil are spilled into the sea. The Deepwater Horizon accident in the Gulf of Mexico in 2010 and the Prestige accident off the coast of Galicia in 2002 are examples of such incidents. During the recovery and cleanup phase, information about slick properties, such as the thickness distribution and the oil volume fraction, is requested. Deliberate man-made slicks are often smaller in size but are nevertheless frequently occurring. Biogenic slicks and natural seeps are also known to resemble oil spills in mono-polarization SAR imagery. Therefore, knowledge about the slick type and its origin is useful input to decisionmakers. This type of information could limit the number of false alarms as well as expensive actions taken by authorities.

Hence, beyond simply detecting the slicks, a demand for additional information is recognized. This has triggered the scientific community to explore polarimetric SAR systems and their potential in:

- Discriminating man-made mineral slick types from monomolecular biogenic slicks and

- Characterizing slicks, e.g. to obtain knowledge on slick thickness and infer oil/water mixing

The ocean is a nonstationary medium, and oil slicks are affected by weathering processes over time. Weathering processes include spreading, drift, evaporation, dissolution, natural dispersion, emulsification, sedimentation, biodegradation and photooxidation (Kotova et al. 1998). This can cause significant variability in the signatures of oil pollutants in SAR imagery. The detection and characterization capabilities of SAR systems also show a dependence on imaging geometry and SAR system properties, e.g. incidence angles and sensor frequency. Norway is one of the few countries allowing oil discharges for the purpose of equipment and procedure testing. To gain increased knowledge in the field of oil spill remote sensing by SAR, controlled large-scale oil-on-water experiments are currently conducted in the North Sea. These experiments are excellent opportunities to create realistic study cases while having access to key parameters such as the oil types released, meteorological conditions and the age of the spills. This chapter describes the lesson learned from the Norwegian oil-on-water experiments in 2011 and 2012, seen from a polarimetric SAR remote sensing perspective.

For polarimetric SAR systems, when Bragg scattering is dominant, the cross-polarization term could be considered negligible. This assumption is made in the Freeman-Durden decomposition, where the first component models an untilted and slightly rough first-order Bragg surface. In the context of marine slicks, the strength of polarimetric SAR systems seems indeed to lie in the relation between the co-polarization coefficients (VV and $\mathrm{HH}$ ) of the scattering matrix. The standard deviation of the co-polarized phase difference (CPD) is described as an important feature, which adds discrimination power to the problem of distinguishing oil spills from biogenic look-alikes (Migliaccio et al. 2009b). The co-polarization ratio is another feature that is sensitive to the dielectric properties of the surface (Minchew et al. 2012), and its potential is currently investigated with respect to discriminating oil from lookalikes in the marginal ice zone (Brekke et al. 2014). Solberg recently gave a literature review on remote sensing of oil pollution, where a brief summary of SAR and some additional polarimetric descriptors is included (Solberg 2012).

\subsubsection{Methodology}

\subsubsection{Oil-on-Water Experiments}

The NOFO conducts an oil-on-water exercise once a year in the North Sea. The purpose of these exercises is to improve the national oil spill preparedness. In 2011 and in 2012, the controlled discharges at sea provided a unique opportunity to acquire satellite data of various oil types and collect other auxiliary information about the oil spills. The substances were released from ships involved in the exercises, and SAR data was collected from European, American, Canadian and Indian satellites. Meteorological conditions were logged, and other data such as photos and infra-red 
(IR) measurements were collected from vessels, aerostats and aircrafts.

\subsubsection{Releases of Mineral and Biogenic Oil Types into the Sea Water}

Kotova et al. (Kotova et al. 1998) emphasizes the importance of weathering processes, as they influence oil spills physicochemical properties and detectability in SAR images. The processes that play the most important role for oil spill detection are evaporation, emulsification and dispersion. Lighter components of the oil will evaporate to the atmosphere. The rate of evaporation is dependent on oil type, thickness of the spill, wind speed and sea temperature. Emulsification of crude oil (crude oil is a naturally occurring substance and has been extracted, but no further processing is done) refers to the process where water droplets become incorporated in the oil and are estimated based on water uptake as a function of the wind exposure of the actual oil type. Dispersion is an important factor in deciding the lifetime of an oil spill, and it is strongly dependent on the sea state. While evaporation, dispersion and dissolution make the oil on the surface disappear, emulsification of the oil increase the viscosity and volume of the pollutant and promote its persistence (Reed et al. 1999). In the oil-on-water experiments taking place at the Frigg field, we have released both crude oil and water-in-oil emulsion to create realistic mineral oil slicks. Figure 6.8 shows crude (Balder) oil forming into a large slick on the ocean surface in 2011.

One look-alike phenomenon frequently discussed in the literature is natural biogenic slicks. Biogenic slicks are surface films consisting of surface-active compounds, originating from marine plants or animals. The main producers of natural surface films are algae and some bacteria. Surface-active organic compounds have one hydrophobic part and one hydrophilic part, i.e. strong tendencies both towards and against water. Hence, the molecules are spontaneously arranged at the air-water interface with the hydrophobic part up in the air and the hydrophilic part down in the water. This forms a very thin and so-called monomolecular film, which is only one molecule thick $(\sim 2.4-2.7 \mathrm{~nm})$. In contrast, crude oil spills mainly consist of chemicals with exclusively hydrophobic character. Depending on the amount and viscosity of the oil and on the environmental conditions, crude oil spills will spread out over time. However, the final thickness still remains orders of magnitude larger than that of monomolecular films $(\mu \mathrm{m}, \mathrm{mm}$ and even $\mathrm{cm}$ for freshly spilled oil) (Hühnerfuss 2006). The two slick types will have different viscoelastic properties and induce
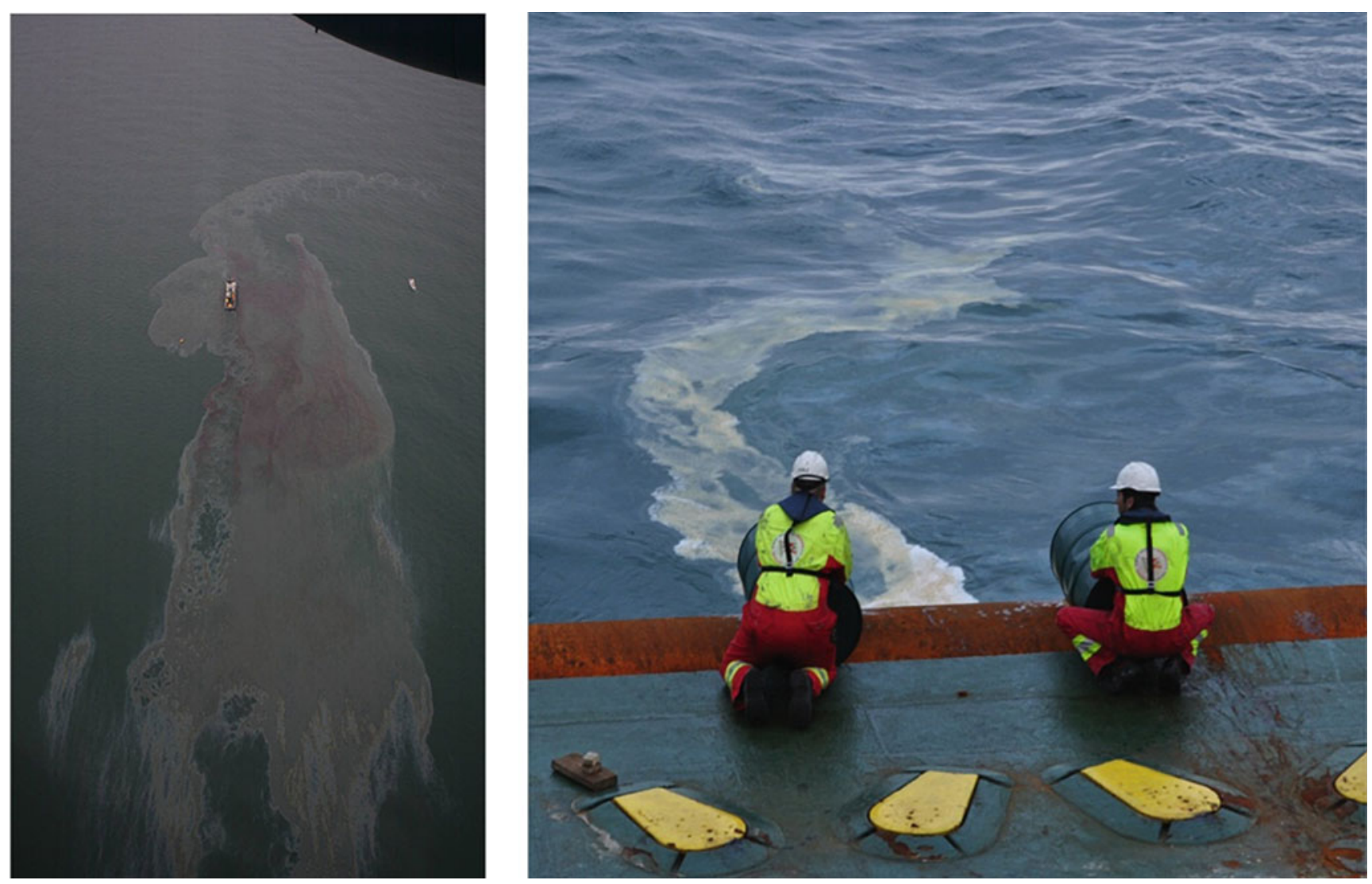

Fig. 6.8 Left: The crude oil slick formed in June 2011. Vessels participating in the exercise are visible. (Photo: Kustbevakningen. Right: Release of palm oil ongoing at the Frigg field in 2011. Photo: Stine Skrunes) 
different damping on the ocean waves (Hühnerfuss 2006). It is difficult to distinguish between mineral oil spills and natural slicks in mono-polarization SAR data. As a third substance applied in the oil-on-water experiments, we have chosen to use palm oil as a biogenic slick simulator. The palm oils viscoelastic behaviour is similar to the one of natural biogenic oil films. Figure 6.8 shows a photo of the release of 400 litres of palm oil in 2011.

\subsubsection{Analysis of Multi-polarization SAR Data}

The methodology applied in the analysis of the SAR datasets includes the following steps:

- Computation of multi-polarization features

- Masking of open sea water

- Unsupervised classification of the remaining slick pixels

The output from the classification is finally discussed in relation to the known in situ slick characteristics.

A quality investigation of SAR data recorded by Radarsat2 and TerraSAR-X during the 2011 experiments has also been conducted. The analysis revealed that the signal over oil slicks in the cross-polarization channels ( $\mathrm{VH}$ and $\mathrm{HV}$ ) often fluctuates around the noise floor (Skrunes et al. 2012a). A threshold of $-6 \mathrm{~dB}$ is proposed in Minchew et al. (2012)), where a study based on the airborne UAVSAR (L-band) system was undertaken. This requirement cannot be met by current spaceborne systems, such as Radarsat-2 and TerraSAR-X, as the signal level in the cross-polarization channels is fairly low over marine slicks. Hence, for the remainder of this chapter, we limit our attention to the co-polarization scattering coefficients ( $\mathrm{VV}$ and $\mathrm{HH}$ ), which have a stronger signal-to-noise ratio.

A selected set of multi-polarization features is applied as input to the unsupervised classification procedure. The names of the features considered are presented in Table 6.4, while more detailed definitions can be found in Skrunes et al. $(2015,2012 b)$. The features are either processed from the mean covariance matrix or from target decomposition theorems. Some of them are well-known multipurpose descriptors. Others are in the literature specifically proposed

Table 6.4 Features applied in this study

\begin{tabular}{l|l}
\hline$\#$ & Feature \\
\hline 1 & Entropy $\mathrm{H}$ \\
\hline 2 & Anisotropy $\mathrm{A}^{\prime}$ \\
\hline 3 & Mean scattering angle $\bar{\alpha}$ \\
\hline 4 & Covariance scaling factor $\mu$ \\
\hline 5 & Co-polarization power ratio $\gamma_{C O}$ \\
\hline 7 & Standard deviation of co-polarized phase difference $\sigma_{\phi C O}$ \\
\hline 8 & Magnitude of co-polarization correlation coefficient $\rho_{C O}$ \\
\hline & Real part of co-polarization correlation $r_{C O}$ \\
\hline
\end{tabular}

for oil slick studies. Several of the features were originally developed for the full scattering matrix case. To comply with the discussion on data quality above, these features are here adapted to dual-polarization (only involving the $\mathrm{VV}$ and $\mathrm{HH}$ scattering coefficients, respectively).

\subsubsection{Experimental Results}

The selected test site is the Frigg field in the North Sea, northwest of Stavanger. SAR measurements of the oil slicks artificially created in 2011 and 2012 have been successfully collected from spaceborne sensors. An overview of some of the datasets is given in Table 6.5.

An investigation of the features presented in Table 6.4 shows a varying degree of separability between the slickcovered areas and the ambient clean sea. Figure 6.9 presents some of the features listed in Table 6.4, i.e. the entropy, the covariance scaling factor and the real part of the co-polarization correlation. The features are computed from one of the quad-polarimetric Radarsat-2 fine-resolution scene recorded in 2011 (see Table 6.5). We can see that some of these features exhibit clear differences within the plant oil slick as compared to values within the mineral oil slicks. Particular regions within individual slicks are also more pronounced than other regions of the same slick.

The intensity image, in the bottom part of Fig. 6.9, shows the VV channel with labels indicating the slick types. As we can see, all slicks are clearly visible despite fairly low-wind speed at the time of recording. The bottom right image shows an unsupervised classification of the three different oil slicks. The classification result indicates a potential for discrimination between mineral oil and biogenic slicks. We can also observe zones along the edges of the mineral slicks. This zoning may be related to changes in physical parameters, e.g. the thickness distribution of the oil. Aerial photos of the crude oil, taken approximately $1 \mathrm{~h}$ before the satellite passed, reveal internal thickness variations corresponding to the Bonn Agreement Oil Appearance Code (BAOAC) (Skrunes et al. 2012b; Areal-surveillance - Cooperation on aerial surveillance over the North Sea area. In: Bonn Agreement Counter Pollution Manual 2005). This supports our SAR-based observations.

\subsubsection{Comparison with Single/Dual Polarization Data}

The simplest type of SAR systems transmits on one polarization and receives on the same (like) polarization. Current operational oil slick monitoring services are mainly based on mono-polarization SAR products (VV or $\mathrm{HH}$ polarization). Both support vector machines (SVM) and Bayesian 
Table 6.5 Test sites and corresponding radar and validation data selected for the generation of showcases on observation of oil pollution at sea

\begin{tabular}{l|l|l|l}
\hline Sensor & Date and pass & Mode and pol. & Oil slicks \\
\hline Radarsat-2 & 8 June 2011 descending & Fine quad-polarization & Emulsion and plant oil \\
\hline TerraSAR-X & 8 June 2011 descending & Strip map, HH and VV & Emulsion \\
\hline TerraSAR-X & 8 June 2011 ascending & Strip map, HH and VV & Emulsion, plant oil and crude oil \\
\hline Radarsat-2 & 8 June 2011 ascending & Fine quad-polarization & Emulsion, plant oil and crude oil \\
\hline Cosmo-SkyMed & 14 June 2012 ascending & PingPong, HH and VV & Emulsion and oleyl alcohol (OLA) or plant oil \\
\hline Cosmo-SkyMed & 14 June 2012 descending & PingPong, HH and VV & Emulsion and plant oil \\
\hline Radarsat-2 & 15 June 2012 descending & Fine quad-polarization & Emulsion and plant oil \\
\hline TerraSAR-X & 15 June 2012 ascending & Strip map, HH and VV & Emulsion \\
\hline Radarsat-2 & 15 June 2012 ascending & Fine quad-polarization & Emulsion \\
\hline Cosmo-SkyMed & 15 June 2012 ascending & PingPong, HH and VV & Emulsion \\
\hline
\end{tabular}
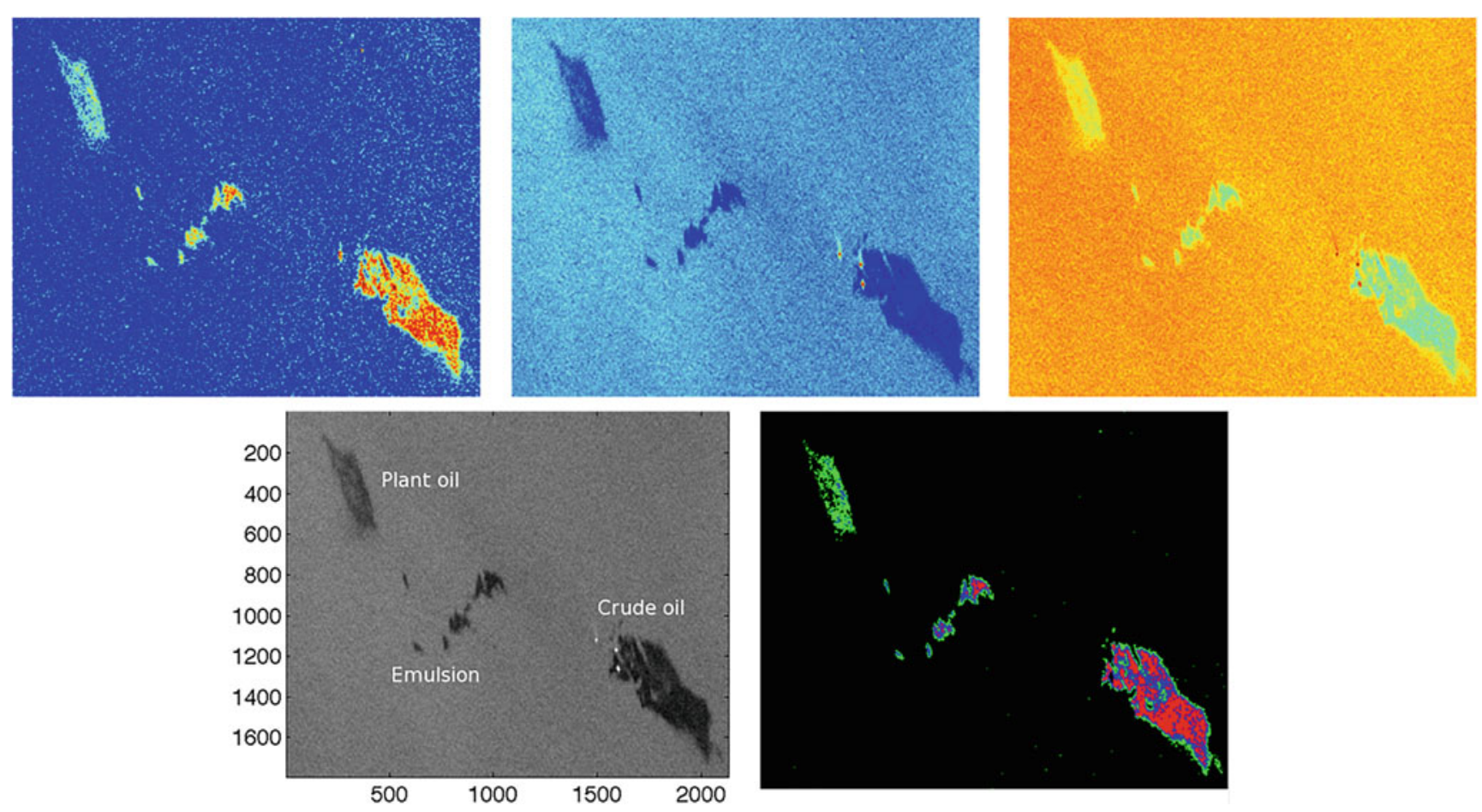

Fig. 6.9 Top row: entropy (left), covariance scaling factor (middle) and co-polarization correlation (right). Bottom row left: VV intensity image from June 2011 at a wind speed of $1-3 \mathrm{~m} / \mathrm{s}$. RADARSAT-2 data and products (C) MDA LTD. (2011) - All Rights Reserved. Bottom row

right: an unsupervised K-means classification based on the set of multi-polarization features visualized in the top row. The open water is masked out (black)

classification schemes have previously been proposed for oil slick detection and oil spill versus look-alike discrimination based on Radarsat-2 ScanSAR or ENVISAT ASAR Wide Swath SAR scenes (Brekke and Solberg 2008). These algorithms are trained and tested on large data sets with reasonable good performance. However, it is shown that oil spills can be hard to separate from look-alikes when only utilizing mono-polarization SAR images. From the monopolarization intensity or amplitude products, the information we can extract about the slicks are mainly related to the geometry and shape of the segmented region, properties of the backscatter level of the spot and the surroundings, spot contextual information and texture.

True polarimetric SAR modes are necessary to be able take advantage of the technology discussed in this chapter. Hence, the previous alternating polarization (AP) mode of ENVISAT or the current PingPong (PP) mode of CosmoSkyMed is not ideal for oil slick characterization. These modes suffer from a time lag between the transmissions of the horizontal and vertical polarized signals. The delay is long enough to cause an incoherent imaging over sea surfaces (Nunziata et al. 2011). These data products can simply be described as images, as they do not include the relative phase between the two received channels. Techniques applicable to these data types are primarily restricted to ratios or differences of their respective images. 
With the launch of Advanced Land Observing Satellite (ALOS) in 2006 and TerraSAR-X and Radarsat-2 in 2007, coherent dual- and quad-polarimetric SAR measurements became available from space. The scattering matrix holds all the complex scattering coefficients that describe the marine target. The scattering coefficients are measured at different linear combinations of send and received polarizations ( $\mathrm{VV}, \mathrm{HH}, \mathrm{VH}$ and $\mathrm{HV})$. The polarimetric SAR systems extend our capabilities to characterize the slicks. It provides us with a mean to investigate the physical scattering characteristics of the surface medium. However, due to limitations in the current technology, only narrow swath modes are available.

In compact polarimetry, one polarization is transmitted, and two orthogonal polarizations are received, together with their relative phase. With this technique, one realizes some of the benefits of quad-polarimetric SAR systems, but not all. An advantage is an extended coverage. However, with compact polarimetry one only gets access to a $2 \times 2$ coherency matrix, whereas quad-polarimetric systems measure the covariance matrix (Raney 2011). Compact polarimetry for oil slick detection has been investigated by simulating compact polarization from Radarsat-2 quad-polarimetric SAR data (Salberg et al. 2012; Shirvany et al. 2012; Nunziata et al. 2015).

\subsubsection{Discussion on the Role of Polarization, on the Maturity of the Application and Conclusions}

We conclude that polarimetric SAR measurements have an improved ability to discriminate between mineral oil slicks and look-alike phenomena, as well as a potential for slick characterization. Interesting internal slick variations are observed, which may be related to differences in thickness or oil volume fraction.

However, we would like to emphasize that, beyond simply detection, a sufficiently high signal-to-noise ratio is necessary to succeed in any physicochemical slick information extraction by polarimetric SAR. Currently, only the VV and HH channels from satellites such as Radarsat- 2 and TerraSAR-X can be utilized for oil slick characterization, while some airborne sensors (e.g. the UAVSAR) have a low enough noise floor to allow exploitation of the whole scattering matrix, which also includes the cross-polarization channels. We would therefore like to emphasize that the choice of SAR sensor properties, such as the noise floor level and the selection of incidence angles, needs to be carefully considered when designing new missions aiming for oil pollution monitoring.

To lift the application described in this chapter to an operational stage, our aim is first to test the techniques on a wider range of data samples, including a wider spectre of metrological conditions, water-in-oil mixtures and surfactants, and second to build up a training dataset that could be used as input to a supervised classifier.

\subsection{Ship Detection}

\subsubsection{Methodology}

The aim of this section is to present a comparison of ship detectors exploiting quad-, dual- and single-polarimetric data over a dataset where a ground survey was carried out during the acquisition. A theoretical presentation of the algorithms exploited for ship detection is included in the previous sections and introductory chapters.

\subsubsection{Test Sites and Data Sets}

Test sites and corresponding radar and validation data sets selected for the generation of showcases on ship detection are summarized in Table 6.6.

\subsubsection{Brief Polarimetric Analysis of the Data}

All the algorithms consider an initial multi-look of $1 \times 5$ (range $\mathrm{x}$ azimuth) pixels applied on the elements of the covariance matrix. This makes the pixel more squared on the ground. Subsequently, a boxcar filter of $3 \times 3$ pixels is employed to further reduce the pixels statistical variation. Clearly, the samples are not all independent each other, and the equivalent number of looks (ENL) returned by such averaging is $\mathrm{ENL}=15$.

The aim of this section is to evaluate the L-band polarimetric behaviour of the sea in this scene. An easy way to display (part of) the information contained in quadpolarimetric acquisitions is by using a Pauli RGB colour composite image. The Pauli scattering vector is defined as $\mathbf{k}_{\mathbf{p}}$, whose first component represents odd bounce (as surface scattering or trihedral corner reflectors), the second is an even bounce (as dihedrals corner reflectors horizontally oriented),

Table 6.6 Test sites and corresponding radar and validation data selected for the generation of showcases on ship detection

\begin{tabular}{l|l|l}
\hline Application/Product & Test site - radar data & Reference data \\
\hline \multirow{2}{*}{ Ship detection } & Tokyo Bay, Japan & NDA reference data (video camera, ground radar, AIS) \\
& ALOS-PalSAR & \\
\hline
\end{tabular}


and the third is a dihedral with the corner 45 degree oriented along the Line of Sight (please note, in case of absence of target orientations, the latter is often exploited as an indicator of volume scattering). Figure 6.10 presents the RGB image of the test area with the overlapped "ground-surveyed" targets.

In this section, the visual interpretation is used as a benchmark to compare the algorithms with the results that could be provided by a SAR image analyst. In the following, green indicates detected targets, while red is for missing vessels. The circles are targets observed in the ground survey and visible in the RGB image, while the rectangles are vessels present in the survey but invisible in the RGB. The number beside the rectangles indicates that more vessels are closed to each other in the same area. The green curve delineates an area where seaweed farms (laver) are present. Please note there are also other coastal areas where laver cultivations are present, but they are not listed in the survey. The video camera and the ground radar were located close to the NDA (National Defence Academy) indicated by a black circle.

In the Pauli RGB [Fig. 6.10a], the sea is clearly distinguishable from other features in the scene due to its blue colour. The Bragg scattering model can be used to approximate the scattering from the sea surface at this frequency. An exception is when the sea state is particularly calm (or generally when the gravitational and capillary waves are dampened) leading to a backscattering proximal to the noise floor. In the image, many point targets in the sea area appear to present a clearly distinguishable polarimetric behaviour (i.e. different colours). In order to have an intuitive idea regarding possible advantages of using quad/polarimetric data, Fig. $6.10 \mathrm{~b}$ presents the intensity of the HV channel (cross-polarization). The latter is often exploited to perform ship detection since, accordingly to the Bragg model, the cross-polarization should be negligible on the sea, therefore increasing the contrast between ships and sea. However, only using HV the visual discrimination between ships and sea becomes harder.

Further details regarding the sea polarimetric behaviour can be obtained applying the Cloude-Pottier decomposition. Figure 6.11 presents the resulting entropy and averaged $\alpha$ angle. As expected, the sea exhibits relatively low values of entropy indicating one dominant scattering mechanism. The averaged $\alpha$ is also small, indicating surface scattering. Finally, it is interesting to notice that in some sea areas, especially exhibiting low backscattering, the values of entropy and $\alpha$ increase. This is mainly due to noise effects.

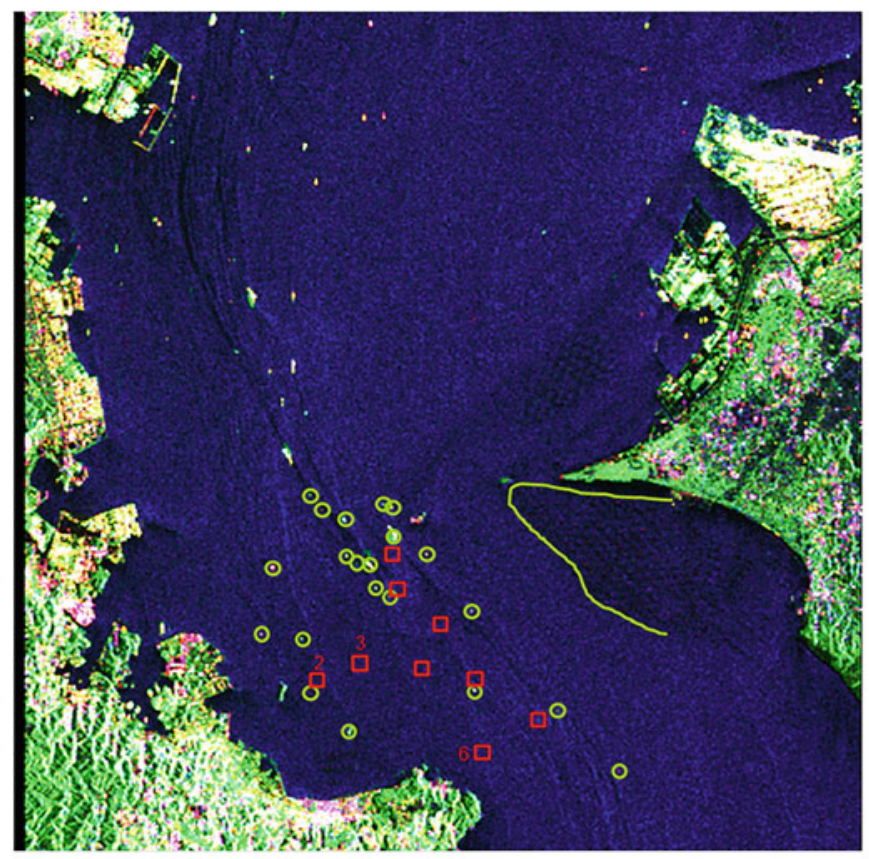

(a) Pauli RGB

Fig. 6.10 RGB Pauli and HV intensity of the surveyed area, ALOSPALSAR (JAXA), Tokyo Bay $(35,294451 ; 139,785816)$. Green circles, targets present in the ground survey and visible in the RGB; red rectangles, vessels present in the ground survey but completely invisible in the RGB. The green curve delineates an area with seaweed (laver)

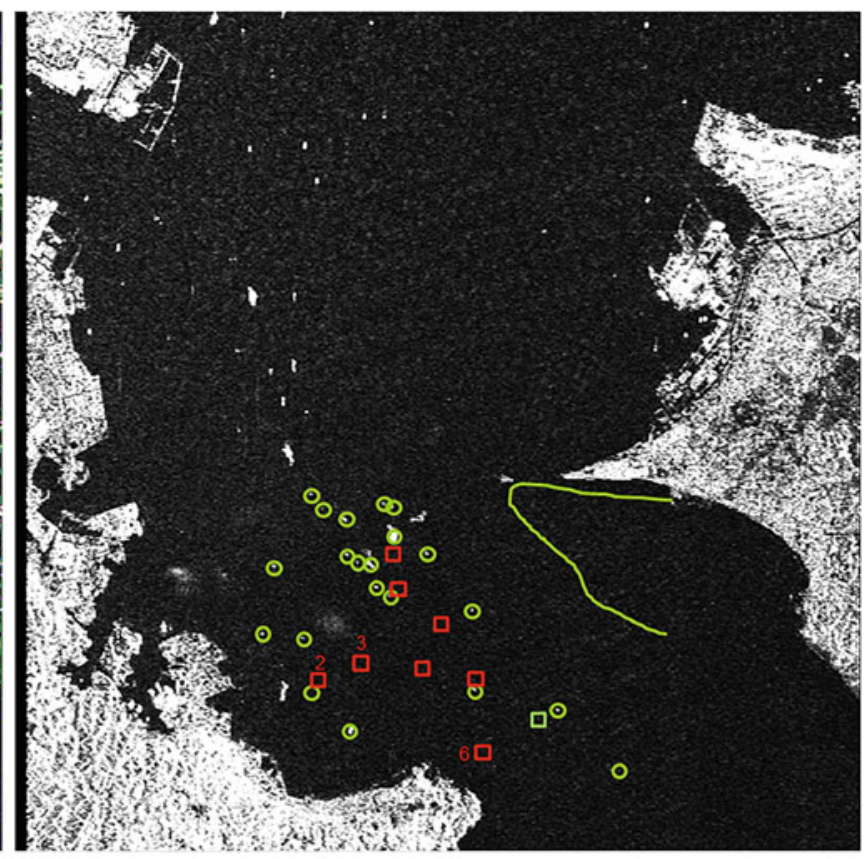

(b) HV intensity

cultivations. The video camera and the ground radar were located close to the NDA (National Defence Academy) indicated by a black circle. Incidence angle: 24degrees. Multi-look: 1x5. Image size: $1000 \times 1000$ pixels, $\sim 30 \times 30 \mathrm{~km}$ 


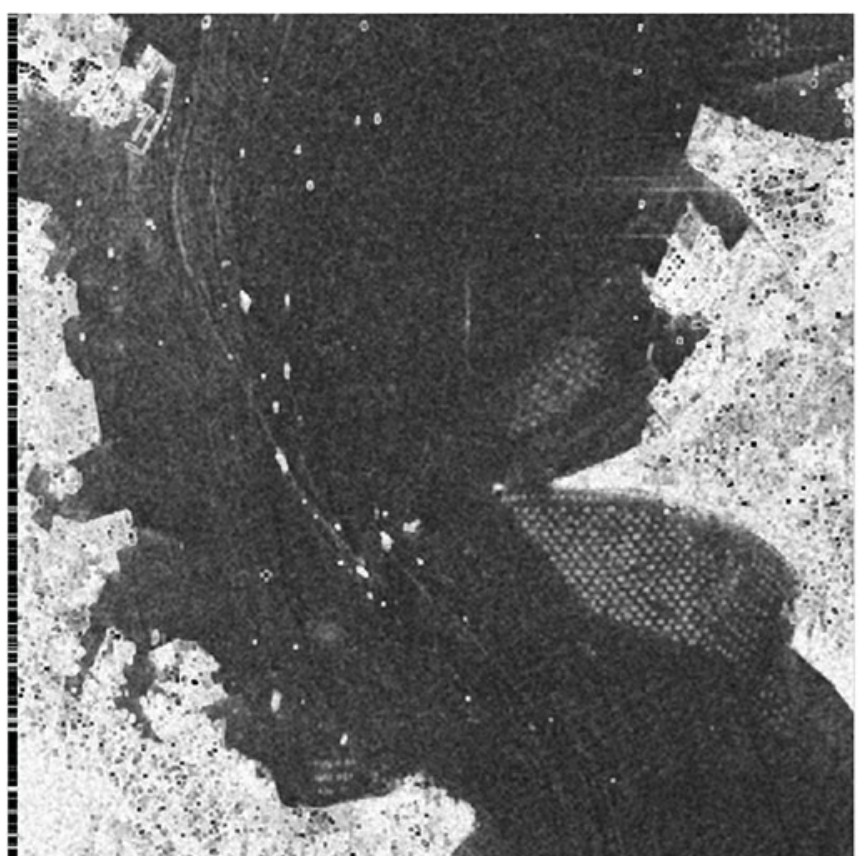

(a) Entropy

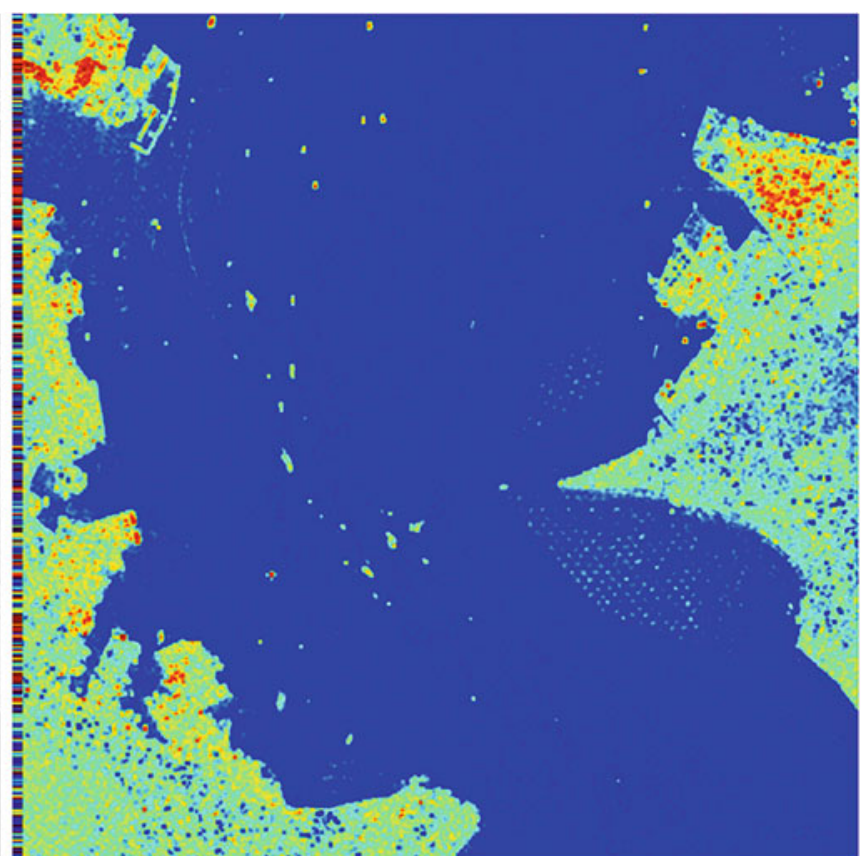

(b) Averaged $\alpha$

Fig. 6.11 Entropy and averaged $\alpha$ angle from the Cloude-Pottier decomposition. (a) Entropy, scaled between 0 and 1; (b) averaged $\alpha$, scaled between 0 and $\pi / 2$, blue-red (rainbow) colour bar

\subsubsection{Detection with Quad-Polarimetric Data}

The quad-polarimetric detectors compared in this section are the geometrical perturbation-polarimetric notch filter (PG-PNF) (Marino 2013), the polarimetric match filter (PMF) (Novak et al. 1989), the Liu et al. detector (Liu et al. 2005) and the polarimetric entropy.

Figure 6.12 presents the detection masks for each detector. The same circles and rectangles shapes extracted by the visual interpretation are represented on each detection masks in order to have a benchmark for algorithm comparison. The window size of the boxcar filter is $3 \times 3$ (after the $1 \times 5$ multilook). The training for the detectors (i.e. the definition of the sea clutter backscattering) is performed manually choosing a representative area in the dataset. An operative solution may consider guard windows; however this is not presented here because it will generate a strong dependency of the results on the dimension of the selected guard window, which may obstruct a proper performance assessment. More details on the detector parameters are provided in the caption of Fig. 6.12. The results are summarized in Table 6.7, in the following only few comments are presented.

\subsubsection{Detection}

Interestingly, all the quad-polarimetric algorithms are able to detect the vessels visible in the RGB image except for the entropy that misses one. Moreover, they can detect one vessel identified in the survey but very hardly visible in the RGB.

\subsubsection{Missing Detection}

Several vessels were missing in the detection masks and visual interpretation. These are mainly small vessels, supposedly made of fibre glass. A finer sensor's spatial resolution may be a way to try to retrieve small targets. However, if a vessel is composed of materials almost transparent to the electromagnetic wave (e.g. absence of large metallic parts), it may still be physically impossible to observe it.

\subsubsection{Seaweed}

All the algorithms seem to be able to identify part of the laver seaweed farm. This is composed of nets lying on the sea surface. Entropy provides the best laver detection; it is followed by the PMF and the GP-PNF. Their detectability is physically based on the fact that the nets damp the Bragg resonant waves leading to a non-Bragg scattering. This is why the entropy is a good candidate to detect them. Please note that in the scene, other coastal areas present laver cultivations and are not indicated in the ground survey (but they can be detected by the entropy). Finally, Liu et al. is missing the most of the nets.

\subsubsection{False Alarms}

Unfortunately, the ground survey that accompanies the SAR data is not accurate enough to allow a reliable estimation. For instance, a vessel detected by the SAR and invisible to the video/radar (perhaps because too far from the NDA buildings) would be listed as false alarm. However, for 


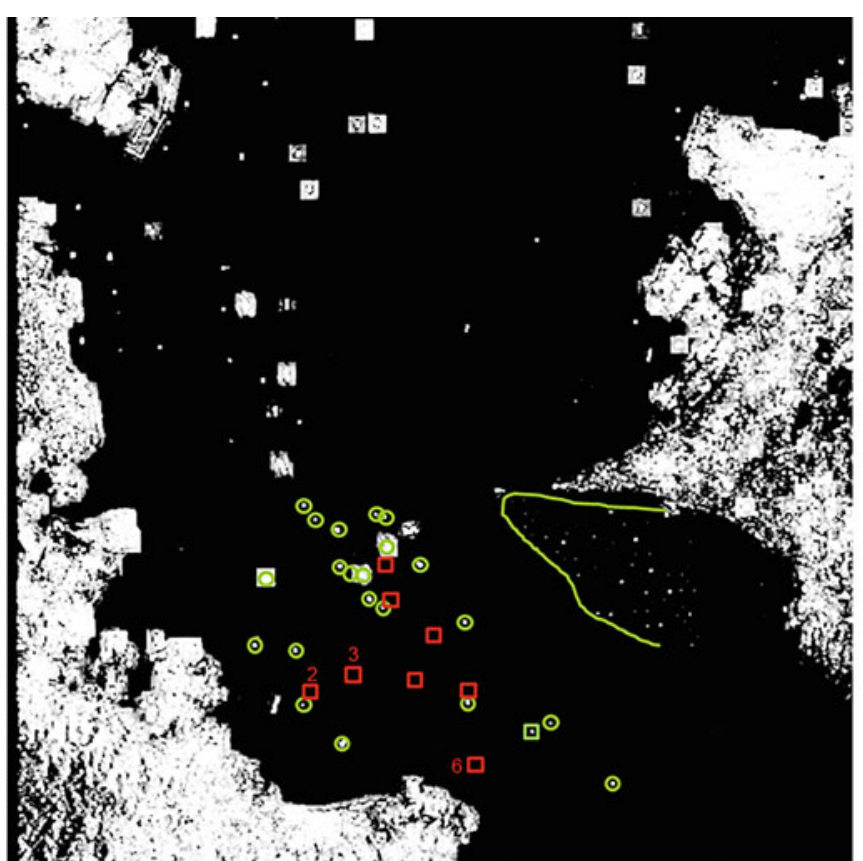

(a) GP-PNF

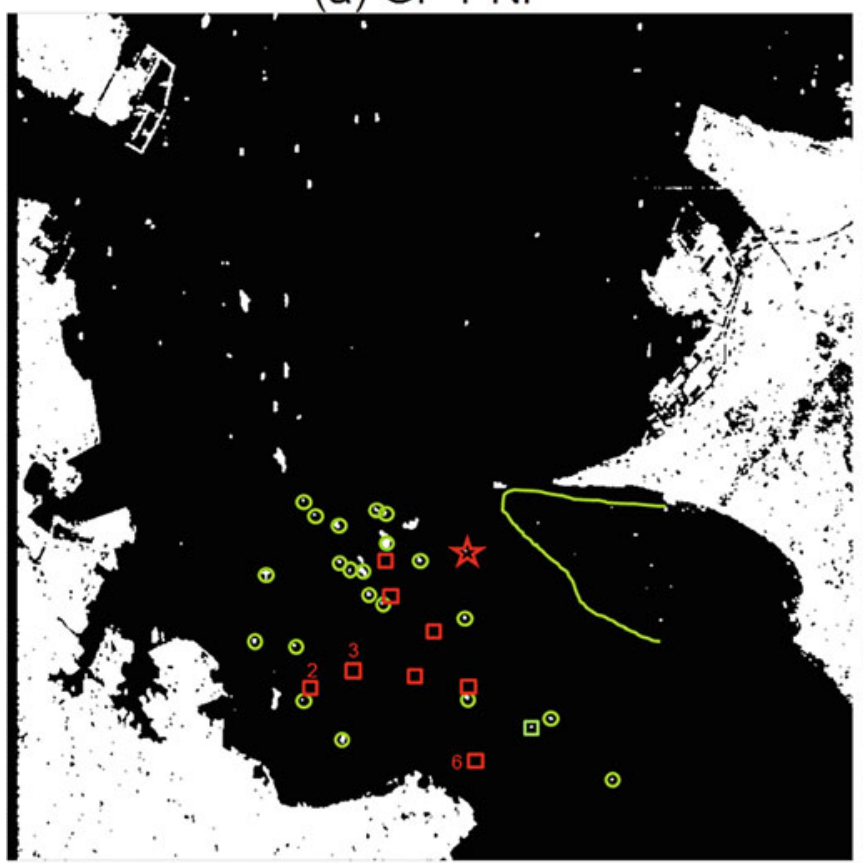

(c) Liu et al.

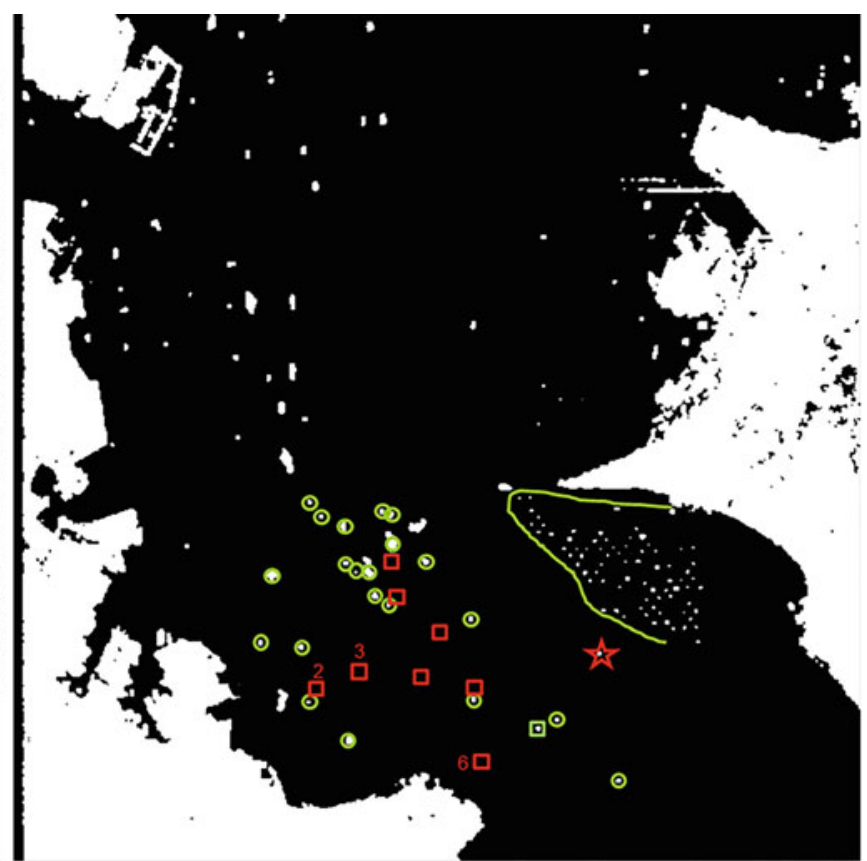

(b) PMF

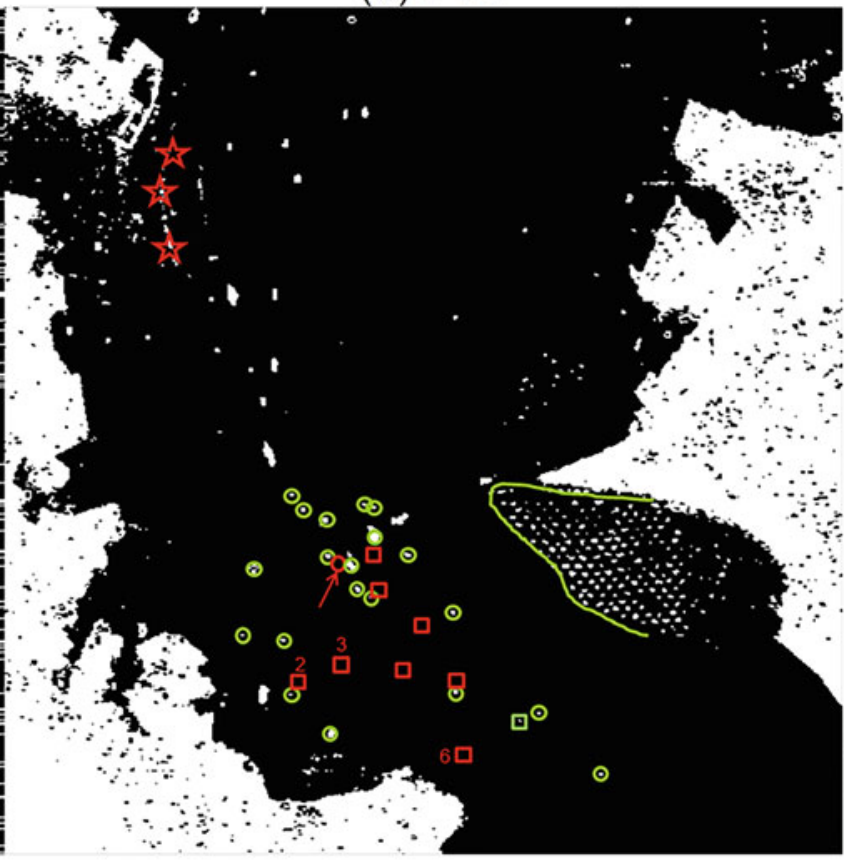

(d) Entropy

Fig. 6.12 Detection with quad-polarimetric data: (a) GP-PNF $\left(\operatorname{Re} R=10^{-4}\right.$ and $\left.T=0.98\right)$; (b) PMF $(T=4)$; (c) Liu et al. $\left(P_{f}=10^{-4}\right)$; (d) entropy $(T=0.5)$. Green, detected vessels; red, missing vessels. Red arrows: missing vessels that can be visually identified. Stars are supposed false alarms

Table 6.7 Detection results for the algorithms tested

\begin{tabular}{l|l|l|l|l|l|l|l}
\hline Algorithms & Visual RGB & GP-PNF & PMF & Liu et al. & Entropy & Symmetry & HV \\
\hline Detected & 21 & 22 & 22 & 22 & 21 & 14 & 18 \\
\hline Missed & $9(17)$ & $8(16)$ & $8(16)$ & $8(16)$ & $9(17)$ & $16(24)$ \\
\hline False alarms & 0 & 0 & 1 & 1 & Several & 0 & $12(20)$ \\
\hline
\end{tabular}


what is observed in the test area, the GP-PNF does not present any false alarm, while the PMF and Liu et al. have one false alarm each. They are indicated in the images with stars. This result shows the robustness of such detectors. The entropy has no false alarms in the area close to NDA; however, many detections are located in areas exhibiting low backscattering. Some of them correspond to ships wakes and therefore can be easily defined as false alarms. A more thorough treatment of false alarms is provided in the final section when the receiver operating characteristic curves are presented.

\subsubsection{Dual- and Single-Polarimetric Detectors}

In this section, the results with the dual-polarimetric VV/VH symmetry detector (Nunziata et al. 2012b) and a CFAR test on the $\mathrm{HV}$ intensity exploiting the K-distribution (Crisp 2004) is presented. In this detection test, the threshold on the HV intensity is set solving numerically the integral for $P_{f}=10^{-5}$ (exploiting a K-distribution). The threshold for the symmetry detector is 0.1 , since this was shown to be a robust threshold selection over other datasets. As for the other detectors, the boxcar window is $3 \times 3$ after multi-look.

Figure 6.13 presents the detection masks for these two detectors, while the results are summarized in Table 6.7. It is evident that the detection performance is reduced substantially. Compared to the 22 vessels detected by the quad-pol

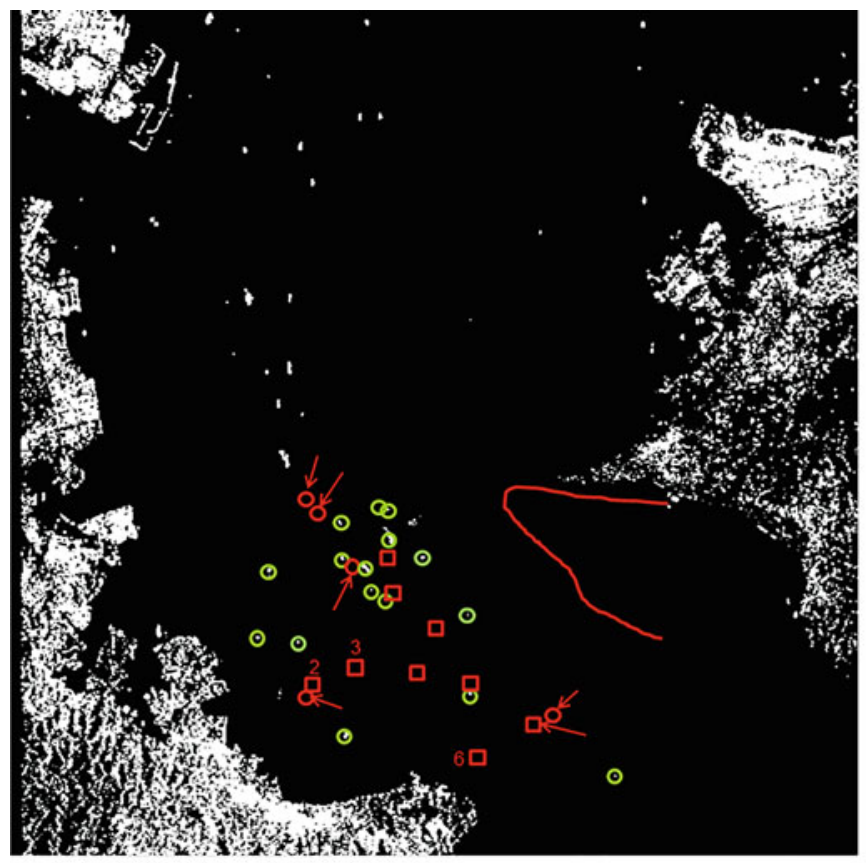

(a) Symmetry algorithms, the HV intensity misses 4, and the symmetry misses 8 vessels. This numbers can be converted in relative degradation of the $P_{d}$ obtaining $18 \%$ for the $\mathrm{HV}$ and $36 \%$ for the symmetry detector. The absolute degradations (when all the surveyed vessels are considered) are $13 \%$ and $26 \%$. In particular, some ships visible in the RGB Pauli image are lost. Other tests were performed changing slightly the threshold in order to ensure the right setting of the detectors parameters. However, the number of detections increased only slightly. In the next section, an analysis is performed to assess the impact of a bad threshold selection to the lower detection performance. The area with seaweed farms is completely missing in the detection masks.

\subsubsection{Receiving Operating Characteristics}

The detection masks presented in the previous section help in understanding the detection capabilities in a more operational test, where one threshold is fixed. However, the threshold selection may be not optimal, and the poor performance of one algorithm may be just caused by a bad threshold selection. For this reason, it is valuable to assess the algorithms performance independently of the threshold selection. This can be accomplished plotting $P_{d}$ against $P_{f}$ and generating the so-called receiver operating characteristic (ROC) curves (Kay 1993). In this way, the problem of optimally selecting the threshold is de-coupled with assessing the best detector.

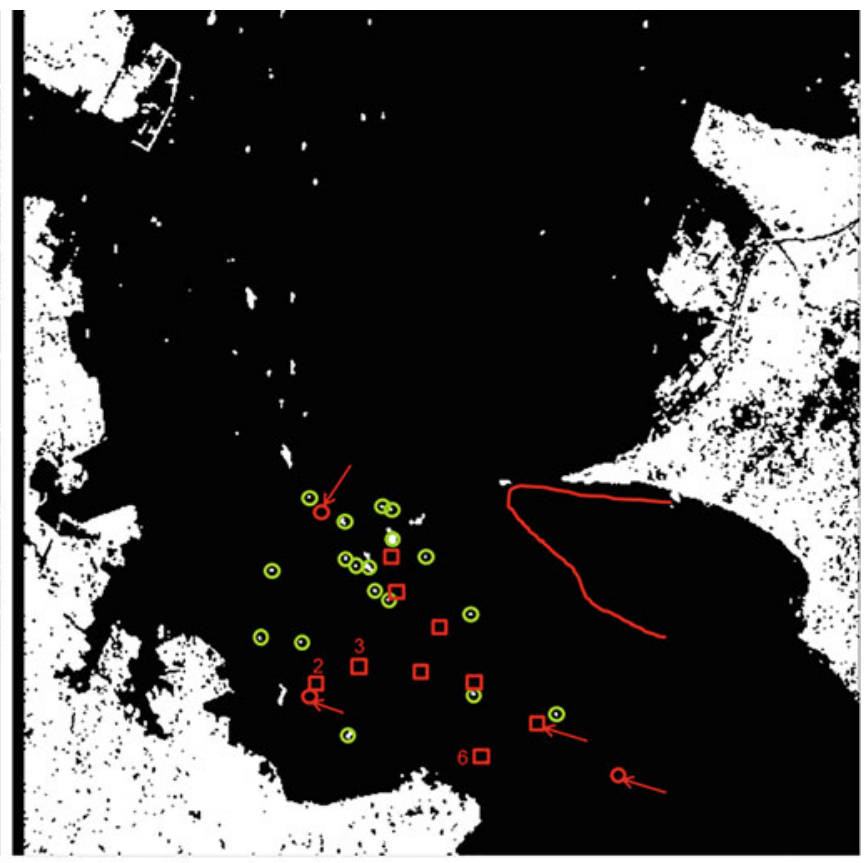

(b) HV intensity

Fig. 6.13 Detection with dual- and single-polarimetric data: (a) symmetry detector $(T=0.1)$; (b) HV intensity $\left(P_{f}=10^{-5}\right)$. Green, detected vessels; red, missing vessels. Red arrows: missing vessels that can be visually identified. Stars are supposed false alarms 


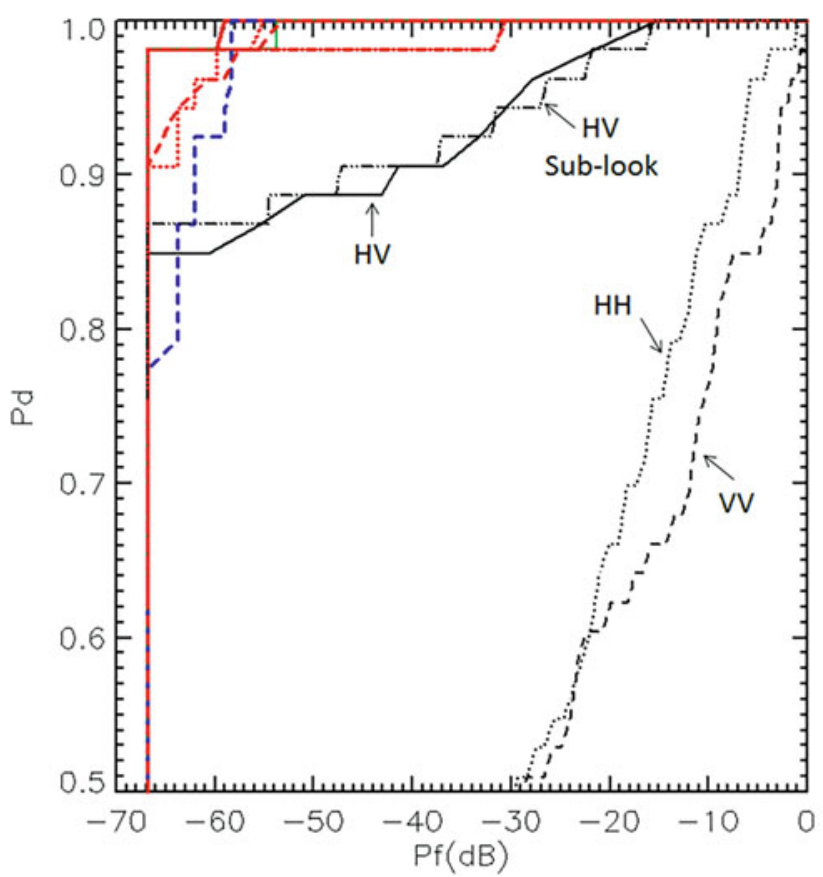

Fig. 6.14 ROC curves for the different detectors: red solid, GP-PNF quad-pol; red dotted, Cloude-Pottier entropy; red dashed, PMF; red squares, Liu et al.; blue dashed, symmetry VV/VH; green solid, dualpol HH/VV GP-PNF; green diamonds, dual-pol HH/HV GP-PNF; black

$P_{d}$ is calculated only for visible vessels, since we were not able to assess the exact location of the pixels representing the missing vessels (i.e. the analysis is restricted to vessels that have some backscattering). In order to find a meaningful value for $P_{f}$, a large area in the dataset outside Tokyo Bay is selected where no apparent vessels were present.

Figure 6.14 shows the ROC curves for the different polarimetric detectors. As a comparison also the single-channel detectors using the intensity of $\mathrm{HV}, \mathrm{HH}$ and $\mathrm{VV}$ are considered. Finally, in order to provide a more complete analysis a detector based on spectral (or sub-look) analysis of SAR data is presented (in the plot, it is indicated with HV sub-look, and a more complete name is sub-aperture cross-correlation magnitude). Details regarding the latter detector are not presented here for the sake of brevity, but they can be found in Brekke et al. (2013)). In very few words, this is a single-channel detector aiming at increasing the contrast between sea clutter and vessels because sea areas in different nonoverlapping portions of the image spectrum are uncorrelated. The sub-look detector tested here considered two nonoverlapping portions of the range spectrum with a $3 \times 3$ window.

Finally, two more detectors are considered in the ROC analysis. The GP-PNF is executed feeding the algorithm with only dual-polarimetric data (HH/VV and HH/HV). The idea behind this analysis is that keeping the same detector and feeding it with fewer images give a clear indication of how the loss of information impacts the detection performance.

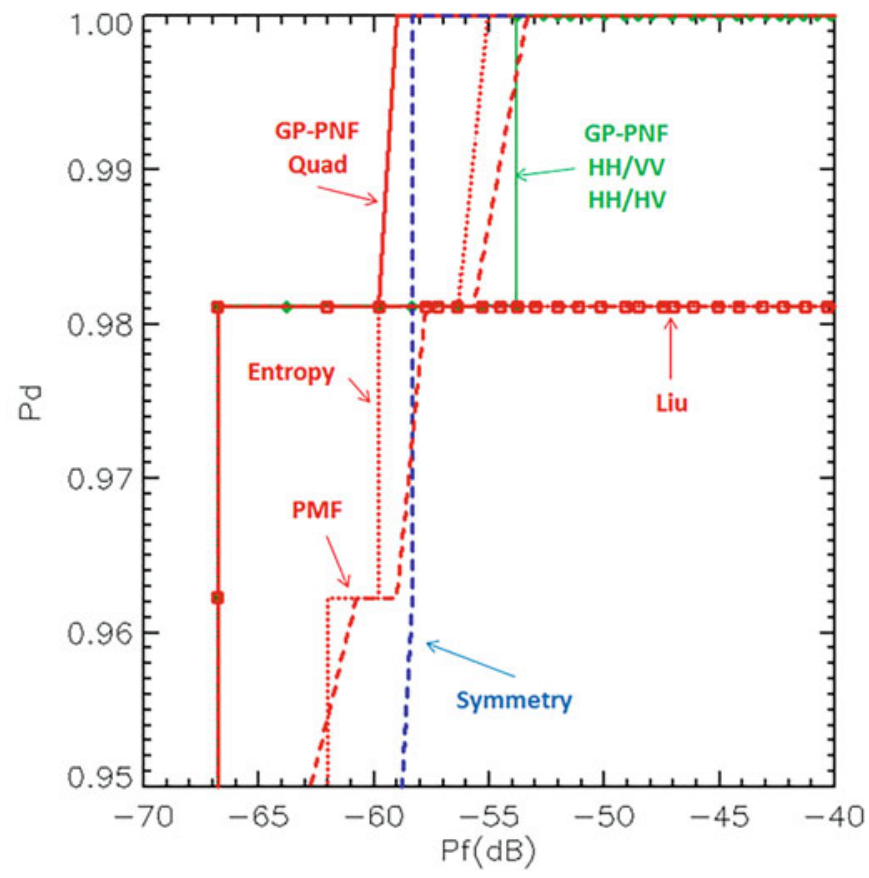

solid, HV intensity; black dotted, HH intensity; black dashed, VV intensity; black dot dash, cross-correlation of sub-look images in HV. (b) Presents a zoom of (a) in the upper left area. Best detection is in the upper left area of the plot

As a final remark, the ROC curves presented here are different from the ones showed in [39] because the area used for estimating the false alarms is different. In [39], a region presenting a large area affected by azimuth ambiguities (coming from the nearby Tokyo) was selected in order to test the sensitivity of the detectors to such artefact. Here, the comparison between different polarimetric modes is investigated, and therefore including artefact may mask part of the differences (since it is never possible to obtain high $P_{d}$ and low $P_{f}$ due to the detection of the ambiguities).

Analysing the ROC curves, few conclusions can be drawn:

1. The best detectors seem to be the quad-polarimetric ones (red curves), followed by the dual-polarimetric ones (blue and green curves).

2. Among the single-polarimetric channels, the HV seems to be the best, followed by HH and finally VV. This can be found in the literature; however, there may be situations where the co-polarized channels may be more beneficial for detection (it depends on sea state and incidence angle).

3. The sub-look processing seems to not improve the HV detection in this test. A reason may be that the sea background is already quite low, and therefore the loss of resolution (due to sub-looking) is not paid back with a significantly better contrast. 
4. The symmetry detector presents a very good ROC curve (not largely inferior to quad-pol detectors), but its $P_{d}$ from the previous analysis was quite low. This is probably due to a wrong selection of the threshold, and therefore it should be possible to improve the detection mask with an algorithm able to select the threshold locally (or at least based on the specific dataset).

\subsubsection{Discussion on the Role of Polarimetry and Conclusions}

This section presented the comparison of quad-, dual- and single-pol detectors over a dataset acquired by ALOSPALSAR and kindly provided by JAXA. The test area is Tokyo Bay, Japan, and during the acquisition, a ground survey was conducted. The latter was carried out with a video camera and a ground radar (in X-band) located beside the Nation Defence Academy (NDA) at $100 \mathrm{~m}$ over the sea level. The availability of such information allowed the evaluation of quantitative results. The quad-pol detectors tested here are the geometrical perturbation-polarimetric notch filter (GP-PNF), the polarimetric match filter (PMF), the Liu et al. and the entropy, while the dual-pol algorithm is the symmetry detector, and the single pol is a CFAR test on the HV intensity exploiting the K-distribution. Besides, a visual inspection was attempted on the RGB Pauli and the HV intensity images to provide a benchmark for the comparison of the other detectors.

The results showed that the quad-pol algorithms are able to detect 22 vessels (i.e. all the vessels visible in the RGB plus one), while the symmetry and HV suffer from more missed detections (respectively 14 and 18 vessels detected) which return a degradation of performances respectively of $13 \%$ and $26 \%$. The false alarms are low for all the detectors tested at exception of the entropy detector which is not suitable in areas presenting low backscattering. Finally, a ROC curve analysis is performed with the purpose of assessing the detector performances independently of the threshold selection.

Some specific comments on the results are as follows:

1. Initially, it is interesting to notice that the use of quadpolarimetry is helpful to improve the detection capability even in the quite straightforward case of human interpretation. In Fig. 5.2 it can be observed that a smaller amount of vessels is distinguishable from the sea clutter in the HV intensity image alone.

2. All the quad-pol detectors perform in similar ways in the context of detection capability. This is an indicator that in this experiment, the main driver for the detection performance is quad-polarimetry (compared to dual-polarimetry) more than the specific detector or the selection of its parameters. Clearly, in other datasets a larger difference between the quad-pol detectors could be observed.

3. Analysing the ROC curves, the difference between quadand single-pol detectors is large on this dataset, especially for the case of the co-polarizations. In the case of $\mathrm{HV}$, a $P_{d}>0.95$ can only be reached at the expenses of a $P_{f}>10^{-3}$ (which is not appropriate for many applications).

\subsubsection{Acknowledgement}

The authors would like to thank the Japanese Aerospace Exploration Agency (JAXA) for the kind provision of the ALOS-PALSAR data.

\subsection{Summary (Table 6.8)}

Table 6.8 Summary of presented application, methods and preferred system configurations for ocean monitoring

\begin{tabular}{l|l|l}
\hline Application & Methods and used frequency (P/L/C/X) & Radar data preference/requirements/comments \\
\hline Oil observation & PolSAR features from T/C matrix for detection (L/X) & $\begin{array}{l}\text { No preferred frequency } \\
\text { Careful choice of incidence angle and noise floor } \\
\text { for system design }\end{array}$ \\
\cline { 2 - 3 } & $\begin{array}{l}\text { Unsupervised classification based on PolSAR features for } \\
\text { classification of oil types (C/X) }\end{array}$ & No preferred frequency \\
\hline \multirow{2}{*}{$\begin{array}{l}\text { Metallic targets and ship } \\
\text { detection }\end{array}$} & $\begin{array}{l}\text { PolSAR detectors based on T/C matrix for metallic target } \\
\text { detection (C) }\end{array}$ & \\
\cline { 2 - 3 } & PolSAR detectors based on T/C matrix for ship detection (L) & \\
\hline
\end{tabular}




\section{References}

(2005) Aerial-surveillance - Cooperation on aerial surveillance over the North Sea area. In: Bonn Agreement Counter Pollution Manual

Brekke C, Solberg AHS (2008) Classifiers and confidence estimation for oil spill detection in ENVISAT ASAR images. IEEE Geosci Remote Sens Lett 5:65-69

Brekke C, Anfinsen SN, Larsen Y (2013) Subband extraction strategies in ship detection with the subaperture cross-correlation magnitude. IEEE Geosci Remote Sens Lett 10:786-790

Brekke C, Holt B, Jones C, Skrunes S (2014) Discrimination of oil spills from newly formed sea ice by synthetic aperture radar. Remote Sens Environ 4:1-14

Crisp DJ (2004) The state-of-the-art in ship detection in synthetic aperture radar imagery. Defence science and technology organisation, DSTO-RR-0272. [online]. http://www.dtic.mil/cgibin/GetTRDoc?AD=ada426096

Ferrara G, Migliaccio M, Nunziata F, Sorrentino A (2011) GK-based observation of metallic targets at sea in full-resolution SAR data: a multipolarization study. IEEE J Ocean Eng 36:195-204

Hühnerfuss H (2006) Basic physicochemical principles of monomolecular sea slicks and crude oil spills. In: Gade M, Hühnerfuss H, Korenowski GM (eds) Marine surface films. Springer, Berlin/ Heidelberg

Jones CE, Minchew B, Holt B, Hensley S (2011) Studies of the deepwater horizon oil spill with the UAVSAR radar. Geophys Monogr Ser 195:33-50

Kay SM (1993) Fundamentals of statistical signal processing. Prentice Hall

Kotova LA, Espedal HA, Johannessen OM (1998) Oil spill detection using spaceborne SAR: a brief review. In: Proceedings of 1998 International Symposium on Remote Sensing of the Environment

Liu C, Vachon PW, Geling GW (2005) Improved ship detection with airborne polarimetric SAR data. Can J Remote Sens 31:122-131

Marino A (2013) A notch filter for ship detection with polarimetric SAR data. IEEE J Sel Top App Earth Obs Remote Sens 6:1219-1232

Migliaccio M, Nunziata F (2014) On the exploitation of polarimetric SAR data to map damping properties of the deepwater horizon oil spill. Int J Remote Sens 35:3499-3519

Migliaccio M, Gambardella A, Nunziata F, Shimada M, Isoguchi O (2009a) The PALSAR polarimetric mode for sea oil slick observation. IEEE Trans Geosci Remote Sens 47:4032-4041

Migliaccio M, Nunziata F, Gambardella A (2009b) On the co-polarised phase difference for oil spill observation. Int J Remote Sens 30:1587-1602

Migliaccio M, Nunziata F, Montuori A, Brown CE (2011a) Marine added-value products by RADARSAT-2 fine quad-polarization mode. Can J Remote Sens 37:441-450

Migliaccio M, Nunziata F, Montuori A, Li X, Pichel W (2011b) A multifrequency polarimetric SAR processing chain to observe oil fields in the Gulf of Mexico. IEEE Trans Geosci Remote Sens 49:4729-4737

Minchew B, Jones CE, Holt B (2012) Polarimetric analysis of backscatter from the deepwater horizon oil spill using L-band synthetic aperture radar. IEEE Trans Geosci Remote Sens 50:3812-3830
Novak LM, Sechtin MB, Cardullo MJ (1989) Studies of target detection algorithms which use polarimetric radar data. IEEE Trans Aerosp Electron Syst 25:150-165

Nunziata F, Migliaccio M (2013) On the COSMO-SkyMed PingPong mode to observe metallic targets at sea. IEEE J Ocean Eng 38:71-79

Nunziata F, Gambardella A, Migliaccio M (2008) On the Mueller scattering matrix for SAR oil slick observation. IEEE Geosci Remote Sens Lett 5:691-695

Nunziata F, Migliaccio M, Gambardella A (2011) Pedestal height for oil spill observation. IET Radar Sonar Navigation 5:103-110

Nunziata F, Gambardella A, Migliaccio M (2012a) A unitary Muellerbased view of polarimetric SAR oil slick observation. Int J Remote Sens 33:6403-6425

Nunziata F, Migliaccio M, Brown CE (2012b) Reflection symmetry for polarimetric observation of man-made metallic targets at sea. IEEE $\mathbf{J}$ Ocean Eng 37:384-394

Nunziata F, Migliaccio M, Li X (2015) Sea oil slick observation using hybrid-polarity SAR architecture. IEEE J Ocean Eng 40:426-440

Raney RK (2011) A perspective on compact polarimetry. IEEE Geosci Remote Sens Newslett 160:12-18

Reed M et al (1999) Oil spill modeling towards the close of the 20th century: overview of the state of the art. Spill Sci Technol Bull $5: 3-16$

Salberg AB, Rudjord, Ø, Solberg AHS (2012) Oil spill detection in compact polarimetry SAR images. Paper presented at ESA SeaSAR 2012

Shirvany R, Chabert M, Tourneret JY (2012) Ship and oil-spill detection using the degree of polarization in linear and hybrid/compact dualpol SAR. IEEE J Sel Top Appl Earth Obs Remote Sens 5:885-892

Skrunes S, Brekke C, Eltoft T (2012a) An experimental study on oil spill characterization by multi-polarization SAR. In: Proceedings of 2012 European Conference on Synthetic Aperture Radar (EUSAR)

Skrunes S, Brekke C, Eltoft T (2012b) A comprehensive analysis of polarimetric features for oil spill characterisation. Paper presented at ESA SeaSAR 2012

Skrunes S, Brekke C, Eltoft T, Kudryavtsev V (2015) Comparing nearcoincindent $\mathrm{C}$ - and X-band SAR acquisitions of marine oil spills. IEEE Trans Geosci Remote Sens 53:1958-1975

Solberg AHS (2012) Remote sensing of ocean oil-spill pollution. Proc IEEE 100:2931-2945

Touzi R, Charbonneau F (2002) Characterization of target symmetric scattering using polarimetric SARs. IEEE Trans Geosci Remote Sens 40:2507-2516

Velotto D, Migliaccio M, Nunziata F, Lehner S (2011) Dual-polarized TerraSAR-X data for oil-spill observation. IEEE Trans Geosci Remote Sens 49:4751-4762

Wenguang W, Fei L, Peng W, Jun W (2010) Oil spill detection from polarimetric SAR image. In: Proceedings of IEEE 2010 International Conference on Acoustic Speech Signal Processing (ICASSP)

Yeremy M, Campbell JWM, Mattar K, Potter T (2001) Ocean surveillance with polarimetric SAR. Can J Remote Sens 27:328-344

Zhang B, Perrie W, Li X, Pichel GP (2011) Mapping Sea surface oil slicks using RADARSAT-2 quad-polarization SAR image. Geophys Res Lett 38:1-5

Open Access This chapter is licensed under the terms of the Creative Commons Attribution 4.0 International License (http://creativecommons.org/ licenses/by/4.0/), which permits use, sharing, adaptation, distribution and reproduction in any medium or format, as long as you give appropriate credit to the original author(s) and the source, provide a link to the Creative Commons license and indicate if changes were made.

The images or other third party material in this chapter are included in the chapter's Creative Commons license, unless indicated otherwise in a credit line to the material. If material is not included in the chapter's Creative Commons license and your intended use is not permitted by statutory regulation or exceeds the permitted use, you will need to obtain permission directly from the copyright holder. 


\section{Appendix: Test Sites and Data Sets}

\section{Forest Applications}

\section{Remningstorp Forest}

The Remningstorp test site $\left(58^{\circ} 30^{\prime} \mathrm{N}, 13^{\circ} 40^{\prime} \mathrm{E}\right)$ covers about 1200 ha of productive forest land. It is classified as hemiboreal, and the dominant tree species are Norway spruce, Scots pine, and birch. Till is the main soil type. The area is fairly flat with topographic variations between 120 and $145 \mathrm{~m}$ above sea level. Location and views of the Remningstorp test site are shown in Fig. 1.

In spring 2007, ground measurements of every single tree with a diameter at breast height $(\mathrm{dbh})$ larger than $5 \mathrm{~cm}$ were carried out in 11 plots having an extent of $80 \mathrm{~m}$ by $80 \mathrm{~m}$ for 4 of them and $20 \mathrm{~m}$ by $50 \mathrm{~m}$ for the others. Additionally, six plots of $80 \mathrm{~m}$ by $80 \mathrm{~m}$ extent are available from fall 2006 . Laser scanning was performed in April 2007 and covered the main area of 400 ha where the ground measurement campaign took place. With support from in situ data (field plots) available from 2004 and 2005, the laser scanning data were processed into a stem volume map with $10 \mathrm{~m}$ grid from which biomass estimates were calculated using biomass expansion factors. In addition, a digital elevation model and a digital surface model were also derived based on the laser data set.

\section{Krycklan Forest}

The Krycklan site is located in central Sweden $\left(64^{\circ} 10^{\prime} \mathrm{N}\right.$, $20^{\circ} 01^{\prime}$ E) (see Fig. 1) and represents a typical Scandinavian boreal forest. It is a managed forest with a mean forest height of $18 \mathrm{~m}$ and a mean biomass level of $90 \mathrm{t} / \mathrm{ha}$. The maximum measured forest height is $28 \mathrm{~m}$ with a biomass of $220 \mathrm{t} / \mathrm{ha}$. The forest is dominated by coniferous tree species (Norway spruce and Scots pine) with fractions of birch. The site has a hilly topography characterized by moderate slopes and a height variation between $20 \mathrm{~m}$ and $400 \mathrm{~m}$ amsl. The bedrock consists almost entirely of gneiss. The dominating soil is moraine, with variations in thickness.

\section{Traunstein Forest}

The Traunstein test site represents a temperate mixed mountain forest and is located in southeastern Germany $\left(47^{\circ} 51^{\prime} \mathrm{N}\right.$, $12^{\circ} 29^{\prime}$ E) (see Fig. 2 for some views). Dominant tree species are European spruce and silver fir accompanied with small fractions of European beech. It is a managed forest composed of even-aged stands which cover forest heights from $10 \mathrm{~m}$ to $40 \mathrm{~m}$. Mean biomass level is on the order of $210 \mathrm{t} / \mathrm{ha}$ while some old forest stands can reach biomass levels up to $600 \mathrm{t} /$ ha. Compared to other managed forests in this ecological zone (mean biomass of $121 \mathrm{t} / \mathrm{ha}$ ), the biomass values of Traunstein forest are significantly higher. Typical for the pre-alpine character of the test site is the fairly flat relief disturbed by some steep slopes embedded in a hilly landscape.

Traunstein test site has already been used for more than 100 years for forest growth and forest yield studies from the chair of forest yield science of the "Technische Universität München" (Technical University of Munich, TUM) and therefore well documented with high precision ground measurements. In total, data from three extensive ground inventory surveys are available $(2003,2008,2013)$. Ground surveys are done by means of inventory plot measurements on a grid basis. For this, a $100 \mathrm{~m}$ by $100 \mathrm{~m}$ grid was laid over the test site with an inventory point at each cross section. Each plot is circular with a radius of $12.62 \mathrm{~m}$ and represents an area of $500 \mathrm{~m}^{2}$. In total, around 220 inventory plots are measured. Most important measurement parameters of the inventory survey are tree height, $\mathrm{dbh}$, and tree species information (needed for biomass estimation). In 2008 and 2012, laser scanning flights were performed (July 2008) to provide complete forest height measurements all over the test site. 

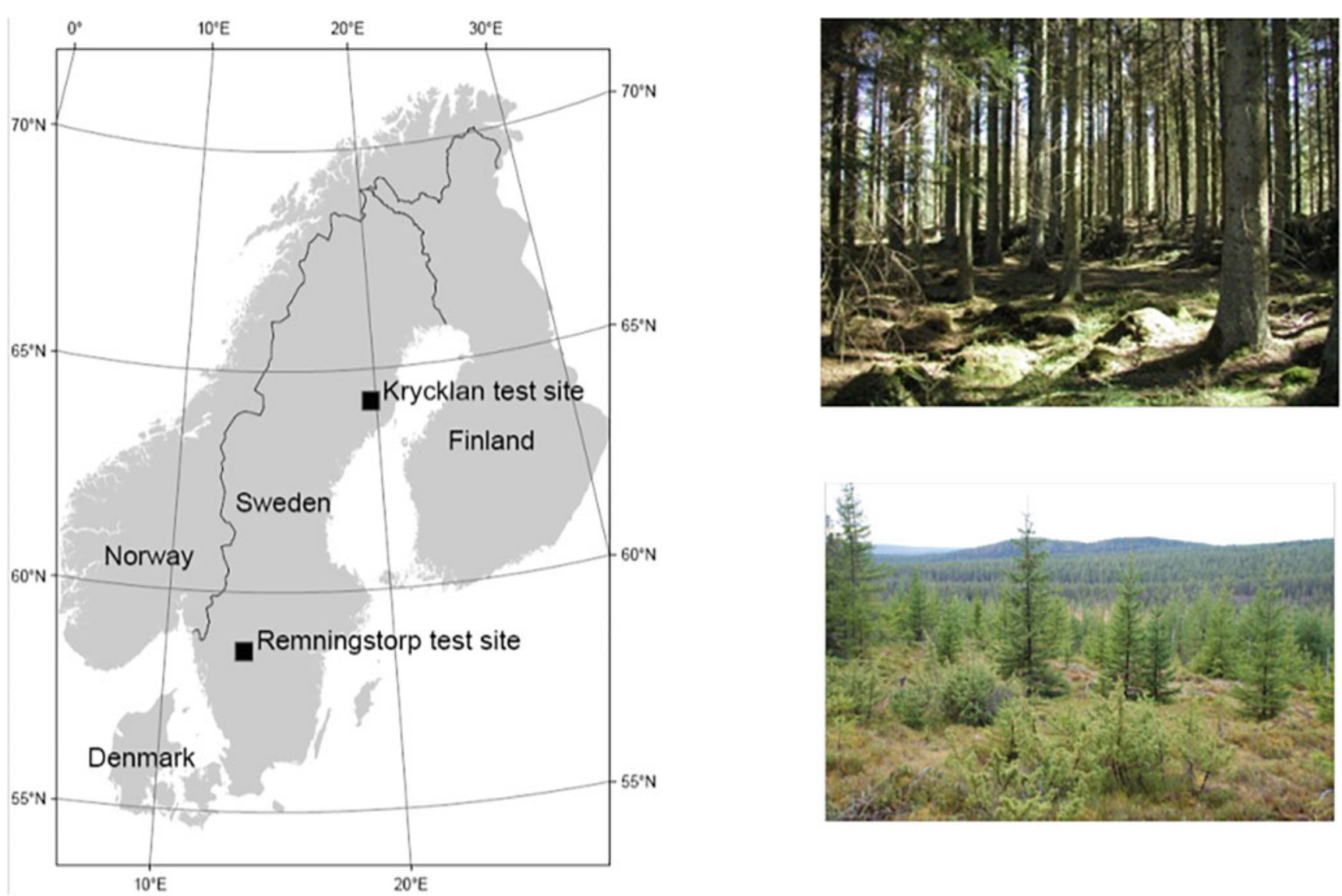

Fig. 1 Locations and views of the Remningstorp test site
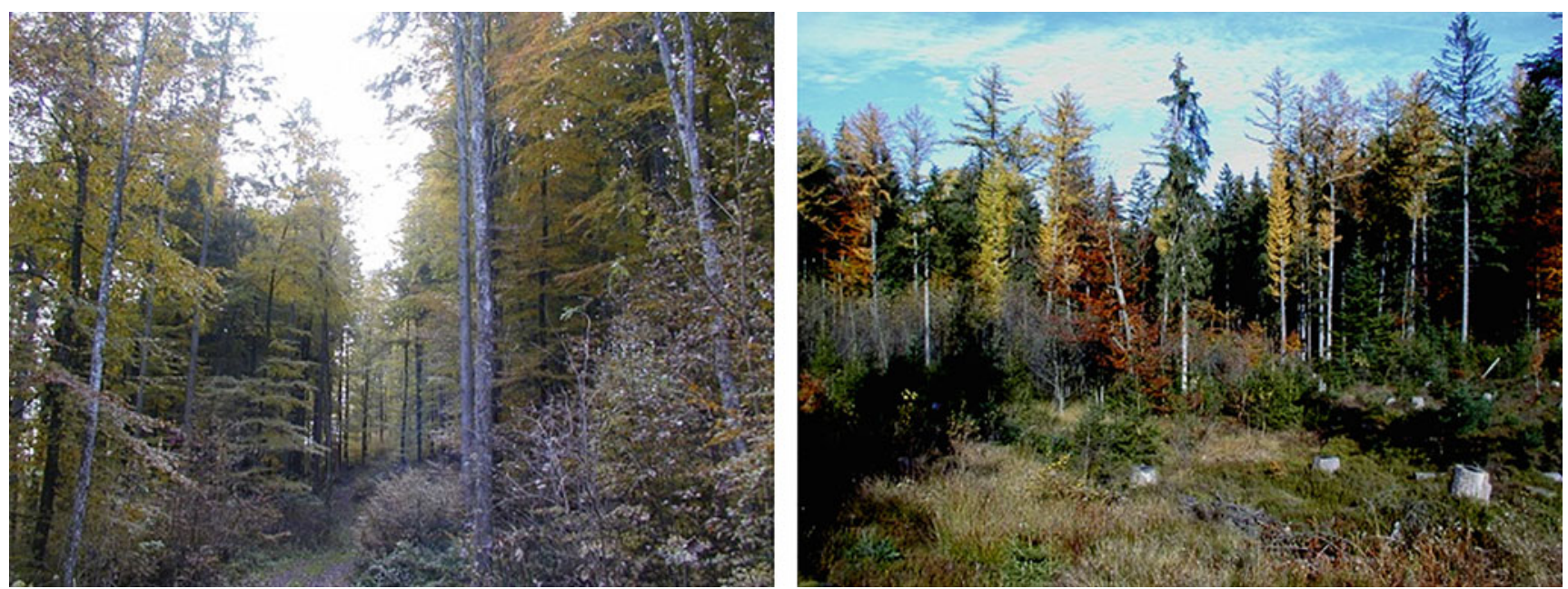

Fig. 2 Two views of the Traunstein forest

Laser scanning data provided a digital elevation model and a digital surace model, from which a forest height map (top height) was derived.

\section{Keg River Forest}

The center geo-coordinates of the Keg River forest are recorded at $57^{\circ} 35^{\prime} \mathrm{N}, 117^{\circ} 45^{\prime} \mathrm{W}$. The study area is about 


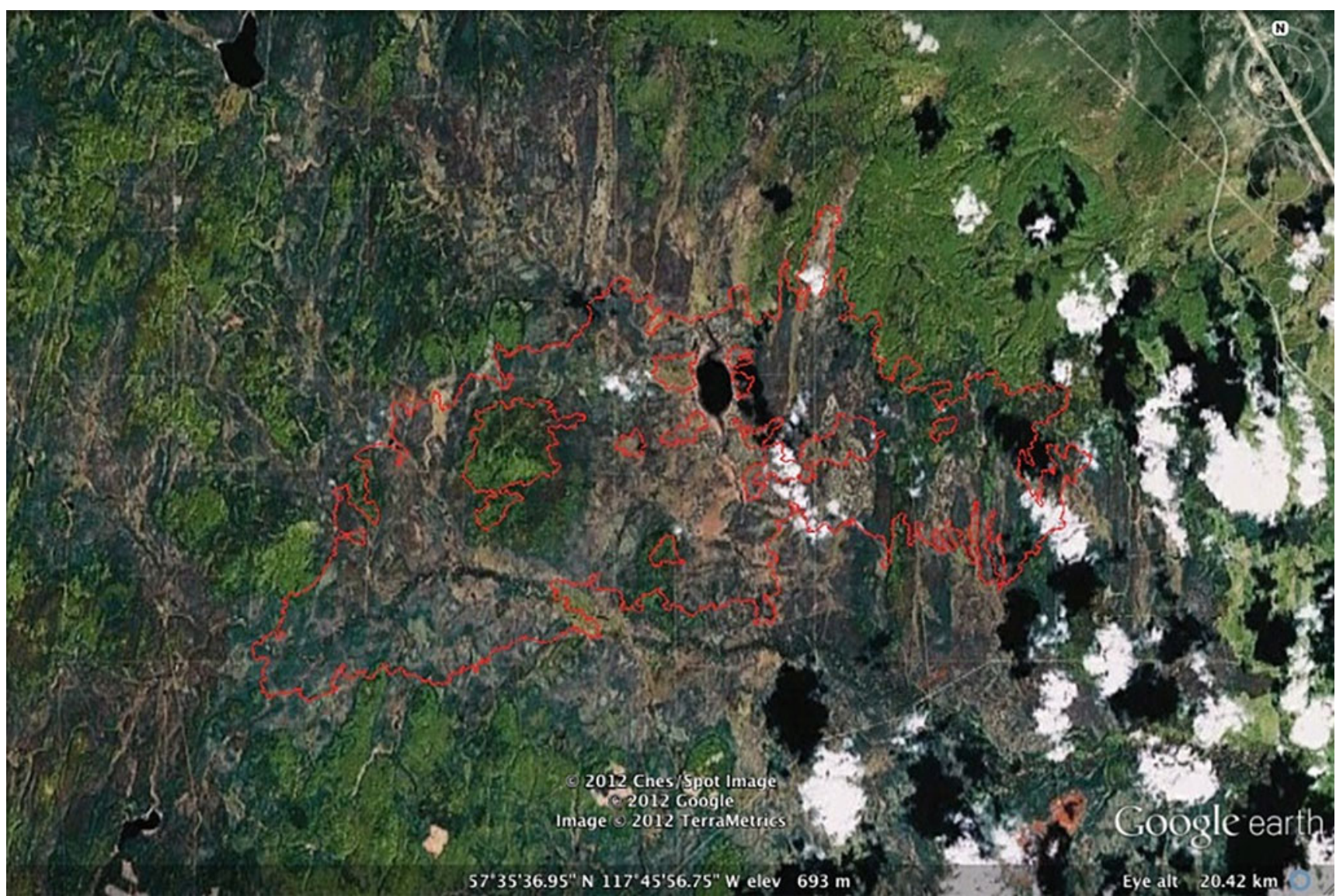

Fig. 3 Fire scar polygon over the Keg River Forest, Alberta, Canada

$900 \mathrm{~km}^{2}$ and the elevation of the site ranges from 400 to $800 \mathrm{~m}$ above sea level. This region is dominated by coniferous forest species, demonstrated in a forest land cover map of Canada produced by the Earth Observation for Sustainable Development (EOSD) of Forests. This area is known with its extensive fire history with multiple fires in every decade since 1950. A latest wild forest fire in this region occurred in 2002, which burned approximately 4830 ha. The Alberta historical wild fire GIS polygons, representing collections based on fire years since 2000, are available from the Government of Alberta. A fire scar polygon in the Keg River region, digitized from aerial photos in 2002, was extracted from this GIS dataset and is shown in Fig. 3 superimposed on a Google Earth image.

\section{Mawas Forest}

The Mawas site is an Indonesian forest conservation area located in Central Kalimantan $\left(2^{\circ} 09^{\prime} \mathrm{S}, 114^{\circ} 27^{\prime} \mathrm{E}\right)$. It lays over a flat topography including several large (ombrogenous) peat domes and is covered by tropical peat swamp forest types. Forest height varies gradually from relatively tall
$(30 \mathrm{~m})$ and dense forest at the edges toward small $(15 \mathrm{~m}$ or lower) and open forest at the center of a dome with biomass levels from 20 to $250 \mathrm{t} / \mathrm{ha}$. The forest is still marked by strong logging activities carried out in the early 1990s last century. In August 2007, lidar measurements were performed with a swath width of about $300 \mathrm{~m}$ along a $22-\mathrm{km}$-long strip. The spatial resolution is of 3-4 m, dependent on the amount of returning samples; the pixel density decreases from the center (nadir) to the corners of the image. From the Lidar raw data, forest height and ground terrain digital elevation models have been derived.

\section{Guaviare Forest}

The Guaviare study site corresponds to an inland tropical forest area located in the Colombian Amazon $\left(2^{\circ} 30^{\prime} \mathrm{N}\right.$, $72^{\circ} 30^{\prime} \mathrm{W}$ ). The area is characterized by a flat terrain, welldrained soils, and flooding occurs seasonally in main rivers. The Guaviare site is a colonization area were land cover types are changing creating a dynamic landscape mosaic with four main vegetation cover types: primary forest, secondary forest, recently deforested (burnt) areas, and grasslands. 
Recently deforested areas are those where forest has been recently cut, and the remnants have been burnt after a short period of drying. Usually crops are planted shortly after burning, while big branches, trunks, and stumps of big trees remain present for some time. For the identification and description of the land cover types, direct field observations on vegetation and terrain characteristics like drainage, flooding and slopes, were made at 124 locations.

\section{Agriculture and Wetlands Applications}

\section{Carman, Casselman, and Indian Head}

The Carman (Manitoba, $49^{\circ} 63^{\prime} \mathrm{N}, 98^{\circ} 00^{\prime} \mathrm{W}$ ) and Indian Head (Saskatchewan, $50^{\circ} 19^{\prime} \mathrm{N}, 103^{\circ} 24^{\prime} \mathrm{W}$ ) sites are located in Canada's western prairie region where the cropping mix is diverse and fields are large (20-60 ha). These sites are dominated by production of cereals (primarily wheat, barley, and oats) and oilseeds (canola, flaxseed, sunflower, and soybean). Typical field rotation is cereal crop alternating with oilseeds/pulses. In contrast to the western site, the eastern Casselman site (Ontario, $45^{\circ} 22^{\prime} \mathrm{N}, 74^{\circ} 58^{\prime}$ W) is characterized by small elongated fields ( 15 ha on average) and a simple crop mix. Here annual crops include corn, soybeans, and spring wheat, as well as perennial cover crops of pasture and forage. For all sites, only one crop is grown during the relatively short April to September season.

Indian Head is the main test site for the applications presented in Chap. 3. A sample RADARSAT-2 RGB composite image over this area is shown in Fig. 4. Indian Head has been one of the three locations included in the ESA-funded AgriSAR2009 campaign.

The ground data collection was conducted in 2009 by the Indian Head Agriculture Research Facility (IHARF) and the University of Regina. Available ground data consist of two sets:

- An intensive detailed survey of representative crops in a small number of fields

- A more extensive, but less detailed, survey of crops over the entire test site
Fig. 4 A sample RADARSAT2 composite image over Indian Head

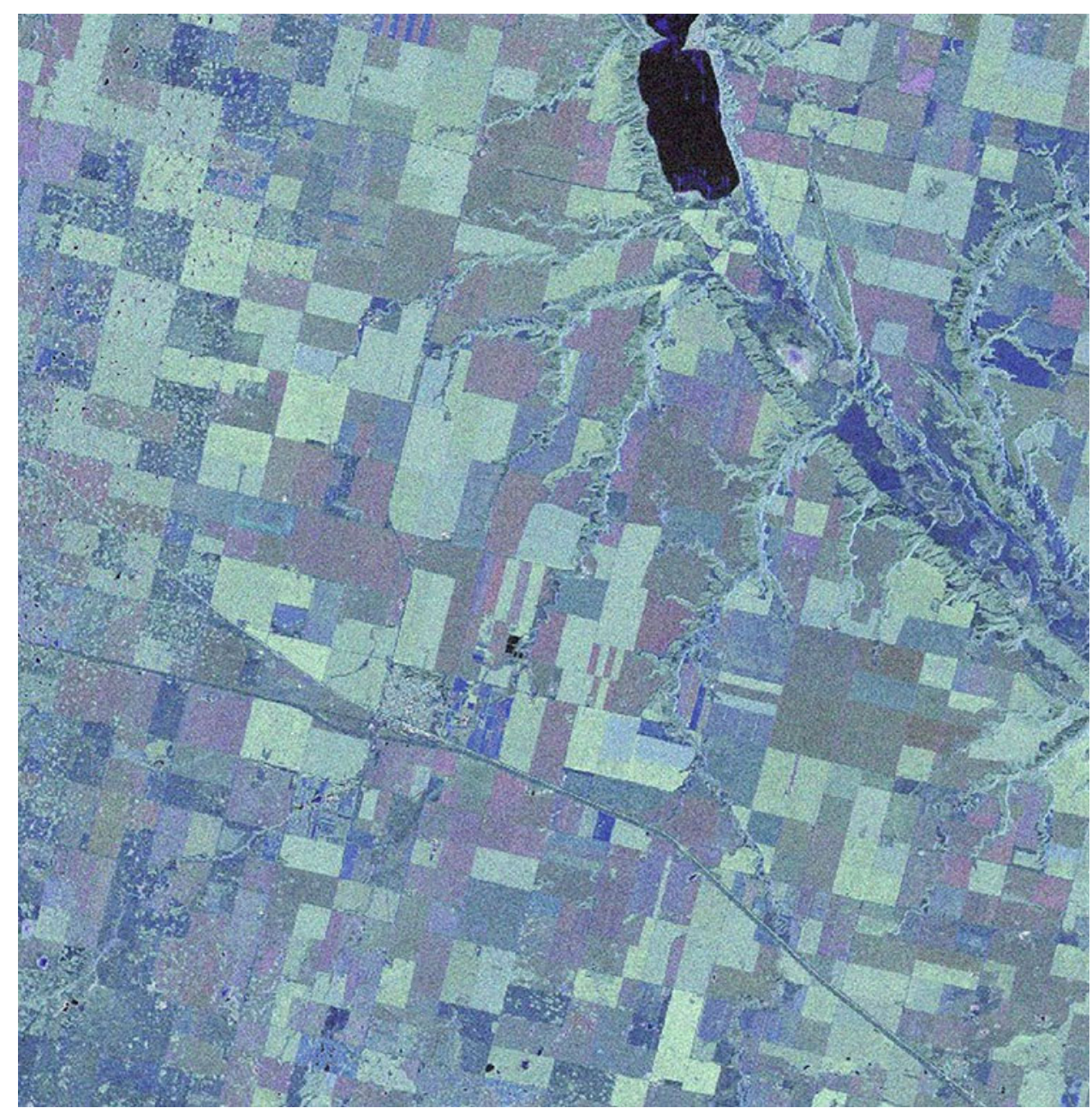


The intensive database comprises the following records gathered in 52 locations, of 14 different parcels, and a total of 6 different crop types. Each point record has the following attributes:

- Crop type

- UTM coordinates

- Growth stage or phenology (multiple dates)

- NDVI (multiple dates)

- LAI (multiple dates)

- Photos (multiple dates)

- Grain yield

The objective of the extensive survey was to record crops grown in all fields within the area of interest. A total of 609 fields covering $90 \%$ of the area of interest were surveyed, including the 14 intensive survey fields. Sixteen different crop types were monitored in this campaign. The following information was gathered for each field:

- Crop type

- Stubble type

- Row orientation (degrees)

- Row spacing (cm)

- Area (ha)

Besides these measurements, the following relevant information is also available:

- Daily meteorology information was acquired during the whole campaign from the Environment Canada weather station at Indian Head: temperature, humidity, rainfall, wind.

- Volumetric soil moisture was automatically measured during the campaign (see Fig. 5). Instrumentation consisted of four soil moisture probes attached to a data logger. The probes were placed in the soil at $5 \mathrm{~cm}$ depth. The data logger recorded hourly values from the four probes during 2 months. Additional soil moisture measurements at $20 \mathrm{~cm}$ depth were recorded separately every few days.

\section{DEMMIN}

The Durable Environmental Multidisciplinary Monitoring Information Network (DEMMIN) is a well-established agricultural test site located close to the town of Demmin $\left(53^{\circ} 54^{\prime} 18^{\prime \prime} \mathrm{N}, 13^{\circ} 02^{\prime} 38^{\prime \prime} \mathrm{E}\right), 150 \mathrm{~km}$ north of Berlin (Germany), that has been regularly used since 1999 for remote sensing studies involving both airborne and groundtruth measurements. Diverse crops such as winter wheat, winter barley, winter rape, maize, and sugar beet are cultivated in this area, where the dominant soil texture is a variation between loamy sand to strong loamy sand.

The AgriSAR 2006 campaign was an ESA conducted campaign carried out in 2006, and it was focused on the Görmin farm, located north-eastern part of the DEMMIN area. Figure 6 displays the Görmin test site. From April to August, detailed ground data, airborne SAR, and optical measurements, as well as satellite images, were collected in the framework of the preparatory activities for Sentinel-1.

The large size of agricultural fields, the variety of crops, the short time interval between acquisitions of ground-truth and radar measurements, and the coverage of the whole season are important features to consider the suitability of these data sets for demonstrations purposes. The main drawback, however, is the reduced number of fields, especially for classification purposes.
Fig. 5 Volumetric soil moisture as a function of time recorded in Indian Head

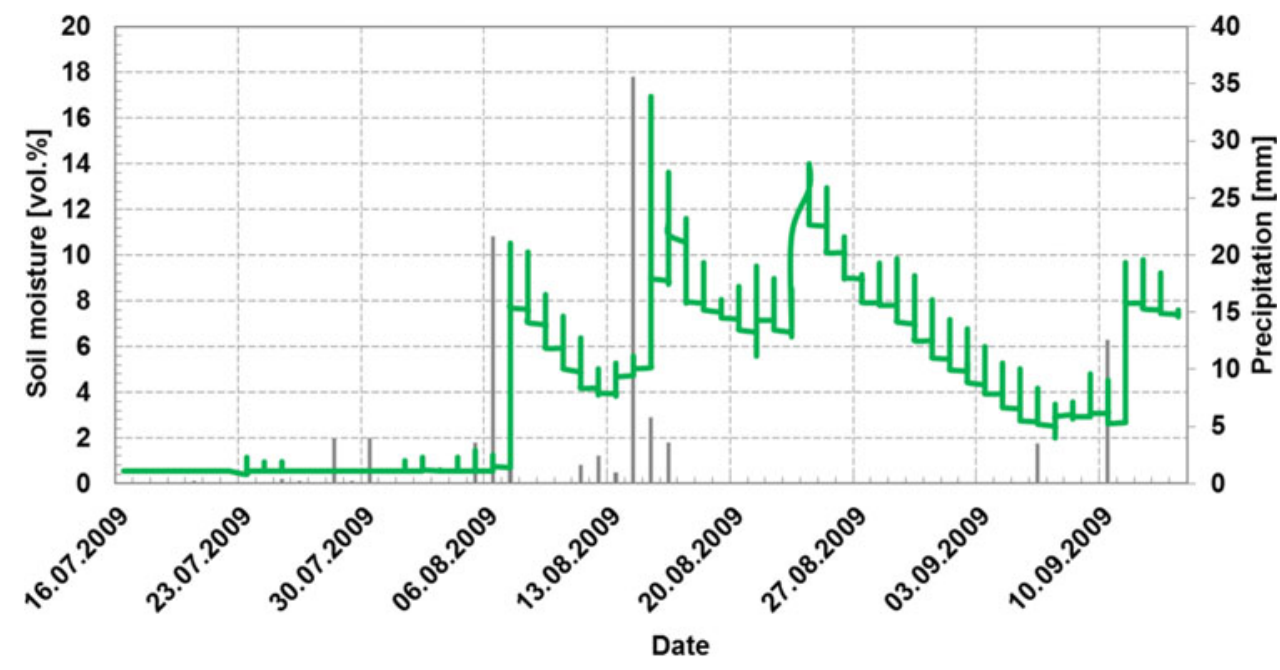




\section{AGRISAR 2006}

\section{Field crops}
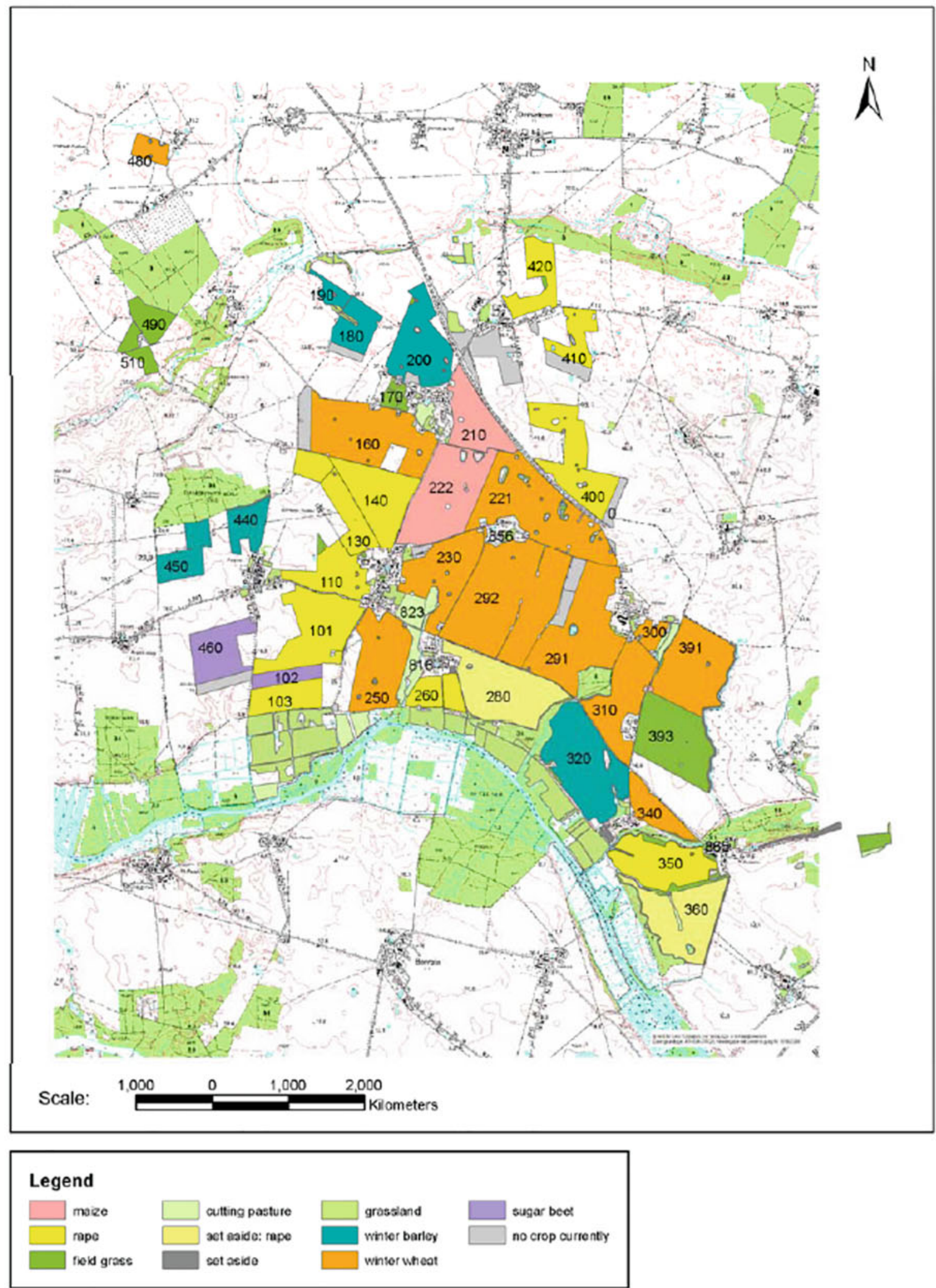

Fig. 6 A land use map of the Görmin test site 
The estimation of soil and vegetation parameters was performed by means of two procedures throughout the project:

- Continuous measurements performed simultaneously to the radar flights

- Intensive measurements performed on three dates during the AGRISAR2006 campaign

In case of continuous measurements, a number of bio- and geophysical parameters were sampled simultaneously with the radar flights. A total of 16 ground measurements campaigns were conducted by the German Remote Sensing Data Center of DLR (DLR-DFD), the Leibnitz-Zentrum für Agrarlandschaftsforschung (ZALF), GEO-Informatik, and University of Kiel with around 13 people collecting vegetation and soil parameters.

These parameters are listed below:

- Phenology stage

- Leaf Area Index (LAI) and vegetation height

- Crop density (plants $/ \mathrm{m}^{2}$ )

- Row distance

- Crop coverage

- Biomass (wet/dry)

- Plant water content

- Chlorophyll (SPAD)

- Volumetric soil moisture at two different depths $(0-5 \mathrm{~cm}$, $5-10 \mathrm{~cm})$

- Surface roughness (photogrammetric)

For the volumetric soil moisture, three gravimetric measurements per sampling unit were averaged for each depth. Standard deviations are also provided and rain events and (slight) topography variations were also recorded and are available as well. An analysis on data quality was performed and the variation coefficient (VC) was calculated for this purpose for each sample unit, depth, and date. Most of the data sets exhibit a variation less than $10 \%$ on average and outliers are easily identified in the time series of $\mathrm{VC}$ as well as the possible sources of error.

The soil moisture was also monitored by means of two TDR stations that were deployed in winter rape and sugar beet fields. Soil moisture was sampled at $5 \mathrm{~cm}$ and $10 \mathrm{~cm}$ at three locations per station with a 10 min sampling period. During the calibration stage of TDR probes, slight overestimations of water content of $3.8 \%$ and $3.45 \%$ were detected on the measurements.

Soil surface roughness measurements were acquired by using photogrammetric image matching techniques. The photogrammetric system allows a quantitative evaluation of quality of the delivered data due to high accurate control points that were installed on the system.
In case of intensive measurements, three campaigns of 1 week were carried out by different institutions in order to obtain additional soil, vegetation, weather, and atmosphere samples. Concerning the soil moisture estimation, permanent registering soil moisture station and TDR probes were used for such a purpose. In the subsequent quality analysis, soil moisture mean and standard deviation were computed for each field and the effect of local slopes over the monitored fields was also taken into account.

In case of soil roughness, 13 roughness profiles were acquired using the ESA laser profiler over bare fields, a wheat field at an early stage. There are 6 profiles $20 \mathrm{~m}$ long, 1 profile $15 \mathrm{~m}$-long, 2 profiles $10 \mathrm{~m}$-long, and 4 profiles $5 \mathrm{~m}$-long. The profiles were acquired along two directions, i.e., perpendicular and parallel to the row directions and standard deviation values are provided.

Optical images were also acquired by two optical systems, the INTA'S AHS and the ITRES CASI system installed in the CASA aircraft from the Spanish Air force, and the CHRIS/ PROBA spaceborne sensor.

\section{Foulum}

Foulum test site (Denmark, $56^{\circ} 29^{\prime} \mathrm{N}, 9^{\circ} 35^{\prime}$ E) contains a large number of agricultural fields with different crops, as well as several lakes, forests, areas with natural vegetation, grasslands, and urban areas. The area is relatively flat, and corrections of the local incidence angle due to terrain slope are therefore, as a first approximation, not necessary. The crop types present in the area are for spring crops, namely, beets, peas, potatoes, maize, spring barley, and oats, and for winter crops, namely, rye, winter barley, winter wheat, winter rape, and grass. The forest areas consist primarily of two types of coniferous forest, i.e., Norway spruce and Caucasian fir. A land cover map was established for the test site including more than 350 test areas.

\section{The Pleine Fougères Test Site}

The Pleine Fougères area in France $\left(48.31^{\circ} \mathrm{N}, 1.15^{\circ} \mathrm{W}\right)$ corresponds to the lower valley of the Couesnon river and presents five marshes. Two of them (Sougéal marsh and Mesnil marsh) have been studied in the framework of a research project conducted by the University of Rennes-1 and other local institutions. This wetland zone is protected by the RAMSAR community agreement and also by ZNIEFF, ZICO, Natura2000, and ZPS. A composite Radarsat-2 image over an orthophoto of the area is shown in Fig. 7.

A ground campaign was carried out during 2010 in order to measure volumetric soil moisture using time-domain reflectometry (TDR) instruments along four transects. 


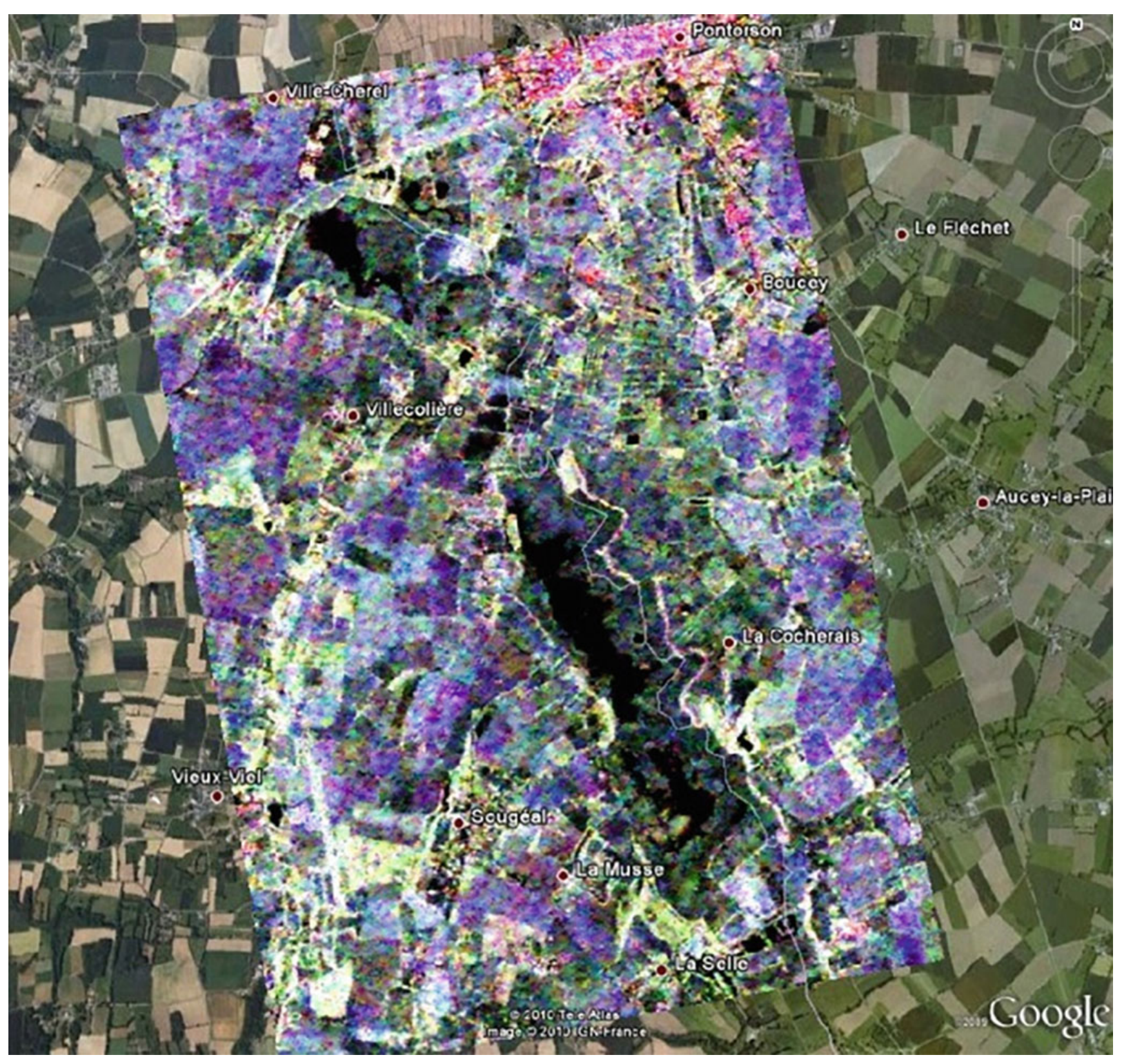

Fig. 7 Radarsat-2 composite image over an orthophoto of the Pleine Fougères test site

Moisture along transects is measured at two different depths $(3.5 \mathrm{~cm}$ and $12 \mathrm{~cm})$. The following data are also available in the framework of the mentioned project:

- Precise surface elevation data gathered with a groundbased Lidar

- Aerial photographs of the site, coincident with the radar acquisitions, for delineation validation

- Ground inventories and maps with the vegetation species present in the area

- Meteorological information

\section{Western Everglades}

The Shark River Slough test site is located in the western Everglades in Florida (FL, USA). The Everglades subtropical wetlands in South FL consist of a wide, shallow, and slow sheet-flow environment. The sheet flow begins in Lake Okeechobee and flows southward to the Everglades wetlands. Artificial changes, such as construction of canals and levees in the past half century, have damaged significantly the flow and ecology of the natural wetland system. At present, the Everglades wetland is composed of managed 
wetlands in the northern section, which is controlled by a series of man-made structures as levees and hydrological gates, and naturally flown wetlands in the southern area, where the original wetland sheet flow has been preserved. In order to maintain the hydrology and ecology of these fragile wetlands, the Everglades wetlands are monitored by a large number of water-level gauges.

The southwestern section of the wetland is located in the Everglades National Park. This area comprises of various wetland environments across the transition between freshwater and saltwater wetlands as shown in. The northeastern part of the study area consists of freshwater herbaceous vegetation, whereas the southwestern area consists of saltwater woody vegetation (mangroves). The shorter scrub is developed in the transition zone between freshwater and saltwater comparing with taller and denser mangrove forest in the saltwater wetland (the vegetation map over the Everglades can be found at http://fcelter.fiu.edu/data/GIS). The optical color composite image of Landsat-7 ETM+ (http://www.landcover.org/data/landsat) shown in Fig. 8 describes clearly two different types of vegetation.

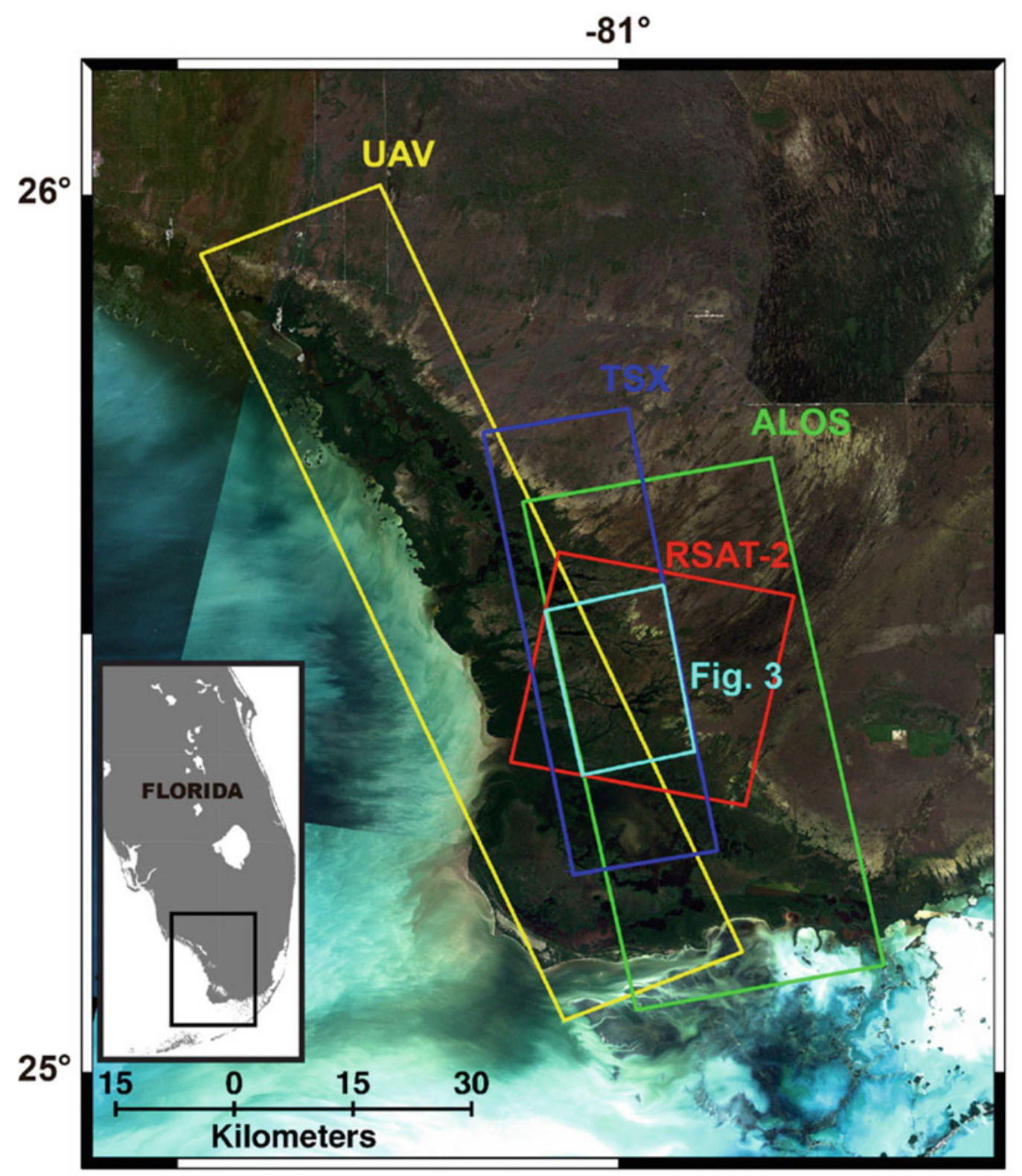

Fig. 8 Location map of the study area in the western Everglades, using a Landsat ETM+ optical color composite (http://www.landcover.org/ data/landsat). The frames mark the swath locations of data acquired by three SAR satellites (X-band TerraSAR-X, C-band Radarsat-2, and
L-band ALOS PALSAR) and one airborne UAVSAR (L-band SAR). The aqua frame indicates the location of a common area to all three satellite acquisitions 


\section{Canada Peatlands: La Baie Des Mines and Wapusk National Park}

La Baie de Mines peatland is a 700 ha raised boreal peat dome located $12 \mathrm{~km}$ west of Trois-Rivières, Canada. Figure 9 presents the peatland classification based on forest inventory, which was provided by the Ministère des Ressources Naturelles et de la Faune du Québec. The site includes four main wetland classes: shrub bog, treed bog, poor sedge fen, and swamp. Shrub bogs and poor sedge fens, which cannot be discriminated with optical sensors (Ikonos), are regrouped in the open peatland class of Fig. 9.

The Wapusk National Park (WNP) is located in the Hudson Bay Lowlands. The Hudson Bay Lowlands contain the most extensive wetlands and thickest peat deposits in Canada. Figure 9 presents the classification of the peatland study site (near the Roberge lake) extracted from Brook's wetland classification. The latter was generated using Landsat-5 TM mosaic and extensive ground field data collected at 600 sites throughout the WNP. The area includes five main wetland classes: lichen melt pond bog, peat plateau bog, litchen spruce bog, sedge bulrush poor fen, and sedge rich fen.

\section{Cryosphere Applications}

\section{The Austfonna Ice Cap}

An area of interest for land ice observation is the Austfonna ice-cap, in the Nordaustlandet island $\left(79^{\circ} 48^{\prime} \mathrm{N}, 22^{\circ} 24^{\prime} \mathrm{E}\right)$, located North-East of the Svalbard archipelago (Norway). On this island, several ice caps and glaciers cover an area of $11,150 \mathrm{~km}^{2}$ and represent one of the largest land ice masses outside of the Antarctic and Greenland ice sheets. Austfonna possesses unique features requiring further investigation including a subpolar thermal regime and the widespread presence of superimposed ice. Moreover, several glaciers in drainage basins have been observed to surge. An additional advantage of studying the Austfonna ice cap is its simple dome-shaped topography, which significantly facilitates the analysis. In particular two sites have been chosen for this study (Fig. 10): one is located in the firn area, very close to the summit of the ice cap (here, referred to as Summit); the second one is in the superimposed ice zone, close to the Etonbreen outlet glacier (Glacier). Airborne SAR acquisitions over both sites were carried out during the ESA IceSAR 2007 campaign.
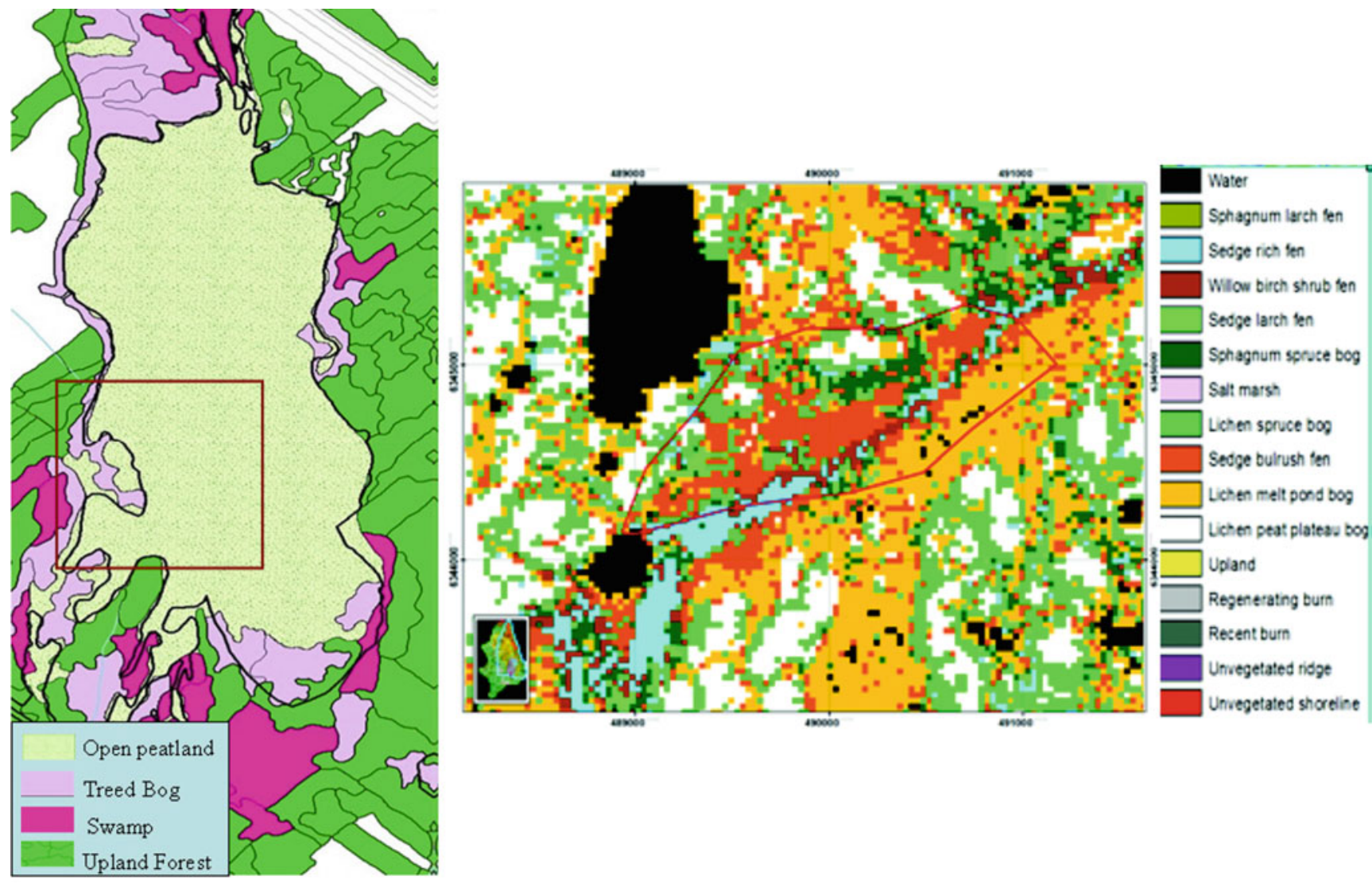

Fig. 9 Left panel: classification of La Baie des Mines based on forest inventory. Right panel: Brook's classification of the peatland areas of the Wapusk National Park 


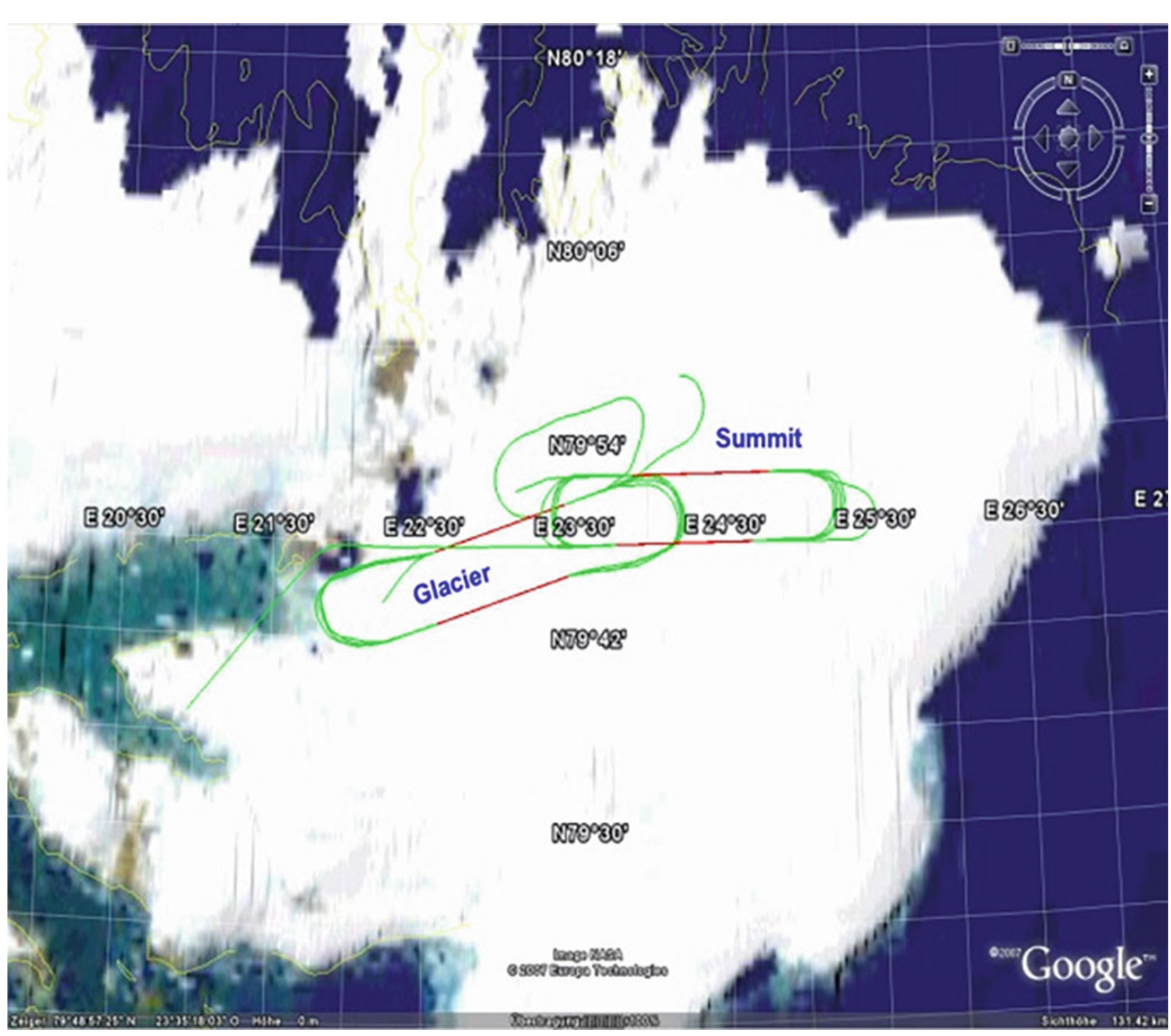

Fig. 10 Locations of Summit and Glacier test sites and overview of the SAR flight trajectories during the IceSAR 2007 campaign

Ground measurements on Austfonna were collected in spring 2007 by a team from the University of Oslo (UiO) and the Norwegian Polar Institute (NPI) as part of calibration and validation activities for the CryoSat-2 satellite. Snow stratigraphy, neutron probe, and ground penetrating radar data were collected during a two-week period spanning the end of April and beginning of May prior to spring melt. Meteorological data for the March and April 2007 acquisition period and limited measurements from 2004-2007 are also available.

\section{Fram Strait, Storfjord, and Barents Sea}

The test sites for the sea ice measurements need to fulfil certain conditions with regard to the position of different sea ice types. The three chosen test sites are all located around the Svalbard archipelago (Norway). The first location is in the Barents Sea, east of the Barentsøya and Edgeøya islands; the second site is located in the Storfjorden, between Spitsbergen and Edgeøya; the Fram strait, a passage from the Arctic Ocean to the Greenland Sea and Norwegian Sea, between Greenland and Spitsbergen, was chosen as third test site (see Fig. 11). SAR images were acquired over these three locations during the ESA IceSAR 2007 campaign.

\section{Sodankylae}

The Sodankylae test site $\left(67^{\circ} 25^{\prime} \mathrm{N}, 26^{\circ} 35^{\prime} \mathrm{E}\right)$ is located in the heart of Lapland, in Finland. The territory is crossed by the rivers Kitinen and Luiro. The topography of the region is 


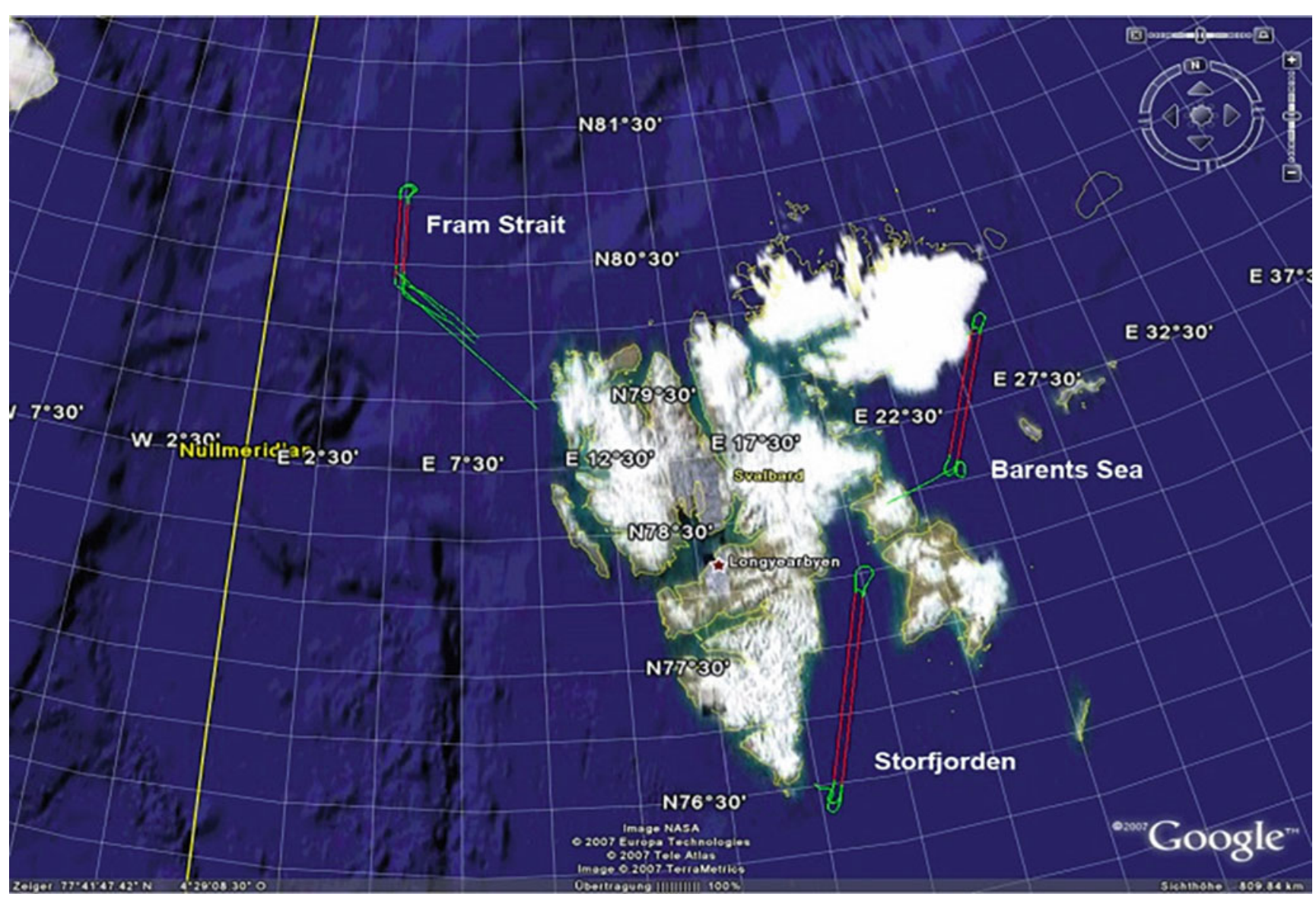

Fig. 11 Locations of Fram Strait, Storfjorden, and Barents Sea test sites and IceSAR 2007 flight trajectories

very smooth with only small relieves of altitude seldom higher than $400 \mathrm{~m}$. It is mainly a forested area populated by pines with an understory of lichens, mosses, and small shrubs over a sandy soil. The ground is usually covered by snow from October until the middle of May, and the uppermost soil layers are frozen during the wintertime. A view of the Sodankylae test site is reported in Fig. 12.

Ground measurements collected during the NoSREx (Nordic Snow Radar Experiment) campaign during the winters 2009/10 and 2010/11 are available. The experiment was conducted by the FMI (Finnish Meteorological Institute) and supported by ESA. The main reference points were collected over three areas, the IAO automatic and manual station, as well as over a bog site. Over this reference points, the main parameter that were collected are snow depth, snow density, grain size, snow water equivalent, soil temperature, and air temperature. In addition two reference points in the forest have been identified.

\section{Churchill}

The test site of Churcill $\left(58^{\circ} 46^{\prime} \mathrm{N}, 94^{\circ} 10^{\prime} \mathrm{W}\right)$ takes its name from the homonymous town on the shore of Hudson Bay in Manitoba, Canada. The landscape is characterized by shallow soils due to a combination of subsurface permafrost and rock formation. The south and central part of Manitoba includes numerous lakes and rivers that flow northeast into the bay. An expanse of almost uninhabited tundra surrounds the port of Churchill.

Ground measurements collected during the CASIX experiment (October 2010 - March 2011) are available. The location of the CASIX experiment is shown in Fig. 13. The campaign was conducted by the University of Waterloo, supported by the ESA and NSERC (National Sciences and Engineering Research Council of Canada). The dataset includes $\mathrm{X}$ - and Ku-band scatterometers data and field in situ measurements of snow properties over different 
Fig. 12 View of Sodankylae test site
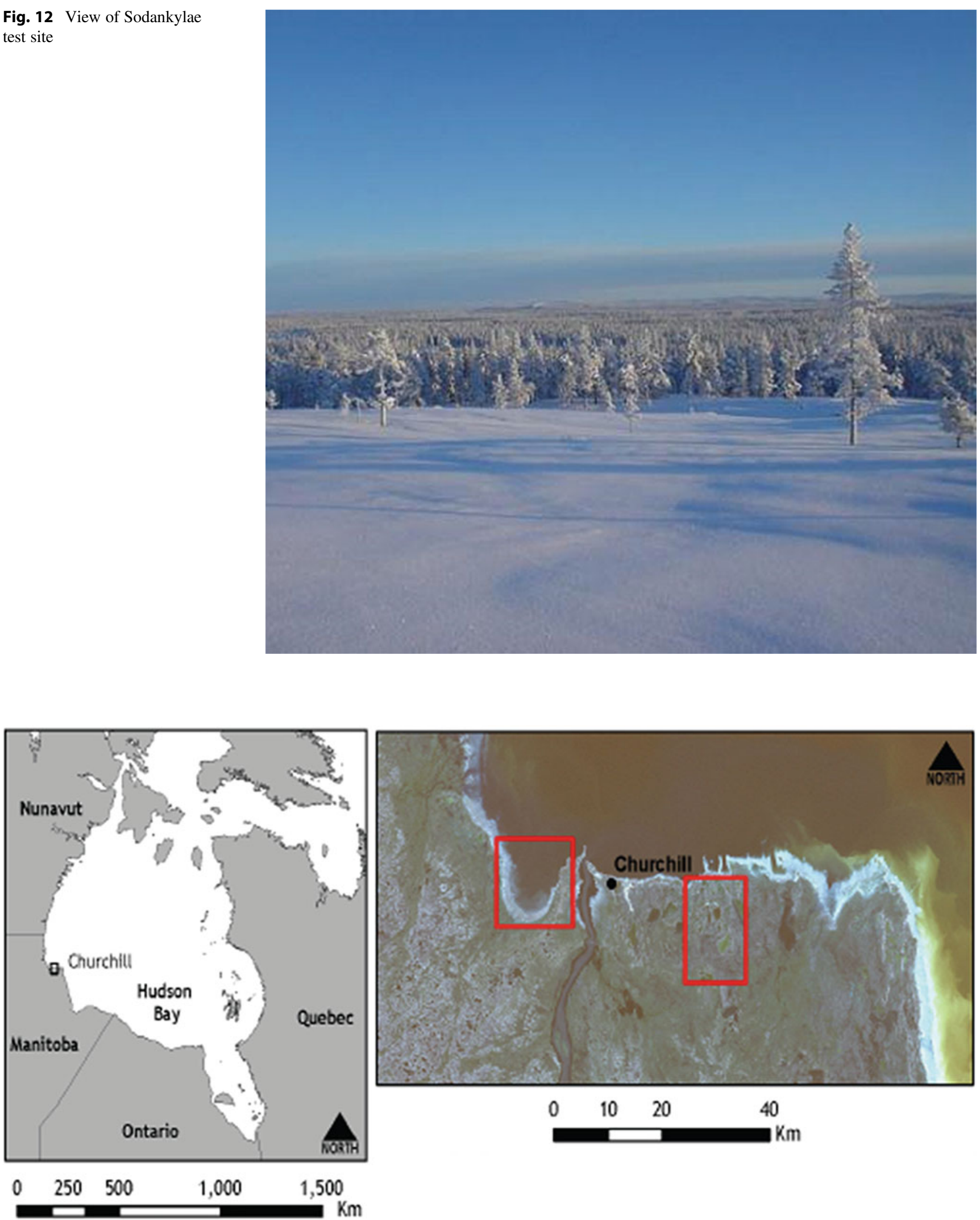

Fig. 13 Location of the Churchill test sites for the CASIX campaign 


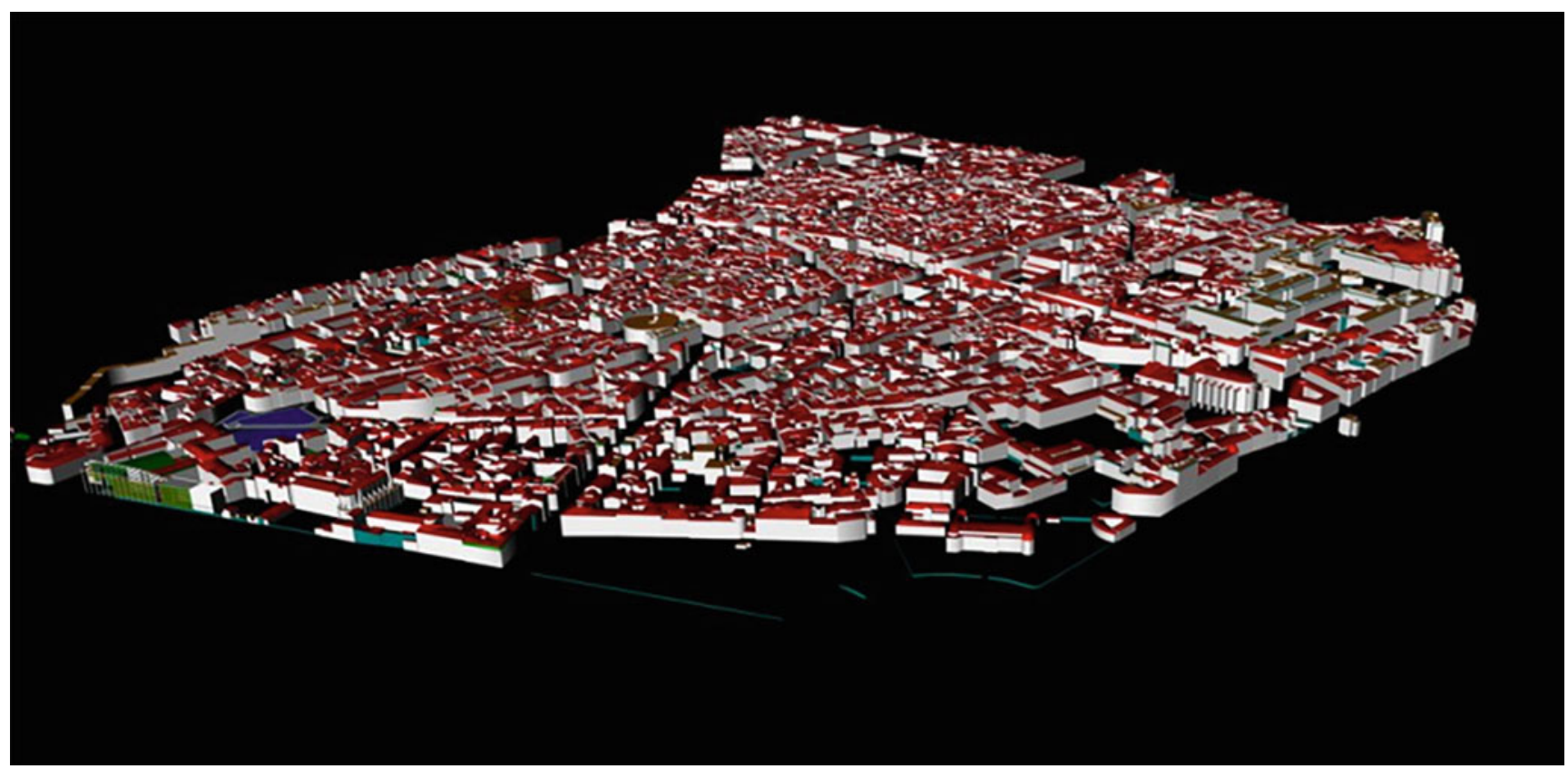

Fig. 14 Representation of ground truth available on two Toulouse districts

scenarios. The references points are located on two lakes and are also covering forest and tundra area. Over this reference points, the main parameter that were collected are snow depth, snow density, grain size, snow water equivalent, soil temperature, and air temperature.

\section{Urban Applications}

\section{Toulouse}

Toulouse is a city in southwestern France. The Toulouse metropolitan area is the fifth largest in France and contains low residential districts and some industrial places. It is one of the bases of the European aerospace industry.

As cadastral registers from French cities such as Toulouse are easily obtainable, precise ground truth can be deducted (see Fig. 14 for an example). It yields that a classification map from the ground can be used to estimate classification performance (soil, vegetation, etc.). Moreover, 3-D representation of these cities is also available and may be used in the third produce (3-D Rendering). A specific ground truth over Toulouse is available. It consists in a 3-D representation of the city. It allows us to have not only the footprints of each building but also their individual heights.

\section{San Francisco}

San Francisco is the financial, cultural, and transportation center of the San Francisco Bay Area, a region of 7.15 million people. The San Francisco Bay is well known to polarimetry field, as one of the first fully polarimetric images have been acquired by NASA/JPL AIRSAR L-band instrument California, USA, from 1988. This previous airborne data set contains four-looked coherency matrices, with a pixel resolution of about $10 \mathrm{~m} \times 10 \mathrm{~m}$. This data set has been from this date extensively used for performance evaluation and presented in the literature of polarimetric SAR. Now quad polarimetric data are available with satellite TerraSAR-X and with better resolution $(3 \mathrm{~m})$.

A description of building footprints and their elevation can be found in a shapefile format on the website https://data. sfgov.org. The file is organized as a structure containing a list of polygonal element. These polygons define the footprint of each building on the ground, and for each element, the minimum and maximum elevation data are given. These data were created using a conversion tool for 3-D shapefile using the ArcGIS 3-D conversion tool "Multipatch Footprint". Original 3-D data were derived from Pictometry, Inc.

\section{Murcia and Barcelona}

Murcia is a major city in southeastern Spain. It has been selected as a test site due to its high deformation rate and the set of extensometers deployed along the city that provide validation data. Subsidence has occurred in the metropolitan area of Murcia City (SE Spain) as a result of soil consolidation due to piezometric level depletion caused by excessive pumping of groundwater. In fact a piezometric level decline between 5 and $15 \mathrm{~m}$ was measured on this layer during recent 
drought periods: 1980-1983, 1993-1995, and 2005-2008. After the second drought period, ground subsidence was triggered causing damages in buildings and other structures with an estimated cost of 50 million Euros, generating a significant social impact. Geology data are available with the distribution of the compressible thickness and the maximum groundwater depletion in the period 2004-2008.

Another significant test site in Spain that has been used for observing deformations is the city of Barcelona. Nowadays Barcelona is being affected by new underground infrastructures construction. In particular, a new metro line that will connect the city with the airport is generating subsidence at different areas. Several hazards have been related to these activities, such as the collapse of a block in El Carmel neighborhood in 2005 or damages on a building in the South of the city.

\section{Ocean Applications}

\section{Gulf of Mexico}

The Gulf of Mexico is one of the largest basins in the world, located south of the USA and north of Mexico. It serves an important role in the worldwide economy due to its oil fields that are at the core of the petrochemical industry of the USA. These oil rigs pose an important environmental risk in the case of disasters and accidents, such as the recent massive Deepwater Horizon oil spill, which is considered the largest offshore spill in US history. In addition to such disasters/ accidents, the Gulf of Mexico is prone to hurricanes. During such events, man-made metallic infrastructures, such as oil rigs, can be wrecked or destroyed, accompanied by release of oil at sea.

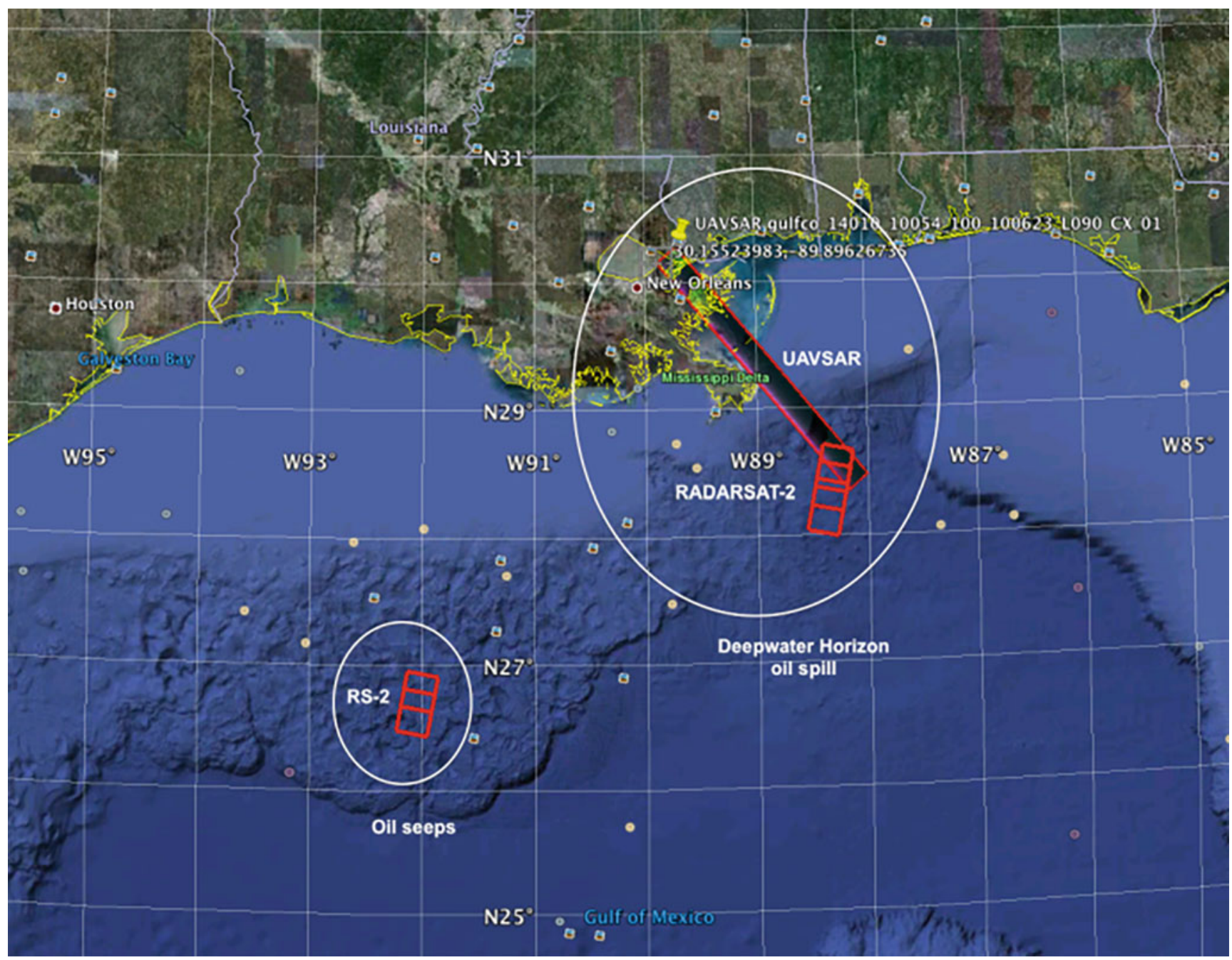

Fig. 15 Location and area coverage of the Gulf of Mexico test site where both RADARSAT-2 and UAVSAR full-polarimetric SAR data have been collected. Test sites concern both oil rigs/ships and oil slicks/oil seeps 
Moreover, Gulf of Mexico is also very rich in oil seepages. Recent studies have suggested that seepage rates in the Gulf of Mexico are much higher than reported in previous NRC studies. By using submarines and remote sensing at least 63 new individual seeps have been identified; see for instance the one shown in Fig. 15.

There are thousands of oil and gas rigs a few miles away from the Gulf of Mexico coast running from Texas to Louisiana and the state of Mississippi. A recent estimate indicates that there are approximately, in June 2010, 3445 oil and gas structures in the Gulf of Mexico, producing, in October $2010,29 \%$ of the oil and $13 \%$ of the natural gas produced in the USA. A map of oil and gas rigs is provided by Minerals Management Service (MMS), http://www.gomr. mms.gov/homepg/pubinfo/repcat/arcinfo/index.html.
The Gulf of Mexico has been recently involved in the Deepwater Horizon accident. On 20 April 2010, a fire broke out on the Transocean Deepwater Horizon oil rig under lease to BP (British Petroleum), with 126 people on board. On 22 April 2010, the rig sank. Safeguards set in place to automatically cap the oil well in case of catastrophe did not work as expected. A first estimate of about five million barrels already makes this accident the world's largest accidental oil spill. It is surpassed only by the intentional 1991 Gulf War spill in Kuwait. Many airborne and satellite missions imaged the polluted area by using different instruments, e.g., http://www.noaa.gov/deepwaterhorizon. Moreover, during the period of the oil spill, there were numerous daily overflights by multiple aerial platforms to provide information on extent and oil properties for first responders, e.g., http://www.epa.gov/bpspill/epa.html. 